\title{
biomolecules
}

Oxidative

Stress in Health

and Disease

Edited by

Michael Breitenbach and Peter Eckl

Printed Edition of the Special Issue Published in Biomolecules 
Michael Breitenbach and Peter Eckl (Eds.)

\section{Oxidative Stress in Health and Disease}

\section{MDPI}


This book is a reprint of the Special Issue "Oxidative Stress and Oxygen Radicals" that appeared in the online, open access journal, Biomolecules (ISSN 2218-273X) in 2015 (available at: http://www.mdpi.com/journal/biomolecules/special_issues/oxygen-radicals).

\section{Guest Editors}

Michael Breitenbach and Peter Eckl

University of Salzburg

Austria

Editorial Office

MDPI AG

Klybeckstrasse 64

Basel, Switzerland

Publisher

Shu-Kun Lin

Assistant Editor

Rongrong Leng

\section{Edition 2016}

MDPI $・$ Basel $\bullet$ Beijing $・$ Wuhan $・$ Barcelona

ISBN 978-3-03842-173-3 (Hbk)

ISBN 978-3-03842-174-0 (PDF)

Articles in this volume are Open Access and distributed under the Creative Commons Attribution license (CC BY), which allows users to download, copy and build upon published articles even for commercial purposes, as long as the author and publisher are properly credited, which ensures maximum dissemination and a wider impact of our publications. The book taken as a whole is (C) 2016 MDPI, Basel, Switzerland, distributed under the terms and conditions of the Creative Commons by Attribution (CC BY-NC-ND) license (http://creativecommons.org/licenses/by-nc-nd/4.0/). 


\section{Table of Contents}

List of Contributors VII

About the Guest Editors XI

Preface XIII

Introduction to Oxidative Stress in Biomedical and Biological Research

Reprinted from: Biomolecules 2015, 5(2), 1169-1177

http://www.mdpi.com/2218-273X/5/2/1169 XV

\section{Ivan Verrastro, Sabah Pasha, Karina Tveen Jensen, Andrew R. Pitt and Corinne M. Spickett}

Mass Spectrometry-Based Methods for Identifying Oxidized Proteins in Disease: Advances and Challenges

Reprinted from: Biomolecules 2015, 5(2), 378-411

http://www.mdpi.com/2218-273X/5/2/378 1

Michael Breitenbach, Manuela Weber, Mark Rinnerthaler, Thomas Karl and Lore Breitenbach-Koller

Oxidative stress in Fungi: Its Function in Signal Transduction, Interaction with Plant Hosts, and Lignocellulose Degradation

Reprinted from: Biomolecules 2015, 5(2), 318-342

http://www.mdpi.com/2218-273X/5/2/318 37

\section{Marina Makrecka-Kuka, Gerhard Krumschnabel and Erich Gnaiger}

High-Resolution Respirometry for Simultaneous Measurement of Oxygen and Hydrogen Peroxide Fluxes in Permeabilized Cells, Tissue Homogenate and Isolated Mitochondria Reprinted from: Biomolecules 2015, 5(3), 1319-1338 http://www.mdpi.com/2218-273X/5/3/1319.

\section{Adelheid Weidinger and Andrey V. Kozlov}

Biological Activities of Reactive Oxygen and Nitrogen Species: Oxidative Stress versus Signal Transduction

Reprinted from: Biomolecules 2015, 5(2), 472-484

http://www.mdpi.com/2218-273X/5/2/472 
Andrea Müllebner, Rudolf Moldzio, Heinz Redl, Andrey V. Kozlov and

\section{J. Catharina Duvigneau}

Heme Degradation by Heme Oxygenase Protects Mitochondria but Induces ER Stress via Formed Bilirubin

Reprinted from: Biomolecules 2015, 5(2), 679-701

http://www.mdpi.com/2218-273X/5/2/679 .97

Mark Rinnerthaler, Johannes Bischof, Maria Karolin Streubel, Andrea Trost and

\section{Klaus Richter}

Oxidative Stress in Aging Human Skin

Reprinted from: Biomolecules 2015, 5(2), 545-589

http://www.mdpi.com/2218-273X/5/2/545

\section{Nikolaus Bresgen and Peter M. Eckl}

Oxidative Stress and the Homeodynamics of Iron Metabolism

Reprinted from: Biomolecules 2015, 5(2), 808-847

http://www.mdpi.com/2218-273X/5/2/808 169

Peter Steinbacher and Peter Eckl

Impact of Oxidative Stress on Exercising Skeletal Muscle

Reprinted from: Biomolecules 2015, 5(2), 356-377

http://www.mdpi.com/2218-273X/5/2/356

\section{Anton Hermann, Guzel F. Sitdikova and Thomas M. Weiger}

Oxidative Stress and Maxi Calcium-Activated Potassium (BK) Channels

Reprinted from: Biomolecules 2015, 5(3), 1870-1911

http://www.mdpi.com/2218-273X/5/3/1870

\section{Nikolaus Netzer, Hannes Gatterer, Martin Faulhaber, Martin Burtscher,}

Stephan Pramsohler and Dominik Pesta

Hypoxia, Oxidative Stress and Fat

Reprinted from: Biomolecules 2015, 5(2), 1143-1150

http:/www.mdpi.com/2218-273X/5/2/1143

\section{Kerstin Nowotny, Tobias Jung, Annika Höhn, Daniela Weber and Tilman Grune}

Advanced Glycation End Products and Oxidative Stress in Type 2 Diabetes Mellitus

Reprinted from: Biomolecules 2015, 5(1), 194-222 


\section{Gabriel Piedrafita, Markus A Keller and Markus Ralser}

The Impact of Non-Enzymatic Reactions and Enzyme Promiscuity on Cellular Metabolism during (Oxidative) Stress Conditions

Reprinted from: Biomolecules 2015, 5(3), 2101-2122

http://www.mdpi.com/2218-273X/5/3/2101

Walter Stoiber, Astrid Obermayer, Peter Steinbacher and Wolf-Dietrich Krautgartner

The Role of Reactive Oxygen Species (ROS) in the Formation of Extracellular Traps (ETs) in Humans

Reprinted from: Biomolecules 2015, 5(2), 702-723

http://www.mdpi.com/2218-273X/5/2/702 340

\section{Alessandra da Silva Dantas, Alison Day, Mélanie Ikeh, Iaroslava Kos, Beatrice Achan and Janet Quinn}

Oxidative Stress Responses in the Human Fungal Pathogen, Candida albicans

Reprinted from: Biomolecules 2015, 5(1), 142-165

http://www.mdpi.com/2218-273X/5/1/142

H. P. Vasantha Rupasinghe, Mannfred M. A. Boehm, Satvir Sekhon-Loodu, Indu Parmar, Bob Bors and Andrew R. Jamieson

Anti-Inflammatory Activity of Haskap Cultivars is Polyphenols-Dependent

Reprinted from: Biomolecules 2015, 5(2), 1079-1098

http:/www.mdpi.com/2218-273X/5/2/1079. .388

Rudolf J. Schaur, Werner Siems, Nikolaus Bresgen and Peter M. Eckl

4-Hydroxy-nonenal-A Bioactive Lipid Peroxidation Product

Reprinted from: Biomolecules 2015, 5(4), 2247-2337

http:/www.mdpi.com/2218-273X/5/4/2247. 409

Index .505 



\section{List of Contributors}

Beatrice Achan Institute for Cell and Molecular Biosciences, Faculty of Medical Sciences, Newcastle University, Newcastle upon Tyne NE2 4HH, UK.

Johannes Bischof Department of Cell Biology, Division of Genetics, University of Salzburg, Salzburg 5020, Austria.

Mannfred M. A. Boehm Department of Environmental Sciences, Faculty of Agriculture, Dalhousie University, Truro, NS B2N 5E3, Canada.

Bob Bors Department of Plant Sciences, University of Saskatchewan, Saskatoon, SK S7N 5A8, Canada.

Michael Breitenbach Department of Cell Biology, Division of Genetics, University of Salzburg, Salzburg 5020, Austria.

Lore Breitenbach-Koller Department of Cell Biology, Division of Genetics, University of Salzburg, Salzburg 5020, Austria.

Nikolaus Bresgen Division of Genetics, Department of Cell Biology, University of Salzburg, Hellbrunnerstasse 34, 5020 Salzburg, Austria.

Martin Burtscher Department of Sport Science, Faculty for Sports Science and Psychology, University of Innsbruck, Innsbruck 6020, Austria.

Alessandra da Silva Dantas Departamento de Biologia Celular e Genética, Instituto de Biologia Roberto Alcantara Gomes, Universidade do Estado do Rio de Janeiro (UERJ), Rio de Janeiro 20550-013, Brazil.

Alison Day Institute for Cell and Molecular Biosciences, Faculty of Medical Sciences, Newcastle University, Newcastle upon Tyne NE2 4HH, UK.

J. Catharina Duvigneau Institute for Medical Biochemistry, Veterinary University Vienna, Veterinaerplatz 1, 1210 Vienna, Austria.

Peter Eckl Division of Genetics, Department of Cell Biology, University of Salzburg, Hellbrunnerstasse 34, 5020 Salzburg, Austria.

Martin Faulhaber Department of Sport Science, Faculty for Sports Science and Psychology, University of Innsbruck, Innsbruck 6020, Austria.

Hannes Gatterer Department of Sport Science, Faculty for Sports Science and Psychology, University of Innsbruck, Innsbruck 6020, Austria.

Erich Gnaiger OROBOROS INSTRUMENTS, Innsbruck 6020, Austria; Daniel Swarovski Research Laboratory, Department of Visceral, Transplant and Thoracic Surgery, Medical University of Innsbruck, Innsbruck 6020, Austria. 
Tilman Grune Department of Molecular Toxicology, German Institute of Human Nutrition Potsdam-Rehbruecke, Arthur-Scheunert-Allee 114-116, 14558 Nuthetal, Germany.

Anton Hermann Department of Cell Biology, Division of Cellular and Molecular Neurobiology, University of Salzburg, Salzburg 5020, Austria.

Annika Höhn Department of Molecular Toxicology, German Institute of Human Nutrition Potsdam-Rehbruecke, Arthur-Scheunert-Allee 114-116, 14558 Nuthetal, Germany.

Mélanie Ikeh Institute for Cell and Molecular Biosciences, Faculty of Medical Sciences, Newcastle University, Newcastle upon Tyne NE2 4HH, UK.

Andrew R. Jamieson Agriculture and Agri-Food Canada, Kentville, NS B4N 1J5, Canada.

Karina Tveen Jensen School of Life and Health Sciences, Aston University, Aston Triangle, Birmingham, B4 7ET, UK.

Tobias Jung Department of Molecular Toxicology, German Institute of Human Nutrition Potsdam-Rehbruecke, Arthur-Scheunert-Allee 114-116, 14558 Nuthetal, Germany.

Thomas Karl Department of Cell Biology, Division of Genetics, University of Salzburg, Salzburg 5020, Austria.

Markus A. Keller Department of Biochemistry, University of Cambridge, 80 Tennis Court Rd, Cambridge CB2 1GA, UK.

Iaroslava Kos Institute for Cell and Molecular Biosciences, Faculty of Medical Sciences, Newcastle University, Newcastle upon Tyne NE2 4HH, UK.

Andrey V. Kozlov Ludwig Boltzmann Institute for Experimental and Clinical Traumatology, AUVA Research Center, Donaueschingenstraße 13, 1200 Vienna, Austria.

Wolf-Dietrich Krautgartner Biomedical Ultrastructure Research Group, Department of Cell Biology, University of Salzburg, Hellbrunnerstrasse 34, Salzburg A-5020, Austria.

Gerhard Krumschnabel OROBOROS INSTRUMENTS, Innsbruck 6020, Austria.

Marina Makrecka-Kuka Laboratory of Pharmaceutical Pharmacology, Latvian Institute of Organic Synthesis, Riga LV1006, Latvia.

Rudolf Moldzio Institute for Medical Biochemistry, Veterinary University Vienna, Veterinaerplatz 1, 1210 Vienna, Austria.

Andrea Müllebner Institute for Medical Biochemistry, Veterinary University Vienna, Veterinaerplatz 1, 1210 Vienna, Austria.

Nikolaus Netzer Department of Sport Science, Faculty for Sports Science and Psychology, University of Innsbruck, Innsbruck 6020, Austria; Hermann Buhl Institute for Hypoxia and Sleep Medicine Research, Bad Aibling 83043, Germany; Department Medicine, Division Sports Medicine, University Hospitals Ulm, Ulm 89081, Germany.

Kerstin Nowotny Department of Molecular Toxicology, German Institute of Human Nutrition Potsdam-Rehbruecke, Arthur-Scheunert-Allee 114-116, 14558 Nuthetal, Germany. 
Astrid Obermayer Biomedical Ultrastructure Research Group, Department of Cell Biology, University of Salzburg, Hellbrunnerstrasse 34, Salzburg A-5020, Austria.

Indu Parmar Department of Environmental Sciences, Faculty of Agriculture, Dalhousie University, Truro, NS B2N 5E3, Canada.

Sabah Pasha School of Life and Health Sciences, Aston University, Aston Triangle, Birmingham, B4 7ET, UK.

Dominik Pesta Department of Sport Science, Faculty for Sports Science and Psychology, University of Innsbruck, Innsbruck 6020, Austria.

Gabriel Piedrafita Department of Biochemistry, University of Cambridge, 80 Tennis Court Rd, Cambridge CB2 1GA, UK.

Andrew R. Pitt School of Life and Health Sciences, Aston University, Aston Triangle, Birmingham, B4 7ET, UK.

Stephan Pramsohler Hermann Buhl Institute for Hypoxia and Sleep Medicine Research, Bad Aibling 83043, Germany.

Janet Quinn Institute for Cell and Molecular Biosciences, Faculty of Medical Sciences, Newcastle University, Newcastle upon Tyne NE2 4HH, UK.

Markus Ralser Department of Biochemistry, University of Cambridge, 80 Tennis Court Rd, Cambridge CB2 1GA, UK; The Francis Crick Institute, Mill Hill Laboratory, The Ridgeway, London NW1 7AA, UK.

Heinz Redl Ludwig Boltzmann Institute for Experimental and Clinical Traumatology, Donaueschingenstraße 13, 1200 Vienna, Austria.

Klaus Richter Department of Cell Biology, Division of Genetics, University of Salzburg, Salzburg 5020, Austria.

Mark Rinnerthaler Department of Cell Biology, Division of Genetics, University of Salzburg, Salzburg 5020, Austria.

H. P. Vasantha Rupasinghe Department of Environmental Sciences, Faculty of Agriculture, Dalhousie University, Truro, NS B2N 5E3, Canada.

Rudolf J. Schaur Institute of Molecular Biosciences, University of Graz, Heinrichstrasse 33a, 8010 Graz, Austria.

Satvir Sekhon-Loodu Department of Environmental Sciences, Faculty of Agriculture, Dalhousie University, Truro, NS B2N 5E3, Canada.

Werner Siems Institute for Medical Education, KortexMed GmbH, Hindenburgring 12a, 38667 Bad Harzburg, Germany.

Guzel F. Sitdikova Department of Physiology of Man and Animals, Kazan Federal University, Kazan 420008, Russia. 
Corinne M. Spickett School of Life and Health Sciences, Aston University, Aston Triangle, Birmingham, B4 7ET, UK.

Peter Steinbacher Department of Cell Biology, University of Salzburg, A-5020 Salzburg, Austria; Biomedical Ultrastructure Research Group, Department of Cell Biology, University of Salzburg, Hellbrunnerstrasse 34, Salzburg A-5020, Austria.

Walter Stoiber Biomedical Ultrastructure Research Group, Department of Cell Biology, University of Salzburg, Hellbrunnerstrasse 34, Salzburg A-5020, Austria.

Maria Karolin Streubel Department of Cell Biology, Division of Genetics, University of Salzburg, Salzburg 5020, Austria.

Andrea Trost Department of Ophthalmology and Optometry, Paracelsus Medical University, Muellner Hauptstrasse 48, 5020 Salzburg, Austria.

Ivan Verrastro School of Life and Health Sciences, Aston University, Aston Triangle, Birmingham, B4 7ET, UK.

Daniela Weber Department of Molecular Toxicology, German Institute of Human Nutrition Potsdam-Rehbruecke, Arthur-Scheunert-Allee 114-116, 14558 Nuthetal, Germany.

Manuela Weber Department of Cell Biology, Division of Genetics, University of Salzburg, Salzburg 5020, Austria.

Adelheid Weidinger Ludwig Boltzmann Institute for Experimental and Clinical Traumatology, AUVA Research Center, Donaueschingenstraße 13, 1200 Vienna, Austria.

Thomas M. Weiger Department of Cell Biology, Division of Cellular and Molecular Neurobiology, University of Salzburg, Salzburg 5020, Austria. 


\section{About the Guest Editors}

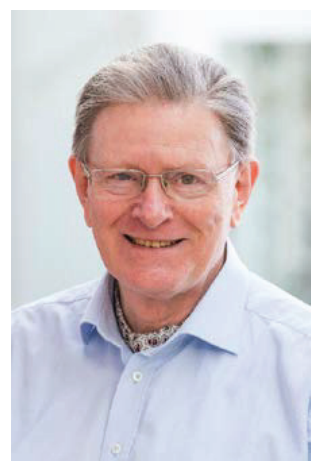

Michael Breitenbach is a Professor of Molecular Genetics Emeritus at the University of Salzburg, Austria. He is the author of over 200 research articles in the field of yeast genetics, aging research and the biology of oxidative stress. Over the last few years his group has intensively studied the physiological role of reactive oxygen species produced by a newly discovered NADPH oxidase of yeast. He is well recognized by the international yeast genetics community where he has represented his home country Austria over the course of 30 years. He was elected to the rank of AAAS fellow in 2011. Collaborations in the field of yeast genetics and molecular biology extend to Australia, the United Kingdom, USA, Italy and Germany.

Photograph: Neumayr/LEO

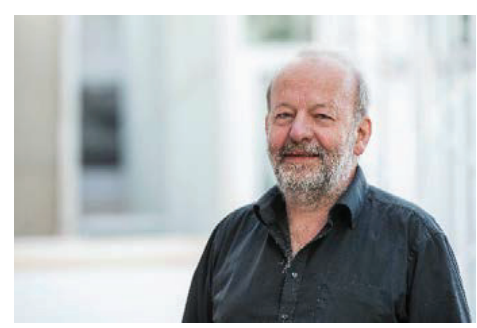

Peter M. Eckl is the Professor of Cell Biology at the University of Salzburg, Austria. He is author of numerous publications in the fields of oxidative stress, lipid peroxidation, antioxidants, apoptosis, cell biology and environmental toxicology, and he is a member of the editorial board of several related scientific journals. Based on his achievements on the toxicological properties of lipid peroxidation products, he also served as chairman of the HNE-Club, a special interest group of the Society for Free Radical Research. Apart from his scientific pursuits, he is coordinating several European projects related to quality assurance and quality development in Kosovo and Albania.

Photograph: Neumayr/LEO 



\section{Preface}

It is a pleasure for me to express some thoughts on this monograph entitled "Oxidative Stress in Health and Disease", and to congratulate Drs. Breitenbach and Eckl for their effort to convene international experts to address specific research areas on this topic. The surge of interest in research on oxidative stress has been bewildering, and it is useful, from time to time, to update and to compile current knowledge. Enormous recent advances in technology have permitted detailed study of redox processes at the molecular level. Improved analytical capabilities enabled identification of protein and lipid redox modifications in the biological setting, which have been put into context of physiological and pathophysiological conditions in the various chapters of this book.

The introductory Editorial provides a knowledgeable presentation of oxidative stress and redox signaling and their molecular basis. The focus of the book is on attempts to bring together chemistry/biochemistry and specific biological and biomedical applications. These range from fungi to human disease states and organ physiology. Skin, skeletal muscle and fat are in focus, as well as subcellular aspects of signal transduction with emphasis on mitochondria and endoplasmic reticulum. All these areas of research have profited from better understanding of the underlying molecular events. However, much has to be learned still on the role of oxidative stress in disease states. It is the question of cause versus consequence. Progress on this issue will lay the groundwork for refined and specific redox medicine.

I hope the book will provide useful insight and will guide future research.

Helmut Sies

Düsseldorf, January 2016 



\title{
Introduction to Oxidative Stress in Biomedical and Biological Research
}

\section{Michael Breitenbach and Peter Eckl}

\begin{abstract}
Oxidative stress is now a well-researched area with thousands of new articles appearing every year. We want to give the reader here an overview of the topics in biomedical and basic oxidative stress research which are covered by the authors of this thematic issue. We also want to give the newcomer a short introduction into some of the basic concepts, definitions and analytical procedures used in this field.
\end{abstract}

Reprinted from Biomolecules. Cite as: Breitenbach, M.; Eckl, P. Introduction to Oxidative Stress in Biomedical and Biological Research. Biomolecules 2015, 5, 1169-1177.

The term "stress" was first used in the biomedical literature as a description of hyperactivity in the hormone system, in particular concerning the corticosteroids of the adrenal cortex [1]. The author nicely summarized some 20 years later [2] how the idea of stress, stress response, and homeostasis as a dynamic equilibrium gradually developed into a highly useful idea in general physiology and the study of diseases. He saw "stress" primarily as a factor causing disease, and even today, as exemplified by this thematic issue, modern stress research is still largely concerned with pathomechanisms of human disease. Today we know that in many of those stress sitations, in fact redox processes play a major role.

The concept of physiological stress in general for a long time was ill-defined in physicochemical terms, it was "fuzzy" like the concept of oxidative stress. It took decades before a clearer picture could be established by delineating the molecular mechanisms of stress generation, stress defense and stress signaling.

The term "oxidative stress" was coined only 30 years ago [3]. In a recent review article [4] the history and development of this scientific concept is vividly described. The concept is based on earlier work by Selye (loc. cit), and inspired by early publications related to oxygen toxicity, often connected with the problem of aging [5-7], the metabolism of oxygen (and other) radicals in biological systems [8], the gradual development of our understanding of mitochondrial physiology [9,10], "mitochondrial" aging research [11,12], and the study of redox imbalance in cells and organisms [13]. Redox imbalance according to one definition is another name for oxidative stress which is based on the Nernst equation taking into account all the redox couples present in the cell or in the different cellular subcompartments [14]. Another more practical and operational definition of oxidative stress is given by Lushchak: "Oxidative stress is a situation when steady-state ROS concentration is transiently or chronically enhanced, disturbing cellular metabolism and its regulation and damaging cellular constituents" [15].

The yeast system of molecular genetics is ideally suited to study the relationship between different kinds of stress (heat stress, oxidative stress by different oxidants like $\mathrm{H}_{2} \mathrm{O}_{2}$, paraquat, and diamide, osmotic stress, salt stress, etc.) by studying the reaction of the cells to low inducing stress conditions by transcriptomic and proteomic methods. This was beautifully 
shown by the group of Ian Dawes in a series of papers [16-19]. The adapted cells after the initial treatment are more resistant to high "killing" stresses than without the hormetic conditioning. These experiments led in part to very surprising results. On the one hand, the adaptive reaction of the yeast cell included not only resistance to the same primary stress that was initially applied, but also to other seemingly unrelated stresses. An example is given by cross-reactivity to conditioning by oxidants and by heat. On the other hand, this cross-reactivity was by no means universal. Also the direct genomic reactions to a primary stress were not universal as shown by the transcriptome. Therefore, the concept of "generalized stress" or a "generalized stress reaction" is certainly an overstatement, if not a mistaken concept altogether, although the idea of a generalized stress response is still to be found in the literature [20]. Also, different oxidants did not overlap in the genomic reaction and the resistance which they induced [16]. There is every reason to believe that a similarly complicated system of cross-reactivity and cross-adaptation also exists in higher cells. However, this was never tested in detail due to the greater technical difficulties in higher cell systems.

Herrmann and Dick have recently summarized the main routes of research in redox biology, which now seem to be of pre-eminent importance [21]. Figure 1 shows a grapical representation of these fields of research.

Presently, an exponentially increasing number of scientific articles and books are appearing which are dealing with oxidative stress in one way or the other. To give an overview and a few examples, we are listing here the numbers of publications, as of May 2015, and give a few typical examples.

In the last two years alone, 59 books appeared dealing with oxidative stress. The large majority of these books are medical, treating oxidative stress in connection with a large number of different specific diseases (for instance: cancer, neurodegeneration in Alzheimer's or Parkinson's disease, arteriosclerosis, etc.), sources of nutritional antioxidants for the prevention of disases, pro-oxidant actions of antioxidants, and in anti-aging medicine, and so on. Only a minority deals with other topics such as oxidative stress in plants, or in veterinary medicine. Looking at the titles of these books, it becomes clear that in some cases concepts are desribed for the public which have no firm founding in science. To give the most prominent example, there are books and articles about the anti-aging effects of many antioxidative plant-derived compounds, although clinical trials did generally not show the promised healing or rejuvenation effects [22]. In the last few years, no fewer than five international research journals (Free Radical Research, Free Radicals in Biology and Medicine, Redox Reports, Antioxidant Redox Signaling, Redox Biology) dealing with oxidative stress were launched, as mentioned by Helmut Sies in his recent review article [4]. Over 1,990,000 hits in Google scholar use the term oxidative stress. Furthermore, there are 627,000 results on lipid peroxidation and 1,840,000 on lipid oxidation, the major downstream targets of oxidative stress. In PubMed, in just under 32,000 articles oxidative stress is named in the title of the papers, of which close to 3500 are review articles. 


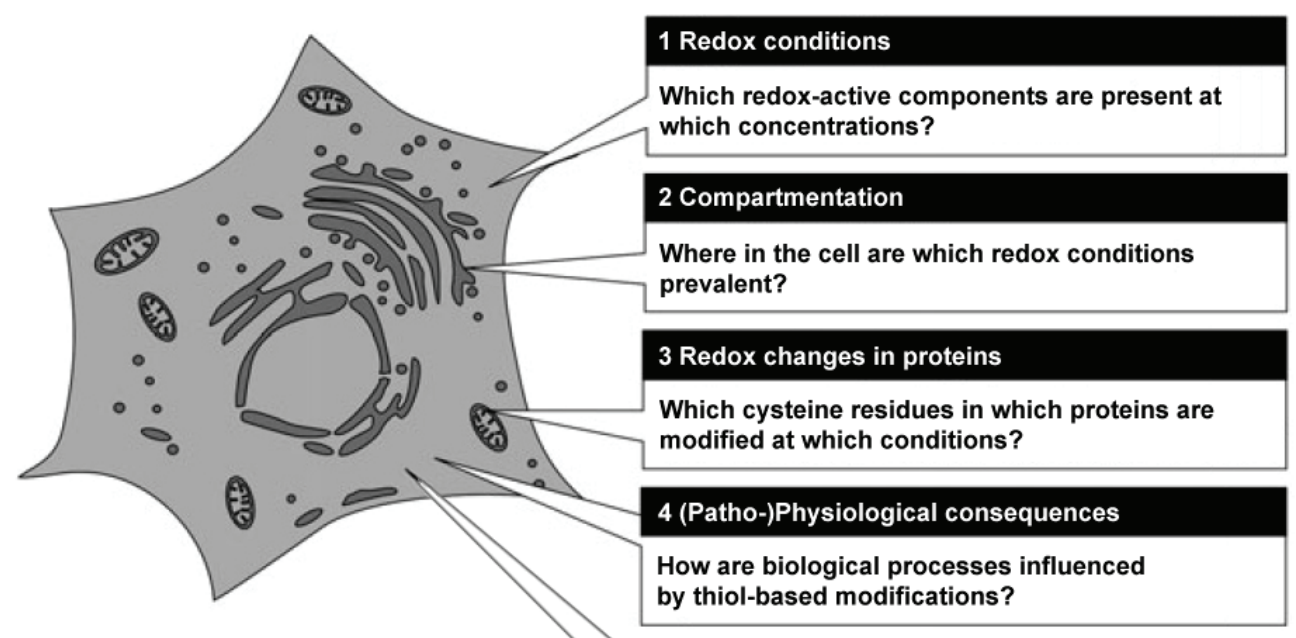

5 Signaling

What is the mechanism and interrelation of oxidative stress buildup, adaptation, and signaling?

Figure 1. Central questions in redox biology. Source: Herrmann and Dick, Biological Chemistry 393, 999-1004 (2012) modified, with permission.

For the present thematic issue, we have assembled an internationally well-recognized team of contributing authors, who are not clinicians, but as biologists work in biomedical science and are presenting here an important and new aspect of oxidative stress research, related either to a specific medical problem, or contributing to the basic understanding of oxidative stress.

Reliability of research in redox biology depends very much on the reliability of the anlytical methods used, and progress in the field very often was enabled by the development of new analytical methods. Two of the chapters in this thematic issue deal with key innovations in methodology that are at present still further improved every year. First of all, modern methods in protein mass spectrometry have made it possible to study the oxidation of individual cysteine residues as shown in the chapter by Verrastro et al. The redox cycle of SH groups in proteins can either activate or inactivate catalytic SH groups or fine-tune the catalytic activity. When the SH groups concerned act as a redox sensor they undergo a redox cycle starting with the formation of a sulfenic acid as described also in the chapter by Breitenbach et al. Analyzing the oxidation state of those $\mathrm{SH}$ groups on specific proteins throughout the "cysteine proteome" [23] using high throughput mass spectrometry methods opens up a huge field of research into the regulatory network of the cell. This is exemplified, for instance in excellent work from the laboratory of Ursula Jakob on the proteomic analysis of the cellular redox interaction network [24].

Another methodological advance concerns respirometry. Being able to measure with high precision the oxygen metabolism of mitochondria in small samples is a key technological advance which is treated by Makrecka-Kuka et al. Oxidative stress in a number of diseases and in aging can profoundly change such mitochondrial parameters as the $\mathrm{P} / \mathrm{O}$ ratio or the 
respiratory control ratio (RCR). Such measurements are required with high precision and, in a clinical setting, with high speed. They can help to define such highly dangerous disease states as ischemia reperfusion injury in stroke and heart infarction, and even more in septic shock, as shown in the chapter by Weidinger and Kozlov.

Being able to measure superoxide, the primary product of oxygen toxicity, with high sensitivity and selectivity is a key requirement of redox biology. This is possible through the development of reliable chemiluminescence and electron spin resonance methods, as described elsewhere [14,25].

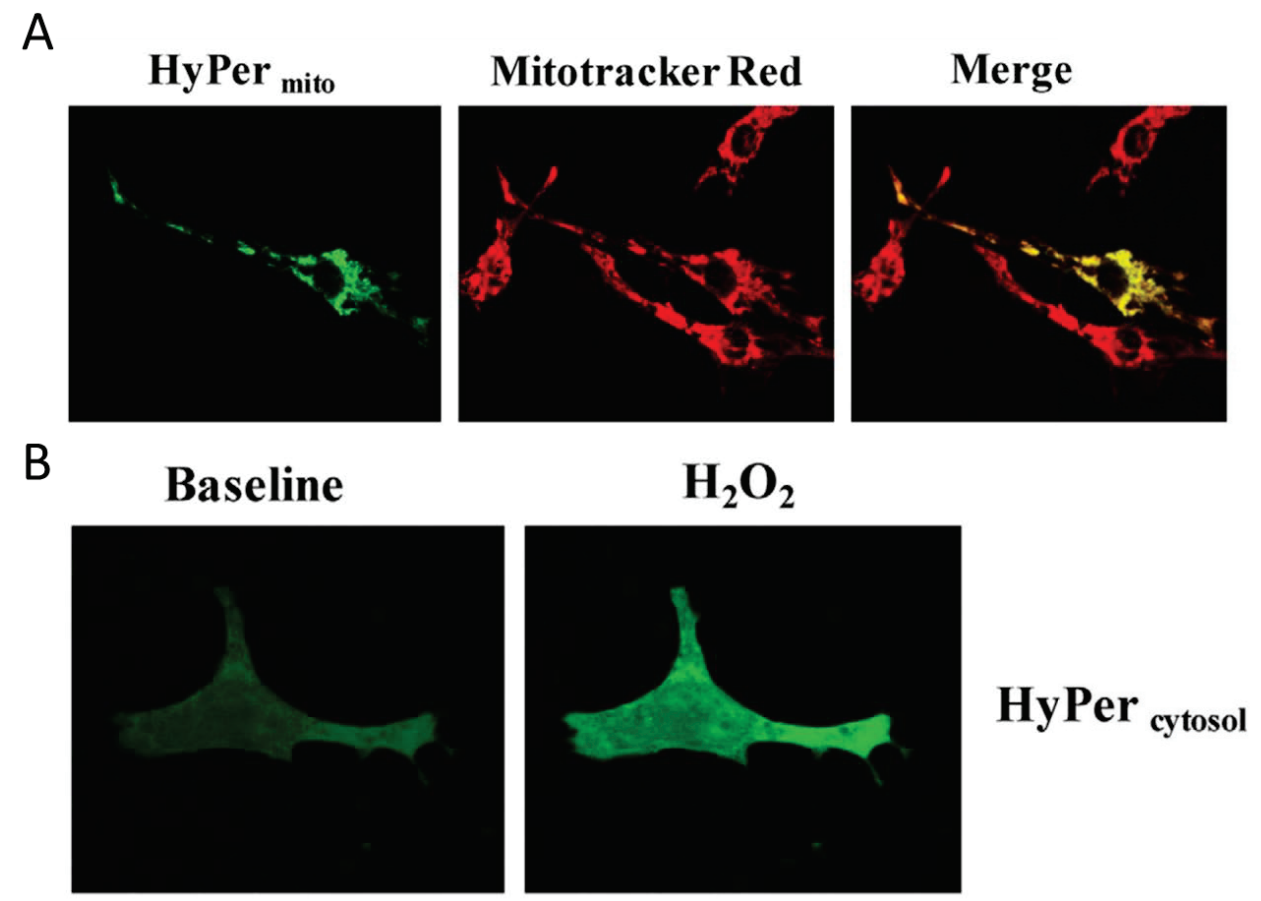

Figure 2. Visualization of $\mathrm{H}_{2} \mathrm{O}_{2}$ in subcellular compartments. (A) Neonatal rat ventricular cardiomyocytes transfected with the genetically encoded mitochondria

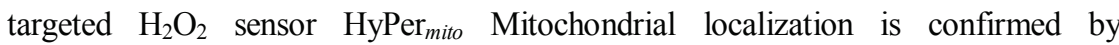
colocalization with the mitochondrial dye Mitotracker Red. Cells were analyzed after monoamine oxidase ativation with dopamine; (B) Neonatal cardiomyocytes

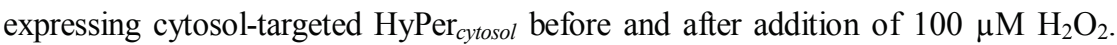
Source: Kaludercic et al., Frontiers in Physiology, 5, article 285 (2014) with permission.

Another and very important series of methodological advances in the last few years are redox sensitive fluorescent tags or dyes that enable to study the localization and quantification of redox active compounds, and in fact the redox potential in different subcellular compartments in vivo [21,26-28]. These studies make it very clear that redox-active micro-domains exist in the known subcellular compartments, such as mitochondria and the 
ER, and even in the cytoplasm of cells. A whole series of protein reporters based on the bacterial $\mathrm{H}_{2} \mathrm{O}_{2}$ sensitive HyPer protein domain has been developed (reviewed in [26]). An alternative method uses roGFP and related constructs to measure the redox potential in subcellular domains [29,30]. Figure 2 shows an example of the $\mathrm{H}_{2} \mathrm{O}_{2}$ detected by mitochondrial HyPer in heart muscle cells metabolically stimulated by dopamine.

The fluorescence strictly co-localizes with the mitochondria-specific Mitotracker dye, while in a control experiment cytoplasmic $\mathrm{H}_{2} \mathrm{O}_{2}$ is detected with a different HyPer construct which localizes strictly to the cytoplasm of the cell. In summary, these relatively recent results clearly show the compartmentation of redox-active compounds and signals in vivo in eukaryotic cells.

Müllebner et al. in their chapter present a bioanalytical method to measure bilirubin, an important antioxidative molecule in liver cell systems, which is relevant in studying heme degradation by heme oxygenase taking place in the liver under oxidative stress.

Oxidative stress in aging in general and in aging human skin in particular is treated here by Rinnerthaler et al. It is now nearly universally accepted that oxidative stress is not only associated with but also plays a major role in the aging processes of all cells [31].

The metabolism of heme and of iron in the cell is essential for survival of every cell, because iron, like copper, is a redox-active transition metal needed for the most basic reactions of cellular metabolism. The transition between ferric and ferrous iron ions, just like the one between cupric and cuprous copper ions is within the range of the biological redox potential of the cell and is important for the reactions catalyzed. On the other hand, the ferrous state in iron and the cuprous state in copper can take part in the Haber-Weiss and Fenton reactions thereby creating $\mathrm{OH}$ radicals, the most dangerous reactive oxygen species known. Therefore, in living cells, these metal ions are usually bound by chelating ligands which prohibit the dangerous reactions, and their redox transitions and the transport of the ions into the cell are tightly regulated. This is described in detail in the chapter by Bresgen and Eckl. Likewise, heme degradation, which is a physiologically necessary process, is tightly regulated and avoids the production of free iron ions. The physiological problems related to this process and its importance for liver diseases is presented by Müllebner et al.

Oxidative stress as a consequence of increased activity of muscle and of other tissues is a topic of high relevance to sports medicine and to the study of exercise in hypoxia. Steinbacher and Eckl discuss the effects of oxidative stress in muscle training including the effect of ROS and ROS derived lipid peroxidation products as signaling substances to increase the production of defense enzymes and antioxidants and the effects on gene expression in muscle that lead to adaptation and beneficial health effects of training.

The influence of oxidative stress on the ion flux activity of calcium-activated potassium channels in nerve and muscle are reviewed by Hermann et al. This constitutes an important link of oxidative stress with the activity of the central nervous system.

Netzer et al. discuss the effects of hypoxia on the metabolism of fat cells. Hypoxia can under certain circumstances induce oxidative stress, and it can exert a positive influence on fat cell metabolism to counteract insulin resistance in type 2 diabetes. Recently, the hypothesis 
was presented by J. D. Watson that type 2 diabetes might be a "redox disease" [32], however, as far as we know Watson's suggested clinical trials in this direction are not yet under way.

Diabetes type 2 clearly leads to all the molecular markers of oxidative stress in combination with other disease markers that have more directly to do with the increased level of free glucose in serum and in the cells. Nowotny et al. give a comprehensive discussion of advanced glycation end products (AGEs) in type 2 diabetes, treating the synthesis as well as the role in the pathomechanism of these highly complex products of irregular oxidative sugar metabolism.

The influence of oxidative stress and other stress situations on a global shift in the metabolic network of the cell was investigated and is presented here by the group of Ralser (Piedrafita et al.). They show that under oxidative stress not only the familiar shift from glycolysis to the pentose phosphate shunt takes place, but also a large number of "unwanted" side reactions which must be accounted for and to some degree repaired to ensure survival of the cell.

Oxidative stress is encountered by cells after bacterial infection and in a state of inflammation [33] and is in fact part of the primary innate immune defense of the body, including also the well known oxidative burst of macrophages and monocytes. Stoiber et al. show that oxidative stress in such a situation is also involved in the formation of so-called extracellular DNA traps which can help killing of bacteria.

Oxidative stress plays a very important role in the interaction of the human body with the common parasite Candida albicans. This fungal commensal organism can in special cases (immune deficiency) develop into a life-threatening systemic infectious disease. The strategies used by $C$. albicans to evade the oxidative burst of human macrophages and the strategies used by macrophages to mitigate the oxidative stress response (including filamentation) in the fungus are treated in the chapter by Da Silva Dantas et al.

The group of Jamieson is presenting an account of anti-inflammatory substances from the fruits of haskap (Lonicera caerulea) from Canada, which were tested in a human cell culture assay after pro-inflammatory stimulation with lipopolysaccharide (Rupasinghe et al.).

An important chapter of this thematic issue deals with what one could call second messengers of oxidative stress, namely lipid oxidation and the biological effects of aldehydic lipid oxidation products, among which 4-hydroxynonenal (HNE) appears to be the most important, since it contributes to both physiological and pathophysiological mechanisms. For example, mitochondrial uncoupling is dependent upon HNE, as are other cellular reactions such as proliferation and apoptosis. Schaur and co-authors give a concise overview on its formation, chemical ractions with macromolecules in the cell, and the consequences thereof.

To conclude this introductory chapter we are coming back to the still fragmentary studies of molecular mechanisms of both the primary response of a cell to oxidative stress and to the adaptive response that enables a cell to survive after a change of the metabolic makeup, or alternatively, the final mechanism of apoptotic or necrotic programmed cell death. These processes require a signaling cascade that is only in outline known today. However, oxidative signaling is mentioned or treated in part by nearly all chapters of this thematic issue. We will briefly describe the role of peroxiredoxin, NADPH oxidases and $\mathrm{H}_{2} \mathrm{O}_{2}$ signaling to 
transcription factors without going into detail, as this topic is treated in the chapter by Breitenbach et al. There is increasing evidence that in human cells the signal created by plasma membrane receptors for peptide hormones and cytokines, like EGF [34], insulin [35,36] or PDGF [37], is transmitted by $\mathrm{H}_{2} \mathrm{O}_{2}$ to phosphotyrosine phosphatase $1 \mathrm{~B}$ (PTP1B). In this way, the catalytic cysteine $\mathrm{SH}$ group of PTP1B is oxidized and inactivated. This leads to an increase in the phosphorylation state of the target proteins. Peroxiredoxin takes part in this reaction. It is the quantitatively most important detoxifying enzyme for $\mathrm{H}_{2} \mathrm{O}_{2}$ and organic hydroperoxides, but it is also a highly regulated module helping in transmission of the $\mathrm{H}_{2} \mathrm{O}_{2}$ signal. This is achieved by reversible hyperoxidation of the peroxidative $\mathrm{SH}$ of peroxiredoxin thus creating a strong but highly localized feed-forward reaction which increases the local $\mathrm{H}_{2} \mathrm{O}_{2}$ concentration needed for signal transmission. Compartmentation of signaling is important to avoid oxidative damage in the cell and is achieved by localization of NADPH oxidase [38] and by localization of $\mathrm{H}_{2} \mathrm{O}_{2}$ [39] which was shown by the HyPer method mentioned above. They showed that internally created $\mathrm{H}_{2} \mathrm{O}_{2}$ acts locally and does not easily diffuse to the whole cytoplasmic space. This signal leads to phosphorylation and nuclear transfer of the relevant transcription factors which are different in yeast cells and human cells [40]. Examples are Yap1 in yeast cells and NF- $\kappa \mathrm{B}$ in human cells. Alternatively, nuclear transfer and transcription activity can also be regulated by direct oxidation of the transcription factors. One of the target genes of NF- $\kappa \mathrm{B}$ is Nox4, the NADPH oxidase which is functional in this signaling cascade [41]. Obviously, many questions concerning this signaling cascade remain open at present. But we believe that the relationship between oxidative stress defense and oxidative signaling by $\mathrm{H}_{2} \mathrm{O}_{2}$ is one of the most exciting topics in oxidative stress research in years to come.

\section{Acknowledgments}

The authors are grateful for financial support by FWF grant No. P26713 (to Michael Breitenbach) and to Mark Rinnerthaler and Thomas Karl for their help with preparation of the figures, finalization and submission of this paper; to Mssrs. Walter de Gruyter Ltd. (Berlin, Germany) for permission to use Figure 1 and to Nina Kaludercic for permission to use Figure 2.

\section{Conflicts of Interest}

The authors declare no conflict of interest.

\section{References}

1. Selye, H. A Syndrome produced by Diverse Nocuous Agents. Nature 1936, 138, 32.

2. Selye, H. Stress and disease. Science 1955, 122, 625-631.

3. Sies, H. Oxidative Stress; Academic Press: London, UK, 1985; p. 507.

4. Sies, H. Oxidative stress: A concept in redox biology and medicine. Redox Biol. 2015, 4C, 180-183. 
5. Gerschman, R.; Gilbert, D.L.; Nye, S.W.; Dwyer, P.; Fenn, W.O. Oxygen poisoning and $\mathrm{x}$-irradiation: A mechanism in common. Science 1954, 119, 623-626.

6. Szilard, L. On the nature of the aging process. Proc. Natl. Acad. Sci. USA 1959, 45, 30-45.

7. Orgel, L.E. The maintenance of the accuracy of protein synthesis and its relevance to ageing. Proc. Natl. Acad. Sci. USA 1963, 49, 517-521.

8. Harman, D. Aging: A theory based on free radical and radiation chemistry. J. Gerontol. 1956, 11, 298-300.

9. Racker, E. Mechanisms of energy transformations. Annu. Rev. Biochem. 1977, 46, 1006-1014.

10. Mitchell, P. Coupling of phosphorylation to electron and hydrogen transfer by a chemi-osmotic type of mechanism. Nature 1961, 191, 144-148.

11. Harman, D. The biologic clock: The mitochondria? J. Am. Geriatr. Soc. 1972, 20, 145-147.

12. Chance, B.; Sies, H.; Boveris, A. Hydroperoxide metabolism in mammalian organs. Physiol. Rev. 1979, 59, 527-605.

13. Schafer, F.Q.; Buettner, G.R. Redox environment of the cell as viewed through the redox state of the glutathione disulfide/glutathione couple. Free Radic. Biol. Med. 2001, 30, 1191-1212.

14. Aung-Htut, M.T.; Ayer, A.; Breitenbach, M.; Dawes, I.W. Oxidative stresses and ageing. Subcell. Biochem. 2012, 57, 13-54.

15. Lushchak, V.I. Free radicals, reactive oxygen species, oxidative stress and its classification. Chem. Biol. Interact. 2014, 224C, 164-175.

16. Jarolim, S.; Ayer, A.; Pillay, B.; Gee, A.C.; Phrakaysone, A.; Perrone, G.G.; Breitenbach, M.; Dawes, I.W. Saccharomyces cerevisiae genes involved in survival of heat shock. G3 2013, 3, 2321-2333.

17. Thorpe, G.W.; Reodica, M.; Davies, M.J.; Heeren, G.; Jarolim, S.; Pillay, B.; Breitenbach, M.; Higgins, V.J.; Dawes, I.W. Superoxide radicals have a protective role during h2o2 stress. Mol. Biol. Cell 2013, 24, 2876-2884.

18. Ayer, A.; Sanwald, J.; Pillay, B.A.; Meyer, A.J.; Perrone, G.G.; Dawes, I.W. Distinct redox regulation in sub-cellular compartments in response to various stress conditions in saccharomyces cerevisiae. PLoS ONE 2013, 8, e65240.

19. Ayer, A.; Fellermeier, S.; Fife, C.; Li, S.S.; Smits, G.; Meyer, A.J.; Dawes, I.W.; Perrone, G.G. A genome-wide screen in yeast identifies specific oxidative stress genes required for the maintenance of sub-cellular redox homeostasis. PLoS ONE 2012, 7, e44278.

20. Horne, S.D.; Chowdhury, S.K.; Heng, H.H. Stress, genomic adaptation, and the evolutionary trade-off. Front. Genet. 2014, doi:10.3389/fgene.2014.00092.

21. Herrmann, J.M.; Dick, T.P. Redox biology on the rise. Biol. Chem. 2012, 393, 999-1004.

22. Goodman, M.; Bostick, R.M.; Kucuk, O.; Jones, D.P. Clinical trials of antioxidants as cancer prevention agents: Past, present, and future. Free Radic. Biol. Med. 2011, 51, 1068-1084. 
23. Jones, D.P.; Go, Y.M. Mapping the cysteine proteome: Analysis of redox-sensing thiols. Curr. Opin. Chem. Biol. 2011, 15, 103-112.

24. Thamsen, M.; Jakob, U. The redoxome: Proteomic analysis of cellular redox networks. Curr. Opin. Chem. Biol. 2011, 15, 113-119.

25. Benov, L.; Sztejnberg, L.; Fridovich, I. Critical evaluation of the use of hydroethidine as a measure of superoxide anion radical. Free Radic. Biol. Med. 1998, 25, 826-831.

26. Kaludercic, N.; Deshwal, S.; di Lisa, F. Reactive oxygen species and redox compartmentalization. Front. Physiol. 2014, 5, 285.

27. Maulucci, G.; Pani, G.; Labate, V.; Mele, M.; Panieri, E.; Papi, M.; Arcovito, G.; Galeotti, T.; de Spirito, M. Investigation of the spatial distribution of glutathione redox-balance in live cells by using fluorescence ratio imaging microscopy. Biosens. Bioelectron. 2009, 25, 682-687.

28. Lopez-Mirabal, H.R.; Winther, J.R. Redox characteristics of the eukaryotic cytosol. Biochim. Biophys. Acta 2008, 1783, 629-640.

29. Hanson, G.T.; Aggeler, R.; Oglesbee, D.; Cannon, M.; Capaldi, R.A.; Tsien, R.Y.; Remington, S.J. Investigating mitochondrial redox potential with redox-sensitive green fluorescent protein indicators. J. Biol. Chem. 2004, 279, 13044-13053.

30. Sugiura, K.; Nagai, T.; Nakano, M.; Ichinose, H.; Nakabayashi, T.; Ohta, N.; Hisabori, T. Redox sensor proteins for highly sensitive direct imaging of intracellular redox state. Biochem. Biophys. Res. Commun. 2015, 457, 242-248.

31. Jones, D.P. Redox theory of aging. Redox Biol. 2015, 5, 71-79.

32. Watson, J.D. Type 2 diabetes as a redox disease. Lancet 2014, 383, 841-843.

33. Salzano, S.; Checconi, P.; Hanschmann, E.M.; Lillig, C.H.; Bowler, L.D.; Chan, P.; Vaudry, D.; Mengozzi, M.; Coppo, L.; Sacre, S.; et al. Linkage of inflammation and oxidative stress via release of glutathionylated peroxiredoxin-2, which acts as a danger signal. Proc. Natl. Acad. Sci. USA 2014, 111, 12157-12162.

34. Lee, S.R.; Kwon, K.S.; Kim, S.R.; Rhee, S.G. Reversible inactivation of protein-tyrosine phosphatase 1B in A431 cells stimulated with epidermal growth factor. J. Biol. Chem. 1998, 273, 15366-15372.

35. Mahadev, K.; Zilbering, A.; Zhu, L.; Goldstein, B.J. Insulin-stimulated hydrogen peroxide reversibly inhibits protein-tyrosine phosphatase $1 \mathrm{~B}$ in vivo and enhances the early insulin action cascade. J. Biol. Chem. 2001, 276, 21938-21942.

36. Salmeen, A.; Andersen, J.N.; Myers, M.P.; Meng, T.C.; Hinks, J.A.; Tonks, N.K.; Barford, D. Redox regulation of protein tyrosine phosphatase $1 \mathrm{~B}$ involves a sulphenyl-amide intermediate. Nature 2003, 423, 769-773.

37. Meng, T.C.; Fukada, T.; Tonks, N.K. Reversible oxidation and inactivation of protein tyrosine phosphatases in vivo. Mol. Cell 2002, 9, 387-399.

38. Chen, K.; Kirber, M.T.; Xiao, H.; Yang, Y.; Keaney, J.F., Jr. Regulation of ros signal transduction by NADPH oxidase 4 localization. J. Cell Biol. 2008, 181, 1129-1139.

39. Mishina, N.M.; Tyurin-Kuzmin, P.A.; Markvicheva, K.N.; Vorotnikov, A.V.; Tkachuk, V.A.; Laketa, V.; Schultz, C.; Lukyanov, S.; Belousov, V.V. Does cellular hydrogen peroxide diffuse or act locally? Antioxid. Redox Signal. 2011, 14, 1-7. 
40. Marinho, H.S.; Real, C.; Cyrne, L.; Soares, H.; Antunes, F. Hydrogen peroxide sensing, signaling and regulation of transcription factors. Redox Biol. 2014, 2, 535-562.

41. Jiang, F.; Liu, G.S.; Dusting, G.J.; Chan, E.C. NADPH oxidase-dependent redox signaling in TGF-beta-mediated fibrotic responses. Redox Biol. 2014, 2, 267-272. 




\title{
Mass Spectrometry-Based Methods for Identifying Oxidized Proteins in Disease: Advances and Challenges
}

\author{
Ivan Verrastro, Sabah Pasha, Karina Tveen Jensen, Andrew R. Pitt and Corinne M. Spickett
}

Abstract: Many inflammatory diseases have an oxidative aetiology, which leads to oxidative damage to biomolecules, including proteins. It is now increasingly recognized that oxidative post-translational modifications (oxPTMs) of proteins affect cell signalling and behaviour, and can contribute to pathology. Moreover, oxidized proteins have potential as biomarkers for inflammatory diseases. Although many assays for generic protein oxidation and breakdown products of protein oxidation are available, only advanced tandem mass spectrometry approaches have the power to localize specific oxPTMs in identified proteins. While much work has been carried out using untargeted or discovery mass spectrometry approaches, identification of oxPTMs in disease has benefitted from the development of sophisticated targeted or semi-targeted scanning routines, combined with chemical labeling and enrichment approaches. Nevertheless, many potential pitfalls exist which can result in incorrect identifications. This review explains the limitations, advantages and challenges of all of these approaches to detecting oxidatively modified proteins, and provides an update on recent literature in which they have been used to detect and quantify protein oxidation in disease.

Reprinted from Biomolecules. Cite as: Verrastro, I.; Pasha, S.; Jensen, K.T.; Pitt, A.R.; Spickett, C.M. Mass Spectrometry-Based Methods for Identifying Oxidized Proteins in Disease: Advances and Challenges. Biomolecules 2015, 5, 378-411.

\section{Introduction to Protein Oxidation}

Many diseases have an oxidative aetiology resulting from activation of the immune system, mitochondrial dysfunction or environmentally-induced oxidative stress. Oxidative modification of proteins can have multiple effects, such as loss of enzymatic activity, functional alterations, loss of structural integrity, and protein aggregation [1]. Various different reactive and oxidizing species exist and vary in their reactivity to protein residues and sites. Metal-catalysed oxidation depends on the formation of hydroxyl radicals through Fenton chemistry; hydroxyl radicals are highly reactive and able to modify almost any site through hydrogen abstraction and peroxide formation, often leading to backbone fragmentation. The most susceptible side chains in proteins are the sulfur-containing cysteine and methionine side chains; the reactivity of cysteine with hydrogen peroxide depends on the pKa of the thiol group as the thiolate anion is a better nucleophile. Cysteine can also react with reactive nitrogen species to form nitrosothiols (Figure 1). Other residues that are commonly oxidized include histidine, proline, lysine and arginine, where hydroxylation or formation of aldehydes or ketones may occur. Reactive nitrogen compounds derived from peroxynitrite are often both nitrating and oxidizing. Sites susceptible to nitration include tyrosine (forming 3-nitrotyrosine) and tryptophan. Hypohalites can also react with aromatic residues to form halogenated products such as 3-chloro and 3-bromotyrosine [2]. 


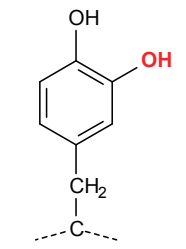

3-Hydroxytyrosine

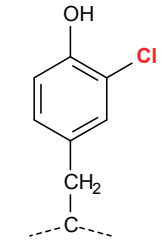

3-Chlorotyrosine

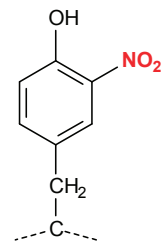

3-Nitrotyrosine

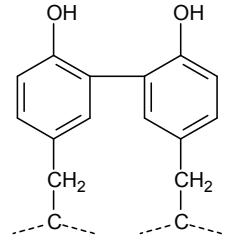

Dityrosine

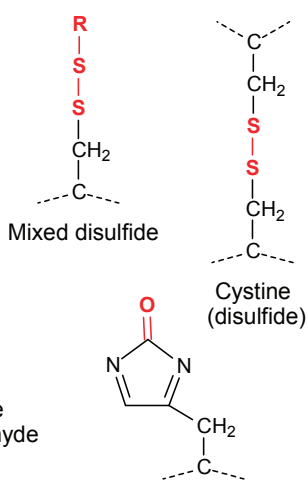

2-oxo-histidine

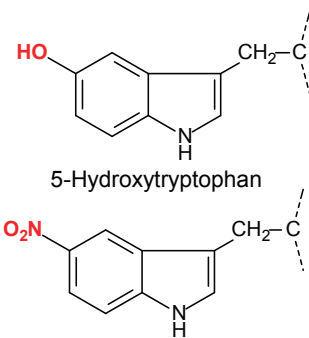

5-Nitrotryptophan
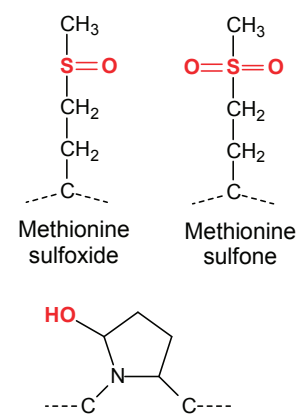

2-Hydroxyproline

Figure 1. Structures of oxidized residues most commonly detected and studied by mass spectrometry. In mixed disulfides, R can be cysteine or glutathione (glutathionylation).

Protein oxidation is often measured as a marker of oxidative damage and cellular stress, and a wide variety of methods exist, varying from simple global methods to specific approaches to detecting individual modified residues [3]. A commonly measured modification is carbonyl formation, which can occur on lysine, arginine, serine, threonine and proline residues following metal-catalysed oxidation or attack by hypochlorous acid. Carbonyl groups react with DNPH (2,4-dinitrophenylhydrazine) and other aldehyde reaction probes such as $N$-aminooxymethylcarbonylhydrazino- $D$-biotin, offering potential for colorimetric detection or selective enrichment approaches. Anti-DNP antibodies form the basis of carbonyl-focused western blotting ("oxy-blotting") and ELISAs [3,4]. Total digestion followed by HPLC, LC-MS or LC-MS/MS has been used to investigate a wide range of oxidized amino acids [5], but these approaches do not provide information on the specific protein that has been modified, or the exact site of modification.

Mass spectrometry has been used for many years for identification and characterization of proteins, and is arguably the most informative method for determining oxidative modification of proteins currently available. This article gives an overview of advances and limitations of LC-MS/MS approaches for detecting specific non-enzymatic oxidative modifications to proteins, and summarizes their recent application in studies of disease.

\section{Overview of Mass Spectrometry Methods for Protein Oxidation Analysis}

Mass spectrometry measures the mass-to-charge ratio $(\mathrm{m} / \mathrm{z})$ of ionized analytes, and as oxidative modifications alter the chemical composition of a protein, they change the $\mathrm{m} / \mathrm{z}$ ratio of the intact 
protein and of the residues where the oxidation occurred; thus, MS is a powerful method for detecting oxidative post-translational modifications (oxPTMs) [2]. Mass spectrometry approaches for the analysis of proteins, both native or oxidized, have advanced substantially in recent years, and can essentially be divided into "top-down", which involves analysis of intact proteins and their fragmentation within the mass spectrometer, and "bottom-up" analysis, in which proteins are enzymatically digested to a peptide mixture before being introduced to the instrument (Figure 2). The latter is by far the more common method, as it is extensively used in proteomics studies to sequence and identify proteins in biological samples, and has been extended to investigate protein oxidation. However, while identification of proteins using search engines to match experimental MS data against protein sequence databases is now routine, the analysis of post-translational modification, including oxidative modifications, continues to be extremely challenging. Consequently, there is a continual search for methodologies that facilitate identification of oxPTMs. This has led to the development of targeted mass spectrometry routines that search for peptides containing ions that are diagnostic for the presence of an oxidative modification, such as chlorotyrosine or methionine sulfoxide. Alternatively, the use of chemical reagents that react with oxidative modifications, which can be used as tags to label modified peptides or proteins, can facilitate both enrichment and detection and has seen significant recent development; carbonyl-reactive probes are a major focus of this approach. For all of these methods, an overarching aim is to be able to quantify the level of oxPTM, either in absolute terms or relative to the level of total protein. Advances in these different strategies are described in more detail in the following sections.

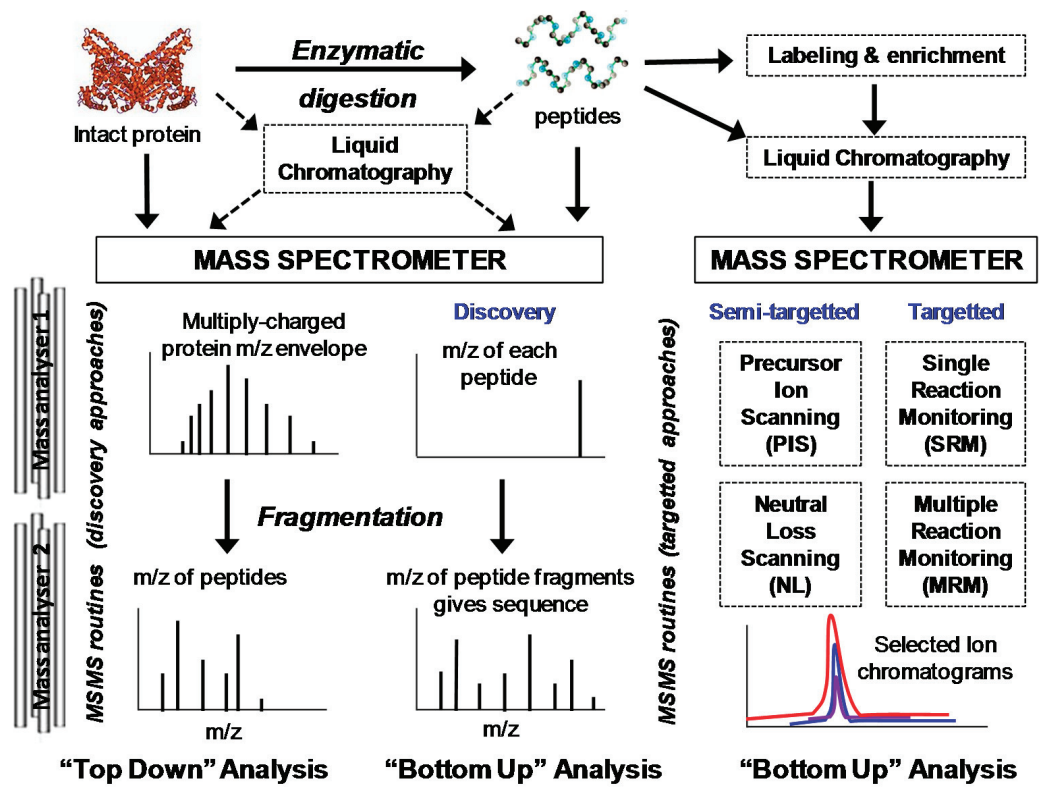

Figure 2. Summary of advanced methods for identification of proteins and oxPTMs. Labeling and enrichment can also be carried out at the protein level, but this approach is less common. 


\subsection{Sample Preparation and Digestion}

An important practical consideration for any study of protein oxidation is how to minimize oxidative artefacts caused by sample processing. Bottom-up strategies usually involve digestion in solution or one or two-dimensional gel electrophoresis followed by in-gel digestion; both methods have been shown to introduce artefacts such as methionine, cysteine or tryptophan oxidation [6], so care is needed to minimise exposure to air and in the interpretation of results. Adventitious oxidation, such as artefactual $S$-thiolation of reactive, surface-exposed cysteine residues, has also been identified as a problem in top-down MS [7].

Protein digestion strategies for bottom-up approaches depend to a great extent on the type of experiment, but there are important considerations for mapping oxPTMs. Where comprehensive mapping of oxidative modifications of proteins is the aim and as close to complete sequence coverage as possible is required, or for studying modifications of specific residues within a protein where trypsin does not yield an appropriate peptide for MS, it is often necessary to use alternative proteases to the commonly used trypsin that cleave at different residues, or even combinations of proteases. Many alternative proteases with orthogonal activities to trypsin have been used successfully in recent years, including chymotrypsin (large hydrophobic), Asp-N (N-terminal to asp), Glu-C (N-terminal to Asp and Glu) and others [8-10]. Selective proteolytic cleavage may also be used to help to identify oxidative modifications; for example, AspN or GluC also cleave at cysteine sulfonic acid and trypsin at aminoethylcysteine [11], and pepsin can be used at low $\mathrm{pH}$, which minimizes disulphide interchange [12]. New digestion methods that may help to minimize sample handling, and thus adventitious oxidation, include in-line digestion where the sample is passed through a column of beads coated with trypsin, which digests the proteins as they flow through $[13,14]$. MS friendly surfactant additives, such as ProteaseMax (Promega) [15,16] and Rapigest (Waters) [17], and on-membrane [18] or in-pellet digestion [15] have all been shown to improve sample digestion efficiency and recovery of peptides, and thus give increased sequence coverage and may improve coverage of modifications; in one study, 1000 S-glutathionylated sites on proteins were identified using in-pellet digestion [19].

\subsection{Enrichment and Separation}

In addition to limiting adventitious oxidation as mentioned in the previous section, it can also be useful to stabilize labile oxidative modifications that are genuinely sample-derived. Moreover, chemical labeling of modifications offers the possibility of enrichment by tag-specific binding systems, thus reducing the complexity of samples and facilitating detection of the oxPTM of interest. Affinity enrichment methods are useful for oxPTMs directly, using antibodies to the modification or chemical tag, or other resin-based capture agents, or based on chemical reactivity (for example thiopropyl sepharose [20]). However, significant care and accurate quantification, as well as appropriate experimental protocols, are necessary to minimise non-specific interactions with the solid support and identify these in the subsequent data analysis [21,22]. Immunoenrichment with antibodies against oxPTMs (for example anti-nitrotyrosine antibodies) has been used to enrich proteins from biological samples [23], although this is not always successful [24] and can introduce 
a high background of immunoglobulins and other proteins into the sample. In addition, the lack of specificity in immunoenrichment exhibited by many antibodies can significantly compromise this approach.

Overall there are many different chemical labeling and enrichment strategies for detecting and quantifying oxidations. The biotin-switch method has been developed to detect reversible cysteine oxidations such as disulfides, sulfenic acids (-SOH) and $S$-nitrosothiols (-SNOs) [25]. The principle is that free thiols are first blocked with an alkylating agent (e.g., iodoacetamide), then the oxidative modifications are selectively reduced; for example, using DTT for disulfides, ascorbate for SNOs or arsenite for sulfenic acids, followed by biotinylation with a thiol-reactive biotin reagent. This allows enrichment by avidin affinity capture [26]. Careful consideration also needs to be given to the protocols for these approaches to ensure residual reducing, oxidising or alkylating agents are properly quenched, or that a significant excess of reagent is used, at each step. The limitations of this approach are the low throughput and difficulty in localising the modification by MS, as ionisation efficiency and peptide fragmentation are often compromised by biotinylation. Recently, some of these issues have been resolved and a quantitative method developed using commercially available iodoacetyl tandem mass tag (iodoTMT) reagents, as described in Section 6 [27]. An alternative method developed for the enrichment of SNOs is the use of organic mercury columns, which involves covalent bond formation between the SNO and mercury. The modified proteins can be digested while bound on the column before elution and MS analysis [28]. Protein carbonyl groups are reactive and can also be labelled by nucleophilic reagents and linked to biotin for enrichment [29]. DNPH is a well-established carbonyl-label and has the advantage that it can act as the matrix for matrix-assisted laser desorption/ionisation (MALDI-) MS, which provides increased specificity and sensitivity for carbonyl-containing peptides and eliminates the need for upstream enrichment. DNPH-labelled peptides can also be analysed with data-dependent acquisition methods with ESI-MS [30]. This technique has recently been applied to a proteome-wide study of protein carbonyl groups generated by mild oxidation; 210 carbonylated proteins were identified with a total of 643 carbonyl locations detected in the HeLa cell proteome [31].

Chemical tagging approaches have also been used to detect the formation of protein bound nitrotyrosine; the initial step is reduction of the nitro group to an amine, which is more amenable to tagging. A wide variety of reduction, labeling and enrichment methods, for example using $N$-succinimidyl-S-acetylthioacetate [32] or dansyl chloride [33], have been reported, and reviewed recently [34]. Usually this approach is reported to give an improvement in selectivity, and indeed enrichment steps have often been regarded as essential. Several of the tags can also be used as reporters in subsequent MS analysis [33].

Developments in LC-separation are also desirable in order to reduce sample loading and improve separation of proteins and peptides. Gel eluted liquid fraction entrapment electrophoresis (GELFrEE) integrates gel electrophoresis separation within reverse phase LC, and eliminates the need for prior electrophoresis and sample processing before injection into the LC [35]. It has been applied to the detection of nitrotyrosine using the increased hydrophilicity of aminotyrosine (formed by reduction of nitrotyrosine with dithionite) and concomitant shift in chromatographic elution of modified peptides on reverse phase HPLC [36]. In contrast, for top-down studies the 
favoured method is Capillary Zonal Electrophoresis (CZE), which allows lower sample loading and has higher separation efficiency than reverse phase HPLC [37,38]. This can facilitate detection of oxidation in complex clinical samples, where protein concentration may be limited.

\subsection{Intact Protein and Top-Down Analysis}

Intact protein analysis, where MS is used to determine the mass of the intact protein and changes in mass can be indicative of modifications to the protein structure, is a well-established approach. Both MALDI and ESI have been used, although ESI is the more common method as it is generally able to give mass accuracies better than 1 in 10,000 on most instruments. This accuracy is due to the protein acquiring more than one charge during ionization, usually many different charges, giving rise to a number of peaks in the spectrum (since MS measures mass-to-charge ratio, each different charge state will give rise to a separate peak in the spectrum) [39]. This set of peaks can be used to help reduce errors in the calculation of the mass, and this has been enhanced further by the ability of high resolution instruments to separate the individual isotopic peaks for even very large proteins, enabling the analysis of larger proteins including antibodies [40] and even protein complexes [41]. However, this multiple charging means that only a limited number of proteins or different protein species can be present in the sample before signals start to overlap and deconvolution becomes more difficult. This method can provide useful information on the total load of modifications on an individual protein molecule [42], and has been applied successfully to detecting methionine oxidation [43], glutathionylated haemoglobin [44] and electrophilic modifications [45], although for small modifications, high-resolution instruments such as Q-TOFs, Orbitraps or FT-ICR MS are really needed for accurate determination of multiple different forms. However, in order to determine the site of modification, either bottom-up or top-down methods are needed. Top-down MS is an emerging platform that involves fragmentation of the intact protein within the mass spectrometer, and analysis of the large fragments produced. It requires high-resolution mass spectrometers and alternative fragmentation technologies that are not available on all instruments. It is currently limited in sensitivity and struggles to deal with complex samples, but has great potential for mapping protein oxidation [46-48]. Most top-down studies have been conducted in vitro with low molecular weight proteins, although more recently a range of $30-80 \mathrm{kDa}$ proteins in a whole cell lysate of $P$. aeruginosa have been analysed [38]. The top-down approach has the advantage of providing additional information on the relative occupancy of oxidation and relationships of oxidised residues to one another in the whole protein $[46,49,50]$. For example, methionine oxidation and nitrotyrosine have been detected and quantified in calmodulin following incubation with lipopolysaccharide (LPS)-activated macrophage lysate [50]. The oxidation of multiple methionine residues has also been quantified using top-down approaches in filgrastim, a granulocyte colony-stimulating factor, to determine the effects of methionine oxidation on biopharmaceutical shelf life [49]. However, despite these reports, the methodology is still some way short of being applied to the detection of protein oxidation in disease. 


\subsection{Bottom-Up Analysis}

Bottom-up proteomics differs from top-down analysis in that the proteins are digested to peptides as mentioned in Section 2.1. Specific labeling and enrichment strategies can be implemented at this stage, as described in the previous section, although label-free methods are more common in standard proteomics. In all bottom-up methods, quantification is restricted to the peptide level, and cannot be used to infer relationships between oxidations on different peptides within an individual protein.

Bottom-up protein analysis is most commonly conducted by LC-MS/MS using either untargeted analysis (often referred to as a shotgun or discovery approach, and described further in Section 3), or semi-targeted/targeted approaches [51], which are described in Section 4. The former is most common, but the limitations of this approach for detecting oxidative modifications lie in the automated selection of the peptides to be fragmented, which tends to be those that give strongest signals in the preliminary MS scan, whereas oxidized peptides are typically present at very low abundance [52]. Hence there has been significant effort recently in developing targeted and semi-targeted methods that depend on scanning for reporter ions in the MS or MS/MS spectra that are diagnostic for the presence of an oxidative modification. This requires some prior knowledge, at least of the fragmentation characteristics of the oxidative modification, and for some methods additionally the specific peptide modified.

These mass spectrometry-based methods can be implemented either in label-free or label-dependent strategies. Label-free approaches are widely used in standard proteomic analysis, and even for analysis of oxidatively modified proteins they have the advantage of less sample manipulation. With regard to identification of oxidative modifications, label-dependent methods usually refer to modification-specific chemical tagging, in contrast to the isotope-labeling techniques used for more generic quantification, although a few studies have combined these methods for quantifying modifications (Section 5). Label-dependent MS approaches often take advantage of reporter ions from the label to indicate the presence of a modification in a peptide, which can then be targeted for further analysis, and are discussed further in Section 4.

\section{Untargeted Mass Spectrometry and "Discovery" Approaches}

A large proportion of proteomics and MS methods are focused on identification and quantification of specific proteins in diverse samples, in order to understand proteomic changes in disease or other conditions. Analysis of oxidative modifications in proteins represents a much smaller field, and although specific methodologies are being developed and utilized, much research is still carried out using untargeted approaches (Figure 2).

\subsection{Use of Search Engines for MS Data and Analysis of oxPTMs}

LC-MS/MS experiments generate very large datasets that are difficult to manually analyse, and consequently many statistical search engines have been recently introduced or further developed for identification of proteins from MS/MS data; some of the most commonly used examples are Mascot, PEAKS, Sequest, ProteinPilot, Tandem, Ommsa and Phenyx [53-56] (Table 1). While 
generally these programmes work very well for identifying proteins, more issues arise when trying to identify oxPTMs, at least using standard parameter settings [57], and some of the advantages and disadvantages that have been observed are identified in Table 1.

Table 1. Comparison of the advantages and disadvantages of the most commonly used search engines for peptide and protein identification.

\begin{tabular}{|c|c|c|c|}
\hline $\begin{array}{l}\text { Search } \\
\text { Engine }\end{array}$ & Method & Advantages & Disadvantages \\
\hline Mascot & $\begin{array}{l}\text { Uses a probability modelling algorithm } \\
\text { and protein database searching. Matches } \\
\text { experimental peptide and fragment ion } \\
\text { masses to ones generated in silico } \\
\text { from databases. }\end{array}$ & $\begin{array}{l}\text { User-friendly interface. Provides } \\
\text { an error-tolerant search facility. } \\
\text { Sophisticated but complex data } \\
\text { export possibilities. }\end{array}$ & $\begin{array}{l}\text { Very reliant on user input for } \\
\text { correct identification of } \\
\text { oxPTMs, otherwise false } \\
\text { positives and negatives occur. }\end{array}$ \\
\hline Sequest & $\begin{array}{l}\text { Uses an algorithm based on a cross } \\
\text { correlation function, plus protein data base } \\
\text { searching. Matches experimental peptide } \\
\text { and fragment ion masses to ones generated } \\
\text { in silico from databases. }\end{array}$ & $\begin{array}{l}\text { User-friendly interface. Provides } \\
\text { an error-tolerant search facility. }\end{array}$ & $\begin{array}{l}\text { Very reliant on user input for } \\
\text { correct identification of } \\
\text { oxPTMs, otherwise false } \\
\text { positives and negatives occur. }\end{array}$ \\
\hline ProteinPilot & $\begin{array}{l}\text { Uses a sequence tag method plus protein } \\
\text { database searching. }\end{array}$ & $\begin{array}{l}\text { User-friendly interface. Potentially } \\
\text { better at identifying unsuspected } \\
\text { modifications. }\end{array}$ & $\begin{array}{l}\text { If the initial sequence tag is } \\
\text { incorrectly identified, the } \\
\text { experimental peptide will not be } \\
\text { matched to the correct peptide. } \\
\text { Long analysis run times. }\end{array}$ \\
\hline pMatch & $\begin{array}{l}\text { Spectral library searching against } \\
\text { experimentally-derived data. }\end{array}$ & $\begin{array}{l}\text { Has been reported to be better } \\
\text { at identifying PTMs, and } \\
\text { specifically at coping with the } \\
\text { unusual fragmentation of } \\
\text { peptides caused by PTMs. }\end{array}$ & $\begin{array}{l}\text { Since this method uses a } \\
\text { spectral library, the peptide } \\
\text { will only be identified if the } \\
\text { spectra are available in the } \\
\text { spectral library. }\end{array}$ \\
\hline $\begin{array}{c}\text { MS } \\
\text { Amanda }\end{array}$ & $\begin{array}{l}\text { Based on a binomial distribution function. } \\
\text { Protein data base searching. Matches } \\
\text { experimental peptide and fragment ion } \\
\text { masses to ones generated in silico } \\
\text { from databases. }\end{array}$ & $\begin{array}{l}\text { Reported to be better at identifying } \\
\text { peptides of higher } m / z \text { than } \\
\text { Mascot and Sequest. }\end{array}$ & $\begin{array}{l}\text { Very reliant on user input for } \\
\text { correct identification of } \\
\text { oxPTMs, otherwise false } \\
\text { positives and negatives occur. }\end{array}$ \\
\hline
\end{tabular}

Many search engines offer the possibility of including a wide variety of oxPTMs as variable modifications [58], but the number of modifications that can be searched in parallel is usually limited to 3-4 to minimize false positive identifications [59], which can be limiting when heterogeneous oxidation has occurred. oxPTMs also add additional complications to searching data. For example, the spectrum of a peptide containing methionine sulfoxide will include a neutral loss of $64 \mathrm{Da}$ $\left(-\mathrm{CH}_{3} \mathrm{SOH}\right)$, which complicates interpretation of the spectrum and sequencing, although this can be improved using alternative fragmentation methods [60]. Protein Pilot works in a different way to Mascot, Sequest and MS Amanda [61], and is less affected by these constraints, so may have advantages for the analysis of complex oxPTMs. It is based on the Paragon algorithm and uses small sequence tags generated by de novo sequence analysis of parts of the experimental data. The 
sequence tag is searched against a protein database and any sequence in the database that matches the sequence tag is investigated for a fit to the experimental data set, in an iterative approach. All possible PTMs are allowed for in the error-tolerant mode. This has the advantage of being a nonstatistical method in which a sequence can be constructed with the inclusion of a wide variety of oxPTMs, but if the initial sequence tag was incorrectly identified, the final peptide will also be incorrect, and the large search space results in a time-consuming process. Mascot also incorporates an error-tolerant search function that has been substantially developed in recent releases, and does not require a list of anticipated modifications; in this mode all possible PTMs are tested against the theoretical peptide and fragment ion masses, and the PMT that gives the best match to the experimental data is reported as a match. Again, this increases the search time and tends also to increase the false positive rate.

A new search engine, MS Amanda, has been specifically designed for high resolution instruments; it has a different scoring function for identification of the peptides and has been reported to identify many more peptides than both Mascot and Sequest, including ones with multiple modifications [62]. The resulting increase in sequence coverage could help in identification of oxPTMs, although the algorithm is still limited by the same issues. An entirely different approach to identifying peptides that is gaining popularity and may help to overcome the limitations of searching for oxPTMs involves spectral library searching using a search tool such as pMatch [63]. This method compares the experimental MS data with previously acquired spectra in a spectral library using an open search. This allows the search engine to search for unknown and unspecified modifications, but depends on them being present and correctly identified in the library.

While statistical software is often used to detect oxidative modifications, comparisons of the results from different search engines are rarely performed. Dorfer et al. compared the ability of several search engines to identify proteins and post translation modifications [62], while Moskovitz used three search engines to detect and localize methionine oxidations [64]. In both instances validation by manual de novo sequencing was not performed; this makes it difficult to determine which search engine is the most reliable for determining the presence and localisation of oxidative modifications.

\subsection{The Importance of Data Validation}

In view of the potential pitfalls described above, it is essential to validate the MS/MS data by de novo sequencing to demonstrate both the presence and location of the modification in the sequence (Figure 3), despite it being time-consuming. This process has been reviewed previously [65] and guides to support different levels of expertise are available, e.g., [66]. The software packages to facilitate de novo sequencing are continually being developed, such as computer aided manual validation software (CAMV), which is compatible with iTRAQ-labeling quantification experiments and has been reported to remove approximately $10 \%$ of false positives [67]. Other software packages that aid in manual validation include PepNovo ${ }^{+}$, PEAKS, pNovo, MS-GFDB. UniNovo is reported to be best for manual validation of Orbitrap MS data [68]. Open source software to improve the user interface of packages such as $\mathrm{PepNovo}^{+}$is also available [69]. A new approach to de novo sequencing by combining data from bottom-up and top-down MS approaches has recently been reported to achieve high sequence coverage and accuracy [70]. These tools are important, as more 
widespread use of de novo sequencing to validate oxPTMs is needed to ensure that correct relationships between oxPTMs and disease are being deciphered.

\section{a) oxPTM identification from search engine}

\section{MS/MS Fragmentation of GQSIDDMIPAQK}

Found in KCRM_MOUSE in SwissProt, Creatine kinase M-type OS=Mus musculus

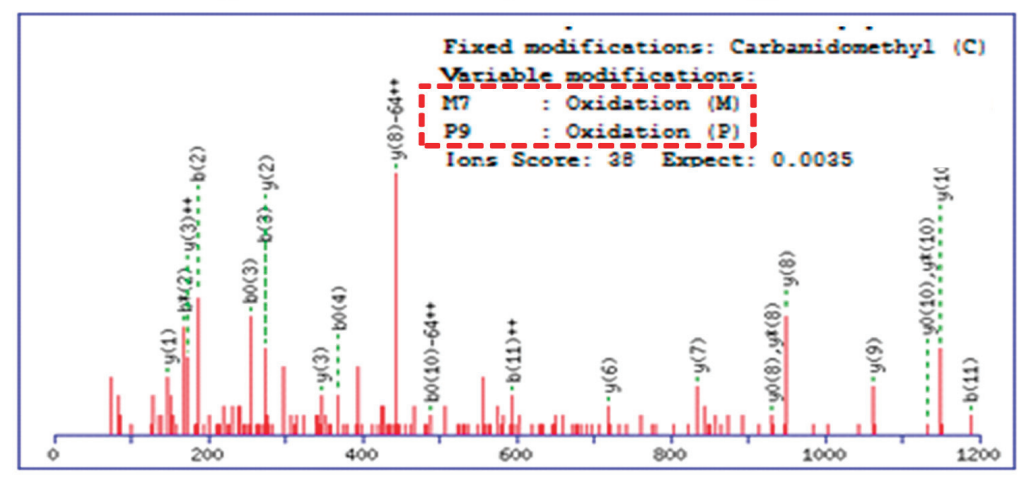

\section{b) oxPTM identification from de novo sequencing}

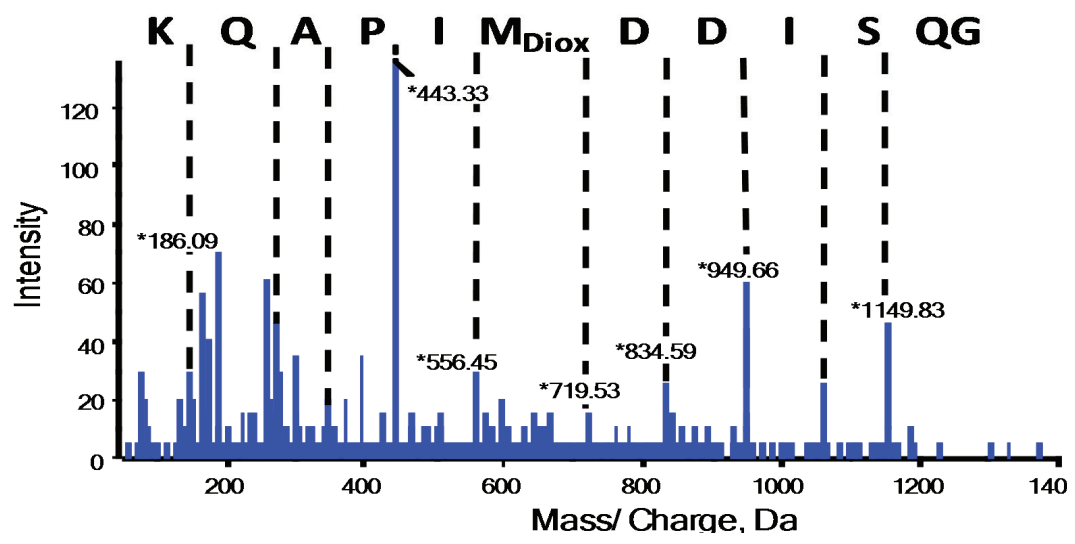

Figure 3. Incorrectly assigned oxidation to proline using a probability-based search engine. (a) Search engine identified 2 modifications on one peptide: methionine-7 mono-oxidation and proline-9 oxidation; (b) de novo sequencing showed that methionine is dioxidised.

\section{Reporter Ion-Based Methodologies}

The term reporter ion refers to the formation of ions that are diagnostic for the specific analyte or type of analyte of interest, usually product ions from the fragmentation of the peptide. Reporter ions have been used in label-free analysis where a sufficiently unique fragment of an oxidized residue has been identified; alternatively, several oxidation-specific chemical probes that are MS compatible and give diagnostic fragmentations have also been developed. In semi-targeted methods, 
the fragmentation products are diagnostic but the precursors are unknown (Sections 4.1 and 4.2), whereas in fully targeted routines both the precursor ion and fragment ions are used as reporters (Section 4.3). In all of these approaches, specificity for oxPTMs is improved compared to untargeted analysis and relative quantification can be achieved using the relevant precursor ions for oxidized and native peptides.

\subsection{Semi-Targeted MS/MS Analysis}

Neutral loss scanning (NL) and precursor ion scanning (PIS) are two MS/MS routines that enable classes of molecular ions to be identified based on a structural feature with a characteristic fragmentation pattern. In precursor ion scanning the second analyser is fixed to detect a specific fragment ion and scans for precursor ions that generate this product ion upon fragmentation. For analysis of oxidized peptides, immonium ions from oxidized residues have been the most commonly reported reporter. For example, there have been several reports of the use of the nitrotyosine immonium ion at $m / z 181.1$ [71], and chlorotyrosine ( $m / z 170.1)$, hydroxytyrosine $(\mathrm{m} / \mathrm{z} 152.1 \mathrm{Da})$, and hydroxytryptophan $(m / z 175.1 \mathrm{Da})$ have also been tested [42]. However, for each of these isobaric ions from fragmentation of non-modified peptides, false positives have been reported; this is particularly a problem on low-resolution instruments where the interfering ions are not resolved from the target fragment ion $[52,59,72]$. One approach developed to overcome this problem is a further fragmentation step to yield a more unique combination of MS/MS and $\mathrm{MS}^{3}$ (MS/MS/MS, a further fragmentation of a selected ion generated in the MS/MS analysis) diagnostic ions; this has been reported for chlorotyrosine, nitrotyrosine, hydroxytyrosine and hydroxytryptophan in model proteins and cell lysates using an indirect scanning routine [42,73]. Greater specificity can also be obtained using higher-resolution instruments, as has been reported for nitrotyrosine [73,74].

In neutral loss scanning, the diagnostic fragment is uncharged and is monitored by scanning in both analysers with a mass offset corresponding to the mass of the fragment. This methodology has been used to identify the presence of oxidized methionine, which has a characteristic neutral loss of 64 $\mathrm{Da}$ (corresponding to methanesulfenic acid, $\mathrm{CH}_{3} \mathrm{SOH}$ ) [75], and has been applied to measure in oxidation of calmodulin [76]. Oxidized cysteine residues fragment in a similar way with different neutral losses depending on the extent of modification, as reviewed recently [2]. Schiff base and Michael adducts of 4-hydroxynonenal with nucleophilic residues can also be monitored by neutral losses of $138 \mathrm{Da}$ and $156 \mathrm{Da}$ respectively, and has been demonstrated in plasma proteins [77].

In addition to these label-free semi-targeted methods, label-dependent approaches have been reported. For example, carbonyl-containing proteins or peptides can be labeled with DNPH to form hydrazone adducts, which can be analysed in negative ion mode based on a precursor-like scan for diagnostic fragments at $m / z 152.0,163.1$ and 179.0 [30]. One of the advantages of this technique is the elimination of the need for upstream enrichment during sample preparation, as demonstrated in its application to analysis of oxidized proteins in bovine serum albumin and extracts of HeLa cells [30]. 


\subsection{Narrow-Window Extracted Ion Chromatograms}

An alternative strategy that has been developed recently as a result of the increasing availability of high-resolution mass spectrometers involves generating extracted ion chromatograms (XICs) of accurate mass reporter ions from MS/MS data [73]. One study used this technique to quantify the levels of $S$-glutathionylation in haemoglobin F subunits, as evidence of oxidative stress in premature infants [44]. However, this strategy has limitations in complex samples where the likelihood of isobaric peptides is higher. Alternatively, XICs of diagnostic product ions can be used to mine data for oxidative modifications. Recently, this approach was utilized for reporter fragments of nitrotyrosine, chlorotyrosine, allysine and for adducts of oxidised phospholipids with proteins [73]. The use of a very narrow mass window $(0.05 \mathrm{Da})$ extracted ion chromatogram allowed exclusion of many false positive signals from isobaric ions. An advantage of this method is that existing data can be mined retrospectively for other modifications, as long as a unique reporter ion can be identified, but a disadvantage is that it involves significant manual processing [59,73].

\subsection{Targeted Methods of Analysis}

Fully-targeted MS/MS approaches involve two related techniques: single reaction monitoring (SRM) and multiple reaction monitoring (MRM), where both the precursor ion and product ion masses are fixed for the analyte of interest [78]. OxMRM, which combines MRM with protein purification and labeling of oxidised cysteine residues with isotope labeled $N$-ethylmaleimide, has been reported to improve sensitivity [79]. Although these targeted approaches are not a discovery strategy as prior knowledge of analytes is required, they represent the most accurate available MS-based quantification tool and can be conveniently used in hypothesis-driven studies upon optimization of chromatographic and mass spectrometric features; further developments of the rapidly developing PeptideAtlas to include modifications may greatly extend their utility [80].

\section{Quantification of (ox)PTMs}

In order to obtain meaningful data on protein oxidation in biological or clinical samples, it is crucial to be able to obtain accurate quantitative information about the oxPTMs and their relative abundance both within and between samples. Quantitative proteomics strategies can be generally divided into label-free and label-based approaches. Label-free techniques rely on comparisons of the abundance of the analyte ion intensities directly, with appropriate normalization, whereas label-based approaches rely on metabolic or chemical labelling of samples with differentially stable isotope labelled reagents and comparison of the ion intensities from these.

\subsection{Label-Free Methods of Quantification}

Label-free methods are becoming the most popular for relative quantification, as they are relatively easy to implement and a number of free, open source software packages are available for analysis. However, label-free strategies also need to be used with care when analysing oxPTMs, as these modifications will affect both peptide ionisation efficiency and MS/MS fragmentation pattern, 
complicating any comparative analysis. Hence, great care needs to be taken in comparing ion intensities between any given peptide and its modified form, especially when the modification removes (e.g., lysine to $\alpha$-aminoadipic semialdehyde) or introduces (e.g., cysteine to cysteine sulfonic acid) ionizable groups, or alters polar residue composition; using this approach to determine a percentage modification can only be semi-quantitative at best, as changes can be very marked. For example, we recently reported a 2.6 fold increase in relative signal intensity on nitration of a peptide [81]. Using the loss of the native peptide ion intensity could be an alternative, but only where there is significant modification, as quantification accuracy is rarely better than $10 \%$. The use of tags that improve ionization, for example the iTRAQ (isobaric tags for relative and absolute quantification) label discussed below, may help to improve this, although relative quantification of the same modified peptide between samples generated under different conditions, or using absolute quantification with a labeled peptide such as in the "protein-AQUA" strategy [82] also discussed below, are the only reliable methods.

The two fundamental strategies currently used in label free quantification are spectral counting and feature-based quantification. The different methods have been reviewed elsewhere [83]. Methods based on spectral counting rely on the number of identified MS/MS spectra corresponding to a given protein as a measure of protein relative abundance. While spectral counting has been used effectively in investigations of protein expression changes, including those induced by oxidative stress $[84,85]$, it is focused on protein-level quantification and is not well suited for the specific analysis of oxPTMs (or many other PTMs) due to their often relatively low stoichiometry and abundance. Feature-based quantification methods rely on the comparison of summed peak intensities for each peptide in each LC-MS run, following software alignment the different LC-MS runs so that the same features are aligned in each data set. With the increasing interest in label-free methods, a new generation of software solutions capable of processing large amount of high resolution data have recently become available, including Progenesis QI (Non Linear Dynamics, Newcastle upon Tyne, UK), msInspect/AMT [86], MAxQuant [87], Rosetta Elucidator (Rosetta Biosoftware, Seattle, WA, USA), OpenMS [88] and Superhirn [89]. Although the use of label-free analysis for biomarker discovery in biological samples has been reported [90], few studies have reported the use of label-free software based methods for the quantitative determination of specific oxPTMs. As for spectral counting, a limitation has been that the methods are generally focused on protein-level quantification, and the identification and quantification of individual PTMs has been challenging; however, this is improving, and the latest versions of many of the programmes now incorporate specific methods for highlighting PTMs. In one recent study, reversibly oxidized cysteines in the membrane proteins of human erythrocytes have been quantified using a robust computational software-based approach and validated by matching the modified peptides against Protein Data Bank entries [91].

\subsection{Label-Dependent Methods of Quantification}

Label-dependent methods rely on the incorporation of isotope labels into the peptides prior to mass spectrometry analysis. Isotope labels can be introduced at various stages of the experimental 
workflow, depending on type of sample and MS approach. The following section will concentrate on methods specific for oxPTMs.

The use of chemical or enzymatic methods to incorporate the isotopic label after protein digestion has been implemented effectively in a wide range of studies to detect and quantify oxPTMs. One of the first was ICAT (isotope coded affinity tags), and its cleavable version cICAT, which are commercially available cysteine-specific tags based on an iodoacetamide (IAM)-based thiol-reactive group, and also carry an affinity tag for the enrichment of tagged peptides. ICAT has been effectively used to quantify evidence of cysteine oxidation in complex protein mixtures [92,93]. It has the advantage that enrichment can improve the depth of the analysis, but a significant disadvantage is that the presence of the ICAT tag can affect the quality of MS/MS data [94]. Other reagents have been recently developed for cysteine oxidation analysis. Isotope-labeled $N$-ethylmaleimide (NEM) has been used in a targeted MS approach to monitor the redox status of reversibly oxidized cysteines and the detection and analysis of cysteine disulfide bonds [95], and IAM based strategies are now being further developed for the detection and quantification of protein $S$-nitrosothiols (SNOs, recently reviewed in [92]). The recently commercially available iodoacetyl tandem mass tag (iodoTMT) six-plex reagent has been used for MS identification and quantification of SNOs [27,96], as well as other cysteine oxidations such as glutathionylation, nitrosoglutathione, and disulfides [96]. The TMT isobaric tags have been adapted recently for the comparison of the relative abundance or cysteine site occupancy by SNOs and sulfenic acids [97], and exploited for the detection of SNOs in LPS-stimulated microglial cells [27].

The use of iTRAQ, which labels primary amino groups and was developed for general quantification studies, has been extended for analysis of oxPTMs [19]. In combination with NEM-based thiol-blockade, iTRAQ has been used to identify the redox-sensitive reversibly-oxidized cysteines in proteins and to quantitatively assess the oxidation states of individual cysteine residues [98]. iTRAQ has recently been modified to detect other oxPTMs including protein carbonylation [99] and to selectively label and quantify 3-nitrotyrosine, both alone and in combination with precursor isotopic labeling [100]. Promising results in the detection of other oxPTMs have also been generated using specific enzymatic reactions to place the isotope tag at specific amino acid groups. For example, enzyme-catalysed $\mathrm{O}^{18}$-based labeling has been successfully used for accurate quantification of oxidized methionine [101].

An extension of label-dependent methods is absolute rather than relative quantification, which can be particularly valuable for clinical biomarker analysis. The most commonly used method is AQUA [82], where a stable isotope-labeled version of the peptide of interest is synthesized and used as an internal standard, but this has not yet been applied to oxPTMs. iTRAQ-labeled internal standards have also been recently used in combination with targeted MS approaches to quantify evidence of proteolytic post translational modifications such as proteolytic cleavage [102] or phosphorylation, but again this method has yet to be applied in oxPTM analysis.

\section{Applications in Vivo and in Disease}

OxPTMs can be classified either as reversible modifications, most commonly the lower oxo-forms of cysteine and methionine, or irreversible modifications, including cysteine sulfonic 
acid, methionine sulfone, and most oxidation products of other residues. The reversible oxPTMs have generated much interest, as evidence is emerging for their role in redox signaling [2]. An increasing number of proteins have been found to be regulated by reversible oxidation of cysteine to sulfenate and disulfide forms $[103,104]$, and this has been shown to contribute to physiological control of signaling pathways governing cell fate, such as apoptosis, proliferation or inflammatory processes [105]. Some of the best known examples include protein tyrosine phosphatases such as PTP1B, apoptosis signal-regulating kinase (ASK-1), caspases and peroxiredoxin [106,107]. Other more recently discovered redox-regulated proteins include the nuclear signalling protein HMGB1 [108] and Hsp33 [109]. These enzymes contain thiolates that are particularly susceptible to oxidation by hydrogen peroxide, which can be generated for example by NADPH oxidases following activation of growth factor or other receptors. The role of SNOs in enzyme regulation and signalling is also gaining recognition [110], as in studies on mitochondrial complex I [111]. Interestingly, there is growing support for the concept that tyrosine nitration has a role to play in protein redox signaling [112,113]. While often these are normal, physiological processes, there is also evidence that they can be dysregulated in disease or aging, and there have been some excellent reviews on this topic recently $[103,114]$, including the application of mass spectrometry to support these studies $[113,115]$. Consequently, the following sections focus instead on examples of stable and irreversible modifications to proteins in specific diseases and their potential as biomarkers.

\subsection{Considerations for Clinical Sample Type in oxPTM Analysis}

Despite advances in technology, the determination of oxPTMs in biological and clinical samples remains challenging owing to sample complexity, low abundance of the modifications, and potential for adventitious oxidation [116]. The low abundance of modifications often encountered in vivo means that many studies are initiated by in vitro analysis of highly modified proteins. These often bear little relationship to the low levels of oxidative modification encountered in clinical samples (e.g., nitration [117]), which means they are relatively poor models for physiological protein modification. This is compounded by the poor quantification of some methods, for example in carbonyl and glycation analysis [116,118,119].

The type and abundance of oxPTMs is dependent on the sample type. The main sources of clinical samples for proteomics are body fluids and tissue extracts. Urine and blood are by far the most widely studied fluids, owing to the relative ease of their acquisition. Although urine can be obtained non-invasively in large volumes and is known to contain a more than 1500 different proteins [120], their concentration is too low for routine detection of oxPTMs. Consequently, there have been more studies of free oxidized amino acids as markers of protein oxidation. Additionally, collection urine is more susceptible to adventitious oxidation during the excretory process. Another non-invasive biological material is exhaled breath condensate, which contains a variety of proteins and has potential for early diagnosis of lung cancer [121]. In asthma patients, exhaled breath condensate has been found by targeted mass spectrometry-based methods to contain free 3-nitrotyrosine $[122,123]$. Plasma is a better source of concentrated proteins (more than 490 proteins have been resolved [124]), and abundant plasma proteins such as albumin [125] and fibrinogen [126] are often investigated. Protein analysis can be achieved using very small volumes of blood, for example from pinpricks, 
especially if combined with novel approaches such as paper-spray mass spectrometry [127], although this has not as yet been applied to oxPTMs.

A limitation of plasma is that it reports on the systemic status rather than being disease or organ-specific; consequently, it can be desirable to study protein oxidation in other body fluids. For example, cerebrospinal fluids have been used to detect products of protein oxidation in Alzheimer's disease patients $[128,129]$, and synovial fluids have been used for the detection of free and protein-bound 3 nitrotyrosine in osteoarthritis [130]. Protein oxidation has also been detected in saliva, seminal fluid, and amniotic fluid [131-133]. Ultimately, information about protein damage in organs requires the use of tissue biopsies to assess the local level of oxidation. Mass spectrometry-based procedures have been used on tissue biopsies of tumours [134] and virus-infected tissues [135], and oxidized proteins have been reported in surgical biopsies of diseased human tissues such as heart [136] and brain [137] tissue. Even with the small sample amounts obtained by needle biopsies, modern approaches and high-resolution instruments can profile proteins [138]. MALDI imaging has recently been used for proteomic analysis of needle-core biopsied human pancreatic tumour tissue spotted on microarrays, and evidence of protein oxidation was reported [139].

\subsection{MS Analysis of Protein Oxidation in Disease}

A major driver for analysing protein oxidation in biological or human samples is to determine their importance in disease [140]. This has two potential benefits: an improved understanding of their role or mechanism in the pathological condition, and the identification of improved biomarkers for diagnosis. Especially for development of clinical biomarkers, much research has been done on the analysis of oxidatively modified amino acids, such free nitrotyrosine or chlorotyrosine, oxidized tryptophan products, advanced glycation endproducts (AGEs), lipoxidation adducts, and thiol-containing compounds, and many well-established targeted MS methods are available [141-149]. Although these methods are very useful for gaining an overview of global oxidative damage, they do not yield information on the target proteins that have been modified or localize the modification on the protein. The desire for greater mechanistic insight has led to the development of the MS methods described in the previous sections, and in recent years the application of both label-free and label-dependent mass spectrometry methods to clinical analysis has grown exponentially.

This section will summarize important findings and provide an update on the analysis of oxidized proteins in disease. In some studies, elevated levels of oxidized proteins and oxPTMs in disease were observed, suggesting their potential as biomarkers, and these findings are summarized in Table 2. Although protein identification data for the protein-bound oxPTM(s) detected is provided in all these articles, it is important to note that not all of them report site-specific information about the modifications, and this limits the confidence of the oxPTM analysis. The oxPTMs that have most often been associated with human disease onset are protein carbonyls, 3-nitrotyrosine, 3-chlorotyrosine, dityrosine, cysteic acid, cysteine disulfide bonds, cysteine $S$-glutathionylation, cysteine $S$-nitrosylation, methionine sulfoxide and methionine sulfone. 


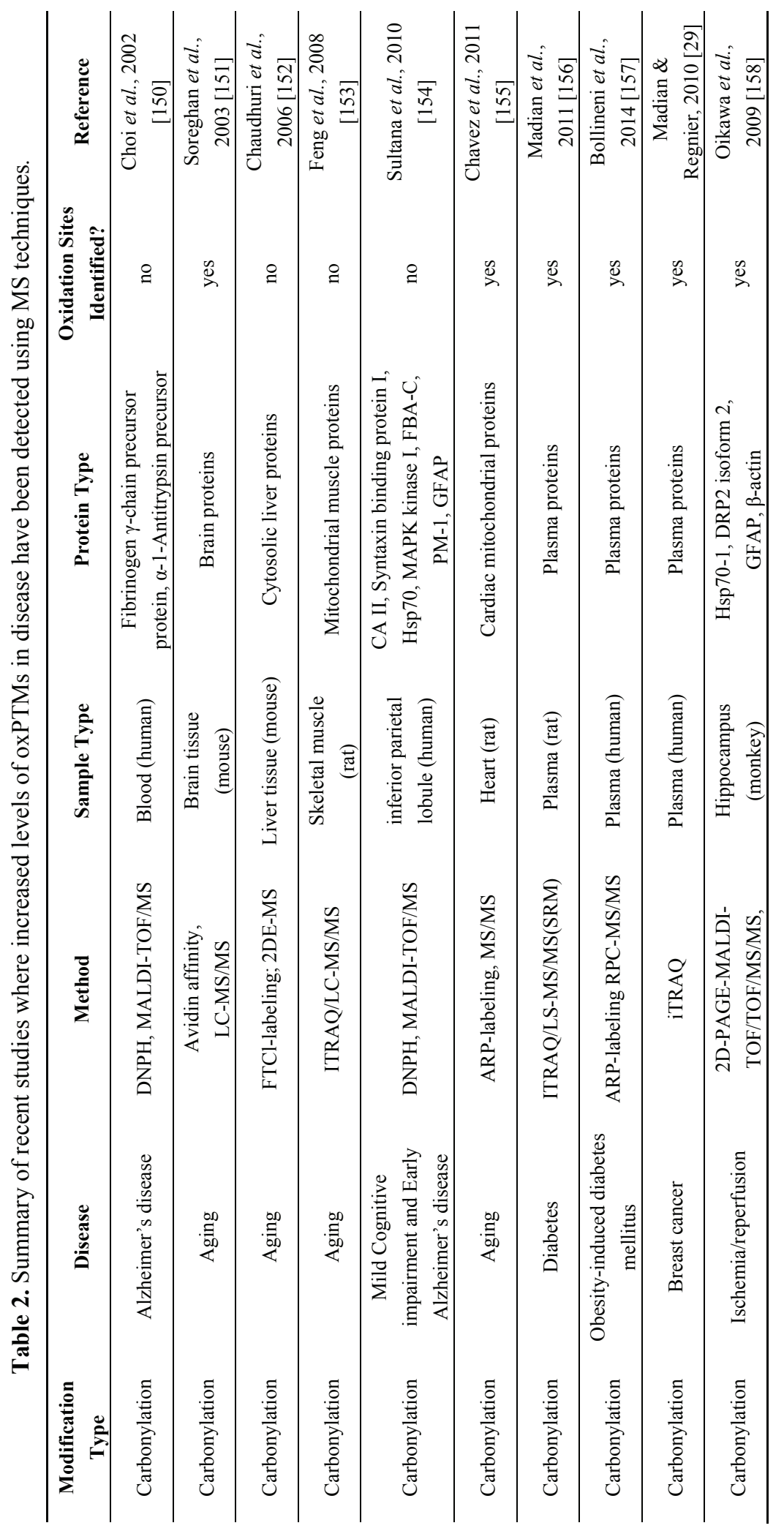




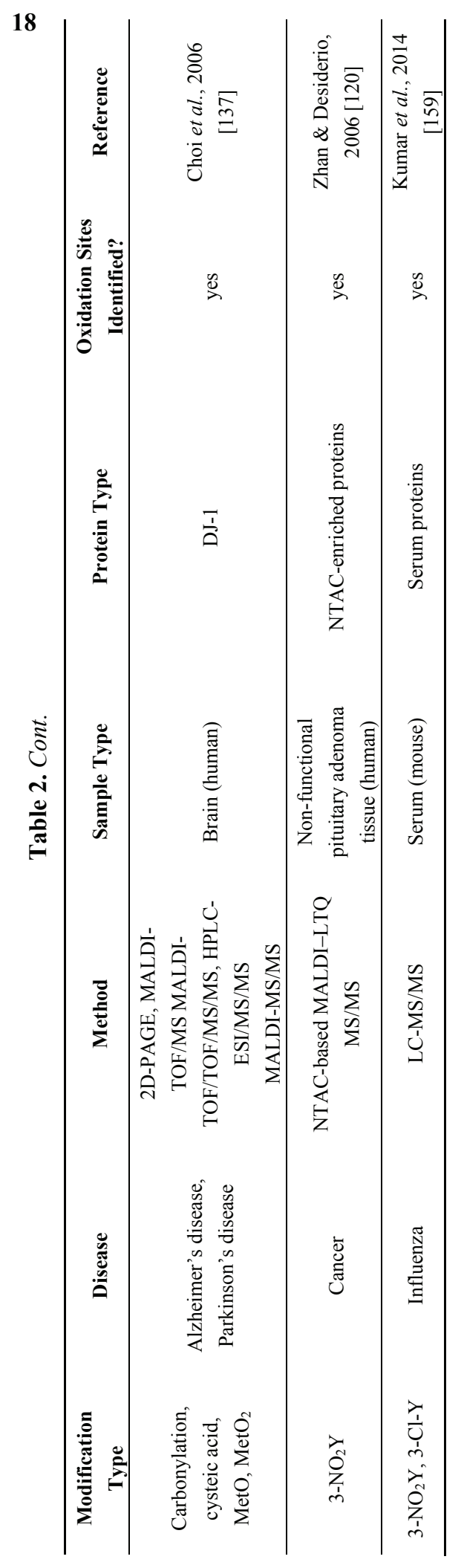


Protein carbonyl formation is one of the most studied and well-established markers of oxidative stress-related human diseases [160]; usually chemical tagging for enrichment is used, as described in Section 2.2. Many clinical and disease-related investigations used untargeted MS or MS/MS methods to analyse gel spots from 2D-electrophoresis of DNPH-derivatised proteins techniques; this identifies the proteins present in gel spots that have been identified as carbonyl-containing by immuno-staining, but it is important to remember that unless the modification has been localized on the proteins of interest by MS/MS analysis and ideally by de novo sequencing, the identification of carbonyl-modified proteins is tentative. Using such approaches, evidence of increased levels of protein carbonyls have been detected in tissues from patients Alzheimer's disease [132,150,154,161], and in aged rat skeletal muscle with quantification by iTRAQ based-methods [153].

In other studies, the DNPH label or other chemical tag has been further utilized for targeted MS/MS analysis. For example, protein carbonylation sites have been determined and validated in rat cardiac mitochondrial proteins using aldehyde/keto reactive probes (ARP) and avidin-based affinity enrichment coupled with LC-MS/MS [155]. The methodology was subsequently applied to study adducts of reactive lipid aldehydes in hearts of young and old rats, and interestingly the level of hydroxyhexanal-modified proteins was higher in mitochondria from young animals, in line with the concept that these mitochondria contain higher levels of omega-3 (n-3) fatty acids. On the other hand, the location and increased levels of carbonyls have been reported in proteins of aged mouse brain [151]. Ischaemia/reperfusion is known to cause oxidative stress, and increased carbonyl modification of Hsp70 and several neuron-specific proteins have been observed in monkey hippocampus [158]. Bollineni et al. used the carbonyl-reactive probe $O$-(biotinylcarbazoylmethyl)hydroxylamine followed by avidin affinity chromatography to demonstrate differences in the profiles of carbonyl-containing proteins in plasma of obese subjects and patients with type 2 diabetes [157]. The carbonyl status of 35 different proteins has also been mapped in diabetic rat plasma, and was found to increase significantly in 11 of them [156]. This group also investigated carbonyl-containing proteins in plasma of breast cancer patients and found that they were strongly associated with the breast cancer type-1 susceptibility protein Brca1 [162]. These studies built on a high through-put methodology incorporating carbonyl-labeling and iTRAQ for quantifying protein carbonyl analysis in human plasma [29].

The redox processes of cysteine, both reversible and irreversible, are also of emerging clinical relevance $[163,164]$. Reversible cysteine oxidation has been found using proteomics approaches in the skeletal muscle of aged rats [165]. Untargeted MS/MS approaches have been used to provide evidence of irreversible cysteine oxidation in different proteins in brain tissue of patients with Alzheimer's or Parkinson's diseases $[137,161,166]$. Cysteine SNOs have been also linked to aging and Alzheimer's disease, according to a proteomics study on human brain samples [167]. Interestingly, cysteine SNO formation has been reported in mouse models of ischaemia/reperfusion injury using SNO-RAC ( $S$-nitrosothiols resin affinity capture) in combination with label-free based quantification [168]. Ischemia/reperfusion was also found to cause reversible oxidation of cysteine in heart tissue of mice using Redox-ICAT for quantification by MS/MS [169]. A similar approach 
has been used to study redox switches in liver mitochondrial protein samples during cadmium toxicity in rats [170].

Methionine oxidation has been much less studied than cysteine oxidation, but evidence is emerging for links to a number of human pathologies, including Alzheimer's [171] and Parkinson's diseases [172]. Protein-bound methionine sulfoxide (MetO) was found to be elevated in the plasma of diabetic patients [173] as well as in the brain tissues of patients affected by Alzheimer's and Parkinson's disease.

Oxidatively modified tyrosines have also been proposed as consistent biomarker of several inflammatory and chronic human pathologies [174]. One of the most studied markers of peroxynitrite-mediated damage in MS-based studies is 3-nitrotyrosine. As with protein carbonyl formation, many studies have utilized anti-nitrotyrosine antibodies for immunoblotting of 2D gels before analysis of gel spots by MALDI peptide fingerprinting or untargeted MS/MS methods, and the same limitations apply. In this way, elevated levels of protein-bound 3-nitrotyrosine have been detected in proteins from brain tissue of Alzheimer's disease patients [175] and in serum and colon during inflammatory bowel disease [176]. Using more rigorous MS approaches, sites of nitrotyrosine formation were identified on high density lipoprotein (HDL) and found to be increased during atherosclerosis [177]. Site-specific signatures of nitrotyrosine and chlorotyrosine in HDL by neutrophil extracellular trap enzymes have been observed in systemic lupus erythematosus (SLE) [178]. In human pituitary non-functional adenoma, nine nitro-proteins were identified using a nitrotyrosine affinity column (NTAC); the nitration sites were localized to functional domains of the proteins and it was suggested that might contribute to pathogenesis [120]. Interestingly, using MS-based strategies elevated levels of protein-bound 3-chlorotyrosine have been recently detected in mouse models of influenza [159], as well as in the clinical samples of inflammatory bowel disease [176], atherosclerosis [179], SLE [178] and post-myocardial infarction [180], providing evidence for the formation of chlorinating species in these inflammatory conditions.

\section{Conclusions and Perspectives}

Oxidative modifications of proteins and the regulation of signalling by oxPTMs are highly topical areas of increasingly recognized importance in biomedical science, and the increased levels of several oxPTMs in inflammatory diseases offer potential as biomarkers for the development of new diagnostics. It is clear that MS-based strategies have greatly underpinned the increase in knowledge in this area, and are confidently expected to continue to do so. The chemical enrichment and labelling approaches, together with the advanced MS/MS routines described, provide very powerful though time-consuming tools for investigating the relationships between specific oxidative modifications of proteins and mechanisms of disease pathology. There are many advances that are also helping to provide new information. MS imaging promises to be able to provide MS-based histology for mapping oxidative modifications across tissues, and although it has been used for mapping oxidised lipids [181], it has not yet been used to any significant degree for proteins. The availability, albeit at significant cost, of stable isotope-labelled animals (e.g., stable isotope labelling in mammals; SILAM) [182,183] may also provide a powerful tool for studying systemic or tissue specific oxidative stress and signalling. The use of genetic knockouts is a well-established method for 
unlocking cellular biochemical mechanisms and their roles in disease, and with the introduction of new technologies such as CRISPRi [184], it is set to become one of the key technologies for studies on pathology and for both mechanistic studies and validation. This has not yet been as widely exploited in the redox field as in others, or for studying redox biology in mammals as much as in plants, but promising results have been obtained from a range of studies (e.g., [185-187]). Kinetic and systems modelling has become well established in systems biology, and this is also now being applied to redox studies using MS and other data to build dynamic and predictive models that can help to understand the underlying biological processes complex regulatory dynamics of steady-state levels of protein oxidation [188].

However, there are still many challenges. It is essential to understand that analysis of oxPTMs involves non-standard proteomics methodology, and an important message of this review is that there are many potential pitfalls in the analysis of MS/MS data, which can lead to erroneous identifications of oxPTMs and conclusions. Consequently, it is essential to understand the requirements and limitations of the techniques used, and select appropriate approaches to address the research question. Although novel methods continue to be developed, their translation to early diagnosis tools for clinical settings continues to be difficult, owing to factors such as lack of well-established validation protocols for oxPTMs, the wide variety of methodologies, and complex data analysis [189]. In the meantime, the scientific community will continue to benefit from the advances in methodology and applications described in this article.

\section{Acknowledgments}

Corinne M. Spickett, Andrew R. Pitt and Karina Tveen Jensen would like to acknowledge the Proxomics Project funded by the Engineering and Physical Sciences Research Council, UK, EP/I017887/1 Cross-Disciplinary Research Landscape Award.

\section{Author Contributions}

Ivan Verrastro, Sabah Pasha and Karina Tveen Jensen carried out most of the literature searches, wrote the first drafts of the manuscript, and generated the tables and Figures 2 and 3. Corinne Spickett and Andrew Pitt were responsible for preparing the final versions of the manuscript and Figure 1.

\section{Abbreviations}

DNPH

DTT

ESI

HDL

HPLC

ICAT

iTRAQ

LC-MS or LC-MS/MS 2,4-dinitrophenylhydrazine

dithiotreitol

electrospray ionization

high density lipoprotein

high performance liquid chromatography

isotope coded affinity tags

isobaric tags for relative and absolute quantification

Liquid chromatography coupled to mass spectrometry

or tandem mass spectrometry 


$\begin{array}{ll}\text { LPS } & \text { lipopolysaccharide } \\ \text { MALDI } & \text { matrix-assisted laser desorption/ionisation } \\ \text { MRM } & \text { multiple reaction monitoring } \\ \text { MS } & \text { mass spectrometry } \\ \text { MS/MS } & \text { tandem mass spectrometry } \\ \text { oxPTM } & \text { oxidative post-translational modification } \\ \text { PTM } & \text { post-translational modification } \\ \text { SLE } & \text { systemic lupus erythematosus } \\ \text { SNO } & \text { S-nitrosothiol } \\ \text { XIC } & \text { extracted ion chromatogram }\end{array}$

\section{Conflicts of Interest}

The authors declare no conflict of interest.

\section{References}

1. Droge, W. Free radicals in the physiological control of cell function. Physiol. Rev. 2002, 82, 47-95.

2. Spickett, C.M.; Pitt, A.R. Protein oxidation: Role in signalling and detection by mass spectrometry. Amino Acids 2012, 42, 5-21.

3. Shacter, E. Quantification and significance of protein oxidation in biological samples. Drug Metab. Rev. 2000, 32, 307-326.

4. Domingues, R.M.; Domingues, P.; Melo, T.; Perez-Sala, D.; Reis, A.; Spickett, C.M. Lipoxidation adducts with peptides and proteins: Deleterious modifications or signaling mechanisms? J. Proteomics. 2013, 92, 110-131.

5. Ahmed, N.; Thornalley, P.J. Quantitative screening of protein biomarkers of early glycation, advanced glycation, oxidation and nitrosation in cellular and extracellular proteins by tandem mass spectrometry multiple reaction monitoring. Biochem. Soc. Trans. 2003, 31, 1417-1422.

6. Perdivara, I.; Deterding, L.J.; Przybylski, M.; Tomer, K.B. Mass spectrometric identification of oxidative modifications of tryptophan residues in proteins: Chemical artifact or post-translational modification? J. Am. Soc. Mass Spectrom. 2010, 21, 1114-1117.

7. Auclair, J.R.; Salisbury, J.P.; Johnson, J.L.; Petsko, G.A.; Ringe, D.; Bosco, D.A.; Agar, N.Y.R.; Santagata, S.; Durham, H.D.; Agar, J.N. Artifacts to avoid while taking advantage of top-down mass spectrometry based detection of protein $S$-thiolation. Proteomics 2014, 14, 1152-1157.

8. Choudhary, G.; Wu, S.L.; Shieh, P.; Hancock, W.S. Multiple enzymatic digestion for enhanced sequence coverage of proteins in complex proteomic mixtures using capillary LC with ion trap MS/MS. J. Proteome Res. 2003, 2, 59-67.

9. Mohammed, S.; Lorenzen, K.; Kerkhoven, R.; van Breukelen, B.; Vannini, A.; Cramer, P.; Heck, A.J. Multiplexed proteomics mapping of yeast RNA polymerase II and III allows near-complete sequence coverage and reveals several novel phosphorylation sites. Anal. Chem. 2008, 80, 3584-3592. 
10. Swaney, D.L.; Wenger, C.D.; Coon, J.J. Value of using multiple proteases for large-scale mass spectrometry-based proteomics. J. Proteome Res. 2010, 9, 1323-1329.

11. Table 2. List of proteases commonly used for fragmenting proteins. Cold Spring Harbor Protocols 2007, doi:10.1101/pdb.tab2ip13.

12. Moulaei, T.; Stuchlik, O.; Reed, M.; Yuan, W.; Pohl, J.; Lu, W.; Haugh-Krumpe, L.; O'Keefe, B.R.; Wlodawer, A. Topology of the disulfide bonds in the antiviral lectin scytovirin. Protein Sci. 2010, 19, 1649-1661.

13. Xiao, P.; Lv, X.; Wang, S.; Iqbal, J.; Qing, H.; Li, Q.; Deng, Y. An aptamer-based trypsin reactor for on-line protein digestion with electrospray ionization tandem mass spectrometry. Anal. Biochem. 2013, 441, 123-132.

14. Li, Y.; Wojcik, R.; Dovichi, N.J. A replaceable microreactor for on-line protein digestion in a two-dimensional capillary electrophoresis system with tandem mass spectrometry detection. J. Chromatogr. A 2011, 1218, 2007-2011.

15. Duan, X.; Young, R.; Straubinger, R.M.; Page, B.; Cao, J.; Wang, H.; Yu, H.; Canty, J.M.; $\mathrm{Qu}$, J. A straightforward and highly efficient precipitation/on-pellet digestion procedure coupled with a long gradient nano-lc separation and orbitrap mass spectrometry for label-free expression profiling of the swine heart mitochondrial proteome. J. Proteome Res. 2009, 8, 2838-2850.

16. Saveliev, S.V.; Woodroofe, C.C.; Sabat, G.; Adams, C.M.; Klaubert, D.; Wood, K.; Urh, M. Mass spectrometry compatible surfactant for optimized in-gel protein digestion. Anal. Chem. 2013, 85, 907-914.

17. Yu, Y.Q.; Gilar, M.; Kaska, J.; Gebler, J.C. A rapid sample preparation method for mass spectrometric characterization of n-linked glycans. Rapid Commun. Mass Spectrom. 2005, 19, 2331-2336.

18. Luque-Garcia, J.L.; Neubert, T.A. On-membrane tryptic digestion of proteins for mass spectrometry analysis. Methods Mol. Biol. 2009, 536, 331-341.

19. Su, D.; Gaffrey, M.J.; Guo, J.; Hatchell, K.E.; Chu, R.K.; Clauss, T.R.; Aldrich, J.T.; Wu, S.; Purvine, S.; Camp, D.G.; et al. Proteomic identification and quantification of S-glutathionylation in mouse macrophages using resin-assisted enrichment and isobaric labeling. Free Radic. Biol. Med. 2014, 67, 460-470.

20. Guo, J.; Gaffrey, M.J.; Su, D.; Liu, T.; Camp, D.G., 2nd; Smith, R.D.; Qian, W.J. Resin-assisted enrichment of thiols as a general strategy for proteomic profiling of cysteine-based reversible modifications. Nat. Protoc. 2014, 9, 64-75.

21. Rees, J.S.; Lilley, K.S. Method for suppressing non-specific protein interactions observed with affinity resins. Methods 2011, 54, 407-412.

22. Trinkle-Mulcahy, L.; Boulon, S.; Lam, Y.W.; Urcia, R.; Boisvert, F.M.; Vandermoere, F.; Morrice, N.A.; Swift, S.; Rothbauer, U.; Leonhardt, H.; et al. Identifying specific protein interaction partners using quantitative mass spectrometry and bead proteomes. J. Cell. Biol. 2008, 183, 223-239. 
23. Pignatelli, B.; Li, C.Q.; Boffetta, P.; Chen, Q.; Ahrens, W.; Nyberg, F.; Mukeria, A.; Bruske-Hohlfeld, I.; Fortes, C.; Constantinescu, V.; et al. Nitrated and oxidized plasma proteins in smokers and lung cancer patients. Cancer Res. 2001, 61, 778-784.

24. Kim, J.K.; Lee, J.R.; Kang, J.W.; Lee, S.J.; Shin, G.C.; Yeo, W.S.; Kim, K.H.; Park, H.S.; Kim, K.P. Selective enrichment and mass spectrometric identification of nitrated peptides using fluorinated carbon tags. Anal. Chem. 2011, 83, 157-163.

25. Burgoyne, J.R.; Eaton, P.; Enrique, C.; Lester, P. Chapter 15-A rapid approach for the detection, quantification, and discovery of novel sulfenic acid or S-nitrosothiol modified proteins using a biotin-switch method. In Methods in Enzymology; Academic Press: Waltham, MA, USA, 2010; Volume 473, pp. 281-303.

26. Jaffrey, S.R.; Snyder, S.H. The biotin switch method for the detection of S-nitrosylated proteins. Sci. STKE 2001, doi:10.1126/stke.2001.86.pl1.

27. Qu, Z.; Meng, F.; Bomgarden, R.D.; Viner, R.I.; Li, J.; Rogers, J.C.; Cheng, J.; Greenlief, C.M.; Cui, J.; Lubahn, D.B.; et al. Proteomic quantification and site-mapping of S-nitrosylated proteins using isobaric iodotmt reagents. J. Proteome Res. 2014, 13, 3200-3211.

28. Doulias, P.T.; Raju, K.; Greene, J.L.; Tenopoulou, M.; Ischiropoulos, H. Mass spectrometry-based identification of S-nitrosocysteine in vivo using organic mercury assisted enrichment. Methods 2013, 62, 165-170.

29. Madian, A.G.; Regnier, F.E. Profiling carbonylated proteins in human plasma. J. Proteome Res. 2010, 9, 1330-1343.

30. Bollineni, R.; Hoffmann, R.; Fedorova, M. Identification of protein carbonylation sites by two-dimensional liquid chromatography in combination with MALDI- and ESI-MS. J. Proteomics 2011, 74, 2338-2350.

31. Bollineni, R.C.; Hoffmann, R.; Fedorova, M. Proteome-wide profiling of carbonylated proteins and carbonylation sites in HeLa cells under mild oxidative stress conditions. Free Radic. Biol. Med. 2014, 68, 186-195.

32. Zhang, Q.; Qian, W.J.; Knyushko, T.V.; Clauss, T.R.; Purvine, S.O.; Moore, R.J.; Sacksteder, C.A.; Chin, M.H.; Smith, D.J.; Camp, D.G., II; et al. A method for selective enrichment and analysis of nitrotyrosine-containing peptides in complex proteome samples. J. Proteome Res. 2007, 6, 2257-2268.

33. Amoresano, A.; Chiappetta, G.; Pucci, P.; D'Ischia, M.; Marino, G. Bidimensional tandem mass spectrometry for selective identification of nitration sites in proteins. Anal. Chem. 2007, 79, 2109-2117.

34. Houée-Lévin, C.; Bobrowski, K.; Horakova, L.; Karademir, B.; Schöneich, C.; Davies, M.J.; Spickett, C.M. Exploring oxidative modifications of tyrosine: An update on mechanisms of formation, advances in analysis and biological consequences. Free Radic. Res. 2015, 49, doi:10.3109/10715762.2015.1007968

35. Tran, J.C.; Doucette, A.A. Gel-eluted liquid fraction entrapment electrophoresis: An electrophoretic method for broad molecular weight range proteome separation. Anal. Chem. 2008, 80, $1568-1573$. 
36. Ghesquiere, B.; Colaert, N.; Helsens, K.; Dejager, L.; Vanhaute, C.; Verleysen, K.; Kas, K.; Timmerman, E.; Goethals, M.; Libert, C.; et al. In vitro and in vivo protein-bound tyrosine nitration characterized by diagonal chromatography. Mol. Cell. Proteomics 2009, 8, 2642-2652.

37. Han, X.; Wang, Y.; Aslanian, A.; Fonslow, B.; Graczyk, B.; Davis, T.N.; Yates, J.R., 3rd. In-line separation by capillary electrophoresis prior to analysis by top-down mass spectrometry enables sensitive characterization of protein complexes. J. Proteome Res. 2014, 13, 6078-6086.

38. Li, Y.; Compton, P.D.; Tran, J.C.; Ntai, I.; Kelleher, N.L. Optimizing capillary electrophoresis for top-down proteomics of 30-80 kda proteins. Proteomics 2014, 14, $1158-1164$.

39. Chait, B.T.; Kent, S.B. Weighing naked proteins: Practical, high-accuracy mass measurement of peptides and proteins. Science 1992, 257, 1885-1894.

40. Bondarenko, P.V.; Second, T.P.; Zabrouskov, V.; Makarov, A.A.; Zhang, Z. Mass measurement and top-down hplc/ms analysis of intact monoclonal antibodies on a hybrid linear quadrupole ion trap-orbitrap mass spectrometer. J. Am. Soc. Mass Spectrom. 2009, 20, 1415-1424.

41. Heck, A.J.; van den Heuvel, R.H. Investigation of intact protein complexes by mass spectrometry. Mass Spectrom. Rev. 2004, 23, 368-389.

42. Mouls, L.; Silajdzic, E.; Haroune, N.; Spickett, C.M.; Pitt, A.R. Development of novel mass spectrometric methods for identifying HOCL-induced modifications to proteins. Proteomics 2009, 9, 1617-1631.

43. Luo, S.; Uehara, H.; Shacter, E. Taurine chloramine-induced inactivation of cofilin protein through methionine oxidation. Free Radic. Biol. Med. 2014, 75, 84-94.

44. Ehrmann, D.C.; Rose, K.; Calcutt, M.W.; Beller, A.B.; Hill, S.; Rogers, T.J.; Steele, S.D.; Hachey, D.L.; Aschner, J.L. Glutathionylated gammag and gammaa subunits of hemoglobin F: A novel post-translational modification found in extremely premature infants by LC-MS and nanoLC-MS/MS. J. Mass Spectrom. 2014, 49, 178-183.

45. Carini, M.; Regazzoni, L.; Aldini, G. Mass spectrometric strategies and their applications for molecular mass determination of recombinant therapeutic proteins. Curr. Pharm. Biotechnol. 2011, 12, 1548-1557.

46. Ansong, C.; Wu, S.; Meng, D.; Liu, X.W.; Brewer, H.M.; Kaiser, B.L.D.; Nakayasu, E.S.; Cort, J.R.; Pevzner, P.; Smith, R.D.; et al. Top-down proteomics reveals a unique protein S-thiolation switch in salmonella typhimurium in response to infection-like conditions. Proc. Natl. Acad. Sci. USA 2013, 110, 10153-10158.

47. Zhang, H.; Ge, Y. Comprehensive analysis of protein modifications by top-down mass spectrometry. Circ. Cardiovasc. Genet. 2011, doi:10.1161/CIRCGENETICS.110.957829.

48. Scotcher, J.; Clarke, D.J.; Mackay, C.L.; Hupp, T.; Sadler, P.J.; Langridge-Smith, P.R.R. Redox regulation of tumour suppressor protein p53: Identification of the sites of hydrogen peroxide oxidation and glutathionylation. Chem. Sci. 2013, 4, 1257-1269.

49. Holzmann, J.; Hausberger, A.; Rupprechter, A.; Toll, H. Top-down ms for rapid methionine oxidation site assignment in filgrastim. Anal. Bioanal. Chem. 2013, 405, 6667-6674. 
50. Lourette, N.; Smallwood, H.; Wu, S.; Robinson, E.W.; Squier, T.C.; Smith, R.D.; Pasa-Tolic, L. A top-down LC-fticr MS-based strategy for characterizing oxidized calmodulin in activated macrophages. J. Am. Soc. Mass Spectrom. 2010, 21, 930-939.

51. Domon, B.; Aebersold, R. Options and considerations when selecting a quantitative proteomics strategy. Nat. Biotechnol. 2010, 28, 710-721.

52. Stevens, S.M., Jr.; Prokai-Tatrai, K.; Prokai, L. Factors that contribute to the misidentification of tyrosine nitration by shotgun proteomics. Mol. Cell. Proteomics. 2008, 7, 2442-2451.

53. Craig, R.; Beavis, R.C. Tandem: Matching proteins with tandem mass spectra. Bioinformatics 2004, 20, 1466-1467.

54. Eng, J.K.; McCormack, A.L.; Yates, J.R. An approach to correlate tandem mass spectral data of peptides with amino acid sequences in a protein database. J. Am. Soc. Mass Spectrom. 1994, 5, 976-989.

55. Geer, L.Y.; Markey, S.P.; Kowalak, J.A.; Wagner, L.; Xu, M.; Maynard, D.M.; Yang, X.; Shi, W.; Bryant, S.H. Open mass spectrometry search algorithm. J. Proteome Res. 2004, 3, 958-964.

56. Ma, B.; Zhang, K.; Hendrie, C.; Liang, C.; Li, M.; Doherty-Kirby, A.; Lajoie, G. Peaks: Powerful software for peptide de novo sequencing by tandem mass spectrometry. Rapid Commun. Mass Spectrom. 2003, 17, 2337-2342.

57. Silva, A.M.N.; Vitorino, R.; Domingues, M.R.M.; Spickett, C.M.; Domingues, P. Post-translational modifications and mass spectrometry detection. Free Radic. Biol. Med. 2013, 65, 925-941.

58. Perkins, D.N.; Pappin, D.J.; Creasy, D.M.; Cottrell, J.S. Probability-based protein identification by searching sequence databases using mass spectrometry data. Electrophoresis 1999, 20, 3551-3567.

59. Spickett, C.M.; Reis, A.; Pitt, A.R. Use of narrow mass-window, high-resolution extracted product ion chromatograms for the sensitive and selective identification of protein modifications. Anal. Chem. 2013, 85, 4621-4627.

60. Srikanth, R.; Wilson, J.; Bridgewater, J.D.; Numbers, J.R.; Lim, J.; Olbris, M.R.; Kettani, A.; Vachet, R.W. Improved sequencing of oxidized cysteine and methionine containing peptides using electron transfer dissociation. J. Am. Soc. Mass Spectrom. 2007, 18, 1499-1506.

61. Shilov, I.V.; Seymour, S.L.; Patel, A.A.; Loboda, A.; Tang, W.H.; Keating, S.P.; Hunter, C.L.; Nuwaysir, L.M.; Schaeffer, D.A. The paragon algorithm, a next generation search engine that uses sequence temperature values and feature probabilities to identify peptides from tandem mass spectra. Mol. Cell. Proteomics 2007, 6, 1638-1655.

62. Dorfer, V.; Pichler, P.; Stranzl, T.; Stadlmann, J.; Taus, T.; Winkler, S.; Mechtler, K. MS Amanda, a universal identification algorithm optimized for high accuracy tandem mass spectra. J. Proteome Res. 2014, 13, 3679-3684.

63. Ye, D.; Fu, Y.; Sun, R.X.; Wang, H.P.; Yuan, Z.F.; Chi, H.; He, S.M. Open MS/MS spectral library search to identify unanticipated post-translational modifications and increase spectral identification rate. Bioinformatics 2010, 26, i399-i406. 
64. Moskovitz, J. Detection and localization of methionine sulfoxide residues of specific proteins in brain tissue. Protein Pept. Lett. 2014, 21, 52-55.

65. Steen, H.; Mann, M. The abc's (and xyz's) of peptide sequencing. Nat. Rev. Mol. Cell Biol. 2004, 5, 699-711.

66. Medzihradszky, K.F.; Chalkley, R.J. Lessons in de novo peptide sequencing by tandem mass spectrometry. Mass Spectrom. Rev. 2013, 34, 43-63.

67. Curran, T.G.; Bryson, B.D.; Reigelhaupt, M.; Johnson, H.; White, F.M. Computer aided manual validation of mass spectrometry-based proteomic data. Methods 2013, 61, 219-226.

68. Jeong, K.; Kim, S.; Pevzner, P.A. Uninovo: A universal tool for de novo peptide sequencing. Bioinformatics 2013, 29, 1953-1962.

69. Muth, T.; Weilnbock, L.; Rapp, E.; Huber, C.G.; Martens, L.; Vaudel, M.; Barsnes, H. Denovogui: An open source graphical user interface for de novo sequencing of tandem mass spectra. J. Proteome Res. 2014, 13, 1143-1146.

70. Liu, X.; Dekker, L.J.; Wu, S.; Vanduijn, M.M.; Luider, T.M.; Tolic, N.; Kou, Q.; Dvorkin, M.; Alexandrova, S.; Vyatkina, K.; et al. De novo protein sequencing by combining top-down and bottom-up tandem mass spectra. J. Proteome Res. 2014, 13, 3241-3248.

71. Petersson, A.S.; Steen, H.; Kalume, D.E.; Caidahl, K.; Roepstorff, P. Investigation of tyrosine nitration in proteins by mass spectrometry. J. Mass Spectrom. 2001, 36, 616-625.

72. Abello, N.; Kerstjens, H.A.; Postma, D.S.; Bischoff, R. Protein tyrosine nitration: Selectivity, physicochemical and biological consequences, denitration, and proteomics methods for the identification of tyrosine-nitrated proteins. J. Proteome Res. 2009, 8, 3222-3238.

73. Tveen-Jensen, K.; Reis, A.; Mouls, L.; Pitt, A.R.; Spickett, C.M. Reporter ion-based mass spectrometry approaches for the detection of non-enzymatic protein modifications in biological samples. J. Proteomics 2013, 92, 71-79.

74. Li, B.; Held, J.M.; Schilling, B.; Danielson, S.R.; Gibson, B.W. Confident identification of 3-nitrotyrosine modifications in mass spectral data across multiple mass spectrometry platforms. J. Proteomics 2011, 74, 2510-2521.

75. Guan, Z.Q.; Yates, N.A.; Bakhtiar, R. Detection and characterization of methionine oxidation in peptides by collision-induced dissociation and electron capture dissociation. J. Am. Soc. Mass Spectrom. 2003, 14, 605-613.

76. Galeva, N.A.; Esch, S.W.; Williams, T.D.; Markille, L.M.; Squier, T.C. Rapid method for quantifying the extent of methionine oxidation in intact calmodulin. J. Am. Soc. Mass Spectrom. 2005, 16, 1470-1480.

77. Rauniyar, N.; Prokai, L. Isotope-coded dimethyl tagging for differential quantification of posttranslational protein carbonylation by 4-hydroxy-2-nonenal, an end-product of lipid peroxidation. J. Mass Spectrom. 2011, 46, 976-985.

78. Cox, D.M.; Zhong, F.; Du, M.; Duchoslav, E.; Sakuma, T.; McDermott, J.C. Multiple reaction monitoring as a method for identifying protein posttranslational modifications. J. Biomol. Technol. 2005, 16, 83-90. 
79. Held, J.M.; Danielson, S.R.; Behring, J.B.; Atsriku, C.; Britton, D.J.; Puckett, R.L.; Schilling, B.; Campisi, J.; Benz, C.C.; Gibson, B.W. Targeted quantitation of site-specific cysteine oxidation in endogenous proteins using a differential alkylation and multiple reaction monitoring mass spectrometry approach. Mol. Cell Proteomics 2010, 9, 1400-1410.

80. Deutsch, E.W.; Lam, H.; Aebersold, R. Peptideatlas: A resource for target selection for emerging targeted proteomics workflows. EMBO Rep. 2008, 9, 429-434.

81. Valim, L.R.; Davies, J.A.; Jensen, K.T.; Guo, R.; Willison, K.R.; Spickett, C.M.; Pitt, A.R.; Klug, D.R. Identification and relative quantification of tyrosine nitration in a model peptide using two-dimensional infrared spectroscopy. J. Phys. Chem. B 2014, 118, 12855-12864.

82. Gerber, S.A.; Rush, J.; Stemman, O.; Kirschner, M.W.; Gygi, S.P. Absolute quantification of proteins and phosphoproteins from cell lysates by tandem ms. Proc. Natl. Acad. Sci. USA 2003, 100, 6940-6945.

83. Nahnsen, S.; Bielow, C.; Reinert, K.; Kohlbacher, O. Tools for label-free peptide quantification. Mol. Cell Proteomics 2013, 12, 549-556.

84. Turk, R.; Piras, C.; Kovacic, M.; Samardzija, M.; Ahmed, H.; de Canio, M.; Urbani, A.; Mestric, Z.F.; Soggiu, A.; Bonizzi, L.; et al. Proteomics of inflammatory and oxidative stress response in cows with subclinical and clinical mastitis. J. Proteomics 2012, 75, 4412-4428.

85. Vogel, C.; Silva, G.M.; Marcotte, E.M. Protein expression regulation under oxidative stress. Mol. Cell Proteomics 2011, doi:10.1074/mcp.M111.009217.

86. May, D.; Fitzgibbon, M.; Liu, Y.; Holzman, T.; Eng, J.; Kemp, C.J.; Whiteaker, J.; Paulovich, A.; McIntosh, M. A platform for accurate mass and time analyses of mass spectrometry data. J. Proteome Res. 2007, 6, 2685-2694.

87. Cox, J.; Mann, M. Maxquant enables high peptide identification rates, individualized p.P.B.range mass accuracies and proteome-wide protein quantification. Nat. Biotechnol. 2008, 26, 1367-1372.

88. Sturm, M.; Bertsch, A.; Gropl, C.; Hildebrandt, A.; Hussong, R.; Lange, E.; Pfeifer, N.; Schulz-Trieglaff, O.; Zerck, A.; Reinert, K.; et al. Openms-An open-source software framework for mass spectrometry. BMC Bioinform. 2008, doi:10.1186/1471-2105-9-163.

89. Mueller, L.N.; Rinner, O.; Schmidt, A.; Letarte, S.; Bodenmiller, B.; Brusniak, M.-Y.; Vitek, O.; Aebersold, R.; Müller, M. Superhirn-A novel tool for high resolution LC-MS-based peptide/protein profiling. Proteomics 2007, 7, 3470-3480.

90. Fischer, R.; Trudgian, D.C.; Wright, C.; Thomas, G.; Bradbury, L.A.; Brown, M.A.; Bowness, P.; Kessler, B.M. Discovery of candidate serum proteomic and metabolomic biomarkers in ankylosing spondylitis. Mol. Cell Proteomics 2012, doi:10.1074/mcp.M111.013904.

91. Zaccarin, M.; Falda, M.; Roveri, A.; Bosello-Travain, V.; Bordin, L.; Maiorino, M.; Ursini, F.; Toppo, S. Quantitative label-free redox proteomics of reversible cysteine oxidation in red blood cell membranes. Free Radic. Biol. Med. 2014, 71, 90-98.

92. Leonard, S.E.; Carroll, K.S. Chemical "omics" approaches for understanding protein cysteine oxidation in biology. Curr. Opin. Chem. Biol. 2011, 15, 88-102. 
93. Sethuraman, M.; McComb, M.E.; Huang, H.; Huang, S.; Heibeck, T.; Costello, C.E.; Cohen, R.A. Isotope-coded affinity tag (ICAT) approach to redox proteomics: Identification and quantitation of oxidant-sensitive cysteine thiols in complex protein mixtures. J. Proteome Res. 2004, 3, 1228-1233.

94. Zhao, Y.; Lee, W.N.; Xiao, G.G. Quantitative proteomics and biomarker discovery in human cancer. Expert Rev. Proteomics 2009, 6, 115-118.

95. Scotcher, J.; Bythell, B.J.; Marshall, A.G. Unequivocal determination of site-specific protein disulfide bond reduction potentials by top-down FTICR MS: Characterization of the N- and C-terminal redox-active sites in human thioredoxin 1. Anal. Chem. 2013, 85, 9164-9172.

96. Pan, K.T.; Chen, Y.Y.; Pu, T.H.; Chao, Y.S.; Yang, C.Y.; Bomgarden, R.D.; Rogers, J.C.; Meng, T.C.; Khoo, K.H. Mass spectrometry-based quantitative proteomics for dissecting multiplexed redox cysteine modifications in nitric oxide-protected cardiomyocyte under hypoxia. Antioxid. Redox Signal. 2014, 20, 1365-1381.

97. Wojdyla, K.; Williamson, J.; Roepstorff, P.; Rogowska-Wrzesinska, A. The SNO/SOH TMT strategy for combinatorial analysis of reversible cysteine oxidations. J. Proteomics 2015, 113, 415-434.

98. McDonagh, B.; Martinez-Acedo, P.; Vazquez, J.; Padilla, C.A.; Sheehan, D.; Barcena, J.A. Application of iTRAQ reagents to relatively quantify the reversible redox state of cysteine residues. Int. J. Proteomics 2012, 2012, 514847.

99. Palmese, A.; de Rosa, C.; Chiappetta, G.; Marino, G.; Amoresano, A. Novel method to investigate protein carbonylation by iTRAQ strategy. Anal. Bioanal. Chem. 2012, 404, 1631-1635.

100. Robinson, R.A.; Evans, A.R. Enhanced sample multiplexing for nitrotyrosine-modified proteins using combined precursor isotopic labeling and isobaric tagging. Anal. Chem. 2012, 84, 4677-4686.

101. Liu, H.; Ponniah, G.; Neill, A.; Patel, R.; Andrien, B. Accurate determination of protein methionine oxidation by stable isotope labeling and LC-MS analysis. Anal. Chem. 2013, 85, 11705-11709.

102. Fahlman, R.P.; Chen, W.; Overall, C.M. Absolute proteomic quantification of the activity state of proteases and proteolytic cleavages using proteolytic signature peptides and isobaric tags. J. Proteomics 2014, 100, 79-91.

103. Knoefler, D.; Leicher, L.I.O.; Thamsen, M.; Cremers, C.M.; Reichmann, D.; Gray, M.J.; Wholey, W.Y.; Jakob, U. About the dangers, costs and benefits of living an aerobic lifestyle. Biochem. Soc. Trans. 2014, 42, 917-921.

104. Forman, H.J.; Ursini, F.; Maiorino, M. An overview of mechanisms of redox signaling. J. Mol. Cell. Cardiol. 2014, 73, 2-9.

105. Wani, R.; Nagata, A.; Murray, B.W. Protein redox chemistry: Post-translational cysteine modifications that regulate signal transduction and drug pharmacology. Front. Pharmacol. 2014, doi:10.3389/fphar.2014.00224. 
106. Forman, H.J.; Fukuto, J.M.; Torres, M. Redox signaling: Thiol chemistry defines which reactive oxygen and nitrogen species can act as second messengers. Am. J. Physiol. Cell. Physiol. 2004, 287, C246-C256.

107. Karplus, P.A. A primer on peroxiredoxin biochemistry. Free Radic. Biol. Med. 2015, 80, 183-190.

108. Venereau, E.; Casalgrandi, M.; Schiraldi, M.; Antoine, D.J.; Cattaneo, A.; De Marchis, F.; Liu, J.; Antonelli, A.; Preti, A.; Raeli, L.; et al. Mutually exclusive redox forms of HMGB1 promote cell recruitment or proinflammatory cytokine release. J. Exp. Med. 2012, 209, 1519-1528.

109. Enescu, M.; Kassim, R.; Ramseyer, C.; Cardey, B. Theoretical insights into the mechanism of redox switch in heat shock protein Hsp33. J. Biol. Inorg. Chem. 2015, doi:10.1007/s00775-015-1240-z.

110. Iyer, A.K.; Rojanasakul, Y.; Azad, N. Nitrosothiol signaling and protein nitrosation in cell death. Nitric Oxide 2014, 42, 9-18.

111. Chouchani, E.T.; Methner, C.; Nadtochiy, S.M.; Logan, A.; Pell, V.R.; Ding, S.; James, A.M.; Cocheme, H.M.; Reinhold, J.; Lilley, K.S.; et al. Cardioprotection by S-nitrosation of a cysteine switch on mitochondrial complex I. Nat. Med. 2013, 19, 753-759.

112. Bottari, S.P. Protein tyrosine nitration: A signaling mechanism conserved from yeast to man. Proteomics 2015, 15, 185-187.

113. Yeo, W.S.; Kim, Y.J.; Kabir, M.H.; Kang, J.W.; Kim, K.P. Mass spectrometric analysis of protein tyrosine nitration in aging and neurodegenerative diseases. Mass Spectrom. Rev. 2015, 34, 166-183.

114. Murphy, E.; Kohr, M.; Menazza, S.; Nguyen, T.; Evangelista, A.; Sun, J.; Steenbergen, C. Signaling by S-nitrosylation in the heart. J. Mol. Cell Cardiol. 2014, 73, 18-25.

115. Torta, F.; Usuelli, V.; Malgaroli, A.; Bachi, A. Proteomic analysis of protein S-nitrosylation. Proteomics 2008, 8, 4484-4494.

116. Thornalley, P.J.; Rabbani, N. Detection of oxidized and glycated proteins in clinical samples using mass spectrometry-A user's perspective. Biochim. Biophys. Acta 2014, 1840, 818-829.

117. Sacksteder, C.A.; Qian, W.J.; Knyushko, T.V.; Wang, H.X.; Chin, M.H.; Lacan, G.; Melega, W.P.; Camp, D.G.; Smith, R.D.; Smith, D.J.; et al. Endogenously nitrated proteins in mouse brain: Links to neurodegenerative disease. Biochemistry 2006, 45, 8009-8022.

118. Luo, S.; Wehr, N.B. Protein carbonylation: Avoiding pitfalls in the 2,4dinitrophenylhydrazine assay. Redox Rep. 2009, 14, 159-166.

119. Augustyniak, E.; Adam, A.; Wojdyla, K.; Rogowska-Wrzesinska, A.; Willetts, R.; Korkmaz, A.; Atalay, M.; Weber, D.; Grune, T.; Borsa, C.; et al. Validation of protein carbonyl measurement: A multi-centre study. Redox Biol. 2014, 4, 149-157.

120. Zhan, X.; Desiderio, D.M. Nitroproteins from a human pituitary adenoma tissue discovered with a nitrotyrosine affinity column and tandem mass spectrometry. Anal. Biochem. 2006, 354, 279-289. 
121. Conrad, D.H.; Goyette, J.; Thomas, P.S. Proteomics as a method for early detection of cancer: A review of proteomics, exhaled breath condensate, and lung cancer screening. J. Gen. Int. Med. 2008, 23, 78-84.

122. Larstad, M.; Soderling, A.S.; Caidahl, K.; Olin, A.C. Selective quantification of free 3-nitrotyrosine in exhaled breath condensate in asthma using gas chromatography/tandem mass spectrometry. Nitric Oxide 2005, 13, 134-144.

123. Tsikas, D. Analytical methods for 3-nitrotyrosine quantification in biological samples: The unique role of tandem mass spectrometry. Amino Acids 2012, 42, 45-63.

124. Anderson, N.L.; Anderson, N.G. The human plasma proteome: History, character, and diagnostic prospects. Mol. Cell Proteomics 2002, 1, 845-867.

125. Colombo, G.; Clerici, M.; Giustarini, D.; Rossi, R.; Milzani, A.; Dalle-Donne, I. Redox albuminomics: Oxidized albumin in human diseases. Antioxid. Redox Signal. 2012, 17, $1515-1527$.

126. Martinez, M.; Weisel, J.W.; Ischiropoulos, H. Functional impact of oxidative posttranslational modifications on fibrinogen and fibrin clots. Free Radic. Biol. Med. 2013, 65, 411-418.

127. Ren, Y.; Wang, H.; Liu, J.J.; Zhang, Z.P.; McLuckey, M.N.; Ouyang, Z. Analysis of biological samples using paper spray mass spectrometry: An investigation of impacts by the substrates, solvents and elution methods. Chromatographia 2013, 76, 1339-1346.

128. Ahmed, N.; Ahmed, U.; Thornalley, P.J.; Hager, K.; Fleischer, G.; Munch, G. Protein glycation, oxidation and nitration adduct residues and free adducts of cerebrospinal fluid in Alzheimer's disease and link to cognitive impairment. J. Neurochem. 2005, 92, 255-263.

129. Korolainen, M.A.; Nyman, T.A.; Nyyssonen, P.; Hartikainen, E.S.; Pirttila, T. Multiplexed proteomic analysis of oxidation and concentrations of cerebrospinal fluid proteins in Alzheimer's disease. Clin. Chem. 2007, 53, 657-665.

130. Radabaugh, M.R.; Nemirovskiy, O.V.; Misko, T.P.; Aggarwal, P.; Mathews, W.R. Immunoaffinity liquid chromatography-tandem mass spectrometry detection of nitrotyrosine in biological fluids: Development of a clinically translatable biomarker. Anal. Biochem. 2008, $380,68-76$.

131. Aydemir, B.; Onaran, I.; Kiziler, A.R.; Alici, B.; Akyolcu, M.C. The influence of oxidative damage on viscosity of seminal fluid in infertile men. J. Androl. 2008, 29, 41-46.

132. Perluigi, M.; di Domenico, F.; Fiorini, A.; Cocciolo, A.; Giorgi, A.; Foppoli, C.; Butterfield, D.A.; Giorlandino, M.; Giorlandino, C.; Schinina, M.E.; et al. Oxidative stress occurs early in down syndrome pregnancy: A redox proteomics analysis of amniotic fluid. Proteomics Clin. Appl. 2011, 5, 167-178.

133. Bahar, G.; Feinmesser, R.; Shpitzer, T.; Popovtzer, A.; Nagler, R.M. Salivary analysis in oral cancer patients: DNA and protein oxidation, reactive nitrogen species, and antioxidant profile. Cancer 2007, 109, 54-59.

134. Kim, H.K.; Reyzer, M.L.; Choi, I.J.; Kim, C.G.; Kim, H.S.; Oshima, A.; Chertov, O.; Colantonio, S.; Fisher, R.J.; Allen, J.L.; et al. Gastric cancer-specific protein profile identified using endoscopic biopsy samples via maldi mass spectrometry. J. Proteome Res. 2010, 9, 4123-4130. 
135. Diamond, D.L.; Jacobs, J.M.; Paeper, B.; Proll, S.C.; Gritsenko, M.A.; Carithers, R.L., Jr.; Larson, A.M.; Yeh, M.M.; Camp, D.G., II; Smith, R.D.; et al. Proteomic profiling of human liver biopsies: Hepatitis $\mathrm{C}$ virus-induced fibrosis and mitochondrial dysfunction. Hepatology 2007, 46, 649-657.

136. Canton, M.; Menazza, S.; Sheeran, F.L.; Polverino de Laureto, P.; di Lisa, F.; Pepe, S. Oxidation of myofibrillar proteins in human heart failure. J. Am. Coll. Cardiol. 2011, 57, 300-309.

137. Choi, J.; Sullards, M.C.; Olzmann, J.A.; Rees, H.D.; Weintraub, S.T.; Bostwick, D.E.; Gearing, M.; Levey, A.I.; Chin, L.S.; Li, L. Oxidative damage of DJ-1 is linked to sporadic parkinson and Alzheimer diseases. J. Biol. Chem. 2006, 281, 10816-10824.

138. Shapiro, J.P.; Biswas, S.; Merchant, A.S.; Satoskar, A.; Taslim, C.; Lin, S.L.; Rovin, B.H.; Sen, C.K.; Roy, S.; Freitas, M.A. A quantitative proteomic workflow for characterization of frozen clinical biopsies: Laser capture microdissection coupled with label-free mass spectrometry. J. Proteomics 2012, 77, 433-440.

139. Djidja, M.C.; Claude, E.; Snel, M.F.; Francese, S.; Scriven, P.; Carolan, V.; Clench, M.R. Novel molecular tumour classification using MALDI-mass spectrometry imaging of tissue micro-array. Anal. Bioanal. Chem. 2010, 397, 587-601.

140. Reuter, S.; Gupta, S.C.; Chaturvedi, M.M.; Aggarwal, B.B. Oxidative stress, inflammation, and cancer: How are they linked? Free Radic. Biol Med 2010, 49, 1603-1616.

141. Kato, Y.; Dozaki, N.; Nakamura, T.; Kitamoto, N.; Yoshida, A.; Naito, M.; Kitamura, M.; Osawa, T. Quantification of modified tyrosines in healthy and diabetic human urine using liquid chromatography/tandem mass spectrometry. J. Clin. Biochem. Nutr. 2009, 44, 67-78.

142. Svatikova, A.; Wolk, R. Circulating free nitrotyrosine in obstructive sleep apnea. Am. J. Physiol. Regul. Integr. Comp. Physiol. 2004, 55905, 284-287.

143. Oriolli, M.; Aldini, G.; Benfatto, M.C.; Facino, R.M.; Carini, M. Hne michael adducts to histidine and histidine-containing peptides as biomarkers of lipid-derived carbonyl stress in urines: Lc-MS/NIS profiling in zucker obese rats. Anal. Chem. 2007, 79, 9174-9184.

144. Hui, Y.; Wong, M.; Zhao, S.S.; Love, J.A.; Ansley, D.M.; Chen, D.D. A simple and robust LC-MS/MS method for quantification of free 3-nitrotyrosine in human plasma from patients receiving on-pump CABG surgery. Electrophoresis 2012, 33, 697-704.

145. Kuligowski, J.; Torres-Cuevas, I.; Quintas, G.; Rook, D.; van Goudoever, J.B.; Cubells, E.; Asensi, M.; Lliso, I.; Nunez, A.; Vento, M.; et al. Assessment of oxidative damage to proteins and DNA in urine of newborn infants by a validated upLC-MS/MS approach. PLOS ONE 2014, 9, e93703.

146. Nemirovskiy, O.V.; Radabaugh, M.R.; Aggarwal, P.; Funckes-Shippy, C.L.; Mnich, S.J.; Meyer, D.M.; Sunyer, T.; Rodney Mathews, W.; Misko, T.P. Plasma 3-nitrotyrosine is a biomarker in animal models of arthritis: Pharmacological dissection of iNOS' role in disease. Nitric Oxide 2009, 20, 150-156. 
147. Johnson, J.M.; Strobel, F.H.; Reed, M.; Pohl, J.; Jones, D.P. A rapid LC-FTMS method for the analysis of cysteine, cystine and cysteine/cystine steady-state redox potential in human plasma. Clin. Chim. Acta 2008, 396, 43-48.

148. Murdaugh, L.S.; Wang, Z.; Del Priore, L.V.; Dillon, J.; Gaillard, E.R. Age-related accumulation of 3-nitrotyrosine and nitro-A2E in human Bruch's membrane. Exp. Eye Res. 2010, $90,564-571$.

149. Winyard, P.G.; Ryan, B.; Eggleton, P.; Nissim, A.; Taylor, E.; lo Faro, M.L.; Burkholz, T.; Szabo-Taylor, K.E.; Fox, B.; Viner, N.; et al. Measurement and meaning of markers of reactive species of oxygen, nitrogen and sulfur in healthy human subjects and patients with inflammatory joint disease. Biochem. Soc. Trans. 2011, 39, 1226-1232.

150. Choi, J.; Malakowsky, C.A.; Talent, J.M.; Conrad, C.C.; Gracy, R.W. Identification of oxidized plasma proteins in Alzheimer's disease. Biochem. Biophys. Res. Commun. 2002, 293, $1566-1570$.

151. Soreghan, B.A.; Yang, F.; Thomas, S.N.; Hsu, J.; Yang, A.J. High-throughput proteomic-based identification of oxidatively induced protein carbonylation in mouse brain. Pharm. Res. 2003, 20, 1713-1720.

152. Chaudhuri, A.R.; de Waal, E.M.; Pierce, A.; van Remmen, H.; Ward, W.F.; Richardson, A. Detection of protein carbonyls in aging liver tissue: A fluorescence-based proteomic approach. Mech. Ageing Dev. 2006, 127, 849-861.

153. Feng, J.; Xie, H.; Meany, D.L.; Thompson, L.V.; Arriaga, E.A.; Griffin, T.J. Quantitative proteomic profiling of muscle type-dependent and age-dependent protein carbonylation in rat skeletal muscle mitochondria. J. Gerontol. A Biol. Sci. Med. Sci. 2008, 63, 1137-1152.

154. Sultana, R.; Perluigi, M.; Newman, S.F.; Pierce, W.M.; Cini, C.; Coccia, R.; Butterfield, D.A. Redox proteomic analysis of carbonylated brain proteins in mild cognitive impairment and early Alzheimer's disease. Antioxid. Redox Signal. 2010, 12, 327-336.

155. Chavez, J.D.; Wu, J.; Bisson, W.; Maier, C.S. Site-specific proteomic analysis of lipoxidation adducts in cardiac mitochondria reveals chemical diversity of 2-alkenal adduction. J. Proteomics 2011, 74, 2417-2429.

156. Madian, A.G.; Myracle, A.D.; Diaz-Maldonado, N.; Rochelle, N.S.; Janle, E.M.; Regnier, F.E. Differential carbonylation of proteins as a function of in vivo oxidative stress. J. Proteome Res. 2011, 10, 3959-3972.

157. Bollineni, R.C.; Fedorova, M.; Bluher, M.; Hoffmann, R. Carbonylated plasma proteins as potential biomarkers of obesity induced type 2 diabetes mellitus. J. Proteome Res. 2014, 13, 5081-5093.

158. Oikawa, S.; Yamada, T.; Minohata, T.; Kobayashi, H.; Furukawa, A.; Tada-Oikawa, S.; Hiraku, Y.; Murata, M.; Kikuchi, M.; Yamashima, T. Proteomic identification of carbonylated proteins in the monkey hippocampus after ischemia-reperfusion. Free Radic. Biol. Med. 2009, 46, 1472-1477. 
159. Kumar, Y.; Liang, C.; Limmon, G.V.; Liang, L.; Engelward, B.P.; Ooi, E.E.; Chen, J.; Tannenbaum, S.R. Molecular analysis of serum and bronchoalveolar lavage in a mouse model of influenza reveals markers of disease severity that can be clinically useful in humans. PLOS ONE 2014, 9, e86912.

160. Dalle-Donne, I.; Aldini, G.; Carini, M.; Colombo, R.; Rossi, R.; Milzani, A. Protein carbonylation, cellular dysfunction, and disease progression. J. Cell. Mol. Med. 2006, 10, 389-406.

161. Choi, J.; Rees, H.D.; Weintraub, S.T.; Levey, A.I.; Chin, L.S.; Li, L. Oxidative modifications and aggregation of $\mathrm{Cu}, \mathrm{Zn}$-superoxide dismutase associated with Alzheimer and Parkinson diseases. J. Biol. Chem. 2005, 280, 11648-11655.

162. Madian, A.G.; Diaz-Maldonado, N.; Gao, Q.; Regnier, F.E. Oxidative stress induced carbonylation in human plasma. J. Proteomics 2011, 74, 2395-2416.

163. Go, Y.M.; Jones, D.P. Cysteine/cystine redox signaling in cardiovascular disease. Free Radic. Biol. Med. 2011, 50, 495-509.

164. Sanders, L.H.; Greenamyre, J.T. Oxidative damage to macromolecules in human parkinson disease and the rotenone model. Free Radic. Biol. Med. 2013, 62, 111-120.

165. Sharov, V.S.; Dremina, E.S.; Galeva, N.A.; Williams, T.D.; Schoneich, C. Quantitative mapping of oxidation-sensitive cysteine residues in SERCA in vivo and in vitro by HPLC-electrospray-tandem MS: Selective protein oxidation during biological aging. Biochem. J. 2006, 394, 605-615.

166. Choi, J.; Levey, A.I.; Weintraub, S.T.; Rees, H.D.; Gearing, M.; Chin, L.S.; Li, L. Oxidative modifications and down-regulation of ubiquitin carboxyl-terminal hydrolase L1 associated with idiopathic Parkinson's and Alzheimer's diseases. J. Biol. Chem. 2004, 279, 13256-13264.

167. Riederer, I.M.; Schiffrin, M.; Kovari, E.; Bouras, C.; Riederer, B.M. Ubiquitination and cysteine nitrosylation during aging and Alzheimer's disease. Brain Res. Bull. 2009, 80, 233-241.

168. Kohr, M.J.; Aponte, A.; Sun, J.; Gucek, M.; Steenbergen, C.; Murphy, E. Measurement of S-nitrosylation occupancy in the myocardium with cysteine-reactive tandem mass tags: Short communication. Circ. Res. 2012, 111, 1308-1312.

169. Kumar, V.; Kleffmann, T.; Hampton, M.B.; Cannell, M.B.; Winterbourn, C.C. Redox proteomics of thiol proteins in mouse heart during ischemia/reperfusion using ICAT reagents and mass spectrometry. Free Radic. Biol. Med. 2013, 58, 109-117.

170. Go, Y.M.; Roede, J.R.; Orr, M.; Liang, Y.; Jones, D.P. Integrated redox proteomics and metabolomics of mitochondria to identify mechanisms of CD toxicity. Toxicol. Sci. 2014, 139, 59-73.

171. Schoneich, C. Methionine oxidation by reactive oxygen species: Reaction mechanisms and relevance to Alzheimer's disease. Biochim. Biophys. Acta 2005, 1703, 111-119.

172. Glaser, C.B.; Yamin, G.; Uversky, V.N.; Fink, A.L. Methionine oxidation, alpha-synuclein and Parkinson's disease. Biochim. Biophys. Acta 2005, 1703, 157-169.

173. Brock, J.W.; Jenkins, A.J.; Lyons, T.J.; Klein, R.L.; Yim, E.; Lopes-Virella, M.; Carter, R.E.; Research, G.; Thorpe, S.R.; Baynes, J.W. Increased methionine sulfoxide content of ApoA-I in type 1 diabetes. J. Lipid Res. 2008, 49, 847-855. 
174. Tsimikas, S. In vivo markers of oxidative stress and therapeutic interventions. Am. J. Cardiol. 2008, 101, 34D-42D.

175. Castegna, A.; Thongboonkerd, V.; Klein, J.B.; Lynn, B.; Markesbery, W.R.; Butterfield, D.A. Proteomic identification of nitrated proteins in Alzheimer's disease brain. J. Neurochem. 2003, 85, 1394-1401.

176. Knutson, C.G.; Mangerich, A.; Zeng, Y.; Raczynski, A.R.; Liberman, R.G.; Kang, P.; Ye, W.; Prestwich, E.G.; Lu, K.; Wishnok, J.S.; et al. Chemical and cytokine features of innate immunity characterize serum and tissue profiles in inflammatory bowel disease. Proc. Natl. Acad. Sci. USA 2013, 110, E2332-E2341.

177. Pennathur, S.; Bergt, C.; Shao, B.; Byun, J.; Kassim, S.Y.; Singh, P.; Green, P.S.; McDonald, T.O.; Brunzell, J.; Chait, A.; et al. Human atherosclerotic intima and blood of patients with established coronary artery disease contain high density lipoprotein damaged by reactive nitrogen species. J. Biol. Chem. 2004, 279, 42977-42983.

178. Smith, C.K.; Vivekanandan-Giri, A.; Tang, C.; Knight, J.S.; Mathew, A.; Padilla, R.L.; Gillespie, B.W.; Carmona-Rivera, C.; Liu, X.; Subramanian, V.; et al. Neutrophil extracellular trap-derived enzymes oxidize high-density lipoprotein: An additional proatherogenic mechanism in systemic lupus erythematosus. Arthritis Rheumatol. 2014, 66, 2532-2544.

179. Shao, B.; Pennathur, S.; Heinecke, J.W. Myeloperoxidase targets apolipoprotein A-I, the major high density lipoprotein protein, for site-specific oxidation in human atherosclerotic lesions. J. Biol. Chem. 2012, 287, 6375-6386.

180. Paton, L.N.; Mocatta, T.J.; Richards, A.M.; Winterbourn, C.C. Increased thrombin-induced polymerization of fibrinogen associated with high protein carbonyl levels in plasma from patients post myocardial infarction. Free Radic. Biol. Med. 2010, 48, 223-229.

181. Sparvero, L.J.; Amoscato, A.A.; Kochanek, P.M.; Pitt, B.R.; Kagan, V.E.; Bayir, H. Mass-spectrometry based oxidative lipidomics and lipid imaging: Applications in traumatic brain injury. J. Neurochem. 2010, 115, 1322-1336.

182. Zanivan, S.; Krueger, M.; Mann, M. In vivo quantitative proteomics: The silac mouse. Methods Mol. Biol. 2012, 757, 435-450.

183. McClatchy, D.B.; Liao, L.; Park, S.K.; Xu, T.; Lu, B.; Yates III, J.R. Differential proteomic analysis of mammalian tissues using silam. PLOS ONE 2011, 6, e16039.

184. Gilbert, L.A.; Larson, M.H.; Morsut, L.; Liu, Z.; Brar, G.A.; Torres, S.E.; Stern-Ginossar, N.; Brandman, O.; Whitehead, E.H.; Doudna, J.A.; et al. CRISPR-mediated modular RNA-guided regulation of transcription in eukaryotes. Cell 2013, 154, 442-451.

185. Ho, Y.S.; Xiong, Y.; Ho, D.S.; Gao, J.; Chua, B.H.; Pai, H.; Mieyal, J.J. Targeted disruption of the glutaredoxin 1 gene does not sensitize adult mice to tissue injury induced by ischemia/reperfusion and hyperoxia. Free Radic. Biol. Med. 2007, 43, 1299-1312.

186. Putker, M.; Vos, H.R.; van Dorenmalen, K.; de Ruiter, H.; Duran, A.G.; Snel, B.; Burgering, B.M.; Vermeulen, M.; Dansen, T.B. Evolutionary acquisition of cysteines determines FOXO paralog-specific redox signaling. Antioxid. Redox Signal. 2015, 22, 15-28. 
187. Rivera, J.; Sobey, C.G.; Walduck, A.K.; Drummond, G.R. Nox isoforms in vascular pathophysiology: Insights from transgenic and knockout mouse models. Redox Rep. 2010, 15, 50-63.

188. Adimora, N.J.; Jones, D.P.; Kemp, M.L. A model of redox kinetics implicates the thiol proteome in cellular hydrogen peroxide responses. Antioxid. Redox Signal. 2010, 13, 731-743.

189. Coon, J.J.; Zurbig, P.; Dakna, M.; Dominiczak, A.F.; Decramer, S.; Fliser, D.; Frommberger, M.; Golovko, I.; Good, D.M.; Herget-Rosenthal, S.; et al. CE-MS analysis of the human urinary proteome for biomarker discovery and disease diagnostics. Proteomics Clin. Appl. 2008, 2, 964-973. 


\title{
Oxidative Stress in Fungi: Its Function in Signal Transduction, Interaction with Plant Hosts, and Lignocellulose Degradation
}

\author{
Michael Breitenbach, Manuela Weber, Mark Rinnerthaler, Thomas Karl and \\ Lore Breitenbach-Koller
}

\begin{abstract}
In this review article, we want to present an overview of oxidative stress in fungal cells in relation to signal transduction, interaction of fungi with plant hosts, and lignocellulose degradation. We will discuss external oxidative stress which may occur through the interaction with other microorganisms or plant hosts as well as internally generated oxidative stress, which can for instance originate from NADPH oxidases or "leaky" mitochondria and may be modulated by the peroxiredoxin system or by protein disulfide isomerases thus contributing to redox signaling. Analyzing redox signaling in fungi with the tools of molecular genetics is presently only in its beginning. However, it is already clear that redox signaling in fungal cells often is linked to cell differentiation (like the formation of perithecia), virulence (in plant pathogens), hyphal growth and the successful passage through the stationary phase.
\end{abstract}

Reprinted from Biomolecules. Cite as: Breitenbach, M.; Weber, M.; Rinnerthaler, M.; Karl, T.; Breitenbach-Koller, L. Oxidative Stress in Fungi: Its Function in Signal Transduction, Interaction with Plant Hosts, and Lignocellulose Degradation. Biomolecules 2015, 5, 318-342.

\section{Introduction: Definition of ROS and Oxidative Stress}

Reactive oxygen and nitrogen species (ROS and RNS; often called RONS by a joint generic name) occur in living cells as a consequence of the metabolism of atmospheric oxygen. Most of these molecules are comparatively short-lived and highly reactive, comprising radical as well as non-radical molecular species including singlet oxygen, the superoxide radical anion, hydrogen peroxide, the hydroxyl radical, nitric oxide, peroxynitrite, and other noxious chemical agents derived from the ones just mentioned [1]. A useful overview of the chemistry and biology of those molecules is given by Winterbourn [2]. They cause detrimental chemical changes in proteins, lipids, polysaccharides, DNA, RNA, and even in small metabolites. However, some RONS through the adaptive processes taking place in millions of years of biological evolution, are now being used and active as signaling substances and for metabolic reactions based on radical chemistry which are needed for life and are not per se detrimental. ROS are also key players in the interaction of fungi with plant hosts and in the degradation of dead plant materials in the soil.

Living cells are chemically and osmotically isolated from their surroundings creating a electrochemical potential gradient across their plasma membrane, which is necessary for life. The distribution of oxidizing and reducing metabolites in the cell and in the medium creates an inside redox potential of $-310 \mathrm{mV}$ relative to the hydrogen electrode under physiological conditions in nearly all living cells [1]. This redox potential is homeostatically controlled by an elaborate system of checks and balances. Deviations from the normal value are tolerated only for a very short time. 
If they are maintained for some longer time we speak of "oxidative stress" (positive deviation from the normal mean value) or even "reductive stress" (negative deviation from the normal mean value). Both deviations can cause cell death by apoptosis and other processes of programmed cell death like necrosis, which in yeasts and fungi have been studied during the last 15 years, starting with the seminal papers of Madeo et al. 1997 [1,3]. Although not absolutely clarified, there is growing evidence that also necrosis is programmed. Therefore the expression of "programmed necrosis" was coined [4]. Strictly speaking, oxidative stress in a cell or cellular compartment is defined by the concentrations of the reduced and oxidized forms of all redox-active metabolites by applying the Nernst equation. In reality, it is often difficult to determine all relevant oxidants and reductants, some of them do not readily participate in redox reactions due to kinetic reasons, and redox exchange between different subcellular compartments further complicates this picture. Another, less rigorous but practically applicable definition of oxidative stress is given by Lushchak [5]: "The situation when due to some reasons the steady-state ROS concentration is acutely or chronically increased leading to oxidative modification of cellular constituents resulting in disturbance of cellular metabolism and regulatory pathways, particularly ROS-based has been called oxidative stress".

The main "redox-buffer" of the cell is the glutathione system, which mediated by a large number of interlinked enzymatic redox systems, can remove ROS and some of their important reaction products like organic hydroperoxides (Figure 1). The number of redox enzymes taking part in these processes even in yeast is over 100 [1] and perhaps 5 times higher in mammalian cells. One key intermediate in these processes is peroxiredoxin, a universal redox protein which we want to describe in more detail below. The necessary reduction equivalents for redox homeostasis are in all cases ultimately supplied by NADPH, which in turn is mainly produced by reducing NADP through the pentose phosphate cycle, one of the oldest metabolic pathways in living cells on earth [6].

The basic biochemistry of oxidative stress and defense against it in fungal cells has been expertly described in recent years [1,5,7-11]. These review aticles include discussions of adaptation to oxidative stress at the level of transcription, postsynthetic modification of proteins, and metabolic reconfiguration and we want to refer the reader to these articles. The basic biochemistry of oxidative stress defense and adaptation is in many respects similar in yeast and in mammalian cells. The main pathways of oxidative stress defense are pictured in Figure 1. We will concentrate here on three topics which in the last few years are increasingly discussed in relation to oxidative stress in fungi: ROS as signal transduction molecules, the role of ROS in the interaction of fungi with plant hosts, and in the degradation of lignocellulose.

Signaling by ROS is a presently highly active field of investigation in mammals, plants and eukaryotic microorganisms [12-14]. It becomes more and more clear that the proximate signal-transducing molecule is $\mathrm{H}_{2} \mathrm{O}_{2}$ [12-16] which for the signaling purpose is mostly produced by NADPH oxidases in conjunction with superoxide dismutases (SODs) [16]. We will in the following text give an overview of the oxidative stress created by NADPH oxidases and other metabolic reactions in fungal cells, the role of peroxiredoxins in redox signaling, and the ocurrence and functions of fungal NADPH oxidases. 


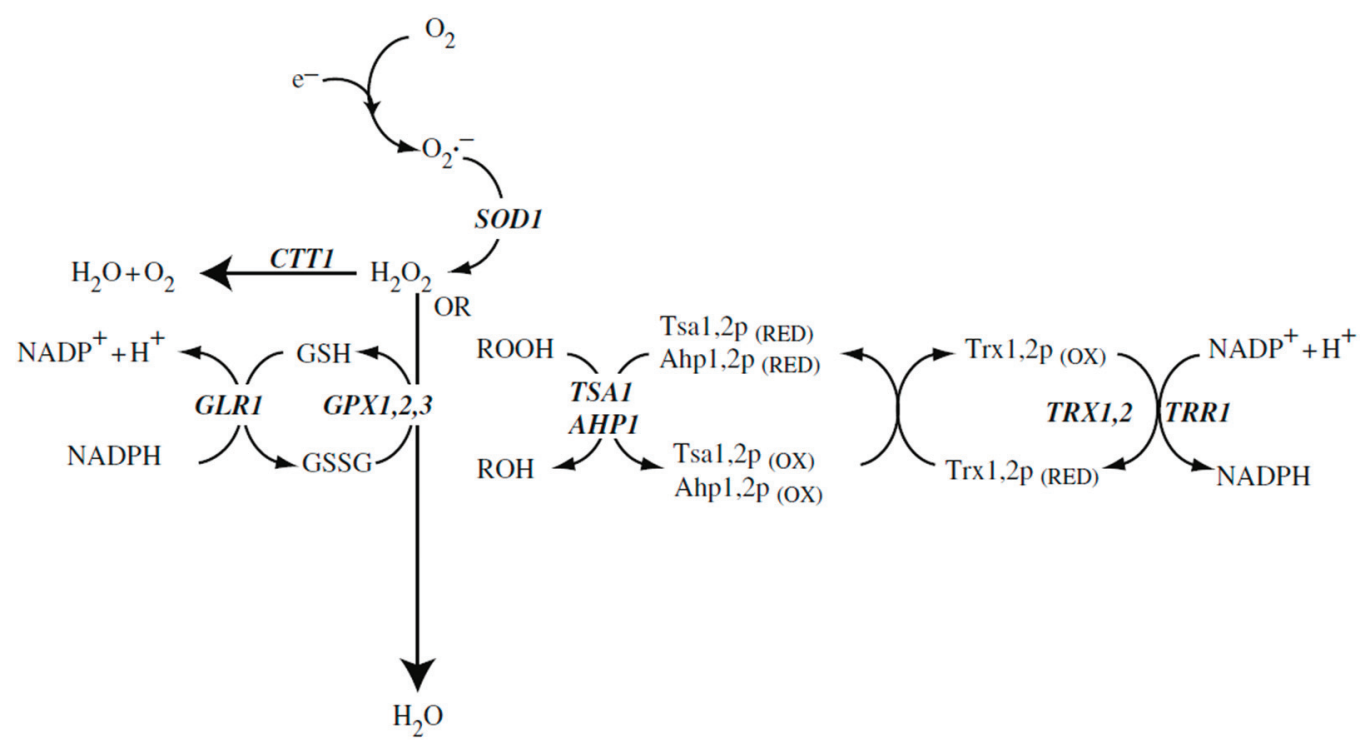

Figure 1. Reactive oxygen species and antioxidant defence systems in the cytosol. The main reactive oxygen species include the superoxide anion radical, hydrogen peroxide, and organic peroxides $(\mathrm{ROOH})$ that are detoxified to water via the $\mathrm{Cu}, \mathrm{Zn}$-superoxide dismutase, catalase or glutathione systems. Hydrogen peroxide and organic peroxides $(\mathrm{ROOH})$ can also be detoxified to an alcohol $(\mathrm{ROH})$ by the thioredoxin system. Gene designations are the ones of $S$. cerevisiae. SOD1: $\mathrm{Cu}, \mathrm{Zn}$-superoxide dismutase; CTT1: catalase T; GSH: reduced form of glutathione; GSSG: disulfide form of glutathione; GPX1,2,3: the three glutathione peroxidases of $S$. cerevisiae; GLR1: glutathione redutase; TSA1, AHP1: peroxiredoxins; TRX1,2: thioredoxins; TRR1: thioredoxin reductase (after Aung-Htut et al. 2012 [1]; with modifications).

\section{Peroxiredoxins}

We have decided to review the structure, function, and physiological significance of this class of redox-active enzymes, because they are among the most important oxidative stress defense systems in all cells and have recently been shown to also take part in redox signaling through $\mathrm{H}_{2} \mathrm{O}_{2}$ in eukaryotes. The peroxiredoxins constitute a protein superfamily which has been rather highly conserved throughout evolution [17,18]. In yeast cells as well as in other fungal cells, peroxiredoxins are the quantitatively most abundant redox defense proteins and in some cases make up about $1 \%$ of the soluble proteins of the cell [19]. Figure 1 shows the involvement of the yeast peroxiredoxins in the predominant ROS detoxification pathway in the cytoplasm of the cell. Two other pathways are active in addition to the peroxidredoxin pathway: The cytoplasmic catalase (encoded by the gene, CTT1) which is specific for $\mathrm{H}_{2} \mathrm{O}_{2}$ and dismutates $\mathrm{H}_{2} \mathrm{O}_{2}$ to $\mathrm{H}_{2} \mathrm{O}$ and $\mathrm{O}_{2}$, and the glutathione peroxidase, which is also specific for $\mathrm{H}_{2} \mathrm{O}_{2}$, but reduces it directly to $\mathrm{H}_{2} \mathrm{O}$ mediated by the glutathione (GSH) cycle. The oxidized form of glutathione (GSSG) is ultimately re-reduced using NADPH. We are mentioning in parenthesis that NADPH is the predominant supplier of reduction 
equivalents in all living cells [6]. The third pathway, based on peroxiredoxins (Figure 1) can reduce a broad spectrum of ROS including $\mathrm{H}_{2} \mathrm{O}_{2}$, alkyl hydroperoxides, lipid hydroperoxides, NO, peroxynitrite, and "unwanted" disulfide bridges in oxidized proteins. The immediate redox partner of peroxiredoxins is thioredoxin, which via thioredoxin reductase is re-reduced, depending again on NADPH as a supply of redution equivalents [1]. This pathway is not only the most abundant at the protein level, it also shows a very high intrinsic enzymatic activity of about $10^{7} \mathrm{M}^{-1} \mathrm{sec}^{-1}$ [19]. This is necessary for efficient detoxification given the high toxicity of the peroxides which are scavenged.

Nevertheless, $\mathrm{H}_{2} \mathrm{O}_{2}$ is a preferred signaling substance in eukaryotic cells, as will be discussed below. Toxic peroxides occur in multiple cellular compartments. For efficient detoxification, all these compartments must contain the peroxiredoxin system, as evidenced by the peroxiredoxin isoforms encoded in the yeast and human genome.

In yeast, five isoforms encoded by independent genes are found [1,20]: three of them are found in the cytoplasm (AHP1, TSA2, TSA1) of which TSA1 is the most important one as evidenced by the strong oxidant hypersensitivity of the corresponding deletion mutant, which is not complemented by the presence of the other isoforms [21-23] unless Tsa2 is expressed from an artificial genetic construct using Tsa2 controlled by the the Tsa1 promoter. Efficient protection from oxidative stress requires not only the right enzymatic activity (which Tsa2 exerts) but also a sufficient level of expression [20]. Two peroxiredoxin isoforms are even found in the nucleus (Dot5) and in the mitochondria (Prx1), respectively [23,24], showing that the detoxification of ROS by this system is important in these subcellular compartments [25]. Tsa1 activity has been shown to be necessary for suppressing genomic instability in the yeast $S$. cerevisiae [20]. This would logically point to the fact that oxidative stress defense in the cytoplasm somehow exerts influence on the redox reactions taking place in the nucleus. However, the mechanism which is at work here has not been investigated.

Six isoforms have been characterized in human cells [26], of which one (PrxI) is the ortholog of Tsaland can functionally replace it as shown by genetic experiments [26]. The human isoforms are named PrxI to PrxVI. PrxI and II are 2Cys peroxiredoxins located in the cytoplasm, PrxVI is a 1Cys isoform also located in the cytoplasm. PrxIII is a 2Cys enzyme located exclusively in mitochondria, and PrxV is an atypical 2Cys peroxiredoxin located in the cytoplasm, mitochondria and peroxisomes. Finally, PrxIV is a 2Cys peroxiredoxin located in the ER which functionally interacts with PDI (protein disulfide isomerase) in the redox reaction forming the disulfide bonds of proteins destined for the secretory pathway. This latter function of the PrxIV protein seems to be specific for the mammalian system and to be still undiscovered or absent from fungal cells. 


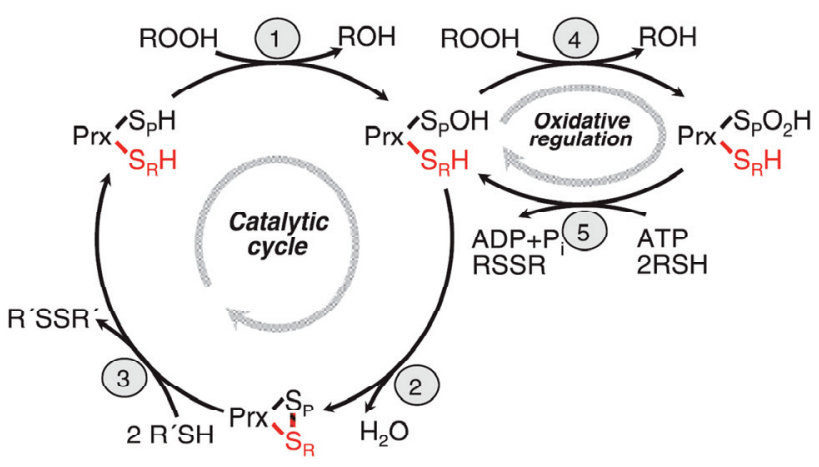

Figure 2. Reaction cycle of eukaryotic 2-Cys peroxidredoxins. Step 1: The peroxidatic cysteine is oxidized to the sulfenic acid and concomitantly substrate $(\mathrm{ROOH})$ is reduced to ROH. Step 2: The sulfenic acid form of the peroxidatic cysteine reacts with the resolving cysteine in the other subunit of the dimer, forming a disulfide bridge and releasing water. Step3: The disulfide form reacts with its redox partner, thioredoxin yielding the fully reduced form which can start a new reaction cycle. Step 4: The sulfenic acid form of the protein is further oxidized by substrate to the sulfinic acid form which is catalytically inactive and forms a chaperonine. Step 5: The sulfinic acid form is reduced back to the sulfenic acid form. Its redox partner is sulfiredoxin which is this reaction forms a diulfide bridge. The reaction is energy dependent and consumes ATP which is hydrolyzed to ADP and $\mathrm{P}_{\mathrm{i}}$. See text for further discussion of the reaction cycle, in particular the structural transition to form a chaperonine, and the local unfolding of the structure (after Hall et al. 2009 [19]; with modifications).

The catalytic cycle of eukaryotic peroxiredoxins involves a number of peculiarities explaining its regulation, recognition of multiple substrates, and role in $\mathrm{H}_{2} \mathrm{O}_{2}$ signaling. The protein undergoes physiologically important local unfolding during its reaction cycle [19,24,27]. We are discussing here only the 2 Cys peroxiredoxins. The enzymes are active as homodimers associated in a head to tail arrangement where each subunit contains two unequal important cysteine residues, named the peroxidatic (catalytic) cysteine $\mathrm{C}_{\mathrm{p}}$, and the resolving cysteine, $\mathrm{C}_{\mathrm{r}}$. In the ground state, the protein is fully reduced (both cysteines in $\mathrm{SH}$ form) and fully folded (FF), the two cysteines which will from an intersubunit disulfide bond are still separated by about $14 \AA$, and the $\mathrm{C}_{\mathrm{p}}$ residue is well shielded by a C-terminal helix and the GGLG loop. These two structural motifs occur only in eukaryotic peroxiredoxins which have developed an additional signal transmission function, but not in the prokaryotic peroxiredoxins. In the first step of the reaction cycle (Figure 2) both Cp residues of the homodimer are oxidized to the sulfenic acid level and the substrate is reduced (typically from $\mathrm{ROOH}$ to $\mathrm{ROH}$ ), in the second step, local unfolding takes place with respect to the C-terminal helix and the GGLG loop. This unfolding enables the $\mathrm{C}_{\mathrm{p}}$ of one subunit to approach $\mathrm{C}_{\mathrm{r}}$ of the other one and formation of the disulfide bond with release of water. The -S-S- form is still locally unfolded (LU) and can now react with the redox partner, thioredoxin (also with other redox patner proteins which are not well known at present), to reform the ground state (fully reduced and FF). We 
assume that the local unfolding is essential for the catalytic activity, both by enabling formation of the intermediate disulfide form and by enabling the protein to interat with so many different substrates, but also with different interaction partner proteins.

The intermediate sulfenic acid state can be further oxidized by a second molecule of $\mathrm{H}_{2} \mathrm{O}_{2}$ (or other oxidized substrates). This is relatively easy to achieve leading to the hyperoxidized state containing the sulfinic acid form of $\mathrm{C}_{\mathrm{p}}$. This form of the enzyme which is fully folded is catalytically inactive, and attains a new structure and activity. It is a decamer (a ring of five dimers) and, moreover, several of the rings form a hollow cylinder which is an efficient chaperonine re-folding misfolded proteins after inserting them into the hollow chamber. The sulfinic acid form can be reduced back to the sulfenic acid by the enzyme, sulfiredoxin, a reaction which needs the help of NADPH and ATP [28]. The physiological significance of this process in signal transmission will be discussed in the next paragraphs. Even a further oxidation of the sulfinic acid form to the sulfonic acid form has been observed. However, this process is thought to be irreversible in vivo.

\section{Protein Disulfide Isomerase (PDI) in Oxidative Stress and Signaling}

Protein disulfide isomerase (PDI) and the isoforms of this enzyme, like peroxidredoxins, are highly abundant redox proteins of eukaryotic (and prokaryotic) cells which are located in the endoplasmic reticulum (ER) of eukaryotic cells and are deeply involved in the creation and regulation of oxidative stress in mammalian as well as in fungal cells. Excellent reviews exist describing the structure and function of PDI [29-31]. These authors consider the ER as the main source of oxidative stress in the cell which can be produced as a consequence of the unfolded protein response (UPR). The direct source of ROS (in particular $\mathrm{H}_{2} \mathrm{O}_{2}$ ) in this mechanism is ERO1 (an essential gene in yeast which has been studied intensively in relation to UPR) and supplies oxidation equivalents to PDI.

The primary function of the enzymatic pathway involving PDI (in S. cerevisiae the isoforms Pdi1, Eps1, Eug1, Mpd1 and Mpd2) and Erolis threefold: (i) oxidation of cysteine SH groups during attainment of the correct folding of secreted proteins in the ER; (ii) reduction of disulfide bridges which are incorrectly formed; and, (iii), isomerization of disulfide bridges which are often formed in the incorrect place in multi-cysteine secreted proteins. The ultimate sources of oxidation and reduction are molecular oxygen which is transformed to $\mathrm{H}_{2} \mathrm{O}_{2}$ by Ero1, and NADPH, respectively. Not suprisingly, PDI protein domains are members of the thioredoxin fold superfamily. They can interact in redox reactions with a number of additional partner proteins [29]. PDI seems to play a central role in induction of apoptosis [30,32,33] which includes a signaling pathway consisting of pro-apototic and anti-apoptotic modules. It is unclear at present how the adaptive (i.e., cell survival) and apoptotic (i.e., cell death) branches of this pathway are balanced. Very unexpected steps are included, like externalization of PDI from the ER to extramitochondrial membranes [29]. At present, a conclusive picture of the role of PDI in oxidative stress in fungi is not yet emerging. However, there can be no doubt that in the near future more interesting facts about the function of PDI in oxidative stress and signaling will be discovered, using the well known fungal model systems $S$. cerevisiae and $S$. pombe. 


\section{Signaling through Hydrogen Peroxide and the Function of Peroxiredoxins as Modulators of Signaling}

We would like to start this part of the chapter by giving examples of signaling through ROS or $\mathrm{H}_{2} \mathrm{O}_{2}$ in those cases where information about the signaling mechanism and the signaling partners is available. As has been stressed before, mammalian cells are in this respect much better known than fungal cells. However the mammalian examples can tell us what we can possibly expect. Another good example is supplied by $S$. pombe, a fungal system that is not closely related to $S$. cerevisiae. Both examples provide possible roadmarks for which to look in $S$. cerevisiae and other fungi.

In those rare cases where detailed information about the fungal systems is available, we can point out the differences to mammalian cell systems. In principle, redox signaling can be divided into three different possible and partly documented mechanisms [19] discussed in the examples given below.

Example 1: In this example from mammalian cells, an extracellular signal which is not itself a redox signal, is transformed into a redox signal in the cell addressing phosphotyrosine phosphatase 1B (PTP1B) [34,35]. An excellent review including PTP1B was published recently [13]. Generally, PTPs in their active site carry a low $\mathrm{pK}_{\mathrm{a}}$ cysteine $\mathrm{SH}$ group which is prone to oxidation [36]. In the case described here, the non-redox signal (epidermal growth factor, EGF) is amplified and transmuted (discussed below) into a redox signal $\left(\mathrm{H}_{2} \mathrm{O}_{2}\right)$, which transiently inactivates PTP1B by sulfenylation at the catalytic cysteinyl SH group of the enzyme [37]. This in turn leads to an increase in the tyrosine phosphorylation state of epidermal growth factor receptor (EGFR) which is a tyrosine kinase capable of autophosphorylation. This amounts to a feed-forward amplification of the signal. In this case, compartimentation of the $\mathrm{H}_{2} \mathrm{O}_{2}$ signal is reached because on binding of EGF to the EGFR, the latter is through endocytotic vesicular transport moved to the ER where it stimulates the ER-located Nox4 (in the relevant epithelial cell cultures) to produce $\mathrm{H}_{2} \mathrm{O}_{2}$. The hydrogen peroxide originating from Nox 4 in turn sulfenylates the receptor tyrosine kinase as well as the PTP1B. The former is activated by sulfenylation while the latter is inactivated. Both effects lead to a strong feed-forward reaction. Production of Nox4 is transcriptionally controlled in this system by the EGFR signaling pathway. Although this system is in our view one of the best described in mammalian cell $\mathrm{H}_{2} \mathrm{O}_{2}$ signaling, many open questions obviously remain. Above all, it is unclear how the signal created in the ER is further transmitted reaching ultimately the trancription machinery in the nucleus. It is known that EGFR activates c-myc and CREB. An equally important open question is the eventual down-regulation of this powerful signaling. This awaits further research in the future. As the key mechanistic steps occur in the ER, it is quite probable that the ER-located peroxiredoxin, PrxIV, and the ER-located PDI play a significant yet still unknown role in this process. Of note, the only $S$. cerevisiae NADPH oxidase identified biochemically so far, resides in the ER, so that the suggested signaling function of YNO1 in reorganization of the actin cytoskeleton during the cell cycle [38] could in part follow the mechanistic model described above.

Other examples from mammalian cells can be found in the published literature [36,39-41]. 
Example 2: In this example, which has been mostly studied in yeast, a redox signal from outside is amplified in the cell to stimulate a defense response to oxidative stress by formation of a disulfide covalent linkage of peroxiredoxin to a partner protein resulting in a downstream transcriptional response [19]. Typically, as a result, a trancription factor forms an internal disulfide bridge which results in blocking nuclear export, transfer to the nucleus and activation of the downstream oxidative stress response genes. The example was discovered in S. pombe [42]. The transcription factor governing the low level of oxidative stress defense reaction in this yeast is Pap1, the structural and functional homolog of the well-known $S$. cerevisiae trancription factor Yap1 which governs oxidative stress response. In $S$. pombe, Pap1 is under control by the $\mathrm{H}_{2} \mathrm{O}_{2}$ sensor Tpx1, one of the $S$. pombe peroxidredoxins. A similar disulfide bond formation in S. cerevisae is indirect, with the primary $\mathrm{H}_{2} \mathrm{O}_{2}$ sensor being the glutathione peroxidase, Gpx3, which then in turn via a disulfide cascade creates a disulfide bond on Yap1. A second pathway depends in a similar way on oxidation of Sty1, a MAPkinase (MAPK), activating the downstream transcription factor Atf1, which is likewise involved in oxidative stress response. This pathway is important for survival of high levels of $\mathrm{H}_{2} \mathrm{O}_{2}$, while the Papl pathway is involved in adaptation to low levels of $\mathrm{H}_{2} \mathrm{O}_{2}$ [42]. Of note, these important $S$. pombe redox signaling pathways have up to now only been studied using external $\mathrm{H}_{2} \mathrm{O}_{2}$. The important question is, of course, what is the still unknown internal source of $\mathrm{H}_{2} \mathrm{O}_{2}$ for signaling. Does $S$. pombe display redox signaling also in cases where the primary signal is not external oxidative stress? Other examples from $S$. pombe exist but are not described in detail here due to space restrictions [43].

Example 3: In this example the authors [19,27] propose their floodgate model (more appropriately called the adjustable buffer model) for the role of the yeast peroxiredoxin, Tsal, in the response to an $\mathrm{H}_{2} \mathrm{O}_{2}$ signal. Tsal deficiency [44] as well as increased unregulated activity [45] result in accelerated mother cell-specific and chronological aging in S. cerevisiae. The floodgate model is presently a very attractive one. The experimental findings are consistent with the model however still without detailed proof of the molecular mechanism in fungi.

The eukaryotic members of the peroxiredoxin protein family (but apparently not the prokaryotic ones) in addition to their defense function play an important role in hydrogen peroxide signaling, and suprisingly also as chaperones (when hyperoxidized), and are regulated mainly by the redox state of their active site cysteines, but also through phosphorylation [46] and other post-synthetic modifications like glutathionylation, and through a large number of partner proteins [47]. A general overview of redox-based modulation of signal transduction by peroxidredoxins is given by Janssen-Heiniger et al. [48] and Park et al. [47].

We want to discuss and make it plausible why $\mathrm{H}_{2} \mathrm{O}_{2}$, a molecule exerting considerable oxidative damage in cells, has nevertheless been chosen by "Mother Nature", as a signaling substance. Signaling through $\mathrm{H}_{2} \mathrm{O}_{2}$ works well in eukaryotic cells (little is known about bacteria), because $\mathrm{H}_{2} \mathrm{O}_{2}$ is a stable non-radical substance occuring naturally through normal metabolic reactions that displays a sufficient half-life to be able to migrate (diffuse) for a few microns within the cell. $\mathrm{H}_{2} \mathrm{O}_{2}$ is electrically neutral and could diffuse through lipid bilayer membranes, however, in real life it is passing membranes bymeans of the aquaporin channels [49]. $\mathrm{H}_{2} \mathrm{O}_{2}$ is synthesized by "regular" metabolic reactions, by "leaky" electron transfer, but also by special reactions designed for the sole 
purpose of creating ROS. Several reactions come to mind and will be enumerated here, of which one (NADPH oxidases) will be discussed in more detail. It is reactive chemically towards its target proteins with the typical reaction being oxidation of a target $\mathrm{SH}$ group which is a reversible reaction. It acts locally (see below) without destroying cellular components outside the target area. It can be readily destroyed if no longer useful by a number of detoxification pathways (see Figure 1) which is essential for every signaling substance. Eukaryotic peroxidredoxins are involved in both the destruction of $\mathrm{H}_{2} \mathrm{O}_{2}$ and in its transient stabilization through hyperoxidation of the peroxidatic sulfhydryl group (Figure 2).

Prokaryotic peroxiredoxins cannot be inactivated by high hydrogen peroxide [24]. This resistance to high hydrogen peroxide is accompanied by absence of the flexibility of the C-terminus and around the resolving Cys. Therefore, the prokaryotic enzymes are not physiologically inactivated by hydrogen peroxide like the eukaryotic ones. Probably this means that the prokaryotic peroxiredoxins are not involved in signaling, only in oxidative stress defense.

Peroxiredoxin is a secondary modulator of eukaryotic hydrogen peroxide signaling. The producer of $\mathrm{H}_{2} \mathrm{O}_{2}$ is (among other enzymes) typically an NADPH oxidase, in conjunction with a superoxide dismutase (SOD). The two enzymes may be tightly linked in the cell $[16,40]$. In a localized burst of $\mathrm{H}_{2} \mathrm{O}_{2}$, peroxiredoxin is locally hyperoxidized, as already mentioned above, leading to inactivation as a peroxidase and enhancement of the $\mathrm{H}_{2} \mathrm{O}_{2}$ signal and to a new function as a chaperonine.

The target of the $\mathrm{H}_{2} \mathrm{O}_{2}$ signal is most often a phosphotyrosine phosphatase (PTP) [36], as has been described above in Example 1, but is not yet investigated in detail in fungal organisms. PTPs in turn influence protein phosphorylation through protein kinases, which are often key modules of cellular signaling. In order to do this, a certain minimum local concentration of $\mathrm{H}_{2} \mathrm{O}_{2}$ must be attained, which is around $10 \mathrm{mM}$ while the typical, maximum bulk concentration of $\mathrm{H}_{2} \mathrm{O}_{2}$ measured in higher cells is about $0.1 \mu \mathrm{M}$ (in resting cells) and $0.7 \mu \mathrm{M}$ (after stimulation through a signal), four to five orders of magnitude lower [50]. Bulk intracellular $\mathrm{H}_{2} \mathrm{O}_{2}$ concentration above $0.7 \mu \mathrm{M}$ lead to apoptosis [50]. It is, therefore, clear that the burst of signaling $\mathrm{H}_{2} \mathrm{O}_{2}$ must be strictly confined in time and space in the cell, unless unwanted oxidative stress reactions are elicited in the cell. This is achieved by the peroxidredoxin system which is the principal hydrogen peroxide degrading system in the cytoplasm. It efficiently removes the signaling substance after the signal has been transmitted, and it does not do it as long as peroxiredoxin is transiently inactivated by oxdizing the catalytic SH group to the inactive sulfinic acid state as mentioned above. Sulfiredoxin (Srx) reduces the sulfinic acid back to the thiol in an ATP and thioredoxin or glutathione-dependent reaction, thereby completing the oxidative regulation cycle of peroxiredoxin [51-55]. This means [19] the reversible opening and closing of a gate or buffer for $\mathrm{H}_{2} \mathrm{O}_{2}$.

\section{Metabolic Reactions Generating $\mathrm{H}_{2} \mathrm{O}_{2}$}

Several sources of $\mathrm{H}_{2} \mathrm{O}_{2}$ in fungal cells (and in higher cells) have been found. There are several obvious possibilities for the production of $\mathrm{H}_{2} \mathrm{O}_{2}$ in the metabolism of fungal cells. They have been listed in the literature: glyoxal oxidase and aryl alcohol oxidase [56], and the combined action of one electon transfer to oxygen in the respiratory chain of mitochondria in conjunction with 
superoxide dismutase (MnSOD) [57,58]. A further source of $\mathrm{H}_{2} \mathrm{O}_{2}$ is the combined action of NADPH oxidases with SOD which is highly regulated in space and time [16,40]. For the occurrence of NADPH oxidases in fungi, the reader is referred to the discussion of "Fungal NADPH Oxidases" below. Another source of $\mathrm{H}_{2} \mathrm{O}_{2}$ is PDI (protein disulfide isomerase), which in fungal cells like in all eukaryotes occurs in the endoplasmic reticulum and may be quantitatively more important for ROS production than the mitochondria [29,31]. The role of NADPH oxidases in signaling has up to now not been researched intensively in fungal systems as much as it has been in mammalian cells.

\section{Fungal NADPH Oxidases and Their Function in Cell Differentiation}

The first report of a fungal NADPH oxidase goes back to Lara-Ortiz et al. [59]. In the beginning of research on fungal NADPH oxidases, the only well-known example of an NADPH oxidase (NOX enzyme) was the human enzyme, NOX2, which is located in the plasma membrane of macrophages/monocytes and plays an important role in non-specific defense against bacterial and fungal infections. Therefore, research concentrated on true orthologs of NOX2 which were expected to exist in fungal cells [60-62]. This means that at that time researchers were trying to find not only orthologs of the human defense enzyme but also orthologs of its regulatory subunits, which was in part misleading because the fungal NADPH oxidases reside in different branches of the evolutionary tree of IMR (integral membrane reductase) enzymes [63-65] and are also regulated in different ways - compare the part on sequence-based evolutionary trees of fungal NADPH oxidases. Human Nox2, also called gp91 ${ }^{\text {phox }}$, is located in the plasma membrane and regulated at the enzyme level by regulatory subunits which are cytoplasmic in unstimulated cells but transferred to the plasma membrane after stimulation of the macrophage cells. More than six regulatory interaction partners of pg91phox are known in the macrophage (and also in other human cell types), of which only two share homology with corresponding regulatory subunits of NADPH oxidase enzymes in fungi: NoxR (corresponding to p67 phox) and the small GTPase, rac [62]. The function of NoxR in fungi is underscored by the mutant phenotype, for instance in A. nidulans, which is similar to the phenotype of the NoxA deletion, resulting in deficiency of cleistothecia formation. However, members of the NoxR family of fungi occur also in species that do not obviously contain orthologs of the classical fungal NADPH oxidases, and therefore presumably have additional new functions unrelated to Noxs. In filamentous fungi, the two classical protein families, NoxA and NoxB (in some fungi called Nox1 and Nox2) both show interaction with NoxR. Additional regulatory subunits are assumed to exist (based on the recognizable protein interaction domain on NoxR). Interactors were genetically identified in Epichloe festucae and found to be BemA and $\mathrm{Cdc} 24$, homologs of which are known to be involved in polarity establishment in fungal cells, and consequently in hyphal growth [66]. The physiological functions of the two enzymes, NoxA and NoxB, were found by analyzing the phenotype of the corresponding deletion mutants, and in some cases by screening for extragenic suppressors and by studying the action of NADPH oxidase inhibitors (for instance, diphenylideneiodonium chloride). In all cases, the mutant phenotypes were either a defect in cell differentiation (cleistothecia formation), so that these strains were female-sterile (NoxA deficiency, [62]), a defect in spore germination (Noxb deficiency, $[67,68])$, or a defect in the symbiotic interaction with a host plant in the case of plant 
parasitic or mutualistic fungi (NoxA deficiency [69]). The filamentous fungi studied most carefully in this respect were Aspergillus nidulans [59], Podospora anserinum [67,68,70], Neurospora crassa [71], Epichloe festucae [66,69], and others. In none of these cases, the molecular details of signaling which would explain the mutant phenotypes, are known [62]. However these findings are clearly in line with the general idea that ROS in fungi serve both as sources of oxidative stress, defence purposes (in the interaction with plants), and signaling to induce cell differentiation.

The third classical NADPH oxidase of fungi, NoxC (also called Nox3 in some species), does not interact with NoxR, carries two or more EF-hand calcium ion binding domains (like human Nox5) pointing to calcium regulation, is sequence-wise not closely related to either NoxA or NoxB, and functionally only known in exceptional cases, mostly in plant pathogenic fungi [62].

NADPH oxidases are without exception located in biological membranes. They comprise 6 or 7 transmembrane helices, and produce superoxide in a vectorial way so that superoxide (in the example of the macrophage enzyme) is produced on the extracellular side of the plasma membrane and molecular oxygen and NADPH are consumed in the cytoplasm. The unique reaction catalyzed by NADPH oxidases is in need for three different cofactors: NADPH, FAD, and (two different) b-type cytochromes, as well as the substrate, dioxygen (Figure 3). The reaction equation can be summarized as: $\mathrm{NADPH}+2 \mathrm{O}_{2} \rightarrow \mathrm{NADP}^{+}+2 \mathrm{O}_{2}{ }^{-}+\mathrm{H}^{+}$.

Superoxide itself is a well known oxygen radical which produces severe oxidative stress and oxidative damage to nearly all cellular components mostly through the formation of follow-up products, the most important of which is the highly reactive $\mathrm{OH}$ radical. In the defense reactions of macrophages, NADPH oxidase cooperates with myeloperoxidase and other peroxidases, forming a set of very highly active bactericidal compounds, like the hypochlorite anion, peroxonitrite, and others.

However, the picture of NADPH oxidases as a class of defense enzymes has been greatly changed and enlarged in recent decades and our present view of this class of enzymes now includes catalysis of specialized chemical reactions and also signal transduction. One example is synthesis of the biologically active form of the hormone, thyroxine, by DUOX2 and a thyroidal peroxidase in the thyroid gland in a radical reaction using iodide and $\mathrm{H}_{2} \mathrm{O}_{2}[60,61]$. Signal transduction is another more general and more important new function of NADPH oxidases based on the production of ROS as signaling compounds which can signal cell proliferation but also cell differentiation [13]. The best available evidence for a signaling ROS exists for hydrogen peroxide in human cells as well as fungal cells. Signaling by NADPH oxidases in fungi $[62,67]$ was studied in detail in connection with cell differentiation in Aspergillus [59], Podospora [70], and Neurospora [71]. These examples relate to the formation of fruiting bodies needed for sexual reproduction, spore germination or interaction with a plant host and without exception concern mutations in the classical fungal NADPH oxidases NoxA, NoxB, and NoxC or the regulatory fungal NOX subunit, NoxR, of filamentous fungi (see also the cladogram given in Figure 4). The mutant phenotypes are pronounced, leading, for instance, to female sterile mycelia. However, there is presently no information available on the molecular mechanisms which would explain how these fungal NADPH oxidases or the ROS produced by them are involved in the physiological cell differentiation in fruiting bodies. 


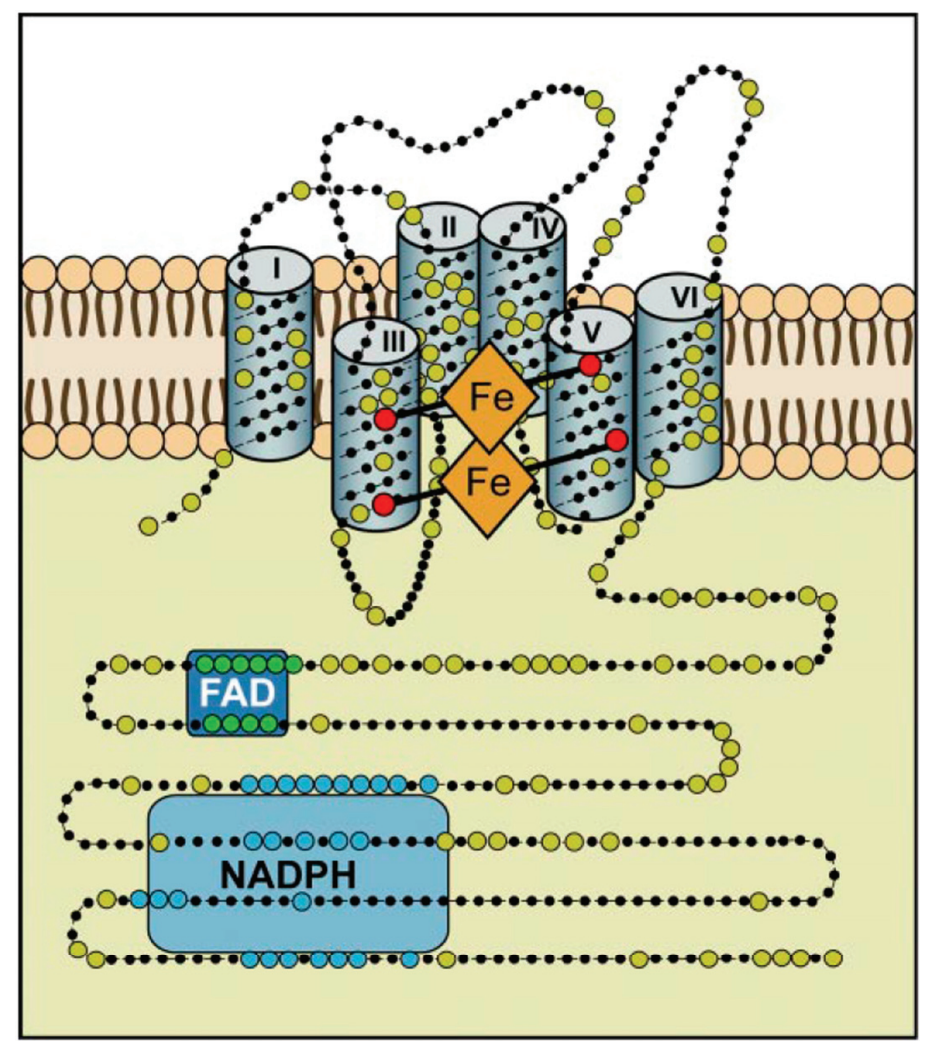

Figure 3. Hypothetical structure of a typical NADPH oxidase. This structural model is based on bioinformatics, cell fractionation and biochemical data concerning the human Nox enyzmes (NOX1, 2, 3, and 4); no crystallographic or NMR structural data are available yet. Nox enzymes comprise typically about 500 amino acids and are exclusively located in lipid bylayer membranes, like the plasma membrane or the ER membrane. Large dots are highly conserved amino acids. The reaction center transferring a single electron to oxygen is the upper b-type heme in this scheme. The enzyme consists of six transmembrane helices. The two b-type hemes are coordinated with histidine residues between helices III and V. The enzyme contains binding sequences for NADPH as well as for FAD in its cytoplasmic tail (after Bedard and Krause 2007 [60]).

The NADPH oxidase of $S$. cerevisiae, Ynol [38] is not closely related to the classical fungal NADPH oxidases NoxA, B, and C, is located in the ER and was studied by in vitro biochemical activity determination. The deletion of the gene confers no defect in cell differentiation, but leads to hypersensitivity to antibiotics inhibiting the actin cytoskeleton. Subsequent work [16] showed that Yno1 is directly coupled to the superoxide dismutase, SOD1, so that the desired signaling substance $\mathrm{H}_{2} \mathrm{O}_{2}$ is tightly controlled in space (and time) leaving no possibility for the primary product, superoxide, to engage in other, deleterious or unwanted metabolic pathways. The topic of ROS 
sequestration is actively researched in many experimental systems, also in higher cells. Toledano and co-workers [40] argue that hydrogen peroxide as a signaling molecule requires protection of those proteins which are not immediately involved in the signaling process. The physiological endpoint found by Reddi and Culotta [16] is the regulation of mitochondrial respiration depending on carbon source and growth phase. This work and the work by Leadsham et al. [72] clearly shows that Ynol's function becomes most obvious when yeast cells use up glucose and reach the point of diauxie. Under these conditions, certain respiratory defective mutants induce a burst of ROS which is under control of Ras2 activity leading to apoptosis, however, is completely abolished in the Yno1 deletion mutant. This finding would indicate a function for Ynol in controlled cell death of yeast.

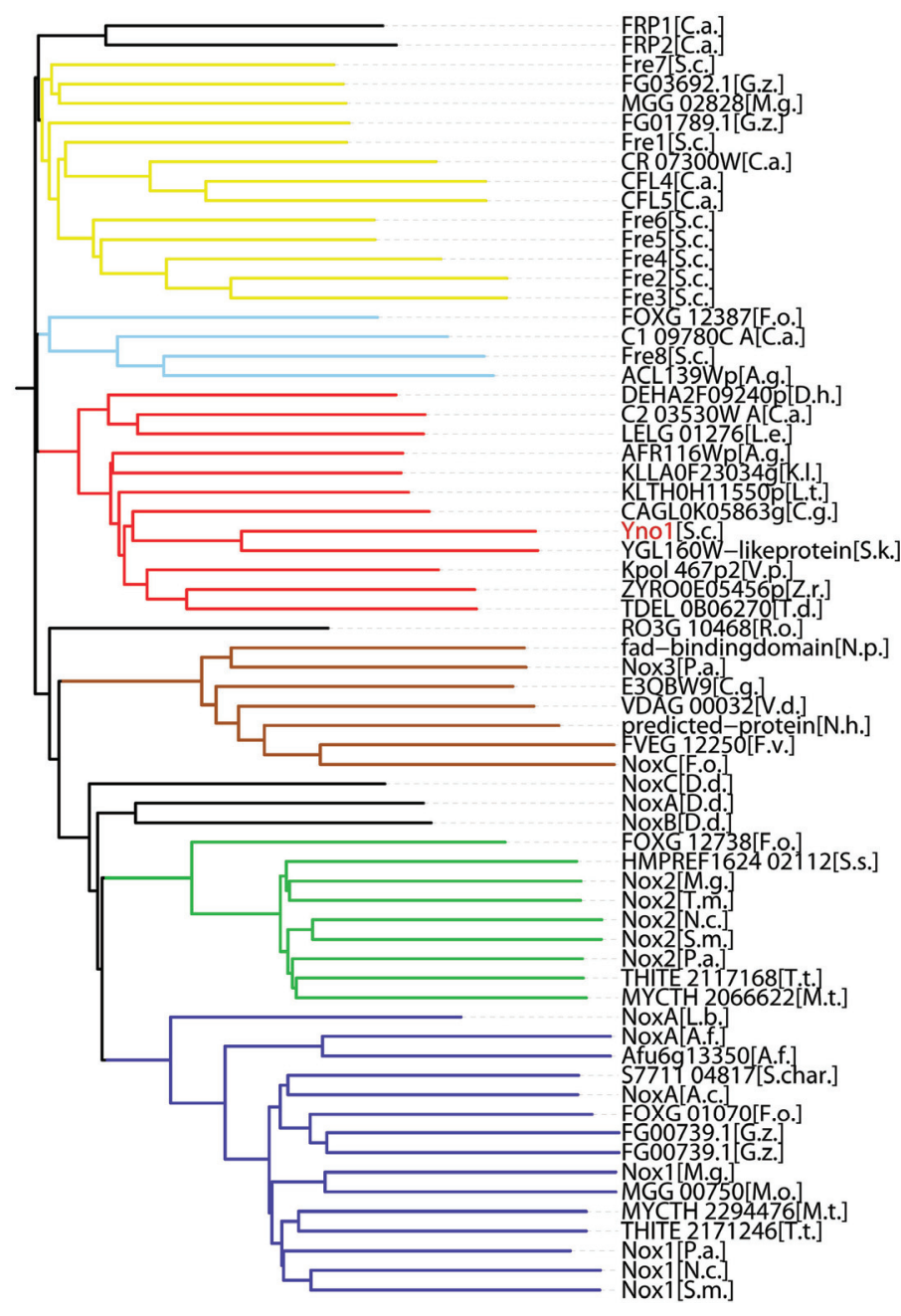

Figure 4. Phylogenetic relationships among the fungal members of the IMR (integral membrane reductase) protein superfamily (see also text for a discussion). 
The cladogram shown was calculated (Vector $\mathrm{NTI}^{\circledR}$ Software Package, Life Technologies, Carlsbad, CA, USA) using 60 representative fungal IMR protein sequences available in the sequence databanks (http://www.ncbi.nlm.nih.gov/gene) by December 2014. The length from the (calculated) origin of the tree to each protein sequence is proportional to the number of amino acid exchanges. The resulting phylogenetic tree is clearly divided into six subfamilies which are separated from each other by deep valleys. In addition, a small number of outliers are shown, which are not members of the six subfamilies. They are discussed in the text. Starting from the bottom, the subfamilies are: blue: NoxA or Nox1 (please note that there is no agreed unified nomenclature and researchers working with different fungal species have invented different names for the enzymes), in some but by no means all proteins of the NoxA group, in vitro biochemical experiments have shown NADPH oxidase activity-the same is true for NoxB and NoxC; green: NoxB or Nox2; brown: NoxC or Nox3; red: the subfamily of Yno1 homologs, as discussed in the text, in vitro biochemical activity measurements clearly show NADPH oxidase activity for the founding member, S. cerevisiae Ynol (shown in red), the other (non-cerevisiae) members of the group were not tested biochmically, but they could be NADPH oxidases due to their close sequence similartiy to Yno1; light blue: the Fre8 group, S.c. Fre8 (but not the other FRE genes) showed weak NADPH oxidase activity, the other three members were not tested; yellow: the ferric reductase subfamily, some, but by no means all of the members were tested for biochemical activity reducing ferric iron complexes during the iron uptake process in $S$. cerevisiae, the subfamily members from other fungi were mostly not tested.

Only the gene names used in the sequence databases are used in the figure.

Abbreviated species names (in alphabetical order) in the cladogram:

A. g. Ashbya gossypii

A. c. Acremonium chrysogenum

A. f. Aspergillus fumigatus

C. a. Candida albicans

C. g. Candida glabrata

D. d. Dictyostelium discoideum

D. h. Debaryomyces hansenii

F. o. Fusarium oxysporum

F. v. Fusarium verticillioides

G. z. Gibberella zeae

K. l. Kluyveromyces lactis

L. e. Lodderomyces elongisporus]

L. b. Laccaria bicolor

L. t. Lachancea thermotolerans

M. g. Magnaporthe grisea

M. o. Magnaporthe oryzae

M. t. Myceliophthora thermophila

N. c. Neurospora crassa

$N$. h. Nectria haematococca

N. p. Neofusicoccum parvum 
P. a. Podospora anserina

R. o. Rhizopus oryzae

S. c. Saccharomyces cervisiae

S. char. Stachybotrys chartarum

S. k. Saccharomyces kudriavzevii

S. m. Sordaria macrospora

S. s. Sporothrix schenckii

T.d. Torulaspora delbrueckii

T. m. Togninia minima

T. t. Thielavia terrestris

V.d. Verticillium dahliae

V.p. Vanderwaltozyma polyspora

Z. r. Zygosaccharomyces rouxii

NADPH oxidases produce superoxide as a primary product, which is in many cases in living cells is the source of deleterious reactive oxygen species (ROS). Strict compartmentalization as well as regulation of enzyme activity and "channeling" of the radical through immediate interaction with SOD have led to the modern picture of NADPH oxidases involved in signaling. Some of the known examples of ROS signaling in fungi are presented in the present paper. It is attempted to cover the literature up to 2014. However, it also seems clear that the same NADPH oxidase systems whose primary role is signaling, can, under certain pathological conditions, be also a source of oxidative stress. This was first discovered and described in mammalian cells [73].

\section{Sequence-Based Phylogenies of IMR Proteins}

We now come to the question of sequence-based phylogenies of the NOX/IMR protein superfamily in fungi in relation to the biochemical function of these enzymes. IMR (integral membrane protein) is an acronym coined by Grissa et al. [63] which encompasses membrane proteins of similar sequences including NADPH oxidases and ferric reductases, and very probably enzymes with further still undiscoverd biochemical activities. There appear to be two well-separated branches in the sequence-based phylogeny of this protein superfamily as calculated by Grissa et al. [63], with all the true NOXes known at the time falling into one branch of this phylogeny and the ferric reductases (integral membrane reductases) into a separate one. However, we showed by in vitro biochemical methods that YNO1, located in the published phylogeny in subfamily XVII of IMR proteins together with FRE8 (showing only a small superoxide producing capacity), is a bona fide NADPH oxidase, while the other FRE genes of yeast (FRE1 through FRE7) are not [38]. We cannot, therefore escape the conclusion that at least one subfamily of IMRs, which is attributed to the ferric reductase branch by Grissa et al. [63], codes not for ferric reducase gene products, but for a biochemically proven NADPH oxidase.

This is further documented in Figure 4. To illustrate the YNO1 subfamily as defined in Figure 4 even more, we have systematically compared the YNO1 sequence over its whole length with close relatives from C. albicans and C. glabrata, as well as with its $S$. cerevisae paralogs located in the FRE subfamily. Yno1 and the best match from C. glabrata (CAGL0K05863g) share 40.9\% 
identity and $69.9 \%$ similarity. By the same criteria, Ynoland its paralog Fre1 share only 18.2\% identity and $47.8 \%$ similarity. By the way, Ynol shares only a weak identity and similarity with the typical members of the fungal NoxA, B, and C proteins. Apparently, the same biochemical activity, NADPH oxidase, can be reached in several quite different subfamilies of the large IMR protein superfamily.

It would be interesting to compare the three-dimensional (3D) structures of NOX and ferric reductase enzymes with the one of Ynol to see if, perhaps, the Ynol structure is more closely related to the NOXes than to the ferric reductases. Protein structures generally show a stronger conservation and correlation with function, than sequence alone. Unfortunately, X-ray crystallography has so far not yielded any structures of the membrane-bound IMR proteins. The argument by Lalucque and Silar [74] linking fungal NOX enzymes with multicellularity would correlate well with $S$. cerevisiae and $S$. pombe having no NOX enzymes (based on sequence criteria alone), while $P$. anserina, $A$. nidulans, and many other filamentous fungi do contain coding sequences for NOXA, $\mathrm{B}$, and/or C enzymes. However, the monocellular $S$. cerevisiae yeast does contain a bona fide NOX enzyme, so the generalization that Noxes are enzymes of multicellularity [74] is certainly no longer true. One could, however, argue that $S$. cerevisiae is a close relative of a multicellular filamentous fungal plant parasite (A. gossypii) and was probably derived from an ancestral plant parasite in the not too distant evolutionary history [75] which could possibly explain the presence of a bona fide NADPH oxidase in this yeast.

Very highly similar sequences to the one of Ynol exist in C. albicans as well as in C. glabrata (see Figure 4). C. albicans is a dimorphic human pathogen and hyphal growth seems to be necessary for pathogenicity. The phenotype of the homozygous deletion mutant of the C. albicans Yno1 ortholog (C2 03530W A) is currently being investigated and should shed additional light at the physiological functions of this group of fungal NADPH oxidase enzymes.

\section{Oxygen Radicals in the Interaction of Plants with Their Fungal Symbionts and Parasites}

A newly emerging field is the long-distance signal tranduction in plants interacting with their fungal symbionts and parasites and likewise the response elicited in the fungal cell during the successful invasion leading to mutualistic or parasitic interaction with the plant. This phenomenon will be viewed here only from the perspectice of the fungal cell.

The mechanism of signal transduction in plants is only now beginning to be understood. ROS and calcium ions play a major role in this process [76]. In this field, a major aim is to gain knowledge about plant NADPH oxidases which are the most important sources of ROS for signal transduction [77]. For instance, in A. thaliana 10 different Rboh (respiratory burst oxidase homologue) genes exist which are called Atrboh and have specialized functions for the life cycle and defense of this plant. A more specialized update was given by Torres et al. [78] concerning ROS signaling of the plant in response to pathogens.

A finding that is fascinating and not understood at all at the present time is that although plant defense relies heavily on NADPH oxidases, the so-called Rboh enzymes, the fungi also need NADPH oxidases for the successful invasion of and interaction with the plant host. In most cases, the NoxA, B or C enzymes are concerned. 
A good example is the infection of rice leaves by Magnaporthe grisea. Both the NoxA and the NoxB genes of the fungus are needed for the succesful penetration, in particular for the formation of the appressorium. The respective deletion mutants of the two Nox genes have lost virulence and the defect is in the infection process [79].

Another example is known in somewhat more molecular detail. NoxA activation through rac is required to establish a mutualistic symbiotic association between Epichloe festucae and its host, the perennial ryegrass Lolium perenne $[66,69,80,81]$. The fungal hyphae grow in the extracellular space of the plant. In the absence of NoxA, in planta, the hyphae overgrow and branch and eventually kill the plant, which shows stunted growth. If the fungus is grown on agar plates in the absence of plants or plant materials, no strong phenotype of the NoxA deletion mutation is shown. Obviously, the mutualistic relationship between plant and fungus requires a delicate balance of redox signaling which is not nearly understood at present.

\section{ROS Production and the Degradation of Lignocellulose by Fungi}

In a short review article [82], the current status of biofuel production from lignocellulose is described. The US this year opened the first factories which produce bioethanol from cellulose (corn stover) in large quantities (more than 300 million liters per year at their present capacity). However, the first step is still the energy demanding conversion of cellulose and hemicellulose to sugars by purely chemical and physical means (heat and $\mathrm{NaOH}$ ). The true breakthrough will be the biological conversion by fungi or fungal enzymes (white rot fungus, and others) which can degrade lignocellulose at room temperature and physiological $\mathrm{pH}$. This process consists of radical reactions and is absolutely dependent on the enzymes, lignin peroxidase and laccase, and on ROS produced in the fungi by glyoxal oxidase, aryl alcohol oxidase, and NADPH oxidases. These processes are up to now poorly known to mycologists and biotechnologists but are under intensive research presently. If we know more about the natural processes, another big challenge will be the design of a commercial process based on the degradation of lignocellulose.

Surprisingly, both the degradation of lignin and of cellulose which are covalently linked in wood (through hemicellulose), require oxidative steps. Both extracellular and intracellular enzymatic reactions are required.

Cellobiose dehydrogenase (reviewed in Baldrian and Valaskova [83]) is described as an example of oxidative degradation. The disaccharide is oxidized at the reducing end $\mathrm{C} 1$ atom to the corresponding gluconolactone, and is subsequently converted to the open chain gluconic acid. The enzyme uses $\mathrm{FADH}_{2}$ and b-type cytochrome as redox co-enzymes and interacts with cytochrome c and/or quinones in one electron transfer reactions. The ultimate source of oxidation equivalents for this reaction is presumably $\mathrm{H}_{2} \mathrm{O}_{2}$.

The degradation and separation of lignin from cellulose in biotechnological processes presently is still done chemically. It serves the purpose of making cellulose more accessible to the enzymes degrading it. The degradation of lignin in nature is cost-efficiently performed by basidiomycete white rot fungi, and Phanerochaete chrysosporium is well researched with respect to this process [84]. 
Laccases are an important class of enzymes that are needed in plants for lignin synthesis and in fungi for lignin degradation [84]. Laccases are extracellular agents catalyzing crucial steps in lignin degradation by fungi. These well studied $4 \mathrm{Cu}$ enzymes ("blue enzymes") reduce dioxygen to water in one-electron steps creating phenolic radicals (for instance semihydroquinones) leading to cleavage of C-C bonds in the phenylpropanoid subunits of lignin (so far shown only using soluble lignin model compounds), thereby cleaving the lignin macromolecule to smaller molecules. Laccases accomplish lignin degradation in conjunction with the peroxidases discussed in the next paragraph, but they are not per se essential for ligin degradation, as for instance the white rot fungus Phanerochaete chrysosporium does not contain a recognizable laccase-encoding gene in its genome sequence. The action of laccases creates considerable oxidative stress in the vicinity of fungal cells growing on wood. $\mathrm{H}_{2} \mathrm{O}_{2}$ is not involved in the known mechanism of the laccase reaction.

\section{Lignin Peroxidases (LiP) and Manganese Peroxidases (MnP)}

Apparently these peroxidases play an essential role in the extracellular degradation of lignin by fungi, as suggested by genetic and genomic data [84]. Peroxidases are heme proteins and employ $\mathrm{H}_{2} \mathrm{O}_{2}$ as a substrate, form an oxo-ferryl compound (recognized spectroscopically) in a first step, thereby reducing $\mathrm{H}_{2} \mathrm{O}_{2}$ to $\mathrm{H}_{2} \mathrm{O}$; and a compound $\mathrm{II}$ in a second step, in which one electron is transferred to an aromatic non-phenolic structure of lignin. Thus, degradation by a radical mechanism is started. Most frequently, bonds in the side chains of phenylpropanoid units are broken leading to a variety of small molecule products (examples are a number of benzoic acid derivatives).

Manganese peroxidases (MnPs) also use $\mathrm{H}_{2} \mathrm{O}_{2}$ as a substrate, but oxidize $\mathrm{Mn}^{2+}$ to $\mathrm{Mn}^{3+}$, a highly oxidizing diffusible reagent which is believed to help degradation of sterically hindered moieties of lignin. A third type of extracelllular peroxidases (versatile peroxidases or "novel peroxidases", NoP) have also been found but are less well researched. The genome of $P$. chrysosporium contains 10LiP, $5 \mathrm{MnP}$ and one NoP-encoding genes [85].

\section{The Use of Fenton Chemistry by Wood-Degrading Fungi}

It is remarkable that processes like the Fenton and Haber-Weiss reactions are employed in the life cycle of fungi growing on wood, which produce highly toxic and aggressive molecular products like the hydroxyl radical and are avoided as much as possible in the cellular metabolism of prokaryotes as well as eukaryotes. Consider the careful avoidance of free ferrous ions in living cells, which are complexed in cellullar stores where they cannot take part in the unwanted production of oxygen radicals. The same applies to the $\mathrm{Cu}^{+}$ions which in a similar way can support the Fenton reaction [86]. In the case of the degradation of wood by fungi, the "strong" chemistry of the Fenton reaction seems to be a way to break up lignin and recycle biologically the enormous amount of dead biomass of woody plants. The process is of course extracellular and highly controlled (see below). A detailed knowledge of this process and its regulation would be highly desirable for the development of the "second generation" of biofuels which requires the degradation and fermentation of the non-edible waste parts of plants, like for instance corn stover. 
In a further step to be taken in the future, even the woody parts of trees which remain in millions of tons in the timber industry processing of trees, could be used.

The strategy to use Fenton chemistry for lignin degradation is mainly used by the basidiomycete brown rot fungi, which express less extracellular peroxidases than the white rot fungi and digest mainly the cellulose part of wood. The fungi which were mainly studied in this respect are Gloeophyllum trabeum [87] and Postia placenta [88]. In order to produce sufficient activity and amounts of hydroxyl radicals, these fungi have invented two additional strategies: First, the secretion of either one or both of two quinone compounds which are efficient redox cyclers capable of one-electron transfer reactions and necessary for the extracellular generation of superoxide for the Fenton process: these are 2,5-dimethoxy-1,4-benzoquinone (2,5 DMBQ) and 4,5-dimethoxy1,2-benzoquinone (4,5 DMBQ). Both compounds are produced as downstream metabolites of lignin degradation $[87,89]$. Second, these fungi secrete oxalic acid (also produced from lignin degradation metabolites) which chelates iron in the extracellular space [90]. The chelated $\mathrm{Fe}^{2+}$ ions are susceptible to oxidation and support the key Fenton reaction with $\mathrm{H}_{2} \mathrm{O}_{2}$. The process is depicted in Figure 4 of Bugg et al. [84]. A very similar but quantitatively less important Fenton process is used also by white rot fungi [91].

All of the processes described so far, with the single exception of the laccase reaction, require $\mathrm{H}_{2} \mathrm{O}_{2}$. The known sources of $\mathrm{H}_{2} \mathrm{O}_{2}$ in fungal cells have been listed above in the part on Metabolic Reactions Generating $\mathrm{H}_{2} \mathrm{O}_{2}$.

\section{Further Degradation Products of Lignocellulose}

Enzymatic breakdown of cellulose and hemicellulose is straightforward leading to glucose and other monomeric sugars and sugar derived metabolites (like gluconic acid, see above). These metabolites are used as carbon sources by the wood-rotting fungi, if no other carbon sources are available, and are extremely valuable for use in a new generation of bio-ethanol production facilities [82]. Lignin degradation is thought to be (at least in part) necessary to enable the attack of lignocellulose by extracelllular cellulases.

The processes which can solubilize and liberate small molecules representing partial structures of lignin are well researched and include a large variety of structures which are all rationally derived from the phenylpropane building blocks of this irregular and cross-linked polymer [84]. Most important are benzoic acid, benzaldehyde, cinnamic acid, substituted biphenyls, substituted diphenyl ethers, acetophenone, and many others, justifying the attempts to create novel bio-refinery processes and extracting industrially valuable chemicals. However, these downstream processes are not the subject of the present review paper.

\section{Conclusions}

We have, in this study, attempted to give an overview of three special aspects of oxidative stress in fungi: ROS as signaling molecules and NADPH oxidases as a major source of ROS; the role of ROS in the interaction of fungi with their plant hosts; and the extracellular degradation of lignocellulose by fungi utilizing ROS for this purpose. In the kingdom fungi, like in animals and 
plants, oxidative stress is both a substantial challenge for cellular survival that must be overcome by appropriate defense systems, but also something that can be used for pro-survival purposes in the specific situations of fungal cells. Several highly developed uses of oxygen radicals, ROS and the generation of oxidative stress come to mind and were reviewed above:

(i) The use of ROS (in this case $\mathrm{H}_{2} \mathrm{O}_{2}$ ) for intracellular signaling in the decision between growth and proliferation on the one hand, and growth arrest and cell differentiation on the other. This kind of signaling has to be compared with $\mathrm{H}_{2} \mathrm{O}_{2}$ signaling in mammalian cells, where much more information is available and the parallels but also the differences between the two signaling systems must be clarified.

(ii) The interaction of fungi with their plant hosts, both in parasitic and symbiotic relationships.

(iii) The degradation of lignocellulose, which is an environmental process of overriding importance for homeostasis in the biosphere. Lignocellulose can only be degraded in nature if oxygen radicals attack this highly resistant polymeric structure. The process must therefore take place in the extracellular space and the cells which use this process for supplying carbon sources for growth must, on the other hand, protect themselves against its detrimental consequences.

While it was traditionally thought that one electron transfer processes occurring erroneously in the mitochondrial respiratory chain are the main or only intracellular source of ROS (apart from such specialized systems as mammalian macrophages), it is now clear that ROS (superoxide radical anion and $\mathrm{H}_{2} \mathrm{O}_{2}$ ) are formed "on purpose" in every cell, by NADPH oxidases. We have therefore included a discussion of the present state of research on fungal NADPH oxidases.

Defense systems against oxidative stress are numerous and highly conserved in all eukaryotic and even prokaryotic cells. Many of the molecules, used normally for defense, can also play a role in signaling. As foremost examples we have discussed here the function of peroxiredoxins and of protein disulfide isomerases in fungal cells.

Taken together, the study of fungal oxidative stress, its use in the life cycle of fungi and its importance for cycles of matter in the biosphere, are a presently intensively researched topic and will be so even more in the future.

\section{Acknowledgments}

We aregrateful for financial support to the Austrian Science fund FWF, project number P26713 (to Michael Breitenbach) and to the OeAD, project number (CZ 10/2014) (to Mark Rinnerthaler).

\section{Author Contributions}

Michael Breitenbach, Manuela Weber, Mark Rinnerthaler, Thomas Karl and Lore BreitenbachKoller designed the structure of this review article and collected the literature; Michael Breitenbach wrote the frist draft; Mark Rinnerthaler prepared the figures and helped writing the final version; Manuela Weber inserted all references; Michael Breitenbach, Manuela Weber, Mark Rinnerthaler, Thomas Karl and Lore Breitenbach-Koller discussed the results and conclusions presented in this paper. 


\section{Conflicts of Interest}

The authors declare no conflict of interest.

\section{References}

1. Aung-Htut, M.T.; Ayer, A.; Breitenbach, M.; Dawes, I.W. Oxidative stresses and ageing. Subcell. Biochem. 2012, 57, 13-54.

2. Winterbourn, C.C. Reconciling the chemistry and biology of reactive oxygen species. Nat. Chem. Biol. 2008, 4, 278-286.

3. Madeo, F.; Frohlich, E.; Frohlich, K.U. A yeast mutant showing diagnostic markers of early and late apoptosis. J. Cell Biol. 1997, 139, 729-734.

4. Galluzzi, L.; Vanden Berghe, T.; Vanlangenakker, N.; Buettner, S.; Eisenberg, T.; Vandenabeele, P.; Madeo, F.; Kroemer, G. Programmed necrosis from molecules to health and disease. Int. Rev. Cell Mol. Biol. 2011, 289, 1-35.

5. Lushchak, V.I. Adaptive response to oxidative stress: Bacteria, fungi, plants and animals. Comp. Biochem. Physiol. C Toxicol. Pharmacol. 2011, 153, 175-190.

6. Stincone, A.; Prigione, A.; Cramer, T.; Wamelink, M.M.; Campbell, K.; Cheung, E.; Olin-Sandoval, V.; Gruening, N.M.; Krueger, A.; Tauqeer Alam, M.; et al. The return of metabolism: Biochemistry and physiology of the pentose phosphate pathway. Biol. Rev. Camb. Philos. Soc. 2014, doi:10.1111/brv.12140.

7. Herrero, E.; Ros, J.; Belli, G.; Cabiscol, E. Redox control and oxidative stress in yeast cells. Biochim. Biophys. Acta 2008, 1780, 1217-1235.

8. Ikner, A.; Shiozaki, K. Yeast signaling pathways in the oxidative stress response. Mutat. Res. 2005, 569, 13-27.

9. Leiter, E.; Gonzalez, A.; Erdei, E.; Casado, C.; Kovacs, L.; Adam, C.; Olah, J.; Miskei, M.; Molnar, M.; Farkas, I.; et al. Protein phosphatase z modulates oxidative stress response in fungi. Fungal. Genet. Biol. 2012, 49, 708-716.

10. Moye-Rowley, W.S. Regulation of the transcriptional response to oxidative stress in fungi: Similarities and differences. Eukaryot. Cell 2003, 2, 381-389.

11. Lushchak, V.I. Oxidative stress in yeast. Biochemistry 2010, 75, 281-296.

12. D'Autreaux, B.; Toledano, M.B. Ros as signalling molecules: Mechanisms that generate specificity in ROS homeostasis. Nat. Rev. Mol. Cell Biol. 2007, 8, 813-824.

13. Marinho, H.S.; Real, C.; Cyrne, L.; Soares, H.; Antunes, F. Hydrogen peroxide sensing, signaling and regulation of transcription factors. Redox Biol. 2014, 2, 535-562.

14. Suzuki, N.; Miller, G.; Morales, J.; Shulaev, V.; Torres, M.A.; Mittler, R. Respiratory burst oxidases: The engines of ROS signaling. Curr. Opin. Plant Biol. 2011, 14, 691-699.

15. Oakley, F.D.; Abbott, D.; Li, Q.; Engelhardt, J.F. Signaling components of redox active endosomes: The redoxosomes. Antioxid. Redox Signal. 2009, 11, 1313-1333.

16. Reddi, A.R.; Culotta, V.C. Sod1 integrates signals from oxygen and glucose to repress respiration. Cell 2013, 152, 224-235. 
17. Nelson, K.J.; Knutson, S.T.; Soito, L.; Klomsiri, C.; Poole, L.B.; Fetrow, J.S. Analysis of the peroxiredoxin family: Using active-site structure and sequence information for global classification and residue analysis. Proteins 2011, 79, 947-964.

18. Nelson, K.J.; Parsonage, D. Measurement of peroxiredoxin activity. Curr. Protoc. Toxicol. 2011, doi:10.1002/0471140856.tx0710s49.

19. Hall, A.; Karplus, P.A.; Poole, L.B. Typical 2-Cys peroxiredoxins-Structures, mechanisms and functions. FEBS J. 2009, 276, 2469-2477.

20. Iraqui, I.; Kienda, G.; Soeur, J.; Faye, G.; Baldacci, G.; Kolodner, R.D.; Huang, M.E. Peroxiredoxin Tsal is the key peroxidase suppressing genome instability and protecting against cell death in Saccharomyces cerevisiae. PLoS Genet. 2009, 5, e1000524.

21. MacDiarmid, C.W.; Taggart, J.; Kerdsomboon, K.; Kubisiak, M.; Panascharoen, S.; Schelble, K.; Eide, D.J. Peroxiredoxin chaperone activity is critical for protein homeostasis in zinc-deficient yeast. J. Biol. Chem. 2013, 288, 31313-31327.

22. Okada, N.; Ogawa, J.; Shima, J. Comprehensive analysis of genes involved in the oxidative stress tolerance using yeast heterozygous deletion collection. FEMS Yeast Res. 2014, 14, 425-434.

23. Park, S.G.; Cha, M.K.; Jeong, W.; Kim, I.H. Distinct physiological functions of thiol peroxidase isoenzymes in Saccharomyces cerevisiae. J. Biol. Chem. 2000, 275, 5723-5732.

24. Wood, Z.A.; Poole, L.B.; Karplus, P.A. Peroxiredoxin evolution and the regulation of hydrogen peroxide signaling. Science 2003, 300, 650-653.

25. Thorpe, G.W.; Fong, C.S.; Alic, N.; Higgins, V.J.; Dawes, I.W. Cells have distinct mechanisms to maintain protection against different reactive oxygen species: Oxidative-stress-response genes. Proc. Natl. Acad. Sci. USA 2004, 101, 6564-6569.

26. Rhee, S.G.; Woo, H.A.; Kil, I.S.; Bae, S.H. Peroxiredoxin functions as a peroxidase and a regulator and sensor of local peroxides. J. Biol. Chem. 2012, 287, 4403-4410.

27. Nystrom, T.; Yang, J.; Molin, M. Peroxiredoxins, gerontogenes linking aging to genome instability and cancer. Genes Dev. 2012, 26, 2001-2008.

28. Jonsson, T.J.; Lowther, W.T. The peroxiredoxin repair proteins. Subcell. Biochem. 2007, 44, $115-141$.

29. Laurindo, F.R.; Pescatore, L.A.; Fernandes Dde, C. Protein disulfide isomerase in redox cell signaling and homeostasis. Free Radic. Biol. Med. 2012, 52, 1954-1969.

30. Ron, D.; Walter, P. Signal integration in the endoplasmic reticulum unfolded protein response. Nat. Rev. Mol. Cell Biol. 2007, 8, 519-529.

31. Wilkinson, B.; Gilbert, H.F. Protein disulfide isomerase. Biochim. Biophys. Acta 2004, 1699, $35-44$.

32. Rutkowski, D.T.; Kaufman, R.J. That which does not kill me makes me stronger: Adapting to chronic ER stress. Trends Biochem. Sci. 2007, 32, 469-476.

33. Tabas, I.; Ron, D. Integrating the mechanisms of apoptosis induced by endoplasmic reticulum stress. Nat. Cell Biol. 2011, 13, 184-190.

34. Chen, K.; Craige, S.E.; Keaney, J.F., Jr. Downstream targets and intracellular compartmentalization in Nox signaling. Antioxid. Redox Signal. 2009, 11, 2467-2480. 
35. Chen, K.; Kirber, M.T.; Xiao, H.; Yang, Y.; Keaney, J.F., Jr. Regulation of ROS signal transduction by NADPH oxidase 4 localization. J. Cell Biol. 2008, 181, 1129-1139.

36. Den Hertog, J.; Groen, A.; van der Wijk, T. Redox regulation of protein-tyrosine phosphatases. Arch. Biochem. Biophys. 2005, 434, 11-15.

37. Paulsen, C.E.; Truong, T.H.; Garcia, F.J.; Homann, A.; Gupta, V.; Leonard, S.E.; Carroll, K.S. Peroxide-dependent sulfenylation of the EGFR catalytic site enhances kinase activity. Nat. Chem. Biol. 2012, 8, 57-64.

38. Rinnerthaler, M.; Buttner, S.; Laun, P.; Heeren, G.; Felder, T.K.; Klinger, H.; Weinberger, M.; Stolze, K.; Grousl, T.; Hasek, J.; et al. Yno1p/Aim14p, a NADPH-oxidase ortholog, controls extramitochondrial reactive oxygen species generation, apoptosis, and actin cable formation in yeast. Proc. Natl. Acad. Sci. USA 2012, 109, 8658-8663.

39. Ostman, A.; Frijhoff, J.; Sandin, A.; Bohmer, F.D. Regulation of protein tyrosine phosphatases by reversible oxidation. J. Biochem. 2011, 150, 345-356.

40. Toledano, M.B.; Planson, A.G.; Delaunay-Moisan, A. Reining in $\mathrm{H}_{2} \mathrm{O}_{2}$ for safe signaling. Cell 2010, 140, 454-456.

41. Tonks, N.K. Redox redux: Revisiting PTPs and the control of cell signaling. Cell 2005, 121, $667-670$.

42. Vivancos, A.P.; Castillo, E.A.; Biteau, B.; Nicot, C.; Ayte, J.; Toledano, M.B.; Hidalgo, E. A cysteine-sulfinic acid in peroxiredoxin regulates $\mathrm{H}_{2} \mathrm{O}_{2}$-sensing by the antioxidant Pap1 pathway. Proc. Natl. Acad. Sci. USA 2005, 102, 8875-8880.

43. Day, A.M.; Brown, J.D.; Taylor, S.R.; Rand, J.D.; Morgan, B.A.; Veal, E.A. Inactivation of a peroxiredoxin by hydrogen peroxide is critical for thioredoxin-mediated repair of oxidized proteins and cell survival. Mol. Cell 2012, 45, 398-408.

44. Lee, J.H.; Park, J.W. Role of thioredoxin peroxidase in aging of stationary cultures of Saccharomyces cerevisiae. Free Radic. Res. 2004, 38, 225-231.

45. Timmermann, B.; Jarolim, S.; Russmayer, H.; Kerick, M.; Michel, S.; Kruger, A.; Bluemlein, K.; Laun, P.; Grillari, J.; Lehrach, H.; et al. A new dominant peroxiredoxin allele identified by whole-genome re-sequencing of random mutagenized yeast causes oxidant-resistance and premature aging. Aging 2010, 2, 475-486.

46. Woo, H.A.; Yim, S.H.; Shin, D.H.; Kang, D.; Yu, D.Y.; Rhee, S.G. Inactivation of peroxiredoxin I by phosphorylation allows localized $\mathrm{H}_{2} \mathrm{O}_{2}$ accumulation for cell signaling. Cell 2010, 140, 517-528.

47. Park, J.; Lee, S.; Lee, S.; Kang, S.W. 2-Cys peroxiredoxins: Emerging hubs determining redox dependency of mammalian signaling networks. Int. J. Cell Biol. 2014, doi:10.1155/2014/715867.

48. Janssen-Heininger, Y.M.; Mossman, B.T.; Heintz, N.H.; Forman, H.J.; Kalyanaraman, B.; Finkel, T.; Stamler, J.S.; Rhee, S.G.; van der Vliet, A. Redox-based regulation of signal transduction: Principles, pitfalls, and promises. Free Radic. Biol. Med. 2008, 45, 1-17.

49. Miller, E.W.; Dickinson, B.C.; Chang, C.J. Aquaporin-3 mediates hydrogen peroxide uptake to regulate downstream intracellular signaling. Proc. Natl. Acad. Sci. USA 2010, 107, $15681-15686$. 
50. Stone, J.R.; Yang, S. Hydrogen peroxide: A signaling messenger. Antioxid. Redox Signal. 2006, 8, 243-270.

51. Jeong, W.; Bae, S.H.; Toledano, M.B.; Rhee, S.G. Role of sulfiredoxin as a regulator of peroxiredoxin function and regulation of its expression. Free Radic. Biol. Med. 2012, 53, 447-456.

52. Jonsson, T.J.; Tsang, A.W.; Lowther, W.T.; Furdui, C.M. Identification of intact protein thiosulfinate intermediate in the reduction of cysteine sulfinic acid in peroxiredoxin by human sulfiredoxin. J. Biol. Chem. 2008, 283, 22890-22894.

53. Moon, J.C.; Kim, G.M.; Kim, E.K.; Lee, H.N.; Ha, B.; Lee, S.Y.; Jang, H.H. Reversal of 2-Cys peroxiredoxin oligomerization by sulfiredoxin. Biochem. Biophys. Res. Commun. 2013, 432, 291-295.

54. Roussel, X.; Bechade, G.; Kriznik, A.; Van Dorsselaer, A.; Sanglier-Cianferani, S.; Branlant, G.; Rahuel-Clermont, S. Evidence for the formation of a covalent thiosulfinate intermediate with peroxiredoxin in the catalytic mechanism of sulfiredoxin. J. Biol. Chem. 2008, 283, 22371-22382.

55. Roussel, X.; Kriznik, A.; Richard, C.; Rahuel-Clermont, S.; Branlant, G. Catalytic mechanism of sulfiredoxin from Saccharomyces cerevisiae passes through an oxidized disulfide sulfiredoxin intermediate that is reduced by thioredoxin. J. Biol. Chem. 2009, 284, 33048-33055.

56. Hernandez-Ortega, A.; Ferreira, P.; Martinez, A.T. Fungal Aryl-alcohol oxidase: A peroxide-producing flavoenzyme involved in lignin degradation. Appl. Microbiol. Biotechnol. 2012, 93, 1395-1410.

57. Murphy, M.P. How mitochondria produce reactive oxygen species. Biochem. J. 2009, 417, 1-13.

58. Pan, Y. Mitochondria, reactive oxygen species, and chronological aging: A message from yeast. Exp. Gerontol. 2011, 46, 847-852.

59. Lara-Ortiz, T.; Riveros-Rosas, H.; Aguirre, J. Reactive oxygen species generated by microbial NADPH oxidase NoxA regulate sexual development in Aspergillus nidulans. Mol. Microbiol. 2003, 50, 1241-1255.

60. Bedard, K.; Krause, K.H. The Nox family of ROS-generating NADPH oxidases: Physiology and pathophysiology. Physiol. Rev. 2007, 87, 245-313.

61. Bedard, K.; Lardy, B.; Krause, K.H. Nox family NADPH oxidases: Not just in mammals. Biochimie 2007, 89, 1107-1112.

62. Takemoto, D.; Tanaka, A.; Scott, B. NADPH oxidases in fungi: Diverse roles of reactive oxygen species in fungal cellular differentiation. Fungal. Genet. Biol. 2007, 44, 1065-1076.

63. Grissa, I.; Bidard, F.; Grognet, P.; Grossetete, S.; Silar, P. The Nox/Ferric reductase/ferric reductase-like families of eumycetes. Fungal Biol. 2010, 114, 766-777.

64. Kawahara, B.T.; Quinn, M.T.; Lambeth, J.D. Molecular evolution of the reactive oxygen-generating NADPH oxidase (Nox/Duox) family of enzymes. BMC Evol. Biol. 2007, doi:10.1186/1471-2148-7-109.

65. Kawahara, T.; Lambeth, J.D. Molecular evolution of phox-related regulatory subunits for NADPH oxidase enzymes. BMC Evol. Biol. 2007, doi:10.1186/1471-2148-7-178. 
66. Kayano, Y.; Tanaka, A.; Akano, F.; Scott, B.; Takemoto, D. Differential roles of NADPH oxidases and associated regulators in polarized growth, conidiation and hyphal fusion in the symbiotic fungus epichloe festucae. Fungal. Genet. Biol. 2013, 56, 87-97.

67. Aguirre, J.; Rios-Momberg, M.; Hewitt, D.; Hansberg, W. Reactive oxygen species and development in microbial eukaryotes. Trends Microbiol. 2005, 13, 111-118.

68. Malagnac, F.; Lalucque, H.; Lepere, G.; Silar, P. Two NADPH oxidase isoforms are required for sexual reproduction and ascospore germination in the filamentous fungus Podospora anserina. Fungal Genet. Biol. 2004, 41, 982-997.

69. Tanaka, A.; Takemoto, D.; Hyon, G.S.; Park, P.; Scott, B. NoxA activation by the small gtpase raca is required to maintain a mutualistic symbiotic association between Epichloe festucae and Perennial ryegrass. Mol. Microbiol. 2008, 68, 1165-1178.

70. Brun, S.; Malagnac, F.; Bidard, F.; Lalucque, H.; Silar, P. Functions and regulation of the Nox family in the filamentous fungus Podospora anserina: A new role in cellulose degradation. Mol. Microbiol. 2009, 74, 480-496.

71. Cano-Dominguez, N.; Alvarez-Delfin, K.; Hansberg, W.; Aguirre, J. NADPH oxidases Nox-1 and Nox-2 require the regulatory subunit Nor-1 to control cell differentiation and growth in Neurospora crassa. Eukaryot. Cell 2008, 7, 1352-1361.

72. Leadsham, J.E.; Sanders, G.; Giannaki, S.; Bastow, E.L.; Hutton, R.; Naeimi, W.R.; Breitenbach, M.; Gourlay, C.W. Loss of cytochrome C oxidase promotes Ras-dependent ROS production from the ER resident NADPH oxidase, Ynolp, in yeast. Cell Metab. 2013, 18, 279-286.

73. Kleikers, P.W.; Wingler, K.; Hermans, J.J.; Diebold, I.; Altenhofer, S.; Radermacher, K.A.; Janssen, B.; Gorlach, A.; Schmidt, H.H. NADPH oxidases as a source of oxidative stress and molecular target in ischemia/reperfusion injury. J. Mol. Med. 2012, 90, 1391-1406.

74. Lalucque, H.; Silar, P. NADPH oxidase: An enzyme for multicellularity? Trends Microbiol. 2003, 11, 9-12.

75. Prillinger, H.; Schweigkofler, W.; Breitenbach, M.; Briza, P.; Staudacher, E.; Lopandic, K.; Molnar, O.; Weigang, F.; Ibl, M.; Ellinger, A. Phytopathogenic filamentous (Ashbya, Eremothecium) and dimorphic fungi (Holleya, Nematospora) with needle-shaped ascospores as new members within the Saccharomycetaceae. Yeast 1997, 13, 945-960.

76. Gilroy, S.; Suzuki, N.; Miller, G.; Choi, W.G.; Toyota, M.; Devireddy, A.R.; Mittler, R. A tidal wave of signals: Calcium and ROS at the forefront of rapid systemic signaling. Trends Plant Sci. 2014, 19, 623-630.

77. Marino, D.; Dunand, C.; Puppo, A.; Pauly, N. A burst of plant NADPH oxidases. Trends Plant. Sci. 2012, 17, 9-15.

78. Torres, M.A.; Jones, J.D.; Dangl, J.L. Reactive oxygen species signaling in response to pathogens. Plant Physiol. 2006, 141, 373-378.

79. Egan, M.J.; Wang, Z.Y.; Jones, M.A.; Smirnoff, N.; Talbot, N.J. Generation of reactive oxygen species by fungal NADPH oxidases is required for rice blast disease. Proc. Natl. Acad. Sci. USA 2007, 104, 11772-11777. 
80. Tanaka, A.; Cartwright, G.M.; Saikia, S.; Kayano, Y.; Takemoto, D.; Kato, M.; Tsuge, T.; Scott, B. Proa, a transcriptional regulator of fungal fruiting body development, regulates leaf hyphal network development in the epichloe festucae-lolium perenne symbiosis. Mol. Microbiol. 2013, 90, 551-568.

81. Tanaka, H.; Yoshida, G.; Baba, Y.; Matsumura, K.; Wasada, H.; Murata, J.; Agawa, M.; Itakura, S.; Enoki, A. Characterization of a hydroxyl-radical-producing glycoprotein and its presumptive genes from the white-rot basidiomycete Phanerochaete chrysosporium. J. Biotechnol. 2007, 128, 500-511.

82. Service, R.F. Renewable energy. Cellulosic ethanol at last? Science 2014, doi:10.1126/science.345.6201.1111.

83. Baldrian, P.; Valaskova, V. Degradation of cellulose by basidiomycetous fungi. FEMS Microbiol. Rev. 2008, 32, 501-521.

84. Bugg, T.D.; Ahmad, M.; Hardiman, E.M.; Rahmanpour, R. Pathways for degradation of lignin in bacteria and fungi. Nat. Prod. Rep. 2011, 28, 1883-1896.

85. Levasseur, A.; Piumi, F.; Coutinho, P.M.; Rancurel, C.; Asther, M.; Delattre, M.; Henrissat, B.; Pontarotti, P.; Asther, M.; Record, E. Foly: An integrated database for the classification and functional annotation of fungal oxidoreductases potentially involved in the degradation of lignin and related aromatic compounds. Fungal Genet. Biol. 2008, 45, 638-645.

86. Halliwell, B. Oxidative stress and neurodegeneration: Where are we now? J. Neurochem. 2006, 97, 1634-1658.

87. Jensen, K.A., Jr.; Houtman, C.J.; Ryan, Z.C.; Hammel, K.E. Pathways for extracellular fenton chemistry in the brown rot basidiomycete Gloeophyllum trabeum. Appl. Environ. Microbiol. 2001, 67, 2705-2711.

88. Vanden Wymelenberg, A.; Gaskell, J.; Mozuch, M.; Sabat, G.; Ralph, J.; Skyba, O.; Mansfield, S.D.; Blanchette, R.A.; Martinez, D.; Grigoriev, I., et al. Comparative transcriptome and secretome analysis of wood decay fungi Postia placenta and Phanerochaete chrysosporium. Appl. Environ. Microbiol. 2010, 76, 3599-3610.

89. Suzuki, M.R.; Hunt, C.G.; Houtman, C.J.; Dalebroux, Z.D.; Hammel, K.E. Fungal hydroquinones contribute to brown rot of wood. Environ. Microbiol. 2006, 8, 2214-2223.

90. Espejo, E.; Agosin, E. Production and degradation of oxalic acid by brown rot fungi. Appl. Environ. Microbiol. 1991, 57, 1980-1986.

91. Gomez-Toribio, V.; Garcia-Martin, A.B.; Martinez, M.J.; Martinez, A.T.; Guillen, F. Induction of extracellular hydroxyl radical production by white-rot fungi through quinone redox cycling. Appl. Environ. Microbiol. 2009, 75, 3944-3953. 


\title{
High-Resolution Respirometry for Simultaneous Measurement of Oxygen and Hydrogen Peroxide Fluxes in Permeabilized Cells, Tissue Homogenate and Isolated Mitochondria
}

\section{Marina Makrecka-Kuka, Gerhard Krumschnabel and Erich Gnaiger}

\begin{abstract}
Whereas mitochondria are well established as the source of ATP in oxidative phosphorylation (OXPHOS), it is debated if they are also the major cellular sources of reactive oxygen species (ROS). Here we describe the novel approach of combining high-resolution respirometry and fluorometric measurement of hydrogen peroxide $\left(\mathrm{H}_{2} \mathrm{O}_{2}\right)$ production, applied to mitochondrial preparations (permeabilized cells, tissue homogenate, isolated mitochondria). The widely used $\mathrm{H}_{2} \mathrm{O}_{2}$ probe Amplex Red inhibited respiration in intact and permeabilized cells and should not be applied at concentrations above $10 \mu \mathrm{M} . \mathrm{H}_{2} \mathrm{O}_{2}$ fluxes were generally less than $1 \%$ of oxygen fluxes in physiological substrate and coupling states, specifically in permeabilized cells. $\mathrm{H}_{2} \mathrm{O}_{2}$ flux was consistently highest in the Complex II-linked LEAK state, reduced with CI\&II-linked convergent electron flow and in mitochondria respiring at OXPHOS capacity, and were further diminished in noncoupled mitochondria respiring at electron transfer system capacity. Simultaneous measurement of mitochondrial respiration and $\mathrm{H}_{2} \mathrm{O}_{2}$ flux requires careful optimization of assay conditions and reveals information on mitochondrial function beyond separate analysis of ROS production.
\end{abstract}

Reprinted from Biomolecules. Cite as: Makrecka-Kuka, M.; Krumschnabel, G.; Gnaiger, E. High-Resolution Respirometry for Simultaneous Measurement of Oxygen and Hydrogen Peroxide Fluxes in Permeabilized Cells, Tissue Homogenate and Isolated Mitochondria. Biomolecules 2015, $5,1319-1338$.

\section{Introduction}

Mitochondrial reactive oxygen species (ROS) production contributes to both physiological and pathological processes and is essential in cell life and death decisions [1]. At low concentrations ROS promote the adaptation of cells to stress conditions by the regulation of oxidative metabolism, cellular differentiation and autophagy, thus supporting cell survival. Cellular concentrations of ROS are tightly regulated by cellular antioxidant defense systems. When the cellular antioxidant capacity is overwhelmed, ROS concentrations may increase dramatically and the resulting oxidative stress can cause substantial cell damage and ultimately cell death. Hence, both oxidative stress originating from mitochondrial activity and mitochondrial dysfunction ensuing from related oxidative damage have been shown to play important roles in aging and the pathogenesis of various disease states such as ischemia, neurodegeneration, diabetes and atherosclerosis (reviewed in [2-4]).

The magnitude of mitochondrial (mt) ROS production depends on the tissue type, the substrates metabolized, and the site of the mitochondrial electron transfer system (ETS) involved [5]. For the ETS it is quite generally agreed that Complexes I and III, but also the electron-transferring flavoprotein and glycerophosphate dehydrogenase complexes are the main sites of ROS production, 
particularly under conditions of high mt-membrane potential (reviewed in [6,7]). The primary chemical species of ROS produced by mitochondrial activity appears to be the superoxide anion, most of which is immediately converted to $\mathrm{H}_{2} \mathrm{O}_{2}$ by mitochondrial superoxide dismutase (MnSOD). ROS formation, therefore, can be detected with probes sensitive to $\mathrm{H}_{2} \mathrm{O}_{2}$. Hydrogen peroxide is comparatively stable and, due to its membrane permeability, accessible to such probes. Most of the studies investigating mtROS formation have been performed on isolated mitochondria, as methods employed in animals or cultured cells are considered to not provide accurate and quantitative results [8]. It is important, however, to relate these measurements to ROS production of mitochondria within their physiological microenvironment of the cell under actual in vivo conditions [9]. Furthermore, measurements of ROS formation are typically conducted in fluorometer cuvettes using buffers optimized for this purpose and generally not identical with the medium applied for determination of mitochondrial respiratory activity. This makes it impossible to accurately correlate ROS formation and mitochondrial energetics under identical conditions imposing a major uncertainty in fluorometric experiments. Continuous measurements of mitochondrial ROS production reported so far were almost always restricted to rather limited time periods of no more than $15 \mathrm{~min}$ [10-14] during which it is hardly possible to accurately evaluate multiple mitochondrial substrate and coupling states.

To overcome some of these limitations, we have recently characterized experimental and technical conditions required for the simultaneous determination of mitochondrial oxygen and $\mathrm{H}_{2} \mathrm{O}_{2}$ fluxes using the OROBOROS O2k-Fluorometer based on the Oxygraph-2k for high-resolution respirometry and the O2k-Fluo LED2-Module for the detection of $\mathrm{H}_{2} \mathrm{O}_{2}$ by Amplex ${ }^{\circledR}$ UltraRed [15]. In the present study we extended this approach to show applications for investigating $\mathrm{H}_{2} \mathrm{O}_{2}$ flux in permeabilized HEK $293 \mathrm{~T}$ cells, mouse brain homogenate, and isolated mouse heart mitochondria as experimental models, with application of substrate-uncoupler-inhibitor titration (SUIT) protocols to interrogate sequentially different substrate and coupling states (Table 1).

\section{Results and Discussion}

\subsection{Effect of Amplex Red on Respiration of Intact and Permeabilized HEK 293T Cells}

The use of Amplex Red or Amplex UltraRed (AmR) at concentrations up to $50 \mu \mathrm{M}$ is suggested by commercial suppliers for the determination of $\mathrm{H}_{2} \mathrm{O}_{2}$ production, but much lower concentrations down to $1 \mu \mathrm{M}$ have been applied successfully [12-14,20,21]. Since some fluorescence probes, e.g., mitochondrial membrane potential sensitive dyes (safranin, rhodamine), inhibit mitochondrial respiration [22,23], we considered it advisable to check for such undesired effects and to evaluate the optimal concentration of AmR prior to the actual experimental series. The dose-dependent effect of AmR on mitochondrial respiration is shown in Figures 1 and 2 for intact and permeabilized HEK 293 T cells. 
Table 1. Definitions of Substrate States and Coupling States used to Characterize Mitochondrial Energetics.

\begin{tabular}{|c|c|}
\hline Abbreviation & Definition \\
\hline CI-linked & $\begin{array}{l}\text { The Complex I-linked substrate state is induced in mt-preparations by addition of } \\
\text { NADH-generating substrates. }\end{array}$ \\
\hline CII-linked & $\begin{array}{l}\text { The Complex II-linked substrate state is induced in mt-preparations by addition of } \\
\text { succinate and rotenone (Complex I inhibitor). }\end{array}$ \\
\hline CI\&II-linked & $\begin{array}{l}\text { The Complex I\&II-linked substrate state is induced in mt-preparations by addition of } \\
\text { NADH-generating substrates (CI-linked) in combination with succinate (CII-linked). } \\
\text { This physiological substrate combination is required for partial reconstitution of TCA } \\
\text { cycle function and convergent electron-input into the Q-junction to compensate for } \\
\text { metabolite depletion into the incubation medium. An additive effect of convergent } \\
\text { CI\&II-linked electron flow is observed in most types of mitochondria. }\end{array}$ \\
\hline$R$ & $\begin{array}{l}\text { In the intact cell, ROUTINE respiration or ROUTINE activity in the physiological } \\
\text { coupling state } R \text { is controlled by cellular energy demand, energy turnover and the degree } \\
\text { of coupling to phosphorylation of ADP (intrinsic uncoupling and pathological } \\
\text { dyscoupling; [16]). }\end{array}$ \\
\hline$L$ & $\begin{array}{l}\text { LEAK respiration or LEAK oxygen flux compensating for proton leak, proton slip, } \\
\text { cation cycling and electron leak, is a dissipative component of respiration which is not } \\
\text { available for performing biochemical work and thus related to heat production. LEAK } \\
\text { respiration is measured in state } L \text {, in the presence of reducing substrate(s), but absence } \\
\text { of ADP (theoretically, absence of inorganic phosphate presents an alternative), or after } \\
\text { enzymatic inhibition of the phosphorylation system. In this non-phosphorylating resting } \\
\text { state, the electrochemical proton gradient is increased to a maximum, exerting feedback } \\
\text { control by depressing oxygen flux to a level determined mainly by the proton leak and the } \\
\mathrm{H}^{+} / \mathrm{O}_{2} \text { ratio [17]. }\end{array}$ \\
\hline$P$ & $\begin{array}{l}\text { OXPHOS capacity is the respiratory capacity of mitochondria in the ADP-activated state } \\
\text { of oxidative phosphorylation, at saturating concentrations of ADP, inorganic phosphate, } \\
\text { oxygen, and defined reduced substrates [17]. It thus differs from State } 3 \text { respiration } \\
\text { which } \\
\text { is respiration of isolated coupled mitochondria in the presence of high ADP and Pi } \\
\text { concentrations [18]. ADP concentrations applied in State } 3 \text { are not necessarily } \\
\text { saturating, whereas OXPHOS capacity is measured at saturating concentrations of ADP } \\
\text { and Pi. }\end{array}$ \\
\hline $\boldsymbol{E}$ & $\begin{array}{l}\text { The mitochondrial electron transfer system (ETS) transfers electrons from externally } \\
\text { supplied reduced substrates to oxygen. It consists of the membrane-bound ETS (mETS) } \\
\text { with enzyme complexes located in the inner mt-membrane, mt-matrix dehydrogenases } \\
\text { generating NADH, and the transport systems involved in metabolite exchange across the } \\
\text { mt-membranes [19]. ETS capacity is max. } \mathrm{O}_{2} \text { flux at optimum uncoupler concentration. }\end{array}$ \\
\hline
\end{tabular}


Intact HEK 293T cells
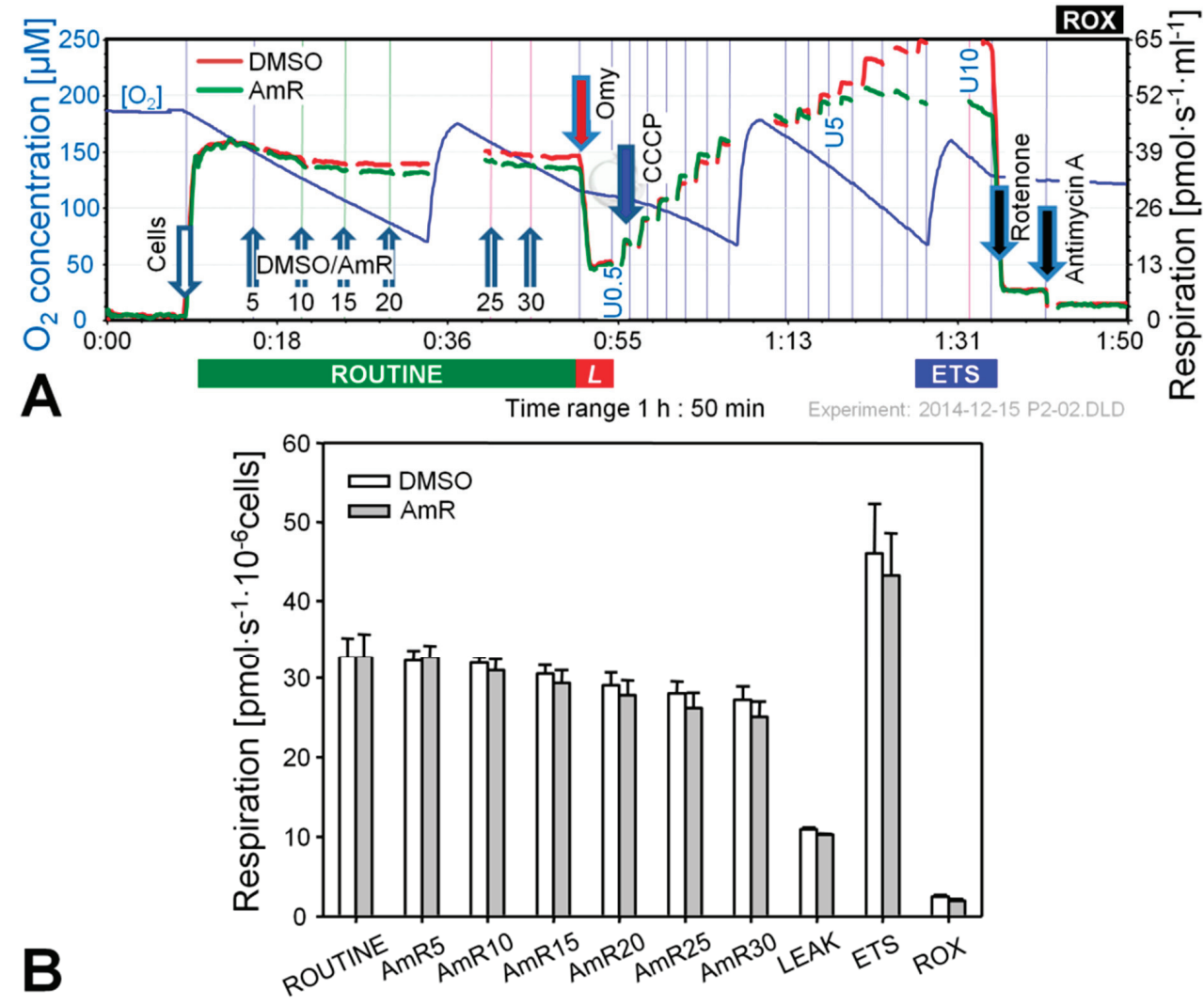

Figure 1. Effect of Amplex UltraRed (AmR) on respiration of intact HEK 293T cells. (A) Representative respiratory experiment with AmR or carrier DMSO titrated in the ROUTINE state. Oxygen concentration (blue plot; left $Y$-axis $[\mu \mathrm{M}]$ ) is shown for one chamber, whereas oxygen fluxes per chamber volume (red and green plots; right $Y$-axis $\left[\mathrm{pmol} \cdot \mathrm{s}^{-1} \cdot \mathrm{mL}^{-1}\right]$ ) are depicted for both $\mathrm{O} 2 \mathrm{k}$-chambers operated simultaneously. The horizontal bar denotes the respiratory states, ROUTINE; LEAK state, L; progressively uncoupled states in which ETS capacity is reached at maximum flux; and residual oxygen consumption, ROX. Numbers indicate final AmR concentrations $[\mu \mathrm{M}]$; U0.5, U5 and U10 indicate final uncoupler concentrations $[\mu \mathrm{M}]$, added in $0.5 \mu \mathrm{M}$ steps between 0.5 and $5 \mu \mathrm{M}$ and $1 \mu \mathrm{M}$ steps between 5 and $10 \mu \mathrm{M}$. Discontinuities of the plots are due to removal of sections with artifacts arising from titrations or re-oxygenations; (B) Oxygen flow expressed as respiration per million cells $\left[\mathrm{pmol} \cdot \mathrm{s}^{-1} \cdot 10^{-6}\right.$ cells], mean $\pm \mathrm{SE}$ of $N=3-5$ independent cultures, each measured in duplicate $(n=2)$. 

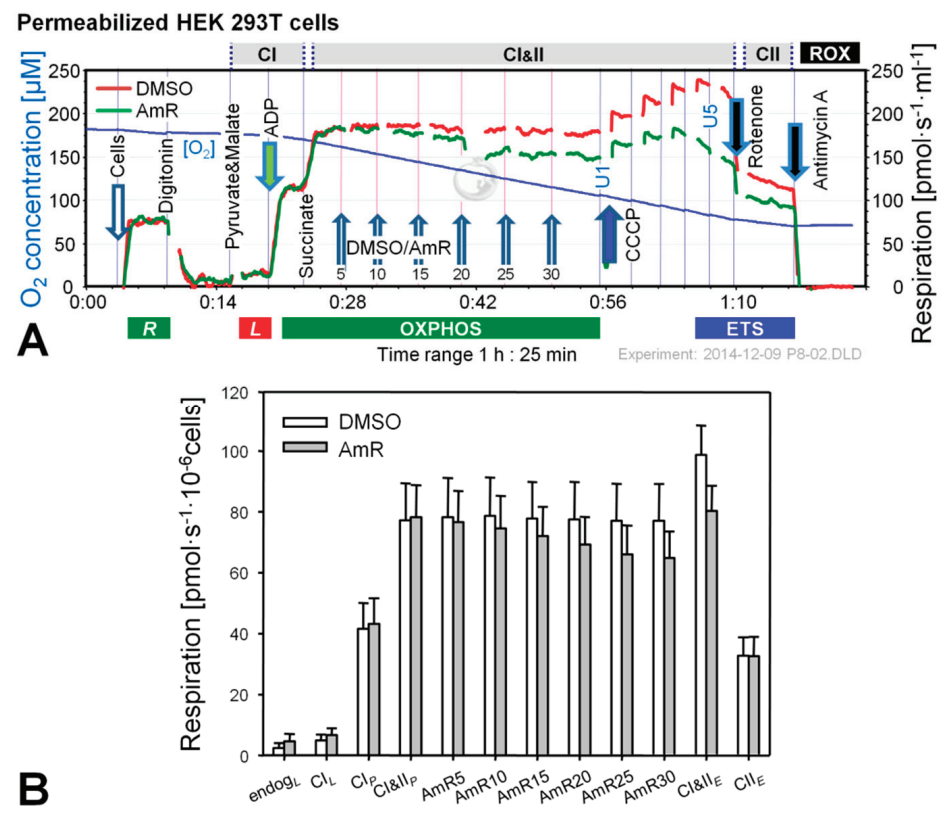

Figure 2. Effect of AmR on respiration of permeabilized HEK 293T cells. (A) Representative experiment with AmR (green plot) or carrier DMSO (red plot) titrated in the CI\&II-linked OXPHOS state. Substrate states and coupling states are shown by horizontal bars. Numbers indicate final Amp concentrations $[\mu \mathrm{M}]$; U1 and U5 indicate final uncoupler concentrations $[\mu \mathrm{M}]$ added in $1 \mu \mathrm{M}$ steps; (B) Oxygen flow $\left[\mathrm{pmol} \cdot \mathrm{s}^{-1} \cdot 10^{-6}\right.$ cells], mean $\pm \mathrm{SE}$ of $N=5$ independent cultures, each measured in duplicate $(n=2)$.

In the experiments with intact cells titrations with AmR were conducted in the ROUTINE state of respiration. ROUTINE respiration was not completely stable in controls for the duration of the carrier titrations (DMSO) which lasted approximately $35 \mathrm{~min}$. Titration of AmR caused a slight but non-significant further reduction of ROUTINE respiration by up to $8 \%$ at $30 \mu \mathrm{M}$ compared to time-matched controls (Figure 1B). Subsequent addition of oligomycin induced an immediate inhibition of respiration, and LEAK respiration was indistinguishable between both groups. To obtain a measure for ETS, i.e., the maximal capacity of the electron transfer system under the conditions examined, a step-by-step titration with uncoupler CCCP was performed showing that AmR caused a slight but variable, on average insignificant reduction of ETS capacity. Residual oxygen consumption, ROX, obtained after inhibition of Complexes I and III by rotenone (Rot) and antimycin A (Ama), respectively, was identical in controls and AmR-treated cells. Taken together, these results suggest that AmR may be used at concentrations up to $30 \mu \mathrm{M}$ to determine $\mathrm{H}_{2} \mathrm{O}_{2}$ production in intact HEK 293T cells with minor side effects on respiration. Exposure of these cells to $20 \mu \mathrm{M}$ AmR for more than $45 \mathrm{~min}$, however, caused about 13\% inhibition of ROUTINE respiration [24], suggesting that AmR concentrations and exposure time should be limited. The inhibitory effect depends on the medium used. Since the sensitivity of the AmR assay was rather low in DMEM, we applied Dulbecco's phosphate-buffered saline, in 
which case excellent assay sensitivity was associated with seriously compromised respiration, both with (up to $50 \%$ inhibition) and without $\mathrm{AmR}$ (up to $20 \%$ inhibition). A brief literature survey indicates that $\mathrm{AmR}$ concentrations and media applied are highly variable, ranging from $1 \mu \mathrm{M} \mathrm{AmR}$ in PBS for the study of permeabilized C2C12 myoblasts and myotubes [25], $10 \mu \mathrm{M}$ AmR in MiR05 for primary human skeletal myotubes [26], to $50 \mu \mathrm{M} \mathrm{AmR}$ in various phosphate-buffered or bicarbonate-buffered saline media investigating N27 cells [27], A549 lung epithelial cells [20], HaCaT keratinocytes [28], or HEK 293T cells [29], or mitochondria prepared from H9c2 rat cardiac myocytes [29] or from HepG2 cells [30]. In the absence of adequate controls it is not clear to which extent these treatments might have affected the results on $\mathrm{H}_{2} \mathrm{O}_{2}$ production. Therefore, careful optimization is required both with regard to AmR concentrations and the incubation media for quality control of measurements on $\mathrm{H}_{2} \mathrm{O}_{2}$ production in cells.

Experiments with permeabilized cells were performed in MiR05 [31] and AmR was titrated in the CI\&II-linked OXPHOS state, i.e., in the presence of pyruvate, malate and succinate at saturating concentration of ADP (Figure 2). Respiration in controls was not affected by the carrier DMSO. In contrast, AmR inhibited CI\&II-linked OXPHOS capacity in a dose-dependent manner, resulting in $15 \% \pm 7 \%$ inhibition at $30 \mu \mathrm{M}$ (mean $\pm \mathrm{SD}, N=5$ ). Similarly, CI\&II-linked ETS capacity was reduced by $19 \% \pm 8 \%$, with a shift to a lower optimum uncoupler concentration compared to controls. CII-linked ETS capacity was not affected, possibly indicating that AmR inhibition occurred at CI, which is highly sensitive to agents damaging mitochondria $[22,32,33]$. In order to minimize such side effects, the AmR concentration was reduced to $10 \mu \mathrm{M}$ for experiments with permeabilized cells.

\section{2. $\mathrm{O}_{2}$ and $\mathrm{H}_{2} \mathrm{O}_{2}$ Flow in Permeabilized HEK 293 T Cells: Dependence on Substrate and}

\section{Coupling State}

$\mathrm{O}_{2}$ and $\mathrm{H}_{2} \mathrm{O}_{2}$ fluxes were determined in permeabilized HEK 293T cells in a sequence of respiratory substrate and coupling states using pyruvate\&malate (PM; CI), pyruvate\&malate\&succinate (PMS; CI\&II) or succinate with Rot (S(Rot)) as respiratory substrates (Figure 3). Results are summarized in Figure 4 and Table 2. The $\mathrm{H}_{2} \mathrm{O}_{2} / \mathrm{O}$ flux ratio is frequently applied to evaluate the relative importance of $\mathrm{H}_{2} \mathrm{O}_{2}$ production at different respiratory states [34,35].

After permeabilization of the cell membranes with digitonin, addition of PM as substrates supporting CI-linked LEAK respiration $\left(\mathrm{CI}_{L}\right)$ induced a moderate increase in respiration (Figure $3 \mathrm{~A}$ ). ADP added at a saturating concentration stimulated respiration about 5 times (CI-linked OXPHOS capacity, $\left.\mathrm{CI}_{P}\right)$. Succinate induced convergent CI\&II-linked OXPHOS (CI\&II $P$ ), at a 2.2-fold higher level compared to $\mathrm{CI}_{P}$. CCCP at optimum concentration elevated respiration further, showing a significant apparent excess ETS capacity in these cells. Subsequently Rot and Ama were added to obtain ROX. Despite these dramatic differences in oxygen fluxes in different respiratory states, alteration in $\mathrm{H}_{2} \mathrm{O}_{2}$ fluxes were comparatively small. When total observed $\mathrm{H}_{2} \mathrm{O}_{2}$ flux was corrected for the background chemical flux obtained in the absence of cells, the net fluxes were negative. This may indicate the ROS scavenging capacity introduced with the cells, as will be discussed below. Thus, even the most pronounced relative change in $\mathrm{H}_{2} \mathrm{O}_{2}$ flux induced by inhibition of CIII (Ama) after ETS was close to the chemical background. As an alternative approach, the lowest flux detected in each experimental run was subtracted from $\mathrm{H}_{2} \mathrm{O}_{2}$ flux in each respiratory state, expressing $\mathrm{H}_{2} \mathrm{O}_{2}$ flux 
not as an absolute metabolic flux but as a difference, $\Delta \mathrm{H}_{2} \mathrm{O}_{2}$ flux (Figure 4C). The lowest $\mathrm{H}_{2} \mathrm{O}_{2}$ flux was observed in fully uncoupled cells in almost all cases.
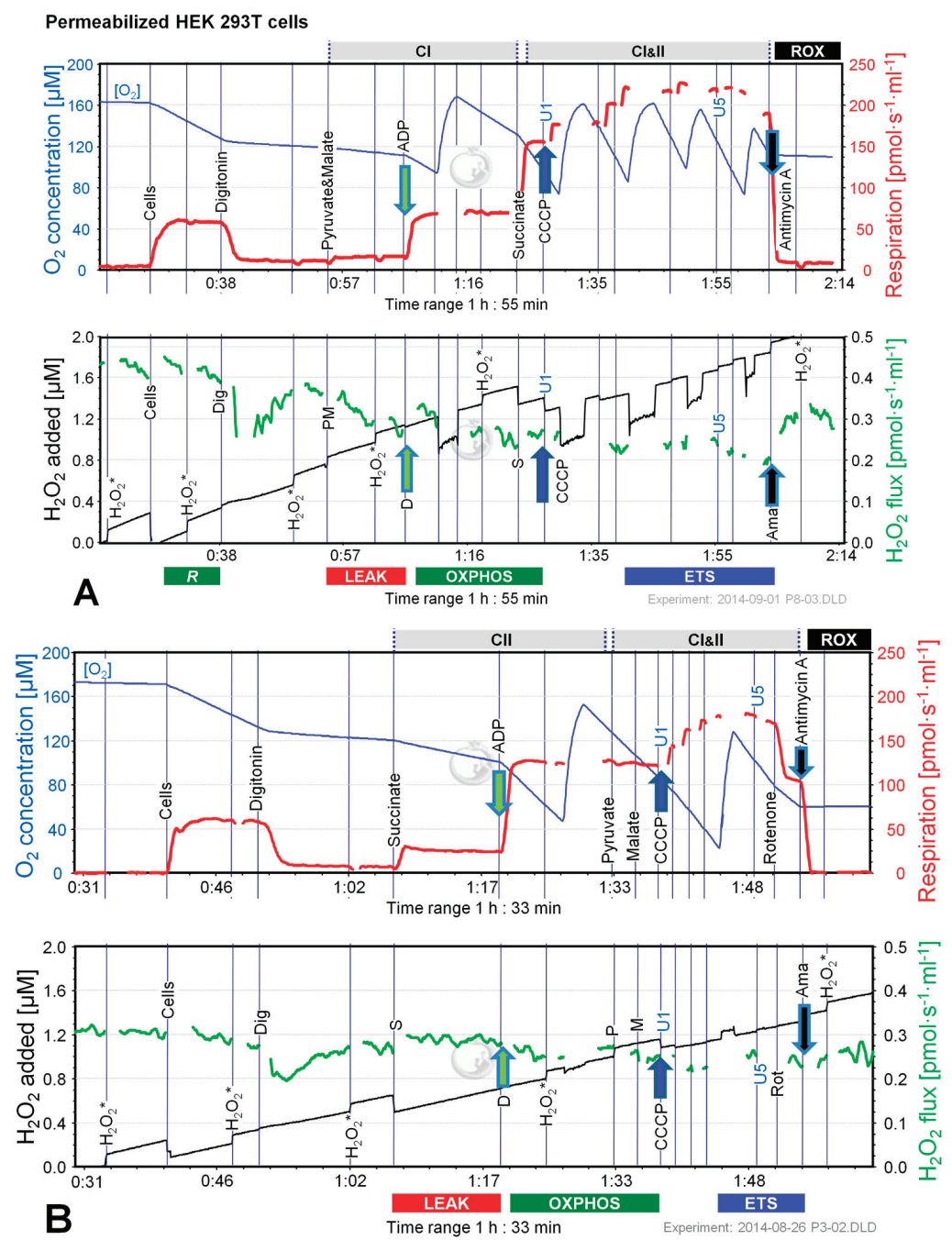

Figure 3. Combined determination of oxygen consumption and $\mathrm{H}_{2} \mathrm{O}_{2}$ flux by O2k-Fluorometry in permeabilized HEK 293T cells. (A) Respiration and fluorescence changes using P and M as initial substrates; (B) Respiration and fluorescence changes using succinate (S) as initial substrate. Respirometric measurements are shown in the upper panels as described in Figure 1. U1 and U5 indicate final uncoupler concentrations $[\mu \mathrm{M}]$ added in $1 \mu \mathrm{M}$ steps. In the lower panels the black plots show the fluorescence signal. $\mathrm{H}_{2} \mathrm{O}_{2}$ * indicates titrations of $0.1 \mu \mathrm{M} \mathrm{H}_{2} \mathrm{O}_{2}$ for calibration, to convert the fluorescence signal to an equivalent $\mathrm{H}_{2} \mathrm{O}_{2}$ concentration (left $Y$-axis $[\mu \mathrm{M}]$ ). Plots are shown on the basis of the first calibration with $\mathrm{H}_{2} \mathrm{O}_{2}$. The positive time derivative yields the volume-specific $\mathrm{H}_{2} \mathrm{O}_{2}$ flux shown as the green plots (right $Y$-axis $\left[\mathrm{pmol} \cdot \mathrm{s}^{-1} \cdot \mathrm{mL}^{-1}\right]$ ). 

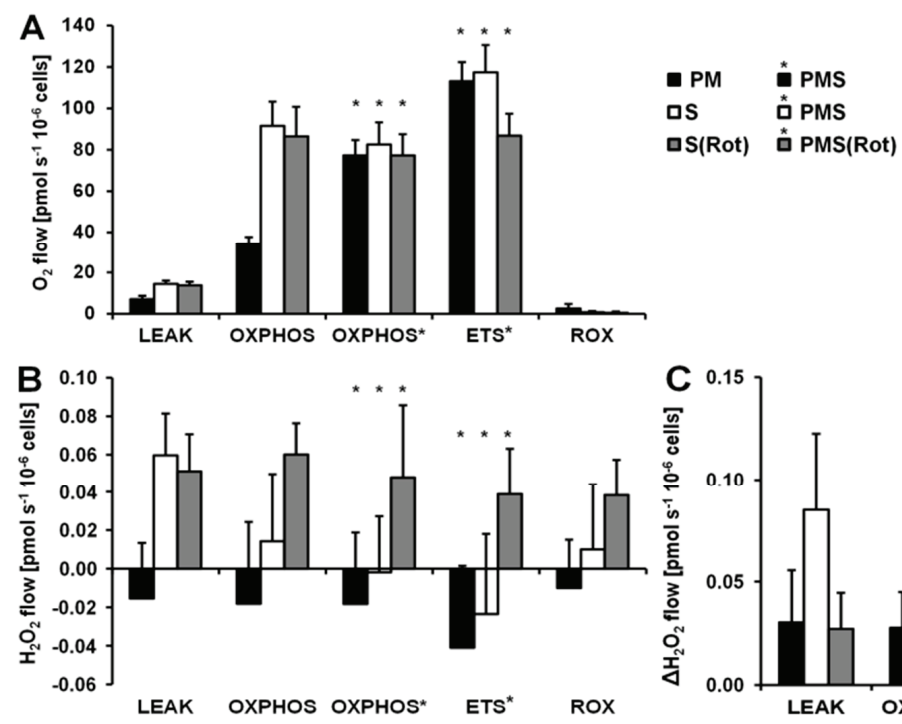

Figure 4. Respiration and $\mathrm{H}_{2} \mathrm{O}_{2}$ flow in permeabilized HEK 293T cells in SUIT protocols using PM (black bars), S (white bars) and S(Rot) (grey bars) as initial substrates. (A) Oxygen flow; (B) $\mathrm{H}_{2} \mathrm{O}_{2}$ flow corrected for the background slope determined in the absence of cells; (C) $\mathrm{H}_{2} \mathrm{O}_{2}$ flow corrected for the lowest observed slope. The fluorescence signals were calibrated using the $\mathrm{H}_{2} \mathrm{O}_{2}$ titrations at the corresponding state (Figure 3). Bars are means $\pm \mathrm{SD}$ of four independent cultures measured in duplicates.

Table 2. $\mathrm{H}_{2} \mathrm{O}_{2} / \mathrm{O}$ flux ratios [\%] as a function of coupling and substrate states in permeabilized HEK cells and mouse brain homogenate.

\begin{tabular}{cccccc}
\hline Substrate & LEAK & OXPHOS & PMS OXPHOS & PMS ETS & ROX \\
\hline \multicolumn{7}{c}{ Permeabilized HEK cells } \\
\hline S & $0.85 \pm 0.34$ & $0.03 \pm 0.08$ & na & na & $7.2 \pm 22.8$ \\
S(Rot) & $0.81 \pm 0.55$ & $0.14 \pm 0.07$ & $0.16 \pm 0.07$ & $0.10 \pm 0.7$ & $7.9 \pm 3.4$ \\
\hline \multicolumn{7}{c}{ Mouse brain homogenate } \\
\hline PM & $1.2 \pm 0.6$ & $0.06 \pm 0.13$ & na & na & $60 \pm 13$ \\
S & $6.3 \pm 0.2$ & $0.05 \pm 0.13$ & $0.05 \pm 0.09$ & na & $26 \pm 6$ \\
S(Rot) & $1.5 \pm 0.2$ & $0.51 \pm 0.08$ & $1.34 \pm 0.09$ & $1.24 \pm 0.06$ & $33 \pm 8$ \\
\hline
\end{tabular}

The $\mathrm{H}_{2} \mathrm{O}_{2} / \mathrm{O}$ flux ratio was calculated as $\mathrm{H}_{2} \mathrm{O}_{2}$ flux $/\left(0.5 \mathrm{O}_{2}\right.$ flux $)$. Means $\pm \mathrm{SD}$ of 4 independent cultures or 3 animals. na-not applicable.

LEAK respiration supported by succinate $\left(\mathrm{CII}_{L}\right)$ was about three times higher than $L$ supported by $\mathrm{PM}\left(\mathrm{CI}_{L}\right)$ and was associated with much higher $\mathrm{H}_{2} \mathrm{O}_{2}$ flux in the absence of rotenone. ADP induced a 6.7-fold increase in respiration $\left(\mathrm{CII}_{P}\right)$ and a concurrent pronounced reduction in $\mathrm{H}_{2} \mathrm{O}_{2}$ flux (Figure $3 \mathrm{~B}$ ). Surprisingly, addition of pyruvate did not stimulate respiration, and malate caused a slight inhibition, such that $\mathrm{CI} \& \mathrm{II} P$ respiration was slightly reduced compared to $\mathrm{CII} P$, whereas $\mathrm{H}_{2} \mathrm{O}_{2}$ flux remained constant. The fact that rotenone was not required to obtain a high CII-linked OXPHOS capacity is 
consistent with a high malic enzyme activity in these cells [36]. Thus, oxaloacetate does not accumulate to concentrations which inhibit succinate dehydrogenase, but pyruvate and further acetyl-CoA are formed from malate, supporting the utilization of oxaloacetate in the citrate synthase reaction $[19,37]$. Formation of NADH and its utilization by $\mathrm{CI}$, therefore, proceeded in the CII-linked OXPHOS state, and CI\&II-linked and CII-linked OXPHOS capacities were not different (Figure 4). However, CI\&II $P$ was limited by the capacity of the phosphorylation system, as shown by the increased ETS capacity with PMS (CI\& $\left.\mathrm{II}_{E}\right)$ which was higher than $\mathrm{CII}_{E}$, indicating the additive effect of convergent electron flow from CI\&II to the Q-junction in these cells [38]. CCCP titration reduced the $\mathrm{H}_{2} \mathrm{O}_{2}$ flux to a level slightly below baseline. As observed in the previous protocol, Ama induced an increase in $\mathrm{H}_{2} \mathrm{O}_{2}$ flux (Figure 4).

Respiration and $\mathrm{H}_{2} \mathrm{O}_{2}$ flux in the LEAK state were similar with $\mathrm{S}$ (Rot) and $\mathrm{S}$ alone (Figure 4). In many other cells and tissues (e.g., rat brain homogenate as shown below), Rot causes a significant reduction of $\mathrm{H}_{2} \mathrm{O}_{2}$ production observed in the CII-linked LEAK state due to inhibition of reversed electron flow to CI [39]. The absence of such an effect is consistent with the effect of malic enzyme on respiration [19]. In contrast to the presence of S alone, addition of ADP to S(Rot) exposed cells did not decrease the $\mathrm{H}_{2} \mathrm{O}_{2}$ flux. The effects of PM and CCCP were relatively small, and the addition of Ama did not stimulate ROS production, in contrast to results obtained with the other protocols (Figure 4).

Taken together, in permeabilized HEK 293T cells the highest $\mathrm{H}_{2} \mathrm{O}_{2}$ flux was observed in the CII-linked LEAK state, amounting to about $0.8 \%$ of oxygen flux (Table 2 ). In comparison, CI-linked $\mathrm{H}_{2} \mathrm{O}_{2}$ flux appears negligible, while inhibition of CI increased $\mathrm{H}_{2} \mathrm{O}_{2}$ production independent of respiratory state. $\mathrm{H}_{2} \mathrm{O}_{2}$ flux in permeabilized HEK $293 \mathrm{~T}$ cells was extremely low under the presently investigated conditions, accounting for less than $0.2 \%$ of $\mathrm{O}_{2}$ flux in the OXPHOS and ETS states (Table 2).

\section{3. $\mathrm{O}_{2}$ and $\mathrm{H}_{2} \mathrm{O}_{2}$ Flow in Permeabilized HEK 293T Cells: Dependence on Cell Density}

Given that estimated $\mathrm{H}_{2} \mathrm{O}_{2}$ fluxes were close to or even below background observed in the absence of cells, the sensitivity of the AmR assay may present a limiting factor at the experimental cell density. By increasing the cell density in the respiration chamber, however, the slopes of the fluorescence signal corrected for the background determined in the absence of cells actually displayed an inverse relation to cell density (Figure 5A). In contrast, when corrected for the slope observed in the presence of digitonin permeabilized cells without substrates added, net $\mathrm{H}_{2} \mathrm{O}_{2}$ fluxes were largely independent of cell density (Figure 5B). Importantly, cell-specific respiration and net $\mathrm{H}_{2} \mathrm{O}_{2}$ flow (per million cells) were independent of cell density (Figure 5C,D). A tentative explanation for these observations is that by increasing the number of cells in the chamber we also enhance the total ROS scavenging capacity associated with the cellular antioxidant systems. In addition, the optical properties are affected by cell density, as shown by the step change of the fluorescence signal upon injection of cells, and by the change of sensitivity when comparing calibrations before and after addition of cells, and after titration of digitonin. For example, sensitivity declined from $0.282 \pm 0.019 \mathrm{~V} / \mu \mathrm{M}$ to $0.260 \pm 0.019 \mathrm{~V} / \mu \mathrm{M}$ after addition of cells ( $n=8$ experiments), from $0.249 \pm 0.033 \mathrm{~V} / \mu \mathrm{M}$ to $0.179 \pm 0.018 \mathrm{~V} / \mu \mathrm{M}$ after adding brain tissue homogenate 
( $n=8$ experiments), and from $0.262 \mathrm{~V} / \mu \mathrm{M}$ to $0.250 \mathrm{~V} / \mu \mathrm{M}$ after injection of mitochondria (means of two experiments). Whereas calibration of the fluorescence signal in the absence of cells is required to obtain the apparent background flux, $\mathrm{H}_{2} \mathrm{O}_{2}$ calibrations are required in the presence of cells and under various respiratory states (Figure 3 ).
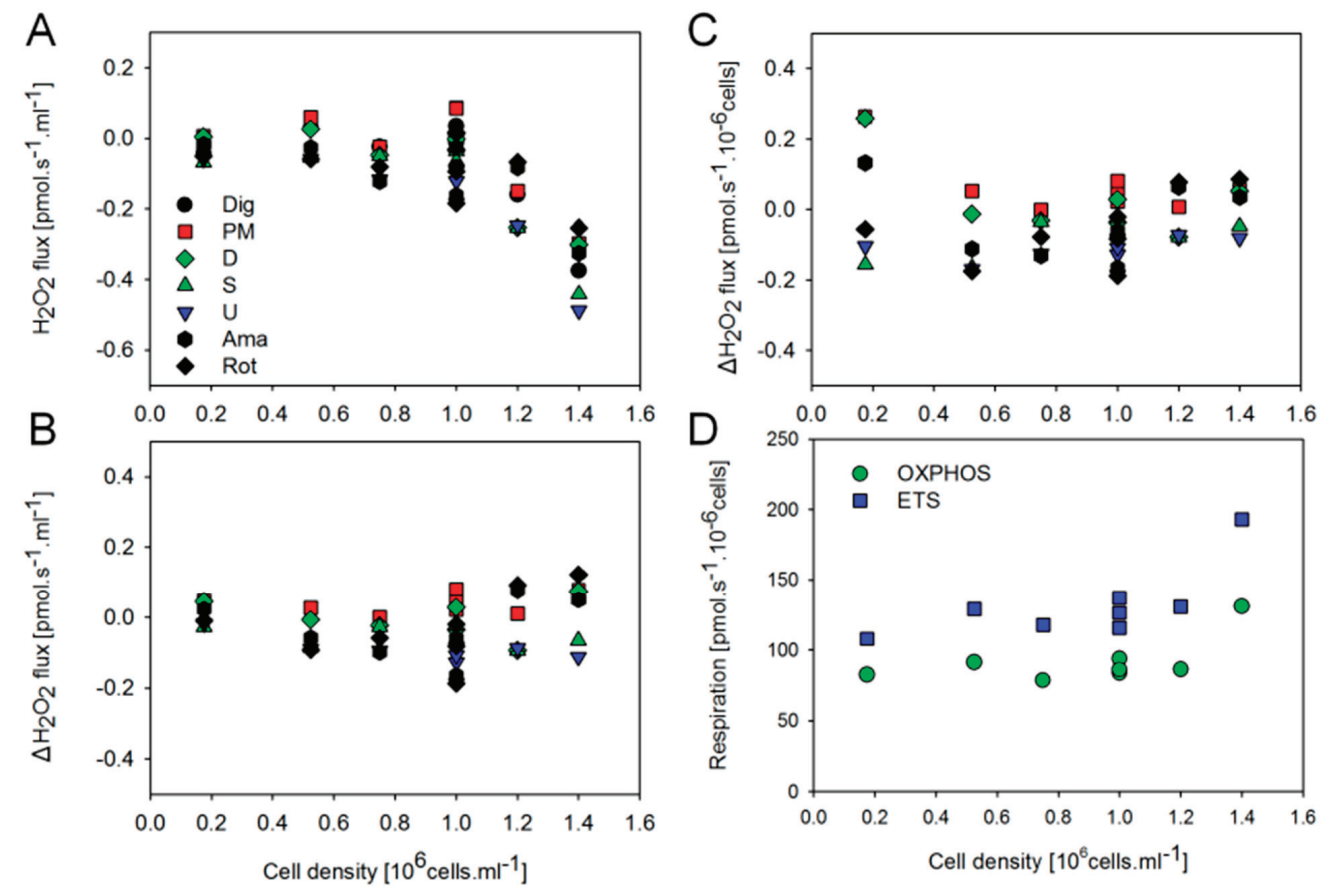

Figure 5. Effect of cell concentration in the $\mathrm{O} 2 \mathrm{k}$-chamber on $\mathrm{H}_{2} \mathrm{O}_{2}$ flux and respiration of permeabilized HEK $293 \mathrm{~T}$ cells. (A) $\mathrm{H}_{2} \mathrm{O}_{2}$ flux (per O2k-chamber volume) corrected for the slope determined in the absence of cells; (B) $\mathrm{H}_{2} \mathrm{O}_{2}$ flux (per O2k-chamber volume) corrected for the slope after addition of digitonin; (C) $\mathrm{H}_{2} \mathrm{O}_{2}$ flow (per million cells) corrected for the slope after addition of digitonin; (D) Respiratory flow (per million cells). $\mathrm{H}_{2} \mathrm{O}_{2}$ fluxes were calculated according to calibrations after addition of digitonin.

\section{4. $\mathrm{O}_{2}$ and $\mathrm{H}_{2} \mathrm{O}_{2}$ Flux in Mouse Brain Homogenate: Dependence on Substrate and Coupling State}

Figure 6 shows representative experiments of respiration and $\mathrm{H}_{2} \mathrm{O}_{2}$ fluxes in mouse brain homogenate with identical SUIT protocols as applied with permeabilized HEK 293T cells (Figure 3). In contrast to permeabilized HEK $293 \mathrm{~T}$ cells (Figure 4), $\mathrm{CII}_{L}$ respiration was lower but $\mathrm{CII}_{P}$ was higher with $\mathrm{S}$ (Rot) compared to $\mathrm{S}$ (Figure 7A). Addition of PM to S induced an increase of respiratory OXPHOS capacity $\left(\mathrm{CI} \& \mathrm{II}_{P}\right)$, despite of the inhibitory effect of malate (Figure $\left.6 \mathrm{~B}\right)$. Consistent with results in permeabilized cells, malate caused a significant decrease of $\mathrm{CII}_{P}$ respiration with S(Rot) (Figure 6C). The inhibitory effect of malate on CII-linked respiration is a general feature of TCA cycle control [40]. 
In contrast to results with permeabilized cells, $\mathrm{H}_{2} \mathrm{O}_{2}$ flux in state $\mathrm{Cl}_{L}$ was well above background in brain homogenate (Figure 6A). As in permeabilized HEK 293T cells, the highest $\mathrm{H}_{2} \mathrm{O}_{2}$ flux was observed in the LEAK state with $\mathrm{S}$ alone (Figure $6 \mathrm{~B}$ ), and $\mathrm{H}_{2} \mathrm{O}_{2}$ flux was significantly reduced in the OXPHOS and ETS state compared to LEAK (Figure 7B). However, addition of Rot caused a significant reduction of $\mathrm{H}_{2} \mathrm{O}_{2}$ flux, as did addition of ADP (Figure 7B). Compared to $\mathrm{CI}_{L}, \mathrm{H}_{2} \mathrm{O}_{2}$ flux in $\mathrm{CII}(\mathrm{Rot})_{L}$ was 2.5 times higher (Figure 7B). No significant difference in $\mathrm{H}_{2} \mathrm{O}_{2}$ flux was noted between LEAK and OXPHOS states with S(Rot). Surprisingly, addition of PM in state CII(Rot) $P$ caused an increase of $\mathrm{H}_{2} \mathrm{O}_{2}$ flux, although Complex I was inhibited by rotenone (Figures 6A and 7B). As expected [41], inhibition of CIII by Ama ( $\mathrm{ROX}$ state) caused an increase in $\mathrm{H}_{2} \mathrm{O}_{2}$ flux in all SUIT protocols (Figures 6 and 7B). $\mathrm{H}_{2} \mathrm{O}_{2}$ flux resulted in similar patterns when corrected for background without homogenate (Figure 7B) or when presented as the difference of flux after subtraction of the minimal observed slope (Figure 7C).

The extraordinarily high $\mathrm{H}_{2} \mathrm{O}_{2} / \mathrm{O}$ flux ratio of $6 \%$ in $\mathrm{CII}_{L}$ without Rot reflects maximal reversed electron transfer under an artificial substrate condition (Table 2). $\mathrm{H}_{2} \mathrm{O}_{2} / \mathrm{O}$ flux ratios between $0.05 \%$ and $1.35 \%$ in OXPHOS and ETS states (Table 2) are consistent with values reported for isolated mitochondria $[1,11,42]$. It is thought that isolation procedures may impact on mitochondrial function (e.g., $[43,44])$. Isolated mitochondrial preparations may represent a selection for particular mitochondrial subpopulations. This is not the case in tissue homogenate or permeabilized tissue preparations. Tissue homogenization can be achieved without injury of the outer mitochondrial membrane using specifically dedicated instruments such as the PBI tissue shredder [45,46]. A more simple glass Potter homogenizer can be used similarly for homogenate preparation of soft tissues.
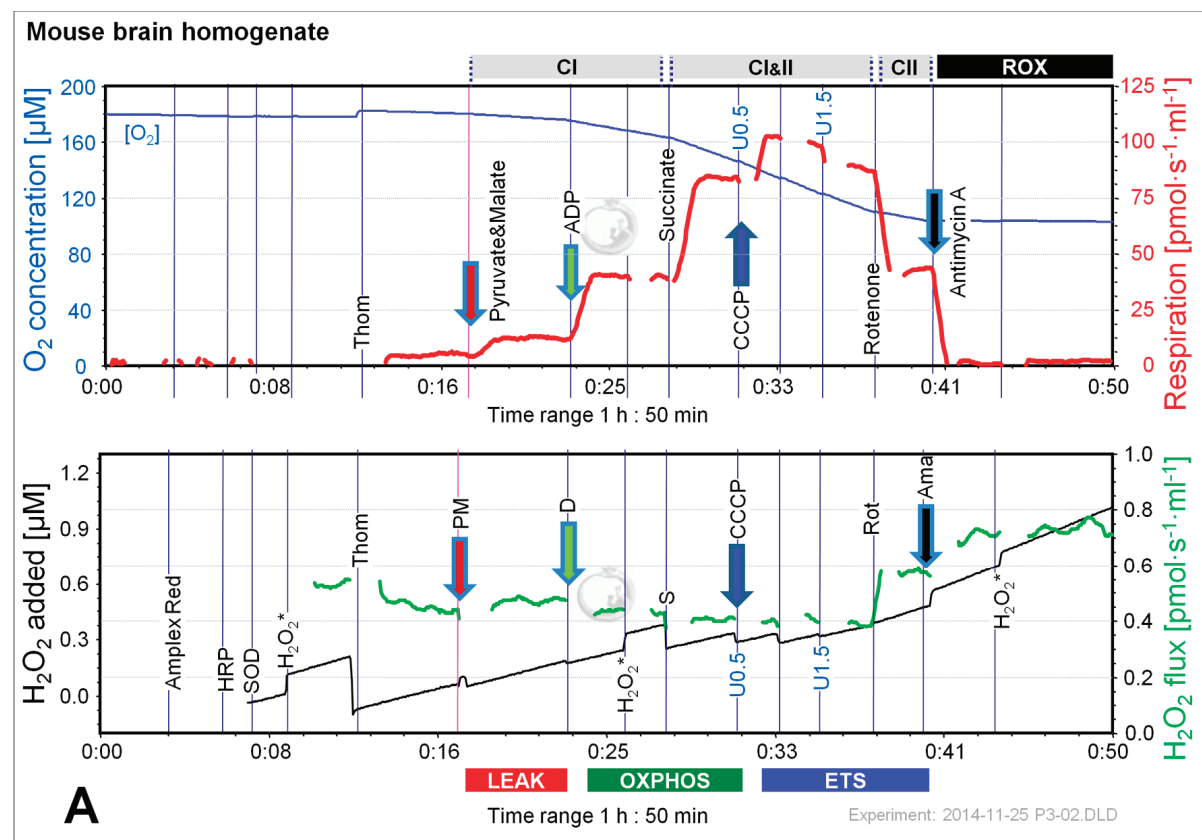

Figure 6. Cont. 

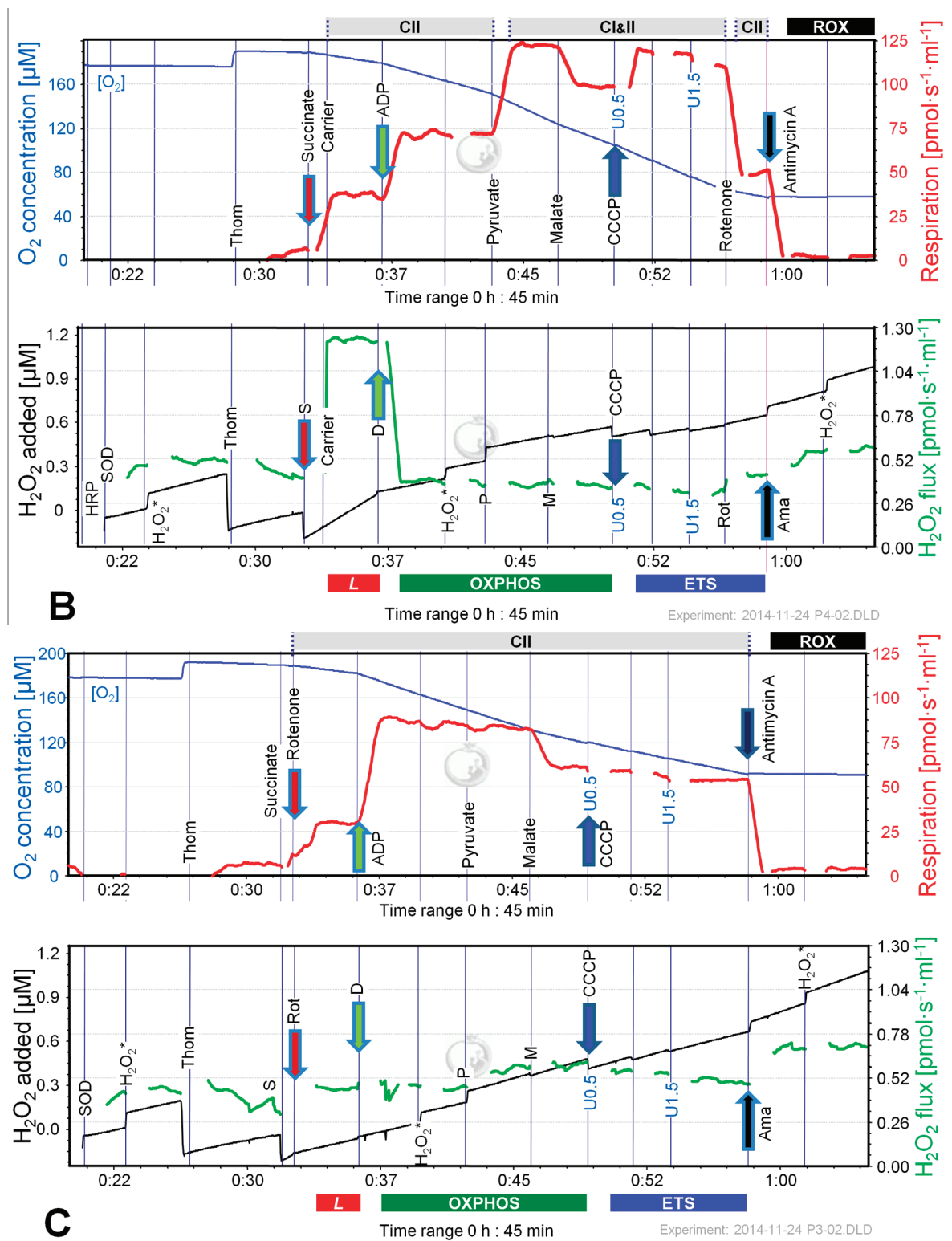

Figure 6. Combined determination of oxygen consumption and $\mathrm{H}_{2} \mathrm{O}_{2}$ flux by O2k-Fluorometry in mouse brain homogenate, using PM (A); S (B); or S(Rot) as initial substrate $(\mathbf{C})$. For details see legend of Figure 3. Carrier denotes ethanol $(1 \mu \mathrm{L})$, which served as the solvent for rotenone and was titrated in parallel to rotenone.

Few comparable data are published on permeabilized cells. From data by Kwak et al. [26] on permeabilized human skeletal myotubes it can be calculated that about $0.9 \%$ of LEAK respiration measured in the presence of a complex substrate mixture was diverted towards $\mathrm{H}_{2} \mathrm{O}_{2}$ production, in 
close agreement with our result on $0.8 \%$ for HEK 293 T cells in the presence of succinate (Table 2). In permeabilized yeast cells the $\mathrm{H}_{2} \mathrm{O}_{2} / \mathrm{O}$ ratio varied between $0.25 \%$ and $2 \%$ depending on the glucose level supplied and the growth phase investigated [47]. The $\mathrm{H}_{2} \mathrm{O}_{2} / \mathrm{O}$ flux ratio determined in a mutant $E$. coli strain was $0.35 \%$ to $0.6 \%$ in different substrate regimes [48].
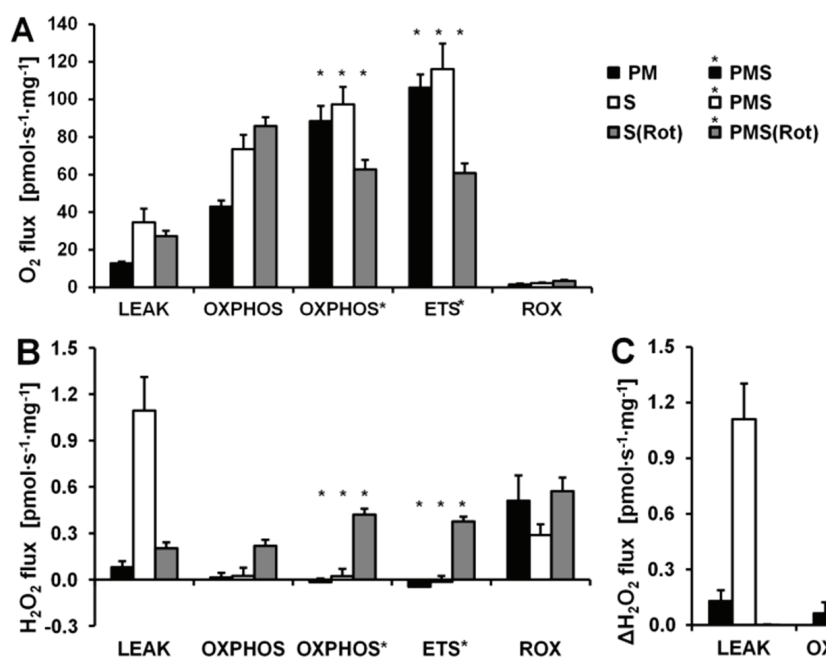

Figure 7. Respiration and $\mathrm{H}_{2} \mathrm{O}_{2}$ flux in mouse brain homogenate in SUIT protocols using PM (black bars), S (white bars) and S(Rot) (grey bars) as initial substrates. (A) Oxygen flux. (B) $\mathrm{H}_{2} \mathrm{O}_{2}$ flux corrected for the slope determined in the absence of homogenate; (C) $\mathrm{H}_{2} \mathrm{O}_{2}$ flux corrected for minimal observed slope. The fluorescence signals were calibrated using the $\mathrm{H}_{2} \mathrm{O}_{2}$ titrations at the corresponding state (Figure 6). Bars are means \pm SD of 3 animals, each measured in duplicate.

\section{5. $\mathrm{O}_{2}$ and $\mathrm{H}_{2} \mathrm{O}_{2}$ Flux in Isolated Cardiac Mitochondria: Dependence on Substrate and}

\section{Coupling State}

Since the most pronounced changes of $\mathrm{H}_{2} \mathrm{O}_{2}$ flux in permeabilized cells and brain homogenate were observed in protocols using $\mathrm{S}$ and $\mathrm{S}(\mathrm{Rot})$, we performed additional experiments applying CII-linked protocols with mitochondria isolated from mouse hearts. Like in mouse brain homogenate, the $\mathrm{H}_{2} \mathrm{O}_{2}$ flux was extremely high in the succinate-supported LEAK state in the absence of Rot, resulting in a $\mathrm{H}_{2} \mathrm{O}_{2} / \mathrm{O}$ flux ratio of nearly $10 \%$, compared to $1.5 \%$ in the presence of Rot (Figure 8). The addition of $\mathrm{ADP}$ to $\mathrm{S}$ (Rot) stimulated respiration and concurrently reduced $\mathrm{H}_{2} \mathrm{O}_{2}$ flux (Figure $8 \mathrm{~B}$ ). In contrast, addition of ADP to $\mathrm{S}$ did not increase respiration, but dramatically diminished $\mathrm{H}_{2} \mathrm{O}_{2}$ flux (Figure 8A). The subsequent addition of $\mathrm{P}$ caused a pronounced increase of respiratory OXPHOS capacity, by removing the inhibitory oxaloacetate and restoring CI\&II-linked TCA cycle activity [19]. Malate exerted an inhibitory effect in the presence and absence of Rot, similar to results with brain homogenate (Figure 6B,C). Uncoupler did not stimulate respiration beyond OXPHOS capacity, indicating that there is no apparent ETS excess capacity in mouse heart in contrast to mouse brain mitochondria. $\mathrm{H}_{2} \mathrm{O}_{2}$ fluxes were slightly elevated by $\mathrm{P}$ in both protocols, largely unresponsive to $\mathrm{M}$, 
and slightly diminished by uncoupler, while Rot and Ama caused a substantial increase of $\mathrm{H}_{2} \mathrm{O}_{2}$ flux (Figure $8 \mathrm{~A}, \mathrm{~B}$ ). $\mathrm{H}_{2} \mathrm{O}_{2} / \mathrm{O}$ flux ratios ranged from $0.04 \%$ in ETS in the absence of Rot to $0.9 \%$ in OXPHOS and ETS in the presence of Rot, consistent with data reported in the literature [5,49].

Isolated mouse heart mitochondria
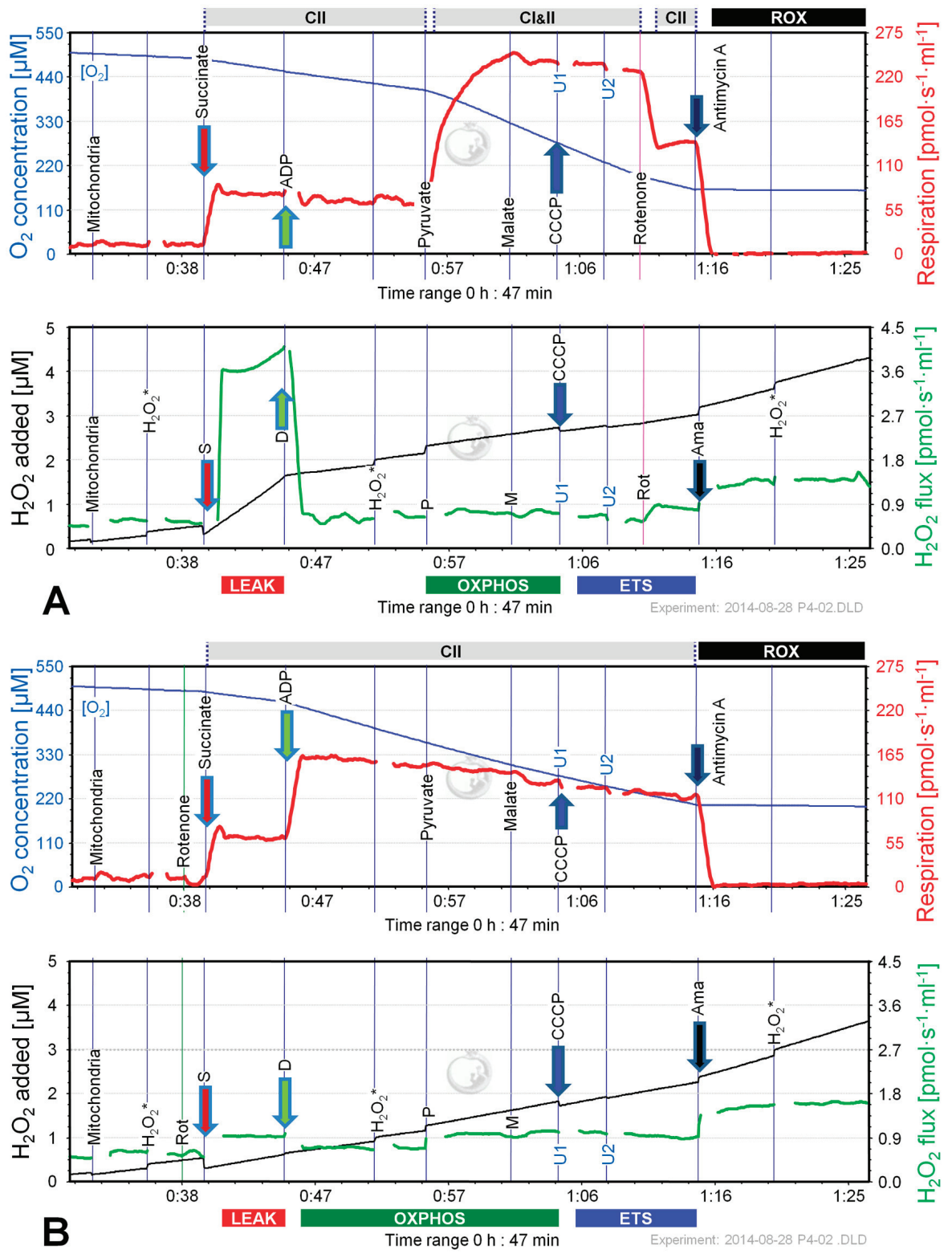

Figure 8. Combined determination of oxygen consumption and $\mathrm{H}_{2} \mathrm{O}_{2}$ production by O2k-Fluorometry in mouse isolated cardiac mitochondria, using S (A); or S(Rot) (B) as initial substrate. For details see legend of Figure 3. 


\section{Experimental Section}

\subsection{Chemicals}

Dulbecco's modified eagle medium (DMEM-low glucose, with L-glutamine) was from PAA Laboratories $\mathrm{GmbH}$, Pasching, Austria, fetal bovine serum from Biowest, Nuaillé, France, and penicillin and streptomycin stocks were from Gibco, Vienna, Austria.

Amplex ${ }^{\circledR}$ UltraRed was obtained from Life Technologies. $\mathrm{H}_{2} \mathrm{O}_{2}$, HRP, SOD, substrates, inhibitors and other chemicals were from Sigma-Aldrich, Acros Organics or Invitrogen [50].

\subsection{High-Resolution Respirometry and O2k-Fluorometry}

The Oxygraph-2k (O2k, OROBOROS Instruments, Innsbruck, Austria) was used for measurements of respiration [50] and combined with the Fluorescence-Sensor Green of the O2k-Fluo LED2-Module for $\mathrm{H}_{2} \mathrm{O}_{2}$ measurement. Up to four $\mathrm{O} 2 \mathrm{k}$ instruments (eight chambers) were used in parallel. Experiments using tissue homogenate and permeabilized cells were performed in MiR05 (110 mM

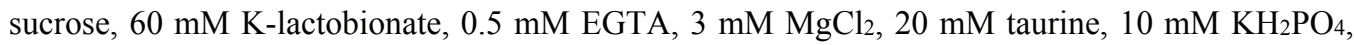
$20 \mathrm{mM}$ HEPES, pH 7.1 at $30{ }^{\circ} \mathrm{C}$, and $0.1 \%$ BSA essentially fatty acid free; [31]). Dulbecco's modified eagle medium supplemented with $10 \%$ fetal bovine serum and 50 units $/ \mathrm{mL}$ penicillin and $50 \mu \mathrm{g} / \mathrm{mL}$ streptomycin was used for measurements on intact cells. All experiments were performed at $37{ }^{\circ} \mathrm{C}$. The medium was reoxygenated when oxygen concentrations reached $80 \mu \mathrm{M}$ unless otherwise indicated.

\subsection{Experimental Procedure}

Respiration of permeabilized cells and tissue homogenate was determined using substrate-uncoupler-inhibitor titration (SUIT) protocols [50] with modifications. Pyruvate and malate $(5 \mathrm{mM}$ and $0.5 \mathrm{mM}$, respectively) or succinate $(10 \mathrm{mM})$ with or without $0.5 \mu \mathrm{M}$ Complex I inhibitor rotenone (Rot) were used to determine Complex I (CI) or Complex II (CII) linked LEAK respiration. ADP was added at $2.5 \mathrm{mM}$ final concentration, which was saturating for oxygen flux to obtain OXPHOS capacity. Additional substrates were added sequentially to reconstitute convergent CI\&II-linked respiration. Titrations with the uncoupler CCCP $(0.5-1 \mu \mathrm{M}$ steps) were performed to determine electron transfer system (ETS) capacity. Rot, if not already present, and Ama ( $2.5 \mu \mathrm{M}$ to inhibit Complex III) were added for determination of residual oxygen consumption (ROX).

Respiration of intact cells was measured applying a coupling control protocol [19]. Up to $300 \mu \mathrm{L}$ of suspended cells were added to the respiration medium. After stabilization of ROUTINE respiration, the ATP-synthase inhibitor oligomycin (Omy, $2 \mu \mathrm{g} / \mathrm{mL}$ ) was added to obtain a measure of LEAK respiration, followed by titration of CCCP to maximum oxygen flux (ETS capacity). Finally, Rot and Ama were added to obtain ROX.

$\mathrm{H}_{2} \mathrm{O}_{2}$ flux was measured simultaneously with respirometry in the O2k-Fluorometer using the $\mathrm{H}_{2} \mathrm{O}_{2}$-sensitive probe Amplex ${ }^{\circledR}$ UltraRed [15]. $10 \mu \mathrm{M}$ Amplex ${ }^{\circledR}$ UltraRed (AmR), $1 \mathrm{U} / \mathrm{mL}$ horse radish peroxidase (HRP) and $5 \mathrm{U} / \mathrm{mL}$ superoxide dismutase (SOD) were added to the chamber. The reaction product between $\mathrm{AmR}$ and $\mathrm{H}_{2} \mathrm{O}_{2}$, catalyzed by $\mathrm{HRP}$, is fluorescent, similar to resorufin. 
Calibrations were performed with $\mathrm{H}_{2} \mathrm{O}_{2}$ repeatedly added at $0.1 \mu \mathrm{M}$ steps as indicated $\left(\mathrm{H}_{2} \mathrm{O}_{2} *\right)$. Volume-specific $\mathrm{H}_{2} \mathrm{O}_{2}$ fluxes were calculated real-time by the DatLab software (OROBOROS INSTRUMENTS, Innsbruck, Austria) from the positive time derivative of the resorufin signal over time (converted to $\mathrm{H}_{2} \mathrm{O}_{2}$ concentration based on the calibrations with $\mathrm{H}_{2} \mathrm{O}_{2}$ ). Only the stable portions of the apparent fluxes were selected and artifacts induced by additions of chemicals or re-oxygenations were excluded.

\subsection{Cell Culture}

Human embryonic kidney cells (HEK 293T, ATCC collection code CRL-1573) were cultured in $10 \mathrm{~cm}^{2}$ culture dishes in DMEM high glucose medium supplemented with additions as indicated above until approximately $90 \%$ confluence was reached. Immediately prior to respirometric assays the cells were washed with PBS, trypsinized and resuspended in MiR05 or DMEM. The final concentration of permeabilized cells in the $\mathrm{O} 2 \mathrm{k}$-chamber was $1.75 \cdot 10^{6} / \mathrm{mL}$ or $1.5-2 \cdot 10^{6} / \mathrm{mL}$ when intact cells were examined. Cells were permeabilized after addition to the respirometer chambers using digitonin at a final concentration of $10 \mu \mathrm{g} / \mathrm{mL}$. This concentration was evaluated in preliminary experiments to achieve full permeabilization of cells to allow for uninhibited access of substrates and ADP to mitochondria without compromising mitochondrial function [50].

\subsection{Preparation of Mouse Brain Homogenate}

Wild-type C57BL/6 mice (age 2-3 months) were housed under standard conditions $\left(21-23{ }^{\circ} \mathrm{C}\right.$, $12 \mathrm{~h}$ light/dark cycle, relative humidity $45 \%-65 \%$ ) with unlimited access to food and water. The experimental procedures were performed in accordance with the guidelines of the European Community as well as local laws and policies.

Animals were sacrificed by cervical dislocation, the skull opened with scissors and the brain removed. Brain cortex was dissected and washed in ice-cold MiR05Cr (MiR05 supplemented with $20 \mathrm{mM}$ creatine). The tissue was transferred to a pre-cooled glass Potter homogenizer and homogenized with 10-15 strokes at medium speed. The resulting homogenate was then kept on ice and used for respirometry without further processing. The final concentration of tissue in the $\mathrm{O} 2 \mathrm{k}$-chamber was $1 \mathrm{mg} / \mathrm{mL}$.

\subsection{Isolation of Mouse Heart Mitochondria}

Wild-type C57BL/6 mice were sacrificed by cervical dislocation and the heart was excised and weighed. The heart was washed in ice-cold BIOPS and minced in $1 \mathrm{~mL}$ of BIOPS. The tissue was transferred to a pre-cooled glass Potter homogenizer with $2 \mathrm{~mL}$ of isolation buffer (225 mM mannitol, $75 \mathrm{mM}$ sucrose, $1 \mathrm{mM}$ EGTA, $2.5 \mathrm{mg} / \mathrm{mL}$ BSA) supplemented with Subtilisin $(0.5 \mathrm{mg} / \mathrm{mL})$. The tissue was homogenized with 6-8 strokes at medium speed. The resulting homogenate was centrifuged for $10 \mathrm{~min}$ at $800 \times \mathrm{g}, 4^{\circ} \mathrm{C}$. Then, the supernatant was transferred to a new tube and centrifuged for $10 \mathrm{~min}$ at $10,000 \times \mathrm{g}, 4^{\circ} \mathrm{C}$. After centrifugation, the supernatant was carefully discarded, the mitochondrial pellet was washed in $2 \mathrm{~mL}$ isolation buffer and resuspended in $100 \mu \mathrm{L}$ of isolation 
buffer. Isolated heart mitochondria were stored on ice until use. $5 \mu \mathrm{L}$ of mitochondrial suspension per chamber were used for each measurement.

\section{Conclusions}

SUIT protocols allow a detailed analysis of mitochondrial fitness in permeabilized tissues and cells, tissue homogenates and isolated mitochondria, extended by combining OXPHOS analysis with measurement of hydrogen peroxide production. Combined measurements provide the basis for quality control to avoid experimental artifacts. AmR inhibited respiration of intact and permeabilized cells and should not be applied at concentrations above $10 \mu \mathrm{M}$ nor during prolonged exposure. The choice of experimental medium is critical and simple media may aggravate the inhibitory effect of AmR. Inhibition of respiration (e.g., by Rot and Ama) exerts an influence on $\mathrm{H}_{2} \mathrm{O}_{2}$ production which is not generally predictable. When increasing the cell density, the cellular ROS scavenging capacity is increased together with the total $\mathrm{H}_{2} \mathrm{O}_{2}$ production, which provides the explanation for our observation that volume-specific $\mathrm{H}_{2} \mathrm{O}_{2}$ production remained constant or even declined with increasing cell density. $\mathrm{H}_{2} \mathrm{O}_{2}$ fluxes were generally less than $1 \%$ of oxygen fluxes in physiological substrate and coupling states. In permeabilized cells only net rates are obtained on $\mathrm{H}_{2} \mathrm{O}_{2}$ production escaping the cellular scavenging systems.

\section{Acknowledgments}

Supported in part by the Tyrolean Government and the European Regional Development Fund (ERDF; K-Regio project; MitoFit; Erich Gnaiger).

Marina Makrecka-Kuka was supported by a travel grant from 7th Framework Programme project InnovaBalt. We thank Mona Fontana-Ayoub for expert technical support.

\section{Author Contributions}

Designed the experiments: Marina Makrecka-Kuka, Gerhard Krumschnabel, and Erich Gnaiger. Performed the experiments: Marina Makrecka-Kuka and Gerhard Krumschnabel. Analyzed the experiments: Marina Makrecka-Kuka, Gerhard Krumschnabel, and Erich Gnaiger. Wrote the paper: Marina Makrecka-Kuka, Gerhard Krumschnabel, and Erich Gnaiger.

\section{Conflicts of Interest}

Gerhard Krumschnabel and Erich Gnaiger are affiliated with OROBOROS INSTRUMENTS.

\section{Abbreviations (See also Table 1)}

$\begin{array}{llll}\text { Ama } & \text { Antimycin A } & \text { Rot } & \text { rotenone } \\ \text { AmR } & \text { Amplex UltraRed } & \text { ROX } & \text { residual oxygen consumption } \\ \text { Dig } & \text { digitonin } & \text { S } & \text { succinate } \\ \text { G } & \text { glutamate } & \text { SOD } & \text { superoxide dismutase } \\ \text { HRP } & \text { horse radish peroxidase } & \text { SUIT } & \text { substrate-uncoupler-inhibitor titration }\end{array}$




$\begin{array}{llll}\mathrm{M} & \text { malate } & \text { Thom } & \text { tissue homogenate } \\ \mathrm{P} & \text { pyruvate } & \mathrm{U} & \text { uncoupler }\end{array}$

\section{References}

1. Sena, L.A.; Chandel, N.S. Physiological roles of mitochondrial reactive oxygen species. Mol. Cell 2012, 48, 158-167.

2. Chen, Q.; Szczepanek, K.; Hu, Y.; Thompson, J.; Lesnefsky, E.J. A deficiency of apoptosis inducing factor (AIF) in Harlequin mouse heart mitochondria paradoxically reduces ROS generation during ischemia-reperfusion. Front. Physiol. 2014, doi:10.3389/fphys.2014.00271.

3. Dai, D.F.; Chiao, Y.A.; Marcinek, D.J.; Szeto, H.H.; Rabinovitch, P.S. Mitochondrial oxidative stress in aging and healthspan. Longev. Healthspan. 2014, doi:10.1186/2046-2395-3-6.

4. Di Lisa, F.; Kaludercic, N.; Carpi, A.; Menabò, R.; Giorgio, M. Mitochondria and vascular pathology. Pharmacol. Rep. 2009, 61, 123-130.

5. Tahara, E.B.; Navarete, F.D.; Kowaltowski, A.J. Tissue-, substrate-, and site-specific characteristics of mitochondrial reactive oxygen species generation. Free Radic. Biol. Med. 2009, 46, 1283-1297.

6. Dröse, S.; Brandt, U. Molecular mechanisms of superoxide production by the mitochondrial respiratory chain. Adv. Exp. Med. Biol. 2012, 748, 145-169.

7. Suski, J.M.; Lebiedzinska, M.; Bonora, M.; Pinton, P.; Duszynski, J.; Wieckowski, M.R. Relation between mitochondrial membrane potential and ROS formation. Methods Mol. Biol. 2012, 810, 183-205.

8. Starkov, A.A. Measurement of mitochondrial ROS production. Methods Mol. Biol. 2010, 648, 245-255.

9. Kowaltowski, A.J.; de Souza-Pinto, N.C.; Castilho, R.F.; Vercesi, A.E. Mitochondria and reactive oxygen species. Free Radic. Biol. Med. 2009, 47, 333-343.

10. Clarke, K.J.; Porter, R.K. Uncoupling protein 1 dependent reactive oxygen species production by thymus mitochondria. Int. J. Biochem. Cell Biol. 2013, 45, 81-89.

11. Malinska, D.; Kudin, A.P.; Debska-Vielhaber, G.; Vielhaber, S.; Kunz, W.S. Quantification of superoxide production by mouse brain and skeletal muscle mitochondria. Methods Enzymol. 2009, 456, 419-437.

12. Tretter, L.; Adam-Vizi, V. Uncoupling is without an effect on the production of reactive oxygen species by in situ synaptic mitochondria. J. Neurochem. 2007, 103, 1864-1871.

13. Tretter, L.; Adam-Vizi, V. High $\mathrm{Ca}^{2+}$ load promotes hydrogen peroxide generation via activation of $\alpha$-glycerophosphate dehydrogenase in brain mitochondria. Free Radic. Biol. Med. 2012, 53, 2119-2130.

14. Tretter, L.; Takacs, K.; Kövér, K.; Adam-Vizi, V. Stimulation of $\mathrm{H}_{2} \mathrm{O}_{2}$ generation by calcium in brain mitochondria respiring on alpha-glycerophosphate. J. Neurosci. Res. 2007, 85, 3471-3479.

15. Krumschnabel, G.; Fontana-Ayoub, M.; Sumbalova, Z.; Heidler, J.; Gauper, K.; Fasching, M.; Gnaiger, E. Simultaneous high-resolution measurement of mitochondrial respiration and hydrogen peroxide production. Methods Mol. Biol. 2015, 1264, 245-261. 
16. Gnaiger, E. Polarographic Oxygen Sensors, the Oxygraph and High-Resolution Respirometry to Assess Mitochondrial Function. In Mitochondrial Dysfunction in Drug-Induced Toxicity; Dykens, J.A., Will, Y., Eds.; John Wiley: Hoboken, NJ, USA, 2008; pp. 327-352.

17. Gnaiger, E. Capacity of oxidative phosphorylation in human skeletal muscle. New perspectives of mitochondrial physiology. Int. J. Biochem. Cell Biol. 2009, 41, 1837-1845.

18. Chance, B.; Williams, G.R. Respiratory enzymes in oxidative phosphorylation: III. The steady state. J. Biol. Chem. 1955, 217, 409-427.

19. Gnaiger, E. Mitochondrial Pathways and Respiratory Control. An Introduction to OXPHOS Analysis, 4th ed.; Mitochondr Physiol Network 19.12, OROBOROS MiPNet Publications: Innsbruck, Austria, 2014; p. 80.

20. Kovács, K.; Erdélyi, K.; Hegedűs, C.; Lakatos, P.; Regdon, Z.; Bai, P.; Haskó, G.; Szabó, E.; Virág, L. Poly(ADP-ribosyl)ation is a survival mechanism in cigarette smoke-induced and hydrogen peroxide-mediated cell death. Free Radic. Biol. Med. 2012, 53, 1680-1688.

21. Marchissio, M.J.; Francés, D.E.; Carnovale, C.E.; Marinelli, R.A. Mitochondrial aquaporin-8 knockdown in human hepatoma HepG2 cells causes ROS-induced mitochondrial depolarization and loss of viability. Toxicol. Appl. Pharmacol. 2012, 264, 246-254.

22. Krumschnabel, G.; Eigentler, A.; Fasching, M.; Gnaiger, E. Use of safranin for the assessment of mitochondrial membrane potential by high-resolution respirometry and fluorometry. Methods Enzymol. 2014, 542, 163-181.

23. Scaduto, R.C., Jr.; Grotyohann, L.W. Measurement of mitochondrial membrane potential using fluorescent rhodamine derivatives. Biophys. J. 1999, 76, 469-477.

24. Krumschnabel, G.; Nydlova, E.; Vrbova, M. OROBOROS INSTRUMENTS, Innsbruck, Austria. Unpublished work, 2015.

25. Wojtala, A.; Bonora, M.; Malinska, D.; Pinton, P.; Duszynski, J.; Wieckowski, M.R. Methods to monitor ROS production by fluorescence microscopy and fluorometry. Methods Enzymol. 2014, 542, 243-262.

26. Kwak, H.B.; Thalacker-Mercer, A.; Anderson, E.J.; Lin, C.T.; Kane, D.A.; Lee, N.S.; Cortright, R.N.; Bamman, M.M.; Neufer, P.D. Simvastatin impairs ADP-stimulated respiration and increases mitochondrial oxidative stress in primary human skeletal myotubes. Free Radic. Biol. Med. 2012, 52, 198-207.

27. Cantu, D.; Fulton, R.E.; Drechsel, D.A.; Patel, M. Mitochondrial aconitase knockdown attenuates paraquat-induced dopaminergic cell death via decreased cellular metabolism and release of iron and $\mathrm{H}_{2} \mathrm{O}_{2}$. J. Neurochem. 2011, 118, 79-92.

28. Zhao, B.; Summers, F.A.; Mason, R.P. Photooxidation of Amplex Red to resorufin: Implications of exposing the Amplex Red assay to light. Free Radic. Biol. Med. 2012, 53, 1080-1087.

29. Suhane, S.; Kanzaki, H.; Arumugaswami, V.; Murali, R.; Ramanujan, V.K. Mitochondrial NDUFS3 regulates the ROS-mediated onset of metabolic switch in transformed cells. Biol. Open. 2013, 2, 295-305.

30. Lee, S.; Tak, E.; Lee, J.; Rashid, M.A.; Murphy, M.P.; Ha, J.; Kim, S.S. Mitochondrial $\mathrm{H}_{2} \mathrm{O}_{2}$ generated from electron transport chain complex I stimulates muscle differentiation. Cell Res. 2011, 21, 817-834. 
31. Gnaiger, E.; Kuznetsov, A.V.; Schneeberger, S.; Seiler, R.; Brandacher, G.; Steurer, W.; Margreiter, R. Mitochondria in the Cold. In Life in the Cold; Heldmaier, G., Klingenspor, M., Eds.; Springer: Heiderlberg, Germany, 2000; pp. 431-442.

32. Borutaite, V.; Toleikis, A.; Brown, G.C. In the eye of the storm: mitochondrial damage during heart and brain ischaemia. FEBS J. 2013, 280, 4999-5014.

33. Fassone, E.; Rahman, S. Complex I deficiency: Clinical features, biochemistry and molecular genetics. J. Med. Genet. 2012, 49, 578-590.

34. Hoffman, D.L.; Brookes, P.S. Oxygen sensitivity of mitochondrial reactive oxygen species generation depends on metabolic conditions. J. Biol. Chem. 2009, 284, 16236-16245.

35. Pham, T.; Loiselle, D.; Power, A.; Hickey, A.J. Mitochondrial inefficiencies and anoxic ATP hydrolysis capacities in diabetic rat heart. Am. J. Physiol. Cell Physiol. 2014, 307, C499-C507.

36. Henry, O.; Jolicoeur, M.; Kamen, A. Unraveling the metabolism of HEK-293 cells using lactate isotopomer analysis. Bioprocess Biosyst. Eng. 2011, 34, 263-267.

37. Sauer, L.A.; Dauchy, R.T.; Nagel, W.O.; Morris, H.P. Mitochondrial malic enzymes. Mitochondrial $\mathrm{NAD}(\mathrm{P})^{+}$-dependent malic enzyme activity and malate-dependent pyruvate formation are progression-linked in Morris hepatomas. J. Biol. Chem. 1980, 255, 3844-3848.

38. Gnaiger, E. P/E from Mouse to Man. Available online: http://wiki.oroboros.at/index.php/ OXPHOS_control_ratio\#P.2FE_from_mouse_to_man (accessed on 5 June 2015).

39. Loschen, G.; Flohe, L.; Chance, B. Respiratory chain linked $\mathrm{H}_{2} \mathrm{O}_{2}$ production in pigeon heart mitochondria. FEBS Lett. 1971, 18, 261-264.

40. Sumbalova, Z.; Vancova, O.; Krumschnabel, G.; Gnaiger, E. Optimization of malate concentration for high-resolution respirometry: Mitochondria from rat liver and brain. Mitochondr. Physiol. Network 2014, 19.13, 37.

41. Bleier, L.; Dröse, S. Superoxide generation by complex III: From mechanistic rationales to functional consequences. Biochim. Biophys. Acta 2013, 1827, 1320-1331.

42. Murphy, M.P. How mitochondria produce reactive oxygen species. Biochem. J. 2009, 417, 1-13.

43. Picard, M.; Taivassalo, T.; Gouspillou, G.; Hepple, R.T. Mitochondria: Isolation, structure and function. J. Physiol. 2011, 589, 4413-4421.

44. Picard, M.; Taivassalo, T.; Ritchie, D.; Wright, K.J.; Thomas, M.M.; Romestaing, C.; Hepple, R.T. Mitochondrial structure and function are disrupted by standard isolation methods. PLoS ONE 2011, 6, e18317.

45. Eigentler, A.; Fontana-Ayoub, M.; Gnaiger, E. O2k-Fluorometry: HRR and $\mathrm{H}_{2} \mathrm{O}_{2}$ production in mouse cardiac tissue homogenate. Mitochondr. Physiol. Network 2013, 18, 1-6.

46. Gross, V.S.; Greenberg, H.K.; Baranov, S.V.; Carlson, G.M.; Stavrovskaya, I.G.; Lazarev, A.V.; Kristal, B.S. Isolation of functional mitochondria from rat kidney and skeletal muscle without manual homogenization. Anal. Biochem. 2011, 418, 213-223.

47. Tahara, E.B.; Barros, M.H.; Oliveira, G.A.; Netto, L.E.; Kowaltowski, A.J. Dihydrolipoyl dehydrogenase as a source of reactive oxygen species inhibited by caloric restriction and involved in Saccharomyces cerevisiae aging. FASEB J. 2007, 21, 274-283.

48. Seaver, L.C.; Imlay, J.A. Are respiratory enzymes the primary sources of intracellular hydrogen peroxide? J. Biol. Chem. 2004, 279, 48742-48750. 
49. Chen, Y.R.; Zweier, J.L. Cardiac mitochondria and reactive oxygen species generation. Circ. Res. 2014, 114, 524-537.

50. Pesta, D.; Gnaiger, E. High-resolution respirometry: OXPHOS protocols for human cells and permeabilized fibers from small biopsies of human muscle. Methods Mol. Biol. 2012, 810, $25-58$. 


\title{
Biological Activities of Reactive Oxygen and Nitrogen Species: Oxidative Stress versus Signal Transduction
}

\author{
Adelheid Weidinger and Andrey V. Kozlov
}

\begin{abstract}
In the past, reactive oxygen and nitrogen species (RONS) were shown to cause oxidative damage to biomolecules, contributing to the development of a variety of diseases. However, recent evidence has suggested that intracellular RONS are an important component of intracellular signaling cascades. The aim of this review was to consolidate old and new ideas on the chemical, physiological and pathological role of RONS for a better understanding of their properties and specific activities. Critical consideration of the literature reveals that deleterious effects do not appear if only one primary species (superoxide radical, nitric oxide) is present in a biological system, even at high concentrations. The prerequisite of deleterious effects is the formation of highly reactive secondary species (hydroxyl radical, peroxynitrite), emerging exclusively upon reaction with another primary species or a transition metal. The secondary species are toxic, not well controlled, causing irreversible damage to all classes of biomolecules. In contrast, primary RONS are well controlled (superoxide dismutase, catalase), and their reactions with biomolecules are reversible, making them ideal for physiological/pathophysiological intracellular signaling. We assume that whether RONS have a signal transducing or damaging effect is primarily defined by their quality, being primary or secondary RONS, and only secondly by their quantity.
\end{abstract}

Reprinted from Biomolecules. Cite as: Weidinger, A.; Kozlov, A.V. Biological Activities of Reactive Oxygen and Nitrogen Species: Oxidative Stress versus Signal Transduction. Biomolecules 2015, 5 , $472-484$.

\section{Introduction}

Reactive oxygen and nitrogen species (RONS) include two classes of chemically-reactive molecules containing oxygen (reactive oxygen species, ROS) and nitrogen (reactive nitrogen species, RNS). Both classes are referred to as RONS. The majority of RONS carries unpaired electrons and is called free radicals. In mammalians, a major function of specialized enzymes, such as NADPH-oxidase, myeloperoxidase and nitric oxide synthase (NOS), is the generation of RONS. The controlled generation of RONS in the extracellular space by these enzymes was developed evolutionarily as part of the innate immune system to kill bacteria. However, an overwhelming release of RONS may also induce deleterious effects, causing damage to host biological structures. Another group of enzymes release RONS intracellularly as a byproduct of metabolic processes. For instance, superoxide $\left(\mathrm{O}_{2}{ }^{--}\right)$is released as a byproduct of mitochondrial respiration and monooxygenase activity of cytochrome p450. Intracellular RONS, as well as excessive release of extracellular RONS were thought to induce deleterious effects, causing oxidative damage to different kinds of biomolecules. These processes are referred to as "oxidative stress".

Oxidative stress is believed to significantly contribute to the development of a number of diseases, particularly age-related diseases. However, more and more evidence suggest that intracellular 
generation of RONS is an important component of intracellular signaling cascades regulating several physiological functions, such as regulation of vascular tone, insulin synthesis, activation of hypoxia-inducible factor (HIF), cell proliferation, differentiation and migration. It took over 50 years for a clear understanding of the chemical basis of free radical/RONS biology to emerge. In the following 50 years, studies on the biological effects of free radicals with biological targets were undertaken. The aim of this review is to put together old and recent ideas on the chemical and pathophysiological role of RONS for a better understanding of their properties and specific activities. This review is predominantly based on selected reviews, elaborating on different aspects of RONS activity and thought to be a guide through a large body of literature existing on this topic.

\section{Chemical Basics}

The current knowledge on RONS biology is based on studies of free radical reactions conducted more than 100 years ago. Free radicals are defined as molecules having an unpaired electron. Their physical properties are similar to those of free electrons, giving a signal at $g=2.0023$ in the electron paramagnetic resonance spectrum [1]. The chemical mechanisms underlying the formation and toxicity of free radicals were proposed by the British chemist Henry J. H. Fenton in 1894 and later developed by the Austrian chemist Joseph Weiss and the German chemist and Nobel Prize winner Fritz Haber in 1934. Henry J. H. Fenton showed that the formation of toxic hydroxyl radicals $\left({ }^{\circ} \mathrm{OH}\right)$ from hydrogen peroxide $\left(\mathrm{H}_{2} \mathrm{O}_{2}\right)$ is catalyzed by iron ions, called the "Fenton reaction" ([2], reviewed in [3]). He pointed out that iron ions are necessary to form toxic ${ }^{\bullet} \mathrm{OH}$ radicals. Joseph Weiss and Fritz Haber discovered that $\mathrm{O}_{2}{ }^{-}$can be converted into $\mathrm{H}_{2} \mathrm{O}_{2}$ and further to ${ }^{\bullet} \mathrm{OH}$, called the Haber-Weiss reaction ([4], reviewed in [5,6]). This reaction shows that one free radical can give rise to another secondary radical. Already in those days, the transformation of one $\operatorname{ROS}\left(\mathrm{O}_{2}{ }^{--}\right)$to another $\left({ }^{\bullet} \mathrm{OH}\right)$ was associated with the presence of iron ions as a catalyst (reviewed in [7]). Later, other transition metals, such as copper ions, were shown to generate toxic RONS.

Another important step in understanding the biological function of RONS was the discovery of free radical chain reactions. This was done in 1935 by the Russian chemist and Nobel Prize winner Nikolai Semenov. He described four types of free radical reactions, namely initiation, propagation, branching and termination [8]. The same reactions occur in biological membranes upon pathological conditions and are termed lipid peroxidation, the major mechanism of oxidative damage to biological membranes. Importantly, the branching chain reaction of lipid peroxidation, the cleavage of lipid peroxides, is catalyzed by ferrous ions similar to the Fenton reaction, which is a cleavage of $\mathrm{H}_{2} \mathrm{O}_{2}$ to ${ }^{\circ} \mathrm{OH}$ by ferrous ions. The branching chain reaction between lipid peroxides and iron ions accelerates lipid peroxidation [9], again suggesting that iron ions are the prerequisite for the toxic effects of lipid peroxidation.

In the $1950 \mathrm{~s}$, researchers started to associate free radical chemistry with biomedical questions. It has been suggested that most of the damaging effects of oxygen in living systems are due to the formation of free radicals (reviewed in [10]). This assumption promoted the application of the knowledge of free radical chemistry to biological systems. 


\section{Oxidative Stress}

However, in 1968, a major breakthrough in the field of free radical biology was done by Irvin Fridovich who discovered superoxide dismutase (SOD), a specific enzyme catalyzing the transition of $\mathrm{O}_{2}{ }^{--}$into $\mathrm{H}_{2} \mathrm{O}_{2}$ ([11,12], reviewed in [13]). A few years later, Chance and coauthors reported that mitochondria are the key generator of $\mathrm{O}_{2}{ }^{--}$in cells ([14], reviewed in [15]). These two findings are crucial, as they show that free radicals, on the one hand, are produced in biological systems, and on the other hand, there is an enzymatic mechanism regulating their concentration. This clearly suggests that free radicals occur in biological systems and probably have a specific function. Since then, numerous studies have been performed to understand the biological function of free radicals.

Until the mid-1970s, the literature almost exclusively refers to free radicals. Later, it became evident that not only free radicals, but also non-radical products, such as $\mathrm{H}_{2} \mathrm{O}_{2}$ or hypochlorous acid ( $\mathrm{HOCl}$ ), which are also powerful oxidizing agents, participate in free radical reactions (reviewed in [16]). To take into account both the radical and the non-radical species, the more general term "reactive oxygen species" (ROS) was introduced. Later, nitric oxide (NO*) and peroxynitrite $\left(\mathrm{ONOO}^{-}\right)$were also shown to interact with ROS, and all of these species were termed RONS. Primarily, the toxic properties of RONS were of interest. It was shown that an excessive generation of RONS damaged almost all classes of biomolecules, such as lipids [17], proteins [18] and DNA (reviewed in $[19,20]$ ). In the 1970s and 1980s, the term "oxidative stress" was used for these deleterious processes. Later, "oxidative stress" was defined by the German biochemist Helmut Sies as an imbalance between oxidants and antioxidants in favor of the oxidants, potentially leading to damage ([21], reviewed in [22]). Evolutionarily, the induction of oxidative stress was possibly developed as an important part of the innate immune system as a defense mechanism against bacteria [23]. However, it was also shown that RONS, produced by the immune system, can also damage host cells [24].

Careful observation of oxidative damage reactions of biomolecules shows that primary RONS, such as $\mathrm{O}_{2}{ }^{\bullet-}, \mathrm{H}_{2} \mathrm{O}_{2}$ or $\mathrm{NO}^{\bullet}$, in most cases reversibly react with the target molecules. $\mathrm{NO}^{\bullet}$, for instance, reversibly binds to heme proteins, whereas $\mathrm{O}_{2}{ }^{--}$reacts with proteins, changing their redox state without damage to their structure. For instance, the reaction between $\mathrm{O}_{2}{ }^{\bullet-}$ and cytochrome c results in the reduction of heme, which is used to detect $\mathrm{O}_{2}{ }^{--}$[25]. The damage is predominantly associated with secondary RONS, such as ${ }^{\bullet} \mathrm{OH}, \mathrm{ONOO}^{-}$and $\mathrm{HOCl}$ [26-28]. All of these toxic species are formed if more than one reactive species is present. Two major reactions leading to the formation of toxic RONS are: (i) the Fenton reaction between ferrous ions and $\mathrm{H}_{2} \mathrm{O}_{2}$ yielding ${ }^{\bullet} \mathrm{OH}$; and (ii) the reaction of $\mathrm{O}_{2}{ }^{--}$with $\mathrm{NO}^{\bullet}$ yielding $\mathrm{ONOO}^{-}$. Furthermore, the formation of $\mathrm{HOCl}$, formed in an enzymatic reaction from $\mathrm{H}_{2} \mathrm{O}_{2}$ and $\mathrm{Cl}^{-}$, is associated with damage to host tissues [28]. In addition, iron ions can directly react with organic peroxides, inducing lipid peroxidation. Moreover, the presence of iron and copper ions induces double-strand breaks of DNA, a DNA damage that is difficult to repair (reviewed in [29]). Some anticancer drugs are based on mechanisms causing double-strand breaks of DNA catalyzed by transition metals [30]. Oxidative damage to proteins is often associated with the reaction between amino acids and $\mathrm{ONOO}^{-}$, resulting in the formation of nitrated amino acids, such as nitrotyrosine. $\mathrm{ONOO}^{-}$is a secondary 
RONS formed in the reaction between $\mathrm{NO}^{\bullet}$ and $\mathrm{O}_{2}{ }^{\bullet-}$. $\mathrm{ONOO}^{-}$and $\mathrm{NO}^{\bullet}$ may have quite opposite biological effects. For instance, $\mathrm{NO}^{\bullet}$ has an inhibitory effect on lipid peroxidation, while $\mathrm{ONOO}^{-}$ activates this process (reviewed in [31]). Another important regulatory mechanism based on the interaction of $\mathrm{NO}^{\bullet}$ and $\mathrm{O}_{2}{ }^{\bullet-}$ is driven by decreased $\mathrm{NO}^{\bullet}$ levels, rather than by increased $\mathrm{ONOO}^{-}$ levels (reviewed in [32,33]). The interaction between $\mathrm{NO}^{\bullet}$ and $\mathrm{O}_{2}{ }^{--}$might also result in vasoconstriction by inactivation of prostacyclin synthase (reviewed in [34]).

The data described above suggest that primary $\operatorname{ROS}\left(\mathrm{O}_{2}{ }^{--}, \mathrm{NO}^{\bullet}, \mathrm{H}_{2} \mathrm{O}_{2}\right)$ only have a weak damaging potential, whereas secondary RONS are more toxic. Primary species are well controlled by SOD, catalase and NO synthases, while secondary species are less controllable, since there is no specific enzymatic system controlling their levels.

Interestingly, $\mathrm{O}_{2}{ }^{\circ-}$-controlling systems are different inside the cells and in extracellular fluids. SOD, the intracellular enzyme for removing $\mathrm{O}_{2}{ }^{--}$, and the extracellular SOD (ecSOD) produce potentially dangerous $\mathrm{H}_{2} \mathrm{O}_{2} . \mathrm{H}_{2} \mathrm{O}_{2}$ itself is relatively inactive, but can lead to the formation of toxic ${ }^{\bullet} \mathrm{OH}$. In contrast, ceruloplasmin in the blood inactivates $\mathrm{O}_{2}{ }^{--}$, yielding $\mathrm{H}_{2} \mathrm{O}$ [35]. However, extracellular SOD, which mainly occurs in tissue ([36,37]; reviewed in [38,39]), can also be found in plasma under specific pathological conditions [40] and contribute to the elimination of $\mathrm{O}_{2}{ }^{\circ-}$. The fact that SOD, not ceruloplasmin, occurs in cells indirectly suggests that $\mathrm{H}_{2} \mathrm{O}_{2}$ may have a physiological function inside cells, but not in extracellular fluids. The data gathered in the last few decades suggest that primary RONS formed in mitochondria $\left(\mathrm{O}_{2}{ }^{\bullet-}\right.$ and $\left.\mathrm{H}_{2} \mathrm{O}_{2}\right)$ and $\mathrm{NO}^{\bullet}$ are associated with intracellular signaling cascades. Since NO-mediated signaling pathways have already been extensively reviewed ([41-47]), in this review, we focus on non-NO-mediated signaling pathways.

\section{Signaling}

There is a solid body of literature supporting the essential role of mitochondrial ROS in intracellular signaling. The data on the involvement of mitochondrial ROS in intracellular signaling pathways related to inflammation have been summarized in recent reviews [48-50]. In the last few years, the role of ROS in positive and negative regulation of insulin signaling has also been intensively studied and reviewed [51]. Furthermore, the role of mitochondrial ROS in activation of HIF has been intensively studied and debated (reviewed in [52,53]). In addition, the role of ROS has been demonstrated for NF-kB-dependent gene transcription and a number of other signaling cascades (reviewed in [54]). Notably, most of the publications on oxidative stress referred to specific types of RONS involved in oxidative damage, whereas data on signaling are predominantly addressed to ROS and RONS in general. This led to a large knowledge gap on the mechanisms of intracellular signaling concerning RONS, since is not clear whether all or only specific RONS contribute to these signaling pathways. In the following section, we will focus on studies where specific types of RONS contributing to intracellular signaling cascades were determined. 


\section{Superoxide Radical}

A number of reports suggest $\mathrm{O}_{2}{ }^{--}$as part of intracellular signaling cascades. This species is predominantly produced by mitochondrial complexes I and III (reviewed in [55]). Evidence of the involvement of mitochondrial $\mathrm{O}_{2}{ }^{\bullet-}$ in intracellular signaling cascades can be shown by:

1. Alteration of mitochondrial function, particularly of complexes I and III, by pharmacological or genetic modulation, which has an effect on signaling pathways.

2. The correlation of a certain $\mathrm{O}_{2}{ }^{--}$level with an effective signaling cascade.

3. Application of mitochondria-targeted antioxidants (mtAOX) or radical scavengers has an effect on signaling pathways.

4. Genetic manipulation of mitochondrial SOD and cytoplasmic SOD decreases the efficiency of specific signaling cascades.

Mitochondrial $\mathrm{O}_{2}{ }^{\bullet-}$, the primary mitochondrial ROS, was often associated with the regulation of inflammatory pathways, such as activation of the inflammasome, regulation of inflammatory cytokines synthesis and mechanisms of innate immunity. The involvement of mitochondrial $\mathrm{O}_{2}{ }^{--}$in the activation of the inflammasome was suggested by Zhou et al. [56]. The authors showed that specific inhibition of mitochondrial complexes I and III, the major sources of ROS in mitochondria, significantly diminished the activation of the "nucleotide-binding domain, leucine-rich family and pyrin domain containing 3" (NLRP3) inflammasome, suggesting that mitochondrial $\mathrm{O}_{2}{ }^{--}$is involved in this signaling cascade. Another important feature of inflammation is the release of cytokines. Bulua et al. [57] showed that LPS-stimulated IL-6 production could be reduced by treatment with MitoQ, a mitochondria-targeted radical scavenger. This effect was coincident with increased levels of mitochondrial $\mathrm{O}_{2}{ }^{-}$, suggesting a key role of $\mathrm{O}_{2}{ }^{--}$in this signaling pathway. Weidinger et al. demonstrated that mitochondria-targeted antioxidants reduce the expression of IL-6 and iNOS in a model of systemic inflammatory response induced by LPS [58]. In leukocytes, Kröller-Schön [59] showed that elevated mtROS formation activated NADPH-oxidase at the posttranslational level. Inhibition of the mitochondrial permeability transition pore, which is supposed to facilitate the transport of $\mathrm{O}_{2}{ }^{\bullet-}$ from mitochondria to the cytoplasm, prevented activation of NADPH-oxidase. In contrast, the deficiency of mitochondrial SOD intensified the stimulation of NADPH-oxidase, suggesting that this process is mediated by $\mathrm{O}_{2}{ }^{\bullet-}$, rather than by $\mathrm{H}_{2} \mathrm{O}_{2}$. Applying specific mitochondrial inhibitors and direct detection of mtROS, Dikalov et al. suggested that mtROS, presumably $\mathrm{O}_{2}{ }^{\bullet-}$, are able to activate NADPH-oxidase via activation of protein kinase C (PKC) [49]. These data suggest that mitochondrial $\mathrm{O}_{2}{ }^{--}$orchestrate cellular ROS production upon inflammation. The same group has shown that stimulation of endothelial cells with angiotensin II elevates mitochondrial $\mathrm{O}_{2}{ }^{--}$levels and simultaneously the activity of NADPH-oxidase in this non-immune cell type [60]. However, treatment with mitoTEMPO, a mitochondria-targeted antioxidant, or overexpression of mitochondrial SOD captured $\mathrm{O}_{2}{ }^{-{ }^{-}}$and decreased the activation of vascular NADPH oxidases [60]. NADPH oxidases, in turn, may regulate important cellular processes, such as cell migration [61], differentiation [62] and proliferation [63] (reviewed in [64]). These data again suggest that $\mathrm{O}_{2}{ }^{--}$rather than $\mathrm{H}_{2} \mathrm{O}_{2}$ is involved in this signaling cascade. However, in the past, it was believed that $\mathrm{O}_{2}{ }^{\bullet-}$ does not participate in signaling, as it cannot exit mitochondria 
due to its polarity. Consequently, $\mathrm{H}_{2} \mathrm{O}_{2}$ formed from $\mathrm{O}_{2}{ }^{\bullet-}$ was considered a necessary intermediate of $\mathrm{O}_{2}{ }^{\bullet-}$-mediated actions. $\mathrm{H}_{2} \mathrm{O}_{2}$ is a nonpolar molecule and can easily diffuse through the membranes. Recently, however, the situation has shifted. $\mathrm{O}_{2}{ }^{--}$has been shown to leave mitochondria via the mitochondrial permeability transition pore [65] and anion channels [66]. We also showed that $\mathrm{O}_{2}{ }^{--}$can be released from mitochondria by using $\mathrm{O}_{2}{ }^{--}$-sensitive spin probes and electron spin resonance spectroscopy [67]. These data strongly support the postulation that $\mathrm{O}_{2}{ }^{\bullet-}$ can directly contribute to intracellular signal transduction pathways.

Nevertheless, other groups propose $\mathrm{H}_{2} \mathrm{O}_{2}$ being the RONS-based messenger in intracellular signaling cascades, as well. In contrast to $\mathrm{O}_{2}{ }^{\bullet-}, \mathrm{H}_{2} \mathrm{O}_{2}$ is a neutral molecule and relatively inactive. Thus, $\mathrm{H}_{2} \mathrm{O}_{2}$ is able to cover relatively large distances, up to several cell diameters, before it reacts with its target or is catabolized [68]. Therefore, it is considered as a suitable ROS-dependent signaling component.

\section{Hydrogen Peroxide}

In the literature, the impact of $\mathrm{H}_{2} \mathrm{O}_{2}$ on intracellular signaling is supported by the following evidence:

1. Exogenous $\mathrm{H}_{2} \mathrm{O}_{2}$ has a direct effect on signaling cascades.

2. The $\mathrm{H}_{2} \mathrm{O}_{2}$ level correlates with the effectiveness of intracellular signal transduction.

3. Genetic manipulation of catalase has an effect on signaling.

4. Upregulation of MnSOD and/or $\mathrm{Cu} / \mathrm{ZnSOD}$ activates signaling.

Treatment with $\mathrm{H}_{2} \mathrm{O}_{2}$ increased the proliferation of endothelial cells in a study of Chen et al., suggesting that $\mathrm{H}_{2} \mathrm{O}_{2}$ directly interferes with pathways regulating proliferation [69]. Wang et al. [70] showed that overexpression of the mitochondria-targeted catalase construct suppressed vascular endothelial growth factor (VEGF)-induced cell migration, suggesting the involvement of $\mathrm{H}_{2} \mathrm{O}_{2}$ in the regulation of cell migration. Schmidt et al. [71] demonstrated that overexpression of catalase in cell lines caused a deficiency in the activation of NF- $\mathrm{\kappa B}$ in response to tumor necrosis factor (TNF), while the catalase inhibitor, aminotriazole, restored the induction of $\mathrm{NF}-\kappa \mathrm{B}$. Overexpression of $\mathrm{Cu} / \mathrm{Zn}$-dependent SOD elevated NF- $\mathrm{KB}$ activation. These data suggest $\mathrm{H}_{2} \mathrm{O}_{2}$ rather than $\mathrm{O}_{2}{ }^{--}$as the mediator of NF-kB pathway activation. Brunelle et al. [72] demonstrated that overexpressing glutathione peroxidase or catalase, but not SOD, stabilized HIF-1 in cells, suggesting that $\mathrm{H}_{2} \mathrm{O}_{2}$ acts as signaling molecule in the process of HIF-1 regulation. West et al. [73] showed that overexpressing catalase in mitochondria results in impaired bacterial killing by leukocytes, suggesting the predominant role of $\mathrm{H}_{2} \mathrm{O}_{2}$. Hoarau et al. [74] demonstrated that $\mathrm{H}_{2} \mathrm{O}_{2}$ plays an essential role in the development of $\beta$-cells, as it activates the ERK1/2 pathway. Other studies on $\mathrm{H}_{2} \mathrm{O}_{2}$-mediated signaling are summarized in several reviews [75-77].

\section{Secondary RONS}

The majority of papers on RONS-mediated intracellular signaling suggest either $\mathrm{O}_{2}{ }^{-{ }^{-}}$or $\mathrm{H}_{2} \mathrm{O}_{2}$ as the major signaling molecule. Only a few studies suggest that signaling molecules may be derivatives of $\mathrm{H}_{2} \mathrm{O}_{2}$. Garlid et al. [78] studied ROS-mediated opening of mitochondrial ATP-sensitive 
potassium channels and suggested an unknown derivative of $\mathrm{H}_{2} \mathrm{O}_{2}$ as a contributor to this pathway. Others suggested that $\mathrm{HOCl}$ may participate in extracellular, but not in intracellular signaling, for instance by interaction with TGF- $\beta 1$ [79]. A few more publications can be found on peroxynitrite-mediated signaling (reviewed in [80,81]). It has been assumed that peroxynitrite has an impact on pathways, which, under physiological conditions, are regulated by tyrosine phosphorylation and dephosphorylation. $\mathrm{ONOO}^{-}$causes tyrosine nitration, which blocks the respective signaling cascades. Tyrosine nitration seems to have a significant impact on a number of pathways, such as MAP kinase, STAT3, ERK and PKC-mediated pathways (reviewed in [80,81]). The fact that $\mathrm{ONOO}^{-}$irreversibly binds to proteins has a pathological impact on cellular function, rather than contributing to physiological intracellular signaling. This suggests that the biological impact of primary and secondary RONS is different. Primary RONS are predominantly associated with signaling, whereas secondary RONS are associated with oxidative stress. Primary RONS are regulated by SOD, catalase and peroxidases and have a specific physiological function for the regulation of intracellular signaling. The secondary RONS were evolutionarily developed for extracellular actions, predominantly as part of the innate immune system for killing bacteria. The intracellular release of such secondary RONS leads to deleterious consequences, as these are catalytically highly active, without a reliable control system for intracellular levels. We assume that, in evolution, the primary species were developed for intracellular physiological signaling and the secondary species for extracellular actions, such as killing of bacteria. However, at the same time, these species are able to cause damage to the cell.

\section{Conclusions}

We assume that whether RONS have a beneficial or deleterious effect is primarily defined by their quality, being primary or secondary RONS, and only secondly by their quantity (Figure 1). Therefore, we think that the common statement that at low concentrations ROS regulate physiological processes and at high concentrations are deleterious is not completely correct. Critical consideration of the existing literature shows that deleterious effects, termed as oxidative stress, do not appear if only one primary species is present in a biological system, even at high concentrations. To develop deleterious effects, a primary species reacts with another or a transition metal, yielding highly reactive secondary species, such as $\mathrm{ONOO}^{-}$or ${ }^{\bullet} \mathrm{OH}$. The secondary RONS are catalytically very active, not tightly controlled and consequently may not act as signal transducers. In contrast, primary RONS are well controlled; their reactions with targets are reversible; and they do not damage target molecules. This makes them ideal for intracellular signaling processes. Unfortunately, the majority of papers on signaling refers to RONS without specifying their types. In this review, we highlight an approach allowing one to distinguish the contribution of different RONS. This can be used to define the origin of RONS contributing to intracellular signaling cascades in future studies. 


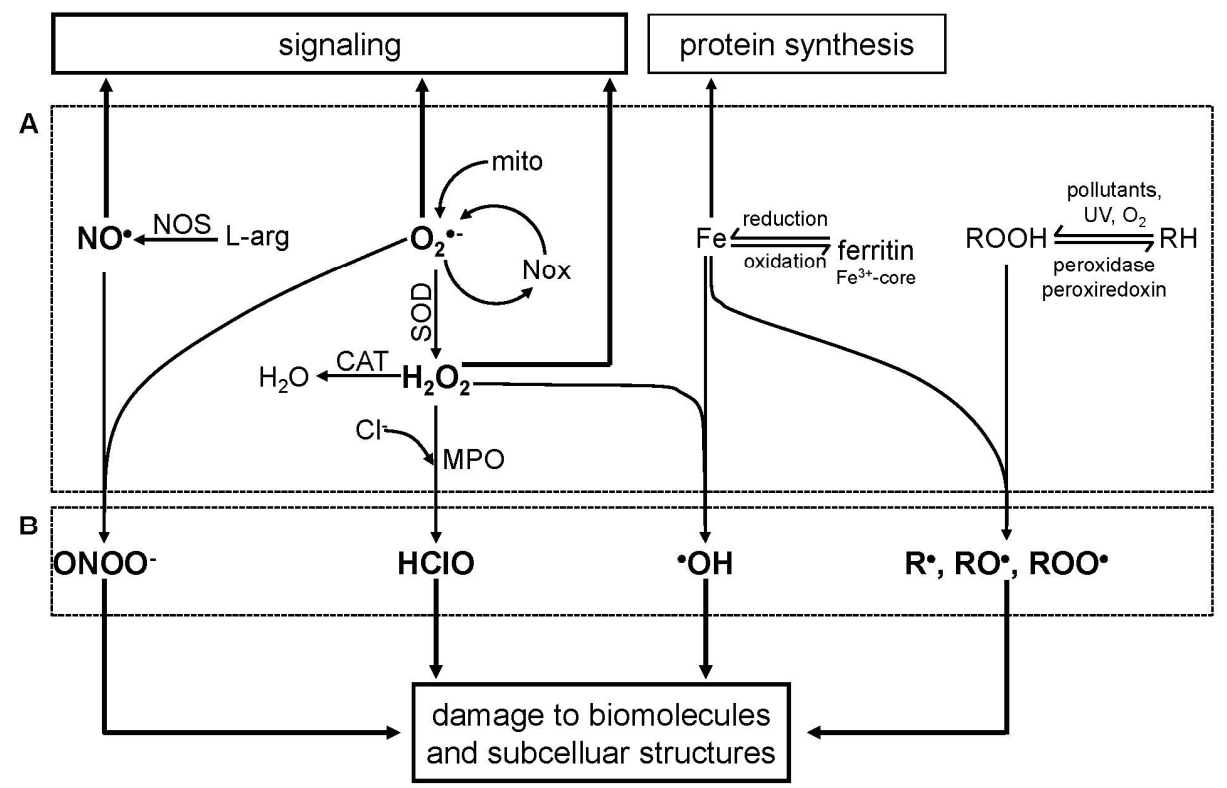

Figure 1. Scheme illustrating physiological and pathophysiological reactions of different reactive species. A, primary reactive species $\left(\mathrm{NO}^{\bullet}, \mathrm{O}_{2}{ }^{--}, \mathrm{Fe}, \mathrm{ROOH}\right)$ and the products of the interaction of two identical reactive species (dismutation of $\mathrm{O}_{2}$ to $\mathrm{H}_{2} \mathrm{O}_{2}$ ) and transition metals (reactive oxygen, nitrogen and metal species $=$ RONMS). B, secondary products of reactions between two different RONMS. Primary products predominantly contribute to physiological processes (e.g., signaling, protein synthesis); secondary products exert deleterious effects on diverse cell functions. Abbreviations: NO, nitric oxide; $\mathrm{O}_{2}{ }^{--}$, superoxide; Fe, iron; $\mathrm{ROOH}$, lipid peroxide; $\mathrm{H}_{2} \mathrm{O}_{2}$ hydrogen peroxide; $\mathrm{RH}$, non-oxidized lipid; $\mathrm{R}^{\bullet}$, $\mathrm{RO}^{\bullet}$, ROO`, lipid radicals; NOS, nitric oxide synthase; L-arg, L-arginine; $\mathrm{ONOO}^{-}$, peroxynitrite; NOX, NADPH oxidase; mito, mitochondria; SOD, superoxide dismutase; CAT, catalase; $\mathrm{H}_{2} \mathrm{O}$, water $\mathrm{Cl}^{-}$, chloride ion; MPO, myeloperoxidase; $\mathrm{HClO}$, hypochlorous acid; ${ }^{\circ} \mathrm{OH}$, hydroxyl radical; UV, ultraviolet radiation.

\section{Acknowledgments}

The authors thank Asmita Banerjee for assistance in the preparation of this manuscript. Adelheid Weidinger was supported by FFG (Austria, COMET K-Project 825329, BioPersMed) and by FWF (Austria, Project P211221-B11).

\section{Conflicts of Interest}

The authors declare no conflict of interest. 


\section{References}

1. Wertz, J.E.; Bolton, J.R. Electron Spin Resonance: Elementary Theory and Practical Applications; John Wiley \& Sons: New York, NY, USA, 1972.

2. Fenton, H.J.H. Oxidation of tartaric acid in presence of iron. J. Chem. Soc. Trans. 1894, 65, 899-910.

3. Koppenol, W.H. The centennial of the Fenton reaction. Free Radic. Biol. Med. 1993, 15, 645-651.

4. Haber, F.; Weiss, J. The catalytic decomposition of hydrogen peroxide. Proc. R. Soc. 1934, 147, 332-351.

5. Koppenol, W.H. The Haber-Weiss cycle-70 Years later. Redox Rep. 1994, 6, 229-234.

6. Kehrer, J.P. The Haber-Weiss reaction and mechanisms of toxicity. Toxicology 2000, 149, 43-50.

7. Ullrich, V.; Kissner, R. Redox signaling: Bioinorganic chemistry at its best. J. Inorg. Biochem. 2006, 100, 2079-2086.

8. Semenov, N.N. Some Problems in Chemical Kinetics and Reactivity; Princeton University Press: Princeton, NJ, USA, 1959.

9. Vladimirov, Y.A.; Olenev, V.; Suslova, T.; Cheremisina, Z. Lipid peroxidation in mitochondrial membrane. Adv. Lipid Res. 1980, 17, 173-249.

10. Gilbert, D.L. Oxygen and Living Processes: An Interdisciplinary Approach; Springer: New York, NY, USA, 1981.

11. McCord, J.M.; Fridovich, I. The reduction of cytochrome c by hypoxanthine and xanthine oxidase. Biochem. J. 1968, 243, 5753-5760.

12. Mccord, J.M.; Fridovich, I. Superoxide dismutase: An enzymic function for erythrocuprein (hemocuprein). J. Biol. Chem. 1969, 244, 6049-6055.

13. Fridovich, I. Superoxide anion radical, superoxide dismutases, and related matters. J. Biol. Chem. 1997, 272, 18515-18517.

14. Boveris, B.A.; Oshino, N.; Chance, B. The cellular production of hydrogen peroxide. Biochem. J. 1972, 128, 617-630.

15. Chance, B.; Sies, H.; Boveris, A. Hydroperoxide metabolism in mammalian organs. Physiol. Rev. 1979, 59, 527-605.

16. Halliwell, B. Free radicals, reactive oxygen species and human disease: A critical evaluation with special reference to atherosclerosis. Br. J. Exp. Pathol. 1989, 70, 737-757.

17. Azizova, O.A.; Panasenko, O.; Volnov, T.; Vladimirov, Y.A. Free radical lipid oxidation affects cholesterol transfer between lipoproteins and erythrocytes. Free Radic. Biol. Med. 1989, 7, 251-257.

18. Lyras, L.; Perry, R.H.; Perry, E.K.; Ince, P.G.; Jenner, A.; Jenner, P.; Halliwell, B. Oxidative damage to proteins, lipids, and DNA in cortical brain regions from patients with dementia with Lewy bodies. J. Neurochem. 1998, 71, 302-312.

19. Halliwell, B.; Aruoma, O.I. DNA damage by oxygen-derived species. FEBS Lett. 1991, 281, 9-19. 
20. Cadet, J.; Douki, T.; Ravanat, J.-L. Oxidatively generated base damage to cellular DNA. Free Radic. Biol. Med. 2010, 49, 9-21.

21. Sies, H. Oxidative stress: Introductory remarks. In Oxidative Stress; Academic Press: London, UK, 1985; pp. 1-8.

22. Sies, H. Oxidative stress: Oxidants and antioxidants. Exp. Physiol. 1997, 82, 291-295.

23. Rosen, H.; Klebanoff, S.J.; Wang, Y.; Brot, N.; Heinecke, J.W.; Fu, X. Methionine oxidation contributes to bacterial killing by the myeloperoxidase system of neutrophils. Proc. Natl. Acad. Sci. USA 2009, 106, 18686-18691.

24. Schwab, L.; Goroncy, L.; Palaniyandi, S.; Gautam, S.; Triantafyllopoulou, A.; Mocsai, A.; Reichardt, W.; Karlsson, F.J.; Radhakrishnan, S.V.; Hanke, K.; et al. Neutrophil granulocytes recruited upon translocation of intestinal bacteria enhance graft-versus-host disease via tissue damage. Nat. Med. 2014, 20, 648-654.

25. Arthur, M.; Kowalski-Saunders, P.; Gurney, S.; Tolcher, R.; Bull, F.; Wright, R. Reduction of ferricytochrome $\mathrm{C}$ may underestimate superoxide production by monocytes. J. Immunol. Methods 1987, 98, 63-69.

26. Cadet, J.; Wagner, J.R. Oxidatively generated base damage to cellular DNA by hydroxyl radical and one-electron oxidants: Similarities and differences. Arch. Biochem. Biophys. 2014, $557,47-54$.

27. Salgo, M.G.; Squadrito, G.L.; Pryor, W.A. Peroxynitrite causes apoptosis in rat thymocytes. Biochem. Biophys. Res. Commun. 1995, 215, 1111-1118.

28. Panasenko, O.M.; Evgina, S.A.; Driomina, E.S.; Sharov, V.S.; Sergienko, V.I.; Vladimirov, Y.A. Hypochlorite induces lipoproteins and lipid peroxidation in blood phospholipid liposomes. Free Radic. Biol. Med. 1995, 19, 133-140.

29. Chevion, M. A site-specific mechanism for free radical induced biological damage: The essential role of redox-active transition metals. Free Radic. Biol. Med. 1988, 5, 27-37.

30. Roy, B.; Hecht, S.M. Hairpin DNA sequences bound strongly by bleomycin exhibit enhanced double-strand cleavage. J. Am. Chem. Soc. 2014, 136, 4382-4393.

31. Violi, F.; Milite, M.T.; Medica, I.C.; La, Á. Nitric oxide and its role in lipid peroxidation. Diabetes Metab. Res. Rev. 1999, 15, 283-288.

32. Cai, H.; Harrison, D.G. Endothelial dysfunction in cardiovascular disease. The role of oxidant stress. Circ. Res. 2000, 87, 840-844.

33. Yung, L.M.; Leung, F.P.; Yao, X.; Chen, Z.-Y.; Huang, Y. Reactive oxygen species in vascular wall. Cardiovasc. Hematol. Disord. Drug Targets 2006, 6, 1-19.

34. Schildknecht, S.; Ullrich, V. Peroxynitrite as regulator of vascular prostanoid synthesis. Arch. Biochem. Biophys. 2009, 484, 183-189.

35. Samokyszyns, V.M.; Miller, D.M.; Reif, D.W.; Austq, S.D. Inhibition of superoxide and ferritin-dependent lipid peroxidation by ceruloplasmin. J. Biol. Chem. 1989, 264, 21-26.

36. Marklund, S.L. Extracellular superoxide dismutase and other superoxide dismutase isoenzymes in tissues from nine mammalian species. Biochem. J. 1984, 222, 649-655.

37. Marklund, S.L. Extracellular superoxide dismutase in human tissues and human cell lines. J. Clin. Invest. 1984, 74, 1398-1403. 
38. Fattman, C.L.; Schaefer, L.M.; Oury, T.D. Extracellular superoxide dismutase in biology and medicine. Free Radic. Biol. Med. 2003, 35, 236-256.

39. Fukai, T.; Ushio-Fukai, M. Superoxide dismutases: Role in redox signaling, vascular function, and diseases. Antioxid. Redox Signal. 2011, 15, 1583-1606.

40. Sandstrom, J.; Nilsson, P.; Karlsson, K.; Marklund, S.L. 10-Fold increase in human plasma extracellular superoxide dismutase content caused by a mutation in heparin-binding domain. J. Biol. Chem. 1994, 269, 19163-19166.

41. Stamler, J.S.; Carolina, N. Redox signaling: Nitrosylation and related target interactions of nitric oxide. Cell 1994, 76, 931-936.

42. Foster, M.W.; McMahon, T.J.; Stamler, J.S. S-nitrosylation in health and disease. Trends Mol. Med. 2003, 9, 160-168.

43. Guikema, B.; Lu, Q.I.; Heuil, D.J. Chemical considerations and biological selectivity of protein nitrosation: Implications for NO-mediated signal transduction. Antioxid. Redox Signal. 2005, 7, 593-606.

44. Ahmed, K.A.; Sawa, T.; Akaike, T. Protein cysteine S-guanylation and electrophilic signal transduction by endogenous nitro-nucleotides. Amino Acids 2011, 41, 123-130.

45. Piantadosi, C.A. Regulation of mitochondrial processes by protein S-nitrosylation. Biochim. Biophys. Acta 2012, 1820, 712-721.

46. Sha, Y.; Marshall, H.E. S-nitrosylation in the regulation of gene transcription. Biochim. Biophys. Acta 2012, 1820, 701-711.

47. Sawa, T.; Zaki, M.H.; Okamoto, T.; Akuta, T.; Tokutomi, Y.; Kim-Mitsuyama, S.; Ihara, H.; Kobayashi, A.; Yamamoto, M.; Fujii, S.; et al. Protein S-guanylation by the biological signal 8-nitroguanosine 3',5'-cyclic monophosphate. Nat. Chem. Biol. 2007, 3, 727-735.

48. Daiber, A. Redox signaling (cross-talk) from and to mitochondria involves mitochondrial pores and reactive oxygen species. Biochim. Biophys. Acta 2010, 1797, 897-906.

49. Dikalov, S.I.; Nazarewicz, R.R. Angiotensin II-induced production of mitochondrial reactive oxygen species: Potential mechanisms and relevance for cardiovascular disease. Antioxid. Redox Signal. 2013, 19, 1085-1094.

50. Schulz, E.; Wenzel, P.; Münzel, T.; Daiber, A. Mitochondrial redox signaling: Interaction of mitochondrial reactive oxygen species with other sources of oxidative stress. Antioxid. Redox Signal. 2014, 20, 308-324.

51. Bashan, N.; Kovsan, J.; Kachko, I.; Ovadia, H.; Rudich, A. Positive and negative regulation of insulin signaling by reactive oxygen and nitrogen species. Physiol. Rev. 2009, 89, 27-71.

52. Cash, T.P.; Pan, Y.; Simon, M.C. Reactive oxygen species and cellular oxygen sensing. Free Radic. Biol. Med. 2007, 43, 1219-1225.

53. Klimova, T.; Chandel, N.S. Mitochondrial complex III regulates hypoxic activation of HIF. Cell Death Differ. 2008, 15, 660-666.

54. Thannickal, V.J.; Fanburg, B.L. Reactive oxygen species in cell signaling. Am. J. Physiol. Lung Cell. Mol. Physiol. 2000, 279, L1005-L1028.

55. Murphy, M.P. How mitochondria produce reactive oxygen species. Biochem. J. 2009, 417, 1-13. 
56. Zhou, R.; Yazdi, A.S.; Menu, P.; Tschopp, J. A role for mitochondria in NLRP3 inflammasome activation. Nature 2011, 469, 221-225.

57. Bulua, A.C.; Simon, A.; Maddipati, R.; Pelletier, M.; Park, H.; Kim, K.-Y.; Sack, M.N.; Kastner, D.L.; Siegel, R.M. Mitochondrial reactive oxygen species promote production of proinflammatory cytokines and are elevated in TNFR1-associated periodic syndrome (TRAPS). Exp. Med. 2011, 208, 519-533.

58. Weidinger, A.; Müllebner, A.; Paier-Pourani, J.; Banerjee, A.; Miller, I.; Lauterböck, L.; Duvigneau, J.C.; Skulachev, V.P.; Redl, H.; Kozlov, A.V.; et al. Vicious inducible nitric oxide synthase-mitochondrial reactive oxygen species cycle accelerates inflammatory response and causes liver injury in rats. Antioxid. Redox Signal. 2015, 22, 572-586.

59. Kröller-Schön, S.; Steven, S.; Kossmann, S.; Scholz, A.; Daub, S.; Oelze, M.; Xia, N.; Hausding, M.; Mikhed, Y.; Zinssius, E.; et al. Molecular mechanisms of the crosstalk between mitochondria and NADPH oxidase through reactive oxygen species-studies in white blood cells and in animal models. Antioxid. Redox Signal. 2014, 20, 247-266.

60. Dikalova, A.E.; Bikineyeva, A.T.; Budzyn, K.; Nazarewicz, R.R.; McCann, L.; Lewis, W.; Harrison, D.G.; Dikalov, S.I. Therapeutic targeting of mitochondrial superoxide in hypertension. Circ. Res. 2010, 107, 106-116.

61. Ullevig, S.; Zhao, Q.; Lee, C.F.; Seok Kim, H.; Zamora, D.; Asmis, R. NADPH oxidase 4 mediates monocyte priming and accelerated chemotaxis induced by metabolic stress. Arterioscler. Thromb. Vasc. Biol. 2012, 32, 415-426.

62. Li, J.; Stouffs, M.; Serrander, L.; Banfi, B.; Bettiol, E.; Charnay, Y.; Steger, K.; Krause, K.-H.; Jaconi, M.E. The NADPH oxidase NOX4 drives cardiac differentiation: Role in regulating cardiac transcription factors and map kinase activation. Mol. Biol. Cell 2006, 17, 3978-3988.

63. Crosas-Molist, E.; Bertran, E.; Sancho, P.; López-Luque, J.; Fernando, J.; Sánchez, A.; Fernández, M.; Navarro, E.; Fabregat, I. The NADPH oxidase NOX4 inhibits hepatocyte proliferation and liver cancer progression. Free Radic. Biol. Med. 2014, 69, 338-347.

64. Crestani, B.; Besnard, V.; Boczkowski, J. Signalling pathways from NADPH oxidase-4 to idiopathic pulmonary fibrosis. Int. J. Biochem. Cell Biol. 2011, 43, 1086-1089.

65. Hou, Y.; Ghosh, P.; Wan, R.; Ouyang, X.; Cheng, H.; Mattson, M.; Cheng, A. Permeability transition pore-mediated mitochondrial superoxide flashes. Neurobiol. Aging 2014, 35, 975-989.

66. Lustgarten, M.S.; Bhattacharya, A.; Muller, F.L.; Jang, Y.C.; Shimizu, T.; Shirasawa, T.; Richardson, A.; van Remmen, H. Complex I generated, mitochondrial matrix-directed superoxide is released from the mitochondria through voltage dependent anion channels. Biochem. Biophys. Res. Commun. 2012, 422, 515-521.

67. Piskernik, C.; Haindl, S.; Behling, T.; Gerald, Z.; Kehrer, I.; Redl, H.; Kozlov, A.V. Antimycin A and lipopolysaccharide cause the leakage of superoxide radicals from rat liver mitochondria. Biochim. Biophys. Acta 2008, 1782, 280-285.

68. Chen, K. Beyond LDL oxidation: ROS in vascular signal transduction. Free Radic. Biol. Med. 2003, 35, 117-132. 
69. Chen, K.; Thomas, S.R.; Albano, A.; Murphy, M.P.; Keaney, J.F. Mitochondrial function is required for hydrogen peroxide-induced growth factor receptor transactivation and downstream signaling. J. Biol. Chem. 2004, 279, 35079-35086.

70. Wang, Y.; Zang, Q.S.; Liu, Z.; Wu, Q.; Maass, D.; Dulan, G.; Shaul, P.W.; Melito, L.; Frantz, D.E.; Kilgore, J.A.; et al. Regulation of VEGF-induced endothelial cell migration by mitochondrial reactive oxygen species. Am. J. Physiol. 2011, 301, C695-C704.

71. Schmidt, K.N.; Amstad, P.; Cerutti, P.; Baeuerle, P.A. The roles of hydrogen peroxide and superoxide as messengers in the activation of transcription factor NF-кB. Chem. Biol. 1995, 2, $13-22$.

72. Brunelle, J.K.; Bell, E.L.; Quesada, N.M.; Vercauteren, K.; Tiranti, V.; Zeviani, M.; Scarpulla, R.C.; Chandel, N.S. Oxygen sensing requires mitochondrial ROS but not oxidative phosphorylation. Cell Metab. 2005, 1, 409-414.

73. West, A.P.; Brodsky, I.E.; Rahner, C.; Woo, D.K.; Erdjument-Bromage, H.; Tempst, P.; Walsh, M.C.; Choi, Y.; Shadel, G.S.; Ghosh, S.; et al. TLR signalling augments macrophage bactericidal activity through mitochondrial ROS. Nature 2011, 472, 476-480.

74. Hoarau, E.; Chandra, V.; Rustin, P.; Scharfmann, R.; Duvillie, B. Pro-oxidant/antioxidant balance controls pancreatic $\beta$-cell differentiation through the ERK1/2 pathway. Cell Death Dis. 2014, doi:10.1038/cddis.2014.441.

75. Henriksen, E.J. Effects of $\mathrm{H}_{2} \mathrm{O}_{2}$ on insulin signaling the glucose transport system in mammalian skeletal muscle. Methods Enzymol. 2013, 528, 269-278.

76. Marinho, H.S.; Real, C.; Cyrne, L.; Soares, H.; Antunes, F. Hydrogen peroxide sensing, signaling and regulation of transcription factors. Redox Biol. 2014, 2, 535-562.

77. Bretón-Romero, R.; Lamas, S. Hydrogen peroxide signaling in vascular endothelial cells. Redox Biol. 2014, 2, 529-534.

78. Garlid, A.O.; Jaburek, M.; Jacobs, J.P.; Garlid, K.D. Mitochondrial reactive oxygen species: Which ROS signals cardioprotection? Am. J. Physiol. Heart Circ. Physiol. 2013, 305, H960-H968.

79. Feng, X.-X.; Liu, M.; Yan, W.; Zhou, Z.-Z.; Xia, Y.-J.; Tu, W.; Li, P.-Y.; Tian, D.-A. $\beta 3$ Integrin promotes TGF- $\beta 1 / \mathrm{H}_{2} \mathrm{O}_{2} / \mathrm{HOCl}$-mediated induction of metastatic phenotype of hepatocellular carcinoma cells by enhancing TGF- $\beta 1$ signaling. PLOS ONE 2013, 8, e79857.

80. Klotz, L.-O.; Schroeder, P.; Sies, H. Peroxynitrite signaling: Receptor tyrosine kinases and activation of stress-responsive pathways. Free Radic. Biol. Med. 2002, 33, 737-743.

81. Szabó, C.; Ischiropoulos, H.; Radi, R. Peroxynitrite: Biochemistry, pathophysiology and development of therapeutics. Nat. Rev. 2007, 6, 662-680. 


\title{
Heme Degradation by Heme Oxygenase Protects Mitochondria but Induces ER Stress via Formed Bilirubin
}

\author{
Andrea Müllebner, Rudolf Moldzio, Heinz Redl, Andrey V. Kozlov and \\ J. Catharina Duvigneau
}

\begin{abstract}
Heme oxygenase (HO), in conjunction with biliverdin reductase, degrades heme to carbon monoxide, ferrous iron and bilirubin (BR); the latter is a potent antioxidant. The induced isoform HO-1 has evoked intense research interest, especially because it manifests anti-inflammatory and anti-apoptotic effects relieving acute cell stress. The mechanisms by which HO mediates the described effects are not completely clear. However, the degradation of heme, a strong pro-oxidant, and the generation of BR are considered to play key roles. The aim of this study was to determine the effects of BR on vital functions of hepatocytes focusing on mitochondria and the endoplasmic reticulum (ER). The affinity of BR to proteins is a known challenge for its exact quantification. We consider two major consequences of this affinity, namely possible analytical errors in the determination of HO activity, and biological effects of BR due to direct interaction with protein function. In order to overcome analytical bias we applied a polynomial correction accounting for the loss of BR due to its adsorption to proteins. To identify potential intracellular targets of BR we used an in vitro approach involving hepatocytes and isolated mitochondria. After verification that the hepatocytes possess $\mathrm{HO}$ activity at a similar level as liver tissue by using our improved post-extraction spectroscopic assay, we elucidated the effects of increased HO activity and the formed BR on mitochondrial function and the ER stress response. Our data show that BR may compromise cellular metabolism and proliferation via induction of ER stress. ER and mitochondria respond differently to elevated levels of BR and HO-activity. Mitochondria are susceptible to hemin, but active HO protects them against hemin-induced toxicity. BR at slightly elevated levels induces a stress response at the ER, resulting in a decreased proliferative and metabolic activity of hepatocytes. However, the proteins that are targeted by BR still have to be identified.
\end{abstract}

Reprinted from Biomolecules. Cite as: Müllebner, A.; Moldzio, R.; Redl, H.; Kozlov, A.V.; Duvigneau, J.C. Heme Degradation by Heme Oxygenase Protects Mitochondria but Induces ER Stress via Formed Bilirubin. Biomolecules 2015, 5, 679-701.

\section{Introduction}

Heme oxygenase $(\mathrm{HO})$, residing at the endoplasmic reticulum membrane, is the rate-limiting enzyme in the degradation of heme, yielding equivalent amounts of carbon monoxide (CO), ferrous iron $\left(\mathrm{Fe}^{2+}\right)$, and biliverdin (BV). BV is subsequently reduced to bilirubin (BR) by the cytosolic BV reductase (BVR). Stressful conditions lead to an increase in HO activity due to induction of HO-1 [1], a member of the heat shock protein family (HSP32). Up-regulation of HO in the liver is caused by multiple stimuli that include cytokines, bacterial toxins, hypoxia, and increased amounts of the HO substrate, protoheme IX (heme). HO-1 was shown to mediate tissue protection, since its inhibition increased tissue injury, while tissues were protected when HO-1 was upregulated prior to 
an acute experimental insult [2]. The cytoprotective effects of HO-1 are partly attributed to the degradation of excessive free heme and partly to the generation of the heme degradation products $\mathrm{CO}$ and $\mathrm{BR}$, which are able to mimic HO-1-mediated effects [3-5]. Although BR was found to exert anti-oxidant activity, which together with BV effectively protects the water/membrane interface [6,7], it is not clear to what extent BR formed in the HO reaction may contribute to the protection against heme-induced oxidative damage to subcellular structures.

Besides its relevance as diagnostic marker for liver diseases [8], BR was for a long time considered a waste product of heme degradation. However, elevated levels of unconjugated BR are able to induce cytotoxic effects, which are well documented for developing neuronal cells [9-12]. Free unconjugated BR was found to unbalance the redox homeostasis [13], or to affect the mitochondrial membrane integrity and to induce apoptosis [14]. The liver is one of the organs with a relatively high $\mathrm{HO}$ activity and involved in the elimination of BR. Thus, especially under conditions of elevated HO activity BR levels may exceed physiologic levels.

Determination of $\mathrm{HO}$ activity by means of BR quantification following the classical photometric extraction assay [15-17] is sensitive to higher protein concentrations making direct comparison between different sample types difficult. Thus, improvements of these assays should take the high affinity of lipophilic BR to proteins into account. Additionally, this affinity suggests that functional interaction with lipid and protein-rich structures, such as mitochondria or ER, are likely to occur. Increased levels of BR are formed during enhanced HO activity [5] and may target intracellular structures. However, it is not clear whether such an interaction would contribute to protective effects of the $\mathrm{HO}$ reaction or whether it may compromise cellular function and thereby limit the cytoprotective properties of the HO reaction. In order to approach this topic we addressed the following questions using rat liver, cultured hepatocytes and isolated mitochondria as in vitro systems:

(1) How to account for the amount of BR that is adsorbed by protein and thus not considered when applying the classical photometric extraction assay for the determination of HO activity?

(2) Are the in vitro model systems suitable to investigate the effects of BR that is released following $\mathrm{HO}$ reaction?

(3) Does the HO reaction rescue hepatic mitochondria from hemin-mediated toxicity?

(4) Is the anti-oxidative property of BR involved in the protective effect of $\mathrm{HO}$ towards mitochondria?

(5) How does BR formation relate to the metabolic activity and the proliferative response of cultured hepatocytes under conditions of accelerated HO activity?

(6) Which subcellular structure in the hepatocyte is sensitive to increased levels of BR?

\section{Aims of This Study}

This study aimed at determining the potential limits of the protective range of the HO reaction in liver cells due to the formation of BR. In contrast to previous reports we focus this study more on the biological/analytical impact of the high affinity of BR to proteins in the liver. We consider two major consequences of the high affinity, namely errors in the determined quantity of BR as a measure 
for $\mathrm{HO}$ activity and the direct interaction of BR with mitochondria and ER. These effects were investigated using rat livers, cultured hepatocytes, and isolated mitochondria.

\section{Results and Discussion}

At increased protein concentrations the precise quantification of BR is a problem, because BR may adsorb to proteins, as known for albumin [18]. In the first part of the study we focused on the improvement of the $\mathrm{HO}$ assay, since determination of $\mathrm{HO}$ activity using BR quantification was compromised by higher protein concentrations [19].

\subsection{Protein Adsorption of BR and Subsequent Interference with the Quantification Can Be Corrected Using a Polynomial that Accounts for the Protein Amount Present in the Assay}

Quantification of BR, the end product from the $\mathrm{HO} / \mathrm{BVR}$ reaction, is least laborious and therefore the most frequently used approach to determine $\mathrm{HO}$ activity $[15,16]$. BV and its reduction product $\mathrm{BR}$ are components exclusively formed by the HO/BVR system and generally tissue or cell homogenates possess sufficient BVR activity assuring the complete conversion of BV to BR and thus allowing the determination of BR by means of HPLC [20] or by photo spectroscopy [15,17].

Although extraction of BR from the aqueous phase into an organic solvent [17] significantly enhances the sensitivity of the assay, because BR is the only component absorbing around $450 \mathrm{~nm}$ in the organic extract, the load of unspecific protein has to be reduced, as it was shown to interfere with the assay [19]. Therefore generally microsomal-enriched fractions are prepared, which contain less protein [21]. However, the use of microsomal preparations bears the risk of partially losing $\mathrm{HO}$ activity. It was shown that pathogenic stimuli may induce translocation of HO-1 into the cell nucleus [22] or into mitochondria [23]. This translocation increased the enzymatic activity to convert heme in the target compartment [23], while the activity in the microsomal fraction decreased [22].

The problem to correctly quantify BR can be solved in two ways. Either BR calibration curves are used, which contain the same amount of protein, as was the case in a recently presented study for the determination of BR by ELISA [24], or the effect of protein adsorption has to be considered using a mathematical approach. The latter has the advantage of circumventing the laborious and time-consuming preparation of appropriate calibration curves. However, both approaches allow an improved comparison of the capacities of cells or tissues to convert heme, since preparation steps that may introduce biases are reduced.

To quantify BR formed by the HO reaction we used calibration curves which we obtained by adding known amounts of BR to an equivalent amount of assay buffer followed by extraction into chloroform (Figure 1A). When adding protein the amount of BR extractable from the buffer decreased in a non-linear fashion (Figure 1B). At constant protein concentrations, however, the relation between input BR and extractable BR remained linear (Figure 1C). Therefore it was possible to develop a polynomial for calculating a correction factor $\mathrm{f}$, which takes into account the adsorption of BR to protein, which is dependent on the amount $\mathrm{x}$ of protein. 
The corrected BR amount is: $b_{\text {corr }}=b r \times f$

$\mathrm{br}=\mathrm{BR}$ concentration (calculated from the calibration curve using the differential OD)

$\mathrm{f}=-0.076 \times \mathrm{x}^{2}+0.704 \times \mathrm{x}+1.027$

$\mathrm{x}=$ protein content present in the assay in $\mathrm{mg}$

Using this equation, we were able to achieve a nearly linear relationship between the amount of BR formed in the reaction and the amount of tissue homogenate subjected to the assay for determination of HO-activity (Figure 1D). The data presented in Figure 1 show that the high affinity of BR to proteins may result in underestimation of HO-activity, which can be corrected using the polynomial. In addition to improvement of the analytical procedure determining HO activity, this result stimulated us to explore the biological impact of a presumed interaction of BR with intracellular protein. Since BR is formed by the HO/BVR reaction within the cell, in close vicinity to the ER, we focused our studies on the effects of BR on mitochondria and ER, structures that are rich in protein and membrane lipids.

\subsection{BRL3A Cells Have Similar HO Activities as Liver Tissue}

We first verified the suitability of the hepatocyte line BRL3A regarding its $\mathrm{HO}$ activity, since we aimed at studying the effects of BR formed by the $\mathrm{HO}$ reaction in a cell culture model. In the liver different cell types contribute to the activity of $\mathrm{HO}$, composed of the activity of both enzymes, HO-1 and HO-2. Under physiological conditions the determined HO activity nearly exclusively consists of the activity of HO-2, while an increase accounts for the induction of HO-1, which occurs in all liver cells to different degrees [25]. Since the amount of BR formed depends on the level of the HO activity, we first examined whether BRL3A cells would be able to convert heme at comparable rates as homogenized liver. Cells were cultured and treated with various amounts of hemin, that is protoporphyrin IX containing ferric iron, or vehicle for $16 \mathrm{~h}$, and examined for HO activity as described in the Materials and Methods section (Figure 2). Basal levels of HO activity (Figure 2, grey bars) were similar to those found in homogenates obtained from livers of control rats (dashed line in Figure 2). An incubation for $16 \mathrm{~h}$ with varying concentrations of hemin resulted in a dose dependent increase in $\mathrm{HO}$ activity, indicative for HO-1 induction (Figure 2, black bars).

These findings show that BRL3A cells are suitable to study the role of $\mathrm{HO}$ and the effects mediated by the products of heme degradation. 

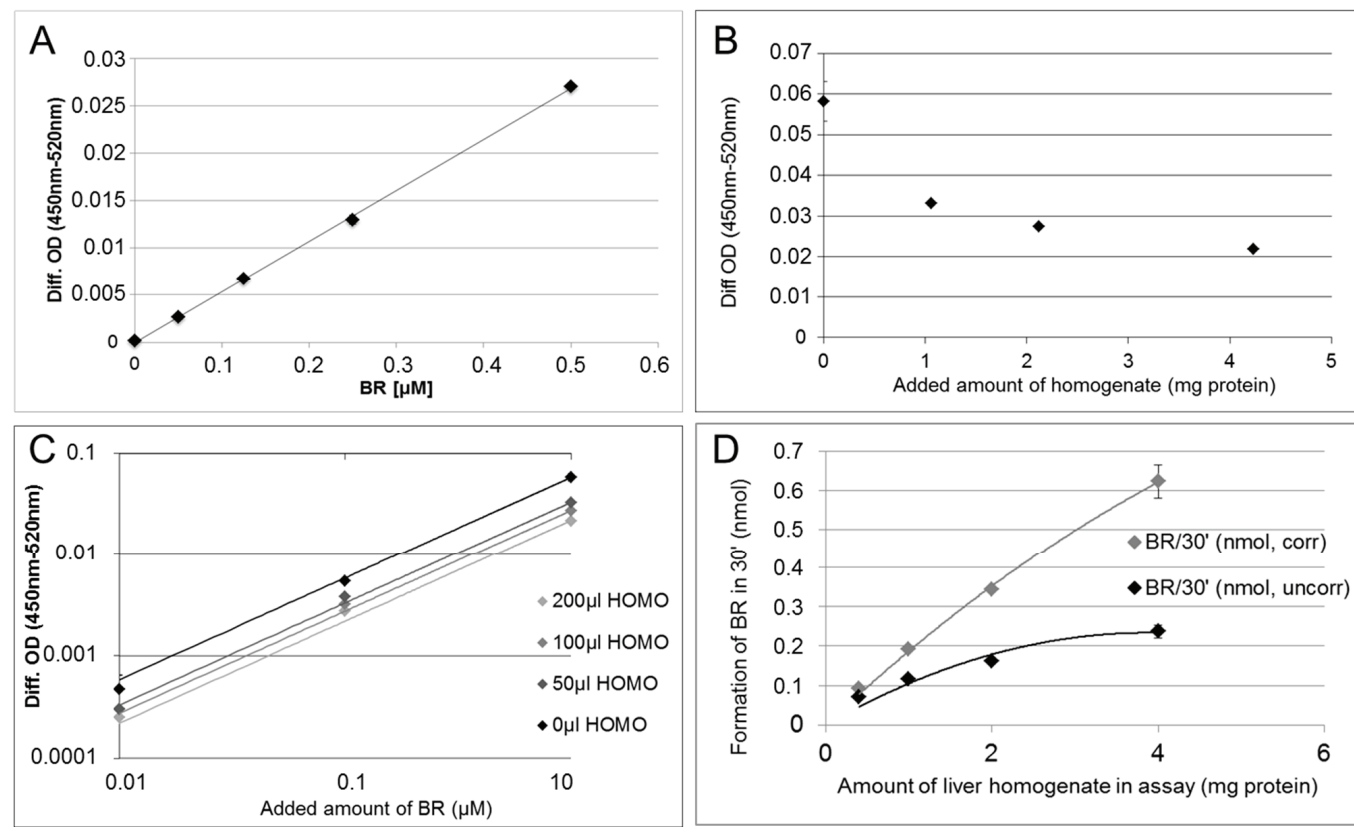

Figure 1. The tight interaction of BR with protein leads to underestimation of enzyme activities using the classical HO-assay. (A) Relationship between input and extractable amount of BR from HO-assay buffer (amount of BR was calculated using OD at $450 \mathrm{~nm}$ corrected for background OD at $520 \mathrm{~nm}$ (Diff. OD)) was linear (no protein added); (B) Presence of protein (liver homogenate: HOMO) in assay buffer supplemented with BR $(1 \mu \mathrm{M})$ decreased the extractable amount of BR (Diff. OD); (C) Relationship between input and extractable amount of BR $(0.01-1 \mu \mathrm{M})$ from HO-assay buffer (Diff. OD) was linear at constant protein concentration (added tissue homogenate (HOMO) with constant protein concentration of $10 \mathrm{mg}$ protein $/ \mathrm{mL}$ ); (D) Using the polynomial for correcting the $\mathrm{BR}$ amount, the activity of $\mathrm{HO}$ (formation of BR/30 min) depended nearly linearly on the amount of liver homogenate used for the assay.

\subsection{HO Reaction Rescues Mitochondria from Hemin-Mediated Impairment of Respiration and}

\section{Subsequent Fragmentation}

We next questioned whether HO in BRL3A cells would protect mitochondria of BRL3A cells against hemin-induced toxicity via formed BR. It is known that mitochondria are particularly sensitive to increased intracellular levels of heme [26]. Although heme may reach much higher concentrations, we used levels that have been reported previously as the intracellular threshold for inducing heme toxicity [26].

Heme toxicity is based on the oxidative modification and consequent damage exerted to membranes and associated proteins when exceeding critical levels. Heme induces HO-1 by directly interacting with the heme-sensitive transcription factors $\mathrm{BACH} 1$ and $\mathrm{BACH} 2$ [27], resulting in its subsequent degradation by the up-regulated HO activity. HO may provide protection in two ways, 
either by consumption of heme or by the release of BR, exerting putative anti-oxidative properties, or by both. In the following experiments we examined the effect of $\mathrm{HO}$ on hemin-mediated impairment of mitochondrial function. BRL3A cells were treated with $20 \mu \mathrm{M}$ hemin and mitochondria were visualized using JC-1 (Figure 3), as a measure for membrane potential (Figure 3A1-C1). Membrane potential initially decreased in cells treated with hemin, and this effect was most pronounced when its degradation was inhibited by zinc protoprophyrin IX (ZnIXPP), a competitive inhibitor of $\mathrm{HO}$ (Figure 3D). Continuous inhibition of hemin degradation resulted in an increased mitochondrial fragmentation (Figure 3C1). Cells treated with hemin or vehicle alone did not show any effects on their mitochondria (Figure 3A,B).

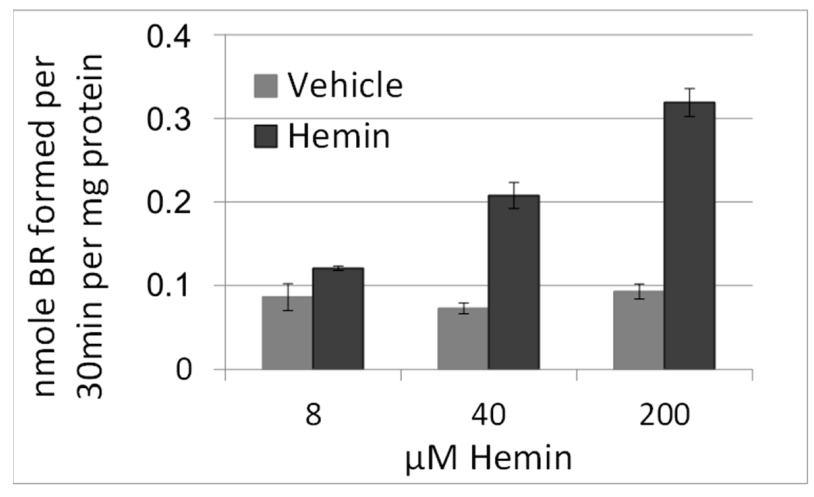

Figure 2. HO activity in hepatocytes after treatment with hemin. Cells were treated with vehicle (DMSO, grey bars) or hemin $(8,40,200 \mu \mathrm{M}$, black bars) for $16 \mathrm{~h}$. HO activity was determined in homogenized cells as described in the Materials and Methods section. The capacity to convert hemin increased in function to the concentration present in medium. HO activity, expressed as capacity of $1 \mathrm{mg}$ cell protein to produce BR was similar to that obtained in the liver of control rats $(n=5$, dashed line). Data are given as means $( \pm \mathrm{SD})$ obtained from one experiment with $n=2$ replicates.

In order to rule out the possibility that the inhibitor itself may have caused mitochondrial dysfunction, we incubated isolated mitochondria with either hemin or ZnIXPP at various concentrations and examined respiration in terms of oxygen consumption (Figure 4).

We found that isolated liver mitochondria responded with a significant decrease of oxygen consumption at a concentration of $20 \mu \mathrm{M}$ hemin (Figure 4), which is in line with our previous cell culture experiment. In contrast to hemin, ZnIXPP did not affect mitochondrial respiration, suggesting that iron ions play the principal role in the induction of mitochondrial dysfunction. Thus, our results show that functional HO in parenchymal liver cells protects mitochondria against hemin-mediated respiratory dysfunction. It is possible that the heme degradation products contribute to this effect, although initial levels present are presumably too low, as the levels of hemin are still high. However, longer incubation time may raise BR until reaching effective concentration. 

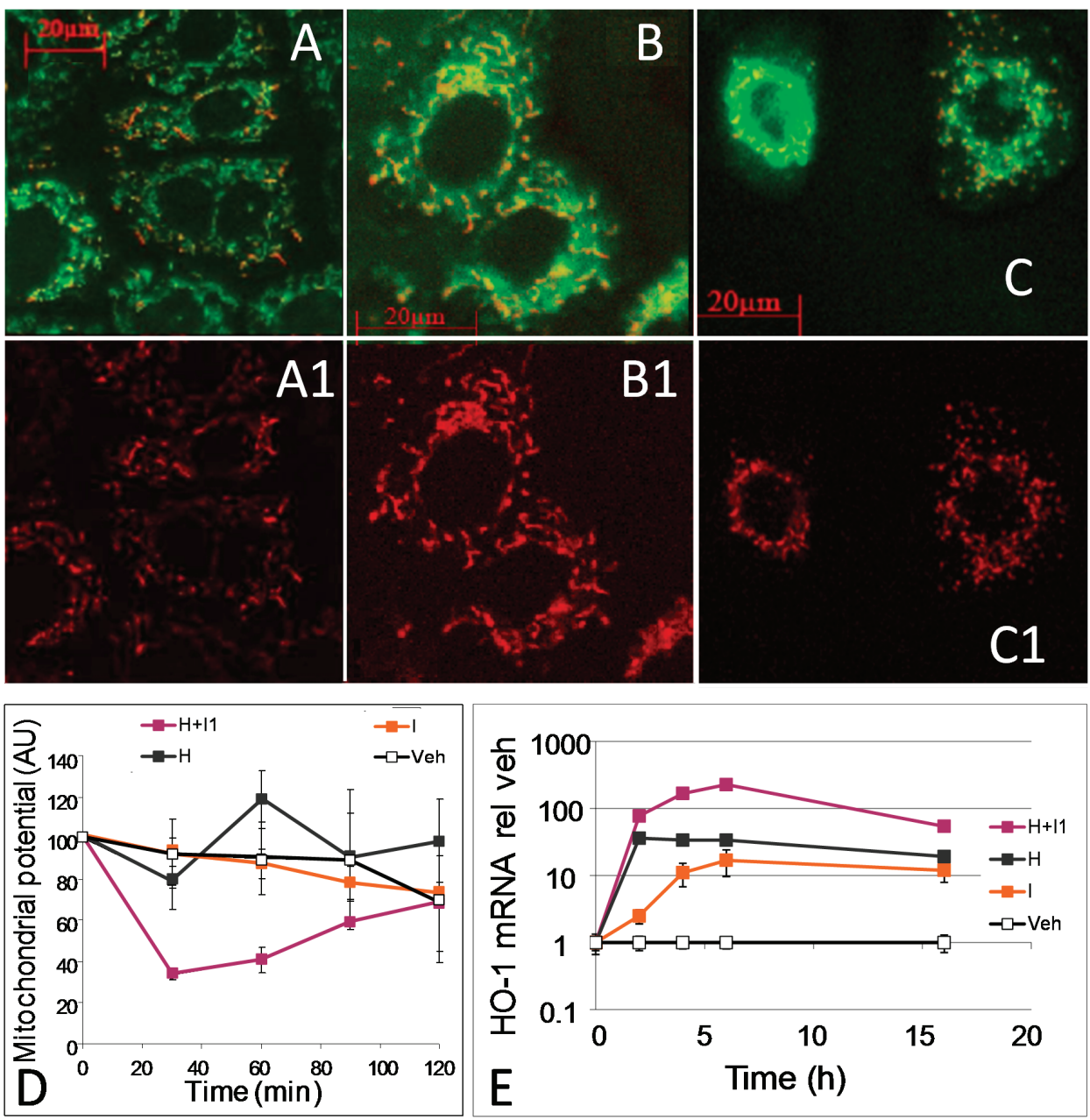

Figure 3. Effect of hemin and zinc protoprophyrin IX on mitochondrial function and morphology in hepatocytes. Liver cells (BRL3A) were incubated with JC-1 for $30 \mathrm{~min}$, thereafter vehicle (DMSO, A) or hemin $(20 \mu \mathrm{M}) ;(\mathbf{B}, \mathbf{C}))$ was added. Zinc protoporphyrin (ZnIXPP), a competitive inhibitor of $\mathrm{HO}(0.2 \mu \mathrm{M})$; was added $10 \mathrm{~min}$ before $(\mathbf{C})$. Cells were analyzed after $2 \mathrm{~h}$. A1, B1 and $\mathbf{C 1}$ show energized mitochondria (fluorescence at $590 \mathrm{~nm}$ ). Competitive inhibition of HO by ZnIXPP resulted in delayed fragmentation of mitochondria (C1); (D) Effect of hemin (H, grey line), HO-inhibitor ZnIXPP (I, orange line), and hemin plus inhibitor $(\mathrm{H}+\mathrm{I} 1$, violet line) in the concentrations indicated above on quantification of mitochondrial potential (intensity of background normalized JC-1 fluorescence (emission at $590 \mathrm{~nm}$ ) was used as a parameter for mitochondrial potential); (E) Effect of hemin (H, grey line), HO-inhibitor ZnIXPP (I, orange line), and hemin plus inhibitor $(\mathrm{H}+\mathrm{I}$, violet line) in the concentrations indicated above on HO-1 mRNA expression in BRL3A cells determined by qPCR. Data are given as means $( \pm \mathrm{SD})$ obtained from one representative experiment using $n=4$ replicates. 


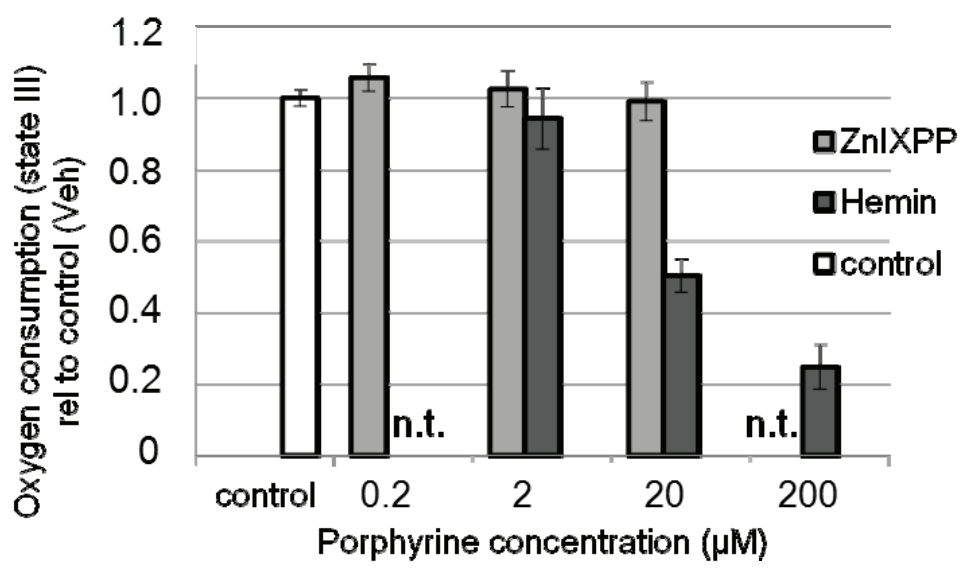

Figure 4. Hemin compromizes respiration of mitochondria. Isolated mitochondria were supplemented with succinate/rotenon to promote respiration of complex II. Transition to state III was induced by adding ADP and oxygen consumption was determined immediately after adding vehicle, hemin in the following concentrations: 2, 20 and $200 \mu \mathrm{M}$. In order to exclude effects of the HO inhibitor we also tested ZnIXPP (HO inhibitor) in the following concentrations: $0.2,2$ and $20 \mu \mathrm{M}$. Concentrations not tested are indicated (n.t.). State III respiration is indicated relative to the control (vehicle alone, set to 1). Data are given as means $( \pm \mathrm{SD})$ obtained from one experiment with $n=5$ replicates.

\subsection{Bilirubin Does not Prevent Hemin-Induced Repression of Respiration in Liver Mitochondria}

In order to understand whether BR is able to mediate the preservation of mitochondrial function seen in cells with functional $\mathrm{HO}$, we used isolated mitochondria treated with hemin and BR simultaneously (Figure 5). As was shown before (Figures 3 and 4), a nearly immediate dose-dependent decrease of oxygen consumption occurred in mitochondria treated with increasing amounts of hemin. Addition of BR at physiological concentrations tended to further decrease mitochondrial respiration, however, without being significant. Additionally, BR was not able to restore the hemin-mediated depression of respiration (Figure 5). This suggests that in cells treated with hemin, the removal of heme by $\mathrm{HO}$ and not the release of $\mathrm{BR}$, mediates the protection of mitochondria.

Interestingly, others showed that BR was able to modulate membrane integrity and redox status [14] of mitochondria, to modulate cytochrome c oxidase activity [28], and to induce apoptotic cell death, which involves mitochondrial pathways [14,29,30], without reporting on changes of respiratory parameters. Thus BR may target other cellular functions that are more sensitive to BR.

\subsection{Formation of BR and Excretion to the Cell Culture Medium Is Accelerated in Response to hemin, but Decreases Cell Proliferation Rate}

We next questioned how much BR is produced by BRL3A cells that are cultured in the presence of hemin, and whether an increased amount of BR extracted from the cell culture medium would 
reflect the underlying capacity to convert hemin determined ex vivo (see Figure 2). The presence of $\mathrm{BR}$ in the cell culture medium may represent an additional measure for $\mathrm{HO}$ activity (in-situ $\mathrm{HO}$ activity), provided that $\mathrm{BR}$ is not degraded. BR was extracted from medium and extracted into $\mathrm{CHCl}_{3}$, and quantified by means of photo spectroscopy using calibration curves.

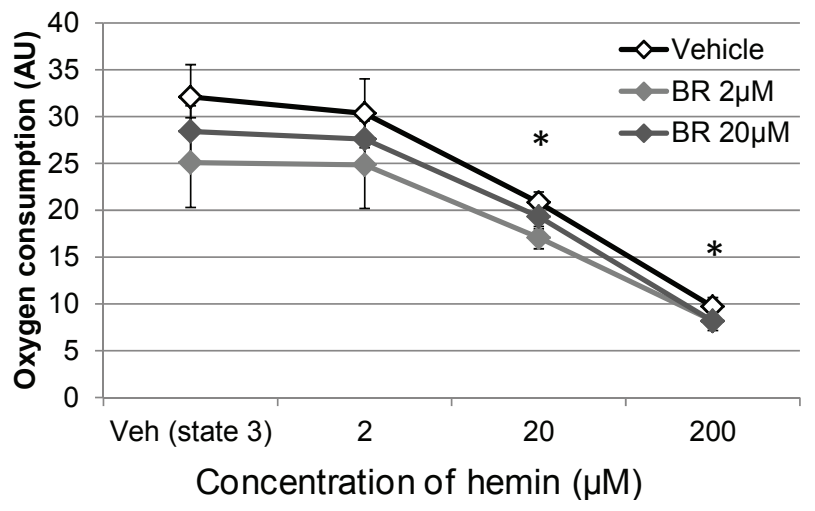

Figure 5. BR was not able to prevent hemin-mediated decrease of mitochondrial respiration. Liver mitochondria were isolated as described in the Materials and Methods section and treated with BR in the indicated concentration or with DMSO (Veh). State 3 respiration of complex II was induced by adding ADP and effects on oxygen consumption were determined after adding hemin in the indicated concentrations. Data are given as means $( \pm \mathrm{SD})$, obtained from one experiment with $n=2$ (control)/4 (BR) replicates, indicating significant differences $(*)$ to the control (Veh state 3 ).

Cells treated with $20 \mu \mathrm{M}$ hemin responded with increasing BR production, compared to control (Figure 6A). We next questioned whether BR production and excretion into the medium would reflect the $\mathrm{HO}$ activity determined ex vivo. If so, we would expect that an amount of $20 \mathrm{nmol}$ hemin should be converted to $20 \mathrm{nmol}$ BR by $1 \mathrm{mg}$ BRL3A cell protein within $66 \mathrm{~h}$. Considering a fully upregulated HO-1 (approximately within $12 \mathrm{~h}$ ) we expected 1.6 nmole BR to be produced. However in the time period between $12 \mathrm{~h}$ and $24 \mathrm{~h}$ we were able to extract only 0.16 nmole BR per mg cell protein (Figure 6B). Although unbound BR may freely diffuse through cell membranes [31], once bound to albumin, a part will be redirected into hepatocytes via vesicular uptake [32]. Furthermore, it was shown that BR may be oxidized by cytochrome P450 2A [33,34], and a part of BR may have been conjugated. Therefore an unknown amount of BR has possibly escaped from quantification. Although the appearance of BR in the cell culture may not properly represent the underlying HO activity in situ, the data show that BR formation occurs much slower than expected. With increasing BR concentrations however, the proliferation of the producing cells slowed down, reaching only $80 \%$ of the cell number of the control, verified by estimation of the underlying cell number at each time point (Figure 6A, inset Y-axis). Due to the tight interaction of BR it is possible, that the newly formed BR modulates cell function by binding to suitable proteins. Bilirubin was reported to inhibit proliferation in several cell types [35-37]. 


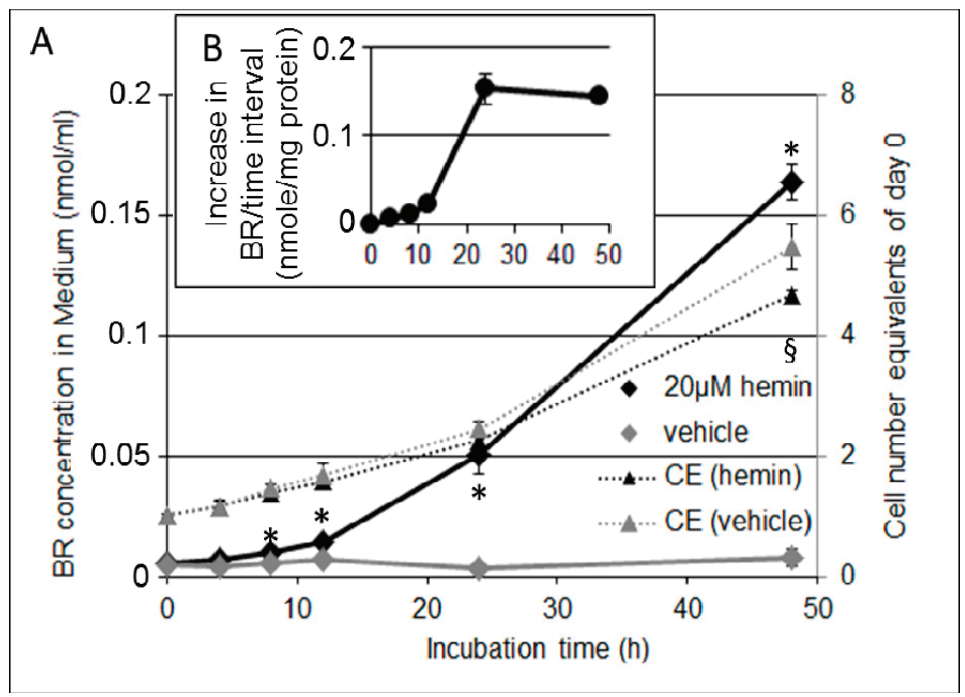

Figure 6. Production of BR by liver cells (BRL3A) as a function of the incubation time. (A) Cells were treated with vehicle (DMSO, grey symbols) or hemin $(20 \mu \mathrm{M}$, black symbols) for the indicated time. BR was extracted from medium (diamonds; solid lines). Underlying cell number equivalents (CE, inset $\mathrm{Y}$-axis) were determined (triangles) using crystal violet assay and expressed relative to the values of day 0 (dotted lines). After $48 \mathrm{~h}$, equivalents of hemin-treated cells were significantly lower compared to the vehicle-control (§); (B) Hemin treatment increased the total amount of BR that was newly formed (nmole) per time interval when calculated per mg cell protein. Protein content of cell samples was determined at $48 \mathrm{~h}$ using the Bradford method and extrapolated from cell equivalents for each time point. Data are given as means $( \pm \mathrm{SD})$, obtained from one experiment with $n=4$ replicates.

To elucidate the role of BR in regulating proliferation of BRL3A cells we incubated cells with varying concentrations of unconjugated BR and determined cell number (crystal violet assay; [38-40]) and metabolic activity (MTT assay; [41]) at different time points (12 h and $48 \mathrm{~h}$; Figure 7). Considering that an increase in $\mathrm{HO}$ would only slowly increase physiologic levels of BR, we used physiologic concentrations of BR, which range between 5 and $32 \mu \mathrm{M}$ in human serum [42] and about half as much in rodents [43], of which around 4\% appears as water-soluble glucuronides [44]. We found that physiologic levels of BR $(4-20 \mu \mathrm{M})$ decreased the proliferation rate about $20 \%$, but affected the metabolic activity to a much higher degree $(50 \%$ activity after $48 \mathrm{~h}$ at the highest BR concentration tested).

\subsection{BR Increases Expression of Markers for ER Stress and Unfolded Protein Response}

Decreased metabolic activity is frequently interpreted as a decrease in mitochondrial energy provision. However, also compromised ER function may lead to decreased cell proliferation rates, especially as a response to ER stressing agents [45]. 
A

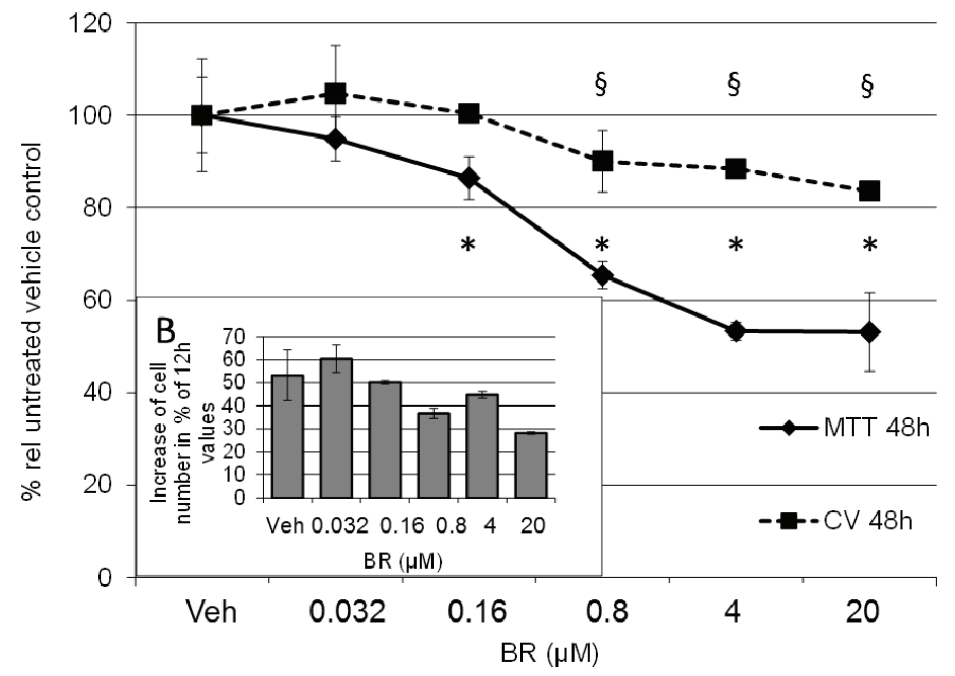

Figure 7. BR results in decreased proliferation and metabolic activity of BRL 3A cells. (A) Cells were treated with vehicle (Veh) or BR in the concentrations indicated, and incubated for $48 \mathrm{~h}$. Cell number (squares and dotted line) was determined using crystal violet assay, and expressed in \% relative to the vehicle control (CV $48 \mathrm{~h}$ ). Data are given as means $( \pm \mathrm{SD})$ obtained from two experiments with $n=2$ replicates. Metabolic activity of treated cells was determined by MTT assay (diamonds and full line). Data are given as means $( \pm \mathrm{SD}$ ) obtained from one experiment with $n=4$ replicates (MTT $48 \mathrm{~h}$ ). Significant differences to the control are indicated as $(*, \S)$; (B) Determination of cell numbers within one experiment at consecutive time points (12 h and $48 \mathrm{~h}$ ) showed a decreased proliferation rate in the presence of BR. Data are given as \% increase relative to the values determined at $12 \mathrm{~h}$.

We have found that BR concentrations reduced the metabolic activity of BRL3A indicative for enhanced cell stress. It is known that induction of ER stress decelerates growth rate, involving sXBP1 [46] and promotes apoptosis via CHOP [47,48]. We therefore analyzed the expression of markers for ER stress, X-Box binding protein 1 (XBP1), glucose regulated protein 78 (GRP78) HO-1, CRBP homologous protein (CHOP), and interleukin 6 (IL6) as a marker for an inflammatory response, in BR-treated BRL3A cells. Already after $8 \mathrm{~h}$ at concentrations between $4 \mu \mathrm{M}$ and $20 \mu \mathrm{M}$ BR elicited an ER stress response (Figure 8), which was accompanied by elevated levels of the XBP1 splice variant, a typical ER-stress marker [49]. Additionally, we determined increased levels of IL6, suggesting onset of an inflammatory response, a pathologic reaction mediated by classical ER stressors [50]. Our data indicate that BR may affect proper function of ER. BR may induce protein mis-folding and aggregation due to its particular chemical properties. We showed that BR, which is newly formed in the $\mathrm{HO}$ reaction, tightly binds to proteins. At higher concentrations BR is known to lead to aggregates which are favored at lower $\mathrm{pH}$ [51]. Additionally, BR is able to associate with 
calcium [52], which is high in the ER lumen, and to precipitate with other amphiphilic compounds. This phenomenon is known to occur in the bile leading to the formation of pigmented gallstones containing calcium bilirubinate [53]. In neuronal cells, both mitochondrial and ER function are sensitive to elevated concentrations of BR [54]. In parenchymal hepatocytes, in contrast, BR affects primarily the ER. If the concept remains valid that BR works as a potent anti-oxidant within the cell, an elevated level of BR is supposed to disturb the finely tuned redox equilibrium. However, it is well possible that under conditions of excessive oxidative stress BR would help to reinstall a disturbed equilibrium.

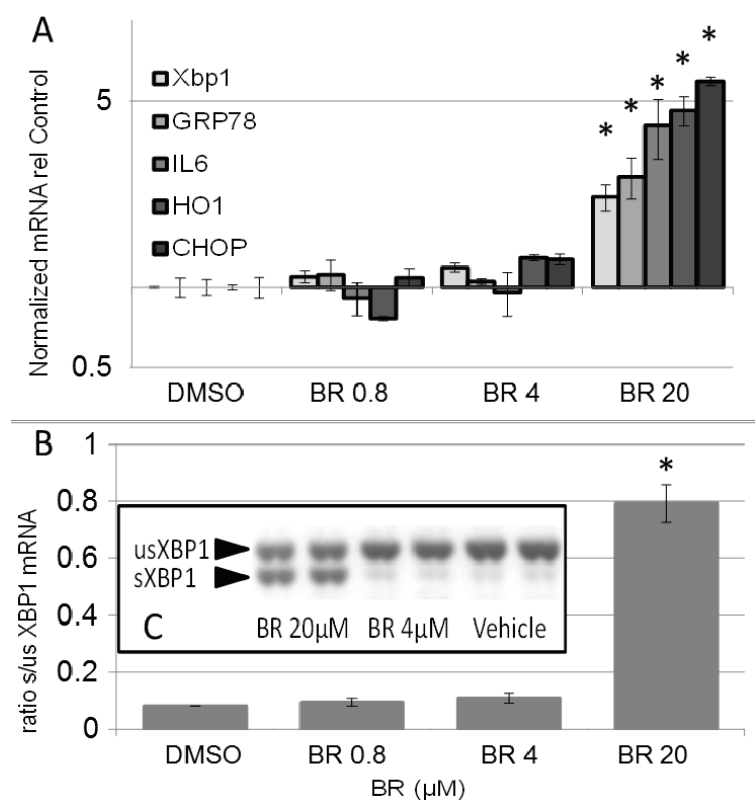

Figure 8. Physiologic range of BR induces ER stress response in BRL 3A cells. (A) Cells were treated with vehicle (DMSO) or BR in the concentrations indicated, and incubated for $8 \mathrm{~h}$. RNA was extracted and expression of cell stress markers X-Box binding protein 1 (XBP1), glucose regulated protein 78 (GRP78), interleukin 6 (IL6), HO-1, CRBP homologous protein (CHOP) and the internal reference genes (cyclophilin A, hypoxanthine ribosyltransferase, glycerinaldehyde dehydrogenase) used for basket normalisation was determined by means of real-time PCR. Target mRNA was normalized to the internal references and calculated relative to the vehicle control (DMSO); (B) BR induced unconventional XBP1 splicing. PCR products were separated using electrophoresis and visualized by ethidium bromide staining. PCR products consisting of spliced (sXBP1) and unspliced variants (usXBP1) were quantified by means of densitometry using the public domain Scion Image program (http://www.scioncorp.com/), and intensities were expressed as a ratio (spliced to unspliced isoforms). Data are given as means $( \pm \mathrm{SD})$ obtained from one experiment with $n=2$ replicates, indicating significant differences $(*)$. 
Thus, we cannot answer the question, whether the observed changes would be beneficial under pathologic conditions, or not. It was found, however, that treatment of obese mice with BR over a longer period, relieved signs of metabolic diseases [55]. Interestingly, in this pathologic model, markers for ER stress decreased. Further studies are needed to clarify the significance of BR-mediated interaction with the ER and the induction of a stress response.

\section{Experimental Section}

\subsection{Chemicals}

All reagents were obtained from Sigma-Aldrich (Vienna, Austria) unless otherwise noted. All porphyrins were dissolved in DMSO and used as a 500× stock solution.

\subsection{Cell Culture}

The adherently growing Buffalo rat liver cell line (BRL3A, European Collection of Cell Cultures, Salisbury, UK) was cultivated in Coon's F-12 medium with 5\% FCS (PAA, Linz, Austria). At a confluency of $70 \%-80 \%$ cells were passaged using $0.25 \%$ trypsin/EDTA and diluted $1 / 10$ for further culture. Maximal six consecutive passages were used.

\subsection{Animals}

Rats were injected with lipopolysaccharide (LPS) at a dose of $8 \mathrm{mg} / \mathrm{kg}$ (i.v.). Adult male Sprague-Dawley rats weighing $280 \pm 21$ g (Animal Research Laboratories, Himberg, Austria) were divided into two groups: a control group receiving saline i.v., and a group receiving $8 \mathrm{mg}$ lipopolysaccharide/kg i.v. (LPS; E. coli 026:B6, Difco, Detroit, MI, USA). At different time points $(0,2,4,8$, and $12 \mathrm{~h}(n=3 / 6))$, the animals were killed; liver tissue was taken for analytical examination, aliquoted and stored at $-80{ }^{\circ} \mathrm{C}$ until analysis. All animals received humane care according to the criteria outlined in the "Guide for the Care and Use of Laboratory Animals" prepared by the National Academy of Sciences and published by the National Institutes of Health (NIH publication 86-23, revised 1985).

\subsection{Determination of Cell Number by Crystal Violet Assay}

BRL3A cells were seeded at a density of $5-10 \times 10^{4} / \mathrm{mL}$ in 24 -wells using Coon's F-12 medium (5\% FCS). The next day medium was exchanged for medium containing hemin $(20 \mu \mathrm{M})$ or BR $(0.032 \mu \mathrm{M}, 0.16 \mu \mathrm{M}, 0.8 \mu \mathrm{M}, 4 \mu \mathrm{M}$, and $20 \mu \mathrm{M})$ or vehicle (DMSO). Cells were incubated for the given time points ( $4 \mathrm{~h}, 8 \mathrm{~h}, 12 \mathrm{~h}, 24 \mathrm{~h}$ and $48 \mathrm{~h}$ or $12 \mathrm{~h}$ and $48 \mathrm{~h}$ ). Thereafter culture medium was removed, cells were washed $3 \times$ with PBS and fixed with $4 \%$ paraformaldehyde in PBS. After washing $3 \times$ with $\mathrm{dH}_{2} \mathrm{O}$ cells were stained using crystal violet staining solution $(0.5 \%$ in ethanol $(10 \%))$ and incubated for $15 \mathrm{~min}$. Thereafter cells were washed three times, plates were dried, and kept in the dark until analysis. In each well $500 \mu \mathrm{L}$ acetic acid (10\%) was added. Stained cells were solubilized by pipetting. One hundred $\mu \mathrm{L}$ of the solution was transferred into a 96-well plate, and extinction at $590 \mathrm{~nm}$ was determined using a plate reader. 


\subsection{Determination of Metabolic Activity by MTT Assay}

BRL3A cells were prepared as described above in Section 4.4. The next day medium was exchanged for medium containing BR $(0.032 \mu \mathrm{M}, 0.16 \mu \mathrm{M}, 0.8 \mu \mathrm{M}, 4 \mu \mathrm{M}$, and $20 \mu \mathrm{M})$ or vehicle (DMSO). Cells were incubated for $48 \mathrm{~h}$. Six hour prior to the end of the experiment, medium was exchanged for MTT-containing medium $(0.5 \mathrm{mg} / \mathrm{mL})$ which was freshly prepared. Following a $6 \mathrm{~h}$ incubation period, supernatant was aspirated and formazan crystals were dissolved in sterile DMSO (same volume as the culture medium) by incubating at $37^{\circ} \mathrm{C}$ for $30 \mathrm{~min}$. After shaking, $100 \mu \mathrm{L}$ aliquots were transferred into 96 -wells and the absorbance was read at $550 \mathrm{~nm}$ using a plate reader.

\subsection{Cellular Heme Oxygenase Activity by Determination of BR Production in Medium}

For determination of BR production, cells were plated in 6-well plates at a density of $5 \times 10^{4} / \mathrm{mL}$. The next day medium was supplemented with $0.5 \%$ hemin solution (solved in DMSO) to a final concentration of $12.5 \mu \mathrm{g} / \mathrm{mL}$ (corresponding to $20 \mu \mathrm{M}$ ). At each time point $(4 \mathrm{~h}, 8 \mathrm{~h}, 12 \mathrm{~h}, 24 \mathrm{~h}$, and $48 \mathrm{~h}$ ) an aliquot of $200 \mu \mathrm{L}$ medium was removed and supplemented with $100 \mu \mathrm{L}$ saturated $\mathrm{KCl}$ and 2 $\mathrm{mL} \mathrm{CHCl}_{3}$. After vortexing $(3 \times 30 \mathrm{~s})$ and centrifugation $(250 \times \mathrm{g})$ the organic phase was harvested, and bilirubin concentration was determined using photo spectroscopy (U-3000, Hitachi, Tokyo, Japan). The samples were repeatedly ( 3 times) scanned between 600 and $380 \mathrm{~nm}$ using the following settings: slit: $2 \mathrm{~nm}, 120 \mathrm{~nm} / \mathrm{min}$, PMT: autogain, high resolution, and the difference in absorption between 450 and $520 \mathrm{~nm}$ determined. Samples were run in triplicates and obtained values were averaged. Calculation of the formed bilirubin was obtained using a standard calibration curve. This standard was generated by adding known amounts of bilirubin to Coon's F12 medium supplemented with $5 \% \mathrm{FCS}$, followed by the subsequent extraction of bilirubin. $\mathrm{HO}$ activity was calculated as nmole bilirubin formed per $\mathrm{ml}$ per $30 \mathrm{~min}$.

\subsection{Laser Scanning Microscopy}

BRL3A cells were grown in Lab-Tek two-chambered cover glasses (Nalge Nunc, Rochester, NY, USA) with cell culture medium (Coon's F-12 medium). For confocal microscopic investigations, cells were stained with JC-1 ( $2 \mu \mathrm{M}$ MitoProbe, Invitrogen, Carlsbad, CA, USA). After $20 \mathrm{~min}$, cells were treated with $\mathrm{Zn}(\mathrm{II})$ protoporphyrin IX $(0.1 ; 1 ; 10 \mathrm{mg} / \mathrm{L}$; Frontier Scientific, Logan, UT, USA). Subsequently hemin was added at a concentration of $12.5 \mathrm{mg} / \mathrm{L}$. Control cells were treated with DMSO (vehicle control). Thereafter cells were washed with Coon's F-12 medium. Fluorescence of JC-1 at $590 \mathrm{~nm}$ was used as parameter for mitochondrial potential. Imaging was performed with an inverted confocal microscope (LSM 510, Zeiss, Oberkochen, Germany) and 63× oil immersion objective. Image analysis was performed with the histogram toolbar (LSM 510, Zeiss). Regions of interests were marked manually and total fluorescence intensity was defined as mean $\mathrm{x}$ area + area $x$ threshold. 


\subsection{Gene Expression}

RNA was isolated from BRL3A treated with BR $(0.8 \mu \mathrm{M}, 4 \mu \mathrm{M}, 20 \mu \mathrm{M})$ for $8 \mathrm{~h}$ and processed as described elsewhere [56]. Primer sequences used for amplification are given in Table 1. Primer sequences for XBP-1 were newly designed (and amplification efficiency was verified by dilution series (accessory information is given in the Appendix Figure A1, Tables A1 and A2). Expression of target genes was measured using a $\mathrm{CFX}^{\mathrm{TM}}$ (Bio-Rad, Hercules, CA, USA). Each reaction contained SYBR ${ }^{\circledR}$ green I as reporter $(0.5 \times)$, iTaq $^{\mathrm{TM}}$ polymerase $^{\mathrm{TM}}(0.625 \mathrm{U} /$ reaction; BioRad $)$, the primers $(250 \mathrm{nmol} / \mathrm{L}$ each, Invitrogen) with a final concentration of $200 \mu \mathrm{mol} / \mathrm{L} \mathrm{dNTP} \mathrm{(each)} \mathrm{and} 3$ $\mathrm{mmol} / \mathrm{L} \mathrm{MgCl} 2$ in the provided reaction buffer with a final volume of $12 \mu \mathrm{L}$. Data were collected in the regression mode and calculated against an internal standard (IS) consisting of pooled cDNA samples of all experiments. We used a modified comparative $\Delta \Delta \mathrm{Cq}$ method. First the gene specific Cqs were subtracted from the mean Cq of the IS obtained for the same gene giving rise to $\Delta \mathrm{Cq}$. The values were then subtracted from the normalization factor, which was calculated by averaging the $\Delta$ Cqs of the internal reference genes (cyclophilin A, hypoxanthinribosyl transferase, glycerinaldehyde dehydrogenase) of the same sample $(\Delta \Delta \mathrm{Cq})$. The obtained $\Delta \Delta \mathrm{Cq}$ values of the replicates were averaged and expressed as $2^{-\Delta \Delta \mathrm{Cq}}$ in fold changes relative to the IS.

Table 1. Primers used for analysis of gene expression by real-time PCR.

\begin{tabular}{|c|c|c|c|c|}
\hline & Accession & Sense Primer & Antisense Primer & Source \\
\hline XBP-1 & NM_001004210.2 & gag tcc aag ggg aat gga gt & aca ggg tcc aac ttg tcc ag & Designed for this study \\
\hline GRP78 & S63521 & gtt ctg ctt gat gtg tgt cc & ttt ggt cat tgg tga tgg tg & {$[57]$} \\
\hline IL6 & NM_012589.1 & ccg gag agg aga ctt cac ag & aca gtg cat cat cge tgt tc & {$[58]$} \\
\hline HO-1 & NM_012580.2 & cca gcc aca cag cac tac & gcg gtc tta gec tct tct $g$ & {$[59]$} \\
\hline CHOP & NM_024134.2 & ttg ggg gca cct ata tct ca & ctc ctt cag tcg ctg ttt cc & {$[60]$} \\
\hline $\begin{array}{c}\text { GAPD } \\
\mathrm{H}\end{array}$ & M17701 & cat gec gcc tgg aga aac ctg cca & tgg get ggg tgg tcc agg ggt ttc & {$[61]$} \\
\hline HPRT & NM_012583 & ctc atg gac tga tta tgg aca gga c & gca ggt cag caa aga act tat agc c & {$[62]$} \\
\hline Cyc & M19533 & tat ctg cac tgc caa gac tga gtg & ctt ctt get ggt ctt gcc att cc & {$[62]$} \\
\hline
\end{tabular}

\subsection{Determination of Unconventional Splicing of XBP1}

For the quantitative determination of the spliced variant of XBP- 1 mRNA, $10 \mu \mathrm{L}$ from the PCR reaction product were separated on a $2 \%$ agarose gel and after staining with ethidium bromide visualized by $300 \mathrm{~nm}$ UV transillumination. Density of both products, the unspliced and the spliced variant, was quantified via computer assisted densitometric scanning using the public domain Scion Image program (http://www.scioncorp.com/), and the ratio of the spliced to the unspliced variant was determined in each sample.

\subsection{Heme Oxygenase Activity of Liver Tissue}

Liver tissue was homogenized 1:10 (gram tissue/mL buffer) in a buffer containing $300 \mathrm{mM}$ sucrose, $20 \mathrm{mM}$ TRIS and $2 \mathrm{mM}$ EDTA at a $\mathrm{pH}$ of 7.4. Approximately $1 \mathrm{mg}$ of protein was added to a reaction mixture containing $500 \mathrm{nmole} \mathrm{NADPH}$ in a $100 \mathrm{mM}$ potassium phosphate buffer with 
$1 \mathrm{mM}$ EDTA (pH: 7.4), supplemented by 20 nmoles of hemin. The mixture was incubated under constant agitation in darkness for $30 \mathrm{~min}$ at $37{ }^{\circ} \mathrm{C}$. Afterwards, the reaction was stopped by transferring the samples on ice. After addition of $1 / 5$ volume of saturated $\mathrm{KCl}$, the formed bilirubin was extracted into chloroform ( $4 \times$ the assay volume). Samples were then processed as described in Section 4.6. Samples were run in duplicates and obtained values were averaged and corrected for the absorption measured in corresponding samples incubated at $0{ }^{\circ} \mathrm{C}$. Calculation of the formed bilirubin was obtained using a standard calibration curve. This standard was generated by adding known amounts of bilirubin to a pool of tissue homogenate followed by the subsequent extraction of bilirubin. Protein concentration of liver homogenate was determined using Coomassie Brilliant Blue [63]. HO activity was corrected for the BR lost due to adsorption by proteins using the correction factor described in Section 3.1 and calculated as nmole bilirubin formed per mg protein per $30 \mathrm{~min}$.

\subsection{Heme Oxygenase Activity of BRL3A Cells}

BRL3A cells were seeded at a density of $5-10 \times 10^{4} / \mathrm{mL}$ in 6 -well plates using Coon's F-12 medium (5\% FCS). The next day medium was exchanged for medium containing hemin $(20 \mu \mathrm{M})$ or vehicle (DMSO). Cells were incubated for $16 \mathrm{~h}$. Medium was discarded, cell layer was washed once with prewarmed PBS and the cells were detached by adding $800 \mu \mathrm{L}$ of $0.25 \%$ trypsin/EDTA. After complete detachment cell suspension of each well was transferred into $5 \mathrm{~mL}$ vials containing $4 \mathrm{ml}$ culture medium to stop the trypsin activity. Cells were gently pelleted $(400 \times g, \mathrm{RT})(10 \mathrm{~min})$ and supernatant was aspirated. The tube was then placed in liquid nitrogen to snap freeze and stored at $-80{ }^{\circ} \mathrm{C}$ until being used. For the determination of $\mathrm{HO}$ activity, the cell pellet was quickly unfrozen and dissolved in $60 \mu \mathrm{L}$ buffer containing $300 \mathrm{mM}$ sucrose, $20 \mathrm{mM}$ TRIS and $2 \mathrm{mM}$ EDTA at a pH of 7.4. Approximately $0.3 \mathrm{mg}$ of protein $(50 \mu \mathrm{L})$ was added to a reaction mixture containing 500 nmole NADPH in a $100 \mathrm{mM}$ potassium phosphate buffer with $1 \mathrm{mM}$ EDTA (assay buffer, $\mathrm{pH}$ 7.4), supplemented by 20 nmoles of hemin. The mixture was incubated under constant agitation in darkness for $30 \mathrm{~min}$ at $37{ }^{\circ} \mathrm{C}$. Afterwards, the reaction was stopped by transferring the samples on ice. After addition of $1 / 5$ volume of saturated $\mathrm{KCl}$, the formed bilirubin was extracted into chloroform ( $4 \times$ the assay volume). Samples were then processed as described in Section 4.6. Samples were run in duplicates and obtained values were averaged and corrected for the absorption measured in solvent alone. Calculation of the formed bilirubin was obtained using regression analysis of standard calibration curves. These standards were generated by adding known amounts of bilirubin to assay buffer followed by the subsequent extraction of bilirubin. Protein concentration of liver homogenate was determined using Coomassie Brilliant Blue [63]. HO activity was corrected for the BR lost due to adsorption by proteins using the correction factor described in Section 3.1 and calculated as nmole bilirubin formed per $\mathrm{mg}$ protein per $30 \mathrm{~min}$.

\subsection{Preparation of Liver Mitochondria}

Sprague-Dawley rats weighing $280 \pm 21 \mathrm{~g}$ (Animal Research Laboratories, Himberg, Austria) were euthanized by decapitation. The protocol was approved by the City Government of Vienna, Austria, 
and all experiments were performed under the conditions described in the Guide for the Care and Use of Laboratory Animals of the National Institutes of Health. Immediately after decapitation, liver was extracted and placed in ice-cold sucrose buffer ( $0.25 \mathrm{M}$ sucrose, $10 \mathrm{mM}$ Tris-HCl, $1 \mathrm{mM}$ EDTA, 0.1\% ethanol, $\mathrm{pH}=7.4$ ), diced and rinsed with the same buffer to remove remaining blood. After blotting dry with paper, the liver pieces were weighed and the same buffer was added in a ratio of 1:6 liver/buffer (w/v) and homogenized using a Potter-Elvehjem homogenizer. Rat liver mitochondria (RLM) were prepared as described previously [64] and stored at $0{ }^{\circ} \mathrm{C}$ for $4-5 \mathrm{~h}$ in a buffer containing $0.25 \mathrm{M}$ sucrose, $10 \mathrm{mM}$ TRIS-HCl, $0.5 \mathrm{mM}$ EDTA (pH 7.2), and $0.5 \mathrm{~g} / \mathrm{L}$ essentially fatty-acid-free bovine serum albumin.

\subsection{Hepatic Mitochondrial Function}

Respiratory parameters of mitochondria isolated from control and LPS-treated rats were determined with a Clark-type oxygen electrode (OROBOROS Ltd, Innsbruck, Austria). Rat liver mitochondria $(0.5 \mathrm{mg} / \mathrm{mL})$ were incubated in a buffer consisting of $105 \mathrm{mM} \cdot \mathrm{KCl}, 20 \mathrm{mM}$ TRIS-HCl, $1 \mathrm{mM}$ diethylenetriaminepentaacetic acid, $5 \mathrm{mM} \cdot \mathrm{KH}_{2} \mathrm{PO}_{4}$, and $1 \mathrm{mg} / \mathrm{mL}$ fatty acid-free

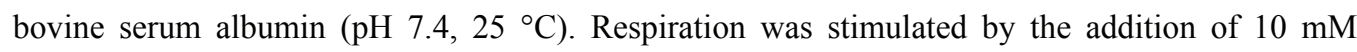
succinate in the presence of rotenone $(1 \mu \mathrm{g} / \mathrm{mL}$; complex II). The transition to state 3 respiration was induced by addition of $200 \mu \mathrm{M}$ ADP and used as parameter for ATP synthesis.

\subsection{Data Analysis and Statistics}

Data processing and graphics were made using Excel or SPSS 15 (SPSS Inc., Chicago, IL, USA). Data from experiments performed with cells and isolated mitochondria were analyzed by one-way ANOVA followed by LSD post hoc test using SPSS. Data from experiments using liver tissues obtained from animals were subjected to non-parametric analysis using Kruskal-Wallis. Differences to the control were considered significant when $p<0.05$, and are indicated. The numbers of independent samples (n) are indicated in figure legends.

\section{Conclusions}

We found that $\mathrm{HO}$ activity can be determined in each type of sample by the modified photometric extraction assay when the adsorption of BR to protein is accounted for. Additionally this shows that $\mathrm{BR}$ that is newly formed by the HO reaction may tightly adsorb to intracellular protein, and thereby modulate the function of sensitive target structures. We found that $\mathrm{HO}$ protected mitochondria from hemin-induced toxicity. BR at concentrations that were only slightly higher than the physiological concentrations was capable of inhibiting cell metabolism and proliferation and inducing a stress response at the ER. In BRL3A cells the primary target modulated by BR was the ER, which indicates that $\mathrm{HO}$ may modulate ER function via newly formed BR. 


\section{Acknowledgments}

The authors thank Annika Cronstedt-Fell, Jasmin Weeger, and Carmen Konzett for skilled assistance in performing and analyzing cell culture experiments. The technical support of Susanne Haindl, performing the experiments with isolated mitochondria, is greatly acknowledged. The study was supported by FWF (Austria, project P211221-B11 assigned to Andrey V. Kozlov).

\section{Author Contributions}

Andrea Müllebner planned the experiments, performed qPCR experiments, analyzed data, wrote first version and revised the manuscript. Rudolf Moldzio performed viability assays and analyzed the data. Heinz Redl designed part of the study and supervised the animal experiments. Andrey V. Kozlov and J. Catharina Duvigneau designed the study, performed experiments with mitochondria, optimized the assay for the determination of the heme oxygenase activity and contributed to the writing and editing of the manuscript.

\section{Abbreviations}

\section{Appendix}

$\begin{array}{ll}\text { BR } & \text { Bilirubin } \\ \text { BV } & \text { Biliverdin } \\ \text { Diff. OD } & \text { background corrected optical density }\end{array}$

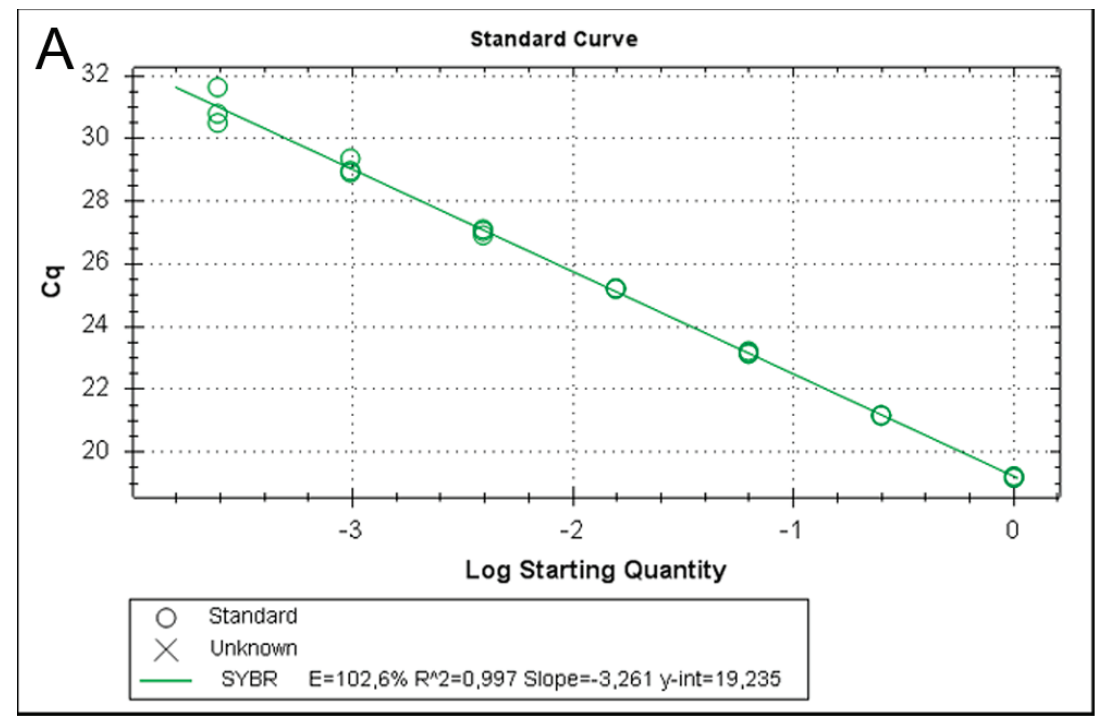

Figure A1. Cont. 


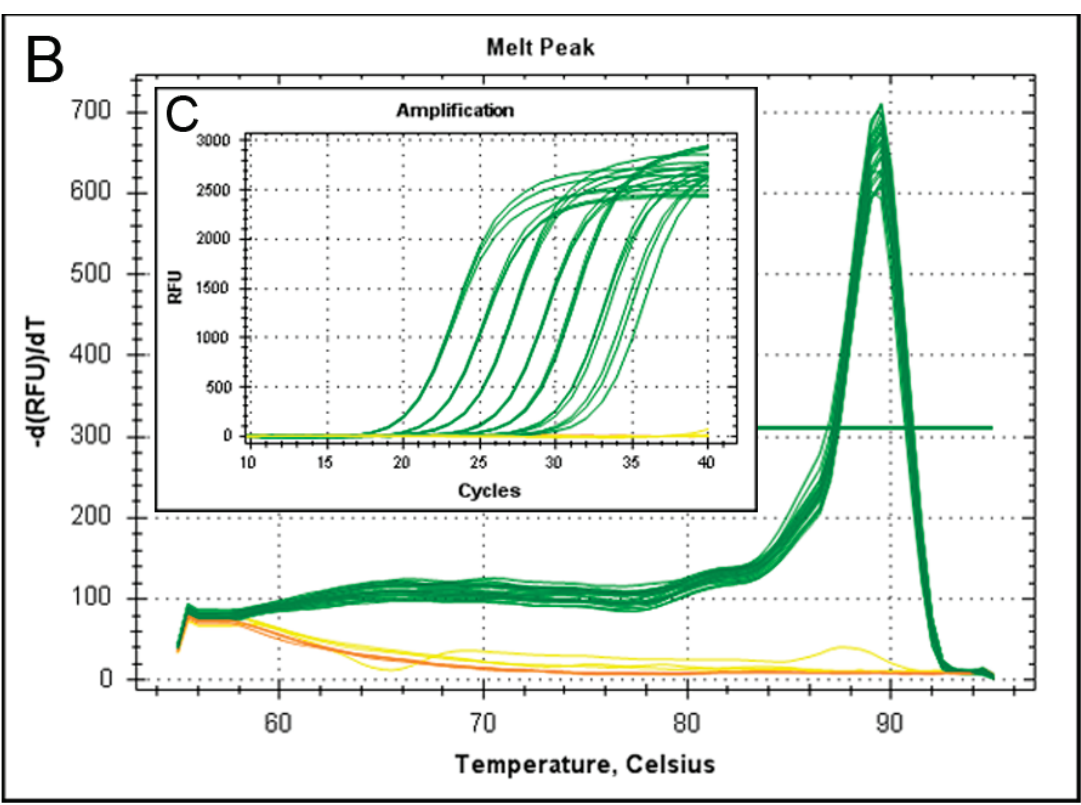

Figure A1. The suitability of the newly designed primers was verified in separate experiments by performing dilution series using the PCR products (Table A2) as well as dilution series of a cDNA pool (A). In melt curve (B) and amplification plots (B) samples are shown in green while controls (no reverse transcription control and no template control) are shown in yellow and orange respectively.

Table A1. Information about Intron-spanning primers.

\begin{tabular}{ccccccc}
\hline Target & $\begin{array}{c}\text { Accession } \\
\text { number }\end{array}$ & $\begin{array}{c}\text { Start on } \\
\text { plus strand }\end{array}$ & $\begin{array}{c}\text { Stop on } \\
\text { plus strand }\end{array}$ & $\begin{array}{c}\text { Product- } \\
\text { length (bp) }\end{array}$ & Exon junctions in & $\begin{array}{c}\text { Intron size } \\
\text { (bp) }\end{array}$ \\
\hline XBP-1, transcript variant & \multirow{2}{*}{ NM_001004210.1 } & 435 & 454 & \multirow{2}{*}{ Forward Primer } & $\sim 300$ \\
1, mRNA (usXBP-1) & 630 & 611 & & Product & $\sim 740$ \\
XBP-1, transcript variant 2, & NM_001271731.1 & 454 & 473 & \multirow{2}{*}{ Forward Primer } & $\sim 300$ \\
mRNA (sXBP-1) & & 623 & 604 & & Product & $\sim 740$ \\
\hline
\end{tabular}

Table A2. Optimized protocol and validation studies using amplificate dilution series

\begin{tabular}{ccccccc}
\hline Target & $\begin{array}{c}\text { Annealing } \\
\mathbf{t e m p}\left({ }^{\circ} \mathbf{C}\right) / \text { time } \\
(\mathbf{s e c})\end{array}$ & $\begin{array}{c}\text { Extension } \\
\operatorname{temp}\left({ }^{\circ} \mathbf{C}\right) / \text { time } \\
(\mathbf{s e c})\end{array}$ & $\begin{array}{c}\Delta \mathbf{C t} \\
(\mathbf{R T}+\mathbf{t o} \mathbf{R T}-)\end{array}$ & slope & $\begin{array}{c}\text { Correlation-Coefficient } \\
\left(\text { Pearson) } \mathbf{R}^{2}\right.\end{array}$ & $\begin{array}{c}\text { Verified dynamic } \\
\text { range }\end{array}$ \\
\hline $\mathrm{XBP}-1$ & $65 / 30$ & $72 / 20$ & not detected & -3.537 & 0.997 & $10^{5}$ \\
\hline
\end{tabular}

\section{Conflicts of Interest}

The authors declare no conflict of interest. 


\section{References}

1. Alam, J.; Cook, J.L. Transcriptional regulation of the heme oxygenase-1 gene via the stress response element pathway. Curr. Pharm. Des. 2003, 9, 2499-2511.

2. Kubulus, D.; Mathes, A.; Pradarutti, S.; Raddatz, A.; Heiser, J.; Pavlidis, D.; Wolf, B.; Bauer, I.; Rensing, H. Hemin arginate-induced heme oxygenase 1 expression improves liver microcirculation and mediates an anti-inflammatory cytokine response after hemorrhagic shock. Shock 2008, 29, 583-590.

3. Wang, W.W.; Smith, D.L.; Zucker, S.D. Bilirubin inhibits iNOS expression and NO production in response to endotoxin in rats. Hepatology 2004, 40, 424-433.

4. Yamaguchi, T.; Horio, F.; Hashizume, T.; Tanaka, M.; Ikeda, S.; Kakinuma, A.; Nakajima, H. Bilirubin is oxidized in rats treated with endotoxin and acts as a physiological antioxidant synergistically with ascorbic acid in vivo. Biochem. Biophys. Res. Commun. 1995, 214, 11-19.

5. Zelenka, J.; Muchova, L.; Zelenkova, M.; Vanova, K.; Vreman, H.J.; Wong, R.J.; Vitek, L. Intracellular accumulation of bilirubin as a defense mechanism against increased oxidative stress. Biochimie 2012, 94, 1821-1827.

6. Jansen, T.; Hortmann, M.; Oelze, M.; Opitz, B.; Steven, S.; Schell, R.; Knorr, M.; Karbach, S.; Schuhmacher, S.; Wenzel, P.; et al. Conversion of biliverdin to bilirubin by biliverdin reductase contributes to endothelial cell protection by heme oxygenase-1-evidence for direct and indirect antioxidant actions of bilirubin. J. Mol. Cell Cardiol. 2010, 49, 186-195.

7. Stocker, R. Antioxidant activities of bile pigments. Antioxid. Redox. Signal. 2004, 6, 841-849.

8. Fevery, J. Bilirubin in clinical practice: A review. Liver Int. 2008, 28, 592-605.

9. Amit, Y.; Boneh, A. Bilirubin inhibits protein kinase $\mathrm{C}$ activity and protein kinase $\mathrm{C}$-mediated phosphorylation of endogenous substrates in human skin fibroblasts. Clin. Chim. Acta 1993, 223, 103-111.

10. Chuniaud, L.; Dessante, M.; Chantoux, F.; Blondeau, J.P.; Francon, J.; Trivin, F. Cytotoxicity of bilirubin for human fibroblasts and rat astrocytes in culture. Effect of the ratio of bilirubin to serum albumin. Clin. Chim. Acta 1996, 256, 103-114.

11. Fernandes, A.; Falcao, A.S.; Silva, R.F.; Gordo, A.C.; Gama, M.J.; Brito, M.A.; Brites, D. Inflammatory signalling pathways involved in astroglial activation by unconjugated bilirubin. J. Neurochem. 2006, 96, 1667-1679.

12. Vitek, L.; Ostrow, J.D. Bilirubin chemistry and metabolism; harmful and protective aspects. Curr. Pharm. Des. 2009, 15, 2869-2883.

13. Qaisiya, M.; Coda Zabetta, C.D.; Bellarosa, C.; Tiribelli, C. Bilirubin mediated oxidative stress involves antioxidant response activation via Nrf2 pathway. Cell Signal. 2014, 26, 512-520.

14. Rodrigues, C.M.; Sola, S.; Brites, D. Bilirubin induces apoptosis via the mitochondrial pathway in developing rat brain neurons. Hepatology 2002, 35, 1186-1195.

15. McCoubrey, W.K., Jr. Detection of heme oxygenase 1 and 2 proteins and bilirubin formation. Curr. Protoc. Toxicol. 2001, doi:10.1002/0471140856.tx0903s00.

16. Sunderman, F.W., Jr.; Downs, J.R.; Reid, M.C.; Bibeau, L.M. Gas-chromatographic assay for heme oxygenase activity. Clin. Chem. 1982, 28, 2026-2032. 
17. Tenhunen, R. Method for microassay of microsomal heme oxygenase activity. Anal. Biochem. 1972, 45, 600-607.

18. Novak, M.; Polacek, K.; Melichar, V. Competition between bilirubin and non-esterified fatty acids for binding to albumin. Biol. Neonat. 1962, 4, 310-315.

19. McNally, S.J.; Ross, J.A.; James, G.O.; Wigmore, S.J. Optimization of the paired enzyme assay for heme oxygenase activity. Anal. Biochem. 2004, 332, 398-400.

20. Ryter, S.W.; Tyrrell, R.M. An HPLC method to detect heme oxygenase activity. Curr. Protoc. Toxicol. 2001, doi:10.1002/0471140856.tx0906s05.

21. Vreman, H.J.; Stevenson, D.K. Heme oxygenase activity as measured by carbon monoxide production. Anal. Biochem. 1988, 168, 31-38.

22. Lin, Q.; Weis, S.; Yang, G.; Weng, Y.H.; Helston, R.; Rish, K.; Smith, A.; Bordner, J.; Polte, T.; Gaunitz, F.; et al. Heme oxygenase-1 protein localizes to the nucleus and activates transcription factors important in oxidative stress. J. Biol. Chem. 2007, 282, 20621-20633.

23. Converso, D.P.; Taille, C.; Carreras, M.C.; Jaitovich, A.; Poderoso, J.J.; Boczkowski, J. HO-1 is located in liver mitochondria and modulates mitochondrial heme content and metabolism. FASEB J. 2006, 20, 1236-1238.

24. Rucker, H.; Amslinger, S. Identification of heme oxygenase-1 stimulators by a convenient ELISA-based bilirubin quantification assay. Free Radic. Biol. Med. 2015, 78, 135-146.

25. Bauer, I.; Wanner, G.A.; Rensing, H.; Alte, C.; Miescher, E.A.; Wolf, B.; Pannen, B.H.; Clemens, M.G.; Bauer, M. Expression pattern of heme oxygenase isoenzymes 1 and 2 in normal and stress-exposed rat liver. Hepatology 1998, 27, 829-838.

26. Nath, K.A.; Grande, J.P.; Croatt, A.J.; Likely, S.; Hebbel, R.P.; Enright, H. Intracellular targets in heme protein-induced renal injury. Kidney Int. 1998, 53, 100-111.

27. Sun, J.; Hoshino, H.; Takaku, K.; Nakajima, O.; Muto, A.; Suzuki, H.; Tashiro, S.; Takahashi, S.; Shibahara, S.; Alam, J.; et al. Hemoprotein Bach1 regulates enhancer availability of heme oxygenase-1 gene. EMBO J. 2002, 21, 5216-5224.

28. Malik, S.G.; Irwanto, K.A.; Ostrow, J.D.; Tiribelli, C. Effect of bilirubin on cytochrome C oxidase activity of mitochondria from mouse brain and liver. BMC Res. Notes 2010, doi:10.1186/1756-0500-3-162.

29. Keshavan, P.; Schwemberger, S.J.; Smith, D.L.; Babcock, G.F.; Zucker, S.D. Unconjugated bilirubin induces apoptosis in colon cancer cells by triggering mitochondrial depolarization. Int. J. Cancer 2004, 112, 433-445.

30. Paradisi, F.; Graziano, L. Mitochondrial swelling induced by unconjugated bilirubin in vitro. Experientia 1973, 29, 1376-1377.

31. Zucker, S.D.; Goessling, W.; Hoppin, A.G. Unconjugated bilirubin exhibits spontaneous diffusion through model lipid bilayers and native hepatocyte membranes. J. Biol. Chem. 1999, 274, 10852-10862.

32. Pascolo, L.; Del, V.S.; Koehler, R.K.; Bayon, J.E.; Webster, C.C.; Mukerjee, P.; Ostrow, J.D.; Tiribelli, C. Albumin binding of unconjugated $[3 \mathrm{H}]$ bilirubin and its uptake by rat liver basolateral plasma membrane vesicles. Biochem. J. 1996, 316, 999-1004. 
33. Bu-Bakar, A.; Arthur, D.M.; Aganovic, S.; Ng, J.C.; Lang, M.A. Inducible bilirubin oxidase: A novel function for the mouse cytochrome P450 2A5. Toxicol. Appl. Pharmacol. 2011, 257, $14-22$.

34. De, M.F.; Lord, G.A.; Kee, L.C.; Pons, N. Bilirubin degradation by uncoupled cytochrome P450. Comparison with a chemical oxidation system and characterization of the products by high-performance liquid chromatography/electrospray ionization mass spectrometry. Rapid Commun. Mass. Spectrom. 2006, 20, 1209-1217.

35. Ollinger, R.; Bilban, M.; Erat, A.; Froio, A.; McDaid, J.; Tyagi, S.; Csizmadia, E.; Graca-Souza, A.V.; Liloia, A.; Soares, M.P.; et al. Bilirubin: A natural inhibitor of vascular smooth muscle cell proliferation. Circulation 2005, 112, 1030-1039.

36. Taille, C.; Almolki, A.; Benhamed, M.; Zedda, C.; Megret, J.; Berger, P.; Leseche, G.; Fadel, E.; Yamaguchi, T.; Marthan, R.; et al. Heme oxygenase inhibits human airway smooth muscle proliferation via a bilirubin-dependent modulation of ERK1/2 phosphorylation. J. Biol. Chem. 2003, 278, 27160-27168.

37. Tell, G.; Gustincich, S. Redox state, oxidative stress, and molecular mechanisms of protective and toxic effects of bilirubin on cells. Curr. Pharm. Des. 2009, 15, 2908-2914.

38. Gillies, R.J.; Didier, N.; Denton, M. Determination of cell number in monolayer cultures. Anal. Biochem. 1986, 159, 109-113.

39. Kueng, W.; Silber, E.; Eppenberger, U. Quantification of cells cultured on 96-well plates. Anal. Biochem. 1989, 182, 16-19.

40. Tremezaygues, L.; Seifert, M.; Tilgen, W.; Reichrath, J. 1,25-dihydroxyvitamin $\mathrm{D}_{3}$ protects human keratinocytes against UV-B-induced damage: In vitro analysis of cell viability/proliferation, DNA-damage and -repair. Dermatoendocrinology 2009, 1, 239-245.

41. Edwards, V.; Markovic, E.; Matisons, J.; Young, F. Development of an in vitro reproductive screening assay for novel pharmaceutical compounds. Biotechnol. Appl. Biochem. 2008, 51, 63-71.

42. Berk, P.D.; Korenblat, K.M. Approach to the Patient with Jaundice or Abnormal Liver Test Results. In Cecil Medicine; Goldman, L., Ausiello, D., Eds.; Elsevier: Philadelphia, PA, USA, 2011.

43. Wolford, S.T.; Schroer, R.A.; Gohs, F.X.; Gallo, P.P.; Brodeck, M.; Falk, H.B.; Ruhren, R. Reference range data base for serum chemistry and hematology values in laboratory animals. J. Toxicol. Environ. Health 1986, 18, 161-188.

44. Arias, I.M.; Alter, H.J.; Boyer, J.L.; Cohen, D.E.; Fausto, N.; Schafritz, D.A.; Wollkoff, A.W. The Liver, Biology and Pathobiology; Wiley: Chinchester, UK, 2009; pp. 0-1216.

45. Yoshida, J.; Ishibashi, T.; Nishio, M. Antiproliferative effect of $\mathrm{Ca}^{2+}$ channel blockers on human epidermoid carcinoma A431 cells. Eur. J. Pharmacol. 2003, 472, 23-31.

46. Thorpe, J.A.; Schwarze, S.R. IRE1alpha controls cyclin A1 expression and promotes cell proliferation through XBP-1. Cell Stress Chaperones. 2010, 15, 497-508.

47. Gu, X.; Li, K.; Laybutt, D.R.; He, M.L.; Zhao, H.L.; Chan, J.C.; Xu, G. Bip overexpression, but not $\mathrm{CHOP}$ inhibition, attenuates fatty-acid-induced endoplasmic reticulum stress and apoptosis in HepG2 liver cells. Life Sci. 2010, 87, 724-732. 
48. Pfaffenbach, K.T.; Gentile, C.L.; Nivala, A.M.; Wang, D.; Wei, Y.; Pagliassotti, M.J. Linking endoplasmic reticulum stress to cell death in hepatocytes: Roles of C/EBP homologous protein and chemical chaperones in palmitate-mediated cell death. Am. J. Physiol. Endocrinol. Metab. 2010, 298, E1027-E1035.

49. Van, S.A.; van't Wout, E.F.; Stolk, J.; Hiemstra, P.S. A quantitative method for detection of spliced X-box binding protein-1 (XBP1) mRNA as a measure of endoplasmic reticulum (ER) stress. Cell Stress Chaperones 2012, 17, 275-279.

50. Zhang, K.; Shen, X.; Wu, J.; Sakaki, K.; Saunders, T.; Rutkowski, D.T.; Back, S.H.; Kaufman, R.J. Endoplasmic reticulum stress activates cleavage of CREBH to induce a systemic inflammatory response. Cell 2006, 124, 587-599.

51. Brodersen, R. Bilirubin Solubility and interaction with albumin and phospholipid. J. Biol. Chem. 1979, 254, 2364-2369.

52. Van der Veere, C.N.; Schoemaker, B.; van der, M.R.; Groen, A.K.; Jansen, P.L.; Oude Elferink, R.P. Rapid association of unconjugated bilirubin with amorphous calcium phosphate. J. Lipid. Res. 1995, 36, 1697-1707.

53. Suzuki, N. On bilirubin-metal complex compounds in relation to black pigments of gallstones. Tohoku J. Exp. Med. 1966, 90, 195-205.

54. Calligaris, R.; Bellarosa, C.; Foti, R.; Roncaglia, P.; Giraudi, P.; Krmac, H.; Tiribelli, C.; Gustincich, S. A transcriptome analysis identifies molecular effectors of unconjugated bilirubin in human neuroblastoma SH-SY5Y cells. BMC Genomics 2009, doi:10.1186/1471-2164-10-543.

55. Dong, H.; Huang, H.; Yun, X.; Kim, D.S.; Yue, Y.; Wu, H.; Sutter, A.; Chavin, K.D.; Otterbein, L.E.; Adams, D.B.; et al. Bilirubin increases insulin sensitivity in leptin-receptor deficient and diet-induced obese mice through suppression of ER stress and chronic inflammation. Endocrinology 2014, 155, 818-828.

56. Weidinger, A.; Mullebner, A.; Paier-Pourani, J.; Banerjee, A.; Miller, I.; Lauterbock, L.; Duvigneau, J.C.; Skulachev, V.P.; Redl, H.; Kozlov, A.V. Vicious inducible nitric oxide synthase-mitochondrial reactive oxygen species cycle accelerates inflammatory response and causes liver injury in rats. Antioxid. Redox Signal. 2015, 22, 572-586.

57. Althausen, S.; Paschen, W. Homocysteine-induced changes in mRNA levels of genes coding for cytoplasmic- and endoplasmic reticulum-resident stress proteins in neuronal cell cultures. Mol. Brain Res. 2000, 84, 32-40.

58. Weidinger, A.; Dungel, P.; Perlinger, M.; Singer, K.; Ghebes, C.; Duvigneau, J.C.; Mullebner, A.; Schafer, U.; Redl, H.; Kozlov, A.V. Experimental data suggesting that inflammation mediated rat liver mitochondrial dysfunction results from secondary hypoxia rather than from direct effects of inflammatory mediators. Front. Physiol. 2013, doi:10.3389/fphys.2013.00138.

59. Di, F.C.; Marfella, R.; Cuzzocrea, S.; Piegari, E.; Petronella, P.; Giugliano, D.; Rossi, F.; D'Amico, M. Hyperglycemia in streptozotocin-induced diabetic rat increases infarct size associated with low levels of myocardial HO-1 during ischemia/reperfusion. Diabetes 2005, 54, 803-810. 
60. Nurnberger, S.; Miller, I.; Duvigneau, J.C.; Kavanagh, E.T.; Gupta, S.; Hartl, R.T.; Hori, O.; Gesslbauer, B.; Samali, A.; Kungl, A.; et al. Impairment of endoplasmic reticulum in liver as an early consequence of the systemic inflammatory response in rats. Am. J. Physiol. Gastrointest. Liver Physiol. 2012, 303, G1373-G1383.

61. Zhu, L.J.; Altmann, S.W. mRNA and 18S-RNA coapplication-reverse transcription for quantitative gene expression analysis. Anal. Biochem. 2005, 345, 102-109.

62. Peinnequin, A.; Mouret, C.; Birot, O.; Alonso, A.; Mathieu, J.; Clarencon, D.; Agay, D.; Chancerelle, Y.; Multon, E. Rat pro-inflammatory cytokine and cytokine related mRNA quantification by real-time polymerase chain reaction using SYBR green. BMC Immunol. 2004, doi:10.1186/1471-2172-5-3.

63. Bradford, M.M. A rapid and sensitive method for the quantitation of microgram quantities of protein utilizing the principle of protein-dye binding. Anal Biochem. 1976, 72, 248-254.

64. Staniek, K.; Nohl, $\mathrm{H} . \mathrm{H}_{2} \mathrm{O}_{2}$ detection from intact mitochondria as a measure for one-electron reduction of dioxygen requires a non-invasive assay system. Biochim. Biophys. Acta 1999, 1413, $70-80$. 


\title{
Oxidative Stress in Aging Human Skin
}

\section{Mark Rinnerthaler, Johannes Bischof, Maria Karolin Streubel, Andrea Trost and Klaus Richter}

\begin{abstract}
Oxidative stress in skin plays a major role in the aging process. This is true for intrinsic aging and even more for extrinsic aging. Although the results are quite different in dermis and epidermis, extrinsic aging is driven to a large extent by oxidative stress caused by UV irradiation. In this review the overall effects of oxidative stress are discussed as well as the sources of ROS including the mitochondrial ETC, peroxisomal and ER localized proteins, the Fenton reaction, and such enzymes as cyclooxygenases, lipoxygenases, xanthine oxidases, and NADPH oxidases. Furthermore, the defense mechanisms against oxidative stress ranging from enzymes like superoxide dismutases, catalases, peroxiredoxins, and GSH peroxidases to organic compounds such as L-ascorbate, $\alpha$-tocopherol, beta-carotene, uric acid, CoQ10, and glutathione are described in more detail. In addition the oxidative stress induced modifications caused to proteins, lipids and DNA are discussed. Finally age-related changes of the skin are also a topic of this review. They include a disruption of the epidermal calcium gradient in old skin with an accompanying change in the composition of the cornified envelope. This modified cornified envelope also leads to an altered anti-oxidative capacity and a reduced barrier function of the epidermis.
\end{abstract}

Reprinted from Biomolecules. Cite as: Rinnerthaler, M.; Bischof, J.; Streubel, M.K.; Trost, A.; Richter, K. Oxidative Stress in Aging Human Skin. Biomolecules 2015, 5, 545-589.

\section{Introduction: The Skin as a Model for the "ROS-Aging" Connection}

Aging research has focused on a central finding that dates back to the year 1956. In this year Denham Harman proposed that reactive oxygen species (ROS) accumulate over time and are a main contributor to the aging process [1]. This concept was broadened 16 years later by Harman himself by identifying mitochondria as the main source of ROS, forming the basis for the mitochondrial free radical theory of aging [2]. Especially in the last decade, serious doubts arose that ROS are in fact the most important components that are fueling aging [3]. For example it was shown that $\mathrm{SOD}^{+/-}$mice had a clear increase in the ROS load but a quite normal lifespan [4]. Even the opposite was observed. In several cases it could be demonstrated that an increase in oxidative stress led to an increase in lifespan (summarized in more detail by Ristow and Schmeisser in [5]). However, if the free radical theory of aging holds true in any organ of the human body it is in the skin. Not only is the ROS load in this organ higher than in any other organ, but in many cases a clear correlation between the ROS originating from external and internal insults and a pro-aging effect can be found. A characteristic of this organ is also the fact that extrinsic aging is at least as important as intrinsic aging.

Intrinsic aging is described as a result of genetic factors and corporal changes that occur/appear during the normal aging process, whereas extrinsic aging focuses on aging process accelerated by environmental influences [6]. It was proposed that only three percent of all aging factors have a 
genetic background [7]. Aging leads to a thinning of epidermal as well as dermal skin layers. The skin also loses sensibility due to decreased production of sex hormones and a decreased number of nerve-endings. In addition the skin gets dryer and gradually loses the function to serve as a first barrier against the environment [8-10]. In contrast, focusing on extrinsic aging, it seems that the skin gets thicker and completely changes its composition [10,11]. Extrinsic aging is synonymous with photoaging as UV-radiation has severe consequences for exposed skin. However, there are many more environmental factors influencing skin aging, as discussed later [12].

\section{The Aging Process in the Dermis}

Human skin essentially consists of two layers: The epidermis on the outside and the dermis below, both are attached to each other via the basal lamina. Aging has a quite different appearance depending if either dermis or epidermis is considered. In the dermis the disruption of the extracellular matrix plays the most obvious role which is true for intrinsic as well as extrinsic aging. The results are fine wrinkles due to the reduction of collagen, elastic fibers, and hyaluronic acid.

In the context of intrinsic as well as extrinsic aging the disruption of the extracellular matrix plays an important role. Enzymes in the extracellular matrix (ECM) are responsible for the processing of elastic fibers, collagens, and proteoglycans $[13,14]$. Elastic fibers are structures of fibrillin-rich microfibrils, glycoproteins, elastins, and other different proteins [15]. Fibrillins and fibrillary collagens are glycoproteins with modifications of different branched oligosaccharides whereas proteoglycans consist of one long, unbranched glycosaminoglycan side chain. These glycoproteins are connected to each other with hyaluronic acid and build up a dermal network [16]. Long collagen fibrils from collagen I and III are interwoven and form an intra-dermal net which is anchored to the dermal-epidermal junction by collagen VII [16-18].

In the course of intrinsic aging collagen and elastic fibers stay intact but are further apart forming a wider-mashed network [16]. During extrinsic aging the skin dramatically loses collagen I, III and VII [19,20]. The long collagen fibrils, elastic fibers, glycoproteins and glycosaminoglycans are no longer interwoven to form a functional network but form an unorganised dermal-spreaded agglomeration [16]. This disruption is further aggravated by elastases produced by neutrophils that migrate to the dermis after inflammation or UV exposure [21] and by the activation of matrix metalloproteases (MMPs). Especially MMP1, 2, 3 and 9 are heavily involved in the degradation of the dermal extracellular matrix [22]. Collagen can only be cleaved by MMP1 and later on completely degraded by MMPs 2, 3 and 9. MMPs 2 and 9 are also able to degrade elastic fibers $[23,24]$. During the aging process these MMPs are upregulated while their inhibitors, namely TIMP1 and 3, are downregulated [24,25].

During photoaging this degradation is significantly accelerated by a process called ECM turnover [26]. UV-irradiation, especially UVA and UVB results in the production of ROS as well as the activation of cell surface receptors [27] leading to an activation of MAP-kinase p38, JNK (c-Jun amino-terminal kinase) and ERK (extracellular signal-regulated kinase) and the recruitment of c-Fos and c-Jun. This leads to the expression of the transcription factor activator protein 1 (AP-1) resulting in the expression of MMP1, 3 and 9 [28] in fibroblasts and keratinocytes [26]. AP-1 also 
inhibits TGF- $\beta$ which is responsible for collagen production [29]. The AP-1 mediated MMP expression leads to an increased degradation of the ECM. This process is reinforced by the production of ROS also resulting in the activation of MAP kinases and in addition leading to the expression of $\mathrm{NF}-\kappa \mathrm{B}$.

Interestingly NF- $\mathrm{\kappa B}$ and $\mathrm{AP}-1$ are also important for the balance between proliferation and apoptosis [30]. An imbalance of these two processes, especially in context with proto-oncogenes like c-Fos [31] may be heavily involved in carcinogenesis in aged human skin [32,33]. This is especially relevant for senescent skin cells [24].

In addition to aging it is well known that UV-irradiation induces mutations in the tumor suppressor p53 leading to actinic keratosis in a first step, that can further develop to skin cancers [34]. UV-irradiation of skin also leads to IGF-1 expression and secretion in dermal fibroblasts, which in turn stimulate the epidermal IGF-1 receptor (IGF-1R) in keratinocytes. Upon IGF-1R activation, keratinocytes get more resistant to UVB-irradiation, and do not undergo apoptosis, but stop the cell cycle with the side effect of becoming senescent. The loss of IGF-1 in aged skin leads to a higher apoptotic rate, but the surviving cells do not become senescent, which contributes together with oxidative stress to an increase in the formation of squamous cell carcinomas and basal cell epitheliomas in aged skin [35-38]. Another age-associated skin disease is vitiligo-similar appearance. This disease is a result of damaged melanocytes leading to white spots (idiopathic guttatehypomelanosis) [39] as well as lighter-tanned skin [40,41], visible only in aged patients. This hypopigmentation is a result of a ROS-imbalance in the skin [30,42] leading to an impairment of either the differentiation and survival of melanocytes or the melanin transport to keratinocytes, especially in aged and photoaged skin [38,40-42].

\section{The Epidermis: The Process of Cornification and Aging}

\subsection{The Cornified Envelope Formation}

Histologically different layers in the epidermis can be distinguished. The stratum basale consists of the stem cells that are attached to the basal membrane that seperates the dermis from the epidermis. Following the stratum basale, the stratum spinosum, the stratum lucidum (in palms and soles), the stratum granulosum, stratum corneum and stratum disjunctum can be seen.

The process of cornification in human skin is a step-by-step process involving the crosslinking of various proteins. This extensive crosslinking leads to the formation of a multi-protein complex in the outermost layer of the epidermis. The first step of the cornification process takes place in a layer located above the stratum basale, the stratum spinosum. Three important proteins are expressed here: envoplakin [43], periplakin [44] and involucrin [45]. The two plakins form a complex with involucrin [46], creating a platform for subsequent crosslinking of further proteins [47]. The next step in the cornification process leads to the formation of lamellar bodies by the Golgi apparatus in the stratum spinosum and the stratum granulosum [48,49]. These granules are characterized by an enveloping lipid layer consisting of a multitude of lipids like glucosylceramides and sphingomyelins [50]. Within the lamellar bodies several enzymes like lipid processing enzymes, antimicrobial peptides, proteases and protease inhibitors as well as proteins like corneodesmosin, an 
adhesive protein, can be found [50-54]. After an influx of calcium into the keratinocytes [55] the lamellar bodies fuse with the plasma membrane. This leads to a replacement of phospholipid in the lipid bilayer with $\omega$-OH-ceramides. These ceramides are further crosslinked with the periplakin-envoplakin-involucrin complex via transglutaminase 1 [56]. The calcium-dependent transglutaminase 1 [57] is responsible for the attachment of this complex to the lipid bilayer via $\mathrm{N} \varepsilon-(\gamma$ glutamyl) lysine (isopeptide) bonds [58]. The main component of the cornified envelope though is loricrin. Loricrin is highly expressed in the stratum granulosum [56] and is packed into granules directly after translation due to its high insolubility [59]. Transglutaminase 1 and 3 are responsible for the crosslinking of all loricrin proteins between each other and for crosslinking loricrin to a family of proteins called small proline rich repeat proteins (SPRRs). These proteins are very hydrophilic and help to increase the solubility of loricrin [60-62]. Our own published data indicates clearly that the addition of calcium to primary keratinocytes dramatically increases the expression of most of the SPRRs and loricrin. The loricrin-SPRR aggregate is then attached to the periplakin-envoplakin-involucrin scaffold at the cell membrane [56].

The cornification process proceeds by the attachment of various other proteins to the multi-protein complex at the cell membrane. One of these proteins is the calcium-regulated filaggrin [63]. Filaggrin is well known for bundling keratins into macrofibrils giving rise to the typical flattened shape of corneocytes [64]. The membrane-associated desmosomal keratins 1 and 10 begin to replace the pre-existing keratin 5 and 14 intermediate filament bundles (KIFs) aggregating them into tight bundles. This causes a significant change in the shape of the cells by changing cytoskeletal properties and cell-cell interactions [56]. Another calcium signal is then needed for the bundling of keratins into tonofilaments [65]. Other proteins attached to the cornified envelope complex are S100 protein family members. Some of the members of this protein family serve as substrates for transglutaminase $1[61,66]$.

In a last step of cornification, the late cornified envelope proteins (LCE) are attached to the protein-lipid complexes [67]. Some of the members react and attach "group-wise" in response to external stimuli like calcium [68]. The resulting "cornified" cell now consists of a mega-protein-lipid skeleton and has degraded its nucleus, mitochondria and other organelles. Its ultimate fate is to build the barrier function of the skin and finally it is shed as a dead corneocyte [69].

\subsection{The Calcium Dependence of the Cornified Envelope Formation}

Calcium has an important role during the cornification process. It is not just an "on-off principle" since calcium regulates the expression of genes in a dose-dependent manner. Keratinocytes need low calcium concentrations for the renewal and division of stem cells and transit amplifying cells, whereas higher concentrations are needed for differentiation. To ensure the right concentration of calcium at the respective epidermal layer the epidermis has built up a calcium gradient. The low calcium concentration found in the stratum basale gradually increases through the stratum spinosum until reaching its peak in the stratum granulosum. In the outermost layer, the stratum corneum, the calcium concentration sharply declines [70-72]. Basically most, if not all steps discussed above are strictly calcium dependent [73]. During the aging process the calcium gradient collapses and the composition of the cornified envelope changes drastically [72]. 
Our data show that epidermis obtained from young and middle-aged foreskin samples has a clear calcium peak in the stratum granulosum. Epidermis from old donors on the other hand has lost its peak and has an equal distribution of calcium in all epidermal layers [72]. Denda et al. were able to show the same rearrangement of calcium in epidermal layers of aged facial skin [74]. This leads to a changed composition of the cornified envelope: main components like loricrin and filaggrin are significantly down-regulated on the transcriptional and translational level while other components like envoplakin, periplakin, and involucrin show no transcriptional regulation in aged skin. The loss of loricrin and filaggrin seems to be compensated by increased levels of SPRRs since the expression of nearly all members of this family, with the exception of SPRR2G, are upregulated. This may represent a rescue mechanism to maintain the function and integrity of the cornified envelope. Transglutaminases, important for the cohesion of the protein-lipid complex, show constantly higher transcription levels in young skin than in old skin. Furthermore LCEs, some of them being substrates for transglutaminases, show either an up- or down-regulation during aging. Calcium-sensitive group 3 LCEs are upregulated during aging while group 2 LCEs are transcriptionally downregulated $[68,72]$. However, all S100 proteins are up-regulated during human skin aging [72]. Important in this context is the upregulation of S100A8 as it plays a role in the epidermal defense against oxidative stress. Besides this pronounced change in the calcium distribution and the resulting change in the composition of the cornified envelope, the transcription of many other genes [75] and such important regulators as miRNAs [76] are also altered during the aging process in the skin.

\section{ROS Production in the Skin}

The sources of ROS, enzymatic as well as non-enzymatic, in the cell are manifold. Enzymes that are ROS producing, on purpose or as a byproduct, include the mitochondrial electron transport chain, NADPH oxidases, xanthine oxidoreductase (XOR), several peroxisomal oxidases, enzymes of the cytochrome P450 family, cyclooxygenases, and lipoxygenases. All possible sources of ROS, the whole anti-oxidative system of the skin as well as all cellular damages are summarized in Figure 1.

\subsection{Mitochondrial ROS Production}

The electron transport chain resides in the inner mitochondrial membrane. The electrons are fed into complex I via NADH and into complex II via $\mathrm{FADH}_{2}$, then transferred to complex III and finally to complex IV. In complex IV (the cytochrome c oxidase) the electrons are finally deposited at molecular oxygen resulting in the production of $\mathrm{H}_{2} \mathrm{O}$. However, before the electrons reach complex IV they can leak prematurely to $\mathrm{O}_{2}$ at complex I and III, leading to the formation of superoxide instead of water $[2,77,78]$. It is estimated that no less than $1 \%-2 \%$ of all oxygen consumed leads to the formation of superoxide [79]. The contribution of mitochondria to the production of ROS in the skin is only substantial in the stem cells, but further on is small compared to other organs, because during the cornification process the keratinocytes degrade all their organelles including the nucleus, mitochondria, peroxisomes, and the endoplasmic reticulum [80,81]. The 
importance of mitochondria in the aging process independently of the production of ROS is summarized elsewhere [82-84].

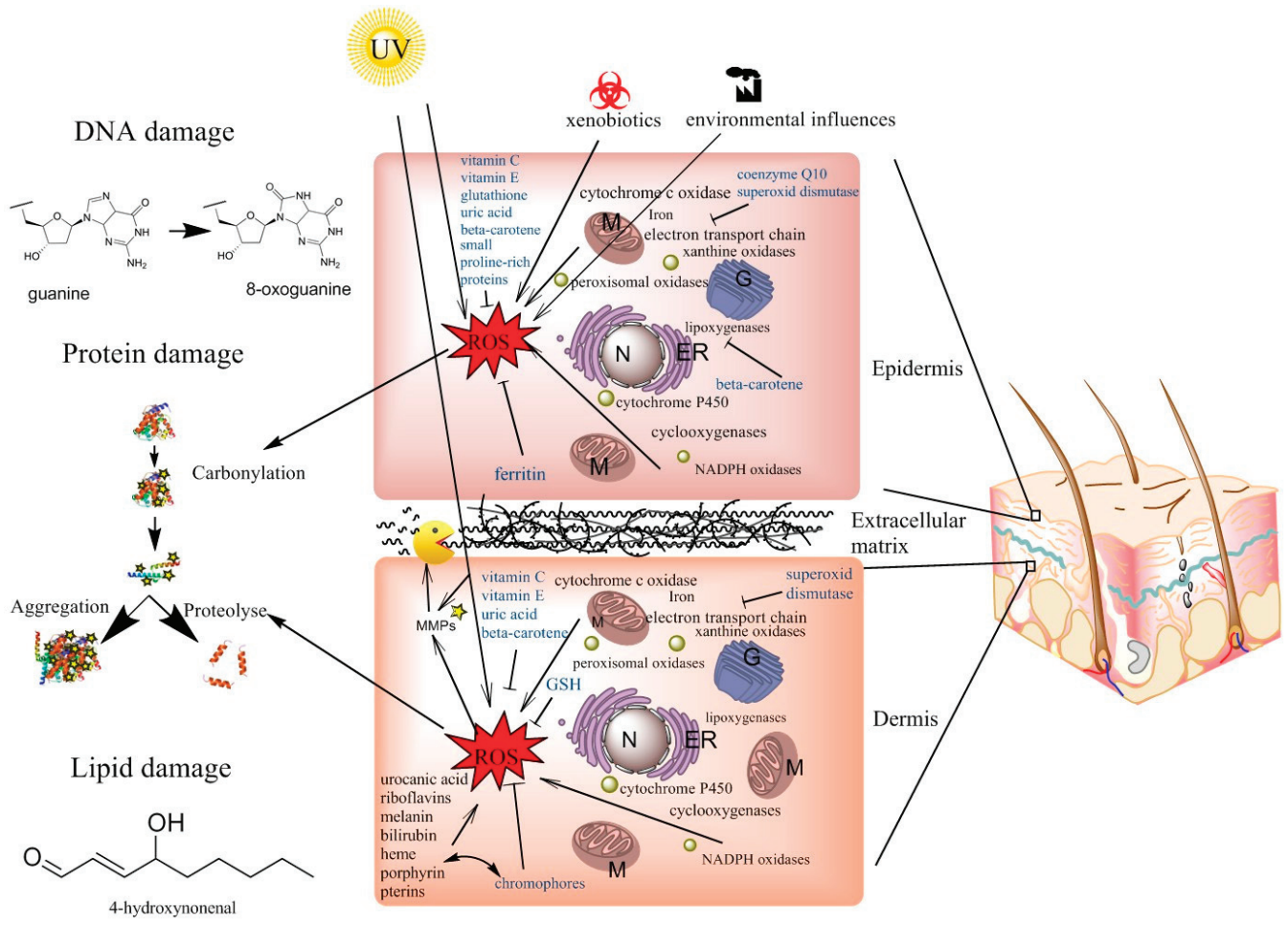

Figure 1. Schematic of the interplay between different ROS sources and the anti-oxidative systems in the skin. All ROS sources discussed in this manuscript are exemplified in black letters: cytochrome c oxidase, ETC (electron transport chain), iron ions, xanthine oxidase, peroxisomal oxidases, lipoxygenases, cytochrome P450, cyclooxygenases, NADPH oxidases, UV-radiation, xenobiotics and several chromophores that lead to ROS. Examples of anti-oxidative systems are given in blue letters: vitamin C and E, GSH (glutathione), GSH peroxidases, uric acid, beta-carotene, the SPRR2 (small proline rich repeat) family, SOD (superoxide dismutase), CoQ10 (coenzyme Q) and ferritin. Generally it has to be stated that only the SPRR2 proteins are specific for the epidermis, but the concentration of most anti-oxidants is much higher in the epidermis than in the dermis. Possible outcomes of oxidative damage to the cells (damage to DNA, proteins and lipids) are shown on the left-hand side of the figure. (M) stands for mitochondria, (ER) for endoplasmic reticulum, (N) for nucleus and $(\mathrm{G})$ for Golgi apparatus.

\subsection{Peroxisomal ROS Production}

It is still commonly assumed that mitochondria are the main contributors to ROS production in the cell, but increasing knowledge in the last decade led to the conclusion that the endoplasmic 
reticulum as well as the peroxisomes produce as much or even more ROS than mitochondria [85]. Peroxisomes, especially, are filled with a wide variety of enzymes, mainly flavoenzymes/oxidoreductases, that are supposed to produce hydrogen peroxide as a byproduct. All these enzymes are either involved in the $\beta$-oxidation of fatty acids, D-amino acid catabolism and anabolism, glyoxylate/dicarboxylate metabolism or the production of the autophagy stimulating and life prolonging substance spermidine. These enzymes are namely Acyl-CoA oxidase 1, 2, and 3, D-amino acid oxidase, D-aspartate oxidase, L-pipecolic acid oxidase, L- $\alpha$-hydroxyacid oxidase 1 and 2, and the polyamine oxidase [85]. Furthermore, peroxisomes not only produce ROS hydrogen peroxide, but similar to mitochondria have the capacity to form superoxide $\left(\mathrm{O}_{2}{ }^{-}\right)$. The $\mathrm{O}_{2}{ }^{-}$mainly originates from the enzyme xanthine oxidase and seems to be important during ischemia reperfusion injury $[86,87]$. Xanthine oxidase is found in the cytosol as well as in the peroxisomes and is the terminal enzyme and therefore key player in purine degradation. The two reactions that are catalyzed by this enzyme are the hydroxylation of hypoxanthine to xanthine and the hydroxylation of xanthine to urate [88]. Besides reactive oxygen radicals, a cell can also produce reactive nitrogen species that are not discussed in detail in the course of this review. The hemeprotein nitric-oxide synthetase catalyses the oxidation of L-arginine leading to nitric oxide (NO). In the absence of this amino acid no nitric oxide but superoxide is formed [89].

\subsection{ROS Production in the Endoplasmic Reticulum}

Oxygen radicals are not only produced, in mitochondria and peroxisomes, but also in the endoplasmic reticulum. The main contributors to ROS production in this organelle are members of the cytochrome P450 family and the combination of the protein disulfide isomerase PDI and the endoplasmic reticulum oxidoreductin-1 (ERO1). The protein PDI induces the formation of disulfide bonds in receptor proteins during the folding process. The isomerase gets reduced in this process and is regenerated by the oxidoreductin ERO1. The reduced protein ERO1 finally transfers the electron via the cofactor FAD to molecular oxygen. Incomplete transfer can lead to the production of superoxide $[90,91]$.

The cytochrome P450 family, mainly found in the ER, is responsible for the detoxification of xenobiotics or lipophilic compounds, mainly by increasing the water solubility of these substances. For this process electrons are transferred from NADPH to cytochrome P450 via the cytochrome P450 reductase, finally leading to the hydroxylation of xenobiotics. A leaky transfer of electrons can result in the formation of oxygen radicals, especially superoxide [92,93]. The main P450 enzymes that are expressed in the skin are cytochromes 1A1, 1B1, 2B6, 2D6, 2E1, 3A4, and 3 A5 $[94,95]$.

\subsection{ROS Production in Membranes and in the Cytosol}

Even membranes harness the power to produce reactive oxygen species. This is due to the activity of NADPH oxidases. Electrons are passed on from NADPH over FAD and two b-type hemes to the final acceptor $\mathrm{O}_{2}$, resulting in the formation of superoxide. In contrast to all other ROS sources discussed so far the superoxide produced this way in membranes is not the byproduct 
of catalytic processes, but superoxide is actively produced as a signaling molecule or as a "weapon" against invading microorganisms [96]. These enzymes can be found in different membranes such as the plasma membrane, the ER or mitochondria [96-99].

Finally, the cytosol has the capacity to produce ROS as a byproduct of the arachidonic acid metabolism. The enzymes cyclooxygenase and lipoxygenase both use arachidonic acid as a substrate to synthesize prostaglandin $\mathrm{H} 2$ and the leukotrienes, respectively. Both enzymes have the capacity to produce superoxide in the presence of NADH or NADPH [100]. The levels of arachidonic acid are relatively low in the skin, but increase in inflammatory skin diseases such as psoriasis, atopic dermatitis, and eventually aging [101,102].

The reaction of oxygen with iron ions additionally contributes to the production of ROS in the cytosol and all organelles of cells. The toxic effect of iron ions relies on the reaction of superoxide with ferric iron, resulting in the formation of ferrous iron. This process is called the Haber-Weiss reaction. In the following Fenton reaction the ferrous iron reacts with hydrogen peroxide, which on the one hand regenerates ferric iron and on the other hand produces the very reactive hydroxyl radical $\left(\mathrm{OH}^{*}\right)$, and hydroxide $\left(\mathrm{OH}^{-}\right)$. Both are more harmful for the cell than superoxide [103,104].

The skin is at the interface between the body and the environment and is therefore in constant contact with pollutants, xenobiotics, and UV irradiation. These exogenous factors represent the main contributor to the formation of ROS in human skin, therefore being very specific for this organ. All these factors are summarized under the term exogenous ROS. Additionally, alcohol intake, false nutrition, and physiological and mechanical stress are believed to contribute to this kind of exogenous mediated ROS production $[105,106]$. In addition the skin is also one of the very few organs that are in direct contact with atmospheric oxygen.

\subsection{Photoaging or UV-Induced ROS}

UV-irradiation especially leads to the genesis of ROS that are in turn main contributors to the aging of skin. To stress the importance of UV irradiation and the resulting ROS formation on the aging process of the skin the term "photoaging" has been coined. ROS production is mainly driven by UVA, in the range of $320-400 \mathrm{~nm}$. The UVB light does not have the capacity to penetrate to the deeper sections of the epidermis. The UVA light induces different changes in the dermis and these seem to be mainly responsible for the process and progression of photoaging [107]. In fact, these dermal alterations are better studied than the changes in the epidermis. UVA light penetrating the skin, is on its way absorbed by cellular chromophores. These cellular chromophores involve components like urocanic acid, riboflavins, melanin, bilirubin, heme, porphyrin, and pterins, but not DNA [106,108]. These photosensitizers absorb photons/energy leading to an excited state of the chromophores called the singlet excited state. Following this initial reaction two reactions can take place: a falling back to the ground state with the emission of either heat or fluorescence or second an intersystem crossing leading to a triplet excited state. This triplet excited state is only an intermediate state that can react with both DNA and molecular oxygen resulting in either modification of DNA or production of ROS such as superoxide, hydroxyl radical, singlet oxygen, or hydrogen peroxide $[108,109]$, which in turn leads to cellular damage discussed later in this review. The process of photoaging also effects DNA, especially the mtDNA. It was demonstrated 
that UV-irradiation leads to a"common", 4977 bp long deletion of mtDNA [110] that contributes to an increased ROS production of mitochondria. This increased ROS production leads to increased levels of mtDNA damage and has in this way the potential to start a vicious cycle.

Besides UVA, UVB also contributes to photoaging. However, due to its limited penetration ability UVB acts only on epidermal cells but not on the dermis [107] and leads to damage in keratinocytes and melanocytes. Nevertheless, UVA mediated epidermal damage affects the subjacent dermis. Moreover, the near-infrared light also has an effect on the epidermis as well as the dermis. It was shown that infrared light is absorbed by the mitochondrial ETC, especially at complex IV, leading to an increased leakage of ROS into the mitochondrial matrix. Krutman and Schroeder [107] introduced the term of the "defective powerhouse model". In this model UV as well as infrared light leads to an impaired energy production by dermal mitochondria that alters the morphology and function of the skin via retrograde signaling. Although the minor penetration ability of UVB is an advantage for skin aging, it has the big disadvantage that it does not act via photosensitizers but can directly damage the cell and DNA [106].

Amongst a variety of xenobiotics and pollutants that have the capacity to induce ROS production in the skin, polycyclic aromatic hydrocarbons are of special interest. These planar aromatic compounds are found in coal, oil, and tar and are especially dangerous after burning [111]. After absorbing the energy from light these substances reach a photo-activated state and react in subsequent processes with molecular oxygen under production of ROS [112].

\section{Anti-Oxidative Capacities of the Skin}

\subsection{Anti-Oxidative Properties of the Cornified Envelope}

To cope with these many sources of ROS the skin has developed sophisticated and in part very skin-specific anti-oxidative mechanisms. Most of the anti-oxidants show in fact a higher concentration in the epidermis than in the dermis [113]. This correlates well with the fact that the ROS load is higher in the epidermis than in the dermis. The epidermis is built up in a very gradual way and displays an increasing calcium concentration from the stratum basale to the stratum granulosum where a peak is reached. Also the cornified envelope gradually increases in its density. The formation of the cornified envelope starts in the stratum spinosum and is fully assembled in the stratum corneum. An epidermal concentration gradient is also found in the case of anti-oxidants, especially the low-molecular-weight ones. Vitamin C, vitamin E, glutathione, ubiquinol, and uric acid are detectable in the startum corneum, but their concentration increases steeply towards deeper cell-layers of the stratum corneum [105,114]. These comparably low concentrations of non-enzymatic and lipophilic anti-oxidants in the outer layers of the stratum corneum are possible, because the cornified envelope itself has anti-oxidative capabilities. These anti-oxidative capabilities of the cornified envelope rely on the SPRR proteins. Members of this protein family are not only rich in prolins but have an over-proportional enrichment incysteines. Therefore these proteins can quench ROS by forming intramolecular disulfide bonds. Interestingly these anti-oxidative properties were mainly found for the SPRR2 subfamily. This fact can be explained by different accessibilities of the cysteine residues of these cornified envelope proteins [113]. According to 
Harman's idea [2] ROS levels increase in the aging process. Indeed, we have found that the CE is dramatically altered in the aging process. Based on our own work [72] we argued that the loss of loricrin is compensated by increased levels of SPRRs. The biggest changes were found for the SPRR2 subfamily. In the light of the anti-oxidative capacities of the cornified envelope this increase in SPRRs during the aging process represents a valid tool to cope with the increasing ROS levels during aging. Below the stratum corneum another, upside-down gradient of anti-oxidative substances and enzymes is found. In this gradient the highest concentrations of enzymes and anti-oxidants are found in the stratum granulosum constantly declining towards the stratum basale [115]. In this way the suprabasal cells have lower ROS levels and are protected against UVB-induced apoptosis [116]. The importance of the CE as an anti-oxidant/UV barrier is also stressed by the fact that UV can completely deplete the stratum corneum of anti-oxidants/vitamins [117]. Therefore only the remaining CE proteins (mainly SPRR2 subfamily) can exert their anti-oxidative properties and protect the epidermal cells.

\subsection{The Non-Enzymatic Anti-Oxidants Vitamin C, Vitamin E, Beta-Carotene and CoQ10}

The strong anti-oxidant L-ascorbate/vitamin C cannot be synthesized by primates and therefore has to be taken up with food [118]. The water soluble vitamin $\mathrm{C}$ itself is an electron donor and is used as a cofactor for enzymatic reactions such as the crosslinking of collagen. Vitamin $\mathrm{C}$ is very prominent and the most abundant of all anti-oxidants [106]. In addition this anti-oxidant can react with a potential dangerous free radical and can donate its electron. In this way vitamin $\mathrm{C}$ itself is oxidized and forms so called "semidehydroascorbic acid". The big advantage of the resulting radical is that it is stable and comparably unreactive. This radical can either be reduced back or can react further to dehydroascorbic acid [119]. It was shown that vitamin $\mathrm{C}$ has a strong effect on photoaged skin, most probably by quenching ROS that originate from UV-irradiation [120]. It was found that the amount of ascorbate decreases in both intrinsic skin aging as well as extrinsic aged/photoaged skin [121].

The second vitamin with anti-oxidative capacities is $\alpha$-tocopherol/vitamin E. Vitamin E is more than one compound, but the most important one in humans is $\alpha$-tocopherol. Similar to vitamin $\mathrm{C}$ $\alpha$-tocopherol has a very important photoprotective and anti-photoaging role in the skin [122]. In contrast to the water soluble vitamin $\mathrm{C}$, vitamin $\mathrm{E}$ is lipophilic and is found in animal membranes. It can be nutritionally supplied by plant oils. The anti-oxidant $\alpha$-tocopherol is highly important because it can stop ongoing lipid peroxidation, by the reduction of the lipid peroxyl radical to hydroperoxide. In the course of this detoxification process this anti-oxidant loses a proton and is itself transformed into a radical. However, the $\alpha$-tocopheroxyl radical is not very reactive and is further on detoxified by ascorbate, glutathione, or enzymes [123]. Recent literature also indicates that $\alpha$-tocopherol exerts its photoprotective and anti-aging functions not only via its anti-oxidative role but also due to its role as activator/mediator of different signaling pathways. It has, for example, been shown that the protein kinase $\mathrm{C}$ pathway is affected by vitamin $\mathrm{E}$ [124]. In the aging process the levels of $\alpha$-tocopherol are unaffected in the dermis, whereas a clear decrease of this anti-oxidant was observed in the epidermis [121]. Cutaneous application of vitamin E ameliorates 
photoaging, decreases lipid peroxidation and furthermore also reduces photocarcinogenesis, MMP-1 transcription levels and thymine dimer formation [106].

Beta-Carotene is produced by plants and bacteria and also has to be taken up by food. This substance is a provitamin for retinol. In addition it has been shown that this precursor of vitamin A has a huge effect on skin aging and photoaging by either scavenging radicals or inhibiting lipoxygenases that are capable of producing ROS as discussed above [125,126]. Beta-carotene, a typical skin carotene, is anti-oxidative because the peroxyl radical is directly added to its backbone forming an epoxide that is decomposed afterwards [127].

The next anti-oxidant discussed here is somehow ambiguous. Uric acid on the one hand is the final product of the degradation of purines and is created by an enzyme that itself is capable of producing ROS as discussed above. On the other hand it is an anti-oxidant. Similar to ascorbate, uric acid is a reductant for ROS and can scavenge radicals such as hydroxyl radicals, singlet oxygen, and oxo-heme oxidants. By absorbing one electron, uric acid itself is transformed into a radical, although not very reactive [128]. It was also shown that uric acid is the main anti-oxidant in serum [129]. Therefore the contribution to the anti-oxidative capacity of the skin is comparably low as the skin has a low blood supply. Moreover, it was demonstrated that the extracellular urate is a potent anti-oxidant but acts as a pro-oxidant within the cell [130].

The last enzyme-free anti-oxidant discussed in this review is CoQ10. CoQ10 is known because of its contribution to the mitochondrial ETC. Ubiquinone is reduced to ubisemiquinone and ubiquinol at complex I and II and oxidized back to ubiquinone at complex III [131]. Besides this important contribution to the ETC, ubiqinone has also been described as an anti-oxidant. The lipid soluble $\mathrm{CoQ} 10 \mathrm{H} 2$ is a chain breaker in lipid peroxidation and protects lipids from lipid peroxidation [132]. In comparison to vitamins $\mathrm{C}$ and $\mathrm{E}$, ubiquinone seems to be ineffective in photoprotection [132].

\subsection{The Importance of Superoxide Dismutases, Catalases, Glutathione Peroxidases, Ferritin, and Peroxiredoxins in Quenching ROS}

Among the most prominent enzymes that can handle reactive oxygen species are the superoxide dismutases. These enzymes "dismutate" superoxide to hydrogen peroxide [133]. In mammals three isoforms can be distinguished that differ in their localization. The enzyme SOD1 is found in the cytosol and nucleus and has $\mathrm{Cu} / \mathrm{Zn}$ as cofactor, SOD2 is found in mitochondria to dismutate superoxide originating from the mitochondrial ETC and binds $\mathrm{Mn}^{2+}$, and SOD3 is found in the extracellular space harboring the metal ions $\mathrm{Cu} / \mathrm{Zn}$ in its active center [134]. In the first half-reaction the electron from the superoxide radical is transferred to the metal ion in the active center thereby reducing it. The superoxide itself is oxidized to $\mathrm{O}_{2}$. In the second half-reaction the reduced metal in the superoxide enzymes is reoxidized by transferring the electron to superoxide resulting in the formation of hydrogen peroxide [135]. All three human superoxide dismutases have a huge impact on aging skin. Generally a deletion of superoxide dismutase is lethal as demonstrated in mice, but with SOD mimetics life can be prolonged for several weeks. Sod $1^{-1}$ mice show a clear skin atrophy that is also observed in aged individuals [136,137]. In case of SOD2 deletions the phenotypes are even more dramatic. UV irradiation leads to the above discussed mtDNA deletions 
and results in a burst of radicals from the defective mitochondrial ETC. Not surprisingly, UV irradiation results in a dose dependent increase in SOD2 mRNA levels in wildtype mice [138]. Although SOD2 overexpression had no obvious life prolonging effects [139], distinct skin aging phenoytpes were observed in Sod $2^{-/-}$mice. These phenotypes comprise a thinning of the epidermis, a clear atrophy of the dermal connective tissue, a reduced amount of procollagen I, and an atrophy of the subcutaneous fat tissue [140-142]. The SOD3 enzyme is expressed in the dermis as well as in the epidermis. By harboring a heparin-binding domain this enzyme is in close contact with the extracellular matrix and cell surfaces. In contrast to SOD1 and SOD2 very high doses of UV are needed to induce the expression of SOD3. Therefore the role of SOD3 in the skin is unclear although it has been shown that SOD3 is involved in skin inflammation and its expression is reduced in psoriasis $[134,143]$.

A very prominent enzyme that detoxifies hydrogen peroxide is the peroxisomal localized catalase [144]. This enzyme consists of four identical polypeptide chains, each harboring a heme group [145]. In a first step hydrogen peroxide reacts with the heme group leading to an oxoferryl porphyrin cation radical and a water molecule. The so called compound I is very active and reacts immediately with a second hydrogen peroxide molecule producing water and molecular oxygen and regenerating the original prosthetic heme group [146]. The catalase enzyme is very prominently expressed in the skin, especially in the stratum corneum. The amount of catalase exceeds the amount of superoxide dismutases. Inside the stratum corneum a gradient of activity, with a decreasing activity towards the surface of the skin, was detected [147,148]. In the aging process the activity of this enzyme is altered with a widening gap between the dermis and epidermis. Thus, catalase activity decreases in the dermis and increases in the epidermis of aged and photoaged skin. Because the ROS load of the cells, especially in the epidermis, increases with aging, increasing catalase activity is reasonable, whereas the reduction of catalase in the dermis remains mysterious [148,149]. A remarkable experiment showed that by targeting the peroxisomal catalase to mitochondria a statistical significant increase in medium and maximum lifespan was found in mice [150].

A main contributor to the anti-oxidative potential of the cell is the tripeptide glutathione GSH, harboring a special gamma peptide linkage. This peptide is synthesized in a two-step process. The first step is performed by the gamma glutamylcysteine synthetase, the second step by the glutathione synthetase. The GSH acts as an anti-oxidant because of its thiol group. In the course of the process GSH is oxidized by reactive oxygen radicals and forms a dimer with another activated GSH via formation of a disulfidic bond (GSSG). GSH can be recovered in a reducing step by the glutathione reductase consuming NADPH [151]. GSH not only detoxifies ROS, but can also regenerate oxidized $\alpha$-tocopherol and retinol [106]. In aged mice it was shown that both, the absolute amount of GSSG as well as the GSSG:GSH ratio strongly increases in the dermis in comparison to young skin [152]. In photoaged skin the concentrations of glutathione are reduced, but this effect could be compensated by an increased activity of the glutathione reductase [121]. It is estimated that in aged skin the concentration of anti-oxidants is strongly decreased, in line with this the levels of $\alpha$-tocopherol, ascorbate and GSH have been shown to be reduced by $70 \%$ [121]. The function and (inter)action of all anti-oxidants is deeply interwoven to keep the redox state in 
the skin tissue in balance. For example, vitamin $\mathrm{C}$ can reduce oxidized $\alpha$-tocopherol and is itself oxidized; glutathione in turn can rescue vitamin E and the resulting GSSG is converted into GSH again by the glutathione reductase enzyme [106].

Beside its role as an anti-oxidant, GSH is also a cofactor for enzymatic reactions. The glutathione peroxidase is an enzyme that fulfills two tasks: reduce hydrogen peroxide to water and stop lipid peroxidation. In humans, eight glutathione peroxidases are known, five of them containing selenium as a co-factor. In a first step, the peroxide, either lipid or hydrogen, oxidizes the enzyme bound $\mathrm{Se}$, thereby forming $\mathrm{SeOH}$. In a next step the enzyme reacts with a thiol group in GSH resulting in the formation of a selenylsulfide bond between the enzyme and the glutathione. A reaction with a second GSH regenerates the enzyme and GSSG is formed [153,154]. Alterations in the enzyme activities in aging skin and photoaged skin have not yet been characterized, however a targeted disruption of the glutathione peroxidase 4 in mice displayed severe skin phenotypes, like hyperplasia of the epidermis, dermal inflammation, increased rates of lipid peroxidation, and higher levels of the cyclooxygenase-2 [155].

As already mentioned, free iron ions are a constant threat for the cell because the Fenton reaction is capable of starting a vicious cycle of ROS production in the cell, ultimately leading to its death. Therefore the cell has to conceal the iron ions very carefully. This iron storage is achieved by the protein ferritin. The protein consists of 24 subunits forming a sphere that surrounds the iron. The iron is only stored in its Fe(III) form as ferrihydrite and upon its release it has to be reduced to the $\mathrm{Fe}$ (II) form. The 24 subunits can be divided into two subtypes: the heavy (H)-type and the light (L)-type. The L-type is involved in the core-formation, the H-type in the Fe(II) oxidation [156]. Ferritin is primarily stored in the cytosol, although mitochondrial and nuclear forms are also known. The iron release is also dependent on lysosomal ferritin degradation [157]. Ferritin seems to be an important tool in the regulation of the redox homeostasis especially after UV irradiation. The highest concentrations of ferritin in the skin are found in the stratum basale [158]. The levels of ferritin in the epidermis are around three to seven fold higher than in the dermis. After UV irradiation, especially UVA, the levels of ferritin in the dermis as well as epidermis, strongly increase, indicating a potential anti-oxidative mechanism to stop ROS production in cells after disturbing the redox homeostasis $[158,159]$. However, the combination of iron storage, ferritin, and UV irradiation also has detrimental potential. It was demonstrated that UVA irradiation of primary dermal fibroblasts induces an immediate degradation of ferritin in the lysosomes, followed by a release of iron ions into the cytosol accompanied by a burst of ROS [160]. The acceleration of skin aging in females after the menopause was also attributed to iron and ferritin. In females there are two ways to get rid of excessive iron: menstruation and desquamation. After the menopause the excessive iron ions are stored in the skin via ferritin and this could contribute to an increase in ROS levels that accelerates the aging process in the skin [161].

The last class of enzymes that have anti-oxidative capacities and are discussed in this review are the peroxiredoxins. In mammals six isoforms were identified, whereas 2-Cys enzymes (PRDX1-5) and 1-Cys enzymes (PRDX6) can be distinguished [162]. In the following, only the 2-Cys enzymes will be discussed. A peroxide substrate reacts with a conserved cysteine in the active center of these enzymes leading to the formation of a sulfenic acid residue. This is followed by a reaction of 
the sulfenic acid with a second cysteine (therefore the term 2-Cys enzyme), thereby forming an intra-molecular disulfide bond [151]. The enzyme is regenerated by a flavoprotein disulfide reductase such as the thioredoxin reductase [162]. More details of the function of this enzyme can be found in the Chapter "Oxidative stress in fungi" in the same issue. High levels of PRXD1-3 were found especially in the epidermis but also in the dermis of rats. Similar to the calcium distribution, PRDX1 and PRDX2 were found with increasing concentrations towards the stratum granulosum. PRDX3 showed the opposite distribution. The highest concentration was found in the stratum basale, the lowest concentration in the stratum granulosum [163]. The peroxiredoxins seem to be very important in the detoxification of ROS originating from UV irradiation. UVB-irradiation induced the expression of PRXD2, UVA the expression of PRDX1 [163,164]. Overexpression of PRDX6 leads to significantly reduced levels of oxidized lipids in mice and results in a reduced rate of UVB and UVA induced apoptosis, whereas loss of PRDX6 leads to an increased skin tumor rate $[165,166]$. There is also growing evidence that an increase of activity of peroxiredoxins has great potential in increasing lifespan [167].

\subsection{The Anti-Oxidant Treatment Paradox}

An increase in ROS levels over time is a common feature of all human tissues and especially of the skin. Therefore many attempts were made to quench these ROS by topical treatment of the skin or supplementation with anti-oxidants, in the hope to improve or even rejuvenate aged skin. But the results are very controversial and are heavily disputed in the literature. Just recently it was demonstrated that a mixture of alpha hydroxy acids, vitamins B3, C, and E applied on facial skin dramatically improves the quality of the epidermis and dermis including the smoothening of wrinkles and the refinement of skin texture without side effects [168]. A similar effect was found on treating aged and photodamaged skin with a special combination of several anti-oxidants consisting of resveratrol, baicalin, and vitamin E. These antioxidants were partially sufficient to rejuvenate aged skin [169]. Resveratrol was shown to stimulate the Nrf2 pathway in skin leading to an increase in the GSH content and improvement of skin quality [170]. Also CoQ10 conjugated with nanoparticles (to improve the skin permeability) seems to have a beneficial effect on skin quality [171,172]. Some additional substances that seem to have a positive effect on aged skin, especially the epidermis, are summarized in Lorencini et al. [173]. Though many studies promote the use of antioxidants for preventing skin aging, others warn of potential side effects. Treatment of various model organisms with vitamin $\mathrm{C}$ gave a broad variety of results ranging from a prolonged lifespan to "no effect" [174]. Surprisingly, no beneficial, statistically significant effect on lifespan elongation was found for food supplementation with vitamin E [175,176]. In the small rodent Microtus agrestis supplementation with vitamin $\mathrm{C}$ and $\mathrm{E}$ led to a remarked reduction of lipid peroxidation, as expected, but significantly reduced the lifespan of this organism [176,177]. Also CoQ10 can lead to both, an increased (mice, nematodes) or decreased ( $S$. cerevisiae) lifespan [176,178-180]. Recent literature also warns of the excessive use of vitamins. Oral administration of beta-carotene, vitamins $\mathrm{E}$ and $\mathrm{A}$ in humans seems to lead to a higher mortality rate [181] or increased risk of diseases [182]. Quite surprisingly it was shown that increased ROS levels and increased oxidative damage can even lead to an increase in lifespan [183,184]. This 
controversy can be explained by the fact that ROS are not exclusively detrimental for cells, but can even be beneficial. There is growing evidence that ROS, especially hydrogen peroxide, have an important role in cells as a second messenger $[96,185]$. Therefore it is not desirable to quench away all the ROS, because this influences the ROS homeostasis of the cell with such detrimental effects as promoting tumor formation [186]. A beneficial effect on skin aging by a treatment with antioxidants can onlybe achieved if the original ROS level of healthy cells is preserved.

\section{Protein Oxidation, Lipofuscin and AGEs}

ROS originating from all sources discussed in the previous chapters basically affect all compartments of the cell. While also attacking DNA and lipids, the modification and potential subsequent aggregation of proteins poses a major problem for cells. As described above, especially UV exposure significantly increases ROS levels in skin cells [187,188]. It has been shown that protein oxidation and detectable unfolding occurs after only 30 min of UV exposure in human skin [189]. Continued UV irradiation accompanied by ROS production over longer periods of time is especially problematic and results in more pronounced modifications like the aggregation of unfolded proteins [190].

ROS can modify proteins directly or indirectly. Indirect attacks come from secondary by-products. An example of this is the protein backbone fragmentation that occurs after previously oxidized glucose binds to amino groups. This may be interesting in the context of diabetes mellitus since there is evidence that this disease is accompanied by increased oxidative stress. Backbone fragmentation could partially account for the tissue damage associated with it [191,192].

Direct ROS protein modificationsare reported at the backbone, at amino-acid side chains or by the formation of carbonyls. Hydroxyl-radicals $\left({ }^{\circ} \mathrm{OH}\right)$ may initiate backbone damage by abstracting hydrogen atoms from the $\alpha$-carbon of polypeptide chains. This initiates a chain of reactions that ultimately lead to the formation of alkoxyl derivatives resulting in spontaneous cleavage of the derivate itself [193]. Several amino acid residues are more susceptible to oxidative modifications than others. Examples of that are histidine, leucine, methionine, and cysteine as well as phenylalanine, tyrosine, and tryptophan. Only modifications of the sulfur-containing amino acids methionine and cysteine are reversible, for example by the enzymes glutaredoxin 1, thioredoxin, and methionine sulfoxide reductases [194-196].

\subsection{Protein Carbonyls}

As mentioned above modifications of other amino acids form more stable products like carbonyl groups [197]. For example, oxidation of protein side chains containing proline, arginine, lysine and threonine results in the formation of carbonyl (CO) groups (aldehydes and ketones). These modifications are therefore irreversible and serve as early markers of oxidative stress [198]. The stability of these protein modifications has led to the general opinion that they are more than markers though. The introduction of carbonyl groups induces conformational changes of the polypeptide chain and leads to partial or total inactivation. This would make protein carbonyls main contributors to the detrimental effects arising from oxidative stress. At the very least the 
increased formation of protein carbonyls may overburden the cell's repair/degradation system resulting in an accumulation of more modified and inactivated proteins [199]. Suntanned epidermis shows a considerably higher amount of carbonylated proteins than epidermis that is rarely exposed to sun and therefore UV-irradiation [200]. Besides UV exposure acrolein, a component of cigarette smoke, was shown to play a significant role in protein carbonylation, as shown in keratinocyte cell culture experiments [201]. Investigating the level of carbonyl groups in keratins in human skin, Thiele et al. were able to show that they were significantly higher in the stratum corneum as compared to deeper layers of the epidermis [202]. Considering that the stratum corneum is the first barrier between the body and the environment, these findings are not surprising. In this context it is also worth mentioning that proteins can be more or less susceptible to carbonylation. The reasons for this are not clear at the moment, but possibly include immediate proximity to ROS generating sites or the presence of transition metals in these proteins [203]. Additionally it has been proposed that carbonylation may play a role in ROS signaling [204].

\subsection{The Process of Protein Oxidation}

As described above, sustained UV-exposure leads to a higher extent of protein oxidation. This increased protein oxidation has also been reported in aged tissue and senescent cells. One reason for the high amount of oxidative damage in old cells is that they have been exposed to a multitude of stressors and have accumulated oxidative damages to DNA, proteins and lipids. This accumulation of oxidative damaged DNA, protein and lipids in turn results in an increased ROS production, leading to a negative feedback loop. Moreover, the ability of aged cells to efficiently clear ROS is impaired [205,206], resulting in an imbalance between the production and the clearance of ROS where more and more proteins begin to aggregate. Postmitotic cells are not able to dilute their protein aggregates by cell division [207]. Jung et al. were able to show that the amount of oxidized proteins is much higher in in vitro aged, senescent fibroblast cells compared to young cells. Experiments with long term exposure to $\mathrm{H}_{2} \mathrm{O}_{2}$ led to increased levels of protein oxidation in young fibroblasts. Interestingly this exposure was not sufficient to increase the already much higher protein oxidation levels of senescent fibroblasts [208]. In line with these findings, a significant increase in protein oxidation was reported in human patients' papillary dermis and in the stratum corneum after daily UVB exposure for 10 subsequent days with a solar simulator, mimicking UV exposure of a typical summer vacation [189]. In general it seems that most epidermal layers are less affected than dermal layers, most probably due to the high amount of anti-oxidants in the epidermis [209] and the anti-oxidative functions provided by SPRRs [113]. This was confirmed in vitro with both, fibroblast and keratinocyte cell cultures. Fibroblasts showed a significantly higher amount of protein oxidation compared to keratinocytes when exposed to UVA/UVB irradiation [189].

The process of protein oxidation can be divided into various stages according to the severity of modifications. At first, only slight oxidations take place leading to a marginally reduced enzyme activity of affected proteins or changes in thermostability. In this stage no extensive unfolding takes place [210] and the cell is still able to counteract by repairing the damages. An important protein in this context is thioredoxin, the cells major protein disulfide reductase. Among other things it has an important role in the protection against oxidative stress since it prevents intra- and 
intermolecular formation of disulfide bonds which would otherwise lead to the inactivation and/or aggregation of these proteins [194]. Thioredoxin is also able to function as an electron donor for thioredoxin peroxidases or peroxiredoxins. Next to glutathione peroxidases and catalase, these enzymes are able to catalyze the reduction of $\mathrm{H}_{2} \mathrm{O}_{2}$, a major contributor to cellular oxidative stress [211].

The next stage is marked by increased protein oxidation. Due to high amounts of ROS or other sources of "protein modifiers" and the inability of the cell to clear these damages, various protein modifications are able to accumulate. The chemically modified proteins completely lose their activity and unfold extensively. Due to the unfolding proteins begin to cross-link and form small aggregates. At this point the proteins either become targets for degradation by the proteasome [197,212] or can be refolded and rescued by heat shock proteins. It is unclear how the cell decides to either refold or degrade these proteins since both possibilities have their advantages [213,214]. The inner proteolytic chamber of the proteasome is mainly accessible to unfolded proteins, a typical fate of oxidized proteins. As long as the protein is not extensively oxidized it is an ideal substrate for the $20 \mathrm{~S}$ proteasome [213]. In contrast to this, the main substrates for the $26 \mathrm{~S}$ proteasome are not unfolded but ubiquitinated proteins. It is a major key player in the unfolded protein response (UPR) initiated by the ER [215-217]. Disassembly of the $26 \mathrm{~S}$ proteasome during age or prolonged stress leads to an increase in $20 \mathrm{~S}$ proteasome abundance. While this may lead to an increase in the degradation of un-ubiquitinated oxidized proteins or smaller protein aggregates, ubiquitinated proteins begin to accumulate triggering different stress responses including lysosomal uptake [218,219]. On the other hand inactivation of the $20 \mathrm{~S}$ proteasome by UVA in dermal fibroblasts leads to an activation of activator protein-1 (Ap-1), controlling MMP-1 (matrixmetalloprotease-1) expression [220]. As mentioned above MMP-1 is responsible for increased extracellular protein degradation [221,222].

The last stage of protein modification is marked by extensive oxidation of proteins, their complete unfolding and covalent crosslinking of several proteins [223-226]. Oxidized, unfolded proteins begin to form extensive, insoluble aggregates due to interactions between the exposed hydrophobic residues [213] and later on form covalent bonds. Not only are these aggregates poor substrates for the proteasome due to their sheer size, they are also able to cause proteasomal inhibition [227,228]. A reason for that may be the extensive crosslinking of the various proteins in the aggregate. The proteasome cannot degrade these aggregates due to steric/mechanic inhibition and remains bound to the structure, unavailable for other substrates [229]. In addition to that, the proteasome loses efficiency in age resulting in even more accumulation of modified proteins [230,231]. It was demonstrated that the proteasomal activity decreases significantly beginning in middle aged ( $60 \pm 8$ years) skin samples, especially in the dermis. Surprisingly, no further decrease in proteasome activity was observed in older skin donors (90 years) [232].

\subsection{Lipofuscin}

Continued elevated ROS levels eventually lead to the formation of large protein-lipid aggregates known as lipofuscin. These structures can be detected in nearly all types of cells including fibroblasts and keratinocytes [197,233,234]. Lipofuscin (meaning "dark fat") appears as 
yellow-brownish material in the light microscope and shows autofluorescence over a broad spectrum [207]. It is also known as "ceroid" or "age pigment" $[235,236]$. The term "age pigment" is especially interesting in the context of the skin since the formation of lipofuscin gradually increases with the age of the individual. In old individuals lipofuscin has accumulated excessively and becomes visible with the naked eye, hence the term "age pigment" [236,237]. Lipofuscin eventually also incorporates lipids and forms protein-lipid clusters, consisting of $30 \%-70 \%$ proteins and $20 \%-50 \%$ lipids [238]. In very old individuals lipofuscin clusters begin to incorporate sugar residues [239]. Lipofuscin clusters have the capability to bind various metals like copper, zinc, manganese, calcium and iron [240] as well as metal-containing proteins like ferritin [241]. These metals can amount to $2 \%$ of the final volume of the lipofuscin cluster [212] and the irreversible binding of these metals makes these clusters another redox-active site for the generation of radicals such as the hydroxyl radical ( $\left.{ }^{\circ} \mathrm{OH}\right)$ [242]. This massive radical formation catalyzing structure in the cell and the inhibition of the proteasome start a vicious cycle leading to even higher amounts of oxidized proteins and eventually protein aggregates [208]. In motor neurons lipofuscin can occupy up to $75 \%$ of the cell's volume decreasing its functionality and later on leading to apoptosis [243].

As mentioned before, mitochondria, the endoplasmatic reticulum and peroxisomes are main contributors to intracellular ROS levels and mainly target the cytosol. The same can be said for externally applied ROS contributors like xenobiotics and UV-irradiation that play a big role in skin. The oxidation of cytosolic proteins could be a kind of "buffer" before nuclear proteins are oxidized [244,245]. It has to be mentioned though that chronic or repeated UV and ROS exposure still leads to the accumulation of nuclear protein oxidation that is able to "block" the nuclear proteasome. An oxidation of nuclear proteins has been shown in senescent fibroblasts and young fibroblasts chronically stressed with appropriate chemicals like $\mathrm{H}_{2} \mathrm{O}_{2}$ or paraquat [208]. It looks like the carbonylated proteins in the nucleus are either excluded quite efficiently or are not sufficient for the formation of lipofuscin. It has been shown in fibroblasts that most of the lipofuscin can be found in the cytosol with a major amount of it inside the lysosomal lumen [208]. Lysosomes are primarily responsible for the removal and degradation of lipofuscin and contain a high amount of hydrolytic enzymes such as proteases, nucleases, lipases and phosphatases [246]. Surprisingly it has been proposed that besides inhibiting the proteasome, lipofuscin may also be able to inhibit lysosomal proteases [227]. It is also not quite clear if the lysosome itself may play a role in the formation of larger lipofuscin clusters. Extensive cross-linking between proteins in aggregates could also take place inside lysosomes, making them mandatory for the formation of advanced protein-lipid clusters. However Höhn et al. were able to show that lipofuscin also forms in dermal fibroblasts when blocking the lysosomal uptake. The formation of lipofuscin was accompanied by elevated levels of ROS [247].

One of the proposed uptake mechanisms into the lysosome is macroautophagy [248], leading to the establishment of the term "aggrephagy" [249]. During macroautophagy, portions of the cytosol as well as organelles are swallowed by a double-membrane vesicle called autophagosome. The autophagosome begins to form at an isolated membrane called phagophore although it is still not clear what the origin of this membrane is. The autophagosomal membrane then fuses with the lysosomal membrane. Its cargo is then released into the lysosomal lumen for degradation. The 
fusion of the two membranes also results in a bigger lysosomal compartment [250,251]. In addition to macroautophagy, microautophagy [252] as well as chaperone-mediated autophagy [253,254] are possible candidates for the removal of oxidized proteins and lipofuscin. While microautophagy involves the direct uptake of cytosolic components and organelles into lysosmes [255] chaperone-mediated autophagy specifically targets proteins containing the sequence signature "KFERQ" [256].

Autophagic uptake and transport to the lysosome is the most efficient way of removing/containing oxidized proteins after aggregates have formed. This is especially important for old, postmitotic cells which cannot dilute their protein aggregates through cell division [207] and where the proteasome has lost efficiency and is even more inhibited by the formation of lipofuscin [230,231]. Nevertheless, the progressive oxidation of proteins severely impacts the cell's metabolism, even in younger cells. Since protein aggregates provide a redox-active surface the increased generation of ROS also elevates the oxidation levels of DNA and lipids. In addition several disorders are known or were proposed to be associated with protein aggregation. Examples are neurological disorders like Alzheimer's and Huntington's [257,258] as well as skin disorders like cutaneous amyloidosis and Darier disease $[259,260]$.

\subsection{Advanced Glycation End Products}

The formation of structures known as advanced glycation end products (AGE) is another problematic process that can be significantly accelerated by oxidative stress. AGEs originate from the non-enzymatic glycation reaction between sugars and proteins, nucleic acids or lipids. The starting point of AGE formation is the Maillard reaction in which carbonyl groups of sugars react with proteins, lipids, or nucleic acids resulting in an unstable Schiff base [261]. Reorganization then leads to the formation of more stable ketoamins (Amadori product). While Schiff bases and Amadori products are reversible they have the ability to react with amino, sulfhydryl, and guanidine groups in proteins [262]. These reactions form protein adducts and protein crosslinks and give rise to AGEs [263]. In addition further oxidation by ROS or oxidative breakdown can lead to more diverse products called advanced glycation end products [264].

AGEs are a very heterogeneous group of molecules and can either be ingested through food consumption or formed inside the cell [265]. It has been confirmed that AGE deposits are accompanied by autofluorescence of the skin depending on their composition and the aging process in general [266] and that they are related to several diseases [267,268]. Interestingly the cell has specific receptors for AGEs (RAGE). Stimulation of these receptors leads to an activation of several pathways cumulating in the activation of the transcription factor nuclear factor kappa-B (NFKB). This factor increases the transcription of pro-inflammatory genes and RAGE themselves leading to a vicious cycle [269]. It was shown that RAGEs are highly expressed on mRNA as well as on protein level in fibroblasts and keratinocytes and that expression was increased in sun-exposed skin [270].

Since AGEs are able to react with a great variety of biomolecules, consequences of their formation are manifold [271]. Examples are collagen crosslinks that lead to decreased flexibility [272], the modification of intracellular proteins like cytokeratin 10 in keratinocytes [273] 
and functional alterations in low-density lipoprotein [274]. AGEs are also heavily connected to oxidative stress $[275,276]$. RAGE signaling can directly induce oxidative stress by decreasing the activity of superoxide dismutase (SOD) or indirectly by reducing cellular anti-oxidant defenses [269,277]. Due to induction of fibroblast activation, the crosslinking of collagen and the increase in metalloproteinase production (MMP 1, 2, and 9) AGEs severely affect the dermis [278]. Concerning the epidermis it was proposed that AGEs impair the migratory and proliferation abilities of keratinocytes in vitro [279]. In addition to these problems there is evidence that the removal of AGEs poses a big problem for the cell. As mentioned before AGE accumulation correlates well with the age of the individual [266] and seems to be resistant to proteolytic degradation [280]. There are enzymes in place to counter the genesis of AGEs [271]. One such enzyme is glyoxylase I which removes $\alpha$-dicarbonyl compounds, another starting point for AGEs. Unfortunately though the decreased activity of this enzyme has been reported during aging [281]. All of these facts paint a very complex picture of the origin and impact of advanced glycation end products. The mutual interactions between AGEs and ROS, induced for example by UV irradiation, make it a challenging area that still needs a lot of further investigation.

\section{Oxidative Stress, DNA, Cancer and Senescence}

\subsection{DNA Mutation}

Besides the already discussed "common" mtDNA deletion, nuclear DNA is also affected by ROS. Of great importance for the oxidation of DNA is the hydroxyl radical originating from the Fenton reaction. The reaction of ROS with other free radicals leads to a multitude of DNA base products that are potentially mutagenic. These DNA base products are summarized in great detail in Cook et al. [282]. The most familiar of these ROS-induced DNA alterations is 8-oxo-2'deoxyguanosine which pairs with an adenine as well as with a cytosine resulting in a GC to TA transition [283]. It was shown that this mutation accumulates specifically during aging in skin and basically all tissues studied [284-286]. Besides the modification of DNA bases, ROS also induce single-strand breaks and to a lesser degree double strand breaks [287]. UV irradiation not only induces ROS production in skin cells and in this way leads to DNA damage but can directly affect DNA. Especially UVB irradiation leads to the formation of cyclobutane pyrimidine dimers (CPD) and (6-4) photoproducts (both from thymine or cytosine bases). C-C dimers as well as the less abundant but more mutagenic (6-4) photoproducts induce a GC to TA transition, [288-290]. The (6-4) photoproducts are removed primarily by the nucleotide excision repair (NER), whereas the most important repair mechanism for cyclobutane pyrimidine dimers is not the NER but the CPD photolyase. Another photolyase exists for the (6-4) photoproducts [291-294]. The ROS induced small DNA damages such as 8-oxo-2'deoxyguanosine, abasic sites and tymine glycol are predominantely repaired by the base excision repair (BER) and the very rare double strand breaks by non-homologous end joining [285,295]. Defects in the nucleotide excision repair lead to severe human diseases that have one thing in common: a segmental premature aging phenotype. In sum 11 diseases were identified that are based on defects in the NER. Besides Cockayne syndrome and trichothiodystrophy, the skin disease Xeroderma pigmentosum has to be named. Xeroderma pigmentosum does not only 
lead to segmental progeria but also to a dramatic increase in sun sensitivity and an increased incidence of sun-induced skin cancer [296,297]. During the aging process a clear decline in the NER as well as the BER could be observed. Sauvaigo et al. tested the repair capacities of primary fibroblasts obtained from three different age groups: young adults (mean age: 25 years), middle-aged adults (mean age: 46 years) and old adults (mean age: 65). The capacity of NER in repairing cyclobutane pyrimidine dimers clearly decreased for the old individuals. Surprisingly the capacity of BER to repair DNA damage seems to decrease much earlier. The repair efficiency for 8-oxo-2'deoxyguanosine, abasic sites and thymine glycol was dramatically reduced in the old as well as the middle aged group [284]. This decrease in repair capacity could explain the increased prevalence of old people for skin cancer.

\subsection{Cancer}

Superoxide is very often considered as the "primary" ROS, because it can be metabolized to other even more reactive radicals such as the hydroxyl radical [298]. The hydroxyl radical can react with the guanosine in the nucleotide chain under formation of 8-oxo-2'deoxyguanosine [299]. In this context it is not surprising that ROS are important factors in tumor development and high levels of 8-oxo-2'deoxyguanosine are found in a multitude of cancers [298,300-304]. The three most frequent skin cancers are basal cell cancer, squamous cell cancer and melanoma. In $90 \%$ of all squamous cell carcinoma and $50 \%$ of all basal cell carcinomas a mutation in the tumor suppressor p53 can be found [305]. In fact the most prominent mutation in p53 is a $G$ to $T$ transversion that could be a result of an oxidative modification of a guanine [306-310]. It is considered that a mutation in p53 leads to an inability of the cells to induce apoptosis, increasing the risk for a cancerous transformation [305,311]. Besides mutations in p53 mutations in Rb, adenomatous polyposis coli (APC) and patched (PTCH) also promote tumor formation [312,313]. ROS are not only involved in initiating tumor formation but also in its progression. It was found that tumor cells produce a high dosage of $\mathrm{H}_{2} \mathrm{O}_{2}$ that could be a key factor in tumor progression [306,314,315]. It was proposed that hydrogen peroxide is a second messenger that is capable of activating molecular switches. Examples of these switches are the redox sensitive Mitogen Activated Protein Kinases and other protein kinases: ERK1/2 (the extracellular signal-regulated kinases), JNK1/2/3 (the c-Jun NH2-terminal kinases), the p38 mitogen-activated kinases, P13K/Akt (phosphoinositide 3-kinase/serine-threonine kinase) and PKB (protein kinase B) [306,316-318]. All these pathways are known to regulate cell proliferation and migration. In general it can be stated that addition of ROS to cell cultures increases cell proliferation [319,320]. Other redox sensitive "switches" are transcription factors such as AP-1, NF- $\mathrm{BB}$, NF-E2 related factor-1 (Nfr1), NF-E2 related factor-2 (Nrf2) and Egr1 (early growth response 1) [306,321,322] that modulate gene expression [298] which could contribute to cell proliferation and migration. In addition, ROS can initiate release of calcium from ER-localized stores. This release of calcium then activates PKC (protein kinase C) which is also involved in cell migration, apoptosis, proliferation and cytoskeletal reorganisation [298,323,324].

An irreversible alteration to a cell signaling pathway is the most common reason for the genesis of cancer, in the skin and elsewhere [325]. It was demonstrated that ROS are essential factors in 
melanomas. After melanocyte transformation the melanosomes are disorganized and, instead of scavenging, they promote ROS formation. These ROS were then shown to activate proto-oncogene pathways [326].

\subsection{Senescence}

One of the most powerful "tools" of a cell against a tumorgenic transformation is senescence [327-329]. An activation of the senescence program leads to an irreversible cell-cycle arrest in the $\mathrm{G}_{1}$-phase [330,331]. Keratinocytes and melanocytes, as well as fibroblast can become senescent [332]. Senescence associated beta-galactosidase, a marker of senescence, is found increasingly in aged tissues and aged skin [333]. In the skin. UV-radiation massively induces premature senescence and could in this way contribute to skin aging and photoaging [334]. The senescent cells stay alive, but start to change their behavior. They develop a very specific secretome, summarized as the "senescence-associated secretory phenotype". In the dermis, senescent fibroblast also activate matrix metalloproteinases and express less matrix metalloproteinase inhibitors and extracellular matrix components like collagen [25,335,336]. Finally, senescent skin cells die by a mechanism that is either described as apoptosis or autophagic programmed cell death [337,338]. Senescence is initiated after such dramatic incidences as severe DNA damage, critical telomere shortening, oxidative stress and hyperactive oncogenic RAS [339-342]. In this context ROS can fulfil a dual role. At low concentration ROS can stimulate the proliferation of cells (discussed above), at high concentrations ROS seem to be involved in the induction of growth arrest of cells [343,344]. This replicative senescence phenotype can be either achieved by the $\mathrm{p} 53 / \mathrm{p} 21$ or $\mathrm{p} 16 / \mathrm{Rb}$ pathway [338]. Retinoblastoma protein $(\mathrm{Rb})$ is responsible for the transition from the $\mathrm{G}_{1}$ to $\mathrm{S}$ phase. This transition is dependent on the phosphorylation state of $\mathrm{Rb}$. $\mathrm{Rb}$ has the ability to bind to members of the transcription factor family E2F. Upon phosphorylation of this tumor supressor E2F transcription factors are released and the cell can pass from the $\mathrm{G}_{1}$ to the $\mathrm{S}$ phase. Rb can either be phosphorylated by the cycline dependent Kinase CDK4 or CDK6. The oxidative stress sensors in this regulatory pathway are the redox sensitive kinases ERK1/2 and p38. Their activation leads to an increased expression of p16. Overexpression of p16 was shown to induce senescence, whereas a p16 knock down was associated with an inhibition of RAS-mediated senescence. p16 is an inhibitor of several cycline dependent kinases. An activation of p16 via Erk1/2 and p38 leads to an inhibition of the cylcine-dependent kinases that are not able to phosphorylate $\mathrm{Rb}$ anymore. The tumor suppressor $\mathrm{Rb}$ is then in a complex with the E2F transcription factors, resulting in an inhibition of E2F target genes and a cell cycle arrest [343,345-351].

The second way to senescence leads through the tumor suppressor p53. p53 can either be activated by DNA damage or by oxidative stress. In healthy cells p53 has a short half-life. p53 is bound by Mdm2 that initiates its transport out of the nucleus resulting in its degradation. DNA damage leads to an activation of ATM (Ataxia telangiectasia mutated)/ATR (ataxia telangiectasia and Rad3-related) protein kinases via DNA damage response elements resulting in a p53 activation. The response to oxidative stress is mediated via p38 that directly phosphorylates p53. Phosphorylated p53 is no longer exported from the nucleus and initiates the transcription of genes involved in apoptosis and cell-cycle arrest. The main candidate for the cell cycle arrest is p21 that 
is a very potent cyclin-dependent kinase inhibitor acting on CDK2 and CDK4 [343,349,352-355]. In keratinocytes a special pathway exists to cope with the high UVB dosage. The UVB response of keratinocytes is tightly connected with the insulin-like growth factor-1 receptor. Activation of this receptor leads to ROS production and expression of p21 via p53 [334]. In more detail, senescence can be divided into acute senescence (like wound healing, development and injury repair) and chronic senescence (like aging) as described in van Deursen 2014 [343].

\section{Oxidative Stress and Lipids}

ROS molecules originating from different sources in the cell have the capacity to induce the lipid peroxidation process. This chain reaction starts with a reaction of a ROS molecule with polyunsaturated fatty acids. In a first step hydrogen atoms are removed from methyl groups of these lipids forming a lipid radical. In the next step a reaction with molecular oxygen takes place leading to a peroxyl radical. A reaction of this radical with another polyunsaturated fatty acid leads to a lipid peroxide and a new radical starting a chain reaction [356]. Oxidation of lipids is in discussion as being involved in human diseases such as atherosclerosis and cancer [357]. The most prominent end product of lipid peroxidation is 4-hydroxynonenal. The complex biology of 4-hydroxynonenal is treated in detail in the chapter by Jörg Schaur et al. elsewhere in this volume. Among other things the application of 4-hydroxynonenal to keratinocytes started a multitude of cellular responses such as an up-regulation of anti-oxidative enzymes (heme oxygenase-1 (HO-1), catalase, NADPH:quinone oxidoreductase (NQO1), and glutathione S-transferases), activation of several kinases such as Erk1/2, JNK and p38 and PI3 and a translocation of the transcription factor Nrf2 to the nucleus [358]. This is of special importance for the aging process because the level of 4-hydroxynonenal increases with aging leading to the cellular responses discussed above. In addition it was shown that in aged human fibroblasts derived from facial skin, 4-hydroxynonenal reacts with macromolecules, leading to the formation of hydroxynonenal modified proteins [359].

\section{Conclusions}

This chapter aims to give an overview of the role of oxidative stress in the general pathology and in the aging process of skin. In the epidermis a very pronounced phenotype is the disruption of the calcium gradient that results in a changed composition of the cornified envelope and changes in gene expression of other calcium dependent proteins of which only the S100 proteins shall be named. A reduced barrier function of aged skin is the major result. The aging process in the dermis is quite different, has other causes, and leads to other phenotypes. The process is driven by the activation of matrix metalloproteases, and the resulting degradation of the extracellular matrix components, especially collagens and elastic fibers. These ECM changes lead to the appearance of fine wrinkles. The aging process in the skin is driven by reactive oxygen species to an extent that is not attained in any other organ. 
This review summarizes the most important contributors to ROS production that are common to all organs:

1. the mitochondrial electron transport chain

2. peroxisomal localized enzymes involved in the $\beta$-oxidation of fatty acids, the glyoxylate/dicarboxylate metabolism and the xanthine oxidase, involved in purine catabolism

3. the endoplasmic reticulum, localized enzymes, protein disulfide isomerase, and endoplasmic reticulum oxidoreductin-1 ERO1 as well as members of the cytochrome P450 family

4. the enzymes cyclooxygenase and lipoxygenase involved in the arachidonic acid metabolism, the Fenton reaction and the membrane localized NADPH oxidase family

Because the skin is at the interface between the exterior and the interior, external factors also contribute to ROS production in the skin. Besides xenobiotics and pollutants the main factor amplifying the photoaging process in the skin is UV irradiation.

Typical defense mechanisms against this high ROS burden are enzymes such as superoxide dismutases, catalase, peroxiredoxins, GSH peroxidases, as well as non-enzymatic components such as L-ascorbate, $\alpha$-tocopherol, beta-carotene, uric acid, CoQ10, and the whole glutathione system. The skin is equipped with several of these enzymes, including enzymatic and non-enzymatic antioxidants. In addition there are water-soluble antioxidants like glucose, pyruvate, and bilirubin as well as lipid-soluble antioxidants like $\alpha$-tocopherol, ubiquinol-10, lycopene, and $\beta$-carotene present. Interestingly the predominant antioxidants found in skin cells are nonenzymatic. This includes L-ascorbic acid, GSH, and uric acid [360].

In contrast to the dermis the epidermis has higher levels of catalase, glutathione peroxidase, glutathione reductase, and ascorbic acid. In addition the stratum corneum is equipped with Vitamins $\mathrm{C}$ and $\mathrm{E}$ as well as uric acid [361-363]. Another defense mechanism against ROS is the SPRR protein family, which is also highly present in the stratum corneum. These proteins are able to efficiently quench ROS by forming intramolecular disulfide bonds.

The highest concentrations of antioxidant enzymes (and the SPRR proteins) can be found in the outermost layer of the epidermis. This is due to the fact that the $\mathrm{O}_{2}$ partial pressure is higher at the surface presenting another source for ROS. Unquenched ROS molecules lead to the formation of protein carbonyls, advanced glycation end products, lipofuscins, start the process of lipid peroxidation and lead to pronounced DNA damage (especially 8-oxo-2'deoxyguanosine).

\section{Acknowledgments}

We are grateful for financial support to the OeAD, project number (CZ 10/2014) (to Mark Rinnerthaler).

\section{Author Contributions}

Mark Rinnerthaler and Klaus Richter designed the structure of this review article. Mark Rinnerthaler, Klaus Richter, Johannes Bischof, Maria Karolin Streubel and Andrea Trost collected the literature and wrote the paper. 


\section{Conflicts of Interest}

The authors declare no conflict of interest.

\section{References}

1. Harman, D. Aging-A theory based on free-radical and radiation-chemistry. J. Gerontol. 1956, 11, 298-300.

2. Harman, D. The biologic clock: The mitochondria? J. Am. Geriatr. Soc. 1972, 20, 145-147.

3. Lapointe, J.; Hekimi, S. When a theory of aging ages badly. Cell. Mol. Life Sci. 2010, 67, 1-8.

4. Hekimi, S. How genetic analysis tests theories of animal aging. Nat. Genet. 2006, 38, 985-991.

5. Ristow, M.; Schmeisser, S. Extending life span by increasing oxidative stress. Free Radic. Biol. Med. 2011, 51, 327-336.

6. Farage, M.A.; Miller, K.W.; Elsner, P.; Maibach, H.I. Intrinsic and extrinsic factors in skin ageing: A review. Int. J. Cosmet. Sci. 2008, 30, 87-95.

7. Poljsak, B.; Dahmane, R.G.; Godic, A. Intrinsic skin aging: The role of oxidative stress. Acta Dermatovenerol. Alp. Pannonica Adriat. 2012, 21, 33-36.

8. Tsutsumi, M.; Denda, M. Paradoxical effects of beta-estradiol on epidermal permeability barrier homeostasis. Br. J. Dermatol. 2007, 157, 776-779.

9. Zouboulis, C.C.; Chen, W.C.; Thornton, M.J.; Qin, K.; Rosenfield, R. Sexual hormones in human skin. Horm. Metab. Res. 2007, 39, 85-95.

10. Zouboulis, C.C.; Makrantonaki, E. Clinical aspects and molecular diagnostics of skin aging. Clin. Dermatol. 2011, 29, 3-14.

11. Farage, M.A.; Miller, K.W.; Elsner, P.; Maibach, H.I. Characteristics of the aging skin. Adv. Wound care 2013, 2, 5-10.

12. Vierkotter, A.; Krutmann, J. Environmental influences on skin aging and ethnic-specific manifestations. Dermato Endocrinol. 2012, 4, 227-231.

13. Ritz-Timme, S.; Laumeier, I.; Collins, M.J. Aspartic acid racemization: Evidence for marked longevity of elastin in human skin. Br. J. Dermatol. 2003, 149, 951-959.

14. Shapiro, S.D.; Endicott, S.K.; Province, M.A.; Pierce, J.A.; Campbell, E.J. Marked longevity of human lung parenchymal elastic fibers deduced from prevalence of d-aspartate and nuclear weapons-related radiocarbon. J. Clin. Invest. 1991, 87, 1828-1834.

15. Kielty, C.M.; Sherratt, M.J.; Shuttleworth, C.A. Elastic fibers. J. Cell Sci. 2002, 115, 2817-2828.

16. Naylor, E.C.; Watson, R.E.; Sherratt, M.J. Molecular aspects of skin ageing. Maturitas 2011, $69,249-256$.

17. Graham, H.K.; Hodson, N.W.; Hoyland, J.A.; Millward-Sadler, S.J.; Garrod, D.; Scothern, A.; Griffiths, C.E.; Watson, R.E.; Cox, T.R.; Erler, J.T.; et al. Tissue section AFM: In situ ultrastructural imaging of native biomolecules. Matrix Biol. 2010, 29, 254-260.

18. Keene, D.R.; Sakai, L.Y.; Lunstrum, G.P.; Morris, N.P.; Burgeson, R.E. Type VII collagen forms an extended network of anchoring fibrils. J. Cell Biol. 1987, 104, 611-621. 
19. Craven, N.M.; Watson, R.E.; Jones, C.J.; Shuttleworth, C.A.; Kielty, C.M.; Griffiths, C.E. Clinical features of photodamaged human skin are associated with a reduction in collagen VII. Br. J. Dermatol. 1997, 137, 344-350.

20. Talwar, H.S.; Griffiths, C.E.; Fisher, G.J.; Hamilton, T.A.; Voorhees, J.J. Reduced type I and type III procollagens in photodamaged adult human skin. J. Investig. Dermatol. 1995, 105, 285-290.

21. Labat-Robert, J.; Fourtanier, A.; Boyer-Lafargue, B.; Robert, L. Age dependent increase of elastase type protease activity in mouse skin. Effect of UV-irradiation. J. Photochem. Photobiol. B Biol. 2000, 57, 113-118.

22. Birkedal-Hansen, H.; Moore, W.G.; Bodden, M.K.; Windsor, L.J.; Birkedal-Hansen, B.; DeCarlo, A.; Engler, J.A. Matrix metalloproteinases: A review. Crit. Rev. Oral Biol. Med. 1993, 4, 197-250.

23. Berton, A.; Godeau, G.; Emonard, H.; Baba, K.; Bellon, P.; Hornebeck, W.; Bellon, G. Analysis of the ex vivo specificity of human gelatinases $\mathrm{a}$ and $\mathrm{b}$ towards skin collagen and elastic fibers by computerized morphometry. Matrix Biol. 2000, 19, 139-148.

24. Jenkins, G. Molecular mechanisms of skin ageing. Mech. Ageing Dev. 2002, 123, 801-810.

25. Millis, A.J.; Hoyle, M.; McCue, H.M.; Martini, H. Differential expression of metalloproteinase and tissue inhibitor of metalloproteinase genes in aged human fibroblasts. Exp. Cell Res. 1992, 201, 373-379.

26. Kohl, E.; Steinbauer, J.; Landthaler, M.; Szeimies, R.M. Skin ageing. J. Eur. Acad. Dermatol. 2011, 25, 873-884.

27. Lavker, R.M.; Veres, D.A.; Irwin, C.J.; Kaidbey, K.H. Quantitative assessment of cumulative damage from repetitive exposures to suberythemogenic doses of uva in human skin. Photochem. Photobiol. 1995, 62, 348-352.

28. Fisher, G.J.; Datta, S.C.; Talwar, H.S.; Wang, Z.Q.; Varani, J.; Kang, S.; Voorhees, J.J. Molecular basis of sun-induced premature skin ageing and retinoid antagonism. Nature 1996, 379, 335-339.

29. Quan, T.; He, T.; Voorhees, J.J.; Fisher, G.J. Ultraviolet irradiation induces Smad7 via induction of transcription factor AP-1 in human skin fibroblasts. J. Biol. Chem. 2005, 280, 8079-8085.

30. Bickers, D.R.; Athar, M. Oxidative stress in the pathogenesis of skin disease. J. Investig. Dermatol. 2006, 126, 2565-2575.

31. Seshadri, T.; Campisi, J. Repression of c-Fos transcription and an altered genetic program in senescent human fibroblasts. Science 1990, 247, 205-209.

32. Campisi, J. Aging and cancer: The double-edged sword of replicative senescence. J. Am. Geriatr. Soc. 1997, 45, 482-488.

33. Campisi, J. Cancer, aging and cellular senescence. In Vivo 2000, 14, 183-188.

34. Tsatsou, F.; Trakatelli, M.; Patsatsi, A.; Kalokasidis, K.; Sotiriadis, D. Extrinsic aging: UV-mediated skin carcinogenesis. Dermato Endocrinol. 2012, 4, 285-297.

35. Samarasinghe, V.; Madan, V. Nonmelanoma skin cancer. J. Cutan. Aesthet. Surg. 2012, 5, 3-10. 
36. Bhandari, P.R.; Pai, V.V. Novel medical strategies combating nonmelanoma skin cancer. Indian J. Dermatol. 2014, 59, 531-546.

37. Epstein, J.H. Photocarcinogenesis, skin cancer, and aging. J. Am. Acad. Dermatol. 1983, 9, 487-502.

38. Lewis, D.A.; Travers, J.B.; Somani, A.K.; Spandau, D.F. The IGF-1/IGF-1R signaling axis in the skin: A new role for the dermis in aging-associated skin cancer. Oncogene 2010, 29, $1475-1485$.

39. Wallace, M.L.; Grichnik, J.M.; Prieto, V.G.; Shea, C.R. Numbers and differentiation status of melanocytes in idiopathic guttate hypomelanosis. J. Cutan. Pathol. 1998, 25, 375-379.

40. Quevedo, W.C.; Szabo, G.; Virks, J. Influence of age and UV on the populations of dopa-positive melanocytes in human skin. J. Investig. Dermatol. 1969, 52, 287-290.

41. Sarin, K.Y.; Artandi, S.E. Aging, graying and loss of melanocyte stem cells. Stem Cell Rev. 2007, 3, 212-217.

42. Passi, S.; Grandinetti, M.; Maggio, F.; Stancato, A.; de Luca, C. Epidermal oxidative stress in vitiligo. Pigment Cell Res. 1998, 11, 81-85.

43. Ruhrberg, C.; Hajibagheri, M.A.; Simon, M.; Dooley, T.P.; Watt, F.M. Envoplakin, a novel precursor of the cornified envelope that has homology to desmoplakin. J. Cell Biol. 1996, 134, 715-729.

44. Ruhrberg, C.; Hajibagheri, M.A.; Parry, D.A.; Watt, F.M. Periplakin, a novel component of cornified envelopes and desmosomes that belongs to the plakin family and forms complexes with envoplakin. J. Cell Biol. 1997, 139, 1835-1849.

45. Watt, F.M.; Green, H. Involucrin synthesis is correlated with cell size in human epidermal cultures. J. Cell Biol. 1981, 90, 738-742.

46. DiColandrea, T.; Karashima, T.; Maatta, A.; Watt, F.M. Subcellular distribution of envoplakin and periplakin: Insights into their role as precursors of the epidermal cornified envelope. J. Cell Biol. 2000, 151, 573-586.

47. Sevilla, L.M.; Nachat, R.; Groot, K.R.; Klement, J.F.; Uitto, J.; Djian, P.; Maatta, A.; Watt, F.M. Mice deficient in involucrin, envoplakin, and periplakin have a defective epidermal barrier. J. Cell Biol. 2007, 179, 1599-1612.

48. Grayson, S.; Johnson-Winegar, A.G.; Wintroub, B.U.; Isseroff, R.R.; Epstein, E.H., Jr.; Elias, P.M. Lamellar body-enriched fractions from neonatal mice: Preparative techniques and partial characterization. J. Investig. Dermatol. 1985, 85, 289-294.

49. Chapman, S.J.; Walsh, A. Membrane-coating granules are acidic organelles which possess proton pumps. J. Investig. Dermatol. 1989, 93, 466-470.

50. Raymond, A.A.; Gonzalez de Peredo, A.; Stella, A.; Ishida-Yamamoto, A.; Bouyssie, D.; Serre, G.; Monsarrat, B.; Simon, M. Lamellar bodies of human epidermis: Proteomics characterization by high throughput mass spectrometry and possible involvement of Clip-170 in their trafficking/secretion. Mol. Cell. Proteomics 2008, 7, 2151-2175.

51. Braff, M.H.; di Nardo, A.; Gallo, R.L. Keratinocytes store the antimicrobial peptide cathelicidin in lamellar bodies. J. Investig. Dermatol. 2005, 124, 394-400. 
52. Galliano, M.F.; Toulza, E.; Gallinaro, H.; Jonca, N.; Ishida-Yamamoto, A.; Serre, G.; Guerrin, M. A novel protease inhibitor of the alpha2-macroglobulin family expressed in the human epidermis. J. Biol. Chem. 2006, 281, 5780-5789.

53. Madison, K.C.; Sando, G.N.; Howard, E.J.; True, C.A.; Gilbert, D.; Swartzendruber, D.C.; Wertz, P.W. Lamellar granule biogenesis: A role for ceramide glucosyltransferase, lysosomal enzyme transport, and the golgi. J. Investig. Dermatol. Sympos. Proc. 1998, 3, 80-86.

54. Serre, G.; Mils, V.; Haftek, M.; Vincent, C.; Croute, F.; Reano, A.; Ouhayoun, J.P.; Bettinger, S.; Soleilhavoup, J.P. Identification of late differentiation antigens of human cornified epithelia, expressed in re-organized desmosomes and bound to cross-linked envelope. J. Investig. Dermatol. 1991, 97, 1061-1072.

55. Denda, M.; Fuziwara, S.; Inoue, K. Influx of calcium and chloride ions into epidermal keratinocytes regulates exocytosis of epidermal lamellar bodies and skin permeability barrier homeostasis. J. Investig. Dermatol. 2003, 121, 362-367.

56. Kalinin, A.; Marekov, L.N.; Steinert, P.M. Assembly of the epidermal cornified cell envelope. J. Cell Sci. 2001, 114, 3069-3070.

57. Kalinin, A.E.; Idler, W.W.; Marekov, L.N.; McPhie, P.; Bowers, B.; Steinert, P.M.; Steven, A.C. Co-assembly of envoplakin and periplakin into oligomers and $\mathrm{Ca}^{2+}$-dependent vesicle binding: Implications for cornified cell envelope formation in stratified squamous epithelia. J. Biol. Chem. 2004, 279, 22773-22780.

58. Marekov, L.N.; Steinert, P.M. Ceramides are bound to structural proteins of the human foreskin epidermal cornified cell envelope. J. Biol. Chem 1998, 273, 17763-17770.

59. Ishida-Yamamoto, A.; Eady, R.A.; Watt, F.M.; Roop, D.R.; Hohl, D.; Iizuka, H. Immunoelectron microscopic analysis of cornified cell envelope formation in normal and psoriatic epidermis. J. Histochem. Cytochem 1996, 44, 167-175.

60. Ahvazi, B.; Boeshans, K.M.; Idler, W.; Baxa, U.; Steinert, P.M. Roles of calcium ions in the activation and activity of the transglutaminase 3 enzyme. J. Biol. Chem. 2003, 278, 23834-23841.

61. Eckert, R.L.; Sturniolo, M.T.; Broome, A.M.; Ruse, M.; Rorke, E.A. Transglutaminase function in epidermis. J. Investig. Dermatol. 2005, 124, 481-492.

62. Steinert, P.M.; Marekov, L.N. Initiation of assembly of the cell envelope barrier structure of stratified squamous epithelia. Mol. Biol. Cell 1999, 10, 4247-4261.

63. Hohl, D.; Lichti, U.; Breitkreutz, D.; Steinert, P.M.; Roop, D.R. Transcription of the human loricrin gene in vitro is induced by calcium and cell density and suppressed by retinoic acid. J. Investig. Dermatol. 1991, 96, 414-418.

64. Proksch, E.; Brandner, J.M.; Jensen, J.M. The skin: An indispensable barrier. Exp. Dermatol. 2008, 17, 1063-1072.

65. Whitfield, J.M. Calcium in Cell Cycles and Cancer; 2nd ed.; CRC Press: Boca Raton, FL, USA, 1995; p. 242. 
66. Robinson, N.A.; Lapic, S.; Welter, J.F.; Eckert, R.L. S100a11, s100a10, annexin I, desmosomal proteins, small proline-rich proteins, plasminogen activator inhibitor-2, and involucrin are components of the cornified envelope of cultured human epidermal keratinocytes. J. Biol. Chem. 1997, 272, 12035-12046.

67. Marshall, D.; Hardman, M.J.; Nield, K.M.; Byrne, C. Differentially expressed late constituents of the epidermal cornified envelope. Proc. Natl. Acad. Sci. USA 2001, 98, 13031-13036.

68. Jackson, B.; Tilli, C.M.; Hardman, M.J.; Avilion, A.A.; MacLeod, M.C.; Ashcroft, G.S.; Byrne, C. Late cornified envelope family in differentiating epithelia-Response to calcium and ultraviolet irradiation. J. Investig. Dermatol. 2005, 124, 1062-1070.

69. Eckhart, L.; Declercq, W.; Ban, J.; Rendl, M.; Lengauer, B.; Mayer, C.; Lippens, S.; Vandenabeele, P.; Tschachler, E. Terminal differentiation of human keratinocytes and stratum corneum formation is associated with caspase-14 activation. J. Investig. Dermatol. 2000, 115, 1148-1151.

70. Mauro, T.; Bench, G.; Sidderas-Haddad, E.; Feingold, K.; Elias, P.; Cullander, C. Acute barrier perturbation abolishes the $\mathrm{Ca}^{2+}$ and $\mathrm{K}^{+}$gradients in murine epidermis: Quantitative measurement using pixe. J. Investig. Dermatol. 1998, 111, 1198-1201.

71. Menon, G.K.; Elias, P.M.; Lee, S.H.; Feingold, K.R. Localization of calcium in murine epidermis following disruption and repair of the permeability barrier. Cell Tissue Res. 1992, 270, 503-512.

72. Rinnerthaler, M.; Duschl, J.; Steinbacher, P.; Salzmann, M.; Bischof, J.; Schuller, M.; Wimmer, H.; Peer, T.; Bauer, J.W.; Richter, K. Age-related changes in the composition of the cornified envelope in human skin. Exp. Dermatol. 2013, 22, 329-335.

73. Rinnerthaler, M.; Streubel, M.K.; Bischof, J.; Richter, K. Skin aging, gene expression and calcium. Exp. Gerontol. 2014, doi:10.1016/j.exger.2014.09.015.

74. Denda, M.; Tomitaka, A.; Akamatsu, H.; Matsunaga, K. Altered distribution of calcium in facial epidermis of aged adults. J. Investig. Dermatol. 2003, 121, 1557-1558.

75. Lener, T.; Moll, P.R.; Rinnerthaler, M.; Bauer, J.; Aberger, F.; Richter, K. Expression profiling of aging in the human skin. Exp. Gerontol. 2006, 41, 387-397.

76. Hackl, M.; Brunner, S.; Fortschegger, K.; Schreiner, C.; Micutkova, L.; Muck, C.; Laschober, G.T.; Lepperdinger, G.; Sampson, N.; Berger, P.; et al. miR-17, miR-19b, miR-20a, and miR-106a are down-regulated in human aging. Aging Cell 2010, 9, 291-296.

77. Chance, B.; Sies, H.; Boveris, A. Hydroperoxide metabolism in mammalian organs. Physiol. Rev. 1979, 59, 527-605.

78. Muller, F.L.; Lustgarten, M.S.; Jang, Y.; Richardson, A.; van Remmen, H. Trends in oxidative aging theories. Free Radic. Biol. Med. 2007, 43, 477-503.

79. Turrens, J.F. Mitochondrial formation of reactive oxygen species. J. Physiol. 2003, 552, 335-344.

80. Holbrook, K.A. Biologic structure and function-Perspectives on morphologic approaches to the study of the granular layer keratinocyte. J. Investig. Dermatol. 1989, 92, S84-S104. 
81. Jeon, S.; Djian, P.; Green, H. Inability of keratinocytes lacking their specific transglutaminase to form cross-linked envelopes: Absence of envelopes as a simple diagnostic test for lamellar ichthyosis. Proc. Natl. Acad. Sci. USA 1998, 95, 687-690.

82. Breitenbach, M.; Rinnerthaler, M.; Hartl, J.; Stincone, A.; Vowinckel, J.; Breitenbach-Koller, H.; Ralser, M. Mitochondria in ageing: There is metabolism beyond the ROS. Fems Yeast Res. 2014, 14, 198-212.

83. Aung-Htut, M.T.; Lam, Y.T.; Lim, Y.L.; Rinnerthaler, M.; Gelling, C.L.; Yang, H.Y.; Breitenbach, M.; Dawes, I.W. Maintenance of mitochondrial morphology by autophagy and its role in high glucose effects on chronological lifespan of saccharomyces cerevisiae. Oxid. Med. Cell Longev. 2013, doi:10.1155/2013/636287.

84. Klinger, H.; Rinnerthaler, M.; Lam, Y.T.; Laun, P.; Heeren, G.; Klocker, A.; Simon-Nobbe, B.; Dickinson, J.R.; Dawes, I.W.; Breitenbach, M. Quantitation of (a)symmetric inheritance of functional and of oxidatively damaged mitochondrial aconitase in the cell division of old yeast mother cells. Exp. Gerontol. 2010, 45, 533-542.

85. Fransen, M.; Nordgren, M.; Wang, B.; Apanasets, O. Role of peroxisomes in ROS/RNSmetabolism: Implications for human disease. Biochim. Biophys. Acta Mol. Basis Dis. 2012, 1822, 1363-1373.

86. Kuppusamy, P.; Zweier, J.L. Characterization of free radical generation by xanthine oxidase. Evidence for hydroxyl radical generation. J. Biol. Chem 1989, 264, 9880-9884.

87. Granger, D.N.; Hollwarth, M.E.; Parks, D.A. Ischemia-reperfusion injury: Role of oxygen-derived free radicals. Acta Physiol. Scand. Suppl. 1986, 548, 47-63.

88. Harrison, R. Structure and function of xanthine oxidoreductase: Where are we now? Free Radic. Biol. Med. 2002, 33, 774-797.

89. Stuehr, D.; Pou, S.; Rosen, G.M. Oxygen reduction by nitric-oxide synthases. J. Biol. Chem 2001, 276, 14533-14536.

90. Benham, A.M.; van Lith, M.; Sitia, R.; Braakman, I. Ero1-PDI interactions, the response to redox flux and the implications for disulfide bond formation in the mammalian endoplasmic reticulum. Philos. Trans. R. Soc. Lond. B Biol. Sci. 2013, doi:10.1098/rstb.2011.0403.

91. Bhandary, B.; Marahatta, A.; Kim, H.R.; Chae, H.J. An involvement of oxidative stress in endoplasmic reticulum stress and its associated diseases. Int J. Mol. Sci. 2012, 14, 434-456.

92. Bae, Y.S.; Oh, H.; Rhee, S.G.; Do Yoo, Y. Regulation of reactive oxygen species generation in cell signaling. Mol. Cells 2011, 32, 491-509.

93. Gorsky, L.D.; Koop, D.R.; Coon, M.J. On the stoichiometry of the oxidase and monooxygenase reactions catalyzed by liver microsomal cytochrome P-450. Products of oxygen reduction. J. Biol. Chem. 1984, 259, 6812-6817.

94. Yengi, L.G.; Xiang, Q.; Pan, J.M.; Scatina, J.; Kao, J.; Ball, S.E.; Fruncillo, R.; Ferron, G.; Wolf, C.R. Quantitation of cytochrome p450 mrna levels in human skin. Analytical Biochem. 2003, 316, 103-110.

95. Baron, J.M.; Holler, D.; Schiffer, R.; Frankenberg, S.; Neis, M.; Merk, H.F.; Jugert, F.K. Expression of multiple cytochrome P450 enzymes and multidrug resistance-associated transport proteins in human skin keratinocytes. J. Investig. Dermatol. 2001, 116, 541-548. 
96. Rinnerthaler, M.; Buttner, S.; Laun, P.; Heeren, G.; Felder, T.K.; Klinger, H.; Weinberger, M.; Stolze, K.; Grousl, T.; Hasek, J.; et al. Yno1p/Aim14p, a NADPH-oxidase ortholog, controls extramitochondrial reactive oxygen species generation, apoptosis, and actin cable formation in yeast. Proc. Natl. Acad. Sci. USA 2012, 109, 8658-8663.

97. Block, K.; Gorin, Y.; Abboud, H.E. Subcellular localization of Nox4 and regulation in diabetes. Proc. Natl. Acad. Sci. USA 2009, 106, 14385-14390.

98. Krause, K.H. Tissue distribution and putative physiological function of Nox family NADPH oxidases. Jpn. J. Infect Dis. 2004, 57, S28-S29.

99. Nauseef, W.M. Biological roles for the Nox family NADPH oxidases. J. Biol. Chem. 2008, 283, 16961-16965.

100. Kukreja, R.C.; Kontos, H.A.; Hess, M.L.; Ellis, E.F. Superoxide generation by prostaglandin synthetase and lipoxygenase in presence of NADH or NADPH. Fed. Proc. 1986, 45, 451-451.

101. Ruzicka, T.; Simmet, T.; Peskar, B.A.; Ring, J. Skin levels of arachidonic acid-derived inflammatory mediators and histamine in atopic-dermatitis and psoriasis. J. Investig. Dermatol. 1986, 86, 105-108.

102. Ziboh, V.A.; Miller, C.C.; Cho, Y.H. Metabolism of polyunsaturated fatty acids by skin epidermal enzymes: Generation of antiinflammatory and antiproliferative metabolites. Am. J. Clin. Nutr. 2000, 71, 361s-366s.

103. Barb, W.G.; Baxendale, J.H.; George, P.; Hargrave, K.R. Reactions of ferrous and ferric ions with hydrogen peroxide. Nature 1949, 163, 692-694.

104. Barbusinski, K. Fenton reaction-Controversy concerning the chemistry. Ecol. Chem. Eng. 2009, 16, 347-358.

105. Poljsak, B.; Dahmane, R. Free radicals and extrinsic skin aging. Dermatol. Res. Pract. 2012, doi:10.1155/2012/135206.

106. Chen, L.; Hu, J.Y.; Wang, S.Q. The role of antioxidants in photoprotection: A critical review. J. Am. Acad. Dermatol. 2012, 67, 1013-1024.

107. Krutmann, J.; Schroeder, P. Role of mitochondria in photoaging of human skin: The defective powerhouse model. J. Investig. Dermatol. Symp. Proc. 2009, 14, 44-49.

108. Prasad, A.; Pospisil, P. Ultraweak photon emission induced by visible light and ultraviolet a radiation via photoactivated skin chromophores: In vivo charge coupled device imaging. J. Biomed. Opt. 2012, doi:10.1117/1.JBO.17.8.085004.

109. Wondrak, G.T.; Jacobson, M.K.; Jacobson, E.L. Endogenous UVA-photosensitizers: Mediators of skin photodamage and novel targets for skin photoprotection. Photochem. Photobiol. Sci. 2006, 5, 215-237.

110. Berneburg, M.; Gattermann, N.; Stege, H.; Grewe, M.; Vogelsang, K.; Ruzicka, T.; Krutmann, J. Chronically ultraviolet-exposed human skin shows a higher mutation frequency of mitochondrial DNA as compared to unexposed skin and the hematopoietic system. J. Investig. Dermatol. 1997, 109, 425-425. 
111. Siddens, L.K.; Larkin, A.; Krueger, S.K.; Bradfield, C.A.; Waters, K.M.; Tilton, S.C.; Pereira, C.B.; Lohr, C.V.; Arlt, V.M.; Phillips, D.H.; et al. Polycyclic aromatic hydrocarbons as skin carcinogens: Comparison of benzo [a]pyrene, dibenzo[def,p]chrysene and three environmental mixtures in the FVB/N mouse. Toxicol. Appl. Pharmacol. 2012, 264, 377-386.

112. Fu, P.P.; Xia, Q.S.; Sun, X.; Yu, H.T. Phototoxicity and environmental transformation of polycyclic aromatic hydrocarbons (PAHs)-light-induced reactive oxygen species, lipid peroxidation, and DNA damage. J. Environ. Sci. Health Part C Environ. Carcinog. Ecotoxicol. Rev. 2012, 30, 1-41.

113. Vermeij, W.P.; Alia, A.; Backendorf, C. ROS quenching potential of the epidermal cornified cell envelope. J. Investig. Dermatol. 2011, 131, 1435-1441.

114. Weber, S.U.; Thiele, J.J.; Cross, C.E.; Packer, L. Vitamin C, uric acid, and glutathione gradients in murine stratum corneum and their susceptibility to ozone exposure. J. Investig. Dermatol. 1999, 113, 1128-1132.

115. Schafer, M.; Werner, S. The cornified envelope: A first line of defense against reactive oxygen species. J. Investig. Dermatol. 2011, 131, 1409-1411.

116. Schafer, M.; Dutsch, S.; Keller, U.A.D.; Navid, F.; Schwarz, A.; Johnson, D.A.; Johnson, J.A.; Werner, S. Nrf2 establishes a glutathione-mediated gradient of uvb cytoprotection in the epidermis. Gene Dev. 2010, 24, 1045-1058.

117. Thiele, J.J.; Traber, M.G.; Packer, L. Depletion of human stratum corneum vitamin E: An early and sensitive in vivo marker of UV induced photo-oxidation. J. Investig. Dermatol. 1998, 110, 756-761.

118. Colven, R.M.; Pinnell, S.R. Topical vitamin C in aging. Clin. Dermatol. 1996, 14, 227-234.

119. Padayatty, S.J.; Katz, A.; Wang, Y.H.; Eck, P.; Kwon, O.; Lee, J.H.; Chen, S.L.; Corpe, C.; Dutta, A.; Dutta, S.K.; et al. Vitamin C as an antioxidant: Evaluation of its role in disease prevention. J. Am. Coll. Nutr. 2003, 22, 18-35.

120. Humbert, P.G.; Haftek, M.; Creidi, P.; Lapiere, C.; Nusgens, B.; Richard, A.; Schmitt, D.; Rougier, A.; Zahouani, H. Topical ascorbic acid on photoaged skin. Clinical, topographical and ultrastructural evaluation: Double-blind study vs. Placebo. Exp. Dermatol. 2003, 12, 237-244.

121. Rhie, G.; Shin, M.H.; Seo, J.Y.; Choi, W.W.; Cho, K.H.; Kim, K.H.; Park, K.C.; Eun, H.C.; Chung, J.H. Aging- and photoaging-dependent changes of enzymic and nonenzymic antioxidants in the epidermis and dermis of human skin in vivo. J. Investig. Dermatol. 2001, $117,1212-1217$.

122. Nachbar, F.; Korting, H.C. The role of vitamin-E in normal and damaged skin. J. Mol. Med. 1995, 73, 7-17.

123. Rubbo, H.; Radi, R.; Anselmi, D.; Kirk, M.; Barnes, S.; Butler, J.; Eiserich, J.P.; Freeman, B.A. Nitric oxide reaction with lipid peroxyl radicals spares alpha-tocopherol during lipid peroxidation-Greater oxidant protection from the pair nitric oxide/alpha-tocopherol than alpha-tocopherol/ascorbate. J. Biol. Chem. 2000, 275, 10812-10818.

124. Engin, K.N. Alpha-tocopherol: Looking beyond an antioxidant. Mol. Vis. 2009, 15, 855-860. 
125. Bayerl, C. Beta-carotene in dermatology: Does it help? Acta Dermatovenerol. Alp. Pannon. Adriat. 2008, 17, 160-162.

126. BarNatan, R.; Lomnitski, L.; Sofer, Y.; Segman, S.; Neeman, I.; Grossman, S. Interaction between beta-carotene and lipoxygenase in human skin. Int. J. Biochem. Cell Biol. 1996, 28, 935-941.

127. Liebler, D.C.; McClure, T.D. Antioxidant reactions of beta-carotene: Identification of carotenoid-radical adducts. Chem. Res. Toxicol. 1996, 9, 8-11.

128. Maples, K.R.; Mason, R.P. Free-radical metabolite of uric-acid. J. Biol. Chem. 1988, 263, 1709-1712.

129. Ames, B.N.; Cathcart, R.; Schwiers, E.; Hochstein, P. Uric-acid provides an antioxidant defense in humans against oxidant-caused and radical-caused aging and cancer-A hypothesis. Proc. Natl. Acad. Sci. USA 1981, 78, 6858-6862.

130. Sautin, Y.Y.; Johnson, R.J. Uric acid: The oxidant-antioxidant paradox. Nucleosides Nucleotides Nucleic Acids 2008, 27, 608-619.

131. Green, D.E.; Tzagoloff, A. The mitochondrial electron transfer chain. Arch. Biochem. Biophys. 1966, 116, 293-304.

132. Littarru, G.P.; Tiano, L. Bioenergetic and antioxidant properties of coenzyme Q10: Recent developments. Mol. Biotechnol. 2007, 37, 31-37.

133. McCord, J.M.; Fridovich, I. Superoxide dismutase. An enzymic function for erythrocuprein (hemocuprein). J. Biol. Chem. 1969, 244, 6049-6055.

134. Kwon, M.J.; Kim, B.; Lee, Y.S.; Kim, T.Y. Role of superoxide dismutase 3 in skin inflammation. J. Dermatol. Sci. 2012, 67, 81-87.

135. Rulisek, L.; Ryde, U. Structure of reduced and oxidized manganese superoxide dismutase: A combined computational and experimental approach. J. Phys. Chem. B 2006, 110, 11511-11518.

136. Murakami, K.; Inagaki, J.; Saito, M.; Ikeda, Y.; Tsuda, C.; Noda, Y.; Kawakami, S.; Shirasawa, T.; Shimizu, T. Skin atrophy in cytoplasmic sod-deficient mice and its complete recovery using a vitamin c derivative. Biochem. Biophys. Res. Commun. 2009, 382, 457-461.

137. Shibuya, S.; Ozawa, Y.; Watanabe, K.; Izuo, N.; Toda, T.; Yokote, K.; Shimizu, T. Palladium and platinum nanoparticles attenuate aging-like skin atrophy via antioxidant activity in mice. PLOS ONE 2014, 9, e109288.

138. Poswig, A.; Wenk, J.; Brenneisen, P.; Wlaschek, M.; Hommel, C.; Quel, G.; Faisst, K.; Dissemond, J.; Briviba, K.; Krieg, T.; et al. Adaptive antioxidant response of manganese-superoxide dismutase following repetitive uva irradiation. J. Investig. Dermatol. 1999, 112, 13-18.

139. Jang, Y.C.; Perez, V.I.; Song, W.; Lustgarten, M.S.; Salmon, A.B.; Mele, J.; Qi, W.; Liu, Y.; Liang, H.; Chaudhuri, A.; et al. Overexpression of MN superoxide dismutase does not increase life span in mice. J. Gerontol. A Biol. Sci. Med. Sci. 2009, 64, 1114-1125.

140. Velarde, M.C.; Flynn, J.M.; Day, N.U.; Melov, S.; Campisi, J. Mitochondrial oxidative stress caused by Sod 2 deficiency promotes cellular senescence and aging phenotypes in the skin. Aging 2012, 4, 3-12. 
141. Treiber, N.; Maity, P.; Singh, K.; Kohn, M.; Keist, A.F.; Ferchiu, F.; Sante, L.; Frese, S.; Bloch, W.; Kreppel, F.; et al. Accelerated aging phenotype in mice with conditional deficiency for mitochondrial superoxide dismutase in the connective tissue. Aging Cell 2011, 10, 912-912.

142. Treiber, N.; Maity, P.; Singh, K.; Ferchiu, F.; Wlaschek, M.; Scharffetter-Kochanek, K. The role of manganese superoxide dismutase in skin aging. Dermato Endocrinol. 2012, 4, 232-235.

143. Wagener, F.A.; Carels, C.E.; Lundvig, D.M. Targeting the redox balance in inflammatory skin conditions. Int. J. Mol. Sci. 2013, 14, 9126-9167.

144. Loew, O. A new enzyme of general occurrence in organismis. Science 1900, 11, 701-702.

145. Fita, I.; Silva, A.M.; Murthy, M.R.N.; Rossmann, M.G. The refined structure of beef-liver catalase at $2.5 \AA$ Å resolution. Acta Crystallogr. Sect. B Struct. Sci. 1986, 42, 497-515.

146. Alfonso-Prieto, M.; Biarnes, X.; Vidossich, P.; Rovira, C. The molecular mechanism of the catalase reaction. J. Am. Chem. Soc. 2009, 131, 11751-11761.

147. Giacomoni, P.U.; Declercq, L.; Hellemans, L.; Maes, D. Aging of human skin: Review of a mechanistic model and first experimental data. IUBMB Life 2000, 49, 259-263.

148. Hellemans, L.; Corstjens, H.; Neven, A.; Declercq, L.; Maes, D. Antioxidant enzyme activity in human stratum corneum shows seasonal variation with an age-dependent recovery. J. Investig. Dermatol. 2003, 120, 434-439.

149. Shin, M.H.; Rhie, G.E.; Kim, Y.K.; Park, C.H.; Cho, K.H.; Kim, K.H.; Eun, H.C.; Chung, J.H. $\mathrm{H}_{2} \mathrm{O}_{2}$ accumulation by catalase reduction changes map kinase signaling in aged human skin in vivo. J. Investig. Dermatol. 2005, 125, 221-229.

150. Schriner, S.E.; Linford, N.J.; Martin, G.M.; Treuting, P.; Ogburn, C.E.; Emond, M.; Coskun, P.E.; Ladiges, W.; Wolf, N.; van Remmen, H.; et al. Extension of murine life span by overexpression of catalase targeted to mitochondria. Science 2005, 308, 1909-1911.

151. Aung-Htut, M.T.; Ayer, A.; Breitenbach, M.; Dawes, I.W. Oxidative stresses and ageing. Subcell. Biochem. 2012, 57, 13-54.

152. Lopez-Torres, M.; Shindo, Y.; Packer, L. Effect of age on antioxidants and molecular markers of oxidative damage in murine epidermis and dermis. J. Investig. Dermatol. 1994, $102,476-480$.

153. Flohe, L.; Toppo, S.; Cozza, G.; Ursini, F. A comparison of thiol peroxidase mechanisms. Antioxid. Redox Signal. 2011, 15, 763-780.

154. Li, F.; Liu, J.; Rozovsky, S. Glutathione peroxidases reaction intermediate selenenic acid is stabilized by the protein microenvironment. Free Radic. Biol. Med. 2014, 76, 127-135.

155. Sengupta, A.; Lichti, U.F.; Carlson, B.A.; Cataisson, C.; Ryscavage, A.O.; Mikulec, C.; Conrad, M.; Fischer, S.M.; Hatfield, D.L.; Yuspa, S.H. Targeted disruption of glutathione peroxidase 4 in mouse skin epithelial cells impairs postnatal hair follicle morphogenesis that is partially rescued through inhibition of cox-2. J. Investig. Dermatol. 2013, 133, 1731-1741.

156. Harrison, P.M.; Arosio, P. The ferritins: Molecular properties, iron storage function and cellular regulation. Biochim. Biophys. Acta 1996, 1275, 161-203. 
157. Asano, T.; Komatsu, M.; Yamaguchi-Iwai, Y.; Ishikawa, F.; Mizushima, N.; Iwai, K. Distinct mechanisms of ferritin delivery to lysosomes in iron-depleted and iron-replete cells. Mol. Cell Biol. 2011, 31, 2040-2052.

158. Applegate, L.A.; Scaletta, C.; Panizzon, R.; Frenk, E. Evidence that ferritin is uv inducible in human skin: Part of a putative defense mechanism. J. Investig. Dermatol. 1998, 111, 159-163.

159. Applegate, L.A.; Frenk, E. Oxidative defense in cultured human skin fibroblasts and keratinocytes from sun-exposed and non-exposed skin. Photodermatol. Photoimmunol. Photomed. 1995, 11, 95-101.

160. Pourzand, C.; Watkin, R.D.; Brown, J.E.; Tyrrell, R.M. Ultraviolet a radiation induces immediate release of iron in human primary skin fibroblasts: The role of ferritin. Proc. Natl. Acad. Sci. USA 1999, 96, 6751-6756.

161. Pelle, E.; Jian, J.; Zhang, Q.; Muizzuddin, N.; Yang, Q.; Dai, J.; Maes, D.; Pernodet, N.; Yarosh, D.B.; Frenkel, K.; et al. Menopause increases the iron storage protein ferritin in skin. J. Cosmet. Sci. 2013, 64, 175-179.

162. Wood, Z.A.; Schroder, E.; Robin Harris, J.; Poole, L.B. Structure, mechanism and regulation of peroxiredoxins. Trends Biochem. Sci. 2003, 28, 32-40.

163. Lee, S.C.; Chae, H.Z.; Lee, J.E.; Kwon, B.D.; Lee, J.B.; Won, Y.H.; Ahn, K.Y.; Kim, Y.P. Peroxiredoxin is ubiquitously expressed in rat skin: Isotype-specific expression in the epidermis and hair follicle. J. Investig. Dermatol. 2000, 115, 1108-1114.

164. Ito, T.; Kimura, S.; Seto, K.; Warabi, E.; Kawachi, Y.; Shoda, J.; Tabuchi, K.; Yamagata, K.; Hasegawa, S.; Bukawa, H.; et al. Peroxiredoxin I plays a protective role against uva irradiation through reduction of oxidative stress. J. Dermatol. Sci. 2014, 74, 9-17.

165. Rolfs, F.; Huber, M.; Gruber, F.; Bohm, F.; Pfister, H.J.; Bochkov, V.N.; Tschachler, E.; Dummer, R.; Hohl, D.; Schafer, M.; et al. Dual role of the antioxidant enzyme peroxiredoxin 6 in skin carcinogenesis. Cancer Res. 2013, 73, 3460-3469.

166. Kumin, A.; Huber, C.; Rulicke, T.; Wolf, E.; Werner, S. Peroxiredoxin 6 is a potent cytoprotective enzyme in the epidermis. Am. J. Pathol. 2006, 169, 1194-1205.

167. Nystrom, T.; Yang, J.S.; Molin, M. Peroxiredoxins, gerontogenes linking aging to genome instability and cancer. Gene Dev. 2012, 26, 2001-2008.

168. Tran, D.; Townley, J.P.; Barnes, T.M.; Greive, K.A. An antiaging skin care system containing alpha hydroxy acids and vitamins improves the biomechanical parameters of facial skin. Clin. Cosmet. Investig. Dermatol. 2015, 8, 9-17.

169. Farris, P.; Yatskayer, M.; Chen, N.; Krol, Y.; Oresajo, C. Evaluation of efficacy and tolerance of a nighttime topical antioxidant containing resveratrol, baicalin, and vitamin e for treatment of mild to moderately photodamaged skin. J. Drugs Dermatol. 2014, 13, 1467-1472.

170. Soeur, J.; Eilstein, J.; Lereaux, G.; Jones, C.; Marrot, L. Skin resistance to oxidative stress induced by resveratrol: From Nrf2 activation to gsh biosynthesis. Free Radic. Biol. Med. 2015, 78, 213-223. 
171. Lohan, S.B.; Bauersachs, S.; Ahlberg, S.; Baisaeng, N.; Keck, C.M.; Muller, R.H.; Witte, E.; Wolk, K.; Hackbarth, S.; Roder, B.; et al. Ultra-small lipid nanoparticles promote the penetration of coenzyme Q10 in skin cells and counteract oxidative stress. Eur. J. Pharm. Biopharm. 2015, 89, 201-207.

172. Schwarz, J.C.; Baisaeng, N.; Hoppel, M.; Low, M.; Keck, C.M.; Valenta, C. Ultra-small NLC for improved dermal delivery of coenyzme Q10. Int. J. Pharm. 2013, 447, 213-217.

173. Lorencini, M.; Brohem, C.A.; Dieamant, G.C.; Zanchin, N.I.; Maibach, H.I. Active ingredients against human epidermal aging. Ageing Res. Rev. 2014, 15, 100-115.

174. Pallauf, K.; Bendall, J.K.; Scheiermann, C.; Watschinger, K.; Hoffmann, J.; Roeder, T.; Rimbach, G. Vitamin C and lifespan in model organisms. Food Chem. Toxicol. 2013, 58, 255-263.

175. Ernst, I.M.; Pallauf, K.; Bendall, J.K.; Paulsen, L.; Nikolai, S.; Huebbe, P.; Roeder, T.; Rimbach, G. Vitamin E supplementation and lifespan in model organisms. Ageing Res. Rev. 2013, 12, 365-375.

176. Sadowska-Bartosz, I.; Bartosz, G. Effect of antioxidants supplementation on aging and longevity. Biomed. Res. Int. 2014, doi:10.1155/2014/404680.

177. Selman, C.; McLaren, J.S.; Collins, A.R.; Duthie, G.G.; Speakman, J.R. Deleterious consequences of antioxidant supplementation on lifespan in a wild-derived mammal. Biol. Lett. 2013, 9, doi:10.1098/rsbl.2013.0432.

178. Lam, Y.T.; Stocker, R.; Dawes, I.W. The lipophilic antioxidants alpha-tocopherol and coenzyme Q10 reduce the replicative lifespan of saccharomyces cerevisiae. Free Radic. Biol. Med. 2010, 49, 237-244.

179. Shetty, R.A.; Forster, M.J.; Sumien, N. Coenzyme Q(10) supplementation reverses age-related impairments in spatial learning and lowers protein oxidation. Age 2013, 35, $1821-1834$.

180. Ishii, N.; Senoo-Matsuda, N.; Miyake, K.; Yasuda, K.; Ishii, T.; Hartman, P.S.; Furukawa, S. Coenzyme Q10 can prolong C. Elegans lifespan by lowering oxidative stress. Mech. Ageing Dev. 2004, 125, 41-46.

181. Bjelakovic, G.; Nikolova, D.; Gluud, L.L.; Simonetti, R.G.; Gluud, C. Antioxidant supplements for prevention of mortality in healthy participants and patients with various diseases. Cochrane Database Syst. Rev. 2012, doi:10.1002/14651858.CD007176.pub2.

182. Marosz, A.; Chlubek, D. The risk of abuse of vitamin supplements. Ann. Acad. Medicae Stetin. 2014, 60, 60-64.

183. Shokolenko, I.N.; Wilson, G.L.; Alexeyev, M.F. Aging: A mitochondrial DNA perspective, critical analysis and an update. World J. Exp. Med. 2014, 4, 46-57.

184. Bjelakovic, G.; Nikolova, D.; Gluud, C. Antioxidant supplements and mortality. Curr. Opin. Clin. Nutr. Metab. Care 2014, 17, 40-44.

185. D'Autreaux, B.; Toledano, M.B. ROS as signalling molecules: Mechanisms that generate specificity in ROS homeostasis. Nat. Rev. Mol. Cell Biol. 2007, 8, 813-824.

186. Sayin, V.I.; Ibrahim, M.X.; Larsson, E.; Nilsson, J.A.; Lindahl, P.; Bergo, M.O. Antioxidants accelerate lung cancer progression in mice. Sci. Transl. Med. 2014, 6, 221 ra215. 
187. Heck, D.E.; Vetrano, A.M.; Mariano, T.M.; Laskin, J.D. UVB light stimulates production of reactive oxygen species-Unexpected role for catalase. J. Biol. Chem. 2003, 278, 22432-22436.

188. Jaeger, J.B.; Pillai, E.D.; Jaeger, T.D.; Duncan, M.A. Ultraviolet and infrared photodissociation of $\mathrm{si}^{+}(\mathrm{C} 6 \mathrm{H} 6)_{(\mathrm{n})}$ and $\mathrm{si}^{+}(\mathrm{C} 6 \mathrm{H} 6)_{(\mathrm{n})}$ Ar clusters. J. Phys. Chem. A 2005, 109, 2801-2808.

189. Sander, C.S.; Chang, H.; Salzmann, S.; Muller, C.S.L.; Ekanayake-Mudiyanselage, S.; Elsner, P.; Thiele, J.J. Photoaging is associated with protein oxidation in human skin in vivo. J. Investig. Dermatol. 2002, 118, 618-625.

190. Squier, T.C. Oxidative stress and protein aggregation during biological aging. Exp. Gerontol. 2001, 36, 1539-1550.

191. Hunt, J.V.; Dean, R.T.; Wolff, S.P. Hydroxyl radical production and autoxidative glycosylation-Glucose autoxidation as the cause of protein damage in the experimental glycation model of diabetes-mellitus and aging. Biochem. J. 1988, 256, 205-212.

192. Wolff, S.P.; Wang, G.M.; Spector, A. Pro-oxidant activation of ocular reductants. 1. Copper and riboflavin stimulate ascorbate oxidation causing lens epithelial cytotoxicity in vitro. Exp. Eye Res. 1987, 45, 777-789.

193. Stadtman, E.R. Protein oxidation and aging. Free Radic. Res. 2006, 40, 1250-1258.

194. Arner, E.S.; Holmgren, A. Physiological functions of thioredoxin and thioredoxin reductase. Eur. J. Biochem. 2000, 267, 6102-6109.

195. Fernandes, A.P.; Holmgren, A. Glutaredoxins: Glutathione-dependent redox enzymes with functions far beyond a simple thioredoxin backup system. Antioxid. Redox Signal. 2004, 6, 63-74.

196. Moskovitz, J. Methionine sulfoxide reductases: Ubiquitous enzymes involved in antioxidant defense, protein regulation, and prevention of aging-associated diseases. Biochim. Biophys. Acta Proteins Proteom 2005, 1703, 213-219.

197. Hohn, A.; Konig, J.; Grune, T. Protein oxidation in aging and the removal of oxidized proteins. J. Proteomics 2013, 92, 132-159.

198. Dalle-Donne, I.; Rossi, R.; Giustarini, D.; Milzani, A.; Colombo, R. Protein carbonyl groups as biomarkers of oxidative stress. Clin. Chim. Acta 2003, 329, 23-38.

199. Dalle-Donne, I.; Aldini, G.; Carini, M.; Colombo, R.; Rossi, R.; Milzani, A. Protein carbonylation, cellular dysfunction, and disease progression. J. Cell Mol. Med. 2006, 10, 389-406.

200. Thiele, J.J.; Traber, M.G.; Re, R.; Espuno, N.; Yan, L.J.; Cross, C.E.; Packer, L. Macromolecular carbonyls in human stratum corneum: A biomarker for environmental oxidant exposure? FEBS Lett. 1998, 422, 403-406.

201. Avezov, K.; Reznick, A.Z.; Aizenbud, D. Time and dose effects of cigarette smoke and acrolein on protein carbonyl formation in hacat keratinocytes. Adv. Exp. Med. Biol. 2015, 849, 57-64.

202. Thiele, J.J.; Schroeter, C.; Hsieh, S.N.; Podda, M.; Packer, L. The antioxidant network of the stratum corneum. Curr. Probl. Dermatol. 2001, 29, 26-42. 
203. Maisonneuve, E.; Ducret, A.; Khoueiry, P.; Lignon, S.; Longhi, S.; Talla, E.; Dukan, S. Rules governing selective protein carbonylation. PLOS ONE 2009, 4, e7269.

204. Wong, C.M.; Marcocci, L.; Liu, L.L.; Suzuki, Y.J. Cell signaling by protein carbonylation and decarbonylation. Antioxid. Redox Signal. 2010, 12, 393-404.

205. Baraibar, M.A.; Friguet, B. Changes of the proteasomal system during the aging process. Prog. Mol. Biol. Transl. Sci. 2012, 109, 249-275.

206. Grune, T.; Merker, K.; Jung, T.; Sitte, N.; Davies, K.J.A. Protein oxidation and degradation during postmitotic senescence. Free Radic. Biol. Med. 2005, 39, 1208-1215.

207. Gray, D.A.; Woulfe, J. Lipofuscin and aging: A matter of toxic waste. Sci. Aging Knowl. Environ. 2005, doi:10.1126/sageke.2005.5.re1.

208. Jung, T.; Hohn, A.; Catalgol, B.; Grune, T. Age-related differences in oxidative protein-damage in young and senescent fibroblasts. Arch. Biochem. Biophys. 2009, 483, 127-135.

209. Thiele, J.J.; Dreher, F.; Packer, L. Antioxidant defense systems in skin (reprinted from cosmeceuticals: Drugs vs. Cosmetics, pg 145-187, 2000). J. Toxicol. Cutaneous. Ocul. Toxicol. 2002, 21, 119-160.

210. Naskalski, J.W.; Bartosz, G. Oxidative modifications of protein structures. Adv. Clin. Chem. 2000, 35, 161-253.

211. Kang, S.W.; Chae, H.Z.; Seo, M.S.; Kim, K.; Baines, I.C.; Rhee, S.G. Mammalian peroxiredoxin isoforms can reduce hydrogen peroxide generated in response to growth factors and tumor necrosis factor-alpha. J. Biol. Chem. 1998, 273, 6297-6302.

212. Grune, T.; Merker, K.; Sandig, G.; Davies, K.J.A. Selective degradation of oxidatively modified protein substrates by the proteasome. Biochem. Biophys. Res. Commun. 2003, 305, 709-718.

213. Pacifici, R.E.; Kono, Y.; Davies, K.J. Hydrophobicity as the signal for selective degradation of hydroxyl radical-modified hemoglobin by the multicatalytic proteinase complex, proteasome. J. Biol. Chem. 1993, 268, 15405-15411.

214. Buchberger, A.; Bukau, B.; Sommer, T. Protein quality control in the cytosol and the endoplasmic reticulum: Brothers in arms. Mol. Cell 2010, 40, 238-252.

215. Ding, Q.; Dimayuga, E.; Keller, J.N. Proteasome regulation of oxidative stress in aging and age-related diseases of the CNS. Antioxid. Redox Signal. 2006, 8, 163-172.

216. Hiller, M.M.; Finger, A.; Schweiger, M.; Wolf, D.H. ER degradation of a misfolded luminal protein by the cytosolic ubiquitin-proteasome pathway. Science 1996, 273, 1725-1728.

217. Malhotra, J.D.; Kaufman, R.J. Endoplasmic reticulum stress and oxidative stress: A vicious cycle or a double-edged sword? Antioxid. Redox Signal. 2007, 9, 2277-2293.

218. Hohn, A.; Grune, T. Lipofuscin: Formation, effects and role of macroautophagy. Redox Biol. 2013, 1, 140-144.

219. Kawaguchi, Y.; Kovacs, J.J.; McLaurin, A.; Vance, J.M.; Ito, A.; Yao, T.P. The deacetylase HDAC6 regulates aggresome formation and cell viability in response to misfolded protein stress. Cell 2003, 115, 727-738. 
220. Catalgol, B.; Ziaja, I.; Breusing, N.; Jung, T.; Hohn, A.; Alpertunga, B.; Schroeder, P.; Chondrogianni, N.; Gonos, E.S.; Petropoulos, I.; et al. The proteasome is an integral part of solar ultraviolet a radiation-induced gene expression. J. Biol. Chem. 2009, 284, 30076-30086.

221. Watanabe, H.; Shimizu, T.; Nishihira, J.; Abe, R.; Nakayama, T.; Taniguchi, M.; Sabe, H.; Ishibashi, T.; Shimizu, H. Ultraviolet a-induced production of matrix metalloproteinase-1 is mediated by macrophage migration inhibitory factor (MIF) in human dermal fibroblasts. J. Biol. Chem. 2004, 279, 1676-1683.

222. Kim, S.; Lee, Y.; Lee, D.H.; Kim, Y.; Cho, K.H.; Chung, J.H. Basal and UV-induced MMP-1 expression are inhibited by p53 in human dermal fibroblasts. Exp. Dermatol. 2008, 17, 939-945.

223. Reinke, L.A.; Kotake, Y.; Moore, D.R.; Nanji, A.A. Free radical formation during ketamine anesthesia in rats: A cautionary note. Free Radic. Biol. Med. 1998, 24, 1002-1006.

224. Valko, M.; Morris, H.; Cronin, M.T. Metals, toxicity and oxidative stress. Curr. Med. Chem. 2005, 12, 1161-1208.

225. Spiteller, G. The important role of lipid peroxidation processes in aging and age dependent diseases. Mol. Biotechnol. 2007, 37, 5-12.

226. Requena, J.R.; Fu, M.X.; Ahmed, M.U.; Jenkins, A.J.; Lyons, T.J.; Thorpe, S.R. Lipoxidation products as biomarkers of oxidative damage to proteins during lipid peroxidation reactions. Nephrol. Dial. Transplant. 1996, 11, 48-53.

227. Sitte, N.; Huber, M.; Grune, T.; Ladhoff, A.; Doecke, W.D.; Von Zglinicki, T.; Davies, K.J.A. Proteasome inhibition by lipofuscin/ceroid during postmitotic aging of fibroblasts. FASEB J. 2000, 14, 1490-1498.

228. Carrard, G.; Bulteau, A.L.; Petropoulos, I.; Friguet, B. Impairment of proteasome structure and function in aging. Int. J. Biochem. Cell B 2002, 34, 1461-1474.

229. Hohn, A.; Jung, T.; Grimm, S.; Catalgol, B.; Weber, D.; Grune, T. Lipofuscin inhibits the proteasome by binding to surface motifs. Free Radic. Biol. Med. 2011, 50, 585-591.

230. Grune, T.; Jung, T.; Merker, K.; Davies, K.J.A. Decreased proteolysis caused by protein aggregates, inclusion bodies, plaques, lipofuscin, ceroid, and "aggresomes" during oxidative stress, aging, and disease. Int. J. Biochem. Cell Biol. 2004, 36, 2519-2530.

231. Poppek, D.; Grune, T. protein oxidation and proteolysis during cellular senescence. Z. Gerontol. Geriatr. 2004, 37, 175-183.

232. Koziel, R.; Greussing, R.; Maier, A.B.; Declercq, L.; Jansen-Durr, P. Functional interplay between mitochondrial and proteasome activity in skin aging. J. Investig. Dermatol. 2011, 131, 594-603.

233. Katz, M.L.; Robison, W.G. What is lipofuscin? Defining characteristics and differentiation from other autofluoreseent lysosomal storage bodies. Arch. Gerontol. Geriat 2002, 34, $169-184$.

234. Soroka, Y.; Ma’or, Z.; Leshem, Y.; Verochovsky, L.; Neuman, R.; Bregegere, F.M.; Milner, Y. Aged keratinocyte phenotyping: Morphology, biochemical markers and effects of dead sea minerals. Exp. Gerontol. 2008, 43, 947-957. 
235. Alpert, M.; Jacobowitz, D.; Marks, B.H. A simple method for the demonstration of lipofuscin pigment. J. Histochem. Cytochem. 1960, 8, 153-158.

236. Brunk, U.T.; Terman, A. Lipofuscin: Mechanisms of age-related accumulation and influence on cell function. Free Radic. Biol. Med. 2002, 33, 611-619.

237. Szweda, P.A.; Camouse, M.; Lundberg, K.C.; Oberley, T.D.; Szweda, L.I. Aging, lipofuscin formation, and free radical-mediated inhibition of cellular proteolytic systems. Ageing Res. Rev. 2003, 2, 383-405.

238. Double, K.L.; Dedov, V.N.; Fedorow, H.; Kettle, E.; Halliday, G.M.; Garner, B.; Brunk, U.T. The comparative biology of neuromelanin and lipofuscin in the human brain. Cell Mol. Life Sci. 2008, 65, 1669-1682.

239. Benavides, S.H.; Monserrat, A.J.; Farina, S.; Porta, E.A. Sequential histochemical studies of neuronal lipofuscin in human cerebral cortex from the first to the ninth decade of life. Arch. Gerontol. Geriatr. 2002, 34, 219-231.

240. Jolly, S.S.; Brownstein, S.; Jordan, D.R.; Munro, S.M.; Keystone, E.C. Scleritis in a patient with limited wegener's granulomatosis and takayasu's arteritis. Can. J. Ophthalmol. 1995, 30, 371-373.

241. Winterbourn, C.C.; Vile, G.F.; Monteiro, H.P. Ferritin, lipid peroxidation and redox-cycling xenobiotics. Free Radic. Res. Commun. 1991, 12, 107-114.

242. Wardman, P.; Candeias, L.P. Fenton chemistry: An introduction. Radiat. Res. 1996, 145, 523-531.

243. Yin, D.Z. Biochemical basis of lipofuscin, ceroid, and age pigment-like fluorophores. Free Radic. Biol. Med. 1996, 21, 871-888.

244. Jung, T.; Engels, M.; Klotz, L.O.; Kroncke, K.D.; Grune, T. Nitrotyrosine and protein carbonyls are equally distributed in HT22 cells after nitrosative stress. Free Radic. Biol. Med. 2007, 42, 773-786.

245. Pompella, A.; Cambiaggi, C.; Dominici, S.; Paolicchi, A.; Tongiani, R.; Comporti, M. Single-cell investigation by laser scanning confocal microscopy of cytochemical alterations resulting from extracellular oxidant challenge. Histochem. Cell Biol. 1996, 105, 173-178.

246. Ohkuma, S.; Poole, B. Fluorescence probe measurement of intralysosomal ph in living cells and perturbation of $\mathrm{pH}$ by various agents. Proc. Natl. Acad. Sci. USA 1978, 75, 3327-3331.

247. Hohn, A.; Sittig, A.; Jung, T.; Grimm, S.; Grune, T. Lipofuscin is formed independently of macroautophagy and lysosomal activity in stress-induced prematurely senescent human fibroblasts. Free Radic. Biol. Med. 2012, 53, 1760-1769.

248. Cuervo, A.M. Autophagy and aging-When "all you can eat" is yourself. Sci. Aging Knowl. Environ. 2003, doi:10.1126/sageke.2003.36.pe25.

249. Overbye, A.; Fengsrud, M.; Seglen, P.O. Proteomic analysis of membrane-associated proteins from rat liver autophagosomes. Autophagy 2007, 3, 300-322.

250. Guo, Y.; Chang, C.; Huang, R.; Liu, B.; Bao, L.; Liu, W. Ap1 is essential for generation of autophagosomes from the trans-golgi network. J. Cell Sci. 2012, 125, 1706-1715.

251. Mizushima, N.; Komatsu, M. Autophagy: Renovation of cells and tissues. Cell 2011, 147, 728-741. 
252. Li, W.W.; Li, J.; Bao, J.K. Microautophagy: Lesser-known self-eating. Cell Mol. Life Sci. 2012, 69, 1125-1136.

253. Kaushik, S.; Cuervo, A.M. Chaperone-mediated autophagy. Methods Mol. Biol. 2008, 445, 227-244.

254. Cuervo, A.M.; Wong, E. Chaperone-mediated autophagy: Roles in disease and aging. Cell Res. 2014, 24, 92-104.

255. Mijaljica, D.; Prescott, M.; Devenish, R.J. Microautophagy in mammalian cells: Revisiting a 40-year-old conundrum. Autophagy 2011, 7, 673-682.

256. Dice, J.F. Peptide sequences that target cytosolic proteins for lysosomal proteolysis. Trends Biochem. Sci. 1990, 15, 305-309.

257. Sorolla, M.A.; Rodriguez-Colman, M.J.; Tamarit, J.; Ortega, Z.; Lucas, J.J.; Ferrer, I.; Ros, J.; Cabiscol, E. Protein oxidation in huntington disease affects energy production and vitamin B6 metabolism. Free Radic. Biol. Med. 2010, 49, 612-621.

258. Irvine, G.B.; El-Agnaf, O.M.; Shankar, G.M.; Walsh, D.M. Protein aggregation in the brain: The molecular basis for Alzheimer's and Parkinson's diseases. Mol. Med. 2008, 14, 451-464.

259. Borowicz, J.; Gillespie, M.; Miller, R. Cutaneous amyloidosis. Skinmed 2011, 9, 96-100.

260. Wang, Y.; Bruce, A.T.; Tu, C.; Ma, K.; Zeng, L.; Zheng, P.; Liu, Y. Protein aggregation of SERCA2 mutants associated with darier disease elicits ER stress and apoptosis in keratinocytes. J. Cell Sci. 2011, 124, 3568-3580.

261. Maillard, L.C. The action of amino acids on sugar; the formation of melanoidin by a methodic route. Cr. Hebd. Acad. Sci. 1912, 154, 66-68.

262. Lo, T.W.C.; Westwood, M.E.; Mclellan, A.C.; Selwood, T.; Thornalley, P.J. Binding and modification of proteins by methylglyoxal under physiological conditions-A kinetic and mechanistic study with $\mathrm{N}$-alpha-acetylarginine, $\mathrm{N}$-alpha-acetylcysteine, and $\mathrm{N}$-alpha-acetyllysine, and bovine serum-albumin. J. Biol. Chem. 1994, 269, 32299-32305.

263. Ahmed, N. Advanced glycation endproducts-Role in pathology of diabetic complications. Diabetes Res. Clin. Pract. 2005, 67, 3-21.

264. Goldberg, T.; Cai, W.J.; Peppa, M.; Dardaine, V.; Baliga, B.S.; Uribarri, J.; Vlassara, H. Advanced glycoxidation end products in commonly consumed foods. J. Am. Diet. Assoc. 2004, 104, 1287-1291.

265. Fleming, T.H.; Humpert, P.M.; Nawroth, P.P.; Bierhaus, A. Reactive metabolites and age/rage-mediated cellular dysfunction affect the aging process-A mini-review. Gerontology 2011, 57, 435-443.

266. Beisswenger, P.J.; Howell, S.; Mackenzie, T.; Corstjens, H.; Muizzuddin, N.; Matsui, M.S. Two fluorescent wavelengths, $440_{\mathrm{ex}} / 520_{\mathrm{em} \mathrm{nm}}$ and $370_{\mathrm{ex}} / 440_{\mathrm{em}} \mathrm{nm}$, reflect advanced glycation and oxidation end products in human skin without diabetes. Diabetes Technol. Ther. 2012, 14, 285-292.

267. Hyogo, H.; Yamagishi, S. Advanced glycation end products (ages) and their involvement in liver disease. Curr. Pharm. Des. 2008, 14, 969-972.

268. Prasad, A.; Bekker, P.; Tsimikas, S. Advanced glycation end products and diabetic cardiovascular disease. Cardiol. Rev. 2012, 20, 177-183. 
269. Bierhaus, A.; Humpert, P.M.; Morcos, M.; Wendt, T.; Chavakis, T.; Arnold, B.; Stern, D.M.; Nawroth, P.P. Understanding rage, the receptor for advanced glycation end products. J. Mol. Med. 2005, 83, 876-886.

270. Lohwasser, C.; Neureiter, D.; Weigle, B.; Kirchner, T.; Schuppan, D. The receptor for advanced glycation end products is highly expressed in the skin and upregulated by advanced glycation end products and tumor necrosis factor-alpha. J. Investig. Dermatol. 2006, 126, 291-299.

271. Gkogkolou, P.; Bohm, M. Advanced glycation end products: Key players in skin aging? Dermato Endocrinol. 2012, 4, 259-270.

272. Sajithlal, G.B.; Chithra, P.; Chandrakasan, G. Advanced glycation end products induce crosslinking of collagen in vitro. Biochim. Biophys. Acta Mol. Basis Dis. 1998, 1407, 215-224.

273. Kawabata, K.; Yoshikawa, H.; Saruwatari, K.; Akazawa, Y.; Inoue, T.; Kuze, T.; Sayo, T.; Uchida, N.; Sugiyama, Y. The presence of N-epsilon-(carboxymethyl) lysine in the human epidermis. Biochim. Biophys. Acta Proteins Proteom 2011, 1814, 1246-1252.

274. Bucala, R.; Mitchell, R.; Arnold, K.; Innerarity, T.; Vlassara, H.; Cerami, A. Identification of the major site of apolipoprotein-B modification by advanced glycosylation end-products blocking uptake by the low-density-lipoprotein receptor. J. Biol. Chem. 1995, 270, 10828-10832.

275. Lal, M.A.; Brismar, H.; Eklof, A.C.; Aperia, A. Role of oxidative stress in advanced glycation end product-induced mesangial cell activation. Kidney Int. 2002, 61, 2006-2014.

276. Yamagishi, S.; Maeda, S.; Matsui, T.; Ueda, S.; Fukami, K.; Okuda, S. Role of advanced glycation end products (ages) and oxidative stress in vascular complications in diabetes. Biochim. Biophys. Acta Gen. Subj. 2012, 1820, 663-671.

277. Loughlin, D.T.; Artlett, C.M. Precursor of advanced glycation end products mediates ER-stress-induced caspase-3 activation of human dermal fibroblasts through $\mathrm{NAD}(\mathrm{P}) \mathrm{H}$ oxidase 4. PLOS ONE 2010, 5, e11093.

278. Pageon, H.; Bakala, H.; Monnier, V.M.; Asselineau, D. Collagen glycation triggers the formation of aged skin in vitro. Eur. J. Dermatol. 2007, 17, 12-20.

279. Zhu, P.; Yang, C.; Chen, L.H.; Ren, M.; Lao, G.J.; Yan, L. Impairment of human keratinocyte mobility and proliferation by advanced glycation end products-modified BSA. Arch. Dermatol. Res. 2011, 303, 339-350.

280. Deuther-Conrad, W.; Franke, S.; Sommer, M.; Henle, T.; Stein, G. Differences in the modulating potential of advanced glycation end product (age) peptides versus age proteins. Kidney Int. Suppl. 2001, 78, S63-S66.

281. Ramasamy, R.; Vannucci, S.J.; Yan, S.S.; Herold, K.; Yan, S.F.; Schmidt, A.M. Advanced glycation end products and rage: A common thread in aging, diabetes, neurodegeneration, and inflammation. Glycobiology 2005, 15, 16R-28R.

282. Cooke, M.S.; Evans, M.D.; Dizdaroglu, M.; Lunec, J. Oxidative DNA damage: Mechanisms, mutation, and disease. FASEB J. 2003, 17, 1195-1214. 
283. Kasai, H.; Chung, M.H.; Jones, D.S.; Inoue, H.; Ishikawa, H.; Kamiya, H.; Ohtsuka, E.; Nishimura, S. 8-hydroxyguanine, a DNA adduct formed by oxygen radicals: Its implication on oxygen radical-involved mutagenesis/carcinogenesis. J. Toxicol. Sci. 1991, 16, 95-105.

284. Sauvaigo, S.; Caillat, S.; Odin, F.; Nkengne, A.; Bertin, C.; Oddos, T. Effect of aging on DNA excision/synthesis repair capacities of human skin fibroblasts. J. Investig. Dermatol. 2010, 130, 1739-1741.

285. Kunisada, M.; Sakumi, K.; Tominaga, Y.; Budiyanto, A.; Ueda, M.; Ichihashi, M.; Nakabeppu, Y.; Nishigori, C. 8-oxoguanine formation induced by chronic uvb exposure makes OGG1 knockout mice susceptible to skin carcinogenesis. Cancer Res. 2005, 65, 6006-6010.

286. Nie, B.; Gan, W.; Shi, F.; Hu, G.X.; Chen, L.G.; Hayakawa, H.; Sekiguchi, M.; Cai, J.P. Age-dependent accumulation of 8-oxoguanine in the DNA and RNA in various rat tissues. Oxid. Med. Cell Longev. 2013, doi:10.1155/2013/303181.

287. Caldecott, K.W. Single-strand break repair and genetic disease. Nat. Rev. Genet. 2008, 9 , 619-631.

288. Tewari, A.; Sarkany, R.P.; Young, A.R. Uva1 induces cyclobutane pyrimidine dimers but not 6-4 photoproducts in human skin in vivo. J. Investig. Dermatol. 2012, 132, 394-400.

289. Ziegler, A.; Jonason, A.; Simon, J.; Leffell, D.; Brash, D.E. Tumor suppressor gene mutations and photocarcinogenesis. Photochem. Photobiol. 1996, 63, 432-435.

290. Otoshi, E.; Yagi, T.; Mori, T.; Matsunaga, T.; Nikaido, O.; Kim, S.T.; Hitomi, K.; Ikenaga, M.; Todo, T. Respective roles of cyclobutane pyrimidine dimers, (6-4)photoproducts, and minor photoproducts in ultraviolet mutagenesis of repair-deficient xeroderma pigmentosum a cells. Cancer Res. 2000, 60, 1729-1735.

291. Todo, T.; Ryo, H.; Borden, A.; Lawrence, C.; Sakaguchi, K.; Hirata, H.; Nomura, T. Non-mutagenic repair of (6-4) photoproducts by (6-4) photolyase purified from drosophila melanogaster. Mutat. Res. DNA Repair. 1997, 385, 83-93.

292. Mitchell, D.L.; Nairn, R.S. The biology of the (6-4) photoproduct. Photochem. Photobiol. 1989, 49, 805-819.

293. Mu, D.; Tursun, M.; Duckett, D.R.; Drummond, J.T.; Modrich, P.; Sancar, A. Recognition and repair of compound DNA lesions (base damage and mismatch) by human mismatch repair and excision repair systems. Mol. Cell. Biol. 1997, 17, 760-769.

294. Sancar, A. Structure and function of DNA photolyase. Biochemistry 1994, 33, $2-9$.

295. Pandel, R.; Poljsak, B.; Godic, A.; Dahmane, R. Skin photoaging and the role of antioxidants in its prevention. ISRN Dermatol. 2013, doi:10.1155/2013/930164.

296. Andressoo, J.O.; Mitchell, J.R.; de Wit, J.; Hoogstraten, D.; Volker, M.; Toussaint, W.; Speksnijder, E.; Beems, R.B.; van Steeg, H.; Jans, J.; et al. An xpd mouse model for the combined xeroderma pigmentosum/cockayne syndrome exhibiting both cancer predisposition and segmental progeria. Cancer Cell 2006, 10, 121-132.

297. Niedernhofer, L.J.; Bohr, V.A.; Sander, M.; Kraemer, K.H. Xeroderma pigmentosum and other diseases of human premature aging and DNA repair: Molecules to patients. Mech. ageing Dev. 2011, 132, 340-347. 
298. Klaunig, J.E.; Kamendulis, L.M.; Hocevar, B.A. Oxidative stress and oxidative damage in carcinogenesis. Toxicol. Pathol. 2010, 38, 96-109.

299. Cadet, J.; Douki, T.; Pouget, J.P.; Ravanat, J.L. Singlet oxygen DNA damage products: Formation and measurement. Methods Enzymol. 2000, 319, 143-153.

300. Kumar, B.; Koul, S.; Khandrika, L.; Meacham, R.B.; Koul, H.K. Oxidative stress is inherent in prostate cancer cells and is required for aggressive phenotype. Cancer Res. 2008, 68, 1777-1785.

301. Weiss, J.M.; Goode, E.L.; Ladiges, W.C.; Ulrich, C.M. Polymorphic variation in hogg1 and risk of cancer: A review of the functional and epidemiologic literature. Mol. Carcinog. 2005, 42, 127-141.

302. Diakowska, D.; Lewandowski, A.; Kopec, W.; Diakowski, W.; Chrzanowska, T. Oxidative DNA damage and total antioxidant status in serum of patients with esophageal squamous cell carcinoma. Hepatogastroenterology 2007, 54, 1701-1704.

303. Tanaka, H.; Fujita, N.; Sugimoto, R.; Urawa, N.; Horiike, S.; Kobayashi, Y.; Iwasa, M.; Ma, N.; Kawanishi, S.; Watanabe, S.; et al. Hepatic oxidative DNA damage is associated with increased risk for hepatocellular carcinoma in chronic hepatitis C. Br. J. Cancer. 2008, 98, 580-586.

304. Ishikawa, K.; Takenaga, K.; Akimoto, M.; Koshikawa, N.; Yamaguchi, A.; Imanishi, H.; Nakada, K.; Honma, Y.; Hayashi, J. Ros-generating mitochondrial DNA mutations can regulate tumor cell metastasis. Science 2008, 320, 661-664.

305. Brash, D.E.; Ziegler, A.; Jonason, A.S.; Simon, J.A.; Kunala, S.; Leffell, D.J. Sunlight and sunburn in human skin cancer: P53, apoptosis, and tumor promotion. J. Investig. Dermatol. Symp. Proc. 1996, 1, 136-142.

306. Waris, G.; Ahsan, H. Reactive oxygen species: Role in the development of cancer and various chronic conditions. J. Carcinog. 2006, doi:10.1186/1477-3163-5-14.

307. Brash, D.E.; Rudolph, J.A.; Simon, J.A.; Lin, A.; McKenna, G.J.; Baden, H.P.; Halperin, A.J.; Ponten, J. A role for sunlight in skin cancer: UV-induced p53 mutations in squamous cell carcinoma. Proc. Natl. Acad. Sci. USA 1991, 88, 10124-10128.

308. Hollstein, M.; Sidransky, D.; Vogelstein, B.; Harris, C.C. P53 mutations in human cancers. Science 1991, 253, 49-53.

309. Harris, C.C.; Hollstein, M. Clinical implications of the p53 tumor-suppressor gene. New Engl. J. Med. 1993, 329, 1318-1327.

310. Reid, T.M.; Loeb, L.A. Effect of DNA-repair enzymes on mutagenesis by oxygen free radicals. Mutat. Res. 1993, 289, 181-186.

311. Ichihashi, M.; Ueda, M.; Budiyanto, A.; Bito, T.; Oka, M.; Fukunaga, M.; Tsuru, K.; Horikawa, T. UV-induced skin damage. Toxicology 2003, 189, 21-39.

312. Levitt, N.C.; Hickson, I.D. Caretaker tumour suppressor genes that defend genome integrity. Trends Mol. Med. 2002, 8, 179-186. 
313. Hahn, H.; Wicking, C.; Zaphiropoulous, P.G.; Gailani, M.R.; Shanley, S.; Chidambaram, A.; Vorechovsky, I.; Holmberg, E.; Unden, A.B.; Gillies, S.; et al. Mutations of the human homolog of drosophila patched in the nevoid basal cell carcinoma syndrome. Cell 1996, 85, 841-851.

314. Szatrowski, T.P.; Nathan, C.F. Production of large amounts of hydrogen peroxide by human tumor cells. Cancer Res. 1991, 51, 794-798.

315. Olinski, R.; Jaruga, P.; Zastawny, T.H. Oxidative DNA base modifications as factors in carcinogenesis. Acta Biochim. Polonica 1998, 45, 561-572.

316. Zhou, S.; Kachhap, S.; Sun, W.; Wu, G.; Chuang, A.; Poeta, L.; Grumbine, L.; Mithani, S.K.; Chatterjee, A.; Koch, W.; et al. Frequency and phenotypic implications of mitochondrial DNA mutations in human squamous cell cancers of the head and neck. Proc. Natl. Acad. Sci. USA 2007, 104, 7540-7545.

317. Liu, L.Z.; Hu, X.W.; Xia, C.; He, J.; Zhou, Q.; Shi, X.; Fang, J.; Jiang, B.H. Reactive oxygen species regulate epidermal growth factor-induced vascular endothelial growth factor and hypoxia-inducible factor-1alpha expression through activation of akt and p70s6k1 in human ovarian cancer cells. Free Radic. Biol. Med. 2006, 41, 1521-1533.

318. Mehdi, M.Z.; Azar, Z.M.; Srivastava, A.K. Role of receptor and nonreceptor protein tyrosine kinases in $\mathrm{H}_{2} \mathrm{O}_{2}$-induced $\mathrm{PKB}$ and ERK1/2 signaling. Cell Biochem. Biophys. 2007, 47, 1-10.

319. Frenkel, K. Carcinogen-mediated oxidant formation and oxidative DNA damage. Pharmacol. Ther. 1992, 53, 127-166.

320. Fiorani, M.; Cantoni, O.; Tasinato, A.; Boscoboinik, D.; Azzi, A. Hydrogen peroxide-and fetal bovine serum-induced DNA synthesis in vascular smooth muscle cells: Positive and negative regulation by protein kinase C isoforms. Biochim. Biophys. Acta 1995, 1269, 98-104.

321. Kensler, T.W.; Wakabayashi, N.; Biswal, S. Cell survival responses to environmental stresses via the keap1-Nrf2-are pathway. Annu. Rev. Pharmacol. Toxicol. 2007, 47, 89-116.

322. Pantano, C.; Reynaert, N.L.; van der Vliet, A.; Janssen-Heininger, Y.M. Redox-sensitive kinases of the nuclear factor-kappab signaling pathway. Antioxid. Redox Signal. 2006, 8, 1791-1806.

323. Wu, W.S. The signaling mechanism of ROS in tumor progression. Cancer Metastasis Rev. 2006, 25, 695-705.

324. Frank, G.D.; Eguchi, S. Activation of tyrosine kinases by reactive oxygen species in vascular smooth muscle cells: Significance and involvement of egf receptor transactivation by angiotensin II. Antioxid. Redox Signal. 2003, 5, 771-780.

325. de Gruijl, F.R.; van Kranen, H.J.; Mullenders, L.H. UV-induced DNA damage, repair, mutations and oncogenic pathways in skin cancer. J. Photochem. Photobiol. B Biol. 2001, 63, 19-27.

326. Fruehauf, J.P.; Trapp, V. Reactive oxygen species: An achilles' heel of melanoma? Expert Rev. Anticancer Ther. 2008, 8, 1751-1757.

327. Braig, M.; Lee, S.; Loddenkemper, C.; Rudolph, C.; Peters, A.H.F.M.; Schlegelberger, B.; Stein, H.; Dorken, B.; Jenuwein, T.; Schmitt, C.A. Oncogene-induced senescence as an initial barrier in lymphoma development. Nature 2005, 436, 660-665. 
328. Chen, Z.B.; Trotman, L.C.; Shaffer, D.; Lin, H.K.; Dotan, Z.A.; Niki, M.; Koutcher, J.A.; Scher, H.I.; Ludwig, T.; Gerald, W.; et al. Crucial role of p53-dependent cellular senescence in suppression of pten-deficient tumorigenesis. Nature 2005, 436, 725-730.

329. Courtois-Cox, S.; Williams, S.M.G.; Reczek, E.E.; Johnson, B.W.; McGillicuddy, L.T.; Johannessen, C.M.; Hollstein, P.E.; MacCollin, M.; Cichowski, K. A negative feedback signaling network underlies oncogene-induced senescence. Cancer Cell 2006, 10, 459-472.

330. Campisi, J.; di Fagagna, F.D. Cellular senescence: When bad things happen to good cells. Nat. Rev. Mol. Cell Biol. 2007, 8, 729-740.

331. Collado, M.; Blasco, M.A.; Serrano, M. Cellular senescence in cancer and aging. Cell 2007, 130, 223-233.

332. Cristofalo, V.J.; Lorenzini, A.; Allen, R.G.; Torres, C.; Tresini, M. Replicative senescence: A critical review. Mech. Ageing Dev. 2004, 125, 827-848.

333. Dimri, G.P.; Lee, X.H.; Basile, G.; Acosta, M.; Scott, C.; Roskelley, C.; Medrano, E.E.; Linskens, M.; Rubelj, I.; Pereirasmith, O.; et al. A biomarker that identifies senescent human-cells in culture and in aging skin in vivo. Proc. Natl. Acad. Sci. USA 1995, 92, 9363-9367.

334. Lewis, D.A.; Yi, Q.F.; Travers, J.B.; Spandau, D.F. Uvb-induced senescence in human keratinocytes requires a functional insulin-like growth factor-1 receptor and p53. Mol. Biol. Cell 2008, 19, 1346-1353.

335. Coppe, J.P.; Patil, C.K.; Rodier, F.; Sun, Y.; Munoz, D.P.; Goldstein, J.; Nelson, P.S.; Desprez, P.Y.; Campisi, J. Senescence-associated secretory phenotypes reveal cell-nonautonomous functions of oncogenic ras and the p53 tumor suppressor. PLoS Biol. 2008, 6, 2853-2868.

336. Shelton, D.N.; Chang, E.; Whittier, P.S.; Choi, D.; Funk, W.D. Microarray analysis of replicative senescence. Curr. Biol. 1999, 9, 939-945.

337. Gosselin, K.; Deruy, E.; Martien, S.; Vercamer, C.; Bouali, F.; Dujardin, T.; Slomianny, C.; Houel-Renault, L.; Chelli, F.; de Launoit, Y.; et al. Senescent keratinocytes die by autophagic programmed cell death. Am. J. Pathol. 2009, 174, 423-435.

338. Deruy, E.; Nassour, J.; Martin, N.; Vercamer, C.; Malaquin, N.; Bertout, J.; Chelli, F.; Pourtier, A.; Pluquet, O.; Abbadie, C. Level of macroautophagy drives senescent keratinocytes into cell death or neoplastic evasion. Cell Death Dis. 2014, 5, e1577.

339. Blackburn, E.H. Telomeres and telomerase: Their mechanisms of action and the effects of altering their functions. FEBS Lett. 2005, 579, 859-862.

340. Sedelnikova, O.A.; Horikawa, I.; Zimonjic, D.B.; Popescu, N.C.; Bonner, W.M.; Barrett, J.C. Senescing human cells and ageing mice accumulate DNA lesions with unrepairable double-strand breaks. Nat. Cell Biol. 2004, 6, 168-170.

341. Toussaint, O.; Medrano, E.E.; von Zglinicki, T. Cellular and molecular mechanisms of stress-induced premature senescence (sips) of human diploid fibroblasts and melanocytes. Exp. Gerontol. 2000, 35, 927-945. 
342. Serrano, M.; Lin, A.W.; McCurrach, M.E.; Beach, D.; Lowe, S.W. Oncogenic RAS provokes premature cell senescence associated with accumulation of p53 and p16(Ink4a). Cell 1997, $88,593-602$.

343. Van Deursen, J.M. The role of senescent cells in ageing. Nature 2014, 509, 439-446.

344. Von Zglinicki, T. Oxidative stress shortens telomeres. Trends Biochem. Sci. 2002, 27, 339-344.

345. Bond, J.; Jones, C.; Haughton, M.; DeMicco, C.; Kipling, D.; Wynford-Thomas, D. Direct evidence from siRNA-directed "knock down" that p16(Ink4a) is required for human fibroblast senescence and for limiting RAS-induced epithelial cell proliferation. Exp. Cell Res. 2004, 292, 151-156.

346. McConnell, B.B.; Starborg, M.; Brookes, S.; Peters, G. Inhibitors of cyclin-dependent kinases induce features of replicative senescence in early passage human diploid fibroblasts. Curr. Biol. 1998, 8, 351-354.

347. Maehara, K.; Yamakoshi, K.; Ohtani, N.; Kubo, Y.; Takahashi, A.; Arase, S.; Jones, N.; Hara, E. Reduction of total E2F/DP activity induces senescence-like cell cycle arrest in cancer cells lacking functional PRB and p53. J. Cell Biol. 2005, 168, 553-560.

348. Udayakumar, T.; Shareef, M.M.; Diaz, D.A.; Ahmed, M.M.; Pollack, A. The E2F1/RB and p53/MDM2 pathways in DNA repair and apoptosis: Understanding the crosstalk to develop novel strategies for prostate cancer radiotherapy. Semin. Radiat. Oncol. 2010, 20, 258-266.

349. Sherr, C.J.; McCormick, F. The RB and p53 pathways in cancer. Cancer Cell 2002, 2, $103-112$

350. Jenkins, N.C.; Liu, T.; Cassidy, P.; Leachman, S.A.; Boucher, K.M.; Goodson, A.G.; Samadashwily, G.; Grossman, D. The p16(Ink4a) tumor suppressor regulates cellular oxidative stress. Oncogene 2011, 30, 265-274.

351. Meloche, S.; Pouyssegur, J. The ERK1/2 mitogen-activated protein kinase pathway as a master regulator of the G1- to S-phase transition. Oncogene 2007, 26, 3227-3239.

352. Bulavin, D.V.; Saito, S.; Hollander, M.C.; Sakaguchi, K.; Anderson, C.W.; Appella, E.; Fornace, A.J. Phosphorylation of human p53 by p38 kinase coordinates N-terminal phosphorylation and apoptosis in response to UV radiation. EMBO. J. 1999, 18, 6845-6854.

353. Sancar, A.; Lindsey-Boltz, L.A.; Unsal-Kacmaz, K.; Linn, S. Molecular mechanisms of mammalian DNA repair and the DNA damage checkpoints. Annu. Rev. Biochem. 2004, 73, 39-85.

354. Zhao, R.B.; Gish, K.; Murphy, M.; Yin, Y.X.; Notterman, D.; Hoffman, W.H.; Tom, E.; Mack, D.H.; Levine, A.J. Analysis of p53-regulated gene expression patterns using oligonucleotide arrays. Gene Dev. 2000, 14, 981-993.

355. Giaccia, A.J.; Kastan, M.B. The complexity of p53 modulation: Emerging patterns from divergent signals. Gene Dev. 1998, 12, 2973-2983.

356. Marnett, L.J. Lipid peroxidation-DNA damage by malondialdehyde. Mutat. Res. 1999, 424, 83-95.

357. Yin, H.Y.; Porter, N.A. New insights regarding the autoxidation of polyunsaturated fatty acids. Antioxid. Redox Signal. 2005, 7, 170-184. 
358. Zheng, R.J.; Heck, D.E.; Mishin, V.; Black, A.T.; Shakarjian, M.P.; Kong, A.N.T.; Laskin, D.L.; Laskin, J.D. Modulation of keratinocyte expression of antioxidants by 4-hydroxynonenal, a lipid peroxidation end product. Toxicol. Appl. Pharmacol. 2014, 275, $113-121$.

359. Jorgensen, P.; Milkovic, L.; Zarkovic, N.; Waeg, G.; Rattan, S.I.S. Lipid peroxidation-derived 4-hydroxynonenal-modified proteins accumulate in human facial skin fibroblasts during ageing in vitro. Biogerontology 2014, 15, 105-110.

360. Thiele, J.; Barland, O.C.; Ghadially, R.; Elias, P.M. Permeability and antioxidant barriers in aged epidermis. In Skin Aging; Springer: Berlin/Heidelberg, Germany, 2006; pp. 65-79.

361. Shindo, Y.; Witt, E.; Packer, L. Antioxidant defense mechanisms in murine epidermis and dermis and their responses to ultraviolet light. J. Investig. Dermatol. 1993, 100, 260-265.

362. Shindo, Y.; Witt, E.; Han, D.; Tzeng, B.; Aziz, T.; Nguyen, L.; Packer, L. Recovery of antioxidants and reduction in lipid hydroperoxides in murine epidermis and dermis after acute ultraviolet radiation exposure. Photodermatol. Photoimmunol. Photomed. 1994, 10, 183-191.

363. Shindo, Y.; Witt, E.; Han, D.; Epstein, W.; Packer, L. Enzymic and non-enzymic antioxidants in epidermis and dermis of human skin. J. Investig. Dermatol. 1994, 102, 122-124. 


\title{
Oxidative Stress and the Homeodynamics of Iron Metabolism
}

\author{
Nikolaus Bresgen and Peter M. Eckl
}

\begin{abstract}
Iron and oxygen share a delicate partnership since both are indispensable for survival, but if the partnership becomes inadequate, this may rapidly terminate life. Virtually all cell components are directly or indirectly affected by cellular iron metabolism, which represents a complex, redox-based machinery that is controlled by, and essential to, metabolic requirements. Under conditions of increased oxidative stress-i.e., enhanced formation of reactive oxygen species (ROS)-however, this machinery may turn into a potential threat, the continued requirement for iron promoting adverse reactions such as the iron $/ \mathrm{H}_{2} \mathrm{O}_{2}$-based formation of hydroxyl radicals, which exacerbate the initial pro-oxidant condition. This review will discuss the multifaceted homeodynamics of cellular iron management under normal conditions as well as in the context of oxidative stress.
\end{abstract}

Reprinted from Biomolecules. Cite as: Bresgen, N.; Eckl, P.M. Oxidative Stress and the Homeodynamics of Iron Metabolism. Biomolecules 2015, 5, 808-847.

\section{Systemic and Cellular Iron Transfers}

Ferric iron or iron contained in heme is absorbed by intestinal enterocytes via heme carrier proteins (HCP1) [1], the divalent metal transporter DMT1 (SLC11A2) [2,3] or the integrin-mobilferrin pathway [4,5] (a review on intestinal iron absorption is given in [6,7]). The absorbed iron is then released from the enterocytes to the bloodstream as transferrin-bound iron (TBI) via ferroportin (see below). Under physiological conditions, the bulk of iron enters the cell bound as TBI via transferrin-receptor (TfR) mediated endocytosis followed by endosomal iron liberation. However, resorption of non-transferrin bound iron (NTBI) from the bloodstream may also occur either via DMT-1, the zinc transporter Zip14 (SLC39A14) [8,9] or specific citrate binding sites [10-12]. Notably, the serum content of labile NTBI is very low under normal conditions but may rise substantially in diseased states, such as thalassemia, where the high NTBI level-essentially caused by repeated blood transfusion - is considered to cause disease-related oxidative stress [13-18]. Similarly, serum ferritin which may serve as iron carrier too [19,20] and is also increased under certain pathological conditions, such as inflammation and cancer [21], can also be endocytosed [22-25] upon binding to distinct ferritin receptors [26-30], TIM-2 [31,32], Scara5 [33] as well as the TfR itself [34]. Finally, heme-bound iron will enter the cells via HCP1 [1] and tissue macrophages will also "ingest iron" upon phagocytosis of aged cells such as erythrocytes or via the haptoglobin/CD 163 or hemopexin/CD91 mediated uptake of hemoglobin or heme [35] and deliver the recycled iron back to the bloodstream, which is indispensable for the maintenance of systemic iron homeostasis [36].

In contrast to several ways of cellular iron uptake, only two mechanisms of cellular iron release are known. Usually, iron release from a cell occurs via ferroportin (Fpn) [37-41] a membrane bound iron exporter, which is controlled by hepatocyte derived hepcidin [42,43], the hepcidin 
activity itself being regulated by the serine protease matriptase-2 [44,45]. The ferroportin-released iron is then directly transferred to transferrin by aid of the multi-copper ferroxidases hephaestin and caeruloplasmin [39,46-48]. Fpn-based iron release from enterocytes or macrophages is essential to systemic iron homeostasis, hepcidin acting as negative regulator of Fpn counteracting systemic iron overload [49]. Hypoxic conditions lower hepcidin expression and thus promote iron absorption [50,51], the negative regulation of hepcidin exerted by hypoxia inducible factor-1 [52] playing an important physiological role in the adaptation to increased altitudes [53]. Notably, apart from its role in systemic iron homeostasis, the Fpn-based iron release mimics the effect of iron chelators, such as desferrioxamine (DFO), by counteracting iron-based oxidative stress [54]. Supportive to this, reduced (or absent) ferroportin activity (e.g., upon hepcidin overexpression or mutation of the ferroportin gene) results in cellular iron overload $[55,56]$. Notably, mutation of the Fpn gene causes the so-called "ferroportin disease"-with symptoms of tissue iron overload reminiscent to hemochromatosis - however, at a less critical clinical manifestation [55]. In particular, unlike the hepatocytic iron overload seen in hemochromatosis, ferroportin disease patients show no hepatic iron accumulation. Noteworthy, serum ferritin levels increase in patients suffering from ferroportin disease [55], the secretion of iron loaded ferritin presumably protecting from hepatic iron overload.

Hence the relase of iron-loaded ferritin could represent a non-orthodox mechanism to avoid iron overload in cells that do not express Fpn or Fpn is inhibhited by high hepcidin levels which is also accompanied by increased serum ferritin levels [56]. The exact mechanism by which ferritin is secreted remains elusive, however, it has been shown that ferritin can be released via exocytosis in an iron dependent mode [57,58] and the release by secretory lysosomes has also been proposed [59]. Moreover, ferritin transcytosis has also been suggested [60]. Evidence exists that ferritin serves iron shuttling between cells including a presumptive role as iron transporter across "barriers" such as the blood-brain-barrier (BBB) or the placental brush border (PBB) $[19,26,29,61,62]$. The findings that uptake of extracellular ferritin may serve haemoglobin synthesis in erythroid precursor cells [24] and the use of ferritin (and not transferrin) as major iron source in oligodendrocytes [63] support this assumption. Furthermore, the ferritin content of serum correlates with total body iron stores [21,64] and is increasing upon dietary iron supplementation [65] and also with age $[66,67]$. In addition, several diseased states are accompanied by pathological changes of serum ferritin levels such as anemia-based hypoferritinemia [68,69] and the hyperferritinemia frequently associated with infection, inflammation and malignancy [21,64,68,70,71], which potentially complicates serum ferritin-based assessment of the body iron status [72]. Albeit this points at a role of extracellular ferritin in cellular and systemic iron homeodynamics and evidence is increasing for a participation of serum ferritins in systemic stress responses (see Section 2.2), our understanding of the biological significance of ferritin secretion and uptake still is incomplete.

\section{Cellular Iron Compartmentalization}

Metabolic requirements focus on proper iron supply for the mitochondrial synthesis of heme and iron-sulfur (Fe-S) clusters, functional groups which are indispensable for cell function and serve as central determinants of cellular iron "handling". Conflicting with the strict demand for iron availability, free "labile", redox-active ferrous iron is prone to generate highly reactive ${ }^{\bullet} \mathrm{OH}$-radicals by reacting 
with $\mathrm{H}_{2} \mathrm{O}_{2}$ in the Fenton reaction eventually causing oxidative cell damage. Thus, intracellular iron is compartmentalized into distinct "cellular labile iron pools" which communicate via secure, protein-based iron shuttles (Figure 1).

Upon receptor-mediated endocytosis, iron will initially locate to the endo-/lysosomal compartment (ELC) from where it is forwarded to the cytosol via distinct iron transporters DMT-1, Zip14 or TRPML1 (mucolipin 1) [3,73-76]. With respect to the continuous need for iron, cytosolic ferritin will serve as dynamic iron buffer, which is essential to a steady-state of intracellular iron availability. Like ferroportin, ferritin will also counterbalance a transient iron overload by sequestering an excess of $\mathrm{Fe}^{2+}$ and thus confer antioxidant and cytoprotective functions [77,78]. However, most of the imported iron will be delivered immediately to the "users", in particular mitochondria, which may involve a bypass of cytosolic iron buffering (see below).

About $0.2 \%-5 \%$ of the total cellular iron is considered as transiently mobile, non-protein bound low molecular weight redox-active iron which together with chelatable protein-bound iron defines the dynamic, intracellular "labile" iron pool (LIP) encompassing compartment specific LIPs of the cytosol (CLIP - also including nuclear labile iron), the mitochondria (MLIP) and the endo-/lysosomal compartment (ELIP) in total containing about 6-16 $\mu \mathrm{M}$ iron, mainly as $\mathrm{Fe}^{2+}$ [79-84]. Iron is shuttled between these pools by several distinct mechanisms: (i) distinct donor-acceptor exchanges (e.g., iron uptake and release to and from ferritin); (ii) transfer of iron across membranes by iron transporters such as DMT-1, TRPML1, Zip14 (ELIP $\rightarrow$ CLIP); and (iii) iron "binding" to mitoferrin, paraferritin (see below) (CLIP $\rightarrow$ MLIP) and ferritin (CLIP $\rightarrow$ ELIP via autophagy) $[79,85,86]$. As mentioned, endosomal iron (ELIP) may also be transferred directly to mitochondria by a "kiss and run" mechanism, iron containing endosomes or iron containing vesicles docking to the outer mitochondrial membrane and passing the iron over to mitoferrin [87-91]. Notably, the latter mechanism circumvents the CLIP and allows an efficient direct transfer of iron to mitochondria which may be beneficial under physiologic conditions but could become critical under conditions of iron overload [90]. Transfers involving free ferrous iron represent a constant hazard of Fenton-reaction derived oxidative stress, which holds particularly true under conditions of iron overload and increased oxidative stress.

Lysosomal processing of iron loaded ferritin either upon autophagy or heterophagic uptake of "serum ferritin" represents such a situation where adequate coupling of the liberated ferrous iron to adequate acceptors is mandatory to avoid an excess of redox-active iron in the lysosomes. It has been shown that autophagy of apoferritin protects lysosomes from iron overload and exerts cytoprotective properties [92-94]. On the other hand, ferritin heterophagy may stimulate lysosomal stress and resulting growth adverse responses $[95,96]$. 


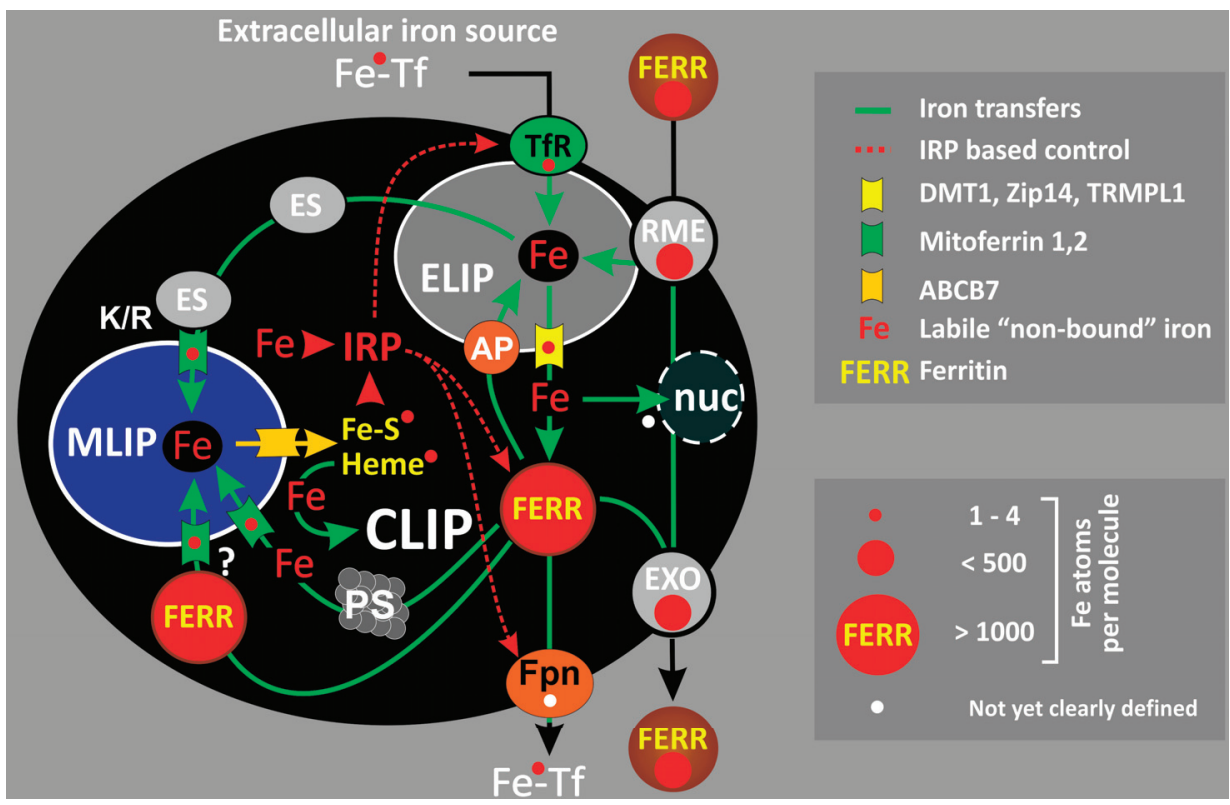

Figure 1. Cellular iron flux. Iron is transferred between communicating "labile iron pools" of the endo-/lysosomal system (ELIP), the cytosol (CLIP) and the mitochondria (MLIP). The ELIP represents the main entry site for extracellular iron such as transferrin bound iron (Fe-Tf) taken up via the TfR. Alternatively, iron containing serum ferritin may also enter the ELIP via receptor-mediated endocytosis (RME). Iron can exit from the ELIP via specific channels (DMT-1, Zip14, TRPML1) and is buffered in the cytosol (CLIP) by ferritin. Iron release from ferritin can occur via proteasomal degradation (PS) or lysosomal digestion upon autophagy (AP). Transfer of iron to the MLIP involves the iron transporter mitoferrin. The shuttling of "endosomal iron" (ES) to mitochondria by a "kiss and run" mechanism $(\mathrm{K} / \mathrm{R})$ as well as a hypothetical direct iron uptake from cytosolic ferritin has also been proposed. Note that iron can be buffered in the MLIP by mitochondrial ferritin. Ferroportin (Fpn) serves iron exit, the exact transfer mechanism not yet being resolved [39]. Fe-S clusters and heme, released from the mitochondria via $\mathrm{ABCB} 7$ transporter as well as labile, non-bound cytosolic iron serve as iron sensors for cytosolic IRPs. IRPs regulate the cellular labile iron pool via translational control of several iron-metabolism related proteins such as TfR, ferritin and ferroportin (Fpn). Alternative iron fluxes include heme-oxygenase 1 mediated iron liberation as well as ferritin endocytosis, transcytosis and exocytosis (EXO). The uptake of heme and extracellular NTBI is not shown. Red circles symbolize the relative iron binding capacities. 


\subsection{Endo-/Lysosomal Iron-ELIP}

Intestinal iron absorption as by enterocytes occurs separately for $\mathrm{Fe}^{2+}$ and $\mathrm{Fe}^{3+}$ via the divalent iron transporter DMT-1, which also serves the uptake of ferrous NTBI in other cell types and the $\beta 3$-integrin-mobilferrin pathway [5]. $\beta 3$-integrin forms a large protein complex together with mobilferrin also incorporating DMT-1, which shows ferrireductase activity, and due to its size has been named paraferritin [97,98]. Intracellular paraferritin is considered to serve the shuttling of iron to mitochondria, which may also apply to iron derived from transferrin [6]. Different from this, iron adsorption of TBI occurs via TfR mediated endocytosis, the transferrin/TfR complex locating to the endosomal compartment where the acidic milieu supports the release of transferrin bound $\mathrm{Fe}^{3+}$. The iron-free apotransferrin/TfR complex is then recycled to the cell membrane where it dissociates, both components serving further iron acquisition. Inside the acidified endo-/lysosomal compartment, the liberated ferric iron is reduced to the ferrous state by the endosomal ferrireductase Steap3 [99,100], which is highly expressed in iron-rich cells, such as hepatocytes and macrophages [101]. The ferrous iron is then released from the endosomal compartment to the cytosol either via DMT-1 or the lysosomal iron channel TRPML1, a type IV mucolipidosis-associated protein also termed mucolipin-1 [73,76]. Interestingly, impaired TRPML1 function provokes a severe disturbance of cellular iron homeostasis, marked by cytosolic $\mathrm{Fe}^{2+}$ depletion and lysosomal $\mathrm{Fe}^{2+}$ overload, accompanied by the lysosomal accumulation of the indigestible lipid-protein oxidation product lipofuscin, sensitizing lysosomes to oxidative stress, which is causal to hereditary mucolipidosis and other lysosomal storage disorders involved in neurodegenerative diseases [73,102-105].

Notably, compared to transferrin which provides two iron ions $\left(\mathrm{Fe}^{3+}\right)$ per molecule, the uptake of serum ferritin via receptor mediated endocytosis expands the lysosomal labile iron pool more substantially since serum ferritins, albeit considered iron poor, may contain about $160-500 \mathrm{Fe}^{3+}$ ions per molecule [106,107]. Moreover, TfR-based iron import is regulated by cellular iron concentrations while this is not the case for the uptake of ferritin via asialoglycoprotein receptors [108]. Besides iron import from the environment, the lysosomal iron pool may also expand upon organelle recycling by macroautophagy of iron-rich mitochondria (mitophagy) and peroxisomes (pexophagy) as well as lysosome recycling itself [109-112], which represents a particularly critical issue during damage induced reparative autophagy [113,114]. Furthermore, macroautophagy of cytosolic iron-loaded ferritin and iron liberation by lysosomal processing will also expand the ELIP [115].

With respect to the different iron input routes feeding the ELIP, iron release from the lysosome is pivotal to the maintenance of proper levels of lysosomal labile iron. Moreover, the pathogenic effect of TRPML1 failure emphasizes the necessity for a stringent ELIP control, especially in post-mitotic cells such as neurons. Several reports have shown the dependence of lysosomal stability on lysosomal iron load [92,94,116-120]. Critical to this, the $\mathrm{Fe}^{2+}$-based ${ }^{\bullet} \mathrm{OH}$ formation is fostered by pro-oxidant conditions, which stimulates lysosomal lipid peroxidation and consequently increases the susceptibility to lysosomal membrane permeability (LMP) and subsequent cell death [117,118,121-128]. Evidence is increasing that cells are able to antagonize lysosomal iron overload and the oxidative stress derived thereof by transferring antioxidants, including apoferritin, 
to the ELC by macroautophagy $[93,94,129]$. Moreover, the pro-oxidant conditions arising from LMP have a stimulatory effect on de novo ferritin synthesis [94,130]. Summarizing, the dynamics of the ELIP serve as "rheostat" of cellular iron flux, which in context with the pro-/antioxidant balance couples redox control to lysosome stability and cell integrity.

\subsection{Ferritin-CLIP}

As stated above, ferritin-based iron-buffering is crucial to cellular integrity in particular under conditions of increased oxidative stress. Albeit ferritin has a maximum storage capacity of $4500 \mathrm{Fe}^{3+}$ ions per ferritin molecule [78], biological ferritin samples may contain less iron $\left(<2000 \mathrm{Fe}^{3+} /\right.$ ferritin molecule), which holds particularly true for secreted, serum ferritins as already stated [106,107,131]. Ferritin is a multimeric protein with a molecular weight of about $450 \mathrm{kDa}$, composed of 24 heavy ( $\mathrm{H}$ chain of 21-23 kDa) and light ( $\mathrm{L}$ chain of 19-21 kDa) subunits arranged in a hollow sphere conformation [132]. Several tissues-specific isoforms have been described which vary in the H:L chain ratio and with respect to their pI are classified as basic, L-rich (e.g., liver, spleen) or acidic, H-rich (e.g., muscle, heart, brain) isoferritins [132-134].

Contrasting the low sequence homology (in mammalians about $\sim 54 \%$ ), the ferritin $\mathrm{L}$ and $\mathrm{H}$ chain show a remarkably high conformational homology [132]. Iron enters the ferritin molecule in the ferrous state via three-fold channels. The entrance is facilitated by the chaperone PCBP1 (Poly-r(C)-Binding Protein 1) [135] and involves oxidation by the ferroxidase activity of the $\mathrm{H}$-chain, a process which also consumes $\mathrm{H}_{2} \mathrm{O}_{2}[132,136]$. The oxidized iron is then shuttled to the inner cavity of the ferritin molecule where it is stored in a mineralized form, the nucleation process aided by the L-chain [137,138]. Iron exit from ferritin (recently reviewed in [139]) involves the reduction of the mineralized $\mathrm{Fe}^{3+}$, however, may also involve electron transfers, gated pores as well as direct iron release mechanisms, which are not based on reduction (reviewed in [140]). Small reducing molecules such as $\mathrm{O}_{2}{ }^{\bullet-}$, ascorbate or the ascorbate radical [132] and $\mathrm{NO}{ }^{\bullet}$ (see below), but also sulfide [141] and hydroxydopamine (6-OHDA) [142] are able to mobilize iron from intact ferritin in vitro. Ionizing radiation together with ascorbate may also trigger $\mathrm{O}_{2}{ }^{\bullet-}$-dependent iron release which expands the LIP in post mortem tumor tissue [143]. Ferritin degradation appears to play an important role in iron release from ferritin in vivo [144-147], which can be stimulated by iron chelators [148] and oxidative stress [149]. Notably, while iron chelators stimulate autophagy and lysosomal ferritin processing, iron release triggered by ferroportin precedes proteasomal digest of the "iron-depleted" ferritin $[42,145,148]$. Thus, iron liberation from ferritin may follow different context-dependent routes serving either cellular iron release (proteasome) or the refueling of a rapidly emptied CLIP (autophagy/ELC). Considering the above discussed risk of lysosomal destabilization arising from ferritin autophagy in oxidatively stressed cells, CLIP depletion under prooxidant conditions will become a potent, cytotoxic challenge when the cell is not adequately equipped with antioxidants including newly synthesized apoferritin.

Emphasizing the antioxidant properties of ferritin, overexpression of human H-chain ferritin confers solid protection against oxidative stress [136]. It has been shown that iron mediated lipid peroxidation is suppressed by recombinant $\mathrm{H}$ and $\mathrm{L}$ ferritins in vitro, which requires both the ferroxidase as well as iron mineralization activity [150]. While L-chain rich isoforms stably 
incorporate iron at increased cellular iron levels [151], the higher ferroxidase activity of $\mathrm{H}$ chain-rich ferritins allows a faster and more efficient iron sequestration, which also improves the antioxidant and cyto-protective potential under conditions of oxidative stress $[78,132,152]$. Thus, with respect to the coordinated regulation of iron oxidation and iron storage, the ferritin H:L ratio plays an important role in tissue specific iron regulation and antioxidant defenses [152], which probably affects the aging process too since the life-span in Saccharomyces cerevisiae and Caenorhabditis elegans is extended upon (over)expression of the human ferritin L-chain [153].

Cellular ferritin levels are regulated primarily at the translational level via m-RNA binding proteins IRP-1 and IRP-2 described below. Nevertheless, the genes for the ferritin $\mathrm{H}$ and L chain contain binding sites for $\mathrm{NF}-\mathrm{\kappa B} / \mathrm{Rel}$ and elements with similarity to AP-1, which mediate transcriptional control of the H-chain by the oncogenes c-Jun and c-Fos [154,155]. Moreover, antioxidant response elements (ARE) located in the promoter region of both ferritin genes allow binding of Nrf2 and junD [156-158], which also links transcriptional control of ferritin to cellular stress management. Thus, ferritin synthesis can be regulated in an iron independent mode for instance by inflammatory cytokines (e.g., TNF- $\alpha$, IFN $\gamma$, IL-1 $\beta$, IL-6-preferentially stimulating H-chain expression) and oxidative stress, which confers cytoprotection [58,159-169]. Strikingly, p53 affects ferritin synthesis too either as negative regulator on the transcriptional level supposedly weakening cellular antioxidant defense in favor of promoting apoptosis [170] or by upregulating ferritin biosynthesis which supports cell survival due to an increased iron sequestration [171]. In addition, experimental evidence exists that the iron controlled synthesis of the ferritin $\mathrm{H}$ and $\mathrm{L}$ chain occurs independently of each other and is modulated by cellular oxygen levels at the transcriptional as well as translational level [172-176]. Hence, ferritin synthesis is regulated by multiple elements of transcriptional and translational control, which supports a tight, dynamic linkage of iron buffering and the oxidant balance to cell cycle and cell death control.

The stimulation of ferritin synthesis by inflammatory cytokines can be paralleled by enhanced ferritin secretion [58]. In accordance with this, serum ferritin levels are increased in inflammatory contexts rendering ferritin an acute phase reactant [177-181]. It is assumed that this elevation of serum ferritin counteracts iron mediated oxidative stress in the inflamed tissue and, in the case of bacterial infection, probably also limits iron availability for bacterial growth [159,177,178,180,182]. Since proinflammatory cytokines preferentially promote ferritin H-chain expression [169], acute phase associated serum ferritins likely represent H-chain rich isoforms [180] which differ from the L-type ferritin found in serum under normal conditions [21]. Therefore, the inflammation related switch towards $\mathrm{H}$ chain-rich serum ferritins which reveal an improved antioxidant activity corresponds well with the enhanced synthesis of $\mathrm{H}$ chain-rich, cyto-protective ferritin in oxidatively stressed cells $[136,173]$. Hence, similar to ferritin mediated iron buffering in the CLIP, ferritin secretion may expand systemic iron buffering capacity in particular under pro-oxidant conditions.

Finally, it should not be neglected that heme-containing proteins, despite the minimal iron binding capacity of protoporphyrin, also affect cellular iron homeodynamics. While heme-biosynthesis lowers the MLIP (discussed in the next section) [183], heme-degradation by heme-oxygenase I (HO-I) [184] potentially adds iron to the CLIP which may occur in the cytosol but alternatively could also locate to the ELIP in macrophages following erythrocyte 
phagocytosis $[185,186]$. Free heme sensitizes cells for cell death especially under pro-inflammatory, pro-oxidant conditions in an iron dependent mode [187-189]. Thus, heme degradation by HO-I will confer cyto-protection provided the released iron is efficiently sequestered by ferritin [190]. In line with this, HO-I and ferritin are concomitantly upregulated in cells exposed to oxidative stress [191,192], and cytosolic iron overload caused by an excessive, HO-I-based heme-degradation such as seen in malaria may also be antagonized by the up-regulation of H-ferritin [193].

\subsection{The Mitochondrial Iron Pool-MLIP}

Complementary to ELC and ferritin-based iron handling, proper mitochondrial iron homeostasis is indispensable for cellular iron management in particular by controlling the synthesis of heme and Fe-S clusters [194-197], functional groups which are essential to the functionality of numerous proteins including those participating in energy production via the respiratory chain. Intimately connected with this, the MLIP also affects the synthesis of Fe-S cluster containing iron regulatory protein-1 (IRP-1) and by this directly interferes with IRP-based translational control of proteins involved in iron management, in particular ferritin, the transferrin receptor, ferroportin and DMT-1. Thus, the iron flux between the CLIP and the MLIP has to be tightly coordinated and is also regulated by a feedback mechanism based on Fe-S cluster synthesis and IRP-1 activity. The MLIP is fueled from the CLIP as well as directly from the ELIP as explained above. Albeit the knowledge on iron shuttling across the outer mitochondrial membrane is still incomplete, the endosomal supply via a "kiss and run" mechanism could involve a distinct endosome-mitochondria interaction and the existence of specific mitochondrial ferritin binding sites has been proposed too [85,198]. In contrast, it has been demonstrated that the iron transfer across the inner mitochondrial membrane is mediated by the mitochondrial iron carrier mitoferrin 1,2 (Mfr1,2) [87,199,200], which may involve the regulation via Mfr protein stability as suggested by the interaction of Mfr1 with the mitochondrial inner membrane ATP-binding cassette transporter Abcb19 in erythroblasts [201]. Notably, Mfr1 and 2 are not regulated by IRPs but other cis-acting elements [202]. Whatever mechanism is responsible for mitochondrial iron uptake, it has to be strictly controlled since any disturbance of the MLIP will critically affect cellular iron management. This is exemplified by pathological conditions caused by mitochondrial iron mismanagement including Friedreich's Ataxia (caused by frataxin deficiency - see below), erythropoietic protoporphyria (a disorder marked by ferrochelatase deficiency and impaired heme synthesis) or sideroblastic anemia (where disrupted heme synthesis alters mitochondrial ferritin levels, the enriched mitochondria resulting in ringed sideroblasts) reviewed in [203-206].

Of special relevance, an expansion of the MLIP will also occur when Fe-S clusters (e.g., contained in mitochondrial dehydratases such as aconitase) are oxidized by superoxide $\left(\mathrm{O}_{2}{ }^{\bullet-}\right)$ formed upon electron leakage from the respiratory chain (reviewed in [207]). Moreover, albeit mitochondrial superoxide dismutase (MnSOD) will detoxify $\mathrm{O}_{2}{ }^{\bullet}$, the dismutase reaction will generate $\mathrm{H}_{2} \mathrm{O}_{2}$, which readily reacts with "labile" ferrous iron leading to the generation of $\bullet \mathrm{OH}$ radicals and subsequent lipid peroxidation in the mitochondrial compartment. Notably, cytochrome c oxidase which catalyzes the electron transfer in complex IV of the respiratory chain is inhibited by 
the lipid peroxidation product 4-hydroxy-nonenal (HNE) [208]. Thus an expanded MLIP at inadequate antioxidant defenses will promote mitochondrial damage with severe consequences. Indeed, Halliwell (1992) has pointed at this adverse effect of SOD-activity, which may occur under certain pathologic conditions [209]. Therefore, the MLIP has to be maintained at an optimum balance which sufficiently serves metabolic needs but does not "fuel" Fenton reaction-based oxidative damage. Moreover, degradation of iron overloaded, damaged mitochondria in autophagolysosomes will amplify the effect of mitochondrial oxidative stress by stimulating lysosomal iron overload as illustrated above [210,211].

It has to be noted that no distinct iron exporter has been identified in mitochondria, the release of newly synthesized heme and Fe-S clusters to the cytosol representing the only way that the MLIP can be lowered. Fe-S clusters are transferred by mitochondrial ATP-binding cassette proteins Atm1, ABC protein 3 and ABCB7 (the human ortholog of yeast Atm1), which has been shown to counteract mitochondrial iron overload [212-215]. Correspondingly, deficiency of such proteins will increase the MLIP, which has been shown for yeast Atm1 raising the mitochondrial iron content by about 30 -fold [216] and also holds true for ABCB7 deficiency in humans causing the rare hereditary disease X-linked sideroblastic anemia and ataxia (XLSA/A) [214,217]. The way that heme is transported to the cytosol remains to be clarified; however, the involvement of a hemecarrier protein, such as heme binding protein-1 (p22 HBP), has been suggested [87,218]. The lack of distinct iron exporters emphasizes the mitochondrial compartment as "bottleneck in iron metabolism" the incoming iron being either directly consumed for Fe-S cluster- and heme synthesis or stored in mitochondria-resident proteins, such as mitochondrial ferritin (see below).

\subsection{Mitochondrial Iron Usage_Frataxin and Mitochondrial Ferritin}

Frataxin (Ftx; Yfhlp in yeast; CyaY in bacteria; reviewed in $[219,220]$ ) represents an iron binding protein of high relevance to mitochondrial function and integrity. Ftx can form multimeric complexes of different size, which can bind between 50-3000 iron ions (depending on the species and grade of multimerization) in its ferric state, although the iron binding capacity of Ftx may be much lower in vivo [221,222]. Opposite to ferritin, which is synthesized as iron-free apoferritin, assembly of Ftx multimers depends on iron [221].

Ftx has gained substantial interest over the last decade since mutations of the Ftx gene are causal to the autosomal recessive disease Friedreich's Ataxia (FRDA) [205,206,223,224], most patients showing strongly reduced levels of Ftx mRNA [223,225] and protein [226]. The disease is marked by severe neurological manifestation, as well as pathological changes of the skeleton (scoliosis), pancreas (diabetes mellitus) and heart. In fact, cardiomyopathy and cachexia represent the most frequent cause of death in FRDA patients; for a detailed review on FRDA see [205] and recent advances in Ftx research are compiled in [219]. From the biochemical point of view, FRDA is marked by iron accumulation and lipofuscin deposits [227]. In particular, FRDA is accompanied by a reduced content of mitochondrial Fe-S cluster containing proteins and a loss of aconitase activity [228], which points at the primary function of Ftx, acting as "iron chaperone" in providing iron for the scaffold protein ISCU (iron-sulfur cluster forming unit) which is essential to mitochondrial Fe-S cluster biosynthesis [229-231]. Ftx also serves the transfer of iron to 
mitochondrial membrane associated ferrochelatase [232], a Fe-S cluster containing protein which catalyzes the final step of heme biosynthesis, the transfer of iron to protoporphyrin IX [233,234].

Albeit Ftx primarily serves ISCU formation, further functions include mitochondrial iron trafficking as well as mitochondrial redox and ROS control [87,235]. Ftx-deletion in fibroblasts yields a characteristic cellular FRDA phenotype, including mitochondrial iron deposits, reduced Fe-S enzyme activity and degenerating mitochondria [229]. Also, neurons of Prp-CreER ${ }^{\mathrm{T}}$ mice, a mouse model for FRDA, show aberrant autophagy, large vacuoles and lipofuscin accumulation [236]. Notably, malfunctioning mitochondria are recycled by mitophagy, which promotes lipofuscinogenesis and renders lysosomes unstable when occurring in excess [237,238]. Hence, Ftx-deficiency may also hamper ELC function and the autophagic process. Pointing at secondary effects of Ftx deficiency in FRDA, markers for lipid peroxidation (malondialdehyde) and oxidative damage (8-hydroxy-2'-deoxyguanosine) are increased in urine and blood of FRDA patients [239-241]. This indicates the onset of iron derived, free radical-based oxidant events upon loss of Ftx activity which may be detrimental to mitochondrial function and will also affect the whole cell. In fact, Ftx deficiency can promote ROS generation in mitochondria (i.e., formation of $\bullet \mathrm{OH}$ by the Fenton reaction), which is accompanied by oxidative mitochondrial damage and the upregulation of ferritin gene expression via nitric oxide (NO) signaling [242,243]. Furthermore, Ftx deficiency also sensitizes cells to oxidative stress $[239,244]$, which may involve both mitochondria and the ELC as discussed above. Taken together, Ftx represents an important regulator of mitochondrial and cellular iron homeodynamics in particular under conditions of iron overload and oxidative stress. It is tempting to consider Ftx as mitochondrial iron buffer, which similar to cytosolic ferritin provides "antioxidant" properties. However, different from the yeast Ftx knock-out mutant $\Delta Y$ fh1 [245] and Ftx-deleted mammalian cells, mitochondrial iron deposits have neither been found in FRDA patients [246] nor in Ftx-deficient Prp-CreER ${ }^{\mathrm{T}}$ mice [247]. Also, Ftx oligomerization is not critical to Ftx function in vivo [229]. Hence, Ftx may not serve MLIP iron buffering in vivo [246,248], but support cell integrity and iron homeodynamics by its participation in ISCU/Fe-S assembly for IRP-1 synthesis.

Another mitochondrial iron binding protein - mitochondrial ferritin (mtFER) — was discovered about a decade ago [249] (reviewed in [250]). mtFer, encoded by an intronless gene shows homology to H-ferritin and also bears a ferroxidase center [251]. Albeit mtFER ferroxidase activity and iron uptake kinetics show some differences to cytosolic ferritins, mtFER is also arranged as 24-subunit homopolymer, which efficiently oxidizes and sequesters ferrous iron [252,253]. In contrast to cytosolic ferritin, however, translation of mtFER is not under the control of iron since the mtFER-mRNA lacks iron regulatory elements (IRE), the 5' UTR being mutated to a leader sequence which mediates mitochondrial targeting [249]. Also different from cytosolic ferritin, mtFER-mRNA has been found at high abundance only in testis and spermatozoa, lower amounts in the brain, kidneys, pancreas (islets of Langerhans) and thymus but is absent from tissues with high iron storage function such as liver and spleen [249,251,254]. Of pathological relevance, mtFER is increased in erythroblast mitochondria of patients suffering from sideroblastic anemia [203] an erythrocyte phenotype (ring sideroblasts), which is also found in XLSA/A patients, caused by ABCB7 deficiency-based mitochondrial iron accumulation (see above). Although the exact role of 
mtFER in iron metabolism remains to be defined in detail it has been shown that overexpression of mtFER in tumor cells leads to iron relocation from the cytosol to the mitochondria provoking mitochondrial iron accumulation and cytosolic iron depletion which abrogates ferritin synthesis and stimulates TfR production [254-256]. Overexpression of $\mathrm{mtFer}$ in tumor cells also modulates cellular ROS levels and-like Ftx deficiency-increases the sensitivity to oxidative stress leading to the onset of apoptosis. Notably, this "toxic" effect of mtFER overexpression is considered to result from increased lysosomal degradation of iron overloaded mitochondria which leads to a shift of redox-active iron in the lysosomes, oxidative stress and the enhanced consumption of antioxidants [257,258]. Furthermore, mtFER as well as cytosolic ferritin containing deposits are found in mitochondria of Ftx-deficient cardiomyocytes of FRDA patients, which points at the role of iron/ferritin derived mitochondrial damage in cardiomyocyte cell death [259]. On the contrary, enhanced expression of mtFER may also rescue Yfh1 deficient yeast cells as well as Ftx deficient Hela cells from mitochondrial dysfunction and confer protection from iron mediated oxidative injury [260-262].

Based on this, it is obvious that mitochondrial iron binding proteins by representing "guardians" of the MLIP provide an essential control of general, cellular integrity. It cannot be ruled out that endocytosed ferritin is transferred to the ELC and ferritin containing endosomes may also directly attach to mitochondria according to a "kiss and run" mechanism providing iron to the mitochondria as suggested by Ulvik [85]. If so, the interaction of internalized ferritin with mitochondria would likely result in the same outcome as seen upon mtFER overexpression: enhanced oxidative stress and free radical-based organelle damage, which eventually triggers apoptotic cell death.

\section{Iron Regulatory Proteins (IRP-1, IRP-2)}

\subsection{IRP-1: Redox-Based Control of Cellular Iron Homeodynamics}

Several proteins which show redox-based properties contain $\mathrm{Fe}-\mathrm{S}$ clusters of the types [4Fe-4S], [3Fe-4S] and [2Fe-2S]. For example, these include ferrochelatase, ferredoxin (providing electron transfer for cytochrome P-450 activity), aldehyde oxidase 1 (AOX1), xanthine dehydrogenase/xanthine oxidase, glutaredoxin 2 (a GSH-dependent oxidoreductase), nuclear proteins involved in DNA and RNA metabolism and DNA repair, and proteins of the mitochondrial respiratory chain participating in the assembly of the electrochemical gradient [263] (a recent review on the sensory and regulatory functions of Fe-S proteins is given in [264]). Of special relevance, Fe-S cluster bearing iron regulatory protein 1 (IRP-1) exerts a dual function. In the presence of "iron replete" [4Fe-4S] clusters, IRP-1 shows cytosolic aconitase activity (c-aconitase; ACO1) catalyzing the citrate to isocitrate interconversion in the cytosol, which regulates cellular NADPH levels [265,266]. However, low cytosolic iron levels as well as pro-oxidant regimens promote cluster disassembly, which abolishes c-aconitase activity but uncovers IRP-1 mRNA binding properties [265]. IRP-1 binds with high affinity to IREs marked by a stem-loop located at the 5' and 3' UTR of mRNAs encoding iron-regulatory proteins, in particular ferritin and Fpn (5' UTR located IRE) as well as TfR, DMT-1 and eALAS (erythroid 5-aminolevulinate synthase producing the heme precursor 5-aminolaevulinic acid) with an IRE at the 3' UTR [267,268]. 
Hence, the Fe-S cluster dependent control of IRP-1 activity serves as redox-active iron sensor which links translational control to cellular iron homeodynamics. Importantly, the Fe-S cluster-based IRP-1/IRE interaction either represses or induces translation of the target mRNAs, thus allowing a precise, efficient control of intracellular iron fluxes: CLIP depletion will favor cluster disassembly and promote IRP-1 RNA binding which inhibits ferritin (and ferroportin) synthesis but triggers TfR (and DMT-1) synthesis resulting in enhanced iron uptake and limited iron sequestration [269]. At increased iron levels, IRP-1 RNA binding activity declines, the IRE release allowing enhanced iron buffering and limited iron influx.

Hence, the continuous control of cellular iron fluxes by the specific IRP-1 activity participates in ELIP, CLIP, and indirectly also MLIP iron balance. Notably, the fact that Fe-S clusters are synthesized in the mitochondria emphasizes the significance of the MLIP to cellular iron homeodynamics. Indeed, Fe-S clusters may report iron loading of the MLIP since a hindrance of Fe-S cluster synthesis due to ISCU inactivation excessively shifts iron transfer to mitochondria which depletes the CLIP and increases IRP-1 RNA binding activity resulting in a disturbance of cellular iron homeodynamics [270].

\subsection{Fe-S Cluster Oxidation}

IRP-1 activity is modulated by ROS, which modify Fe-S cluster conformation, including cluster destabilization, and can also lead to IRP-1 degradation, connecting iron management and metabolic activity to cellular ROS production [271-275]. Oxidation of [4Fe-4S] clusters by $\mathrm{O}_{2}{ }^{\bullet-}$ and $\mathrm{H}_{2} \mathrm{O}_{2}$ yields the [3Fe-4S]-IRP-1 conformation, which lacks aconitase activity (Equations (1) and (3)), the reuptake of $\mathrm{Fe}^{2+}$ restoring the [4Fe-4S]-IRP conformation (Equation (4)) and aconitase activity, which is supported by sulfhydryls such as glutathione (GSH) (Equation (5)) [273,276-279].

$$
\begin{aligned}
& {[4 \mathrm{Fe}-4 \mathrm{~S}]^{2+}+\mathbf{O}_{2}{ }^{\bullet-}+2 \mathrm{H}^{+} \rightarrow[3 \mathrm{Fe}-4 \mathrm{~S}]^{+}+\mathbf{F e}^{\mathbf{2 +}}+\mathbf{H}_{2} \mathbf{O}_{2}} \\
& {[4 \mathrm{Fe}-4 \mathrm{~S}]^{2+}+\mathbf{H}_{2} \mathbf{O}_{2} \rightarrow[4 \mathrm{Fe}-4 \mathrm{~S}]^{3+}+\mathrm{OH}^{-}+\bullet \mathbf{O H}} \\
& {[4 \mathrm{Fe}-4 \mathrm{~S}]^{3+} \rightarrow[3 \mathrm{Fe}-4 \mathrm{~S}]^{+}+\mathbf{F e}^{2+}} \\
& {[3 \mathrm{Fe}-4 \mathrm{~S}]^{+}+\mathrm{e}^{-} \rightarrow[3 \mathrm{Fe}-4 \mathrm{~S}]^{\circ}+\mathrm{Fe}^{2+} \rightarrow[4 \mathrm{Fe}-4 \mathrm{~S}]^{2+}} \\
& {[3 \mathrm{Fe}-4 \mathrm{~S}]^{+}+\mathrm{Fe}^{2+}+\mathrm{GSH} \rightarrow[4 \mathrm{Fe}-4 \mathrm{~S}]^{2+}+1 / 2 \mathrm{GSSG}+\mathrm{H}^{+}} \\
& {[4 \mathrm{Fe}-4 \mathrm{~S}]^{2+}+\mathbf{H}_{2} \mathrm{O}_{2} \rightarrow[4 \mathbf{F e}-4 \mathrm{~S} / \mathrm{O}]^{2+}+\mathrm{H}_{2} \mathrm{O}} \\
& {[4 \mathrm{Fe}-4 \mathrm{~S} / \mathrm{O}]^{2+}+\mathrm{H}^{+} \rightarrow[3 \mathrm{Fe}-4 \mathrm{~S}]^{+}+\mathrm{Fe}^{3+}+\mathrm{OH}^{-}} \\
& \mathrm{Fe}^{2+}+\mathbf{H}_{2} \mathrm{O}_{2} \rightarrow \mathrm{Fe}^{3+}+\mathrm{OH}^{-}+\bullet \mathbf{O H}
\end{aligned}
$$

Notably, albeit cluster oxidation by $\mathrm{H}_{2} \mathrm{O}_{2}$ could theoretically generate ${ }^{\bullet} \mathrm{OH}$ radicals (Equation (2)), it is more likely that the oxidation of [4Fe-4S] by $\mathrm{H}_{2} \mathrm{O}_{2}$ leads to ferryl-radical [4Fe- $\left.4 \mathrm{~S} / \mathrm{O}\right]^{2+}$ clusters (Equation (6a)) from which [3Fe-4S] $]^{+}$clusters are derived (Equation (6b)) [277]. Nevertheless, $\mathrm{Fe}^{2+}$ and $\mathrm{H}_{2} \mathrm{O}_{2}$ represent harmful byproducts of $\mathrm{Fe}-\mathrm{S}$ cluster oxidation (Equations (1) and (3)) which may generate ${ }^{\bullet} \mathrm{OH}$ radicals in the Fenton reaction (Equation (7)). Indeed, Fe-S oxidation-based $\bullet \mathrm{OH}$ radical formation represents a potent killing mechanism in bacteria, which is supposed to underlie the $\mathrm{H}_{2} \mathrm{O}_{2}$-based antimicrobial defenses used by higher organisms [257,277]. 
Moreover, inactivation of mitochondrial aconitase by $\mathrm{O}_{2}{ }^{\bullet-}$ mediated $\mathrm{Fe}-\mathrm{S}$ cluster oxidation causes necrotic cell death of embryonic rat cortical cells [276] which may be connected with the interruption of energy metabolism (mitochondrial aconitase - $\mathrm{ACO} 2$ - is a key enzyme of the TCA cycle) and Fenton-reaction-based ${ }^{\bullet} \mathrm{OH}$ formation as stated above. With respect to the abundance of $\mathrm{Fe}-\mathrm{S}$ cluster containing proteins of the respiratory chain, enhanced ${ }^{\bullet} \mathrm{OH}$ formation in fact could be of considerable relevance since it may directly exert protein damage and also stimulate lipid peroxidation (LPO), aldehydic LPO metabolites such as malondialdehyde or HNE leading to mitochondrial malfunction, instability and cellular collapse [208,280-286]. For instance, HNE may form adducts with cysteine residues of the cubane Fe-S cluster and the catalytic center of ACO2 which substantially lowers the enzymatic activity [287] and could also interfere with the RNA binding properties.

Moderate cytosolic Fe-S cluster oxidation changing the cluster conformation to the [3Fe-4S] state will abolish IRP-1 c-aconitase activity but this not necessarily is sufficient to induce IRP-1 mRNA binding activity [288]. Enhanced pro-oxidant conditions, however, will stimulate cluster decomposition, which promotes IRP-1 RNA binding, an effect which is of particular relevance to iron metabolism in cultured cells exposed to increased, non-physiologic oxygen concentrations [265]. Interestingly, IRP-1 RNA binding is stimulated by extracellular $\mathrm{H}_{2} \mathrm{O}_{2}$ while the endogenous, cytosolic $\mathrm{H}_{2} \mathrm{O}_{2}$ production shows no comparable effect [289,290]. This suggests the involvement of additional, Fe-S cluster independent, mechanisms controlling IRP-1 activity. Indeed, IRP-1 contains a phosphorylation site for protein kinase C [291,292], which allows an integration of IRP-1 activity in cellular stress responses. Moreover, phosphorylation of IRP-1 sensitizes for iron-dependent protein degradation and by this controls IRP-1 abundance per se [293]. Similarly, enhanced oxidative stress and massive iron overload also facilitate IRP-1 degradation, which will enhance iron buffering by altered ferritin synthesis at a limited TfR-based iron uptake [294-296]. Albeit this suggests that ROS triggered IRP-1 degradation acts as "emergency break", limiting the labile iron pool under pro-oxidant conditions [267,275,297], IRP-1 degradation not necessarily changes intracellular iron levels [298]. Thus, under conditions of increased oxidative stress, the labile iron pool may be controlled by more than a single IRP-1-based mechanism.

Hence, IRP-1 apparently regulates iron homeodynamics at rather extreme conditions. While IRP-1 degradation restricts the labile iron pool at elevated ROS concentrations, IRP-1 RNA-binding counteracts iron-depletion and stabilizes the LIP under moderately increased oxidative stress, both mechanisms also controllable by additional cellular stress signals via IRP-1 phosphorylation. However, it should not be ignored that stimulation of IRP-1 RNA-binding by moderate ROS attack may also occur under iron-replete conditions which may promote cellular iron overload. Concerning the ELC/ELIP, this inappropriate response is prone to generate lysosomal stress, which alters the susceptibility for lysosome mediated cell death under pro-oxidant conditions [299,300]. Complicating the issue, inactivation of IRP-1 c-aconitase activity by Fe-S cluster oxidation will also weaken antioxidant defenses since IRP-1 c-aconitase activity contributes to both glutathione (GSH) synthesis and NADPH generation which is necessary for the reduction of oxidized glutathione (GSSG) [301-304]. Thus, a cytotoxic condition can readily emerge from mild oxidative 
stress if oxidation of IRP-1 Fe-S clusters leads to an inadequate disturbance of iron homeodynamics and antioxidant defenses.

\subsection{IRP-2}

Similar to IRP-1, iron regulatory protein 2 (IRP-2) also shows RNA-binding properties, however, lacks Fe-S clusters as well as aconitase activity and is regulated via proteasome-mediated degradation [265,305-309]. Among several target mRNAs, IRP-2 shows a preference to bind to ferritin $\mathrm{H}$ and $\mathrm{L}$ chain mRNA which is stabilized by proteasome inhibitors abrogating ferritin synthesis while iron-rich conditions promote proteasomal decomposition of IRP-2 [310-312]. Hypoxia stabilizes IRP-2 RNA binding too, which is also antagonized by iron [307,313,314]. Importantly, at physiologic oxygen concentrations (3\%) IRP control of cellular iron levels is mainly exerted by IRP-2, IRP-1 showing little mRNA binding activity and marginal iron responsiveness [314,315]. On the contrary, at increased tissue oxygen tensions, IRP-2 abundance declines and IRP-1 adopts the role as main iron regulatory protein [314] as discussed above. IRP-2 RNA binding is upregulated by phosphorylation, which, different from IRP-1, is iron dependent and does not increase IRP degradation [293,316]. However, similar to IRP-1, phosphorylation links IRP-2 activity to intra- and extracellular signaling which may serve cell proliferation and differentiation [316]. For instance, it has been shown that IRP-2 couples Jak/Stat5 signaling to TfR expression in erythropoiesis [317]. Also, IRP-2 knock out mice show disturbances of dopamine regulation as well as iron overload and increased ferritin expression in distinct brain areas and it is suggested that iron mismanagement upon loss of IRP-2 control accelerates the aging of dopaminergic neurons [318,319]. As stated above, IRP-2 is considered to be the main regulator of iron metabolism under normal conditions and may compensate for IRP-1 deficiency [320]. However, the responsiveness of IRP-1 and 2 to stress related stimuli, which may involve changes of the phosphorylation state, points at distinct roles of both IRPs in controlling the cellular labile iron pool under stress conditions.

\subsection{NO Signaling and IRP Regulation}

Nitric oxide synthase (NOS) generated nitric oxide $\left(\mathrm{NO}^{\bullet}\right)$ and peroxynitrite $\left(\mathrm{ONOO}^{-}\right)$ derived thereof by reaction with superoxide $\left(\mathrm{NO}^{\bullet}\right.$ and $\mathrm{ONOO}^{-}$representing reactive nitrogen species-RNS) are able to react with $\mathrm{Fe}$ contained in proteins as heme or Fe-S cluster bound iron [321-327]. Of note, $\mathrm{NO}^{\bullet}$ which has a high affinity to iron [328] can mobilize iron from ferritin in a GSH dependent manner [329,330]. In addition, $\mathrm{NO}{ }^{\bullet}$ as well as the nitrosonium cation $\left(\mathrm{NO}^{+}\right)$ can S-nitrosylate thiol groups of proteins including ferritin and IRPs which confers important regulatory functions in iron metabolism including changes of ferritin and TfR synthesis [328,331-335]. NO may also react with ferrous "labile" iron and thiol containing GSH which generates dinitrosyl-iron complexes [336] leading to S-nitrosothiol formation [337,338]. Of special relevance, nitrosylation of $\mathrm{GSH}$ by $\mathrm{NO}^{+}$produces S-nitrosoglutathione (GSNO) [339-343] a potent antioxidant which exerts cytoprotective properties [341,344-350] albeit a hepatocytotoxic effect of GSNO has also been reported [351]. 
Several investigations have addressed the interference of NO-signaling with IRP-1 and 2 activity via $\mathrm{NO}^{\bullet} / \mathrm{ONOO}^{-}-\mathrm{Fe}-\mathrm{S}$ cluster interaction and IRP S-nitrosylation-which may reversibly $\left(\mathrm{NO}^{+}\right)$ or irreversibly $\left(\mathrm{ONOO}^{-}\right)$inhibit IRP-1 aconitase activity, stabilize IRP-1 RNA binding (NO^) or irreversibly modify IRP-1 thus abrogating RNA binding $\left(\mathrm{ONOO}^{-}\right)$and also affect IRP-2 stability [311,325,332,333,352-361]. In particular, the enhanced degradation of IRP-2 mediated by NOS derived $\mathrm{NO}^{\bullet}$ which triggers ferritin synthesis in cells exposed to proinflammatory stimuli points at the importance of NO-signaling in pathophysiological contexts [311,352,357]. However, it has been reported that $\mathrm{NO}^{\bullet}$ can also stabilize IRP-2 probably by LIP interference $[359,361,362]$ while IRP-2 degradation is promoted by $\mathrm{NO}^{+}$mediated nitrosylation [333]. Notably, $\mathrm{NO}^{+}$is able to stimulate ferritin synthesis also in an IRP-2 independent mode [311]. Hence, cellular iron homeodynamics is regulated by nitrogen species based on complex, feedback-regulated mechanisms, the expression of NOS and thus NO levels itself being directly affected by cellular iron levels [363]. Moreover, NO-signaling also allows an intercellular control of iron pools and by this may contribute to cell-cell interaction mediated changes of iron homeodynamics. Albeit is has been shown that macrophages stimulate iron release from target cells [364] it is questionable whether this is mediated by NO, however, evidence exists that NO can limit transferrin/TfR-based iron uptake (discussed in [365]).

\subsection{Additional Regulatory Roles of IRPs}

Recently, additional functions of IRPs have been identified which are more indirectly related to iron metabolism (reviewed in [366]). In particular, it has been shown that IRP-1 also acts as negative translational regulator of hypoxia-inducible factor $2 \alpha$ (HIF2 $\alpha$ ) [367]. This interference affects several downstream targets of HIF $2 \alpha$ such as erythropoietin (EPO) expression and by this erythropoiesis and hepcidin expression [368-372] as well as transcriptional activation of Fpn and DMT-1 [373-375] in addition to the IRP-1/mRNA-based regulation. Since HIF2 $\alpha$, like HIF1 $\alpha$, also affects tumor progression and tumor stem cell function [376,377], IRP-1 could also play a role in tumorigenesis. Moreover, tumor cell proliferation is enhanced upon overexpression of IRP-2 [378], the oncogene c-myc upregulating IRP-2 but repressing H-ferritin [379]. Thus, the role of IRPs may change in the course of neoplastic transformation serving the tumor growth associated reprogramming of iron metabolism [380] and cellular iron homeodynamics.

\section{Iron Homeodynamics under Stress Conditions-A Distinct Role for Ferritin?}

Taken together, iron compartmentalization together with iron-, redox- and stress dependent gene expression (on the transcriptional and translational level) constitutes the framework of cellular iron homeodynamics. Transcriptional control of iron metabolism related genes defines a distinct $m R N A$ signature [381] which is translated into an iron management-related proteome serving the dynamic fine-adjustment of intracellular iron balance. Stress conditions will modulate the cell type and condition (e.g., iron requirement, state of differentiation, proliferation) specific mRNA signature and even more specifically its translation, which is under IRP control. IRP activity by itself is directly (IRP-1) or indirectly (IRP-2) regulated by the LIP/CLIP, which involves regulatory 
feedback loops (e.g., MLIP dependent Fe-S cluster synthesis acting directly on IRP-1) as well as additional stress-related signals (e.g., NO-signaling). Hence, IRPs serve as central guardians of cellular iron homeodynamics and stress tolerance as illustrated in Figure 2.

Under normal conditions iron homeodynamics is predominantly determined by mitochondrial iron consumption (MLIP), IRP-2 serving the dynamic housekeeping adjustment of the LIP. Tightly coordinated with this, IRP-1 c-aconitase activity links the LIP to GSH and NADPH abundance and via this to cellular antioxidant capacity. Oxidative stress markedly interferes with this regulatory network depending on the source (ROS, RNS), severity and persistence of the pro-oxidant stressor. Moderate stress conditions promote Fe-S cluster disassembly and stimulate IRP-1 RNA binding which fosters iron overload. When antioxidant defenses are inadequate, this could aggravate the pro-oxidant condition especially with respect to the lysosomal and mitochondrial compartment. On the other hand, severe or chronic states of increased oxidative stress will lead to enhanced IRP degradation (IRP-1 and 2) which promotes iron (LIP) depletion due to elevated ferritin synthesis and reduced TfR-based iron import. Complicated by the concomitant decline of IRP-1 c-aconitase activity, which interrupts refueling of the antioxidant pools, this pro-oxidant condition readily will become incompatible with cell survival. Therefore, oxidative stress, especially when persistent, demands specific adaptations of iron management that support proper LIP control and continued iron supply for metabolic needs.

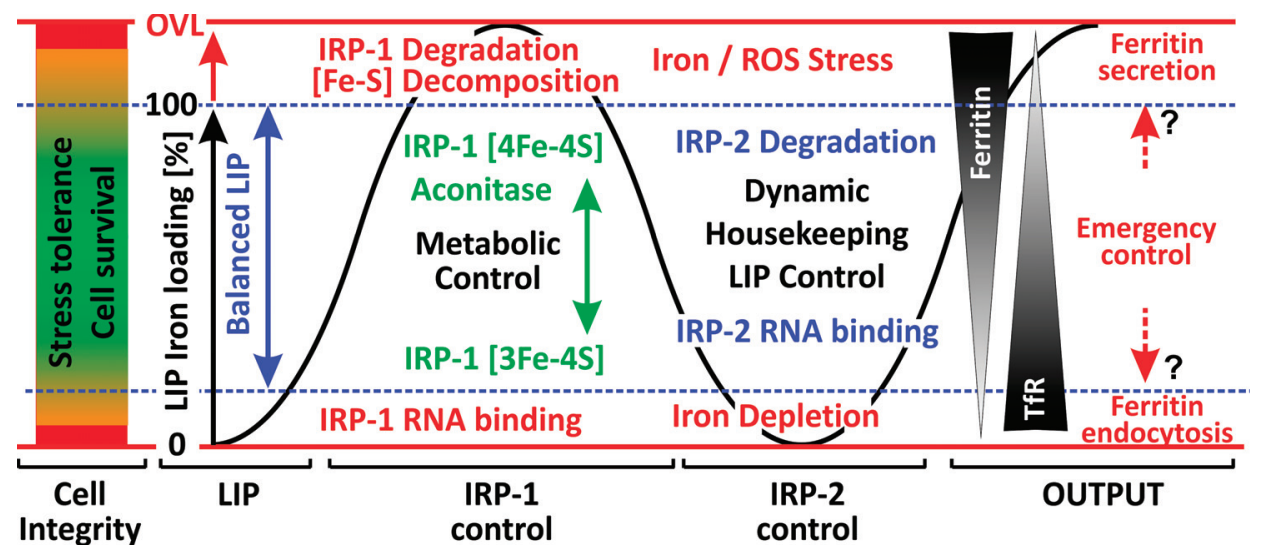

Figure 2. Iron homeodynamics and stress conditions. Cell integrity and stress tolerance demands a balanced LIP between 0 (iron depletion) and 100\% (maximum loading). Under normal conditions (blue range) the LIP is controlled by IRP-2 abundance, IRP-1 preferentially exerting c-aconitase activity depending on Fe-S cluster conformation. Moderately enhanced oxidative stress will promote Fe-S cluster decomposition (see p. 820 ), while severe pro-oxidant regimens as well as iron overload (OVL) lead to IRP-1 degradation. Iron import via TfR and ferritin-based iron buffering control the LIP in opposite directions depending on the actual iron content and oxidant conditions (Output). It is hypothesized that ferritin exo- and endocytosis serve the "emergency control" of the LIP under conditions of either severe (or chronic) iron overload and oxidative stress or massive iron depletion, respectively. 
Moreover, severe ROS attack on iron-loaded ferritin could become a further considerable threat when the "safely stored iron" is liberated rendering the LIP (CLIP) uncontrollable due to an impaired iron buffering capacity. Hypothetically, ferritin/iron-rich cells such as hepatocytes and macrophages as well could face the risk of irreversible ferritin-iron/ROS derived damage by releasing iron loaded ferritin under oxidative stress which would rapidly lower the tenuous iron burden. The increase of serum ferritin associated with hepatic iron overload in ferroportin disease [55] provides support to this assumption. Similarly, in macrophages ferritin release could compensate the heme degradation-based iron charging of the CLIP/ferritin - a notion that fits with the finding that serum ferritin is mainly derived from macrophages [59] — which may increase under oxidative stress [382]. Furthermore, primary hepatocytes release ferritin in vitro in particular at initial culture stages [383,384], the secreted ferritin exerting an iron-dependent cytotoxic effect [95]. It cannot be excluded that this also reflects an attempt of the freshly isolated cells to mitigate the cell isolation derived pro-oxidant condition by emptying their intracellular iron buffer. On the contrary, it should not be neglected that serum ferritin may deliver at least 100 times more iron per molecule than transferrin. Thus, endocytosis of serum ferritin could counteract iron undersupply in cells with enhanced iron needs such as oligodendrocytes and erythroid precursor cells $[24,63]$.

\section{Conclusions}

In conclusion, cellular iron homeodynamics is based on a well-orchestrated interaction of iron uptake, intracellular transport, iron storage, usage and export, which is embedded in cellular metabolic and surveillance control. Under normal conditions this machinery provides a dynamic response to changing iron requirements and iron supply allowing the constant fueling of intracellular iron metabolism. Under stress conditions, this orchestration changes in order to maintain homeodynamics and protect the cell from severe destabilization. Potentially, this may also involve distinct emergency control mechanism such as the release or uptake of ferritin to and from the extracellular environment, the possible existence of such alternative pathways remaining to be defined.

\section{Abbreviations}

$\begin{array}{ll}\text { CLIP } & \text { cytosolic labile iron pool } \\ \text { DMT1 } & \text { divalent metal transporter } \\ \text { ELC } & \text { endo-/lysosomal compartment } \\ \text { ELIP } & \text { endo-/lysosomal labile iron pool } \\ \text { Fpn } & \text { ferroportin } \\ \text { Ftx } & \text { frataxin } \\ \text { HNE } & \text { 4-hydroxy-nonenal } \\ \text { FRDA } & \text { Friedreich's ataxia } \\ \text { IRE } & \text { iron regulatory elements } \\ \text { IRP } & \text { iron regulatory protein } \\ \text { ISCU } & \text { iron-sulfur cluster forming unit }\end{array}$




$\begin{array}{ll}\text { LIP } & \text { labile iron pool } \\ \text { LMP } & \text { lysosomal membrane permeability } \\ \text { MLIP } & \text { mitochondrial labile iron pool } \\ \text { mtFER } & \text { mitochondrial ferritin } \\ \text { NTBI } & \text { non-transferrin bound iron } \\ \text { RME } & \text { receptor mediated endocytosis } \\ \text { RNS } & \text { reactive nitrogen species } \\ \text { ROS } & \text { reactive oxygen species } \\ \text { TBI } & \text { transferrin-bound iron } \\ \text { TfR } & \text { transferrin receptor } \\ \text { TRPML1 } & \text { type IV mucolipidosis-associated protein }\end{array}$

\section{Conflicts of Interest}

The authors declare no conflict of interest.

\section{References}

1. Shayeghi, M.; Latunde-Dada, G.O.; Oakhill, J.S.; Laftah, A.H.; Takeuchi, K.; Halliday, N.; Khan, Y.; Warley, A.; McCann, F.E.; Hider, R.C.; et al. Identification of an intestinal heme transporter. Cell 2005, 122, 789-801.

2. Garrick, M.D.; Dolan, K.G.; Horbinski, C.; Ghio, A.J.; Higgins, D.; Porubcin, M.; Moore, E.G.; Hainsworth, L.N.; Umbreit, J.N.; Conrad, M.E.; et al. DMT1: A mammalian transporter for multiple metals. Biometals 2003, 16, 41-54.

3. Andrews, N.C. The iron transporter DMT1. Int. J. Biochem. Cell Biol. 1999, 31, 991-994.

4. Garrick, M.D.; Garrick, L.M. Cellular iron transport. Biochim. Biophys. Acta Gen. Subj. 2009, 1790, 309-325.

5. Conrad, M.E.; Umbreit, J.N.; Moore, E.G.; Hainsworth, L.N.; Porubcin, M.; Simovich, M.J.; Nakada, M.T.; Dolan, K.; Garrick, M.D. Separate pathways for cellular uptake of ferric and ferrous iron. Am. J. Physiol. Gastrointest. Liver Physiol. 2000, 279, G767-G774.

6. Conrad, M.E.; Umbreit, J.N. Pathways of iron absorption. Blood Cells Mol. Dis. 2002, 29, 336-355.

7. Fuqua, B.K.; Vulpe, C.D.; Anderson, G.J. Intestinal iron absorption. J. Trace Elem. Med. Biol. 2012, 26, 115-119.

8. Liuzzi, J.P.; Aydemir, F.; Nam, H.; Knutson, M.D.; Cousins, R.J. Zip14 (Slc39a14) mediates non-transferrin-bound iron uptake into cells. Proc. Natl. Acad. Sci. USA 2006, 103, 13612-13617.

9. Pinilla-Tenas, J.J.; Sparkman, B.K.; Shawki, A.; Illing, A.C.; Mitchell, C.J.; Zhao, N.; Liuzzi, J.P.; Cousins, R.J.; Knutson, M.D.; Mackenzie, B. Zip14 is a complex broad-scope metal-ion transporter whose functional properties support roles in the cellular uptake of zinc and nontransferrin-bound iron. Am. J. Physiol. Cell Physiol. 2011, 301, C862-C871. 
10. Graham, R.M.; Morgan, E.H.; Baker, E. Characterisation of citrate and iron citrate uptake by cultured rat hepatocytes. J. Hepatol. 1998, 29, 603-613.

11. Baker, E.; Baker, S.M.; Morgan, E.H. Characterisation of non-transferrin-bound iron (ferric citrate) uptake by rat hepatocytes in culture. Biochim. Biophys. Acta Gen. Subj. 1998, 1380, 21-30.

12. Graham, R.M.; Morgan, E.H.; Baker, E. Ferric citrate uptake by cultured rat hepatocytes is inhibited in the presence of transferrin. Eur. J. Biochem. 1998, 253, 139-145.

13. Cighetti, G.; Duca, L.; Bortone, L.; Sala, S.; Nava, I.; Fiorelli, G.; Cappellini, M.D. Oxidative status and malondialdehyde in $\beta$-thalassaemia patients. Eur. J. Clin. Investig. 2002, 32, 55-60.

14. Porter, J.B.; Garbowski, M. The Pathophysiology of Transfusional Iron Overload. Hematol. Oncol. Clin. N. Am. 2014, 28, 683-701.

15. De Luca, C.; Filosa, A.; Grandinetti, M.; Maggio, F.; Lamba, M.; Passi, S. Blood antioxidant status and urinary levels of catecholamine metabolites in beta-thalassemia. Free Radic. Res. 1999, 30, 453-462.

16. Esposito, B.P.; Breuer, W.; Sirankapracha, P.; Pootrakul, P.; Hershko, C.; Cabantchik, Z.I. Labile plasma iron in iron overload: redox activity and susceptibility to chelation. Blood 2003, 102, 2670-2677.

17. Hershko, C.; Link, G.; Cabantchik, I. Pathophysiology of iron overload. Ann. NY Acad. Sci. 1998, 850, 191-201.

18. Gutteridge, J.M.; Rowley, D.A.; Griffiths, E.; Halliwell, B. Low-molecular-weight iron complexes and oxygen radical reactions in idiopathic haemochromatosis. Clin. Sci. 1985, 68, $463-467$.

19. Sibille, J.C.; Kondo, H.; Aisen, P. Interactions between isolated hepatocytes and Kupffer cells in iron metabolism: A possible role for ferritin as an iron carrier protein. Hepatology 1988, 8 , 296-301.

20. Sibille, J.C.; Kondo, H.; Aisen, P. Uptake of ferritin and iron bound to ferritin by rat hepatocytes: Modulation by apotransferrin, iron chelators and chloroquine. Biochim. Biophys. Acta 1989, 1010, 204-209.

21. Wang, W.; Knovich, M.A.; Coffman, L.G.; Torti, F.M.; Torti, S.V. Serum ferritin: Past, present and future. Biochim. Biophys. Acta 2010, 1800, 760-769.

22. Sibille, J.C.; Ciriolo, M.; Kondo, H.; Crichton, R.R.; Aisen, P. Subcellular localization of ferritin and iron taken up by rat hepatocytes. Biochem. J. 1989, 262, 685-688.

23. Kalgaonkar, S.; Lonnerdal, B. Receptor-mediated uptake of ferritin-bound iron by human intestinal Caco-2 cells. J. Nutr. Biochem. 2009, 20, 304-311.

24. Gelvan, D.; Fibach, E.; Meyron-Holtz, E.G.; Konijn, A.M. Ferritin uptake by human erythroid precursors is a regulated iron uptake pathway. Blood 1996, 88, 3200-3207.

25. Meyron-Holtz, E.G.; Fibach, E.; Gelvan, D.; Konijn, A.M. Binding and uptake of exogenous isoferritins by cultured human erythroid precursor cells. Br. J. Haematol. 1994, 86, 635-641.

26. Hulet, S.W.; Hess, E.J.; Debinski, W.; Arosio, P.; Bruce, K.; Powers, S.; Connor, J.R. Characterization and distribution of ferritin binding sites in the adult mouse brain. J. Neurochem. 1999, 72, 868-874. 
27. Liao, Q.K.; Kong, P.A.; Gao, J.; Li, F.Y.; Qian, Z.M. Expression of ferritin receptor in placental microvilli membrane in pregnant women with different iron status at mid-term gestation. Eur. J. Clin. Nutr. 2001, 55, 651-656.

28. Mack, U.; Storey, E.L.; Powell, L.W.; Halliday, J.W. Characterization of the binding of ferritin to the rat liver ferritin receptor. Biochim. Biophys. Acta 1985, 843, 164-170.

29. Takami, M.; Mizumoto, K.; Kasuya, I.; Kino, K.; Sussman, H.H.; Tsunoo, H. Human placental ferritin receptor. Biochim. Biophys. Acta Gen. Subj. 1986, 884, 31-38.

30. Hulet, S.W.; Heyliger, S.O.; Powers, S.; Connor, J.R. Oligodendrocyte progenitor cells internalize ferritin via clathrin-dependent receptor mediated endocytosis. J. Neurosci. Res. 2000, 61, 52-60.

31. Chen, T.T.; Li, L.; Chung, D.-H.; Allen, C.D.C.; Torti, S.V.; Torti, F.M.; Cyster, J.G.; Chen, C.-Y.; Brodsky, F.M.; Niemi, E.C.; et al. TIM-2 is expressed on B cells and in liver and kidney and is a receptor for H-ferritin endocytosis. J. Exp. Med. 2005, 202, 955-965.

32. Todorich, B.; Zhang, X.; Slagle-Webb, B.; Seaman, W.E.; Connor, J.R. Tim-2 is the receptor for H-ferritin on oligodendrocytes. J. Neurochem. 2008, 107, 1495-1505.

33. Li, J.Y.; Paragas, N.; Ned, R.M.; Qiu, A.; Viltard, M.; Leete, T.; Drexler, I.R.; Chen, X.; Sanna-Cherchi, S.; Mohammed, F.; et al. Scara5 is a ferritin receptor mediating non-transferrin iron delivery. Dev. Cell 2009, 16, 35-46.

34. Li, L.; Fang, C.J.; Ryan, J.C.; Niemi, E.C.; Lebron, J.A.; Björkman, P.J.; Arase, H.; Torti, F.M.; Torti, S.V.; Nakamura, M.C.; et al. Binding and uptake of H-ferritin are mediated by human transferrin receptor-1. Proc. Natl. Acad. Sci. USA 2010, 107, 3505-3510.

35. Thomsen, J.H.; Etzerodt, A.; Svendsen, P.; Moestrup, S.K. The haptoglobin-CD163-heme oxygenase-1 pathway for hemoglobin scavenging. Oxid. Med. Cell. Longev. 2013, doi:10.1155/2013/523652.

36. Nairz, M.; Schroll, A.; Demetz, E.; Tancevski, I.; Theurl, I.; Weiss, G. "Ride on the ferrous wheel"-The cycle of iron in macrophages in health and disease. Immunobiology 2014, 220, 280-294.

37. Abboud, S.; Haile, D.J. A Novel mammalian iron-regulated protein involved in intracellular iron metabolism. J. Biol. Chem. 2000, 275, 19906-19912.

38. McKie, A.; Barlow, D. The SLC40 basolateral iron transporter family (IREG1/ferroportin/MTP1). Pflüg. Arch. 2004, 447, 801-806.

39. Musci, G.; Polticelli, F.; Bonaccorsi di Patti, M.C. Ceruloplasmin-ferroportin system of iron traffic in vertebrates. World J. Biol. Chem. 2014, 5, 204-215.

40. Donovan, A.; Lima, C.A.; Pinkus, J.L.; Pinkus, G.S.; Zon, L.I.; Robine, S.; Andrews, N.C. The iron exporter ferroportin/Slc40a1 is essential for iron homeostasis. Cell Metab. 2005, 1, 191-200.

41. Donovan, A.; Brownlie, A.; Zhou, Y.; Shepard, J.; Pratt, S.J.; Moynihan, J.; Paw, B.H.; Drejer, A.; Barut, B.; Zapata, A.; et al. Positional cloning of zebrafish ferroportin1 identifies a conserved vertebrate iron exporter. Nature 2000, 403, 776-781. 
42. Nemeth, E.; Tuttle, M.S.; Powelson, J.; Vaughn, M.B.; Donovan, A.; Ward, D.M.; Ganz, T.; Kaplan, J. Hepcidin regulates cellular iron efflux by binding to ferroportin and inducing its internalization. Science 2004, 306, 2090-2093.

43. Ganz, T. Hepcidin and iron regulation, 10 years later. Blood 2011, 117, 4425-4433.

44. Folgueras, A.R.; de Lara, F.M.; Pendás, A.M.; Garabaya, C.; Rodríguez, F.; Astudillo, A.; Bernal, T.; Cabanillas, R.; López-Otín, C.; Velasco, G. Membrane-bound serine protease matriptase-2 (Tmprss6) is an essential regulator of iron homeostasis. Blood 2008, 112, 2539-2545.

45. Du, X.; She, E.; Gelbart, T.; Truksa, J.; Lee, P.; Xia, Y.; Khovananth, K.; Mudd, S.; Mann, N.; Moresco, E.M.Y.; et al. The serine protease TMPRSS6 is required to sense iron deficiency. Science 2008, 320, 1088-1092.

46. Harris, Z.L.; Durley, A.P.; Man, T.K.; Gitlin, J.D. Targeted gene disruption reveals an essential role for ceruloplasmin in cellular iron efflux. Proc. Natl. Acad. Sci. USA 1999, 96, 10812-10817.

47. Vulpe, C.D.; Kuo, Y.-M.; Murphy, T.L.; Cowley, L.; Askwith, C.; Libina, N.; Gitschier, J.; Anderson, G.J. Hephaestin, a ceruloplasmin homologue implicated in intestinal iron transport, is defective in the sla mouse. Nat. Genet. 1999, 21, 195-199.

48. Vashchenko, G.; MacGillivray, R. Multi-copper oxidases and human iron metabolism. Nutrients 2013, 5, 2289-2313.

49. Nicolas, G.; Bennoun, M.; Devaux, I.; Beaumont, C.; Grandchamp, B.; Kahn, A.; Vaulont, S. Lack of hepcidin gene expression and severe tissue iron overload in upstream stimulatory factor 2 (USF2) knockout mice. Proc. Natl. Acad. Sci. USA 2001, 98, 8780-8785.

50. Nicolas, G.; xEb; Chauvet, C.; Viatte, L.; Danan, J.L.; Bigard, X.; Devaux, I.; Beaumont, C.; Kahn, A.; Vaulont, S. The gene encoding the iron regulatory peptide hepcidin is regulated by anemia, hypoxia, and inflammation. J. Clin. Invest. 2002, 110, 1037-1044.

51. Chaston, T.B.; Matak, P.; Pourvali, K.; Srai, S.K.; McKie, A.T.; Sharp, P.A. Hypoxia inhibits hepcidin expression in HuH7 hepatoma cells via decreased SMAD4 signaling. Am. J. Physiol. Cell Physiol. 2011, 300, C888-C895.

52. Peyssonnaux, C.; Zinkernagel, A.S.; Schuepbach, R.A.; Rankin, E.; Vaulont, S.; Haase, V.H.; Nizet, V.; Johnson, R.S. Regulation of iron homeostasis by the hypoxia-inducible transcription factors (HIFs). J. Clin. Invest. 2007, 117, 1926-1932.

53. Hintze, K.J.; McClung, J.P. Hepcidin: A critical regulator of iron metabolism during hypoxia. Adv. Hematol. 2011, doi:10.1155/2011/510304.

54. Song, N.; Wang, J.; Jiang, H.; Xie, J. Ferroportin1 and hephaestin overexpression attenuate iron-induced oxidative stress in MES23.5 dopaminergic cells. J. Cell. Biochem. 2010, 110, 1063-1072.

55. Pietrangelo, A. The ferroportin disease. Blood Cells Mol. Dis. 2004, 32, 131-138.

56. Nemeth, E.; Valore, E.V.; Territo, M.; Schiller, G.; Lichtenstein, A.; Ganz, T. Hepcidin, a putative mediator of anemia of inflammation, is a type II acute-phase protein. Blood 2003, 101, 2461-2463. 
57. Ghosh, S.; Hevi, S.; Chuck, S.L. Regulated secretion of glycosylated human ferritin from hepatocytes. Blood 2004, 103, 2369-2376.

58. Tran, T.N.; Eubanks, S.K.; Schaffer, K.J.; Zhou, C.Y.; Linder, M.C. Secretion of ferritin by rat hepatoma cells and its regulation by inflammatory cytokines and iron. Blood 1997, 90, 4979-4986.

59. Cohen, L.A.; Gutierrez, L.; Weiss, A.; Leichtmann-Bardoogo, Y.; Zhang, D.-L.; Crooks, D.R.; Sougrat, R.; Morgenstern, A.; Galy, B.; Hentze, M.W.; et al. Serum ferritin is derived primarily from macrophages through a nonclassical secretory pathway. Blood 2010, 116, 1574-1584.

60. Van Deurs, B.; Von Bulow, F.; Moller, M. Vesicular transport of cationized ferritin by the epithelium of the rat choroid plexus. J. Cell Biol. 1981, 89, 131-139.

61. Fisher, J.; Devraj, K.; Ingram, J.; Slagle-Webb, B.; Madhankumar, A.B.; Liu, X.; Klinger, M.; Simpson, I.A.; Connor, J.R., Ferritin: A novel mechanism for delivery of iron to the brain and other organs. Am. J. Physiol. Cell Physiol. 2007, 293, C641-C649.

62. Meyron-Holtz, E.; Moshe-Belizowski, S.; Cohen, L. A possible role for secreted ferritin in tissue iron distribution. J. Neural Transm. 2011, 118, 337-347.

63. Todorich, B.; Zhang, X.; Connor, J.R. H-ferritin is the major source of iron for oligodendrocytes. Glia 2011, 59, 927-935.

64. Knovich, M.A.; Storey, J.A.; Coffman, L.G.; Torti, S.V.; Torti, F.M. Ferritin for the clinician. Blood Rev. 2009, 23, 95-104.

65. Casgrain, A.; Collings, R.; Harvey, L.J.; Hooper, L.; Fairweather-Tait, S.J. Effect of iron intake on iron status: A systematic review and meta-analysis of randomized controlled trials. Am. J. Clin. Nutr. 2012, 96, 768-780.

66. Yip, R. Changes in iron metabolism with age. In Iron Metabolism in Health and Disease; Brock, J.H., Halliday, J.W., Pippard, M.J., Powell, L.W.E., Eds.; WB Saunders: London, UK, 1994; pp. 428-448.

67. Finch, C.A.; Bellotti, V.; Stray, S.; Lipschitz, D.A.; Cook, J.D.; Pippard, M.J.; Huebers, H.A. Plasma ferritin determination as a diagnostic tool. West. J. Med. 1986, 145, 657-663.

68. Ferraro, S.; Mozzi, R.; Panteghini, M. Revaluating serum ferritin as a marker of body iron stores in the traceability era. Clin. Chem. Lab. Med. 2012, 50, 1911-1916.

69. Karlsson, T.; Sjöö, F.; Kedinge-Cyrus, B.; Bäckström, B. Plasma soluble transferrin receptor assay when screening for iron-deficiency in an unselected population of elderly anaemic patients. J. Intern. Med. 2010, 267, 331-334.

70. Alkhateeb, A.A.; Connor, J.R. The significance of ferritin in cancer: Anti-oxidation, inflammation and tumorigenesis. Biochim. Biophys. Acta Rev. Cancer 2013, 1836, 245-254.

71. Fan, K.; Gao, L.; Yan, X. Human ferritin for tumor detection and therapy. Wiley Interdiscip. Rev. Nanomed. Nanobiotechnol. 2013, 5, 287-298.

72. Fairweather-Tait, S.J.; Wawer, A.A.; Gillings, R.; Jennings, A.; Myint, P.K. Iron status in the elderly. Mech. Ageing Dev. 2014, 136-137, 22-28. 
73. Dong, X.-P.; Cheng, X.; Mills, E.; Delling, M.; Wang, F.; Kurz, T.; Xu, H. The type IV mucolipidosis-associated protein TRPML1 is an endolysosomal iron release channel. Nature 2008, 455, 992-996.

74. Jenkitkasemwong, S.; Wang, C.-Y.; Mackenzie, B.; Knutson, M. Physiologic implications of metal-ion transport by ZIP14 and ZIP8. Biometals 2012, 25, 643-655.

75. Zhao, N.; Gao, J.; Enns, C.A.; Knutson, M.D. ZRT/IRT-like protein 14 (ZIP14) promotes the cellular assimilation of iron from transferrin. J. Biol. Chem. 2010, 285, 32141-32150.

76. Zeevi, D.A.; Frumkin, A.; Bach, G. TRPML and lysosomal function. Biochim. Biophys. Acta 2007, 1772, 851-858.

77. Berberat, P.O.; Katori, M.; Kaczmarek, E.; Anselmo, D.; Lassman, C.; Ke, B.; Shen, X.; Busuttil, R.W.; Yamashita, K.; Csizmadia, E.; et al. Heavy chain ferritin acts as an antiapoptotic gene that protects livers from ischemia reperfusion injury. FASEB J. 2003, 17, 1724-1726.

78. Arosio, P.; Ingrassia, R.; Cavadini, P. Ferritins: A family of molecules for iron storage, antioxidation and more. Biochim. Biophys. Acta Gen. Subj. 2009, 1790, 589-599.

79. Breuer, W.; Shvartsman, M.; Cabantchik, Z.I. Intracellular labile iron. Int. J. Biochem. Cell Biol. 2008, 40, 350-354.

80. Kruszewski, M. Labile iron pool: The main determinant of cellular response to oxidative stress. Mutat. Res. Fundam. Mol. Mech. Mutagen. 2003, 531, 81-92.

81. Petrat, F.; de Groot, H.; Sustmann, R.; Rauen, U. The chelatable iron pool in living cells: A methodically defined quantity. Biol. Chem. 2002, 383, 489-502.

82. Rauen, U.; Springer, A.; Weisheit, D.; Petrat, F.; Korth, H.-G.; de Groot, H.; Sustmann, R. Assessment of chelatable mitochondrial iron by using mitochondrion-selective fluorescent iron indicators with different iron-binding affinities. ChemBioChem 2007, 8, 341-352.

83. Cabantchik, Z.I.; Kakhlon, O.; Epsztejn, S.; Zanninelli, G.; Breuer, W. Intracellular and extracellular labile iron pools. Adv. Exp. Med. Biol. 2002, 509, 55-75.

84. Petrat, F.; de Groot, H.; Rauen, U. Subcellular distribution of chelatable iron: A laser scanning microscopic study in isolated hepatocytes and liver endothelial cells. Biochem. J. 2001, 356, 61-69.

85. Ulvik, R.J. Relevance of ferritin-binding sites on isolated mitochondria to the mobilization of iron from ferritin. Biochim. Biophys. Acta 1982, 715, 42-51.

86. Sohn, Y.S.; Breuer, W.; Munnich, A.; Cabantchik, Z.I. Redistribution of accumulated cell iron: A modality of chelation with therapeutic implications. Blood 2008, 111, 1690-1699.

87. Richardson, D.R.; Lane, D.J.R.; Becker, E.M.; Huang, M.L.-H.; Whitnall, M.; Rahmanto, Y.S.; Sheftel, A.D.; Ponka, P. Mitochondrial iron trafficking and the integration of iron metabolism between the mitochondrion and cytosol. Proc. Natl. Acad. Sci. USA 2010, 107, 10775-10782.

88. Richardson, D.; Ponka, P.; Vyoral, D. Distribution of iron in reticulocytes after inhibition of heme synthesis with succinylacetone: Examination of the intermediates involved in iron metabolism. Blood 1996, 87, 3477-3488. 
89. Ponka, P. Tissue-Specific regulation of iron metabolism and heme synthesis: Distinct control mechanisms in erythroid cells. Blood 1997, 89, 1-25.

90. Shvartsman, M.; Kikkeri, R.; Shanzer, A.; Cabantchik, Z.I. Non-transferrin-bound iron reaches mitochondria by a chelator-inaccessible mechanism: Biological and clinical implications. Am. J. Physiol. Cell Physiol. 2007, 293, C1383-C1394.

91. Zhang, A.-S.; Sheftel, A.D.; Ponka, P. Intracellular kinetics of iron in reticulocytes: Evidence for endosome involvement in iron targeting to mitochondria. Blood 2005, 105, 368-375.

92. Kurz, T.; Gustafsson, B.; Brunk, U.T. Intralysosomal iron chelation protects against oxidative stress-induced cellular damage. FEBS J. 2006, 273, 3106-3117.

93. Garner, B.; Roberg, K.; Brunk, U.T. Endogenous ferritin protects cells with iron-laden lysosomes against oxidative stress. Free Radic. Res. 1998, 29, 103-114.

94. Kurz, T.; Gustafsson, B.; Brunk, U.T. Cell sensitivity to oxidative stress is influenced by ferritin autophagy. Free Radic. Biol. Med. 2011, 50, 1647-1658.

95. Bresgen, N.; Jaksch, H.; Lacher, H.; Ohlenschläger, I.; Uchida, K.; Eckl, P.M. Iron mediated oxidative stress plays an essential role in ferritin induced cell death. Free Radic. Biol. Med. 2010, 48, 1347-1357.

96. Krenn, M.A.; Schürz, M.; Teufl, B.; Uchida, K.; Eckl, P.M.; Bresgen, N. Ferritin stimulated lipid peroxidation, lysosomal leak and macroautophagy promote lysosomal "metastability" in primary hepatocytes determining in vitro cell survival. Free Radic. Biol. Med. 2015, 80, 45-58.

97. Umbreit, J.N.; Conrad, M.E.; Moore, E.G.; Desai, M.P.; Turrens, J. Paraferritin: A protein complex with ferrireductase activity is associated with iron absorption in rats. Biochemistry 1996, 35, 6460-6469.

98. Umbreit, J.N.; Conrad, M.E.; Hainsworth, L.N.; Simovich, M. The ferrireductase paraferritin contains divalent metal transporter as well as mobilferrin. Am. J. Physiol. Gastrointest. Liver Physiol. 2002, 282, G534-G539.

99. Ohgami, R.S.; Campagna, D.R.; Greer, E.L.; Antiochos, B.; McDonald, A.; Chen, J.; Sharp, J.J.; Fujiwara, Y.; Barker, J.E.; Fleming, M.D. Identification of a ferrireductase required for efficient transferrin-dependent iron uptake in erythroid cells. Nat. Genet. 2005, $37,1264-1269$.

100. Ohgami, R.S.; Campagna, D.R.; McDonald, A.; Fleming, M.D. The Steap proteins are metalloreductases. Blood 2006, 108, 1388-1394.

101. Zhang, F.; Tao, Y.; Zhang, Z.; Guo, X.; An, P.; Shen, Y.; Wu, Q.; Yu, Y.; Wang, F. Metalloreductase Steap3 coordinates the regulation of iron homeostasis and inflammatory responses. Haematologica 2012, 97, 1826-1835.

102. Jalanko, A.; Braulke, T. Neuronal ceroid lipofuscinoses. Biochim. Biophys. Acta Mol. Cell Res. 2009, 1793, 697-709.

103. Terman, A.; Brunk, U.T. Lipofuscin. Int. J. Biochem. Cell Biol. 2004, 36, 1400-1404. 
104. Bassi, M.T.; Manzoni, M.; Monti, E.; Pizzo, M.T.; Ballabio, A.; Borsani, G. Cloning of the gene encoding a novel integral membrane protein, mucolipidin-and identification of the two major founder mutations causing mucolipidosis type IV. Am. J. Hum. Genet. 2000, 67, 1110-1120.

105. Sun, M.; Goldin, E.; Stahl, S.; Falardeau, J.L.; Kennedy, J.C.; Acierno, J.S., Jr.; Bove, C.; Kaneski, C.R.; Nagle, J.; Bromley, M.C.; et al. Mucolipidosis type IV is caused by mutations in a gene encoding a novel transient receptor potential channel. Hum. Mol. Genet. 2000, 9, 2471-2478.

106. Watanabe, K.; Yamashita, Y.; Ohgawara, H.; Sekiguchi, M.; Satake, N.; Orino, K.; Yamamoto, S. Iron content of rat serum ferritin. J. Vet. Med. Sci. 2001, 63, 587-589.

107. Worwood, M.; Dawkins, S.; Wagstaff, M.; Jacobs, A. The purification and properties of ferritin from human serum. Biochem. J. 1976, 157, 97-103.

108. Adams, P.C.; Chau, L.A. Uptake of ferritin by isolated rat hepatocytes. Effect of metabolic inhibitors and iron. Clin. Investig. Med. 1993, 16, 15-21.

109. Maejima, I.; Takahashi, A.; Omori, H.; Kimura, T.; Takabatake, Y.; Saitoh, T.; Yamamoto, A.; Hamasaki, M.; Noda, T.; Isaka, Y.; et al. Autophagy sequesters damaged lysosomes to control lysosomal biogenesis and kidney injury. EMBO J. 2013, 32, 2336-2347.

110. Ezaki, J.; Kominami, E.; Ueno, T. Peroxisome degradation in mammals. IUBMB Life 2011, 63, 1001-1008.

111. Ding, W.X.; Yin, X.M. Mitophagy: Mechanisms, pathophysiological roles, and analysis. Biol. Chem. 2012, 393, 547-564.

112. Tolkovsky, A.M. Mitophagy. Biochim. Biophys. Acta Mol. Cell Res. 2009, 1793, 1508-1515.

113. Persson, H.L.; Kurz, T.; Eaton, J.W.; Brunk, U.T. Radiation-induced cell death: Importance of lysosomal destabilization. Biochem. J. 2005, 389, 877-884.

114. Bergamini, E. Autophagy: A cell repair mechanism that retards ageing and age-associated diseases and can be intensified pharmacologically. Mol. Asp. Med. 2006, 27, 403-410.

115. Zhang, Y.; Mikhael, M.; Xu, D.; Li, Y.; Soe-Lin, S.; Ning, B.; Li, W.; Nie, G.; Zhao, Y.; Ponka, P. Lysosomal proteolysis is the primary degradation pathway for cytosolic ferritin and cytosolic ferritin degradation is necessary for iron exit. Antioxid. Redox Signal. 2010, 13, 999-1009.

116. Kurz, T.; Brunk, U.T. Autophagy of HSP70 and chelation of lysosomal iron in a non-redox-active form. Autophagy 2009, 5, 93-95.

117. Yu, Z.; Persson, H.L.; Eaton, J.W.; Brunk, U.T. Intralysosomal iron: A major determinant of oxidant-induced cell death. Free Radic. Biol. Med. 2003, 34, 1243-1252.

118. Persson, H.L.; Nilsson, K.J.; Brunk, U.T. Novel cellular defenses against iron and oxidation: Ferritin and autophagocytosis preserve lysosomal stability in airway epithelium. Redox Rep. 2001, 6, 57-63.

119. Antunes, F.; Cadenas, E. Cellular titration of apoptosis with steady state concentrations of $\mathrm{H}_{2} \mathrm{O}_{2}$ : Submicromolar levels of $\mathrm{H}_{2} \mathrm{O}_{2}$ induce apoptosis through Fenton chemistry independent of the cellular thiol state. Free Radic. Biol. Med. 2001, 30, 1008-1018. 
120. Antunes, F.; Cadenas, E.; Brunk, U.T. Apoptosis induced by exposure to a low steady-state concentration of $\mathrm{H}_{2} \mathrm{O}_{2}$ is a consequence of lysosomal rupture. Biochem. J. 2001, 356, 549-555.

121. Kroemer, G.; Jaattela, M. Lysosomes and autophagy in cell death control. Nat. Rev. Cancer 2005, 5, 886-897.

122. Nilsson, E.; Ghassemifar, R.; Brunk, U.T. Lysosomal heterogeneity between and within cells with respect to resistance against oxidative stress. Histochem. J. 1997, 29, 857-865.

123. Zdolsek, J.M.; Svensson, I. Effect of reactive oxygen species on lysosomal membrane integrity. A study on a lysosomal fraction. Virchows Arch. B 1993, 64, 401-406.

124. Persson, H.L.; Yu, Z.; Tirosh, O.; Eaton, J.W.; Brunk, U.T. Prevention of oxidant-induced cell death by lysosomotropic iron chelators. Free Radic. Biol. Med. 2003, 34, 1295-1305.

125. Yu, Z.; Eaton, J.W.; Persson, H.L. The radioprotective agent, amifostine, suppresses the reactivity of intralysosomal iron. Redox Rep. 2003, 8, 347-355.

126. Ollinger, K.; Brunk, U.T. Cellular injury induced by oxidative stress is mediated through lysosomal damage. Free Radic. Biol. Med. 1995, 19, 565-574.

127. Maiuri, M.C.; Zalckvar, E.; Kimchi, A.; Kroemer, G. Self-eating and self-killing: Crosstalk between autophagy and apoptosis. Nat. Rev. Mol. Cell Biol. 2007, 8, 741-752.

128. Brunk, U.T.; Neuzil, J.; Eaton, J.W., Lysosomal involvement in apoptosis. Redox Rep. 2001, 6, 91-97.

129. Baird, S.K.; Kurz, T.; Brunk, U.T. Metallothionein protects against oxidative stress-induced lysosomal destabilization. Biochem. J. 2006, 394, 275-283.

130. Ghosh, M.; Carlsson, F.; Laskar, A.; Yuan, X.M.; Li, W. Lysosomal membrane permeabilization causes oxidative stress and ferritin induction in macrophages. FEBS Lett. 2011, 585, 623-629.

131. Arosio, P.; Yokota, M.; Drysdale, J.W. Characterization of serum ferritin in iron overload: Possible identity to natural apoferritin. Br. J. Haematol. 1977, 36, 199-207.

132. Harrison, P.M.; Arosio, P. The ferritins: Molecular properties, iron storage function and cellular regulation. Biochim. Biophys. Acta 1996, 1275, 161-203.

133. Arosio, P.; Adelman, T.G.; Drysdale, J.W. On ferritin heterogeneity. Further evidence for heteropolymers. J. Biol. Chem. 1978, 253, 4451-4458.

134. Bomford, A.; Berger, M.; Lis, Y.; Williams, R. The iron content of human liver and spleen isoferritins correlates with their isoelectric point and subunit composition. Biochem. Biophys. Res. Commun. 1978, 83, 334-341.

135. Shi, H.; Bencze, K.Z.; Stemmler, T.L.; Philpott, C.C. A cytosolic iron chaperone that delivers iron to ferritin. Science 2008, 320, 1207-1210.

136. Zhao, G.; Arosio, P.; Chasteen, N.D. Iron(II) and hydrogen peroxide detoxification by human H-chain ferritin. An EPR spin-trapping study. Biochemistry 2006, 45, 3429-3436.

137. Levi, S.; Yewdall, S.J.; Harrison, P.M.; Santambrogio, P.; Cozzi, A.; Rovida, E.; Albertini, A.; Arosio, P. Evidence of H- and L-chains have co-operative roles in the iron-uptake mechanism of human ferritin. Biochem. J. 1992, 288, 591-596. 
138. Papaefthymiou, G.C. The Mossbauer and magnetic properties of ferritin cores. Biochim. Biophys. Acta 2010, 1800, 886-897.

139. Linder, M. Mobilization of stored iron in mammals: A Review. Nutrients 2013, 5, 4022-4050.

140. Watt, R.K.; Hilton, R.J.; Graff, D.M. Oxido-reduction is not the only mechanism allowing ions to traverse the ferritin protein shell. Biochim. Biophys. Acta 2010, 1800, 745-759.

141. Cassanelli, S.; Moulis, J. Sulfide is an efficient iron releasing agent for mammalian ferritins. Biochim. Biophys. Acta 2001, 1547, 174-182.

142. Double, K.L.; Maywald, M.; Schmittel, M.; Riederer, P.; Gerlach, M. In vitro studies of ferritin iron release and neurotoxicity. J. Neurochem. 1998, 70, 2492-2499.

143. Moser, J.C.; Rawal, M.; Wagner, B.A.; Du, J.; Cullen, J.J.; Buettner, G.R. Pharmacological ascorbate and ionizing radiation (IR) increase labile iron in pancreatic cancer. Redox Biol. 2014, 2, 22-27.

144. Kidane, T.Z.; Sauble, E.; Linder, M.C. Release of iron from ferritin requires lysosomal activity. Am. J. Physiol. Cell Physiol. 2006, 291, C445-C455.

145. De Domenico, I.; Vaughn, M.B.; Li, L.; Bagley, D.; Musci, G.; Ward, D.M.; Kaplan, J. Ferroportin-mediated mobilization of ferritin iron precedes ferritin degradation by the proteasome. EMBO J. 2006, 25, 5396-5404.

146. Konijn, A.M.; Glickstein, H.; Vaisman, B.; Meyron-Holtz, E.G.; Slotki, I.N.; Cabantchik, Z.I. The cellular labile iron pool and intracellular ferritin in K562 cells. Blood 1999, 94, 2128-2134.

147. Truty, J.; Malpe, R.; Linder, M.C. Iron prevents ferritin turnover in hepatic cells. J. Biol. Chem. 2001, 276, 48775-48780.

148. De Domenico, I.; Ward, D.M.; Kaplan, J. Specific iron chelators determine the route of ferritin degradation. Blood 2009, 114, 4546-4551.

149. Mehlhase, J.; Sandig, G.; Pantopoulos, K.; Grune, T. Oxidation-induced ferritin turnover in microglial cells: Role of proteasome. Free Radic. Biol. Med. 2005, 38, 276-285.

150. Cozzi, A.; Santambrogio, P.; Levi, S.; Arosio, P. Iron detoxifying activity of ferritin. Effects of $\mathrm{H}$ and $\mathrm{L}$ human apoferritins on lipid peroxidation in vitro. FEBS Lett. 1990, 277, 119-122.

151. Levi, S.; Santambrogio, P.; Cozzi, A.; Rovida, E.; Corsi, B.; Tamborini, E.; Spada, S.; Albertini, A.; Arosio, P. The role of the L-chain in ferritin iron incorporation. Studies of homo and heteropolymers. J. Mol. Biol. 1994, 238, 649-654.

152. Arosio, P.; Levi, S. Ferritin, iron homeostasis, and oxidative damage. Free Radic. Biol. Med. 2002, 33, 457-463.

153. Chen, C.; Dewaele, S.; Braeckman, B.; Desmyter, L.; Verstraelen, J.; Borgonie, G.; Vanfleteren, J.; Contreras, R. A high-throughput screening system for genes extending life-span. Exp. Gerontol. 2003, 38, 1051-1063.

154. Tsuji, Y.; Akebi, N.; Lam, T.; Nakabeppu, Y.; Torti, S.; Torti, F. FER-1, an enhancer of the ferritin $\mathrm{H}$ gene and a target of E1A-mediated transcriptional repression. Mol. Cell. Biol. 1995, $15,5152-5164$. 
155. Kwak, E.L.; Larochelle, D.A.; Beaumont, C.; Torti, S.V.; Torti, F.M. Role for NF-kappa B in the regulation of ferritin $\mathrm{H}$ by tumor necrosis factor-alpha. J. Biol. Chem. 1995, 270, $15285-15293$.

156. Hintze, K.J.; Theil, E.C. DNA and mRNA elements with complementary responses to hemin, antioxidant inducers, and iron control ferritin-L expression. Proc. Natl. Acad. Sci. USA 2005, $102,15048-15052$.

157. Tsuji, Y. JunD activates transcription of the human ferritin $\mathrm{H}$ gene through an antioxidant response element during oxidative stress. Oncogene 2005, 24, 7567-7578.

158. Pietsch, E.C.; Chan, J.Y.; Torti, F.M.; Torti, S.V. Nrf2 mediates the induction of ferritin H in response to xenobiotics and cancer chemopreventive dithiolethiones. J. Biol. Chem. 2003, 278, 2361-2369.

159. Rogers, J.T.; Bridges, K.R.; Durmowicz, G.P.; Glass, J.; Auron, P.E.; Munro, H.N. Translational control during the acute phase response. Ferritin synthesis in response to interleukin-1. J. Biol. Chem. 1990, 265, 14572-14578.

160. Ikegami, Y.; Inukai, K.; Imai, K.; Sakamoto, Y.; Katagiri, H.; Kurihara, S.; Awata, T.; Katayama, S. Adiponectin upregulates ferritin heavy chain in skeletal muscle cells. Diabetes 2009, 58, 61-70.

161. Pham, C.G.; Bubici, C.; Zazzeroni, F.; Papa, S.; Jones, J.; Alvarez, K.; Jayawardena, S.; De Smaele, E.; Cong, R.; Beaumont, C.; et al. Ferritin heavy chain upregulation by NF-kappaB inhibits TNFalpha-induced apoptosis by suppressing reactive oxygen species. Cell 2004, 119, 529-542.

162. Pham, C.G.; Papa, S.; Bubici, C.; Zazzeroni, F.; Franzoso, G. In the crosshairs: NF-кB targets the JNK signaling cascade. Curr. Med. Chem. Anti Inflamm. Anti Allergy Agents 2005, 4, 569-576.

163. Tsuji, Y.; Miller, L.L.; Miller, S.C.; Torti, S.V.; Torti, F.M. Tumor necrosis factor-alpha and interleukin 1-alpha regulate transferrin receptor in human diploid fibroblasts. Relationship to the induction of ferritin heavy chain. J. Biol. Chem. 1991, 266, 7257-7261.

164. Miller, L.L.; Miller, S.C.; Torti, S.V.; Tsuji, Y.; Torti, F.M. Iron-independent induction of ferritin $\mathrm{H}$ chain by tumor necrosis factor. Proc. Natl. Acad. Sci. USA 1991, 88, 4946-4950.

165. Fahmy, M.; Young, S.P. Modulation of iron metabolism in monocyte cell line U937 by inflammatory cytokines: Changes in transferrin uptake, iron handling and ferritin mRNA. Biochem. J. 1993, 296, 175-181.

166. Hirayama, M.; Kohgo, Y.; Kondo, H.; Shintani, N.; Fujikawa, K.; Sasaki, K.; Kato, J.; Niitsu, Y. Regulation of iron metabolism in HepG2 cells: A possible role for cytokines in the hepatic deposition of iron. Hepatology 1993, 18, 874-880.

167. Sato, H.; Yamaguchi, M.; Bannai, S. Regulation of ferritin synthesis in macrophages by oxygen and a sulfhydryl-reactive agent. Biochem. Biophys. Res. Commun. 1994, 201, 38-44.

168. Qi, Y.; Dawson, G. Hypoxia specifically and reversibly induces the synthesis of ferritin in oligodendrocytes and human oligodendrogliomas. J. Neurochem. 1994, 63, 1485-1490.

169. Torti, F.M.; Torti, S.V. Regulation of ferritin genes and protein. Blood 2002, 99, 3505-3516. 
170. Faniello, M.C.; Di Sanzo, M.; Quaresima, B.; Baudi, F.; di Caro, V.; Cuda, G.; Morrone, G.; del Sal, G.; Spinelli, G.; Venuta, S.; et al. p53-Mediated downregulation of H ferritin promoter transcriptional efficiency via NF-Y. Int. J. Biochem. Cell Biol. 2008, 40, 2110-2119.

171. Zhang, F.; Wang, W.; Tsuji, Y.; Torti, S.V.; Torti, F.M. Post-transcriptional modulation of iron homeostasis during p53-dependent growth arrest. J. Biol. Chem. 2008, 283, 33911-33918.

172. Sammarco, M.C.; Ditch, S.; Banerjee, A.; Grabczyk, E. Ferritin L and H subunits are differentially regulated on a post-transcriptional level. J. Biol. Chem. 2008, 283, 4578-4587.

173. Tsuji, Y.; Ayaki, H.; Whitman, S.P.; Morrow, C.S.; Torti, S.V.; Torti, F.M. Coordinate transcriptional and translational regulation of ferritin in response to oxidative stress. Mol. Cell. Biol. 2000, 20, 5818-5827.

174. Cairo, G.; Tacchini, L.; Pogliaghi, G.; Anzon, E.; Tomasi, A.; Bernelli-Zazzera, A. Induction of ferritin synthesis by oxidative stress. J. Biol. Chem. 1995, 270, 700-703.

175. Cairo, G.; Tacchini, L.; Recalcati, S.; Azzimonti, B.; Minotti, G.; Bernelli-Zazzera, A. Effect of reactive oxygen species on iron regulatory protein activity. Ann. NY Acad. Sci. 1998, 851, 179-186.

176. Muckenthaler, M.; Richter, A.; Gunkel, N.; Riedel, D.; Polycarpou-Schwarz, M.; Hentze, S.; Falkenhahn, M.; Stremmel, W.; Ansorge, W.; Hentze, M.W. Relationships and distinctions in iron-regulatory networks responding to interrelated signals. Blood 2003, 101, 3690-3698.

177. Koorts, A.M.; Viljoen, M. Ferritin and ferritin isoforms II: Protection against uncontrolled cellular proliferation, oxidative damage and inflammatory processes. Arch. Physiol. Biochem. 2007, 113, 55-64.

178. Koc, M.; Taysi, S.; Sezen, O.; Bakan, N. Levels of some acute-phase proteins in the serum of patients with cancer during radiotherapy. Biol. Pharm. Bull. 2003, 26, 1494-1497.

179. Maria, T.G.; Vasileios, K.E.; Panagiotis, P.S.; Kostas, S.N. Changes of acute-phase protein levels in the serum of lung cancer patients following radiotherapy. Int. J. Clin. Exp. Med. 2013, $6,50-56$.

180. Koorts, A.M.; Viljoen, M. Acute Phase Proteins: Ferritin and Ferritin Isoforms, Acute Phase Proteins-Regulation and Functions of Acute Phase Proteins. In Acute Phase Proteins-Regulation and Functions of Acute Phase Proteins; Francesco, V., Ed.; InTech: Rijeka, Croatia, 2011; pp. 153-184.

181. Breda, L.; Nozzi, M.; De Sanctis, S.; Chiarelli, F. Laboratory tests in the diagnosis and follow-up of pediatric rheumatic diseases: An update. Semin. Arthritis Rheum. 2010, 40, 53-72.

182. Rogers, J.; Lacroix, L.; Durmowitz, G.; Kasschau, K.; Andriotakis, J.; Bridges, K. The Role of Cytokines in the Regulation of Ferritin Expression. In Progress in Iron Research; Hershko, C., Konijn, A., Aisen, P., Eds.; Springer: Berlin/Heidelberg, Germany, 1994; Volume 356, pp. 127-132.

183. Ajioka, R.S.; Phillips, J.D.; Kushner, J.P. Biosynthesis of heme in mammals. Biochim. Biophys. Acta Mol. Cell Res. 2006, 1763, 723-736.

184. Wegiel, B.; Nemeth, Z.; Correa-Costa, M.; Bulmer, A.C.; Otterbein, L.E. Heme oxygenase-1: A metabolic nike. Antioxid. Redox Signal. 2013, 20, 1709-1722. 
185. Soe-Lin, S.; Apte, S.S.; Andriopoulos, B.; Andrews, M.C.; Schranzhofer, M.; Kahawita, T.; Garcia-Santos, D.; Ponka, P. Nramp1 promotes efficient macrophage recycling of iron following erythrophagocytosis in vivo. Proc. Natl. Acad. Sci. USA 2009, 106, 5960-5965.

186. Soe-Lin, S.; Apte, S.S.; Mikhael, M.R.; Kayembe, L.K.; Nie, G.; Ponka, P. Both Nramp1 and DMT1 are necessary for efficient macrophage iron recycling. Exp. Hematol. 2010, 38, 609-617.

187. Seixas, E.; Gozzelino, R.; Chora, Â.; Ferreira, A.; Silva, G.; Larsen, R.; Rebelo, S.; Penido, C.; Smith, N.R.; Coutinho, A.; et al. Heme oxygenase-1 affords protection against noncerebral forms of severe malaria. Proc. Natl. Acad. Sci. USA 2009, 106, 15837-15842.

188. Larsen, R.; Gozzelino, R.; Jeney, V.; Tokaji, L.; Bozza, F.A.; Japiassu, A.M.; Bonaparte, D.; Cavalcante, M.M.; Chora, A.; Ferreira, A.; et al. A central role for free heme in the pathogenesis of severe sepsis. Sci. Transl. Med. 2010, doi:10.1126/scitranslmed.3001118.

189. Gozzelino, R.; Jeney, V.; Soares, M.P. Mechanisms of cell protection by heme oxygenase-1. Annu. Rev. Pharmacol. Toxicol. 2010, 50, 323-354.

190. Gozzelino, R.; Soares, M.P. Coupling heme and iron metabolism via ferritin H chain. Antioxid. Redox Signal. 2013, 20, 1754-1769.

191. Vile, G.F.; Basu-Modak, S.; Waltner, C.; Tyrrell, R.M. Heme oxygenase 1 mediates an adaptive response to oxidative stress in human skin fibroblasts. Proc. Natl. Acad. Sci. USA 1994, 91, 2607-2610.

192. Vile, G.F.; Tyrrell, R.M., Oxidative stress resulting from ultraviolet A irradiation of human skin fibroblasts leads to a heme oxygenase-dependent increase in ferritin. J. Biol. Chem. 1993, 268, 14678-14681.

193. Gozzelino, R.; Andrade, B.B.; Larsen, R.; Luz, N.F.; Vanoaica, L.; Seixas, E.; Coutinho, A.; Cardoso, S.; Rebelo, S.; Poli, M.; et al. Metabolic adaptation to tissue iron overload confers tolerance to malaria. Cell Host Microbe 2012, 12, 693-704.

194. Hamza, I.; Dailey, H.A. One ring to rule them all: Trafficking of heme and heme synthesis intermediates in the metazoans. Biochim. Biophys. Acta Mol. Cell Res. 2012, 1823, $1617-1632$.

195. Lill, R.; Hoffmann, B.; Molik, S.; Pierik, A.J.; Rietzschel, N.; Stehling, O.; Uzarska, M.A.; Webert, H.; Wilbrecht, C.; Mühlenhoff, U. The role of mitochondria in cellular iron-sulfur protein biogenesis and iron metabolism. Biochim. Biophys. Acta Mol. Cell Res. 2012, 1823, 1491-1508.

196. Lill, R.; Mühlenhoff, U. Maturation of iron-sulfur proteins in eukaryotes: Mechanisms, connected processes, and diseases. Annu. Rev. Biochem. 2008, 77, 669-700.

197. Lill, R.; Mühlenhoff, U. Iron-sulfur-protein biogenesis in eukaryotes. Trends Biochem. Sci. 2005, 30, 133-141.

198. Ulvik, R.J. Ferritin iron as substrate for synthesis of protoheme in intact rat liver mitochondria. FEBS Lett. 1981, 132, 281-284.

199. Shaw, G.C.; Cope, J.J.; Li, L.; Corson, K.; Hersey, C.; Ackermann, G.E.; Gwynn, B.; Lambert, A.J.; Wingert, R.A.; Traver, D.; et al. Mitoferrin is essential for erythroid iron assimilation. Nature 2006, 440, 96-100. 
200. Paradkar, P.N.; Zumbrennen, K.B.; Paw, B.H.; Ward, D.M.; Kaplan, J. Regulation of mitochondrial iron import through differential turnover of mitoferrin 1 and mitoferrin 2 . Mol. Cell. Biol. 2009, 29, 1007-1016.

201. Chen, W.; Paradkar, P.N.; Li, L.; Pierce, E.L.; Langer, N.B.; Takahashi-Makise, N.; Hyde, B.B.; Shirihai, O.S.; Ward, D.M.; Kaplan, J.; et al. Abcb10 physically interacts with mitoferrin-1 (Slc25a37) to enhance its stability and function in the erythroid mitochondria. Proc. Natl. Acad. Sci. USA 2009, 106, 16263-16268.

202. Amigo, J.D.; Yu, M.; Troadec, M.-B.; Gwynn, B.; Cooney, J.D.; Lambert, A.J.; Chi, N.C.; Weiss, M.J.; Peters, L.L.; Kaplan, J.; et al. Identification of distal cis-regulatory elements at mouse mitoferrin loci using zebrafish transgenesis. Mol. Cell. Biol. 2011, 31, 1344-1356.

203. Cazzola, M.; Invernizzi, R.; Bergamaschi, G.; Levi, S.; Corsi, B.; Travaglino, E.; Rolandi, V.; Biasiotto, G.; Drysdale, J.; Arosio, P. Mitochondrial ferritin expression in erythroid cells from patients with sideroblastic anemia. Blood 2003, 101, 1996-2000.

204. Casanova-Gonzalez, M.J.; Trapero-Marugan, M.; Jones, E.A.; Moreno-Otero, R. Liver disease and erythropoietic protoporphyria: A concise review. World J. Gastroenterol. 2010, 16, 4526-4531.

205. Koeppen, A.H. Friedreich's ataxia: Pathology, pathogenesis, and molecular genetics. J. Neurol. Sci. 2011, 303, 1-12.

206. Koeppen, A.H.; Mazurkiewicz, J.E., Friedreich ataxia: Neuropathology revised. J. Neuropathol. ExpNeurol. 2013, 72, 78-90.

207. Liochev, S.I.; Fridovich, I. Superoxide and iron: Partners in crime. IUBMB Life 1999, 48, $157-161$.

208. Chen, J.; Schenker, S.; Frosto, T.A.; Henderson, G.I. Inhibition of cytochrome c oxidase activity by 4-hydroxynonenal (HNE): Role of HNE adduct formation with the enzyme subunits. Biochim. Biophys. Acta Gen. Subj. 1998, 1380, 336-344.

209. Halliwell, B.; Gutteridge, J.M.C. Biologically relevant metal ion-dependent hydroxyl radical generation An update. FEBS Lett. 1992, 307, 108-112.

210. Terman, A.; Gustafsson, B.; Brunk, U.T. Mitochondrial damage and intralysosomal degradation in cellular aging. Mol. Aspects Med. 2006, 27, 471-482.

211. Terman, A.; Gustafsson, B.; Brunk, U.T. The lysosomal-mitochondrial axis theory of postmitotic aging and cell death. Chem. Biol. Interact. 2006, 163, 29-37.

212. Kispal, G.; Csere, P.; Prohl, C.; Lill, R. The mitochondrial proteins Atm1p and Nfs $1 \mathrm{p}$ are essential for biogenesis of cytosolic Fe/S proteins. EMBO J. 1999, 18, 3981-3989.

213. Mitsuhashi, N.; Miki, T.; Senbongi, H.; Yokoi, N.; Yano, H.; Miyazaki, M.; Nakajima, N.; Iwanaga, T.; Yokoyama, Y.; Shibata, T.; et al. MTABC3, a novel mitochondrial ATP-binding cassette protein involved in iron homeostasis. J. Biol. Chem. 2000, 275, 17536-17540.

214. Bekri, S.; Kispal, G.; Lange, H.; Fitzsimons, E.; Tolmie, J.; Lill, R.; Bishop, D.F. Human ABC7 transporter: Gene structure and mutation causing X-linked sideroblastic anemia with ataxia with disruption of cytosolic iron-sulfur protein maturation. Blood 2000, 96, 3256-3264.

215. Csere, P.; Lill, R.; Kispal, G. Identification of a human mitochondrial ABC transporter, the functional orthologue of yeast Atm1p. FEBS Lett. 1998, 441, 266-270. 
216. Kispal, G.; Csere, P.; Guiard, B.; Lill, R. The ABC transporter Atmlp is required for mitochondrial iron homeostasis. FEBS Lett. 1997, 418, 346-350.

217. Koc, S.; Harris, J.W. Sideroblastic anemias: Variations on imprecision in diagnostic criteria, proposal for an extended classification of sideroblastic anemias. Am. J. Hematol. 1998, 57, 1-6.

218. Taketani, S.; Adachi, Y.; Kohno, H.; Ikehara, S.; Tokunaga, R.; Ishii, T. Molecular characterization of a newly identified heme-binding protein induced during differentiation of urine erythroleukemia cells. J. Biol. Chem. 1998, 273, 31388-31394.

219. Martelli, A.; Napierala, M.; Puccio, H. Understanding the genetic and molecular pathogenesis of Friedreich's ataxia through animal and cellular models. Dis. Model. Mech. 2012, 5, 165-176.

220. Pastore, A.; Puccio, H. Frataxin: A protein in search for a function. J. Neurochem. 2013, 126, 43-52.

221. Adamec, J.; Rusnak, F.; Owen, W.G.; Naylor, S.; Benson, L.M.; Gacy, A.M.; Isaya, G. Iron-dependent self-assembly of recombinant yeast frataxin: implications for Friedreich ataxia. Am. J. Hum. Genet. 2000, 67, 549-562.

222. Layer, G.; Ollagnier-de Choudens, S.; Sanakis, Y.; Fontecave, M. Iron-Sulfur cluster biosynthesis: characterization of Escherichia coli $\mathrm{CYaY}$ as an iron donor for the assembly of [2Fe-2S] clusters in the scaffold IscU. J. Biol. Chem. 2006, 281, 16256-16263.

223. Campuzano, V.; Montermini, L.; Molto, M.D.; Pianese, L.; Cossee, M.; Cavalcanti, F.; Monros, E.; Rodius, F.; Duclos, F.; Monticelli, A.; et al. Friedreich's ataxia: Autosomal recessive disease caused by an intronic GAA triplet repeat expansion. Science 1996, 271, $1423-1427$.

224. Koutnikova, H.; Campuzano, V.; Foury, F.; Dolle, P.; Cazzalini, O.; Koenig, M. Studies of human, mouse and yeast homologues indicate a mitochondrial function for frataxin. Nat. Genet. 1997, 16, 345-351.

225. Cossee, M.; Campuzano, V.; Koutnikova, H.; Fischbeck, K.; Mandel, J.-L.; Koenig, M.; Bidichandani, S.I.; Patel, P.I.; Molte, M.D.; Canizares, J.; et al. Frataxin fracas. Nat. Genet. 1997, 15, 337-338.

226. Campuzano, V.; Montermini, L.; Lutz, Y.; Cova, L.; Hindelang, C.; Jiralerspong, S.; Trottier, Y.; Kish, S.J.; Faucheux, B.; Trouillas, P.; et al. Frataxin is reduced in Friedreich ataxia patients and is associated with mitochondrial membranes. Hum. Mol. Genet. 1997, 6, 1771-1780.

227. Lamarche, J.B.; Cote, M.; Lemieux, B. The cardiomyopathy of Friedreich's ataxia morphological observations in 3 cases. Can. J. Neurol. Sci. 1980, 7, 389-396.

228. Rotig, A.; de Lonlay, P.; Chretien, D.; Foury, F.; Koenig, M.; Sidi, D.; Munnich, A.; Rustin, P. Aconitase and mitochondrial iron-sulphur protein deficiency in Friedreich ataxia. Nat. Genet. 1997, 17, 215-217.

229. Schmucker, S.; Martelli, A.; Colin, F.; Page, A.; Wattenhofer-Donzé, M.; Reutenauer, L.; Puccio, H. Mammalian Frataxin: An essential function for cellular viability through an interaction with a preformed ISCU/NFS1/ISD11 iron-sulfur assembly complex. PLOS ONE 2011, 6, e16199. 
230. Adinolfi, S.; Iannuzzi, C.; Prischi, F.; Pastore, C.; Iametti, S.; Martin, S.R.; Bonomi, F.; Pastore, A. Bacterial frataxin $\mathrm{CyaY}$ is the gatekeeper of iron-sulfur cluster formation catalyzed by IscS. Nat. Struct. Mol. Biol. 2009, 16, 390-396.

231. Tsai, C.-L.; Barondeau, D.P. Human frataxin Is an Allosteric Switch That Activates the Fe-S Cluster Biosynthetic Complex. Biochemistry 2010, 49, 9132-9139.

232. Yoon, T.; Cowan, J.A. Frataxin-mediated Iron Delivery to ferrochelatase in the final step of heme biosynthesis. J. Biol. Chem. 2004, 279, 25943-25946.

233. Sellers, V.M.; Wu, C.-K.; Dailey, T.A.; Dailey, H.A. Human ferrochelatase: Characterization of Substrate-iron binding and proton-abstracting residues. Biochemistry 2001, 40, 9821-9827.

234. Wu, C.-K.; Dailey, H.A.; Rose, J.P.; Burden, A.; Sellers, V.M.; Wang, B.-C. The $2.0 \AA$ structure of human ferrochelatase, the terminal enzyme of heme biosynthesis. Nat. Struct. Mol. Biol. 2001, 8, 156-160.

235. Busi, M.V.; Gomez-Casati, D.F. Exploring frataxin function. IUBMB Life 2012, 64, 56-63.

236. Simon, D.; Seznec, H.; Gansmuller, A.; Carelle, N.; Weber, P.; Metzger, D.; Rustin, P.; Koenig, M.; Puccio, H. Friedreich ataxia mouse models with progressive cerebellar and sensory ataxia reveal autophagic neurodegeneration in dorsal root ganglia. J. Neurosci. 2004, 24, 1987-1995.

237. Kurz, T.; Terman, A.; Brunk, U.T. Autophagy, ageing and apoptosis: The role of oxidative stress and lysosomal iron. Arch. Biochem. Biophys. 2007, 462, 220-230.

238. Terman, A.; Kurz, T.; Navratil, M.; Arriaga, E.A.; Brunk, U.T. Mitochondrial turnover and aging of long-lived postmitotic cells: The mitochondrial-lysosomal axis theory of aging. Antioxid. Redox Signal. 2010, 12, 503-535.

239. Bradley, J.L.; Homayoun, S.; Hart, P.E.; Schapira, A.H.V.; Cooper, J.M. Role of oxidative damage in Friedreich's ataxia. Neurochem. Res. 2004, 29, 561-567.

240. Emond, M.; Lepage, G.; Vanasse, M.; Pandolfo, M. Increased levels of plasma malondialdehyde in Friedreich ataxia. Neurology 2000, 55, 1752-1753.

241. Schulz, J.B.; Dehmer, T.; Schöls, L.; Mende, H.; Hardt, C.; Vorgerd, M.; Bürk, K.; Matson, W.; Dichgans, J.; Beal, M.F.; et al. Oxidative stress in patients with Friedreich ataxia. Neurology 2000, 55, 1719-1721.

242. Lefevre, S.; Sliwa, D.; Rustin, P.; Camadro, J.-M.; Santos, R. Oxidative stress induces mitochondrial fragmentation in frataxin-deficient cells. Biochem. Biophys. Res. Commun. 2012, 418, 336-341.

243. Martin, M.; Colman, M.J.R.; Gómez-Casati, D.F.; Lamattina, L.; Zabaleta, E.J. Nitric oxide accumulation is required to protect against iron-mediated oxidative stress in frataxin-deficient Arabidopsis plants. FEBS Lett. 2009, 583, 542-548.

244. Calmels, N.; Schmucker, S.; Wattenhofer-Donzé, M.; Martelli, A.; Vaucamps, N.; Reutenauer, L.; Messaddeq, N.; Bouton, C.; Koenig, M.; Puccio, H. The First cellular models based on frataxin missense mutations that reproduce spontaneously the defects associated with Friedreich ataxia. PLOS ONE 2009, 4, e6379. 
245. Babcock, M.; de Silva, D.; Oaks, R.; Davis-Kaplan, S.; Jiralerspong, S.; Montermini, L.; Pandolfo, M.; Kaplan, J. Regulation of mitochondrial iron accumulation by Yfh1p, a putative homolog of frataxin. Science 1997, 276, 1709-1712.

246. Martelli, A.; Puccio, H. Dysregulation of cellular iron metabolism in Friedreich ataxia: From primary iron-sulfur cluster deficit to mitochondrial iron accumulation. Front. Pharmacol. 2014, doi:10.3389/fphar.2014.00130.

247. Afford, S.C.; Randhawa, S.; Eliopoulos, A.G.; Hubscher, S.G.; Young, L.S.; Adams, D.H. CD40 activation induces apoptosis in cultured human hepatocytes via induction of cell surface fas ligand expression and amplifies Fas-mediated hepatocyte death during allograft rejection. J. Exp. Med. 1999, 189, 441-446.

248. Seguin, A.; Sutak, R.; Bulteau, A.-L.; Garcia-Serres, R.; Oddou, J.-L.; Lefevre, S.; Santos, R.; Dancis, A.; Camadro, J.-M.; Latour, J.-M.; et al. Evidence that yeast frataxin is not an iron storage protein in vivo. Biochim. Biophys. Acta Mol. Basis Dis. 2010, 1802, 531-538.

249. Levi, S.; Corsi, B.; Bosisio, M.; Invernizzi, R.; Volz, A.; Sanford, D.; Arosio, P.; Drysdale, J. A human mitochondrial ferritin encoded by an intronless gene. J. Biol. Chem. 2001, 276, 24437-24440.

250. Gao, G.; Chang, Y.-Z. Mitochondrial ferritin in the regulation of brain iron homeostasis and neurodegenerative diseases. Front. Pharmacol. 2014, doi:10.3389/fphar.2014.00019.

251. Levi, S.; Arosio, P. Mitochondrial ferritin. Int. J. Biochem. Cell Biol. 2004, 36, 1887-1889.

252. Langlois d'Estaintot, B.; Santambrogio, P.; Granier, T.; Gallois, B.; Chevalier, J.M.; Precigoux, G.; Levi, S.; Arosio, P. Crystal structure and biochemical properties of the human mitochondrial ferritin and its mutant Ser144Ala. J. Mol. Biol. 2004, 340, 277-293.

253. Bou-Abdallah, F.; Santambrogio, P.; Levi, S.; Arosio, P.; Chasteen, N.D. Unique iron binding and oxidation properties of human mitochondrial ferritin: A comparative analysis with human H-chain ferritin. J. Mol. Biol. 2005, 347, 543-554.

254. Drysdale, J.; Arosio, P.; Invernizzi, R.; Cazzola, M.; Volz, A.; Corsi, B.; Biasiotto, G.; Levi, S. Mitochondrial ferritin: A new player in iron metabolism. Blood Cells Mol. Dis. 2002, 29, 376-383.

255. Corsi, B.; Cozzi, A.; Arosio, P.; Drysdale, J.; Santambrogio, P.; Campanella, A.; Biasiotto, G.; Albertini, A.; Levi, S. Human mitochondrial ferritin expressed in HeLa cells incorporates iron and affects cellular iron metabolism. J. Biol. Chem. 2002, 277, 22430-22437.

256. Nie, G.; Sheftel, A.D.; Kim, S.F.; Ponka, P. Overexpression of mitochondrial ferritin causes cytosolic iron depletion and changes cellular iron homeostasis. Blood 2005, 105, 2161-2167.

257. Lu, Z.; Nie, G.; Li, Y.; Soe-Lin, S.; Tao, Y.; Cao, Y.; Zhang, Z.; Liu, N.; Ponka, P.; Zhao, B. Overexpression of mitochondrial ferritin sensitizes cells to oxidative stress via an iron-mediated mechanism. Antioxid. Redox Signal. 2009, 11, 1791-1803.

258. Santambrogio, P.; Erba, B.G.; Campanella, A.; Cozzi, A.; Causarano, V.; Cremonesi, L.; Galli, A.; Della Porta, M.G.; Invernizzi, R.; Levi, S. Overexpression of mitochondrial ferritin affects the JAK2/STAT5 pathway in K562 cells and causes mitochondria iron accumulation. Haematologica 2011, 96, 1424-1432. 
259. Michael, S.; Petrocine, S.; Qian, J.; Lamarche, J.; Knutson, M.; Garrick, M.; Koeppen, A. Iron and iron-responsive proteins in the cardiomyopathy of Friedreich's ataxia. Cerebellum 2006, 5, 257-267.

260. Campanella, A.; Rovelli, E.; Santambrogio, P.; Cozzi, A.; Taroni, F.; Levi, S. Mitochondrial ferritin limits oxidative damage regulating mitochondrial iron availability: Hypothesis for a protective role in Friedreich ataxia. Hum. Mol. Genet. 2009, 18, 1-11.

261. Zanella, I.; Derosas, M.; Corrado, M.; Cocco, E.; Cavadini, P.; Biasiotto, G.; Poli, M.; Verardi, R.; Arosio, P. The effects of frataxin silencing in HeLa cells are rescued by the expression of human mitochondrial ferritin. Biochim. Biophys. Acta Mol. Basis Dis. 2008, 1782, 90-98.

262. Campanella, A.; Isaya, G.; O'Neill, H.A.; Santambrogio, P.; Cozzi, A.; Arosio, P.; Levi, S. The expression of human mitochondrial ferritin rescues respiratory function in frataxin-deficient yeast. Hum. Mol. Genet. 2004, 13, 2279-2288.

263. Sheftel, A.; Stehling, O.; Lill, R. Iron-sulfur proteins in health and disease. Trends Endocrinol. Metab. 2010, 21, 302-314.

264. Mettert, E.L.; Kiley, P.J. Fe-S proteins that regulate gene expression. Biochim. Biophys. Acta Mol. Cell Res. 2015, 1853, 1284-1293.

265. Rouault, T.A. The role of iron regulatory proteins in mammalian iron homeostasis and disease. Nat. Chem. Biol. 2006, 2, 406-414.

266. Koh, H.-J.; Lee, S.-M.; Son, B.-G.; Lee, S.-H.; Ryoo, Z.Y.; Chang, K.-T.; Park, J.-W.; Park, D.-C.; Song, B.J.; Veech, R.L.; et al. Cytosolic NADP ${ }^{+}$-dependent isocitrate dehydrogenase plays a key role in lipid metabolism. J. Biol. Chem. 2004, 279, 39968-39974.

267. Wallander, M.L.; Leibold, E.A.; Eisenstein, R.S. Molecular control of vertebrate iron homeostasis by iron regulatory proteins. Biochim. Biophys. Acta Mol. Cell Res. 2006, 1763, 668-689.

268. Walden, W.E.; Selezneva, A.I.; Dupuy, J.; Volbeda, A.; Fontecilla-Camps, J.C.; Theil, E.C.; Volz, K. Structure of dual function iron regulatory protein 1 complexed with ferritin IRE-RNA. Science 2006, 314, 1903-1908.

269. Eisenstein, R.S.; Ross, K.L. Novel roles for iron regulatory proteins in the adaptive response to iron deficiency. J. Nutr. 2003, 133, 1510S-1516S.

270. Tong, W.-H.; Rouault, T.A. Functions of mitochondrial ISCU and cytosolic ISCU in mammalian iron-sulfur cluster biogenesis and iron homeostasis. Cell Metab. 2006, 3, 199-210.

271. Pantopoulos, K.; Hentze, M.W. Rapid responses to oxidative stress mediated by iron regulatory protein. EMBO J. 1995, 14, 2917-2924.

272. Caltagirone, A.; Weiss, G.; Pantopoulos, K. Modulation of cellular iron metabolism by hydrogen peroxide: Effects of $\mathrm{H}_{2} \mathrm{O}_{2}$ on the expression and function of iron-responsive element-containing mRNAs in B6 fibroblasts. J. Biol. Chem. 2001, 276, 19738-19745.

273. Gardner, P.R.; Raineri, I.; Epstein, L.B.; White, C.W. Superoxide radical and iron modulate aconitase activity in mammalian cells. J. Biol. Chem. 1995, 270, 13399-13405.

274. Rouault, T.A.; Klausner, R.D. Iron-sulfur clusters as biosensors of oxidants and iron. Trends Biochem. Sci. 1996, 21, 174-177. 
275. Cairo, G.; Recalcati, S.; Pietrangelo, A.; Minotti, G. The iron regulatory proteins: Targets and modulators of free radical reactions and oxidative damage. Free Radic. Biol. Med. 2002, 32, 1237-1243.

276. Patel, M.; Day, B.J.; Crapo, J.D.; Fridovich, I.; McNamara, J.O. Requirement for superoxide in excitotoxic cell death. Neuron 1996, 16, 345-355.

277. Jang, S.; Imlay, J.A. Micromolar intracellular hydrogen peroxide disrupts metabolism by damaging iron-sulfur enzymes. J. Biol. Chem. 2007, 282, 929-937.

278. Flint, D.H.; Tuminello, J.F.; Emptage, M.H. The inactivation of Fe-S cluster containing hydro-lyases by superoxide. J. Biol. Chem. 1993, 268, 22369-22376.

279. Kennedy, M.C.; Emptage, M.H.; Dreyer, J.L.; Beinert, H. The role of iron in the activation-inactivation of aconitase. J. Biol. Chem. 1983, 258, 11098-11105.

280. Singh, S.P.; Niemczyk, M.; Saini, D.; Awasthi, Y.C.; Zimniak, L.; Zimniak, P. Role of the electrophilic lipid peroxidation product 4-hydroxynonenal in the development and maintenance of obesity in mice. Biochemistry 2008, 47, 3900-3911.

281. Yarian, C.S.; Rebrin, I.; Sohal, R.S. Aconitase and ATP synthase are targets of malondialdehyde modification and undergo an age-related decrease in activity in mouse heart mitochondria. Biochem. Biophys. Res. Commun. 2005, 330, 151-156.

282. McLain, A.L.; Szweda, P.A.; Szweda, L.I. alpha-Ketoglutarate dehydrogenase: A mitochondrial redox sensor. Free Radic. Res. 2011, 45, 29-36.

283. Yang, J.H.; Yang, E.S.; Park, J.W. Inactivation of $\mathrm{NADP}^{+}$-dependent isocitrate dehydrogenase by lipid peroxidation products. Free Radic. Res. 2004, 38, 241-249.

284. Lashin, O.M.; Szweda, P.A.; Szweda, L.I.; Romani, A.M.P. Decreased complex II respiration and HNE-modified SDH subunit in diabetic heart. Free Radic. Biol. Med. 2006, 40, 886-896.

285. Begriche, K.; Igoudjil, A.; Pessayre, D.; Fromenty, B. Mitochondrial dysfunction in NASH: Causes, consequences and possible means to prevent it. Mitochondrion 2006, 6, 1-28.

286. Pessayre, D.; Mansouri, A.; Haouzi, D.; Fromenty, B. Hepatotoxicity due to mitochondrial dysfunction. Cell Biol. Toxicol. 1999, 15, 367-373.

287. Liu, Q.; Simpson, D.C.; Gronert, S. Carbonylation of mitochondrial aconitase with 4-hydroxy2-(E)-nonenal: Localization and relative reactivity of addition sites. Biochim. Biophys. Acta Proteins Proteomics 2013, 1834, 1144-1154.

288. Brazzolotto, X.; Gaillard, J.; Pantopoulos, K.; Hentze, M.W.; Moulis, J.-M. Human cytoplasmic aconitase (iron regulatory protein 1) is converted into its [3Fe-4S] form by hydrogen peroxide in vitro but is not activated for iron-responsive element binding. J. Biol. Chem. 1999, 274, 21625-21630.

289. Pantopoulos, K.; Hentze, M.W. Activation of iron regulatory protein-1 by oxidative stress in vitro. Proc. Natl. Acad. Sci. USA 1998, 95, 10559-10563.

290. Pantopoulos, K.; Mueller, S.; Atzberger, A.; Ansorge, W.; Stremmel, W.; Hentze, M.W. Differences in the regulation of iron regulatory protein-1 (IRP-1) by extra- and intracellular oxidative stress. J. Biol. Chem. 1997, 272, 9802-9808. 
291. Brown, N.M.; Anderson, S.A.; Steffen, D.W.; Carpenter, T.B.; Kennedy, M.C.; Walden, W.E.; Eisenstein, R.S. Novel role of phosphorylation in Fe-S cluster stability revealed by phosphomimetic mutations at Ser-138 of iron regulatory protein 1. Proc. Natl. Acad. Sci. USA 1998, 95, $15235-15240$.

292. Eisenstein, R.S.; Tuazon, P.T.; Schalinske, K.L.; Anderson, S.A.; Traugh, J.A. Iron-responsive element-binding protein. Phosphorylation by protein kinase C. J. Biol. Chem. 1993, 268, 27363-27370.

293. Clarke, S.L.; Vasanthakumar, A.; Anderson, S.A.; Pondarre, C.; Koh, C.M.; Deck, K.M.; Pitula, J.S.; Epstein, C.J.; Fleming, M.D.; Eisenstein, R.S. Iron-responsive degradation of iron-regulatory protein 1 does not require the Fe-S cluster. EMBO J. 2006, 25, 544-553.

294. Neonaki, M.; Graham, D.C.; White, K.N.; Bomford, A. Down-regulation of liver iron-regulatory protein 1 in haemochromatosis. Biochem. Soc. Trans. 2002, 30, 726-728.

295. Goessling, L.S.; Daniels-McQueen, S.; Bhattacharyya-Pakrasi, M.; Lin, J.J.; Thach, R.E. Enhanced degradation of the ferritin repressor protein during induction of ferritin messenger RNA translation. Science 1992, 256, 670-673.

296. Minotti, G.; Ronchi, R.; Salvatorelli, E.; Menna, P.; Cairo, G. Doxorubicin irreversibly inactivates iron regulatory proteins 1 and 2 in cardiomyocytes. Cancer Res. 2001, 61, 8422-8428.

297. Cairo, G.; Recalcati, S. Iron-regulatory proteins: Molecular biology and pathophysiological implications. Expert Rev. Mol. Med. 2007, 9, 1-13.

298. Starzyński, R.R.; Lipiński, P.; Drapier, J.-C.; Diet, A.; Smuda, E.; Bartłomiejczyk, T.; Gralak, M.A.; Kruszewski, M. Down-regulation of iron regulatory protein 1 activities and expression in superoxide dismutase 1 knock-out mice is not associated with alterations in iron metabolism. J. Biol. Chem. 2005, 280, 4207-4212.

299. Terman, A.; Kurz, T. Lysosomal iron, iron chelation, and cell death. Antioxid. Redox Signal. 2013, 18, 888-898.

300. Terman, A.; Kurz, T.; Gustafsson, B.; Brunk, U.T. Lysosomal labilization. IUBMB Life 2006, 58, 531-539.

301. McGahan, M.C.; Harned, J.; Mukunnemkeril, M.; Goralska, M.; Fleisher, L.; Ferrell, J.B. Iron alters glutamate secretion by regulating cytosolic aconitase activity. Am. J. Physiol. Cell Physiol. 2005, 288, C1117-C1124.

302. Lee, S.H.; Jo, S.H.; Lee, S.M.; Koh, H.J.; Song, H.; Park, J.W.; Lee, W.H.; Huh, T.L. Role of $\mathrm{NADP}^{+}$-dependent isocitrate dehydrogenase $\left(\mathrm{NADP}^{+}-\mathrm{ICDH}\right)$ on cellular defence against oxidative injury by $\gamma$-rays. Int. J. Radiat. Biol. 2004, 80, 635-642.

303. Conrad, M.; Sato, H. The oxidative stress-inducible cystine/glutamate antiporter, system $\mathrm{X}_{\mathrm{c}}$ : Cystine supplier and beyond. Amino Acids 2012, 42, 231-246.

304. Lall, M.M.; Ferrell, J.; Nagar, S.; Fleisher, L.N.; McGahan, M.C. Iron regulates L-cystine uptake and glutathione levels in lens epithelial and retinal pigment epithelial cells by its effect on cytosolic aconitase. Investig. Ophthalmol. Vis. Sci. 2008, 49, 310-319.

305. Guo, B.; Yu, Y.; Leibold, E.A. Iron regulates cytoplasmic levels of a novel iron-responsive element-binding protein without aconitase activity. J. Biol. Chem. 1994, 269, 24252-24260. 
306. Samaniego, F.; Chin, J.; Iwai, K.; Rouault, T.A.; Klausner, R.D. Molecular characterization of a second iron-responsive element binding protein, iron regulatory protein 2. Structure, function, and post-translational regulation. J. Biol. Chem. 1994, 269, 30904-30910.

307. Hanson, E.S.; Foot, L.M.; Leibold, E.A. Hypoxia post-translationally activates iron-regulatory protein 2. J. Biol. Chem. 1999, 274, 5047-5052.

308. Guo, B.; Phillips, J.D.; Yu, Y.; Leibold, E.A. Iron regulates the intracellular degradation of iron regulatory protein 2 by the proteasome. J. Biol. Chem. 1995, 270, 21645-21651.

309. Iwai, K.; Drake, S.K.; Wehr, N.B.; Weissman, A.M.; LaVaute, T.; Minato, N.; Klausner, R.D.; Levine, R.L.; Rouault, T.A. Iron-dependent oxidation, ubiquitination, and degradation of iron regulatory protein 2: Implications for degradation of oxidized proteins. Proc. Natl. Acad. Sci. USA 1998, 95, 4924-4928.

310. Ke, Y.; Sierzputowska-Gracz, H.; Gdaniec, Z.; Theil, E.C. Internal loop/bulge and hairpin loop of the iron-responsive element of ferritin mRNA contribute to maximal iron regulatory protein 2 binding and translational regulation in the iso-iron-responsive element/iso-iron regulatory protein family. Biochemistry 2000, 39, 6235-6242.

311. Kim, S.; Ponka, P. Nitrogen monoxide-mediated control of ferritin synthesis: Implications for macrophage iron homeostasis. Proc. Natl. Acad. Sci. USA 2002, 99, 12214-12219.

312. Ke, Y.; Wu, J.; Leibold, E.A.; Walden, W.E.; Theil, E.C. Loops and bulge/loops in iron-responsive element isoforms influence iron regulatory protein binding. J. Biol. Chem. 1998, 273, 23637-23640.

313. Hanson, E.S.; Rawlins, M.L.; Leibold, E.A. Oxygen and iron regulation of iron regulatory protein 2. J. Biol. Chem. 2003, 278, 40337-40342.

314. Meyron-Holtz, E.G.; Ghosh, M.C.; Rouault, T.A. Mammalian tissue oxygen levels modulate iron-regulatory protein activities in vivo. Science 2004, 306, 2087-2090.

315. Chen, O.S.; Schalinske, K.L.; Eisenstein, R.S. Dietary iron intake modulates the activity of iron regulatory proteins and the abundance of ferritin and mitochondrial aconitase in rat liver. J. Nutr. 1997, 127, 238-248.

316. Schalinske, K.L.; Eisenstein, R.S. Phosphorylation and activation of both iron regulatory proteins 1 and 2 in HL-60 cells. J. Biol. Chem. 1996, 271, 7168-7176.

317. Kerenyi, M.A.; Grebien, F.; Gehart, H.; Schifrer, M.; Artaker, M.; Kovacic, B.; Beug, H.; Moriggl, R.; Müllner, E.W. Stat5 regulates cellular iron uptake of erythroid cells via IRP-2 and TfR-1. Blood 2008, 112, 3878-3888.

318. Salvatore, M.F.; Fisher, B.; Surgener, S.P.; Gerhardt, G.A.; Rouault, T. Neurochemical investigations of dopamine neuronal systems in iron-regulatory protein 2 (IRP-2) knockout mice. Mol. Brain Res. 2005, 139, 341-347.

319. Grabill, C.; Silva, A.C.; Smith, S.S.; Koretsky, A.P.; Rouault, T.A. MRI detection of ferritin iron overload and associated neuronal pathology in iron regulatory protein-2 knockout mice. Brain Res. 2003, 971, 95-106. 
320. Meyron-Holtz, E.G.; Ghosh, M.C.; Iwai, K.; LaVaute, T.; Brazzolotto, X.; Berger, U.V.; Land, W.; Ollivierre-Wilson, H.; Grinberg, A.; Love, P.; et al. Genetic ablations of iron regulatory proteins 1 and 2 reveal why iron regulatory protein 2 dominates iron homeostasis. EMBO J. 2004, 23, 386-395.

321. Drapier, J.-C. Interplay between NO and [Fe-S] Clusters: Relevance to biological systems. Methods 1997, 11, 319-329.

322. Hausladen, A.; Fridovich, I. Superoxide and peroxynitrite inactivate aconitases, but nitric oxide does not. J. Biol. Chem. 1994, 269, 29405-29408.

323. Castro, L.; Rodriguez, M.; Radi, R. Aconitase is readily inactivated by peroxynitrite, but not by its precursor, nitric oxide. J. Biol. Chem. 1994, 269, 29409-29415.

324. Radi, R. Peroxynitrite, a Stealthy Biological Oxidant. J. Biol. Chem. 2013, 288, 26464-26472.

325. Soum, E.; Brazzolotto, X.; Goussias, C.; Bouton, C.; Moulis, J.-M.; Mattioli, T.A.; Drapier, J.-C. Peroxynitrite and nitric oxide differently target the iron-sulfur cluster and amino acid residues of human iron regulatory protein 1. Biochemistry 2003, 42, 7648-7654.

326. Kim, Y.-M.; Bergonia, H.A.; Müller, C.; Pitt, B.R.; Watkins, W.D.; Lancaster, J.R. Loss and degradation of enzyme-bound heme induced by cellular nitric oxide synthesis. J. Biol. Chem. 1995, 270, 5710-5713.

327. Hentze, M.W.; Kühn, L.C. Molecular control of vertebrate iron metabolism: mRNA-based regulatory circuits operated by iron, nitric oxide, and oxidative stress. Proc. Natl. Acad. Sci. USA 1996, 93, 8175-8182.

328. Stamler, J.S.; Singel, D.J.; Loscalzo, J. Biochemistry of nitric oxide and its redox-activated forms. Science 1992, 258, 1898-1902.

329. Watts, R.N.; Richardson, D.R. The mechanism of nitrogen monoxide (NO)-mediated iron mobilization from cells. Eur. J. Biochem. 2002, 269, 3383-3392.

330. Watts, R.N.; Richardson, D.R. Nitrogen monoxide (NO) and glucose: Unexpected links between energy metabolism and NO-mediated iron mobilization from cells. J. Biol. Chem. 2001, 276, 4724-4732.

331. Lee, M.; Arosio, P.; Cozzi, A.; Chasteen, N.D. Identification of the EPR-active iron-nitrosyl complexes in mammalian ferritins. Biochemistry 1994, 33, 3679-3687.

332. Kim, S.; Wing, S.S.; Ponka, P. S-nitrosylation of IRP2 regulates its stability via the ubiquitin-proteasome pathway. Mol. Cell. Biol. 2004, 24, 330-337.

333. Mikhael, M.; Kim, S.F.; Schranzhofer, M.; Lin, S.S.; Sheftel, A.D.; Mullner, E.W.; Ponka, P. Iron regulatory protein-independent regulation of ferritin synthesis by nitrogen monoxide. FEBS J. 2006, 273, 3828-3836.

334. Lipton, S.A.; Choi, Y.-B.; Pan, Z.-H.; Lei, S.Z.; Chen, H.-S.V.; Sucher, N.J.; Loscalzo, J.; Singel, D.J.; Stamler, J.S. A redox-based mechanism for the neuroprotective and neurodestructive effects of nitric oxide and related nitroso-compounds. Nature 1993, 364, 626-632.

335. Stamler, J.S. Redox signaling: Nitrosylation and related target interactions of nitric oxide. Cell 1994, 78, 931-936. 
336. Vanin, A.F. Dinitrosyl iron complexes and S-nitrosothiols are two possible forms for stabilization and transport of nitric oxide in biological systems. Biochemistry 1998, 63, 782-793.

337. Boese, M.; Mordvintcev, P.I.; Vanin, A.F.; Busse, R.; Mülsch, A. S-nitrosation of serum albumin by dinitrosyl-iron complex. J. Biol. Chem. 1995, 270, 29244-29249.

338. Bosworth, C.A.; Toledo, J.C.; Zmijewski, J.W.; Li, Q.; Lancaster, J.R. Dinitrosyliron complexes and the mechanism(s) of cellular protein nitrosothiol formation from nitric oxide. Proc. Natl. Acad. Sci. USA 2009, 106, 4671-4676.

339. Gaston, B.; Reilly, J.; Drazen, J.M.; Fackler, J.; Ramdev, P.; Arnelle, D.; Mullins, M.E.; Sugarbaker, D.J.; Chee, C.; Singel, D.J. Endogenous nitrogen oxides and bronchodilator S-nitrosothiols in human airways. Proc. Natl. Acad. Sci. USA 1993, 90, 10957-10961.

340. Gow, A.J.; Buerk, D.G.; Ischiropoulos, H. A novel reaction mechanism for the formation of S-nitrosothiol in vivo. J. Biol. Chem. 1997, 272, 2841-2845.

341. Hogg, N.; Singh, R.J.; Kalyanaraman, B. The role of glutathione in the transport and catabolism of nitric oxide. FEBS Lett. 1996, 382, 223-228.

342. Keszler, A.; Zhang, Y.; Hogg, N. Reaction between nitric oxide, glutathione, and oxygen in the presence and absence of protein: How are S-nitrosothiols formed? Free Radic. Biol. Med. 2010, 48, 55-64.

343. Wink, D.A.; Nims, R.W.; Darbyshire, J.F.; Christodoulou, D.; Hanbauer, I.; Cox, G.W.; Laval, F.; Laval, J.; Cook, J.A. Reaction kinetics for nitrosation of cysteine and glutathione in aerobic nitric oxide solutions at neutral $\mathrm{pH}$. Insights into the fate and physiological effects of intermediates generated in the NO/O2 reaction. Chem. Res. Toxicol. 1994, 7, 519-525.

344. Martínez-Ruiz, A.; Lamas, S. Signalling by NO-induced protein S-nitrosylation and S-glutathionylation: Convergences and divergences. Cardiovasc. Res. 2007, 75, 220-228.

345. Rauhala, P.; Lin, A.M.-Y.; Chiueh, C.C. Neuroprotection by S-nitrosoglutathione of brain dopamine neurons from oxidative stress. FASEB J. 1998, 12, 165-173.

346. Rauhala, P.; Parameswarannay Mohanakumar, K.; Sziraki, I.; Lin, A.M.Y.; Chiueh, C.C. S-nitrosothiols and nitric oxide, but not sodium nitroprusside, protect nigrostriatal dopamine neurons against iron-induced oxidative stress in vivo. Synapse 1996, 23, 58-60.

347. Struck, A.T.; Hogg, N.; Thomas, J.P.; Kalyanaraman, B. Nitric oxide donor compounds inhibit the toxicity of oxidized low-density lipoprotein to endothelial cells. FEBS Lett. 1995, 361, 291-294.

348. Wink, D.A.; Cook, J.A.; Pacelli, R.; DeGraff, W.; Gamson, J.; Liebmann, J.; Krishna, M.C.; Mitchell, J.B. The effect of various nitric oxide-donor agents on hydrogen peroxide-mediated toxicity: A direct correlation between nitric oxide formation and protection. Arch. Biochem. Biophys. 1996, 331, 241-248.

349. Chiueh, C.C.; Rauhala, P. The redox pathway of S-nitrosoglutathione, glutathione and nitric oxide in cell to neuron communications. Free Radic. Res. 1999, 31, 641-650.

350. Chiueh, C.C. Neuroprotective Properties of Nitric Oxide. Ann. NY Acad. Sci. 1999, 890, 301-311. 
351. Meloche, B.A.; O'Brien, P.J. S-nitrosyl glutathione-mediated hepatocyte cytotoxicity. Xenobiotica 1993, 23, 863-871.

352. Kim, S.; Ponka, P. Effects of interferon- $\gamma$ and lipopolysaccharide on macrophage iron metabolism are mediated by nitric oxide-induced degradation of iron regulatory protein 2 . J. Biol. Chem. 2000, 275, 6220-6226.

353. Kim, S.; Ponka, P. Control of transferrin receptor expression via nitric oxide-mediated modulation of iron-regulatory protein 2. J. Biol. Chem. 1999, 274, 33035-33042.

354. Kennedy, M.C.; Antholine, W.E.; Beinert, H. An EPR Investigation of the products of the reaction of cytosolic and mitochondrial aconitases with nitric oxide. J. Biol. Chem. 1997, 272, 20340-20347.

355. Bouton, C.; Raveau, M.; Drapier, J.-C. Modulation of Iron regulatory protein functions. J. Biol. Chem. 1996, 271, 2300-2306.

356. Drapier, J.C.; Hirling, H.; Wietzerbin, J.; Kaldy, P.; Kuhn, L.C. Biosynthesis of nitric oxide activates iron regulatory factor in macrophages. EMBO J. 1993, 12, 3643-3649.

357. Recalcati, S.; Taramelli, D.; Conte, D.; Cairo, G. Nitric oxide-mediated induction of ferritin synthesis in $\mathrm{j} 774$ macrophages by inflammatory cytokines: Role of selective iron regulatory protein-2 downregulation. Blood 1998, 91, 1059-1066.

358. Pantopoulos, K. Iron Metabolism and the IRE/IRP regulatory system: An update. Ann. NY Acad. Sci. 2004, 1012, 1-13.

359. Pantopoulos, K.; Weiss, G.; Hentze, M.W. Nitric oxide and oxidative stress $\left(\mathrm{H}_{2} \mathrm{O}_{2}\right)$ control mammalian iron metabolism by different pathways. Mol. Cell. Biol. 1996, 16, 3781-3788.

360. Mulero, V.; Brock, J.H. Regulation of Iron Metabolism in Murine J774 Macrophages: Role of nitric oxide-dependent and -independent pathways following activation with gamma interferon and lipopolysaccharide. Blood 1999, 94, 2383-2389.

361. Weiss, G.; Goossen, B.; Doppler, W.; Fuchs, D.; Pantopoulos, K.; Werner-Felmayer, G.; Wachter, H.; Hentze, M.W. Translational regulation via iron-responsive elements by the nitric oxide/NO-synthase pathway. EMBO J. 1993, 12, 3651-3657.

362. Wang, J.; Chen, G.; Pantopoulos, K. Nitric oxide inhibits the degradation of IRP2. Mol. Cell. Biol. 2005, 25, 1347-1353.

363. Weiss, G.; Werner-Felmayer, G.; Werner, E.R.; Grünewald, K.; Wachter, H.; Hentze, M.W. Iron regulates nitric oxide synthase activity by controlling nuclear transcription. J. Exp. Med. 1994, 180, 969-976.

364. Hibbs, J.B., Jr.; Taintor, R.R.; Vavrin, Z. Iron depletion: Possible cause of tumor cell cytotoxicity induced by activated macrophages. Biochem. Biophys. Res. Commun. 1984, 123, $716-723$.

365. Richardson, D.R.; Ponka, P. The molecular mechanisms of the metabolism and transport of iron in normal and neoplastic cells. Biochim. Biophys. Acta 1997, 1331, 1-40.

366. Wilkinson, N.; Pantopoulos, K. The IRP/IRE system in vivo: Insights from mouse models. Front. Pharmacol. 2014, doi:10.3389/fphar.2014.00176.

367. Ghosh, M.C.; Zhang, D.-L.; Jeong, S.Y.; Kovtunovych, G.; Ollivierre-Wilson, H.; Noguchi, A.; Tu, T.; Senecal, T.; Robinson, G.; Crooks, D.R.; et al. Deletion of iron 
regulatory protein 1 causes polycythemia and pulmonary hypertension in mice through translational de-repression of HIF2 $\alpha$. Cell Metab. 2013, 17, 271-281.

368. Kapitsinou, P.P.; Liu, Q.; Unger, T.L.; Rha, J.; Davidoff, O.; Keith, B.; Epstein, J.A.; Moores, S.L.; Erickson-Miller, C.L.; Haase, V.H. Hepatic HIF-2 regulates erythropoietic responses to hypoxia in renal anemia. Blood 2010, 116, 3039-3048.

369. Ganz, T. Systemic iron homeostasis. Physiol. Rev. 2013, 93, 1721-1741.

370. Liu, Q.; Davidoff, O.; Niss, K.; Haase, V.H. Hypoxia-inducible factor regulates hepcidin via erythropoietin-induced erythropoiesis. J. Clin. Invest. 2012, 122, 4635-4644.

371. Mastrogiannaki, M.; Matak, P.; Mathieu, J.R.R.; Delga, S.; Mayeux, P.; Vaulont, S.; Peyssonnaux, C. Hepatic hypoxia-inducible factor-2 down-regulates hepcidin expression in mice through an erythropoietin-mediated increase in erythropoiesis. Haematologica 2012, 97 , 827-834.

372. Wilkinson, N.; Pantopoulos, K. IRP1 regulates erythropoiesis and systemic iron homeostasis by controlling HIF2 $\alpha$ mRNA translation. Blood 2013, 122,1658-1668.

373. Taylor, M.; Qu, A.; Anderson, E.R.; Matsubara, T.; Martin, A.; Gonzalez, F.J.; Shah, Y.M. Hypoxia-Inducible factor- $2 \alpha$ mediates the adaptive increase of intestinal ferroportin during iron deficiency in mice. Gastroenterology 2011, 140, 2044-2055.

374. Mastrogiannaki, M.; Matak, P.; Keith, B.; Simon, M.C.; Vaulont, S.; Peyssonnaux, C. HIF-2 $\alpha$, but not HIF-1 $\alpha$, promotes iron absorption in mice. J. Clin. Invest. 2009, 119, 1159-1166.

375. Shah, Y.M.; Matsubara, T.; Ito, S.; Yim, S.-H.; Gonzalez, F.J. Intestinal hypoxia inducible transcription factors are essential for iron absorption following iron deficiency. Cell Metab. 2009, 9, 152-164.

376. Keith, B.; Johnson, R.S.; Simon, M.C. HIF $1 \alpha$ and HIF2 $\alpha$ : Sibling rivalry in hypoxic tumour growth and progression. Nat. Rev. Cancer 2012, 12, 9-22.

377. Li, Z.; Rich, J. Hypoxia and Hypoxia Inducible Factors in Cancer Stem Cell Maintenance. In Diverse Effects of Hypoxia on Tumor Progression; Simon, M.C., Ed.; Springer: Berlin/Heidelberg, Germany, 2010; Volume 345, pp. 21-30.

378. Wang, W.; Deng, Z.; Hatcher, H.; Miller, L.D.; Di, X.; Tesfay, L.; Sui, G.; D’Agostino, R.B.; Torti, F.M.; Torti, S.V. IRP2 regulates breast tumor growth. Cancer Res. 2014, 74, 497-507.

379. Wu, K.-J.; Polack, A.; Dalla-Favera, R. Coordinated regulation of iron-controlling genes, H-Ferritin and IRP2, by c-MYC. Science 1999, 283, 676-679.

380. Torti, S.V.; Torti, F.M. Iron and cancer: More ore to be mined. Nat. Rev. Cancer 2013, 13, 342-355.

381. Templeton, D.M.; Liu, Y. Genetic regulation of cell function in response to iron overload or chelation. Biochim. Biophys. Acta Gen. Subj. 2003, 1619, 113-124.

382. Wesselius, L.J.; Nelson, M.E.; Skikne, B.S. Increased release of ferritin and iron by iron-loaded alveolar macrophages in cigarette smokers. Am. J. Respir. Crit. Care Med. 1994, $150,690-695$. 
383. Bresgen, N.; Ohlenschlager, I.; Wacht, N.; Afazel, S.; Ladurner, G.; Eckl, P.M. Ferritin and FasL (CD95L) mediate density dependent apoptosis in primary rat hepatocytes. J. Cell. Physiol. 2008, 217, 800-808.

384. Bresgen, N.; Rolinek, R.; Hochleitner, E.; Lottspeich, F.; Eckl, P.M. Induction of apoptosis by a hepatocyte conditioned medium. J. Cell. Physiol. 2004, 198, 452-460. 


\section{Impact of Oxidative Stress on Exercising Skeletal Muscle}

\section{Peter Steinbacher and Peter Eckl}

Abstract: It is well established that muscle contractions during exercise lead to elevated levels of reactive oxygen species (ROS) in skeletal muscle. These highly reactive molecules have many deleterious effects, such as a reduction of force generation and increased muscle atrophy. Since the discovery of exercise-induced oxidative stress several decades ago, evidence has accumulated that ROS produced during exercise also have positive effects by influencing cellular processes that lead to increased expression of antioxidants. These molecules are particularly elevated in regularly exercising muscle to prevent the negative effects of ROS by neutralizing the free radicals. In addition, ROS also seem to be involved in the exercise-induced adaptation of the muscle phenotype. This review provides an overview of the evidences to date on the effects of ROS in exercising muscle. These aspects include the sources of ROS, their positive and negative cellular effects, the role of antioxidants, and the present evidence on ROS-dependent adaptations of muscle cells in response to physical exercise.

Reprinted from Biomolecules. Cite as: Steinbacher, P.; Eckl, P. Impact of Oxidative Stress on Exercising Skeletal Muscle. Biomolecules 2015, 5, 356-377.

\section{Introduction}

Skeletal muscle is a highly specialized tissue with excellent plasticity in response to external stimuli such as exercise and training. The repetitive muscle contractions conducted during endurance training lead to a variety of phenotypic and physiological responses. These responses include activation of mitochondrial biogenesis, fiber type transformation and angiogenesis. Together, they increase the muscle's capacity of aerobic metabolism and its resistance to fatigue. High muscle activity also involves a strong increase in reactive oxygen species (ROS) production. These unstable molecules and ions contain oxygen and are extremely reactive due to an unpaired electron. Among these oxygen intermediates are the free radicals superoxide, peroxide and the hydroxyl radicals and other highly reactive oxidants, such as singlet oxygen and hypochlorous acid. They promote oxidation reactions with other molecules, such as proteins, lipids and DNA and can thus be highly detrimental. However, recent research has demonstrated that ROS also have a beneficial role in promoting the adaptive responses of muscle to training.

More than three decades ago it was established that muscle activity leads to an increase in ROS production and concentration of free radicals $[1,2]$. Since then numerous investigations in rodents and humans have confirmed these early observations. Thus, it is generally accepted that single bouts of aerobic or anaerobic exercise, as well as chronic exercise promote the generation of ROS (summarized in the reviews [3,4]; and more recently e.g., [5-7]).

The great interest in this topic also stems from data that show that ROS levels are increased in subjects with aging-related sarcopenia, cardiac reperfusion injuries or muscular diseases, i.e., muscle dystrophies. Thus, it was assumed that exercise-induced ROS are potentially detrimental to 
muscle function and lead to muscle fatigue and muscle atrophy. Hence, many investigations focused on ways to prevent ROS production and accumulation and subsequent oxidative damage during and following physical exercise.

\section{Sources of ROS in Muscle}

It has consistently been shown that muscle activity leads to a strong increase in ROS production [8]. However, there is a large debate about the sources and the extent of ROS that these sources produce. Several potential producers of ROS have been identified in muscle cells which are likely to be activated by different stimuli. Among these are mitochondria, nicotinamide adenine dinucleotide phosphate (NADPH) oxidases (NOXs), phospholipase A2 (PLA2), xanthine oxidase (XO) and lipoxygenases (Figure 1). Some of these are discussed in more detail below. In addition to these intracellular sources, ROS has been shown to be produced from non-muscle sources. Strenuous exercise can elicit muscle injuries, which then lead to the activation of the neutrophils and macrophages via interferon- $\gamma$ (IFN- $\gamma$ ), interleukin-1 (IL-1) and tumor necrosis factor (TNF) (for more detailed information see reviews $[9,10])$. These immune cells excessively produce ROS (oxidative burst), which is a central component of neutrophil defense mechanism. In addition, the exercise-induced increase of catecholamines (adrenaline, noradrenaline, dopamine) also play a role in the generation of ROS [11], as well as ROS derived from endothelium [12] (Figure 1).

\subsection{Mitochondria}

For a long period of time, mitochondria were regarded as the main producer of cellular ROS with an estimated superoxide production rate of approximately $1 \%-4 \%$ of total mitochondrial $\mathrm{O}_{2}$ consumption (see reviews $[8,13,14]$ ). More recent data demonstrate that the production of ROS in mitochondria is by an order of magnitude smaller than originally expected and is approximately $0.15 \%$ [15]. Mitochondria are thought to produce ROS by a leak of single electrons in the respiratory chain in the mitochondrial inner membrane of the contracting muscle cells. Ten different sites of superoxide $/ \mathrm{H}_{2} \mathrm{O}_{2}$ generation have been found as yet in mammalian mitochondria [16,17]. Superoxide production mainly occurs from complexes I (NADH dehydrogenase) and III (coenzyme Q and cytochrome $\mathrm{C}$ oxidoreductase) of the electron transport chain $[18,19]$. New findings also identify complex II (succinate dehydrogenase) as a major source of superoxide production [16]. Using isolated mitochondria, the contribution of each site to total $\mathrm{H}_{2} \mathrm{O}_{2}$ production has recently been quantified and shown to strongly depend on the substrate being oxidized [20]. At rest, $\mathrm{H}_{2} \mathrm{O}_{2}$ was predominantly produced from the quinol site in complex I (site IQ) and flavin site in complex II (site $\mathrm{II}_{\mathrm{F}}$ ), followed by sites $\mathrm{I}_{\mathrm{F}}$ and $\mathrm{III}_{\mathrm{Q}}$. Under conditions that mimic mild and intense aerobic exercise, total production is much less and the low capacity site $\mathrm{I}_{\mathrm{F}}$ dominates [20]. 


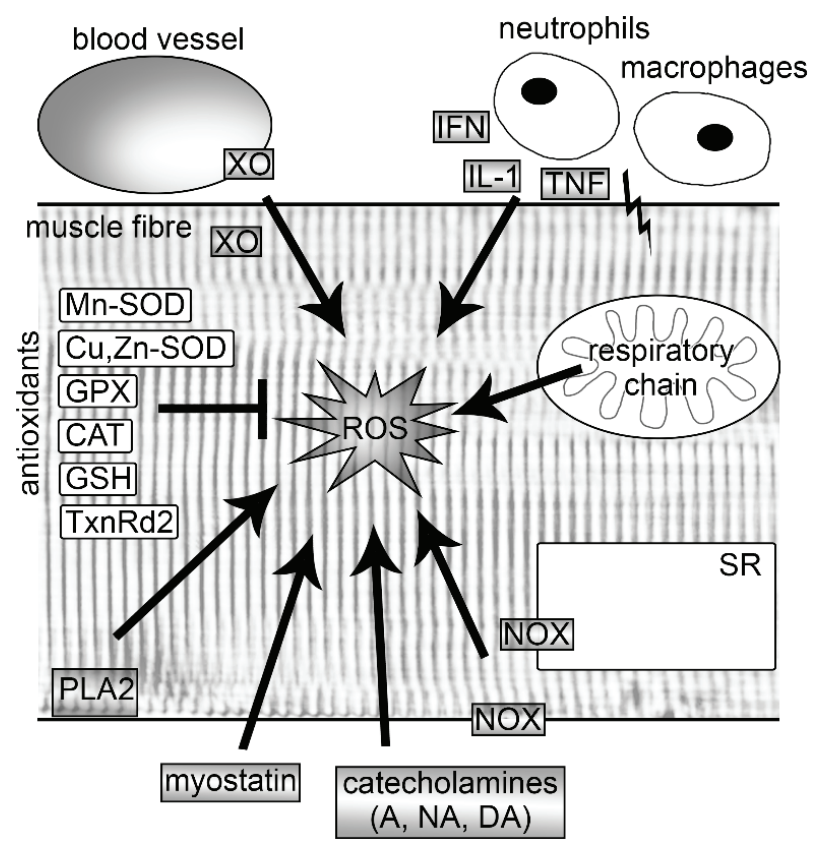

Figure 1. Sources of reactive oxygen species (ROS) and endogenous antioxidants in skeletal muscle fibers. Following exercise, ROS are produced endogenously by mitochondria, NOXs, PLA2 and XO. In addition, exercise increases ROS production also in activated neutrophils and macrophages, endothelia of blood vessels and by catecholamines. Regular exercise leads to an increase of endogenous antioxidants, which are able to neutralize free radicals. A, adrenaline; CAT, catalase; $\mathrm{Cu}, \mathrm{Zn}-\mathrm{SOD}$, copper-zinc superoxide dismutase; DA, dopamine; GPX, glutathione peroxidase; GSH, glutathione; IFN, interferon $\gamma$; IL-1, interleukin-1; Mn-SOD, manganese superoxide dismutase; NA, noradrenaline; NOX, nicotinamide adenine dinucleotide phosphate oxidase; PLA2, phospholipase A2; SR, sarcoplasmic reticulum; TNF, tumor necrosis factor; TxnRd2, thioredoxin reductase $2 ; \mathrm{XO}$, xanthine oxidase.

\subsection{NADPH Oxidases}

NADPH oxidases (NOXs) are flavoprotein enzymes that are activated by calcium, free fatty acids, protein-protein interactions and posttranslational modifications and use NADPH as electron donors $[21,22]$. They are transmembrane proteins in the transverse tubules and the sarcoplasmic reticulum and transport electrons across biological membranes to reduce oxygen to superoxide or $\mathrm{H}_{2} \mathrm{O}_{2}$ [21,22]. It was shown that NOX family members contribute to cytosolic superoxide production in skeletal muscle both at rest and during contractile activity to a larger extent than mitochondria [23-25]. ROS generated by NOXs activates ryanodine receptors (RyR), which leads to an intracellular $\mathrm{Ca}^{2+}$ release [26-28]. More recently, it was found that insulin induces ROS generation through NOX activation and that this ROS increase is required for the intracellular $\mathrm{Ca}^{2+}$ rise mediated by inositol triphosphate $\left(\mathrm{IP}_{3}\right)$ receptors [29]. 


\subsection{Xanthine Oxidase}

Xanthine oxidase (XO) is a cytosolic molybdoflavoenzyme that is recognized as a key enzyme in purine catabolism in which it catalyzes the hydroxylation of hypoxanthine to xanthine and of xanthine to uric acid [30]. In muscle, XO is present in the cytosol but also in the associated endothelial cells [8]. Upon contraction, XO activity is significantly increased and leads to increased lipid peroxidation, protein oxidation, muscle damage and edema [31]. During intense exercise in which large amounts of ATP are consumed, hypoxanthine and xanthine levels are rising and serve as substrates for XO to generate ROS [32]. Interestingly, ROS generated by XO appears to be involved in the regulation of exercise-induced mitochondrial biogenesis via peroxisome proliferator-activated receptor- $\gamma$ coactivator- $1 \alpha(\mathrm{PGC}-1 \alpha)[33]$.

\subsection{Myostatin}

Recently, it was demonstrated that myostatin, a blocker of muscle differentiation, is capable of signaling ROS production via canonical Smad3, nuclear factor (NF)- $\kappa B$ and TNF- $\alpha$ in muscle cells [34]. In the absence of $\operatorname{Smad3}$, myostatin induces ROS production through the activation of p38 and ERK mitogen-activated protein kinase (MAPK) pathways mediated via TNF- $\alpha$, IL-6, NOX and XO [35].

\subsection{Phospholipase A2}

Enzymes of the phospholipase A2 (PLA2) family also contribute to intra- and extracellular ROS increase during muscle contraction. They cleave arachidonic acid from phospholipids in the plasma membrane, sarcoplasmic reticulum or mitochondrial membranes. Arachidonic acid is an important lipid-signaling molecule and is a substrate for lipoxygenases for the production of ROS [36]. In addition, the cytosolic PLA2 enzyme has been demonstrated to increase ROS by stimulating NOXs [37]. Human muscle is known to contain approximately 15 different PLA2 isoforms that are either $\mathrm{Ca}^{2+}$-sensitive or $\mathrm{Ca}^{2+}$-insensitive [38]. The $\mathrm{Ca}^{2+}$-independent and dependent enzymes are supposed to produce ROS under resting and activity conditions, respectively [39].

\section{Effects of ROS on Force Generation and Muscle Atrophy}

In unfatigued muscle, intracellular ROS appear to be essential for normal force generation. Low-level ROS supplementation even increases force production [40]. A stronger increase of ROS due to intense exercise leads to a variety of adaptations of the muscle cells. Dependent upon the ROS concentration, duration of ROS exposure and training status of the individual, ROS can have beneficial and detrimental effects (Figure 2). Thus, a single bout of exhaustive exercise has been shown to cause oxidative damage in untrained persons while in trained subjects, no such effects are observed due to an increased resistance of such persons to oxidative stress [41]. Strong increases in ROS after strenuous exercise, aging and/or disease (e.g., chronic heart failure, COPD, cancer) can cause contractile dysfunction and muscle atrophy, which both promote muscle weakness and fatigue $[3,42]$. 


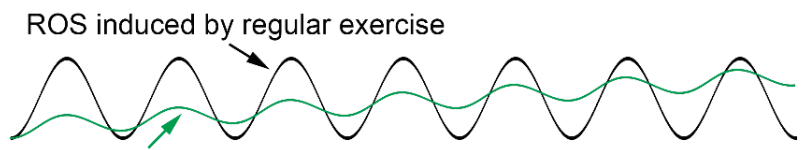

level of adaptation

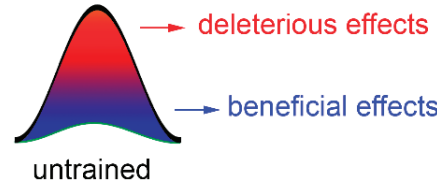

deleterious effects

muscle weakness and fatigue (contractile dysfunction, muscle protein degradation)

DNA mutations

lipid peroxidation mitochondrial dysfunction

apoptosis/necrosis

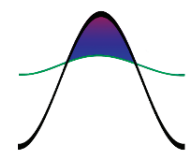

trained

beneficial effects

antioxidant capacity

mitochondrial biogenesis

insulin sensitivity

cytoprotection

aerobic capacity

Figure 2. Deleterious and beneficial effects of exercise-induced ROS increase. Exercise produces ROS and whether they are beneficial or detrimental to health is dependent upon the ROS concentration, duration of ROS exposure and training status of the individual. A single bout of exhaustive exercise leads to strong increases of ROS, which cannot be buffered by endogenous antioxidants, particularly in untrained individuals. This results in severe oxidative damage, including muscle weakness and fatigue, DNA mutations, lipid peroxidation, mitochondrial dysfunction and apoptosis/necrosis. Trained persons have a higher level of adaptation and less health risks. ROS produced during regular exercise continuously increase the level of adaptation by improving antioxidant capacity, mitochondrial biogenesis, insulin sensitivity, cytoprotection and aerobic capacity of skeletal muscle.

\subsection{Contractile Dysfunction}

Contractile dysfunction may result from oxidative modifications of a variety of proteins in diverse intracellular components $[43,44]$. However, our understanding of the processes involved is still limited and many data are equivocal. In the sarcoplasmic reticulum, the ryanodine receptor (RyR), which is the $\mathrm{Ca}^{2+}$ release channel, was shown to be oxidized by ROS and it was hypothesized to contribute to muscle fatigue [26-28]. However, other work demonstrated that this oxidation resulted only in increased $\mathrm{Ca}^{2+}$-induced $\mathrm{Ca}^{2+}$ release, whereas the $\mathrm{Ca}^{2+}$ release triggered by action potentials was not affected $[45,46]$. From this it was inferred that ROS-mediated effects on $\mathrm{Ca}^{2+}$ release in the sarcoplasmic reticulum are unlikely to contribute to muscle fatigue. This goes in line with recent findings that demonstrate that the mitochondrial antioxidant SS-31 restored the decrease in sarcoplasmic reticulum $\mathrm{Ca}^{2+}$ release while force recovery was not improved [47].

Similar uncertainty surrounds the effect of ROS on the force generating myofilaments. Initial data have shown that brief exposure to low concentrations of $\mathrm{H}_{2} \mathrm{O}_{2}$ increased force by $27 \%$, while a 
longer exposure results in force decline [48]. By contrast, a short exposure of skinned fibers to 10 $\mathrm{mM} \mathrm{H} \mathrm{O}_{2}$ had no effect on maximum force [45], while longer exposure to $50 \mathrm{mM} \mathrm{H}_{2} \mathrm{O}_{2}$ inhibited contractility [49]. Although the exact mechanisms are unknown, it is generally assumed that changes in force generation are the result of changes in the myofibrillar $\mathrm{Ca}^{2+}$ sensitivity $[8,44,47]$. In this regard, it was most recently suggested that troponin I, which is involved in sensing the intracellular $\mathrm{Ca}^{2+}$ levels, is a target of ROS. Oxidized cysteine residues of troponin I can react with the antioxidant glutathione, which helps protect the molecule from oxidative stress and make the contractile apparatus much more sensitive to $\mathrm{Ca}^{2+}[50]$. However, it must be mentioned that ROS may also lead to changes in the contractile proteins. In this regard, it was demonstrated that $\mathrm{H}_{2} \mathrm{O}_{2}$ is able to modify the S1 fragment in the myosin head, which then leads to a restriction of the myosinactin dynamics in the presence of ATP [49].

\subsection{Muscle Atrophy}

Besides their effects on the contractile kinetics, ROS are also able to modulate various signaling pathways, such as calcium, protein tyrosine kinases and phosphatases, serine/threonine kinases, and phospholipases [51]. This then leads to changes in gene expression, cell function, metabolism or cell damage. Chronic oxidative stress is associated with an increase in protein loss and muscle atrophy. High ROS levels cause a sustained activation of NF- $\kappa \mathrm{B}$ and of FoxO which then activate two muscle-specific E3 ubiquitin ligases, atrogin-1 or muscle atrophy F-box (MAFbx) and muscle RING (Really Interesting New Gene)-finger protein 1 (MuRF-1) [52]. MAFbx and MuRF-1 then degrade various proteins, such as titin, nebulin, troponin, myosin-binding protein $\mathrm{C}$, myosin light chains 1 and 2 and myosin heavy chain [53,54]. Recently, it was demonstrated that excessive oxidative stress also enhances the transcription factor $\mathrm{C} / \mathrm{EBP}$ homology protein $(\mathrm{CHOP})$. This transcription factor also enhances expression of MuRF1, which again results in increased protein degradation [35].

\section{Antioxidants in Muscle}

\subsection{Enzymatic and Nonenzymatic Antioxidants}

Muscle activity increases ROS but simultaneously also the body's antioxidant defense system. These molecules are able to neutralize free radicals by accepting the unpaired electron and thereby inhibit the oxidation of other molecules. Depending on the oxygen consumption rate, cells constitutively express different levels of antioxidant enzymes, including mitochondrial antioxidant manganese superoxide dismutase (Mn-SOD, SOD2), cytosolic copper-zinc superoxide dismutase (Cu, Zn-SOD, SOD1), glutathione peroxidase (GPX) and catalase (CAT), and the nonenzymatic antioxidant glutathione (GSH) [55] (Figure 1).

GSH is the most abundant nonprotein thiol in cells with intracellular concentrations of 1-15 $\mathrm{mM} \mathrm{[56].} \mathrm{It} \mathrm{plays} \mathrm{a} \mathrm{major} \mathrm{role} \mathrm{in} \mathrm{the} \mathrm{detoxification} \mathrm{of} \mathrm{electrophilic} \mathrm{xenobiotics,} \mathrm{such} \mathrm{as}$ chemical carcinogens, environmental pollutants, and the inactivation of endogenous $\alpha, \beta$-unsaturated aldehydes, quinones, epoxides, and hydroperoxides, which are formed as secondary metabolites during oxidative stress via members of the glutathione transferase family [57]. It also protects from oxidative stress by reducing hydrogen peroxide and organic peroxides levels via a reaction 
catalyzed by GSH peroxidase thus keeping the intracellular environment in the reduced state $[55,58]$. In addition, GSH is a substrate for dehydroascorbate reductase enabling the recycling of ascorbic acid, and it is a scavenger of hydroxyl radicals and singlet oxygen [59].

The aforementioned enzymatic reactions lead to the oxidation of GSH to glutathione disulfide (GSSG). This molecule can inactivate a number of enzymes by reacting with protein thiols leading to the formation of mixed disulfides (e.g., [60]). To avoid damage to intracellular constituents, GSSG is efficiently reduced to GSH by glutathione reductase (GR) utilizing NADPH. This action of GR is also very important during and after exercise in which a substantial amount of GSH is oxidized due to the elevated ROS levels to keep the GSH/GSSG ratio constant thereby maintaining homeostasis. Furthermore, exercising skeletal muscle appears to increase GSH import from plasma [61,62], and liver can synthesize GSH de novo and supply it [58]. But also increased muscle glutathione synthetase activities have been observed after treadmill training [63]. These exercise responses are tissue- and fiber-specific [64].

The antioxidant enzymes SOD, CAT and GPX are the primary defense against ROS generated during exercise and increase in response to exercise $[65,66]$. Recent work identified thioredoxin reductase-2 (TxnRd2) as another key player to decrease the exercise-induced content of mitochondrial $\mathrm{H}_{2} \mathrm{O}_{2}$ in skeletal muscle [67]. The same authors have shown that TxnRd2 is also able to control mitochondrial $\mathrm{H}_{2} \mathrm{O}_{2}$ levels after a high-fat, high-sucrose diet in the heart but not in skeletal muscle. Antioxidant enzyme levels vary considerably with respect to muscle fiber types, i.e., type I muscle fibers possess higher activity of all antioxidant enzymes than the type IIA and type IIB fibers [68].

\subsection{Adaptive Responses to Exercise}

In general, it was found that there is an exercise-induced increase in antioxidant protein levels and antioxidant activity. Thus, endurance training in rats leads to an increase in Mn-SOD, GPX and CAT, while the data on $\mathrm{Cu}, \mathrm{Zn}-\mathrm{SOD}$ are somewhat less clear [69-73]. Note that from the above studies, it is likely that upregulation of these antioxidants is muscle- and/or fiber type-specific. Many studies have shown that even an acute bout of exercise increases SOD activity in skeletal muscle ([74-77]; for review see [55]), and it has further been shown that $\mathrm{Cu}, \mathrm{Zn}-\mathrm{SOD}$ and $\mathrm{Mn}-\mathrm{SOD}$ contents are increased. While the $\mathrm{Cu}, \mathrm{Zn}-\mathrm{SOD}$ enzyme activity gradually returns to resting levels within three days, Mn-SOD activity and protein content continues to increase in the post-exercise period [78]. GPX activity after acute exercise on the other hand appears to depend on the muscle type, i.e., GPX activity was increased a day after an acute bout of treadmill running to exhaustion in rat soleus but not tibialis muscle [78], and CAT activity appears not to be altered by acute exercise [65].

ROS generated by acute exercise can lead to increased lipid peroxidation as measured by the formation of malondialdehyde [79]. Interestingly, this effect was only found in liver and fast skeletal muscle in the sedentary group, whereas the endurance-trained group did not show increases in lipid peroxidation after exercise. Lipid peroxidation generates a vast number of oxidative lipid breakdown products for which more or less specific tests are available (for review see [59]. Some investigators determined plasma isoprostane levels in athletes performing either a $50 \mathrm{~km}$ 
ultramarathon [80] or exercise for $2.5 \mathrm{~h}$ on a treadmill [81]. Peak levels of isoprostanes were found directly post-exercise, followed by a return to baseline within one day or one hour, respectively. Other investigators found increased levels of pentane in the breath after exercise [1], increased levels of lipid hydroperoxides [82], conjugated dienes [83] and oxidative DNA damage as measured by 8-hydroxydeoxyguanosine [84] or single cell gel electrophoresis [85]. The latter investigation gave results similar to those of Alessio and Goldfarb [79], namely a significant reduction of oxidative DNA damage in the trained compared to the untrained group indicating that exercise training causes an adaptive response to elevated oxidative stress by increased antioxidant enzyme activity. Powers et al. [86] studied the influence of training and observed significantly increased SOD activity in the soleus following exercise up to $60 \mathrm{~min} / \mathrm{d}$. Conversely, training induced significant increases in GPX activity in slow gastrocnemius only, and the magnitude of the GPX increase was directly related to exercise duration but relatively independent of intensity. However, CAT activity was not increased in any muscle with training. In addition, Radák et al. [87] observed decreased DNA damage and increased DNA repair levels as well as resistance against oxidative stress of proteins in aged rat skeletal muscle upon training. The obviously paradoxical situation that increased exercise-induced oxidative stress causes beneficial effects is interpreted in terms of hormesis - beneficial effects of potentially harmful agents - which apart from providing an adaptation to the damaging agent provide also systemic beneficial effects, including improved physiological function, decreased incidence of disease and a higher quality of life [88]. However, the beneficial effects appear to depend upon the duration of the exercise. While a single bout of exercise is suggested to lead to a limited adaptive response, regular exercise appears to gradually increase the level of adaptation by the repeated activation of antioxidant genes and proteins [32]. These authors hypothesize that it is the increased ROS level that is the important stimulus for the muscle cells to adapt to chronic exercise. The improved capabilities to decrease ROS may then provide a better protection from ROS during subsequent trainings but also attenuate the aging process and promote health with increased functional capacities [32]. Therefore, exercise is very similar to the adaptive ischemic preconditioning response [89]. Restoration of perfusion to ischemic organs results in increased ROS levels that can lead to tissue damage, myocardial infarction and stroke. However, such deleterious effects can be avoided by short intermittent bouts of reperfusion in which the transiently elevated ROS levels are important mediators of a cardioprotective response $[89,90]$.

Important mediators of the adaptive responses are the adenosine monophosphate-activated protein kinase (AMPK), the transcription factors NF- $\mathrm{kB}$, together with $\mathrm{p} 38 \mathrm{MAPK}$, and members of the FoxO transcription factor family [91-93]. At low ROS levels, they promote adaptation by increasing gene expression of antioxidant enzymes, such as Mn-SOD and $\mathrm{Cu}, \mathrm{Zn}-\mathrm{SOD}$, CAT and GPX1 [94]. High antioxidant capacities then diminish the deleterious effects of subsequent increases in ROS [95]. In addition, products of radical reactions are also suggested to be the mediator of this adaptation. Of special interest in this context are lipid peroxidation products, in particular 4-hydroxynonenal (HNE), which has been shown to both induce DNA damage [96] but also to be involved in the regulation of cell proliferation and growth as well as necrotic or apoptotic cell death by its marked ability to modulate several major pathways of cell signaling and, 
consequently, gene expression (for review see [97]). With respect to antioxidant gene expression it has been shown to be one of the most effective activators of nuclear factor erythroid-derived 2-like 2 (Nrf2) [98] which on stimulation dissociates from its cytoplasmic inhibitor Keap1, translocates to the nucleus and transactivates antioxidant-responsive elements (ARE)-dependent genes [99]. In addition, HNE has been demonstrated to cause mitochondrial uncoupling and thus protection from ROS specifically via the induction of the uncoupling proteins UCP1, UCP2 and UCP3 and the adenine nucleotide translocase [100].

\subsection{Exogenous Antioxidants and Exercise}

Apart from the endogenous antioxidants, which are obviously regulated by exercise, exogenous antioxidants such as vitamin C, E, and carotenoids are taken up with the food or are used as dietary supplements. The question therefore arises whether such supplements can be considered beneficial during exercise. To address this question, Ristow et al. [73] investigated the effects of a diet supplemented with vitamin $\mathrm{C}$ and $\mathrm{E}$ on exercise-induced insulin sensitivity as measured by glucose infusion rates during a hyperinsulinemic, euglycemic clamp in previously untrained and pre-trained healthy young men. Interestingly, exercise was found to increase parameters of insulin sensitivity (including adiponectin) only in the absence of antioxidants in both previously untrained and pretrained individuals. This was paralleled by increased expression of ROS-sensitive transcriptional regulators of insulin sensitivity and ROS defense capacity, peroxisome proliferator-activated receptor $\gamma$ (PPAR $\gamma$ ) and PPAR $\gamma$ coactivators PGC- $1 \alpha$ and PGC-1 $\beta$ only in the absence of antioxidants. Molecular mediators of endogenous ROS defense (Mn-SOD, Cu, Zn-SOD and GPX) were also induced by exercise, and this effect was again blocked by antioxidant supplementation. The authors concluded that exercise-induced oxidative stress ameliorates insulin resistance and causes an adaptive response promoting endogenous antioxidant defense capacity and that supplementation with antioxidants may preclude these health-promoting effects of exercise in humans. It was demonstrated that exercise causes an activation of mitogen-activated protein kinases (MAPKs: p38, ERK 1 and ERK 2), which in turn activates nuclear factor $\kappa \mathrm{B}(\mathrm{NF}-\kappa \mathrm{B})$ in rat gastrocnemius muscle and consequently the expression of important enzymes associated with defense against ROS (SOD) and adaptation to exercise - endothelial nitric oxide synthase (eNOS) and inducible nitric oxide synthase (iNOS) [101-103]. The expression of these enzymes can be inhibited by allopurinol, an inhibitor of XO indicating also that the prevention of ROS formation causes an inhibition of an adaptive response. The authors therefore conclude that in all likelihood, antioxidant supplements should not be recommended before training as they interfere with muscle cell adaptation. Thus, physical exercise is considered a double-edged sword: when practiced strenuously it causes oxidative stress and cell damage; in this case application of antioxidants may be helpful. But when practiced in moderation, it increases the expression of antioxidant enzymes and thus should be considered an antioxidant [101,103]. Supportive evidence for this assumption comes from studies on physical overtraining. Margonis et al. [104] examined the responses of oxidative stress biomarkers to a resistance training protocol of progressively increased and decreased volume/intensity in male test persons and observed significantly increased levels of urinary isoprostanes (7-fold), serum levels of thiobarbituric acid reactive substances (TBARS), 
protein carbonyls, CAT, GPX, and GSSG and significantly decreased levels of GSH, the GSH/GSSG ratio, and total antioxidant capacity in blood serum of over-trained individuals. Similarly, Palazzetti et al. [105] investigated the effects of overloaded training (OT) with athletes exercising for a duathlon before and after a four week OT and found that at rest conditions, OT induced an increased plasma GPX activity and a decreased plasma total antioxidant status, while OT resulted in higher exercise-induced variations of blood GSH/GSSG ratios, TBARS levels and decreased total antioxidant status in exercise conditions indicating that OT could compromise the antioxidant defense mechanisms. By comparing the oxidative stress response in control athletes and athletes with overtraining syndrome Tanskanen et al. [106] were further able to show that exercise to exhaustion led to an increase in oxygen radical absorbance (antioxidant) capacity and malondialdehyde in the controls but not in the over-trained athletes. Instead, over-trained athletes showed negative correlations between oxygen radical absorbance capacity at rest and protein carbonyls after exhaustive exercise indicating that increased oxidative stress may play a role in the pathophysiology of overtraining syndrome. Although these observations are not yet conclusive they indicate that adaptation to exercise is limited and that its protective effect can be exceeded leading to oxidative stress that cannot be dealt with by the endogenous antioxidant system. Whether it is helpful to apply exogenous antioxidants under such conditions as suggested still has to be elucidated.

\section{Training-Induced Muscular Adaptation, PGC-1 $\alpha$ and ROS}

In addition to the above-described effects of exercise on contents and activities of antioxidant enzymes, regularly performed exercise in the form of endurance training leads to well described adaptations of the cardiovascular and muscular system. Important responses at the intramyocellular level include increases in size and number of mitochondria as well as such in the activities of oxidative enzymes [107-109]. In support of the increased oxidation of fatty acids, the content of intramyocellular lipid is also elevated [110]. Endurance exercise is also known to improve insulin sensitivity and muscular glucose uptake [108,111]. Recent research has demonstrated that ROS also have a beneficial role in promoting these adaptive responses of muscle to training.

\subsection{Role of $P G C$-1 $\alpha$ in Exercise}

In rodents and humans, it has been demonstrated that peroxisome proliferator-activated receptor gamma coactivator-1 alpha (PGC-1 $\alpha$ ) is a key regulator of the exercise-induced changes of muscle fibers towards a slow phenotype, as well as in the protection from muscle atrophy [108,112,113]. Several studies have shown that PGC-1 $\alpha$ is upregulated after high-intensity training [114-118]. Activation of PGC- $1 \alpha$ is likely to occur by phosphorylation of the PGC- $1 \alpha$ protein by p38 MAPK together with NF-kB [119], both of which are known to be activated by ROS [91,92]. PGC-1 $\alpha$ has been demonstrated to regulate lipid and carbohydrate metabolism, and to improve the oxidative capacity of the muscle fibers by increasing the amount and activity of mitochondria through upregulation of nuclear respiratory factors (NRF-1, 2) and mitochondrial transcription factor A (TFAM) $[120,121]$. Furthermore, PGC-1 $\alpha$ regulates genes involved in the determination of muscle fiber type. Overexpression of PGC-1 $\alpha$ increases the proportion of oxidative type I fibers [122] 
while PGC-1 $\alpha$ knock-out (KO) mice exhibit a shift from oxidative type I and IIA toward glycolytic type IID/X and IIB fibers [123]. This regulatory diversity of PGC-1 $\alpha$ is enabled by its broad binding capacity to transcription factors in various signaling pathways. PGC-1 $\alpha$ has multiple binding sites for the interactions with diverse coactivators. A domain between amino acids 200 and 400 interacts with the nuclear receptors PPAR $\gamma$ and NRF-1 [124], which are considered as master regulators of mitochondrial biogenesis [125]. PGC-1 $\alpha$ binds to and activates the transcriptional function of NRF-1 on the promoter for TFAM, a direct regulator of mitochondrial DNA replication and transcription [120]. Another domain that predominantly binds to nuclear hormone receptors such as ERR- $\alpha$, PPARs, RXR, glucocorticoid receptor, HNF4, and probably others, is an LXXLL sequence in the N-terminal region of PGC-1 $\alpha$ [124]. This sequence is necessary for the coactivation of the nuclear receptor liver $\mathrm{x}$ receptor $\alpha(\mathrm{LXR} \alpha)$ [126]. The transcription complex of LXR $\alpha$ and PGC-1 $\alpha$ then activates fatty acid synthase (FAS), a multifunctional enzyme that catalyzes all reactions required for the de novo biosynthesis of lipid [127]. The binding site of the nuclear receptor estrogen-related receptor $\alpha(E R R-\alpha)$ is also in the LXXLL region of PGC-1 $\alpha$ [124]. The transcription complex formed by ERR- $\alpha$ and PGC- $1 \alpha$ induces the expression of vascular endothelial growth factor (VEGF), a potent stimulator of angiogenesis [128,129]. Between amino acids 400 to 500 of the PGC-1 $\alpha$ protein is the binding site for myocyte enhancer factor 2 (MEF2). This transcription factor is a key regulator of slow muscle identity [130]. MEF2 proteins are activated through the calcium-regulated calcineurin signaling pathway [130,131]. When overexpressed, MEF2C promotes the formation of slow fibers, thus enhancing running endurance in mice [132]. Genetic deletion of $M e f 2 c$ has been shown to block activity-dependent (exercise-induced) fast-to-slow fiber type transition [132]. This is in line with the proposed role of PGC-1 $\alpha$ in such transitions. Muscle-specific overexpression of PGC- $1 \alpha$ has been shown to evoke a transition of glycolytic type II in oxidative type I fibers [122]. This shift is initiated by the formation of a PGC- $1 \alpha / \mathrm{MEF} 2$ transcription complex, which then activates the expression of slow muscle genes [133]. Handschin et al. [123] have shown that PGC-1 $\alpha$ deficient mice display a significant shift from slow oxidative type I and fast oxidative IIA toward fast glycolytic type IIX and IIB fibers, resulting in a reduced endurance capacity.

\subsection{PGC-1 $\alpha$ Regulates ROS Defense}

It has been shown that oxidative stress increases the expression of PGC-1 $\alpha$ [134]. Similarly, depleting the endogenous antioxidant glutathione augments exercise-mediated induction of PGC-1 $\alpha$ expression [135]. Upregulation of PGC-1 $\alpha$ possibly involves the transcription factor Cre-binding protein (CREB) [136]. PGC-1 $\alpha$ then induces an increase of ROS-detoxifying enzymes, including GPX1 and Mn-SOD [136]. Only recently, new light has been shed on the molecular mechanisms involved in antioxidant activation. Therefore, it is highly likely that PGC-1 $\alpha$ binds to ERR- $\alpha$ and activates the $\mathrm{NAD}^{+}$-dependent histone deacetylase silent information regulator 3 (SIRT3) in the mitochondrial matrix [137]. SIRT3 is also known to regulate ROS production by directly binding and deacetylating mitochondrial complex I and II [138,139]. Previous studies have also shown that SIRT3 is able to deacetylate the mitochondrial enzyme Mn-SOD, thereby promoting its antioxidative activity [140-142]. Thus, it appears that PGC- $1 \alpha$ is a powerful suppressor of ROS production mainly by upregulation of antioxidant expression. Correspondingly, it was demonstrated 
that PGC-1 $\alpha$ knock-out (KO) mice have reduced expression levels of Mn-SOD, Cu, Zn-SOD and GPX1 and are thus more sensitive to oxidative stressors [136]. By contrast, overexpression of PGC-1 $\alpha$ enhances antioxidant defense by upregulation of Mn-SOD expression and a higher catalase activity [143]. Further, PGC-1 $\alpha$ increases the expression of uncoupling proteins 2 and 3 (UCP2, UCP3) and thereby concomitantly reduces mitochondrial ROS production [144].

\section{Conclusions}

There is rapidly growing evidence that ROS have both positive and negative effects in contracting skeletal muscle cells. The deleterious effects such as a reduction of force generation and increased muscle atrophy appear to occur particularly after non-regular strenuous exercise, while regular training has positive effects by influencing cellular processes that lead to increased expression of antioxidants. These molecules then provide a better protection from ROS during subsequent trainings. However, a diet supplemented with exogenous antioxidants such as vitamins appears to prevent health-promoting effects of physical exercise in humans. The exercise-induced production of ROS may also be an important signal to activate PGC- $1 \alpha$, a key player in the adaption of muscle cells to exercise.

\section{Author Contributions}

Both authors contributed interactively to explore the background literature and to the writing procedure. Peter Steinbacher took responsibility for preparing the final version of the manuscript.

\section{Abbreviations}

$\begin{array}{ll}\text { AMPK } & \text { adenosine monophosphate-activated protein kinase } \\ \text { ARE } & \text { antioxidant-responsive element } \\ \text { CAT } & \text { catalase } \\ \text { CHOP } & \text { C/EBP homology protein } \\ \text { CREB } & \text { Cre-binding protein } \\ \mathrm{Cu}, \mathrm{Zn} \text {-SOD } & \text { copper-zinc superoxide dismutase } \\ \text { eNOS } & \text { endothelial nitric oxide synthase } \\ \text { ERR- } \alpha & \text { estrogen-related receptor } \alpha \\ \text { FAS } & \text { fatty acid synthase } \\ \text { GPX } & \text { glutathione peroxidase } \\ \text { GR } & \text { glutathione reductase } \\ \text { GSH } & \text { glutathione } \\ \text { GSSG } & \text { glutathione disulfide } \\ \text { HNE } & \text { 4-hydroxynonenal } \\ \text { IFN- } \gamma & \text { interferon- } \gamma \\ \text { IL-1 } & \text { interleukin-1 } \\ \text { iNOS } & \text { inducible nitric oxide synthase } \\ \text { LXR } \alpha & \text { nuclear receptor liver x receptor } \alpha\end{array}$




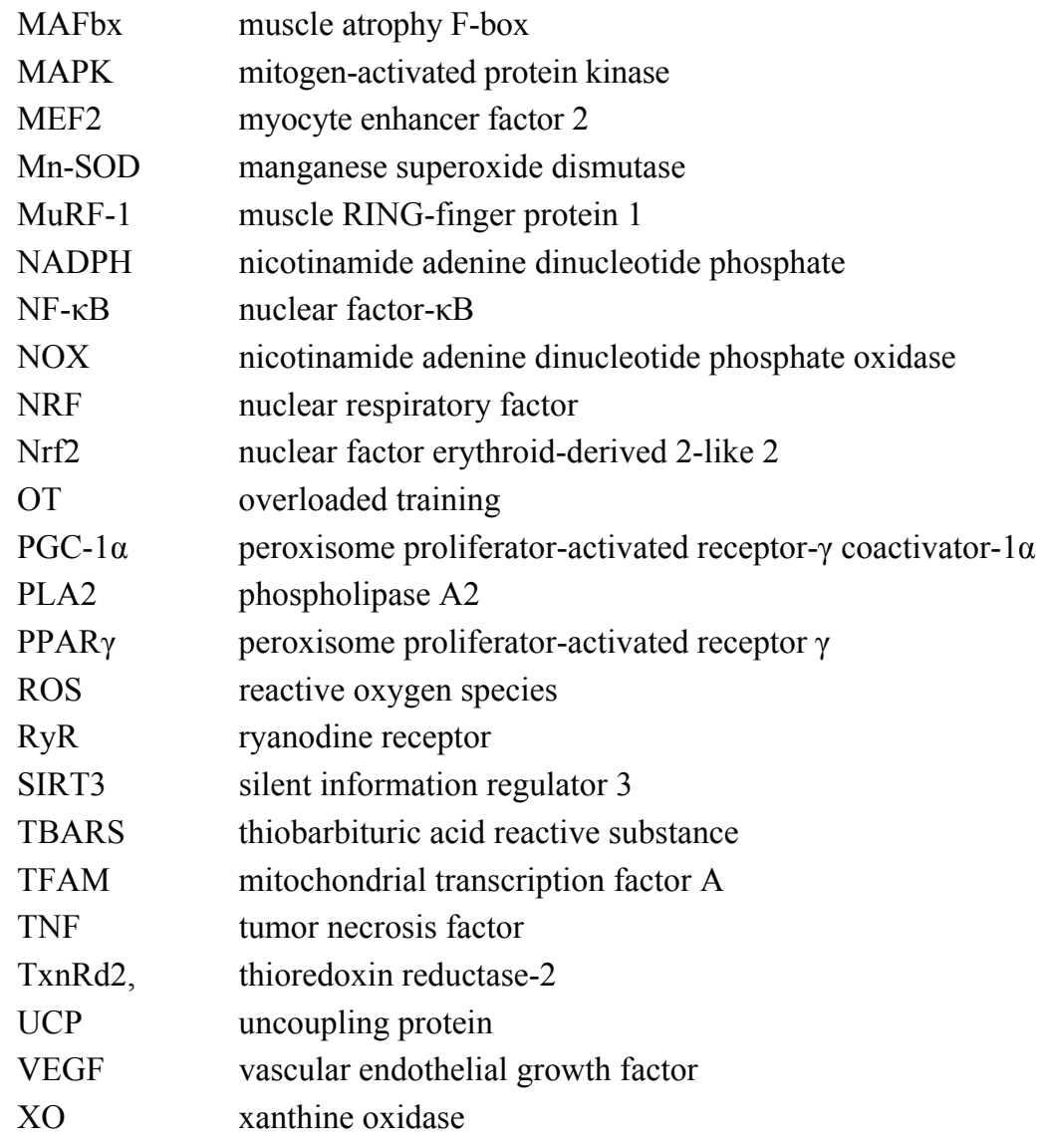

\section{Conflicts of Interest}

The authors declare no conflict of interest.

\section{References}

1. Dillard, C.J.; Litov, R.E.; Savin, W.M.; Dumelin, E.E.; Tappel, A.L. Effects of exercise, vitamin E, and ozone on pulmonary function and lipid peroxidation. J. Appl. Physiol. Respir. Environ. Exerc. Physiol. 1978, 45, 927-932.

2. Davies, K.J.; Quintanilha, A.T.; Brooks, G.A.; Packer, L. Free radicals and tissue damage produced by exercise. Biochem. Biophys. Res. Commun. 1982, 107, 1198-1205.

3. Vollaard, N.B.; Shearman, J.P.; Cooper, C.E. Exercise-induced oxidative stress: Myths, realities and physiological relevance. Sports Med. 2005, 35, 1045-1062.

4. Fisher-Wellman, K.; Bloomer, R.J. Acute exercise and oxidative stress: A 30 year history. Dyn. Med. 2009, doi:10.1186/1476-5918-8-1. 
5. Sahlin, K.; Shabalina, I.G.; Mattsson, C.M.; Bakkman, L.; Fernström, M.; Rozhdestvenskaya, Z.; Enqvist, J.K.; Nedergaard, J.; Ekblom, B.; Tonkonogi, M. Ultraendurance exercise increases the production of reactive oxygen species in isolated mitochondria from human skeletal muscle. J. Appl. Physiol. 2010, 108, 780-787.

6. Hey-Mogensen, M.; Højlund, K.; Vind, B.F.; Wang, L.; Dela, F.; Beck-Nielsen, H.; Fernström, M.; Sahlin, K. Effect of physical training on mitochondrial respiration and reactive oxygen species release in skeletal muscle in patients with obesity and type 2 diabetes. Diabetologia 2010, 53, 1976-1985.

7. Ghosh, S.; Lertwattanarak, R.; Lefort, N.; Molina-Carrion, M.; Joya-Galeana, J.; Bowen, B.P.; Garduno-Garcia Jde, J.; Abdul-Ghani, M.; Richardson, A.; DeFronzo, R.A.; et al. Reduction in reactive oxygen species production by mitochondria from elderly subjects with normal and impaired glucose tolerance. Diabetes 2011, 60, 2051-2060.

8. Powers, S.K.; Jackson, M.J. Exercise-induced oxidative stress: Cellular mechanisms and impact on muscle force production. Physiol. Rev. 2008, 88, 1243-1276.

9. Peake, J.; Suzuki, K. Neutrophil activation, antioxidant supplements and exercise-induced oxidative stress. Exerc. Immunol. Rev. 2004, 10, 129-141.

10. Moylan, J.S.; Reid, M.B. Oxidative stress, chronic disease, and muscle wasting. Muscle Nerve 2007, 35, 411-429.

11. Gomes, E.C.; Silva, A.N.; de Oliveira, M.R. Oxidants, antioxidants, and the beneficial roles of exercise-induced production of reactive species. Oxid. Med. Cell Longev. 2012, 2012, 756132.

12. Duarte, J.A.; Appell, H.J.; Carvalho, F.; Bastos, M.L.; Soares, J.M. Endothelium-derived oxidative stress may contribute to exercise-induced muscle damage. Int. J. Sports Med. 1993, $14,440-443$.

13. Murphy, M.P. How mitochondria produce reactive oxygen species. Biochem. J. 2009, 417, $1-13$.

14. Brand, M.D. The sites and topology of mitochondrial superoxide production. Exp. Gerontol. 2010, 45, 466-472.

15. St-Pierre, J.; Buckingham, J.A.; Roebuck, S.J.; Brand, M.D. Topology of superoxide production from different sites in the mitochondrial electron transport chain. J. Biol. Chem. 2002, 277, 44784-44790.

16. Perevoshchikova, I.V.; Quinlan, C.L.; Orr, A.L.; Gerencser, A.A.; Brand, M.D. Sites of superoxide and hydrogen peroxide production during fatty acid oxidation in rat skeletal muscle mitochondria. Free Radic. Biol. Med. 2013, 61, 298-309.

17. Hey-Mogensen, M.; Goncalves, R.L.; Orr, A.L.; Brand, M.D. Production of superoxide $/ \mathrm{H}_{2} \mathrm{O}_{2}$ by dihydroorotate dehydrogenase in rat skeletal muscle mitochondria. Free Radic. Biol. Med. 2014, 72, 149-155.

18. Barja, G. Mitochondrial oxygen radical generation and leak: Sites of production in states 4 and 3, organ specificity, and relation to aging and longevity. J. Bioenerg. Biomembr. 1999, 31, $347-366$. 
19. Muller, F.L.; Liu, Y.; van Remmen, H. Complex III releases superoxide to both sides of the inner mitochondrial membrane. J. Biol. Chem. 2004, 279, 49064-49073.

20. Goncalves, R.L.; Quinlan, C.L.; Perevoshchikova, I.V.; Hey-Mogensen, M.; Brand, M.D. Sites of superoxide and hydrogen peroxide production by muscle mitochondria assessed ex vivo under conditions mimicking rest and exercise. J. Biol. Chem. 2015, 290, 209-227.

21. Bedard, K.; Krause, K.H. The NOX family of ROS-generating NADPH oxidases: Physiology and pathophysiology. Physiol. Rev. 2007, 87, 245-313.

22. Brandes, R.P.; Weissmann, N.; Schröder, K. Nox family NADPH oxidases: Molecular mechanisms of activation. Free Radic. Biol. Med. 2014, 76, 208-226.

23. Xia, R.; Webb, J.A.; Gnall, L.L.; Cutler, K.; Abramson, J.J. Skeletal muscle sarcoplasmic reticulum contains a NADH-dependent oxidase that generates superoxide. Am. J. Physiol. Cell Physiol. 2003, 285, C215-C221.

24. Shkryl, V.M.; Martins, A.S.; Ullrich, N.D.; Nowycky, M.C.; Niggli, E.; Shirokova, N. Reciprocal amplification of ROS and $\mathrm{Ca}^{2+}$ signals in stressed mdx dystrophic skeletal muscle fibers. Pflugers Arch. 2009, 458, 915-928.

25. Sakellariou, G.K.; Vasilaki, A.; Palomero, J.; Kayani, A.; Zibrik, L.; McArdle, A.; Jackson, M.J. Studies of mitochondrial and nonmitochondrial sources implicate nicotinamide adenine dinucleotide phosphate oxidase(s) in the increased skeletal muscle superoxide generation that occurs during contractile activity. Antioxid. Redox Signal. 2013, 18, 603-621.

26. Cherednichenko, G.; Zima, A.V.; Feng, W.; Schaefer, S.; Blatter, L.A.; Pessah, I.N. NADH oxidase activity of rat cardiac sarcoplasmic reticulum regulates calcium-induced calcium release. Circ. Res. 2004, 94, 478-486.

27. Espinosa, A.; Leiva, A.; Peña, M.; Müller, M.; Debandi, A.; Hidalgo, C.; Carrasco, M.A.; Jaimovich, E. Myotube depolarization generates reactive oxygen species through $\mathrm{NAD}(\mathrm{P}) \mathrm{H}$ oxidase; ROS-elicited $\mathrm{Ca}^{2+}$ stimulates ERK, CREB, early genes. J. Cell Physiol. 2006, 209, 379-388.

28. Hidalgo, C.; Sánchez, G.; Barrientos, G.; Aracena-Parks, P. A transverse tubule NADPH oxidase activity stimulates calcium release from isolated triads via ryanodine receptor type 1 S-glutathionylation. J. Biol. Chem. 2006, 281, 26473-26482.

29. Espinosa, A.; García, A.; Härtel, S.; Hidalgo, C.; Jaimovich, E. NADPH oxidase and hydrogen peroxide mediate insulin-induced calcium increase in skeletal muscle cells. J. Biol. Chem. 2009, 284, 2568-2575.

30. Harrison, R. Structure and function of xanthine oxidoreductase: Where are we now? Free Radic. Biol. Med. 2002, 33, 774-797.

31. Judge, A.R.; Dodd, S.L. Xanthine oxidase and activated neutrophils cause oxidative damage to skeletal muscle after contractile claudication. Am. J. Physiol. Heart Circ. Physiol. 2004, 286, H252-H256.

32. Radák, Z.; Zhao, Z.; Koltai, E.; Ohno, H.; Atalay, M. Oxygen consumption and usage during physical exercise: The balance between oxidative stress and ROS-dependent adaptive signaling. Antioxid. Redox Signal. 2013, 18, 1208-1246. 
33. Kang, C.; Chung, E.; Diffee, G.; Ji, L.L. Exercise training attenuates aging-associated mitochondrial dysfunction in rat skeletal muscle: Role of PGC-1 $\alpha$. Exp. Gerontol. 2013, 48, 1343-1350.

34. Sriram, S.; Subramanian, S.; Sathiakumar, D.; Venkatesh, R.; Salerno, M.S.; McFarlane, C.D.; Kambadur, R.; Sharma, M. Modulation of reactive oxygen species in skeletal muscle by myostatin is mediated through NF-кB. Aging Cell 2011, 10, 931-948.

35. Sriram, S.; Subramanian, S.; Juvvuna, P.K.; Ge, X.; Lokireddy, S.; McFarlane, C.D.; Wahli, W.; Kambadur, R.; Sharma, M. Myostatin augments muscle-specific ring finger protein-1 expression through an NF- $\mathrm{B}$ independent mechanism in SMAD3 null muscle. Mol. Endocrinol. 2014, 28, 317-330.

36. Zuo, L.; Christofi, F.L.; Wright, V.P.; Bao, S.; Clanton, T.L. Lipoxygenase-dependent superoxide release in skeletal muscle. J. Appl. Physiol. 2004, 97, 661-668.

37. Zhao, X.; Bey, E.A.; Wientjes, F.B.; Cathcart, M.K. Cytosolic phospholipase A2 (cPLA2) regulation of human monocyte NADPH oxidase activity. cPLA2 affects translocation but not phosphorylation of p67(phox) and p47(phox). J. Biol. Chem. 2002, 277, 25385-25392.

38. Ježek, J.; Jaburek, M.; Zelenka, J.; Ježek, P. Mitochondrial phospholipase A2 activated by reactive oxygen species in heart mitochondria induces mild uncoupling. Physiol. Res. 2010, 59, 737-747.

39. Gong, M.C.; Arbogast, S.; Guo, Z.; Mathenia, J.; Su, W.; Reid, M.B. Calcium-independent phospholipase A2 modulates cytosolic oxidant activity and contractile function in murine skeletal muscle cells. J. Appl. Physiol. 2006, 100, 399-405.

40. Smith, M.A.; Reid, M.B. Redox modulation of contractile function in respiratory and limb skeletal muscle. Respir. Physiol. Neurobiol. 2006, 151, 229-241.

41. Radák, Z.; Taylor, A.W.; Ohno, H.; Goto, S. Adaptation to exercise-induced oxidative stress: From muscle to brain. Exerc. Immunol. Rev. 2001, 7, 90-107.

42. Reid, M.B. Free radicals and muscle fatigue: Of ROS, canaries, and the IOC. Free Radic. Biol. Med. 2008, 44, 169-179.

43. Allen, D.G.; Lamb, G.D.; Westerblad, H. Skeletal muscle fatigue: Cellular mechanisms. Physiol. Rev. 2008, 88, 287-332.

44. Lamb, G.D.; Westerblad, H. Acute effects of reactive oxygen and nitrogen species on the contractile function of skeletal muscle. J. Physiol. 2011, 589, 2119-2127.

45. Lamb, G.D.; Posterino, G.S. Effects of oxidation and reduction on contractile function in skeletal muscle fibres of the rat. J. Physiol. 2003, 546, 149-163.

46. Posterino, G.S.; Cellini, M.A.; Lamb, G.D. Effects of oxidation and cytosolic redox conditions on excitation-contraction coupling in rat skeletal muscle. J. Physiol. 2003, 547, 807-823.

47. Cheng, A.J.; Bruton, J.D.; Lanner, J.T.; Westerblad, H. Antioxidant treatments do not improve force recovery after fatiguing stimulation of mouse skeletal muscle fibres. J. Physiol. 2015, 593, 457-472. 
48. Andrade, F.H.; Reid, M.B.; Allen, D.G.; Westerblad, H. Effect of hydrogen peroxide and dithiothreitol on contractile function of single skeletal muscle fibres from the mouse. J. Physiol. 1998, 509, 565-575.

49. Prochniewicz, E.; Spakowicz, D.; Thomas, D.D. Changes in actin structural transitions associated with oxidative inhibition of muscle contraction. Biochemistry 2008, 47, 11811-11817.

50. Mollica, J.P.; Dutka, T.L.; Merry, T.L.; Lamboley, C.R.; McConell, G.K.; McKenna, M.J.; Murphy, R.M.; Lamb, G.D. S-glutathionylation of troponin I (fast) increases contractile apparatus $\mathrm{Ca}^{2+}$ sensitivity in fast-twitch muscle fibres of rats and humans. J. Physiol. 2012, 590, 1443-1463.

51. Jackson, M.J.; Papa, S.; Bolaños, J.; Bruckdorfer, R.; Carlsen, H.; Elliott, R.M.; Flier, J.; Griffiths, H.R.; Heales, S.; Holst, B.; et al. Antioxidants, reactive oxygen and nitrogen species, gene induction and mitochondrial function. Mol. Aspects Med. 2002, 23, 209-285.

52. Gumucio, J.P.; Mendias, C.L. Atrogin-1, MuRF-1, and sarcopenia. Endocrine 2013, 43, 12-21.

53. Witt, S.H.; Granzier, H.; Witt, C.C.; Labeit, S. MURF-1 and MURF-2 target a specific subset of myofibrillar proteins redundantly: Towards understanding MURF-dependent muscle ubiquitination. J. Mol. Biol. 2005, 350, 713-722.

54. Cohen, S.; Brault, J.J.; Gygi, S.P.; Glass, D.J.; Valenzuela, D.M.; Gartner, C.; Latres, E.; Goldberg, A.L. During muscle atrophy, thick, but not thin, filament components are degraded by MuRF1-dependent ubiquitylation. J. Cell Biol. 2009, 185, 1083-1095.

55. Ji, L.L. Antioxidants and oxidative stress in exercise. Proc. Soc. Exp. Biol. Med. 1999, 222, 283-292.

56. Smith, C.V.; Jones, D.P.; Guenther, T.M.; Lash, L.H.; Lauterburg, B.H. Compartmentation of glutathione: Implications for the study of toxicity and disease. Toxicol. Appl. Pharmacol. 1996, 140, 1-12.

57. Hayes, J.D.; Flanagan, J.U.; Jowsey, I.R. Glutathione transferases. Ann. Rev. Pharmacol. Toxicol. 2005, 45, 51-88.

58. Meister, A.; Anderson, M.E. Glutathione. Annu. Rev. Biochem. 1983, 52, 711-760.

59. Halliwell, B.; Gutteridge, J. Free Radicals in Biology and Medicine; Oxford University Press: New York, NY, USA, 2007.

60. Bellomo, G.; Mirabelli, F.; DiMonte, D.; Richelmi, P.; Thor, H.; Orrenius, C.; Orrenius, S. Formation and reduction of glutathione-protein mixed disulfides during oxidative stress. A study with isolated hepatocytes and menadione (2-methyl-1,4-naphthoquinone). Biochem. Pharmacol. 1987, 36, 1313-1320.

61. Ji, L.L.; Fu, R.; Mitchell, E.W. Glutathione and antioxidant enzymes in skeletal muscle: Effects of fiber type and exercise intensity. J. Appl. Physiol. 1992, 73, 1854-1859.

62. Lew, H.; Pyke, S.; Quintanilha, A. Changes in the glutathione status of plasma, liver and muscle following exhaustive exercise in rats. FEBS Lett. 1985, 185, 262-266.

63. Marin, E.; Kretzschmar, M.; Arokoski, J.; Hänninen, O.; Klinger, W. Enzymes of glutathione synthesis in dog skeletal muscles and their response to training. Acta Physiol. Scand. 1993, 147, 369-373. 
64. Leeuwenburgh, C.; Hollander, J.; Leichtweis, S.; Griffiths, M.; Gore, M.; Ji, L.L. Adaptations of glutathione antioxidant system to endurance training are tissue and muscle fiber specific. Am. J. Physiol. 1997, 272, R363-R369.

65. Ji, L.L. Exercise and oxidative stress: Role of the cellular antioxidant systems. Exerc. Sport Sci. Rev. 1995, 23, 135-166.

66. Sen, C.K. Oxidants and antioxidants in exercise. J. Appl. Physiol. 1995, 79, 675-686.

67. Fisher-Wellman, K.H.; Mattox, T.A.; Thayne, K.; Katunga, L.A.; la Favor, J.D.; Neufer, P.D.; Hickner, R.C.; Wingard, C.J.; Anderson, E.J. Novel role for thioredoxin reductase-2 in mitochondrial redox adaptations to obesogenic diet and exercise in heart and skeletal muscle. J. Physiol. 2013, 591, 3471-3486.

68. Ji, L.L. Modulation of skeletal muscle antioxidant defense by exercise: Role of redox signaling. Free Radic. Biol. Med. 2008, 44, 142-152.

69. Powers, S.K.; Criswell, D.; Lawler, J.; Martin, D.; Ji, L.L.; Herb, R.A.; Dudley, G. Regional training-induced alterations in diaphragmatic oxidative and antioxidant enzymes. Respir. Physiol. 1994, 95, 227-237.

70. Gore, M.; Fiebig, R.; Hollander, J.; Leeuwenburgh, C.; Ohno, H.; Ji, L.L. Endurance training alters antioxidant enzyme gene expression in rat skeletal muscle. Can. J. Physiol. Pharmacol. 1998, 76, 1139-1145.

71. Hollander, J.; Fiebig, R.; Gore, M.; Bejma, J.; Ookawara, T.; Ohno, H.; Ji, L.L. Superoxide dismutase gene expression in skeletal muscle: Fiber-specific adaptation to endurance training. Am. J. Physiol. 1999, 277, R856-R862.

72. Lambertucci, R.H.; Levada-Pires, A.C.; Rossoni, L.V.; Curi, R.; Pithon-Curi, T.C. Effects of aerobic exercise training on antioxidant enzyme activities and mRNA levels in soleus muscle from young and aged rats. Mech. Ageing Dev. 2007, 128, 267-275.

73. Ristow, M.; Zarse, K.; Oberbach, A.; Klöting, N.; Birringer, M.; Kiehntopf, M.; Stumvoll, M.; Kahn, C.R.; Blüher, M. Antioxidants prevent health-promoting effects of physical exercise in humans. Proc. Natl. Acad. Sci. USA 2009, 106, 8665-8670.

74. Ji, L.L.; Fu, R. Responses of glutathione system and antioxidant enzymes to exhaustive exercise and hydroperoxide. J. Appl. Physiol. 1992, 72, 549-554.

75. Lawler, J.M.; Powers, S.K.; Visser, T.; van Dijk, H.; Kordus, M.J.; Ji, L.L. Acute exercise and skeletal muscle antioxidant and metabolic enzymes: Effects of fiber type and age. Am. J. Physiol. 1993, 265, R1344-R1350.

76. Hollander, J.; Fiebig, R.; Gore, M.; Ookawara, T.; Ohno, H.; Ji, L.L. Superoxide dismutase gene expression is activated by a single bout of exercise in rat skeletal muscle. Pflugers Arch. 2001, 442, 426-434.

77. Hitomi, Y.; Watanabe, S.; Kizaki, T.; Sakurai, T.; Takemasa, T.; Haga, S.; Ookawara, T.; Suzuki, $\mathrm{K}$.; Ohno, H. Acute exercise increases expression of extracellular superoxide dismutase in skeletal muscle and the aorta. Redox. Rep. 2008, 13, 213-226.

78. Radák, Z.; Asano, K.; Inoue, M.; Kizaki, T.; Oh-Ishi, S.; Suzuki, K.; Taniguchi, N.; Ohno, H. Superoxide dismutase derivative reduces oxidative damage in skeletal muscle of rats during exhaustive exercise. J. Appl. Physiol. 1995, 79, 129-135. 
79. Alessio, H.M.; Goldfarb, A.H. Lipid peroxidation and scavenger enzymes during exercise: Adaptive response to training. J. Appl. Physiol. 1988, 64, 1333-1336.

80. Mastaloudis, A.; Leonard, S.W.; Traber, M.G. Oxidative stress in athletes during extreme endurance exercise. Free Radic. Biol. Med. 2001, 31, 911-922.

81. Steensberg, A.; Morrow, J.; Toft, A.D.; Bruunsgaard, H.; Pedersen, B.K. Prolonged exercise, lymphocyte apoptosis and F2-isoprostanes. Eur. J. Appl. Physiol. 2002, 87, 38-42.

82. Ashton, T.; Rowlands, C.C.; Jones, E.; Young, I.S.; Jackson, S.K.; Davies, B.; Peters, J.R. Electron spin resonance spectroscopic detection of oxygen-centred radicals in human serum following exhaustive exercise. Eur. J. Appl. Physiol. Occup. Physiol. 1998, 77, 498-502.

83. Vasankari, T.; Kujala, U.; Heinonen, O.; Kapanen, J.; Ahotupa, M. Measurement of serum lipid peroxidation during exercise using three different methods: Diene conjugation, thiobarbituric acid reactive material and fluorescent chromolipids. Clin. Chim. Acta 1995, 234, 63-69.

84. Umegaki, K.; Daohua, P.; Sugisawa, A.; Kimura, M.; Higuchi, M. Influence of one bout of vigorous exercise on ascorbic acid in plasma and oxidative damage to DNA in blood cells and muscle in untrained rats. J. Nutr. Biochem. 2000, 11, 401-407.

85. Niess, A.M.; Hartmann, A.; Grünert-Fuchs, M.; Poch, B.; Speit, G. DNA damage after exhaustive treadmill running in trained and untrained men. Int. J. Sports Med. 1996, 17, 397-403.

86. Powers, S.K.; Criswell, D.; Lawler, J.; Ji, L.L.; Martin, D.; Herb, R.A.; Dudley, G. Influence of exercise and fiber type on antioxidant enzyme activity in rat skeletal muscle. Am. J. Physiol. 1994, 266, R375-R380.

87. Radák, Z.; Naito, H.; Kaneko, T.; Tahara, S.; Nakamoto, H.; Takahashi, R.; Cardozo-Pelaez, F.; Goto, S. Exercise training decreases DNA damage and increases DNA repair and resistance against oxidative stress of proteins in aged rat skeletal muscle. Pflugers Arch. 2002, 445, 273-278.

88. Radák, Z.; Chung, H.Y.; Goto, S. Systemic adaptation to oxidative challenge induced by regular exercise. Free Radic. Biol. Med. 2008, 44, 153-159.

89. Alleman, R.J.; Katunga, L.A.; Nelson, M.A.M.; Brown, D.A.; Anderson, E.J. The "Goldilocks Zone" from a redox perspective-Adaptive $v s$. deleterious responses to oxidative stress in striated muscle. Front. Physiol. 2014, doi:10.3389/fphys.2014.00358.

90. Kalogeris, T.; Bao, Y.; Korthuis, R.J. Mitochondrial reactive oxygen species: A double edged sword in ischemia/reperfusion vs. preconditioning. Redox Biol. 2014, 2, 702-714.

91. Dodd, S.L.; Gagnon, B.J.; Senf, S.M.; Hain, B.A.; Judge, A.R. Ros-mediated activation of NF-kappaB and Foxo during muscle disuse. Muscle Nerve 2010, 41, 110-113.

92. Derbre, F.; Ferrando, B.; Gomez-Cabrera, M.C.; Sanchis-Gomar, F.; Martinez-Bello, V.E.; Olaso-Gonzalez, G.; Diaz, A.; Gratas-Delamarche, A.; Cerda, M.; Viña, J. Inhibition of xanthine oxidase by allopurinol prevents skeletal muscle atrophy: Role of p38 MAPKinase and E3 ubiquitin ligases. PLOS ONE 2012, 7, e46668. 
93. Kröller-Schön, S.; Jansen, T.; Hauptmann, F.; Schüler, A.; Heeren, T.; Hausding, M.; Oelze, M.; Viollet, B.; Keaney, J.F., Jr.; Wenzel, P.; et al. a1AMP-activated protein kinase mediates vascular protective effects of exercise. Arterioscler. Thromb. Vasc. Biol. 2012, 32, 1632-1641.

94. Morgan, M.J.; Liu, Z.G. Crosstalk of reactive oxygen species and NF-кB signaling. Cell Res. 2011, 21, 103-115.

95. McArdle, F.; Spiers, S.; Aldemir, H.; Vasilaki, A.; Beaver, A.; Iwanejko, L.; McArdle, A.; Jackson, M.J. Preconditioning of skeletal muscle against contraction-induced damage: The role of adaptations to oxidants in mice. J. Physiol. 2004, 561, 233-244.

96. Eckl, P.M.; Ortner, A.; Esterbauer, H. Genotoxic properties of 4-hydroxyalkenals and analogous aldehydes. Mutat. Res. 1993, 290, 183-192.

97. Poli, G.; Schaur, R.J.; Siems, W.G.; Leonarduzzi, G. 4-hydroxynonenal: A membrane lipid oxidation product of medicinal interest. Med. Res. Rev. 2008, 28, 569-631.

98. Ishii, T.; Itoh, K.; Ruiz, E.; Leake, D.S.; Unoki, H.; Yamamoto, M.; Mann, G.E. Role of Nrf2 in the regulation of $\mathrm{CD} 36$ and stress protein expression in murine macrophages: Activation by oxidatively modified LDL and 4-hydroxynonenal. Circ. Res. 2004, 94, 609-616.

99. Levonen, A.L.; Landar, A.; Ramachandra, A.; Cease, E.K.; Dickinson, D.A.; Zanoni, G.; Morrow, J.D.; Darley-Usmar, V.M. Cellular mechanisms of redox cell signalling: Role of cysteine modification in controlling antioxidant defences in response to electrophilic lipid oxidation products. Biochem. J. 2004, 378, 373-382.

100. Echtay, K.S.; Esteves, T.C.; Pakay, J.L.; Jekabsons, M.B.; Lambert, A.J.; Portero-Otín, M.; Pamplona, R.; Vidal-Puig, A.J.; Wang, S.; Roebuck, S.J.; et al. A signalling role for 4-hydroxy-2-nonenal in regulation of mitochondrial uncoupling. EMBO J. 2003, 22, 4103-4110.

101. Gomez-Cabrera, M.C.; Borrás, C.; Pallardó, F.V.; Sastre, J.; Ji, L.L.; Viña, J. Decreasing xanthine oxidase-mediated oxidative stress prevents useful cellular adaptations to exercise in rats. J. Physiol. 2005, 567, 113-120.

102. Gomez-Cabrera, M.C.; Domenech, E.; Viña, J. Moderate exercise is an antioxidant: Upregulation of antioxidant genes by training. Free Radic. Biol. Med. 2008, 44, 126-131.

103. Ji, L.L.; Gomez-Cabrera, M.C.; Steinhafel, N.; Viña, J. Acute exercise activates nuclear factor (NF)-kappaB signaling pathway in rat skeletal muscle. FASEB J. 2004, 18, 1499-1506.

104. Margonis, K.; Fatouros, I.G.; Jamurtas, A.Z.; Nikolaidis, M.G.; Douroudos, I.; Chatzinikolaou, A.; Mitrakou, A.; Mastorakos, G.; Papassotiriou, I.; Taxildaris, K.; et al. Oxidative stress biomarkers responses to physical overtraining: Implications for diagnosis. Free Radic. Biol. Med. 2007, 43, 901-910.

105. Palazzetti, S.; Richard, M.J.; Favier, A.; Margaritis, I. Overloaded training increases exercise-induced oxidative stress and damage. Can. J. Appl. Physiol. 2003, 28, 588-604.

106. Tanskanen, M.; Atalay, M.; Uusitalo, A. Altered oxidative stress in overtrained athletes. J. Sports Sci. 2010, 28, 309-317. 
107. Irrcher, I.; Adhihetty, P.J.; Sheehan, T.; Joseph, A.M.; Hood, D.A. PPAR $\gamma$ coactivator-1 $\alpha$ expression during thyroid hormone- and contractile activity-induced mitochondrial adaptations. Am. J. Physiol. Cell Physiol. 2003, 284, C1669-C1677.

108. Holloszy, J.O. Regulation by exercise of skeletal muscle content of mitochondria and GLUT4. J. Physiol. Pharmacol. 2008, 59, 5-18.

109. Yan, Z.; Okutsu, M.; Akhtar, Y.N.; Lira, V.A. Regulation of exercise-induced fiber type transformation, mitochondrial biogenesis, and angiogenesis in skeletal muscle. J. Appl. Physiol. 2011, 110, 264-274.

110. Van Loon, L.J.C.; Goodpaster, B.H. Increased intramuscular lipid storage in the insulin-resistant and endurance-trained state. Pflugers Arch. 2006, 451, 606-616.

111. Hawley, J.A.; Lessard, S.J. Exercise training-induced improvements in insulin action. Acta Physiol. 2008, 192, 127-135.

112. Olesen, J.; Kiilerich, K.; Pilegaard, H. PGC-1 $\alpha$-mediated adaptations in skeletal muscle. Pflugers Arch. 2010, 460, 153-162.

113. Kang, C.; Ji, L.L. Role of PGC-1 $\alpha$ in muscle function and aging. J. Sport Health Sci. 2013, 2, 81-86.

114. Pilegaard, H.; Osada, T.; Andersen, L.T.; Helge, J.W.; Saltin, B.; Neufer, P.D. Substrate availability and transcriptional regulation of metabolic genes in human skeletal muscle during recovery from exercise. Metabolism 2005, 54, 1048-1055.

115. Krämer, D.K.; Ahlsen, M.; Norrbom, J.; Jansson, E.; Hjeltnes, N.; Gustafsson, T.; Krook, A. Human skeletal muscle fibre type variations correlate with PPAR $\alpha, \operatorname{PPAR} \delta$ and PGC-1 $\alpha$ mRNA. Acta Physiol. 2006, 188, 207-216.

116. Mathai, A.S.; Bonen, A.; Benton, C.R.; Robinson, D.L.; Graham, T.E. Rapid exercise-induced changes in PGC-1 $\alpha$ mRNA and protein in human skeletal muscle. J. Appl. Physiol. 2008, 105, 1098-1105.

117. Little, J.P.; Safdar, A.; Bishop, D.; Tarnopolsky, M.A.; Gibala, M.J. An acute bout of high-intensity interval training increases the nuclear abundance of PGC- $1 \alpha$ and activates mitochondrial biogenesis in human skeletal muscle. Am. J. Physiol. Regul. Integr. Comp. Physiol. 2011, 300, R1303-R1310.

118. Cobley, J.N.; Bartlett, J.D.; Kayani, A.; Murray, S.W.; Louhelainen, J.; Donovan, T.; Waldron, S.; Gregson, W.; Burniston, J.G.; Morton, J.P.; et al. PGC-1 $\alpha$ transcriptional response and mitochondrial adaptation to acute exercise is maintained in skeletal muscle of sedentary elderly males. Biogerontology 2012, 13, 621-631.

119. Wright, D.C.; Han, D.H.; Garcia-Roves, P.M.; Geiger, P.C.; Jones, T.E.; Holloszy, J. Exercise-induced mitochondrial biogenesis begins before the increase in muscle PGC-1 $\alpha$ expression. J. Biol. Chem. 2007, 282, 194-199.

120. Wu, Z.; Puigserver, P.; Andersson, U.; Zhang, C.; Adelmant, G.; Mootha, V.; Troy, A.; Cinti, S.; Lowell, B.; Scarpulla, R.C.; et al. Mechanisms controlling mitochondrial biogenesis and respiration through the thermogenic coactivator PGC-1. Cell 1999, 98, 115-124. 
121. Koves, T.R.; Li, P.; An, J.; Akimoto, T.; Slentz, D.; Ilkayeva, O.; Dohm, G.L.; Yan, Z.; Newgard, C.B.; Muoio, D.M. Peroxisome proliferator-activated receptor- $\gamma$ co-activator $1 \alpha$-mediated metabolic remodeling of skeletal myocytes mimics exercise training and reverses lipid-induced mitochondrial inefficiency. J. Biol. Chem. 2005, 280, 33588-33598.

122. Lin, J.; Wu, H.; Tarr, P.T.; Zhang, C.Y.; Wu, Z.; Boss, O.; Michael, L.F.; Puigserver, P.; Isotani, E.; Olson, E.N.; et al. Transcriptional co-activator PGC-1 $\alpha$ drives the formation of slow-twitch muscle fibres. Nature 2002, 418, 797-801.

123. Handschin, C.; Chin, S.; Li, P.; Liu, F.; Maratos-Flier, E.; Lebrasseur, N.K.; Yan, Z.; Spiegelman, B.M. Skeletal muscle fiber-type switching, exercise intolerance, and myopathy in PGC-1 $\alpha$ muscle-specific knock-out animals. J. Biol. Chem. 2007, 282, 30014-30021.

124. Puigserver, P.; Spiegelman, B.M. Peroxisome proliferator-activated receptor- $\gamma$ coactivator $1 \alpha$ (PGC-1 $\alpha$ ): Transcriptional coactivator and metabolic regulator. Endocr. Rev. 2003, 24, 78-90.

125. Scarpulla, R.C.; Vega, R.B.; Kelly, D.P. Transcriptional integration of mitochondrial biogenesis. Trends Endocrinol. Metab. 2012, 23, 459-466.

126. Oberkofler, H.; Schraml, E.; Krempler, F.; Patsch, W. Potentiation of liver X receptor transcriptional activity by peroxisome-proliferator-activated receptor $\gamma$ co-activator $1 \alpha$. Biochem. J. 2003, 371, 89-96.

127. Summermatter, S.; Baum, O.; Santos, G.; Hoppeler, H.; Handschin, C. Peroxisome proliferator-activated receptor $\gamma$ coactivator $1 \alpha(\mathrm{PGC}-1 \alpha)$ promotes skeletal muscle lipid refueling in vivo by activating de novo lipogenesis and the pentose phosphate pathway. J. Biol. Chem. 2010, 285, 32793-32800.

128. Arany, Z.; Foo, S.Y.; Ma, Y.; Ruas, J.L.; Bommi-Reddy, A.; Girnun, G.; Cooper, M.; Laznik, D.; Chinsomboon, J.; Rangwala, S.M.; et al. HIF-independent regulation of VEGF and angiogenesis by the transcriptional coactivator PGC-1 $\alpha$. Nature 2008, 451, 1008-1012.

129. Chinsomboon, J.; Ruas, J.; Gupta, R.K.; Thom, R.; Shoag, J.; Rowe, G.C.; Sawada, N.; Raghuram, S.; Arany, Z. The transcriptional coactivator PGC-1 $\alpha$ mediates exercise-induced angiogenesis in skeletal muscle. Proc. Natl. Acad. Sci. USA 2009, 106, 21401-21406.

130. Chin, E.R.; Olson, E.N.; Richardson, J.A.; Yang, Q.; Humphries, C.; Shelton, J.M.; Wu, H.; Zhu, W.; Bassel-Duby, R.; Williams, R.S. A calcineurin-dependent transcriptional pathway controls skeletal muscle fiber type. Genes Dev. 1998, 12, 2499-2509.

131. Wu, H.; Naya, F.J.; McKinsey, T.A.; Mercer, B.; Shelton, J.M.; Chin, E.R.; Simard, A.R.; Michel, R.N.; Bassel-Duby, R.; Olson, E.N.; et al. MEF2 responds to multiple calcium-regulated signals in the control of skeletal muscle fiber type. EMBO J. 2000, 19, 1963-1973.

132. Potthoff, M.J.; Wu, H.; Arnold, M.A.; Shelton, J.M.; Backs, J.; McAnally, J.; Richardson, J.A.; Bassel-Duby, R.; Olson, E.N. Histone deacetylase degradation and MEF2 activation promote the formation of slow-twitch myofibers. J. Clin. Investig. 2007, 117, 2459-2467.

133. Handschin, C.; Rhee, J.; Lin, J.; Tarr, P.T.; Spiegelman, B.M. An autoregulatory loop controls peroxisome proliferator-activated receptor $\gamma$ coactivator $1 \alpha$ expression in muscle. Proc. Natl. Acad. Sci. USA 2003, 100, 7111-7116. 
134. Kang, C.; O’Moore, K.M.; Dickman, J.R.; Ji, L.L. Exercise activation of muscle peroxisome proliferator-activated receptor-gamma coactivator-1 $\alpha$ signaling is redox sensitive. Free Radic. Biol. Med. 2009, 47, 1394-1400.

135. Strobel, N.A.; Matsumoto, A.; Peake, J.M.; Marsh, S.A.; Peternelj, T.T.; Briskey, D.; Fassett, R.G.; Coombes, J.S.; Wadley, G.D. Altering the redox state of skeletal muscle by glutathione depletion increases the exercise-activation of PGC-1 $\alpha$. Physiol. Rep. 2014, 2, e12224.

136. St-Pierre, J.; Drori, S.; Uldry, M.; Silvaggi, J.M.; Rhee, J.; Jäger, S.; Handschin, C.; Zheng, K.; Lin, J.; Yang, W.; et al. Suppression of reactive oxygen species and neurodegeneration by the PGC-1 transcriptional coactivators. Cell 2006, 127, 397-408.

137. Kong, X.; Wang, R.; Xue, Y.; Liu, X.; Zhang, H.; Chen, Y.; Fang, F.; Chang, Y. Sirtuin 3, a new target of PGC-1 $\alpha$, plays an important role in the suppression of ROS and mitochondrial biogenesis. PLoS ONE 2010, 5, e11707.

138. Ahn, B.H.; Kim, H.S.; Song, S.; Lee, I.H.; Liu, J.; Vassilopoulos, A.; Deng, C.X.; Finkel, T. A role for the mitochondrial deacetylase Sirt3 in regulating energy homeostasis. Proc. Natl. Acad. Sci. USA 2008, 105, 14447-14452.

139. Finley, L.W.; Haas, W.; Desquiret-Dumas, V.; Wallace, D.C.; Procaccio, V.; Gygi, S.P.; Haigis, M.C. Succinate dehydrogenase is a direct target of sirtuin 3 deacetylase activity. PLoS ONE 2011, 6, e23295.

140. Qiu, X.; Brown, K.; Hirschey, M.D.; Verdin, E.; Chen, D. Calorie restriction reduces oxidative stress by SIRT3-mediated SOD2 activation. Cell Metab. 2010, 12, 662-667.

141. Tao, R.; Coleman, M.C.; Pennington, J.D.; Ozden, O.; Park, S.H.; Jiang, H.; Kim, H.S.; Flynn, C.R.; Hill, S.; Hayes McDonald, W.; et al. Sirt3-mediated deacetylation of evolutionarily conserved lysine 122 regulates MnSOD activity in response to stress. Mol. Cell 2010, 40, 893-904.

142. Chen, Y.; Zhang, J.; Lin, Y.; Lei, Q.; Guan, K.L.; Zhao, S.; Xiong, Y. Tumour suppressor SIRT3 deacetylates and activates manganese superoxide dismutase to scavenge ROS. EMBO Rep. 2011, 12, 534-541.

143. Wenz, T.; Rossi, S.G.; Rotundo, R.L.; Spiegelman, B.M.; Moraes, C.T. Increased muscle PGC-1 $\alpha$ expression protects from sarcopenia and metabolic disease during aging. Proc. Natl. Acad. Sci. USA 2009, 106, 20405-20410.

144. St-Pierre, J.; Lin, J.; Krauss, S.; Tarr, P.T.; Yang, R.; Newgard, C.B.; Spiegelman, B.M. Bioenergetic analysis of peroxisome proliferator-activated receptor $\gamma$ coactivators $1 \alpha$ and $1 \beta$ (PGC-1 $\alpha$ and PGC-1 $\beta$ ) in muscle cells. J. Biol. Chem. 2003, 278, 26597-26603. 


\section{Oxidative Stress and Maxi Calcium-Activated Potassium (BK) Channels}

\section{Anton Hermann, Guzel F. Sitdikova and Thomas M. Weiger}

Abstract: All cells contain ion channels in their outer (plasma) and inner (organelle) membranes. Ion channels, similar to other proteins, are targets of oxidative impact, which modulates ion fluxes across membranes. Subsequently, these ion currents affect electrical excitability, such as action potential discharge (in neurons, muscle, and receptor cells), alteration of the membrane resting potential, synaptic transmission, hormone secretion, muscle contraction or coordination of the cell cycle. In this chapter we summarize effects of oxidative stress and redox mechanisms on some ion channels, in particular on maxi calcium-activated potassium (BK) channels which play an outstanding role in a plethora of physiological and pathophysiological functions in almost all cells and tissues. We first elaborate on some general features of ion channel structure and function and then summarize effects of oxidative alterations of ion channels and their functional consequences.

Reprinted from Biomolecules. Cite as: Hermann, A.; Sitdikova, G.F.; Weiger, T.M. Oxidative Stress and Maxi Calcium-Activated Potassium (BK) Channels. Biomolecules 2015, 5, 1870-1911.

\section{Introduction}

Ion channels play a pivotal role for the functioning of any cell in the animal as well as in the plant kingdom. An important class of ion channels is the family of potassium $\left(\mathrm{K}^{+}\right)$channels, they are not only in charge of the membrane resting potential or the repolarization of the action potentials, but also control cell proliferation or transmitter/hormone release, to name a few. A subgroup of $\mathrm{K}^{+}$ channels are the so called calcium $\left(\mathrm{Ca}^{2+}\right)$ activated $\mathrm{K}^{+}$channels which need either an increase of $\mathrm{Ca}^{2+}$ at their intracellular face to open or a combination of $\mathrm{Ca}^{2+}$ and voltage to function properly. Maxi $\mathrm{Ca}^{2+}$ activated $\mathrm{K}^{+}$channels, also named BK channels which constitute a subgroup of $\mathrm{Ca}^{2+}$ activated $\mathrm{K}^{+}$channels, are in the focus of our review. These channels modulate a number of physiological events, like blood pressure, smooth muscle relaxation or electrical tuning of hair cells in the cochlea and have a leading role in many pathophysiological conditions such as epilepsy or the behavioral response to alcohol, to give only a few examples. Oxidative stress on the other side is a physiological byproduct of any aerobic metabolic process and as such common for cells to deal with. Oxidation modulates many pathways in the cell including activity of ion channels like BK channels. The modulatory actions of oxidative stress on BK channels will be in the focal point of this paper. First, in Section 2, we will address general properties of ion channels and in particular the qualities of BK channels. In Section 3, we will specify what we mean by the term oxidative stress, while Section 4 will deal with the impact of redox modulation of BK channels. At the end, we will discuss these findings in the light of their clinical relevance and conclude with perspectives and vistas. 


\section{2. $\mathrm{Ca}^{2+}$-Activated $\mathrm{K}^{+}$Channels $\left(\mathrm{K}_{\mathrm{Ca}}\right)$}

\subsection{General Introduction to Ion Channels}

The task of cell membranes (plasma/organelles) is to separate cellular compartments, such as extra- and intracellular or extra- and intra-organelle areas to allow for proper performance of biochemical processes. Since the lipid bilayer membranes are almost impermeable for ions, tunnel proteins are inserted to allow for communication between compartments for electrical and chemical signaling. In addition membranes themselves are sites for processing biochemical reactions. Readers familiar with ion channels may omit this section on general features of ion channels.

Since cellular ion channels form minute pores across membranes they are suitable for the passage of ions, in particular monovalent sodium $\left(\mathrm{Na}^{+}\right), \mathrm{K}^{+}$, protons $\left(\mathrm{H}^{+}\right)$, the divalent cation $\mathrm{Ca}^{2+}$ or the anion chloride $\left(\mathrm{Cl}^{-}\right)$. These ions are differently concentrated across cell membranes and various mechanisms are involved in the separation of ions, i.e., (a) active transport systems, which directly consume energy (adenosine triphosphate, ATP) to transport ions against a concentration gradient; (b) transport systems which do not directly consume ATP but use the concentration gradient across the membrane as driving force; or (c) the redistribution of ions due to fixed negative charges provided mostly by intracellular proteins (Donnan equilibrium). In essence, this creates an electro-chemical gradient for each ion which provides for a driving force since for example at the membrane resting potential, endowed in all living cells, none of the ions is at equilibrium [1].

Most ion channels are gated, which means they contain an intrinsic mechanism which allows for either a closed or open configuration. Once the pore is open ions can flow according to their electrochemical gradient and as moving charges produce a current which provides a potential difference across the membrane. Hence, in difference to our technical current which is brought about by a flow of electrons, living cells use ions as charge carriers.

An enormous amount of knowledge about the structure and function of ion channels has been gathered over the last decades. This development was based on the seminal work by Hodgkin and Huxley (1952) [2]. They provided the basis for proper measurement of membrane potentials and they measured the ionic current flow across cell membranes by application of the "voltage clamp" technique which inspired generations of scientists. Only about 30 years later Neher and Sackmann (1981) [3], by installing the "patch clamp" method, succeeded to measure the flow of ions through single channels in native cell membranes. This technique, in fact allows us to visualize a protein at work. Again, 20 years later, MacKinnon and his group (1998) [4] succeeded to combine molecular-, electrophysiological techniques and structural analysis to develop a 3-dimensional scheme of an ion channel and its functions. It is clear now that there are many more types of ion channels than previously imagined. Although not all details concerning ion channels have been resolved to date, however, the knowledge gathered by scientists on structure and function over the last decades is overwhelming.

In general, ion channel proteins consist of hydrophobic amino acid $\alpha$-helices inserted into the membrane lipid bilayer connected by hydrophilic amino acid linkers. Cation channels usually contain four domains with two, four, or seven $\alpha$-helix segments. In some channels amino acids built one continuous fibrous structure (i.e., $\mathrm{Na}^{+}-, \mathrm{Ca}^{2+}$ channels), while others consist of four separate domains 
which form a tetrameric channel within the membrane (i.e., $\mathrm{K}^{+}$channels). In most channels " $\alpha$-subunits" form the pore for the passage of ions. Attached to the $\alpha$-subunits are in most cases a variety of other proteins — called auxiliary subunits $(\beta, \gamma, \varepsilon, \delta)[5]$, or special channel specific proteins, and/or enzymes, such as kinases, phosphatases or heme [5,6]. To classify ion channels by their mechanism of activation (gating) has been considered as a proper means.

The structure and function of ion channels has been summarized in several reviews [7-11]. The today's extensive realm of ion channel structure and function is brought about by the separation into many special topics, such as for $\mathrm{Na}^{+}, \mathrm{Ca}^{2+}, \mathrm{K}^{+}, \mathrm{Cl}^{-}$[12-16] channels. The field of ion channels developed immensely and we therefore limit our brief introduction to maxi calcium activated $\mathrm{K}^{+}$or BK channels which are the main focus of this review. $\mathrm{K}^{+}$channels can be devoid of auxiliary subunits but usually have various types of $\beta$-subunits, i.e., BK channels consist of four types of $\beta$ subunits $(\beta 1-\beta 4)$, and a set of $\gamma$-subunits (Figure 1). These subunits are of particular interest because of their potential to diversify the transport properties and distribution of channels in various cells and tissues. Attached to the $\alpha$-subunits these auxiliary subunits cause a variety of different effects on channel gating, on current kinetics or conductance, on trafficking the $\alpha$-subunit to the cell membrane, link to intracellular cytoskeleton and extracellular matrix proteins, modulation of channel expression or they provide for binding of drugs.

In general, the activity of ion channels which composes the ion current and hence the potential across the membrane is governed by three factors: the number of active channels $(\mathrm{N})$, the channels in the open state ( $\mathrm{Po}=$ open probability) and the single channel current (i). This can be summarized by the relationship: $\mathrm{I}_{\text {tot }}=\mathrm{N}$ Po $\mathrm{i}$, where $\mathrm{I}_{\text {tot }}$ is the total current, $\mathrm{N}$ is the number of functional channels and $\mathrm{i}$ is the single channel current. In most cases Po is modulated by ligands or drugs.

\subsection{Types of $K_{C a}$ Channels}

$\mathrm{K}_{\mathrm{Ca}}$ membrane permeability was first described from experiments using erythrocytes where the presence of internal $\mathrm{Ca}^{2+}$ increased the permeability of the cell plasma membrane to $\mathrm{K}^{+}$[17]. It took more than a decade to transfer this idea to other cells and to further investigate this phenomenon in more detail. To date a variety of $\mathrm{K}_{\mathrm{Ca}}$ channels have been described in a great variety of excitable and non-excitable cells of many species and are mainly defined by their biophysical and pharmacological properties.

The $\mathrm{K}_{\mathrm{Ca}}$ channel family contains eight members according to the sequence homology of transmembrane hydrophobic segments [18] with four subfamilies: BK (or $\mathrm{K}_{\mathrm{Ca}} 1.1$, Slo1, Maxi-K, KCNMA1), SK (KCa2.1 (SK1, KCNN1), KCa2.2 (SK2, KCNN2), KCa2.3 (SK3, KCNN3), IK (Kсa3.1 (IKCa1, SK4, KCNN4) and other subfamilies (KCa4.1 (KCNT1), $\mathrm{K}_{\mathrm{Ca}} 4.2$ (SLICK, Slo2.1, KCNT2), $\mathrm{K}_{\mathrm{Ca}}$ 5.1 (Slo3, KCNU1). These channels generally consist of four $\alpha$-subunits with six membrane-spanning $\alpha$-helices (segments) per domain (S1-S6) containing the $\mathrm{K}^{+}$pore. Both, $\mathrm{N}$ - and C-terminal are usually at the internal, cytosolic side of cells. In addition, Slo 1 (BK) and Slo 3 channels have one more transmembrane helix ( $\mathrm{S} 0$ ) which locates the N-terminus to the external side (see Figure 1 and section $\mathrm{BK}$ channels). Not all of these channels are responsive to $\mathrm{Ca}^{2+}$ - since some are activated by $\mathrm{Na}^{+}$(Slo 2 ) or $\mathrm{Cl}^{-}$(Slo 3 ). 


\subsection{Maxi Calcium-Activated $K^{+}$Channels (BK)}

BK channels are expressed by a single gene. The conductance of single BK channels is up to $300 \mathrm{pS}$ and hence comprises the largest single-channel conductance of all $\mathrm{K}^{+}$channels. To date a vast amount of information has been gathered regarding their biophysical, structural and functional, physiological, pathophysiological and pharmacological properties (recently reviewed in [19-37]). BK channels are usually synergistically activated by both- $\mathrm{Ca}^{2+} / \mathrm{Mg}^{2+}-$ metal ions and by membrane voltage. The channels can be also activated in the absence of $\mathrm{Ca}^{2+}$ but then require an extremely large depolarization of $100-200 \mathrm{mV}$ which is not physiologically relevant but important for the understanding of some biophysical properties of these channels. BK channel $\alpha$-subunits consist of a total of seven transmembrane segments with a S0 segment preceding the six transmembrane segments (S1-S6). This renders the N-terminus (amino terminal) at the extracellular side of the membrane (Figure 1). The transmembrane segments (S1-S4) consist of various charged amino acid residues which confer voltage sensitivity to the channels. The S0 segment appears mainly required for interaction of $\alpha$-subunits with auxiliary $\beta$-subunits as well as for targeting the channels to the plasma membrane. As in other voltage dependent $\mathrm{K}^{+}$channels, four pore-forming loops between the segments S5-S6 of each $\alpha$-subunit configure the ion selectivity filter. The large C-terminus (carboxyl terminal) comprises about two thirds of the $\alpha$-subunit protein and contains various binding sites for kinases, phosphatases and negatively charged $\mathrm{Ca}^{2+}$ binding sites as well as two so called RCK-domains (regulatory domain of $\mathrm{K}^{+}$conductance) (Figure 1).

Multiple splice variants of the $\alpha$-subunit have been identified resulting in a great variety of channel properties in various cell types [38]. Through alternative splicing the pore forming $\alpha$-subunit contains at its C-terminus a cysteine-rich 59-amino-acid insert between RCK domains called stress-axis regulated exon (STREX). STREX exon expression is under physiological conditions initiated by the stress-axis adrenocorticotropic hormone [39]. STREX inserts cause BK channels to activate at more negative potentials, enhance activation and decrease deactivation which leads to increased repetitive firing of action potentials. STREX inserts can also be artificially produced by growing cells in phenol red which causes a significant increase in channel sensitivity to inhibition by oxidation [40]. Phenol red, which is routinely used as a $\mathrm{pH}$ indicator in tissue culture media, bears structural resemblance to some nonsteroidal estrogens and has significant estrogenic activity [41]. Further findings indicate that maxi-K channel transcripts are differentially spliced by $17 \beta$-estradiol, which may contribute to changes in isoform expression [42] and may also lead to STREX expression in GH3 cells which carry estrogen receptors [43].

Specific blockers of the channels are tetraethylammonium (TEA) at submillimolar concentrations [45,46], iberiotoxin (from scorpion) or paxilline (mycotoxin from Penicillium paxilli) $[47,48]$ ). BK channels can be expressed together with other types of $\mathrm{K}_{\mathrm{Ca}}$ channels (SK or IK) [49] or in particular with $\mathrm{Ca}^{2+}$ channels [50,51] which makes them important modulators of electrical discharge activity and synaptic transmission. $\mathrm{Ca}^{2+}$ as a major player in cellular signaling is involved in a large variety of cell functions and links cell excitation to cell metabolism and gene expression. 


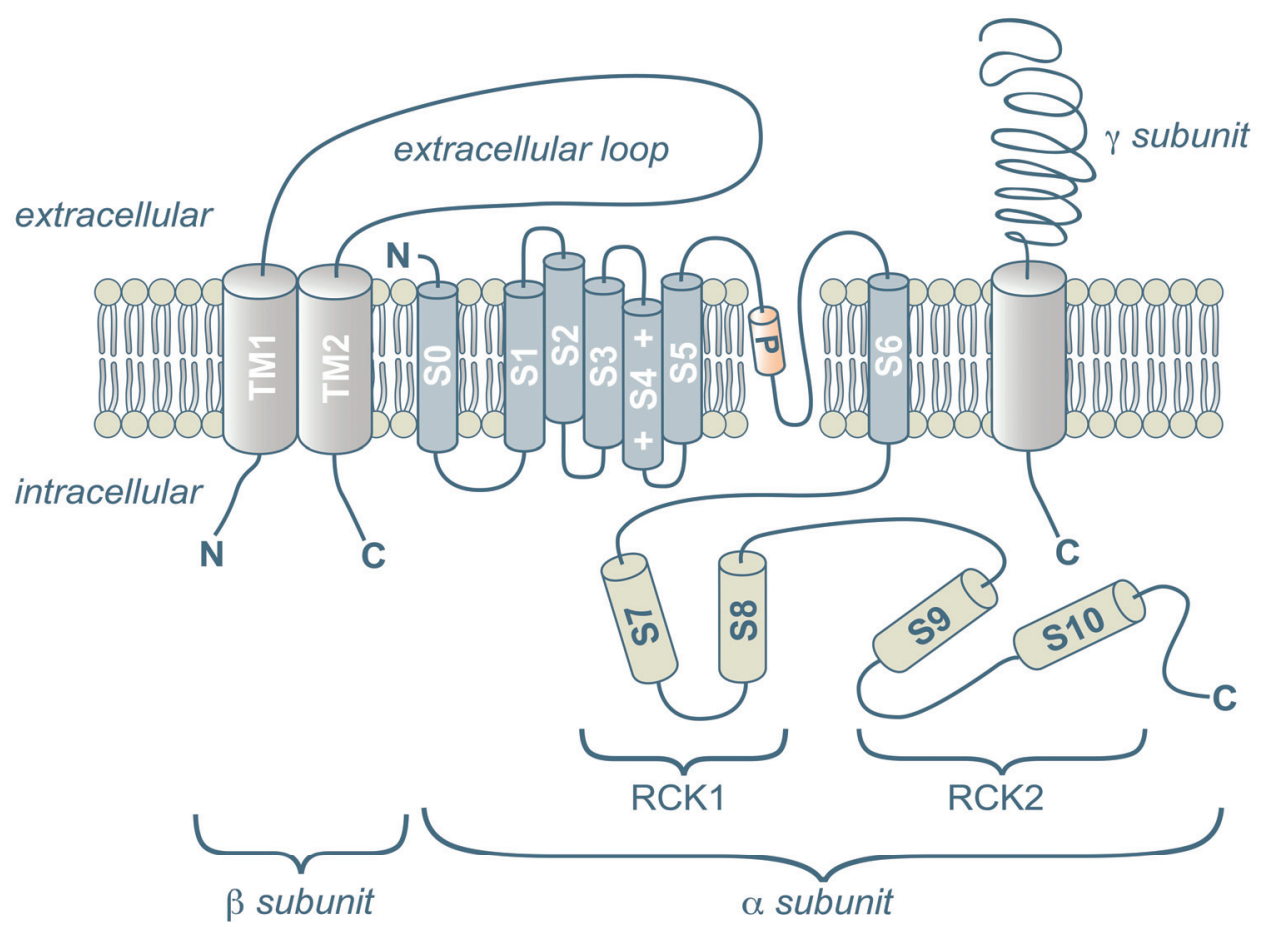

Figure 1. Schematic structure and membrane topology of maxi calcium-activated potassium (BK) channel $\alpha, \beta$ and $\gamma$ subunits (adapted from [44]). See text for a discussion of the structure.

BK channels are associated and modulated by a wide variety of intra- and extracellular factors, such as auxiliary subunits $(\beta, \gamma)$, Slobs (slo binding protein), phosphorylation, gasotransmitter action (nitrosylation, carboxylation, sulfhydration) or by redox mechanisms [11,44,52-67]. The BK $\alpha$-subunits assemble 1:1 with four different auxiliary types of $\boldsymbol{\beta}$-subunits $(\beta 1, \beta 2, \beta 3$ or $\beta 4)$ (Figure 1). $\beta$-subunits are members of the protein superfamily of oxidoreductases $[68,69]$ which contain 191-235 amino acids [70-73]. BK $\beta$-subunits consist of two transmembrane segments (TM1, TM2) with both, the $\mathrm{N}$-terminal and the $\mathrm{C}$-terminal located intracellularly. In different tissues four different genes can be expressed, as in smooth muscle, adrenal chromaffin cells or in neurons. $\beta$-subunits are involved in modifying voltage sensitivity, current kinetics and/or pharmacological properties of BK channels $[65,70,74]$. $\beta$-subunits are also responsible for tissue specificity, they can alter channel activity by activation of protein kinases, confer hormone (estradiol) activation, and alter toxin binding to the channels [75]. They are involved in current inactivation by a flexible N-terminal chain and ball structure which blocks the channel's pore [76,77]. In the brain, beta 4-subunits for example, inhibit BK channel activation and slow down channel kinetics [65,78-80], confer resistance to peptide blockers such as charybdotoxin and iberiotoxin $[65,81,82]$ or protect against temporal lobe seizures [79].

Various leucine-rich repeat containing proteins (LRRCxx), have been identified and designated as auxiliary $\gamma$-subunits (Figure 1) $[66,83,84]$. These proteins, which are clearly distinct from 
$\beta$-subunits, interact with BK channel $\alpha$-subunits. Various types of $\gamma$-subunits cause in the absence of $\mathrm{Ca}^{2+}$ a negative shift of up to $\sim 140 \mathrm{mV}$ in the voltage dependence of the BK channel activation, i.e., the channels open at voltages near the membrane resting potential. This way they exert interesting physiological functions in a great variety of tissues, such as the nervous system, skeletal muscle, and adrenal glands. For example, in fetal nervous tissue using knock-down studies, $\gamma 1$-subunits appear to participate in governing neuronal excitability in the early development of the brain [83] and in rat cerebral arterial smooth muscle LRRC26 constitutes functional $\gamma$-subunits involved in vaso-regulation [84]. In non-excitable cells, such as in salivary glands, prostate, testes or the airway epithelia, the hyperpolarizing actions of $\gamma$-subunits provide for an increase of the driving force for $\mathrm{Ca}^{2+}$ via non-voltage dependent (ligand gated) $\mathrm{Ca}^{2+}$ channels. It was suggested that $\gamma$-subunits may offer therapeutic potential targets as BK channel openers for the development of new BK channel-activating drugs in the treatment of various diseases [66].

A further class of proteins, called Slo binding proteins (Slobs) can attach to and modulate BK channels [85-88]. Slob 57, for example, shifts the voltage dependence to more depolarized voltages and causes faster closure of the channels [87]. Furthermore, Slob exerts a diurnal cycle in vivo [89] indicating that BK channel activity changes as a function of day time imparting circadian rhythmicity to neurons. Other Slobs like Slob71 or Slob 53, shift the voltage dependence to less depolarized voltages but have no effect on channel kinetics [88].

Some BK channels can be activated by stretch or pressure. These stretch-activated BK channels (SAKCaC) $[90,91]$ are expressed in a variety of tissues such as in myocytes or neurons and modulate vascular smooth muscle tone and endocrine cell secretion. A STREX insert between RCK1 and RCK2 domains at the channel's C-terminal $\alpha$-subunit is indicated to confer stretch sensitivity to the channels [90,92]. However, other BK channels lacking the STREX insert still remain sensitive to membrane stretch suggesting that additional structures of the channel may be responsible for mechanical coupling to the cell membrane [93].

\subsection{Function of BK Channels in Norm and Pathology}

BK channels contribute to various functions, such as controlling electrical discharge activity of nerve and muscle cells. Since the opening of $\mathrm{K}^{+}$channels drives the membrane potential towards the $\mathrm{K}^{+}$equilibrium potential, this will result in hyperpolarization of the membrane resting potential which will lead a nerve cell away from excitation. On the other hand, it may speed up the repolarization of action potentials, which makes their duration shorter and more action potentials per time can be generated. At the synapse the duration of the excitation, i.e., duration of the action potential is translated into the amount of opening of $\mathrm{Ca}^{2+}$ channels, influx of $\mathrm{Ca}^{2+}$ into the cell and an increase of the internal $\mathrm{Ca}^{2+}$ concentration which directly relates to the quantity of transmitter release or hormone secretion [94]. Similar mechanisms play a role in vaso-regulation, auditory tuning of the hair cells, in erectile processes and participate in mediating drug actions such as ethanol or acetaldehyde. In circadian rhythm generation BK channels are expressed in neurons of the supra chiasmatic nucleus during night and are removed during the day causing silencing or excitation, respectively [95]. Targeting of BK channels to appropriate membranes is important for the proper functioning of cells and organelles. Trafficking to and expression of BK channels in the plasma membrane has been 
found to be regulated by distinct splicing motifs located within the intracellular C-terminal RCK domains [96]. In particular a splice variant that excluded these motifs prevented cell surface expression of BK channels and suggests that such a mechanism impacts physiology and pathophysiology.

BK channels are not only present in the plasma membrane of cells but are also located in the membranes of cellular organelles such as mitochondria, nucleus, endoplasmic reticulum or the Golgi apparatus (reviewed in [97]). Information on the function and regulation of BK channel in organelles is at its infancy. A recent report indicates that nuclear BK channels (nBK) regulate gene expression of hippocampal neurons in a synaptic activity-evoked, $\mathrm{Ca}^{2+}$ dependent manner, suggesting that $\mathrm{nBK}$ channels play an important role as modulator and molecular linker of neuronal activity dependent functions and nuclear $\mathrm{Ca}^{2+}$ [98]. Since most information is available from mitochondria we will briefly discuss these channels in a later section.

Channelopathies are caused by mutations at one or more amino acids of the channel protein which may lead to dysfunction of cells and organs. These mutations can occur at any site of the channel protein, the pore, the voltage sensor, or the inactivation structures, including auxiliary subunits. Channelopathies affect all kinds of channels (voltage-, ligand-, mechano-gated, etc.), and hence are involved in a multitude of disorders, such as epilepsy, stroke, paroxysmal movements, cerebellar ataxia, hearing loss, autism, asthma mental deficiency, myotonia, heart diseases, hypertension, cystic fibrosis, bladder or gastric hypermotility, erectile dysfunctions, etc. (for recent reviews see [25,32,99-102]). BK channel mRNA expression is lower in the prefrontal cortex of schizophrenic, autistic, and mentally retarded persons $[103,104]$. Mutation at the $\alpha$-subunit which is associated with idiopathic generalized epilepsy and paroxysmal dyskinesia [105] appear to result from augmented $\mathrm{Ca}^{2+}$ sensitivity at the RCK1 binding site together with mutations at the brain specific $\beta 4$-subunit. Mutant $\mathrm{BK}$ channels being more sensitive to $\mathrm{Ca}^{2+}$ were found to increase excitability in humans by causing a more rapid repolarization of action potentials which in turn limits the amount of $\mathrm{Ca}^{2+}$ flowing into the cell. An enhanced repolarization favors a faster removal of inactivation from $\mathrm{Na}^{+}$channels and thus lets neurons fire at a higher frequency. A nonfunctional $\beta 4$-subunit which under wild type conditions broadens the actions potential on the other hand also leads to faster action potential repolarization. Eventually this results in an increased discharge of action potentials which may lead to epilepsy, paroxysmal movement disorders, or alcohol dependent initiation of dyskinesias $[79,106]$. Although depletion of BK channels in the brain appears not to be lethal it produces a variety of deficiencies as indicated above.

This introduction to the realm of ion channels as well as BK channels in detail was intended to provide some background for (a) getting to know and summarizing the structure and function of these proteins and for (b) providing a basis for the following elaboration of their modulation by oxidative stress.

\section{Oxidative Stress}

Oxidative stress in general has been introduced by other authors of this special issue, defined, interpreted and generally covered and therefore will not be further discussed. In this section we intend to summarize some aspects of redox effects and oxidative stress on BK channels. Reactive oxygen species (ROS) are involved in a magnitude of oxidative stress modulations but also in the 
physiological regulation of a great variety of proteins and cell functions as outlined in various reviews [34,61,97,107-117]. The sulfur containing cysteine (Cys or C) and/or methionine (Met or M) residues (Figure 2) within proteins are preferred targets of redox modulation, i.e., reversible oxidation/reduction. Cysteines can be oxidized at their thiol groups where two cysteine molecules link to form disulfide bonds internally within the protein or with other proteins. For experiments, cysteine specific reagents 5'5-dithio-bis(2-nitrobenzoic acid) (DTNB), methanethiosulfonate ethylammonium (MTSEA) or p-chloromercuribenzoic acid (PCMB) have been used. Biochemically, disulfide bonds are readily reduced, i.e., by dithiothretiol (DTT) or mercaptoethanol ( $\beta$-ME) to convert them back to thiol cysteines. In vivo reversibility of cysteines is brought about by antioxidants such as the tripeptide glutathione (GSH) or the small protein thioredoxine (TRX) which is present in all organisms [118]. Methionine can be preferentially oxidized by agents such as chloramine-T (Ch-T) under physiological conditions or by $N$-chlorosuccinimid (NCS) [119]. Methionine residues have been hypothesized to function as endogenous antioxidants in proteins [119]. Oxidation leads to polar methionine sulfoxide (Met-O) which can be reversed by methionine sulfoxide reductases (MSRA). Some oxidizing/reducing (ROS) agents are listed in Table 1.
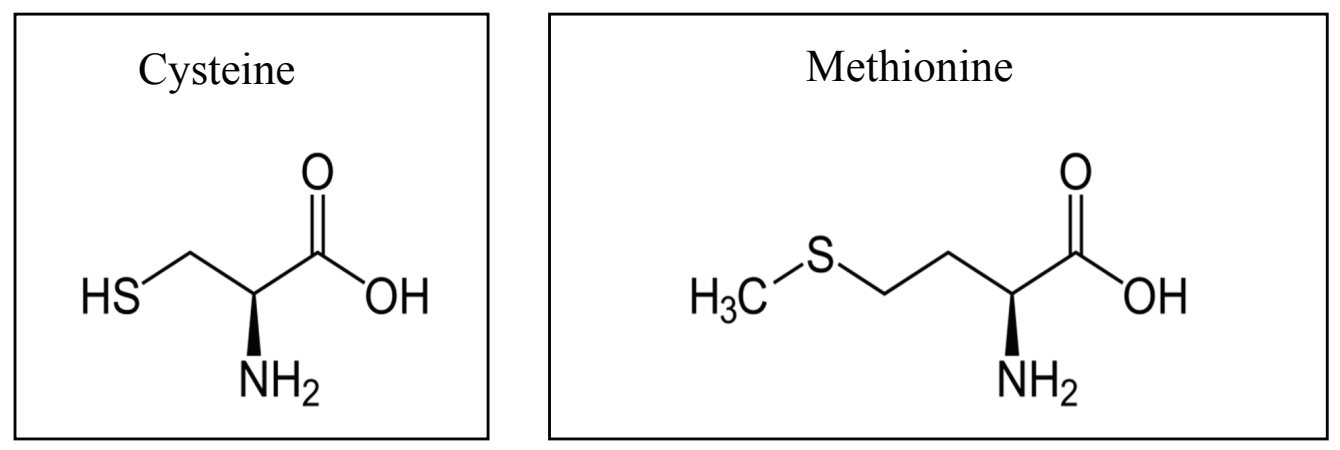

Figure 2. Structure of the sulfur containing amino acids cysteine and methionine.

Since these amino acids are particularly sensitive to redox reactions ROS may act on these targets in an autocrine or paracrine manner, i.e., on the cell itself or in its near vicinity. Other amino acids, such as arginine, lysine, proline, histidine, tryptophan and tyrosine may also provide targets for oxidation. The reactions may proceed extremely fast as with hydroxyl radicals ( $\left.{ }^{\circ} \mathrm{OH}\right)$ with a life-time of a few nanoseconds and a diffusion radius of a few nanometers [130], whereas the weaker radical nitric oxide (NO) has a life span of several seconds and a diffusion radius of several hundred micrometers [145]. Redox mechanisms linked to cell metabolism appear extremely important in the modulation of cellular signaling pathways and for the regulation of ion channels and electrical activity. 
Table 1. Reactive oxygen species (ROS) agents, reducing agents and gasotransmitters that are produced by cells and/or are experimentally used chemicals for modulating BK channels.

\begin{tabular}{|c|c|c|c|}
\hline \multicolumn{4}{|c|}{ Oxidizing/Reducing agents at BK channels } \\
\hline Chemical nomenclature & $\begin{array}{l}\text { Abbrev./ } \\
\text { Formula }\end{array}$ & Company & Notes/literature \\
\hline \multicolumn{4}{|l|}{ Oxidizing agents } \\
\hline Hydrogen peroxide & $\mathbf{H}_{2} \mathbf{O}_{2}$ & $\begin{array}{l}\text { Fisher } \\
\text { Scientific; } \\
\text { Sigma }\end{array}$ & $\begin{array}{l}\text { commonly used oxidizing agent. } \mathrm{H}_{2} \mathrm{O}_{2} \text { is naturally } \\
\text { produced by the cell metabolism, is membrane } \\
\text { permeable and relatively stable }[120,121] \text {. }\end{array}$ \\
\hline Superoxide anion & $\mathbf{O}_{2}^{-\cdot}$ & Sigma & $\begin{array}{l}\text { radical; range of } 10^{-11} \text { to } 10^{-10} \mathrm{M} ;[122] \\
\text { antagonist: superoxide dismutase. }\end{array}$ \\
\hline Glutathione disulfide & GSSG & Sigma & physiological oxidized form of GSH [123]. \\
\hline Chloramine- $\mathrm{T}$ & Ch-T & Sigma & $\begin{array}{l}\text { oxidizes methionine [119]; oxidation leads to } \\
\text { polar methionine sulfoxide (Met-O), reversed by } \\
\text { methionine sulfoxide reductases [109]. }\end{array}$ \\
\hline$N$-chlorosuccinimide & NCS & Sigma & oxidizes methionine [119]. \\
\hline 4,4'-dithiodipyridine & 4,4DTDP & Sigma & {$[124]$} \\
\hline 2,2'-dithiodipyridine & 2,2DTDP & Sigma & {$[125,126]$} \\
\hline $\begin{array}{l}\text { 5'5-dithio-bis } \\
\text { (2-nitrobenzoic acid }\end{array}$ & DTNB & Sigma & cysteine-specific reagent $[109,123,127]$. \\
\hline $\begin{array}{l}\text { (2-Aminoethyl) methan- } \\
\text { ethiosulfonate } \\
\text { hydrochloide }\end{array}$ & MTSEA $^{+}$ & $\begin{array}{l}\text { Toronto } \\
\text { Research }\end{array}$ & $\begin{array}{l}\text { cysteine-specific, covalently modifies free thiol } \\
\text { groups [125]. }\end{array}$ \\
\hline $\begin{array}{l}\text { 2-(trimethylammonium) } \\
\text { ethyl } \\
\text { methanethiosulfonate, } \\
\text { bromide }\end{array}$ & MTSET & $\begin{array}{l}\text { Toronto } \\
\text { Research }\end{array}$ & [128] \\
\hline $\begin{array}{l}\text { p-chloromercuribenzoic } \\
\text { acid }\end{array}$ & рCMB & Sigma & cysteine-specific [109]. \\
\hline $\begin{array}{l}\text { Sodium (2-sulfanatoethyl) } \\
\text { methanethiosulfonate }\end{array}$ & MTSES & $\begin{array}{l}\text { Toronto } \\
\text { Research }\end{array}$ & [128] \\
\hline Thimerosal & & Sigma & $\begin{array}{l}\text { sulfhydryl reagent; its oxidizing ability is related } \\
\text { to the presence of mercury }[124,129] \text {. }\end{array}$ \\
\hline Diamide & & Sigma & {$[125]$} \\
\hline Hydroxyl radicals & $\mathbf{O H}^{\bullet} \bullet$ & & {$[130]$} \\
\hline Peroxynitrite & ONOO $^{-}$ & & $\begin{array}{l}\text { formed at a low rate by the reaction of } \mathrm{NO} \bullet \text { with } \\
\left(\mathrm{O}_{2}^{-\bullet}\right) \text { in a 1:1 stoichiometry for synthesis cf. [131]. }\end{array}$ \\
\hline Rose bengal & $\begin{array}{l}4,5,6,7- \\
\text { tetrachloro- } \\
2^{\prime}, 4^{\prime}, 5^{\prime}, 7^{\prime}- \\
\text { tetraiodo } \\
\text { fluorescein }\end{array}$ & Sigma & $\begin{array}{l}\text { Stain, clinical trials in some cancer therapies; } \\
\text { generates singlet oxygen from triplet oxygen } \\
\text { [132]. }\end{array}$ \\
\hline Normoxia & & & $\begin{array}{l}\text { test solutions equilibrated with room } \\
\text { air }[126,133,134] \text {. }\end{array}$ \\
\hline
\end{tabular}


Table 1. Cont.

\begin{tabular}{|c|c|c|c|}
\hline \multicolumn{4}{|c|}{ Oxidizing/Reducing agents at BK channels } \\
\hline Reducing agents & & & \\
\hline Chemical nomenclature & $\begin{array}{l}\text { Abbrev./ } \\
\text { Formula }\end{array}$ & Company & notes/literature \\
\hline Dithiothreitol & DTT & Sigma & reduction of disulfide links $[124,126,129,135]$. \\
\hline$\beta$-mercaptoethanol & $\beta$-ME & Sigma & {$[125]$} \\
\hline Glutathione & GSH & & Physiological reduced form [136]. \\
\hline $\begin{array}{l}\text { Nicotinamide adenine } \\
\text { dinucleotide hydrate }\end{array}$ & NADH & Sigma & $\begin{array}{l}\text { reduced form of physiological oxidized form } \\
\mathrm{NAD}^{+}[137] \text {. }\end{array}$ \\
\hline Thioredoxine & TRX & Sigma & in reduced form act as oxidoreductases [118]. \\
\hline $\begin{array}{l}\text { Methionine sulfoxide } \\
\text { reductase }\end{array}$ & MSRA & Sigma & converts Met-O to Met [109]. \\
\hline Hypoxia & & & $\begin{array}{l}\text { bubbling of experimental solutions with nitrogen } \\
\text { gas }[126,133,134] .\end{array}$ \\
\hline \multicolumn{4}{|l|}{ Gasotransmitters } \\
\hline $\begin{array}{l}\text { Nitric oxide, gas - donors } \\
\text { Diethylamine NONOate } \\
\text { Sodium nitroprusside } \\
\text { S-Nitroso- } N \text {-Acetyl-D,L- } \\
\text { Penicillamine, etc. }\end{array}$ & $\begin{array}{l}\text { NO } \\
\text { DEA-NO } \\
\text { SNP } \\
\text { SNAP }\end{array}$ & $\begin{array}{l}\text { Alexis } \\
\text { Corp. } \\
\text { Cayman } \\
\text { Sigma }\end{array}$ & $\begin{array}{l}\text { short lived radical (seconds) acts directly or via } \\
\text { the sGC-cGMP-PKG signaling pathway } \\
{[138] \text {. NO range of }\left(10^{-9} \text { to } 10^{-7} \mathrm{M}\right)[124,132]}\end{array}$ \\
\hline $\begin{array}{l}\text { Hydrogen sulfide, gas- } \\
\text { donors } \\
\text { Sodium hydrogen sulfide } \\
\text { Sodium sulfide, } \text { etc. }\end{array}$ & $\begin{array}{l}\mathrm{H}_{2} \mathrm{~S} \\
\mathrm{NaHS} \\
\mathrm{Na}_{2} \mathrm{~S}\end{array}$ & Sigma & {$[60,129,139]$} \\
\hline $\begin{array}{l}\text { Carbon monoxide, gas- } \\
\text { donors } \\
\text { Carbon monoxide } \\
\text { releasing molecules }\end{array}$ & $\begin{array}{l}\text { CO } \\
\text { CORM-A1 } \\
\text { CORM-2 } \\
\text { CORM-3 }\end{array}$ & Sigma & $\begin{array}{l}{[140]} \\
{[141,142]} \\
{[143]} \\
{[144]}\end{array}$ \\
\hline$N$-ethylmaleimide & NEM & Sigma & alkylating agent [128] \\
\hline
\end{tabular}

Human BK (Slo1) channels have 29 cysteines and 30 methionines per $\alpha$-subunit which adds to 116 and 120 residues for each tetrameric complex [113]. Due to conformational properties many of these amino acids are buried within the 3D-structure of the protein and hence are not readily accessible to modifications. Functional consequences of oxidation of cysteine and methionine residues have been reviewed by Sahoo et al. (2014) [113].

\section{Ion Channels and Oxidative Stress}

Ion channels are modulated and regulated by a vast array of mechanisms, drugs and ligands. One of the first recognized and more detailed investigated posttranslational modifications of ion channels was their modulation by phosphorylation. In the human genome more than 500 putative kinase genes 
have been identified [146]. The attachment of phosphate groups to amino acids by protein kinases and the antagonistic action of phosphatases paved the way in our conceptualization of posttranslational modification of ion channel functioning [52,53,59,147-149]. Thereafter the field was open for more modulatory factors from signaling pathways, such as by G-proteins or second messengers, by redox mechanisms, by the action of gasotransmitters, such as nitric oxide (NO), carbon monoxide (CO), or hydrogen sulfide $\left(\mathrm{H}_{2} \mathrm{~S}\right)[1,44,57,59,86,150]$ or more recently by S-acylation (the post-translational attachment of fatty acids to cysteine residues) [151]. Modulation of ion channels is involved in physiological processes such as transmitter release, hormone secretion or muscle contraction to name just a few [11,52,152-155]. Alterations of channels proteins by oxidative stress and its functional consequences constitutes one further way of channel modulation in a great variety of cells and tissues which will be covered in the following sections.

\subsection{BK Channels and Redox Modification/Regulation}

The brain, although it only accounts for $2 \%$ of the body weight, consumes $\sim 20 \%$ of $\mathrm{O}_{2}$ and hence during metabolic activity produces large amounts ROS [156]. High amounts of $\mathrm{O}_{2}$ appear to be needed for the production of ATP which is used to maintain ion homeostasis in the brain in order to run active ion transport to establish transmembrane ion concentration gradients, intracellular neuronal transport systems or the synaptic transmission/neurosecretion machinery.

From brain-derived human BK channels (hslo) expressed in HEK cells (Human Embryonic Kidney cells) DiChiara and Reinhart (1997) [135] provided first evidence that the reducing agent dithiothreitol (DTT) shifted the voltage activation of the channels to more negative potentials and increased current activation as well as channel open probability. These modulations cause the channels to open earlier, i.e., during action potential repolarization and shorten the action potential duration which speeds up repetitive firing. Oxidation, by using hydrogen peroxide $\left(\mathrm{H}_{2} \mathrm{O}_{2}\right)$, had the opposite effect. In contrast, BK channels cloned from Drosophila (dslo) were not modulated by DTT. It was suggested that in hslo proteins disulfide bonds were formed between cysteines - predominantly at the channels' large interior C-terminus region. Soh et al. 2001 [136], reported that in neonatal rat hippocampal neurons the reducing reagent glutathione (GSH) increased BK channel activity whereas its oxidized form (GSSH) had the opposite effect indicating a redox modulatory mechanism when applied to the intracellular side of the cell membrane. On the other hand, after intracellular application of the oxidizing agent DTNB, Gong et al. (2000) [127] and Gao and Fung (2002) [157] reported an increase of open probability and decrease of closing times of BK channels from adult native hippocampal CA1 pyramidal neurons, while the reducing GSH had no apparent effect on the channel activity.

As natural reducing agent, the sulfhydryl specific reagent GSH is present in millimolar concentrations within cells. Depending on the cell type the intracellular concentration of GSH ranges between $1 \mathrm{mM}$ and $10 \mathrm{mM}$ whereof 98\% exist in reduced form [158-160]. GSH rapidly (within 1 minute) in a concentration dependent manner increased BK channel activity in excised patches (a membrane patch which is removed from the cell like an inside out or outside out patch is called excised) of the cell when applied to the intracellular face of the membrane (of non-identified rat hippocampal cells) and this effect was rapidly reversible after wash-out [136]. In its oxidized form 
glutathione (GSSG) inhibited single channel activity which again was readily reversible. Hence, under physiological conditions GSH and GSSG form a redox pair where the ratio of $\mathrm{GSH} / \mathrm{GSH}+\mathrm{GSSG}$ appears responsible for the modulation of channel activity. Evidence suggests that GSH varies between brain regions and is augmented in glia cells compared to neurons [160-162] which makes neurons more susceptible to oxidative stress such as in Parkinson's disease where GSH levels in neurons are decreased [163,164].

Mammalian neurons are highly vulnerable to oxygen $\left(\mathrm{O}_{2}\right)$ deprivation. It has been hypothesized that the resulting depolarization of the membrane resting potential of these cells by hypoxia maybe in part mediated by inhibition of $\mathrm{K}^{+}$-currents. In neocortical neurons of mice hypoxia inhibited BK channel open probability in cell-attached patches but not in the excised inside-out mode [165]. Glutathione and application of DTT increased BK channel open probability. The experimental results suggested that $\mathrm{O}_{2}$ deprivation modulates $\mathrm{BK}$ channel activity by some cytosolic process(es) altering $\mathrm{Ca}^{2+}$ sensitivity resulting from intracellular $\mathrm{pH}$ changes or phosphorylation. These channels were, however, iberiotoxin and charybdotoxin insensitive which makes it questionable if these channels are genuine BK channels or if these channels were associated with $\beta 4$ subunits which causes insensitivity to these toxins [65].

Lewis et al. (2002) [134] reported that the open probability of recombinant BK channel $\alpha$-subunits from human brain, when co-expressed with $\beta 1$ subunits in HEK cells in excised inside out patches, is oxygen-sensitive and reversibly suppressed by hypoxia. The experiments further indicated that the inhibition of channels by hypoxia was voltage-independent, reduced their $\mathrm{Ca}^{2+}$ sensitivity and did not require soluble intracellular factors.

Wyatt and Peers, (1995) [166] described $\mathrm{O}_{2}$-sensitive $\mathrm{K}^{+}$currents in carotid body chemoreceptors cells of neonatal rats. In single channel and perforated patch whole cell recordings the authors reported on charybdotoxin sensitive channels which were inhibited by lowered $\mathrm{PO}_{2}$ and required cytosolic factors for normal functioning as well as $\mathrm{O}_{2}$ sensing. Hypoxia, anoxia or charybdotoxin depolarized the cells suggesting that closure of these channels leads to cell depolarization which is sufficient to activate voltage-gated $\mathrm{Ca}^{2+}$ channels and hence increased transmitter release. Jiang and Haddad (1994) [133] reported $\mathrm{Ca}^{2+}$ and voltage-dependent $\mathrm{K}^{+}$channels of large conductance from rat central neurons in cell free, excised membrane patches, which were blocked by ATP. These channels were reversibly inhibited by hypoxia - but independent of cytosolic factors. Although ATP inhibition of BK channels has been reported [167] their identity as genuine BK channels remained unclear. Furthermore, hypoxia has also been reported to inhibit BK channel activity of rat carotid body type I cells in both whole cell and in excised single channel recording [126]. However, reduction of the channels using DTT increased, whereas oxidation using DTDP decreased channel open probability. From their experiments the authors concluded that hypoxic inhibition is not related to channel reduction and modulation of channels does not require cytoplasmic mediators. Interestingly, $\mathrm{CO}$ reverts hypoxic inhibition of the $\mathrm{BK}$ channels suggesting that $\mathrm{CO}$ binds to the channels or some hemoprotein sensor linked to the channels. In fact, such hemo-proteins have been also postulated for other preparations [168]. Oxidative modulation of $\mathrm{K}^{+}$channels in the nervous system has been recently comprehensively summarized $[113,117]$. 
Perturbation of ion homeostasis is fundamentally involved in causing cell death after ischemia. $\mathrm{Ca}^{2+}$ excitotoxicity, which leads to the release of ROS, generates degrading enzymes or apoptotic factors $[111,169]$. One of the counteracting measures initiated by intracellular $\mathrm{Ca}^{2+}$ accumulation is the activation of BK channels. In excitable cells opening of BK channels causing hyperpolarization of cells provides a negative feedback for $\mathrm{Ca}^{2+}$ influx through voltage dependent $\mathrm{Ca}^{2+}$ channels and hence is involved in the regulation of action potential duration, neurotransmitter release or modulation of excitotoxicity [48,170-172]. Organotypic hippocampal slice cultures in vitro exposed to oxygen and glucose deprivation initiates cell death of CA1, and less of CA3 neurons. Treatment with the BK channel blockers, paxilline or iberiotoxin, increased this effect suggesting that BK channels act as a kind of "emergency brake" in ischemia [173]. Interestingly these treatments also increased the vulnerability of granula cells which are normally resistant to ischemic treatment. This raises the question as to the possible differences in the BK channel setting of these neurons. Accordingly, BK channels are considered as potential molecular targets for neuro-protective therapy in stroke. Using in vivo experimentation the issue was further investigated [174]. Focal ischemia (by cerebral artery occlusion) produced neuronal death in $\mathrm{BK}^{-1-}$ knock-out mice which was significantly increased compared to wild type animals. Organotypic hippocampal slice cultures if exposed to ischemia-like conditions experienced neuronal death in BK knock-out animals which was significantly increased compared to wild type cultures indicating that neuronal BK channels are important for protection against ischemic brain damage [174]. On the other hand, Kulawiak and Szewczyk (2012) [175] reported that inhibition of BK channels by the specific toxin paxilline dose dependently protected hippocampal neurons against glutamate induced cell death. However, iberiotoxin and charybdotoxin were not cytoprotective. From this and further data the authors concluded that the cytoprotective effect of paxilline was not dependent on BK channel inhibition. Although the experiments are not directly comparable to the studies of Liao's group [174], since neuronal death was initiated by different procedures (low oxygen vs high glutamate) the results cause concern about the general mechanism.

Investigation of field excitatory postsynaptic potentials (fEPSPs) revealed that fEPSPs were depressed in an Alzheimer's disease (AD) mouse model compared to age-matched controls [176]. BK channel blockers (charybdotoxin, paxilline) enhanced the fEPSP-potentials giving rise to the notion that impaired $\mathrm{Ca}^{2+}$ homeostasis and/or ROS generation may be considered as the underlying mechanisms.

Preconditioning, a phenomenon in which a non-harmful stress stimulus renders cells tolerant to a following otherwise damaging stimulus (see also below), has been tested in rat cortical neuronal cultures [177,178]. The BK channel opener NS1619, a potent inducer of delayed or immediate neuronal preconditioning, dose-dependently protected cells against toxic insults (oxygen/glucose deprivation, $\mathrm{H}_{2} \mathrm{O}_{2}$, or glutamate excitotoxicity) but protection was not blocked by $\mathrm{BK}$ channel inhibitors. Since NS1619 increases ROS generation, activates the phosphoinositide 3-kinase pathway and inhibits caspase activation it was proposed that it acts cytoprotective via these mechanisms rather than via BK channel activation. In immediate preconditioning modulation of NMDA receptors by ROS and up-regulation of superoxide dismutase activity followed by decreased $\mathrm{Ca}^{2+}$ influx, 
a reduction in oxidative stress upon glutamate exposure independent of BK channel activation was proposed [177].

Vertebrobasilar insufficiency (VBI) represents transient clinical symptoms including vertigo and hearing loss caused by decreased blood supply to the brain. Using whole cell patch clamp experiments at medial vestibular nucleus (MVN) neurons in brain slices Xie et al. (2014) [179] found that brief hypoxia elicits depolarization of the resting membrane potential and an increase of action potential discharge frequency. Furthermore, hypoxia decreased BK-mRNA levels as well as BK channel activity, the latter being alleviated by application of the BK channel opener NS1619. These experiments suggest a neuro-protective role of BK channels and a potential target for treatments in ischemia or stroke.

Hemin, an oxidation product of heme is released from decomposed erythrocytes and appears to play a role during hemorrhagic stroke or brain trauma (indicating intracerebral hemorrhage) [180]. Hemin inhibits BK channels of plasma and mitochondrial membranes [180]. The BK channel opener NS1619 attenuates ROS generation of brain mitochondria under conditions that allow for reverse electron flow [181], which may provide the reason for the different action of NS1619 on mitochondria compared to cells. The addition of hemin inhibits this effect by about $30 \%$ which is comparable to the specific BK channel blocker iberiotoxin [180]. Hence, the inhibition of mtBK channels by hemin may constitute a novel mechanism of neurotoxicity contributing to intracerebral hemorrhage. The authors suggest that an early anti-hemin therapy could help to prevent or diminish cytotoxicity [182].

There are no consistent results on the effects of oxidizing $v s$. reducing agents on BK channel activity. Although there is only one gene for BK channel expression many other factors can influence channel behavior, such as auxiliary subunits being present or absent, or differential splicing, as outlined in the previous section. Furthermore, different recording techniques using either whole cell recordings, where signaling pathways may interfere, $v s$. excised patch recordings, where channel activity is more directly accessed, may play a role. Also, the natures of the oxidizing/reducing compounds and their accessibility to the target site have to be considered.

\subsection{BK Channels and Oxidative Stress at Muscle/Endothelial Cells}

BK channel activity from excised inside-out tracheal myocytes was modified by sulfhydryl redox agents [125]. The reducing agent DTT augmented, whereas the oxidizing agent thimerosal inhibited channel open probability which persisted following wash-out of the drugs but were reversed by counteracting reagents. Alkylation of channel proteins to remove free thiols prevented the action of sulfhydryl altering agents. The experiments suggested that the inhibition by oxidizing compounds is caused by covalent modification of cytosolic channel thiol groups, likely cysteine residues. Glutathione (GSH) at low concentrations also significantly augmented BK channel activity indicating that alterations by the reducing GSH are of physiological relevance [125].

These results were in contrast to findings by other authors. In inside-out patches from isolated smooth muscle cells of rabbit pulmonary arteries, BK channel activity was increased by oxidizing agents such as nicotinamide adenine dinucleotide (NAD), 5'5-dithiobis(2-nitrobenzoic acid) (DTNB) or the oxidized form of glutathione (GSSG), whereas the reducing agents such as dithiothreitol (DTT), 2-hydroxy-1-ethanethiol ( $\beta$-mercaptoethanol, $(\mathrm{BME}))$, the reduced form of nicotinamide adenine 
dinucleotide (NADH) or GSH decreased channel activity [123]. Extracellular application of hydrogen peroxide $\left(\mathrm{H}_{2} \mathrm{O}_{2}\right)$ relaxes porcine coronary arteries by increasing BK channel activity. The effect was observed in the cell attached mode and after excision of the patch to the inside-out mode and was mimicked by arachidonic acid suggesting the involvement of the lipoxygenase signaling pathway [120]. At the same preparation, Hayabuchi et al. (1998) [183] reported that the $\mathrm{H}_{2} \mathrm{O}_{2}$ initiated BK channel dependent vascular relaxation was mediated in part by direct action on the channels and in part by activation of the cGMP signaling pathway. From the experimental results, it appears feasible that both direct redox modulation at BK channel residues and/or indirect modulation occurs via some signaling pathway, which is initiated by redox processes. Explanations for these different findings may be the specificity of the preparations used, the expression of different auxiliary subunits with different redox sensitivity, the effect on the $\mathrm{Ca}^{2+}$ sensitivity directly or via auxiliary subunits per se or the preconditioned status of the channels [184].

Peroxynitrite $\left(\mathrm{ONOO}^{-}\right)$in biology can be produced from the reaction of the superoxide anion radical $\left(\mathrm{O}_{2}^{-\cdot}\right)$ with the nitric oxide radical $\left(\mathrm{NO}^{\circ}\right)$. In rat cerebral artery smooth muscle cells, $\mathrm{ONOO}^{-}$ has been found in whole cell BK current recordings as well as in inside out patches to decrease BK currents, BK channel open probability and channel mean open times, respectively [131]. The effects could be reversed by reduced GSH which also protected BK currents/channels from oxidation. The experimental results are consistent with $\mathrm{ONOO}^{-}$being a contractile agonist of cerebral arteries and myocytes implicating a physiological mechanism in the modulation of vasoconstriction.

Infarct size in heart is profoundly reduced by ischemic preconditioning (IP) - a technique in which brief periods of ischemia precede sustained ischemia and provide tolerance to subsequent damaging insults. BK channels appear to be involved in this mechanism since pharmacological BK channel openers were found to be cardio-protective which was blunted by BK channel blockers, such as iberiotoxin or charybdotoxin [185-187] or after knock-out of the KCNMA1 gene of neurons involved in the regulation of the heart beat [188].

ROS in addition to many other functions also appears involved in altering cellular ion homeostasis [189]. In heart ROS via oxidation of kinases (PKA, PKC, Calcium/Calmodulin Kinase II (CaMKII)) together with dysfunction of the sarcoplasmic reticulum can lead to perturbation of the Ca-homeostasis (which will indirectly affect BK channel activity) and finally cause heart failure.

\subsection{Effects of Oxidative Stress on BK Channels of Epithelial Cells}

In cultured alveolar epithelial A549 cells acute changes of oxygen tension $\left(\mathrm{PO}_{2}\right)$ increased BK channel mean open time whereas chronic changes in $\mathrm{PO}_{2}$ did not affect expression, recruitment or function of BK channels or $\mathrm{Na}^{+}$channel activity [190]. The authors concluded that BK channels serve as oxygen sensors. The mechanism of oxygen sensing was further elaborated by demonstrating that hemoxygenase-2 (HO-2), which is associated to BK channels enhances channel activity in normoxia [191]. It was found that carbon monoxide (CO), which is produced from $\mathrm{O}_{2}$ via $\mathrm{HO}-2$, is a mediator of this function, indicating that HO-2 is an oxygen sensor.

In cultured human epithelial pigment cells, the oxidants, t-butyl hydroperoxide (t-BHP) or thimerosal suppressed BK outward currents recorded in whole cell configuration as well as BK channel open probability in the cell attached mode [192]. Internal application of ceramide prevented the 
oxidizing effect suggesting that BK channel inhibition may involve the intracellular generation of ceramide. In fact, ceramide can be produced in cells via the sphingomyelin pathway or by de novo synthesis $[189,190] . \mathrm{H}_{2} \mathrm{O}_{2}$ and other stressors have been reported to generate ceramide in various cells [193,194] and ceramide per se suppresses BK channel activity [195]. More information on this issue appears an interesting research endeavor.

It remained ambiguous for some time why oxidation in some cases increased, however, in others decreased channel activity. In their publication, Tang et al. (2001) [109] provided an explanation for the oxidative modulation of hSlo (human BK) channels considering the reversible oxidation of the amino acids cysteine and methionine. They showed that the agent chloramine-T (Ch-T), which oxidizes preferentially methionine to methionine sulfoxide $(\operatorname{met}(\mathrm{O}))$ or to $\operatorname{met}\left(\mathrm{O}_{2}\right)$, increased hSlo channel open probability. In contrast cysteine specific reagents, such as DNTB and other oxidizing agents decreased channel activity. Oxidation of these amino acids may also provide an explanation for the run-down of channels after patch excision (loss of channel activity with time), which exposes the channel inside to a more oxidized environment compared to the more reduced, normal intracellular milieu, and may also explain some earlier conflicting results. The authors were able to further provide evidence where at the channels the functional modification may take place. Internal channel blockers tetraethylammonium (TEA) or 1-methyl-4phenyl-1,2,3,6-tetrahydropyridine (MPTP) protected the channels from oxidation by $\mathrm{Ch}-\mathrm{T}$, indicating that residues oxidized by $\mathrm{Ch}-\mathrm{T}$ are located near or within the channel pore and $\beta$-subunits are unlikely to contribute to this process. As outlined by the authors, oxidation of critical amino acids may be clinically relevant. In reperfusion after ischemic conditions the formation of free radicals may affect vascular BK channels. Methionine oxidation causing activation of BK channels may limit $\mathrm{Ca}^{2+}$ entry into cells and serve as neuronal protectant.

Tang et al. (2004) [61] further provided evidence that ROS to a large extent inhibited BK channels $\left(\alpha+\beta\right.$ subunits expressed in HEK cells) by targeting cysteine residues near the intracellular $\mathrm{Ca}^{2+}$ binding site $\left(\mathrm{Ca}^{2+}\right.$ bowl). In particular, a single oxidized cysteine residue, Cys911, prevented $\mathrm{Ca}^{2+}$ sensitivity. Further evidence that the $\alpha$-subunit was the target for oxidation was obtained from experiments where its sole expression was sufficient for the suppression of currents. The authors line out that oxidative stress causing inhibition of BK channel activity and hence impairment of vascular relaxation and blood pressure may have crucial implications in disease and aging.

Auxiliary $\beta$-subunits also contain various amounts of cysteines (4-7) and methionines (3-11). Modifications at these amino acids are therefore amenable to affect channel activity. Zeng et al. (2003) [184] observed that reduction of extracellular disulfide linkages of the $\beta 3$-subunit abolished current rectification and improved charybdotoxin blockade of the channels. The results indicate that the $\beta$-subunit appears to be close to the ion permeation pathway of the $\alpha$-subunit and may regulate the gating mechanism and access of blocking molecules. Physiological consequences may be the dynamic regulation of channel inactivation by oxidative modification of SH groups.

The bovine $\beta$-subunit contains five cysteine residues which are conserved among various species such as rat, dog and humans [196]. Mutagenesis of each of the four cysteines present in the extracellular loop caused a profound reduction of charybdotoxin binding suggesting the generation of disulfide bridges between these residues. Methionines are only present at the intracellular 
$\mathrm{N}$-terminus (3), and one is present at the end of the second transmembrane domain [197]. Oxidation of methionine in BK channel $\alpha$-subunits (hSlo) by chloramine-T induced a leftward shift at the voltage axis to more negative values [109], i.e., the channels are activated already at more negative potentials closer to the membrane resting potential. In the presence of $\beta 1$-subunits and under low intracellular $\mathrm{Ca}^{2+}$ conditions the hyperpolarizing shift was largely augmented, channel open probability increased and deactivation slowed [198]. However, this shift was independent of oxidation of methionine or cysteine residues of $\beta 1$-subunits indicating that this auxiliary subunit has no direct effect but appeared to prime or to amplify the oxidation of the pore forming $\alpha$-subunit residues. As a physiological implication it appeared that in electrically quiet cells at low baseline $\mathrm{Ca}^{2+}$ concentrations $\mathrm{BK}$ channels may have an increased impact on cellular electrical activity and hence influence vascular tone. In a following publication the authors demonstrated that the mutation of three conserved methionines located within the RCK domains (regulators of $\mathrm{K}^{+}$conductance) at the cytoplasmic C-terminus eliminate oxidative sensitivity of the BK channels [199]. Oxidation of at least one of these key methionine residues was sufficient to increase the open probability of the channels and slowing of inactivation at low internal $\mathrm{Ca}^{2+}$. From theoretical considerations the authors proposed a mechanism of conformational changes in the gating ring structure which are transmitted to the voltage sensor domain. The functional effects caused by oxidation may serve as a protective mechanism by driving the cells into a more hyperpolarized, resting state and hence may be advantageous in pathological conditions such as after post-ischemic reperfusion or neurodegenerative disease to prevent neurotoxicity.

\subsection{Oxidative Stress, BK Channels and Vascular Regulation}

BK channels in the vascular system are modulated by agents naturally produced in the body, such as angiotensin II (Ang II), high glucose or arachidonic acid (AA) [200] which is modulated in diabetes by oxidative stress (ROS) $[131,200,201]$. In coronary smooth muscle cells BK channels are activated by arachidonic acid metabolites, in particular prostacyclin 2 (PGI2) [200]. In Zucker diabetic fatty rats channel activation is impaired caused by reduced production of prostacyclin 2 (PGI2) due to reduced PGI2 synthase. As a possible mechanism, ROS formation appeared to be involved. Lu et al. (2006) [201] reported that high glucose reduced BK channel density and channel kinetics by increasing ROS generation, in particular via $\mathrm{H}_{2} \mathrm{O}_{2}$-dependent oxidation of cysteine 911 . In a further study it was shown that caveolae (small 50-100 nanometer invaginations of plasma membrane micro domains containing signaling components in close approximation) play an important role in mediating inhibition of BK channels by ROS [202]. In diabetic rat aortas, cav-1 expression (indicating caveolae) is upregulated and accompanied by an improved physical link between BK channels and the activated angiotensin-1 receptors $\left(\mathrm{AT}_{1} \mathrm{R}\right)$ signaling complex. Angiotensin II (Ang II)-activated AT1R receptors in diabetic rats stimulate enhanced non-phagocytic NAD(P)H oxidase and (NOX1) expression. This leads to an increased ROS-induced redox inhibition of BK channels and also to phosphorylation and nitration of tyrosine residues of the channels. The absence of caveolae (initiated by caveolin-1 (cav-1) knock-down or gene ablation) caused disaggregation of the Ang II-BK channel signaling cascade and preserved BK channel function in diabetes. The molecular mechanisms delineated so far indicate that the composition and spatial arrangement of the signaling system is important for proper functioning of coronary blood flow and 
maybe an interesting target for further pharmacological developments in the treatment of hypertension or atherosclerosis [202].

Another mechanism of BK channel activation of vascular tissue by $\mathrm{H}_{2} \mathrm{O}_{2}$ was presented by Burgoyne et al. (2007) [203] and Zhang et al. (2012) [204]. $\mathrm{H}_{2} \mathrm{O}_{2}$ which serves as an endothelium-derived hyperpolarizing factor (EDHF) causes dilation of human coronary arterioles by activation of BK channels. Cell-attached single channel recordings as well as inside out channel recordings from coronary smooth muscle $\mathrm{BK}$ channels revealed strong evidence that $\mathrm{H}_{2} \mathrm{O}_{2}$ increased channel open probability (Po) which requires intracellular signaling by a mechanism involving disulfide dimerization of the redox sensitive cGMP-dependent protein kinase (PKG-I $\alpha$ ). Hence the $\mathrm{H}_{2} \mathrm{O}_{2}$ mechanism of action appears different to the NO mediated sGC-stimulated cGMP-PKG pathway but acts downstream directly at the PKG-I $\alpha$ level. Release of $\mathrm{H}_{2} \mathrm{O}_{2}$ from endothelial cells appears to play a prominent role as endogenous regulator under normal conditions and in diseased states [204].

There is also evidence that anoxia may act via protein kinase $\mathrm{C}(\mathrm{PKC})$. In Western painted turtle cortical pyramidal neurons anoxia reversibly reduced the open probability of BK channels in the cell-attached mode but not in excised patches [205]. The PKC inhibitor chelerythrine prevented the anoxic effect whereas the PKC activating phorbol ester PMA (phorbol-12-myristate-13-acetate) decreased Po during normoxia. It was concluded that the inhibition of BK channels activity prevents $\mathrm{K}^{+}$efflux, preserves $\mathrm{K}^{+}$homeostasis and hence reduces cellular ATP usage and promotes survival of neurons which enables the animals to adapt to low oxygen levels. Similarly, an anoxia dependent inhibition of $\mathrm{Na}^{+}$currents recorded from rat hippocampal neurons via a PKC dependent mechanism has been reported previously [206].

In cat cerebral arterial muscle cells, hypoxia superfusion produced a transient increase in mean open time of BK channels in excised inside-out patches and this effect was independent of changes of the internal $\mathrm{Ca}^{2+}$ concentration or $\mathrm{pHi}$ [207]. Furthermore, reduced $\mathrm{PO}_{2}$ caused dilation of cerebral arterial segments which was attenuated by tetraethylammonium (TEA). In a following publication Gembremedhin et al. (2008) [208] reported that in rat cerebral arterial muscle cells hypoxia reversibly enhanced BK channel open-state probability in cell-attached patches but this effect was absent in either excised inside-out or outside-out patches. The results further indicated that hypoxia induced the generation of superoxide which caused a reduction in endogenous level of 20-hydroxyeicosatetraenoic acid (20-HETE) that may account for the hypoxia-induced activation of arterial BK channel currents and cerebral vasodilatation.

BK channels, among other $\mathrm{K}$ channels, are associated with decreased uterine vascular tone and an increase of uterine blood flow during pregnancy in order to optimize adequate nutritional supply and tissue oxygenation for the fetus. Chronic hypoxia during gestation by suppression of BK channel function increases uterine vascular tone and decreases uterine blood supply which increases the risk of preeclampsia and retarded fetal growth [209-211]. In addition, expression of BK channels during pregnancy is upregulated but down-regulated during chronic hypoxia. At the same time, protein kinase $\mathrm{C}(\mathrm{PKC})$ activity is increased during hypoxia which inhibits BK channels, whereas under normoxia these effects are reversed [212]. 
Sex steroid hormones (estrogens) regulate uterine vascular tone and uterine blood flow by increasing BK channel density (in particular $\beta 1$-subunits) and activity, whereby also PKC signaling appears an important modulatory mechanism. In contrast, progesterone appears to inhibit BK channels [213] but its contribution to regulating uterine vascular tone needs further investigation. Exposure to hypoxia during pregnancy attenuates the effects of sex steroid hormones/receptors, leading to enhanced PKC activation resulting in the inhibition of BK channel activity and increased pressure-dependent myogenic tone in pregnant uterine arteries [211]. Furthermore, hypoxia-mediated ROS activation during gestation inhibits steroid hormone mediated up-regulation of BK channel activity which may contribute to malfunctions during gestational hypoxia [214]. The evidence suggests that modulation of $\mathrm{K}^{+}$channel activity during pregnancy conveys an important mechanism underlying hypoxia induced uterine vascular dysfunction [215].

\subsection{Mitochondrial BK Channels (mtBK) and Oxygen}

Mitochondria are a major source of ROS generation targeting BK channels [122,216-218]. The inner membrane of mitochondria contains BK channels (mtBK) [219-221] which appear essential in the production of ROS. mtBK channels appear to be inserted into the mitochondrial membrane with the toxin binding sites for charybdotoxin and iberiotoxin exposed to the mitochondrial intermembrane space (accessed by using outside-out patch configuration of the inner mitochondrial membrane). Consequently the $\mathrm{C}$-terminal tail domain including the $\mathrm{Ca}^{2+}$ binding site is localized to the mitochondrial matrix. mtBK channels of cardiac tissue have recently been specified as being encoded by the KCNMAl gene and hence appear to be identified as genuine BK channels [222].

Opening of BK channels allows $\mathrm{K}^{+}$, which is present in a high concentration in the cytosol, to flow into the negatively charged mitochondrial matrix and depolarize the organelle (reviewed in [216]). This reduces the driving force for $\mathrm{Ca}^{2+}$ and hence $\mathrm{Ca}^{2+}$ influx which reduces $\mathrm{Ca}^{2+}$ overload of mitochondria. In fact, mtBK channel activation and $\mathrm{K}^{+}$uptake was reported to confer cytoprotection to heart infarction [221]. Further studies revealed that preconditioned activation of mtBK channels by the channel opener NS-1619 or NS11021 reduces superoxide production and reduces $\mathrm{Ca}^{2+}$ overload which improved $\mathrm{Ca}^{2+}$ homeostasis and redox state after ischemia/reperfusion in isolated guinea pig hearts [122,223-225]. Another mtBK channel opener 12,14-dichlorodehydroabietic acid (diCl-DHAA) was also reported to reduce ischemic injury in rat cardiac myocytes [226]. The steroid, $17 \beta$-estradiol, enhances the activity of cardiac mtBK channels, but only in the presence of the auxiliary $\beta 1$-subunit and increased survival of myocytes under simulated ischemia [227]. In addition the $\beta 1$-subunit appeared to interact with the cytochrome $\mathrm{c}$ oxidase subunit I. These findings may help to improve ischemic diseases such as heart attack in postmenopausal women by applying an estrogen-induced cardio-protective treatment. Furthermore, opening of mtBK channels of brain-derived mitochondria appears to inhibit ROS production suggesting that this may also contribute to the beneficial effects of BK channel openers on neuronal survival [175].

In the heart, BK channels appear absent from the sarcolemma, but the channels are present in mitochondrial membranes (reviewed in [97,228]). The opening of BK channels by hypoxia exerts a cytoprotective effect, which has been attributed to the mechanism mentioned above. In addition $\beta 1$-subunits are highly expressed together with mtBK $\alpha$-subunits that contain two $N$-glycosylation 
sites at their C-terminus which on enzymatic deglycosylation cause activation of BK channels [229,230]. Borchert et al. (2013) [231] reported that the BK channel opener NS11021 or $\mathrm{H}_{2} \mathrm{O}_{2}$ increased survival of cardiomyocytes in simulated ischemia/reperfusion experiments. The cytoprotective effect was abolished by the specific BK channel inhibitor paxilline or tempol, a specific antioxidant. The study indicates that the mechanism for this protection requires ROS signaling suggesting that activation of $\mathrm{mtBK}$ channels protect the cells against injury. Experiments using chronic hypoxic rats exposed to brief intermittent re-oxygenation, attenuated cardio protection possibly by a mechanism involving oxidative stress and suppression of mtBK activity [232].

Mitochondrial BK channels $(\mathrm{mtBK})$ derived from streptozotocin-induced diabetic rat brain incorporated into lipid bilayer membranes were found to exhibit a decreased open probability and conductance [233]. In addition, both BK $\alpha$ - and $\beta 4$ subunits expression was down-regulated. This evidence and an increased production of ROS during diabetic conditions, as proposed by others [201], were suggested to account for the abnormalities in channel gating. In the studies on molecular level, Lu et al. (2006) have shown that C911 is a major molecular target at the channel protein for the redox modulation by high glucose [201]. This body of acquired knowledge may be useful for the development of improved treatment of diabetics.

\subsection{Gasotransmitters and ROS Effects on BK Channels}

In this review, we only briefly outline some aspects of the interaction of BK channels and gasotransmitters. Recent information on this issue has been summarized by [63,117,234]. Nitric oxide (NO) produced by endothelial NO synthase (eNOS) appears to prevent atherosclerosis by interaction with the vascular NO generating system [235]. NADPH oxidases are a major source of ROS leading to various types of vascular pathophysiology. In atherosclerosis bioactivity of NO is decreased by reduced NO synthesis due to an increased NO inactivation.

Endothelial cells by appropriate stimulation (humoral or hemodynamic), release vasodilatory factors, such as NO, whereas vasoconstrictory ROS are generated as byproducts of oxygen metabolism [121]. Single BK channels recorded from in situ renal artery endothelium preparations revealed that NO increased channel open probability by two mechanisms: either by direct action on the channel proteins (probably by nitrosylation) or by indirect action via cGMP (mediated by phosphorylation) [132]. In contrast, $\mathrm{ROS}\left(\mathrm{H}_{2} \mathrm{O}_{2}\right.$ etc.) resulted in irreversible channel inactivation. The authors concluded that BK activation by NO creates a positive feedback by autocrine regulation of endothelial function, whereas ROS inhibits BK channels which impairs hyperpolarization of endothelial cells and consequently prevents vasodilation.

NO inhibits M-currents of rat sensory neurons from trigeminal ganglia [236]. M-type $\mathrm{K}^{+}$channels are subthreshold voltage-gated $\mathrm{K}^{+}$channels of the six TM type (designated $\mathrm{Kv} 7, K C N Q$ gene family) which are inhibited by M1 muscarinic acetylcholine receptors and affect excitability of neurons in the central and peripheral nervous system or cardiac tissue. The authors identified a site of NO action within the cytosolic channel linker between transmembrane domains 2 and 3, which appears also to be a site of oxidative modification by ROS. However, NO and oxidative modifications exhibit opposing effects on M-currents. These channels therefore appear to contain a dynamic redox sensor that is responsible for dynamic M-current modulation by gasotransmitters and ROS and may play a 
role in trigeminal disorders such as headache and migraine. It appears interesting to probe this channel site for different types of gasotransmitters and ROS also at BK channels. Other types of $\mathrm{K}^{+}$channels and oxidative stress are discussed in [112,117,237,238].

In our experiments using GH3 pituitary tumor cells, sodium hydrogen sulfide (NaHS), a $\mathrm{H}_{2} \mathrm{~S}$ donor, increased channel open probability which was prevented by the reducing agent DTT, whereas the oxidizing agent thimerosal increased channel open probability in the presence of $\mathrm{H}_{2} \mathrm{~S}$ [129]. The effect was linked to the reducing action of $\mathrm{H}_{2} \mathrm{~S}$ on sulfhydryl groups of the channel protein. This finding is in concert with a report by Liu et al. 2009 [239] indicating that the $\mathrm{H}_{2} \mathrm{~S}$ donor NaHS prevents postischemic mitochondrial dysfunction by a BK channel dependent mechanism. The development of drugs interfering with $\mathrm{H}_{2} \mathrm{~S}$ signaling might be rewarding in the treatment of mitochondrial linked diseases or to BK channel dependent high blood pressure. Some reagents used to study the modulation of BK channels by gasotransmitters are listed in Table 1 .

\subsection{Oxidative Stress, Proliferation and Various Types of $\mathrm{K}^{+}$Channels}

In pulmonary artery smooth muscle cells various types of $\mathrm{K}^{+}$channels, such as $\mathrm{Kv}, \mathrm{K}_{\mathrm{ir}}, \mathrm{BK}, \mathrm{K}_{2 \mathrm{P}}$, participate in vascular remodeling (proliferation, apoptosis) [240]. In general, loss or inhibition of $\mathrm{K}^{+}$channel function contributes to pulmonary pathogenesis which leads to a decrease of proliferation and inhibition of apoptosis. Hypoxic inhibition of ROS production causing inhibition of $\mathrm{K}^{+}$channels leads to depolarization, opening of $\mathrm{Ca}^{2+}$ channels and augmentation of intracellular $\mathrm{Ca}^{2+}$ which finally results in vasoconstriction of small pulmonary arteries. $\mathrm{K}^{+}$channel expression is transcriptionally regulated and cells need $\mathrm{K}^{+}$channels to proceed through the cell cycle (in particular G1 progression) [240]. Hence, $\mathrm{K}^{+}$channels are appealing therapeutic targets in pulmonary arterial hypertension. Oxidized low-density lipoprotein lysophosphatidylcholine (LPC) increases BK channel open state probability by causing capacitative $\mathrm{Ca}^{2+}$ influx [241]. $\mathrm{Ca}^{2+}$ accumulation increases ROS production that causes reduction of NO generation which promotes proliferation of cultured human endothelial cells. In addition LPC-induced BK activation contributes to increased cGMP levels, if ROS production was prevented by transfection with antisense oligonucleotides against $\mathrm{NAD}(\mathrm{P}) \mathrm{H}$ oxidase. Cancer incidence also appears associated with a great variety of ion channels, in particular $\mathrm{K}^{+}$channelopathies [242-244]. In fact, almost all known types of $\mathrm{K}^{+}$channels have been implicated in oncogenic processes. The dysregulation of $\mathrm{K}^{+}$channel expression (mostly overexpression) and resulting dysfunction of $\mathrm{K}^{+}$channels correlates with dysregulation of proliferation, malignant growth and migration (metastatic spread) of tumorigenic cells. As a general phenomenon, the membrane potential of cancer cells is more depolarized [245] and the resting membrane potential oscillates during the cell cycle, being more depolarized during S and G2 phases [246,247].

Polyamines (mainly putrescine, spermidine and spermine) exhibit a wide array of functions from modulating ion channels, involvement in apoptosis, carcinogenicity, cell proliferation or development. Modulatory properties of polyamines concerning $\mathrm{K}^{+}$channels involved in cell proliferation has been summarized by Weiger and Hermann (2014) [248]. Here we briefly comment on some issues in the context of oxygen impact. Polyamine deficient yeast (Saccharomyces cerevisiae), for example, is very sensitive to oxygen. Polyamine depleted cells accumulate ROS, develop an apoptotic phenotype and die after incubation in polyamine-deficient medium [249]. Addition of spermine caused a marked 
decrease in ROS accumulation and some protection against cell death. The data indicate that part of the function of polyamines is protection of the cells from accumulation of ROS.

Polyamine biosynthesis is increased during cerebral ischemia through induction of ornithine decarboxylase (ODC), a key enzyme for their synthesis. Inhibition of ODC prevents ischemic brain injury [250]. Metabolization of polyamines by polyamine oxidases generates cytotoxic aldehydes and ROS. Polyamines may therefore constitute crucial players in oxidative stress. There is little information on polyamines, ion channels (BK) and oxidative stress which remains an interesting field for future investigations.

\subsection{Oxidative Stress of BK Channels in Tumors}

Tumors are remarkably tolerant to hypoxia whereas normal nerve cells have a high demand on oxygen and rapidly die on hypoxia. This of course raises the question concerning the mechanisms involved. Evidence suggests that the modulation of ion channels and intracellular signaling pathways may be a key in determining cellular tolerance to low oxidative stress [251]. Expression of BK channels is increased in cancerous compared to healthy cells and correlates with the malignancy of the tumors [252,253]. It was hypothesized that differences in the expression of BK channels in tumor cells compared to healthy cells could be the reason for the differences in their response to hypoxia. These findings may provide an approach for novel vistas in cancer therapy.

Hypoxia in a variety of healthy cells in general decreases open probability of BK channels in the plasma membrane (plBK), whereas mtBK channels are opened by hypoxia [254,255]. Studies of normoxic vs. hypoxic conditions of single mtBK and plBK channels from human glioma cells showed that plBK channels were insensitive to hypoxia whereas $\mathrm{mtBK}$ channels open probability was increased during hypoxia [254,256]. Activation of mtBK channels by specific agonists had no effect on cell viability. In contrast, activation of plBK channels by other specific agonists, impaired cell viability of tumor cells $[255,257]$ and this effect was increased by hypoxia [255]. It was suggested that the mechanism leading to cell death/apoptosis in normoxia is based on $\mathrm{Ca}^{2+}$ toxicity, i.e., it is mediated by an increase of cytosolic $\mathrm{Ca}^{2+}$ and activation of calpains $[251,258]$. The effect of hypoxia on mtBK channels appears to have different consequences on cell viability [254]. A cytoprotective effect has been attributed to (a) increased matrix $\mathrm{K}^{+}$, (b) prevention of $\mathrm{Ca}^{2+}$ overload, and c) closing of the MPTP pore. Mitochondrial permeability transition pores (mPTP) close on hypoxia. mPTP in the inner mitochondrial membrane are considered to activate a pathway for the release of pro-apoptotic factors from mitochondria (for further information about this topic the reader is referred to [259]).

\subsection{Clinical Relevance}

Oxidative modulation of BK channels is important not only in order to understand and treat diseased conditions like myocardial infarction or stroke but, as it recently came also into focus, for transplantation surgery. For instance, in the case of lung transplantation the graft is subjected to ischemia followed by reperfusion during routine procedure. During ischemia a number of cellular processes eventually lead to membrane depolarization and ROS generation [260] which in turn favors inflammation and cell death [261]. Noda et al. 2014 [262] found that preconditioning lung grafts with 
inhaled hydrogen, a cytoprotective gaseous signaling molecule, reduced the proinflammatory changes and led to better post-transplant graft function. The molecular mechanism behind this effect is, among others, a stimulation of hemeoxygenase-1 (HO-1) expression by hydrogen [262]. As already discussed above HO-1 activity results in production of CO which then activates BK channels [263]. Active BK channels will cause repolarization of the membrane resting potential and this way may contribute to improved lung graft transplantation. These discoveries again underline the importance and protective role of BK channels. In conclusion, ROS modulation may exert conformational alterations at the channel proteins with two major implications: (1) impairment or dysfunction of channels which may lead to diseases or death, such as in vascular impairment or heart attack, or (2) modifications at channels may lead to physiological modulation of channel functioning, such as in sensing of oxygen tension.

\subsection{Perspectives}

In a recent publication the authors report that a point mutation of a phenylalanine at the F380 position in the S6 transmembrane helix of BK channels greatly obviates channel opening [264]. Based on further functional experiments and molecular dynamic simulations they proposed a model where in the process of channel conduction a hydrophobic ring structure forms that acts as an integration node which affects the interaction between the voltage sensor and the pore. It appears interesting to probe this site for ROS manipulation.

Sulcatone (6-Methyl-5-hepten-2-one (C8H14O)), a prominent volatile component of human body odor that is less abundant in non-human animals, appears to specifically attract some species of female mosquitos (Aedes aegypti) to feed on our blood [265]. A prokaryotic protein with features reminiscent to an ancestral single domain bacterial $\mathrm{K}^{+}$channel protein may have provided the evolutionary blueprint for pentameric ligand-gated ion channels which serve as odorant receptors (Ore4) for sulcatone [266]. Interestingly, sulcatone belongs to the family of oxidoreductases which are known to act on ion channels. It may be speculated that sulcatone acting on $\mathrm{K}^{+}$channels which have been found to alter cellular signaling and are involved in controlling proliferation [248] may be interesting targets for future investigations.

Various types of other $\mathrm{K}^{+}$channels, other than BK channels, such as voltage gated $\mathrm{K}^{+}$channels $\left(\mathrm{KV}_{\mathrm{V}}\right)$, ATP-gated $\mathrm{K}^{+}$channels $\left(\mathrm{K}_{\mathrm{ATP}}\right)$ or $\left(\mathrm{K}_{\mathrm{ir}}\right)$, appear to be involved in vasomodulation $[267,268]$. Only a few other $\mathrm{K}^{+}$channel studies in the context of oxidative stress, from which we may draw interesting information and extend our view, will be covered in this section. In a recent study, Park et al. (2015) [269] report that $\mathrm{H}_{2} \mathrm{O}_{2}$ relaxes rat mesenteric arteries which was reversed by application of the reducing agent dithiothreitol. The vasodilatory effect of $\mathrm{H}_{2} \mathrm{O}_{2}$ was reduced by the voltage gated $\mathrm{K}^{+}$channels $(\mathrm{KV})$ blocker 4-aminopyridine (4-AP) but was resistant to BK and inward rectifier $\mathrm{K}^{+}$channels $\left(\mathrm{K}_{\mathrm{ir}}\right)$ channel blockers. Whole cell patch clamp studies further showed that $\mathrm{K}_{V}$ currents recorded from mesenteric smooth muscle cells were dose-dependently increased by $\mathrm{H}_{2} \mathrm{O}_{2}$ as well as by oxidized glutathione (GSSH) and prevented by glutathione reductase. Further studies showed that reduced glutathione (GSH) is incorporated into the $\mathrm{K}_{V}$ channel protein indicating S-glutathionylation of the channels by $\mathrm{H}_{2} \mathrm{O}_{2}$. Park et al. (2015) [269] now report that by an increased basal level of $\mathrm{H}_{2} \mathrm{O}_{2}$, i.e., under conditions of persistent oxidative stress, $\mathrm{Kv}$ channels were not 
activated, but rather inhibited by the addition of $\mathrm{H}_{2} \mathrm{O}_{2}$. The findings suggest that the actual cellular redox status affects S-glutathionylation of the $\mathrm{K}_{V}$ channels and determines the response of these $\mathrm{K}_{V}$ channels to $\mathrm{H}_{2} \mathrm{O}_{2}$.

Oxidative stress is also a hallmark of vascular disorders such as diabetic retinopathy. In this case ATP-sensitive $\mathrm{K}$ channels $\left(\mathrm{K}_{\mathrm{ATP}}\right)$ activity were found to be increased during exposure of retinal capillaries to $\mathrm{H}_{2} \mathrm{O}_{2}$ [270]. The effect on KATP was boosted by increasing the influx of $\mathrm{Ca}^{2+}$ into microvascular cells and oxidant-induced activation of $\mathrm{Ca}^{2+}$-permeable nonspecific cation channels. Furthermore, it was found that inhibition of $\mathrm{K}_{\mathrm{ATP}}$ channels by the specific $\mathrm{K}_{\text {ATP }}$ blocker glibenclamide significantly lessened $\mathrm{H}_{2} \mathrm{O}_{2}$ induced microvascular cell death. The findings were suggested to provide new targets for pharmacological treatments of retinal microvasculature during oxidative stress.

\section{Conclusions}

In conclusion, the overall status of the present studies indicates that redox modifications of cysteines/methionines cause conformational alterations at the BK channel protein which translate into modulation of channel pore openings (gating) in which interference with the $\mathrm{Ca}^{2+}$ activation mechanism also appears to play a major role. If the target alterations occur at single sites or in combination, whether other amino acid targets bear functional relevance and how these manipulations affect conformational changes of the 3D-protein structure needs further investigation. Primary effects, directly at the channels, and/or secondary effects on signaling pathways, may be both relevant, but have to be clearly separated. BK channel/ROS modulation will certainly be an important target for development of pharmacological agents which function as channel openers/blockers opposing negatively effective redox mechanisms.

\section{Acknowledgments}

This work was supported by travel grants from the Stiftungs- und Förderungsgesellschaft der Universität Salzburg, grants from the University of Salzburg to Guzel F. Sitdikova and by the Russian Scientific Fund No.14-15-00618 to Guzel F. Sitdikova.

\section{Conflicts of Interest}

The authors declare on conflict of interest.

\section{Abbreviations}

AA

ACA

Ach

AD

Ang

4-AP
Arachidonic acid

Acetaldehyde

Acetylcholine

Alzheimer's disease

II angiotensin II

4-aminopyridine 
ATP

$\mathrm{AT}_{1} \mathrm{R}$

$\beta$-ME

BME

BK

$\mathrm{BK}^{-1-}$

CaMKII

cAMP

cav-1

cGMP

CBS

Ch-T

$\mathrm{CO}$

CORM

CREB

CSE

Cys or $\mathrm{C}$

diCl-DHAA

DTT

DTDP

DTNB

EDHF

EET

ER

$\mathrm{EtOH}$

fEPSP

G-protein

GSH

GSSH

20-HETE

$\mathrm{H}_{2} \mathrm{O}_{2}$

$\mathrm{H}_{2} \mathrm{~S}$

HEK

$\mathrm{HO}$

HO-2

HS

hSlo

IK

IP

$\mathrm{K}_{\text {ATP }}$

LPC
Adenosine triphosphate

Angiotensin-1 receptor

Mercaptoethanol

2-hydroxy-1-ethanethiol ( $\beta$-mercaptoethanol)

Big or maxi calcium-activated potassium channel

Knock-out animal

Calcium/Calmodulin Kinase II

Cyclic adenosinemonophosphate

Caveolin-1

Cyclic gCanosinemonophosphate

Cystathionine b-synthase

Chloramine- $\mathrm{T}$

Carbon monoxide

Carbon monoxide releasing molecule

Cyclic AMP response element-binding protein

Cystathionine c-lyase

Cysteine

12,14-dichlorodehydroabietic acid

1,4-dithio-DL-threitol (short: dithiothretiol)

2,2'-dithiodipyridine or 4,4'-dithiodipyridine

5,5'-dithiobis(2-nitrobenzoic acid)

Endothelium-derived hyperpolarizing factor

Epoxyeicosatrienoic acid

Estrogen receptor

Ethanol

Field excitatory postsynaptic potential

Guanosine triphosphate (GTP) binding protein

Glutathione-reduced form

Glutathione-oxidized form

20-hydroxyeicosatetraenoic acid

Hydrogen peroxide

Hydrogen sulfide

Human Embryonic Kidney cells

Heme oxygenase

Hemoxygenase-2

Hydrogen sulfide anion

Human BK channel

Intermediate conductance $\mathrm{K}^{+}$channel

Ischemic preconditioning

Adenosine-triphosphate dependent $\mathrm{K}^{+}$channel

Lysophosphatidylcholine 


\begin{tabular}{|c|c|}
\hline LTP & Long-term potentiation \\
\hline Met or M & Methionine \\
\hline Met-O & Methionine sulfoxide \\
\hline $\mathrm{mM}$ & Milli Molar \\
\hline MPTP & 1-methyl-4phenyl-1,2,3,6-tetrahydropyridine \\
\hline MSRA & Methionine sulfoxide reductases \\
\hline $\mathrm{mtBK}$ & Mitochondrial BK channel \\
\hline MTSEA & Methanethiosulfonate ethylammonium \\
\hline MVN & Medial vestibular nucleus \\
\hline NAD & Nicotinamide adenine dinucleotide \\
\hline NADH & Nicotinamide adenine dinucleotide \\
\hline $\mathrm{NaHS}$ & Sodium hydrogen sulfide \\
\hline $\mathrm{nBK}$ & Nuclear BK channel \\
\hline NCS & $N$-chlorosuccinimid \\
\hline NO & Nitric oxide \\
\hline NOS & NO synthase \\
\hline $\mathrm{nM}$ & Nano Molar \\
\hline NMDA & $N$-methyl D-aspartate \\
\hline $\mathrm{O}_{2}$ & Oxygen \\
\hline $\mathrm{O}_{2}^{-\bullet}$ & Superoxide anion radical \\
\hline$\cdot \mathrm{OH}$ & Hydroxyl radical \\
\hline $\mathrm{ONOO}^{-}$ & Peroxynitrite \\
\hline ODC & Ornithine decarboxylase \\
\hline $\mathrm{P}$ & Pore loop \\
\hline PDE & Phosphodiesterase \\
\hline PGI2 & Prostacyclin 2 (Prostaglandin $\mathrm{I}_{2}$ ) \\
\hline PKA, PKC, PKG & Protein kinase $\mathrm{A}, \mathrm{C}, \mathrm{G}$ \\
\hline plBK & Plasma membrane BK channel \\
\hline PMA & Phorbol-12-myristate-13-acetate \\
\hline $\mathrm{PO}_{2}$ & Oxygen tension \\
\hline Po (Popen) & Open probability of channels \\
\hline PP2A & Protein phosphatase \\
\hline PS & Phosphatidylserine \\
\hline $\mathrm{pS}$ & Piko Siemens \\
\hline PTP & permeability transition pore \\
\hline RCK & Regulatory domain of $\mathrm{K}^{+}$conductance \\
\hline ROS & Reactive oxygen species \\
\hline SK & Small conductance $\mathrm{K}^{+}$channel \\
\hline sGC & Soluble guanylyl cyclase \\
\hline Slob & Slo binding protein \\
\hline STREX & Stress-axis regulated exon \\
\hline
\end{tabular}




$\begin{array}{ll}\text { T } & \text { Transmembrane } \\ \text { t-BHP } & \text { t-butyl hydroperoxide } \\ \text { TEA } & \text { tetraethylammonium } \\ \text { TRX } & \text { Thioredoxine } \\ \text { VBI } & \text { Vertebrobasilar insufficiency }\end{array}$

\section{References}

1. Hille, B. Ion Channels of Excitable Membranes, 3rd ed.; Sinauer: Sunderland, MA, USA, 2001.

2. Hodgkin, A.L.; Huxley, A.F.; Katz, B. Measurements of current-voltage relations in the membrane of the giant axon of Loligo. J. Physiol. 1952, 116, 424-448.

3. Hamill, O.P.; Marty, A.; Neher, E.; Sakmann, B.; Sigworth, F.J. Improved patch-clamp techniques for high-resolution current recording from cells and cell-free membrane patches. Pflüg. Arch. 1981, 391, 85-100.

4. Doyle, D.A.; Cabral, J.M.; Pfuetzner, R.A.; Kuo, A.; Gulbis, J.M.; Cohen, S.L.; Chait, B.T.; MacKinnon, $\mathrm{R}$. The structure of the potassium channel: Molecular basis of $\mathrm{K}^{+}$conduction and selectivity. Science 1998, 280, 69-77.

5. Brueggemann, L.I.; Gentile, S.; Byron, K.L. Social networking among voltage-activated potassium channels. Prog. Mol. Biol. Transl. Sci. 2013, 117, 269-302.

6. Levitan, I.B. Signaling protein complexes associated with neuronal ion channels. Nat. Neurosci. 2006, 9, 305-310.

7. Armstrong, C.M.; Hille, B. Voltage-gated ion channels and electrical excitability. Neuron 1998, 20, 371-380.

8. Yu, F.H.; Yarov-Yarovoy, V.; Gutman, G.A.; Catterall, W.A. Overview of molecular relationships in the voltage-gated ion channel superfamily. Pharmacol. Rev. 2005, 57, 387-395.

9. Bezanilla, F. Voltage-gated ion channels. IEEE Trans. Nanobioscience 2005, 4, 34-48.

10. Long, S.B.; Tao, X.; Campbell, E.B.; MacKinnon, R. Atomic structure of a voltage-dependent $\mathrm{K}^{+}$channel in a lipid membrane-like environment. Nature 2007, 450, 376-382.

11. Dai, S.; Hall, D.D.; Hell, J.W. Supramolecular assemblies and localized regulation of voltage-gated ion channels. Physiol. Rev. 2009, 89, 411-452.

12. Catterall, W.A. Structure and function of voltage-gated ion channels. Annu. Rev. Biochem. 1995, 64, 493-531.

13. Catterall, W.A. Structure and function of voltage-gated sodium channels at atomic resolution. Exp. Physiol. 2014, 99, 35-51.

14. Catterall, W.A. Voltage-gated calcium channels. Cold Spring Harb. Perspect. Biol. 2011, 3, doi:10.1101/cshperspect.a003947.

15. González, C.; Baez-Nieto, D.; Valencia, I.; Oyarzún, I.; Rojas, P.; Naranjo, D.; Latorre, R. K channels: Function-structural overview. Compr. Physiol. 2012, 2, 2087-2149.

16. Stölting, G.; Fischer, M.; Fahlke, C. CLC channel function and dysfunction in health and disease. Front. Physiol. 2014, doi:10.3389/fphys.2014.00378. 
17. Gardos, G. The function of calcium in the potassium permeability of human erythrocytes. Biochim. Biophys. Acta 1958, 30, 653-654.

18. Wei, A.D.; Gutman, G.A.; Aldrich, R.; Chandy, K.G.; Grissmer, S.; Wulff, H. International union of pharmacology. LII. Nomenclature and molecular relationships of calcium-activated potassium channels. Pharmacol. Rev. 2005, 57, 463-472.

19. Ghatta, S.; Nimmagadda, D.; Xu, X.; O’Rourke, S.T. Large-conductance, calcium-activated potassium channels: Structural and functional implications. Pharmacol. Ther. 2006, 110, 103-116.

20. Shipston, M.J. S-acylation dependent post-translational cross-talk regulates large conductance calcium- and voltage- activated potassium (BK) channels. Membr. Physiol. Membr. Biophys. 2014, doi:10.3389/fphys.2014.00281.

21. Salkoff, L.; Butler, A.; Ferreira, G.; Santi, C.; Wei, A. High-conductance potassium channels of the SLO family. Nat. Rev. Neurosci. 2006, 7, 921-931.

22. Cui, J.; Yang, H.; Lee, U.S. Molecular mechanisms of BK channel activation. Cell. Mol. Life Sci. 2009, 66, 852-875.

23. Berkefeld, H.; Fakler, B.; Schulte, U. $\mathrm{Ca}^{2+}$-activated $\mathrm{K}^{+}$channels: From protein complexes to function. Physiol. Rev. 2010, 90, 1437-1459.

24. Cui, J. BK-type calcium-activated potassium channels: Coupling of metal ions and voltage sensing. J. Physiol. 2010, 588, 4651-4658.

25. Lee, U.S.; Cui, J. BK channel activation: Structural and functional insights. Trends Neurosci. 2010, 33, 415-423.

26. Grimm, P.R.; Sansom, S.C. BK channels and a new form of hypertension. Kidney Int. 2010, 78, 956-962.

27. Hill, M.A.; Yang, Y.; Ella, S.R.; Davis, M.J.; Braun, A.P. Large conductance, $\mathrm{Ca}^{2+}$-activated $\mathrm{K}^{+}$channels (BKCa) and arteriolar myogenic signaling. FEBS Lett. 2010, 584, 2033-2042.

28. Wu, Y.; Yang, Y.; Ye, S.; Jiang, Y. Structure of the gating ring from the human large-conductance $\mathrm{Ca}^{2+}$-gated $\mathrm{K}^{+}$channel. Nature 2010, 466, 393-397.

29. Latorre, R.; Morera, F.J.; Zaelzer, C. Allosteric interactions and the modular nature of the voltage- and $\mathrm{Ca}^{2+}$-activated (BK) channel. J. Physiol. 2010, 588, 3141-3148.

30. Hermann, A.; Sitdikova, G.F.; Weiger, T.M. BK Channels-Focus on Polyamines, Ethanol/Acetaldehyde and Hydrogen Sulfide (H2S). In Patch Clamp Technique; Shad Kaneez, F., Ed.; InTech: Rijeka, Croatia, 2012; pp. 109-142.

31. Rothberg, B.S. The BK channel: A vital link between cellular calcium and electrical signaling. Protein Cell 2012, 3, 883-892.

32. N'Gouemo, P. Targeting BK (big potassium) channels in epilepsy. Expert Opin. Ther. Targets 2011, 15, 1283-1295.

33. Hoshi, T.; Pantazis, A.; Olcese, R. Transduction of voltage and $\mathrm{Ca}^{2+}$ signals by Slo1 BK channels. Physiology 2013, 28, 172-189.

34. Toro, L.; Li, M.; Zhang, Z.; Singh, H.; Wu, Y.; Stefani, E. MaxiK channel and cell signalling. Pflüg. Arch. 2014, 466, 875-886. 
35. Ge, L.; Hoa, N.T.; Wilson, Z.; Arismendi-Morillo, G.; Kong, X.-T.; Tajhya, R.B.; Beeton, C.; Jadus, M.R. Big Potassium (BK) ion channels in biology, disease and possible targets for cancer immunotherapy. Int. Immunopharmacol. 2014, 22, 427-443.

36. Kyle, B.D.; Braun, A.P. The regulation of BK channel activity by pre- and post-translational modifications. Front. Physiol. 2014, doi:10.3389/fphys.2014.00316.

37. Yang, H.; Zhang, G.; Cui, J. BK channels: Multiple sensors, one activation gate. Front. Physiol. 2015, doi:10.3389/fphys.2015.00029.

38. Fodor, A.A.; Aldrich, R.W. Convergent evolution of alternative splices at domain boundaries of the BK channel. Annu. Rev. Physiol. 2009, 71, 19-36.

39. Xie, J.; McCobb, D.P. Control of alternative splicing of potassium channels by stress hormones. Science 1998, 280, 443-446.

40. Erxleben, C.; Everhart, A.L.; Romeo, C.; Florance, H.; Bauer, M.B.; Alcorta, D.A.; Rossie, S.; Shipston, M.J.; Armstrong, D.L. Interacting effects of N-terminal variation and strex exon splicing on slo potassium channel regulation by calcium, phosphorylation, and oxidation. J. Biol. Chem. 2002, 277, 27045-27052.

41. Berthois, Y.; Katzenellenbogen, J.A.; Katzenellenbogen, B.S. Phenol red in tissue culture media is a weak estrogen: Implications concerning the study of estrogen-responsive cells in culture. Proc. Natl. Acad. Sci. USA 1986, 83, 2496-2500.

42. Holdiman, A.J.; Fergus, D.J.; England, S.K. 17ß-Estradiol upregulates distinct maxi-K channel transcripts in mouse uterus. Mol. Cell. Endocrinol. 2002, 192, 1-6.

43. Norfleet, A.M.; Thomas, M.L.; Gametchu, B.; Watson, C.S. Estrogen receptor-alpha detected on the plasma membrane of aldehyde-fixed GH3/B6/F10 rat pituitary tumor cells by enzyme-linked immunocytochemistry. Endocrinology 1999, 140, 3805-3814.

44. Hermann, A.; Sitdikova, G.F.; Weiger, T.M. Modulated by Gasotransmitters: BK Channels. In Gasotransmitters: Physiology and Pathophysiology; Hermann, A., Sitdikova, G.F., Weiger, T.M., Eds.; Springer: Heidelberg, Germany, 2012; pp. 163-201.

45. Hermann, A.; Gorman, A.L. External and internal effects of tetraethylammonium on voltage-dependent and Ca-dependent $\mathrm{K}^{+}$currents components in molluscan pacemaker neurons. Neurosci. Lett. 1979, 12, 87-92.

46. Lang, D.G.; Ritchie, A.K. Tetraethylammonium blockade of apamin-sensitive and insensitive $\mathrm{Ca}^{2+}$-activated $\mathrm{K}^{+}$channels in a pituitary cell line. J. Physiol. 1990, 425, 117-132.

47. Zhou, Y.; Lingle, C.J. Paxilline inhibits BK channels by an almost exclusively closed-channel block mechanism. J. Gen. Physiol. 2014, 144, 415-440.

48. Gribkoff, V.K.; Lum-Ragan, J.T.; Boissard, C.G.; Post-Munson, D.J.; Meanwell, N.A.; Starrett, J.E.; Kozlowski, E.S.; Romine, J.L.; Trojnacki, J.T.; Mckay, M.C.; et al. Effects of channel modulators on cloned large-conductance calcium-activated potassium channels. Mol. Pharmacol. 1996, 50, 206-217.

49. Thompson, J.; Begenisich, T. Mechanistic details of BK channel inhibition by the intermediate conductance, $\mathrm{Ca}^{2+}$-activated K channel. Channels 2009, 3, 194-204.

50. Gola, M.; Crest, M. Colocalization of active $\mathrm{KCa}$ channels and $\mathrm{Ca}^{2+}$ channels within $\mathrm{Ca}^{2+}$ domains in helix neurons. Neuron 1993, 10, 689-699. 
51. Issa, N.P.; Hudspeth, A.J. Clustering of $\mathrm{Ca}^{2+}$ channels and $\mathrm{Ca}^{2+}$-activated $\mathrm{K}^{+}$channels at fluorescently labeled presynaptic active zones of hair cells. Proc. Natl. Acad. Sci. USA 1994, 91, 7578-7582.

52. Levitan, I.B. Modulation of ion channels by protein phosphorylation and dephosphorylation. Annu. Rev. Physiol. 1994, 56, 193-212.

53. Levitan, I.B. Modulation of ion channels by protein phosphorylation. How the brain works. Adv. Second Messenger Phosphoprotein Res. 1999, 33, 3-22.

54. Tian, L.; Duncan, R.R.; Hammond, M.S.L.; Coghill, L.S.; Wen, H.; Rusinova, R.; Clark, A.G.; Levitan, I.B.; Shipston, M.J. Alternative splicing switches potassium channel sensitivity to protein phosphorylation. J. Biol. Chem. 2001, 276, 7717-7720.

55. Zhou, X.B.; Arntz, C.; Kamm, S.; Motejlek, K.; Sausbier, U.; Wang, G.X.; Ruth, P.; Korth, M. A molecular switch for specific stimulation of the BKCa channel by cGMP and cAMP kinase. J. Biol. Chem. 2001, 276, 43239-43245.

56. Zhou, X.-B.; Wulfsen, I.; Utku, E.; Sausbier, U.; Sausbier, M.; Wieland, T.; Ruth, P.; Korth, M. Dual role of protein kinase C on BK channel regulation. Proc. Natl. Acad. Sci. USA 2010, 107, 8005-8010.

57. Weiger, T.M.; Hermann, A.; Levitan, I.B. Modulation of calcium-activated potassium channels. J. Comp. Physiol. Neuroethol. Sens. Neural. Behav. Physiol. 2002, 188, 79-87.

58. Alioua, A.; Kumar, Y.; Eghbali, M.; Stefani, E. MaxiK channel partners: Physiological impact. J. Physiol. 2006, 570, 65-72.

59. Hou, S.; Heinemann, S.H.; Hoshi, T. Modulation of BKCa channel gating by endogenous signaling molecules. Physiology 2009, 24, 26-35.

60. Sitdikova, G.F.; Fuchs, R.; Kainz, V.; Weiger, T.M.; Hermann, A. Phosphorylation of BK channels modulates the sensitivity to hydrogen sulfide (H2S). Front. Physiol. 2014, doi:10.3389/fphys. 2014.00431.

61. Tang, X.D.; Garcia, M.L.; Heinemann, S.H.; Hoshi, T. Reactive oxygen species impair Slo1 BK channel function by altering cysteine-mediated calcium sensing. Nat. Struct. Mol. Biol. 2004, 11, 171-178.

62. Tang, G.; Wu, L.; Wang, R. Interaction of hydrogen sulfide with ion channels. Clin. Exp. Pharmacol. Physiol. 2010, 37, 753-763.

63. Wilkinson, W.J.; Kemp, P.J. Carbon monoxide: An emerging regulator of ion channels. J. Physiol. 2011, 589, 3055-3062.

64. Peers, C.; Bauer, C.C.; Boyle, J.P.; Scragg, J.L.; Dallas, M.L. Modulation of ion channels by hydrogen sulfide. Antioxid. Redox Signal. 2012, 17, 95-105.

65. Weiger, T.M.; Holmqvist, M.H.; Levitan, I.B.; Clark, F.T.; Sprague, S.; Huang, W.J.; Ge, P.; Wang, C.; Lawson, D.; Jurman, M.E.; et al. A novel nervous system beta subunit that downregulates human large conductance calcium-dependent potassium channels. J. Neurosci. 2000, 20, 3563-3570.

66. Zhang, J.; Yan, J. Regulation of BK channels by auxiliary $\gamma$ subunits. Membr. Physiol. Membr. Biophys. 2014, doi:10.3389/fphys.2014.00401. 
67. Torres, Y.P.; Granados, S.T.; Latorre, R. Pharmacological consequences of the coexpression of BK channel $\alpha$ and auxiliary $\beta$ subunits. Membr. Physiol. Membr. Biophys. 2014, doi:10.3389/fphys.2014.00383.

68. McCormack, T.; McCormack, K. Shaker $\mathrm{K}^{+}$channel beta subunits belong to an NAD(P)H-dependent oxidoreductase superfamily. Cell 1994, 79, 1133-1135.

69. Gulbis, J.M.; Mann, S.; MacKinnon, R. Structure of a voltage-dependent $\mathrm{K}^{+}$channel beta subunit. Cell 1999, 97, 943-952.

70. Wallner, M.; Meera, P.; Toro, L. Determinant for beta-subunit regulation in high-conductance voltage-activated and $\mathrm{Ca}^{2+}$-sensitive $\mathrm{K}^{+}$channels: An additional transmembrane region at the N terminus. Proc. Natl. Acad. Sci. USA 1996, 93, 14922-14927.

71. Hanner, M.; Schmalhofer, W.A.; Munujos, P.; Knaus, H.G.; Kaczorowski, G.J.; Garcia, M.L. The beta subunit of the high-conductance calcium-activated potassium channel contributes to the high-affinity receptor for charybdotoxin. Proc. Natl. Acad. Sci. USA 1997, 94, 2853-2858.

72. Meera, P.; Wallner, M.; Jiang, Z.; Toro, L. A calcium switch for the functional coupling between alpha (hslo) and beta subunits (KV,Ca beta) of maxi K channels. FEBS Lett. 1996, 382, 84-88.

73. Orio, P.; Rojas, P.; Ferreira, G.; Latorre, R. New disguises for an old channel: MaxiK channel beta-subunits. News Physiol. Sci. 2002, 17, 156-161.

74. Wallner, M.; Meera, P.; Toro, L. Molecular basis of fast inactivation in voltage and $\mathrm{Ca}^{2+}$-activated $\mathrm{K}^{+}$channels: A transmembrane beta-subunit homolog. Proc. Natl. Acad. Sci. USA 1999, 96, 4137-4142.

75. Tseng-Crank, J.; Godinot, N.; Johansen, T.E.; Ahring, P.K.; Strøbaek, D.; Mertz, R.; Foster, C.D.; Olesen, S.P.; Reinhart, P.H. Cloning, expression, and distribution of a $\mathrm{Ca}^{2+}$-activated $\mathrm{K}^{+}$ channel beta-subunit from human brain. Proc. Natl. Acad. Sci. USA 1996, 93, 9200-9205.

76. Bentrop, D.; Beyermann, M.; Wissmann, R.; Fakler, B. NMR structure of the "ball-and-chain" domain of $\mathrm{KCNMB} 2$, the beta 2 -subunit of large conductance $\mathrm{Ca}^{2+}$ - and voltage-activated potassium channels. J. Biol. Chem. 2001, 276, 42116-42121.

77. Xia, X.-M.; Ding, J.P.; Lingle, C.J. Inactivation of BK channels by the NH2 terminus of the beta2 auxiliary subunit: An essential role of a terminal peptide segment of three hydrophobic residues. J. Gen. Physiol. 2003, 121, 125-148.

78. Ha, T.S.; Heo, M.-S.; Park, C.-S. Functional effects of auxiliary beta4-subunit on rat large-conductance $\mathrm{Ca}^{2+}$-activated $\mathrm{K}^{+}$channel. Biophys. J. 2004, 86, 2871-2882.

79. Brenner, R.; Chen, Q.H.; Vilaythong, A.; Toney, G.M.; Noebels, J.L.; Aldrich, R.W. BK channel beta4 subunit reduces dentate gyrus excitability and protects against temporal lobe seizures. Nat. Neurosci. 2005, 8, 1752-1759.

80. Brenner, R.; Jegla, T.J.; Wickenden, A.; Liu, Y.; Aldrich, R.W. Cloning and functional characterization of novel large conductance calcium-activated potassium channel $\beta$ subunits, hKCNMB3 and hKCNMB4. J. Biol. Chem. 2000, 275, 6453-6461.

81. Meera, P.; Wallner, M.; Toro, L. A neuronal beta subunit (KCNMB4) makes the large conductance, voltage- and $\mathrm{Ca}^{2+}$-activated $\mathrm{K}^{+}$channel resistant to charybdotoxin and iberiotoxin. Proc. Natl. Acad. Sci. USA 2000, 97, 5562-5567. 
82. Behrens, R.; Nolting, A.; Reimann, F.; Schwarz, M.; Waldschütz, R.; Pongs, O. hKCNMB3 and hKCNMB4, cloning and characterization of two members of the large-conductance calcium-activated potassium channel beta subunit family. FEBS Lett. 2000, 474, 99-106.

83. Yan, J.; Aldrich, R.W. LRRC26 auxiliary protein allows BK channel activation at resting voltage without calcium. Nature 2010, 466, 513-516.

84. Yan, J.; Aldrich, R.W. BK potassium channel modulation by leucine-rich repeat-containing proteins. Proc. Natl. Acad. Sci. USA 2012, 109, 7917-7922.

85. Schopperle, W.M.; Holmqvist, M.H.; Zhou, Y.; Wang, J.; Wang, Z.; Griffith, L.C.; Keselman, I.; Kusinitz, F.; Dagan, D.; Levitan, I.B. Slob, a novel protein that interacts with the Slowpoke calcium-dependent potassium channel. Neuron 1998, 20, 565-573.

86. Zhou, Y.; Schopperle, W.M.; Murrey, H.; Jaramillo, A.; Dagan, D.; Griffith, L.C.; Levitan, I.B. A dynamically regulated 14-3-3, Slob, and Slowpoke potassium channel complex in Drosophila presynaptic nerve terminals. Neuron 1999, 22, 809-818.

87. Zeng, H.; Weiger, T.M.; Fei, H.; Levitan, I.B. Mechanisms of two modulatory actions of the channel-binding protein Slob on the Drosophila Slowpoke calcium-dependent potassium channel. J. Gen. Physiol. 2006, 128, 583-591.

88. Zeng, H.; Weiger, T.M.; Fei, H.; Jaramillo, A.M.; Levitan, I.B. The amino terminus of Slob, Slowpoke channel binding protein, critically influences its modulation of the channel. J. Gen. Physiol. 2005, 125, 631-640.

89. Jaramillo, A.M.; Zheng, X.; Zhou, Y.; Amado, D.A.; Sheldon, A.; Sehgal, A.; Levitan, I.B. Pattern of distribution and cycling of SLOB, Slowpoke channel binding protein, in Drosophila. BMC Neurosci. 2004, doi:10.1186/1471-2202-5-3.

90. Kawakubo, T.; Naruse, K.; Matsubara, T.; Hotta, N.; Sokabe, M. Characterization of a newly found stretch-activated $\mathrm{KCa}$, ATP channel in cultured chick ventricular myocytes. Am. J. Physiol. 1999, 276, H1827-H1838.

91. Gasull, X.; Ferrer, E.; Llobet, A.; Castellano, A.; Nicolás, J.M.; Palés, J.; Gual, A. Cell membrane stretch modulates the high-conductance $\mathrm{Ca}^{2+}$-activated $\mathrm{K}^{+}$channel in bovine trabecular meshwork cells. Invest. Ophthalmol. Vis. Sci. 2003, 44, 706-714.

92. Tang, Q.Y.; Qi, Z.; Naruse, K.; Sokabe, M. Characterization of a functionally expressed stretch-activated BKca channel cloned from chick ventricular myocytes. J. Membr. Biol. 2003, 196, 185-200.

93. Wang, W.; Huang, H.; Hou, D.; Liu, P.; Wei, H.; Fu, X.; Niu, W. Mechanosensitivity of STREX-lacking BKCa channels in the colonic smooth muscle of the mouse. Am. J. Physiol. Gastrointest. Liver Physiol. 2010, 299, G1231-G1240.

94. Pattillo, J.M.; Yazejian, B.; DiGregorio, D.A.; Vergara, J.L.; Grinnell, A.D.; Meriney, S.D. Contribution of presynaptic calcium-activated potassium currents to transmitter release regulation in cultured Xenopus nerve-muscle synapses. Neuroscience 2001, 102, 229-240.

95. Meredith, A.L.; Wiler, S.W.; Miller, B.H.; Takahashi, J.S.; Fodor, A.A.; Ruby, N.F.; Aldrich, R.W. BK calcium-activated potassium channels regulate circadian behavioral rhythms and pacemaker output. Nat. Neurosci. 2006, 9, 1041-1049. 
96. Chen, L.; Jeffries, O.; Rowe, I.C.M.; Liang, Z.; Knaus, H.-G.; Ruth, P.; Shipston, M.J. Membrane trafficking of large conductance calcium-activated potassium channels is regulated by alternative splicing of a transplantable, acidic trafficking motif in the RCK1-RCK2 linker. J. Biol. Chem. 2010, 285, 23265-23275.

97. Singh, H.; Stefani, E.; Toro, L. Intracellular BK(Ca) (iBK(Ca)) channels. J. Physiol. 2012, 590, 5937-5947.

98. Li, B.; Jie, W.; Huang, L.; Wei, P.; Li, S.; Luo, Z.; Friedman, A.K.; Meredith, A.L.; Han, M.-H.; Zhu, X.-H.; et al. Nuclear BK channels regulate gene expression via the control of nuclear calcium signaling. Nat. Neurosci. 2014, 17, 1055-1063.

99. Nardi, A.; Olesen, S.-P. BK channel modulators: A comprehensive overview. Curr. Med. Chem. 2008, 15, 1126-1146.

100. Rolim, A.L.R.; Lindsey, S.C.; Kunii, I.S.; Fujikawa, A.M.; Soares, F.A.; Chiamolera, M.I.; Maciel, R.M.B.; da Silva, M.R.D. Ion channelopathies in endocrinology: Recent genetic findings and pathophysiological insights. Arq. Bras. Endocrinol. Metabol. 2010, 54, 673-681.

101. Catterall, W.A. Ion channel voltage sensors: Structure, function, and pathophysiology. Neuron 2010, 67, 915-928.

102. Kullmann, D.M.; Waxman, S.G. Neurological channelopathies: New insights into disease mechanisms and ion channel function: Neurological channelopathies. J. Physiol. 2010, 588, 1823-1827.

103. Zhang, L.; Li, X.; Zhou, R.; Xing, G. Possible role of potassium channel, big K in etiology of schizophrenia. Med. Hypotheses 2006, 67, 41-43.

104. Laumonnier, F.; Roger, S.; Guérin, P.; Molinari, F.; M’rad, R.; Cahard, D.; Belhadj, A.; Halayem, M.; Persico, A.M.; Elia, M.; et al. Association of a functional deficit of the BKCa channel, a synaptic regulator of neuronal excitability, with autism and mental retardation. Am. J. Psychiatry 2006, 163, 1622-1629.

105. Lorenz, S.; Heils, A.; Kasper, J.M.; Sander, T. Allelic association of a truncation mutation of the KCNMB3 gene with idiopathic generalized epilepsy. Am. J. Med. Genet. Part B Neuropsychiatr. Genet. 2007, 144, 10-13.

106. Du, W.; Bautista, J.F.; Yang, H.; Diez-Sampedro, A.; You, S.-A.; Wang, L.; Kotagal, P.; Lüders, H.O.; Shi, J.; Cui, J.; et al. Calcium-sensitive potassium channelopathy in human epilepsy and paroxysmal movement disorder. Nat. Genet. 2005, 37, 733-738.

107. Kourie, J.I. Interaction of reactive oxygen species with ion transport mechanisms. Am. J. Physiol. 1998, 275, C1-C24.

108. Hoshi, T.; Heinemann, S.H. Regulation of cell function by methionine oxidation and reduction. J. Physiol. 2001, 531, 1-11.

109. Tang, X.D.; Daggett, H.; Hanner, M.; Garcia, M.L.; McManus, O.B.; Brot, N.; Weissbach, H.; Heinemann, S.H.; Hoshi, T. Oxidative regulation of large conductance calcium-activated potassium channels. J. Gen. Physiol. 2001, 117, 253-274.

110. Annunziato, L.; Pannaccione, A.; Cataldi, M.; Secondo, A.; Castaldo, P.; di Renzo, G.; Taglialatela, M. Modulation of ion channels by reactive oxygen and nitrogen species: A pathophysiological role in brain aging? Neurobiol. Aging 2002, 23, 819-834. 
111. Chinopoulos, C.; Adam-Vizi, V. Calcium, mitochondria and oxidative stress in neuronal pathology. FEBS J. 2006, 273, 433-450.

112. Sesti, F.; Liu, S.; Cai, S.-Q. Oxidation of potassium channels by ROS: A general mechanism of aging and neurodegeneration? Trends Cell Biol. 2010, 20, 45-51.

113. Sahoo, N.; Hoshi, T.; Heinemann, S.H. Oxidative modulation of voltage-gated potassium channels. Antioxid. Redox Signal. 2014, 21, 933-952.

114. Ray, P. D.; Huang, B.-W.; Tsuji, Y. Reactive oxygen species (ROS) homeostasis and redox regulation in cellular signaling. Cell. Signal. 2012, 24, 981-990.

115. Circu, M.L.; Aw, T.Y. Reactive oxygen species, cellular redox systems, and apoptosis. Free Radic. Biol. Med. 2010, 48, 749-762.

116. Bertram, C.; Hass, R. Cellular responses to reactive oxygen species-induced DNA damage and aging. Biol. Chem. 2008, 389, 211-220.

117. Peers, C.; Boyle, J.P. Oxidative modulation of $\mathrm{K}^{+}$channels in the central nervous system in neurodegenerative diseases and aging. Antioxid. Redox Signal. 2015, 22, 505-521.

118. Arnér, E.S.J.; Holmgren, A. Physiological functions of thioredoxin and thioredoxin reductase. Eur. J. Biochem. 2000, 267, 6102-6109.

119. Levine, R.L.; Mosoni, L.; Berlett, B.S.; Stadtman, E.R. Methionine residues as endogenous antioxidants in proteins. Proc. Natl. Acad. Sci. USA 1996, 93, 15036-15040.

120. Barlow, R.S.; White, R.E. Hydrogen peroxide relaxes porcine coronary arteries by stimulating BKCa channel activity. Am. J. Physiol. 1998, 275, H1283-H1289.

121. Gutterman, D.D.; Miura, H.; Liu, Y. Redox modulation of vascular tone: Focus of potassium channel mechanisms of dilation. Arterioscler. Thromb. Vasc. Biol. 2005, 25, 671-678.

122. Stowe, D.F.; Aldakkak, M.; Camara, A.K.S.; Riess, M.L.; Heinen, A.; Varadarajan, S.G.; Jiang, M.-T. Cardiac mitochondrial preconditioning by Big $\mathrm{Ca}^{2+}$-sensitive $\mathrm{K}^{+}$channel opening requires superoxide radical generation. Am. J. Physiol. Heart Circ. Physiol. 2006, 290, H434-H440.

123. Thuringer, D.; Findlay, I. Contrasting effects of intracellular redox couples on the regulation of maxi-K channels in isolated myocytes from rabbit pulmonary artery. J. Physiol. 1997, 500, 583-592.

124. Lang, R.J.; Harvey, J.R.; McPhee, G.J.; Klemm, M.F. Nitric oxide and thiol reagent modulation of $\mathrm{Ca}^{2+}$-activated $\mathrm{K}^{+}$(BKCa) channels in myocytes of the guinea-pig taenia caeci. J. Physiol. 2000, 525, 363-376.

125. Wang, Z.-W.; Nara, M.; Wang, Y.-X.; Kotlikoff, M.I. Redox regulation of large conductance $\mathrm{Ca}^{2+}$-activated $\mathrm{K}^{+}$channels in smooth muscle cells. J. Gen. Physiol. 1997, 110, 35-44.

126. Riesco-Fagundo, A.M.; Pérez-García, M.T.; González, C.; López-López, J.R. O O modulates large-conductance $\mathrm{Ca}^{2+}$-dependent $\mathrm{K}^{+}$channels of rat chemoreceptor cells by a membrane-restricted and CO-sensitive mechanism. Circ. Res. 2001, 89, 430-436.

127. Gong, L.-W.; Gao, T.M.; Huang, H.; Tong, Z. Redox modulation of large conductance calcium-activated potassium channels in CA1 pyramidal neurons from adult rat hippocampus. Neurosci. Lett. 2000, 286, 191-194. 
128. Zhang, G.; Horrigan, F.T. Cysteine modification alters voltage- and $\mathrm{Ca}^{2+}$-dependent gating of large conductance (BK) potassium channels. J. Gen. Physiol. 2005, 125, 213-236.

129. Sitdikova, G.F.; Weiger, T.M.; Hermann, A. Hydrogen sulfide increases calcium-activated potassium (BK) channel activity of rat pituitary tumor cells. Pflüg. Arch. 2010, 459, 389-397.

130. Haugland, R.P.; Spence, M.T.Z.; Johnson, I.D. Handbook of Fluorescent Probes and Research Chemicals; Molecular Probes: Eugene, OR, USA, 1996.

131. Brzezinska, A.K.; Gebremedhin, D.; Chilian, W.M.; Kalyanaraman, B.; Elliott, S.J. Peroxynitrite reversibly inhibits $\mathrm{Ca}^{2+}$-activated $\mathrm{K}^{+}$channels in rat cerebral artery smooth muscle cells. Am. J. Physiol. Heart Circ. Physiol. 2000, 278, H1883-H1890.

132. Brakemeier, S.; Eichler, I.; Knorr, A.; Fassheber, T.; Köhler, R.; Hoyer, J. Modulation of $\mathrm{Ca}^{2+}$-activated $\mathrm{K}^{+}$channel in renal artery endothelium in situ by nitric oxide and reactive oxygen species. Kidney Int. 2003, 64, 199-207.

133. Jiang, C.; Haddad, G.G. Oxygen deprivation inhibits a $\mathrm{K}^{+}$channel independently of cytosolic factors in rat central neurons. J. Physiol. 1994, 481, 15-26.

134. Lewis, A.; Peers, C.; Ashford, M.L.J.; Kemp, P.J. Hypoxia inhibits human recombinant large conductance, $\mathrm{Ca}^{2+}$-activated $\mathrm{K}^{+}$(maxi-K) channels by a mechanism which is membrane delimited and $\mathrm{Ca}^{2+}$ sensitive. J. Physiol. 2002, 540, 771-780.

135. DiChiara, T.J.; Reinhart, P.H. Redox Modulation of hslo $\mathrm{Ca}^{2+}$-Activated $\mathrm{K}^{+}$Channels. J. Neurosci. 1997, 17, 4942-4955.

136. Soh, H.; Jung, W.; Uhm, D.Y.; Chung, S. Modulation of large conductance calcium-activated potassium channels from rat hippocampal neurons by glutathione. Neurosci. Lett. 2001, 298, $115-118$.

137. Bednarczyk, P.; Wieckowski, M.R.; Broszkiewicz, M.; Skowronek, K.; Siemen, D.; Szewczyk, A. Putative structural and functional coupling of the mitochondrial BKCa channel to the respiratory chain. PLoS One 2013, 8, e68125.

138. Moncada, S.; Palmer, R.M.; Higgs, E.A. Nitric oxide: Physiology, pathophysiology, and pharmacology. Pharmacol. Rev. 1991, 43, 109-142.

139. Yoo, D.; Jupiter, R.C.; Pankey, E.A.; Reddy, V.G.; Edward, J.A.; Swan, K.W.; Peak, T.C.; Mostany, R.; Kadowitz, P.J. Analysis of cardiovascular responses to the H2S donors Na2S and NaHS in the rat. Am. J. Physiol. 2015, doi:10.1152/ajpheart.00171.2015.

140. Wang, R.; Wu, L. The chemical modification of KCa channels by carbon monoxide in vascular smooth muscle cells. J. Biol. Chem. 1997, 272, 8222-8226.

141. Basuroy, S.; Leffler, C.W.; Parfenova, H. CORM-A1 prevents blood-brain barrier dysfunction caused by ionotropic glutamate receptor-mediated endothelial oxidative stress and apoptosis. Am. J. Physiol. Cell Physiol. 2013, 304, C1105-C1115.

142. Ryan, M.J.; Jernigan, N.L.; Drummond, H.A.; McLemore, G.R., Jr; Rimoldi, J.M.; Poreddy, S.R.; Gadepalli, R.S.V.; Stec, D.E. Renal vascular responses to CORM-A1 in the mouse. Pharmacol. Res. 2006, 54, 24-29.

143. Soni, H.; Pandya, G.; Patel, P.; Acharya, A.; Jain, M.; Mehta, A.A. Beneficial effects of carbon monoxide-releasing molecule-2 (CORM-2) on acute doxorubicin cardiotoxicity in mice: Role of oxidative stress and apoptosis. Toxicol. Appl. Pharmacol. 2011, 253, 70-80. 
144. Smith, H.; Mann, B.E.; Motterlini, R.; Poole, R.K. The carbon monoxide-releasing molecule, CORM-3 (RU(CO) 3 CL(glycinate)), targets respiration and oxidases in Campylobacter jejuni, generating hydrogen peroxide. IUBMB Life 2011, 63, 363-371.

145. Stadtman, E.R.; Berlett, B.S. Reactive oxygen-mediated protein oxidation in aging and disease. Drug Metab. Rev. 1998, 30, 225-243.

146. Manning, G. Genomic Overview of Protein Kinases. Available online: http://www.wormbook.org (accessed on 20 July 2015).

147. Levitan, I.B. Phosphorylation of ion channels. J. Membr. Biol. 1985, 87, 177-190.

148. Park, K.-S.; Yang, J.-W.; Seikel, E.; Trimmer, J.S. Potassium channel phosphorylation in excitable cells: Providing dynamic functional variability to a diverse family of ion channels. Physiology 2008, 23, 49-57.

149. Ismailov, I.I.; Benos, D.J. Effects of phosphorylation on ion channel function. Kidney Int. 1995, $48,1167-1179$.

150. Hille, B. Modulation of ion-channel function by G-protein-coupled receptors. Trends Neurosci. 1994, 17, 531-536.

151. Shipston, M.J. Ion channel regulation by protein S-acylation. J. Gen. Physiol. 2014, 143, 659-678.

152. Schubert, R.; Nelson, M.T. Protein kinases: Tuners of the BKCa channel in smooth muscle. Trends Pharmacol. Sci. 2001, 22, 505-512.

153. Newton, P.M.; Messing, R.O. Intracellular signaling pathways that regulate behavioral responses to ethanol. Pharmacol. Ther. 2006, 109, 227-237.

154. Lorca, R.A.; Prabagaran, M.; England, S.K. Functional insights into modulation of BKCa channel activity to alter myometrial contractility. Front. Physiol. 2014, doi:10.3389/fphys.2014.00289.

155. Ashcroft, F.M.; Rorsman, P. K(ATP) channels and islet hormone secretion: New insights and controversies. Nat. Rev. Endocrinol. 2013, 9, 660-669.

156. Friedman, J. Why Is the Nervous System Vulnerable to Oxidative Stress? In Oxidative Stress and Free Radical Damage in Neurology, Oxidative Stress in Applied Basic Research and Clinical Practice; Gadoth, N., Göbel, H.H., Eds.; Humana Press: New York, NY, USA, 2011; pp. 19-27.

157. Gao, T.-M.; Fung, M.-L. Decreased large conductance $\mathrm{Ca}^{2+}$-activated $\mathrm{K}^{+}$channel activity in dissociated CA1 hippocampal neurons in rats exposed to perinatal and postnatal hypoxia. Neurosci. Lett. 2002, 332, 163-166.

158. Ji, L.L.; Fu, R.; Mitchell, E.W. Glutathione and antioxidant enzymes in skeletal muscle: Effects of fiber type and exercise intensity. J. Appl. Physiol. 1992, 73, 1854-1859.

159. Meister, A. Glutathione metabolism. Methods Enzymol. 1995, 251, 3-7.

160. Langeveld, C.H.; Schepens, E.; Jongenelen, C.A.; Stoof, J.C.; Hjelle, O.P.; Ottersen, O.P.; Drukarch, B. Presence of glutathione immunoreactivity in cultured neurones and astrocytes. Neuroreport 1996, 7, 1833-1836. 
161. Dringen, R.; Kussmaul, L.; Gutterer, J.M.; Hirrlinger, J.; Hamprecht, B. The glutathione system of peroxide detoxification is less efficient in neurons than in astroglial cells. J. Neurochem. 1999, 72, 2523-2530.

162. Fernandez-Fernandez, S.; Almeida, A.; Bolaños, J.P. Antioxidant and bioenergetic coupling between neurons and astrocytes. Biochem. J. 2012, 443, 3-11.

163. Martin, H.L.; Teismann, P. Glutathione-A review on its role and significance in Parkinson's disease. Fed. Am. Soc. Exp. Biol. 2009, 23, 3263-3272.

164. Smeyne, M.; Smeyne, R.J. Glutathione metabolism and Parkinson's disease. Free Radic. Biol. Med. 2013, 62, 13-25.

165. Liu, H.; Moczydlowski, E.; Haddad, G.G. $\mathrm{O}_{2}$ deprivation inhibits $\mathrm{Ca}^{2+}$-activated $\mathrm{K}^{+}$channels via cytosolic factors in mice neocortical neurons. J. Clin. Invest. 1999, 104, 577-588.

166. Wyatt, C.N.; Wright, C.; Bee, D.; Peers, C. O2-sensitive $\mathrm{K}^{+}$currents in carotid body chemoreceptor cells from normoxic and chronically hypoxic rats and their roles in hypoxic chemotransduction. Proc. Natl. Acad. Sci. USA 1995, 92, 295-299.

167. Clark, A.G.; Hall, S.K.; Shipston, M.J. ATP inhibition of a mouse brain large-conductance K ${ }^{+}$ (mslo) channel variant by a mechanism independent of protein phosphorylation. J. Physiol. 1999, 516, 45-53.

168. López-López, J.R.; González, C. Time course of $\mathrm{K}^{+}$current inhibition by low oxygen in chemoreceptor cells of adult rabbit carotid body. Effects of carbon monoxide. FEBS Lett. 1992, 299, 251-254.

169. Gleichmann, M.; Mattson, M.P. Neuronal calcium homeostasis and dysregulation. Antioxid. Redox Signal. 2011, 14, 1261-1273.

170. Storm, J.F. Action potential repolarization and a fast after-hyperpolarization in rat hippocampal pyramidal cells. J. Physiol. 1987, 385, 733-759.

171. Hu, H.; Shao, L.R.; Chavoshy, S.; Gu, N.; Trieb, M.; Behrens, R.; Laake, P.; Pongs, O.; Knaus, H.G.; Ottersen, O.P.; et al. Presynaptic $\mathrm{Ca}^{2+}$-activated $\mathrm{K}^{+}$channels in glutamatergic hippocampal terminals and their role in spike repolarization and regulation of transmitter release. J. Neurosci. 2001, 21, 9585-9597.

172. Mancini, M.; Soldovieri, M.V.; Gessner, G.; Wissuwa, B.; Barrese, V.; Boscia, F.; Secondo, A.; Miceli, F.; Franco, C.; Ambrosino, P.; et al. Critical role of large-conductance calcium- and voltage-activated potassium channels in leptin-induced neuroprotection of N-methyl-D-aspartate-exposed cortical neurons. Pharmacol. Res. 2014, 87, 80-86.

173. Rundén-Pran, E.; Haug, F.M.; Storm, J.F.; Ottersen, O.P. BK channel activity determines the extent of cell degeneration after oxygen and glucose deprivation: A study in organotypical hippocampal slice cultures. Neuroscience 2002, 112, 277-288.

174. Liao, Y.; Kristiansen, A.-M.; Oksvold, C.P.; Tuvnes, F.A.; Gu, N.; Rundén-Pran, E.; Ruth, P.; Sausbier, M.; Storm, J.F. Neuronal $\mathrm{Ca}^{2+}$-activated $\mathrm{K}^{+}$channels limit brain infarction and promote survival. PLOS ONE 2010, 5, e15601.

175. Kulawiak, B.; Szewczyk, A. Glutamate-induced cell death in HT22 mouse hippocampal cells is attenuated by paxilline, a BK channel inhibitor. Mitochondrion 2012, 12, 169-172. 
176. Ye, H.; Jalini, S.; Mylvaganam, S.; Carlen, P. Activation of large-conductance $\mathrm{Ca}^{2+}$-activated $\mathrm{K}^{+}$channels depresses basal synaptic transmission in the hippocampal CA1 area in APP (swe/ind) TgCRND8 mice. Neurobiol. Aging 2010, 31, 591-604.

177. Gáspár, T.; Domoki, F.; Lenti, L.; Katakam, P.V.G.; Snipes, J.A.; Bari, F.; Busija, D.W. Immediate neuronal preconditioning by NS1619. Brain Res. 2009, 1285, 196-207.

178. Gáspár, T.; Katakam, P.; Snipes, J.A.; Kis, B.; Domoki, F.; Bari, F.; Busija, D.W. Delayed neuronal preconditioning by NS1619 is independent of calcium activated potassium channels. J. Neurochem. 2008, 105, 1115-1128.

179. Xie, H.; Zhang, Y.-Q.; Pan, X.-L.; Wu, S.-H.; Chen, X.; Wang, J.; Liu, H.; Qian, X.-Z.; Liu, Z.-G.; Liu, L.-J. Decreased calcium-activated potassium channels by hypoxia causes abnormal firing in the spontaneous firing medial vestibular nuclei neurons. Eur. Arch. Otorhinolaryngol. 2014, doi:10.1007/s00405-014-3158-4.

180. Tang, X.D.; Xu, R.; Reynolds, M.F.; Garcia, M.L.; Heinemann, S.H.; Hoshi, T. Haem can bind to and inhibit mammalian calcium-dependent Slo1 BK channels. Nature 2003, 425, 531-535.

181. Kulawiak, B.; Kudin, A.P.; Szewczyk, A.; Kunz, W.S. BK channel openers inhibit ROS production of isolated rat brain mitochondria. Exp. Neurol. 2008, 212, 543-547.

182. Augustynek, B.; Kudin, A.P.; Bednarczyk, P.; Szewczyk, A.; Kunz, W.S. Hemin inhibits the large conductance potassium channel in brain mitochondria: A putative novel mechanism of neurodegeneration. Exp. Neurol. 2014, 257, 70-75.

183. Hayabuchi, Y.; Nakaya, Y.; Matsuoka, S.; Kuroda, Y. Hydrogen peroxide-induced vascular relaxation in porcine coronary arteries is mediated by $\mathrm{Ca}^{2+}$-activated $\mathrm{K}^{+}$channels. Heart Vessels 1998, 13, 9-17.

184. Zeng, X.-H.; Xia, X.-M.; Lingle, C.J. Redox-sensitive extracellular gates formed by auxiliary beta subunits of calcium-activated potassium channels. Nat. Struct. Biol. 2003, 10, 448-454.

185. Gribkoff, V.K.; Starrett, J.E.; Dworetzky, S.I.; Hewawasam, P.; Boissard, C.G.; Cook, D.A.; Frantz, S.W.; Heman, K.; Hibbard, J.R.; Huston, K.; et al. Targeting acute ischemic stroke with a calcium-sensitive opener of maxi-K potassium channels. Nat. Med. 2001, 7, 471-477.

186. Shintani, Y.; Node, K.; Asanuma, H.; Sanada, S.; Takashima, S.; Asano, Y.; Liao, Y.; Fujita, M.; Hirata, A.; Shinozaki, Y.; et al. Opening of $\mathrm{Ca}^{2+}$-activated $\mathrm{K}^{+}$channels is involved in ischemic preconditioning in canine hearts. J. Mol. Cell. Cardiol. 2004, 37, 1213-1218.

187. Bentzen, B.H.; Olesen, S.-P.; Rønn, L.C.B.; Grunnet, M. BK channel activators and their therapeutic perspectives. Front. Physiol. 2014, doi:10.3389/fphys.2014.00389.

188. Wojtovich, A.P.; Nadtochiy, S.M.; Urciuoli, W.R.; Smith, C.O.; Grunnet, M.; Nehrke, K.; Brookes, P.S. A non-cardiomyocyte autonomous mechanism of cardioprotection involving the SLO1 BK channel. Peer J. 2013, doi:10.7717/peerj.48.

189. Wagner, S.; Rokita, A.G.; Anderson, M.E.; Maier, L.S. Redox regulation of sodium and calcium handling. Antioxid. Redox Signal. 2013, 18, 1063-1077.

190. Jovanović, S.; Crawford, R.M.; Ranki, H.J.; Jovanović, A. Large conductance $\mathrm{Ca}^{2+}$-activated $\mathrm{K}^{+}$channels sense acute changes in oxygen tension in alveolar epithelial cells. Am. J. Respir. Cell Mol. Biol. 2003, 28, 363-372. 
191. Williams, S.E.J.; Wootton, P.; Mason, H.S.; Bould, J.; Iles, D.E.; Riccardi, D.; Peers, C.; Kemp, P.J. Hemoxygenase-2 is an oxygen sensor for a calcium-sensitive potassium channel. Science 2004, 306, 2093-2097.

192. Sheu, S.-J.; Wu, S.-N. Mechanism of inhibitory actions of oxidizing agents on calcium-activated potassium current in cultured pigment epithelial cells of the human retina. Invest. Ophthalmol. Vis. Sci. 2003, 44, 1237-1244.

193. Mathias, S.; Peña, L.A.; Kolesnick, R.N. Signal transduction of stress via ceramide. Biochem. J. 1998, 335, 465-480.

194. Li, X.; Becker, K.A.; Zhang, Y. Ceramide in redox signaling and cardiovascular diseases. Cell. Physiol. Biochem. 2010, 26, 41-48.

195. Li, P.L.; Zhang, D.X.; Zou, A.P.; Campbell, W.B. Effect of ceramide on KCa channel activity and vascular tone in coronary arteries. Hypertension 1999, 33, 1441-1446.

196. Hanner, M.; Vianna-Jorge, R.; Kamassah, A.; Schmalhofer, W.A.; Knaus, H.G.; Kaczorowski, G.J.; Garcia, M.L. The beta subunit of the high conductance calcium-activated potassium channel. Identification of residues involved in charybdotoxin binding. J. Biol. Chem. 1998, 273, 16289-16296.

197. Knaus, H.G.; Folander, K.; Garcia-Calvo, M.; Garcia, M.L.; Kaczorowski, G.J.; Smith, M.; Swanson, R. Primary sequence and immunological characterization of beta-subunit of high conductance $\mathrm{Ca}^{2+}$-activated $\mathrm{K}^{+}$channel from smooth muscle. J. Biol. Chem. 1994, 269, 17274-17278.

198. Santarelli, L.C.; Chen, J.; Heinemann, S.H.; Hoshi, T. The betal subunit enhances oxidative regulation of large-conductance calcium-activated $\mathrm{K}^{+}$channels. J. Gen. Physiol. 2004, 124, 357-370.

199. Santarelli, L.C.; Wassef, R.; Heinemann, S.H.; Hoshi, T. Three methionine residues located within the regulator of conductance for $\mathrm{K}^{+}(\mathrm{RCK})$ domains confer oxidative sensitivity to large-conductance $\mathrm{Ca}^{2+}$-activated $\mathrm{K}^{+}$channels. J. Physiol. 2006, 571, 329-348.

200. Lu, T.; Wang, X.-L.; He, T.; Zhou, W.; Kaduce, T.L.; Katusic, Z.S.; Spector, A.A.; Lee, H.-C. Impaired arachidonic acid-mediated activation of large-conductance $\mathrm{Ca}^{2+}$-activated $\mathrm{K}^{+}$ channels in coronary arterial smooth muscle cells in Zucker Diabetic Fatty rats. Diabetes 2005, 54, 2155-2163.

201. Lu, T.; He, T.; Katusic, Z.S.; Lee, H.-C. Molecular mechanisms mediating inhibition of human large conductance $\mathrm{Ca}^{2+}$-activated $\mathrm{K}^{+}$channels by high glucose. Circ. Res. 2006, 99, 607-616.

202. Lu, T.; Zhang, D.-M.; Wang, X.-L.; He, T.; Wang, R.-X.; Chai, Q.; Katusic, Z.S.; Lee, H.-C. Regulation of coronary arterial BK channels by caveolae-mediated angiotensin II signaling in diabetes mellitus. Circ. Res. 2010, 106, 1164-1173.

203. Burgoyne, J.R.; Madhani, M.; Cuello, F.; Charles, R.L.; Brennan, J.P.; Schröder, E.; Browning, D.D.; Eaton, P. Cysteine redox sensor in PKGIa enables oxidant-induced activation. Science 2007, 317, 1393-1397. 
204. Zhang, D.X.; Borbouse, L.; Gebremedhin, D.; Mendoza, S.A.; Zinkevich, N.S.; Li, R.; Gutterman, D.D. H2O2-induced dilation in human coronary arterioles: Role of protein kinase $\mathrm{G}$ dimerization and large-conductance $\mathrm{Ca}^{2+}$-activated $\mathrm{K}^{+}$channel activation. Circ. Res. 2012, 110, 471-480.

205. Rodgers-Garlick, C.I.; Hogg, D.W.; Buck, L.T. Oxygen-sensitive reduction in $\mathrm{Ca}^{2+}$-activated $\mathrm{K}^{+}$channel open probability in turtle cerebrocortex. Neuroscience 2013, 237, 243-254.

206. O’Reilly, J.P.; Cummins, T.R.; Haddad, G.G. Oxygen deprivation inhibits $\mathrm{Na}^{+}$current in rat hippocampal neurones via protein kinase C. J. Physiol. 1997, 503, 479-488.

207. Gebremedhin, D.; Bonnet, P.; Greene, A.S.; England, S.K.; Rusch, N.J.; Lombard, J.H.; Harder, D.R. Hypoxia increases the activity of $\mathrm{Ca}^{2+}$-sensitive $\mathrm{K}^{+}$channels in cat cerebral arterial muscle cell membranes. Pflüg. Arch. 1994, 428, 621-630.

208. Gebremedhin, D.; Yamaura, K.; Harder, D.R. Role of 20-HETE in the hypoxia-induced activation of $\mathrm{Ca}^{2+}$-activated $\mathrm{K}^{+}$channel currents in rat cerebral arterial muscle cells. $\mathrm{Am}$. J. Physiol. Heart Circ. Physiol. 2008, 294, H107-H120.

209. Hu, L.-F.; Lu, M.; Hon Wong, P.T.; Bian, J.-S. Hydrogen sulfide: Neurophysiology and neuropathology. Antioxid. Redox Signal. 2011, 15, 405-419.

210. Hu, X.-Q.; Xiao, D.; Zhu, R.; Huang, X.; Yang, S.; Wilson, S.M.; Zhang, L. Chronic hypoxia suppresses pregnancy-induced upregulation of large-conductance $\mathrm{Ca}^{2+}$-activated $\mathrm{K}^{+}$channel activity in uterine arteries. Hypertension 2012, 60, 214-222.

211. Zhu, R.; Xiao, D.; Zhang, L. Potassium channels and uterine vascular adaptation to pregnancy and chronic hypoxia. Curr. Vasc. Pharmacol. 2013, 11, 737-747.

212. Xiao, D.; Zhu, R.; Zhang, L. Gestational hypoxia up-regulates protein kinase C and inhibits calcium-activated potassium channels in ovine uterine arteries. Int. J. Med. Sci. 2014, 11, 886-892.

213. Wong, C.-M.; Tsang, S.-Y.; Yao, X.; Chan, F.L.; Huang, Y. Differential effects of estrogen and progesterone on potassium channels expressed in Xenopus oocytes. Steroids 2008, 73, 272-279.

214. Zhu, R.; Huang, X.; Hu, X.-Q.; Xiao, D.; Zhang, L. Gestational hypoxia increases reactive oxygen species and inhibits steroid hormone-mediated upregulation of $\mathrm{Ca}^{2+}$-activated $\mathrm{K}^{+}$ channel function in uterine arteries. Hypertension 2014, 64, 415-422.

215. Wareing, M. Oxygen sensitivity, potassium channels, and regulation of placental vascular tone. Microcirculation 2014, 21, 58-66.

216. Szewczyk, A.; Skalska, J.; Głab, M.; Kulawiak, B.; Malińska, D.; Koszela-Piotrowska, I.; Kunz, W.S. Mitochondrial potassium channels: From pharmacology to function. Biochim. Biophys. Acta 2006, 1757, 715-720.

217. Szewczyk, A.; Jarmuszkiewicz, W.; Kunz, W.S. Mitochondrial potassium channels. IUBMB Life 2009, 61, 134-143.

218. Kalogeris, T.; Bao, Y.; Korthuis, R.J. Mitochondrial reactive oxygen species: A double edged sword in ischemia/reperfusion vs. preconditioning. Redox Biol. 2014, 2, 702-714.

219. Balderas, E.; Zhang, J.; Stefani, E.; Toro, L. Mitochondrial BKCa channel. Front. Physiol. 2015, doi:10.3389/fphys.2015.00104. 
220. Siemen, D.; Loupatatzis, C.; Borecky, J.; Gulbins, E.; Lang, F. Ca ${ }^{2+}$-activated $\mathrm{K}$ channel of the BK-type in the inner mitochondrial membrane of a human glioma cell line. Biochem. Biophys. Res. Commun. 1999, 257, 549-554.

221. Xu, W.; Liu, Y.; Wang, S.; McDonald, T.; van Eyk, J.E.; Sidor, A.; O’Rourke, B. Cytoprotective role of $\mathrm{Ca}^{2+}$ - activated $\mathrm{K}^{+}$channels in the cardiac inner mitochondrial membrane. Science 2002, 298, 1029-1033.

222. Singh, H.; Lu, R.; Bopassa, J.C.; Meredith, A.L.; Stefani, E.; Toro, L. MitoBK(Ca) is encoded by the Kcnmal gene, and a splicing sequence defines its mitochondrial location. Proc. Natl. Acad. Sci. USA 2013, 110, 10836-10841.

223. Heinen, A.; Camara, A.K.S.; Aldakkak, M.; Rhodes, S.S.; Riess, M.L.; Stowe, D.F. Mitochondrial $\mathrm{Ca}^{2+}$-induced $\mathrm{K}^{+}$influx increases respiration and enhances ROS production while maintaining membrane potential. Am. J. Physiol. Cell Physiol. 2007, 292, C148-C156.

224. Heinen, A.; Aldakkak, M.; Stowe, D.F.; Rhodes, S.S.; Riess, M.L.; Varadarajan, S.G.; Camara, A.K.S. Reverse electron flow-induced ROS production is attenuated by activation of mitochondrial $\mathrm{Ca}^{2+}$-sensitive $\mathrm{K}^{+}$channels. Am. J. Physiol. Heart Circ. Physiol. 2007, 293, H1400-H1407.

225. Bentzen, B.H.; Osadchii, O.; Jespersen, T.; Hansen, R.S.; Olesen, S.-P.; Grunnet, M. Activation of big conductance $\mathrm{Ca}^{2+}$-activated $\mathrm{K}^{+}$channels (BK) protects the heart against ischemia-reperfusion injury. Pflüg. Arch. 2009, 457, 979-988.

226. Sakamoto, K.; Ohya, S.; Muraki, K.; Imaizumi, Y. A novel opener of large-conductance $\mathrm{Ca}^{2+}$-activated $\mathrm{K}^{+}(\mathrm{BK})$ channel reduces ischemic injury in rat cardiac myocytes by activating mitochondrial K(Ca) channel. J. Pharmacol. Sci. 2008, 108, 135-139.

227. Ohya, S.; Kuwata, Y.; Sakamoto, K.; Muraki, K.; Imaizumi, Y. Cardioprotective effects of estradiol include the activation of large-conductance $\mathrm{Ca}^{2+}$-activated $\mathrm{K}^{+}$channels in cardiac mitochondria. Am. J. Physiol. Heart Circ. Physiol. 2005, 289, H1635-H1642.

228. Tano, J.-Y.; Gollasch, M. Calcium-activated potassium channels in ischemia reperfusion: A brief update. Front. Physiol. 2014, doi:10.3389/fphys.2014.00381.

229. Hagen, B.M.; Sanders, K.M. Deglycosylation of the betal-subunit of the BK channel changes its biophysical properties. Am. J. Physiol. Cell Physiol. 2006, 291, C750-C756.

230. Borchert, G.H.; Yang, C.; Kolár, F. Mitochondrial BKCa channels contribute to protection of cardiomyocytes isolated from chronically hypoxic rats. Am. J. Physiol. Heart Circ. Physiol. 2011, 300, H507-H513.

231. Borchert, G.H.; Hlaváčková, M.; Kolář, F. Pharmacological activation of mitochondrial $\mathrm{BK}(\mathrm{Ca})$ channels protects isolated cardiomyocytes against simulated reperfusion-induced injury. Exp. Biol. Med. Maywood 2013, 238, 233-241.

232. Neckár, J.; Borchert, G.H.; Hlousková, P.; Mícová, P.; Nováková, O.; Novák, F.; Hroch, M.; Papousek, F.; Ost'ádal, B.; Kolár, F. Brief daily episode of normoxia inhibits cardioprotection conferred by chronic continuous hypoxia. Role of oxidative stress and BKCa channels. Curr. Pharm. Des. 2013, 19, 6880-6889. 
233. Noursadeghi, E.; Jafari, A.; Saghiri, R.; Sauve, R.; Eliassi, A. Impairment of brain mitochondrial charybdotoxin- and ATP-insensitive BK channel activities in diabetes. Neuromol. Med. 2014, 16, 862-871.

234. Leffler, C.W.; Parfenova, H.; Jaggar, J.H. Carbon monoxide as an endogenous vascular modulator. Am. J. Physiol. Heart Circ. Physiol. 2011, 301, H1-H11.

235. Li, H.; Förstermann, U. Prevention of atherosclerosis by interference with the vascular nitric oxide system. Curr. Pharm. Des. 2009, 15, 3133-3145.

236. Ooi, L.; Gigout, S.; Pettinger, L.; Gamper, N. Triple cysteine module within M-type $\mathrm{K}^{+}$ channels mediates reciprocal channel modulation by nitric oxide and reactive oxygen species. J. Neurosci. 2013, 33, 6041-6046.

237. Liu, Y.; Gutterman, D.D. Oxidative stress and potassium channel function. Clin. Exp. Pharmacol. Physiol. 2002, 29, 305-311.

238. Drews, G.; Düfer, M. Role of K(ATP) channels in $\beta$-cell resistance to oxidative stress. Diabetes Obes. MeTable 2012, 14, 120-128.

239. Liu, Y.; Kalogeris, T.; Wang, M.; Zuidema, M.Y.; Wang, Q.; Dai, H.; Davis, M.J.; Hill, M.A.; Korthuis, R.J. Hydrogen sulfide preconditioning or neutrophil depletion attenuates ischemia-reperfusion-induced mitochondrial dysfunction in rat small intestine. Am. J. Physiol. Gastrointest. Liver Physiol. 2012, 302, G44-G54.

240. Moudgil, R.; Michelakis, E.D.; Archer, S.L. The role of $\mathrm{K}^{+}$channels in determining pulmonary vascular tone, oxygen sensing, cell proliferation, and apoptosis: Implications in hypoxic pulmonary vasoconstriction and pulmonary arterial hypertension. Microcirculation 2006, 13, 615-632.

241. Wolfram Kuhlmann, C.R.; Wiebke Lüdders, D.; Schaefer, C.A.; Kerstin Most, A.; Backenköhler, U.; Neumann, T.; Tillmanns, H.; Erdogan, A. Lysophosphatidylcholine-induced modulation of $\mathrm{Ca}^{2+}$-activated $\mathrm{K}^{+}$channels contributes to ROS-dependent proliferation of cultured human endothelial cells. J. Mol. Cell. Cardiol. 2004, 36, 675-682.

242. Pardo, L.A. Voltage-gated potassium channels in cell proliferation. Physiology 2004, 19, 285-292.

243. Huang, X.; Jan, L.Y. Targeting potassium channels in cancer. J. Cell Biol. 2014, 206, 151-162.

244. Pardo, L.A.; Stühmer, W. The roles of $\mathrm{K}^{+}$channels in cancer. Nat. Rev. Cancer 2014, 14, 39-48.

245. Yang, M.; Brackenbury, W.J. Membrane potential and cancer progression. Front. Physiol. 2013, doi:10.3389/fphys.2013.00185.

246. Barghouth, P.G.; Thiruvalluvan, M.; Oviedo, N.J. Bioelectrical regulation of cell cycle and the planarian model system. Biochim. Biophys. Acta 2015, doi:10.1016/j.bbamem.2015.02.024.

247. Blackiston, D.J.; McLaughlin, K.A.; Levin, M. Bioelectric controls of cell proliferation: Ion channels, membrane voltage and the cell cycle. Cell Cycle 2009, 8, 3519-3528.

248. Weiger, T.M.; Hermann, A. Cell proliferation, potassium channels, polyamines and their interactions: A mini review. Amino Acids 2014, 46, 681-688. 
249. Chattopadhyay, M.K.; Tabor, C.W.; Tabor, H. Polyamine deficiency leads to accumulation of reactive oxygen species in a spe2Delta mutant of Saccharomyces cerevisiae. Yeast Chichester. Engl. 2006, 23, 751-761.

250. Takano, K.; Ogura, M.; Nakamura, Y.; Yoneda, Y. Neuronal and glial responses to polyamines in the ischemic brain. Curr. Neurovasc. Res. 2005, 2, 213-223.

251. Pamenter, M.E.; Haddad, G.G. Do BK channels mediate glioma hypoxia-tolerance? Channels 2014, 8, 176-177.

252. Liu, X.; Chang, Y.; Reinhart, P.H.; Sontheimer, H.; Chang, Y. Cloning and characterization of glioma BK, a novel BK channel isoform highly expressed in human glioma cells. J. Neurosci. 2002, 22, 1840-1849.

253. Sontheimer, H. An unexpected role for ion channels in brain tumor metastasis. Exp. Biol. Med. 2008, 233, 779-791.

254. Cheng, Y.; Gu, X.Q.; Bednarczyk, P.; Wiedemann, F.R.; Haddad, G.G.; Siemen, D. Hypoxia increases activity of the BK-channel in the inner mitochondrial membrane and reduces activity of the permeability transition pore. Cell. Physiol. Biochem. 2008, 22, 127-136.

255. Gu, X.Q.; Pamenter, M.E.; Siemen, D.; Sun, X.; Haddad, G.G. Mitochondrial but not plasmalemmal BK channels are hypoxia-sensitive in human glioma. Glia 2014, 62, 504-513.

256. Gu, X.Q.; Siemen, D.; Parvez, S.; Cheng, Y.; Xue, J.; Zhou, D.; Sun, X.; Jonas, E.A.; Haddad, G.G. Hypoxia increases BK channel activity in the inner mitochondrial membrane. Biochem. Biophys. Res. Commun. 2007, 358, 311-316.

257. Han, X.; Xi, L.; Wang, H.; Huang, X.; Ma, X.; Han, Z.; Wu, P.; Ma, X.; Lu, Y.; Wang, G.; et al. The potassium ion channel opener NS1619 inhibits proliferation and induces apoptosis in A2780 ovarian cancer cells. Biochem. Biophys. Res. Commun. 2008, 375, 205-209.

258. Debska-Vielhaber, G.; Godlewski, M.M.; Kicinska, A.; Skalska, J.; Kulawiak, B.; Piwonska, M.; Zablocki, K.; Kunz, W.S.; Szewczyk, A.; Motyl, T. Large-conductance $\mathrm{K}^{+}$channel openers induce death of human glioma cells. J. Physiol. Pharmacol. 2009, 60, 27-36.

259. Halestrap, A.P. What is the mitochondrial permeability transition pore? J. Mol. Cell. Cardiol. 2009, 46, 821-831.

260. Chatterjee, S.; Nieman, G.F.; Christie, J.D.; Fisher, A.B. Shear stress-related mechanosignaling with lung ischemia: Lessons from basic research can inform lung transplantation. Am. J. Physiol. Lung Cell. Mol. Physiol. 2014, 307, L668-L680.

261. Mohamed, M.S.A. Calcium-activated potassium channels in ischemia-reperfusion: Learning for the clinical application. Pulm. Med. 2015, doi:10.3389/fmed.2015.00021.

262. Noda, K.; Shigemura, N.; Tanaka, Y.; Bhama, J.; D’Cunha, J.; Kobayashi, H.; Luketich, J.D.; Bermudez, C.A. Hydrogen preconditioning during ex vivo lung perfusion improves the quality of lung grafts in rats. Transplantation 2014, 98, 499-506.

263. Dong, D.-L.; Zhang, Y.; Lin, D.-H.; Chen, J.; Patschan, S.; Goligorsky, M.S.; Nasjletti, A.; Yang, B.-F.; Wang, W.-H. Carbon monoxide stimulates the $\mathrm{Ca}^{2+}$-activated big conductance $\mathrm{K}$ channels in cultured human endothelial cells. Hypertension 2007, 50, 643-651. 
264. Carrasquel-Ursulaez, W.; Contreras, G.F.; Sepúlveda, R.V.; Aguayo, D.; González-Nilo, F.; González, C.; Latorre, R. Hydrophobic interaction between contiguous residues in the S6 transmembrane segment acts as a stimuli integration node in the BK channel. J. Gen. Physiol. 2015, 145, 61-74.

265. McBride, C.S.; Baier, F.; Omondi, A.B.; Spitzer, S.A.; Lutomiah, J.; Sang, R.; Ignell, R.; Vosshall, L.B. Evolution of mosquito preference for humans linked to an odorant receptor. Nature 2014, 515, 222-227.

266. Adler, E.M. Of ELIC and evolution. J. Gen. Physiol. 2015, 145, 1-2.

267. Korovkina, V.P.; England, S.K. Molecular diversity of vascular potassium channel isoforms. Clin. Exp. Pharmacol. Physiol. 2002, 29, 317-323.

268. Ko, E.A.; Han, J.; Jung, I.D.; Park, W.S. Physiological roles of $\mathrm{K}^{+}$channels in vascular smooth muscle cells. J. Smooth Muscle Res. 2008, 44, 65-81.

269. Park, S.W.; Noh, H.J.; Sung, D.J.; Kim, J.G.; Kim, J.M.; Ryu, S.-Y.; Kang, K.; Kim, B.; Bae, Y.M.; Cho, H. Hydrogen peroxide induces vasorelaxation by enhancing 4-aminopyridine-sensitive Kv currents through S-glutathionylation. Pflüg. Arch. 2015, 467, 285-297.

270. Fukumoto, M.; Nakaizumi, A.; Zhang, T.; Lentz, S.I.; Shibata, M.; Puro, D.G. Vulnerability of the retinal microvasculature to oxidative stress: Ion channel-dependent mechanisms. Am. J. Physiol. Cell Physiol. 2012, 302, C1413-C1420. 


\title{
Hypoxia, Oxidative Stress and Fat
}

\author{
Nikolaus Netzer, Hannes Gatterer, Martin Faulhaber, Martin Burtscher, \\ Stephan Pramsohler and Dominik Pesta
}

\begin{abstract}
Metabolic disturbances in white adipose tissue in obese individuals contribute to the pathogenesis of insulin resistance and the development of type 2 diabetes mellitus. Impaired insulin action in adipocytes is associated with elevated lipolysis and increased free fatty acids leading to ectopic fat deposition in liver and skeletal muscle. Chronic adipose tissue hypoxia has been suggested to be part of pathomechanisms causing dysfunction of adipocytes. Hypoxia can provoke oxidative stress in human and animal adipocytes and reduce the production of beneficial adipokines, such as adiponectin. However, time-dose responses to hypoxia relativize the effects of hypoxic stress. Long-term exposure of fat cells to hypoxia can lead to the production of beneficial substances such as leptin. Knowledge of time-dose responses of hypoxia on white adipose tissue and the time course of generation of oxidative stress in adipocytes is still scarce. This paper reviews the potential links between adipose tissue hypoxia, oxidative stress, mitochondrial dysfunction, and low-grade inflammation caused by adipocyte hypertrophy, macrophage infiltration and production of inflammatory mediators.
\end{abstract}

Reprinted from Biomolecules. Cite as: Netzer, N.; Gatterer, H.; Faulhaber, M.; Burtscher, M.; Pramsohler, S.; Pesta, D. Hypoxia, Oxidative Stress and Fat. Biomolecules 2015, 5, 1143-1150.

\section{What Do We Really Know about Oxygen Concentration in White Adipose Tissue?}

In order to understand under which circumstances hypoxia can induce oxidative stress in adipocytes it is useful to consider some basic physical principles. This is important because the perception of oxidative stress and fast reactions in response to hypoxia usually relate to tissues with a high blood perfusion and a solubility coefficient similar to that of water. However, this is different in fat tissue. According to William Henry's law [1], the solubility of oxygen in fat and oil is five times higher than in water. The amount of dissolved oxygen in fat is around $5 \mathrm{mg} / 100 \mathrm{~mL}$, as compared to about $0.9 \mathrm{mg} / 100 \mathrm{~mL}$ for water. However, according to John Scott Haldane's [2] research about the transport of gases in blood and additional calculations of Albert Bühlmann in Zürich 50 years later [3], the saturation and desaturation of adipose tissue with oxygen, which is comparable to that of nerve and brain tissue, takes more time than that of, for example, muscle tissue. For bone, cartilage and tendon tissue, this process takes even longer. Therefore it is difficult to estimate when the effect of transient hypoxia reaches fat tissue and how fast reoxygenation can lead to oxidative stress. The partial pressure of oxygen $\left(\mathrm{pO}_{2}\right)$ of white adipose tissue completely saturated with oxygen in a healthy lean young adult is $55-60 \mathrm{mmHg}$, not far from arterial blood $p \mathrm{O}_{2}$ (Figure 1A). 

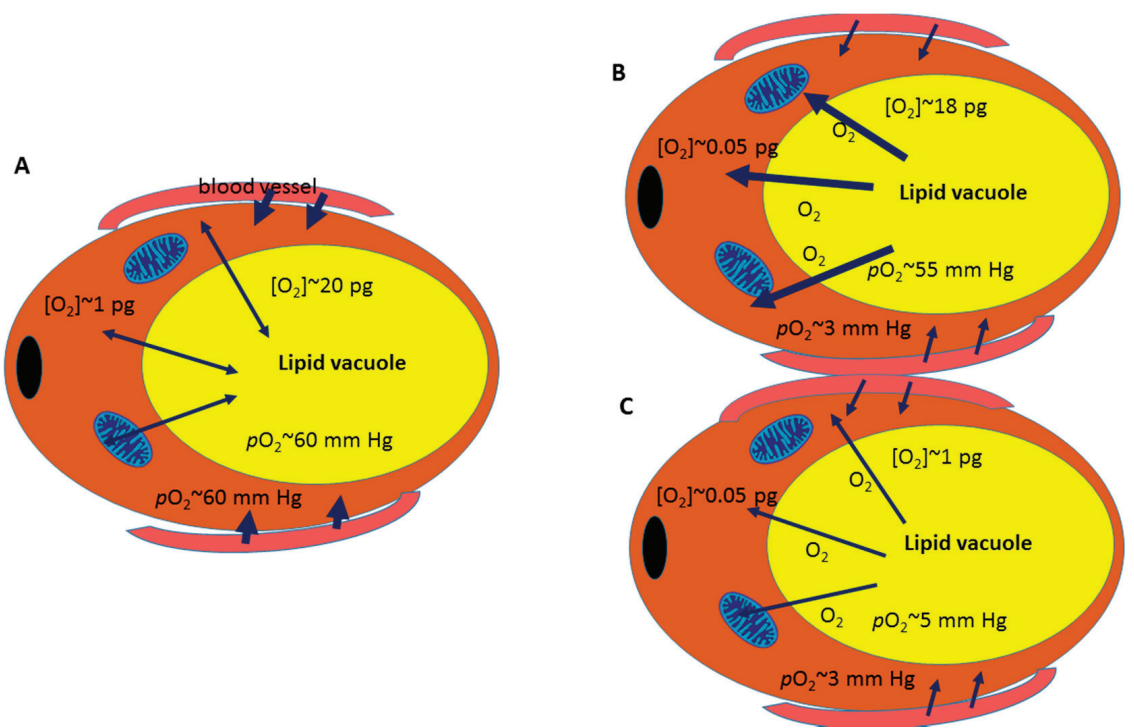

Figure 1. Schematic of oxygen transport in adipocytes: (A) Equilibrium oxygen saturation: during long-term exposure at sea level, no net flux of $\mathrm{O}_{2}$ occurs between the cytosolic and the lipid compartment of the cell as both compartments are in equilibrium due to saturation with $\mathrm{O}_{2}$. (B) Acute Hypoxia: during acute hypoxia, the $p \mathrm{O}_{2}$ in the cytoplasm drops whereas the $p \mathrm{O}_{2}$ in the lipid vacuole lags behind due to the slower equilibration of fat with oxygen. Due to the concentration difference, an outward flux of oxygen from the lipid vacuole into the cytoplasm is expected, which decreases with prolonged hypoxia. (C) Prolonged Hypoxia: with prolonged hypoxic exposure, the outward flux decreases due to faster desaturation of the cytosolic and delayed, but continuous desaturation of the lipid compartment of the cell. Assumptions are based on an adipocyte volume of $500 \mathrm{~nL}$ [4] and a lipid vacuole fraction of $80 \%$ of cell volume.

\section{Hypoxia in White Adipose Tissue: A Question of Time and Dose}

In their publication in the journal Circulation, Goossens and coauthors [5] show that their new method to continuously measure oxygen tension in the interstitium between adipocytes via optochemical microdialysis sensors over several hours gives a better insight into hypoxic reactions of fat cells as compared to assessment with conventional electrochemical sensors. Their results in humans differ somewhat from results obtained with electrochemical sensors, which measure $\mathrm{pO}_{2}$ at one time point only, and their results in humans differ strongly from results obtained from rodents with electrochemical sensors.

Interestingly, these authors found that, unlike what was expected, $p \mathrm{O}_{2}$ in white adipose tissue (interstitium between adipocytes) of obese individuals $(80 \mathrm{mmHg})$ was higher than that in lean individuals $(60 \mathrm{mmHg})$ despite a reduced arterial blood flow. This has been explained by insulin resistance and a lower oxygen consumption of already impaired adipocytes with higher inflammatory markers, such as interleukin (IL)-6, in the obese. 
Nevertheless, taking these results into account, the question arises, when the hypoxic stimulus hits the fat cells and how fast changes in oxygen tension in the cell (the above mentioned measurements reflect $p \mathrm{O}_{2}$ in the interstitium) really occur. In an obese, insulin resistant individual it would take a while until a short period of hypoxia with arterial oxygen desaturation, as can be caused by obstructive sleep apnea, could transform hyperoxic or normoxic adipose tissue into a hypoxic state. However, quick changes from hyperoxia or normoxia to hypoxia would be needed in order to cause oxidative stress.

Another study by Wang et al. [6], on the time-dose response of adipokine secretion, in response to hypoxia in human adipocyte cell culture, also shows that exposure to hypoxia is important in order to determine if oxidative stress occurs in human fat tissue. Wang et al. mimicked hypoxia in the cells for $24 \mathrm{~h}$ using cobalt chloride $\left(\mathrm{CoCl}_{2}\right)$ and then determined hypoxia-inducible factor (HIF)- $1 \alpha$ as an indicator of hypoxia and leptin mRNA, adiponectin, and inflammatory markers, such as tumor necrosis factor (TNF)- $\alpha 1$ and IL-6, among others. As expected, HIF-1 $\alpha$ increased, but this increase was more pronounced in preadipocytes than in adipocytes; the oxidative stress markers, inflammatory markers and leptin also increased with hypoxia while adiponectin decreased, but the time of responses differed markedly between cell types. HIF reached its maximum after $8 \mathrm{~h}$ during the first $24 \mathrm{~h}$ and then slowly decreased until day 14 [6]. The highest level of oxidative stress in accordance to the rise of the antioxidant glutathione occurred in parallel to HIF after $8 \mathrm{~h}$ and subsequently the adipocytes may have adapted to hypoxia and glutathione slowly decreased with HIF. Adiponectin decreased continuously right after the onset of hypoxia, whereas leptin reached its peak with a delay of $16 \mathrm{~h}$. Among the inflammatory markers, which mostly go in accordance with HIF and glutathione, TNF- $\alpha$ already reached its peak after $2 \mathrm{~h}$.

All these data demonstrate how important it is to consider the time response to hypoxia, and question acute effects in adipocytes after seconds of oxygen desaturation, as occurring, for instance, during intermittent hypoxia in obstructive sleep apnea. Not telling, however, how this type of intermittent hypoxia may have accumulative effects. In any case it has to be taken into account that after some hours of hypoxia, adipocytes start to adapt to the hypoxic stimulus accompanied by oxidative stress reduction, and that older adipocytes seem to be somewhat more "relaxed" in their reaction to hypoxia (i.e., they show a delayed response) compared to younger ones.

\section{Lipid Metabolism and Adipose Tissue Hypoxia}

Metabolic disturbances in white adipose tissue in obese individuals contribute to the pathogenesis of insulin resistance and the development of type 2 diabetes mellitus. Impaired insulin action in adipocytes is associated with elevated lipolysis and increased release of free fatty acids leading to ectopic fat deposition in liver and skeletal muscle. Chronic hypoxia has been suggested to be part of pathomechanisms causing dysfunction of adipocytes [7].

In general, chronic hypoxia leads to derangements in lipid metabolism and reduced lipoprotein clearance by decreasing lipoprotein lipase activity in mice [8] and diminished subcutaneous adipose tissue lipolysis by decreased efficiency of beta-adrenergic, growth hormone and parathyroid hormone lipolytic signaling in humans [9]. A similar depression of lipolysis in human adipocytes was seen after induction of pseudo-hypoxia by ablating the adipose prolyl hydroxylase enzyme 2 gene [10]. 
Acute hypoxia, however, was shown to increase lipolysis by activation of adipose protein kinase A via increased epinephrine and norepinephrine release following sympathetic nervous system stimulation [11]. Propranolol treatment markedly reduced lipolysis in this experiment, suggesting that an activated sympathetic nervous system contributed to increased lipolysis. Interestingly, this is in contrast to findings from Larsen et al., who studied the effects of hypoxia on lipolysis in isolated rat myocardial cells and reported that propranolol did not affect the hypoxia-induced increase in lipolysis, which suggests that other, non-adrenergic mechanisms might be at play during hypoxia in cardiac myocytes [12].

In addition to its effects on lipolysis, hypoxia also alters glucose uptake capacity in human adipocytes. Wood et al. showed that glucose transporter (GLUT)-1, GLUT-3, and GLUT-5 gene expression and GLUT-1 protein is increased in human adipocytes in response to hypoxia, and that these changes are accompanied by a hypoxia-induced increase in glucose uptake [13]. In $o b / o b$ mice, adipose tissue hypoxia caused free fatty acid release and inhibited glucose uptake in adipocytes by inhibition of the insulin-signaling pathway [14]. Thus, it seems likely that adipose tissue hypoxia will elicit different metabolic effects in non-obese and obese individuals and likely also in different types of adipose tissue, i.e., central visceral (abdominal) and peripheral subcutaneous (thighs and buttocks) depots.

\section{Blood Flow Regulation in Adipose Tissue}

White adipose tissue is now recognized, not only as a passive storage organ, but also as a highly active metabolic organ. Importantly, all metabolic processes in adipose tissue depend on blood supply. Blood supply and regulation in adipose tissue differ largely from other metabolic organs, such as skeletal muscle or liver [15]. Under fasting conditions, adipose tissue blood flow is mainly regulated by vasodilatory (nitric oxide) and vasoconstrictive (alpha 2-adrenergic and angiotensin II) mechanisms, while $\beta$-adrenergic stimulation becomes important after a meal (postprandially) [15].

Disturbances in the regulation of adipose tissue blood flow have been linked to obesity and insulin-resistance [16]. It has been demonstrated that fasting adipose tissue blood flow is reduced in obese, compared to non-obese, individuals [17]. Whereas exercise training clearly increases blood flow to working muscles, this seems to not be the case for adipose tissue [15]. Since exercise training improves insulin sensitivity (mainly in skeletal muscle), but has only minor effects on adipose tissue, exercise training may improve insulin resistance independent of unaltered adipose tissue blood flow [16]. Although adipose tissue blood flow is predominantly regulated by glucose and insulin, diminished blood flow affects lipid metabolism associated with dyslipidemia in the fasting and postprandial periods [16]. It is the growing fat mass, in particular of the abdominal adipose tissue, which is associated with unfavorable changes in adipose tissue blood flow, and the development of metabolic disorders. When exposed to moderate systemic hypoxia, subcutaneous adipose blood flow at rest is comparable to that in normoxia, however, during exercise in hypoxia, adipose tissue blood flow is reduced compared to normoxic exercise [18]. This may be explained by blood flow redistribution due to the diminished availability of oxygen in skeletal muscle. Taken together, adipose tissue blood flow regulation is a very complex process, which seems to be impaired in obese and/or insulin resistant individuals. Interventions like exercise training and exposure to normobaric or hypobaric hypoxia may 
differently impact on adipose tissue blood flow, adipose tissue oxygen tension, oxidative stress, and related consequences.

\section{Changes in Adipose Tissue Oxygen Tension and Its Consequences}

Several studies report that hypoxia may occur within adipose tissue due to the obesity-associated expansion of adipocytes and a concomitant reduction in capillary density and blood flow [19-21]. However, additionally, contrasting reports exist suggesting increased [5] or unaltered oxygen content [22]. Different techniques for the determination of $\mathrm{pO}_{2}$ might be responsible for the divergent findings and it would be of importance to establish whether hypoxia or hyperoxia occurs within the adipose tissue. In various experiments, adipose tissue was exposed to extremely low or high levels of $p \mathrm{O}_{2}$, as reviewed elsewhere [23]. Both conditions lead to oxidative stress [24,25] and a pro-inflammatory response in adipocytes [23]. Oxidative stress, in addition to other factors (e.g., endoplasmic reticulum stress) was assumed to increase macrophage infiltration into white adipose tissue [26,27], thus causing further inflammation [26]. The inflammatory state induces expression of genes including TNF- $\alpha$, IL-1, IL-6, monocyte chemoattractant protein-1, plasminogen activator inhibitor-1, macrophage migration inhibition factor, inducible nitric oxide synthase, matrix metalloproteinases (MMP) 9, and MMP2 [28]. The mechanistical relation of this gene expression profile is related to activation of NF- $\mathrm{B}$ and HIF-1 $\alpha$ [28]. To sum up, the oxidative stress and the pro-inflammatory state in response to severe hypoxia or hyperoxia may cause dysregulation of adipocytokines, leading to obesity-associated diseases $[20,24,26]$. Furthermore, the increased reactive oxygen species (ROS) secretion into peripheral blood from adipose tissue is involved in induction of insulin resistance in skeletal muscle and adipose tissue, impaired insulin secretion, and pathogenesis of various vascular diseases such as atherosclerosis and hypertension [26]. On the other hand, it was suggested that a transient increase of ROS is important for the insulin signaling pathway and might prevent further lipid storage by suppressing lipogenic genes [26]. As indicated, many findings stem from experiments performed in extremely low or high $\mathrm{pO}_{2}$. Under in vivo $\mathrm{pO}_{2}$ levels $\left(3 \%-11 \% \mathrm{O}_{2}\right)$, conflicting results were found showing positive [5] or negative [29] correlations to the inflammatory status. Additionally, concentrations of $10 \% \mathrm{O}_{2}$ and below increased adipocyte triacylglycerol content and enhanced secretion rates of IL-6 and dipeptidyl-peptidase-4 [30]. Based on the findings of Goossens et al. who found increased $p \mathrm{O}_{2}$ levels that positively correlated with several pro-inflammatory markers and negatively correlated with peripheral insulin sensitivity [5], one might hypothesize that chronic hypoxic exposure might positively affect the inflammatory state. In accordance with these results, mice that were exposed to chronic hypoxia for 21 days $\left(8 \% \mathrm{O}_{2}\right)$ showed decreased adipocyte size, improved mitochondrial function and decreased macrophage infiltration [31]. Furthermore, in obese men, ten nights of hypoxic exposure $\left(15 \% \mathrm{O}_{2}\right)$ led to increased whole-body insulin sensitivity [32] In contrast, it should be outlined that obstructive sleep apnea syndrome, characterized by cycles of severe hypoxia, is considered a risk factor for insulin resistance [33]. Therefore, the dose, duration, and patterns of hypoxic exposure may determine the effects on metabolic and cardiovascular health. 


\section{Conclusions}

Diminished blood flow and metabolic disturbances in white adipose tissue in obese individuals contribute to the pathogenesis of insulin resistance and the development of type 2 diabetes mellitus. Hyperinsulinemia leads to increased lipolysis, ectopic deposition of fat in liver and skeletal muscles decreased glucose uptake into adipocytes by inhibition of the insulin-signaling pathway.

Since vasculature cannot expand with adipocyte hypertrophy, hypoxia, mitochondrial dysfunction, and oxidative stress are important consequences, accompanied by impaired adipokine secretion and inflammation. Hypoperfusion and hypoxia in adipose tissues likely underlie the dysregulated production of adipocytokines and metabolic syndrome in obesity. Nutritional interventions, weight loss, and regular physical activity are the most promising measures to counteract these deleterious effects. Clinically, these countermeasures will likely result in a decrease in fat mass and vasculature remodeling, resulting in increased perfusion of adipose tissue, improved mitochondrial function, and metabolic health.

\section{Acknowledgments}

This work was supported by the Austrian Science Fund (FWF), project number J 3267.

\section{Author Contributions}

Nikolaus Netzer and Dominik Pesta conceived the idea for the manuscript. Nikolaus Netzer, Hannes Gatterer, Martin Faulhaber, Martin Burtscher, Stephan Pramsohler and Dominik Pesta contributed to the writing of the manuscript.

\section{Conflicts of Interest}

The authors declare no conflict of interest.

\section{References}

1. Henry, W. Experiments on the quantity of gases absorbed by water, at different temperatures, and under different pressures. Philos. Trans. R. S. Lond. 1803, 93, 29-276.

2. Haldane, J.S.; Priestley, J.G. Respiration, 2nd ed.; Oxford University Press: London, UK, 1935.

3. Bühlmann, A.A.; Völlm, E.B.; Nussberger, P. Tauchmedizin, 5th ed.; Springer: New York, NK, USA, 2002.

4. Arner, P.; Bernard, S.; Salehpour, M.; Possnert, G.; Liebl, J.; Steier, P.; Buchholz, B.A.; Eriksson, M.; Arner, E.; Hauner, H.; et al. Dynamics of human adipose lipid turnover in health and metabolic disease. Nature 2011, 478, 110-113.

5. Goossens, G.H.; Bizzarri, A.; Venteclef, N.; Essers, Y.; Cleutjens, J.P.; Konings, E.; Jocken, J.W.; Cajlakovic, M.; Ribitsch, V.; Clement, K.; et al. Increased adipose tissue oxygen tension in obese compared with lean men is accompanied by insulin resistance, impaired adipose tissue capillarization, and inflammation. Circulation 2011, 124, 67-76. 
6. Wang, B.; Wood, I.S.; Trayhurn, P. Dysregulation of the expression and secretion of inflammation-related adipokines by hypoxia in human adipocytes. Pflugers Arch. 2007, 455, 479-492.

7. Wood, I.S.; de Heredia, F.P.; Wang, B.; Trayhurn, P. Cellular hypoxia and adipose tissue dysfunction in obesity. Proc. Nutr. Soc. 2009, 68, 370-377.

8. Drager, L.F.; Li, J.; Shin, M.K.; Reinke, C.; Aggarwal, N.R.; Jun, J.C.; Bevans-Fonti, S.; Sztalryd, C.; O’Byrne, S.M.; Kroupa, O.; et al. Intermittent hypoxia inhibits clearance of triglyceride-rich lipoproteins and inactivates adipose lipoprotein lipase in a mouse model of sleep apnoea. Eur. Heart J. 2012, 33, 783-790.

9. De Glisezinski, I.; Crampes, F.; Harant, I.; Havlik, P.; Gardette, B.; Jammes, Y.; Souberbielle, J.C.; Richalet, J.P.; Riviere, D. Decrease of subcutaneous adipose tissue lipolysis after exposure to hypoxia during a simulated ascent of Mt. Everest. Pflugers Arch. 1999, 439, 134-140.

10. Michailidou, Z.; Morton, N.M.; Moreno Navarrete, J.M.; West, C.C.; Stewart, K.J.; Fernandez-Real, J.M.; Schofield, C.J.; Seckl, J.R.; Ratcliffe, P.J. Adipocyte pseudohypoxia suppresses lipolysis and facilitates benign adipose tissue expansion. Diabetes 2015, 64, 733-745.

11. Shin, M.-K.; Jun, J.C.; Bevans-Fonti, S.; Drager, L.F.; Polotsky, V.Y. Acute hypoxia induces lipolysis via activation of the sympathetic nervous system. In Proceedings of the American Thoracic Society 2011 International Conference, Denver, CO, USA, 13-18 May 2011.

12. Larsen, T.S.; Myrmel, T.; Skulberg, A.; Severson, D.L.; Mjos, O.D. Effects of hypoxia on lipolysis in isolated rat myocardial cells. Mol. Cell. Biochem. 1989, 88, 139-144.

13. Wood, I.S.; Wang, B.; Lorente-Cebrian, S.; Trayhurn, P. Hypoxia increases expression of selective facilitative glucose transporters (glut) and 2-deoxy-D-glucose uptake in human adipocytes. Biochem. Biophys. Res. Commun. 2007, 361, 468-473.

14. Yin, J.; Gao, Z.; He, Q.; Zhou, D.; Guo, Z.; Ye, J. Role of hypoxia in obesity-induced disorders of glucose and lipid metabolism in adipose tissue. Am. J. Physiol. Endocrinol. Metab. 2009, 296, E333-E342.

15. Sotornik, R.; Brassard, P.; Martin, E.; Yale, P.; Carpentier, A.C.; Ardilouze, J.L. Update on adipose tissue blood flow regulation. Am. J. Physiol. Endocrinol. Metab. 2012, 302, E1157-E1170.

16. Karpe, F.; Fielding, B.A.; Ilic, V.; Macdonald, I.A.; Summers, L.K.; Frayn, K.N. Impaired postprandial adipose tissue blood flow response is related to aspects of insulin sensitivity. Diabetes 2002, 51, 2467-2473.

17. Blaak, E.E.; van Baak, M.A.; Kemerink, G.J.; Pakbiers, M.T.; Heidendal, G.A.; Saris, W.H. Beta-adrenergic stimulation and abdominal subcutaneous fat blood flow in lean, obese, and reduced-obese subjects. Metabolism 1995, 44, 183-187.

18. Heinonen, I.; Kemppainen, J.; Kaskinoro, K.; Knuuti, J.; Boushel, R.; Kalliokoski, K.K. Capacity and hypoxic response of subcutaneous adipose tissue blood flow in humans. Circ. J. 2014, 78, 1501-1506. 
19. Hosogai, N.; Fukuhara, A.; Oshima, K.; Miyata, Y.; Tanaka, S.; Segawa, K.; Furukawa, S.; Tochino, Y.; Komuro, R.; Matsuda, M.; et al. Adipose tissue hypoxia in obesity and its impact on adipocytokine dysregulation. Diabetes 2007, 56, 901-911.

20. Trayhurn, P. Hypoxia and adipose tissue function and dysfunction in obesity. Physiol. Rev. 2013, 93, 1-21.

21. Trayhurn, P. Hypoxia and adipocyte physiology: Implications for adipose tissue dysfunction in obesity. Annu. Rev. Nutr. 2014, 34, 207-236.

22. Hodson, L.; Humphreys, S.M.; Karpe, F.; Frayn, K.N. Metabolic signatures of human adipose tissue hypoxia in obesity. Diabetes 2013, 62, 1417-1425.

23. Goossens, G.H.; Blaak, E.E. Adipose tissue dysfunction and impaired metabolic health in human obesity: A matter of oxygen? Front. Endocrinol. 2015, doi:10.3389/fendo.2015.00055.

24. Lee, Y.S.; Kim, A.Y.; Choi, J.W.; Kim, M.; Yasue, S.; Son, H.J.; Masuzaki, H.; Park, K.S.; Kim, J.B. Dysregulation of adipose glutathione peroxidase 3 in obesity contributes to local and systemic oxidative stress. Mol. Endocrinol. 2008, 22, 2176-2189.

25. Zhang, L.; Ebenezer, P.J.; Dasuri, K.; Fernandez-Kim, S.O.; Francis, J.; Mariappan, N.; Gao, Z.; Ye, J.; Bruce-Keller, A.J.; Keller, J.N. Aging is associated with hypoxia and oxidative stress in adipose tissue: Implications for adipose function. Am. J. Physiol. Endocrinol. Metab. 2011, 301, E599-E607.

26. Furukawa, S.; Fujita, T.; Shimabukuro, M.; Iwaki, M.; Yamada, Y.; Nakajima, Y.; Nakayama, O.; Makishima, M.; Matsuda, M.; Shimomura, I. Increased oxidative stress in obesity and its impact on metabolic syndrome. J. Clin. Invest. 2004, 114, 1752-1761.

27. Wellen, K.E.; Hotamisligil, G.S. Inflammation, stress, and diabetes. J. Clin. Invest. 2005, 115, 1111-1119.

28. Ye, J. Emerging role of adipose tissue hypoxia in obesity and insulin resistance. Int. J. Obes. 2009, 33, 54-66.

29. Pasarica, M.; Sereda, O.R.; Redman, L.M.; Albarado, D.C.; Hymel, D.T.; Roan, L.E.; Rood, J.C.; Burk, D.H.; Smith, S.R. Reduced adipose tissue oxygenation in human obesity: Evidence for rarefaction, macrophage chemotaxis, and inflammation without an angiogenic response. Diabetes 2009, 58, 718-725.

30. Famulla, S.; Schlich, R.; Sell, H.; Eckel, J. Differentiation of human adipocytes at physiological oxygen levels results in increased adiponectin secretion and isoproterenol-stimulated lipolysis. Adipocyte 2012, 1, 132-181.

31. Van den Borst, B.; Schols, A.M.; de Theije, C.; Boots, A.W.; Köhler, S.E.; Goossens, G.H.; Gosker, H.R. Characterization of the inflammatory and metabolic profile of adipose tissue in a mouse model of chronic hypoxia. J. Appl. Physiol. 2013, 114, 1619-1628.

32. Lecoultre, V.; Peterson, C.M.; Covington, J.D.; Ebenezer, P.J.; Frost, E.A.; Schwarz, J.M.; Ravussin, E. Ten nights of moderate hypoxia improves insulin sensitivity in obese humans. Diabetes Care 2013, 36, e197-e198.

33. Ip, M.S.; Lam, B.; Ng, M.M.; Lam, W.K.; Tsang, K.W.; Lam, K.S. Obstructive sleep apnea is independently associated with insulin resistance. Am. J. Respir. Crit. Care Med. 2002, 165, $670-676$. 


\title{
Advanced Glycation End Products and Oxidative Stress in Type 2 Diabetes Mellitus
}

\author{
Kerstin Nowotny, Tobias Jung, Annika Höhn, Daniela Weber and Tilman Grune
}

\begin{abstract}
Type 2 diabetes mellitus (T2DM) is a very complex and multifactorial metabolic disease characterized by insulin resistance and $\beta$ cell failure leading to elevated blood glucose levels. Hyperglycemia is suggested to be the main cause of diabetic complications, which not only decrease life quality and expectancy, but are also becoming a problem regarding the financial burden for health care systems. Therefore, and to counteract the continually increasing prevalence of diabetes, understanding the pathogenesis, the main risk factors, and the underlying molecular mechanisms may establish a basis for prevention and therapy. In this regard, research was performed revealing further evidence that oxidative stress has an important role in hyperglycemia-induced tissue injury as well as in early events relevant for the development of T2DM. The formation of advanced glycation end products (AGEs), a group of modified proteins and/or lipids with damaging potential, is one contributing factor. On the one hand it has been reported that AGEs increase reactive oxygen species formation and impair antioxidant systems, on the other hand the formation of some AGEs is induced per se under oxidative conditions. Thus, AGEs contribute at least partly to chronic stress conditions in diabetes. As AGEs are not only formed endogenously, but also derive from exogenous sources, i.e., food, they have been assumed as risk factors for T2DM. However, the role of AGEs in the pathogenesis of T2DM and diabetic complications - if they are causal or simply an effect - is only partly understood. This review will highlight the involvement of AGEs in the development and progression of T2DM and their role in diabetic complications.
\end{abstract}

Reprinted from Biomolecules. Cite as: Nowotny, K.; Jung, T.; Höhn, A.; Weber, D.; Grune, T. Advanced Glycation End Products and Oxidative Stress in Type 2 Diabetes Mellitus. Biomolecules 2015, 5, 194-222.

\section{Introduction}

With around 350 million cases in 2014 [1], type 2 diabetes mellitus (T2DM) is one of the most frequent diseases throughout the world. This number is predicted to increase dramatically in the coming years, resulting in serious health and economic challenges. In general, diabetes mellitus is a group of metabolic diseases in which the pancreas is not able to produce insulin, insulin production is not sufficient or cells cannot effectively use this hormone [2]. In T2DM, the body is per se able to produce insulin. However, several processes induce abnormalities in a manner that either hormone production is insufficient or cells are unable to mediate the effects of insulin. As insulin is required for an efficient cellular uptake of glucose to convert it into energy, the ineffectiveness of insulin causes elevated blood glucose levels (hyperglycemia). Hyperglycemia is estimated to be one major factor contributing to diabetic complications including diseases which affect the cardiovascular and nervous system, eyes or kidneys. Every year, 4.9 million people die from diabetes [1] some $50 \%$ of them by cardiovascular complications [3]. To prevent diabetic complications, 
an early diagnosis is very important. However, the high prevalence of T2DM, its morbidity as well as mortality rates are at least partly due to the fact, that most people only recognize this disease in a state when symptoms already occur. This indicates that the diagnostic and therefore therapeutic possibilities are limited at the moment. For therapeutic approaches it is essential to gain further knowledge about the main risk factors and molecular mechanisms in the pathogenesis of T2DM and related complications.

The formation of reactive oxygen species (ROS) is an inevitable byproduct of metabolism. The main source of ROS in mammalian cells is the "dripping" of electrons from the mitochondrial respiratory chain, and their subsequent transfer to molecular oxygen, resulting in the formation of the superoxide anion $\left(\mathrm{O}_{2}{ }^{--}\right)$. Together with hydrogen peroxide $\left(\mathrm{H}_{2} \mathrm{O}_{2}\right)$ and nitric oxide ( $\left.\mathrm{NO}\right)$, superoxide is considered to be one of the main "primary" ROS, forming the bulk variety of other ROS found in cells in further reactions. Due to the highly reducing cellular environment, powerful antioxidative systems are needed, that are capable of scavenging ROS or transforming them into less reactive products. Another task of the cell's antioxidative machinery is the "repair" of already (oxidatively) damaged structures or their degradation. Thus, "three lines of defense" can be defined roughly (as shown in Figure 1): low molecular ROS-scavengers, antioxidative enzymes, and repairing or degrading ones. If both, the antioxidative machinery of a cell is overwhelmed by the present amount of ROS and the cellular redox-signaling is disturbed, the cell is found in a stage defined as "oxidative stress" [4]. Oxidative stress, induced by an abundance of ROS or failure in the antioxidative machinery, is the cause of many pathologies.

There is increasing evidence that oxidative stress also plays a key role in pathological processes observed in T2DM (reviewed in [6-8]). Oxidative stress has long been associated with diabetic complications and more recent studies indicate that oxidative stress is also causal in the development of $\beta$ cell dysfunction and insulin resistance, the two hallmarks of T2DM. Beta cell dysfunction and insulin resistance occur long before blood glucose levels reach the amount defined as prediabetes [9]. Moreover, both processes mediate progression of prediabetes to diabetes so that the prevention of insulin resistance as well as of $\beta$ cell failure is essential to prevent T2DM. Diabetes is related to oxidative stress at least partly to the overproduction of ROS. Under diabetic conditions, there are several sources of ROS described, among them advanced glycation end products (AGEs). AGEs are a group of heterogeneous compounds increasingly formed under hyperglycemic conditions. Due to this and their damaging potential, AGEs have been assumed to be involved in the pathogenesis of T2DM and diabetic complications. Additionally, it was recently proposed that another source of AGEs, the diet, contributes to the development of T2DM (reviewed in [10]). This review will give an overview on the involvement of AGEs in T2DM, in particular in the development of insulin resistance, $\beta$ cell dysfunction and death as well as their role in diabetic complications. 


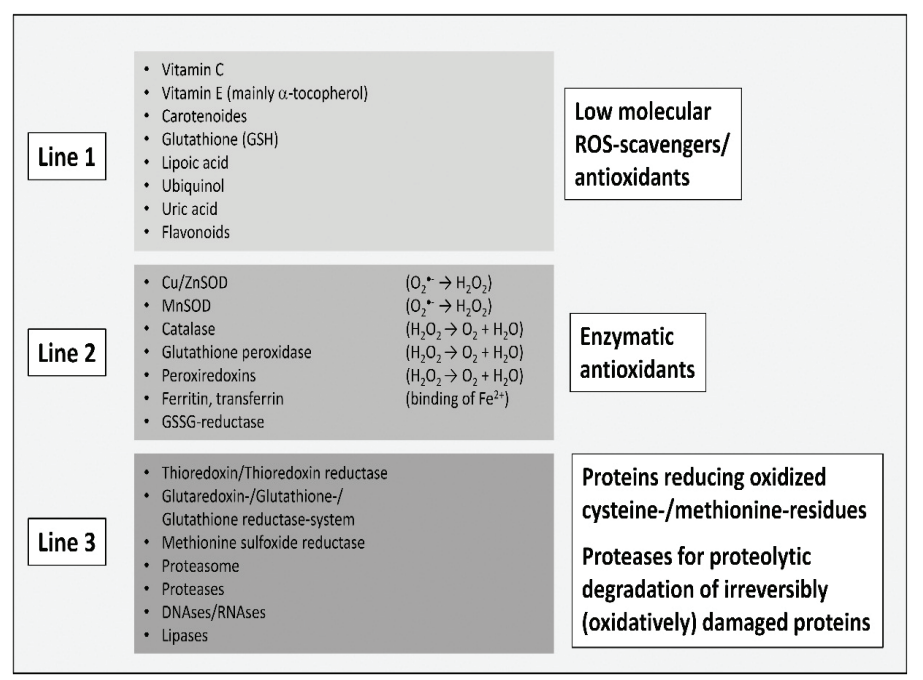

Figure 1. "Three lines of antioxidative defense" in mammalian cells, modified according to [5]. The first line contains low molecular antioxidants that can scavenge ROS/reactive particles in a purely competitive way, preventing damage from cellular structures like proteins, nucleobases and lipids. The reaction products are mostly significantly less reactive and may in several cases be restored by cellular systems (like vitamin $\mathrm{C}$ and tocopherol in a glutathione (GSH)-consuming manner). The most important and abundant low molecular intracellular scavenger is GSH, thus determining the cellular redox-state, defined as the ratio of GSH to its oxidized form, glutathione disulfide (GSSG). Under normal physiological conditions, this ratio is about 1:1000 (GSSG:GSH) or even higher, providing a strongly reducing cellular environment. The second line of defense contains antioxidative enzymes that are able to convert ROS into less reactive particles. This includes the superoxide dismutases $(\mathrm{Cu}, \mathrm{ZnSOD}$ and $\mathrm{MnSOD}$ ) as well as catalase. Further important enzymes are glutathione peroxidases, catalyzing the reaction of peroxides $(\mathrm{R}-\mathrm{OOH})$ to hydroxyls $(\mathrm{R}-\mathrm{OH})$ via GSH-consumption. Besides catalase, glutathione peroxidases are the most important $\mathrm{H}_{2} \mathrm{O}_{2}$-detoxifying enzymes. In this group enzymes are also found, which bind redox-active metals - iron is the most important transition metal in mammalian cells - in an inert form. Otherwise, metals like iron $\left(\mathrm{Fe}^{2+}\right)$ or copper $\left(\mathrm{Cu}^{+}\right)$are able to transfer an electron to $\mathrm{H}_{2} \mathrm{O}_{2}$ (Fenton reaction), releasing both $\mathrm{OH}^{-}$and the highly reactive hydroxyl radical $\left({ }^{\circ} \mathrm{OH}\right) .{ }^{\circ} \mathrm{OH}$ is able to oxidize virtually every organic molecule. The oxidized forms of those metals $\left(\mathrm{Fe}^{3+} / \mathrm{Cu}^{2+}\right)$ are quickly reduced in the cytosolic environment, fuelling the vicious circle. In the last line of defense is a summary of enzymes that are able to restore oxidatively modified amino acids (only methionine and cysteine), thus preventing proteolytic degradation of the whole damaged protein. If repair is not possible, several proteases are available, that can recognize and remove dysfunctional proteins in a proteolytic manner, preventing their intracellular accumulation. The most important one is the proteasomal system, responsible for the degradation of more than $90 \%$ of all (oxidatively) damaged proteins, as well as the cathepsins of the lysosomal system. Other catabolic enzymes might also play some role in these defense lines. 


\section{AGEs in Diabetes: An Overview}

The first link between glycated proteins and diabetes was made in 1968 with the discovery of an altered form of hemoglobin (meanwhile known as $\mathrm{HbA} 1_{c}$ ) in red blood cells of patients with diabetes [11]. It became clear, that glycation occurs predominantly on the $\mathrm{N}$-terminal valine of the $\beta$ chain and that this Amadori product is formed non-enzymatically in a reaction which was before only known to take place in food [12,13]. In this so-called Maillard reaction, the carbonyl group of a reducing sugar reacts with the amino group of a protein, lipid or nucleic acid generating Schiff bases which rearrange to Amadori products (Figure 2). However, Amadori products are relatively unstable so that further consecutive and parallel reactions occur, eventually leading to the formation of irreversible AGEs. The Maillard reaction is the most common pathway known to form AGEs. Not only during all stages of the Maillard reaction, but also as intermediates or byproducts of glucose autoxidation, lipid peroxidation or the polyol pathway, high reactive carbonyl compounds, including glyoxal, methylglyoxal or 3-deoxyglucosone are formed [14-16]. Increased concentrations of glyoxal, methylglyoxal as well as 3-deoxyglucosone have been found in plasma of patients with T2DM [17]. Glyoxal, for example, causes the formation of Ne-(carboxymethyl) lysine (CML) [18] which is at present the best characterized AGE. Further AGEs formed by glyoxal are glyoxal-derived lysyl dimer (GOLD) [19], N $\omega$-(carboxymethyl) arginine (CMA) [20] or S-carboxymethylcysteine [21]. Methylglyoxal causes the generation of, for example, Ne-(carboxyethyl) lysine (CEL) [22], methylglyoxal-derived lysyl dimer (MOLD) [23], argpyrimidine [24] or methylglyoxal-derived hydroimidazolone MG-H1 [25] whereas 3-deoxyglucosone leads to the formation of pyrraline [26], pentosidine [27], imidazolone or also CML [28].

Even if oxidation is not always necessary, many AGEs are generated by a combination of oxidation and glycation so that the formation of so-called glycoxidation products is triggered by oxidative stress [30]. Two important AGEs produced by glycoxidation are pentosidine and CML. The complexity and diversity of AGE formation makes clear why substances belonging to the group of AGEs are so heterogeneous regarding their chemical and physical properties. Some AGEs are fluorescent; a few induce protein cross-linking. There are compounds which show both properties, other AGEs are either fluorescent or cross-linkers. AGE formation occurs intra- as well as extracellularly as part of physiological metabolism. To detect AGE formation in fluids and tissues, AGE-specific fluorescence can be measured. The majority of so far identified AGEs are characterized by fluorescence in the area around an excitation wavelength of $370 \mathrm{~nm}$ and an emission of $440 \mathrm{~nm}$ [24,31,32]. Additionally, pentosidine emits light at $385 \mathrm{~nm}$ when excited at $335 \mathrm{~nm}$ [33]. Further methods for AGE detection used in in vitro and in vivo studies are immunohistochemical staining or enzyme-linked immunosorbent assay (ELISA) using antibodies against different AGEs, for example, CML or pentosidine. However, application of these methods is often limited due to lack of reliable antibodies. More sensitive methods for AGE detection include high performance liquid chromatography (HPLC), gas chromatography or liquid chromatography with different detectors (reviewed in [34]). 


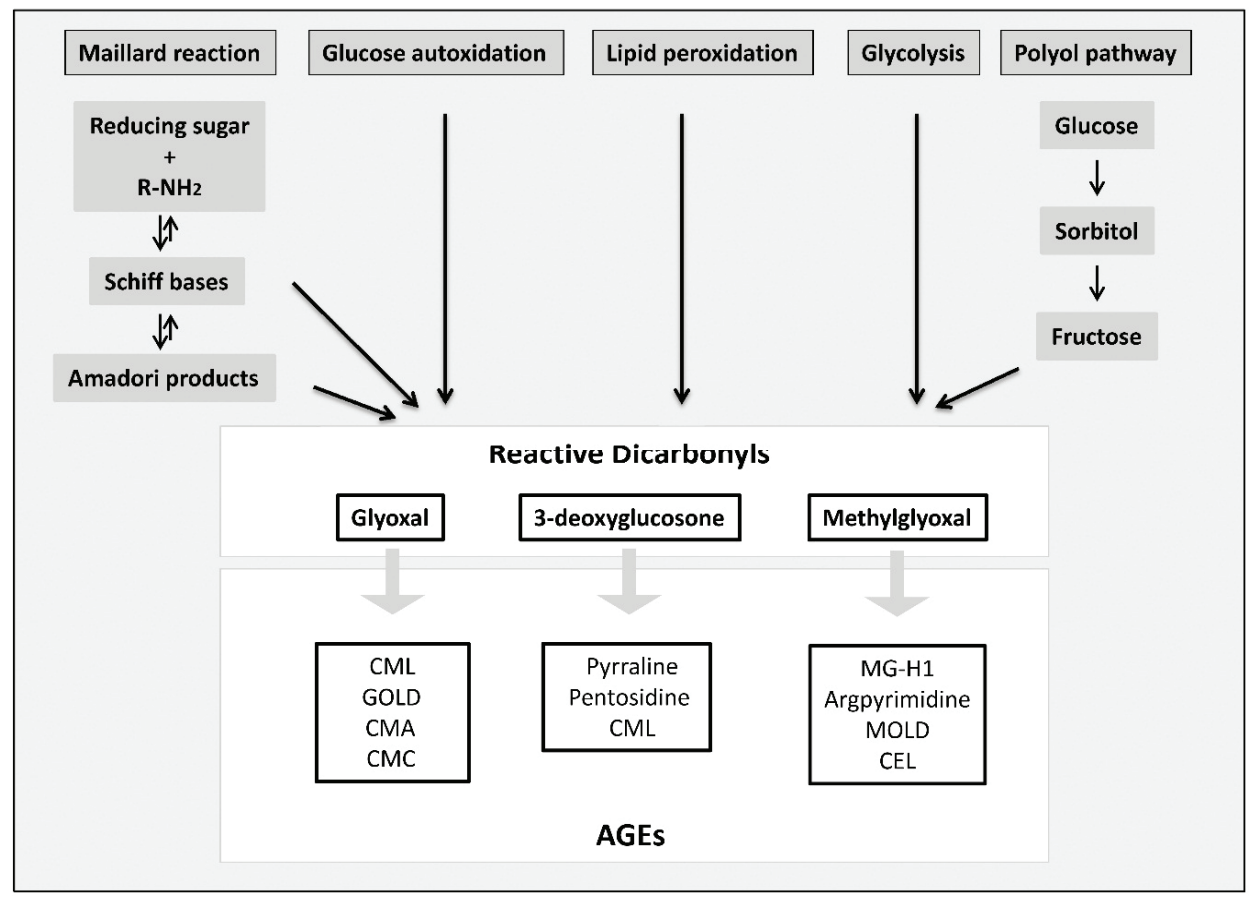

Figure 2. Formation of reactive dicarbonyls and AGEs, modified according to [29]. Reactive dicarbonyls including methylglyoxal, glyoxal and 3-deoxyglucosone are formed through several pathways: the Maillard reaction, the polyol pathway, glycolysis, lipid peroxidation or glucose autoxidation. Dicarbonyl compounds react further to form irreversible products, the so-called AGEs.

In people with diabetes, AGE formation is accelerated due to increased concentration of circulating glucose, AGE precursors and oxidative stress. In serum and tissues of patients with type 1 diabetes mellitus (T1DM) as well as T2DM, increased levels of AGEs, among them CML [35-37], MG-derived hydroimidazolone [38], pentosidine [39] or glucosepane [40,41] have been found. Furthermore, accumulation of AGEs in diabetic tissue was shown to correlate with diabetic complications (reviewed in [42]).

At this point it should be noted, that another source of AGEs, the diet, may contribute to pathological features related to diabetes. Different studies have investigated the AGE content in food items, for example by measuring CML concentrations with ELISA [43] or further ultra pressure liquid chromatography with mass spectrometry detection (UPLC-MS) [44]. That the detection of AGEs with only one method is not sufficient and can result in under- or overestimation of AGEs in food items [34], is demonstrated by comparing these studies. Goldberg et al. reported that food items rich in fat and protein contain particularly high concentrations of CML in contrast to carbohydrate-rich food in which only low CML concentrations were measured. In contrast, $\mathrm{CML}$ detection with UPLC-MS observed highest levels of CML in bread crust and evaporated full-fat milk while lowest CML levels were detected in uncooked minced beef, raw full-fat milk and 
pasteurized skimmed milk. No CML was detected in olive oil. Nevertheless, people are continuously exposed to AGEs through their diet, and this source might even be greater than the amount formed endogenously [45]. Around one-tenth [46] or even more [47] of the consumed AGEs are absorbed in the gut and, therefore, contribute to the body's AGE pool. Studies reported that the dietary uptake of AGEs correlates with serum AGE levels [46,48] and that restriction of food-derived AGEs can lower AGE concentrations in serum [49,50]. There is a growing body of evidence that the amount of food-derived AGEs which contributes to the AGE pool is sufficient to promote the development of T2DM. Studies in humans and mice revealed that an AGE-rich diet elevates AGE concentrations such as MG-H1 also in non-diabetic subjects and increases biomarkers of inflammation and oxidative stress associated with insulin resistance [51,52]. Furthermore, it was shown that a high-AGE diet causes decreased insulin secretion and increased $\beta$ cell death in rats [53]. Taken together, AGEs are proposed to play a role in the development and progression of $\mathrm{T} 2 \mathrm{DM}$ as well as in diabetic complications.

The damaging potential of AGEs results from direct alterations on protein structures and functions due to AGEs per se or the cross-linking effect of some AGEs. AGEs are often found in the extracellular matrix (ECM) and thus modified matrix proteins impair matrix-matrix as well as matrix-cell interactions. This may cause cell death, cell differentiation or reduced cell adhesion and migration. Intracellular proteins are also targets of modifications and AGE formation was shown to impair their functions. Besides direct changes in protein structures and functions, AGE-mediated damage occurs via binding of AGEs to the receptor of advanced glycation end products (RAGE). RAGE belongs to the immunoglobulin superfamily and additionally interacts with a wide range of ligands including S100 calgranulins [54], high mobility group box 1 [55], or Mac-1 [56] which is why RAGE is also classified as a multi-ligand receptor. In recent years the interaction of AGEs with RAGE was studied in vitro demonstrating the activation of Janus kinase, rho-GTPases, extracellular-signal-regulated kinase $1 / 2$ and p38 mitogen-activated protein kinase due to AGE-RAGE interaction [57-60]. It should be pointed out that ligand binding to RAGE activates NAPDH oxidases and thus increases intracellular ROS formation [61,62]. Increased ROS in turn leads to AGE formation, which triggers all described damaging mechanisms mediated by AGEs, but also activates the transcription factor nuclear factor kappa B (NFkB) [63]. Activation of NFkB increases the expression of proinflammatory cytokines such as interleukin 6 [64] and monocyte chemoattractant peptide 1 (MCP-1) [65] as well as RAGE itself [66] thus intensifying the inflammatory response. There are two possibilities to protect tissues against AGE-mediated damage: AGEs are eliminated or cells activate compensatory mechanisms. It has been shown that cells process other AGE receptors which are able to bind extracellular AGEs and mediate their cellular uptake (reviewed in [67]). In cells, AGEs can be degraded by the endosomal-lysosomal system. Cathepsin D and L were identified as two enzymes involved in the detoxification of AGEs [68,69]. After digestion, the AGE degradation products are released and circulate in the bloodstream until renal elimination. One possibility to prevent RAGE-mediated damage, is enabled by the upregulation of advanced glycation end products receptor 1 (AGER1) after AGE exposure. Although AGER1 was first only associated with the AGE turnover, studies observed that its upregulation suppresses RAGE-mediated pathways: AGER1 inhibits the activity of NADPH oxidase and weakens oxidative stress generation 
as well as ROS-mediated signaling [70-73]. Moreover, it has been described that AGER1 is linked to sirtuin1 (SIRT1) [50,52], a NAD ${ }^{+}$-dependent deacetylase. For example, through deacetylation of

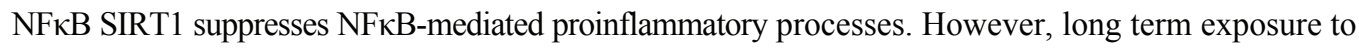
AGEs depletes AGER1 and SIRT1 expression [52] so that the regulation of RAGE signaling fails and oxidative stress and inflammation increase.

\section{Advanced Glycation End Products and Insulin Resistance}

Insulin resistance describes the condition when cells are no longer able to appropriately respond to the hormone insulin, which mediates the uptake of glucose. Although not all persons with insulin resistance develop T2DM, it is one relevant factor which increases the risk to develop diabetes [74]. In turn, genetic as well as environmental factors, especially obesity and lack of physical activity, increase the risk that cells become insulin resistant. There is more and more evidence that AGEs are another risk factor for the development of insulin resistance. For example, by multiple regression analysis Tan et al. reported that AGE levels are independently correlated with insulin resistance in healthy subjects [75]. Insulin resistance was estimated by the homeostatic model assessment index (HOMA-IR) which is based on the calculation of fasting insulin and glucose concentration [76]. Another study by Tahara et al. in which more than 300 non-diabetic persons were examined confirmed that serum AGE levels were independently correlated with the HOMA-IR [77].

The underlying molecular mechanisms leading to AGE-induced insulin resistance are still poorly understood, but some in vitro and in vivo studies were performed to gain knowledge in this research area. Figure 3 summarizes the principle mechanisms of AGE contribution to insulin resistance which have been observed in the described studies. As serum proteins are frequent targets of modifications by sugars and reactive carbonyl compounds such as methylglyoxal and glyoxal, in vitro glycated albumin is often used for cell culture experiments. Glycated albumin was shown to induce the expression of tumor necrosis factor alpha $(\mathrm{TNF} \alpha)$ which suppresses insulin signaling [78]. Furthermore, protein kinase $\mathrm{C}$ alpha $(\mathrm{PKC} \alpha$ ) was reported as a target of glycated human serum albumin leading to increased serine/threonine phosphorylation of insulin receptor substrate (IRS) 1 and 2 but reduced IRS tyrosine phosphorylation. This resulted in impaired insulin signaling (phosphatidylinositol 3-kinase/protein kinase B pathway) and inhibition of insulin-mediated glucose metabolism [79]. As the induction of PKC $\alpha$ via protein tyrosine kinase Src and the inhibition of insulin receptor substrate 1 (IRS1) was mediated by RAGE after the incubation of skeletal muscle cells with glycated albumin, it is assumed that RAGE plays a role in this process [80]. Previous studies already showed that both, TNF $\alpha$ (reviewed in [81]) and proteins of the PKC family $[82,83]$, are involved in insulin resistance. 


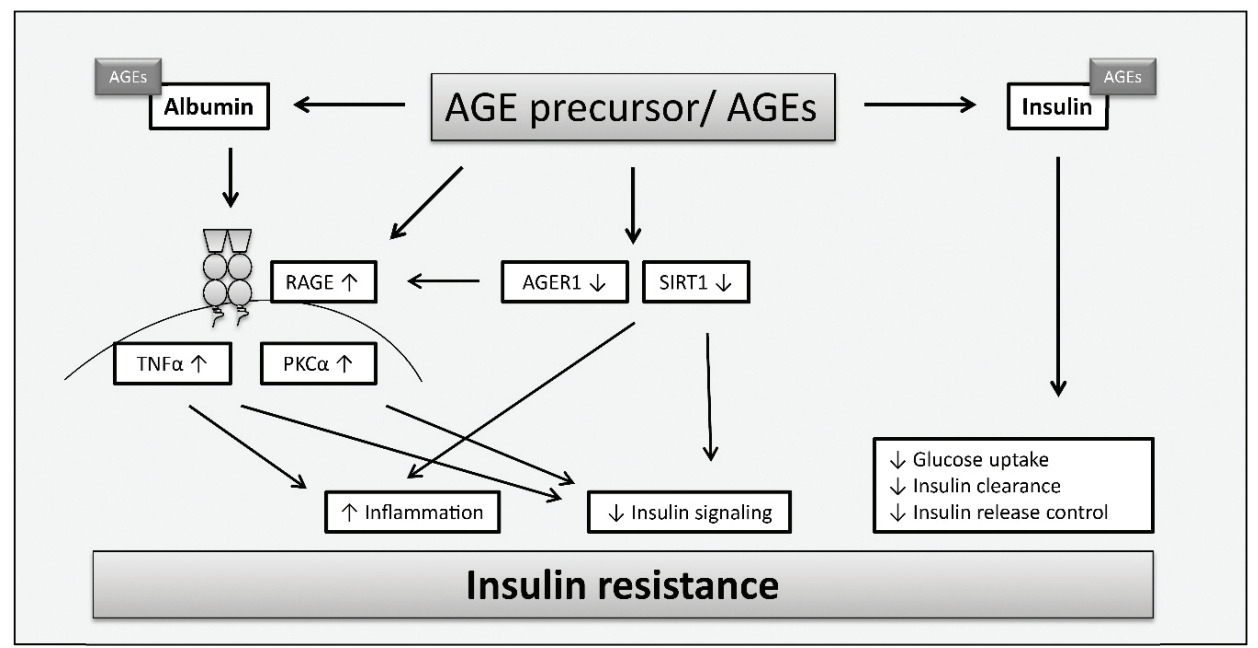

Figure 3. Mechanisms of AGEs leading to insulin resistance in insulin-sensitive tissues according to $[52,78-80,84-86]$. AGEs are involved in mechanisms contributing to insulin resistance due to direct modification of insulin which alters insulin action resulting in impaired glucose uptake, inhibited insulin clearance or further increased insulin secretion. Furthermore, AGEs may contribute to insulin resistance via increased expression of RAGE and reduced expression of AGER1 and SIRT1. AGEs affect insulin signaling and trigger inflammation via stimulation of $P K C \alpha$ and upregulation of TNF $\alpha$. SIRT1 depletion causes changes in insulin signaling and induces inflammation.

Another possible factor contributing to insulin resistance is the direct glycation of insulin. Although insulin has a very short half-life and is not a typical target for modification, glycation sites on insulin have been found in vivo $[85,87]$ as well as when cells and islets were cultured under hyperglycemic conditions $[87,88]$. In vitro, glycation of human insulin was shown to occur in the phenylalanine position in the amino terminus of the insulin $\beta$ chain [89]. Moreover, it was demonstrated that the reaction of glyoxal with insulin results in the formation of N-terminal pyrazinones, products belonging to AGEs [90]. Studies using in vitro prepared monoglycated insulin revealed that glycation affects insulin function. When in vitro monoglycated insulin was infused to mice the glucose lowering potential was around $20 \%$ lower compared to unmodified insulin [84]. Cell culture experiments with isolated muscle and glycated insulin additionally showed that glucose uptake, oxidation and glycogen production is reduced. Monoglycated insulin had similar effects in humans. After infusion to healthy humans, around $70 \%$ more glycated insulin was needed to induce an equal amount of glucose uptake compared to unmodified insulin [85]. The role of glycated insulin in T2DM was supported by the measurement of the modified insulin in the plasma of T2DM subjects which was found to comprise around $9 \%$ of the total insulin level [85]. Furthermore, it was shown that methylglyoxal causes modifications on the arginine residue of the insulin $\beta$ chain decreasing glucose uptake in insulin-sensitive cells including 3T3-L1 adipocytes and L8 skeletal muscle cells [86]. Usually, extracellular insulin concentrations regulate insulin 
secretion from $\beta$ cells, however, methylglyoxal-modified insulin can no longer inhibit insulin secretion. Moreover, Jia et al. reported that methylglyoxal-modified insulin triggers hyperinsulinemia through decreased insulin clearance by liver cells [86].

The potential role of AGEs in insulin resistance is supported by studies examining the influence of antiglycating agents on the manifestation of insulin resistance. The agents pyridoxamine or TM2002 inhibit the formation of AGEs and were shown to improve insulin resistance in rodents which already suffered from diabetes [91] or in which insulin resistance was induced by the administration of methylglyoxal [92]. Antioxidants reduced insulin resistance in the same manner making it difficult to distinguish between oxidative stress and AGE formation but making clear that there is an important link between both factors.

There are studies which indicate that predominantly prooxidative AGEs derived from the diet increase the risk for insulin resistance. Cai et al. reported that a diet enriched with methylglyoxal-modified albumin (MG-diet) and fed to non-obese C57BL6 mice led to weight gain, adiposity and the development of insulin resistance in $\mathrm{F} 3 / \mathrm{MG}^{+}$mice [52]. The MG-diet increased serum AGE levels and AGE-lipids. Furthermore, the chronic AGE intake caused elevated oxidative stress and inflammation and resulted in an insulin-resistant state (increased level of fasting plasma insulin and leptin and reduced adiponectin level). The phenotypical change was mediated by reduced expression of AGER1 and SIRT1 and upregulation of RAGE in skeletal muscle, liver and white adipose tissue as well as an increase in NFKB acetylated p65 in adipocytes. This resulted in changes in insulin signaling and increased inflammation leading to elevated levels of oxidative stress. In this context, another study showed that restriction of AGEs in a cohort of patients with T2DM reduced insulin resistance and increased expression of SIRT1 and AGER1 [50]. Due to its deacetylase activity, SIRT1 seems to have a significant role in insulin actions. On the one hand, SIRT1 is involved in the induction of insulin secretion from $\beta$ cells, on the other hand SIRT1 induces insulin signaling by inhibiting negative regulators or further regulating the activation of IRS1 and 2 and Akt. Moreover, as SIRT1 also regulates inflammatory processes, adiponectin secretion or ROS formation, it indirectly contributes to the development of insulin resistance [93].

\section{The Role of AGEs in $\beta$ Cell Dysfunction and $\beta$ Cell Death}

There is a growing body of evidence that AGEs not only contribute to insulin resistance, but also damage $\beta$ cells directly, leading to impaired functions or even cell death. The cytotoxic potential of AGEs on pancreatic $\beta$ cells was investigated in a number of studies. Lim et al. reported that AGE treatment caused apoptosis in $\beta$ cells [94]. Additionally, they showed that AGEs stimulate ROS production and induce the expression of RAGE. Inhibition of RAGE as well as antioxidant treatment prevented these changes so that AGEs might induce apoptosis via RAGE-induced ROS formation. However, they also demonstrated that proliferation increases after AGE treatment. In contrast, decreased proliferation but also ROS-induced cell death in HIT-T15 cells due to treatment with ribose-modified serum was observed by Viviani et al. [95]. Moreover, Zhu et al. indicated that apoptosis in $\beta$ cells characterized by caspase activation, cytochrome c release and reduced expression of anti-apoptotic bcl2 might be due to RAGE [96]. In the study of Lin et al., INS-1 cells were treated with AGEs resulting in cell apoptosis [97]. They concluded that 
the AGE-induced ROS generation occurs primarily through the mitochondrial electron transport chain but also through stress-related signaling pathways (Jun N-terminal kinase and p38) which activates ROS production via the NAPDH oxidase.

In addition to $\beta$ cell death, most of the studies showed that AGEs affect insulin secretion [95-97]. Further evidence for the AGE-mediated decline in insulin secretion was given by Zhao et al. [98]. They showed that AGEs block the activity of cytochrome c oxidase and production of adenosine triphosphate (ATP) in islets isolated from mice. Impaired insulin secretion increases plasma glucose levels which were accompanied by increased formation of ${ }^{\circ} \mathrm{NO}$ and elevated expression of inducible nitric oxide synthase (iNOS) suggesting that AGEs cause the induction of iNOS so that increasing concentrations of $\mathrm{NO}$ inhibit cytochrome $\mathrm{c}$ oxidase activity and ATP production (Figure 4). ATP is necessary for insulin secretion as ATP causes the shutdown of ATP-sensitive potassium channels leading to membrane depolarization and the influx of $\mathrm{Ca}^{2+}$. Increased intracellular $\mathrm{Ca}^{2+}$-concentrations trigger the exocytosis of insulin granules [99]. Low ATP levels inhibit this process. More recently, Hachiya et al. tested the influence of bovine serum albumin (BSA) modified with glucose and glyceraldehyde on insulin secretion of isolated rat pancreatic islets [100]. Both, glucose-BSA and glyceraldehyde-BSA impaired insulin secretion induced by high concentrations of glucose. However, the authors were not able to detect any changes in the expression of oxidative response genes including iNOS. They concluded the impaired insulin secretion to be due to defects in the tricarboxylic acid cycle (TCA). Moreover, gene expression of the NADH (reduced nicotinamide adenine dinucleotide) shuttle enzymes malate dehydrogenase $1 / 2$ was reduced after AGE treatment. However, as the expression of glycerol phosphate shuttle was unchanged, and inhibition of both have been linked to decreased insulin secretion [101], further investigations must clarify whether AGEs affect NADH shuttle function and thus ATP production. The different results reveal that more research to understand the causal mechanisms leading to impaired insulin secretion needs to be performed.

Another factor contributing to reduced insulin secretion is the decline in insulin gene transcription. In this respect, AGEs are also proposed to play a role (Figure 4). Shu et al. reported the impaired insulin secretion of $\beta$ cells as a result of the downregulation of insulin transcription [102]. They identified that the transcription factor FoxO1 (Forkhead box protein O1) accumulates in the nucleus which in turn decreases the expression of the transcription factor PDX-1 (pancreatic and duodenal homeobox-1) by reducing protein stability. This is in agreement with the study of Puddu et al. who observed that the reduced insulin content in the pancreatic islet cell line HIT-T15 after AGE treatment is linked to reduced expression of PDX-1 and an increase of FoxO1 in the nucleus [103]. They suggested that AGEs decrease phosphorylation of FoxO1, thus, inducing the translocation into the nucleus. In addition, they were able to show that AGEs induce FoxO1 acetylation and that PDX-1 translocates into the cytoplasm. Acetylation of the transcription factor protects against proteasomal degradation, and nucleocytoplasmic translocation of PDX-1 decreases its availability for insulin transcription. For therapeutic approaches, it is important to note that even if hyperglycemia is restored, AGEs can still contribute to $\beta$ cell failure and thus T2DM. Therefore, destroying AGE structures, preventing endogenous AGE formation as well as dietary AGE uptake may all be considered as part of a therapy to completely "remove" sources of $\beta$ cell dysfunction. 


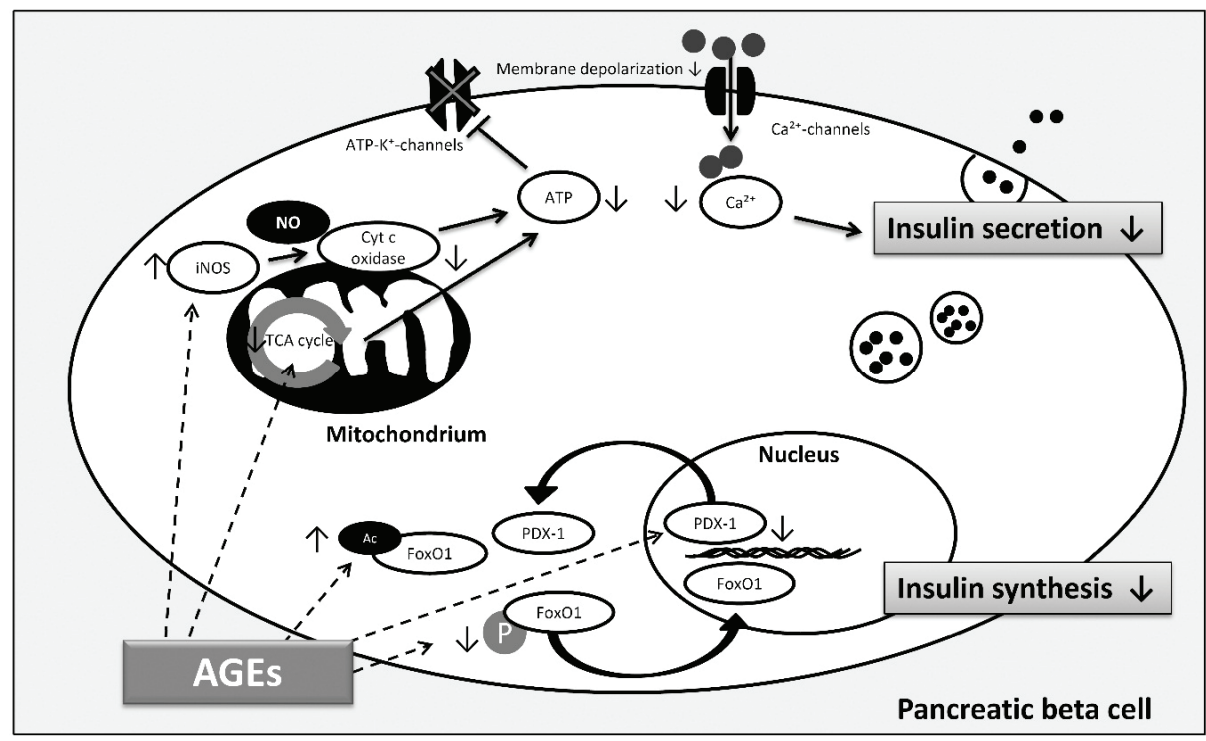

Figure 4. AGE-induced pathways involved in $\beta$ cell dysfunction according to $[98,100,102,103]$. Decreased insulin synthesis and reduced insulin secretion are both involved in $\beta$ cell failure contributing to hyperglycemia. AGEs reduce phosphorylation (P) and induce acetylation (Ac) of FoxO1, thus, FoxO1 translocates into the nucleus and is protected against proteasomal degradation, respectively. In addition, AGEs induce PDX-1 translocation into the cytoplasm and decrease PDX-1 protein expression, finally affecting insulin gene transcription and insulin synthesis. Regarding insulin secretion, AGEs cause inhibition by activation of iNOS and consequent blocking of cytochrome $\mathrm{c}$ oxidase activity and ATP depletion. Moreover, AGEs decrease insulin secretion through alterations in the TCA cycle which limits ATP production. ATP depletion inhibits closure of ATP-dependent potassium channels which leads to reduced membrane depolarization and decrease of intracellular calcium concentration inhibiting insulin secretion. (Arrows illustrate direct interactions; dashed arrows illustrate possible targets of AGEs).

\section{The Role of AGEs in Diabetic Complications}

Diabetics have an increased risk for the development of infections and several diseases including cardiovascular, kidney, eye, nerves and skin diseases. Referring to the International Diabetes Federation, diabetes is the main cause of blindness, amputation of the lower limb, kidney failure and cardiovascular diseases in many countries [2]. Most relevant for the development of diabetic complications is the exposure to hyperglycemia. One of the most important injuries which arise from hyperglycemia is damage to the vascular system. If injury occurs on large or small blood vessel most diabetic diseases can be accordingly grouped into macro- or microvascular complications, respectively. Several mechanisms leading from hyperglycemia to diabetic diseases have been described, among them the formation of AGEs. 
AGE formation and accumulation increasingly occurs under diabetic conditions, and even if glycemic control is restored, AGEs can remain in tissues of diabetic subjects for a long time. Diabetic complications occur in both types of diabetes, T1DM and T2DM, and in general, there is evidence that AGEs accumulating in tissues and serum are associated with diabetic complications [42]. Regarding T2DM, studies were able to show that AGE-levels correlate with diabetic complications including diabetic retinopathy [104-106], nephropathy [107,108] and cardiovascular disease $[35,107,109]$. Most of the studies used glycated serum proteins to investigate their relevance as a marker or even a predictor for diabetic diseases. Frequently, they measured AGE-levels by immunoassay (ELISA, DELFIA) using anti-AGE, anti-CML or anti-hydroimidazolone antibodies. These methods are not very specific and the measurements of plasma AGEs may lead to underestimated associations between AGEs and diabetic complications due to the fact that most AGEs are formed or further accumulate intracellular or in tissues without reaching the circulation. This might explain why, for example, the study of Busch et al. reported no association of CML with cardiovascular and renal outcomes in T2DM patients [110]. However, even by analyzing CML, CEL and pentosidine with liquid chromatography, a more precise technique to measure AGEs, no association between AGEs and prior cardiovascular events was observed in a cross-sectional study [111]. Indeed, more recently the same group published results of a prospective study in which higher plasma levels of CML, CEL and pentosidine were associated with an increased risk of cardiovascular outcome in T2DM patients [112]. Although the causal relationship needs to be further clarified there is increasing evidence observed in these clinical studies that AGEs serve as potential biomarkers for diabetic complications.

\subsection{Modified ECM Proteins and the Relevance for Diabetic Complications}

One main target of increased concentrations of reducing sugars and dicarbonyl compounds found in diabetes is collagen. There are a few studies which investigated the effect of modified collagen on cell functions. According to the subsequent studies, a simplified scheme describing which cell functions are affected by methylglyoxal-modified collagen, contributing to diabetes-related changes related to diabetic complications, is illustrated in Figure 5. Firstly, it was reported that modification of basement membrane collagen type IV by methylglyoxal reduces attachment of vascular endothelial cells and angiogenesis [113]. AGEs such as MG-H1 were found on integrin-binding sites of collagen and were assumed to cause decreased cell attachment. As demonstrated by Chong et al., methylglyoxal modifications of arginine residues in the integrin-binding sequence also reduce collagen binding so that collagen degradation by phagocytosis is impaired, thus, promoting fibrosis [114]. Regarding the collagen turnover, it was shown that collagen modified with glyoxal and methylglyoxal is per se less degradable by proteases due to the formation of cross-links [115]. Yuen et al. observed that methylglyoxal-modified collagen type I inhibits cell adhesion of cardiac fibroblasts while stimulating myofibroblast differentiation and their migration [116]. Further investigations of Talior-Volodarsky et al. reported that myofibroblast differentiation is stimulated by upregulation of $\alpha 11$ integrin expression which was found to be induced by transforming growth factor (TGF) $\beta 2 / \mathrm{Smad} 3$ signaling $[117,118]$. 
Myofibroblasts mediate fibrosis, a pathological response to tissue damage which has been associated with diabetic complication and heart failure due to fibrotic lesions. That modified collagen alters cell-matrix interaction was also shown by Pozzi et al. [119]. On the one hand, cell adhesion and migration but not proliferation of mesangial cells plated on collagen type IV modified with methylglyoxal is inhibited. On the other hand, long term glucose-modified collagen reduces migration and proliferation and increases collagen synthesis. The authors proposed that these findings may explain pathological mechanisms seen in diabetic nephropathy: early proliferation of mesangial cells followed by impaired proliferation, mesangial matrix expansion and mesangial cell dysfunction. In the study of Sassi-Gaha et al. [120], after modification of type I collagen lattices with methylglyoxal and 3-deoxyglucosone, dermal fibroblast were seeded on the modified collagen. For methylglyoxal an increased expression of TGF $\beta 1$, collagen and $\beta 1$ integrin, and decreased expression of the transcription factor Smad7 was shown. The opposite effects were found for fibroblasts grown on 3-deoxyglucosone-modified collagen. To understand the pathological mechanisms mediated by AGEs it is quite important to investigate the effect of different AGE precursors as well as individual AGEs.

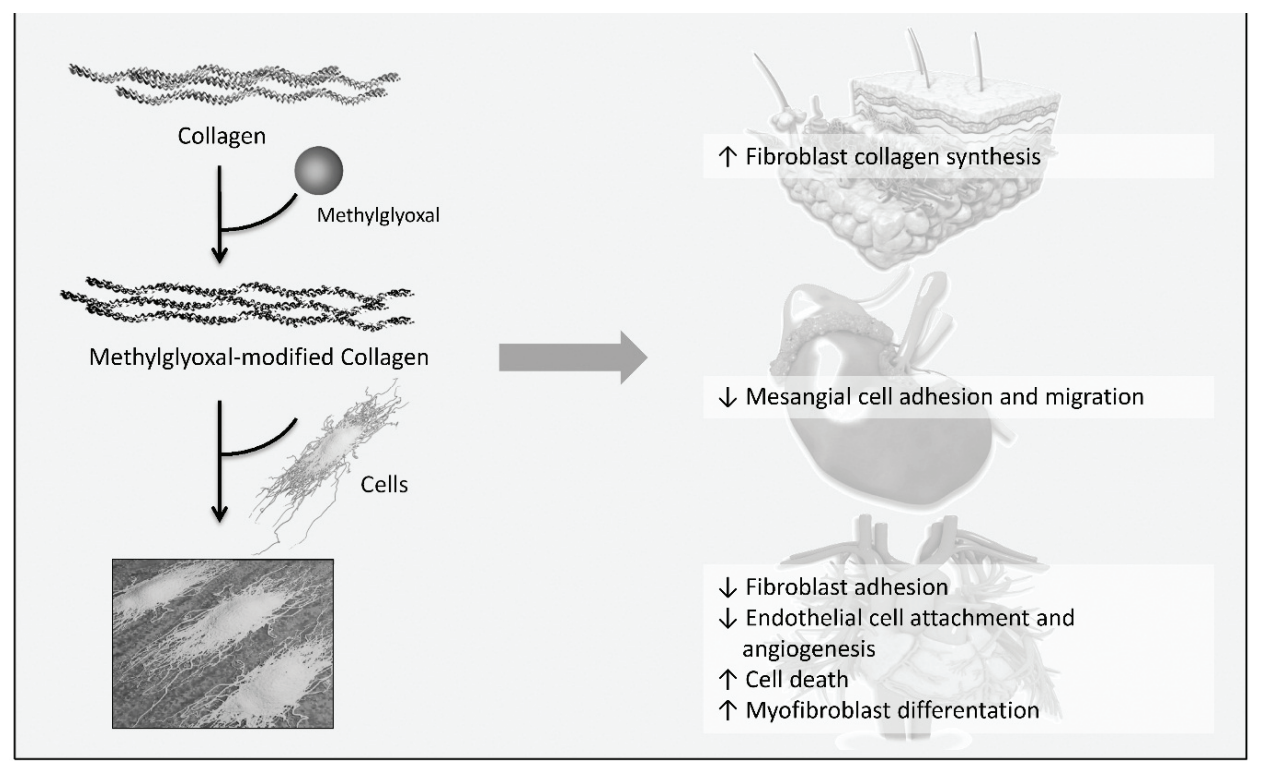

Figure 5. Influence of methylglyoxal-modified collagen on cellular functions relevant to diabetic complications, according to $[113,116,119,120]$. Cells were cultured on methylglyoxal-modified collagen and the effect of AGE-collagen on cellular functions was studied. Dermal fibroblasts grown on modified collagen increase collagen synthesis. Methylglyoxal-modified collagen reduces cell adhesion and migration of mesangial cells, cardiac fibroblast are less adherent. Myofibroblast differentiation is stimulated in cardiac fibroblasts cultured on modified collagen and modifications of basement membrane collagen causes detachment, cell death and reduced angiogenesis of vascular endothelial cells. 
In addition to collagen, AGE formation on further ECM protein including laminin or fibronectin has been studied showing impaired self-assembly and interactions with other matrix components as well as matrix-cell interactions [121-125]. It is assumed that glycation of these ECM proteins, therefore, may contribute to diabetic complications. Just to give an example, bovine retinal pericytes cultured on fibronectin modified with glyoxal and methylglyoxal were shown to induce apoptosis which may contribute to cell loss in diabetic retinopathy [124]. Regarding the development of diabetic neuropathy, glycated laminin and fibronectin reduced neurite outgrowth providing a mechanism for the failure of axonal regeneration and collateral sprouting [125].

\subsection{Ischemic Heart Disease and Atherosclerosis}

As already mentioned, patients with diabetes are at high risk of developing cardiovascular disease [126]. One common type of cardiovascular diseases is ischemic heart disease in which the blood supply to the heart is reduced often due to plaque formation in the arterial wall. The association between AGEs and ischemic heart disease of patients with T2DM was investigated in some clinical studies. Kilhovd et al. reported increased serum AGEs in diabetic persons with ischemic heart disease compared to patients without [35]. This is in agreement with a study in which increased serum AGE concentrations were measured in patients with coronary artery disease compared to diabetic patients without coronary artery disease [109]. Furthermore, serum AGE levels may predict ischemic heart disease mortality in persons with T2DM. Evidence was given in a long-term follow-up study in which serum AGEs, measured by immunoassay using a polyclonal anti-AGE antibody, were predictors for total, cardiovascular as well as coronary mortality in woman with T2DM [127]. Moreover, skin autofluorescence has been strongly related to coronary heart disease and mortality in patients with T2DM [128]. The measurement of skin autofluorescence is a technique with some limitations for AGE analysis: not all AGEs are fluorescent, no specific compound is measured and there is no information on the quantity of AGEs at all. However, skin autofluorescence can be used as a non-invasive method and is associated with AGE accumulation in vivo [129].

Atherosclerosis is the underlying cause of most ischemic heart diseases and it has been suggested that AGEs are involved in the development of atherosclerosis (for more detailed review see [130]). On the one hand it has been shown that AGEs accumulate in atherosclerotic lesions [131,132] and on the other hand studies identified AGE-mediated mechanisms related to endothelial dysfunction, inflammation and lipid modifications. In particular, it was shown that AGEs contribute to endothelial dysfunction through their pro-apoptotic effect on endothelial cells [133,134] and endothelial progenitor cells [135]. Moreover, AGEs stimulate the expression of genes such as MCP-1, intercellular adhesion molecule 1, vascular cell adhesion molecule 1 and plasminogen activator inhibitor 1 [136-141]. This mediates recruitment and adhesion of inflammatory cells to the vessel wall or further inhibition of fibrinolysis. The expression of MCP-1 and adhesion molecules can be induced by endothelin 1, its expression in turn is also stimulated by AGEs [142]. In porcine coronary fibroblasts, it was observed that AGEs induce the mRNA expression of interleukin 6, vascular cell adhesion molecule 1 and MCP-1 followed by increased interleukin secretion and leukocyte adhesion [143]. The atherogenic properties of AGEs could be further attributed to the 
"NO quenching effect of AGEs [144]. Furthermore, AGEs impair the synthesis of NO by decreasing the expression as well as the activity of endothelial ${ }^{\circ} \mathrm{NO}$ synthase $[145,146]$. ${ }^{\circ} \mathrm{NO}$ mediates a series of intracellular effects that lead to endothelial regeneration, vasodilation and inhibition of platelet adhesion and leukocyte chemotaxis and therefore defects in $\mathrm{NO}$ production and activity are supposed to be major mechanisms of endothelial dysfunction and atherosclerosis [147].

To further clarify the role of AGEs in diabetic complications, in vitro and in vivo studies showing AGE-mediated pathomechanisms which contribute to diabetic retino- and nephropathy will be highlighted below.

\subsection{Diabetic Retinopathy}

AGE-albumin-induced pericyte death has been assumed to be caused by AGE-RAGE interaction [148]. Moreover, studies reported that AGEs induce ROS formation and reduce protein kinase B/Akt [124] or further platelet-derived growth factor signaling [149] contributing to reduced pericyte survival. Pericytes are cells which cover capillaries in the retina and their loss is an early event in diabetic retinopathy followed by the formation of acellular capillaries (capillaries devoid of cells), microaneurysms and vascular basement membrane thickening. The interaction of pericyctes with endothelial cells is necessary for the maintenance of the blood-retina barrier [150] so that pericycte loss is also associated with the breakdown of the barrier.

The blood-retina barrier regulates the flux of nutrients, fluids and other blood components into the retina and its breakdown may cause the development of macular edema which is a main cause for vision loss in diabetes. Another mechanism leading to the breakdown of the blood-retinal barrier is the induction of retinal leukostasis, a state of chronic inflammation, which may contribute to endothelial cell death and increased vascular permeability. The involvement of AGEs in this process has been shown by Moore et al. [151]. AGEs induce NFKB DNA binding, intercellular adhesion molecule 1 expression and further leukocyte adhesion to retinal endothelial cells. In vivo, AGE-albumin injection into mice resulted in a breakdown of the blood-retina barrier [151]. Moreover, the breakdown of the blood-retina barrier has been shown to be dependent on VEGF. AGE-upregulation of vascular endothelial growth factor (VEGF) expression was observed after AGE-albumin injection into rodents, as well as after AGE-albumin incubation of cultured retinal cells $[148,152-154]$, leading to vascular permeability and angiogenesis.

\subsection{Diabetic Nephropathy}

Similar to pericytes in the retina, AGEs induce apoptosis and VEGF expression in mesangial cells [155], specialized pericytes which are located in blood vessels of the kidney. Mesangial cells in the glomerulus regulate glomerular filtration and provide structural support [156]. Their loss or further VEGF upregulation contributes to increased vascular permeability associated with hyperfiltration and proteinuria in kidney diseases, suggesting that AGEs play a role here. Furthermore, it has been shown, that AGEs upregulate MCP-1 expression in mesangial cells [155]. MCP-1 is a chemokine which regulates macrophage/monocyte migration and infiltration, therefore, $\mathrm{MCP}-1$ upregulation by AGEs may trigger inflammation in renal tissue. Moreover, AGE-induced expression of TGF $\beta$ was 
demonstrated in vitro $[157,158]$ and as TGF $\beta 1$ upregulation was associated with enhanced expression of ECM proteins this might be an explanation for glomerular hypertrophy in vivo [159]. In this context, studies reported that TGF $\beta$ expression is induced via AGE-RAGE interaction $[160,161]$. Elevated expression of TGF $\beta$ is also known to induce epithelial-to-mesenchymal transition and AGEs were shown to stimulate transdifferentiation in a rat kidney epithelial cell line [161]. Another protein which plays a role in AGE-induced epithelial-to-mesenchymal transition is prosclerotic connective tissue growth factor (CTGF), a target gene of TGF $\beta$ signaling which is elevated in plasma of persons with diabetic nephropathy $[162,163]$. Because AGEs induce epithelial-to-mesenchymal transition and CTGF is a downstream target of TGF $\beta$ it is generally believed that increased CTGF expression is mediated through TGF $/ \mathrm{Smad}$ signaling. More recently, AGE stimulation of tubular CTGF expression was also found to be independent of TGF $\beta / \mathrm{Smad}$ signaling. This pathway is RAGE dependent and leads to the activation of Smad3 through the induction of extracellular-signal-regulated kinase $1 / 2$ and p38 [164].

\section{Conclusions}

Extensive research has been performed in the last years to clarify the impact of AGEs as well as to identify causal pathological mechanisms in the development and progression of T2DM (insulin resistance, $\beta$ cell death and dysfunction) and in diabetic diseases. Increasing concentrations of different AGEs in serum and tissues of diabetic persons have been detected and the association of AGEs with diabetic complications has been shown in several studies suggesting AGEs as biomarkers and even predictors for diabetic complications. Moreover, studies reported a wide range of effects and reactions induced by AGEs which were proposed to play a role in insulin resistance, $\beta$ cell failure and diabetic complications. In general, it was found that AGE formation occurs on extra- and intracellular proteins leading to protein cross-linking, structural and functional changes, e.g., loss of enzyme activity. Modifications on ECM proteins have been shown to affect not only matrix-matrix but especially matrix-cell interactions. Many studies proposed that the damaging effect of AGEs is predominantly induced by RAGE signaling thus increasing ROS formation and inflammatory processes. However, there are also some critical aspects which must be considered when obtaining general conclusions from the data. Many studies used AGEs prepared in vitro by incubating a protein, often albumin, with a reducing sugar or dicarbonyl compound. According to Henle, only a small amount of AGEs is formed in glucose-modified BSA in contrast to Amadori products such as fructoselysine which accounts for about $90 \%$ of the detectable lysine derivatization [45]. Therefore, it is questionable whether the induced biological effects are stimulated by AGEs, Amadori products or even other modification products. Improvement of analytical methods may contribute to more precise results regarding the involvement of AGEs in general but also individual AGE structures. Because it is estimated that the wide range of AGEs is at present only partially identified, this further impedes the research about the biological effects of AGEs. Moreover, the use of different AGE precursors, different concentration and varying target proteins hinders not only comparability of studies but also complicates the understanding of AGE-induced mechanisms. Most frequently, the products are not characterized, the AGE content was measured by unspecific methods, and the individual AGEs 
were not identified. Without knowing the specifity of the products it is difficult to assess the findings observed in these studies and to determine the role of AGEs in several aspects of diabetes.

Another point which should be noted is that the relevance of AGE-RAGE interactions is still controversially discussed [165-167]. Only aggregates of highly modified AGE-proteins which are rare in tissue and body fluids are able to activate RAGE. However, these highly modified proteins can be formed exogenously in thermally processed food, thus the relevance for AGE-RAGE interaction needs to be further clarified. Nevertheless, the reduction of hyperglycemia-induced AGE formation as well as the restriction of AGE-rich food items both present targets for therapeutic approaches against the pathological events in diabetes and associated diseases. Finally, the use of AGEs as biomarkers/predictors for diabetic complications may help to reduce health problems in persons with diabetes.

\section{Abbreviations}

AGEs Advanced glycation end products

AGER1 Advanced glycation end product receptor 1

ATP

Adenosine triphosphate

BSA Bovine serum albumin

CEL Ne-(carboxyethyl) lysine

CMA N $\quad$-(carboxymethyl) arginine

CML Ne-(carboxymethyl) lysine

CTGF Connective tissue growth factor

ELISA Enzyme-linked immunosorbent assay

ECM Extracellular matrix

FoxO1 Forkhead box protein $\mathrm{O} 1$

GOLD Glyoxal-derived lysyl dimer

GSH Glutathione

GSSG Glutathione disulfide

$\mathrm{H}_{2} \mathrm{O}_{2} \quad$ Hydrogen peroxide

HPLC High performance liquid chromatography

iNOS Inducible nitric oxide synthase

IRS Insulin receptor substrate

MCP-1 Monocyte chemoattractant peptide 1

MOLD Methylglyoxal-derived lysyl dimer

$\mathrm{NAD}^{+} \quad$ Oxidized nicotinamide adenine dinucleotide

NADH Reduced nicotinamide adenine dinucleotide

NADPH Reduced nicotinamide adenine dinucleotide phosphate

NFкB Nuclear factor kappa B

NO Nitric oxide

$\mathrm{O}_{2}{ }^{--} \quad$ Superoxide anion

PDX-1 Pancreatic and duodenal homeobox-1

PKC $\alpha \quad$ Protein kinase c alpha

RAGE Receptor of advanced glycation end products 


$\begin{array}{ll}\text { ROS } & \text { Reactive oxygen species } \\ \text { SIRT1 } & \text { Sirtuin1 } \\ \text { T1DM } & \text { Type 1 diabetes mellitus } \\ \text { T2DM } & \text { Type 2 diabetes mellitus } \\ \text { TCA } & \text { Tricarboxylic acid cycle } \\ \text { TNF } \alpha & \text { Tumor necrosis factor alpha } \\ \text { UPLC-MS } & \text { Ultra pressure liquid chromatography with mass spectrometry detection } \\ \text { VEGF } & \text { Vascular endothelial growth factor }\end{array}$

\section{Conflicts of Interest}

The authors declare no conflict of interest.

\section{References}

1. International Diabetes Federation. IDF Diabetes Atlas Update Poster, 6th ed.; International Diabetes Federation: Brussels, Belgium, 2014.

2. International Diabetes Federation. IDF Diabetes Atlas, 6th ed.; International Diabetes Federation: Brussels, Belgium, 2013.

3. Morrish, N.; Wang, S.-L.; Stevens, L.; Fuller, J.; Keen, H. Mortality and causes of death in the who multinational study of vascular disease in diabetes. Diabetologia 2001, 44, S14-S21.

4. Sies, H. Oxidative stress: A concept in redox biology and medicine. Redox Biol. 2015, 4, 180-183.

5. Jung, T.; Catalgol, B.; Grune, T. The proteasomal system. Mol. Asp. Med. 2009, 30, 191-296.

6. Giacco, F.; Brownlee, M. Oxidative stress and diabetic complications. Circ. Res. 2010, 107, 1058-1070.

7. Abdul-Ghani, M.A.; DeFronzo, R.A. Oxidative stress in type 2 diabetes mellitus. In Oxidative stress in aging, Miwa, S., Beckman, K., Muller, F., Eds. Humana Press: Totowa, NJ, USA, 2008; pp. 191-211.

8. Henriksen, E.J.; Diamond-Stanic, M.K.; Marchionne, E.M. Oxidative stress and the etiology of insulin resistance and type 2 diabetes. Free Radic. Biol. Med. 2011, 51, 993-999.

9. Leahy, J.L. Pathogenesis of type 2 diabetes mellitus. Arch. Med. Res. 2005, 36, 197-209.

10. Vlassara, H.; Uribarri, J. Advanced glycation end products (AGE) and diabetes: Cause, effect, or both? Curr. Diab. Rep. 2014, doi:10.1007/s11892-013-0453-1.

11. Rahbar, S. An abnormal hemoglobin in red cells of diabetics. Clin. Chim. Acta 1968, 22, 296-298.

12. Bunn, H.F.; Haney, D.N.; Gabbay, K.H.; Gallop, P.M. Further identification of the nature and linkage of the carbohydrate in hemoglobin Alc. Biochem. Biophys. Res. Commun. 1975, 67, 103-109.

13. Hodge, J.E. Dehydrated foods - Chemistry of browning reactions in model systems. J. Agric. Food Chem. 1953, 1, 928-943.

14. Fu, M.X.; Requena, J.R.; Jenkins, A.J.; Lyons, T.J.; Baynes, J.W.; Thorpe, S.R. The advanced glycation end product, nepsilon-(carboxymethyl)lysine, is a product of both lipid peroxidation and glycoxidation reactions. J. Biol. Chem. 1996, 271, 9982-9986. 
15. Thornalley, P.J.; Langborg, A.; Minhas, H.S. Formation of glyoxal, methylglyoxal and 3-deoxyglucosone in the glycation of proteins by glucose. Biochem. J. 1999, 344, 109-116.

16. Wells-Knecht, K.J.; Zyzak, D.V.; Litchfield, J.E.; Thorpe, S.R.; Baynes, J.W. Mechanism of autoxidative glycosylation: Identification of glyoxal and arabinose as intermediates in the autoxidative modification of proteins by glucose. Biochemistry 1995, 34, 3702-3709.

17. Scheijen, J.L.; Schalkwijk, C.G. Quantification of glyoxal, methylglyoxal and 3-deoxyglucosone in blood and plasma by ultra performance liquid chromatography tandem mass spectrometry: Evaluation of blood specimen. Clin. Chem. Lab. Med. 2014, 52, 85-91.

18. Al-Abed, Y.; Bucala, R. Ne-carboxymethyllysine formation by direct addition of glyoxal to lysine during the maillard reaction. Bioorg. Med. Chem. Lett. 1995, 5, 2161-2162.

19. Wells-Knecht, K.J.; Brinkmann, E.; Baynes, J.W. Characterization of an imidazolium salt formed from glyoxal and N.alpha.-hippuryllysine: A model for maillard reaction crosslinks in proteins. J. Org. Chem. 1995, 60, 6246-6247.

20. Glomb, M.A.; Lang, G. Isolation and characterization of glyoxal-arginine modifications. J. Agric. Food Chem. 2001, 49, 1493-1501.

21. Thorpe, S.R.; Baynes, J.W. Maillard reaction products in tissue proteins: New products and new perspectives. Amino Acids 2003, 25, 275-281.

22. Ahmed, M.U.; Brinkmann Frye, E.; Degenhardt, T.P.; Thorpe, S.R.; Baynes, J.W. N-epsilon-(carboxyethyl)lysine, a product of the chemical modification of proteins by methylglyoxal, increases with AGE in human lens proteins. Biochem. J. 1997, 324, 565-570.

23. Nagaraj, R.H.; Shipanova, I.N.; Faust, F.M. Protein cross-linking by the maillard reaction: Isolation, characterization, and in vivo detection of a lysine-lysine cross-link derived from methylglyoxal. J. Biol. Chem. 1996, 271, 19338-19345.

24. Shipanova, I.N.; Glomb, M.A.; Nagaraj, R.H. Protein modification by methylglyoxal: Chemical nature and synthetic mechanism of a major fluorescent adduct. Arch. Biochem. Biophys. 1997, 344, 29-36.

25. Henle, T.; Walter, A.; Haeßner, R.; Klostermeyer, H. Detection and identification of a protein-bound imidazolone resulting from the reaction of arginine residues and methylglyoxal. Z. Lebensm. Unters. Forsch. 1994, 199, 55-58.

26. Portero-Otin, M.; Nagaraj, R.H.; Monnier, V.M. Chromatographic evidence for pyrraline formation during protein glycation in vitro and in vivo. Biochim. Biophys. Acta 1995, 1247, $74-80$.

27. Dyer, D.; Blackledge, J.; Thorpe, S.; Baynes, J. Formation of pentosidine during nonenzymatic browning of proteins by glucose. Identification of glucose and other carbohydrates as possible precursors of pentosidine in vivo. J. Biol. Chem. 1991, 266, 11654-11660.

28. Jono, T.; Nagai, R.; Lin, X.; Ahmed, N.; Thornalley, P.J.; Takeya, M.; Horiuchi, S. $\mathrm{N} \varepsilon$-(carboxymethyl) lysine and 3-DG-imidazolone are major AGE structures in protein modification by 3-deoxyglucosone. J. Biochem. 2004, 136, 351-358.

29. Singh, R.; Barden, A.; Mori, T.; Beilin, L. Advanced glycation end-products: A review. Diabetologia 2001, 44, 129-146. 
30. Baynes, J.W. Role of oxidative stress in development of complications in diabetes. Diabetes 1991, 40, 405-412.

31. Nakamura, K.; Nakazawa, Y.; Ienaga, K. Acid-stable fluorescent advanced glycation end products: Vesperlysines A, B, and C are formed as crosslinked products in the maillard reaction between lysine or proteins with glucose. Biochem. Biophys. Res. Commun. 1997, 232, 227-230.

32. Pongor, S.; Ulrich, P.C.; Bencsath, F.A.; Cerami, A. Aging of proteins: Isolation and identification of a fluorescent chromophore from the reaction of polypeptides with glucose. Proc. Natl. Acad. Sci. USA 1984, 81, 2684-2688.

33. Sell, D.R.; Monnier, V.M. Structure elucidation of a senescence cross-link from human extracellular matrix. Implication of pentoses in the aging process. J. Biol. Chem. 1989, 264, 21597-21602.

34. Poulsen, M.W.; Hedegaard, R.V.; Andersen, J.M.; de Courten, B.; Bügel, S.; Nielsen, J.; Skibsted, L.H.; Dragsted, L.O. Advanced glycation endproducts in food and their effects on health. Food Chem. Toxicol. 2013, 60, 10-37.

35. Kilhovd, B.K.; Berg, T.J.; Birkeland, K.I.; Thorsby, P.; Hanssen, K.F. Serum levels of advanced glycation end products are increased in patients with type 2 diabetes and coronary heart disease. Diabetes Care 1999, 22, 1543-1548.

36. Schleicher, E.D.; Wagner, E.; Nerlich, A.G. Increased accumulation of the glycoxidation product $\mathrm{N}($ epsilon)-(carboxymethyl) lysine in human tissues in diabetes and aging. J. Clin. Investig. 1997, 99, 457-468.

37. Schalkwijk, C.G.; Baidoshvili, A.; Stehouwer, C.D.A.; van Hinsbergh, V.W.M.; Niessen, H.W.M. Increased accumulation of the glycoxidation product $\mathrm{N} \varepsilon$-(carboxymethyl)lysine in hearts of diabetic patients: Generation and characterisation of a monoclonal anti-CML antibody. Biochim. Biophys. Acta 2004, 1636, 82-89.

38. Kilhovd, B.K.; Giardino, I.; Torjesen, P.A.; Birkeland, K.I.; Berg, T.J.; Thornalley, P.J.; Brownlee, M.; Hanssen, K.F. Increased serum levels of the specific AGE-compound methylglyoxal-derived hydroimidazolone in patients with type 2 diabetes. Metabolism 2003, $52,163-167$.

39. Sell, D.R.; Carlson, E.C.; Monnier, V.M. Differential effects of type 2 (non-insulin-dependent) diabetes mellitus on pentosidine formation in skin and glomerular basement membrane. Diabetologia 1993, 36, 936-941.

40. Biemel, K.M.; Friedl, D.A.; Lederer, M.O. Identification and quantification of major maillard cross-links in human serum albumin and lens protein. Evidence for glucosepane as the dominant compound. J. Biol. Chem. 2002, 277, 24907-24915.

41. Sell, D.R.; Biemel, K.M.; Reihl, O.; Lederer, M.O.; Strauch, C.M.; Monnier, V.M. Glucosepane is a major protein cross-link of the senescent human extracellular matrix. Relationship with diabetes. J. Biol. Chem. 2005, 280, 12310-12315.

42. Monnier, V.M.; Sell, D.R.; Genuth, S. Glycation products as markers and predictors of the progression of diabetic complications. Ann. NY Acad. Sci. 2005, 1043, 567-581. 
43. Goldberg, T.; Cai, W.; Peppa, M.; Dardaine, V.; Baliga, B.S.; Uribarri, J.; Vlassara, H. Advanced glycoxidation end products in commonly consumed foods. J. Am. Diet. Assoc. 2004, 104, 1287-1291.

44. Assar, S.; Moloney, C.; Lima, M.; Magee, R.; Ames, J. Determination of Ne-(carboxymethyl)lysine in food systems by ultra performance liquid chromatography-mass spectrometry. Amino Acids 2009, 36, 317-326.

45. Henle, T. AGEs in foods: Do they play a role in uremia? Kidney Int. Suppl. 2003, S145-S147.

46. Koschinsky, T.; He, C.J.; Mitsuhashi, T.; Bucala, R.; Liu, C.; Buenting, C.; Heitmann, K.; Vlassara, H. Orally absorbed reactive glycation products (glycotoxins): An environmental risk factor in diabetic nephropathy. Proc. Natl. Acad. Sci. USA 1997, 94, 6474-6479.

47. Delgado-Andrade, C.; Tessier, F.J.; Niquet-Leridon, C.; Seiquer, I.; Pilar Navarro, M. Study of the urinary and faecal excretion of nepsilon-carboxymethyllysine in young human volunteers. Amino Acids 2012, 43, 595-602.

48. Vlassara, H.; Cai, W.; Crandall, J.; Goldberg, T.; Oberstein, R.; Dardaine, V.; Peppa, M.; Rayfield, E.J. Inflammatory mediators are induced by dietary glycotoxins, a major risk factor for diabetic angiopathy. Proc. Natl. Acad. Sci. USA 2002, 99, 15596-15601.

49. Uribarri, J.; Peppa, M.; Cai, W.; Goldberg, T.; Lu, M.; He, C.; Vlassara, H. Restriction of dietary glycotoxins reduces excessive advanced glycation end products in renal failure patients. J. Am. Soc. Nephrol. 2003, 14, 728-731.

50. Uribarri, J.; Cai, W.; Ramdas, M.; Goodman, S.; Pyzik, R.; Chen, X.; Zhu, L.; Striker, G.E.; Vlassara, H. Restriction of advanced glycation end products improves insulin resistance in human type 2 diabetes: Potential role of AGER1 and SIRT1. Diabetes Care 2011, 34, 1610-1616.

51. Uribarri, J.; Cai, W.; Peppa, M.; Goodman, S.; Ferrucci, L.; Striker, G.; Vlassara, H. Circulating glycotoxins and dietary advanced glycation endproducts: Two links to inflammatory response, oxidative stress, and aging. J. Gerontol. Ser. A Biol. Sci. Med. Sci. 2007, 62, 427-433.

52. Cai, W.; Ramdas, M.; Zhu, L.; Chen, X.; Striker, G.E.; Vlassara, H. Oral advanced glycation endproducts (AGEs) promote insulin resistance and diabetes by depleting the antioxidant defenses AGE receptor-1 and Sirtuin 1. Proc. Natl. Acad. Sci. USA 2012, 109, 15888-15893.

53. Coughlan, M.T.; Yap, F.Y.; Tong, D.C.; Andrikopoulos, S.; Gasser, A.; Thallas-Bonke, V.; Webster, D.E.; Miyazaki, J.; Kay, T.W.; Slattery, R.M.; et al. Advanced glycation end products are direct modulators of beta-cell function. Diabetes 2011, 60, 2523-2532.

54. Hofmann, M.A.; Drury, S.; Fu, C.; Qu, W.; Taguchi, A.; Lu, Y.; Avila, C.; Kambham, N.; Bierhaus, A.; Nawroth, P.; et al. RAGE mediates a novel proinflammatory axis: A central cell surface receptor for S100/calgranulin polypeptides. Cell 1999, 97, 889-901.

55. Hori, O.; Brett, J.; Slattery, T.; Cao, R.; Zhang, J.; Chen, J.X.; Nagashima, M.; Lundh, E.R.; Vijay, S.; Nitecki, D.; et al. The receptor for advanced glycation end products (RAGE) is a cellular binding site for amphoterin. Mediation of neurite outgrowth and co-expression of RAGE and amphoterin in the developing nervous system. J. Biol. Chem. 1995, 270, 25752-25761. 
56. Chavakis, T.; Bierhaus, A.; Al-Fakhri, N.; Schneider, D.; Witte, S.; Linn, T.; Nagashima, M.; Morser, J.; Arnold, B.; Preissner, K.T.; et al. The pattern recognition receptor (RAGE) is a counterreceptor for leukocyte integrins: A novel pathway for inflammatory cell recruitment. J. Exp. Med. 2003, 198, 1507-1515.

57. Grimm, S.; Ott, C.; Horlacher, M.; Weber, D.; Hohn, A.; Grune, T. Advanced-glycation-endproduct-induced formation of immunoproteasomes: Involvement of RAGE and Jak2/Stat1. Biochem. J. 2012, 448, 127-139.

58. Hirose, A.; Tanikawa, T.; Mori, H.; Okada, Y.; Tanaka, Y. Advanced glycation end products increase endothelial permeability through the RAGE/Rho signaling pathway. FEBS Lett. 2010, 584, 61-66.

59. Tanikawa, T.; Okada, Y.; Tanikawa, R.; Tanaka, Y. Advanced glycation end products induce calcification of vascular smooth muscle cells through RAGE/p38 mapk. J. Vasc. Res. 2009, 46, 572-580.

60. Li, J.H.; Wang, W.; Huang, X.R.; Oldfield, M.; Schmidt, A.M.; Cooper, M.E.; Lan, H.Y. Advanced glycation end products induce tubular epithelial-myofibroblast transition through the RAGE-ERK1/2 MAP kinase signaling pathway. Am. J. Pathol. 2004, 164, 1389-1397.

61. Guimaraes, E.L.; Empsen, C.; Geerts, A.; van Grunsven, L.A. Advanced glycation end products induce production of reactive oxygen species via the activation of NADPH oxidase in murine hepatic stellate cells. J. Hepatol. 2010, 52, 389-397.

62. Zhang, M.; Kho, A.L.; Anilkumar, N.; Chibber, R.; Pagano, P.J.; Shah, A.M.; Cave, A.C. Glycated proteins stimulate reactive oxygen species production in cardiac myocytes: Involvement of Nox2 (gp91phox)-containing NADPH oxidase. Circulation 2006, 113, 1235-1243.

63. Bierhaus, A.; Schiekofer, S.; Schwaninger, M.; Andrassy, M.; Humpert, P.M.; Chen, J.; Hong, M.; Luther, T.; Henle, T.; Kloting, I.; et al. Diabetes-associated sustained activation of the transcription factor nuclear factor-кB. Diabetes 2001, 50, 2792-2808.

64. Libermann, T.A.; Baltimore, D. Activation of interleukin-6 gene expression through the NF-кB transcription factor. Mol. Cell. Biol. 1990, 10, 2327-2334.

65. Ueda, A.; Ishigatsubo, Y.; Okubo, T.; Yoshimura, T. Transcriptional regulation of the human monocyte chemoattractant protein-1 gene: Cooperation of two NF- $\kappa$ B sites and NF- $\mathrm{B} / \mathrm{Rel}$ subunit specificity. J. Biol. Chem. 1997, 272, 31092-31099.

66. Li, J.; Schmidt, A.M. Characterization and functional analysis of the promoter of RAGE, the receptor for advanced glycation end products. J. Biol. Chem. 1997, 272, 16498-16506.

67. Ott, C.; Jacobs, K.; Haucke, E.; Navarrete Santos, A.; Grune, T.; Simm, A. Role of advanced glycation end products in cellular signaling. Redox Biol. 2014, 2, 411-429.

68. Grimm, S.; Ernst, L.; Grotzinger, N.; Hohn, A.; Breusing, N.; Reinheckel, T.; Grune, T. Cathepsin D is one of the major enzymes involved in intracellular degradation of AGE-modified proteins. Free Radic. Res. 2010, 44, 1013-1026.

69. Grimm, S.; Horlacher, M.; Catalgol, B.; Hoehn, A.; Reinheckel, T.; Grune, T. Cathepsins D and $\mathrm{L}$ reduce the toxicity of advanced glycation end products. Free Radic. Biol. Med. 2012, 52, $1011-1023$. 
70. Cai, W.; Torreggiani, M.; Zhu, L.; Chen, X.; He, J.C.; Striker, G.E.; Vlassara, H. AGER1 regulates endothelial cell NADPH oxidase-dependent oxidant stress via PKC-delta: Implications for vascular disease. Am. J. Physiol. Cell Physiol. 2010, 298, C624-C634.

71. Lu, C.; He, J.C.; Cai, W.; Liu, H.; Zhu, L.; Vlassara, H. Advanced glycation endproduct (AGE) receptor 1 is a negative regulator of the inflammatory response to AGE in mesangial cells. Proc. Natl. Acad. Sci. USA 2004, 101, 11767-11772.

72. Vlassara, H.; Cai, W.; Goodman, S.; Pyzik, R.; Yong, A.; Chen, X.; Zhu, L.; Neade, T.; Beeri, M.; Silverman, J.M.; et al. Protection against loss of innate defenses in adulthood by low advanced glycation end products (AGE) intake: Role of the antiinflammatory AGE receptor-1. J. Clin. Endocrinol. Metab. 2009, 94, 4483-4491.

73. Torreggiani, M.; Liu, H.; Wu, J.; Zheng, F.; Cai, W.; Striker, G.; Vlassara, H. Advanced glycation end product receptor-1 transgenic mice are resistant to inflammation, oxidative stress, and post-injury intimal hyperplasia. Am. J. Pathol. 2009, 175, 1722-1732.

74. Zimmet, P.; Alberti, K.G.M.M.; Shaw, J. Global and societal implications of the diabetes epidemic. Nature 2001, 414, 782-787.

75. Tan, K.C.; Shiu, S.W.; Wong, Y.; Tam, X. Serum advanced glycation end products (AGEs) are associated with insulin resistance. Diabetes Metab. Res. Rev. 2011, 27, 488-492.

76. Matthews, D.; Hosker, J.; Rudenski, A.; Naylor, B.; Treacher, D.; Turner, R. Homeostasis model assessment: Insulin resistance and $\beta$-cell function from fasting plasma glucose and insulin concentrations in man. Diabetologia 1985, 28, 412-419.

77. Tahara, N.; Yamagishi, S.; Matsui, T.; Takeuchi, M.; Nitta, Y.; Kodama, N.; Mizoguchi, M.; Imaizumi, T. Serum levels of advanced glycation end products (AGEs) are independent correlates of insulin resistance in nondiabetic subjects. Cardiovasc. Ther. 2012, 30, 42-48.

78. Naitoh, T.; Kitahara, M.; Tsuruzoe, N. Tumor necrosis factor-alpha is induced through phorbol ester-And glycated human albumin-dependent pathway in Thp-1 cells. Cell Signal. 2001, 13, 331-334.

79. Miele, C.; Riboulet, A.; Maitan, M.A.; Oriente, F.; Romano, C.; Formisano, P.; Giudicelli, J.; Beguinot, F.; van Obberghen, E. Human glycated albumin affects glucose metabolism in 16 skeletal muscle cells by impairing insulin-induced insulin receptor substrate (IRS) signaling through a protein kinase C alpha-mediated mechanism. J. Biol. Chem. 2003, 278, 47376-47387.

80. Cassese, A.; Esposito, I.; Fiory, F.; Barbagallo, A.P.; Paturzo, F.; Mirra, P.; Ulianich, L.; Giacco, F.; Iadicicco, C.; Lombardi, A.; et al. In skeletal muscle advanced glycation end products (AGEs) inhibit insulin action and induce the formation of multimolecular complexes including the receptor for AGEs. J. Biol. Chem. 2008, 283, 36088-36099.

81. Borst, S. The role of TNF- $\alpha$ in insulin resistance. Endocrine 2004, 23, 177-182.

82. Nakajima, K.; Yamauchi, K.; Shigematsu, S.; Ikeo, S.; Komatsu, M.; Aizawa, T.; Hashizume, K. Selective attenuation of metabolic branch of insulin receptor down-signaling by high glucose in a hepatoma cell line, Hepg2 cells. J. Biol. Chem. 2000, 275, 20880-20886. 
83. Ravichandran, L.V.; Esposito, D.L.; Chen, J.; Quon, M.J. Protein kinase c- $\zeta$ phosphorylates insulin receptor substrate-1 and impairs its ability to activate phosphatidylinositol 3-kinase in response to insulin. J. Biol. Chem. 2001, 276, 3543-3549.

84. Boyd, A.C.; Abdel-Wahab, Y.H.; McKillop, A.M.; McNulty, H.; Barnett, C.R.; O’Harte, F.P.; Flatt, P.R. Impaired ability of glycated insulin to regulate plasma glucose and stimulate glucose transport and metabolism in mouse abdominal muscle. Biochim. Biophys. Acta 2000, 1523, $128-134$.

85. Hunter, S.J.; Boyd, A.C.; O’Harte, F.P.; McKillop, A.M.; Wiggam, M.I.; Mooney, M.H.; McCluskey, J.T.; Lindsay, J.R.; Ennis, C.N.; Gamble, R.; et al. Demonstration of glycated insulin in human diabetic plasma and decreased biological activity assessed by euglycemic-hyperinsulinemic clamp technique in humans. Diabetes 2003, 52, 492-498.

86. Jia, X.; Olson, D.J.; Ross, A.R.; Wu, L. Structural and functional changes in human insulin induced by methylglyoxal. FASEB J. 2006, 20, 1555-1557.

87. Abdel-Wahab, Y.H.A.; O’Harte, F.P.; Ratcliff, H.; McClenaghan, N.H.; Barnett, C.R.; Flatt, P.R. Glycation of insulin in the islets of langerhans of normal and diabetic animals. Diabetes 1996, 45, 1489-1496.

88. Abdel-Wahab, Y.H.A.; O'Harte, F.P.M.; Barnett, C.R.; Flatt, P.R. Characterization of insulin glycation in insulin-secreting cells maintained in tissue culture. J. Endocrinol. 1997, 152, $59-67$.

89. O’Harte, F.P.; Hojrup, P.; Barnett, C.R.; Flatt, P.R. Identification of the site of glycation of human insulin. Peptides 1996, 17, 1323-1330.

90. Krause, R.; Kühn, J.; Penndorf, I.; Knoll, K.; Henle, T. N-terminal pyrazinones: A new class of peptide-bound advanced glycation end-products. Amino Acids 2004, 27, 9-18.

91. Unoki-Kubota, H.; Yamagishi, S.; Takeuchi, M.; Bujo, H.; Saito, Y. Pyridoxamine, an inhibitor of advanced glycation end product (AGE) formation ameliorates insulin resistance in obese, type 2 diabetic mice. Protein Pept. Lett. 2010, 17, 1177-1181.

92. Guo, Q.; Mori, T.; Jiang, Y.; Hu, C.; Osaki, Y.; Yoneki, Y.; Sun, Y.; Hosoya, T.; Kawamata, A.; Ogawa, S.; et al. Methylglyoxal contributes to the development of insulin resistance and salt sensitivity in sprague-dawley rats. J. Hypertens. 2009, 27, 1664-1671.

93. Liang, F.; Kume, S.; Koya, D. Sirtl and insulin resistance. Nat. Rev. Endocrinol. 2009, 5, $367-373$.

94. Lim, M.; Park, L.; Shin, G.; Hong, H.; Kang, I.; Park, Y. Induction of apoptosis of beta cells of the pancreas by advanced glycation end-products, important mediators of chronic complications of diabetes mellitus. Ann. NY Acad. Sci. 2008, 1150, 311-315.

95. Luciano Viviani, G.; Puddu, A.; Sacchi, G.; Garuti, A.; Storace, D.; Durante, A.; Monacelli, F.; Odetti, P. Glycated fetal calf serum affects the viability of an insulin-secreting cell line in vitro. Metabolism 2008, 57, 163-169.

96. Zhu, Y.; Shu, T.; Lin, Y.; Wang, H.; Yang, J.; Shi, Y.; Han, X. Inhibition of the receptor for advanced glycation endproducts (RAGE) protects pancreatic beta-cells. Biochem. Biophys. Res. Commun. 2011, 404, 159-165. 
97. Lin, N.; Zhang, H.; Su, Q. Advanced glycation end-products induce injury to pancreatic beta cells through oxidative stress. Diabetes Metab. 2012, 38, 250-257.

98. Zhao, Z.; Zhao, C.; Zhang, X.H.; Zheng, F.; Cai, W.; Vlassara, H.; Ma, Z.A. Advanced glycation end products inhibit glucose-stimulated insulin secretion through nitric oxide-dependent inhibition of cytochrome $\mathrm{C}$ oxidase and adenosine triphosphate synthesis. Endocrinology 2009, 150, 2569-2576.

99. Jitrapakdee, S.; Wutthisathapornchai, A.; Wallace, J.C.; MacDonald, M.J. Regulation of insulin secretion: Role of mitochondrial signalling. Diabetologia 2010, 53, 1019-1032.

100. Hachiya, H.; Miura, Y.; Inoue, K.; Park, K.H.; Takeuchi, M.; Kubota, K. Advanced glycation end products impair glucose-induced insulin secretion from rat pancreatic beta-cells. J. Hepatobiliary Pancreat. Sci. 2014, 21, 134-141.

101. Eto, K.; Tsubamoto, Y.; Terauchi, Y.; Sugiyama, T.; Kishimoto, T.; Takahashi, N.; Yamauchi, N.; Kubota, N.; Murayama, S.; Aizawa, T.; et al. Role of NADH shuttle system in glucose-induced activation of mitochondrial metabolism and insulin secretion. Science 1999, 283, 981-985.

102. Shu, T.; Zhu, Y.; Wang, H.; Lin, Y.; Ma, Z.; Han, X. AGEs decrease insulin synthesis in pancreatic beta-cell by repressing $\mathrm{Pdx}-1$ protein expression at the post-translational level. PLOS ONE 2011, 6, e18782.

103. Puddu, A.; Storace, D.; Odetti, P.; Viviani, G.L. Advanced glycation end-products affect transcription factors regulating insulin gene expression. Biochem. Biophys. Res. Commun. 2010, 395, 122-125.

104. Wautier, M.; Massin, P.; Guillausseau, P.; Huijberts, M.; Levy, B.; Boulanger, E.; Laloi-Michelin, M.; Wautier, J. N(carboxymethyl) lysine as a biomarker for microvascular complications in type 2 diabetic patients. Diabetes Metab. 2003, 29, 44-52.

105. Boehm, B.; Schilling, S.; Rosinger, S.; Lang, G.; Lang, G.; Kientsch-Engel, R.; Stahl, P. Elevated serum levels of $\mathrm{N} \varepsilon$-carboxymethyl-lysine, an advanced glycation end product, are associated with proliferative diabetic retinopathy and macular oedema. Diabetologia 2004, 47, 1376-1379.

106. Fosmark, D.S.; Torjesen, P.A.; Kilhovd, B.K.; Berg, T.J.; Sandvik, L.; Hanssen, K.F.; Agardh, C.-D.; Agardh, E. Increased serum levels of the specific advanced glycation end product methylglyoxal-derived hydroimidazolone are associated with retinopathy in patients with type 2 diabetes mellitus. Metabolism 2006, 55, 232-236.

107. Aso, Y.; Inukai, T.; Tayama, K.; Takemura, Y. Serum concentrations of advanced glycation endproducts are associated with the development of atherosclerosis as well as diabetic microangiopathy in patients with type 2 diabetes. Acta Diabetol. 2000, 37, 87-92.

108. Ono, Y.; Aoki, S.; Ohnishi, K.; Yasuda, T.; Kawano, K.; Tsukada, Y. Increased serum levels of advanced glycation end-products and diabetic complications. Diabetes Res. Clin. Pract. 1998, 41, 131-137.

109. Kiuchi, K.; Nejima, J.; Takano, T.; Ohta, M.; Hashimoto, H. Increased serum concentrations of advanced glycation end products: A marker of coronary artery disease activity in type 2 diabetic patients. Heart 2001, 85, 87-91. 
110. Busch, M.; Franke, S.; Wolf, G.; Brandstädt, A.; Ott, U.; Gerth, J.; Hunsicker, L.G.; Stein, G. The advanced glycation end product $\mathrm{N} \varepsilon$-carboxymethyllysine is not a predictor of cardiovascular events and renal outcomes in patients with type 2 diabetic kidney disease and hypertension. Am. J. Kidney Dis. 2006, 48, 571-579.

111. Hanssen, N.M.; Engelen, L.; Ferreira, I.; Scheijen, J.L.; Huijberts, M.S.; van Greevenbroek, M.M.; van der Kallen, C.J.; Dekker, J.M.; Nijpels, G.; Stehouwer, C.D. Plasma levels of advanced glycation endproducts $\mathrm{N} \varepsilon$-(carboxymethyl) lysine, $\mathrm{N} \varepsilon$-(carboxyethyl) lysine, and pentosidine are not independently associated with cardiovascular disease in individuals with or without type 2 diabetes: The hoorn and codam studies. J. Clin. Endocrinol. Metab. 2013, 98, E1369-E1373.

112. Hanssen, N.M.; Beulens, J.W.; van Dieren, S.; Scheijen, J.L.; Spijkerman, A.M.; van der Schouw, Y.T.; Stehouwer, C.D.; Schalkwijk, C.G. Plasma advanced glycation endproducts are associated with incident cardiovascular events in individuals with type 2 diabetes: A case-cohort study with a median follow-up of 10 years (EPIC-NL). Diabetes 2014, 64, 257-265.

113. Dobler, D.; Ahmed, N.; Song, L.; Eboigbodin, K.E.; Thornalley, P.J. Increased dicarbonyl metabolism in endothelial cells in hyperglycemia induces anoikis and impairs angiogenesis by RGD and GFOGER motif modification. Diabetes 2006, 55, 1961-1969.

114. Chong, S.A.; Lee, W.; Arora, P.D.; Laschinger, C.; Young, E.W.; Simmons, C.A.; Manolson, M.; Sodek, J.; McCulloch, C.A. Methylglyoxal inhibits the binding step of collagen phagocytosis. J. Biol. Chem. 2007, 282, 8510-8520.

115. Nowotny, K.; Grune, T. Degradation of oxidized and glycoxidized collagen: Role of collagen cross-linking. Arch. Biochem. Biophys. 2014, 542, 56-64.

116. Yuen, A.; Laschinger, C.; Talior, I.; Lee, W.; Chan, M.; Birek, J.; Young, E.W.; Sivagurunathan, K.; Won, E.; Simmons, C.A.; et al. Methylglyoxal-modified collagen promotes myofibroblast differentiation. Matrix Biol. 2010, 29, 537-548.

117. Talior-Volodarsky, I.; Arora, P.D.; Wang, Y.; Zeltz, C.; Connelly, K.A.; Gullberg, D.; McCulloch, C.A. Glycated collagen induces alpha11 integrin expression through TGF-beta2 and Smad3. J. Cell. Physiol. 2015, in press.

118. Talior-Volodarsky, I.; Connelly, K.A.; Arora, P.D.; Gullberg, D.; McCulloch, C.A. Alpha1 1 integrin stimulates myofibroblast differentiation in diabetic cardiomyopathy. Cardiovasc. Res. 2012, 96, 265-275.

119. Pozzi, A.; Zent, R.; Chetyrkin, S.; Borza, C.; Bulus, N.; Chuang, P.; Chen, D.; Hudson, B.; Voziyan, P. Modification of collagen IV by glucose or methylglyoxal alters distinct mesangial cell functions. J. Am. Soc. Nephrol. 2009, 20, 2119-2125.

120. Sassi-Gaha, S.; Loughlin, D.T.; Kappler, F.; Schwartz, M.L.; Su, B.; Tobia, A.M.; Artlett, C.M. Two dicarbonyl compounds, 3-deoxyglucosone and methylglyoxal, differentially modulate dermal fibroblasts. Matrix Biol. 2010, 29, 127-134.

121. Charonis, A.S.; Tsilibary, E.C.; Saku, T.; Furthmayr, H. Inhibition of laminin self-assembly and interaction with type IV collagen by antibodies to the terminal domain of the long arm. J. Cell Biol. 1986, 103, 1689-1697. 
122. Haitoglou, C.S.; Tsilibary, E.C.; Brownlee, M.; Charonis, A.S. Altered cellular interactions between endothelial cells and nonenzymatically glucosylated laminin/type IV collagen. J. Biol. Chem. 1992, 267, 12404-12407.

123. Cohen, M.P.; Ku, L. Inhibition of fibronectin binding to matrix components by nonenzymatic glycosylation. Diabetes 1984, 33, 970-974.

124. Liu, B.; Bhat, M.; Padival, A.K.; Smith, D.G.; Nagaraj, R.H. Effect of dicarbonyl modification of fibronectin on retinal capillary pericytes. Investig. Ophthalmol. Vis. Sci. 2004, 45, 1983-1995.

125. Duran-Jimenez, B.; Dobler, D.; Moffatt, S.; Rabbani, N.; Streuli, C.H.; Thornalley, P.J.; Tomlinson, D.R.; Gardiner, N.J. Advanced glycation end products in extracellular matrix proteins contribute to the failure of sensory nerve regeneration in diabetes. Diabetes 2009, 58, 2893-2903.

126. Collaboration, E.R.F. Diabetes mellitus, fasting blood glucose concentration, and risk of vascular disease: A collaborative meta-analysis of 102 prospective studies. Lancet 2010, 375, $2215-2222$.

127. Kilhovd, B.K.; Juutilainen, A.; Lehto, S.; Rönnemaa, T.; Torjesen, P.A.; Hanssen, K.F.; Laakso, M. Increased serum levels of advanced glycation endproducts predict total, cardiovascular and coronary mortality in women with type 2 diabetes: A population-based 18 year follow-up study. Diabetologia 2007, 50, 1409-1417.

128. Meerwaldt, R.; Lutgers, H.L.; Links, T.P.; Graaff, R.; Baynes, J.W.; Gans, R.O.B.; Smit, A.J. Skin autofluorescence is a strong predictor of cardiac mortality in diabetes. Diabetes Care 2007, 30, 107-112.

129. Meerwaldt, R.; Graaff, R.; Oomen, P.; Links, T.; Jager, J.; Alderson, N.; Thorpe, S.; Baynes, J.; Gans, R.; Smit, A. Simple non-invasive assessment of advanced glycation endproduct accumulation. Diabetologia 2004, 47, 1324-1330.

130. Del Turco, S.; Basta, G. An update on advanced glycation endproducts and atherosclerosis. BioFactors 2012, 38, 266-274.

131. Kume, S.; Takeya, M.; Mori, T.; Araki, N.; Suzuki, H.; Horiuchi, S.; Kodama, T.; Miyauchi, Y.; Takahashi, K. Immunohistochemical and ultrastructural detection of advanced glycation end products in atherosclerotic lesions of human aorta with a novel specific monoclonal antibody. Am. J. Pathol. 1995, 147, 654-667.

132. Nerlich, A.G.; Schleicher, E.D. Ne-(carboxymethyl)lysine in atherosclerotic vascular lesions as a marker for local oxidative stress. Atherosclerosis 1999, 144, 41-47.

133. Zhou, Y.J.; Wang, J.H.; Zhang, J. Hepatocyte growth factor protects human endothelial cells against advanced glycation end products-induced apoposis. Biochem. Biophys. Res. Communi. 2006, 344, 658-666.

134. Li, Y.; Li, J.; Cui, L.; Lai, Y.; Yao, Y.; Zhang, Y.; Pang, X.; Wang, J.; Liu, X. Inhibitory effect of atorvastatin on AGE-induced HCAEC apoptosis by upregulating HSF-1 protein. Int. J. Biol. Macromol. 2013, 57, 259-264. 
135. Chen, J.; Song, M.; Yu, S.; Gao, P.; Yu, Y.; Wang, H.; Huang, L. Advanced glycation endproducts alter functions and promote apoptosis in endothelial progenitor cells through receptor for advanced glycation endproducts mediate overpression of cell oxidant stress. Mol. Cell. Biochem. 2010, 335, 137-146.

136. Inagaki, Y.; Yamagishi, S.; Okamoto, T.; Takeuchi, M.; Amano, S. Pigment epithelium-derived factor prevents advanced glycation end products-induced monocyte chemoattractant protein-1 production in microvascular endothelial cells by suppressing intracellular reactive oxygen species generation. Diabetologia 2003, 46, 284-287.

137. Vlassara, H.; Fuh, H.; Donnelly, T.; Cybulsky, M. Advanced glycation endproducts promote adhesion molecule (VCAM-1, ICAM-1) expression and atheroma formation in normal rabbits. Mol. Med. 1995, 1, 447-456.

138. Schmidt, A.M.; Hori, O.; Chen, J.X.; Li, J.F.; Crandall, J.; Zhang, J.; Cao, R.; Yan, S.D.; Brett, J.; Stern, D. Advanced glycation endproducts interacting with their endothelial receptor induce expression of vascular cell adhesion molecule-1 (VCAM-1) in cultured human endothelial cells and in mice. A potential mechanism for the accelerated vasculopathy of diabetes. J. Clin. Investig. 1995, 96, 1395-1403.

139. Ishibashi, Y.; Matsui, T.; Takeuchi, M.; Yamagishi, S.-I. Glucagon-like peptide-1 (GLP-1) inhibits advanced glycation end product (AGE)-induced up-regulation of VCAM-1 mRNA levels in endothelial cells by suppressing AGE receptor (RAGE) expression. Biochem. Biophys. Res. Commun. 2010, 391, 1405-1408.

140. Ishibashi, Y.; Matsui, T.; Maeda, S.; Higashimoto, Y.; Yamagishi, S.-I. Advanced glycation end products evoke endothelial cell damage by stimulating soluble dipeptidyl peptidase-4 production and its interaction with mannose 6-phosphate/insulin-like growth factor II receptor. Cardiovasc. Diabetol. 2013, 12, 125-125.

141. Yamagishi, S.; Fujimori, H.; Yonekura, H.; Yamamoto, Y.; Yamamoto, H. Advanced glycation endproducts inhibit prostacyclin production and induce plasminogen activator inhibitor-1 in human microvascular endothelial cells. Diabetologia 1998, 41, 1435-1441.

142. Quehenberger, P.; Bierhaus, A.; Fasching, P.; Muellner, C.; Klevesath, M.; Hong, M.; Stier, G.; Sattler, M.; Schleicher, E.; Speiser, W.; et al. Endothelin 1 transcription is controlled by nuclear factor- $\mathrm{\kappa B}$ in AGE-stimulated cultured endothelial cells. Diabetes 2000, 49, 1561-1570.

143. Zhang, L.; Zalewski, A.; Liu, Y.; Mazurek, T.; Cowan, S.; Martin, J.L.; Hofmann, S.M.; Vlassara, H.; Shi, Y. Diabetes-induced oxidative stress and low-grade inflammation in porcine coronary arteries. Circulation 2003, 108, 472-478.

144. Bucala, R.; Tracey, K.J.; Cerami, A. Advanced glycosylation products quench nitric oxide and mediate defective endothelium-dependent vasodilatation in experimental diabetes. J. Clin. Investig. 1991, 87, 432-438.

145. Xu, B.; Chibber, R.; Ruggiero, D.; Kohner, E.; Ritter, J.; Ferro, A. Impairment of vascular endothelial nitric oxide synthase activity by advanced glycation end products. FASEB J. 2003, 17, 1289-1291. 
146. Chakravarthy, U.; Hayes, R.G.; Stitt, A.W.; McAuley, E.; Archer, D.B. Constitutive nitric oxide synthase expression in retinal vascular endothelial cells is suppressed by high glucose and advanced glycation end products. Diabetes 1998, 47, 945-952.

147. Barbato, J.E.; Tzeng, E. Nitric oxide and arterial disease. J. Vasc. Surg. 2004, 40, 187-193.

148. Yamagishi, S.; Amano, S.; Inagaki, Y.; Okamoto, T.; Koga, K.; Sasaki, N.; Yamamoto, H.; Takeuchi, M.; Makita, Z. Advanced glycation end products-induced apoptosis and overexpression of vascular endothelial growth factor in bovine retinal pericytes. Biochem. Biophys. Res. Commun. 2002, 290, 973-978.

149. Stitt, A.W.; Hughes, S.J.; Canning, P.; Lynch, O.; Cox, O.; Frizzell, N.; Thorpe, S.R.; Cotter, T.G.; Curtis, T.M.; Gardiner, T.A. Substrates modified by advanced glycation end-products cause dysfunction and death in retinal pericytes by reducing survival signals mediated by platelet-derived growth factor. Diabetologia 2004, 47, 1735-1746.

150. Antonetti, D. Eye vessels saved by rescuing their pericyte partners. Nat. Med. 2009, 15, 1248-1249.

151. Moore, T.C.; Moore, J.E.; Kaji, Y.; Frizzell, N.; Usui, T.; Poulaki, V.; Campbell, I.L.; Stitt, A.W.; Gardiner, T.A.; Archer, D.B.; et al. The role of advanced glycation end products in retinal microvascular leukostasis. Investig. Ophthalmol. Vis. Sci. 2003, 44, 4457-4464.

152. Lu, M.; Kuroki, M.; Amano, S.; Tolentino, M.; Keough, K.; Kim, I.; Bucala, R.; Adamis, A.P. Advanced glycation end products increase retinal vascular endothelial growth factor expression. J. Clin. Investig. 1998, 101, 1219-1224.

153. Stitt, A.W.; Bhaduri, T.; McMullen, C.B.; Gardiner, T.A.; Archer, D.B. Advanced glycation end products induce blood-retinal barrier dysfunction in normoglycemic rats. Mol. Cell Biol. Res. Commun. 2000, 3, 380-388.

154. Mamputu, J.C.; Renier, G. Advanced glycation end products increase, through a protein kinase C-dependent pathway, vascular endothelial growth factor expression in retinal endothelial cells. Inhibitory effect of gliclazide. J. Diabetes Complicat. 2002, 16, 284-293.

155. Yamagishi, S.; Inagaki, Y.; Okamoto, T.; Amano, S.; Koga, K.; Takeuchi, M.; Makita, Z. Advanced glycation end product-induced apoptosis and overexpression of vascular endothelial growth factor and monocyte chemoattractant protein-1 in human-cultured mesangial cells. J. Biol. Chem. 2002, 277, 20309-20315.

156. Ghayur, M.N.; Krepinsky, J.C.; Janssen, L.J. Contractility of the renal glomerulus and mesangial cells: Lingering doubts and strategies for the future. Med. Hypotheses Res. 2008, 4, $1-9$.

157. Yamagishi, S.; Inagaki, Y.; Okamoto, T.; Amano, S.; Koga, K.; Takeuchi, M. Advanced glycation end products inhibit de novo protein synthesis and induce TGF-beta overexpression in proximal tubular cells. Kidney Int. 2003, 63, 464-473.

158. Throckmorton, D.C.; Brogden, A.P.; Min, B.; Rasmussen, H.; Kashgarian, M. PDGF and TGF-bold beta mediate collagen production by mesangial cells exposed to advanced glycosylation end products. Kidney Int. 1995, 48, 111-117. 
159. Yang, C.W.; Vlassara, H.; Peten, E.P.; He, C.J.; Striker, G.E.; Striker, L.J. Advanced glycation end products up-regulate gene expression found in diabetic glomerular disease. Proc. Natl. Acad. Sci. USA 1994, 91, 9436-9440.

160. Fukami, K.; Ueda, S.; Yamagishi, S.; Kato, S.; Inagaki, Y.; Takeuchi, M.; Motomiya, Y.; Bucala, R.; Iida, S.; Tamaki, K.; et al. AGEs activate mesangial TGF-beta-Smad signaling via an angiotensin II type I receptor interaction. Kidney Int. 2004, 66, 2137-2147.

161. Oldfield, M.D.; Bach, L.A.; Forbes, J.M.; Nikolic-Paterson, D.; McRobert, A.; Thallas, V.; Atkins, R.C.; Osicka, T.; Jerums, G.; Cooper, M.E. Advanced glycation end products cause epithelial-myofibroblast transdifferentiation via the receptor for advanced glycation end products (RAGE). J. Clin. Investig. 2001, 108, 1853-1863.

162. Burns, W.C.; Twigg, S.M.; Forbes, J.M.; Pete, J.; Tikellis, C.; Thallas-Bonke, V.; Thomas, M.C.; Cooper, M.E.; Kantharidis, P. Connective tissue growth factor plays an important role in advanced glycation end product-induced tubular epithelial-to-mesenchymal transition: Implications for diabetic renal disease. J. Am. Soc. Nephrol. 2006, 17, 2484-2494.

163. Roestenberg, P.; van Nieuwenhoven, F.A.; Wieten, L.; Boer, P.; Diekman, T.; Tiller, A.M.; Wiersinga, W.M.; Oliver, N.; Usinger, W.; Weitz, S., et al. Connective tissue growth factor is increased in plasma of type 1 diabetic patients with nephropathy. Diabetes Care 2004, 27, 1164-1170.

164. Chung, A.C.K.; Zhang, H.; Kong, Y.-Z.; Tan, J.-J.; Huang, X.R.; Kopp, J.B.; Lan, H.Y. Advanced glycation end-products induce tubular CTGF via TGF- $\beta$-independent Smad3 signaling. J. Am. Soc. Nephrol. 2010, 21, 249-260.

165. Thornalley, P.J. Dietary AGEs and ALEs and risk to human health by their interaction with the receptor for advanced glycation endproducts (RAGE)—An introduction. Mol. Nutr. Food Res. 2007, 51, 1107-1110.

166. Ramasamy, R.; Yan, S.F.; Schmidt, A.M. Arguing for the motion: Yes, rage is a receptor for advanced glycation endproducts. Mol. Nutr. Food Res. 2007, 51, 1111-1115.

167. Heizmann, C.W. The mechanism by which dietary AGEs are a risk to human health is via their interaction with RAGE: Arguing against the motion. Mol. Nutr. Food Res. 2007, 51, 1116-1119. 


\title{
The Impact of Non-Enzymatic Reactions and Enzyme Promiscuity on Cellular Metabolism during (Oxidative) Stress Conditions
}

\author{
Gabriel Piedrafita, Markus A. Keller and Markus Ralser
}

\begin{abstract}
Cellular metabolism assembles in a structurally highly conserved, but functionally dynamic system, known as the metabolic network. This network involves highly active, enzyme-catalyzed metabolic pathways that provide the building blocks for cell growth. In parallel, however, chemical reactivity of metabolites and unspecific enzyme function give rise to a number of side products that are not part of canonical metabolic pathways. It is increasingly acknowledged that these molecules are important for the evolution of metabolism, affect metabolic efficiency, and that they play a potential role in human disease-age-related disorders and cancer in particular. In this review we discuss the impact of oxidative and other cellular stressors on the formation of metabolic side products, which originate as a consequence of: (i) chemical reactivity or modification of regular metabolites; (ii) through modifications in substrate specificity of damaged enzymes; and (iii) through altered metabolic flux that protects cells in stress conditions. In particular, oxidative and heat stress conditions are causative of metabolite and enzymatic damage and thus promote the non-canonical metabolic activity of the cells through an increased repertoire of side products. On the basis of selected examples, we discuss the consequences of non-canonical metabolic reactivity on evolution, function and repair of the metabolic network.
\end{abstract}

Reprinted from Biomolecules. Cite as: Piedrafita, G.; Keller, M.A.; Ralser, M. The Impact of Non-Enzymatic Reactions and Enzyme Promiscuity on Cellular Metabolism during (Oxidative) Stress Conditions. Biomolecules 2015, 5, 2101-2122.

\section{Introduction}

During its lifespan, a cell is exposed to many hazards and changes in the environment. These include varying $\mathrm{pH}$ and temperature, osmotic imbalances, radiation, drought, starvation, contact with deleterious or toxic chemicals, or biotic interactions with other species. These stress situations can manifest as an acute pulse, or be sustained over time, and can affect cellular functioning and ultimately impair cell growth, replicative capabilities, and/or promote aging. Stress-induced damage to biological macromolecules such as DNA, proteins and membrane components has been intensively studied and characterized [1-4]. However, also cellular metabolites and the flux through metabolic pathways are affected. The impact of stress on metabolism and its small-molecule cellular constituents has attracted notable attention in areas of chemistry and toxicology $[5,6]$, but only recently research has begun to systemically analyze the wide-ranging consequences at the systems level $[7,8]$. Since metabolism is a basal property of life and a main driving force during evolution, being the closest-to-phenotype cellular attribute, and given the reactive nature of many metabolites, the identification of stress-related metabolic processes appears to be of fundamental importance. 


\section{Chemical Damage to Metabolites: Oxidants and Antioxidants}

One of the most important causes of metabolic damage is unspecific oxidation. Basal levels of oxidizing molecules arise constantly from normal cellular processes involving redox reactions or in the mitochondrial or photosynthetic electron transport chains [9]. Normal levels of oxidants are compensated by cellular metabolism, and being integral part of physiology, are required for biochemical reactions and as signaling molecules [10]. In a range of conditions referred to as "oxidative stress" [11], normal redox homeostasis is however disrupted and the balance between reducing and oxidizing molecules shifts towards the latter. Under oxidative stress, reactive oxygen species (ROS) represent one of the most important groups of cellular oxidants and comprise oxygen-derived molecules such as hydrogen peroxide $\left(\mathrm{H}_{2} \mathrm{O}_{2}\right)$, superoxide anion $\left(\mathrm{O}_{2}{ }^{-}\right)$, or hydroxyl radical $(\cdot \mathrm{HO})$. Non-enzymatic chemical reactivity plays a crucial role in this scenario, as for example in the context of the $\mathrm{Fe}(\mathrm{II})$-catalyzed Fenton reaction, that converts $\mathrm{H}_{2} \mathrm{O}_{2}$ into $\bullet \mathrm{HO}$ [12]. Superoxide anion and hydroxyl radical are highly reactive and indiscriminately damage metabolites, lipids, enzymes and nucleic acids via peroxidation [13-15]. This damage causes "errors" in metabolism [16,17], (Figure 1 (i)) such as the propagation of lipid peroxidation and carbohydrate oxidation [18,19]. In addition to the strong potential of superoxide anion to react with transition metals, it also combines with other chemical species, e.g., nitric oxide, and generates highly oxidant species such as peroxynitrite, causing further damage to lipids, altered nucleobases, and nitration of amino acid residues [20-23]. Under oxidative stress, the functionality of cellular systems declines, increasing the risk of cellular damage, apoptosis, aging, neurodegeneration and cancer [24-26].

Common targets to oxidative damage are amino acids, both in their free and protein integrated forms that are both highly abundant. Especially cysteine (which will be discussed later in the text) and methionine are the most prone to oxidation [27]. When the sulphur of methionine reacts with oxidizing molecules, it can form methionine- $S$ - or methionine- $R$-sulfoxides, whose accumulation has been associated with various diseases and aging [28,29].

Biological systems exploit this property of metabolites and use them as scavengers for ROS. This applies not only to methionine and cysteine, but also to pyruvate, $\alpha$-tocopherol, ascorbic acid, or other amino acids [12,30-32] (Figure 1 (ii)). While free methionine acts as general redox buffer, protein-coded methionine residues absorb oxidizing agents by positioning on the exposed protein surface and surrounding the active site, thereby preventing more severe oxidative damage on targeted proteins $[33,34]$. This oxidation of methionine residues at the protein surface can proceed without significant reduction in enzyme activity, as for example in glutamine synthetase, which is shown extremely resistant to $\mathrm{H}_{2} \mathrm{O}_{2}$ [33]. 


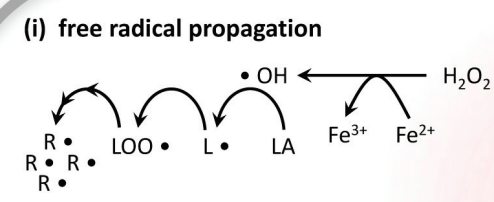

(ii) molecular scavenging

$\mathrm{H}_{2} \mathrm{O} \stackrel{\text { GSH, Met.... }}{\longleftarrow} \mathrm{O}_{2}+\stackrel{\mathrm{Mn}^{2+}}{\mathrm{H}_{2} \mathrm{O}_{2}} \mathrm{O}_{2}$

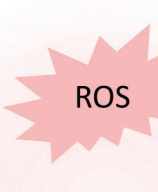

(iii) metabolite repair

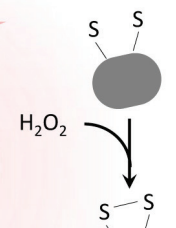

\section{GSSG GSH}
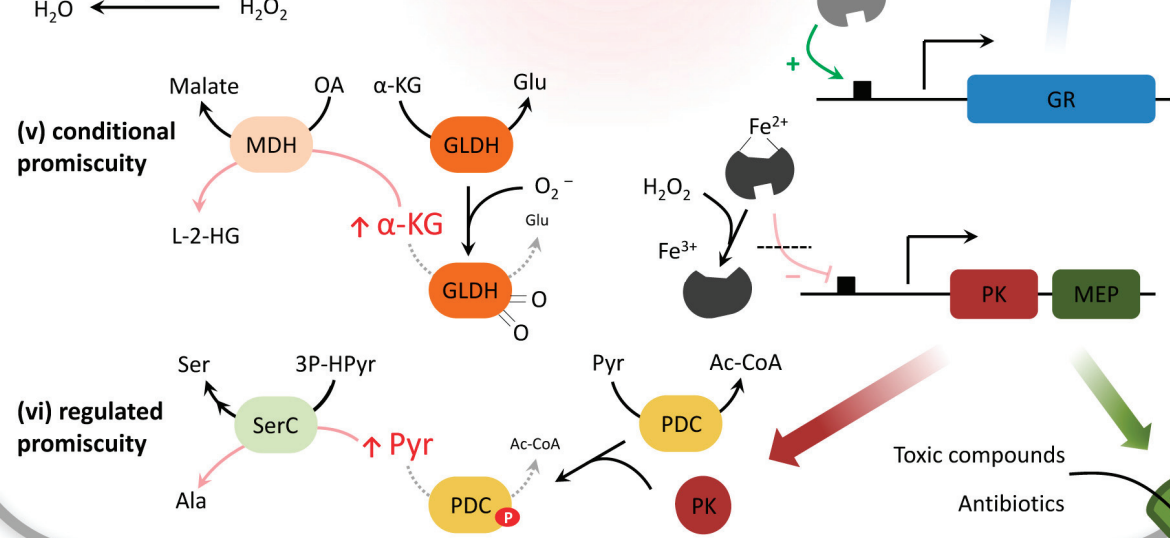

Pyr Ac-CoA

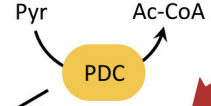

Toxic compounds

Antibiotics

(iv) unspecific efflux

Figure 1. Formation of non-canonical metabolites and repair strategies. Chemical modification of metabolites leads to unwanted reactions, causing damage to both macromolecules and small molecules (i). Molecular scavengers cleanse and channel ROS toward less toxic products (ii). Stress sensors activate genes involved in specific protein-based repair responses (iii); or in unspecific transport processes (iv). As a general outcome, the metabolome reconfigures, affecting metabolite levels and fluxes; this in turn affects the specificity of enzymatic reactions. (v-vi) Selection of relevant examples from metabolism. Abbreviations: ROS, reactive oxygen species; Met, Methionine; GR, glutathione reductase; GSSG, oxidized glutathione or glutathione disulfide; GSH, reduced glutathione; MEP, multidrug efflux pump; LA, lipid; L•, lipid radical; LOO•, lipid peroxyl radical; R - complex radicals; GLDH, glutamate dehydrogenase; $\alpha-\mathrm{KG}, \alpha$-ketoglutarate; Glu, glutamate; MDH, malate dehydrogenase; OA, oxaloacetate; L-2-HG, L-2-hydroxyglutarate; PK, protein kinase; PDC, pyruvate dehydrogenase complex; Pyr, pyruvate; Ac-CoA, acetyl-coenzyme A; SerC, phosphoserine transaminase; 3P-HPyr, 3-phosphohydroxypyruvate; Ser, serine; Ala, alanine. 


\subsection{Not only Protein and DNA Damage, but also Metabolites Can Be Repaired}

In analogy to DNA and protein repair, cells have developed a series of control mechanisms to cope with the oxidized/chemically damaged metabolites, summarized by the term "metabolite repair" [35] or "metabolite-damage control" [36-38]. These involve complementary strategies that either: (i) "repair" the chemical damage to metabolites (e.g., reversion through reduction of an oxidized molecule); (ii) degrade or convert the altered molecules into less harmful products or functional metabolites; or (iii) export the non-canonical metabolite. Some of these mechanisms are of general nature and overlap with the parallel occurring protein repair mechanisms (e.g., glutathione reductase, thioredoxin). Others are specifically targeted against small-molecule derivatives, as for example Glutathione peroxidase $4(\mathrm{GPx} 4)$, which is a protein of the glutathione peroxidase (GPx) family with wide substrate specificity, able to reduce a variety of lipid hydroperoxides, preventing radical propagation through lipid peroxidation $[39,40]$. GPx4 activity obtains its redox potential through the reduced form of the tripeptide glutathione (GSH) [41,42], a molecular scavenger which is regenerated in a NADPH-dependent reaction catalyzed by glutathione reductase (GR) (Figure 1 (iii)). Indeed, most of the repair mechanisms require, directly or indirectly, energy supply in the form of ATP or reducing equivalents [4,15], and many are conditionally activated under stress conditions (Figure 1). The consequence is a sudden change of ATP and NADPH consumption once cells enter a stress situation and its balance requires metabolism to reconfigure.

An illustrative example for specific oxidative stress-related repair concerns methionine. Repair of oxidized methionine does occur when the amino acid is in its free form as well as when it is part of a protein $[43,44]$ (Figure 2). Three enzyme families of methionine sulfoxide reductases, MsrA, MsrB and $\mathrm{MsrC}$, metabolize the derivatives in a stereospecific manner. Of them, MsrA and MsrB are highly conserved and reduce the $S$-sulfoxide and the $R$-sulfoxide residues, respectively, predominantly within a protein context [45]. MsrC (also named $\mathrm{f} R \mathrm{Msr}$ ) is only present in prokaryotes [46] and in some eukaryotes [47], and has its main affinity for free methionine- $R$-sulfoxide. Interestingly, MsrC exhibits no sequence homology with MsrA and MsrB, indicating independent evolutionary origin of these functionally overlapping proteins. In higher plants, where no specific MsrC-like activity exists, the repair function on free methionine- $R$-sulfoxide is efficiently covered by a subtype of MsrB proteins (which would have evolved by segmental duplication) [48], evidencing the evolution of alternative adaptive strategies to cope with a similar type of damage (Figure 2). As a common observation, repair mechanisms appear widely distributed, irrespective of whether metabolic damage proceeds through direct metabolite oxidation, non-enzymatic reactivity, enzyme inactivation, or enzyme promiscuity. Although most of these repair functions are non-essential under normal conditions, the respective mutants commonly show growth defects, lower survival rates or higher mutation frequencies $[36,37,47,49,50]$. In humans, deficiencies in metabolite detoxification systems are related to encephalopathic disorders [51], cancer predisposition [52], drug intolerance [53], or aging and a decreased lifespan [54]. Evidence has also been presented that neuronal clearance of toxic metabolites could be the explanation of why metazoans require sleep [55]. 


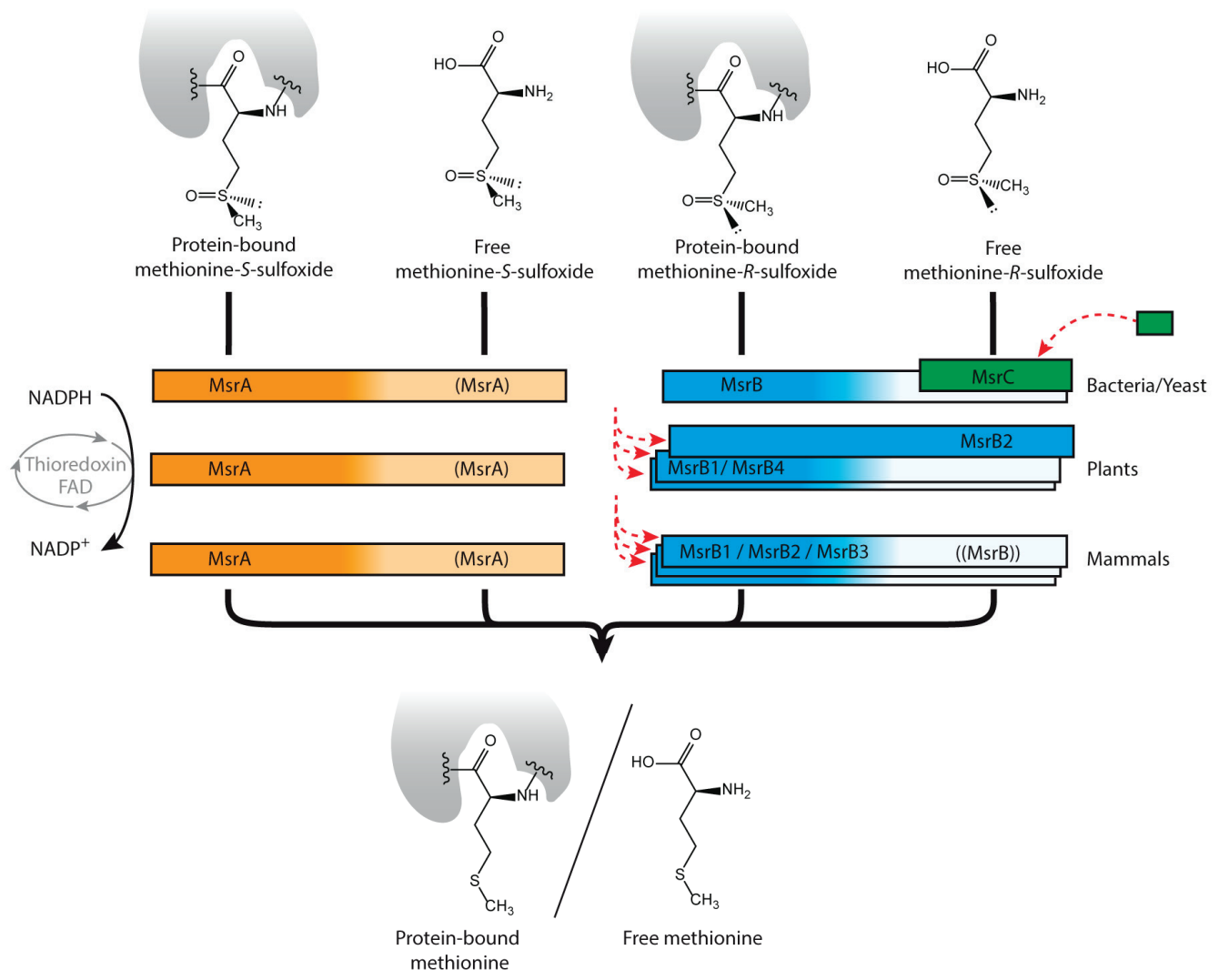

Figure 2. Oxidative damage on methionine and its repair enzymes. Methionine is oxidized to methionine- $S$-sulfoxide and methionine- $R$-sulfoxide derivatives. Three families of methionine sulfoxide reductases (Msr, different colors), revert the process depending on thioredoxin, each being stereo-specific against the $R$ - or the $S$-enantiomeric form. While MsrA and MsrB are highly conserved with preference for protein integrated methionine sulfoxides, MsrC ( $\mathrm{f} R \mathrm{Msr}$ ) is limited to unicellular organisms and reduces free methionine- $R$-sulfoxide. MsrB has evolved by duplication and divergence (red dashed arrows), accounting for coverage of diverse subcellular localizations, and enabling in plants the appearance of a subtype active against the free methionine- $R$-sulfoxide. Color intensities illustrate different strength in catalytic efficiency.

\subsection{Other Forms of Metabolite Damage: Examples of Nucleotide and Cofactor Metabolism}

Metabolism comprises a large diversity of molecules. The spectrum of possible combinatorial reactions and modifications on metabolites extends to a huge space of chemical reactions. Some modifications occur however more frequent than others. For instance, nucleotide metabolism is by its nature composed of many metabolites possessing high structural similarities. A range of abnormal nucleotides is generated as a consequence of spontaneous oxidation [56]. Others arise from unspecific enzymatic activities, as for example by methylation of nucleobases. Several of these 
aberrant compounds with only slight structural differences from the standard bases, can subsequently be recognized by downstream enzymes which propagate the error via incorporation. The mischarge of nucleoside diphosphate (NDP) kinases, which can promiscuously accept a broad range of base-differing substrates, releases abnormal NTPs that cause mispairing and mutagenesis if incorporated into DNA [56,57]. Cells have developed various families of NTP pyrophosphatases that intercept and hydrolyze non-canonical NTPs [37]. For instance, MutT-like proteins of the widespread Nudix superfamily catalyze - with variable degree of substrate specificity - the detoxification of oxo-purine dNTPs generated during oxidative stress, and thereby prevent DNA damage [58-60]. In other cases, substrate promiscuity (which will be discussed in more detail below) is the main contributor to the pool of abnormal nucleotides: dUTPases hydrolyze with high specificity dUTP, discriminating against normal cytosine and thymine nucleobases. dUTP can arise from UDP through a secondary activity of ribonucleotide reductase followed by phosphorylation, or by (catalytic) deamination of a deoxycytidine (tri-)phosphate. Interestingly, this principle of dUTPase activity is present in all three kingdoms of life, but is fulfilled by two mutually exclusive dUTPase families that present no sequence homology, yet being functionally analogous [61,62].

Another quantitatively highly relevant case concerns chemical modification of enzymatic coenzymes, such as NADH or FADH. Hydration of NADH or NADPH is common, occurring either non-enzymatically [63] or as a side reaction of promiscuous enzymes such as glyceraldehyde 3-phosphate dehydrogenase [64]. The resulting $R$ and $S$ hydrated forms of NAD(P)H display high structural similarity with the cofactors but are non-functional and inhibit various dehydrogenase reactions [65]. An ADP/ATP-dependent NAD(P)H dehydratase, widespread in all domains of life, converts the $S$ enantiomeric derivative back to $\mathrm{NAD}(\mathrm{P}) \mathrm{H}$ [63]. Its action is complemented by the catalytic activity of an epimerase that catalyzes the interconversion between the $R$ and $S$ hydrated forms, an enzyme that appears fused to the dehydratase in some species such as Escherichia coli. Interestingly, in higher plants [66,67] and also in mammals [68] both genes possess organellar targeting sequences, allowing the proteins to be directed and co-localize within diverse subcellular compartments.

\section{Non-Enzymatic Metabolic Reactions Are Affected by Stress Conditions}

A second aspect of the susceptibility of metabolism concerns non-enzymatic reactions that occur as part of normal metabolism or through non-specific chemical reactivity but that contribute to canonical metabolic pathways [69,70]. Such non-enzymatic reactions are of high importance for the evolution of metabolism, as exemplified by the metabolism-like non-enzymatic interconversions that can occur between central carbon metabolites, which might have given rise to the early evolution of glycolysis and pentose phosphate pathway [71]. The presence of an enzyme catalyst in modern cells does however not prevent these non-enzymatic chemical reactions to occur in parallel to the enzymatic pathways. Termed Class I non-enzymatic metabolic reactions, a subset of non-enzymatic reactions are of low substrate specificity and can affect multitude of metabolites and modify a series of macromolecules [69]. These include Maillard reactions, unspecific conjugations of amino acids that are accelerated under heat and UV exposure, and also unspecific protein modifications such as protein (poly-)phosphorylation, glycosylation or acylation. The term "underground metabolism" [70] has been suggested to summarize the repertoire of this chemical reactivity, both non-enzymatic and 
enzyme-promiscuous, occurring in parallel to the flux of functional metabolic pathways. This reactivity is distinguishable from other more specific non-enzymatic reactions that are part of the metabolic network, and occur either exclusively non-enzymatically (Class II non-enzymatic metabolic reactions) or in parallel to existing enzyme function (Class III) [69].

Non-enzymatic reactions are dependent on the chemical environment (metal availability, $\mathrm{pH}$, temperature, ionic strength), and must therefore be particularly sensitive to stress conditions. This is well illustrated for the case of non-enzymatic protein acylation: A number of essential, endogenous thioester metabolites, such as acetyl-CoA or succinyl-CoA, can unspecifically cross-acylate protein lysines $[72,73]$. Protein acylation appears most prominent in mitochondria, correlates with the levels of these reactive metabolites and with mitochondrial energy-metabolism, and has been linked to a form of "carbon stress" [74]. Non-enzymatic modification of macromolecules can alter protein function and may require quality-control responses. In this case, the sirtuin family of deacetylase enzymes (which has received notable attention by a potential connection to the benefits arising from a calorie-restriction diet) has been proposed to counteract the potential deleterious effects of non-enzymatic protein acylation and thus to play a role in stress resistance [74]. Similarly, for small molecules, intermediates generated in non-enzymatic reactions can, if accumulated, interfere with metabolic pathways by acting as competitive inhibitors of enzymes, or serve as alternative substrates, and thus need to be cleared.

While specific repair mechanisms might have evolved for non-canonical metabolites which are produced in higher quantities or present strong cytotoxic effects, it is reasonable to assume that not all metabolic side products possess specific clearance mechanisms. This becomes illustrative, as the number of potential chemical reaction products from non-enzymatic reactivity exceeds the number of enzymes encoded in a typical genome by several orders of magnitude. A broad range of metabolites, including especially those for which no specific cleaning enzyme exists [75-77], might however be cleared via unspecific cellular export, which is largely mediated by efflux pumps and transmembrane channels such as multidrug transporters (Figure 1 (iv)). In bacteria, numerous studies report the involvement of membrane transporters in multidrug resistance [78-80]. E. coli's resistance to a high number of compounds is mediated by the outer-membrane pore-forming protein TolC [81]. This transporter acts in concert with the inner membrane TolC-dependent efflux pump AcrB and with cognate periplasmic proteins (e.g., AcrA) to form tripartite trans-periplasmic exporters that push xenobiotics out of the cell $[82,83]$. There is growing evidence suggesting that TolC-mediated extrusion is not limited to xenobiotics [84]: E. coli tolC mutants show lower fitness phenotypes in certain stress conditions [85], accumulate cell-synthesized enterotoxins and potentially-deleterious intermediary metabolites [86], and overexpress key stress response regulators, presumably triggered by the abnormal retention of toxic cellular products [87]. Similar transport mechanisms operate in many other bacteria [80].

\section{Enzymes That Change Substrate Specificity during Stress Conditions}

A third important aspect of metabolism during stress conditions does not concern the direct chemical modification of metabolites, but affects them indirectly by altering enzyme function. Upon damage, several enzymes decrease in substrate specificity and simultaneously increase in promiscuity. 
When enzymes are modified, novel subsidiary activities and interactions can arise concomitantly from the altered proteins, enabling additional metabolic reactions, some of potential physiological significance $[70,88]$. It is a general property of metabolism that most metabolic enzymes are not as specific as sometimes depicted in textbooks, and are in fact error-prone or ambiguous in their function [89,90] (Figure 3) (The concept of "enzyme promiscuity" has been massively used recently, but in different contexts and with different meanings [91]. The classification that we make below follows that by Hult and Berglund [91].).

A) Substrate promiscuity

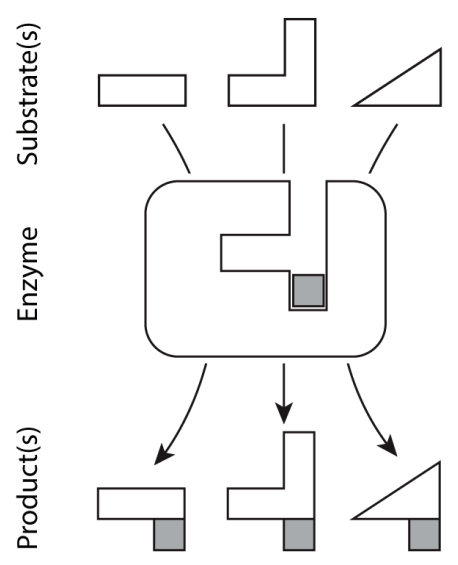

B) Catalytic promiscuity

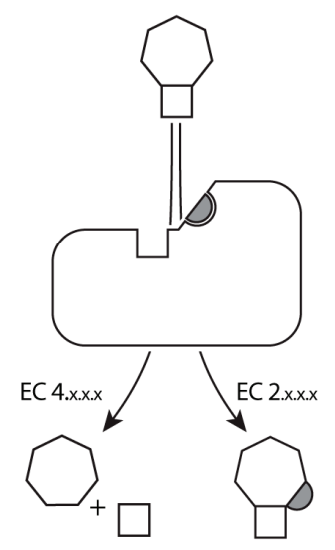

C) Conditional promiscuity

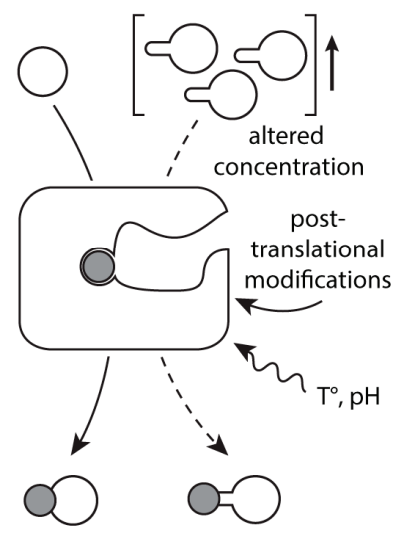

Figure 3. Mechanistic classification of enzyme promiscuity. (A) Substrate promiscuity or multispecificity: A certain enzyme can perform the same catalytic reaction on a diverse set of substrates indistinctly; (B) Catalytic promiscuity: Different chemical transformations are allowed by the same enzyme, according to which this can be classified with various E.C. numbers. (C) Conditional promiscuity: Latent secondary activities of an enzyme might gain activity in response to environmental changes, e.g., due to an increase in the concentration of substrate analogs with lower affinity for the enzyme, or by post-translational signals related with induced conformational changes. The three examples shown are merely illustrative but inspired by transaminase TyrB, cytosine methyltransferase and thymidine kinase, respectively.

Most enzymes accept multiple substrates and possess affinities for a wider range of compounds, a phenomenon referred to as substrate promiscuity (Figure 3A) (Some authors consider that the capacity of an enzyme to catalyze various natural substrates may be more conveniently described by the term "substrate multispecificity" or "cross-reactivity", so that the term "substrate promiscuity" is reserved to adventitious secondary activities different from the one the enzyme has evolved: see $[90,92]$.). A recent example of notable attention is the enzyme TP53-induced glycolysis and apoptosis regulator (TIGAR), an enzyme that possesses an important role in cancer cell metabolism. TIGAR was first identified as fructose-2,6-bisphosphatase (F26BPase) [93], but it accepts multiple 
substrates. TIGAR shows its highest activity as phosphoglycolate-independent 2,3-bisphosphoglycerate phosphatase [94], acting most efficiently on a metabolite whose function is so far unclear.

Other enzymes are able to catalyze more than one type of chemical reaction on a given set of substrates (catalytic promiscuity, Figure 3B). This is the case of certain cytosine methyltransferases that are able to catalyze cytosine methylation as well as cytosine deamination. Cytosine deamination yields thymine, implying that this catalytic activity causes mutations and could play a potential role in tumourogenesis [95].

A classical example of enzymes that possess promiscuous activities that can lead to toxic by-products are malate dehydrogenase (MDH) and isocitrate dehydrogenase (IDH1 and IDH2). $\mathrm{MDH}$ typically catalyzes the interconversion between malate and oxaloacetate, but possesses certain affinity towards $\alpha$-ketoglutarate. Despite the much higher affinity for the canonical substrate, high intracellular levels of $\alpha$-ketoglutarate promote that MDH also produces L-2-hydroxyglutarate (see conditional promiscuity, Figure 1 (v), Figure 3C) [35]. A widely distributed FAD-dependent enzyme, the L-2-hydroxyglutarate dehydrogenase, specifically converts this side-product back into $\alpha$-ketoglutarate [36]. Deleterious mutations in this enzyme in humans are responsible for an inherited metabolic disease called L-2-hydroxyglutaric aciduria, attributed to the accumulation of the aberrant product [51]. An analogous detoxification system exists also for its enantiomer D-2-hydroxyglutarate [96]. This normal metabolic intermediate is overproduced by mutant forms of isocitrate dehydrogenase-1 and -2 (IDH1 and IDH2) [97]. These mutations, preferentially occurring in cancer, alter the enzyme towards an increased production of this metabolite, which is otherwise formed in a side reaction and at a low rate only. D-2-hydroxyglutarate is also found at increased levels in patients suffering from the metabolic syndrome D-2-hydroxyglutaric aciduria [98], a genetically heterogeneous neurometabolic disorder attributed to germline mutations in IDH2 [99] and mutant genotypes of the D-2-hydroxyglutarate dehydrogenase, the enzyme responsible for D-2-hydroxyglutarate repair [98].

Importantly, such secondary activities of enzymes are often latent in normal growth conditions, but can become relevant in response to environmental changes or stresses (conditional promiscuity, Figure 3C), either by: (i) an enhanced bioavailability of a low-affinity substrate analog in comparison with the concentration of the natural substrate; (ii) conformational (allosteric) changes of the enzyme induced by direct exposure to stressors; (iii) conformational changes regulated by specific post-translational modifications exerted by stress-related signaling pathways. Therefore, enzyme promiscuity must be of critical importance for an integral view of metabolic reconfiguration under stress conditions (Figure 1).

An illustrative case is represented by metalloenzymes that exhibit a tendency toward mis-metallation during stress conditions. The activity of most metalloproteins depends on the binding of the correct metal, with a mis-metallation resulting in change of reactivity, or partial to total inactivation. Bacterial ribulose 5-phosphate epimerase (Rpe) and some other enzymes that are canonically iron-dependent, actually accept both iron Fe(II) and manganese Mn(II) as cofactor [100,101]. As pointed out by Cotruvo and Stubbe [102], Fe(II)- and Mn(II)- binding affinities are comparable in these proteins, and thus the discrimination between both metals is roughly determined by the differential bioavailability. While the enzymes are hence bound to Fe(II) under normal cellular 
growth conditions, they appear charged with $\mathrm{Mn}$ (II) under oxidative stress or iron-limiting conditions. This ambiguity is regarded as an adaptive strategy to protect enzymes from irreversible inactivation and severe damage [100] (Table 1): (i) Fe(II) centers are sensitive to ROS and oxidize to $\mathrm{Fe}(\mathrm{III})$, causing enzyme inactivation [101]; (ii) free iron pools react with ROS propagating the toxic free radical formation [103]. Interestingly, the same transcriptional control mechanisms that are responsible to ensure iron sequestration and control during oxidative stress [103] are linked to $\mathrm{Mn}(\mathrm{II})$ import and the promotion of the Mn metabolism [102]. Noteworthy, Mn(II), when bound to phosphate or carbonate, can act as an efficient non-enzymatic catalyst for the superoxide disproportionation at physiological conditions [104], which can in fact rescue yeast superoxide dismutase-knockouts [105], conferring an additional role to $\mathrm{Mn}(\mathrm{II})$ as antioxidant.

\section{Adaptive Promiscuity: When Altered Substrate Specificity Becomes Advantageous}

Despite being an energetic burden to the cell, promiscuity and multi-substrate specificity is an integral biological property in metabolic networks, and is essential as it is necessary for the evolution of metabolism [106]. In some instances, the ability to process multiple substrates is beneficial to cells in stress situations (Table 1). Perhaps one of the most illustrative examples is the genetic code "ambiguity". Translational fidelity, and thus an adequate protein biosynthesis, relies on the accurate aminoacylation of the different tRNA molecules by specific aminoacyl-tRNA synthetases and on unambiguous codon-anticodon pairing within the ribosome. Yet a certain degree of permissiveness is known to exist due to nucleobase wobbling, accounting for the degeneracy in the genetic code. Indeed, numerous exceptions and variants to the standard decoding have been reported in different organisms [107-109]. Netzer et al. [110] demonstrated how the genetic code accuracy can also change within the lifespan of an individual cell, as a consequence of induced changes in the acylation fidelity of aminoacyl-tRNA synthetases. A small but significant amount of methionine (Met) residues (approximately $1 \%$ of all coded Met) misacylate to non-cognate tRNA in normal unstressed mammalian cells. This fraction sharply increases (to approximately 13\%) under ROS-related stress conditions, for example when exposing cultured cells to viral infections, bacterial wall factors or physico-chemical stressors. This results in proteins with high content in mistranslated Met. As discussed above, Met is a biological scavenger of ROS, proposing an adaptive role of methionyl-tRNA synthetase (MetRS) promiscuity and controlled Met-misacylation in coping with oxidative damage (Table 1). A recent study [111] reveals that the modulation of MetRS specificity is mediated by particular serine phosphorylations driven by the protein kinase ERK, which is a common mediator in the signaling response to stress. This represents a further indication about the in vivo protective role of Met-misincorporation for cell survival and growth under ROS stress.

The antioxidant function of Met residues might be also the cause of AUA codon reassignment in mitochondria, from isoleucine (Ile) to Met, which is widespread in vertebrates and also occurs in several invertebrates with a highly aerobic metabolism (like insects) and in some unicellular eukaryotes, including Saccharomyces cerevisiae [112]. With the respiratory chain as continuous source of ROS, one might hypothesize that a permanent redefinition of the genetic code (with one extra codon assigned to Met, and 2 instead of 3 codons for Ile) could have been a more suitable evolutionary adaptation in mitochondria than an inducible mechanism to tune promiscuous 
methionylation activities on non-methionyl tRNAs. Integral proteins of the mitochondrial inner membrane are overloaded with Met on their exposed surfaces (a classical preferential position for Ile residues), and in this way a significant containment of oxidative damage is achieved [112].

Table 1. Possible physiological benefits of various promiscuous and redundant activities induced in response to stress or environmental changes.

\begin{tabular}{lllll}
\hline Stress & Enzyme & Induced Activity & Alleged Function & Reference \\
\hline Oxidative stress & Mammalian MetRS & $\begin{array}{l}\text { Mismethionylation of } \\
\text { non-cognate tRNAs }\end{array}$ & $\begin{array}{l}\text { Protect enzymes from } \\
\text { inactivation }\end{array}$ & {$[110]$} \\
\hline Biotic interaction & $\begin{array}{l}\text { SerRS/LeuRS } \\
\text { from Candida }\end{array}$ & $\begin{array}{l}\text { Misleucinylation } \\
\text { of Ser-tRNA }\end{array}$ & $\begin{array}{l}\text { Increased invasivity, } \\
\text { immune invisibility }\end{array}$ & {$[113]$} \\
\hline $\begin{array}{l}\text { Biotic interaction, } \\
\text { anaerobiosis, } \\
\text { starvation... }\end{array}$ & Bacterial transaminases & $\begin{array}{l}\text { Redundancy in Ala \& } \\
\text { Lys biosynthesis }\end{array}$ & Robust peptidoglycan & {$[114]$} \\
\hline Oxidative stress & $\begin{array}{l}\text { Bacterial Pentosephosphate } \\
\text { epimerase (Rpe) }\end{array}$ & $\begin{array}{l}\text { Mismetallation } \\
\text { with Mn(II) }\end{array}$ & Protect enzymes from & {$[102]$} \\
\hline
\end{tabular}

Another case in which ambiguous tRNA acylation has been proven beneficial is that of the fungus Candida albicans [115] (Table 1). Here the codon CUG can be translated both as a leucine (Leu) and as a serine (Ser) (with 5\% and 95\% frequency, respectively). This duality arises from the capacity of two aminoacyl-tRNA synthetases (LeuRS and SerRS) to recognize the particular unique serine tRNA (CAG) and allows statistically distributed populations of different mistranslated proteins from the same gene. This increase in phenotypic diversity is exploited in pathogenicity [113]. In fact, genes encoding cell surface adhesins are especially rich in CUG codons: partial CUG mistranslation as Leu results in morphological variations and heterogeneity in the cell wall that modify Candida interactions with the host microenvironment. In particular, experimental evidence shows that adherence capacities improve while recognition by the hosts immune system decreases [113], both being related with Candida virulence.

\section{Changing Activity and Metabolic Switches: Stress Situations That Affect Flux Distribution}

Enzyme activities are tunable and the degree of affinity and/or efficiency $\left(K_{\mathrm{m}}, k_{\text {cat }}\right)$ for different metabolites may change according to conditions [91] (Figure 3C). This also applies during the individual lifetime of an enzyme, e.g., due to age related conformational alterations [116]. As a consequence, aside from the immediate effects of protein damage on the enzymes natural function and its promiscuous activities, the inactivation of a metabolic enzyme commonly involves an adaptation in total metabolic flux and/or results in a redistribution of flux toward other pathways. Due to its redox capacity, cysteine plays a pivotal role in many enzyme catalytic mechanisms, and the redox sensitivity of this amino acid renders several enzymes vulnerable to redox imbalances and ROS accumulation $[27,117,118]$. Interestingly, this redox sensitivity is exploited by cells to intensify metabolic protection in stress situations. In yeast, two enzymes of glycolysis, glyceraldehyde-3-phosphate dehydrogenase (GAPDH) and pyruvate kinase (PK), have been shown to be sensitive to oxidation, leading to a rapidly induced (in seconds-scale) but temporal glycolytic 
block $[119,120]$. Termed the glycolysis/PPP transition, the oxidative part of the pentose phosphate pathway (PPP) that is more redox resistant, increases in activity, resulting in enhanced NADPH production under the conditions where this cofactor is required by the anti-oxidant machinery $[119,121]$. The inactivation of GAPDH during oxidative stress is not only an adverse side reaction; the enzyme possesses evolved structural features that compete for free $\mathrm{H}_{2} \mathrm{O}_{2}$ to assure rapid inactivation when levels of this metabolite rise [122]). Increased PPP activity and stress resistance augmentation in yeast are also observed when the activities of triosephosphate isomerase [123] and pyruvate kinase $[124,125]$ are reduced by genetic modification, implying that increased NADPH production in the oxidative PPP is a recurrent consequence to blocks in glycolysis. Recently, time-resolved metabolomics has revealed this mechanism to be conserved in mammalian skin cells, revealing however that the mechanistic trigger of the glycolytic/PPP transition is not yet understood [126]. In this context, it is however important to keep in mind that the contribution of distinct metabolic pathways, including the oxidative PPP, to the total NADPH pool differs between yeast and mammalian cell types, and even between tissues [127], so that the individual cells and organisms may distribute NADPH production in a different manner.

Another oxidant-induced change in metabolic flux distribution is connected to the polyamine metabolites spermine, spermidine and putrescine. These polycations are implicated in a multitude of cellular functions, including the stress response [128]. Treatment with peroxides affects the intracellular concentrations of spermidine and putrescine in correlation with the expression of the polyamine transporter TPO1 [129]. Yeast cells overexpressing this transporter are sensitive to oxidants and are unable to induce canonical stress response proteins, such as HSP70 or HSP104. Effects of the transporter overexpression on metabolite levels are only detected upon application of the oxidant; stress-induced transport of these metabolites thus appears critical for the interaction of the metabolome and the proteome in stress situations [129]. In this respect, polyamine catabolism has attracted attention as a potential contributor to cytotoxicity, with $\mathrm{H}_{2} \mathrm{O}_{2}$ being a product of polyamine oxidation [130]. Studies in human breast cancer cell lines demonstrate the involvement of the polyamine degradative enzyme spermine oxidase SMO (PAOh1) in the production of $\mathrm{H}_{2} \mathrm{O}_{2}$ upon induction by a spermine analogue [131]. Overexpressing SMO leads to sub-lethal DNA damage and this confers sensitivity to radiation $[132,133]$.

\section{Evolution and the Impact of Metabolic Inaccuracy on the Genotype to Phenotype Relationship}

Carbonell et al. [134] implies that promiscuous enzymes are widespread in the phylogenetic tree of life and appear statistically more shared across species (allegedly more ancient) than specific ones, consistent with the hypothesis of an evolutionary trend toward the specialization of enzymes [135,136]. The different levels of specificity of current metabolic enzymes would have evolved by segmental duplication of previously promiscuous protein-coding genes. Enzymes would diverge at different rates depending on trade-offs and selective pressures over their particular function and the environmental context [106]. Interestingly, the content in promiscuous and unspecific enzymes differs between organisms and correlates with their ecology; taxonomic groups facing stronger environmental changes possess more promiscuous enzymes [134]. Recently, evidence has 
been provided that a fraction of latent enzymatic side activities maps to functional products of the existing metabolic network, and confers advantage under novel growth environments [137].

Also metabolite repair - potentially in the form of much more rudimentary mechanisms - might date back to the very early stages of evolution and could already have been important for the development of metabolism during the origin of life [138], as first metabolic networks likely required mechanisms to achieve a minimally robust functioning [139]. During subsequent evolution, non-enzymatic reactions and enzyme promiscuity did contribute to the increase in the phenotypic variability of a cell. Persisting in modern cells, metabolic ambiguity is to be added to the sources of variation at the transcriptional and translational levels, required to understand the genotype-to-phenotype relationship (Figure 4): During the flow of information from gene over protein to metabolite, errors and variability are propagated, increasing the spectrum of phenotypes to be reached from the same genotype. Whereas most of these variants may involve a fitness cost for a well-adapted organism, flexibility facilitates adaptation when conditions change (Figure 4).

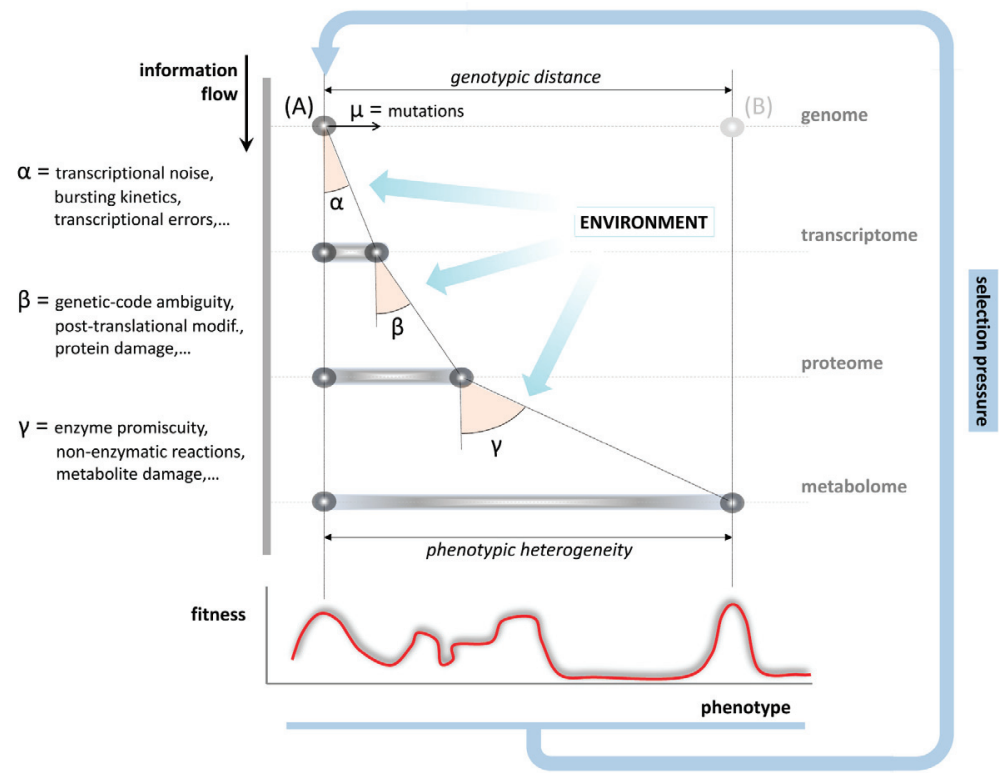

Figure 4. Impact of metabolic inaccuracy on the genotype-to-phenotype relationship. Biological processes, including transcription, translation and metabolism are not totally accurate. During the gene-protein-metabolite information flow, errors generated at each operational layer that are not compensated (depicted as angles $\alpha, \beta, \gamma$ ) propagate, overall shaping the phenotypic space to be achieved from the same genotype (variation in the $\mathrm{x}$ axis). Phenotypic variability arising due to error propagation can be enhanced by environmental stress conditions and enables a wide space of positions in the fitness landscape (red curve), all of which contribute to define the selection pressure over the given genotype (A). Promiscuity, noise, and unspecificity may thus facilitate the physiological adaptation to changing conditions, which would otherwise require the evolution to a different genotype (B). 


\section{Conclusions}

With the advent of systems biology and analytical techniques that allow analyzing multiple cellular metabolites in parallel, it has become clear that metabolism is constituted by a highly dynamic network. In this short review, we discuss examples that reveal the impact of stress conditions on metabolites, metabolic reactions and metabolic flux. Non-enzymatic reactions, chemical reactivity between metabolites, and enzyme promiscuity are inevitable consequence of the chemical properties of essential metabolites, and mechanistic and structural constraints of the participating enzymes. In stress conditions, these factors play synergetic roles in the propagation of errors and damage within metabolism, but also form the basis to reconfigure metabolism so that cells can adapt to changing or stress-inducing environments [90,140].

Metabolism in stress situations appears to be mainly affected by: (i) direct chemical modification of metabolites; or (ii) damage on enzymes that change their substrate specificity and/or catalytic activity. Temporary blockage of metabolic pathways translates as changes in the metabolic flux distribution. Although stress situations appear to be deleterious in the first instance, they have been exploited by evolution to extend the metabolic network, and to develop dynamic response mechanism to cope with changes in the environment. This includes either the use of promiscuous metabolites in signaling, so that repair and damage responses are initiated and function as stress sensors [10,27,141], or direct defense systems that involve stress-induced redistribution of the metabolic flux [70,119,120]. Finally, enzyme conditional promiscuity acts as a reservoir of new functions that can evolve into tunable mechanisms [142], with some of the non-canonical metabolites turning out to be useful [70,106,143-145]. Metabolism during stress conditions thus changes in activity, structure and complexity; and contributes to evolution, phenotype, and survival in stress situations.

\section{Acknowledgments}

We thank our lab-members for critical discussion. Work in the Ralser lab is funded from the Wellcome Trust (RG 093735/Z/10/Z), the ERC (Starting grant 260809). Markus A. Keller is supported by the Austrian Science Funds by an Erwin Schrödinger postdoctoral fellowship (FWF, J 3341). Markus Ralser is a Wellcome Trust Research Career Development and Wellcome-Beit Prize fellow.

\section{Author Contributions}

Gabriel Piedrafita, Markus A Keller and Markus Ralser have written this Review article.

\section{Conflicts of Interest}

The authors declare no conflict of interest.

\section{References}

1. Van Houten, B. Nucleotide excision repair in Escherichia coli. Microbiol. Rev. 1990, 54, 18-51.

2. Mary, J.; Vougier, S.; Picot, C.R.; Perichon, M.; Petropoulos, I.; Friguet, B. Enzymatic reactions involved in the repair of oxidized proteins. Exp. Gerontol. 2004, 39, 1117-1123. 
3. Perrone, G.G.; Tan, S.-X.; Dawes, I.W. Reactive oxygen species and yeast apoptosis. Biochim. Biophys. Acta 2008, 1783, 1354-1368.

4. Morano, K.A.; Grant, C.M.; Moye-Rowley, W.S. The response to heat shock and oxidative stress in Saccharomyces cerevisiae. Genetics 2012, 190, 1157-1195.

5. Harman, D. Aging: A theory based on free radical and radiation chemistry. J. Gerontol. 1956, 11, 298-300.

6. Sies, H. Biochemistry of oxidative stress. Angew. Chem. Int. Ed. Engl. 1986, 25, 1058-1071.

7. Liu, J.; Litt, L.; Segal, M.R.; Kelly, M.J.S.; Pelton, J.G.; Kim, M. Metabolomics of oxidative stress in recent studies of endogenous and exogenously administered intermediate metabolites. Int. J. Mol. Sci. 2011, 12, 6469-6501.

8. Noctor, G.; Lelarge-Trouverie, C.; Mhamdi, A. The metabolomics of oxidative stress. Phytochemistry 2014, 112, 33-53.

9. Ott, M.; Gogvadze, V.; Orrenius, S.; Zhivotovsky, B. Mitochondria, oxidative stress and cell death. Apoptosis 2007, 12, 913-922.

10. D'Autréaux, B.; Toledano, M.B. ROS as signalling molecules: Mechanisms that generate specificity in ROS homeostasis. Nat. Rev. Mol. Cell Biol. 2007, 8, 813-824.

11. Sies, H. Oxidative stress: A concept in redox biology and medicine. Redox Biol. 2015, 4C, $180-183$.

12. Valko, M.; Morris, H.; Cronin, M.T.D. Metals, toxicity and oxidative stress. Curr. Med. Chem. 2005, 12, 1161-1208.

13. Du, J.; Gebicki, J.M. Proteins are major initial cell targets of hydroxyl free radicals. Int. J. Biochem. Cell Biol. 2004, 36, 2334-2343.

14. Liu, C.-C.; Gebicki, J.M. Intracellular GSH and ascorbate inhibit radical-induced protein chain peroxidation in HL-60 cells. Free Radic. Biol. Med. 2012, 52, 420-426.

15. Davies, K.J. Oxidative stress, antioxidant defenses, and damage removal, repair, and replacement systems. IUBMB Life 2000, 50, 279-289.

16. Halliwell, B.; Gutteridge, J. Free Radicals in Biology and Medicine; 4th ed.; Oxford University Press: Oxford, OX, USA, 2007.

17. Breen, A.P.; Murphy, J.A. Reactions of oxyl radicals with DNA. Free Radic. Biol. Med. 1995, 18, 1033-1077.

18. Gilbert, B.C.; King, D.M.; Thomas, C.B. Radical reactions of carbohydrates. Part 2. An electron spin resonance study of the oxidation of D-glucose and related compounds with the hydroxyl radical. J. Chem. Soc. Perkin Trans. 1981, 2, 1186-1199.

19. Manini, P.; la Pietra, P.; Panzella, L.; Napolitano, A.; d'Ischia, M. Glyoxal formation by Fenton-induced degradation of carbohydrates and related compounds. Carbohydr. Res. 2006, 341, 1828-1833.

20. Arteel, G.E.; Briviba, K.; Sies, H. Protection against peroxynitrite. FEBS Lett. 1999, 445, 226-230.

21. Sawa, T.; Akaike, T.; Maeda, H. Tyrosine nitration by peroxynitrite formed from nitric oxide and superoxide generated by xanthine oxidase. J. Biol. Chem. 2000, 275, 32467-32474. 
22. Klotz, L.-O.; Schroeder, P.; Sies, H. Peroxynitrite signaling: Receptor tyrosine kinases and activation of stress-responsive pathways. Free Radic. Biol. Med. 2002, 33, 737-743.

23. Klotz, L.-O.; Sies, H. Defenses against peroxynitrite: Selenocompounds and flavonoids. Toxicol. Lett. 2003, 140-141, 125-132.

24. Williams, T.I.; Lynn, B.C.; Markesbery, W.R.; Lovell, M.A. Increased levels of 4-hydroxynonenal and acrolein, neurotoxic markers of lipid peroxidation, in the brain in Mild Cognitive Impairment and early Alzheimer's disease. Neurobiol. Aging 2006, 27, 1094-1099.

25. Pamplona, R. Membrane phospholipids, lipoxidative damage and molecular integrity: A causal role in aging and longevity. Biochim. Biophys. Acta 2008, 1777, 1249-1262.

26. Thanan, R.; Oikawa, S.; Hiraku, Y.; Ohnishi, S.; Ma, N.; Pinlaor, S.; Yongvanit, P.; Kawanishi, S.; Murata, M. Oxidative stress and its significant roles in neurodegenerative diseases and cancer. Int. J. Mol. Sci. 2015, 16, 193-217.

27. Cremers, C.M.; Jakob, U. Oxidant sensing by reversible disulfide bond formation. J. Biol. Chem. 2013, 288, 26489-26496.

28. Moskovitz, J. Methionine sulfoxide reductases: Ubiquitous enzymes involved in antioxidant defense, protein regulation, and prevention of aging-associated diseases. Biochim. Biophys. Acta 2005, 1703, 213-219.

29. Stadtman, E.R.; van Remmen, H.; Richardson, A.; Wehr, N.B.; Levine, R.L. Methionine oxidation and aging. Biochim. Biophys. Acta 2005, 1703, 135-140.

30. O’Donnell-Tormey, J.; Nathan, C.F.; Lanks, K.; DeBoer, C.J.; de la Harpe, J. Secretion of pyruvate. An antioxidant defense of mammalian cells. J. Exp. Med. 1987, 165, 500-514.

31. Troxell, B.; Zhang, J.-J.; Bourret, T.J.; Zeng, M.Y.; Blum, J.; Gherardini, F.; Hassan, H.M.; Yang, X.F. Pyruvate protects pathogenic spirochetes from $\mathrm{H}_{2} \mathrm{O}_{2}$ killing. PLoS ONE 2014, 9, e84625.

32. Rose, R.C.; Bode, A.M. Biology of free radical scavengers: An evaluation of ascorbate. FASEB J. 1993, 7, 1135-1142.

33. Levine, R.L.; Mosoni, L.; Berlett, B.S.; Stadtman, E.R. Methionine residues as endogenous antioxidants in proteins. Proc. Natl. Acad. Sci. USA 1996, 93, 15036-15040.

34. Luo, S.; Levine, R.L. Methionine in proteins defends against oxidative stress. FASEB J. 2009, 23, 464-472.

35. Rzem, R.; Vincent, M.-F.; van Schaftingen, E.; Veiga-da-Cunha, M. L-2-hydroxyglutaric aciduria, a defect of metabolite repair. J. Inherit. Metab. Dis. 2007, 30, 681-689.

36. Linster, C.L.; van Schaftingen, E.; Hanson, A.D. Metabolite damage and its repair or pre-emption. Nat. Chem. Biol. 2013, 9, 72-80.

37. Galperin, M.Y.; Moroz, O.V.; Wilson, K.S.; Murzin, A.G. House cleaning, a part of good housekeeping. Mol. Microbiol. 2006, 59, 5-19.

38. Van Schaftingen, E.; Rzem, R.; Marbaix, A.; Collard, F.; Veiga-da-Cunha, M.; Linster, C.L. Metabolite proofreading, a neglected aspect of intermediary metabolism. J. Inherit. Metab. Dis. 2013, 36, 427-434.

39. Ursini, F.; Maiorino, M.; Brigelius-Flohé, R.; Aumann, K.D.; Roveri, A.; Schomburg, D.; Flohé, L. Diversity of glutathione peroxidases. Methods Enzymol. 1995, 252, 38-53. 
40. Labunskyy, V.M.; Hatfield, D.L.; Gladyshev, V.N. Selenoproteins: Molecular pathways and physiological roles. Physiol. Rev. 2014, 94, 739-777.

41. Galano, A.; Alvarez-Idaboy, J.R. Glutathione: Mechanism and kinetics of its non-enzymatic defense action against free radicals. RSC Adv. 2011, 1, 1763-1771.

42. Haenen, G.R.M.M.; Bast, A. Glutathione revisited: A better scavenger than previously thought. Front. Pharmacol. 2014, doi:10.3389/fphar.2014.00260.

43. Stadtman, E.R. Oxidation of free amino acids and amino acid residues in proteins by radiolysis and by metal-catalyzed reactions. Annu. Rev. Biochem. 1993, 62, 797-821.

44. Lee, B.C.; Gladyshev, V.N. The biological significance of methionine sulfoxide stereochemistry. Free Radic. Biol. Med. 2011, 50, 221-227.

45. Boschi-Muller, S.; Olry, A.; Antoine, M.; Branlant, G. The enzymology and biochemistry of methionine sulfoxide reductases. Biochim. Biophys. Acta 2005, 1703, 231-238.

46. Lin, Z.; Johnson, L.C.; Weissbach, H.; Brot, N.; Lively, M.O.; Lowther, W.T. Free methionine- $(R)$-sulfoxide reductase from Escherichia coli reveals a new GAF domain function. Proc. Natl. Acad. Sci. USA 2007, 104, 9597-9602.

47. Le, D.T.; Lee, B.C.; Marino, S.M.; Zhang, Y.; Fomenko, D.E.; Kaya, A.; Hacioglu, E.; Kwak, G.-H.; Koc, A.; Kim, H.-Y.; et al. Functional analysis of free methionine- $R$-sulfoxide reductase from Saccharomyces cerevisiae. J. Biol. Chem. 2009, 284, 4354-4364.

48. Le, D.T.; Tarrago, L.; Watanabe, Y.; Kaya, A.; Lee, B.C.; Tran, U.; Nishiyama, R.; Fomenko, D.E.; Gladyshev, V.N.; Tran, L.-S.P. Diversity of plant methionine sulfoxide reductases B and evolution of a form specific for free methionine sulfoxide. PLOS ONE 2013, 8, e65637.

49. Heurlier, K.; Vendeville, A.; Halliday, N.; Green, A.; Winzer, K.; Tang, C.M.; Hardie, K.R. Growth deficiencies of Neisseria meningitidis $p f s$ and luxS mutants are not due to inactivation of quorum sensing. J. Bacteriol. 2009, 191, 1293-1302.

50. Lyu, L.-D.; Tang, B.-K.; Fan, X.-Y.; Ma, H.; Zhao, G.-P. Mycobacterial MazG safeguards genetic stability via housecleaning of 5-OH-dCTP. PLoS Pathog. 2013, 9, e1003814.

51. Van Schaftingen, E.; Rzem, R.; Veiga-da-Cunha, M. L-2-Hydroxyglutaric aciduria, a disorder of metabolite repair. J. Inherit. Metab. Dis. 2009, 32, 135-142.

52. Nakabeppu, Y. Cellular levels of 8-oxoguanine in either DNA or the nucleotide pool play pivotal roles in carcinogenesis and survival of cancer cells. Int. J. Mol. Sci. 2014, 15, 12543-12557.

53. Marinaki, A.M.; Duley, J.A.; Arenas, M.; Ansari, A.; Sumi, S.; Lewis, C.M.; Shobowale-Bakre, M.; Fairbanks, L.D.; Sanderson, J. Mutation in the ITPA gene predicts intolerance to azathioprine. Nucleosides. Nucleotides Nucleic Acids 2004, 23, 1393-1397.

54. Sakamoto, T.; Maebayashi, K.; Nakagawa, Y.; Imai, H. Deletion of the four phospholipid hydroperoxide glutathione peroxidase genes accelerates aging in Caenorhabditis elegans. Genes Cells 2014, 19, 778-792.

55. Inoué, S.; Honda, K.; Komoda, Y. Sleep as neuronal detoxification and restitution. Behav. Brain Res. 1995, 69, 91-96.

56. Delaney, S.; Jarem, D.A.; Volle, C.B.; Yennie, C.J. Chemical and biological consequences of oxidatively damaged guanine in DNA. Free Radic. Res. 2012, 46, 420-441. 
57. Kamiya, H. Mutagenic potentials of damaged nucleic acids produced by reactive oxygen/nitrogen species: Approaches using synthetic oligonucleotides and nucleotides: Survey and summary. Nucleic Acids Res. 2003, 31, 517-531.

58. Bessman, M.J.; Frick, D.N.; O’Handley, S.F. The MutT proteins or "Nudix" hydrolases, a family of versatile, widely distributed, "housecleaning" enzymes. J. Biol. Chem. 1996, 271, 25059-25062.

59. Sekiguchi, M.; Tsuzuki, T. Oxidative nucleotide damage: Consequences and prevention. Oncogene 2002, 21, 8895-8904.

60. McLennan, A.G. The Nudix hydrolase superfamily. Cell. Mol. Life Sci. 2006, 63, 123-143.

61. Persson, R.; Cedergren-Zeppezauer, E.S.; Wilson, K.S. Homotrimeric dUTPases; structural solutions for specific recognition and hydrolysis of dUTP. Curr. Protein Pept. Sci. 2001, 2, 287-300.

62. Hemsworth, G.R.; González-Pacanowska, D.; Wilson, K.S. On the catalytic mechanism of dimeric dUTPases. Biochem. J. 2013, 456, 81-88.

63. Marbaix, A.Y.; Noël, G.; Detroux, A.M.; Vertommen, D.; van Schaftingen, E.; Linster, C.L. Extremely conserved ATP- or ADP-dependent enzymatic system for nicotinamide nucleotide repair. J. Biol. Chem. 2011, 286, 41246-41252.

64. Oppenheimer, N.J.; Kaplan, N.O. Glyceraldehyde-3-phosphate dehydrogenase catalyzed hydration of the 5-6 double bond of reduced $\beta$-nicotinamide adenine dinucleotide ( $\beta N A D H)$. Formation of $\beta$-6-hydroxy-1,4,5,6-tetrahydronicotinamide adenine dinucleotide. Biochemistry 1974, 13, 4685-4694.

65. Yoshida, A.; Dave, V. Inhibition of NADP-dependent dehydrogenases by modified products of NADPH. Arch. Biochem. Biophys. 1975, 169, 298-303.

66. Niehaus, T.D.; Richardson, L.G.L.; Gidda, S.K.; ElBadawi-Sidhu, M.; Meissen, J.K.; Mullen, R.T.; Fiehn, O.; Hanson, A.D. Plants utilize a highly conserved system for repair of NADH and NADPH hydrates. Plant Physiol. 2014, 165, 52-61.

67. Colinas, M.; Shaw, H.V.; Loubéry, S.; Kaufmann, M.; Moulin, M.; Fitzpatrick, T.B. A pathway for repair of NAD(P)H in plants. J. Biol. Chem. 2014, 289, 14692-14706.

68. Marbaix, A.Y.; Tyteca, D.; Niehaus, T.D.; Hanson, A.D.; Linster, C.L.; van Schaftingen, E. Occurrence and subcellular distribution of the NADPHX repair system in mammals. Biochem. J. 2014, 460, 49-58.

69. Keller, M.A.; Piedrafita, G.; Ralser, M. The widespread role of non-enzymatic reactions in cellular metabolism. Curr. Opin. Biotechnol. 2015, 34, 153-161.

70. D’Ari, R.; Casadesús, J. Underground metabolism. Bioessays 1998, 20, 181-186.

71. Keller, M.A.; Turchyn, A.V.; Ralser, M. Non-enzymatic glycolysis and pentose phosphate pathway-like reactions in a plausible Archean ocean. Mol. Syst. Biol. 2014, doi:10.1002/msb.20145228.

72. Weinert, B.T.; Iesmantavicius, V.; Moustafa, T.; Schölz, C.; Wagner, S.A.; Magnes, C.; Zechner, R.; Choudhary, C. Acetylation dynamics and stoichiometry in Saccharomyces cerevisiae. Mol. Syst. Biol. 2014, doi:10.1002/msb.134766.

73. Wagner, G.R.; Payne, R.M. Widespread and enzyme-independent Ne-acetylation and $\mathrm{N} \varepsilon$-succinylation of proteins in the chemical conditions of the mitochondrial matrix. J. Biol. Chem. 2013, 288, 29036-29045. 
74. Wagner, G.R.; Hirschey, M.D. Nonenzymatic protein acylation as a carbon stress regulated by sirtuin deacylases. Mol. Cell 2014, 54, 5-16.

75. Zhang, Y.; Xiao, M.; Horiyama, T.; Zhang, Y.; Li, X.; Nishino, K.; Yan, A. The multidrug efflux pump MdtEF protects against nitrosative damage during the anaerobic respiration in Escherichia coli. J. Biol. Chem. 2011, 286, 26576-26584.

76. Zhou, K.; Zou, R.; Stephanopoulos, G.; Too, H.-P. Metabolite profiling identified methylerythritol cyclodiphosphate efflux as a limiting step in microbial isoprenoid production. PLOS ONE 2012, 7, e47513.

77. Fung, D.K.C.; Lau, W.Y.; Chan, W.T.; Yan, A. Copper efflux is induced during anaerobic amino acid limitation in Escherichia coli to protect iron-sulfur cluster enzymes and biogenesis. J. Bacteriol. 2013, 195, 4556-4568.

78. Piddock, L.J.V. Clinically relevant chromosomally encoded multidrug resistance efflux pumps in bacteria. Clin. Microbiol. Rev. 2006, 19, 382-402.

79. Nikaido, H.; Zgurskaya, H.I. AcrAB and related multidrug efflux pumps of Escherichia coli. J. Mol. Microbiol. Biotechnol. 2001, 3, 215-218.

80. Li, X.-Z.; Nikaido, H. Efflux-mediated drug resistance in bacteria: An update. Drugs 2009, 69, 1555-1623.

81. Bleuel, C.; Grosse, C.; Taudte, N.; Scherer, J.; Wesenberg, D.; Krauss, G.J.; Nies, D.H.; Grass, $\mathrm{G}$. TolC is involved in enterobactin efflux across the outer membrane of Escherichia coli. J. Bacteriol. 2005, 187, 6701-6707.

82. Du, D.; Wang, Z.; James, N.R.; Voss, J.E.; Klimont, E.; Ohene-Agyei, T.; Venter, H.; Chiu, W.; Luisi, B.F. Structure of the AcrAB-TolC multidrug efflux pump. Nature 2014, 509, $512-515$.

83. Takatsuka, Y.; Chen, C.; Nikaido, H. Mechanism of recognition of compounds of diverse structures by the multidrug efflux pump AcrB of Escherichia coli. Proc. Natl. Acad. Sci. USA 2010, 107, 6559-6565.

84. Poole, K. Bacterial multidrug efflux pumps serve other functions. Microbe 2008, 3, 179-185.

85. Deininger, K.N.W.; Horikawa, A.; Kitko, R.D.; Tatsumi, R.; Rosner, J.L.; Wachi, M.; Slonczewski, J.L. A requirement of TolC and MDR efflux pumps for acid adaptation and GadAB induction in Escherichia coli. PLoS ONE 2011, 6, e18960.

86. Tatsumi, R.; Wachi, M. TolC-dependent exclusion of porphyrins in Escherichia coli. J. Bacteriol. 2008, 190, 6228-6233.

87. Rosner, J.L.; Martin, R.G. Reduction of cellular stress by TolC-dependent efflux pumps in Escherichia coli indicated by BaeSR and CpxARP activation of spy in efflux mutants. J. Bacteriol. 2013, 195, 1042-1050.

88. Nagy, P.; Lechte, T.P.; Das, A.B.; Winterbourn, C.C. Conjugation of glutathione to oxidized tyrosine residues in peptides and proteins. J. Biol. Chem. 2012, 287, 26068-26076.

89. Bar-Even, A.; Noor, E.; Savir, Y.; Liebermeister, W.; Davidi, D.; Tawfik, D.S.; Milo, R. The moderately efficient enzyme: Evolutionary and physicochemical trends shaping enzyme parameters. Biochemistry 2011, 50, 4402-4410.

90. Khersonsky, O.; Tawfik, D.S. Enzyme promiscuity: A mechanistic and evolutionary perspective. Annu. Rev. Biochem. 2010, 79, 471-505. 
91. Hult, K.; Berglund, P. Enzyme promiscuity: Mechanism and applications. Trends Biotechnol. 2007, 25, 231-238.

92. Copley, S.D. Enzymes with extra talents: Moonlighting functions and catalytic promiscuity. Curr. Opin. Chem. Biol. 2003, 7, 265-272.

93. Bensaad, K.; Tsuruta, A.; Selak, M.A.; Vidal, M.N.C.; Nakano, K.; Bartrons, R.; Gottlieb, E.; Vousden, K.H. TIGAR, a p53-inducible regulator of glycolysis and apoptosis. Cell 2006, 126, $107-120$.

94. Gerin, I.; Noël, G.; Bolsée, J.; Haumont, O.; van Schaftingen, E.; Bommer, G.T. Identification of TP53-induced glycolysis and apoptosis regulator (TIGAR) as the phosphoglycolate-independent 2,3-bisphosphoglycerate phosphatase. Biochem. J. 2014, 458, 439-448.

95. Yebra, M.J.; Bhagwat, A.S. A cytosine methyltransferase converts 5-methylcytosine in DNA to thymine. Biochemistry 1995, 34, 14752-14757.

96. Engqvist, M.K.M.; Eßer, C.; Maier, A.; Lercher, M.J.; Maurino, V.G. Mitochondrial 2-hydroxyglutarate metabolism. Mitochondrion 2014, 19, 275-281.

97. Ward, P.S.; Patel, J.; Wise, D.R.; Abdel-Wahab, O.; Bennett, B.D.; Coller, H.A.; Cross, J.R.; Fantin, V.R.; Hedvat, C.V.; Perl, A.E.; et al. The common feature of leukemia-associated IDH1 and IDH2 mutations is a neomorphic enzyme activity converting alpha-ketoglutarate to 2-hydroxyglutarate. Cancer Cell 2010, 17, 225-234.

98. Struys, E.A.; Salomons, G.S.; Achouri, Y.; van Schaftingen, E.; Grosso, S.; Craigen, W.J.; Verhoeven, N.M.; Jakobs, C. Mutations in the D-2-hydroxyglutarate dehydrogenase gene cause D-2-hydroxyglutaric aciduria. Am. J. Hum. Genet. 2005, 76, 358-360.

99. Kranendijk, M.; Struys, E.A.; van Schaftingen, E.; Gibson, K.M.; Kanhai, W.A.; van der Knaap, M.S.; Amiel, J.; Buist, N.R.; Das, A.M.; de Klerk, J.B.; et al. IDH2 mutations in patients with D-2-hydroxyglutaric aciduria. Science 2010, doi:10.1126/science.1192632.

100. Sobota, J.M.; Imlay, J.A. Iron enzyme ribulose-5-phosphate 3-epimerase in Escherichia coli is rapidly damaged by hydrogen peroxide but can be protected by manganese. Proc. Natl. Acad. Sci. USA 2011, 108, 5402-5407.

101. Anjem, A.; Imlay, J.A. Mononuclear iron enzymes are primary targets of hydrogen peroxide stress. J. Biol. Chem. 2012, 287, 15544-15556.

102. Cotruvo, J.A.; Stubbe, J. Metallation and mismetallation of iron and manganese proteins in vitro and in vivo: The class I ribonucleotide reductases as a case study. Metallomics 2012, 4, 1020-1036.

103. Frawley, E.R.; Fang, F.C. The ins and outs of bacterial iron metabolism. Mol. Microbiol. 2014, 93, 609-616.

104. Barnese, K.; Gralla, E.B.; Valentine, J.S.; Cabelli, D.E. Biologically relevant mechanism for catalytic superoxide removal by simple manganese compounds. Proc. Natl. Acad. Sci. USA 2012, 109, 6892-6897.

105. Sanchez, R.J.; Srinivasan, C.; Munroe, W.H.; Wallace, M.A.; Martins, J.; Kao, T.Y.; Le, K.; Gralla, E.B.; Valentine, J.S. Exogenous manganous ion at millimolar levels rescues all known dioxygen-sensitive phenotypes of yeast lacking CuZnSOD. J. Biol. Inorg. Chem. 2005, 10, 913-923. 
106. Copley, S.D. Toward a systems biology perspective on enzyme evolution. J. Biol. Chem. 2012, 287, 3-10.

107. Söll, D. Enter a new amino acid. Nature 1988, 331, 662-663.

108. Prat, L.; Heinemann, I.U.; Aerni, H.R.; Rinehart, J.; O’Donoghue, P.; Söll, D. Carbon source-dependent expansion of the genetic code in bacteria. Proc. Natl. Acad. Sci. USA 2012, 109, 21070-21075.

109. Li, L.; Boniecki, M.T.; Jaffe, J.D.; Imai, B.S.; Yau, P.M.; Luthey-Schulten, Z.A.; Martinis, S.A. Naturally occurring aminoacyl-tRNA synthetases editing-domain mutations that cause mistranslation in Mycoplasma parasites. Proc. Natl. Acad. Sci. USA 2011, 108, 9378-9383.

110. Netzer, N.; Goodenbour, J.M.; David, A.; Dittmar, K.A.; Jones, R.B.; Schneider, J.R.; Boone, D.; Eves, E.M.; Rosner, M.R.; Gibbs, J.S.; et al. Innate immune and chemically triggered oxidative stress modifies translational fidelity. Nature 2009, 462, 522-526.

111. Lee, J.Y.; Kim, D.G.; Kim, B.-G.; Yang, W.S.; Hong, J.; Kang, T.; Oh, Y.S.; Kim, K.R.; Han, B.W.; Hwang, B.J.; et al. Promiscuous methionyl-tRNA synthetase mediates adaptive mistranslation to protect cells against oxidative stress. J. Cell Sci. 2014, 127, 4234-4245.

112. Bender, A.; Hajieva, P.; Moosmann, B. Adaptive antioxidant methionine accumulation in respiratory chain complexes explains the use of a deviant genetic code in mitochondria. Proc. Natl. Acad. Sci. USA 2008, 105, 16496-16501.

113. Miranda, I.; Silva-Dias, A.; Rocha, R.; Teixeira-Santos, R.; Coelho, C.; Gonçalves, T.; Santos, M.A.S.; Pina-Vaz, C.; Solis, N.V.; Filler, S.G.; et al. Candida albicans CUG mistranslation is a mechanism to create cell surface variation. MBio 2013, 4, doi:10.1128/mBio.00285-13.

114. Lal, P.B.; Schneider, B.L.; Vu, K.; Reitzer, L. The redundant aminotransferases in lysine and arginine synthesis and the extent of aminotransferase redundancy in Escherichia coli. Mol. Microbiol. 2014, 94, 843-856.

115. Miranda, I.; Rocha, R.; Santos, M.C.; Mateus, D.D.; Moura, G.R.; Carreto, L.; Santos, M.A.S. A genetic code alteration is a phenotype diversity generator in the human pathogen Candida albicans. PLoS ONE 2007, 2, e996.

116. Rothstein, M. Aging and the alteration of enzymes: A review. Mech. Ageing Dev. 1975, 4, 325-338.

117. Van Montfort, R.L.M.; Congreve, M.; Tisi, D.; Carr, R.; Jhoti, H. Oxidation state of the active-site cysteine in protein tyrosine phosphatase 1B. Nature 2003, 423, 773-777.

118. Lee, J.-G.; Baek, K.; Soetandyo, N.; Ye, Y. Reversible inactivation of deubiquitinases by reactive oxygen species in vitro and in cells. Nat. Commun. 2013, doi:10.1038/ncomms2532.

119. Ralser, M.; Wamelink, M.M.; Kowald, A.; Gerisch, B.; Heeren, G.; Struys, E.A.; Klipp, E.; Jakobs, C.; Breitenbach, M.; Lehrach, H.; et al. Dynamic rerouting of the carbohydrate flux is key to counteracting oxidative stress. J. Biol. 2007, doi:10.1186/jbiol61.

120. Ralser, M.; Wamelink, M.M.C.; Latkolik, S.; Jansen, E.E.W.; Lehrach, H.; Jakobs, C. Metabolic reconfiguration precedes transcriptional regulation in the antioxidant response. Nat. Biotechnol. 2009, 27, 604-605. 
121. Stincone, A.; Prigione, A.; Cramer, T.; Wamelink, M.M.C.; Campbell, K.; Cheung, E.; Olin-Sandoval, V.; Gruening, N.-M.; Krueger, A.; Tauqeer Alam, M.; et al. The return of metabolism: Biochemistry and physiology of the pentose phosphate pathway. Biol. Rev. Camb. Philos. Soc. 2015, 90, 927-963.

122. Peralta, D.; Bronowska, A.K.; Morgan, B.; Dóka, É.; van Laer, K.; Nagy, P.; Gräter, F.; Dick, T.P. A proton relay enhances $\mathrm{H}_{2} \mathrm{O}_{2}$ sensitivity of GAPDH to facilitate metabolic adaptation. Nat. Chem. Biol. 2015, 11, 156-163.

123. Ralser, M.; Heeren, G.; Breitenbach, M.; Lehrach, H.; Krobitsch, S. Triose phosphate isomerase deficiency is caused by altered dimerization-Not catalytic inactivity-Of the mutant enzymes. PLoS ONE 2006, 1, e30.

124. Grüning, N.-M.; Du, D.; Keller, M.A.; Luisi, B.F.; Ralser, M. Inhibition of triosephosphate isomerase by phosphoenolpyruvate in the feedback-regulation of glycolysis. Open Biol. 2014, doi:10.1098/rsob.130232.

125. Grüning, N.-M.; Rinnerthaler, M.; Bluemlein, K.; Mülleder, M.; Wamelink, M.M.C.; Lehrach, H.; Jakobs, C.; Breitenbach, M.; Ralser, M. Pyruvate kinase triggers a metabolic feedback loop that controls redox metabolism in respiring cells. Cell Metab. 2011, 14, 415-427.

126. Kuehne, A.; Emmert, H.; Soehle, J.; Winnefeld, M.; Fischer, F.; Wenck, H.; Gallinat, S.; Terstegen, L.; Lucius, R.; Hildebrand, J.; et al. Acute activation of oxidative pentose phosphate pathway as first-line response to oxidative stress in human skin cells. Mol. Cell 2015, doi:10.1016/j.molcel.2015.06.017.

127. Fan, J.; Ye, J.; Kamphorst, J.J.; Shlomi, T.; Thompson, C.B.; Rabinowitz, J.D. Quantitative flux analysis reveals folate-dependent NADPH production. Nature 2014, 510, 298-302.

128. Miller-Fleming, L.; Olin-Sandoval, V.; Campbell, K.; Ralser M. Remaining mysteries of molecular biology: The role of polyamines in the cell. J. Mol. Biol. 2015, doi:10.1016/j.jmb.2015.06.020.

129. Krüger, A.; Vowinckel, J.; Mülleder, M.; Grote, P.; Capuano, F.; Bluemlein, K.; Ralser, M. Tpo1-mediated spermine and spermidine export controls cell cycle delay and times antioxidant protein expression during the oxidative stress response. EMBO Rep. 2013, 14, 1113-1119.

130. Casero, R.A.; Pegg, A.E. Polyamine catabolism and disease. Biochem. J. 2009, 421, 323-338.

131. Pledgie, A.; Huang, Y.; Hacker, A.; Zhang, Z.; Woster, P.M.; Davidson, N.E.; Casero, R.A. Spermine oxidase SMO(PAOh1), not N1-acetylpolyamine oxidase PAO, is the primary source of cytotoxic $\mathrm{H}_{2} \mathrm{O}_{2}$ in polyamine analogue-treated human breast cancer cell lines. J. Biol. Chem. 2005, 280, 39843-39851.

132. Bianchi, M.; Bellini, A.; Cervelli, M.; Degan, P.; Marcocci, L.; Martini, F.; Scatteia, M.; Mariottini, P.; Amendola, R. Chronic sub-lethal oxidative stress by spermine oxidase overactivity induces continuous DNA repair and hypersensitivity to radiation exposure. Biochim. Biophys. Acta 2007, 1773, 774-783.

133. Amendola, R.; Cervelli, M.; Fratini, E.; Sallustio, D.E.; Tempera, G.; Ueshima, T.; Mariottini, P.; Agostinelli, E. Reactive oxygen species spermine metabolites generated from amine oxidases and radiation represent a therapeutic gain in cancer treatments. Int. J. Oncol. 2013, 43, 813-820. 
134. Carbonell, P.; Lecointre, G.; Faulon, J.-L. Origins of specificity and promiscuity in metabolic networks. J. Biol. Chem. 2011, 286, 43994-44004.

135. Jensen, R.A. Enzyme recruitment in evolution of new function. Annu. Rev. Microbiol. 1976, 30, 409-425.

136. Voordeckers, K.; Brown, C.A.; Vanneste, K.; van der Zande, E.; Voet, A.; Maere, S.; Verstrepen, K.J. Reconstruction of ancestral metabolic enzymes reveals molecular mechanisms underlying evolutionary innovation through gene duplication. PLoS Biol. 2012, 10, e1001446.

137. Notebaart, R.A.; Szappanos, B.; Kintses, B.; Pal, F.; Gyorkei, A.; Bogos, B.; Lazar, V.; Spohn, R.; Csorg, B.; Wagner, A.; et al. Network-level architecture and the evolutionary potential of underground metabolism. Proc. Natl. Acad. Sci. USA 2014, 111, 11762-11767.

138. Cornish-Bowden, A.; Cardenas, M.L. Self-organization at the origin of life. J. Theor. Biol. 2008, 252, 411-418.

139. Piedrafita, G.; Montero, F.; Morán, F.; Cárdenas, M.L.; Cornish-Bowden, A. A simple self-maintaining metabolic system: Robustness, autocatalysis, bistability. PLoS Comput. Biol. 2010, doi:10.1371/journal.pcbi.1000872.

140. Tawfik, D.S. Messy biology and the origins of evolutionary innovations. Nat. Chem. Biol. 2010, 6, 692-696.

141. Benoit, R.; Auer, M. A direct way of redox sensing. RNA Biol. 2011, 8, 18-23.

142. Andersson, D.I. Evolving promiscuously. Proc. Natl. Acad. Sci. USA 2011, 108, 1199-1200.

143. Miller, B.G.; Raines, R.T. Identifying latent enzyme activities: Substrate ambiguity within modern bacterial sugar kinases. Biochemistry 2004, 43, 6387-6392.

144. Patrick, W.M.; Quandt, E.M.; Swartzlander, D.B.; Matsumura, I. Multicopy suppression underpins metabolic evolvability. Mol. Biol. Evol. 2007, 24, 2716-2722.

145. Patrick, W.M.; Matsumura, I. A study in molecular contingency: Glutamine phosphoribosylpyrophosphate amidotransferase is a promiscuous and evolvable phosphoribosylanthranilate isomerase. J. Mol. Biol. 2008, 377, 323-336. 


\title{
The Role of Reactive Oxygen Species (ROS) in the Formation of Extracellular Traps (ETs) in Humans
}

\author{
Walter Stoiber, Astrid Obermayer, Peter Steinbacher and Wolf-Dietrich Krautgartner
}

\begin{abstract}
Extracellular traps (ETs) are reticulate structures of extracellular DNA associated with antimicrobial molecules. Their formation by phagocytes (mainly by neutrophils: NETs) has been identified as an essential element of vertebrate innate immune defense. However, as ETs are also toxic to host cells and potent triggers of autoimmunity, their role between pathogen defense and human pathogenesis is ambiguous, and they contribute to a variety of acute and chronic inflammatory diseases. Since the discovery of ET formation (ETosis) a decade ago, evidence has accumulated that most reaction cascades leading to ET release involve ROS. An important new facet was added when it became apparent that ETosis might be directly linked to, or be a variant of, the autophagy cell death pathway. The present review analyzes the evidence to date on the interplay between ROS, autophagy and ETosis, and highlights and discusses several further aspects of the ROS-ET relationship that are incompletely understood. These aspects include the role of NADPH oxidase-derived ROS, the molecular requirements of NADPH oxidase-dependent ETosis, the roles of NADPH oxidase subtypes, extracellular ROS and of ROS from sources other than NADPH oxidase, and the present evidence for ROS-independent ETosis. We conclude that ROS interact with ETosis in a multidimensional manner, with influence on whether ETosis shows beneficial or detrimental effects.
\end{abstract}

Reprinted from Biomolecules. Cite as: Stoiber, W.; Obermayer, A.; Steinbacher, P.; Krautgartner, W.-D. The Role of Reactive Oxygen Species (ROS) in the Formation of Extracellular Traps (ETs) in Humans. Biomolecules 2015, 5, 702-723.

\section{Introduction and Background}

Extracellular traps (ETs) are reticulate formations of extracellular DNA associated with antimicrobial molecules (Figure 1A-D). Their formation, mainly by neutrophils and other cells of the immune system (eosinophils, macrophages, mast cells) in a distinctive process of cell death, has been identified as an important evolutionarily conserved mechanism of vertebrate innate immune defense [1-4]. First described in 2004 in humans [5], the ability of cells to release ETs is now known to occur not only in mammals but is also found in immune cells of birds and fish (e.g., [6-8]). ETs constitute complex three-dimensional web-like scaffolds of DNA strands with dimensions down to $2 \mathrm{~nm}$, the size of individual double helices (Figure 1D) [9]. These scaffolds are decorated with histones and other molecules, including elastase (Figure 1B,D), myeloperoxidase (MPO), bactericidal permeability-increasing protein (BPI), cathepsin G and other proteinases that are all antimicrobially effective [5,9-14]. ETs have been shown to aid the entrapment and/or removal of bacterial, fungal, protist and even platyhelminth pathogens (e.g., [15-19]). They are also formed during viral infections, probably exerting a cell protective role [20,21]. However, the protein components of ETs have also been identified as toxic to host cells (e.g., [20,22]) and as potent 
triggers of autoimmunity [23,24]. Thus, since their discovery [5], ETs have been established in an ambiguous role between pathogen defense and host tissue damage [25].

The generation and release of ETs, specifically by neutrophils, has been shown to be induced by a variety of internal and/or pathogen derived molecular signals. These signals include chemokines with CXC motif such as interleukin 8 (IL-8) $[5,26]$, but also tumor necrosis factor alpha (TNF $\alpha$ ), lipopolysaccharides (LPS), formylated peptides such as $N$-formyl-methionyl-leucyl-phenylalanine (fMLP) (Figure 1A and D), placental syncytiotrophoblast microparticles, antineutrophil cytoplasmic antibodies (ANCAs), and pharmacological agents, such as phorbol myristate acetate (PMA) [2,5,23,24,27]. According to recent evidence, antibody (IgA) binding by phagocyte Fc $\alpha$ RI-receptors [28] and binding of neutrophil FcyRIIIb receptors to immune complexes immobilized in the extracellular matrix [29] are also likely to play an inductive role.

Mechanisms of ET formation (ETosis) have been found to vary in relation to the signaling pathways involved and in the morphological execution of the process, enabling more than one mechanism per cell type. This is well documented for PMA and AMLP induced pathways, and for the polymorphisms of ET formation in neutrophils (see below under Sections 2-8). There is also evidence that some types of ETosis leave the cells viable [30,31], a subject dealt with in more detail in Section 7 below.

Despite this heterogeneity, ETosis is mainly a distinctive process of cell death involving full chromatin decondensation and break-up of the cell membrane. This holds, primarily, for ET formation by neutrophils (NETosis), which is perhaps the best-investigated form of ETosis. Morphological research has identified a standard pattern referred to as the NETotic cascade. The steps of this cascade are well defined in the recent literature (e.g., $[2,32,33]$ ) and describe the progressive change from the undisturbed globular cell, via cytoplasmic and nuclear swelling, vacuolization, membrane protrusion, enzyme binding to DNA, histone citrullination and chromatin decondensation, to terminal membrane rupture and NET release (Figure 1B).

Independent of the mechanisms and cell types from which ETs derive, there is mounting evidence that they strongly contribute to severe acute illness and chronic inflammation when formed in excess or are insufficiently cleared (e.g., [25]). This has led to a surge of research activity into the details of these pathogenic effects, specifically, again, with regard to neutrophil generated ETs (NETs), which appear to be the most abundant.
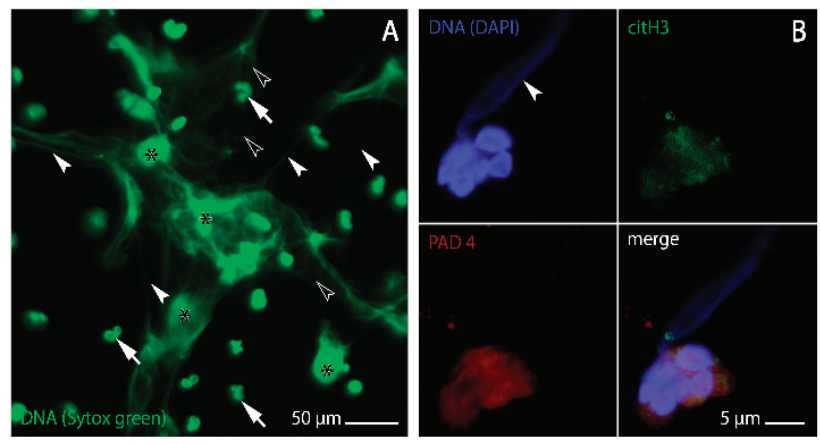

Figure 1. Cont. 

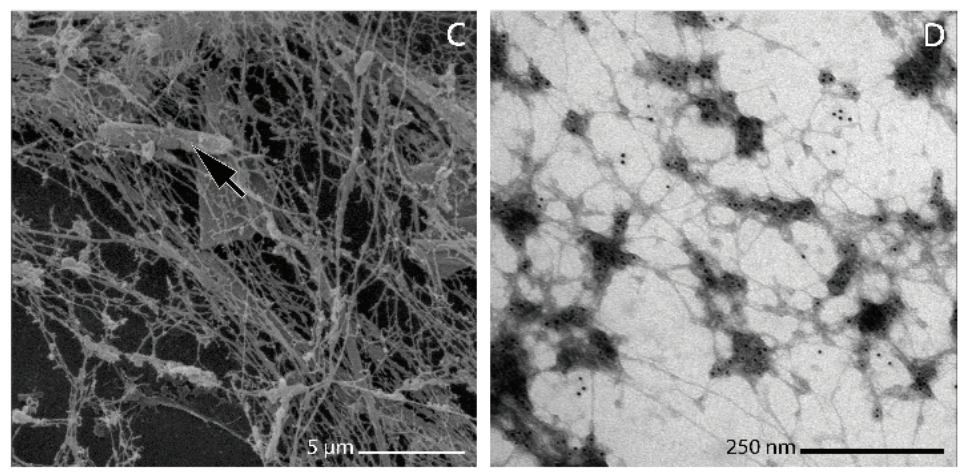

Figure 1. Morphology of NETs and NET-forming human neutrophils as analyzed by confocal laser microscopy (A, B), and scanning and transmission electron microscopy (C and D, respectively). (A) NETs generated in vitro from human neutrophils isolated from whole venous blood using a standard gradient separation medium containing sodium metrizoate and Dextran 500 [34]. NETosis induction by stimulation with $1 \mu \mathrm{M}$ fMLP followed the procedure described by [35]. DNA was stained with sytox ${ }^{\circledR}$ green. At light microscopic resolution, NETs appear as irregular cloudy structures in which dense clusters of brightly stained extracellular DNA (asterisks) merge with more faintly stained dilated areas in which the DNA is more thinly spread and forms a meshwork of threads (white arrowheads). Lobulated nuclei of non-NET forming neutrophils (white arrows) are found within the meshwork as well as outside of it, some being slightly out of focus; (B) elongated plume of NET-DNA (arrowhead) protruding from one of two attached NET-forming neutrophils from the sputum of a patient with chronic obstructive pulmonary disease (COPD). The cells are immunostained for peptidyl arginine deiminase 4 (PAD4, red) and citrullinated histone 3 (citH3, green), DNA is stained with 4',6-diamidino-2-phenylindole (DAPI, blue). Overlapping PAD4 and citH3 staining at nuclear and cytoplasmic sites is characteristic of NET-forming neutrophils (cf. $[9,36])$ and conforms with the observation of [37] that histone $\mathrm{H} 3$ deimination by PAD4 is not entirely confined to the nucleus; (C) bacterium (arrow) entangled in NETs from the sputum of a COPD patient; (D) on-grid preparation of in vitro generated NETs (procedures as described for A above) immunogold stained for the enzyme neutrophil elastase, one of the key protein components of NETs.

\section{ET Formation Is Linked to Reactive Oxygen Species (ROS) and Autophagy}

ROS are a heterogeneous group of oxygen-containing molecules with high chemical reactivity, some being rendered unstable and extremely reactive due to an unpaired electron. This group includes peroxides, hypochlorous acid, hydroxyl radicals, singlet oxygen, and the superoxide anion, among other compounds. Physiological generation of ROS occurs either as byproducts of (redox) reactions in various cell organelles including mitochondria, peroxisomes, and endoplasmic reticulum, or by primary enzyme function, such as with oxidases and oxygenases. Such enzymes have long 
been associated with the respiratory burst of phagocytes (see below), but are now known to occur in virtually every type of cell and tissue [38-40].

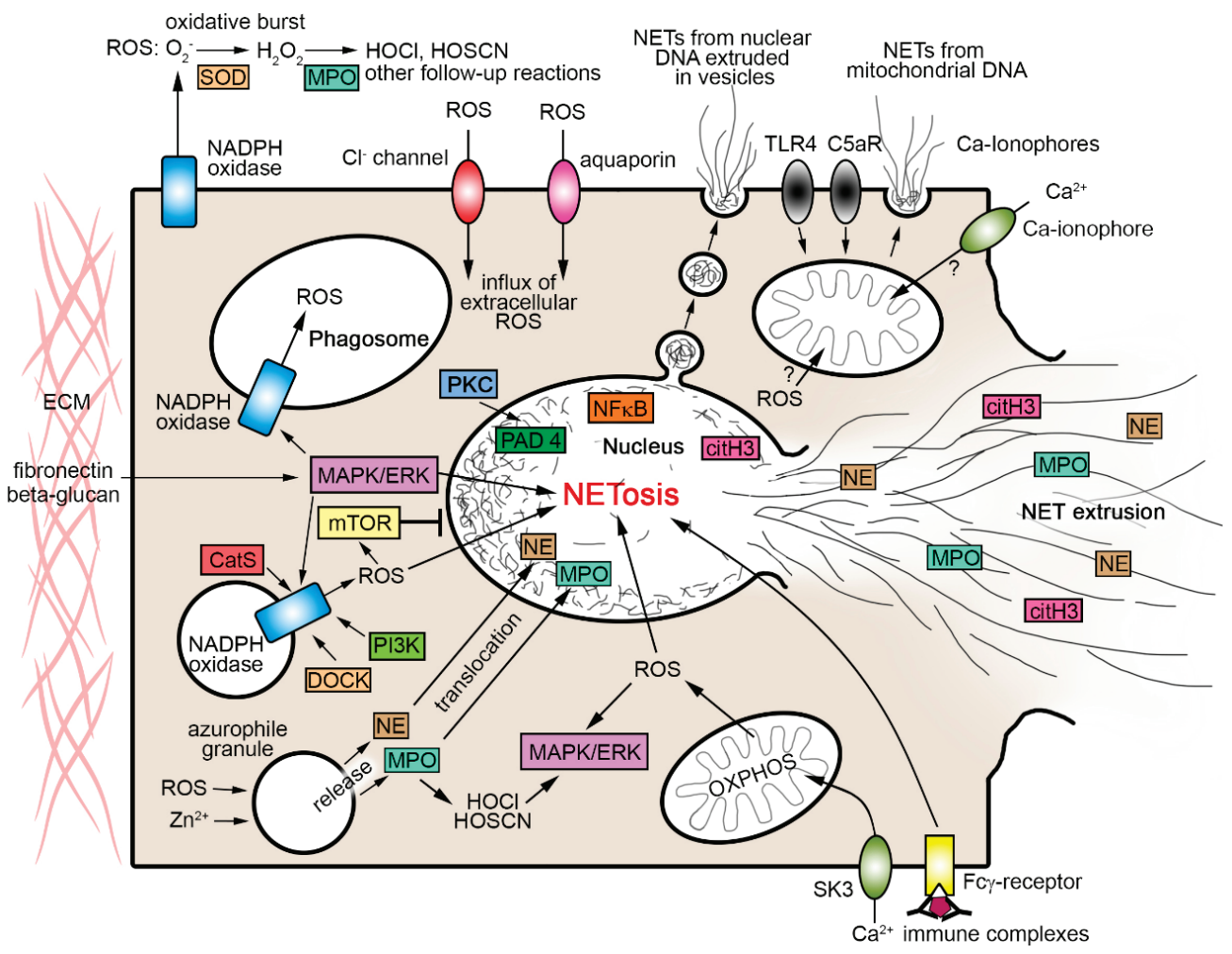

Figure 2. Scheme summarizing pathways of interaction between ROS and NET formation as addressed in the text. Arrows indicate directions of effects. Note that only some of the processes shown can co-occur. CatS cathepsin S, citH3 citrullinated histone H3, C5aR complement component 5a receptor, DOCK dedicator of cytokinesis proteins, ERK extracellular signal-regulated kinases, $\mathrm{HOCl}$ hypochlorous acid, HOCSN hypothiocyanous acid, MASPK mitogen-activated protein kinases, MPO myeloperoxidase, mTOR mammalian target of rapamycin, NE neutrophil elastase, NFkB nuclear factor kappa-light-chain-enhancer of activated B cells, OXPHOS oxidative phosphorylation, PAD4 peptidylarginine deiminase 4, PI3K phosphoinositide-3-kinase, PKC protein kinase C, SK3 small conductance calcium-activated potassium channel 3, SOD superoxide dismutase, TLR4 toll-like receptor 4.

An important finding in recent ET research is that ROS are apparently an integral part of most reaction cascades entailing the release of ETs [3,41,42] (Figure 2). As with the morphological pattern above, the evidence is mainly derived from ET formation by neutrophils (NETosis). Analysis from various perspectives has shown that NETosis involves ROS formation by the multienzyme complex NADPH oxidase (e.g., [1,3,18,28,32,42]) (see below under Section 3). 
Recent research provides strong evidence that NETosis is directly linked to (or is a variant of) the autophagy pathway $[43,44]$. Autophagy is a conserved process of lysosome-mediated intracelluar degradation [45] enabling the routine turnover of proteins and organelles. It further contributes to a wide spectrum of physiological functions including stress response, nutritional starvation management, tumor development and pathogen clearance (e.g., [46-50]). Protein kinase B (PKB/AKT), mammalian/mechanistic target of rapamycin (mTOR), and mitogen-activated protein kinases (MAPK, also known as extracellular signal-regulated kinases, ERK) have long been known to be important regulators of autophagy, mainly in work from tumor biology. Specifically, the phosphatidylinositol 3-kinase (PI3K)/AKT/mTOR signaling pathway is a negative regulator of both autophagy and apoptosis (e.g., [49,51-53]), while MAPK/ERK pathways act as positive autophagy regulators (e.g., $[49,54,55])$.

It is now known that the regulation of autophagy, and especially its association with ETosis, is closely tied to ROS in a multifactorial manner (e.g., [56,57]). Importantly, the level of intracellular ROS determines whether the autophagy reaction ends in NETosis $[1,44,58,59]$. Placed in a wider perspective, this association with NETosis strengthens the view that autophagy is not a "simple" mechanism of cell death alternative to apoptosis, but serves primarily to protect vertebrate cells from various types of harm including that from microbes (cf. [60]). However, the exact ways in which ROS interfere with the signaling network behind autophagy to initiate and/or promote NETosis are still incompletely understood, particularly in relation to how they contribute to the remodeling of the cell interior.

\section{The Role of NADPH Oxidase-Derived ROS in NET Formation}

NADPH (nicotinamide adenine dinucleotide phosphate) oxidases are a family of membrane bound multiprotein enzymes that generate ROS delivered into either extracellular or intracellular compartments. Phagocytes have long been known to express large amounts of NADPH oxidase residing in the plasma membrane and in phagosome membranes (Figure 2), but intermediate or low amounts of the enzyme occur in most — if not in all — mammalian cell types and tissues [40,61,62]. The phagocyte NADPH oxidase consists of a b-type cytochrome-containing transmembrane protein termed gp91 ${ }^{\text {phox }}$ (also known as NOX2, see below), with four further "phox" (phagocytic oxidase) elements (p22 $2^{\text {phox }}, \mathrm{p} 40^{\text {phox }}, \mathrm{p} 47^{\text {phox }}, \mathrm{p} 67^{\text {phox }}$ ) and a guanosine triphosphatase (GTPase), usually belonging to the Ras-related C3 botulinum toxin substrate types Rac1 or Rac2 within the Rho (Ras homologue) family and Ras (rat sarcoma) superfamily of small GTPases. The gp91 phox subunit was found to have several homologues in nonphagocytic NADPH oxidases (NOX). Together with gp91 $1^{\text {phox }}$, these homologues have been pooled in the NOX family, comprising NOX1, NOX2 (= gp91 ${ }^{\text {phox }}$ ), NOX3, NOX4, NOX5, and also DUOX (dual oxidase) 1 and 2. Nonphagocytic NADPH oxidases differ from the phagocytic enzyme in molecular structure, subcellular location and biochemical function [40,62-64].

NADPH oxidases exist in different states of activation (resting, primed, active, or inactive). Signal molecules able to induce subunit assembly and activation include proinflammatory cytokines, lipopolysaccharides, Toll-like receptor (TLR) agonists, and chemical agents such as PMA [65], and are largely the same as those known to induce NETosis. Forming the functional 
enzyme requires the phosphorylation of protein subunits (e.g., p40 phox $)$ by MAPK, and the translocation of cytosolic components to membranes [65-68]. The situation is complicated by the fact that the regulatory pathways of NADPH oxidase assembly vary depending upon the molecular triggers. In neutrophils, this is exemplified by the difference between PMLP and PMA, the former acting via receptor-mediated pathways with downstream protein kinase involvement (e.g., [69,70], see also information on $\mathrm{PI} 3 \mathrm{~K} / \mathrm{AKT} / \mathrm{mTOR}$ pathways below) while the latter operates directly via protein-kinase C (e.g., [71,72]).

Once assembled and activated, NADPH oxidases transfer electrons from cytoplasmic NADPH across biological membranes and couple them to molecular oxygen, thus generating the superoxide radical anion ${ }^{\bullet} \mathrm{O}_{2}^{-}$. This enables a cascade of $\mathrm{ROS}$ generation that continues with the rapid conversion of ${ }^{\bullet} \mathrm{O}_{2}{ }^{-}$.to hydrogen peroxide $\mathrm{H}_{2} \mathrm{O}_{2}$, either spontaneously or catalysed by superoxide dismutase (SOD) [40]. The process may then proceed to the MPO catalysed formation of hypochlorous acid ( $\mathrm{HOCl}$ ) from $\mathrm{H}_{2} \mathrm{O}_{2}$ [73-75]. $\mathrm{HOCl}$ is able, in turn, to re-react with $\mathrm{H}_{2} \mathrm{O}_{2}$ to generate singlet molecular oxygen $\left({ }^{1} \mathrm{O}_{2}\right)$ and peroxyl radicals [76]. Availability of transition metals (specifically iron) may enable the $\mathrm{H}_{2} \mathrm{O}_{2}$ to undergo the Fenton reaction rendering the highly reactive hydroxyl radical $\left({ }^{\bullet} \mathrm{OH}\right)[39,40]$. Further reactions may follow under given conditions, such as the production of nitric oxide (NO) by inducible nitric oxide synthase (iNOS), and may again entail consecutive reactions, e.g., with ${ }^{\bullet} \mathrm{O}_{2}{ }^{-}$to form peroxynitrite $[66,77]$.

It has been recognized for some time that neutrophils and other phagocytes produce large amounts of extracellular ROS (e.g., $\mathrm{H}_{2} \mathrm{O}_{2}$ and superoxide anion ${ }^{\bullet} \mathrm{O}_{2}{ }^{-}$) upon stimulation with a wide variety of agents. Due to a transient rise in oxygen demand, this behavior was originally named respiratory (or oxidative) burst (e.g., [40,78]). Plasma membrane-bound phagocyte NADPH oxidase is commonly thought to be the main source of ROS delivery to the extracellular space during respiratory bursts, and into engulfed phagosomes for microbial killing [65,79,80]. In addition, NADPH oxidase derived ${ }^{\bullet} \mathrm{O}_{2}^{-}$has been shown to promote microbial killing in the phagosome also indirectly by enabling the activation of serin proteases (e.g., cathepsin $\mathrm{G}$ and neutrophil elastase, NE) in neutrophil azurophilic granules via modulation of ion influx and $\mathrm{pH}[14,81,82]$ (there is, however, evidence suggesting that the importance of this mechanism for the microbicidal capacity of the neutrophils is limited [83]).

The circumstances of how NADPH oxidase-derived ROS influence NET formation inside the cell appear complex, and are still not fully conclusive. There is accumulating evidence that NADPH oxidase-derived ROS acting at the intracellular level are capable, and in some cases requisite, to initiate the formation of NETs. Experiments with ${ }^{1} \mathrm{O}_{2}$ scavengers have confirmed singlet oxygen involvement in NADPH oxidase-dependent NET formation in human neutrophils upon stimulation with PMA [74]. Experimental inhibition [32,84] and mutation-caused failure [85] of NADPH oxidase has been shown to prevent NET formation. NADPH oxidase-deficient neutrophils of mutant mice and of humans with chronic granulomatous disease (CGD) are not able to form NETs $[18,32,86]$. However, although recent work has indicated that it is intracellular ROS levels that direct signaling in favor of autophagy/NETosis (see Section 2 above), the details of how this occurs are still not fully understood. Particularly it remains unclear how ROS influence the main features of NETosis, i.e., chromatin decondensation, histone citrullination, binding of enzymes 
to DNA, and membrane rupture. Present research has only provided pieces of a much larger puzzle. There is evidence indicating that in addition to their role in NE activation, ROS enable the release of NE and MPO from the azurophilic granules in the neutrophil cytoplasm. This is a prerequisite for the translocation of these enzymes to the nucleus, where NE aids histone degradation and MPO chromatin relaxation $[13,59,87,88]$. Additionally, ROS act to facilitate the citrullination of histone proteins by peptidyl arginine deiminase type 4 (PAD4) [89]. All these together are thought to promote nucleosome disassembly, chromatin decondensation, and the rupture of intracellular membranes. An upstream requirement of NADPH oxidase-dependent NET formation, and specifically also of PAD4-mediated histone citrullination, seems to be a rise in cytosolic calcium concentration via influx from the ER and/or the extracellular space [37,90].

The work of Parker et al. [91] indicates that the involvement of NADPH oxidase-derived ROS in the regulatory pathways of NET formation, just as the pathways themselves, vary depending upon the inducing molecular stimuli. These authors found that NETosis requires NADPH oxidase-derived ROS when induced with PMA or by bacterial stimulation, but not if the induction occurs via calcium influx mediated by the bacterial calcium ionophore ionomycin (see Section 8 below). Other work confirmed the role of NADPH oxidase-derived ROS in PMA-induced NET release from human neutrophils and demonstrated involvement of the MAPK/ERK pathway [84]. Similarly, MPO was found essential only after PMA induction, while bacteria induced NETs were formed without it [91]. Probably in partial contrast to this, Metzler et al. [92] found that functional MPO is a strict requirement for NET formation when comparing the NET-forming ability of neutrophils from MPO-deficient subjects and healthy donors after stimulation with PMA and opsonized Candida albicans cells. Work investigating NET formation in neutrophilic granulocytes of carp suggests that a stimulus-dependent selective requirement of ROS is an evolutionarily conserved pattern of vertebrate phagocytes [8].

\section{NADPH Oxidase-Dependent NETosis Is a Matter of Delicate Coordination, Depending on Various Co-Factors}

There are several lines of evidence to indicate that the regulatory influences behind the NADPH oxidase-dependent pathway of autophagy/NETosis induction are far from straightforward, and much is still incompletely understood. Perhaps the most fundamental question in this respect is under what circumstances NADPH oxidase-derived ROS signaling promotes autophagy/NETosis rather than cell death through apoptosis? This is because ROS such as $\mathrm{H}_{2} \mathrm{O}_{2}$ have also been found to promote neutrophil apoptosis, e.g., by caspase activation via the sphingolipid ceramide or the lysosomal aspartyl protease cathepsin D [93-95]. Such dual response is evident from recent work on the mouse lung showing that Aspergillus infection in the presence of functional NADPH oxidase enables NETosis while at the same time promoting neutrophil apoptosis [18]. Present knowledge of factors that affect whether NADPH oxidase-derived ROS direct a neutrophil into the autophagy/NETosis pathway is limited. Particular focus may be placed on the following aspects (see also Figure 2): 
- Cathepsin C. Recent work investigating NET formation in patients with Papillon-Lefèvre syndrome suggests that the cycteine protease cathepsin $\mathrm{C}$ interferes with the interplay between NADPH oxidase-derived ROS and NETosis (albeit without entailing a substantial deficit in general immune defense) [83].

- DOCK proteins. A co-regulatory role in NADPH oxidase-dependent NETosis has been established for "dedicator of cytokinesis" (DOCK) proteins. Via their function as activators of Rac GTPases, DOCK proteins are involved in both neutrophil chemotaxis [96] and NADPH oxidase-dependent ROS production, the latter entailing a massive reduction in NET-forming ability in DOCK2 ${ }^{-/-}$individuals and to an almost complete loss of this ability in DOCK $2^{-/} / \mathrm{DOCK}^{-/-}$double deficient individuals in the murine test system [97].

- Zinc. Intracellular zinc ion $\left(\mathrm{Zn}^{2+}\right)$ concentration has been identified as a co-regulator in PMA-induced protein kinase $\mathrm{C}$ mediated NET formation, which depends on the NADPH oxidase-derived ROS [98].

- mTOR-related pathways. Data from in vitro experimentation with human neutrophils using fMLP as inducing agent suggest that a central role in the ROS-mediated regulation toward NETosis is played by pathways involving the mTOR serine/threonine kinase [99]. Specifically the (PI3K/AKT)/mTOR pathway has been confirmed as a ROS sensitive negative regulator of autophagy [57,100,101], and is also gaining attention in relation to autophagy-related NETosis [44,59]. Moreover, also in this case, commitment to autophagy/NETosis was not found to be mandatory, as the ROS mediated impairment of mTOR activity may also terminate in apoptosis [57,102,103], so that additional factors must be assumed to play a role. Relevant regulatory influence in this respect will result from whether the molecular inducers activate NETosis in an mTOR-dependent manner, or along other pathways, as shown for IL-8 [26,27,90].

- Protein kinase C. The observations of Neeli and Radic [104] suggest that the calcium-dependent regulation of histone deimination by PAD4 (see also Section 3 above) is influenced by an intricate antagonism between the alpha and zeta isoforms of protein kinase $\mathrm{C}(\mathrm{PKC})$.

- Extracellular matrix. Important co-factors seem to be located in the extracellular matrix. Recent work on human neutrophil responses to fungal (Candida albicans) infection indicates that ubiquitous matrix constituents such as fibronectin may be attached with a significant role in deciding between respiratory burst behavior and NETosis [105]. This work demonstrates that neutrophils, when exposed solely to Candida-derived beta-glucan, activate ROS production but not NETosis. Simultaneous presence of fibronectin and beta-glucan, by contrast, leads to the suppression of ROS production and to rapid NET generation. The type of NETosis found depends on MAPK/ERK but not on ROS, and exhibits fine structural features that are strikingly similar to those shown for ROS independent Staphylococcus induced NETosis by Pilsczek et al. [31] (see also Section 7 below). This illustrates that extracellular matrix components may exert a more complex influence on the co-regulation of ROS production and NETosis than presently assumed. These results are in close agreement with experimental findings prior to the detection of $(\mathrm{N})$ ETosis. Testing 
neutrophils adhering to substrates including collagen IV, laminin, thrombospondin, heparan sulfate proteoglycan (HSP) and again fibronectin during respiratory bursts primed by PMA and TNF-alpha, Borgquist et al. [106] showed a highly variable ROS production depending upon the extracellular matrix present. Recent work demonstrates that a major role in extracellular matrix effects on ROS-dependent NETosis mediated by NADPH oxidase and MPO is likely to be played by immobilized immune complexes that bind to neutrophil FcyRIIIb receptors [29].

- Microbial-derived substances. A further source of co-factors influencing the regulation of ROS-dependent NETosis is microbial-derived substances. This is demonstrated by recent work showing that gram-positive bacteria derived peptide bacteriocins containing polycyclic thioether amino acids (so-called lantibiotics, such as nisin) enhance levels of NADPH oxidase-derived intracellular ROS and induce NETosis in human neutrophils in a dose-dependent manner [107].

All this strengthens the view that ROS-dependent NETosis in neutrophils is modulated in a complex manner by integration of multiple stimuli (see also Byrd et al. [105]). However, in the light of the results of a recent investigation of NET-forming ability in cathepsin C-deficient individuals (see point on cathepsin above), it may be necessary to add that failure of ROS-mediated NET formation need not entail a substantial deficit in general immune defense [83].

\section{Subtypes of NADPH Oxidase and Extracellular ROS}

In addition to the present incomplete knowledge of co-factors, understanding of the role of ROS in the regulation of subcellular events during NETosis is further complicated by a variety of other parameters, such as the different pathways of NADPH oxidase activation depending on the type of external inducers. These uncertainties are exemplified by the observation that it is still not clear whether neutrophil NADPH oxidase may be divided into two subtypes that are distinct from each other by both their location and the involvement of PI3K in their activation pathways. This divergence has been demonstrated by inhibition experiments with the PI3K inhibitor wortmannin (a fungal steroid metabolite), which reliably blocks neutrophil NADPH oxidase activation if depending on PI3K pathways. It was first shown for activation induced by $N$-formylmethionyl-leucylphenylalanine (fMLP), while PMA-induced activation was found to be resistant to inhibition by wortmannin [108]. The extended experiments of Karlsson et al. [71] then demonstrated that PMA is also able to induce a wortmannin-sensitive (i.e., PI3K-dependent) NADPH oxidase activation in neutrophils, which, however, resulted in only intracellular but not extracellular release of superoxide. Neutrophils were therefore thought to harbour two NADPH oxidase subtypes, one residing in the plasma membrane, the other in the membranes of the so-called specific granules within the neutrophil cyptoplasm. Activation of both subtypes was found to depend on MAPK/ERK kinase and protein phosphatase 1 and/or 2A, while diverging in dependence on PI3K, which was found essential only for the intracellular variant of the enzyme [71]. Such a role of the specific granules in NADPH oxidase-dependent intracellular ROS generation has been further corroborated by Ambruso et al. [109], while more recent work indicates that endosomes commonly assigned to the 
secretory vesicles are also involved [110]. In view of the emerging importance of ROS dependent PI3K-mTOR pathways in the control of NET formation via regulation of autophagy (e.g., [99,103], see also Section 2 above), this is a relevant point of uncertainty that would require further examination.

However, despite such uncertainties, other capacious evidence summarized by Bedard and Krause [40] suggests that all phagocyte/neutrophil NADPH oxidase contains NOX2/gp91 ${ }^{\text {phox }}$ as central functional component, irrespective of localization. The model of NADPH oxidase function presented by these authors does not discriminate between plasma membrane- and granule membrane-localized subtypes of the enzyme. Instead, it depicts a flexible situation in which most NOX2 is, together with $\mathrm{p} 22^{\text {phox }}$, localized in the membranes of intracellular granules as long as the cells maintain a resting state. According to this model, the granules fuse with the plasma membrane only after completion of subunit assembly and activation, thus enabling ROS release to the extracellular compartment. In addition, the model implicates that granule membrane-bound NOX2 may become functional as part of NADPH oxidase intracellularly, without the need for fusion with the surface membrane. This may be requisite for the proposed roles of ROS in signaling cascades for NETosis induction (see also [111]).

Irrespective of whether or not neutrophil ROS release to the extracellular and intracellular compartment is caused by distinct subtypes of NADPH oxidase, it is unclear how extracellular ROS contributes to NETosis induction. As reported above, most work on the role of ROS in NETosis signaling refers, as a matter of course, to intracellular ROS. However, knowledge is incomplete as to the extent to which these ROS originate from outside the cells. While it seems clear that there is an inward passage of superoxide anions ${ }^{\bullet} \mathrm{O}_{2}{ }^{-}$and $\mathrm{H}_{2} \mathrm{O}_{2}$ through anion $\left(\mathrm{Cl}^{-}\right)$and aquaporin channels, respectively [112], it is not known how this transmembrane flux is balanced with ROS generation by intracellular NADPH oxidase (or other sources such as mitochondria). Some progress in this context has been made by the in vitro analyses of Kirchner et al. [41]. Testing the effects of various inhibitors of ROS generating enzymes (NADPH oxidase, SOD, MPO) and mitochondrial electron transport on NETosis in human neutrophils, these authors found that NADPH oxidase- and MPO-derived ROS, but not those from SOD and mitochondria, are important for NET release. This further underpins the potential importance of NADPH oxidase-generated superoxide, and perhaps also of spontaneously formed (not SOD-catalyzed) $\mathrm{H}_{2} \mathrm{O}_{2}$, in NETosis induction. However, as the NADPH oxidase inhibitor diphenyleneiodonium chloride (DPI) used in this work is likely to act similarly on plasma membrane-bound and intracellular NADPH oxidase [113,114], and, more importantly, also on mitochondrial OXPHOS flavoenzymes [115], the questions as to true sources of NETosis inducing ROS remain largely unresolved.

\section{The Role of ROS from Sources other than NADPH Oxidase}

Although much of the present knowledge on the role of ROS in the molecular signaling of ETosis/NETosis has been obtained from NADPH oxidase-dependent pathways, there are several lines of evidence suggesting that ROS contributing to the formation of NETs need not necessarily derive from pathways involving NADPH oxidase. It becomes increasingly evident that there are likely two distinct (main) types of ROS-dependent NETosis: one that requires ROS supply from 
NADPH oxidase and responds to NADPH oxidase blocking, and another that does neither of these, instead relying on other ROS sources (cf. [116]).

Substantial new information has come from work supplying singlet oxygen $\left({ }^{1} \mathrm{O}_{2}\right)$ to neutrophils from CGD patients and healthy humans via application of the photosensitizing agent Photofrin (porfimer sodium). Results indicate that singlet oxygen is likely to be able to induce NET formation independent from NADPH oxidase activation [74].

An alternative important source of ROS in eukarotic cells is mitochondrial oxidative phosphorylation (OXPHOS) complexes. Mitochondria are generally thought to be the largest contributors to intracellular ROS production (e.g., [39]). Work on oxidative stress levels has indicated that this might also apply to neutrophils [117], despite the fact that mature neutrophils are characterized by low mitochondrial content and reduced levels of oxidative phosphorylation [118]. Cytochrome $\mathrm{C}$ from mitochondrial OXPHOS complexes has been shown to support caspase activation for neutrophil apoptosis, the alternative cell fate to autophagy/NETosis [95]. ROS are relevant byproducts of mitochondrial energy supply to respiratory/oxidative bursts [40]. Intracellular ROS may be influenced by the dependence of mitochondrial OXPHOS complexes on NADPH-derived NADH as electron donors, entailing competition for NADPH between mitochondria and NADPH oxidase (e.g., [117]). This competition could be even stronger as NADPH oxidase may be able to utilize NADH as a second electron source in addition to NADPH, even though NOX2-containing phagocyte NADPH oxidases have a preference for the latter [40]. Despite a result to the contrary reported by Kirchner et al. [41], mitochondrial ROS production may thus, albeit in limited form, exert influence on intracellular ROS levels of ET-forming cells, even in neutrophils. This conclusion is in strong agreement with most recent evidence of a NADPH oxidase-independent pathway of NETosis that depends on mitochondrial ROS and is mediated by small conductance calcium-activated potassium channel 3 (SK3) (Figure 2). In contrast to NADPH oxidase-dependent NETosis, this pathway does not essentially require MAPK/ERK activation [116]. Further research will be required to determine the full scope of influence by mitochondrial ROS on the regulation of ROS-dependent NETosis, especially in the context of the close correlation with autophagy that is now apparent (see Section 2 above).

Another source of ROS not directly related to NADPH oxidase that is likely to play a particular role in ETosis-mediated inflammation is MPO. ETosis is certainly a key mechanism of MPO release to the extracellular compartment. The catalytic activity of MPO is partitioned between a halogenation cycle and a peroxidase cycle [119]. It is well known that MPO released by neutrophils during respiratory bursts catalyzes the oxidation of chloride $\left(\mathrm{Cl}^{-}\right)$bromide $\left(\mathrm{Br}^{-}\right)$and thiocyanate $\left(\mathrm{SCN}^{-}\right)$by $\mathrm{H}_{2} \mathrm{O}_{2}$ to hypochlorous acid $(\mathrm{HOCl})$, hypobromous acid $(\mathrm{HOBr})$ and hypothiocyanous acid (HOSCN), respectively. Although MPO itself may become inactivated by $\mathrm{H}_{2} \mathrm{O}_{2}$ [120], these oxidants support the phagocytes' ability to kill pathogens. Both are, directly and via their secondary reactions, able to harm host tissues in various manners, leading to growth arrest, apoptosis or necrosis in a dose dependent manner [73,75,121,122]. Direct molecular influences include inactivation of thiol enzymes (specifically by HOSCN), modification of lipoproteins and perturbation of phosphorylation-dependent signaling pathways such as MAPK/ERK [119-121,123]. The potential feedback effects on the role of MAPK/ERK pathways in the induction of ET formation (see 
Sections 2 and 3) are as yet largely undetermined. It will be a challenge to integrate the finding that extracellular products of MPO are unable to rescue ET formation in MPO-deficient neutrophils [92] into this context.

$\mathrm{HOCl}$ and $\mathrm{HOBr}$ contribute to the formation of ROS and radicals also via various secondary reactions. Both react, for example, with $\mathrm{H}_{2} \mathrm{O}_{2}$ to form singlet oxygen ${ }^{1} \mathrm{O}_{2}$ and peroxyl radicals [76,124,125] (see also Section 3 above). Both also target thiols, thioethers, disulfides, amines and amides, leading to the formation of advanced oxidation products (AOPPs) that interfere with the structure and physiology of cells [119]. AOPP formation with the involvement of extracellular MPO is also likely to include Fenton reactions. They generate hydroxyl radicals $(\mathrm{OH})$ from $\mathrm{H}_{2} \mathrm{O}_{2}$ catalyzed by transition metals (mainly iron) [39,40], accounting for the peroxidation of lipids with unsaturated fatty acyl residues, a long known factor in ROS-induced tissue damage [126-128]. At sites of inflammation, the extracellular iron required to allow for this is likely to be abundantly available from ferritin secretion by macrophages [129-132]. Further radicals derive from the peroxidase cycle of MPO, which performs one electron oxidation of a multitude of organic and inorganic substrates. These include amino acids (tyrosine, tryptophan), thiols, ascorbate, steroid hormones and urate, but also singlet oxygen ${ }^{1} \mathrm{O}_{2}$ and nitric oxide NO [119].

Thus, MPO is not only an important constituent in the intracellular regulation of ET formation (see Section 3) but once extruded, in all probability also a key contributor to extracellular (interstitial) ROS accumulation and phagocyte-mediated tissue injury.

\section{The Role of ROS in Non-Cell Death ETosis}

In addition to the accumulating information on the characteristics of "standard type" ETosis, research over the last decade has also rendered evidence of alternative forms of ET formation. These forms deviate from the "standard type" both mechanistically and in that they may leave the donor cells viable, thus being referred to as non-cell death ETosis. But all these alternative forms have some relationship to ROS.

The earliest example of non-cell death ETosis is provided by Yousefi et al. [133]. This work describes a fast form of ET extrusion by eosinophils, which diverges from the "standard type" by its eruptive ("catapult-like") nature, and also that it utilizes mitochondrial DNA thus avoiding instant cell death. The release of these ETs can be stimulated by IL-5 and LPS and proved sensitive to blocking with diphenyleneiodonium (DPI). Thus, it is likely that the underlying signaling is mediated by membrane bound receptors and depends on ROS. However, the exact circumstances of how this occurs have not been elucidated and may be complex. LPS alone is thought to be unable to activate NADPH oxidase while facilitating activation by subsequent triggers $[2,66]$. DPI has been shown to be not only an effective blocker of NADPH oxidase but also of other NAD(P)-dependent enzymes (such as glucose 6-phosphate dehydrogenase) and mitochondrial OXPHOS flavoenzymes [115]. It is thus unclear from where the ROS involved in this process derive and how they act. Similarly, it is not clear from both the morphological and the chemical points of view, how the mitochondrial DNA is combined with granule proteins to form these ETs, and how the lack of histones in mitochondrial DNA influences their function. 
A second variant of ETosis that leaves the donor cells viable and relies, in all probability, on mitochondrial DNA has been established for human neutrophils in response to treatment with granulocyte/macrophage colony-stimulating factor (GM-CSF) and subsequent TLR4 or complement factor 5a (C5a) receptor stimulation. Also in this case, treatment with DPI leads to a complete block of (N)ET release, and neutrophils from ROS-deficient CGD patients failed to generate such type of ETs [30]. This indicates that the process depends on ROS, but with the same uncertainties/caveats regarding the use of the blocking agent DPI as mentioned for eosinophil ETosis above.

A further type of fast ETosis/NETosis occurring without instant cell death was recently described for human neutrophils in response to stimulation with Staphylococcus aureus bacteria [31]. Similar to eosinophil ETosis [133], this type is rather fast, with NETs being observable as early as 5-10 min after onset of stimulation. In contrast to both eosinophil ETosis [133] and the type of NETosis described by Yousefi et al. [30], the process is not based on the release of mitochondrial DNA. Instead, DNA from nuclear chromatin is extruded via vesicles that bud from the nuclear membranes and rupture after release into the extracellular environment. However, this third variant of ETosis seems to only represent the initial phase of a longer lasting cycle, terminating in "conventional" cell death NETosis. But even if so, it is remarkable that this initial phase appears to be entirely devoid of ROS regulation [31].

\section{ROS-Independent ETosis}

Evidence as to whether there are pathways of NETosis that are entirely independent of ROS appears incomplete and conflicting. Some uncertainty still remains as to the role of ROS from NADPH oxidase or other sources in the induction of NET formation by elevation of cytosolic calcium. Experiments testing the effects of bacterial ionomycin in human promyelocytic leukemia (HL-60) cells and neutrophils strongly suggested that calcium influx-mediated induction of NETosis utilizes a ROS-independent pathway [37,91]. In contrast to this, recent work also investigating calcium ionophore-mediated NETosis, provides evidence for a calcium activated pathway of NETosis that depends on mitochondrial ROS, while being independent of NADPH oxidase-derived ROS [116]. It remains to be clarified how these findings can be integrated.

An ambiguous but inspiring situation has developed regarding the relationship between uric acid (UA) and NETosis depending on NADPH oxidase-derived ROS. UA, an abundant terminal product of vertebrate nitrogen metabolism, can act as an antioxidant but also as a pro-oxidant and pro-inflammatory factor, depending on the particular conditions [134]. This dual nature is clearly reflected in the present literature on the role of UA in mammalian NETosis. Some recent work suggests that UA in the form of monosodium urate (MSU) crystals is a strong inducer of ROS-dependent NETosis, which can be inhibited by anti-oxidants such as butylated hydroxytoluene (BHT), butylated hydroxyanisole (BHA) and ascorbic acid [135]. However, other work testing the effects of non-crystalline UA in solution found a dose-dependent ambivalent influence [136]. Low concentrations of UA $(1 \mathrm{mg} / 100 \mathrm{~mL})$ exerted an inhibitory effect on NADPH oxidase-dependent NET formation, most likely due to the antioxidant potential of UA. High concentrations $(8 \mathrm{mg} / 100 \mathrm{~mL})$, were, by contrast, found to be potent inducers of NETosis. Tests 
with ROS-inhibited control neutrophils and neutrophils from ROS-deficient CGD patients demonstrated that NETosis induction by high UA levels occurs in a NADPH oxidase/ROSindependent manner, with nuclear factor "kappa-light-chain-enhancer" of activated B-cells (NF- $\kappa \mathrm{B})$ playing a role in the signaling pathway [136]. This is consistent with findings that NF- $\mathrm{KB}$ protein accumulates in the nuclei of PMA- or TNF $\alpha$-stimulated neutrophils [137], and that reduced phosphorylation of the NF- $\mathrm{kB}$ p65 subunit by different inhibitors (ASA, BAY-11-7082, and Ro 106-9920) abrogates the formation of NETs [138].

\section{Conclusions}

There is rapidly growing evidence that ROS are able to interact with the formation of ETs in a multidimensional manner (Figure 2). This occurs either directly via the signaling cascades that allow for ET formation and release, or indirectly via influence on other factors that modulate the process. The interaction with ROS is likely to be an important determinant in the regulatory network that determines whether ETosis is beneficial or noxious. The ROS-ETosis interaction will need to be taken into account to understand the characteristics of virtually all kinds of inflammatory disease, and to improve their treatment.

\section{Acknowledgments}

The authors thank John R. Haslett, University of Salzburg, for his continued support during the preparation of this manuscript.

\section{Author Contributions}

All authors contributed interactively to explore the background literature and to the writing procedure. Walter Stoiber and Astrid Obermayer took responsibility for preparing the final version of the manuscript. Astrid Obermayer and Peter Steinbacher prepared the illustrations.

\section{Conflicts of Interest}

The authors declare no conflicts of interest.

\section{References}

1. Von Köckritz-Blickwede, M.; Nizet, V. Innate immunity turned inside-out: Antimicrobial defense by phagocyte extracellular traps. J. Mol. Med. 2009, 87, 775-783.

2. Remijsen, Q.; Kuijpers, T.; Wirawan, E.; Lippens, S.; Vandenabeele, P.; Berghe, T.V. Dying for a cause: NETosis, mechanisms behind an antimicrobial cell death modality. Cell Death Differ. 2011, 18, 581-588.

3. Guimarães-Costa, A.B.; Nascimento, M.T.C.; Wardini, A.B.; Pinto-da-Silva, L.H.; Saraiva, E.M. ETosis: A microbicidal mechanism beyond Cell Death. J. Parasitol. Res. 2012, doi:10.1155/2012/929743. 
4. Goldmann, O.; Medina, E. The expanding world of extracellular traps: Not only neutrophils but much more. Front. Immunol. 2013, doi:10.3389/fimmu.2012.00420.

5. Brinkmann, V.; Reichard, U.; Goosmann, C.; Fauler, B.; Uhlemann, Y.; Weiss, D.S.; Weinrauch, Y.; Zychlinsky, A. Neutrophil extracellular traps kill bacteria. Science 2004, 303, 1532-1535.

6. Chuammitri, P.; Ostojić, J.; Andreasen, C.B.; Redmond, S.B.; Lamont, S.J.; Palić, D. Chicken heterophil extracellular traps (HETs): Novel defense mechanism of chicken heterophils. Vet. Immunol. Immunopathol. 2009, 129, 126-131.

7. Palić, D.; Ostojić, J.; Andreasen, C.B.; Roth, J.A. Fish cast NETs: Neutrophil extracellular traps are released from fish neutrophils. Dev. Comp. Immunol. 2007, 31, 805-816.

8. Pijanowski, L.; Golbach, L.; Kolaczkowska, E.; Scheer, M.; Verburg-van Kemenade, B.M.L.; Chadzinska, M. Carp neutrophilic granulocytes form extracellular traps via ROS-dependent and independent pathways. Fish Shellfish Immunol. 2013, 34, 1244-1252.

9. Obermayer, A.; Stoiber, W.; Krautgartner, W.-D.; Klappacher, M.; Kofler, B.; Steinbacher, P.; Vitkov, L.; Grabcanovic-Musija, F.; Studnicka, M. New aspects on the structure of neutrophil extracellular traps from chronic obstructive pulmonary disease and in vitro generation. PLOS ONE 2014, 9, e97784.

10. Parseghian, M.H.; Luhrs, K.A. Beyond the walls of the nucleus: The role of histones in cellular signaling and innate immunity. Biochem. Cell Biol. 2006, 84, 589-604.

11. Brinkmann, V.; Zychlinsky, A. Beneficial suicide: Why neutrophils die to make NETs. Nat. Rev. Microbiol. 2007, 5, 577-582.

12. Urban, C.F.; Ermert, D.; Schmid, M.; Abu-Abed, U.; Goosmann, C.; Nacken, W.; Brinkmann, V.; Jungblut, P.R.; Zychlinsky, A. Neutrophil extracellular traps contain calprotectin, a cytosolic protein complex involved in host defense against Candida albicans. PLOS Pathog. 2009, 5, e1000639.

13. Papayannopoulos, V.; Metzler, K.D.; Hakkim, A.; Zychlinsky, A. Neutrophil elastase and myeloperoxidase regulate the formation of neutrophil extracellular traps. J. Cell Biol. 2010, 191, 677-691.

14. Reeves, E.P.; Lu, H.; Jacobs, H.L.; Messina, C.G.M.; Bolsover, S.; Gabella, G.; Potma, E.O.; Warley, A.; Roes, J.; Segal, A.W. Killing activity of neutrophils is mediated through activation of proteases by $\mathrm{K}^{+}$flux. Nature 2002, 416, 291-297.

15. Ermert, D.; Zychlinsky, A.; Urban, C. Fungal and bacterial killing by neutrophils. Methods Mol. Biol. 2009, 470, 293-312.

16. Guimaraes-Costa, A.B.; Nascimento, M.T.C.; Froment, G.S.; Soares, R.P.P.; Morgado, F.N.; Conceicao-Silva, F.; Saraiva, E.M. Leishmania amazonensis promastigotes induce and are killed by neutrophil extracellular traps. Proc. Natl. Acad. Sci. USA 2009, 106, 6748-6753.

17. Bonne-Année, S.; Kerepesi, L.A.; Hess, J.A.; Wesolowski, J.; Paumet, F.; Lok, J.B.; Nolan, T.J.; Abraham, D. Extracellular traps are associated with human and mouse neutrophil and macrophage mediated killing of larval Strongyloides stercoralis. Microbes Infect. Inst. Pasteur 2014, 16, 502-511. 
18. Röhm, M.; Grimm, M.J.; D’Auria, A.C.; Almyroudis, N.G.; Segal, B.H.; Urban, C.F. NADPH oxidase promotes neutrophil extracellular trap formation in pulmonary aspergillosis. Infect. Immun. 2014, 82, 1766-1777.

19. Galioto, A.M.; Hess, J.A.; Nolan, T.J.; Schad, G.A.; Lee, J.J.; Abraham, D. Role of eosinophils and neutrophils in innate and adaptive protective immunity to larval strongyloides stercoralis in mice. Infect. Immun. 2006, 74, 5730-5738.

20. Narasaraju, T.; Yang, E.; Samy, R.P.; Ng, H.H.; Poh, W.P.; Liew, A.-A.; Phoon, M.C.; Rooijen, N.; van; Chow, V.T. Excessive neutrophils and neutrophil extracellular traps contribute to acute lung injury of influenza pneumonitis. Am. J. Pathol. 2011, 179, 199-210.

21. Jenne, C.N.; Wong, C.H.Y.; Zemp, F.J.; McDonald, B.; Rahman, M.M.; Forsyth, P.A.; McFadden, G.; Kubes, P. Neutrophils recruited to sites of infection protect from virus challenge by releasing neutrophil extracellular traps. Cell Host Microbe 2013, 13, 169-180.

22. Marcos, V.; Zhou-Suckow, Z.; Yildirim, A.Ö.; Bohla, A.; Hector, A.; Vitkov, L.; Krautgartner, W.D.; Stoiber, W.; Griese, M.; Eickelberg, O.; et al. Free DNA in cystic fibrosis airway fluids correlates with airflow obstruction. Mediators Inflamm. $\mathbf{2 0 1 5}$ doi: $10.1155 / 2015 / 408935$.

23. Sangaletti, S.; Tripodo, C.; Chiodoni, C.; Guarnotta, C.; Cappetti, B.; Casalini, P.; Piconese, S.; Parenza, M.; Guiducci, C.; Vitali, C.; et al. Neutrophil extracellular traps mediate transfer of cytoplasmic neutrophil antigens to myeloid dendritic cells toward ANCA induction and associated autoimmunity. Blood 2012, 120, 3007-3018.

24. Khandpur, R.; Carmona-Rivera, C.; Vivekanandan-Giri, A.; Gizinski, A.; Yalavarthi, S.; Knight, J.S.; Friday, S.; Li, S.; Patel, R.M.; Subramanian, V.; et al. NETs are a source of citrullinated autoantigens and stimulate inflammatory responses in rheumatoid arthritis. Sci. Transl. Med. 2013, doi:10.1126/scitranslmed.3005580.

25. Kaplan, M.J.; Radic, M. Neutrophil extracellular traps: Double-edged swords of innate immunity. J. Immunol. 2012, 189, 2689-2695.

26. Keshari, R.S.; Jyoti, A.; Dubey, M.; Kothari, N.; Kohli, M.; Bogra, J.; Barthwal, M.K.; Dikshit, M. Cytokines induced neutrophil extracellular traps formation: Implication for the inflammatory disease condition. PLOS ONE 2012, 7, e48111.

27. Gupta, A.K.; Hasler, P.; Holzgreve, W.; Gebhardt, S.; Hahn, S. Induction of neutrophil extracellular DNA lattices by placental microparticles and IL-8 and their presence in preeclampsia. Hum. Immunol. 2005, 66, 1146-1154.

28. Aleyd, E.; van Hout, M.W.M.; Ganzevles, S.H.; Hoeben, K.A.; Everts, V.; Bakema, J.E.; van Egmond, M. IgA enhances NETosis and release of neutrophil extracellular traps by polymorphonuclear cells via Fc $\alpha$ receptor I. J. Immunol. 2014, 192, 2374-2383.

29. Behnen, M.; Leschczyk, C.; Möller, S.; Batel, T.; Klinger, M.; Solbach, W.; Laskay, T. Immobilized immune complexes induce neutrophil extracellular trap release by human neutrophil granulocytes via FcyRIIIB and Mac-1. J. Immunol. 2014, 193, 1954-1965.

30. Yousefi, S.; Mihalache, C.; Kozlowski, E.; Schmid, I.; Simon, H.U. Viable neutrophils release mitochondrial DNA to form neutrophil extracellular traps. Cell Death Differ. 2009, 16, 1438-1444. 
31. Pilsczek, F.H.; Salina, D.; Poon, K.K.H.; Fahey, C.; Yipp, B.G.; Sibley, C.D.; Robbins, S.M.; Green, F.H.Y.; Surette, M.G.; Sugai, M.; et al. A novel mechanism of rapid nuclear neutrophil extracellular trap formation in response to Staphylococcus aureus. J. Immunol. 2010, 185, 7413-7426.

32. Fuchs, T.A.; Abed, U.; Goosmann, C.; Hurwitz, R.; Schulze, I.; Wahn, V.; Weinrauch, Y.; Brinkmann, V.; Zychlinsky, A. Novel cell death program leads to neutrophil extracellular traps. J. Cell Biol. 2007, 176, 231-241.

33. Yipp, B.G.; Petri, B.; Salina, D.; Jenne, C.N.; Scott, B.N.V.; Zbytnuik, L.D.; Pittman, K.; Asaduzzaman, M.; Wu, K.; Meijndert, H.C.; et al. Infection-induced NETosis is a dynamic process involving neutrophil multitasking in vivo. Nat. Med. 2012, 18, 1386-1393.

34. Böyum, A. Separation of leukocytes from blood and bone marrow. Introduction. Scand. J. Clin. Lab. Invest. 1968, 97, 7.

35. Brinkmann, V.; Laube, B.; Abed, U.A.; Goosmann, C.; Zychlinsky, A. Neutrophil extracellular traps: How to generate and visualize them. J. Vis. Exp. 2010, 36, 1724-1727.

36. Anzilotti, C.; Pratesi, F.; Tommasi, C.; Migliorini, P. Peptidylarginine deiminase 4 and citrullination in health and disease. Autoimmun. Rev. 2010, 9, 158-160.

37. Neeli, I.; Khan, S.N.; Radic, M. Histone deimination as a response to inflammatory stimuli in neutrophils. J. Immunol. 2008, 180, 1895-1902.

38. Thannickal, V.J.; Fanburg, B.L. Reactive oxygen species in cell signaling. Am. J. Physiol. Lung Cell. Mol. Physiol. 2000, 279, L1005-1028.

39. Holmström, K.M.; Finkel, T. Cellular mechanisms and physiological consequences of redox-dependent signalling. Nat. Rev. Mol. Cell Biol. 2014, 15, 411-421.

40. Bedard, K.; Krause, K.-H. The NOX family of ROS-generating NADPH oxidases: Physiology and pathophysiology. Physiol. Rev. 2007, 87, 245-313.

41. Kirchner, T.; Möller, S.; Klinger, M.; Solbach, W.; Laskay, T.; Behnen, M. The impact of various reactive oxygen species on the formation of neutrophil extracellular traps. Mediators Inflamm. 2012, doi:10.1155/2012/849136.

42. Arazna, M.; Pruchniak, M.P.; Demkow, U. Neutrophil extracellular traps in bacterial infections: Strategies for escaping from killing. Respir. Physiol. Neurobiol. 2013, 187, 74-77.

43. Mitroulis, I.; Kambas, K.; Chrysanthopoulou, A.; Skendros, P.; Apostolidou, E.; Kourtzelis, I.; Drosos, G.I.; Boumpas, D.T.; Ritis, K. Neutrophil extracellular trap formation is associated with IL-1 $\beta$ and autophagy-related signaling in gout. PLOS ONE 2011, 6, e29318.

44. Remijsen, Q.; Berghe, T.V.; Wirawan, E.; Asselbergh, B.; Parthoens, E.; de Rycke, R.; Noppen, S.; Delforge, M.; Willems, J.; Vandenabeele, P. Neutrophil extracellular trap cell death requires both autophagy and superoxide generation. Cell Res. 2011, 21, 290-304.

45. Deter, R.L.; Baudhuin, P.; de Duve, C. Participation of lysosomes in cellular autophagy induced in rat liver by glucagon. J. Cell Biol. 1967, 35, C11-C16.

46. Eskelinen, E.-L. New insights into the mechanisms of macroautophagy in mammalian cells. Int. Rev. Cell Mol. Biol. 2008, 266, 207-247. 
47. Kroemer, G.; Mariño, G.; Levine, B. Autophagy and the integrated stress response. Mol. Cell 2010, 40, 280-293.

48. Levine, B.; Abrams, J. p53: The Janus of autophagy? Nat. Cell Biol. 2008, 10, 637-639.

49. Bowman, C.J.; Ayer, D.E.; Dynlacht, B.D. Foxk proteins repress the initiation of starvation-induced atrophy and autophagy programs. Nat. Cell Biol. 2014, 16, 1202-1214.

50. Leidal, A.M.; Debnath, J. "Doubling down" on the autophagy pathway to suppress tumor growth. Genes Dev. 2014, 28, 1137-1139.

51. Gozuacik, D.; Kimchi, A. Autophagy as a cell death and tumor suppressor mechanism. Oncogene 2004, 23, 2891-2906.

52. Kondo, Y.; Kanzawa, T.; Sawaya, R.; Kondo, S. The role of autophagy in cancer development and response to therapy. Nat. Rev. Cancer 2005, 5, 726-734.

53. Liang, N.; Zhang, C.; Dill, P.; Panasyuk, G.; Pion, D.; Koka, V.; Gallazzini, M.; Olson, E.N.; Lam, H.; Henske, E.P.; et al. Regulation of YAP by mTOR and autophagy reveals a therapeutic target of tuberous sclerosis complex. J. Exp. Med. 2014, 211, 2249-2263.

54. Ogier-Denis, E.; Pattingre, S.; El Benna, J.; Codogno, P. Erk1/2-dependent phosphorylation of Galpha-interacting protein stimulates its GTPase accelerating activity and autophagy in human colon cancer cells. J. Biol. Chem. 2000, 275, 39090-39095.

55. Liu, Y.L.; Lai, F.; Wilmott, J.S.; Yan, X.G.; Liu, X.Y.; Luan, Q.; Guo, S.T.; Jiang, C.C.; Tseng, H.-Y.; Scolyer, R.A.; et al. Noxa upregulation by oncogenic activation of MEK/ERK through CREB promotes autophagy in human melanoma cells. Oncotarget 2014, 5, $11237-11251$.

56. Mitroulis, I.; Kourtzelis, I.; Kambas, K.; Rafail, S.; Chrysanthopoulou, A.; Speletas, M.; Ritis, K. Regulation of the autophagic machinery in human neutrophils. Eur. J. Immunol. 2010, 40, 1461-1472.

57. Scherz-Shouval, R.; Elazar, Z. Regulation of autophagy by ROS: Physiology and pathology. Trends Biochem. Sci. 2011, 36, 30-38.

58. Chargui, A.; Cesaro, A.; Mimouna, S.; Fareh, M.; Brest, P.; Naquet, P.; Darfeuille-Michaud, A.; Hébuterne, X.; Mograbi, B.; Vouret-Craviari, V.; et al. Subversion of autophagy in adherent invasive Escherichia coli-infected neutrophils induces inflammation and cell death. PLOS ONE 2012, 7, e51727.

59. Chargui, A.; El May, M.V. Autophagy mediates neutrophil responses to bacterial infection. Acta Pathol. Microbiol. Immunol. Scand. 2014, 122, 1047-1058.

60. Kroemer, G.; Levine, B. Autophagic cell death: The story of a misnomer. Nat. Rev. Mol. Cell Biol. 2008, 9, 1004-1010.

61. Drummond, G.R.; Selemidis, S.; Griendling, K.K.; Sobey, C.G. Combating oxidative stress in vascular disease: NADPH oxidases as therapeutic targets. Nat. Rev. Drug Discov. 2011, 10, 453-471.

62. Gao, H.-M.; Zhou, H.; Hong, J.-S. NADPH oxidases: Novel therapeutic targets for neurodegenerative diseases. Trends Pharmacol. Sci. 2012, 33, 295-303.

63. Chen, K.; Kirber, M.T.; Xiao, H.; Yang, Y.; Keaney, J.F. Regulation of ROS signal transduction by NADPH oxidase 4 localization. J. Cell Biol. 2008, 181, 1129-1139. 
64. Rabêlo, L.A.; de Souza, V.N.; da Fonseca, L.J.S.; Sampaio, W.O. Redox unbalance: NADPH oxidase as therapeutic target in blood pressure control. Arq. Bras. Cardiol. 2010, 94, 684-693.

65. El-Benna, J.; Dang, P.M.-C.; Gougerot-Pocidalo, M.-A. Priming of the neutrophil NADPH oxidase activation: Role of p47phox phosphorylation and NOX2 mobilization to the plasma membrane. Semin. Immunopathol. 2008, 30, 279-289.

66. Sheppard, F.R.; Kelher, M.R.; Moore, E.E.; McLaughlin, N.J.D.; Banerjee, A.; Silliman, C.C. Structural organization of the neutrophil NADPH oxidase: Phosphorylation and translocation during priming and activation. J. Leukoc. Biol. 2005, 78, 1025-1042.

67. Dang, P.M.-C.; Stensballe, A.; Boussetta, T.; Raad, H.; Dewas, C.; Kroviarski, Y.; Hayem, G.; Jensen, O.N.; Gougerot-Pocidalo, M.-A.; El-Benna, J. A specific p47phox-serine phosphorylated by convergent MAPKs mediates neutrophil NADPH oxidase priming at inflammatory sites. J. Clin. Invest. 2006, 116, 2033-2043.

68. El-Benna, J.; Dang, P.M.-C.; Gougerot-Pocidalo, M.A.; Marie, J.C.; Braut-Boucher, F. p47phox, the phagocyte NADPH oxidase/NOX2 organizer: Structure, phosphorylation and implication in diseases. Exp. Mol. Med. 2009, 41, 217-225.

69. Wittmann, S.; Frohlich, D.; Daniels, S. Characterization of the human fMLP receptor in neutrophils and in Xenopus oocytes. Br. J. Pharmacol. 2002, 135, 1375-1382.

70. Kuiper, J.W.P.; Sun, C.; Magalhães, M.A.O.; Glogauer, M. Rac regulates PtdInsP3 signaling and the chemotactic compass through a redox-mediated feedback loop. Blood 2011, 118, 6164-6171.

71. Karlsson, A.; Nixon, J.B.; McPhail, L.C. Phorbol myristate acetate induces neutrophil NADPH-oxidase activity by two separate signal transduction pathways: Dependent or independent of phosphatidylinositol 3-kinase. J. Leukoc. Biol. 2000, 67, 396-404.

72. Wang, Y.-H.; Shen, Y.-C.; Liao, J.-F.; Lee, C.-H.; Li, C.-H.; Chou, C.-Y.; Liou, K.-T.; Chou, Y.-C. Anti-inflammatory effects of dimemorfan on inflammatory cells and LPS-induced endotoxin shock in mice. Br. J. Pharmacol. 2008, 154, 1327-1338.

73. Klebanoff, S.J. Myeloperoxidase: Friend and foe. J. Leukoc. Biol. 2005, 77, 598-625.

74. Nishinaka, Y.; Arai, T.; Adachi, S.; Takaori-Kondo, A.; Yamashita, K. Singlet oxygen is essential for neutrophil extracellular trap formation. Biochem. Biophys. Res. Commun. 2011, $413,75-79$.

75. Klebanoff, S.J.; Kettle, A.J.; Rosen, H.; Winterbourn, C.C.; Nauseef, W.M. Myeloperoxidase: A front-line defender against phagocytosed microorganisms. J. Leukoc. Biol. 2013, 93, 185-198.

76. Miyamoto, S.; Martinez, G.R.; Rettori, D.; Augusto, O.; Medeiros, M.H.G.; di Mascio, P. Linoleic acid hydroperoxide reacts with hypochlorous acid, generating peroxyl radical intermediates and singlet molecular oxygen. Proc. Natl. Acad. Sci. USA 2006, 103, 293-298.

77. Garcia-Bonilla, L.; Moore, J.M.; Racchumi, G.; Zhou, P.; Butler, J.M.; Iadecola, C.; Anrather, J. Inducible Nitric Oxide Synthase in Neutrophils and Endothelium Contributes to Ischemic Brain Injury in Mice. J. Immunol. 2014, 193, 2531-2537.

78. Forman, H.J.; Torres, M. Reactive oxygen species and cell signaling: Respiratory burst in macrophage signaling. Am. J. Respir. Crit. Care Med. 2002, 166, S4-S8. 
79. Rybicka, J.M.; Balce, D.R.; Khan, M.F.; Krohn, R.M.; Yates, R.M. NADPH oxidase activity controls phagosomal proteolysis in macrophages through modulation of the lumenal redox environment of phagosomes. Proc. Natl. Acad. Sci. USA 2010, 107, 10496-10501.

80. Moriguchi, K.; Ohno, N. Electron microscopic identification of hydrogen peroxide detected in fixed human polymorphonuclear leukocytes during phagocytosis. Okajimas Folia Anat. Jpn. 2014, 90, 97-100.

81. Pham, C.T.N. Neutrophil serine proteases: Specific regulators of inflammation. Nat. Rev. Immunol. 2006, 6, 541-550.

82. Nauseef, W.M. How human neutrophils kill and degrade microbes: An integrated view. Immunol. Rev. 2007, 219, 88-102.

83. Sørensen, O.E.; Clemmensen, S.N.; Dahl, S.L.; Østergaard, O.; Heegaard, N.H.; Glenthøj, A.; Nielsen, F.C.; Borregaard, N. Papillon-Lefèvre syndrome patient reveals species-dependent requirements for neutrophil defenses. J. Clin. Invest. 2014, 124, 4539-4548.

84. Keshari, R.S.; Verma, A.; Barthwal, M.K.; Dikshit, M. Reactive oxygen species-induced activation of ERK and p38 MAPK mediates PMA-induced NETs release from human neutrophils. J. Cell. Biochem. 2013, 114, 532-540.

85. Bianchi, M.; Hakkim, A.; Brinkmann, V.; Siler, U.; Seger, R.A.; Zychlinsky, A.; Reichenbach, J. Restoration of NET formation by gene therapy in CGD controls aspergillosis. Blood 2009, 114, 2619-2622.

86. Bianchi, M.; Niemiec, M.J.; Siler, U.; Urban, C.F.; Reichenbach, J. Restoration of anti-Aspergillus defense by neutrophil extracellular traps in human chronic granulomatous disease after gene therapy is calprotectin-dependent. J. Allergy Clin. Immunol. 2011, 127, $1243-1252$.

87. Papayannopoulos, V.; Staab, D.; Zychlinsky, A. Neutrophil elastase enhances sputum solubilization in cystic fibrosis patients receiving DNase therapy. PLOS ONE 2011, 6, e28526.

88. Branzk, N.; Papayannopoulos, V. Molecular mechanisms regulating NETosis in infection and disease. Semin. Immunopathol. 2013, 35, 513-530.

89. Kawakami, T.; He, J.; Morita, H.; Yokoyama, K.; Kaji, H.; Tanaka, C.; Suemori, S.; Tohyama, K.; Tohyama, Y. Rab27a is essential for the formation of neutrophil extracellular traps (NETs) in neutrophil-like differentiated HL60 cells. PLOS ONE 2014, 9, e84704.

90. Gupta, A.K.; Giaglis, S.; Hasler, P.; Hahn, S. Efficient neutrophil extracellular trap induction requires mobilization of both intracellular and extracellular calcium pools and is modulated by cyclosporine A. PLOS ONE 2014, 9, e97088.

91. Parker, H.; Dragunow, M.; Hampton, M.B.; Kettle, A.J.; Winterbourn, C.C. Requirements for NADPH oxidase and myeloperoxidase in neutrophil extracellular trap formation differ depending on the stimulus. J. Leukoc. Biol. 2012, 92, 841-849.

92. Metzler, K.D.; Fuchs, T.A.; Nauseef, W.M.; Reumaux, D.; Roesler, J.; Schulze, I.; Wahn, V.; Papayannopoulos, V.; Zychlinsky, A. Myeloperoxidase is required for neutrophil extracellular trap formation: Implications for innate immunity. Blood 2011, 117, 953-959. 
93. Conus, S.; Perozzo, R.; Reinheckel, T.; Peters, C.; Scapozza, L.; Yousefi, S.; Simon, H.-U. Caspase- 8 is activated by cathepsin D initiating neutrophil apoptosis during the resolution of inflammation. J. Exp. Med. 2008, 205, 685-698.

94. Lopes, F.; Coelho, F.M.; Costa, V.V.; Vieira, É.L.M.; Sousa, L.P.; Silva, T.A.; Vieira, L.Q.; Teixeira, M.M.; Pinho, V. Resolution of neutrophilic inflammation by $\mathrm{H}_{2} \mathrm{O}_{2}$ in antigen-induced arthritis. Arthritis Rheum. 2011, 63, 2651-2660.

95. Geering, B.; Simon, H.-U. Peculiarities of cell death mechanisms in neutrophils. Cell Death Differ. 2011, 18, 1457-1469.

96. Kunisaki, Y.; Nishikimi, A.; Tanaka, Y.; Takii, R.; Noda, M.; Inayoshi, A.; Watanabe, K.; Sanematsu, F.; Sasazuki, T.; Sasaki, T.; et al. DOCK2 is a Rac activator that regulates motility and polarity during neutrophil chemotaxis. J. Cell Biol. 2006, 174, 647-652.

97. Watanabe, M.; Terasawa, M.; Miyano, K.; Yanagihara, T.; Uruno, T.; Sanematsu, F.; Nishikimi, A.; Côté, J.-F.; Sumimoto, H.; Fukui, Y. DOCK2 and DOCK5 act additively in neutrophils to regulate chemotaxis, superoxide production, and extracellular trap formation. J. Immunol. 2014, 193, 5660-5667.

98. Hasan, R.; Rink, L.; Haase, H. Zinc signals in neutrophil granulocytes are required for the formation of neutrophil extracellular traps. Innate Immun. 2013, 19, 253-264.

99. Itakura, A.; McCarty, O.J.T. Pivotal role for the mTOR pathway in the formation of neutrophil extracellular traps via regulation of autophagy. Am. J. Physiol. Cell Physiol. 2013, 305, C348-C354.

100. Tang, B.; Cai, J.; Sun, L.; Li, Y.; Qu, J.; Snider, B.J.; Wu, S. Proteasome inhibitors activate autophagy involving inhibition of PI3K-Akt-mTOR pathway as an anti-oxidation defense in human RPE cells. PLOS ONE 2014, 9, e103364.

101. Wang, C.; Zhang, X.; Teng, Z.; Zhang, T.; Li, Y. Downregulation of PI3K/Akt/mTOR signaling pathway in curcumin-induced autophagy in APP/PS1 double transgenic mice. Eur. J. Pharmacol. 2014, 740, 312-320.

102. Eom, J.-M.; Seo, M.-J.; Baek, J.-Y.; Chu, H.; Han, S.H.; Min, T.S.; Cho, C.; Yun, C.-H. Alpha-eleostearic acid induces autophagy-dependent cell death through targeting AKT/mTOR and ERK1/2 signal together with the generation of reactive oxygen species. Biochem. Biophys. Res. Commun. 2010, 391, 903-908.

103. Zhang, L.; Wang, H.; Xu, J.; Zhu, J.; Ding, K. Inhibition of cathepsin S induces autophagy and apoptosis in human glioblastoma cell lines through ROS-mediated $\mathrm{PI} 3 \mathrm{~K} / \mathrm{AKT} / \mathrm{mTOR} / \mathrm{p} 70 \mathrm{~S} 6 \mathrm{~K}$ and JNK signaling pathways. Toxicol. Lett. 2014, 228, 248-259.

104. Neeli, I.; Radic, M. Opposition between PKC isoforms regulates histone deimination and neutrophil extracellular chromatin release. Front. Immunol. 2013, doi:10.3389/fimmu.2013.00038.

105. Byrd, A.S.; O’Brien, X.M.; Johnson, C.M.; Lavigne, L.M.; Reichner, J.S. An extracellular matrix-based mechanism of rapid neutrophil extracellular trap formation in response to Candida albicans. J. Immunol. 2013, 190, 4136-4148.

106. Borgquist, J.D.; Quinn, M.T.; Swain, S.D. Adhesion to extracellular matrix proteins modulates bovine neutrophil responses to inflammatory mediators. J. Leukoc. Biol. 2002, 71, 764-774. 
107. Begde, D.; Bundale, S.; Mashitha, P.; Rudra, J.; Nashikkar, N.; Upadhyay, A. Immunomodulatory efficacy of nisin-A bacterial lantibiotic peptide. J. Pept. Sci. 2011, 17, 438-444.

108. Arcaro, A.; Wymann, M.P. Wortmannin is a potent phosphatidylinositol 3-kinase inhibitor: The role of phosphatidylinositol 3,4,5-trisphosphate in neutrophil responses. Biochem. J. 1993, 296, 297-301.

109. Ambruso, D.R.; Cusack, N.; Thurman, G. NADPH oxidase activity of neutrophil specific granules: Requirements for cytosolic components and evidence of assembly during cell activation. Mol. Genet. MeTable 2004, 81, 313-321.

110. Lamb, F.S.; Hook, J.S.; Hilkin, B.M.; Huber, J.N.; Volk, A.P.D.; Moreland, J.G. Endotoxin priming of neutrophils requires endocytosis and NADPH oxidase-dependent endosomal reactive oxygen species. J. Biol. Chem. 2012, 287, 12395-12404.

111. Karlsson, A.; Dahlgren, C. Assembly and activation of the neutrophil NADPH oxidase in granule membranes. Antioxid. Redox Signal. 2002, 4, 49-60.

112. Fisher, A.B. Redox signaling across cell membranes. Antioxid. Redox Signal. 2009, 11, 1349-1356.

113. Morré, D.J. Preferential inhibition of the plasma membrane NADH oxidase (NOX) activity by diphenyleneiodonium chloride with NADPH as donor. Antioxid. Redox Signal. 2002, 4, 207-212.

114. Kim, Y.; Kim, Y.-S.; Kim, D.E.; Lee, J.S.; Song, J.H.; Kim, H.-G.; Cho, D.-H.; Jeong, S.-Y.; Jin, D.-H.; Jang, S.J.; et al. BIX-01294 induces autophagy-associated cell death via EHMT2/G9a dysfunction and intracellular reactive oxygen species production. Autophagy 2013, 9, 2126-2139.

115. Riganti, C.; Gazzano, E.; Polimeni, M.; Costamagna, C.; Bosia, A.; Ghigo, D. Diphenyleneiodonium inhibits the cell redox metabolism and induces oxidative stress. J. Biol. Chem. 2004, 279, 47726-47731.

116. Douda, D.N.; Khan, M.A.; Grasemann, H.; Palaniyar, N. SK3 channel and mitochondrial ROS mediate NADPH oxidase-independent NETosis induced by calcium influx. Proc. Natl. Acad. Sci. USA 2015, 112, 2817-2822.

117. Vitte, J.; Michel, B.F.; Bongrand, P.; Gastaut, J.-L. Oxidative stress level in circulating neutrophils is linked to neurodegenerative diseases. J. Clin. Immunol. 2004, 24, 683-692.

118. Maianski, N.A.; Geissler, J.; Srinivasula, S.M.; Alnemri, E.S.; Roos, D.; Kuijpers, T.W. Functional characterization of mitochondria in neutrophils: A role restricted to apoptosis. Cell Death Differ. 2004, 11, 143-153.

119. Davies, M.J. Myeloperoxidase-derived oxidation: Mechanisms of biological damage and its prevention. J. Clin. Biochem. Nutr. 2011, 48, 8-19.

120. Edwards, S.W.; Nurcombe, H.L.; Hart, C.A. Oxidative inactivation of myeloperoxidase released from human neutrophils. Biochem. J. 1987, 245, 925-928.

121. Pullar, J.M.; Vissers, M.C.; Winterbourn, C.C. Living with a killer: The effects of hypochlorous acid on mammalian cells. IUBMB Life 2000, 50, 259-266. 
122. Lloyd, M.M.; van Reyk, D.M.; Davies, M.J.; Hawkins, C.L. Hypothiocyanous acid is a more potent inducer of apoptosis and protein thiol depletion in murine macrophage cells than hypochlorous acid or hypobromous acid. Biochem. J. 2008, 414, 271-280.

123. Lane, A.E.; Tan, J.T.M.; Hawkins, C.L.; Heather, A.K.; Davies, M.J. The myeloperoxidasederived oxidant HOSCN inhibits protein tyrosine phosphatases and modulates cell signalling via the mitogen-activated protein kinase (MAPK) pathway in macrophages. Biochem. J. 2010, 430, 161-169.

124. Augusto, O.; Miyamoto, S. Oxygen Radicals and Related Species. In Principles of Free Radical Biomedicine; Nova Science Publishers: Hauppauge, NY, USA, 2011; Volume 1, Chapter 2, p. 23.

125. Bauer, G. HOCl-dependent singlet oxygen and hydroxyl radical generation modulate and induce apoptosis of malignant cells. Anticancer Res. 2013, 33, 3589-3602.

126. Mylonas, C.; Kouretas, D. Lipid peroxidation and tissue damage. Vivo Athens Greece 1999, 13, 295-309.

127. Yoon, J.H.; Lee, M.S.; Kang, J.H. Reaction of ferritin with hydrogen peroxide induces lipid peroxidation. BMB Rep. 2010, 43, 219-224.

128. Wu, J.; Teuber, K.; Eibisch, M.; Fuchs, B.; Schiller, J. Chlorinated and brominated phosphatidylcholines are generated under the influence of the Fenton reagent at low pH-a MALDI-TOF MS study. Chem. Phys. Lipids 2011, 164, 1-8.

129. Wesselius, L.J.; Nelson, M.E.; Skikne, B.S. Increased release of ferritin and iron by iron-loaded alveolar macrophages in cigarette smokers. Am. J. Respir. Crit. Care Med. 1994, 150, 690-695.

130. Ponka, P.; Beaumont, C.; Richardson, D.R. Function and regulation of transferrin and ferritin. Semin. Hematol. 1998, 35, 35-54.

131. Yuan, X.-M.; Li, W.; Baird, S.K.; Carlsson, M.; Melefors, O. Secretion of ferritin by iron-laden macrophages and influence of lipoproteins. Free Radic. Res. 2004, 38, 1133-1142.

132. Recalcati, S.; Invernizzi, P.; Arosio, P.; Cairo, G. New functions for an iron storage protein: The role of ferritin in immunity and autoimmunity. J. Autoimmun. 2008, 30, 84-89.

133. Yousefi, S.; Gold, J.A.; Andina, N.; Lee, J.J.; Kelly, A.M.; Kozlowski, E.; Schmid, I.; Straumann, A.; Reichenbach, J.; Gleich, G.J.; Simon, H.-U. Catapult-like release of mitochondrial DNA by eosinophils contributes to antibacterial defense. Nat. Med. 2008, 14, 949-953.

134. Sautin, Y.Y.; Johnson, R.J. Uric acid: The oxidant-antioxidant paradox. Nucleosides Nucleotides Nucleic Acids 2008, 27, 608-619.

135. Schorn, C.; Janko, C.; Krenn, V.; Zhao, Y.; Munoz, L.E.; Schett, G.; Herrmann, M. Bonding the foe-NETting neutrophils immobilize the pro-inflammatory monosodium urate crystals. Front. Immunol. 2012, doi:10.3389/fimmu.2012.00376.

136. Arai, Y.; Nishinaka, Y.; Arai, T.; Morita, M.; Mizugishi, K.; Adachi, S.; Takaori-Kondo, A.; Watanabe, T.; Yamashita, K. Uric acid induces NADPH oxidase-independent neutrophil extracellular trap formation. Biochem. Biophys. Res. Commun. 2014, 443, 556-561. 
137. McDonald, P.P.; Bald, A.; Cassatella, M.A. Activation of the NF- $\kappa$ B pathway by inflammatory stimuli in human neutrophils. Blood 1997, 89, 3421-3433.

138. Lapponi, M.J.; Carestia, A.; Landoni, V.I.; Rivadeneyra, L.; Etulain, J.; Negrotto, S.; Pozner, R.G.; Schattner, M. Regulation of neutrophil extracellular trap formation by anti-inflammatory drugs. J. Pharmacol. Exp. Ther. 2013, 345, 430-437. 


\title{
Oxidative Stress Responses in the Human Fungal Pathogen, Candida albicans
}

\author{
Alessandra da Silva Dantas, Alison Day, Mélanie Ikeh, Iaroslava Kos, Beatrice Achan and \\ Janet Quinn
}

\begin{abstract}
Candida albicans is a major fungal pathogen of humans, causing approximately 400,000 life-threatening systemic infections world-wide each year in severely immunocompromised patients. An important fungicidal mechanism employed by innate immune cells involves the generation of toxic reactive oxygen species (ROS), such as superoxide and hydrogen peroxide. Consequently, there is much interest in the strategies employed by C. albicans to evade the oxidative killing by macrophages and neutrophils. Our understanding of how C. albicans senses and responds to ROS has significantly increased in recent years. Key findings include the observations that hydrogen peroxide triggers the filamentation of this polymorphic fungus and that a superoxide dismutase enzyme with a novel mode of action is expressed at the cell surface of $C$. albicans. Furthermore, recent studies have indicated that combinations of the chemical stresses generated by phagocytes can actively prevent $C$. albicans oxidative stress responses through a mechanism termed the stress pathway interference. In this review, we present an up-date of our current understanding of the role and regulation of oxidative stress responses in this important human fungal pathogen.
\end{abstract}

Reprinted from Biomolecules. Cite as: da Silva Dantas, A.; Day, A.; Ikeh, M.; Kos, I.; Achan, B.; Quinn, J. Oxidative Stress Responses in the Human Fungal Pathogen, Candida albicans. Biomolecules 2015, 5, 142-165.

\section{Candida albicans Is a Major Fungal Pathogen of Humans}

The polymorphic fungus, Candida albicans, is a constituent of the normal human microbiome. This fungus, together with other Candida family members, is present on the skin and in the oral cavity and gastrointestinal and urogenital tracts of most healthy individuals [1,2]. In the healthy host, C. albicans normally exists as a benign commensal organism. However, as an opportunistic pathogen, this fungus can also cause superficial infections, such as oral or vaginal candidiasis, or life-threatening systemic infections [2]. Perturbation of the microbiome through antibiotic usage or mild to severe defects in immune defences, such as in patients with HIV, can result in superficial oral and vaginal infections (thrush), termed oral (OC) and vulvovaginal (VVC) candidiasis, respectively. OC occurs in about $90 \%$ of HIV-infected persons as an AIDS-defining illness [3]. Defective immunity in premature infants and the elderly can also result in OC [4,5]. Significantly, $75 \%$ of women of childbearing age suffer from VVC, $45 \%$ of whom go on to have a least one recurrent infection [6]. Superficial candidiasis can also manifest as chronic infections of the skin and nails, resulting in mucocutaneous candidiasis (CMC) [7]. Although superficial infections are remarkably commonplace, they are non-life threatening and can be easily treated.

In contrast, systemic candidiasis is associated with unacceptably high crude and attributable mortality rates of 42 and 27\%, respectively, despite the availability of antifungal drugs, such as the 
polyenes, azoles and echinocandins. These mortality rates exceed those attributed to sepsis caused by the most aggressive bacterial and viral pathogens [8] and are attributed to difficulties in diagnosing fungal systemic infections and the consequential delays in treatment [9]. Patients who are severely immunocompromised, such as those on immunosuppressive treatments for cancer or transplant surgery, are at risk of systemic candidiasis [10]. In such patients, the innate defence mechanisms, which are vital to prevent invasive disease, are significantly compromised [11]. Consequently, the fungus can survive in the bloodstream and subsequently colonise a number of internal organs [2]. Other risk factors include invasive clinical procedures or trauma, which disrupt the protective anatomical barrier of the mucosa, and the use of venous catheters, which can allow access of the fungus to the bloodstream [10]. Indeed, overall, Candida spp. are the fourth most common nosocomial (hospital acquired) systemic infection in the United States [8]. Clearly, C. albicans poses a significant medical problem, and thus, it is important that we understand what makes this fungus such a successful pathogen.

\section{Reactive Oxygen Species Are a Core Component of the Immune Cell Armoury}

In healthy hosts, the first line of defence against $C$. albicans is through phagocytosis by innate immune cells, including macrophages and neutrophils. A major antimicrobial defence mechanism mounted by these phagocytes is the production of reactive oxygen species (ROS) through a process known as the respiratory burst. Following stimulation by cytokines, phagocytic cells activate the assembly of the NADPH oxidase complex, which results in the generation of superoxide $\left(\mathrm{O}_{2}^{-} \bullet\right)$. Given the potency of the ROS produced by NADPH oxidase [12], activation of this multi-subunit enzyme is tightly regulated. The NADPH oxidase complex consists of the Nox2 (gp91 ${ }^{\text {phox }}$ ) catalytic subunit, the $\mathrm{p} 22^{\text {phox }}$ transmembrane protein and three cytosolic subunits, $\mathrm{p} 47^{\text {phox }}, \mathrm{p} 67^{\text {phox }}$ and $\mathrm{p} 40^{\text {phox }}$. Nox 2 and $\mathrm{p} 22^{\text {phox }}$ make up the membrane-associated cytochrome $b_{558}$ heterodimer. Activation of Nox 2 is dependent on the interaction with the cytosolic components, in particular p6 $7^{\text {phox }}$, which translocate to the membrane following phagocytosis [13]. This interaction is dependent on the binding of the small GTPase Rac to p67 ${ }^{\text {phox }}$, which induces a conformation change in this subunit, thus promoting its interaction with Nox2 [14]. Activation of Nox2 drives the production of superoxide via the NADPH-driven reduction of molecular oxygen. This is generated at an extremely high rate of 5 to $10 \mathrm{nmol}$ per s within the neutrophil phagosome [15], and it has been estimated that approximately $4 \mathrm{~mol} \mathrm{~L}^{-1}$ of $\mathrm{O}_{2}^{-} \cdot$ is produced per bacterium engulfed in the phagocytic vacuole [16]. The superoxide is then dismutated to hydrogen peroxide $\left(\mathrm{H}_{2} \mathrm{O}_{2}\right)$ by superoxide dismutase or to hydroxyl anions $\left(\mathrm{OH}^{-}\right)$and hydroxyl radicals $(\mathrm{OH})$ via the Haber-Weiss reaction. The importance of the NADPH oxidase-mediated respiratory burst as an antimicrobial mechanism is manifested in patients with chronic granulomatous disease (CGD). CGD is a human genetic disorder characterized by a deficiency in the NAPDH oxidase complex and is associated with recurrent and life-threatening bacterial and fungal infections [17]. Significantly, patients with CGD have an increased susceptibility to Candida infections [18]. Interestingly, in addition to the fungicidal roles of ROS, recent work has revealed that the ROS produced by NADPH oxidase also functions to recruit phagocytes to C. albicans infection foci. This NADPH oxidase-regulated recruitment of phagocytes is 
important for efficient phagocytosis, containment of the fungus within the phagocyte and survival of the host [19].

Other toxic chemicals are subsequently derived from the ROS in the phagosome [20]. For example, $\mathrm{H}_{2} \mathrm{O}_{2}$ can react with chloride ions $\left(\mathrm{Cl}^{-}\right)$to form hypochlorous acid $(\mathrm{HOCl})$ in a reaction catalysed by myeloperoxidase (MPO). In addition, the nitric oxide radical generated by the action of the inducible nitric oxide synthase (iNOS) interacts with superoxide to produce the highly toxic peroxynitrite (ONOO) [21]. Recently, work has also revealed that the combination of reactive oxygen species together with the cationic stress generated during phagocyte maturation underlies the potency of phagocytes in C. albicans killing [22]. Thus, phagocytic cells synthesize an array of toxic chemicals that work in combination to promote fungal killing. It is also noteworthy that, in addition to ROS production within the phagosome, phagocytes secrete ROS into the external milieu [23]. Consistent with this, C. albicans cells have been shown to mount an oxidative stress response prior to phagocytosis [24]. Furthermore, C. albicans will also come in contact with ROS produced by $\mathrm{H}_{2} \mathrm{O}_{2}$-producing bacteria in the mouth and gut. Several commensal bacteria, for example Enterococcus faecalis [25] and Lactobacillus species [26], secrete ROS into their surroundings, and this may have an inhibitory effect on the growth of $C$. albicans in host niches, other than the phagosomal environment. Consistent with this, using a Caenorhabditis elegans model of polymicrobial infection, E. faecalis was shown to reduce the virulence of C. albicans [27].

The ROS generated within the phagosome creates a toxic environment that induces oxidative stress in C. albicans. Indeed, exogenous ROS can induce programmed cell death in this fungal pathogen [28]. ROS interact with proteins, lipids and nucleic acids [29], causing irreversible damage to the pathogen. DNA damage caused by ROS can result in chemical base changes, structural alterations, single- and double-strand breaks and cross-linkage. Lipid peroxidation occurs by a free radical chain reaction, which culminates in peroxidation events at many fatty acid side chains, leading to the damage of the cell membrane. ROS reactions with proteins can lead to the formation of protein-protein cross-links, oxidation of the peptide backbone and reversible or irreversible oxidation of amino acid side chains. Although this can be deleterious to protein function, as discussed below, several oxidative stress-sensing proteins are activated by the reversible oxidation of cysteine residues.

\section{Response of Candida albicans to ROS}

\subsection{Transcriptional Responses to ROS}

A well-characterized response of eukaryotic microbes to ROS is the rapid induction of mRNAs that encode oxidative stress detoxification and repair proteins. Interestingly, C. albicans is considerably more resistant to oxidative stress than the benign model yeasts, Schizosaccharomyces pombe and Saccharomyces cerevisiae [30,31]. However, the basis for this resistance does not appear to be due to differences in transcriptional responses to oxidative stress, as all three fungi appear to induce a similar set of core antioxidant genes following exposure to $\mathrm{H}_{2} \mathrm{O}_{2} \quad[32,33]$. These include catalase $(C A T 1)$, glutathione peroxidase $(G P X)$ and superoxide dismutase $(S O D)$ antioxidant-encoding genes, in addition to genes encoding components of the 
glutathione/glutaredoxin (GSH1, TTRI) and thioredoxin (TSA1, TRX1, TRR1) systems, which play critical roles in repairing oxidatively-damaged proteins, protein folding and sulphur metabolism. Such oxidative stress-responsive genes are also induced in C. albicans following exposure to macrophages or neutrophils [34-38], illustrating that this pathogen induces the respiratory burst in these phagocytes. The analyses of GFP-reporter fusions, under the control of oxidative stress-responsive promoters, have also revealed that $C$. albicans is exposed to significant levels of ROS prior to phagocytosis [24]. In contrast, however, oxidative stress responses do not appear to be induced once $C$. albicans cells have established systemic kidney infections [34,39,40]. Thus, inducible oxidative stress responses appear vital for $C$. albicans to survive phagocytosis by innate immune cells, but are seemingly less important for the fungus to develop systemic infections. Indeed, whilst a number of genes encoding key antioxidants (such as CAT1, TRX1, GRX2, SOD1, SOD5) are important for virulence in systemic models of infection [41-45], others (including TSA1, GPXs) are dispensable $[46,47]$.

\subsection{Transcriptional Responses to ROS Are Inhibited in the Presence of Cationic Stress}

In healthy individuals, $C$. albicans cannot evade the oxidative-killing mechanisms mounted by innate immune cells. Such cells prevent infection by employing a battery of toxic chemicals in addition to ROS. For example, phagocytes expose $C$. albicans to cationic fluxes $\left(\mathrm{K}^{+}\right)$and acidification, as well as to superoxide anions $[16,21,48]$. However, as $C$. albicans is resistant to each of these individual stresses in vitro [30,31], a key question, therefore, is: what accounts for the potency of innate immune defences? Although host microenvironments are complex and dynamic, our understanding of $C$. albicans stress responses is based on studies of individual stresses. Significantly, however, recent work has revealed that $C$. albicans is exquisitely sensitive to combinations of oxidative and cationic stresses [49], which are encountered following phagocytosis. Cationic stress can be imposed in vitro by exposure of the fungus to either $\mathrm{NaCl}$ or $\mathrm{KCl}$ and in the phagocyte is caused by increased flux of $\mathrm{K}^{+}$into the phagosome [16]. Strikingly, exposure to cationic stress results in the inhibition of $C$. albicans oxidative stress responses. This phenomenon has been termed "stress pathway interference" [22]. The combinatorial stress-mediated synergistic killing of C. albicans contrasts starkly with the stress cross-protection described in model yeasts, whereby exposure to one stress protects against subsequent exposure to a different stresses [50]. The existence of stress pathway interference was revealed through gene expression analysis in C. albicans. Transcript profiling showed that $\mathrm{H}_{2} \mathrm{O}_{2}$-induced gene expression is severely attenuated, and intracellular ROS levels increase dramatically, following combinatorial oxidative and cationic stress. For example, key antioxidant genes, such as $C A T 1$ encoding catalase and TRR1 encoding thioredoxin reductase, which are significantly induced following $\mathrm{H}_{2} \mathrm{O}_{2}$ stress, fail to be induced following exposure of cells to $\mathrm{H}_{2} \mathrm{O}_{2}$ in the presence of cationic stress [22]. This cationic stress-mediated inhibition of oxidative stress responses appears to be of physiological relevance, as the high fungicidal activity of human neutrophils is dependent on the combinatorial effects of the oxidative burst and cationic fluxes [22]. However, as discussed above, oxidative stress-responsive genes are induced following co-culture of C. albicans with phagocytes [34,35], so how is this reconciled with the combinatorial stress-mediated inactivation of such genes? It has been suggested [22] that the activation of $C$. albicans antioxidant 
genes during interaction with phagocytes may be due to exposure to extracellular ROS prior to engulfment [24]. Furthermore, as exposure of cells to combinatorial oxidative and cationic stresses prevents the normal activation of oxidative stress-responsive genes, this may explain why C. albicans oxidative stress genes are not expressed in certain host niches, such as during systemic infections of the kidney, despite the presence of neutrophil infiltrates [34].

\subsection{Extracellular Antioxidant Enzymes as a Pathogen-Specific Adaptation Mechanism}

As $C$. albicans appears to mount standard transcription responses to oxidative stress, the high level of resistance of this pathogen to ROS could, instead, be related to the evolutionary expansion of the SOD family and the fact that this pathogen expresses SODs and other antioxidant enzymes on the cell surface. C. albicans contains six SOD enzymes distributed between different cellular compartments. Sod1-3 are intracellular enzymes, while Sod4-6 are glycosylphosphatidylinositol (GPI)-anchored cell wall-associated enzymes. The $\mathrm{Cu}$-/Zn-containing Sod1 is induced following phagocytosis and is required for $C$. albicans to resist macrophage-mediated killing [43]. The extracellular Sods also have vital roles in the detoxification of superoxide radicals generated by phagocytes; co-culture of macrophages with C. albicans cells lacking Sod4 and Sod5 leads to massive extracellular ROS accumulation in vitro [23]. Consequently, inactivation of Sod4 and Sod5 results in C. albicans cells that are exquisitely more susceptible to phagocyte-mediated killing [23,35]. Interestingly, the expression of Sod4 and Sod5 is dependent on the morphology of C. albicans, as Sod4 is expressed in yeast cells, whereas Sod5 is a hyphal-induced gene [44,51]. Sod5 is also induced following phagocytosis by neutrophils independently of hyphae formation [35]. Recently, structural analysis of Sod5 revealed that it represents a novel class of superoxide dismutases that only depends on $\mathrm{Cu}$ for activity. Furthermore, it is secreted in its apo-form and can readily capture extracellular copper without the aid of a $\mathrm{Cu}$ chaperone, which rapidly induces activity [52]. It is suggested that this novel mode of activation is uniquely adapted to the host environment, as macrophages release copper in an attempt to kill invading microbes through copper toxicity [53]. In addition to specific Sods, two key peroxidase enzymes have also been found at the cell surface of $C$. albicans; the thiol-specific peroxidase Tsa1 [47,54] and the peroxide detoxifying enzyme catalase [54]. Tsa1 and Cat1 were identified as major plasminogen-binding proteins in isolated cell wall protein preparations [54], and the cell wall localization of Tsal has also been illustrated using fluorescence microscopy [47]. These extracellular mechanisms for protection against ROS likely reflect an adaptation of this pathogenic fungus to prevent the intracellular accumulation of toxic levels of ROS.

\subsection{Morphogenesis as an Oxidative Stress Response}

Following phagocytosis, C. albicans can evade oxidative-killing by macrophages and neutrophils by switching from budding to filamentous cells, which can pierce the phagosomal membrane [37]. Not only does this allow the pathogen to escape, but this also results in the C. albicans-mediated killing of the phagocyte [55]. It has recently been demonstrated that the ability of C. albicans to mount robust oxidative stress responses is vital for this polymorphic pathogen to filament in the phagosome [46,56]. C. albicans mutants that are sensitive to ROS in vitro fail to filament once phagocytosed and, thus, are trapped within the macrophage and unable to evade phagocyte-mediated 
killing. Consistent with the requirement of fungal oxidative stress defences to allow filamentation and macrophage escape, the phagocyte NADPH oxidase is important in inhibiting filamentation in vivo [57]. The ROS produced by the NADPH oxidase also function to recruit phagocytes, thereby increasing phagocytosis and inhibiting filamentation [19]. Thus, the outcome of the battle between $C$. albicans and innate immune cells appears dependent on the NADPH oxidase-regulated functions of the phagocyte and the robustness of the fungal oxidative stress responses.

The mechanisms underlying $C$. albicans filamentation following phagocytosis remain to be fully explored. A recent study reported that the ROS-induced induction of arginine biosynthesis genes is important for hyphal formation following phagocytosis of C. albicans [58]. Moreover, exposure of C. albicans to the $\mathrm{ROS} \mathrm{H}_{2} \mathrm{O}_{2}$ triggers the filamentation of this polymorphic fungus in vitro [42,59]. A close examination of the morphology of these cells revealed that $\mathrm{H}_{2} \mathrm{O}_{2}$-induced filaments are hyperpolarized buds, which are morphologically distinct from hyphae and pseudohyphae filamentous forms [42]. The hyperpolarised bud is a relatively recently characterized filamentous form of C. albicans and is normally associated with either mutations or chemicals that perturb cell cycle progression $[60,61]$. The observation that $\mathrm{H}_{2} \mathrm{O}_{2}$ stimulates hyperpolarised bud formation provided the first example of a physiologically relevant condition that induces this filamentous form of growth in C. albicans. Does exposure of ROS following phagocytosis trigger the formation of hyperpolarised buds allowing this pathogen to pierce the phagocyte membrane and escape? Evidence so far indicates that ROS-stimulated hyperpolarized bud formation may not contribute to $C$. albicans filamentation within the macrophage. For example, ROS-sensitive C. albicans mutants that cannot filament within the macrophage [56] can form $\mathrm{H}_{2} \mathrm{O}_{2}$-induced hyperpolarized buds in vitro [42]. Nonetheless, ROS-stimulated filamentation of $C$. albicans may be beneficial for survival in other host niches, such as the gut, where C. albicans co-exists with ROS-producing bacteria.

\section{Signalling Pathways that Mediate $C$. albicans Responses to ROS}

To date, three signalling pathways have been demonstrated to be directly activated in response to ROS in C. albicans. These include the Cap1 transcription factor, the Hog 1 stress-activated protein kinase and the Rad53 DNA damage checkpoint kinase. Here, we discuss the role and regulation of these pathways in oxidative stress responses in C. albicans. Other signalling pathways not known to be activated by ROS, but which contribute to oxidative stress tolerance in $C$. albicans are also briefly summarized.

\subsection{The Cap1 ROS-Responsive Transcription Factor}

In C. albicans, the Cap1 transcription factor is the major regulator of the oxidative stress-induced transcriptome and proteome, both in vitro [62,63] and ex vivo, following exposure to neutrophils [35]. Cap1 is a bZip transcription factor of the AP-1 family and is closely related to the $S$. cerevisiae Yap1 and $S$. pombe Pap1 proteins, which have well-characterized roles in oxidative stress and multi-drug resistance [64,65]. Similarly, C. albicans cap1 $\Delta$ cells are sensitive to several reactive oxygen species and drugs $[33,66,67]$. Chromatin immunoprecipitation (CHiP) analysis to determine direct targets of Cap1 identified many key antioxidant genes, including CTA1 and TRXI, 
and those involved in the response to drugs, such as $M D R 1$ [68]. Cap1 plays a role in recruiting the Ada2 component of the SAGA/ADA histone acetylase co-activator complex to the promoters of oxidative stress and drug-responsive target genes [69,70]. Cells lacking Ada2 are highly sensitive to ROS, and the oxidative stress-induced transcription of key Cap1 target genes is significantly impaired; therefore, Cap1 recruitment of the SAGA complex appears to be a vital component of the oxidative stress response in C. albicans.

\subsubsection{Regulation of Cap1}

Similar to that reported for S. cerevisiae Yap1, C. albicans Cap1 rapidly accumulates in the nucleus in response to $\mathrm{H}_{2} \mathrm{O}_{2}$ [56,67]. Under non-stressed conditions, Yap1 shuttles between the cytoplasm and the nucleus due to the interaction of a nuclear export sequence (NES), located at the C-terminus of these transcription factors, with the Crm1 nuclear export factor [71]. However, following exposure to $\mathrm{H}_{2} \mathrm{O}_{2}$, Yap1 is activated by oxidation of specific cysteine residues, resulting in disulphide bond formation between two cysteine-rich domains ( $\mathrm{n}-\mathrm{CRD}$ and c-CRD). This triggers a conformational change within Yap1 that masks the NES, thereby preventing its interaction with Crm1. The inability to be recognized by Crm1 leads to the nuclear accumulation of Yap1, the nuclear-dependent phosphorylation of this transcription factor and the induction of Yap1-dependent genes [72]. Conversely, activation of Yap1 is counteracted by the thioredoxins Trx1 and Trx2, which function to reduce oxidised Yap1 [72]. This basic mechanism of regulation is conserved in C. albicans (Figure 1). Mutation of the c-CRD affects Cap1 regulation [67], and Cap1 is rapidly oxidised following exposure to $\mathrm{H}_{2} \mathrm{O}_{2}$ [42]. In addition, following the nuclear accumulation of Cap1, this transcription factor becomes phosphorylated, and the induction of Cap1-dependent genes is observed. Furthermore, as seen in $S$. cerevisiae, thioredoxin functions to reverse the $\mathrm{H}_{2} \mathrm{O}_{2}$-induced oxidation and activation of Cap1 [42].

Fungal AP-1-like transcription factors are not directly oxidised by $\mathrm{H}_{2} \mathrm{O}_{2}$, but instead, specific peroxidase enzymes sense and transduce the $\mathrm{H}_{2} \mathrm{O}_{2}$ signal to these transcription factors (Figure 1). Similar to that observed in $S$. cerevisiae [72], Cap1 oxidation requires Gpx3, a glutathione peroxidase (Gpx)-like enzyme [56]. Studies with Yap1 showed that this transcription factor undergoes multiple oxidation events, with Gpx3 initiating Yap1 oxidation [73-75]. Similarly, multiple oxidized forms of Cap1 are also observed [56]. Gpx3-mediated oxidation of Yap1 and Cap1 also requires a second protein, Ybp1, which binds to and forms a complex with the AP-1-like factors [56,76]. A recent study has provided insight into an additional function of $\mathrm{Ybp} 1$ in both $C$. albicans and $S$. cerevisiae, as Cap1 and Yap 1 are highly unstable in $y b p 1 \Delta$ cells [56]. Ubiquitin-mediated degradation of oxidised AP-1-like factors has recently been shown to be an important regulatory mechanism [77,78]; therefore, Ybp1 binding to the reduced cytoplasmic pools of Yap1 or Cap1 possibly functions to prevent this proteasome-mediated degradation [56]. 
A

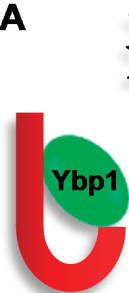

Cap1RED

cytoplasm

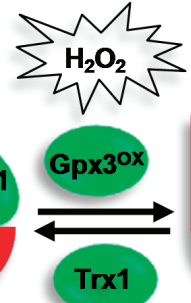

Trx1

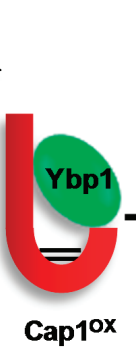

Cap10x

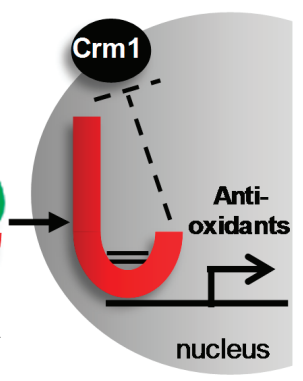

B

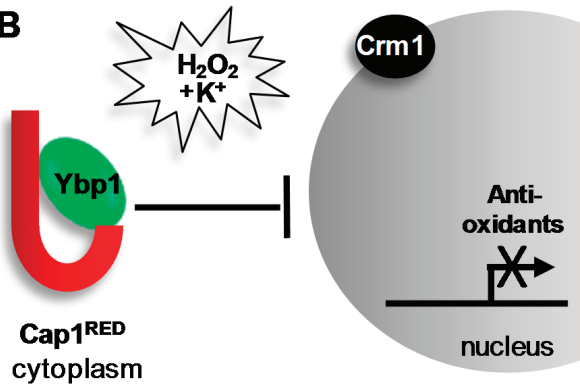

Figure 1. $\mathrm{H}_{2} \mathrm{O}_{2}$-induced activation of Capl is inhibited in the presence of cations. (A) Exposure of C. albicans to $\mathrm{H}_{2} \mathrm{O}_{2}$ promotes the $\mathrm{Gpx} 3 / \mathrm{Ybp} 1$-mediated oxidation and activation the Cap1 transcription factor $\left(\mathrm{Cap} 1^{\mathrm{ox}}\right)$. Cap $1^{\mathrm{ox}}$ can no longer interact with the Crm1 nuclear export factor resulting in its nuclear accumulation, and the subsequent Cap1-dependent induction of genes with antioxidant functions necessary for cell survival. Following cellular adaptation, Cap $1^{\text {ox }}$ is returned to the inactive reduced form $\left(\mathrm{Cap} 1^{\mathrm{RED}}\right.$ ) by thioredoxin (Trx1); (B) Remarkably, when C. albicans cells are exposed to $\mathrm{H}_{2} \mathrm{O}_{2}$ in the presence of cations, Cap1 fails to accumulate in the nucleus and therefore antioxidant gene expression is not induced leading to cell death. This is important as, following phagocytosis, C. albicans is exposed simultaneously to ROS and cationic fluxes. See text for details.

In $S$. cerevisiae, Yap1 functions alongside the $\operatorname{Skn} 7$ response regulator transcription factor, to regulate antioxidant gene expression [79]. An orthologue of Skn7 has been identified in C. albicans [80]. The overall domain architecture is conserved and comprised of a DNA-binding domain, a coiled-coil domain and a receiver domain (analogous to those in response regulator proteins of two-component signal transduction pathways). It is not known whether Skn7 acts alongside Cap1 in C. albicans. However, C. albicans cells lacking Skn7 display increased sensitivity to ROS, including $\mathrm{H}_{2} \mathrm{O}_{2}$, consistent with this transcription factor regulating oxidative stress-induced gene expression [80].

Interestingly, Cap1 fails to be activated following exposure to combinatorial oxidative and cationic stress (Figure 1), which underlies the lack of antioxidant gene expression following this combinatorial stress treatment (Section 3.2). In contrast with that seen following oxidative stress, following combinatorial cationic plus oxidative stress treatments, Cap1 fails to accumulate in the nucleus [22]. Consequently, Cap1 is not phosphorylated, and Cap1-dependent oxidative stress genes are not induced [22]. The impact of stress pathway interference upon Cap1 signalling underlies the potency of combinatorial cationic plus oxidative stress, as ectopic expression of the Cap1-dependent catalase gene, CAT1, rescues the hypersensitivity to the combinatorial stress [22]. However, the mechanism underlying combinatorial stress-mediated inactivation of Cap1 is not known. Cations inhibit catalase function, which results in high levels of intracellular ROS [22]. Whether high levels of ROS result in Cap1 inactivation or whether cations inhibit Cap1 activation in other ways remains to be determined. 


\subsubsection{Role of Cap1 in Virulence}

Loss of Cap1 or its regulators Gpx3 and $\mathrm{Ybp} 1$ attenuates virulence in some, but not all infection models. For example, cells lacking Cap1, Gpx3 or Ybp1 are unable to kill macrophages, due to the inability of these mutant strains to filament following phagocytosis [56]. Consequently, cells lacking Cap1 or its regulators are sensitive to macrophage- and neutrophil-mediated killing [35,56,81]. Cap1, Gpx3 and Ybp1 are also vital for C. albicans virulence in a Galleria mellonella model of infection [56], and Cap1 is important for virulence in a Caenorhabditis elegans infection model in nematode hosts that have a functional NADPH oxidase [81]. In contrast, Cap1, Gpx3 and Ybp1 are dispensable for $C$. albicans virulence in murine systemic infection models [56,81]. Similar findings were reported for $\operatorname{Skn} 7$ [80]. The observation that Cap1 is dispensable for virulence in murine systemic models of infection was unexpected, as certain genes that are induced by Cap1 in response to $\mathrm{H}_{2} \mathrm{O}_{2}$, such as CTA1 and TRX1, are important for C. albicans survival in such models [42,45]. This indicates that Cap1-independent basal levels of such genes may be important for virulence in such models and that Cap1-mediated gene expression is not vital for the establishment of systemic infections.

\subsection{The Hog1 SAPK}

Stress-activated MAPKs are conserved signalling molecules that promote the ability of cells to adapt to environmental change [82]. They are components of a three-tiered core signalling module that comprises the SAPK itself, a MAP kinase kinase (MAPKK) and a MAPKK kinase (MAPKKK). Activation of the MAPKKK results in the phosphorylation and activation of the MAPKK, which, in turn, culminates in the phosphorylation of the SAPK on conserved threonine and tyrosine residues located within the TGY motif in the phosphorylation lip of the catalytic domain. This induces the activation and nuclear accumulation of the kinase [83] and the proline-directed phosphorylation of Ser/Thr residues on diverse substrates, including transcription factors, kinases, cell cycle regulators and membrane proteins, thus eliciting appropriate cellular responses. In C. albicans, Hog 1 is robustly phosphorylated and rapidly accumulates in the nucleus following exposure of cells to $\mathrm{H}_{2} \mathrm{O}_{2}$ [33]. In addition, cells lacking Hog 1 display increased sensitivity to a range of ROS, indicating that Hog 1 activation is a critical component of the oxidative stress response in C. albicans [84,85]. Interestingly, $\mathrm{Hog} 1$ is only activated following exposure of $C$. albicans cells to relatively high levels of $\mathrm{H}_{2} \mathrm{O}_{2}$ compared to the analogous Sty1 SAPK in the model yeast, $S$. pombe. This may reflect an adaption of this pathogenic fungus to restrict Hog1 activation to ROS-rich environments during infection [85]. Despite the increased $\mathrm{H}_{2} \mathrm{O}_{2}$ sensitivity exhibited by hog $1 \Delta$ cells and significant phosphorylation of $\mathrm{Hog} 1$ in response to $\mathrm{H}_{2} \mathrm{O}_{2}$, transcript profiling experiments revealed that $\mathrm{Hog} 1$ is largely dispensable for $\mathrm{H}_{2} \mathrm{O}_{2}$-induced gene expression [33]. Although a small subset of $\mathrm{H}_{2} \mathrm{O}_{2}$-responsive genes were identified that showed Hog1-dependent induction, subsequent analysis failed to identify any genes coding for proteins with known antioxidant functions [33]. This is in contrast with $S$. pombe, where Styl is required for the activation of the core stress genes in response to $\mathrm{H}_{2} \mathrm{O}_{2}$, including genes encoding important antioxidants, such as catalase and glutathione peroxidase [86]. What, therefore, is the role of Hog1 in the C. albicans oxidative stress response if it is not required for the induction 
of antioxidant gene expression? One possibility is that Hog 1 contributes to the oxidative stress response at a post-transcriptional level in $C$. albicans. Indeed, the $S$. pombe Sty1 SAPK has been shown to interact with translation factors [87]. However, Hog 1 does not play a major role in regulating the oxidative stress-induced proteome, although proteomic experiments did indicate that Hog1 might be required to ensure the prolonged expression of some proteins during recovery from $\mathrm{H}_{2} \mathrm{O}_{2}$ stress [88]. Loss of Hog1 has been shown to affect respiratory function [89], although it is unclear whether this underlies the sensitivity of hog $1 \Delta$ cells to ROS. One downstream target of Hog 1 regulated by $\mathrm{H}_{2} \mathrm{O}_{2}$ stress is the Mkc1 cell integrity MAPK. Mkcl is rapidly phosphorylated in response to $\mathrm{H}_{2} \mathrm{O}_{2}$ stress in a Hog1-dependent mechanism, although $\mathrm{Mkc1}$ is not required for cell survival in response to $\mathrm{H}_{2} \mathrm{O}_{2}$ stress [90]. In addition, the Skol transcription factor is a target of the Hog1 SAPK in C. albicans, as this becomes phosphorylated following stress in a Hog1-dependent manner [91]. However, consistent with Hog1 not playing a major role in regulating oxidative stress-induced gene expression, the $\mathrm{H}_{2} \mathrm{O}_{2}$-induced transcriptome is not dependent on Sko1 [92]. Thus, in C. albicans, Hog1 regulation of the oxidative stress response must involve targets in addition to Mkc1 and Sko1 (Figure 2).

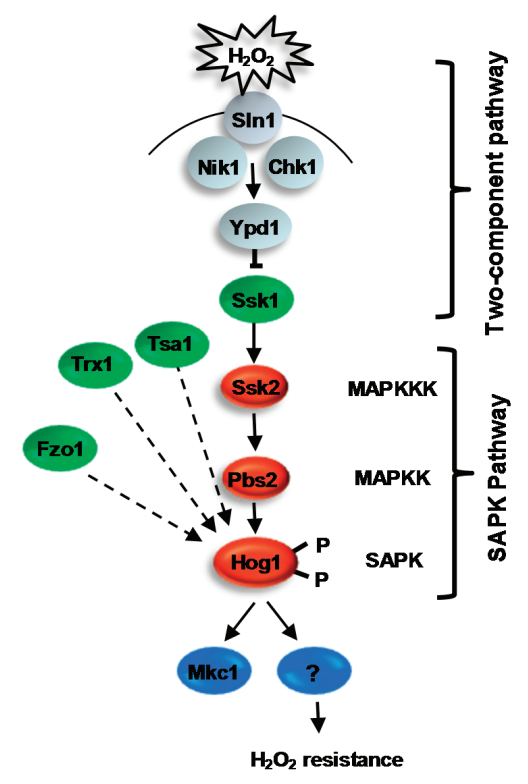

Figure 2. $\mathrm{H}_{2} \mathrm{O}_{2}$-induced activation of the $\mathrm{Hog} 1$ SAPK. In response to $\mathrm{H}_{2} \mathrm{O}_{2}, \mathrm{Hog} 1$ becomes rapidly phosphorylated and accumulates in the nucleus, and $C$. albicans cells lacking $\mathrm{Hog} 1$ are sensitive to oxidative stress. Proteins required for $\mathrm{H}_{2} \mathrm{O}_{2}$-induced activation of Hog1 are shown in green. These include the response regulator Ssk1 (but no other two-component protein), the redox sensitive antioxidants Tsa1 and Trx1, and the mitochondria biogenesis factor Fzol. Following $\mathrm{H}_{2} \mathrm{O}_{2}$-induced activation, $\mathrm{Hog} 1$ phosphorylates the Mkc1 MAPK. However, cells lacking Mkc1 are not sensitive to oxidative stress, suggesting that an, as yet, unknown Hog1 substrate(s), mediates oxidative stress resistance. 


\subsubsection{Regulation of Hog1 in Response to ROS}

Whilst little is known regarding the cellular targets of Hog and the cellular role(s) of this kinase in promoting oxidative stress tolerance, more progress has been made in delineating how $\mathrm{H}_{2} \mathrm{O}_{2}$ signals are relayed to Hog1 (Figure 2). Oxidative stress-induced activation of Hog 1 is entirely dependent on the Pbs2 MAPKK [93], which, in turn, is regulated by a single MAPKKK Ssk2 [94]. Furthermore, a recent study reported that deletion of a mitochondrial biogenesis factor, Fzo1, significantly impairs the $\mathrm{H}_{2} \mathrm{O}_{2}$-induced activation of Hog1 [95]. Thus, intriguingly, functional mitochondria may play an important role in the regulation of the Hog1 pathway in response to oxidative stress. In addition, both two-component related proteins and redox-sensitive antioxidants are necessary for the activation of the Hog1 SAPK in response to oxidative stress, and these will be described in turn.

\subsubsection{Two-Component Mediated Regulation of Hog1}

In the model yeasts, two-component signalling pathways have been shown to play an important role in the sensing and transmission of stress signals to their respective SAPK pathways. Such pathways are comprised of a histidine kinase, an intermediary phosphorelay protein and a response regulator protein. In $S$. cerevisiae, the $S \ln 1$ histidine kinase is inactivated in response to osmotic stress. This halts phosphorelay through the Ypd1 phosphorelay protein, leading to the rapid dephosphorylation of the Ssk1 response regulator. Dephosphorylated Ssk1 is a potent activator of the Ssk2/Ssk22 MAPKKKs in S. cerevisiae, which regulate Hog1 activation [96,97]. In C. albicans, deletion of the analogous SSK1 gene prevents Hog1 activation in response to oxidative stress, and consistent with this, ssk1 $\Delta$ cells are sensitive to oxidative stress [98]. Although Ssk1 is involved in the transmission of oxidative stress signals to Hog1, the identity of the histidine kinase(s) responsible for sensing and signalling oxidative stress signals to Ssk1 in C. albicans remains elusive [99,100]. Of the three histidine kinases present in C. albicans, Chk1 would appear to be the most likely candidate for a potential peroxide-sensing histidine kinase, as this shows significant similarity to the $S$. pombe peroxide-sensing histidine kinases, Mak2 and Mak3 [101,102]. However, deletion of CHK1 alone or in combination with either of the genes encoding the two remaining histidine kinases, SLN1 or NIK1, does not impair $\mathrm{H}_{2} \mathrm{O}_{2}$-induced activation of $\mathrm{Hog} 1$ [100,103]. Hence, it is currently unclear as to which histidine kinase(s) senses oxidative stress and regulates phosphorelay to Ssk1. Moreover, observations that Hog1 activation is seen in cells expressing a non-phosphorylatable Ssk1 mutant [103] or in cells lacking the Ypd1 phosphorelay protein in which Ssk1 is predicted to be unphosphorylated [104] indicate that $\mathrm{Ssk} 1$ may relay $\mathrm{H}_{2} \mathrm{O}_{2}$ signals to $\mathrm{Hog} 1$ in a mechanism independent of two-component signalling. It is also noteworthy that a novel response regulator, named $\mathrm{Crr} 1 / \mathrm{Srr} 1$, has been recently identified that is only present in fungi belonging to the Candida clade [105,106]. Cells lacking Crr1 or expressing a mutant lacking the predicted aspartate phosphorylation site are sensitive to $\mathrm{H}_{2} \mathrm{O}_{2}$ [105]. However, in contrast with Ssk1, Crr1 is not required for the $\mathrm{H}_{2} \mathrm{O}_{2}$-induced activation of $\mathrm{Hog} 1$ [105]. Thus, whilst this novel response regulator mediates the response of C. albicans to $\mathrm{H}_{2} \mathrm{O}_{2}$, it does so in a Hog1-independent manner. Finally, in S. cerevisiae, the transmembrane protein, Sho1, relays osmotic stress signals to the Hog1 SAPK in parallel with the Sln1-mediated two-component signalling pathway [107]. In C. albicans, the analogous Shol protein appears to have been reassigned 
to oxidative stress signalling [100]. However, it is not clear how this is mediated, as Sho1 is not required for ROS-stimulated activation of the Hog1 pathway [100].

\subsubsection{Redox-Sensitive Antioxidant Proteins as Regulators of Hog1}

It is now well recognized that redox-sensitive antioxidant proteins have important sensing and signalling roles in the cellular response to oxidative stress [108]. In C. albicans, the redox-sensitive thioredoxin peroxidase enzyme, Tsa1, is specifically required for $\mathrm{H}_{2} \mathrm{O}_{2}$-induced activation of $\mathrm{Hog} 1$ [42]. This is similar to that previously reported in $S$. pombe, as $\mathrm{H}_{2} \mathrm{O}_{2}$-induced activation of the Sty 1 SAPK also requires the analogous thioredoxin peroxidase enzyme, Tpx1 [109]. In S. pombe, intermolecular disulphide bonds are formed between conserved cysteine residues in Sty 1 and Tpx 1 following $\mathrm{H}_{2} \mathrm{O}_{2}$ stress, which suggests that Tpx1 regulates Styl function directly. However, the mechanism of Tsa1 regulation of Hog 1 in C. albicans may be different, as the conserved peroxidatic cysteine residue of Tsa1, which is essential for Tpx 1 regulation of Sty 1 , is dispensable for Tsa1 regulation of Hog1 [42]. Furthermore, the thioredoxin enzyme, Trx1, which regulates the redox status of Tsa1, is also essential for the relay of oxidative stress signals to the Hog1 SAPK module [42]. eletion of TRX1 or mutation of the catalytic cysteine residues of Trx1 drastically impairs Hog1 phosphorylation in response to $\mathrm{H}_{2} \mathrm{O}_{2}$. However, it would appear that Trx 1 regulates Hog1 independently of Tsa1, as the catalytic cysteine residues of Tsa1, which are reduced by Trx1, are dispensable for Hog1 activation [42]. In mammalian systems, thioredoxin functions as a repressor of the Hog1-related JNK and p38 SAPK signalling cascades [110]. The upstream Ask1 MAPKKK in the mammalian SAPK pathways is activated via cysteine oxidation, and Trx1 negatively regulates this pathway by reducing the oxidized cysteines of Ask1 [111,112]. As Trx1 is a positive regulator of the Hog1 SAPK in C. albicans, it seems unlikely that a similar mechanism is in place. It is also interesting to note that protein tyrosine phosphatases, which are negative regulators of SAPKs, are susceptible to inactivation by oxidation of their catalytic cysteine residue [113]. Whether thioredoxin regulates such phosphatases that dephosphorylate Hog1 in C. albicans, however, remains to be established.

\subsubsection{Role of the Hog1 SAPK in Virulence}

The stress-activated MAPK Hog1 in C. albicans is phosphorylated and accumulates in the nucleus, in response to a range of stresses likely to be encountered in the host, including ROS, osmotic stress and anti-microbial peptides [114]. Cells lacking Hog1 display impaired virulence in a wide range of infection models, including murine systemic and commensal models [114-116], and are more susceptible to killing by macrophages or neutrophils [117]. As Hog1 regulates a number of distinct stress responses, it is difficult to dissect whether it is the role of Hog1 in oxidative stress responses or a different aspect of Hog1 signalling that is important for virulence in these models. Importantly, however, although Hog1 signalling has also been implicated in morphogenetic regulation, mutational analysis has inferred that the importance of $\mathrm{Hog} 1$ in virulence is due to its role in stress protection, rather than its role in repressing the yeast to hyphal transition [115]. 


\subsection{The Rad53 DNA Damage Checkpoint Kinase}

Following exposure to $\mathrm{H}_{2} \mathrm{O}_{2}$, C. albicans forms hyperpolarised buds, which are morphologically distinct from hyphae and pseudohyphae filamentous forms (Section 3.4). Consistent with this, $\mathrm{H}_{2} \mathrm{O}_{2}$-induced hyperpolarized bud formation occurs independently of the key hyphal regulators, Efg 1 and Cph1, and, instead, depends on the activation of the Rad53 DNA damage checkpoint pathway [42,61] (Figure 3). A wide range of genotoxic stresses, including UV, methyl methanesulfonate (MMS) and the ribonucleotide reductase inhibitor hydroxyurea have been shown to activate the Rad53 kinase in C. albicans [61], and loss of Rad53 or upstream regulators of Rad53, prevents hyperpolarised bud formation [61,118]. ROS are also genotoxic agents due to the induction of DNA damage [119], which, in turn, triggers the activation of the Rad53 DNA checkpoint pathway [120]. Indeed, treatment of C. albicans cells with $\mathrm{H}_{2} \mathrm{O}_{2}$ elicits the phosphorylation of Rad53, and cells lacking $R A D 53$ fail to form hyperpolarised buds in response to $\mathrm{H}_{2} \mathrm{O}_{2}$ [42].

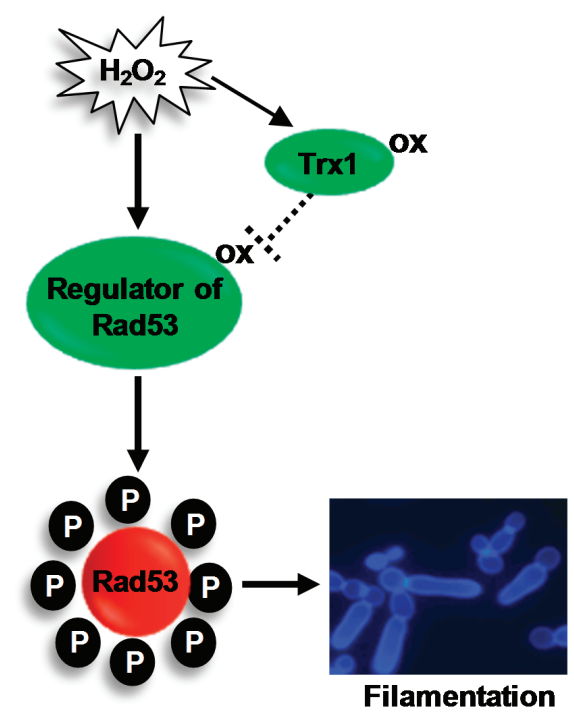

Figure 3. $\mathrm{H}_{2} \mathrm{O}_{2}$-induced activation of $\mathrm{Rad} 53$ triggers filamentation in C. albicans. The redox sensitive oxidoreductase Trx1 inhibits $\mathrm{H}_{2} \mathrm{O}_{2}$-induced activation of the DNA damage checkpoint kinase Rad53. This suggests that a regulator of Rad53 is activated by oxidation, and this active oxidised form is reduced by Trx1. Activation of the DNA damage checkpoint triggers the formation of hyperpolarised buds. See text for details.

Regulation of $\operatorname{Rad} 53$

Regarding the $\mathrm{H}_{2} \mathrm{O}_{2}$-mediated activation of Rad53, a recent study illustrated that $\mathrm{H}_{2} \mathrm{O}_{2}$-induced oxidation, and inactivation, of the thioredoxin protein Trx1 is important for the activation of Rad53 and polarized cell growth [42]. Rad53 is constitutively phosphorylated in cells lacking Trx1, which display a hyperpolarized bud morphology. Conversely, ectopic expression of the thioredoxin reductase gene, TRR1, which reduces oxidized Trx1, inhibited $\mathrm{H}_{2} \mathrm{O}_{2}$-induced filamentation [42]. 
Taken together, these results illustrate that oxidation of $\operatorname{Trx} 1$ following $\mathrm{H}_{2} \mathrm{O}_{2}$ exposure is key in the activation of Rad53 that drives hyperpolarised bud formation. The finding that Trx 1 inhibits Rad53 activation under non-stressed conditions may be conserved in higher eukaryotes, as ectopic expression of thioredoxin inhibits the phosphorylation of the analogous DNA damage checkpoint kinase, Chk2, in mammalian cells [121]. However, the mechanism of Trx1 regulation of Rad53 is unclear. As Trx1 functions to reduce oxidised proteins, an attractive hypothesis is that Rad53, or a regulator of this kinase, is activated by oxidation (Figure 3). In this regard, it is interesting that the human homologue (ATM) of the fungal Tel1 DNA-damage sensing kinase, which regulates Rad53, has recently been shown to be activated by oxidation [122]. Further studies are needed to determine if Tel1 is similarly regulated to mediate $\mathrm{H}_{2} \mathrm{O}_{2}$-induced filamentation in C. albicans.

\subsection{Other Signaling Pathways that Contribute to Oxidative Stress Resistance}

The cAMP/PKA signalling pathway has a negative impact on oxidative stress responses in $C$. albicans. For example, induction of the pathway by inactivation of the phosphodiesterase, Pde2, which degrades cAMP, results in increased sensitivity to $\mathrm{H}_{2} \mathrm{O}_{2}$ [123]. Related to this, farnesol treatment of $C$. albicans cells results in increased resistance to $\mathrm{H}_{2} \mathrm{O}_{2}$, due to the inhibition of the cAMP/PKA signalling pathway [124]. Such changes in resistance are possibly due to changes in the levels of anti-oxidant gene expression [124]; however, the mechanism linking cAMP/PKA to their regulation is unknown.

There is also evidence that the spindle assembly checkpoint is required for $C$. albicans oxidative stress resistance. Cells lacking the spindle checkpoint protein kinase, Mps1, are sensitive to $\mathrm{H}_{2} \mathrm{O}_{2}$ [125] and, similar to other oxidative stress sensitive mutants [56], fail to filament following phagocytosis. Related to this, the spindle assembly checkpoint protein, Mad2, is essential for C. albicans survival in macrophages [126].

\section{Conclusions and Future Perspectives}

In this review, we have summarized the current literature of oxidative stress responses and how they are regulated in the human fungal pathogen C. albicans. This is an important area of research, as oxidative stress adaptation is emerging as an important virulence trait in this, and other, fungal pathogens. Table 1 summarizes studies that have documented the impact of the loss of oxidative stress regulatory proteins or antioxidant enzymes on C. albicans virulence in either a murine systemic infection model or a macrophage/neutrophil phagocyte-survival infection model. From this summary, a number of observations can be made. First of all, when examined, mutants that display an impaired tolerance to oxidative stress show an impaired ability to survive phagocyte killing. This is consistent with previous observations that $C$. albicans mounts a robust transcriptional response to oxidative stress following phagocytosis [34-38]. Secondly, not all oxidative stress-sensitive C. albicans mutants display attenuated virulence in a murine systemic infection model. Perhaps most striking is the observation that the major regulator of anti-oxidant gene expression, Cap1, is dispensable for virulence in such an infection model. This is particularly intriguing, as certain genes, which are dependent on Cap1 for induction following oxidative stress, are important for virulence in systemic 
models. Thirdly, the role of many other oxidative stress-responsive proteins in mediating C. albicans virulence, such as the Rad53-mediated DNA damage checkpoint pathway, have yet to be explored.

Table 1. Summary of the role of oxidative stress-responsive signalling proteins and antioxidant enzymes in C. albicans virulence. The importance of proteins needed for resistance to oxidative stress in mediating $C$. albicans virulence in either a systemic infection model (SIM) or phagocyte infection model (PIM) is indicated; +, important for virulence; -, dispensable for virulence; nd, not determined. For further explanation, see the text.

\begin{tabular}{ccccc}
\hline Protein & Function & SIM & PIM & References \\
\hline Signalling Proteins & & & & \\
Hog1 & Stress-activated protein kinase & + & + & {$[114,115,117]$} \\
Ssk1 & Response regulator & + & + & {$[127,128]$} \\
Cap1 & Transcription factor & - & + & {$[56,81]$} \\
Ybp1 & Cap1 regulator & - & + & {$[56]$} \\
Gpx3 & Cap1 regulator & - & + & {$[56]$} \\
Skn7 & Transcription factor & - & nd & {$[80]$} \\
Signalling Proteins & Phosphodiesterase & + & nd & {$[123]$} \\
Pde2 & Spindle checkpoint & nd & + & {$[125]$} \\
Mps1 & Spindle checkpoint & + & + & {$[126]$} \\
Mad2 & & & & \\
Antioxidant Enzymes & Catalase & + & + & {$[45]$} \\
Cat1 & Thioredoxin & + & nd & {$[42]$} \\
Trx1 & Thioredoxin peroxidase & - & nd & {$[47]$} \\
Tsa1 & Superoxide dismutase & + & + & {$[43]$} \\
Sod1 & Superoxide dismutase & + & + & {$[23,44]$} \\
Sod5 & Glutaredoxin & + & nd & {$[41]$} \\
Grx2 & Glutathione peroxidases & nd & + & {$[46]$} \\
Gpx31-33 & & & & \\
\hline
\end{tabular}

In addition to gaps in our knowledge regarding the relative importance of specific oxidative stress responses in mediating $C$. albicans virulence, there are also additional key questions that remain to be addressed. For example, what is the role of the Hog1 SAPK in mediating oxidative stress resistance in C. albicans, and how is this regulated? This is important, as, although Hog1 is an essential virulence determinant in C. albicans, the conservation with highly related SAPKs in human cells suggests that Hog 1 itself may be unsuitable as a specific antifungal target. Thus, there is much interest in identifying fungal-specific SAPK regulators or substrates, as such proteins hold greater promise for future therapeutic strategies. In addition, the recent findings that $C$. albicans is exquisitely sensitive to combinations of stress that are encountered following phagocytosis represent a new unchartered area in the field of stress signalling. A key question for the future is how do combinations of stress imposed by the phagosome inhibit oxidative stress adaptation and survival of $C$. albicans? Addressing this question is critical to further our understanding of Candida-host interactions during disease progression. 


\section{Acknowledgments}

We thank our friends and colleagues, in particular Al Brown, Lars Erwig, Ken Haynes, Brian Morgan and Elizabeth Veal, for stimulating discussions on oxidative stress signalling mechanisms and fungal pathogenesis. Research in the Janet Quinn lab is funded by the BBSRC BB/K016393/1, an MRC-DTP studentship, the NIHR Newcastle Biomedical Research Centre and the Wellcome Trust Strategic Award for Medical Mycology and Fungal Immunology 097377/Z/11/Z.

\section{Author Contributions}

All authors contributed to the initial draft of this review, which was edited by Janet Quinn.

\section{Conflicts of Interest}

The authors declare no conflict of interest.

\section{References and Notes}

1. Odds, F.C. Candida and Candidosis, 2nd ed.; Bailliere-Tindall: London, UK, 1988.

2. Calderone, R.A.; Clancy, C.J. Candida and Candidiasis; ASM Press: Washington, DC, USA, 2012.

3. Anaissie, E.J.; McGinnis, M.R.; Pfaller, M.A. Clinical Mycology; Churchill Livingstone: London, UK, 2009.

4. Flevari, A.; Theodorakopoulou, M.; Velegraki, A.; Armaganidis, A.; Dimopoulos, G. Treatment of invasive candidiasis in the elderly: A review. Clin. Interv. Aging 2013, 8, 1199-1208.

5. Healy, C.M.; Campbell, J.R.; Zaccaria, E.; Baker, C.J. Fluconazole prophylaxis in extremely low birth weight neonates reduces invasive candidiasis mortality rates without emergence of fluconazole-resistant candida species. Pediatrics 2008, 121, 703-710.

6. Sobel, J.D. Vulvovaginal candidosis. Lancet 2007, 369, 1961-1971.

7. Lilic, D. Unravelling fungal immunity through primary immune deficiencies. Curr. Opin. Microbiol. 2012, 15, 420-426.

8. Morgan, J. Global trends in candidemia: Review of reports from 1995-2005. Curr. Infect. Dis. Rep. 2005, 7, 429-439.

9. Brown, G.D.; Denning, D.W.; Gow, N.A.; Levitz, S.M.; Netea, M.G.; White, T.C. Hidden killers: Human fungal infections. Sci. Transl. Med. 2012, doi:10.1126/scitranslmed.3004404.

10. Koh, A.Y.; Kohler, J.R.; Coggshall, K.T.; van Rooijen, N.; Pier, G.B. Mucosal damage and neutropenia are required for Candida albicans dissemination. PLOS Pathog. 2008, 4, e35.

11. Seider, K.; Heyken, A.; Luttich, A.; Miramon, P.; Hube, B. Interaction of pathogenic yeasts with phagocytes: Survival, persistence and escape. Curr. Opin. Microbiol. 2010, 13, 392-400.

12. Babior, B.M. NADPH oxidase. Curr. Opin. Immunol. 2004, 16, $42-47$.

13. Bedard, K.; Krause, K.H. The NOX family of ROS-generating NADPH oxidases: Physiology and pathophysiology. Physiol. Rev. 2007, 87, 245-313. 
14. Pick, E. Role of the Rho GTPase Rac in the activation of the phagocyte NADPH oxidase: Outsourcing a key task. Small GTPases 2014, 5, e27952.

15. Hampton, M.B.; Kettle, A.J.; Winterbourn, C.C. Involvement of superoxide and myeloperoxidase in oxygen-dependent killing of Staphylococcus aureus by neutrophils. Infect. Immun. 1996, 64, 3512-3517.

16. Reeves, E.P.; Lu, H.; Jacobs, H.L.; Messina, C.G.; Bolsover, S.; Gabella, G.; Potma, E.O.; Warley, A.; Roes, J.; Segal, A.W. Killing activity of neutrophils is mediated through activation of proteases by $\mathrm{K}^{+}$flux. Nature 2002, 416, 291-297.

17. Holland, S.M. Chronic granulomatous disease. Clin. Rev. Allerg. Immunol. 2010, 38, 3-10.

18. Cohen, M.S.; Isturiz, R.E.; Malech, H.L.; Root, R.K.; Wilfert, C.M.; Gutman, L.; Buckley, R.H. Fungal infection in chronic granulomatous disease. The importance of the phagocyte in defense against fungi. Am. J. Med. 1981, 71, 59-66.

19. Brothers, K.M.; Gratacap, R.L.; Barker, S.E.; Newman, Z.R.; Norum, A.; Wheeler, R.T. Nadph oxidase-driven phagocyte recruitment controls Candida albicans filamentous growth and prevents mortality. PLOS Pathog. 2013, 9, e1003634.

20. Brown, A.J.; Haynes, K.; Quinn, J. Nitrosative and oxidative stress responses in fungal pathogenicity. Curr. Opin. Microbiol. 2009, 12, 384-391.

21. Fang, F.C. Antimicrobial reactive oxygen and nitrogen species: Concepts and controversies. Nat. Rev. Microbiol. 2004, 2, 820-832.

22. Kaloriti, D.; Jacobsen, M.; Yin, Z.; Patterson, M.; Tillmann, A.; Smith, D.A.; Cook, E.; You, T.; Grimm, M.J.; Bohovych, I.; et al. Mechanisms underlying the exquisite sensitivity of Candida albicans to combinatorial cationic and oxidative stress that enhances the potent fungicidal activity of phagocytes. MBio 2014, 5, e01334-e01314.

23. Frohner, I.E.; Bourgeois, C.; Yatsyk, K.; Majer, O.; Kuchler, K. Candida albicans cell surface superoxide dismutases degrade host-derived reactive oxygen species to escape innate immune surveillance. Mol. Microbiol. 2009, 71, 240-252.

24. Miramon, P.; Dunker, C.; Windecker, H.; Bohovych, I.M.; Brown, A.J.; Kurzai, O.; Hube, B. Cellular responses of Candida albicans to phagocytosis and the extracellular activities of neutrophils are critical to counteract carbohydrate starvation, oxidative and nitrosative stress. PLOS ONE 2012, 7, e52850.

25. Huycke, M.M.; Abrams, V.; Moore, D.R. Enterococcus faecalis produces extracellular superoxide and hydrogen peroxide that damages colonic epithelial cell DNA. Carcinogenesis 2002, 23, 529-536.

26. Fitzsimmons, N.; Berry, D.R. Inhibition of Candida albicans by lactobacillus acidophilus: Evidence for the involvement of a peroxidase system. Microbios 1994, 80, 125-133.

27. Cruz, M.R.; Graham, C.E.; Gagliano, B.C.; Lorenz, M.C.; Garsin, D.A. Enterococcus faecalis inhibits hyphal morphogenesis and virulence of Candida albicans. Infect. Immun. 2013, 81, 189-200.

28. Phillips, A.J.; Sudbery, I.; Ramsdale, M. Apoptosis induced by environmental stresses and amphotericin B in Candida albicans. Proc. Natl. Acad. Sci. USA 2003, 100, 14327-14332.

29. Halliwell, B. Oxidative stress and cancer: Have we moved forward? Biochem. J. 2007, 401, 1-11. 
30. Jamieson, D.J.; Stephen, D.W.; Terriere, E.C. Analysis of the adaptive oxidative stress response of Candida albicans. FEMS Microbiol. Lett. 1996, 138, 83-88.

31. Nikolaou, E.; Agrafioti, I.; Stumpf, M.; Quinn, J.; Stansfield, I.; Brown, A.J. Phylogenetic diversity of stress signalling pathways in fungi. BMC Evol. Biol. 2009, doi:10.1186/1471-2148-9-44.

32. Enjalbert, B.; Nantel, A.; Whiteway, M. Stress-induced gene expression in Candida albicans: Absence of a general stress response. Mol. Biol. Cell 2003, 14, 1460-1467.

33. Enjalbert, B.; Smith, D.A.; Cornell, M.J.; Alam, I.; Nicholls, S.; Brown, A.J.; Quinn, J. Role of the Hog 1 stress-activated protein kinase in the global transcriptional response to stress in the fungal pathogen Candida albicans. Mol. Biol. Cell 2006, 17, 1018-1032.

34. Enjalbert, B.; MacCallum, D.M.; Odds, F.C.; Brown, A.J. Niche-specific activation of the oxidative stress response by the pathogenic fungus Candida albicans. Infect. Immun. 2007, 75, 2143-2151.

35. Fradin, C.; de Groot, P.; MacCallum, D.; Schaller, M.; Klis, F.; Odds, F.C.; Hube, B. Granulocytes govern the transcriptional response, morphology and proliferation of Candida albicans in human blood. Mol. Microbiol. 2005, 56, 397-415.

36. Fradin, C.; Kretschmar, M.; Nichterlein, T.; Gaillardin, C.; d'Enfert, C.; Hube, B. Stage-specific gene expression of Candida albicans in human blood. Mol. Microbiol. 2003, 47, 1523-1543.

37. Lorenz, M.C.; Bender, J.A.; Fink, G.R. Transcriptional response of Candida albicans upon internalization by macrophages. Eukaryot. Cell 2004, 3, 1076-1087.

38. Rubin-Bejerano, I.; Fraser, I.; Grisafi, P.; Fink, G.R. Phagocytosis by neutrophils induces an amino acid deprivation response in Saccharomyces cerevisiae and Candida albicans. Proc. Natl. Acad. Sci. USA 2003, 100, 11007-11012.

39. Thewes, S.; Kretschmar, M.; Park, H.; Schaller, M.; Filler, S.G.; Hube, B. In vivo and ex vivo comparative transcriptional profiling of invasive and non-invasive Candida albicans isolates identifies genes associated with tissue invasion. Mol. Microbiol. 2007, 63, 1606-1628.

40. Walker, L.A.; Maccallum, D.M.; Bertram, G.; Gow, N.A.; Odds, F.C.; Brown, A.J. Genome-wide analysis of Candida albicans gene expression patterns during infection of the mammalian kidney. Fungal Genet. Biol. 2009, 46, 210-219.

41. Chaves, G.M.; Bates, S.; Maccallum, D.M.; Odds, F.C. Candida albicans GRX2, encoding a putative glutaredoxin, is required for virulence in a murine model. Genet. Mol. Res. 2007, 6, 1051-1063.

42. Da Silva Dantas, A.; Patterson, M.J.; Smith, D.A.; Maccallum, D.M.; Erwig, L.P.; Morgan, B.A.; Quinn, J. Thioredoxin regulates multiple hydrogen peroxide-induced signaling pathways in Candida albicans. Mol. Cell. Biol. 2010, 30, 4550-4563.

43. Hwang, C.S.; Rhie, G.E.; Oh, J.H.; Huh, W.K.; Yim, H.S.; Kang, S.O. Copper- and zinc-containing superoxide dismutase $(\mathrm{Cu} / \mathrm{ZnSOD})$ is required for the protection of Candida albicans against oxidative stresses and the expression of its full virulence. Microbiology 2002, 148, 3705-3713. 
44. Martchenko, M.; Alarco, A.M.; Harcus, D.; Whiteway, M. Superoxide dismutases in Candida albicans: Transcriptional regulation and functional characterization of the hyphal-induced SOD5 gene. Mol. Biol. Cell 2004, 15, 456-467.

45. Wysong, D.R.; Christin, L.; Sugar, A.M.; Robbins, P.W.; Diamond, R.D. Cloning and sequencing of a Candida albicans catalase gene and effects of disruption of this gene. Infect. Immun. 1998, 66, 1953-1961.

46. Miramon, P.; Dunker, C.; Kasper, L.; Jacobsen, I.D.; Barz, D.; Kurzai, O.; Hube, B. A family of glutathione peroxidases contributes to oxidative stress resistance in Candida albicans. Med. Mycol. 2014, 52, 223-239.

47. Urban, C.; Xiong, X.; Sohn, K.; Schroppel, K.; Brunner, H.; Rupp, S. The moonlighting protein tsalp is implicated in oxidative stress response and in cell wall biogenesis in Candida albicans. Mol. Microbiol. 2005, 57, 1318-1341.

48. Steinberg, B.E.; Huynh, K.K.; Grinstein, S. Phagosomal acidification: Measurement, manipulation and functional consequences. Biochem. Soc. Trans. 2007, 35, 1083-1087.

49. Kaloriti, D.; Tillmann, A.; Cook, E.; Jacobsen, M.; You, T.; Lenardon, M.; Ames, L.; Barahona, M.; Chandrasekaran, K.; Coghill, G.; et al. Combinatorial stresses kill pathogenic Candida species. Med. Mycol. 2012, 50, 699-709.

50. Schuller, C.; Brewster, J.L.; Alexander, M.R.; Gustin, M.C.; Ruis, H. The hog pathway controls osmotic regulation of transcription via the stress response element (STRE) of the Saccharomyces cerevisiae CTT1 gene. EMBO J. 1994, 13, 4382-4389.

51. Heilmann, C.J.; Sorgo, A.G.; Siliakus, A.R.; Dekker, H.L.; Brul, S.; de Koster, C.G.; de Koning, L.J.; Klis, F.M. Hyphal induction in the human fungal pathogen Candida albicans reveals a characteristic wall protein profile. Microbiology 2011, 157, 2297-2307.

52. Gleason, J.E.; Galaleldeen, A.; Peterson, R.L.; Taylor, A.B.; Holloway, S.P.; Waninger-Saroni, J.; Cormack, B.P.; Cabelli, D.E.; Hart, P.J.; Culotta, V.C. Candida albicans SOD5 represents the prototype of an unprecedented class of $\mathrm{Cu}$-only superoxide dismutases required for pathogen defense. Proc. Natl. Acad. Sci. USA 2014, 111, 5866-5871.

53. Festa, R.A.; Thiele, D.J. Copper at the front line of the host-pathogen battle. PLOS Pathog. 2012, 8, e1002887.

54. Crowe, J.D.; Sievwright, I.K.; Auld, G.C.; Moore, N.R.; Gow, N.A.; Booth, N.A. Candida albicans binds human plasminogen: Identification of eight plasminogen-binding proteins. Mol. Microbiol. 2003, 47, 1637-1651.

55. Lewis, L.E.; Bain, J.M.; Okai, B.; Gow, N.A.; Erwig, L.P. Live-cell video microscopy of fungal pathogen phagocytosis. J. Vis. Exp. 2013, doi:10.3791/50196.

56. Patterson, M.J.; McKenzie, C.G.; Smith, D.A.; da Silva Dantas, A.; Sherston, S.; Veal, E.A.; Morgan, B.A.; MacCallum, D.M.; Erwig, L.P.; Quinn, J. Ybp1 and Gpx3 signaling in Candida albicans govern hydrogen peroxide-induced oxidation of the Cap1 transcription factor and macrophage escape. Antioxid. Redox Signal. 2013, 19, 2244-2260.

57. Brothers, K.M.; Newman, Z.R.; Wheeler, R.T. Live imaging of disseminated candidiasis in zebrafish reveals role of phagocyte oxidase in limiting filamentous growth. Eukaryot. Cell 2011, 10, 932-944. 
58. Jimenez-Lopez, C.; Collette, J.R.; Brothers, K.M.; Shepardson, K.M.; Cramer, R.A.; Wheeler, R.T.; Lorenz, M.C. Candida albicans induces arginine biosynthetic genes in response to host-derived reactive oxygen species. Eukaryot. Cell 2013, 12, 91-100.

59. Nasution, O.; Srinivasa, K.; Kim, M.; Kim, Y.J.; Kim, W.; Jeong, W.; Choi, W. Hydrogen peroxide induces hyphal differentiation in Candida albicans. Eukaryot. Cell 2008, 7, 2008-2011.

60. Bachewich, C.; Nantel, A.; Whiteway, M. Cell cycle arrest during S or M phase generates polarized growth via distinct signals in Candida albicans. Mol. Microbiol. 2005, 57, 942-959.

61. Shi, Q.M.; Wang, Y.M.; Zheng, X.D.; Lee, R.T.; Wang, Y. Critical role of DNA checkpoints in mediating genotoxic-stress-induced filamentous growth in Candida albicans. Mol. Biol. Cell 2007, 18, 815-826.

62. Kusch, H.; Engelmann, S.; Albrecht, D.; Morschhauser, J.; Hecker, M. Proteomic analysis of the oxidative stress response in Candida albicans. Proteomics 2007, 7, 686-697.

63. Wang, Y.; Cao, Y.Y.; Jia, X.M.; Cao, Y.B.; Gao, P.H.; Fu, X.P.; Ying, K.; Chen, W.S.; Jiang, Y.Y. Caplp is involved in multiple pathways of oxidative stress response in Candida albicans. Free Radic. Biol. Med. 2006, 40, 1201-1209.

64. Moye-Rowley, W.S. Regulation of the transcriptional response to oxidative stress in fungi: Similarities and differences. Eukaryot. Cell 2003, 2, 381-389.

65. Toone, W.M.; Morgan, B.A.; Jones, N. Redox control of AP-1-like factors in yeast and beyond. Oncogene 2001, 20, 2336-2346.

66. Alarco, A.M.; Raymond, M. The bzip transcription factor Cap1p is involved in multidrug resistance and oxidative stress response in Candida albicans. J. Bacteriol. 1999, 181, 700-708.

67. Zhang, X.; de Micheli, M.; Coleman, S.T.; Sanglard, D.; Moye-Rowley, W.S. Analysis of the oxidative stress regulation of the Candida albicans transcription factor, Cap1p. Mol. Microbiol. 2000, 36, 618-629.

68. Znaidi, S.; Barker, K.S.; Weber, S.; Alarco, A.M.; Liu, T.T.; Boucher, G.; Rogers, P.D.; Raymond, M. Identification of the Candida albicans Cap1p regulon. Eukaryot. Cell 2009, 8, 806-820.

69. Ramirez-Zavala, B.; Mogavero, S.; Scholler, E.; Sasse, C.; Rogers, P.D.; Morschhauser, J. SAGA/ADA complex subunit Ada2 is required for Cap1- but not Mrr1-mediated upregulation of the Candida albicans multidrug efflux pump Mdr1. Antimicrob. Agents Chemother. 2014, 58, 5102-5110.

70. Sellam, A.; Askew, C.; Epp, E.; Lavoie, H.; Whiteway, M.; Nantel, A. Genome-wide mapping of the coactivator Ada2p yields insight into the functional roles of SAGA/ADA complex in Candida albicans. Mol. Biol. Cell 2009, 20, 2389-2400.

71. Delaunay, A.; Isnard, A.D.; Toledano, M.B. $\mathrm{H}_{2} \mathrm{O}_{2}$ sensing through oxidation of the Yap1 transcription factor. EMBO J. 2000, 19, 5157-5166.

72. Delaunay, A.; Pflieger, D.; Barrault, M.B.; Vinh, J.; Toledano, M.B. A thiol peroxidase is an $\mathrm{H}_{2} \mathrm{O}_{2}$ receptor and redox-transducer in gene activation. Cell 2002, 111, 471-481. 
73. Okazaki, S.; Tachibana, T.; Naganuma, A.; Mano, N.; Kuge, S. Multistep disulfide bond formation in Yap1 is required for sensing and transduction of $\mathrm{H}_{2} \mathrm{O}_{2}$ stress signal. Mol. Cell. 2007, 27, 675-688.

74. Okazaki, S.; Naganuma, A.; Kuge, S. Peroxiredoxin-mediated redox regulation of the nuclear localization of Yap1, a transcription factor in budding yeast. Antioxid. Redox Signal. 2005, 7, 327-334.

75. Tachibana, T.; Okazaki, S.; Murayama, A.; Naganuma, A.; Nomoto, A.; Kuge, S. A major peroxiredoxin-induced activation of Yap1 transcription factor is mediated by reduction-sensitive disulfide bonds and reveals a low level of transcriptional activation. J. Biol. Chem. 2009, 284, 4464-4472.

76. Veal, E.A.; Ross, S.J.; Malakasi, P.; Peacock, E.; Morgan, B.A. Ybp1 is required for the hydrogen peroxide-induced oxidation of the Yap1 transcription factor. J. Biol. Chem. 2003, 278, 30896-30904.

77. Gulshan, K.; Thommandru, B.; Moye-Rowley, W.S. Proteolytic degradation of the Yap1 transcription factor is regulated by subcellular localization and the E3 ubiquitin ligase Not4. J. Biol. Chem. 2012, 287, 36796-36805.

78. Kitamura, K.; Taki, M.; Tanaka, N.; Yamashita, I. Fission yeast Ubr1 ubiquitin ligase influences the oxidative stress response via degradation of active Pap1 bZIP transcription factor in the nucleus. Mol. Microbiol. 2011, 80, 739-755.

79. Morgan, B.A.; Banks, G.R.; Toone, W.M.; Raitt, D.; Kuge, S.; Johnston, L.H. The Skn7 response regulator controls gene expression in the oxidative stress response of the budding yeast Saccharomyces cerevisiae. EMBO J. 1997, 16, 1035-1044.

80. Singh, P.; Chauhan, N.; Ghosh, A.; Dixon, F.; Calderone, R. Skn7 of Candida albicans: Mutant construction and phenotype analysis. Infect. Immun. 2004, 72, 2390-2394.

81. Jain, C.; Pastor, K.; Gonzalez, A.Y.; Lorenz, M.C.; Rao, R.P. The role of Candida albicans AP-1 protein against host derived ROS in in vivo models of infection. Virulence 2013, 4, 67-76.

82. Smith, D.A.; Morgan, B.A.; Quinn, J. Stress signalling to fungal stress-activated protein kinase pathways. FEMS Microbiol. Lett. 2010, 306, 1-8.

83. Bellon, S.; Fitzgibbon, M.J.; Fox, T.; Hsiao, H.M.; Wilson, K.P. The structure of phosphorylated p38gamma is monomeric and reveals a conserved activation-loop conformation. Structure 1999, 7, 1057-1065.

84. Alonso-Monge, R.; Navarro-Garcia, F.; Roman, E.; Negredo, A.I.; Eisman, B.; Nombela, C.; $\mathrm{Pla}$, J. The Hog 1 mitogen-activated protein kinase is essential in the oxidative stress response and chlamydospore formation in Candida albicans. Eukaryot. Cell 2003, 2, 351-361.

85. Smith, D.A.; Nicholls, S.; Morgan, B.A.; Brown, A.J.; Quinn, J. A conserved stress-activated protein kinase regulates a core stress response in the human pathogen Candida albicans. Mol. Biol. Cell 2004, 15, 4179-4190.

86. Chen, D.; Toone, W.M.; Mata, J.; Lyne, R.; Burns, G.; Kivinen, K.; Brazma, A.; Jones, N.; Bahler, J. Global transcriptional responses of fission yeast to environmental stress. Mol. Biol. Cell 2003, $14,214-229$. 
87. Asp, E.; Nilsson, D.; Sunnerhagen, P. Fission yeast mitogen-activated protein kinase Sty 1 interacts with translation factors. Eukaryot. Cell 2008, 7, 328-338.

88. Yin, Z.; Stead, D.; Walker, J.; Selway, L.; Smith, D.; Brown, A.J.P.; Quinn, J. A proteomic analysis of the salt, cadmium and peroxide stress responses in Candida albicans and the role of the Hog1 Sapk in regulating the stress-induced proteome. Proteomics 2009, 9, 4680-4703.

89. Alonso-Monge, R.; Carvaihlo, S.; Nombela, C.; Rial, E.; Pla, J. The Hog1 MAP kinase controls respiratory metabolism in the fungal pathogen Candida albicans. Microbiology 2009, 155, 413-423.

90. Navarro-Garcia, F.; Eisman, B.; Fiuza, S.M.; Nombela, C.; Pla, J. The MAP kinase Mkc1p is activated under different stress conditions in Candida albicans. Microbiology 2005, 151, 2737-2749.

91. Rauceo, J.M.; Blankenship, J.R.; Fanning, S.; Hamaker, J.J.; Deneault, J.S.; Smith, F.J.; Nantel, A.; Mitchell, A.P. Regulation of the Candida albicans cell wall damage response by transcription factor Sko1 and PAS kinase Psk1. Mol. Biol. Cell 2008, 19, 2741-2751.

92. Alonso-Monge, R.; Roman, E.; Arana, D.M.; Prieto, D.; Urrialde, V.; Nombela, C.; Pla, J. The skol protein represses the yeast-to-hypha transition and regulates the oxidative stress response in Candida albicans. Fungal Genet. Biol. 2010, 47, 587-601.

93. Arana, D.M.; Nombela, C.; Alonso-Monge, R.; Pla, J. The Pbs2 MAP kinase kinase is essential for the oxidative-stress response in the fungal pathogen Candida albicans. Microbiology 2005, 151, 1033-1049.

94. Cheetham, J.; Smith, D.A.; da Silva Dantas, A.; Doris, K.S.; Patterson, M.J.; Bruce, C.R.; Quinn, J. A single MAPKKK regulates the Hog1 MAPK pathway in the pathogenic fungus Candida albicans. Mol. Biol. Cell 2007, 18, 4603-4614.

95. Thomas, E.; Roman, E.; Claypool, S.; Manzoor, N.; Pla, J.; Panwar, S.L. Mitochondria influence Cdr1 efflux pump activity, Hog1-mediated oxidative stress pathway, iron homeostasis, and ergosterol levels in Candida albicans. Antimicrob. Agents Chemother. 2013, 57, 5580-5599.

96. Horie, T.; Tatebayashi, K.; Yamada, R.; Saito, H. Phosphorylated Ssk1 prevents unphosphorylated Ssk1 from activating the Ssk2 mitogen-activated protein kinase kinase kinase in the yeast high-osmolarity glycerol osmoregulatory pathway. Mol. Cell. Biol. 2008, 28, 5172-5183.

97. Posas, F.; Saito, H. Activation of the yeast Ssk2 map kinase kinase kinase by the Ssk1 two-component response regulator. EMBO J. 1998, 17, 1385-1394.

98. Chauhan, N.; Inglis, D.; Roman, E.; Pla, J.; Li, D.; Calera, J.A.; Calderone, R. Candida albicans response regulator gene Ssk1 regulates a subset of genes whose functions are associated with cell wall biosynthesis and adaptation to oxidative stress. Eukaryot. Cell 2003, 2, 1018-1024.

99. Li, D.; Gurkovska, V.; Sheridan, M.; Calderone, R.; Chauhan, N. Studies on the regulation of the two-component histidine kinase gene CHK1 in Candida albicans using the heterologous lacz reporter gene. Microbiology 2004, 150, 3305-3313.

100. Roman, E.; Nombela, C.; Pla, J. The SHOI adaptor protein links oxidative stress to morphogenesis and cell wall biosynthesis in the fungal pathogen Candida albicans. Mol. Cell. Biol. 2005, 25, 10611-10627. 
101. Buck, V.; Quinn, J.; Soto Pino, T.; Martin, H.; Saldanha, J.; Makino, K.; Morgan, B.A.; Millar, J.B. Peroxide sensors for the fission yeast stress-activated mitogen-activated protein kinase pathway. Mol. Biol. Cell 2001, 12, 407-419.

102. Quinn, J.; Malakasi, P.; Smith, D.A.; Cheetham, J.; Buck, V.; Millar, J.B.; Morgan, B.A. Two-component mediated peroxide sensing and signal transduction in fission yeast. Antioxid. Redox Signal. 2011, 15, 153-165.

103. Menon, V.; Li, D.; Chauhan, N.; Rajnarayanan, R.; Dubrovska, A.; West, A.H.; Calderone, R. Functional studies of the Ssk1p response regulator protein of Candida albicans as determined by phenotypic analysis of receiver domain point mutants. Mol. Microbiol. 2006, 62, 997-1013.

104. Mavrianos, J.; Desai, C.; Chauhan, N. Two-component histidine phosphotransfer protein Ypd1 is not essential for viability in Candida albicans. Eukaryot. Cell 2014, 13, 452-460.

105. Bruce, C.R.; Smith, D.A.; Rodgers, D.; da Silva Dantas, A.; MacCallum, D.M.; Morgan, B.A.; Quinn, J. Identification of a novel response regulator, Crr1, that is required for hydrogen peroxide resistance in Candida albicans. PLOS ONE 2011, 6, e27979.

106. Desai, C.; Mavrianos, J.; Chauhan, N. Candida albicans Srr1, a putative two-component response regulator gene, is required for stress adaptation, morphogenesis, and virulence. Eukaryot. Cell 2011, 10, 1370-1374.

107. O'Rourke, S.M.; Herskowitz, I. A third osmosensing branch in Saccharomyces cerevisiae requires the Msb2 protein and functions in parallel with the Sho1 branch. Mol. Cell. Biol. 2002, 22, 4739-4749.

108. Veal, E.A.; Day, A.M.; Morgan, B.A. Hydrogen peroxide sensing and signaling. Mol. Cell 2007, 26, 1-14.

109. Veal, E.A.; Findlay, V.J.; Day, A.M.; Bozonet, S.M.; Evans, J.M.; Quinn, J.; Morgan, B.A. A 2-cys peroxiredoxin regulates peroxide-induced oxidation and activation of a stress-activated MAP kinase. Mol. Cell 2004, 15, 129-139.

110. Saitoh, M.; Nishitoh, H.; Fujii, M.; Takeda, K.; Tobiume, K.; Sawada, Y.; Kawabata, M.; Miyazono, K.; Ichijo, H. Mammalian thioredoxin is a direct inhibitor of apoptosis signal-regulating kinase (ASK) 1. EMBO J. 1998, 17, 2596-2606.

111. Nadeau, P.J.; Charette, S.J.; Landry, J. Redox reaction at ASK1-Cys250 is essential for activation of jnk and induction of apoptosis. Mol. Biol. Cell 2009, 20, 3628-3637.

112. Nadeau, P.J.; Charette, S.J.; Toledano, M.B.; Landry, J. Disulfide bond-mediated multimerization of ASK1 and its reduction by thioredoxin-1 regulate $\mathrm{H}_{2} \mathrm{O}_{2}$-induced c-Jun $\mathrm{NH}_{2}$-terminal kinase activation and apoptosis. Mol. Biol. Cell 2007, 18, 3903-3913.

113. Brandes, N.; Schmitt, S.; Jakob, U. Thiol-based redox switches in eukaryotic proteins. Antioxid. Redox Signal. 2009, 11, 997-1014.

114. Alonso-Monge, R.; Navarro-Garcia, F.; Molero, G.; Diez-Orejas, R.; Gustin, M.; Pla, J.; Sanchez, M.; Nombela, C. Role of the mitogen-activated protein kinase Hog1p in morphogenesis and virulence of Candida albicans. J. Bacteriol. 1999, 181, 3058-3068.

115. Cheetham, J.; MacCallum, D.M.; Doris, K.S.; da Silva Dantas, A.; Scorfield, S.; Odds, F.; Smith, D.A.; Quinn, J. MAPKKK-independent regulation of the Hog1 stress-activated protein kinase in Candida albicans. J. Biol. Chem. 2011, 286, 42002-42016. 
116. Prieto, D.; Roman, E.; Correia, I.; Pla, J. The Hog pathway is critical for the colonization of the mouse gastrointestinal tract by Candida albicans. PLOS ONE 2014, 9, e87128.

117. Arana, D.M.; Alonso-Monge, R.; Du, C.; Calderone, R.; Pla, J. Differential susceptibility of mitogen-activated protein kinase pathway mutants to oxidative-mediated killing by phagocytes in the fungal pathogen Candida albicans. Cell. Microbiol. 2007, 9, 1647-1659.

118. Loll-Krippleber, R.; d'Enfert, C.; Feri, A.; Diogo, D.; Perin, A.; Marcet-Houben, M.; Bougnoux, M.E.; Legrand, M. A study of the DNA damage checkpoint in Candida albicans: Uncoupling of the functions of Rad53 in DNA repair, cell cycle regulation and genotoxic stress-induced polarized growth. Mol. Microbiol. 2014, 91, 452-471.

119. Shockley, A.H.; Doo, D.W.; Rodriguez, G.P.; Crouse, G.F. Oxidative damage and mutagenesis in Saccharomyces cerevisiae: Genetic studies of pathways affecting replication fidelity of 8-oxoguanine. Genetics 2013, 195, 359-367.

120. Leroy, C.; Mann, C.; Marsolier, M.C. Silent repair accounts for cell cycle specificity in the signaling of oxidative DNA lesions. EMBO J. 2001, 20, 2896-2906.

121. Muniyappa, H.; Song, S.; Mathews, C.K.; Das, K.C. Reactive oxygen species-independent oxidation of thioredoxin in hypoxia: Inactivation of ribonucleotide reductase and redox-mediated checkpoint control. J. Biol. Chem. 2009, 284, 17069-17081.

122. Guo, Z.; Kozlov, S.; Lavin, M.F.; Person, M.D.; Paull, T.T. ATM activation by oxidative stress. Science 2010, 330, 517-521.

123. Wilson, D.; Tutulan-Cunita, A.; Jung, W.; Hauser, N.C.; Hernandez, R.; Williamson, T.; Piekarska, K.; Rupp, S.; Young, T.; Stateva, L. Deletion of the high-affinity camp phosphodiesterase encoded by Pde 2 affects stress responses and virulence in Candida albicans. Mol. Microbiol. 2007, 65, 841-856.

124. Deveau, A.; Piispanen, A.E.; Jackson, A.A.; Hogan, D.A. Farnesol induces hydrogen peroxide resistance in Candida albicans yeast by inhibiting the Ras-cyclic AMP signaling pathway. Eukaryot. Cell 2010, 9, 569-577.

125. Kamthan, M.; Nalla, V.K.; Ruhela, D.; Kamthan, A.; Maiti, P.; Datta, A. Characterization of a putative spindle assembly checkpoint kinase Mps1, suggests its involvement in cell division, morphogenesis and oxidative stress tolerance in Candida albicans. PLOS ONE 2014, 9, e101517.

126. Bai, C.; Ramanan, N.; Wang, Y.M.; Wang, Y. Spindle assembly checkpoint component CaMad2p is indispensable for Candida albicans survival and virulence in mice. Mol. Microbiol. 2002, 45, 31-44.

127. Du, C.; Calderone, R.; Richert, J.; Li, D. Deletion of the SSK1 response regulator gene in Candida albicans contributes to enhanced killing by human polymorphonuclear neutrophils. Infect. Immun. 2005, 73, 865-871.

128. Calera, J.A.; Zhao, X.J.; Calderone, R. Defective hyphal development and avirulence caused by a deletion of the SSK1 response regulator gene in Candida albicans. Infect. Immun. 2000, 68, $518-525$. 


\title{
Anti-Inflammatory Activity of Haskap Cultivars Is Polyphenols-Dependent
}

\author{
H. P. Vasantha Rupasinghe, Mannfred M. A. Boehm, Satvir Sekhon-Loodu, Indu Parmar, \\ Bob Bors and Andrew R. Jamieson
}

\begin{abstract}
Haskap (Lonicera caerulea L.) berries have long been used for their health promoting properties against chronic conditions. The current study investigated the effect of Canadian haskap berry extracts on pro-inflammatory cytokines using a human monocytic cell line THP-1 derived macrophages stimulated by lipopolysaccharide. Methanol extracts of haskap from different growing locations in Canada were prepared and characterized for their total phenolic profile using colorimetric assays and liquid chromatography-Mass spectrometry (UPLC-MS/MS). Human THP-1 monocytes were seeded in 24-well plates $\left(5 \times 10^{5} /\right.$ well $)$ and treated with phorbol 12-myristate 13-acetate (PMA, $0.1 \mu \mathrm{g} / \mathrm{mL}$ ) for $48 \mathrm{~h}$ to induce macrophage differentiation. After $48 \mathrm{~h}$, the differentiated macrophages were washed with Hank's buffer and treated with various concentrations of test compounds for $4 \mathrm{~h}$, followed by the lipopolysaccharide (LPS)-stimulation $(18 \mathrm{~h})$. Borealis cultivar showed the highest phenolic content, flavonoid content and anthocyanin content $(p<0.05)$. A negative correlation existed between the polyphenol concentration of the extracts and pro-inflammatory cytokines: Interleukin-6 (IL-6), tumour necrosis factor-alpha (TNF- $\alpha$ ), prostaglandin $\left(\mathrm{PGE}_{2}\right)$, and cyclooxygenase-2 (COX-2) enzyme. Borealis exhibited comparable anti-inflammatory effects to COX inhibitory drug, diclofenac. The results showed that haskap berry polyphenols has the potential to act as an effective inflammation inhibitor.
\end{abstract}

Reprinted from Biomolecules. Cite as: Rupasinghe, H.P.V.; Boehm, M.M.A.; Sekhon-Loodu, S.; Parmar, I.; Bors, B.; Jamieson, A.R. Anti-Inflammatory Activity of Haskap Cultivars Is Polyphenols-Dependent. Biomolecules 2015, 5, 1079-1098.

\section{Introduction}

Free radicals, produced as metabolic process mediators have a tendency to attack biomolecules like DNA, RNA, proteins and lipids [1]. The damage caused by these reactive oxygen species can vary from loss of enzyme function, increased cell permeability, and affected cell signalling causing inflammation, diabetes, and cancer [2-4]. Chronic inflammation is known to contribute to the risk of developing metabolic disease, which has become especially prevalent in recent years $[5,6]$. Inflammation is a pathophysiological response of living tissue to injuries, mediated by macrophages, leukocytes and neutrophils through oxidative species. The inflammation cascade involves various factors and enzymes, mainly cyclooxygenase (COX)-2, tumour necrosis factor-alpha (TNF- $\alpha$ ), interferon-gamma (IFN- $\gamma$ ) among others. However, metabolic syndrome can be amended through addressing obesity, elevated blood sugar and cholesterol, physical exercise, and improving diet [7].

The commonly prescribed non-steroidal anti-inflammatory drugs (NSAIDs) such as aspirin (acetyl salicylic acid), diclofenac (dichloranilino phenylacetic acid) (non-specific COX inhibitor), nimesulide [N-(2-phenyloxy-4-nitrophenyl)methanesulphonamide] (COX-2 specific inhibitor) have 
the ability to inhibit COX enzyme which is responsible for the production of pro-inflammatory prostaglandins and prostacyclins. However, there are different undesirable effects related with these NSAID, mainly causing bleeding and ulceration in gastrointestinal tract and platelet dysfunction by blocking COX-1 derived prostanoids [8]. Hence, there is a growing interest in finding alternative food therapies, as well as new anti-inflammatory nutraceuticals derived from plant-based foods, such as fruit crops.

Phytochemicals present in functional foods such as cool climate berries offer a great hope as an alternative therapy for chronic disorders. Polyphenols in fruits have demonstrated the potential to terminate free radical reactions and many antioxidant and physiological benefits in biological systems [9]. Haskap (Lonicera caerulea L.), also known as blue honeysuckle, honeyberry, or sweet berry honeysuckle, is native to Siberia, China, and Japan. Haskap is fairly new to Canada, with only three major varieties in production, namely Borealis, Indigo Gem, and Tundra [10]. Along with a flavour similar to raspberries, black currants, and blueberries, haskap polyphenols also attenuates nuclear factor (NF)-kappaB dependent signaling pathway and subsequent production of proinflammatory mediators [11]. Our previous study has demonstrated the antioxidant properties and total phenolic content of the Borealis cultivar of haskap to be competitive with other berries [12], thereby suggesting its health promoting potential. Although various in vitro studies have evaluated blue honeysuckle extracts for their antimicrobial, anti-adherence, antioxidant effects, protective activity against ultraviolet B (UVB)-caused injury of keratinocytes (HaCaT cells), lipopolysaccharide-induced inflammation, and gingival fibroblast oxidative damage by lipopolysaccharide $[11,13,14]$, the information on phenolic characterization and biological activity of recently introduced cultivars of Canadian haskap is limited.

Considering the previous reports, the present study was conducted to investigate the sugar, organic acid and polyphenol profiles of different haskap cultivars grown in Canada and to study their anti-inflammatory potential. In this study, human monocytes (THP-1 cells) differentiated macrophages were employed to investigate the anti-inflammatory properties of haskap berry extracts, as macrophages are predominately involved at the initial stage of inflammation process and secreting cell-signalling molecules [15].

\section{Materials and Methods}

\subsection{Plant Material}

Fruits of four haskap cultivars, Berry Blue (BL), Borealis (BR), Tundra (TN) and Indigo Gem (IG) were obtained from LaHave Forests Farm, Blockhouse, NS, Canada; two cultivars Indigo Gem (SAS-IG) and Tundra (SAS-TN) were obtained from the University of Saskatchewan, Saskatoon, SK, Canada and five genotypes, LC12, LC13, LC16, LC23, and LC47, were obtained from Agriculture and Agric-Food Canada, Kentville, NS, Canada. Berry Blue (also known as Czech No. 17) is a Czech cultivar of Russian descent with early maturity and tall plants, often used as a polliniser. The other cultivars (BR, TN, and IG) are sister seedlings bred in Saskatchewan from Blue Velvet $(\mathrm{Kiev} \# 3) \times$ Blue Belle (also known as Tomichka). Kiev \#3 was derived from an open pollinated plant of L. caerulea var. kamtschatica Sevast, gathered from the Kurile Islands. Czech No. 
17 and Tomichka, like most cultivars of Russian descent, were derived mostly from four varieties of L. caerulea L., but were not derived from varieties from Japan [16].

The Kentville haskap samples (L. caerulea L.) were selected from seedlings planted in 2007. The seed came from open pollenated fruit grown in BC, Canada in 2006 from plants originally developed by Dr. Maxine Thompson of the National Clonal Germplasm Repository in Corvallis, Oregon. The BC plants were derived from L. caerulea var. emphyllocalyx Nakai from Hokkaido. The Kentville selections flower and their fruit ripen later than the listed cultivars. The soil in Kentville was a sandy loam of the Berwick series with $\mathrm{pH} 6.8$ and $2.8 \%$ organic matter. Plants grew with no pruning and minimal fertilization and 5 plants were selected based on positive fruit characteristics and designated LC-12, LC-13, LC-16, LC-23, and LC-47. Fruit from these five plants was hand-harvested for analysis on 10 July 2013 . The samples were frozen at $-20{ }^{\circ} \mathrm{C}$ until use.

\subsection{Chemicals}

HPLC grade methanol, acetonitrile, formic acid, Folin-Ciocalteu reagent and dimethylcinnamaldehyde were purchased from Sigma-Aldrich (Oakville, ON, Canada). The liquid chromatography standards were purchased as follows: Cyanidin-3-O-glucoside from Extrasynthese (Genay Cedex, France); phloridzin, phloretin, chlorogenic acid, ferulic acid and caffeic acid from Sigma-Aldrich; catechin, epicatechin, quercetin, quercetin-3-O-galactoside and quercitin-3-Oglucoside from ChromaDex, Inc. (Santa Ana, CA, USA); quercitin-3-O-rhamnoside, quercitin-3-Ogalactoside and anthocyanin standards from Indofine Chemical Company (Hillsborough, NJ, USA). Hydrochloric acid, sulfuric acid, and 96-well microplates were purchased from Fisher Scientific (Ottawa, ON, Canada). Phorbol 12-myristate 13-acetate, diclofenac sodium salt, nimesulide and lipopolysaccharide were obtained from Sigma-Aldrich. COX-2 human ELISA kit was purchased from Enzo Life Sciences, Inc. (Farmingdale, NY, USA). Prostaglandin E2 EIA kit and nitric oxide quantification kit was purchased from Cayman Chemical Company (Ann Arbor, MI, USA). The TNF- $\alpha$ and IL-6 ELISA kits were purchased from BD Biosciences (San Diego, CA, USA).

\subsection{Extraction}

Frozen fruit samples (50 g) were ground and extracted with 100\% methanol $(250 \mathrm{~mL})$ using a blender (Model HBB909, Hamilton Beach Brands Inc., Glen Allen, VA, USA) under semi-dark conditions. The extract was filtered through eight layers of cheese cloth and centrifuged at $4900 \times g$ for $10 \mathrm{~min}$ and supernatant was stored at $-20^{\circ} \mathrm{C}$. Prior to performing the assays the berry extracts were evaporated of methanol under a nitrogen evaporator and stored at $-80{ }^{\circ} \mathrm{C}$ until assays were performed.

\subsubsection{Total Phenolic Content}

Total phenolic content was determined by using the modified Folin-Ciocalteu assay as described elsewhere [12]. Total phenolic content was calculated against a gallic acid calibration curve, and samples diluted when necessary to fit range. Total phenolics were expressed as gallic acid equivalents (GAE) $100 \mathrm{~g}^{-1}$ fresh weight (FW). 


\subsubsection{Total Flavonoid Content}

The total flavonoid assay was based on the aluminum chloride colorimetric method as described by Marinova et al. [17] and modified by Rupasinghe et al. [12]. Results were expressed as mmole quercetin equivalents (QE) $100 \mathrm{~g}^{-1} \mathrm{FW}$. The flavonoid quercetin was used as a standard to produce a calibration curve (range 50-500 $\mathrm{mM}$ ).

\subsubsection{Total Anthocyanin Content}

Total anthocyanin concentration (TAC) in the samples was based on the $\mathrm{pH}$-differential method (AOAC method 2005.02) as previously described [18] and their concentration expressed as mg cyanidin-3-O-glucoside (C3G) per 100 gram FW using a molar extinction coefficient $(\varepsilon)$ 28,000, molecular weight (MW) 484.8 for $\mathrm{C} 3 \mathrm{G}$.

\subsubsection{Total Proanthocyanidin Content}

Total proanthocyanidin content was determined by the 4-dimethylaminocinnamaldehyde (DMAC) assay, as reported by Prior et al. [19] with modifications. DMAC reagent was prepared by adding $10 \mathrm{~mL}$ of acidified methanol to $0.01 \mathrm{~g}$ of DMAC. Then, $150 \mu \mathrm{L}$ of DMAC reagent was added to a 96 -well plate with $50 \mu \mathrm{L}$ of berry extract and read at $640 \mathrm{~nm}$. Samples were standardized against a 1000 ppm catechin stock in 5 dilutions with methanol. Total proanthocyanidin concentration was expressed as mg catechin equivalents (CE) $100 \mathrm{~g}^{-1} \mathrm{FW}$.

\subsubsection{LC-MS/MS Analysis of Specific Polyphenols}

Total monomeric polyphenols were identified and massed by liquid chromatography-Mass spectrometry (LC-MS/MS) analysis as described elsewhere [20]. Analysis was carried out using a Waters H-class UPLC separation module (Waters, Milford, MA, USA) coupled with a Micromass Quattro micro API MS/MS system and controlled with MassLynx V4.0 data analysis system (Micromass, Cary, NC, USA). An Aquity BEH C18 $(100 \mathrm{~mm} \times 2.1 \mathrm{~mm}, 1.7 \mu \mathrm{m})$ column (Waters, Milford, MA, USA) was used.

The analysis of flavonol, flavan-3-ol, phenolic acid, and dihydrochalcone compounds was done by electrospray ionization in negative ion mode (ESI-), with a capillary voltage of $3000 \mathrm{~V}$, nebulizer gas $\left(\mathrm{N}_{2}\right)$ temperature of $375^{\circ} \mathrm{C}$, and flow rate of $0.35 \mathrm{~mL} \cdot \mathrm{min}^{-1}$. Anthocyanin compounds were analysed by electrospray ionization in positive ion mode $(\mathrm{ESI}+)$, with capillary voltage $3500 \mathrm{~V}$, nebulizer gas at $375{ }^{\circ} \mathrm{C}$, and flow rate of $0.35 \mathrm{~mL} \cdot \mathrm{min}^{-1}$. The cone voltage $(25-50 \mathrm{~V})$ was optimized for individual compounds.

\subsubsection{Sugars and Organic Acid Analyses}

Samples were evaporated to remove methanol and dissolved in $100 \%$ deionised water prior to their filtration through a $0.45 \mu \mathrm{m}$ nylon filter (Chromaspec, Brockville, ON, Canada) for analyses of sugars (glucose, fructose and sucrose) and organic acids (lactic, malic, quinic, citric acid) by HPLC using a Waters Alliance 2695 HPLC system connected to a Waters 2414 refractive index (RI) 
detector. A Rezex ROA column $(250 \times 4.6 \mathrm{~mm} ; 8 \mu \mathrm{m}$; Phenomenex, Torrance, CA, USA) was used and the analysis conditions were: Temperature of $40{ }^{\circ} \mathrm{C}$ and $30{ }^{\circ} \mathrm{C}$ for column and detector, respectively; mobile phase of $0.005 \mathrm{~N}$ sulfuric acid in deionised water; run time of $30 \mathrm{~min}$ and isocratic flow rate $0.6 \mathrm{~mL} / \mathrm{min}$. An injector volume of $10 \mu \mathrm{L}$ was used for each run. Four point calibration curves were obtained by using mixed standard solutions of sugars and organic acids at concentration range between 100 and $1000 \mathrm{mg} / \mathrm{L}$. The results are expressed in $\mathrm{mg} 100 \mathrm{~g}^{-1} \mathrm{FW}$.

\subsection{Cell Culture}

The human leukemia monocytes THP-1 (ATCC ${ }^{\circledR}$ TIB202 ${ }^{\mathrm{TM}}$ ) cell line was cultured at $37^{\circ} \mathrm{C}$ with $5 \%$ humidified $\mathrm{CO}_{2}$ in Roswell Park Memorial Institute (RPMI) 1640 media with $0.05 \mathrm{mM}$ of 2-mercaptoethanol and $10 \%$ fetal bovine serum. The cells were seeded in 24-well plates $\left(5 \times 10^{5} /\right.$ well) and treated with phorbol 12-myristate 13-acetate (PMA, $\left.0.1 \mu \mathrm{g} / \mathrm{mL}\right)$ to induce macrophage differentiation. Differentiated macrophages were verified by observing the cell morphology through an inverted phase contrast microscope (Figure A1) (Nikon Eclipse E 100, Nikon, Mississauga, ON, Canada). The differentiated macrophages were washed and treated with various concentrations of extracts for $4 \mathrm{~h}$, followed by LPS $(18 \mathrm{~h})$. The supernatants were collected and stored at $-20{ }^{\circ} \mathrm{C}$ for further analysis. Extracts that were used in cell culture studies were completely dried to remove solvents and reconstituted in DMSO. The controls contain the DMSO concentration equivalent to all the treatments.

\subsubsection{Measurement of Cell Viability}

Cell viability was determined using the 3-(4,5-dimethylthiazol-2-yl)-5-(3carboxymethoxyphenyl)-2-(4-sulfophenyl)-2H-tetrazolium (MTS) assay. THP-1 monocytes differentiated macrophages were cultured in 96 -well tissue culture plate $\left(5 \times 10^{4}\right.$ cells $/$ well $)$. Cells were treated with 50 and $100 \mu \mathrm{g} / \mathrm{mL}$ of haskap extract and incubated with MTS reagent for $4 \mathrm{~h}$. Optical density was measured at $490 \mathrm{~nm}$ with a microplate reader (FLUOstar OPTIMA, BMG Labtech, Durham, NC, USA).

\subsubsection{Measurement of Nitric Oxide}

Products of nitric oxide synthase activity were measured using Cayman's nitrate/nitrite colorimetric assay kit (Cayman Chemical Co., Ann Arbor, MI, USA). Nitrates were converted to nitrites by nitrate reductase and total accumulated nitrites were converted to an azo compound using Griess reagent. The absorbance was read at $540 \mathrm{~nm}$ using the microplate reader.

\subsubsection{Measurement of COX-2 Activity}

The COX-2 concentration was measured using human COX-2 ELISA kit provided by Enzo Life Sciences. Samples were prepared by extracting COX-2 from the cells and standards were prepared, according to instructions of the manufacturer. The plate was incubated at $37^{\circ} \mathrm{C}$ for $1 \mathrm{~h}$, followed by washing steps and labeled antibody was added. The plate was incubated at $4{ }^{\circ} \mathrm{C}$ for $30 \mathrm{~min}$, followed 
by washing steps and addition of substrate solution. The reaction was stopped and absorbance was measured at $450 \mathrm{~nm}$ using a FLUOstar OPTIMA plate reader (BMG Labtech). The concentrations were calculated in reference to the standard curve and presented as the percentage of the inflammation control.

\subsubsection{Measurement of IL- 6 and TNF- $\alpha$}

The concentrations of pro-inflammatory cytokines (TNF- $\alpha$ and IL-6) were measured from culture medium of control and treated cells by enzyme linked immunosorbant assay (ELISA) kit provided by BD Biosciences (Mississauga, ON, Canada). Anti-human monoclonal antibodies coated plates were developed by using detection antibodies and streptavidin-horseradish peroxidase conjugate provided by the manufacturer with each kit, according to the provided instructions. The absorbance was read at $450 \mathrm{~nm}$ using a FLUOstar OPTIMA plate reader (BMG Labtech). The concentrations were calculated against the standard curve and presented as the percentage of the inflammation control.

\subsubsection{Measurement of $\mathrm{PGE}_{2}$}

The concentration of $\mathrm{PGE}_{2}$ released by LPS-stimulated macrophages was determined by acetylcholinesterase $^{\mathrm{TM}}\left(\mathrm{ACE}^{\mathrm{TM}}\right)$ competitive enzyme immunoassay (EIA) kit purchased from Cayman Chemicals (Burlington, ON, Canada). The standards were prepared according to the manufacturer's instructions. The samples $(50 \mu \mathrm{L})$ were added to the designated wells, followed by $\mathrm{PGE}_{2}$-ACE tracer $(50 \mu \mathrm{L})$ and $\mathrm{PGE}_{2}$-monoclonal antibody. The plates were covered for $18 \mathrm{~h}$ at $4{ }^{\circ} \mathrm{C}$. The plates were developed according to the manufacturer's instructions. The absorbance was read at $420 \mathrm{~nm}$ using a FLUOstar OPTIMA plate reader (BMG Labtech).

\subsection{Statistical Analysis}

All measurements were conducted in triplicate with the mean and standard deviation calculated. The normal distribution of the residuals was tested using the Anderson-Darling test. The data was analysed using ANOVA and multiple mean comparison was done using Tukey's student range test ( $t$-test) on SAS version 9.3 for Windows (SAS Institute, Cary, NC, USA). Significant differences were compared using a $p$ value $\leq 0.05$ for all the parameters and correlations recorded with Pearson correlation coefficients.

\section{Results and Discussion}

\subsection{Qualitative Phenolic Composition}

The total phenolic content determined by the Folin-Ciocalteu assay ranged from 634 to $1154 \mathrm{mg}$ GAE $100 \mathrm{~g}^{-1} \mathrm{FW}$ with the mean value of $832 \mathrm{mg}$ GAE $100 \mathrm{~g}^{-1} \mathrm{FW}$ (Table 1). Cultivar Borealis (BR) had the highest phenolic content and was consistent with the previous findings [12]. Tundra collected from both locations (TN and SAS-TN) had the second highest levels of phenolics (953-1015 mg GAE $\left.100 \mathrm{~g}^{-1} \mathrm{FW}, p>0.05\right)$, while the LCs contained the lowest total phenolic content (634-849 mg GAE $100 \mathrm{~g}^{-1}$ ). Previously reported values of phenolic content of $L$. caerulea 
have been 428.1-622.5 mg GAE $100 \mathrm{~g}^{-1} \mathrm{FW}$ by Rupasinghe et al. [12] and 575 to $903 \mathrm{mg}$ GAE $100 \mathrm{~g}^{-1} \mathrm{FW}$ by Rop et al. [21] that support the current data.

Table 1. Effect of location and cultivars on total phenolic, total flavonoid, total proanthocyanidins and total anthocyanidin content of haskap berry.

\begin{tabular}{|c|c|c|c|c|c|}
\hline $\begin{array}{l}\text { Growing } \\
\text { Location }\end{array}$ & Cultivar & $\begin{array}{c}\text { Total Phenolics (mg } \\
\text { GAE/100 g FW) }\end{array}$ & $\begin{array}{c}\text { Total Flavonoids } \\
(\mathrm{mg} \mathrm{QE} / 100 \mathrm{~g} \mathrm{FW})\end{array}$ & $\begin{array}{c}\text { Total Proanthocyanidins } \\
(\mathrm{mg} \mathrm{CE} / 100 \mathrm{~g} \mathrm{FW})\end{array}$ & $\begin{array}{r}\text { Total Anthocyanins } \\
(\mathrm{mg} \text { CGE/100 g FW) }\end{array}$ \\
\hline \multirow[t]{4}{*}{ LaHave farm } & $\mathrm{BL}$ & $755.9 \pm 9.4^{\mathrm{d}, \mathrm{e}, \mathrm{f}}$ & $1156.6 \pm 121.7^{\mathrm{b}, \mathrm{c}}$ & $13.2 \pm 1.0^{\mathrm{c}}$ & $163.0 \pm 10.1^{\mathrm{c}}$ \\
\hline & $\mathrm{BR}$ & $1154.1 \pm 59.7^{\mathrm{a}}$ & $1582.8 \pm 140.5^{\mathrm{a}}$ & $16.3 \pm 0.9^{\mathrm{c}}$ & $314.0 \pm 2.7^{\mathrm{a}}$ \\
\hline & $\mathrm{TN}$ & $952.9 \pm 18.7^{\mathrm{b}, \mathrm{c}}$ & $1260.3 \pm 69.0^{\mathrm{b}, \mathrm{c}}$ & $16.0 \pm 1.0^{\mathrm{c}}$ & $234.4 \pm 2.6^{b}$ \\
\hline & IG & $884.3 \pm 25.0^{\mathrm{c}, \mathrm{d}}$ & $1327.0 \pm 12.1^{\mathrm{b}, \mathrm{c}}$ & $14.4 \pm 0.5^{\mathrm{c}}$ & $246.9 \pm 13.7^{b}$ \\
\hline \multirow[t]{5}{*}{ Kentville } & $\mathrm{LC}-12$ & $849.2 \pm 28.3^{\mathrm{c}, \mathrm{d}}$ & $1035.5 \pm 86.7^{\mathrm{b}, \mathrm{c}}$ & $37.2 \pm 0.9^{b}$ & $164.9 \pm 5.4^{\mathrm{c}}$ \\
\hline & LC-13 & $796.8 \pm 5.2^{\mathrm{d}}$ & $997.0 \pm 32.0^{\mathrm{b}, \mathrm{c}}$ & $41.0 \pm 3.6^{b}$ & $142.5 \pm 9.1^{c}$ \\
\hline & LC-16 & $664.9 \pm 51.4^{\mathrm{e}, \mathrm{f}}$ & $900.7 \pm 16.6^{\mathrm{c}, \mathrm{d}}$ & $52.3 \pm 2.3^{\mathrm{a}}$ & $70.2 \pm 2.2^{\mathrm{d}}$ \\
\hline & LC-23 & $658.1 \pm 0.4^{\mathrm{e}, \mathrm{f}}$ & $956.3 \pm 11.0^{\mathrm{b}, \mathrm{c}}$ & $34.2 \pm 0.8^{b}$ & $120.2 \pm 1.5^{\mathrm{c}, \mathrm{d}}$ \\
\hline & LC-47 & $634.4 \pm 29.9^{\mathrm{f}}$ & $916.5 \pm 65.2^{\mathrm{c}, \mathrm{d}}$ & $47.0 \pm 0.4^{\mathrm{a}, \mathrm{b}}$ & $133.4 \pm 1.2^{\mathrm{c}}$ \\
\hline \multirow[t]{2}{*}{ Saskatchewan } & SAS-IG & $790.3 \pm 68.4^{\mathrm{d}}$ & $1128.5 \pm 54.2^{\mathrm{c}, \mathrm{d}}$ & $19.6 \pm 2.0^{\mathrm{c}}$ & $246.3 \pm 2.8^{b}$ \\
\hline & SAS-TN & $1015.3 \pm 78.2^{\mathrm{a}, \mathrm{b}}$ & $1428.4 \pm 35.1^{\mathrm{a}, \mathrm{b}}$ & $38.5 \pm 0.9^{b}$ & $303.2 \pm 5.5^{\mathrm{a}}$ \\
\hline
\end{tabular}

Results represent the mean $\pm \mathrm{SD}(n=3)$, Tukey's test, $p<0.05$. BL, Berry Blue; BR, Borealis; TN, Tundra; IG, Indigo Gem; SAS-TN, Saskatoon Tundra; SAS-IG, Saskatoon Indigo GemGAE, gallic acid equivalents; QE, quercetin equivalents; CE, catechin equivalents; CGE, cyanidin-3-glucoside equivalents. Significant differences in each column were compared using the $p$ value $\leq 0.05 .{ }^{\text {a-f }}$ Different letters within rows denote significant differences between values as determined by one way ANOVA analysis.

\subsubsection{Total Flavonoid Content}

The total flavonoid contents of the four cultivars varied from 901 to $1583 \mathrm{mg} \mathrm{QE} 100 \mathrm{~g}^{-1} \mathrm{FW}$ with the mean value of $1154 \mathrm{mg}$ QE $100 \mathrm{~g}^{-1} \mathrm{FW}$ (Table 1). BR was again significantly higher in flavonoids as it was in total phenolics, which was followed by Tundra (SAS-TN and TN) and Indigo Gem (IG). Similar to the total phenolics results, the LC breeding lines had the least amount of flavonoids. Additionally, Berry Blue (BL) demonstrated a low flavonoid content of merely $1156 \mathrm{mg}$ QE $100 \mathrm{~g}^{-1} \mathrm{FW}$. The present data shows about two times higher values of TFC for L. caerulea than one of the previous reports ranging between 594 to $699 \mathrm{mg} \mathrm{QE} \mathrm{g}^{-1} \mathrm{FW}$, with Borealis containing the greatest amounts [12]. However, another finding reported much less total flavonoids (30 to $40 \mathrm{mg}$ QE $100 \mathrm{~g}^{-1} \mathrm{FW}$ ) in blue honeysuckle [21]. This, the large variation in flavonoid content may be attributed to differences in cultivars and growing locations and conditions.

\subsubsection{Total Anthocyanin Content}

The total anthocyanin content determined by the $\mathrm{pH}$-differential method was found to have a large variation between 70 to $314 \mathrm{mg} \mathrm{C} 3 \mathrm{G} 100 \mathrm{~g}^{-1} \mathrm{FW}$ (Table 1). BR and SAS-TN were attributed with the highest levels of anthocyanins $(p<0.05)$, while the LCs was found to be significantly lower in these compounds. However, these results were lower than the previously reported values of $1470 \mathrm{mg}$ C3G $100 \mathrm{~g}^{-1}$ for L. caerulea [22]. 


\subsubsection{Total Proanthocyanidin Content}

The total proanthocyanidin content was found to be between 13 and $52 \mathrm{mg} \mathrm{CE} 100 \mathrm{~g}^{-1} \mathrm{FW}$ (Table 1). Interestingly, all the LC samples showed more than twice the higher proanthocyanidin concentration than the other cultivars. While LC47 and LC16 exhibited the highest level of total proanthocyanidins, on the other hand, BL followed by BR, TN and IG showed the least content. Proanthocyanidins are considered as one of the major phenolic groups found in L. caerulea species [23]. However, in contrast to the previous studies [24,25] showing edible honeysuckle with very high proanthocyanidin content $\left(195-772 \mathrm{mg} 100 \mathrm{~g}^{-1} \mathrm{FW}\right)$, the present results demonstrated much lower values. This discrepancy could be attributed to the difference in standards used for calibration, cultivars and geographical locations. At the time of writing, no previously published data existed stating the concentration of proanthocyanidins (for berries of genus Lonicera) expressed as catechin equivalents.

\subsubsection{LC-MS/MS Composition of Haskap Berry Extract}

The phenolic characterization of haskap by LC-MS/MS is given in Table 2. In line with previous studies, cyanidin-3-O-glucoside followed by cyanidin-3-O-rutinoside were the most abundant anthocyanins observed in selected cultivars of haskap [26,27]. BR exhibited the highest cyanidin-3-O-glucoside concentration ( $76 \%$ of the total anthocyanins determined by LCMS), followed by SAS-TN (59\% of the total anthocyanins). On the other hand, TN demonstrated the highest cyanidin-3-O-rutinoside concentration (37\% of the total anthocyanins) among all the investigated cultivars. Consistent with [28], the current study also found trace amounts of petunidin and malvidin. Except for LC samples, quercetin (Q) rutinoside (also called rutin) represented the highest concentrations of $27 \%-88 \%$ of the total flavonols analysed by LCMS. BR presented the highest amount of $24.3 \mathrm{mg} 100 \mathrm{~g}^{-1} \mathrm{FW}$; while LC-13 exhibited the least amount of $6.2 \mathrm{mg} 100 \mathrm{~g}^{-1} \mathrm{FW}$. These results are in agreement with previous studies, which reported a range of 7-17 mg $100 \mathrm{~g}^{-1} \mathrm{FW}$ for rutin $[23,24]$. This was followed by the presence of Q. arabinoside and Q. glucoside; while Q. galactoside was the least present flavonol in the selected haskap cultivars. The Kentville genotypes had lower Q. rutinoside but markedly higher Q. arabinoside (Table 2). This may prove to be a characteristic of L. caerulea var. emphyllocalyx. Among flavan-3-ol monomers, epicatechin and catechin were found most abundant in the selected haskap cultivars representing around $25 \%-77 \%$ and $15 \%-70 \%$ of the total flavan-3-ols, respectively. LC-23 and LC-16 exhibited the highest concentrations of catechin and epicatechin; while BL represented their least amount. Chlorogenic acid was the major phenolic acid observed in the Canadian haskap cultivars with amounts varying between 201-234 mg $100 \mathrm{~g}^{-1} \mathrm{FW}$. Overall, the phenolic content was found to be consistent with a previous report comparing haskap to other berries [12].

Cyanidin-3-O-glucoside has been reported to down-regulate the expression of inducible nitric oxide synthase ( $i \mathrm{NOS}$ ) in mice by suppressing the levels of proinflammatory cytokines (TNF- $\alpha$, IL-6, and IL-1 $\beta$ ) [29], while both rutin and cyanidin-3-O-glucoside have shown to decrease the activity of cytokine mediated transcription factors c-Jun and nuclear factor- $\kappa \mathrm{B}(\mathrm{NF}-\kappa \mathrm{B})$ [30]. Chlorogenic acid is a phenylpropanoid, produced by plants as a stress response in pathogenesis [31] and is an intermediate produced in lignin biosynthesis [32]. This compound has demonstrated an 
ability to reduce levels of NO, expression of COX-2 and $i$ NOS, cytokines TNF- $\alpha$, IL-6, IL-1 $\beta$ as well as impeding the nuclear translocation of NF- $\kappa B$ [33]. Rutin has been reported to reduce TNF- $\alpha$ production and a range of interleukins in mice model [34]. It has also been reported to maintain glucose sensitivity in mice while blocking the development of insulin resistance and preventing macrophage activation [35].
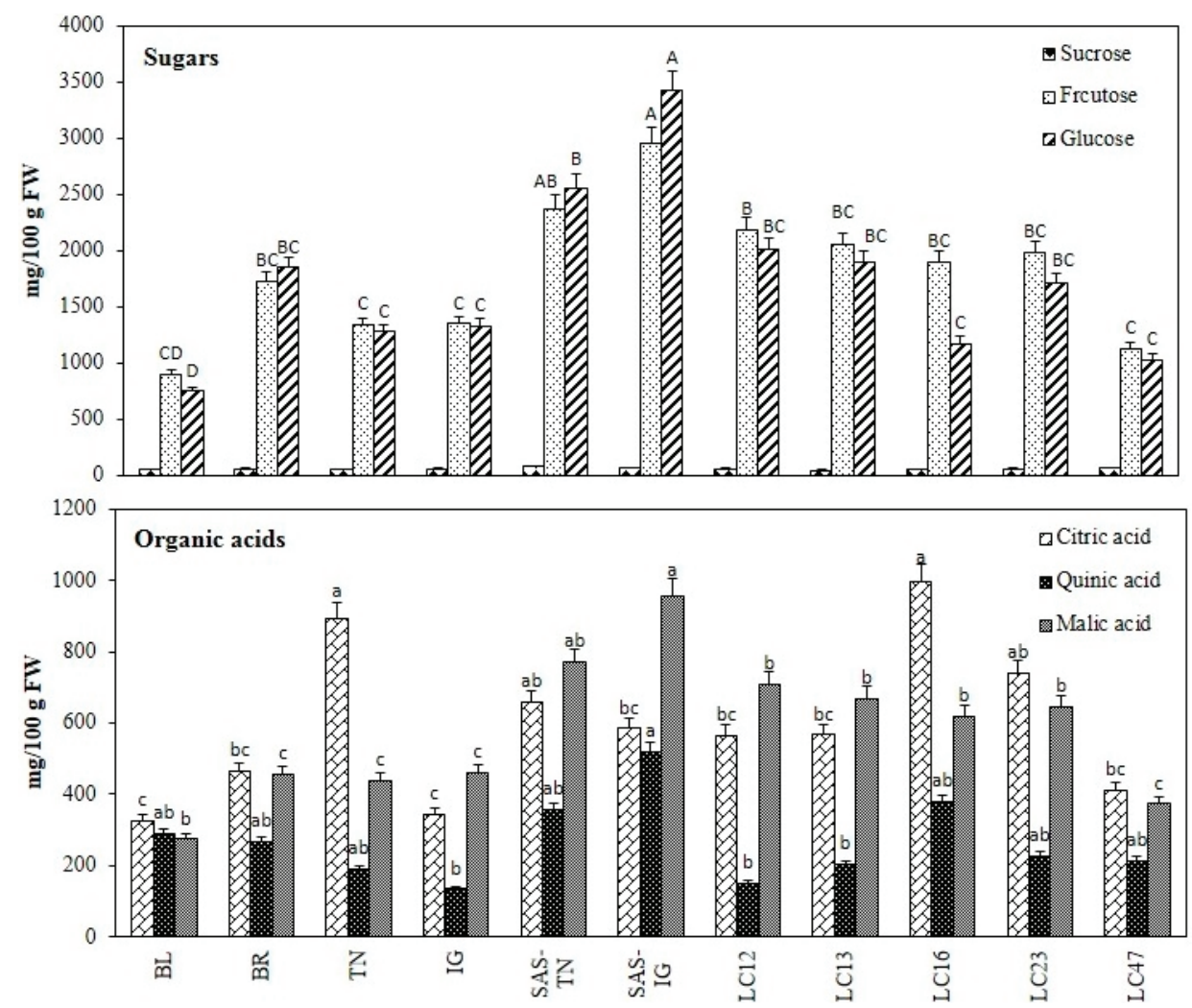

Figure 1. Sugar and organic acid profile of different haskap cultivars. Results represent the mean $\pm \mathrm{SD}(n=3)$, Tukey's test, $p<0.05$. BL, Berry Blue; BR, Borealis; TN, Tundra; IG, Indigo Gem; SAS-TN, Saskatoon Tundra; SAS-IG, Saskatoon Indigo Gem. Significance mean values at $p$ value $\leq 0.05$ : Capital letter grouping $(\mathrm{A}-\mathrm{D})$ is for sugars and small letter grouping $(\mathrm{a}-\mathrm{c})$ is for organic acids. 


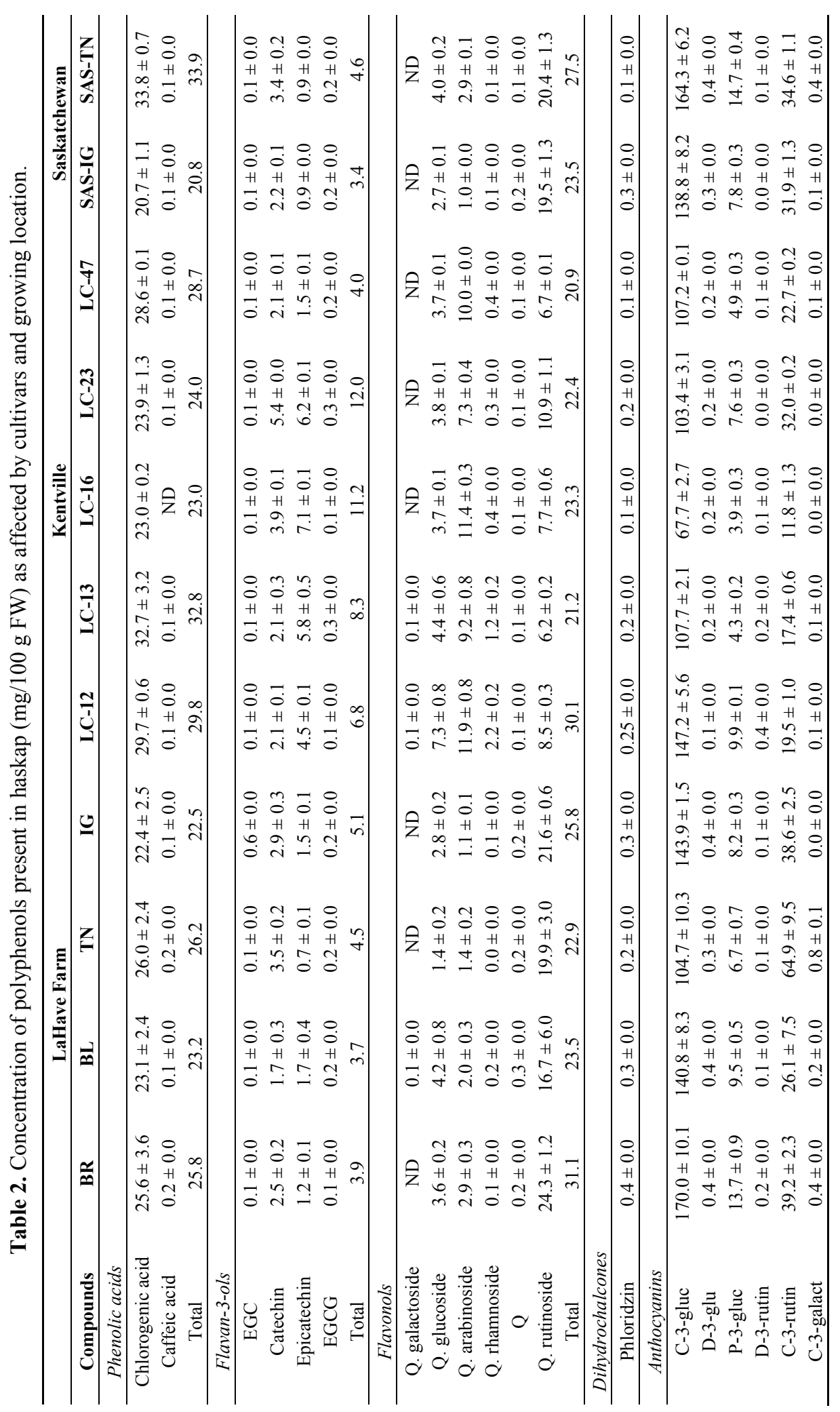




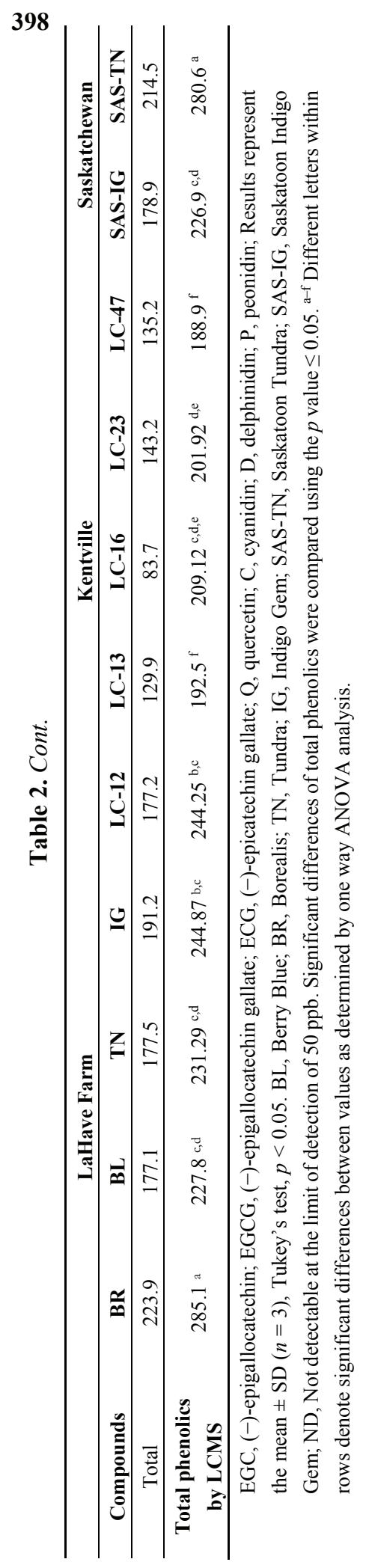




\subsubsection{Sugar and Organic Acid Profile}

Citric, quinic, malic and lactic acid were quantified in the selected haskap cultivars (Figure 1). Citric acid was the predominant organic acid, accounting for $30 \%-58 \%$ of the total organic acid content. These results supported a recent report on $L$. caerulea that showed citric acid representing around $47 \%$ of the total organic acid content in haskap samples [23]. This was closely followed by malic acid content, which ranged between $28 \%-50 \%$ of the total organic acid content. The present study showed the presence of $10 \%-32 \%$ quinic acid with respect to the total organic acid, which was higher than reported by the previous study [23]. In contrast, lactic acid was not detected in all Canadian haskap cultivars. SAS-IG and LC-16 exhibited the highest concentration of organic acid.

The monosaccharides, glucose and fructose, predominated the haskap berries and together accounted for more than $95 \%$ of the total sugars analysed (Figure 1). Trace amounts of sucrose were also identified in the analysed fruits. Similar to the organic acid content, the highest sugar content

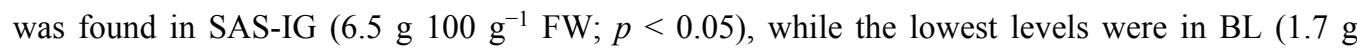

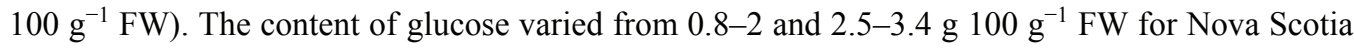
and Saskatoon berries, respectively. Similarly fructose content ranged from 0.9-2.1 and 2.3-2.9 g $100 \mathrm{~g}^{-1} \mathrm{FW}$ for Nova Scotia and Saskatoon fruits, respectively. Consistent with the previously described pattern, the results showed that the cultivars with a high fructose content also had a high glucose content [23].

\subsection{Inhibition of Inflammatory Markers by Haskap Berry Extract}

The working concentrations did not reduce the cell viability significantly. Following LPS stimulation, five markers of inflammation were measured and compared to two common COX inhibitors, diclofenac and nimesulide (Figures 2 and 3). Cultivar BR exhibited the most consistent inhibitory effects. At a concentration of $100 \mu \mathrm{g} / \mathrm{mL}$, BR extract was found to have dose dependent effects on inflammation by inhibiting cytokines TNF- $\alpha$, IL-6, PGE2 and COX-2 to $55 \%, 50 \%, 52 \%$ and $38 \%$, respectively (Figure 2). Furthermore, the concentrations of all five measured inflammatory markers in response to $100 \mu \mathrm{g} / \mathrm{mL}$ haskap extracts were comparable to the performance of diclofenac. Considering that BR was found to have the highest concentration of total phenolics (Tables 1 and 2), the inhibition of inflammatory markers was significantly correlated with total flavonols, total anthocyanins and total phenolics (Table 3). Additionally, Borealis had the highest levels of cyanidin3-O-glucoside and rutin (Table 2).

Extract of TN cultivar berries also showed significant dose dependent effects; at $10 \mu \mathrm{g} / \mathrm{mL}$ all markers except PGE2 were significantly reduced and at $100 \mu \mathrm{g} / \mathrm{mL}$ all parameters, TNF- $\alpha$ and IL-6, $\mathrm{PGE}_{2}$ and COX-2 were further reduced to $68 \%, 70 \%$ and $74 \%$, respectively, and was as effective as diclofenac in inhibiting COX-2 (47\%) and $\mathrm{PGE}_{2}$ (53\%) (Figure 2). Cultivar IG was not an effective inhibitor for TNF- $\alpha$ at $10 \mu \mathrm{g} / \mathrm{mL}$ concentration, however, was comparable to the other three cultivars at $100 \mu \mathrm{g} / \mathrm{mL}$ concentration in reducing the levels of markers (Figure 2). Extract of cultivar BL was consistently the least effective at a concentration of $10 \mu \mathrm{g} / \mathrm{mL}$ and was not able to effectively reduce levels of COX-2 (81\%), TNF- $\alpha(91 \%)$, IL-6 (99\%) $(p<0.05)$ (Figure 2). Furthermore, its inhibitory effects on COX-2, TNF- $\alpha$, and IL- 6 at $100 \mu \mathrm{g} / \mathrm{mL}$ concentration were comparable to that of the other 
three cultivars at $10 \mu \mathrm{g} / \mathrm{mL}$ concentration. The dose dependent effect was not observed on NO inhibition in BR, TN extracts and diclofenac treatments (Figure 3). The extract of BL cultivar reduced NO level by $37 \%$, followed by BR (32\%), IG (30\%), TN (27\%), respectively, compared to LPS treatment at $100 \mu \mathrm{g} / \mathrm{mL}$. Nimesulide was most effective and reduced NO level by $48 \%$ at $100 \mu \mathrm{g} / \mathrm{mL}$. All extracts were comparable to diclofenac in reducing NO level (Figure 3).
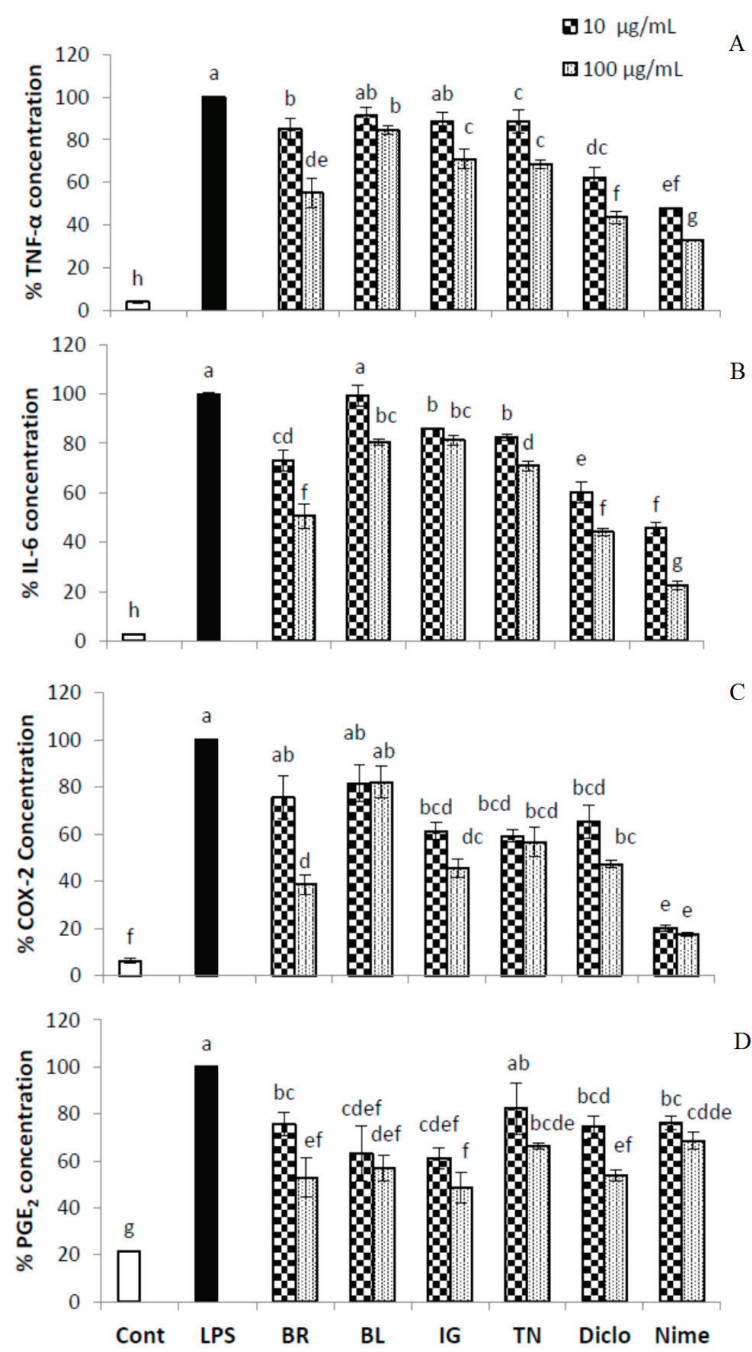

Figure 2. Quantification of TNF- $\alpha$ (A), IL-6 (B), COX-2 (C) and PGE 2 (D) in THP-1 differentiated macrophages. The cells were pretreated with test compounds for $4 \mathrm{~h}$, followed by $18 \mathrm{~h}$ LPS-stimulation. Results represent the mean $\pm \mathrm{SD}(n=3)$, Tukey's test, $p<0.05$. Cont, control; LPS, lipopolysaccharide; BR, borealis; BL, berry blue; IG, indigo gem; TN, tundra; Diclo, diclofenac; Nime, nimesulide. For each bar, different letters a-g represents significance statistical difference using Tukey's $t$-test analysis $(p \leq 0.05)$. The bars with one or more same letter are not statistically different. 


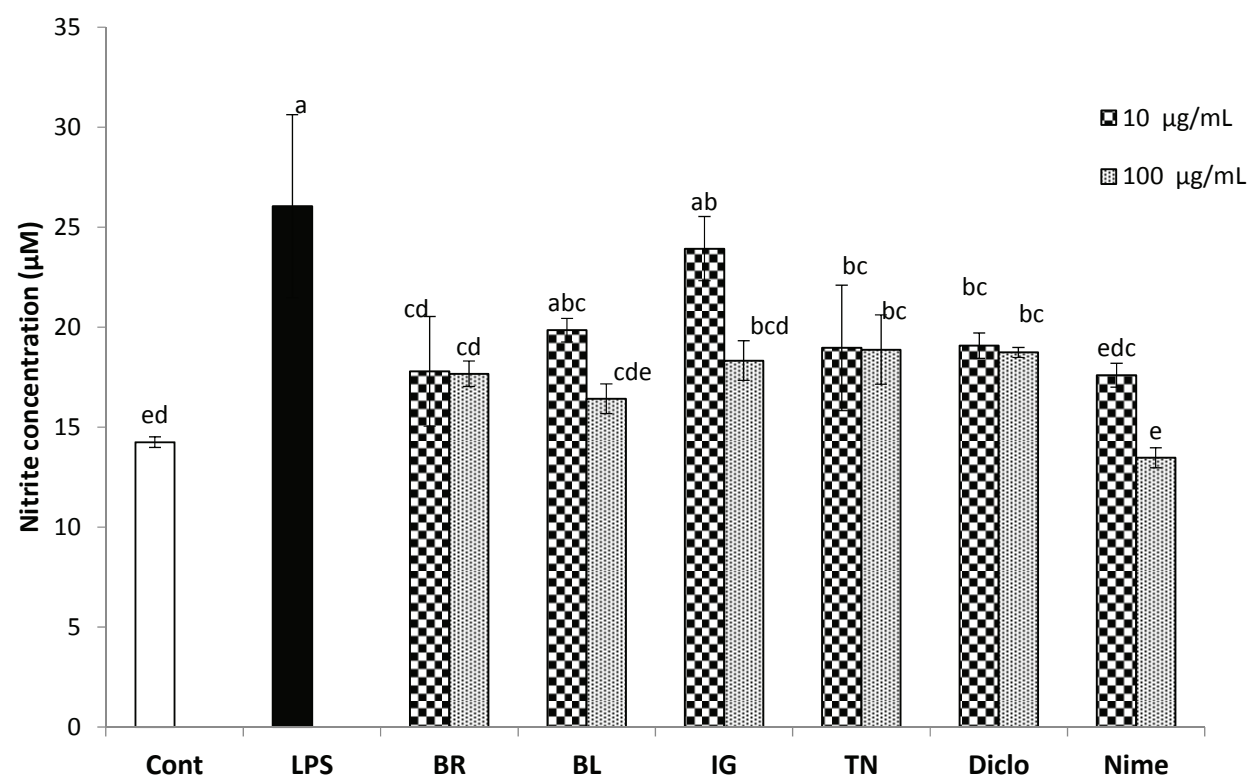

Figure 3. Inhibition of free-radical product (nitric oxide) by haskap berry extract in THP1 differentiated macrophages. The cells were pretreated with test compounds for $4 \mathrm{~h}$, followed by $18 \mathrm{~h}$ LPS-stimulation. Results represent the mean $\pm \mathrm{SD}(n=3)$, Tukey's test, $p<0.05$. Cont, control; LPS, lipopolysaccharide; BR, borealis; BL, berry blue; IG, indigo gem; TN, tundra; Diclo, diclofenac; Nime, nimesulide. For each bar, different letters a-e represents significance statistical difference using Tukey's $t$-test analysis $(p \leq 0.05)$.

Table 3. Correlation between phenolic measurements and five measured parameters of inflammation in LPS-induced macrophages.

\begin{tabular}{cccccc}
\hline Parameters & COX-2 & TNF- $\alpha$ & IL-6 & PGE $_{2}$ & NO \\
\hline Phenolics & $-0.781,0.003$ & $-0.935,0.000$ & $-0.896,0.000$ & $-0.026,0.936$ & $0.324,0.304$ \\
Flavonoids & $-0.742,0.006$ & $-0.781,0.003$ & $-0.723,0.008$ & $-0.186,0.563$ & $0.264,0.406$ \\
Anthocyanins & $-0.728,0.007$ & $-0.831,0.001$ & $-0.629,0.028$ & $0.005,0.988$ & $0.327,0.300$ \\
Proanthocyanidins & $-0.211,0.511$ & $-0.290,0.360$ & $-0.298,0.348$ & $0.459,0.134$ & $0.084,0.796$ \\
\hline
\end{tabular}

Cell contents: Pearson correlation, $p$-value.

LPS is present in the cell wall of gram-negative bacteria and considered to be the most potent stimulator of monocytes and macrophages. Monocytes and macrophages play important roles at the initiation of inflammation and mobilization of the host defence against bacterial infection. LPS-stimulated macrophage activation leads to the secretion of pro-inflammatory signalling molecules known as cytokines [36]. Two common cytokines associated with inflammation are TNF- $\alpha$ and IL-6. TNF- $\alpha$ is an inflammation response protein produced in the body by macrophages, monocytes and adipocytes and endothelial cells. Long term elevated levels of TNF- $\alpha$ have been linked to both Type II diabetes and metabolic syndrome [37]. Reactive oxygen species can activate NF- $\kappa \mathrm{B}$ by releasing inhibitor I $\kappa \mathrm{B}-\alpha$ and activated NF- $\kappa \mathrm{B}$ induces the production of various 
cytokines [38]. The elevation of TNF- $\alpha$ and IL-6 levels are linked to diseases induced by inflammation and are known to stimulate endothelial cells to produce vascular cell adhesion molecules, leading to blood clots and increasing the chances of atherosclerosis [39]. Inflammatory cytokines are known to be associated with COX-2 induction, which plays a role in inflammation, as well as cell growth [40]. COX-2 is a key enzyme attributed to inducing an inflammatory response. It is known to be responsible for the production of pro-inflammatory prostaglandins ( $\left.\mathrm{PGE}_{2}\right)$ at sites of injury [41]. COX-2 has been associated with a range of ailments such as heart disease, joint and muscle pain, and minor headaches. NSAIDS research to treat such symptoms accounts for a multi-billion dollar industry in the United States [42]. The inducible nitric oxide, produced by macrophages induced by TNF- $\alpha$, IL-1 and IL-6, has pro-inflammatory role, whereas e-NO regulates vasodilation is anti-inflammatory in nature. Based on the growing body of evidences, the inhibition of major cytokines like TNF- $\alpha$ and IL- 6 could be the best solution for preventing inflammation related diseases. Inhibition of inflammation appears to be most effective at the level of the two measured cytokines and COX-2 or in other words, the initial steps in the inflammation cascade. This is particularly relevant as it indicates that the haskap extract may be more effective as a preventive rather than responsive treatment to inflammation.

\subsection{Correlation between Phenolics and Inflammatory Parameters}

Overall, the correlation between levels of phenolics of the haskap and the concentrations of COX-2, IL-6, and TNF- $\alpha$ was found to be negative by Pearson's correlation (Table 3). Furthermore, the flavonoids and specifically the anthocyanins showed significant negative correlation with levels of IL-6 and no significant effects were measured on levels of PGE2 or NO. Overall, proanthocyanidins demonstrated no statistically significant effects on the five measured markers of inflammation.

The various epidemiological studies have inversely correlated flavonoid consumption with incidence of stroke, CVD and cancer [42]. Flavonoids are renowned for their free radical scavenging abilities, which are attributed to both their physical structure and chemical activity. As an antioxidant, their primary mode of action is hydrogen atom donation by a hydroxyl group, which stabilizes the target radical to a flavonoid phenoxyl radical [43]. Quercetin, a commonly occurring flavonoid in fruits and vegetables, has been found to inhibit pro-inflammatory cytokine TNF- $\alpha$ by modulating the $\mathrm{NF}-\kappa \mathrm{B}$ transcription factor associated with cytokine expression [44]. Quercetin has also been reported for its benefits in diabetes [45] and hyperglycemia [46].

Anthocyanins are a sub-class of flavonoids and also function as free radical scavengers. Anthocyanins have been reported to inhibit the expression of $i$ NOS responsible for producing nitric oxide [29] by suppressing the levels of pro-inflammatory cytokines earlier in the inflammatory cascade. Similarly, anthocyanins have been shown to modulate transcription factors (c-Jun and $\mathrm{NF}-\kappa \mathrm{B}$ ) responsible for expressing cytokines [30]. This is an exciting prospect because the anti-inflammatory properties of haskap might not be limited to free-radical scavenging, could also be involved in gene expression of the cytokines and enzymes responsible for initiating the inflammatory cascade. 
In summary, we characterized various haskap cultivars for their phenolic composition, sugar and organic profile along with anti-inflammatory properties. Among the selected haskap cultivars, Borealis was found to be the most rich in phenolic content, flavonoid content and anthocyanin content $(p<0.05)$. Cyanidin-3-O-glucoside, cyanidin-3-O-rutinoside, chlorogenic acid and rutin were polyphenols found in the highest concentrations and have been implicated in inhibiting inflammatory transcription factors in previous reports $[13,14]$. The phenolic profile was consistent with a previously published study of haskap [12]. We demonstrated that the extracts of haskap berry, specifically Borealis and Tundra, have markedly suppressed LPS-induced inflammation in an in vitro model of THP-1 derived human macrophages, when compared that with the non-specific COX inhibitor drug, diclofenac. A negative correlation was found between the polyphenols and the major inflammatory mediators. These inflammatory mediators are predominately involved at the initial stages of the inflammatory cascade and, therefore, results suggest that anti-inflammatory compounds could effectively control onset of inflammation. Overall, the results suggest that polyphenols-rich haskap berry has a potential to use as an effective functional food to control inflammation. Recently, Weidinger and colleagues [47] have demonstrated that intracellular signalling pathways mediated by NO and ROS are linked to each other via mitochondrial ROS (mtROS) and form an inducible nitric oxide synthase (iNOS)-mtROS feed-forward loop which intensifies liver failure upon acute inflammation. In general, prospective trials have shown that habitual intake of dietary polyphenols reduces the risk of dementia, stroke and neurodegenerative disorders through a wide spectrum of activities including free radical scavenging, transition metal chelation, activation of survival genes and signalling pathways, regulation of mitochondrial function and modulation of neuroinflammation [48]. Therefore, investigations will be continued to understand the possible mechanisms of haskap polyphenols in regulation of mtROS and other specific targets associated with inflammation. Further investigations are also required to find out the effectiveness of haskap berry polyphenol in animal model systems.

\section{Conclusions}

In conclusion, among the Canadian haskap cultivars assessed, Borealis exhibited the highest phenolic, flavonoid and anthocyanin contents. The polyphenol extracts of haskap could able to suppress the major pro-inflammatory cytokines including IL-6, TNF- $\alpha$ and PGE2 as well as COX-2 enzyme in LPS-stimulated human macrophages in vitro. The results suggest that haskap berry polyphenols need to be further assessed as a natural health product to control inflammation.

\section{Acknowledgements}

This research was funded by Discovery Grant program of the Natural Sciences and Engineering Research Council (NSERC) of Canada. We thank LaHave Forest Farm Inc., Blockhouse, NS, Canada for providing haskap samples. 


\section{Author Contributions}

H. P. Vasantha Rupasinghe is the principal investigator who designed and executed the study. Bob Bors and Andrew R. Jamieson provided the plant materials. Indu Parmar and H. P. Vasantha Rupasinghe performed antioxidant and phenolic characterization studies. Mannfred M. A. Boehm and Satvir Sekhon-Loodu performed the cell culture studies of inflammation. Mannfred M. A. Boehm, Indu Parmar, and Satvir Sekhon-Loodu carried out data analysis and interpretation of the results. All the authors contributed to writing of this manuscript, read and approved the final manuscript.

\section{Appendix}

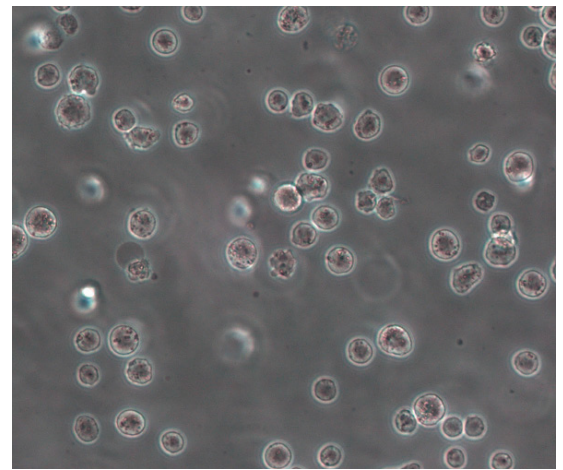

(A)

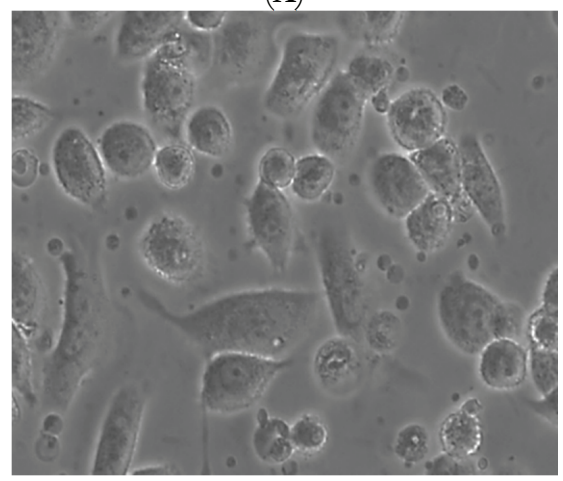

(C)

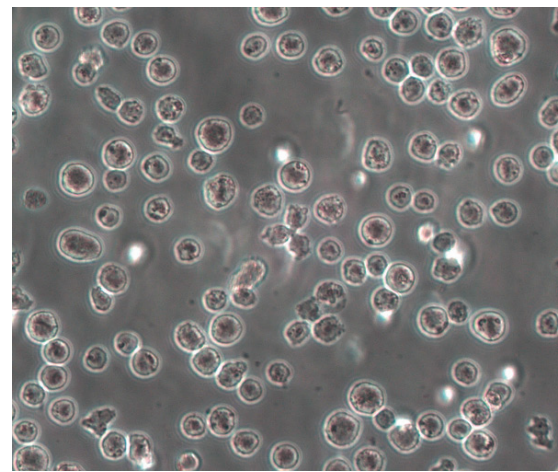

(B)

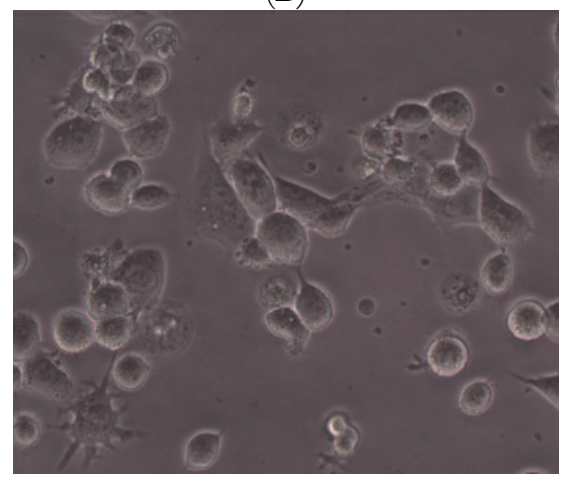

(D)

Figure A1. Morphology of human monocytes (THP-1) and LPS-induced macrophages. Monocytes exist in suspension phase and have spherical shape. Monocytes upon differentiation take adherent phase and form various cell shapes. The undifferentiated monocytes (A), (B) and differentiated macrophages (C), (D) are shown in the figure.

\section{Conflict of Interests}

The authors declare no competing financial interests. 


\section{References}

1. Diplock, A.T.; Charleux, J.L.; Crozier-Willi, G.; Kok, F.J.; Rice-Evans, C.; Roberfroid, M.; Stahl, W.; Vina-Ribes, J. Functional food science and defence against reactive oxidative species. Br. J. Nutr. 1998, 80, S77-S112.

2. Antonicelli, F.; Parmentier, M.; Hirani, N.; Drost, E.; Rahman, I.; Donaldson, K.; MacNee W. LPS stimulation of IL-8 release is inhibited by thiol antioxidant at the transcriptional level in THP-1 macrophage cells. Am. J. Respir. Crit. Care Med. 2000, 161, 1319-1327.

3. Mehta, J.L.; Rasouli, N.; Sinha, A.K.; Molavi, B. Oxidative stress in diabetes, a mechanistic overview of its effects on atherogenesis and myocardial dysfunction. Int. J. Biochem. Cell. Biol. 2006, 38, 794-803.

4. Valko, M.; Rhodes, C.J.; Moncol, J.; Izakovic, M.; Mazur, M. Free radicals; metals and antioxidants in oxidative stress-induced cancer. Chem. Biol. Interact. 2006, 160, 1-40.

5. Prescott, S.L. Early-life environmental determinants of allergic diseases and the wider pandemic of inflammatory non-communicable diseases. J. Allergy Clin. Immunol. 2013, 131, 23-30.

6. Statistics Canada. Canadian Health Measures Survey: Metabolic Syndrome in Canadians. Available online: http://www.statcan.gc.ca/pub/82-625-x/2012001/article/11735-eng.htm\#n1 (accessed on 13 February 2014).

7. Ford, E.S.; Giles, W.H.; Dietz, W.H. Prevalence of the metabolic syndrome among us adults: Findings from the third national health and nutrition examination survey. JAMA 2002, 287, $356-359$.

8. Gautam, R.; Jachak, S.M. Recent developments in anti-inflammatory natural products. Med. Res. Rev. 2009, 29, 767-820.

9. Rupasinghe, H.P.V.; Nair, S.; Robinson, R. Studies in Natural Products Chemistry; Ur Rahman, A., Ed.; Elsevier Science Publishers: Amsterdam, The Netherlands, 2014; Volume 42, pp. 229-266.

10. Bors, B. Breeding of Lonicera caerulea L. for saskatchewan and Canada. In Proceedings of the 1st Virtual International Scientific Conference on Lonicera caerulea L., Saskatoon, SK, Canada, 23 March-23 April 2009; pp. 88-98.

11. Jin, X.H.; Ohgami, K.; Shiratori, K.; Suzuki, Y.; Koyama, Y.; Yoshida, K.; Ilieva, I.; Tanaka, T.; Onoe, K.; Ohno, S. Effects of blue honeysuckle (Lonicera caerulea L.) extract on lipopolysaccharide induced inflammation in vitro and in vivo. Exp. Eye. Res. 2006, 82, 860-867.

12. Rupasinghe, H.P.V.; Yu, L.J.; Bhullar, K.S.; Bors, B. Haskap (Lonicera caerulea): A new berry crop with high antioxidant capacity. Can. J. Plant Sci. 2012, 92, 1311-1317.

13. Palikova, I.; Valentova, K.; Oborna, I.; Ulrichova, J. Protectivity of blue honeysuckle extract against oxidative human endothelial cells and rat hepatocyte damage. J. Agric. Food Chem. 2009, 57, 6584-6589.

14. Zdarilova, A.; Svobodova, A.R.; Chytilova, K.; Simanek, V.; Ulrichova, J. Polyphenolic fraction of Lonicera caerulea L. fruits reduced oxidative stress and inflammatory markers induced by lipopolysaccharide in gingival fibroblasts. Food Chem. Toxicol. 2010, 48, $1555-1561$. 
15. Olefsky, J.M.; Glass, C.K. Macrophages, inflammation, and insulin resistance. Annu. Rev. Physiol. 2010, 72, 219-246.

16. Plekhanova, M.N. Blue honeysuckle (Lonicera caerulea L.)-A new commercial berry crop for temperate climate: Genetic resources and breeding. Acta Hortic. 2000, 538, 159-164.

17. Marinova, D.; Ribarova, F.; Atanassova, M. Total phenolics and total flavonoids in Bulgarian fruits and vegetables. J. Univ. Chem. Tech. Metall. 2005, 40, 255-260.

18. Ratnasooriya, C.; Rupasinghe, H.P.V.; Jamieson, A. Juice quality and polyphenol concentration of fresh fruits and pomace of selected Nova Scotia-grown grape cultivars. Can. J. Plant Sci. 2010, 90, 193-205.

19. Prior, R.L.; Fan, E.; Ji, H.; Howell, A.; Nio, C.; Payne, M.J.; Reed, J. Multi-laboratory validation of a standard method for quantifying proanthocyanidins in cranberry powders. J. Sci. Food Agric. 2010, 90, 1473-1478.

20. Rupasinghe, H.P.V.; Erkan, N.; Yasmin, A. Antioxidant protection of eicosapentaenoic acid and fish oil oxidation by polyphenolic-enriched apple skin extract. J. Agric. Food Chem. 2010, 58, 1233-1239.

21. Rop, O.; Reznicek, V.; Mlcek, J.; Jurikova, T.; Balik, J.; Sochor, J.; Kramarova, D. Antioxidant and radical oxygen species scavenging activities of 12 cultivars of blue honeysuckle fruit. Hortic. Sci. 2011, 38, 63-70.

22. Petrova, V.P. Biochimija Dikorastuščich Plodovo-Jagodnych Rastenij (In Ukraine), 1st ed.; Golovnoe Izdatel'stvo Objedenija, Kijev, Ukraine, 1986; pp. 260-266.

23. Wojdylo, A.; Jauregui, P.N.N.; Carbonell-Barrachina, A.; Oszmianski, J.; Golis, T. Variability of phytochemical properties and content of bioactive compounds in Lonicera caerulea L. var. kamtschatica berries. J. Agric. Food Chem. 2013, 61, 12072-12084.

24. Orincak, J.; Matuskovic, J.; Jurcak, S. Possibilities of Species Lonicera caerulea in Utilization of the Secondary Metabolism in Food and Pharmaceutical Processing, 1st ed.; SPU: Nitra, Slovak, 2003; pp. 210-219.

25. Plekhanova, M.N.; Streltsyna, S.A.; Rostova, N.S. Phenolic compounds in berries of Lonicera subsect. Caerulea species. Plant Res. 1993, 29, 16-25.

26. Andersen, O.M.; Jordheim, M. The anthocyanins. Flavonoids Chemistry, Biochemistry and Applications, 4th ed.; Andersen, O.M., Markham, K.R., Eds.; CRC Press: Boca Raton, FL, USA, 2006; pp. 471-552.

27. Gazdik, Z.; Krska, B.; Adam, V.; Saloun, J.; Jurikova, T.; Reznicek, V.; Horna, A.; Kizek, R. Electrochemical determination of antioxidant potential of some less common fruit species. Sensors 2008, 8, 7564-7570.

28. Bakowska, A.M.; Marianchuk, M.; Kolodziejczyk, P. Survey of bioactive components in Western Canadian berries. Can. J. Physiol. Pharmacol. 2007, 85, 1139-1152.

29. Tsuda, T.; Horia, F.; Osawa, T. Cyanidin 3-O- $\beta$-D-glucoside suppresses nitric oxide production during a zymosan treatment in rats. J. Nutr. Sci. Vitaminol. 2002, 48, 305-310.

30. Karlsen, A.; Retterstol, L.; Laake, P.; Paur, I.; Kjolsrud-Bohn, S.; Sandvik, L.; Blomhoff, R. Anthocyanins inhibit nuclear factor- $\mathrm{BB}$ activation in monocytes and reduce plasma concentrations of pro-inflammatory mediators in healthy adults. J. Nutr. 2007, 137, 1951-1954. 
31. Leiss, K.; Maltese, F.; Choi, Y.H.; Verpoorte, R.; Klinkhamer, P.G.L. Identification of chlorogenic acid as a resistance factor for thrips in chrysanthemum. Plant Physiol. 2009, 150, $1567-1575$.

32. Boerjan, W.; Ralph, J.; Baucher, M. Lignin biosynthesis. Annu. Rev. Plant Biol. 2003, 54, 519-546.

33. Hwang, S.J.; Kim, Y.W.; Park, Y.; Lee, H.J.; Kim, K.W. Anti-inflammatory effects of chlorogenic acid in lipopolysaccharide-stimulated RAW 264.7 cells. Inflamm. Res. 2014, 63, 81-90.

34. Choi, J.K.; Kim, S.H. Rutin suppresses atopic dermatitis and allergic contact dermatitis. Exp. Biol. Med. 2012, 238, 410-417.

35. Gao, M.; Ma, Y.; Liu, D. Rutin suppresses palmitic acids-triggered inflammation in macrophages and blocks high fat diet-induced obesity and fatty liver in mice. Pharm. Res. 2013, 30, 2940-2950.

36. Kim, Y.; So, H.S.; Moon, B.S.; Youn, M.J.; Kim, H.J.; Shin, Y.I.; Moon, S.K.; Song, M.S.; Choi, K.Y.; Song, J.; et al. Sasim attenuates LPS-induced TNF-alpha production through the induction of HO-1 in THP-1 differentiated macrophage-like cells. J. Ethnopharmacol. 2008, $119,122-128$

37. Yang, J.; Park, Y.; Zhang, H.; Gao, X.; Wilson, E.; Zimmer, W.; Abbott, L.; Zhang, C. Role of MCP-1 in tumor necrosis factor- $\alpha$-induced endothelial dysfunction in type 2 diabetic mice. Am. J. Physiol. Heart Circ. Physiol. 2009, 297, H1208-H1216.

38. Rahman, I.; Gilmour, P.S.; Jimenez, L.A.; MacNee, W. Oxidative stress and TNF alpha induce histone acetylation and NF-kappaB/AP-1 activation in alveolar epithelial cells: Potential mechanism in gene transcription in lung inflammation. Mol. Cell Biochem. 2002, 234-235, 239-248.

39. Thilakarathna, S.H.; Rupasinghe, H.P.V. Anti-atherosclerotic effects of fruit bioactive compounds: A review of current scientific evidence. Can. J. Plant Sci. 2012, 92, 407-419.

40. Vane, J.R.; Bakhle, Y.S.; Botting, R.M. Cyclooxygenases 1 and 2. Annu. Rev. Pharmacol. 1998, 38, 97-120.

41. Das, U. Is obesity an inflammatory condition? Nutrition 2001, 17, 953-966.

42. Knekt, P.; Kumpulainen, J.; Jarvinen, R.; Rissanen, H.; Heliovaara, M.; Reunanen, A.; Hakulinen, T.; Aromaa, A. Flavonoid intake and risk of chronic diseases. Am. J. Clin. Nutr. 2002, 76, 560-568.

43. Amic, D.; Davidovic-Amic, D.; Beslo, D.; Rastija, V.; Lucic, B.; Trinajstic, N. SAR and QSAR of the antioxidant activity of flavonoids. Curr. Med. Chem. 2007, 14, 827-845.

44. Dias, A.S.; Porawski, M.; Alonso, M.; Marroni, N.; Collado, P.S.; Gonzalez-Gallego, J. Quercetin decreases oxidative stress, NF-kappa $\beta$ activation, and iNOS overexpression in liver of streptozotocin-induced diabetic rats. J. Nutr. 2005, 135, 2299-2304.

45. Nair, M.P.; Mahajan, S.; Reynolds, J.L.; Aalinkeel, R; Nair, H.; Schwartz, S.A.; Kandaswami, C. The flavonoid quercetin inhibits proinflammatory cytokine (tumor necrosis factor alpha) gene expression in normal peripheral blood mononuclear cells via modulation of the NF-kappa beta system. Clin. Vaccine Immunol. 2006, 13, 319-328. 
46. Jung, M.; Triebel, S.; Anke, T.; Richling, E.; Erkel, G. Influence of apple polyphenols on inflammatory gene expression. Mol. Nutr. Food Res. 2009, 53, 1263-1280.

47. Weidinger, A.; Mullebner, A.; Paier-Pourani, J.; Banerjee, A.; Miller, I.; Lauterbock, L.; Duvigneau, J.C.; Skulachev, V.P.; Redl, H.; Kozlov, A.V. Vicious inducible nitric oxide synthase-mitochondrial reactive oxygen species cycle accelerates inflammatory response and causes liver injury in rats. Antioxid. Redox Signal. 2015, 22, 572-586.

48. Jones, Q.R.D.; Warford, J.; Rupasinghe, H.P.V.; Robertson, G.S. Target-based selection of flavonoids for neurodegenerative disorders. Trends Pharmacol. Sci. 2012, 33, 602-610. 


\section{4-Hydroxy-nonenal-A Bioactive Lipid Peroxidation Product}

\section{Rudolf J. Schaur, Werner Siems, Nikolaus Bresgen and Peter M. Eckl}

Abstract: This review on recent research advances of the lipid peroxidation product 4-hydroxy-nonenal (HNE) has four major topics: I. the formation of HNE in various organs and tissues, II. the diverse biochemical reactions with Michael adduct formation as the most prominent one, III. the endogenous targets of HNE, primarily peptides and proteins (here the mechanisms of covalent adduct formation are described and the (patho-) physiological consequences discussed), and IV. the metabolism of HNE leading to a great number of degradation products, some of which are excreted in urine and may serve as non-invasive biomarkers of oxidative stress.

Reprinted from Biomolecules. Cite as: Schaur, R.J.; Siems, W.; Bresgen, N.; Eck1, P.M. 4-Hydroxy-nonenal-A Bioactive Lipid Peroxidation Product. Biomolecules 2015, 5, 2247-2337.

\section{Table of Contents}

Preface 412

1. Lipid Peroxidation as a Free Radical Amplification Process

2. Structure, Properties and Generation of HNE 417

3. Major Reaction Mechanisms.

3.1. Reactions of the $\mathrm{C}=\mathrm{C}$ Double Bond.... 420

3.1.1. Michael Additions 420

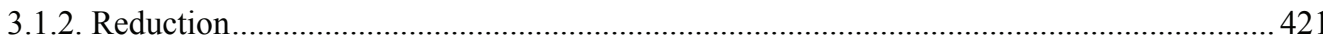

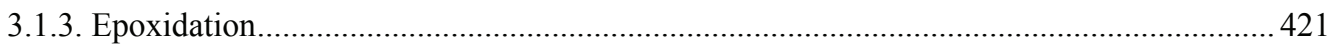

3.2. Reactions of the Carbonyl Group ........................................................................................ 421

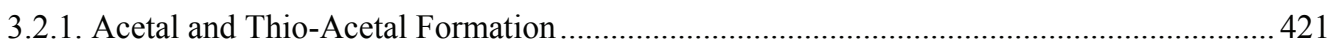

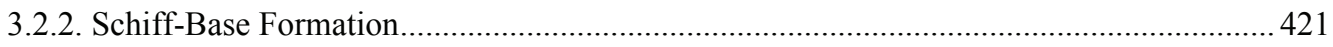

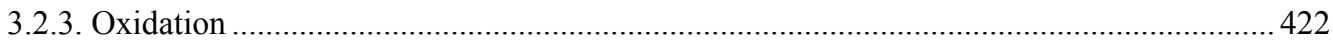

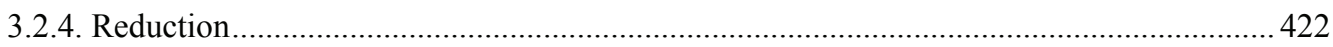

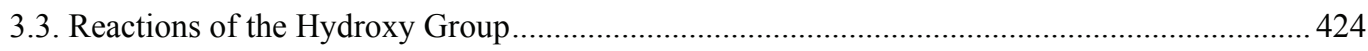

4. Biophysical Effects............................................................................................................................................... 424

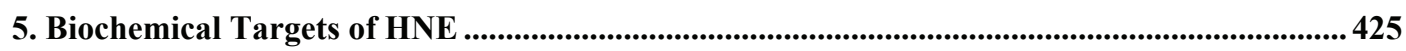

5.1. Reactions with Peptides and Proteins ............................................................................... 425

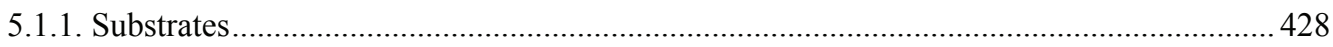

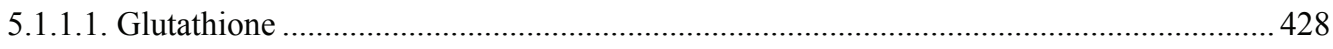

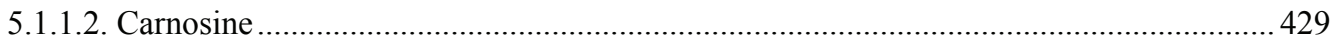

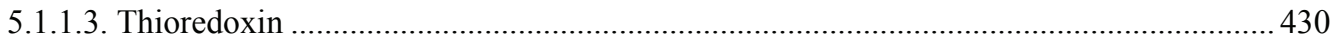




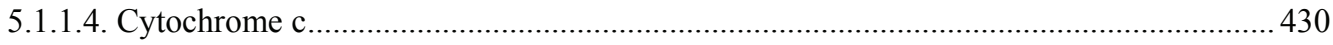

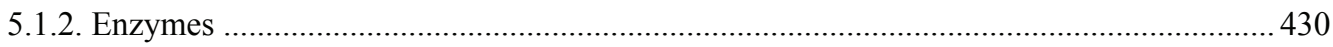

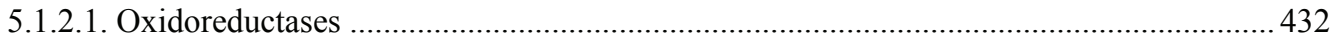

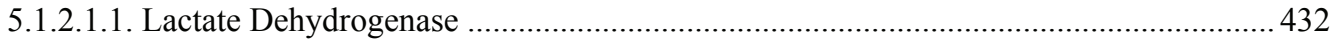

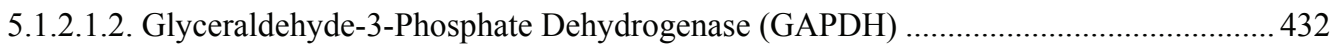

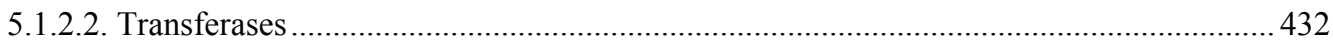

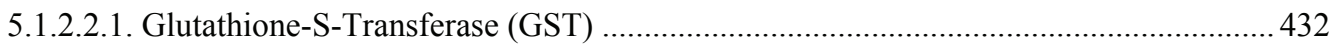

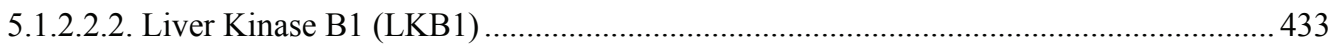

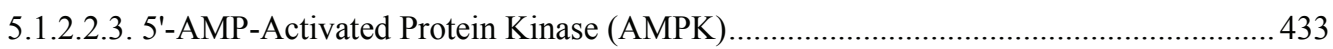

5.1.2.2.4. ZAK Kinase (Sterile Alpha Motif and Leucine Zipper Containing Kinase AZK) ...... 434

5.1.2.2.5. Serine/Threonine-Protein Kinase AKT2 (Proteinkinase B2) ..................................... 434

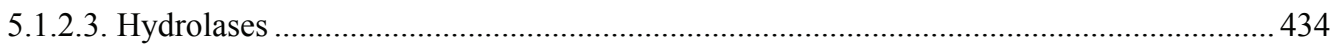

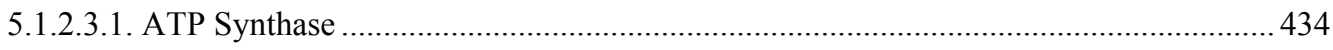

5.1.2.3.2. Phosphatase and Tensin Homolog Deleted on Chromosome 10 (PTEN) ................... 434

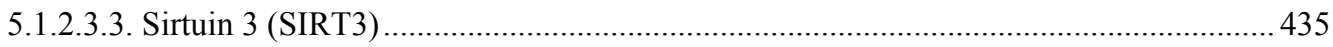

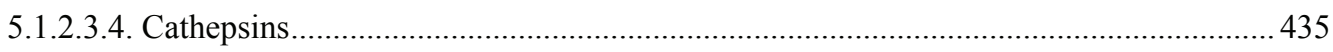

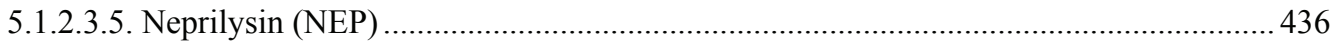

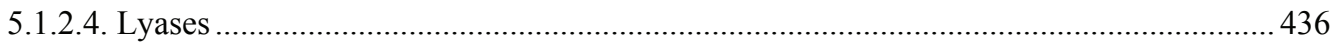

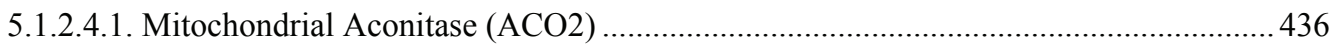

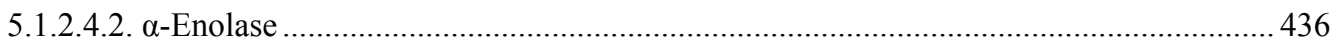

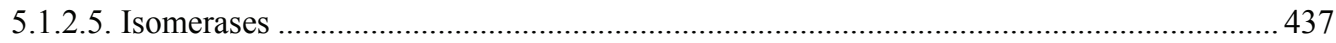

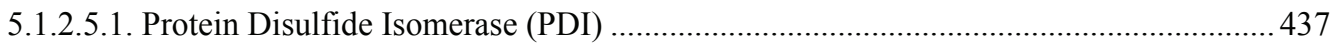

5.1.2.5.2. Peptidyl-Prolyl Cis/Trans-Isomerase A1 (Pin1) ........................................................ 437

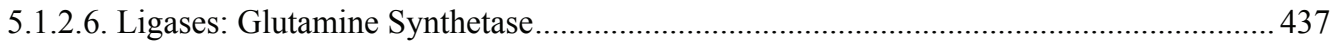

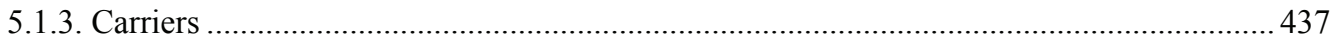

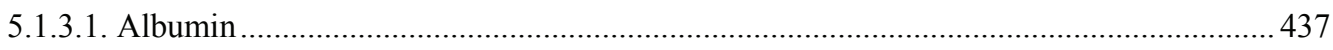

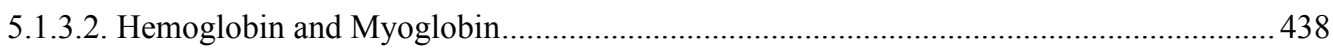

5.1.3.3. Liver and Adipocyte Fatty Acid-Binding Protein (FABP) ......................................... 438

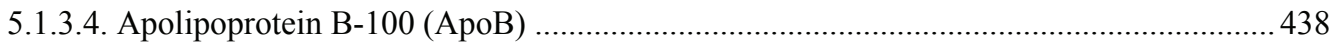

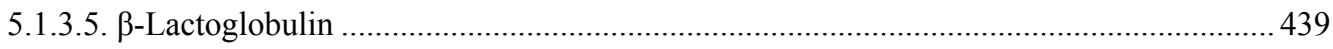

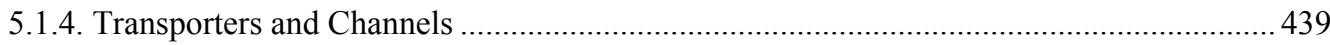

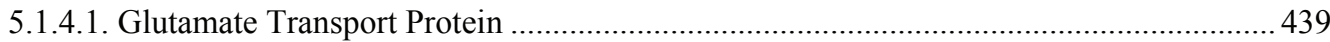




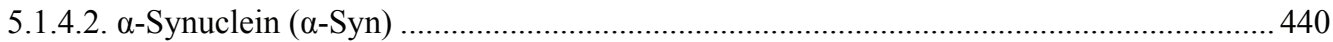

5.1.4.3. Sarco/Endoplasmic Reticulum $\mathrm{Ca}^{2+}$-ATPase (SERCA1a) ............................................... 440

5.1.4.4. Transient Receptor Potential Vanilloid 1 (TRPV1) .................................................... 440

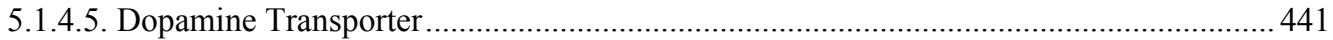

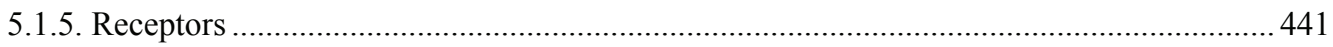

5.1.5.1. Platelet-Derived Growth Factor Receptor- $\beta$ (PDGFR- $\beta$ ) ............................................ 441

5.1.5.2. Lectin-Like Oxidized Low-Density Lipoprotein Receptor-1 (LOX-1)........................... 441

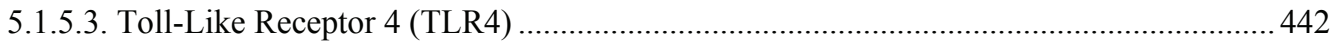

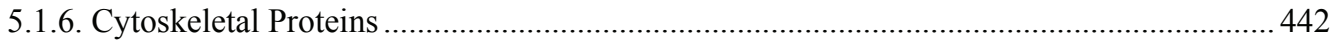

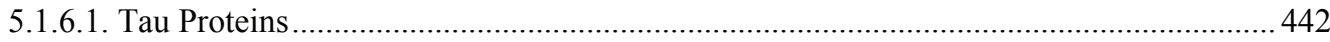

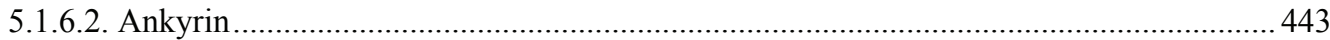

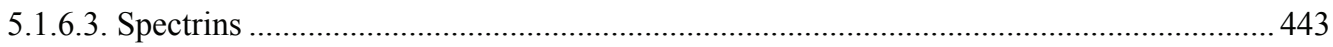

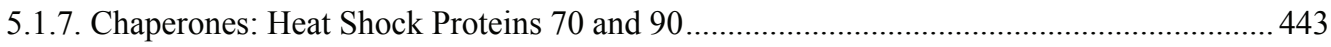

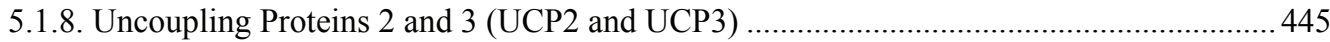

5.1.9. Growth Factors: Platelet-Derived Growth Factor (PDGF)............................................ 446

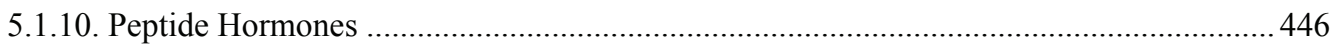

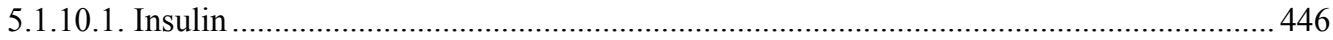

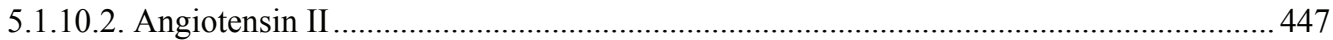

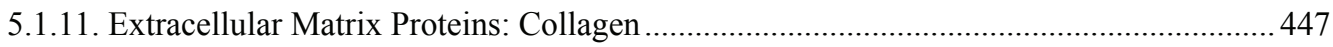

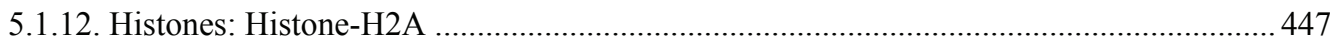

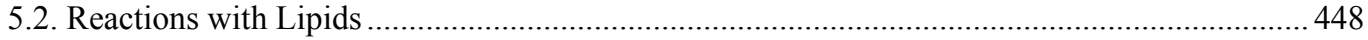

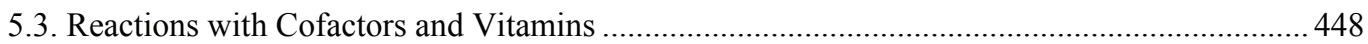

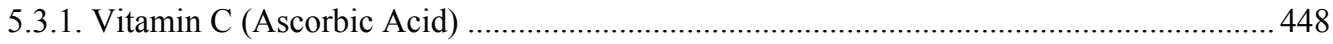

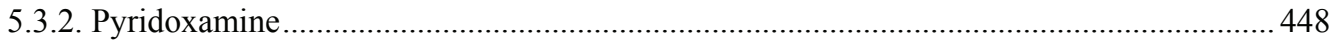

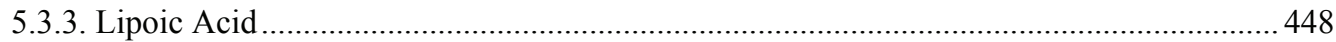

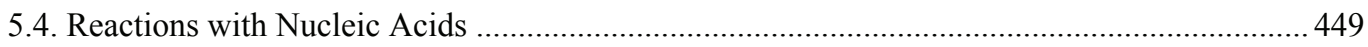

6. Formation of HNE in Mammalian Cells and Tissues ...............................................................451

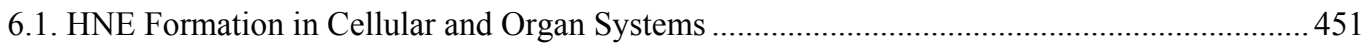

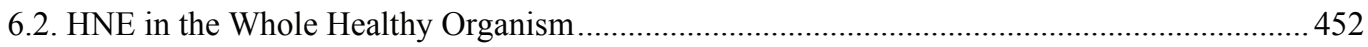

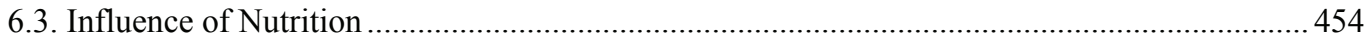

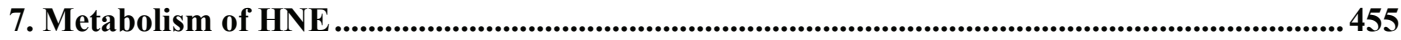

7.1. HNE Metabolism in Mammalian Cells and Organs .............................................................. 457 
7.2. HNE Metabolism in Subcellular Organelles ..................................................................... 458

7.3. HNE Metabolism in Whole Animals and Interorgan Relationships ..................................... 459

7.4. Primary HNE Intermediates_Enzymatic Reactions and Quantitative Results ....................... 460

7.5. Secondary HNE Intermediates_Enzymatic Reactions and Quantitative Results ................... 466

7.6. HNE Metabolism as a Component of the Antioxidative Defense System .............................. 471

7.7. HNE Intermediates as Potential Biomarkers of LPO .................................................................. 472

7.8. Further Medical Applications of HNE Metabolism ............................................................... 472

8. Conclusions .................................................................................................................................. 474

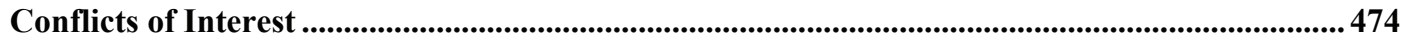

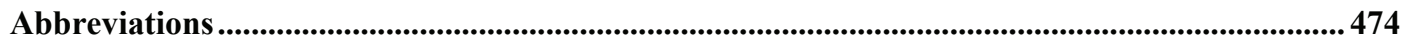

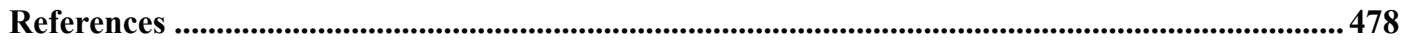

\section{Tables and Figures}

Table 1. HNE concentrations in cells, tissues and organs

Table 2. Maximal velocity of total HNE degradation in cells, subcellular organelles, and perfused organs.

Table 3. Primary HNE metabolites in different cells and tissues after the addition of $100 \mu \mathrm{M} \mathrm{HNE}$ to the biological system.

Figure 1. Idealized representation of the initiation and propagation reactions of lipid peroxidation... 415 Figure 2. Formation of lipid hydroperoxides and cyclic peroxides from arachidonic acid................. 416

Figure 3. Chemical structure of 4-hydroxy-2-trans-nonenal (HNE) ................................................. 417

Figure 4. Overview of the reactions of 4-hydroxy-nonenal with different biomolecules .................... 419

Figure 5. HNE plasma concentration in dependence on age of the blood donor (5 to 90 years) ......... 452

Figure 6. Degradation/metabolism of 4-HNE in rat hepatocytes ...................................................... 456

Figure 7. Identification of HNE and 4-hydroxynonenoic acid (HNA) by isocratic HPLC separation 462 Figure 8. Mass spectrum of dihydroxynonene urethane (HPLC plus MS) with fluorimetric detection

Figure 9. HNE metabolites.

Figure 10. HPLC chromatogram of the isoindol derivative of the HNE-GSH conjugate (reaction product in presence of o-phthalaldehyde)

\section{Preface}

Due to its diverse biochemical reactions and far-reaching biological effects 4-hydroxy-nonenal (HNE) is a fascinating compound, the discovery of which is hidden deep in the scientific literature. When Hermann Esterbauer isolated this aldehyde for the first time a quantitative analysis of its chemical elements was made resulting in values in between those for 4-hydroxy-octenal and 4hydroxy-nonenal (Percent Composition: C 69.19\%, H 10.32\%, O 20.48\%). Since these values 
were closer to those of 4-hydroxy-octenal Esterbauer and his supervisor Schauenstein published it with this chemical formula [1]. Only later the experimental error became evident. Thus, if one searches the literature for 4-hydroxy-nonenal, one would hardly find the paper describing its discovery as a product of lipid peroxidation.

Lipid peroxidation is a prominent feature of oxidative stress (for a review see the classical monograph of Halliwell and Gutteridge [2]), to which reference is made here. Additionally, a multi-author work on lipid peroxidation was edited by Catala [3]. Lipid peroxidation is a free radical amplification process yielding a great variety of bioactive products. One of the most extensively studied examples is HNE, which will be treated in this review. Since several reviews on HNE appeared previously [4-6], the focus of this contribution is directed on recent advances. In a recent review Fritz and Petersen [7] describe the generation and chemical reactivity of HNE and other lipid-derived aldehydes with a special attention to the homeostatic responses to electrophilic stress. Zarkovic et al. [8] describe the pathophysiological relevance of aldehydic protein modifications with special attention to HNE. A recent review of Perluigi et al. [9] describes the role of lipid peroxidation, particularly of HNE-induced protein modification, in neurodegenerative diseases. Furthermore, a review of Riahi et al. [10] addresses the origin and cellular functions of 4-hydroxyalkenals. Forman [11] reviewed the role of HNE and other $\alpha, \beta$-unsaturated aldehydes as well as reactive oxygen species in signal transduction. New concepts and molecular mechanisms in cell signalling by reactive lipid species are discussed by Higdon et al. [12]. Similarly, Dwivedi et al. [13] as well as Ayala et al. [14] describe HNE as a signaling molecule. The chemistry and analysis of HNE was reviewed by Spickett [15]. LoPachin et al. [16] discussed the chemical attributes of HNE and acrolein that determine their toxicities.

A review of Catala [3] provide a synopsis of identified effects of $\mathrm{HNE}$ and other hydroxy-alkenals and oxidized phospholipids on cell signaling, from their intracellular production to their action as intracellular messengers, up to their influence on transcription factors and gene expression. Mattson [17] gave an overview of the roles of HNE in obesity, the metabolic syndrome, and associated vascular and neurodegenerative disorders. Finally, Guichardant and Lagarde [18] reviewed $\mathrm{HNE}$ as well as 4-hydroxy-hexenal and their degradation products as biomarkers of oxidative stress.

\section{Lipid Peroxidation as a Free Radical Amplification Process}

In this chapter a brief overview will be presented on the molecular mechanisms underlying lipid peroxidation. Lipid peroxidation was defined by Tappel [19] as the oxidative deterioration of polyunsaturated lipids. Poly-unsaturated fatty acids are those that contain two or more carbon-carbon double bonds. Initiation of lipid peroxidation can be caused by addition of a reactive species (RS) or, more usually, by hydrogen atom abstraction from a methylene $\left(-\mathrm{CH}_{2}-\right)$ group by a RS; in both cases, a carbon radical results. For example, the hydroxyl radical ${ }^{\bullet} \mathrm{OH}$ can react by addition (Equation (1)) or by $\mathrm{H}^{\bullet}$ abstraction (Equation (2)).

$$
\begin{gathered}
-\mathrm{HC}=\mathrm{CH}-+{ }^{\bullet} \mathrm{OH} \rightarrow-\mathrm{HC}(\mathrm{OH})-{ }^{\bullet} \mathrm{CH}- \\
-\mathrm{CH}_{2-}+{ }^{\bullet} \mathrm{OH} \rightarrow-{ }^{\bullet} \mathrm{CH}-+\mathrm{H}_{2} \mathrm{O}
\end{gathered}
$$


A double bond weakens the bond energy of the $\mathrm{C}-\mathrm{H}$ bonds present on the next carbon atom (the allylic hydrogens), especially when there is a double bond on either side of the $\mathrm{C}-\mathrm{H}$ bond (Figure 1), giving bis-allylic hydrogens.

Hydroxyl radicals readily initiate peroxidation of fatty acids, lipoproteins and membranes. In addition the hydroperoxyl radical $\mathrm{HOO}^{\bullet}$ can abstract $\mathrm{H}$ from some polyunsaturated fatty acids (Equation (3))

$$
\mathrm{HOO}^{\bullet}+\mathrm{CH} \rightarrow \mathrm{HOOH}+\mathrm{C}^{\bullet}
$$

and it can stimulate peroxidation by reaction with preformed lipid hydroperoxides to generate peroxyl radicals [20] (Equation (4)).

$$
\mathrm{HOO}^{\bullet}+\mathrm{ROOH} \rightarrow \mathrm{ROO}^{\bullet}+\mathrm{H}_{2} \mathrm{O}_{2}
$$

The carbon radicals often stabilize by molecular rearrangement to form conjugated dienes (Figure 1).

Under aerobic conditions carbon radicals readily combine with $\mathrm{O}_{2}$ to give a peroxyl radical (Equation (5)).

$$
\mathrm{R}^{\bullet}+\mathrm{O}_{2} \rightarrow \mathrm{ROO}^{\bullet}
$$

Peroxyl radicals can also abstract $\mathrm{H}^{\bullet}$ from an adjacent fatty-acid side-chain (Equation (6)).

$$
\mathrm{ROO}^{\bullet}+\mathrm{CH} \rightarrow \mathrm{ROOH}+\mathrm{C}^{\bullet}
$$

This is the propagation stage of lipid peroxidation. It forms new carbon radicals that can react with $\mathrm{O}_{2}$ to form new peroxyl radicals, and so the chain reaction of lipid peroxidation continues (Figure 1). The $\mathrm{ROO}^{\bullet}$ combines with the $\mathrm{H}^{\bullet}$ that it abstracts to give a lipid hydroperoxide $(\mathrm{ROOH})$. A single initiation event thus has the potential to generate multiple peroxide molecules by a chain reaction.

Cyclic and bicyclic peroxides can also form (Figure 2).

Peroxidized lipids are bioactive. They can have effects on cells similar to those of $\mathrm{H}_{2} \mathrm{O}_{2}$ : low levels can stimulate proliferation, higher levels block proliferation and yet higher ones induce apoptosis and necrosis [21]. Such events occur in atherosclerosis and maybe in the gastrointestinal tract. Products of lipid peroxidation can also have more specific effects. For example, some mimic the actions of platelet activating factor (PAF $=1-O$-alkyl-2-acetyl-sn-glycero-3-phosphocholine). Peroxidation of phosphatidyl-choline (lecithin) can generate fragments that bind to PAF receptors on target cells and exert PAF-like activity. Oxidized PAPC (1-palmitoyl-2-arachidonoylsn-glycero-3-phospho-choline) (ox-PAPC) can stimulate cytokine production in endothelial cells, but can inhibit activation of transcription factor NFKB by endotoxin and subsequent cytokine production, i.e., it can be anti-inflammatory under some circumstances. It can also increase levels of haemoxygenase, a cytoprotective enzyme in endothelial cells. Intraperitoneal injection of ox-PAPC allows mice that had been treated with a normally lethal dose of endotoxin, to survive [22]. Hence several specific lipid oxidation products act as signalling molecules and their actions are not always bad. It can be predicted that more such roles will be found. 


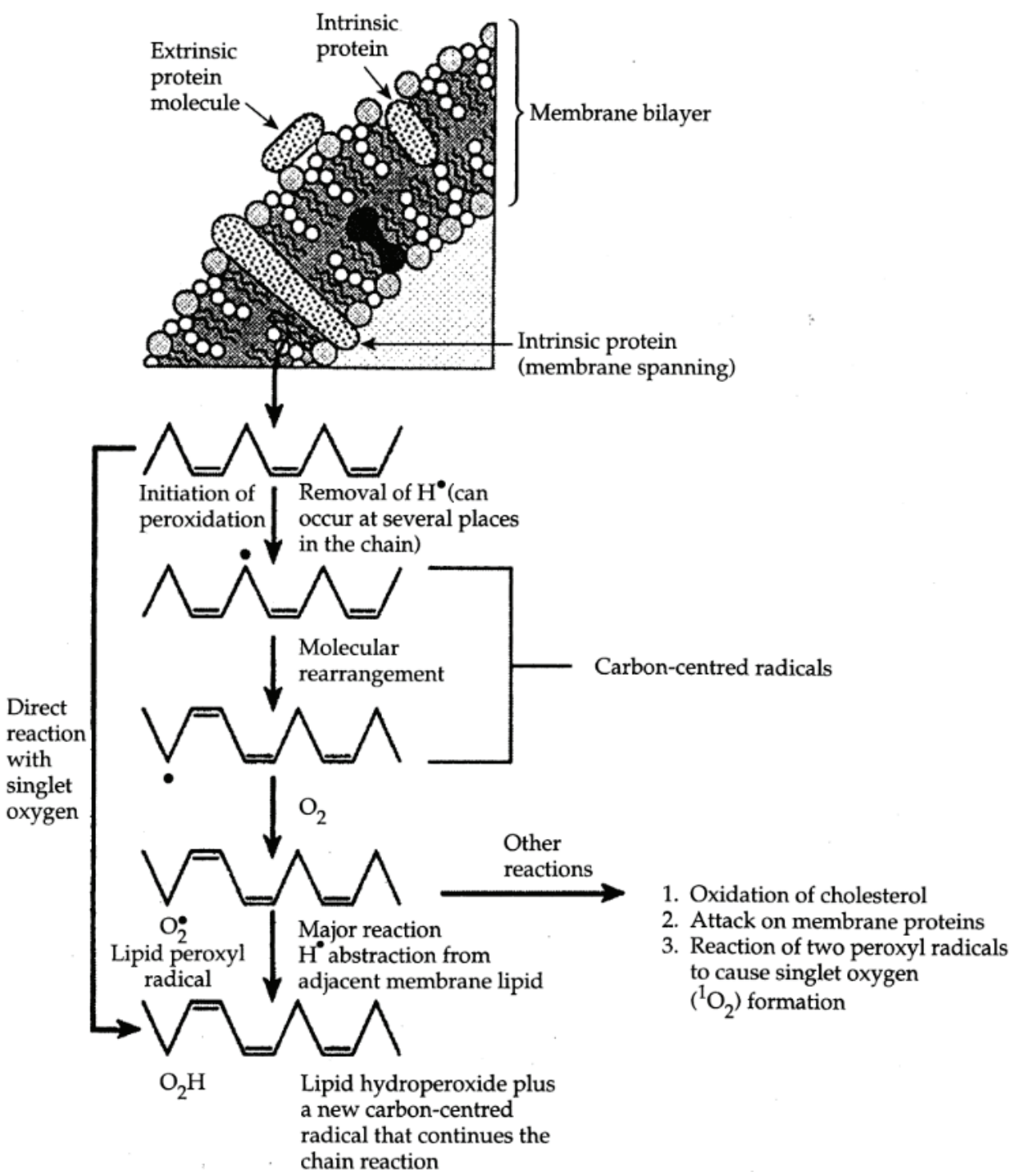

Figure 1. Idealized representation of the initiation and propagation reactions of lipid peroxidation. The peroxidation of fatty acids with three double bonds is shown. Reprinted from [2]. 


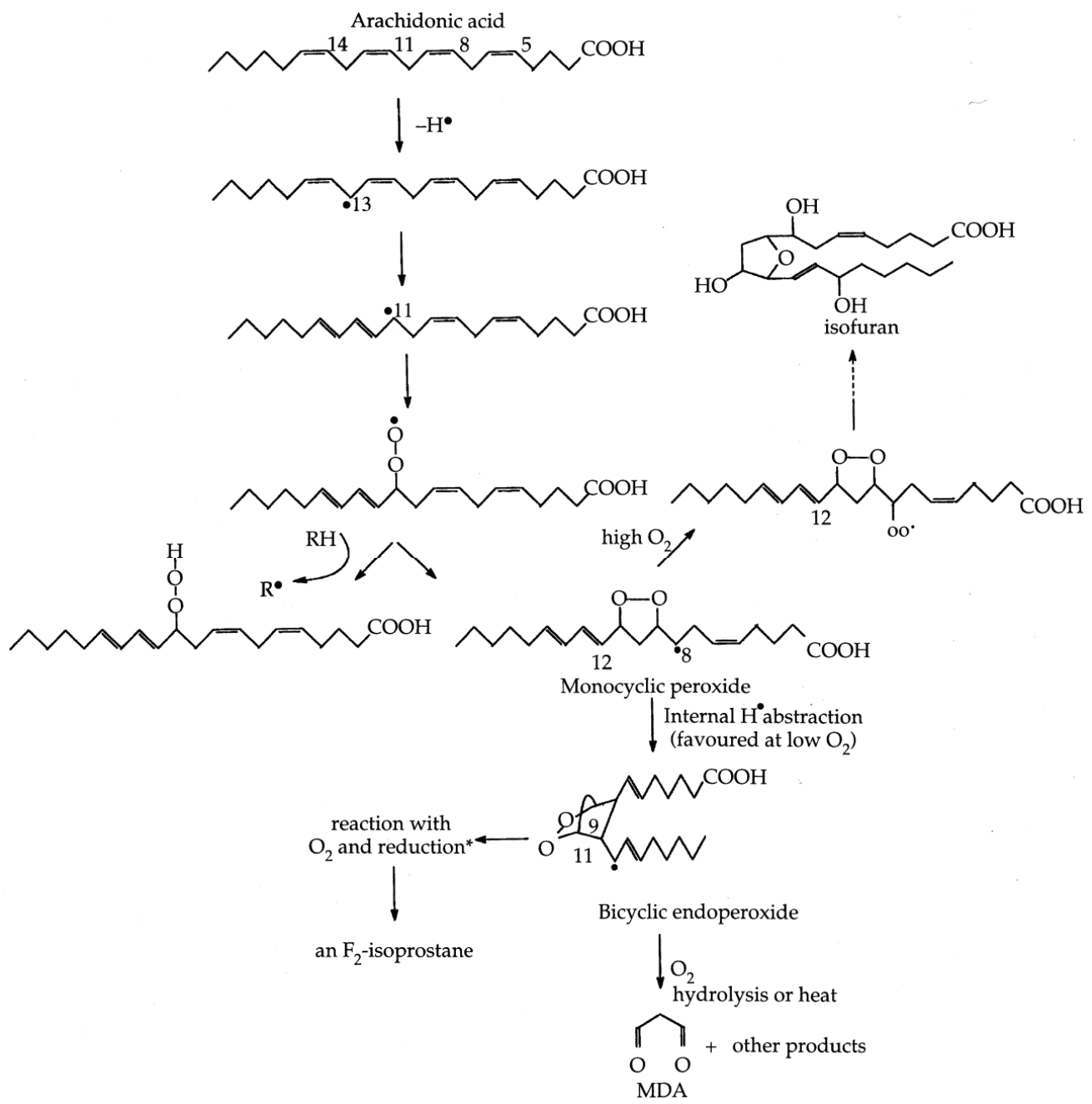

Figure 2. Formation of lipid hydroperoxides and cyclic peroxides from arachidonic acid. The chemistry is complex because abstraction of $\mathrm{H}^{\bullet}$ can occur at different sites; for simplicity we show it only for $\mathrm{C} 13$. The $\mathrm{ROO}^{\bullet}$ radicals formed can generate hydroperoxides or cyclize to monocyclic peroxides. This results when peroxyl radicals attack a double bond in the same chain. Monocyclic peroxides can form bicyclic structures that can give rise to isoprostanes, or react with $\mathrm{O}_{2}$, eventually producing isofurans. Unstable peroxides can decompose to aldehydes such as malondialdehyde. Even products containing bicyclic endoperoxide and cyclic peroxide groups in the same molecule (dioxolane-isoprostanes) can be generated [23]. $\mathrm{O}_{2}$ concentration affects the relative levels of isoprostanes and other products. Reprinted from [2]. 


\section{Structure, Properties and Generation of HNE}

The chemical structure of 4-hydroxy-2-trans-nonenal (4-hydroxy-2E-nonenal, HNE) is shown in Figure 3.

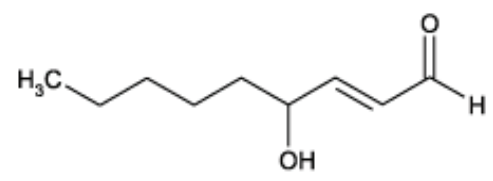

Figure 3. Chemical structure of 4-hydroxy-2-trans-nonenal (HNE).

$\mathrm{HNE}$ is an extraordinarily reactive compound containing three functional groups which in many cases act in concert and help to explain its high reactivity [24]. Most importantly there is a conjugated system of a $\mathrm{C}=\mathrm{C}$ double bond and a $\mathrm{C}=\mathrm{O}$ carbonyl group which provide a partial positive charge to carbon 3 , because this structure contains mobile $\pi$-electrons. The carbonyl oxygen atom is electronegative and can promote the withdrawal of mobile electron density from the beta-carbon atom causing regional electron deficiency. Considering this type of electron polarizability, HNE is considered to be a soft electrophile that preferentially forms 1,4-Michael type adducts with soft nucleophiles. Cysteine sulfhydryl groups are the primary soft nucleophilic targets of HNE while lysine and histidine residues are harder biological nucleophiles [16]. The positive charge on carbon 3 is further increased by the inductive effect of the hydroxy group at carbon 4 . Therefore nucleophilic attack e.g., by thiol or amino groups occurs primarily at carbon 3 and secondarily at the carbonyl carbon 1 .

The relationship between structure and activity has been studied in a toxicity test using L6 muscle cells [25]. The effect of 4-hydroxy-2-alkenals was compared to several chemically related derivatives in order to clarify the physico-chemical requirement of their toxicity in L6 muscle cells. The rank of derivative toxicity was: hydroxy-alkenals $>$ acetal derivatives approximate to 2-alkenals $>$ alkanals and a high correlation was found between toxicity and protein carbonylation. This suggests a cyto-protective effect of nucleophilic scavengers against electrophilic compounds which could be of potential therapeutic benefit in oxidative stress associated diseases.

For the biological effects of HNE it is essential that the lipophilic properties are more pronounced than its hydrophilic properties. Thus HNE tends to concentrate in biomembranes rather than in the aqueous space of cells. HNE can be easily transferred from a membrane to both cytosol and the extracellular space. Studying the behaviour within membranes by using the timedependent fluorescence shift method and molecular dynamics simulations demonstrated the stabilization of HNE in the carbonyl region of a 1-palmitoyl-2-oleoyl-sn-glycero-3-phosphocholine bilayer [26]. Thus, HNE is able to react with cell membrane proteins and lipids albeit stabilization in the membrane is moderate and HNE shuttling to either the extra- or intracellular space occurs in the microsecond range. These characteristics of the HNE-lipid membrane interaction provide a good explanation for the observed reactivity of HNE with proteins inside and outside the cell.

HNE shows chirality at carbon 4 . This might be also biologically relevant. Although HNE is formed in tissues as a racemate, enantiospecific HNE effects have not yet been widely investigated. 
Different cellular responses have been reported for treatment with (R)-HNE, (S)-HNE, or racemic HNE. For instance, (S)-HNE and racemic HNE potently stimulate phosphorylation of Jun kinase and Akt while (R)-HNE is strongest in phosphorylating MAPK. Also, (S)-HNE shows a more pronounced cytotoxicity [27,28] which points at the relevance of HNE enantiomers in cellular responses to HNE. Furthermore, Guéraud et al. [29] have shown, that (R)- and (S)-HNE are enantioselectively metabolised in rats and $\mathrm{d}_{11-4-h y d r o x y-2-(E)-n o n e n a l}$ is conjugated with glutathione in an enantio-selective mode, and exported from the liver [30].

Generally, HNE reacts with some sulfhydryl groups in proteins in a stereoselective manner. This has been characterized in detail by Wakita et al. [31]. Incubation of $N$-acetylcysteine with the Sand R-HNE entantiomer generated HNE-cystein adducts characterized as anomeric isomers by reverse-phase HPLC and NMR. The stereoselective formation of HNE-cyteine adducts was also demonstrated in the redox-regulatory protein thioredoxin, the active site at $\mathrm{Cys}^{32}$ showing a preference for R-HNE cysteine adducts. These findings provide insight into structural aspects of lipid peroxidation product/HNE - based sulfhydryl modification and the chemical characterization of protein S-associated aldehydes in vitro and in vivo. In this context it is worth to mention that an efficient, enantioselective synthesis of (R)- and (S)-HNE has been worked out recently by Komisarski et al. [32].

Of special relevance, HNE and its glutathione conjugates are able to regulate oxidative stress related transcription factors such as NFKB and AP-1 by addressing protein kinase cascade mediated stress signaling. This transcriptional activation leads to an upregulated expression of several genes involved in cell differentiation and cell death control. The mechanisms by which HNE and other lipid aldehydes transduce activation of NFKB signaling pathways have been described by Yadav et al. [33].

Regulatory effects of HNE can be based on purely metabolic mechanisms. Disruption of GSTbased HNE conjugation, resulting in elevated HNE-levels, promotes obesity in mice and the nematode Caenorhabditis elegans suggesting a phylogenetically conserved, HNE-dependent mechanism involved in lipid metabolism [34,35]. In C. elegans, enhanced HNE-conjugation favours a lean phenotype while an inhibition of conjugation or oxidation of HNE yields fat accumulation. Together with the finding that synthetic HNE also stimulates lipid deposition in C. elegans, a causative role of HNE, acting on a metabolic basis in the development of an obese phenotype cannot be ruled out.

HNE is a degradation product of $\omega-6$ polyunsaturated fatty acids, and the different mechanisms of HNE formation have been reviewed recently by Schneider et al. [36] and Spickett [15]. Notably, 4-hydroxy-hexenal (HHE), representing the corresponding hydroxyalkenal derived from $\omega-3$ polyunsaturated fatty acids shares several properties with HNE, but also shows important differences particularly with respect to targets of aldehyde adduct formation and detoxification pathways [37].

HNE formation may follow several radical-dependent oxidative routes involving the generation of hydroperoxides, alkoxyl radicals, epoxides, and fatty acyl cross-linking reactions. Cleavage of the oxidized fatty acyl chain results in HNE formation from the methyl end, and 9-oxo-nonanoic 
acid from the carboxylate or esterified end of the chain, although many other products are also possible.

Furthermore, the enzymatic production of HNE has been described by Wang et al. [38], reporting that Escherichia faecalis, a human intestinal commensal, triggers COX-2 based HNE formation in macrophages.

Finally, HNE is hepato- and nephrotoxic in mammalians [39]. Interestingly, high HNE concentrations $(\geq 20 \mu \mathrm{M})$ are considered to contribute to cardiac ischemia-reperfusion injury, while lower, sublethal aldehyde concentrations $(5 \mu \mathrm{M})$ serve cardioprotection by supporting cellular stress resistance [40]. At low concentrations, HNE stimulates the Nrf2 (NFE2-related factor 2) mediated upregulation of $\gamma$-glutamylcysteine ligase and the core subunit of the high-affinity cystine transporter Xc(-) yielding a 1.45-fold shift of intracellular GSH levels.

\section{Major Reaction Mechanisms}

Essentially, HNE exhibits a high reactivity towards thiol and amino groups which are responsible for most of the biochemical effects of HNE. An overview of the reactions of HNE with different biomolecules is shown in Figure 4.

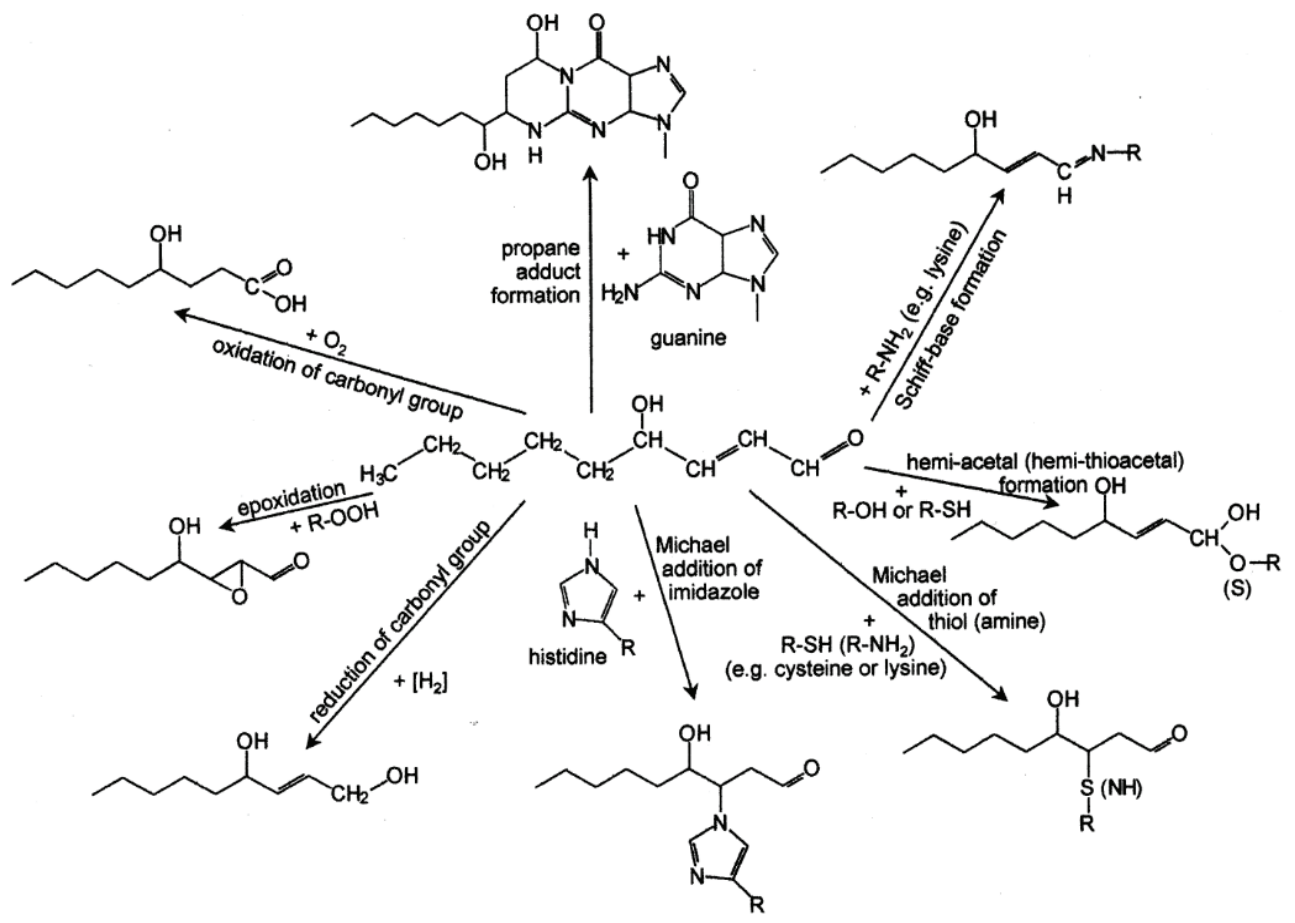

Figure 4. Overview of the reactions of 4-hydroxy-nonenal with different biomolecules. Explanations in the text. Reprinted from [24]. 


\subsection{Reactions of the $C=C$ Double Bond}

\subsubsection{Michael Additions}

In Michael additions a nucleophile, e.g., cysteine or glutathione $(\mathrm{GSH})$, is added to the $\mathrm{C}=\mathrm{C}$ double bond (Figure 4). In some cases the reaction is reversible (Retro Michael cleavage). In case of GSH the reaction velocity is greatly enhanced by the enzyme glutathione-S-transferase (GST) as reviewed by Mannervik (2012) [41]. In particular the GSTA4-4 isoform strongly catalyzes the reaction between HNE and GSH which is due to the high alkenal activity related to C-terminal interactions [42]. The elimination of an excess of HNE by GST activity may confer cytoprotection, however, this can change to the opposite in the presence of interacting factors such as the multidrug resistence protein-1 (MRP1). This is demonstrated by the HNE mediated cytotoxicity seen in MCF7 cells stably expressing MRP1 together with human GST-M1 (GSTM1) [43]. In these cells, the co-expression of GSTM1 and MRP1 leads to increased GSH depletion and formation of protein adducts upon HNE exposure which facilitated a cytotoxic outcome rather than protection.

GSTA4-4 was also used to address the question whether HNE metabolizing enzymes avoid HNE mediated inactivation [44]. Despite marked structural similarities between several GST isoforms (GSTA1-1, GSTA4-4, GSTP1-1) GST A4-4 shows the highest catalytic activity to metabolize HNE but is insensitive to covalent HNE adduct formation.

Similar to thiols amino compounds such as lysine, ethanol-amine, guanine and the imidazole group of histidine can also undergo Michael additions to HNE. For example, the histidine-containing dipeptide carnosine is a target of HNE [45].

As a recent example of Michael adduction of proteins by HNE, the modification of recombinant human phosphatase PTEN (Phosphatase and Tensin homolog) by HNE was studied by Shearn et al. [46] who identified several Michael adducts of HNE on Cys ${ }^{71}$, Cys ${ }^{136}$, Lys ${ }^{147}$, Lys $^{223}$, $\mathrm{Cys}^{250}$, Lys ${ }^{254}$, Lys ${ }^{313}$, Lys ${ }^{327}$ and Lys $^{344}$ by mass analysis (LC/MS/MS). HNE adducts to Cys ${ }^{71}$ adjacent to the active center and Lys $^{327}$ locating to the C2 domain of PTEN may lead to PTEN inhibition via steric hindrance of the active-site pocket or abrogation of PTEN functions associated with the C2 domain. Shearn et al. [46] also propose that Akt2 could be activated by the HNE mediated inhibiton of PTEN which suggests a potential novel mechanism of lipid accumulation initiated by an increased production of reactive aldehydes during chronic ethanol administration.

In rare cases Michael addition reactions of HNE as well as acrolein (ACR) have been observed with aromatic hydroxy groups of polyphenols. The formation of adducts with HNE or ACR was reported for naturally occurring flavan-3-ols, theaflavins, cyanomaclurin, dihydrochalcones and phloretin which showed the most efficient scavenging activity [47]. With respect to this, certain polyphenols could be of benefit in antagonizing "carbonyl stress" via quenching of $\alpha, \beta$-unsaturated aldehydes. Finally, Michael addition of HNE to ascorbic acid has also been described, targeting an activated $\mathrm{C}-\mathrm{H}$ bond followed by ring closure and hemiacetal formation (i.e., addition of a hydroxyl group to a carbonyl group) $[31,48]$.

Several methods are available at present to study Michael adduction by HNE. In particular, polyclonal and monoclonal antibodies (e.g., applied as ELISA) are intensively used to assess Michael-adduct formation of HNE with proteins [49]. Michael addition-type amino acid adducts of 
HNE can also be readily detected by HPLC methodology employing 2-aminopyridine (2-AP) as fluorescent probe [50]. Moreover the relation between protein adduct formation and gene expression is studied by the integration of protein adduction, gene expression, protein-DNA interaction, and protein-protein interaction data following a systemic approach [51].

\subsubsection{Reduction}

The $\mathrm{C}=\mathrm{C}$ double bond can be reduced by an alkenal/one oxidoreductase with $\mathrm{NAD}(\mathrm{P}) \mathrm{H}$ as cofactor to yield 4-hydroxynonanal (Figure 4).This enzyme can metabolize HNE quite efficiently, which is demonstrated by the effect of $\mathrm{NADP}(\mathrm{H})$ overexpression seen in human embyonic kidney cells, efficiently protecting the cells from HNE challenge [52].

\subsubsection{Epoxidation}

As further reaction, epoxidation in presence of a hydroperoxide may introduce an oxirane ring into HNE (Figure 4). Albeit the exact underlying mechanism for this reaction remains to be defined, epoxides are formed by the incubation of HNE with hydrogen peroxide or 9- or 13-linoleic acid hydrogen peroxide at $37{ }^{\circ} \mathrm{C}$ for $24 \mathrm{~h}$ as well as in the presence of a fatty acid (linoleic acid, $\gamma$-linolenic acid, or arachidonic acid) and lipoxygenase [53].

\subsection{Reactions of the Carbonyl Group}

\subsubsection{Acetal and Thio-Acetal Formation}

The reaction of the carbonyl group of HNE with alcohols or thiols is a two step mechanism yielding (thio)acetals via (thio)hemi-actals (Figure 4). Since acetals are much more stable than HNE the reaction of HNE with methanol or ethanol is frequently used in synthetic chemistry for the storage of HNE. The free aldehyde can easily be released again in acidic medium. The hydroxyl group at carbon 4 of HNE can undergo a hemi-acetal formation only as a secondary reaction after a primary reaction such as a Michael addition which confers rotatory freedom to the C2-C3 bond.

\subsubsection{Schiff-Base Formation}

Schiff-base formation with primary amines, e.g., lysine, is a competitive reaction to the Michael addition of amines (Figure 4). This reaction contributes frequently to the crosslinking of proteins by HNE as was observed in mouse sciatic nerves. It has been shown that HNE adducts characterize axons of the mouse sciatic nerve from birth to senility. Using an antibody specific for a lysine-lysine cross-link, Perry et al. [54] examined axonal labeling in these nerves. As demonstrated by immunoblotting, HNE mediated intramolecular cross-linking is restricted to heavy and medium neurofilament subunits, the extent of HNE modification remaining relatively constant over the life span. Furthermore, Schiff-base formation may also have therapeutic implications. This is suggested by the reaction of the HNE carbonyl group with the hydrazine functional group of phenelzine underlying the HNE scavenging effect of this agent in traumatic brain injury [55]. 


\subsubsection{Oxidation}

The oxidation of the HNE carbonyl group producing 4-hydroxy-nonenoic acid is catalyzed by aldehyde dehydrogenase (ALDH), $\mathrm{NAD}^{+}$or $\mathrm{NADP}^{+}$acting as cofactor (Figure 4). Fournet et al., 2013 critically reviewed the existing literature with resepct to the presumptive role of aldehyde dehydrogenases (ALDH1, ALDH2 and ALDH3) in regulating HNE and other endogenous apoptogenic aldehydes (methional, malondialdehyde) showing conflicting results concerning the contribution of different ALDH isoforms to HNE detoxification [56].

ALDH2 may play an important role in neuronal injury, the overexpression or activation in ALDH2 deficient rats providing neuroprotection via enhanced HNE removal [57]. Supportive to this, the neuroprotective effect of protein kinase $\mathrm{C} \varepsilon-\mathrm{a}$ positive regulator of ALDH2 was abolished in ALDH2-knockdown rats. Notably, ALDH2 also contributes to myocardial protection from ischaemia/reperfusion injury which appers to be attributable to HNE detoxification as well as autophagy regulated by AMPK- and Akt-mTOR signalling during ischaemia and reperfusion [58].

Furthermore, the ALDH3 subfamily comprises a group of enzymes which oxidize medium-chain aliphatic and aromatic aldehydes (e.g., fatty and peroxidic aldehydes). ALDH3A1, a phase II drug-metabolizing enzyme is poorly expressed in the normal liver but present at high levels in the lung, stomach, cornea and keratinocytes, cells with a high ALDH3A1 content revealing an increased resistance to the cytostatic and cytotoxic effects of lipidic aldehydes [59]. Transfection studies demonstrated that ALDH3, but not class 1 ALDH, protects from severe GSH depletion and HNE mediated apoptosis in V79 fibroblasts and RAW264.7 macrophages which is associated with reduced levels of HNE protein-adducts [60]. Moreover, HNE serves as substrate for ALDH3 catalyzed oxidation suggesting that the oxidative decomposition of HNE by class 3 ALDH constitutes an important mechanism protecting from HNE toxicity [60]. Notably, this report also showed that the contributions of HNE functional groups to HNE toxicity follows the order aldehyde $\geq \mathrm{C} 2=\mathrm{C} 3$ double bond $>>\mathrm{C} 4$-hydroxyl group. The role of ALDH3A1 in oxidative stress responses was further characterized in a rabbit corneal keratocyte cell line (TRK43) overexpressing ALDH3A1 which confirmed the protective role of ALDH3A1 towards the adverse effects of HNE which may occur under conditions of oxidative stress [61]. Additionally, Yoval-Sanchez et al., 2012 reported that human ALDHs reveal different sensitivities to inactivation by LPO-derived aldehydes, ALDH2 most effciently oxidizing HNE, acrolein, and MDA [62]. Interestingly, while these compounds are also processed by ALDH1A1, ALDH3A1 specifically oxidizes HNE. Of note, ALDH2 is irreversibly inactivated by these LPO-metabolites which may target the ALDH aldehyde and $\mathrm{NAD}^{+}$binding sites. In contrast, ALDH3A1 tolerates concentrations of $\mathrm{HNE}$ or MDA of $20 \mathrm{mM}$. Hence, the pronounced resistance towards inactivation by aldehydic LPO metabolites emphasizes a particular role of ALDH3A1 (and also ALDH1A1) in cellular detoxification of LPO-derived aldehydes [62].

\subsubsection{Reduction}

The opposite reaction, reduction of the carbonyl group, can be catalyzed by alcohol dehydrogenase $(\mathrm{ADH})$ and NADH (Figure 4). Of special relevance, amyloid beta peptide (A $\beta)$ 
binding-alcohol dehydrogenase (ABAD) which is present in the brain metabolizes different substrates including $\beta$-estradiol as well as $n$-isopropanol in addition to its $A \beta$ binding property. In the pathogenesis of Alzheimer disease (AD), the association of mitochondria derived oxidative stress with $\mathrm{A} \beta$ plays a considerable role and the aldehyde detoxification by $\mathrm{ABAD}$ has been suspected to play a particular role. Indeed, in SH-SY5Y neuroblastoma cells, representing a widely used in vitro model of neuronal function and differentiation, $\mathrm{ABAD}$ is able to decompose HNE and to antagonize HNE cytotoxicity wich is suppressed by $A \beta$ [63]. Based on this, it is suggested that the impairment of $\mathrm{ABAD}$ mediated HNE-detoxification by $\mathrm{A} \beta$ contributes to the pathogenesis of $\mathrm{AD}$.

A second enzyme, aldose reductase, preferentially reduces the glutathione conjugate of HNE. Aldose reductase protects against early atherosclerotic lesion formation in apolipoprotein E-null mice by removing HNE [64] and protects also against myocardial ischemia-reperfusion injury in mice [65].

Several studies indicate that a third group of enzymes, namely several members of the aldo-keto reductase (AKR) family are also involved in the metabolism of HNE [66-68]. For instance, AKR1C3 contributes to HNE reduction and thereby protects human SH-SY5Y cells from HNE cytotoxicity. Notably, AKR expression is under the control of Nrf2, a regulatorys effect which may be crucial to the cytoprotection conferred by Nrf2 towards the toxic effects of lipid peroxides. Similarly, AKR7A2 renders V79-4 Chinese hamster lung cells resitant towards HNE cytotoxicity, but fails to have the same effect on the toxicity of crotonaldehyde and acrolein [66]. Overexpression of AKR7A2 in V79 cells lowered HNE genotoxicity, improved the resistance to the redox-cycler menadione by lowering menadione-induced ROS generation and GSH depletion. With resepct to this, it cannot be ruled out that the participation of AKRs like AKR7A2 in cellular detoxifying pathways may also be of relevance to antioxidant defenses in vivo. This is also indicated by the activity of AKR1B10, another AKR isoform which detoxifies HNE and other dietary and lipid-derived $\alpha, \beta$-unsaturated carbonyls at physiological levels [69]. In addition, retinals have been shown to be the best substrate of AKR1B10 [70]. Recombinant rat AKR1B10 (R1B10), derived from the corresponding cDNA isolated from rat brain reduces different aldehydes including HHE, HNE and 4-oxo-2-nonenal as well as $\alpha$-dicarbonyls such as methylglyoxal and 3deoxyglucosone, NADPH serving as preferred coenzyme [71]. 4-oxo-2-nonenal is most efficiently reduced into 4-oxo-2-nonenol by recombinant R1B10 which also lowers its cytotoxic properties. Expression of R1B10 (mRNA) is highest in rat brain and heart, low levels detectable also in other tissues and skin fibroblasts. Similar to AKR7A2, these findings suggest that R1B10 serves antioxidant defenses in rat tissues which also holds true for rat AKR1C15, which is upregulated by HNE and protects endothelial cells from HNE mediated cell damage [72].

In photoreceptor cells a fourth group of HNE reducing enzymes was detected: the retinol dehydrogenases RDH11 and RDH12, which are capable of reducing LPO products such as HNE. As demonstrated by Marchette et al. [73], expression of RDH11 and RDH12 in human embryonic kidney (HEK-293) cells suppressed HNE protein modification and apoptosis triggered by this aldehyde. Moreover, LPO and HNE formation is induced upon exposure of the mouse retina to bright light peroxidation which is accompanied by HNE protein modification in the inner segments 
of the photoreceptor containing RDH11 and RDH12. Interestingly, HNE adduct formation and light-induced photoreceptor apoptosis was suppressed only by RDH12 which suggests a substrate specifity of RDH12 for HNE. From the mechanistical aspect, the protection from the light-induced prooxidant and cytotoxic effects is considered to be based on the reduction of HNE by RDH12 to the nontoxic alcohol 1,4-dihydoxy-nonene. It is worth to note that the mutation of the RDH12 gene is causal to early onset retinal dystrophy Leber congenital amaurosis (LCA), a severe inherited eyedisease with a still incompletely understood pathogenesis.

Finally, the carbonyl group of HNE can also be reduced or oxidized by members of the cytochrome P450 family. For example, the incubation of cytochrome P450 3A4 with $\mathrm{HNE}, \mathrm{O}_{2}$, and NADPH leads to the formation of several compounds including 1,4-dihydroxynonene (DHN) and 4-hydroxy-2-nonenoic acid (HNA) [74]. The reduction reaction is catalyzed also by other P450 isoforms especially (human) P450 2B6, revealing an activity similar to P450 3A4 and P450 1A2, P450 2J2, (mouse) P450 2c29, which are less active. In contrast, human P450 2E1 and rabbit P450 2B4 lack a reducing activity of the carbonyl group.

\subsection{Reactions of the Hydroxy Group}

The formation of cyclic hemi-acetals - as it is known for sugars - as secondary reactions has already been mentioned above. Similarly, the oxidation of HNE to 4-oxo-nonenal (Figure 4) has also been described as a secondary event only.

In addition, catabolic HNE degradation in the liver may also involve phosphorylation of the hydroxy group [75] as well as $\omega$-oxidation, i.e., the oxidation of the methyl group. $\omega$-Oxidation, following oxidation of the carbonyl group is indicated by the presence of intermediates (4-hydroxynonanedioyl-CoA, 4-hydroxyheptanedioyl-CoA and 4-hydroxy-pentanedioyl-CoA) which account for a degradation of HNE from either end of the molecule [76].

\section{Biophysical Effects}

Besides biochemical effects, also biophysical changes of protein and lipid membrane conformation - e.g., loss of phospholipid asymmetry have to be taken into consideration. Using electron paramagnetic resonance spectroscopy in combination with protein-specific spin labeling (2,2,6,6-tetramethyl-4-maleimidopiperidin-1-oxyl), the induction of conformational changes of synaptosomal membrane proteins by physiologically relevant HNE concentrations $(1 \mu \mathrm{M})$ was demonstrated [77].

HNE may also interfere with membrane fluidity, albeit results dealing with this aspect are conflicting. In gerbil synaptosomal membranes, fluidity of the bilayer is increased at higher HNE conentrations (i.e., $50 \mu \mathrm{M}$ ) [77]. On the contrary, the fluidity of hepatic mitochondrial membranes in rats was decreased by HNE and to a lower extent also MDA supposedly as result of a direct interaction of HNE with membrane phospholipids [78]. Also, HNE directly inhibits the dopamine transporter and also decreases $\mathrm{Na}^{+} / \mathrm{K}^{+}$ATPase activity in synaptosomes, the effect on $\mathrm{Na}^{+} / \mathrm{K}^{+}$ ATPase activity most likely resulting from HNE induced loss of membrane fluidity [79]. 


\section{Biochemical Targets of HNE}

The cellular reactions to HNE are strongly dose-dependent. According to recent concepts and supported by transcriptome analysis, low HNE concentrations provide protection from oxidativestress mediated cell damage while increasing HNE doses promote genotoxic effects and initiate apoptosis or severely deregulates cell integrity [80].

The smallest reported biochemical target of HNE is hydrogen sulfide $\left(\mathrm{H}_{2} \mathrm{~S}\right)$ which has been recognized as neuromodulator and gasotransmitter. $\mathrm{H}_{2} \mathrm{~S}$ protects $\mathrm{SH}-\mathrm{SY} 5 \mathrm{Y}$ neuroblastoma cells from $\mathrm{HNE}$ cytotoxicity and HNE-related protein modification leading to the assumption that $\mathrm{H}_{2} \mathrm{~S}$ could represent an important antagonist to "carbonyl stress" arising from aldehydic LPO metabolites [81].

\subsection{Reactions with Peptides and Proteins}

From a quantitative perspective, proteins and peptides represent the most important class of biomolecules which are targeted by HNE. About $1 \%$ to $8 \%$ of the HNE formed in a cell are considered to modify proteins [82] and several investigations have been carried out to identify protein targets of HNE modification [83-85]. For example, more than 1500 proteins were identified as HNE targets in human colon carcinoma cells, 417 of these proteins showing a significant concentration dependent HNE-adduct formation [86]. Another study by Chavez et al. [85] using HNE treated human monocytic THP-1 cells demonstrated distinct, site-specific (His, Cys) HNE modifications of 16 proteins comprising cytoskeletal elements (tubulin $\alpha$-1B chain, $\alpha$-actinin- 4 , vimentin), proteins associated with stress responses and metabolic enzymes (D-3-phosphoglycerate dehydrogenase, aldolase A). Also, heat shock protein Hsp90 as well as the endoplasmic stress response protein, protein disulfide isomerase (PDI) may also become targets of HNE generated by alcohol-induced oxidative stress in rat liver [87]. Another illustrative in vivo example of selective targeting of enzymes involved in glycolysis, energy production as well as $\mathrm{CO}_{2}$ hydration has been described for the ventilatory muscles of rats under the conditions of severe sepsis and strenuous muscle contractions [88]. Under these conditions, HNE adduction of enolase 3b, aldolase and triosephosphate isomerase 1, creatine kinase, carbonic anhydrase III, aconitase 2, dihydrolipoamide dehydrogenase, and electron transfer flavoprotein-beta is detectable. Together these studies account for different sensitivities to HNE protein modification between cellular proteins. In this context it has to be noted that HNE protein adduct formation affects the whole cell while prooxidant regimens such as $\mathrm{H}_{2} \mathrm{O}_{2}$ preferentially trigger cytosolic protein oxidation [89].

Enolase activity is incrementally declining at increasing HNE concentrations in vitro which demonstrates a dose-dependent negative effect of HNE-adduct formation on enzyme activity [88]. Indeed, in most instances protein function is impaired by HNE adduct formation. The aforementioned modification of Hsp70 and Hsp90 in alcoholic liver disease adversely interferes with the protein binding and ATPase activity of these stress response proteins. Moreover, HNE adducts negatively affect the activity of protein disulfide isomerase which is required for the proper establishment of disulfide bonds in protein folding. As a consequence, HNE modification will 
promote the accumulation of damaged or misfolded proteins which is detectable in the liver upon chronic alcohol ingestion [87,90,91].

It is well established that HNE forms adducts with three different side chains in proteins, namely Cys, His and Lys. The differential reactivity of these residues as well as of Arg and Glu for adduct formation with HNE in synthetic poly-aminoacid model compounds has been studied [92]. It turned out that Cys residues displayed by far the highest reactivity; the order of the molar HNE/AA ratio was Cys $(0.6)>>$ His $\left(1 \times 10^{-3}\right)>$ Lys $\left(3 \times 10^{-4}\right)>>\operatorname{Arg}\left(4 \times 10^{-5}\right)$. No reaction of HNE was detected with Glu. Similar results were obtained for the keto-derivative of HNE, 4-oxononenal: (Cys $>$ His $>$ Lys $>$ Arg) with Arg being a target for 4-oxononenal but generally not for HNE [93]. However, one case has been described (cytochrome c), where $\operatorname{Arg}^{38}$ is also modified by HNE besides $\mathrm{His}^{33}$ and $\mathrm{Lys}^{87}$ residues [94]. The above mentioned order does not imply that Cys residues are always the preferential targets of HNE in proteins. Several other factors besides reactivity may determine the adduction site, such as polarity of the microenvironment and accessibility in the tertiary/quarternary structure. For example it has been shown for HNE- and 4-oxo-2-nonenaltreated myoglobin and apomyoglobin, that the latter more "open" protein structure resulted in more extensive modification [95]. Changes in the polarity of the microenvironment may explain, why the presence of Arg in a model peptide increases the reaction rate of Cys for adduct formation with HNE as well as 4-oxononenal by a factor of 5-6 compared to the Cys nucleophile alone [93]. The impact of accessibility and acidity has been shown for actin. Among several Cys residues only Cys ${ }^{374}$ is adducted by HNE because of significant accessible surface and substantial thiol acidity due to the particular microenvironment surrounding $\mathrm{Cys}^{374}$ [96].

Furthermore, the presence of other ligands bound to proteins can strongly influence adduct formation with HNE. The in vitro modification of Cys ${ }^{120}$ of epithelial fatty acid-binding protein by HNE is markedly potentiated by noncovalently bound fatty acids [97].

Adduct formation of HNE with proteins is a rather fast reaction. The half life for the covalent modification of the epithelial fatty acid-binding protein was reported to be $t_{1 / 2}<60 \mathrm{~s}$ in vitro [97]. Adduct formation of HNE with protein thiols can be reversible in the presence of low molecular thiols such as GSH. Physiological (e.g., $4 \mathrm{mM}$ ) concentrations of GSH were capable of removing the HNE adducts from PDI [98]. It is likely this mechanism serves as a protective mechanism against inactivation by HNE and other lipid peroxidation products.

Protein adducts of HNE are physiological constituents of mammalian organisms. Notably these adducts occur in blood, where they increase with age [99]. In an age-dependence study on HNE-adducted serum proteins in Fischer 344 rats albumin, transferrin and immunoglobulins were most prominently adducted by HNE [100]. In a similar study HNE-adducted proteins have also been determined in serum of aging Fischer 344 rats [101]. Among these, 16 are involved in blood coagulation, lipid transport, blood pressure regulation, and protease inhibition.

Recent evidence suggesting a role for protein modification by HNE in the pathogenesis of several diseases has been reviewed in [102]. The precise mechanisms are currently unknown but likely result from adduction of proteins involved in cellular homeostasis or biological signaling. The extent of HNE adduction to proteins can increase dramatically under pathophysiological conditions. e.g., using a heterotropic rat heart transplantation model the formation of protein 
adducts of HNE was studied as a response to cold storage and warm blood reperfusion in the recipient [103]. In hearts submitted to ischemia only, the adduct formation was low $(<1 \%$ of the tissue area). However, transplantation and reperfusion in the recipient increased the amount of protein adducts to about $6 \%$ and it was assumed that this damage negatively affects longterm survival of the transplant.

In a murine model for alcoholic liver disease 414 protein targets for modification by reactive aldehydes (HNE, 4-oxononenal, acrolein, and MDA) were identified by a hydrazide method coupled to a highly sensitive 2-dimensional liquid chromatography tandem mass spectrometry (2D LC-MS/MS or MuDPIT) technique [104].

Also for individual proteins a high degree of HNE adduction has been reported in a condition of oxidative stress. In progressive supranuclear palsy, a neurodegenerative disorder, which may possibly be induced by oxidative stress, two-thirds of total glutathione peroxidase in the cerebrospinal fluid was found to be adducted with HNE. It was assumed that the antioxidant system might be unable to function effectively because of conjugation with HNE [105].

Adduction by HNE tends to make the targeted protein more succeptible to degradation by the proteasomal pathway which is responsible for most intracellular proteolysis. While under normal conditions the proteasomal system is able to remove the bulk of oxidatively damaged and modified proteins, under severe oxidative stress the accumulation of modified proteins occurs. This takes place either due to cross-linking of proteins or due to malfunctioning of the proteolytic machinery of the cell. The efficiency of the removal of HNE-modified proteins clearly depends on the dose of cellular exposure to HNE. Taking into consideration the permanent formation of HNE and its metabolism it has been shown that low levels of HNE-modification are sufficient to increase the proteolytic susceptibility of proteins [106]. Equine ADH treated with a 2-fold molar excess of HNE was degraded by a rabbit reticulocyte lysate system approximately 1.5 -fold faster than the control, while treatment with concentrations up to 100-fold molar excess of HNE were inhibitory to degradation [107].

On the other hand, the proteasome itself is a target for HNE adduction causing inhibition of proteolysis $[108,109]$. In the liver of rats which were fed ethanol for one month HNE-produced presumably by P450 cytochrome 2E1 (CYP2E1) - formed an adduct with the Rpt4 subunit of the $26 \mathrm{~S}$ proteasome [110]. It was proposed that this adduct could impede the association of $19 \mathrm{~S}$ and $20 \mathrm{~S}$ subunits and thus accounts for the observed decrease of proteasomal activity. In accordance with these data a decline in trypsin-like (but not chymotrypsin-like) activity of the proteasome in HepG2 cells overexpressing CYP2E1 was associated with higher levels of HNE-modified proteins compared to control cells [111]. An altered balance between protein modification, ubiquitination, and degradation was proposed for these cells.

Modification of another subunit of the proteasome has also been reported leading to a decline of chymotrypsin-like activity. A catalytic site-specific inhibition of the $\alpha 6 / \mathrm{C} 2$ subunit of the $20 \mathrm{~S}$ proteasome by HNE was observed in liver [112]. Furthermore, the degradation of proteins by a proteasome-independent pathway may also be triggered by adduct formation with HNE as was shown for glyceraldehyde-3-phosphate dehydrogenase in U937 cells [113]. 
The reaction of HNE with proteins is frequently associated with their covalent crosslinking leading to the formation of fluorophores. A major fluorophore has been identified as a lysine-derived dihydro-pyrrole derivative [114]. Finally, it should be mentioned that protein-HNE adducts also represent useful biomarkers of oxidative stress, lipid peroxidation and oxidative homeostasis [115].

In the next chapter several well studied examples of peptides and proteins are described as targets of HNE including synaptic proteins such as synaptosomal-associated protein 25 [116].

\subsubsection{Substrates}

\subsubsection{Glutathione}

Glutathione (GSH), whose concentration in mammalian liver is in the millimolar range, is likely to be the quantitatively most important target of HNE. Several glutathione-S-transferases (GST) such as GSTA4-4 and hGST5.8 are involved in the conjugation of HNE to GSH in humans. The isoenzyme GSTA4-4 with high specificity for HNE has been found in human liver mitochondria. The specific mitochondrial GST activities toward HNE exceeded that observed in liver cytosol. These observations are suggestive of a role of GST in protecting against mitochondrial injury during the secondary phase of oxidative stress, or modulation of HNE-mediated mitochondrial signaling pathways [117].

The conjugation of HNE with glutathione is reversible. Both the spontaneous and the GST-catalyzed retro-Michael cleavages of HNE-GSH are the source of HNE in the urine of rats [118]. Strategies such as irrigation with gamma-glutamyl-cysteine to increase glutathione levels have been suggested as effective means for normalizing pathophysiological HNE levels after traumatic injuries [119].

GSTs (in humans hGSTA4-4 and hGST5.8) and the transporter $76 \mathrm{kDa}$ Ral-binding GTPase activating protein (RLIP76) regulate intracellular concentrations of HNE through a coordinated action in various human cell lines and erythrocytes and catalyzes the ATP-dependent transport of the conjugate into the extracellular space, i.e., a mild stress caused by heat, UV-A, or hydrogen peroxide with no apparent effect on the cells in culture caused a rapid, transient induction of hGST5.8 and RLIP76. These stress preconditioned cells acquired the ability to metabolize and exclude HNE at an accelerated pace and acquired relative resistance to apoptosis by UV and oxidative stress as compared to unconditioned control cells [120].

Patrick et al., 2005 demonstrated that depletion of HNE in hGSTA4-4-transfected adherent HLE B-3 cells results in profound changes in gene expression, phenotypic transformation and immortalization [121]. At the genetic level this transfection caused changes in the expression of genes involved in cell adhesion, cell cycle control, proliferation, cell growth, and apoptosis, which is consistent with the phenotypic changes of the transformed cells. For example, the expression of p53, p21, p16, fibronectin 1, laminin gamma 1, connexin 43, Fas, integrina6, TGF $\alpha$, and c-jun was down-regulated, while the expression of protein kinase $\mathrm{C}$ beta II, c-myc, cyclin-dependent kinase 2 , extracellular signal regulated kinase and transforming growth factor $\beta 1$ was up-regulated [121].

The consequences of severe oxidative stress have also been studied in GSTA4-4 deficient mice. The acute hepatotoxicity of $\mathrm{CCl}_{4}$ in GSTA4- $4^{-/-}$mice was compared with wild type GSTA4-4 ${ }^{+/+}$ 
mice [122]. The results indicated that GSTA4-4 is an important component during the early stages (1-6 h) of cellular defense against oxidative stress and lipid peroxidation, although it is not effective in protecting against the degree of overall cell injury.

In addition chronic hepatotoxicity was studied in mGSTA4 null mice. Mice homozygous for the disrupted mGSTA4 allele were viable and appeared normal except for lower litter size, higher fat content in bones, and greater susceptibility to bacterial infection. The null mice had a lower survival time than wild-type controls when chronically treated with relatively low doses of paraquat. These mice had a reduced ability to conjugate HNE, and had an increased steady-state level of HNE in tissues [123].

The genotoxicity of HNE is highly dependent on cellular GSH status and those GSTs that contribute toward HNE conjugation, including hGSTA4-4. Depletion of GSH in HT29 cells, derived from a human colorectal adenocarcinoma, by BSO (L-buthionine-S,R-sulfoximine) decreased cellular GSH levels by $77 \%$ without significant changes in cell viability. Associated with this decrease was a 2-fold higher level of HNE-induced DNA damage as measured by the comet assay [124].

The glutathione adduct of HNE has signaling properties of its own [125]. For instance, it enhances the peritoneal leukocyte infiltration and stimulates the formation of proinflammatory lipid mediators. Moreover, the reduced form of the glutathione conjugate of HNE (GS-DHN) elicits strong mitogenic signaling in smooth muscle cells. Also, GSH-HNE is not only a product of adipocyte oxidative stress but also an activator of macrophage inflammation [126] and is pro-inflammatory in vivo in mice [127].

\subsubsection{Carnosine}

Carnosine is a dipeptide (beta-alanyl-L-histidine) present in mammalian tissue and in particular at high concentration in skeletal muscle. Growing evidence indicates that it acts as quencher of reactive and cytotoxic carbonyl species including HNE [45,128]. The reaction mechanism was found to be an intra-molecular Michael addition. Two reaction products of carnosine were identified, in a pH-dependent equilibrium: (a) the Michael adduct, stabilized as a 5-member cyclic hemi-acetal and (b) an imine macrocyclic derivative. The adduction of carnosine to HNE thus appears to start with the formation of a reversible $\alpha, \beta$-unsaturated imine, followed by ring closure through an intra-molecular Michael addition [128]. By trapping HNE in stable covalent adducts, carnosine can inhibit HNE-induced protein cross-linking.

The biological role of carnosine as a quencher of $\alpha, \beta$-unsaturated aldehydes was verified by detecting carnosine-HNE adducts in oxidized rat skeletal muscle homogenates [129]. In a therapeutic approach it was shown that dietary carnosine prevents early atherosclerotic lesion formation in apolipoprotein E-null mice [130]. The facile Michael adduct formation of carnosine with 4-hydroxyalkenal species was explored and a sensitive, facile, shotgun lipidomics-based method was developed for quantification of these compounds directly from organic solvent lipid extracts of biological samples [131].

A perspective for the design of novel derivatives, active as exogenous agents able to detoxify carbonyl compounds has been proposed [132]. Histidyl-containing carnosine 
analogues bearing hydrazide or 1,2-diol moieties have been synthesized, some of which have demonstrated higher aldehyde-sequestering efficiency than carnosine and were also efficient in protecting SH-SY5Y neuroblastoma cells and rat hippocampal neurons from HNE-mediated death. The cytoprotective efficacy of these compounds suggests their potential use as therapeutic agents for disorders that involve excessive membrane lipid peroxidation and HNE-mediated neuronal toxicity [133]. Vistoli et al., 2009 described a set of aryl derivatives that are characterized by high stability in human plasma and a quenching activity toward HNE, as a model of reactive carbonyl species, up to threefold greater than D-carnosine [134].

The synthesis and the physicochemical and biological characterization of a series of carnosine amides bearing alkyl substituents on the amido group endowed with different lipophilicity have been described by Bertinaria et al., 2011 [135]. These products can be considered as metabolically stable analogues of carnosine and are worth of additional investigation as potential neuroprotective agents.

\subsubsection{Thioredoxin}

Thioredoxins (TRXs) are scavengers of intracellular ROS and participate in the anti-oxidant system of the retina. Disruption of these systems leads to dysfunction of retinal pigment epithelial (RPE) cells, which then accelerates the development of age-related macular degeneration (AMD). The role of TRXs in the protection against HNE-induced oxidative stress was studied in RPE cells by assessing the effect of TRX overexpression on cell viability, morphology, NFkB expression, and mitochondrial membrane potential [136]. It was found that overexpression of TRXs reduced cell death caused by HNE: HNE caused perinuclear NFאB accumulation, which was absent in TRX-overexpressing cells. Moreover, overexpression of TRXs prevented depolarization of mitochondrial membranes. TRX2 was more effective than TRX1 in maintaining the membrane potential. The difference in the protective effects of these TRXs against oxidative stress may be due to their expression profile. TRX2 was expressed in the mitochondria, while TRX1 was expressed in the cytoplasm. It was therefore suggested that the effect of TRXs on mitochondria may be a key to prevent oxidative stress in RPE cells [137].

\subsubsection{Cytochrome c}

The nature of cytochrome c modification by HNE was investigated in an in vitro study [138]. The overwhelming reaction observed is Michael addition by Lys side-chains in addition to the modification of $\mathrm{His}^{33}$. While the Lys-HNE adducts were generally observed to be reversible, the HNE-His ${ }^{33}$ was found to be stable with half of the formed adduct surviving the denaturation and proteolysis protocols used to generate proteolytic peptides for LC-ESI-MS/MS.

\subsubsection{Enzymes}

When the amino acid targets of HNE are in the active site of an enzyme, the activity is frequently diminished. It was therefore proposed that HNE for example may disrupt the active site of lipoxygenase-1 by forming a Michael adduct with one or more of the three histidines that ligate the iron 
active site [139]. However, the inactivation of enzymes by HNE need not necessarily be due to the modification of the catalytic center but may be due to the selective modification of amino acids primarily located on the surface of the enzyme as has been shown for glyceraldehyde-3-phosphate dehydrogenase (GAPDH). HNE is a potent inhibitor of sulfhydryl enzymes such as the glycolytic enzyme GAPDH. It has therefore been suggested that HNE exerts an inhibitory effect on the enzyme due to the modification of $\mathrm{Cys}^{149}$ at the catalytic site generating the HNE-cysteine Michael addition-type adduct [140]. To test this hypothesis, ESI-MS of tryptic peptides was carried out and identified five peptides, which contained the HNE adducts at $\mathrm{His}^{164}, \mathrm{Cys}^{244}$, $\mathrm{Cys}^{281}$, $\mathrm{His}^{327}$, and Lys $^{331}$ and revealed that both His ${ }^{164}$ and $\mathrm{Cys}^{281}$ were very rapidly modified within 5 min, followed by $\mathrm{Cys}^{244}$ at $15 \mathrm{~min}$ and $\mathrm{His}^{327}$ and $\mathrm{Lys}^{331}$ at $30 \mathrm{~min}$. However, a modification of the catalytic center, Cys $^{149}$, by HNE was not observed [141].

As mentioned, the inhibition of enzymes is in some cases reversible in the presence of thiolcontaining compounds such as glutathione [142,143] or acetylcysteine. For example, cysteine restores the activity of the pyruvate dehydrogenase complex [144] but in most instances irreversible destruction takes place which may also be site-specific [145]. Profound consequences are to be expected when HNE forms adducts both with the substrate and the enzyme of a biochemical reaction as is the case of the TRX/TrxR system. TrxR is most sensitive to inhibition by HNE. This seleno-enzyme regulates redox-sensitive proteins involved in inflammation and carcinogenesis, including ribonucleotide reductase, p53, NFkB, and others. HNE and also its precursor, the 15-lipoxygenase-1 product, 15(S)-hydroperoxy-5,8,11-cis-13-trans-eicosatetraenoic acid, inhibit TrxR with $\mathrm{IC}_{50}=1 \mu \mathrm{M}$ and $13 \mu \mathrm{M}$, respectively, in colo-rectal cancer cells [146]. Besides inhibition of TrxR irreversible inactivation of TRX occurs. A pair of Cys residues $\left(\mathrm{Cys}^{32} / \mathrm{Cys}^{35}\right)$ in Trx and the selenocysteine (Sec) and Cys residues $\left(\mathrm{Cys}^{496} / \mathrm{Sec}^{497}\right)$ in the active site of TrxR are primary targets for HNE modification [147]. Because the TRX system is one of the core regulatory enzymes of cellular functions, it is assumed that inhibition of both TrxR and TRX by HNE provides a possibly novel mechanism for the explanation of its cytotoxic effect and signaling activity, as well as the further damage indirectly caused under oxidative stress conditions.

However, HNE is also an inducer of TrxR [148]. It has been shown to exert an adaptive cytoprotective effect at low concentrations through induction of TrxR 1 via transcriptional activation of Nrf2. As a consequence pretreatment with $\mathrm{HNE}$ at sublethal concentrations significantly protected $\mathrm{PC} 12$ cells rat adrenal pheochromocytoma line, against the subsequent oxidative cell death induced by $\mathrm{H}_{2} \mathrm{O}_{2}$ and 6-hydroxydopamine. Since $\mathrm{HNE}$ also exhibited adaptive protection in human arterial endothelial cells far reaching conclusions were drawn from these findings. It was suggested that this may be a general effect of HNE and may lead to a reappraisal of the eventual role of ROS and LPO in organisms [149]. It has to be determined whether these complex effects of HNE-inhibitor on the one side, inducer on the other - can be explained by tissue-specific responses.

In a few instances activation rather than inhibition of enzymes may occur. Interestingly, in the case of a protease HNE is promoting reactions via four separate mechanisms under pathophysiological conditions: activation and induction of the enzyme, modification of the substrate and inhibition of expression of the substrate [150]. During the destruction of cartilage HNE is prominently produced 
in osteoarthritic (OA) synovial cells. In vitro, HNE binding to matrix metalloproteinase 13 (MMP-13) activated this enzyme, and modification of type II collagen (Col II) by HNE accelerated its degradation by MMP-13. The authors concluded that the increased level of HNE in OA cartilage and the ability of HNE to induce transcriptional and posttranslational modifications of Col II and MMP-13 suggest that HNE could play a role in OA [150].

In the following section several examples of enzymes from different enzyme classes and other proteins with pathophysiological significance are presented as targets of HNE. An overview over the enzymes identified to form adducts with HNE has been presented previously [4].

\subsubsection{Oxidoreductases}

\subsection{Lactate Dehydrogenase}

Lactate dehydrogenase (LDH) activity can regenerate NADH. Mass spectrometric examination revealed that HNE binds with cysteine and histidine residues of $\mathrm{LDH}$ at $\mathrm{pH} 5.6$ and 7.4, and covalent binding of HNE decreases NADH formation [151].

\subsection{Glyceraldehyde-3-Phosphate Dehydrogenase (GAPDH)}

Schlisser et al., 2009 conducted an investigation on organogenesis in mice with the goal to determine whether exposure of CD1 mice to the model teratogen hydroxyurea (HU) on gestation day nine generates region-specific HNE-protein adducts in the embryo, and to identify the proteins targeted [152]. They found out that the formation of HNE-protein adducts was elevated in the caudal region of control embryos and that HU exposure further increased HNE-protein adduct formation in this area. Interestingly, among the eight identified HNE-modified proteins, GAPDH appears to play a specific role since it was shown that HNE adducts reduced GAPDH enzymatic activity by $20 \%$. The authors therefore concluded that GAPDH may play a role in stress response during development.

\subsubsection{Transferases}

\subsection{Glutathione-S-Transferase (GST)}

HNE is not only a substrate for GSTs, but also a modifier under pathophysiological conditions such as Alzheimer's disease (AD), a neurodegenerative disorder characterized pathologically by intracellular inclusions including neurofibrillary tangles and senile plaques. Oxidative stress is associated with the pathogenesis of the disease leading to oxidative modifications of cellular macromulecules such as lipids and proteins [153,154], and an increased formation of HNE [155]. Since the alpha class of GST can detoxify HNE and may interact with the multidrug resistance protein-1 (MRP1) to export the glutathione conjugate of HNE to confer cellular protection. Sultana and Butterfield, 2004 investigated oxidative modifications of GST and MRP1 in AD brain [156]. They found that HNE is covalently bound to GST and MRP1 proteins in excess in AD brain and 
suggested that HNE may be an important mediator of oxidative stress-induced impairment of this detoxifying system which thus may play a role in promoting neuronal cell death.

Besides modification HNE can induce the expression of GST via Nrf2. Nrf2 regulates a battery of antioxidative and phase II drug metabolizing/detoxifying genes via binding to the antioxidant response elements (ARE). Nrf2-ARE signaling plays a central role in protecting cells from a wide spectrum of reactive toxic species including reactive oxygen/nitrogen species (RONS). The role of Nrf2 in regulating the HNE induced gene expression of antioxidant and detoxifying enzymes was studied in HeLa cells [157]. When HeLa cells were treated with HNE, Nrf2 rapidly translocated into the nucleus and increased Nrf2 protein in the nuclear fraction causing a dose-dependent transcriptional activity of ARE followed by the expression of GST A4, AKR1C1 and heme oxygenase-1 (HO-1). Moreover, this induction was attenuated by knocking down Nrf2, highlighting the role of Nrf2 in mediating HNE induced expression of antioxidant and detoxifying genes.

GSTA4-4 has been postulated to be a rigid template that is preorganized for HNE metabolism. However, the combination of high substrate chemoselectivity and low substrate stereoselectivity is intriguing [158]. GSTA4-4 must metabolize both enantiomers of HNE to efficiently detoxify the biologically formed mixture and achieves this by an ideal location of the active site residue Arg 15 enabling the interaction with the 4-hydroxyl group of either HNE enantiomer [158].

\subsection{Liver Kinase B1 (LKB1)}

LKB1 is characterized as a serine/threonine kinase that phosphorylates and activates the AMP-activated protein kinase (AMPK) and 12 other AMPK-related kinases. HNE treatment (10 $\mu \mathrm{M}$ for $1 \mathrm{~h}$ ) of human embryonic kidney 293T (HEK293T) cells expressing LKB1 resulted in the formation of HNE-LKB1 adducts and decreased LKB1 kinase activity but had no effect on the association of LKB1 with its adaptor proteins sterile-20-related adaptor and mouse protein 25. Mutation of LKB1 Lys ${ }^{97}$ residue reduced HNE adduct formation and attenuated the effect of HNE on LKB1 activity. It was therefore concluded that adduction of LKB1 Lys ${ }^{97}$ mediates the inhibitory effect of HNE [159].

\subsection{5'-AMP-Activated Protein Kinase (AMPK)}

AMPK is a major regulator of $\beta$-oxidation, which is altered in ALD. In an in vitro cellular model, AMPK was identified as a direct target of HNE adduction resulting in inhibition of $\mathrm{H}_{2} \mathrm{O}_{2}$ - and 5-aminoimidazole-4-carboxyamide ribonucleoside (AICAR)-induced downstream signaling [160]. Using a murine model of ALD the authors were able to demonstrate that treatment with high concentrations of ethanol resulted in an increase in phosphorylated as well as carbonylated AMPK $\alpha$. Mass spectrometry thereafter identified Michael addition adducts of HNE on Cys ${ }^{130}$, $\mathrm{Cys}^{174}, \mathrm{Cys}^{227}$, and $\mathrm{Cys}^{304}$ on rAMPK $\alpha$ and $\mathrm{Cys}^{225}$ on $\mathrm{rAMPK} \beta$ which by molecular modeling analysis of the adducted sites revealed an inhibition of AMPK by steric hindrance of the active site pocket and inhibition of hydrogen peroxide induced oxidation. 


\subsection{ZAK Kinase (Sterile Alpha Motif and Leucine Zipper Containing Kinase AZK)}

ZAK kinase is a member of the MAPKKK family of signal transduction molecules. It contains an N-terminal kinase catalytic domain, followed by a leucine zipper motif and a sterile-alpha motif (SAM). It mediates gamma radiation signaling leading to cell cycle arrest and the activity of this protein plays a role in cell cycle checkpoint regulation. The protein also has pro-apoptotic activity.

ZAK kinase is adducted by HNE on a conserved, active site-proximal cysteine and the resulting enzyme inhibition generates a negative feedback mechanism that can suppress the activation of JNK pathways normally induced by oxidative stress [161].

\subsection{Serine/Threonine-Protein Kinase AKT2 (Proteinkinase B2)}

The effects of HNE on insulin-dependent stimulation of the AKT2 pathway have been evaluated by Shearn et al., 2011 [162]. The regulatory effect of AKT2 is mediated through serine and/or threonine phosphorylation of a range of downstream substrates. Following HNE treatment, the level of downstream phosphorylation of Akt substrates such as glycogen synthase kinase-3- $\beta$ (GSK3 $\beta$ ) was significantly decreased in the hepatocellular carcinoma cell line HepG2, and this effect was shown to be mediated by Michael addition adducts of HNE with His ${ }^{196}$ and $\mathrm{Cys}^{311}$ of rAkt2 suggesting inhibition of GSK3 $\beta$ peptide binding in the Akt2 substrate binding pocket. This inhibition of Akt by HNE provides a novel mechanism for increased insulin resistance in ALD [162].

\subsubsection{Hydrolases}

\subsection{ATP Synthase}

Oxidative stress has been proposed as a mechanism for impaired beta-cell function in type 2 diabetes. Pancreatic islets from humans with type 2 diabetes were used to study the occurrence of HNE adducts in these cells [163]. The major HNE adduct was a 52-kDa protein seen with four different antibodies (two antibodies against HNE and two other antibodies generated against reactive small aliphatic compounds) that was also seen in islets of nondiabetic humans, rat islets, and insulinoma cells and in mitochondria of various rat tissues. It was identified as the $\beta$-chain of the mitochondrial F-ATP synthase, an enzyme responsible for $95 \%$ of ATP formed in tissues. The ATP synthase $\beta$-subunit has also been identified as a major target in isolated rat liver mitochondria upon HNE exposure [164].

\subsection{Phosphatase and Tensin Homolog Deleted on Chromosome 10 (PTEN)}

The tumor suppressor PTEN is a key regulator of Akt or protein kinase B (Akt/PKB) activation in hepatocytes, and plays a role in the etiology of alcoholic liver disease. PTEN negatively regulates AKT activation via its lipid phosphatase activity. PTEN is a phosphatidylinositol 3-phosphatase catalyzing the removal of the 3-position phosphate from phosphatidylinositol3,4,5-trisphosphate [PtdIns(3,4,5)P-3] to produce phosphatidylinositol-4,5-bisphosphate.

Treatment of both the human hepatocellular carcinoma cell line (HepG2) and primary hepatocytes with subcytotoxic concentrations of HNE resulted in the activation of Akt within 30 min 
as demonstrated by increased phosphorylation of residues $\mathrm{Ser}^{473}$ and $\mathrm{Thr}^{308}$ [165]. This increased phosphorylation is accompanied by a 6-fold increase in total PtdIns(3,4,5)P-3 and an increased immunostaining at the plasma membrane after HNE treatment. However, PTEN lipid phosphatase activity is decreased due to the formation of a single HNE adduct with the active site cysteine in PTEN (Cys $\left.{ }^{124}\right)$.

PTEN has also been used to demonstrate a new methodology to interrogate effects of reactive electrophiles on specific target proteins in cells [166]. In this study a target specific electrophile delivery platform was introduced that ultimately paves the way to interrogate effects of reactive electrophiles on specific target proteins in cells. This new methodology was demonstrated by photoinducible targeted delivery of HNE to the PTEN and Keap1. Covalent conjugation of the HNE-precursor to HaloTag fused to the target proteins enables directed HNE delivery upon photoactivation and allows a more precise determination of the pathophysiological consequences of HNE-induced protein modifications [166]. HaloTag again was designed as a modular protein tagging system that allows different functionalities to be linked onto a single genetic fusion, either in solution, in living cells, or in chemically fixed cells [167]. The protein tag (HaloTag) is a modified haloalkane dehalogenase designed to covalently bind to synthetic ligands (HaloTag ligands). The synthetic ligands comprise a chloroalkane linker attached to a variety of useful molecules, such as fluorescent dyes, affinity handles, or solid surfaces. Covalent bond formation between the protein tag and the chloroalkane linker is highly specific, occurs rapidly under physiological conditions, and is essentially irreversible. The utility of this system for cellular imaging and protein immobilization was demonstrated by analyzing multiple molecular processes associated with NFKB-mediated cellular physiology, including imaging of subcellular protein translocation and capture of protein-protein and protein-DNA complexes [167].

\subsection{Sirtuin 3 (SIRT3)}

SIRT3 is a mitochondrial class III histone deacetylase, which is inhibited by HNE via a thiol-specific modification [168]. HNE covalently modifies rSIRT3 at Cys ${ }^{280}$ altering the conformation of the zinc-binding domain and thus allosterically inhibits SIRT3 activity.

\subsection{Cathepsins}

Since reduced lysosomal capacity may contribute to lipofuscinogenesis and progressive dysfunction of the retinal pigment epithelium (RPE) during the pathogenesis of age-related macular degeneration Krohne et al., 2010 investigated the effects of HNE and MDA on both isolated lysosomes from primary human retinal pigment epithelial cells (RPE) and on cultured RPE cells. Both HNE and MDA inhibited the cysteine proteases cathepsin B and $\mathrm{L}$ at a concentration of $1 \mu \mathrm{M}$ by $88 \%-94 \%$ and this effect was due to HNE and MDA adducts in the active center of these proteases [169]. 


\subsection{Neprilysin (NEP)}

As one of the dominant amyloid- $\beta$ peptide $(A \beta)$ proteases, NEP plays a crucial role in maintaining a physiological balance between $\mathrm{A} \beta$ production and catabolism. Wang et al., 2003 and 2009 showed that NEP is modified by HNE adducts, resulting in decreased activity in the brain of AD patients and cultured SH-SY5Y and H4 APP695wt cells [170,171]. The inactivation of NEP by HNE-adduction was associated with, at least partly, reduced $A \beta$ cleavage and enhanced $A \beta$ accumulation [171]. The HNE-induced modification and inactivation can be prevented by the carbonyl-scavenger $N$-acetylcysteine [172].

\subsubsection{Lyases}

\subsubsection{Mitochondrial Aconitase (ACO2)}

ACO2 is inactivated by HNE. Liu et al., 2013 related the inactivation of ACO2 by HNE to structural features and determined the HNE addition reaction rates using the iTRAQ approach (isobaric Tags for Relative and Absolute Quantitation) [173]. The most reactive sites were Cys ${ }^{358}$, $\mathrm{Cys}^{421}$, and $\mathrm{Cys}^{424}$, the three iron-sulfur cluster-coordinating cysteines, $\mathrm{Cys}^{99}$, the closest non-ligated cysteine to the cluster, and $\mathrm{Cys}^{565}$, which is located in the cleft leading to the active site.

Not only aconitase but also other enzymes of the citric acid cycle are targeted by HNE. Zhao et al., 2014 identified several HNE-modified mitochondrial proteins in mouse heart mitochondria after doxorubicin (DOX) treatment [174]. The majority of the identified proteins were related to mitochondrial energy metabolism. These included proteins in the citric acid cycle and electron transport chain.

\subsubsection{2. $\alpha$-Enolase}

The formation of HNE adducts with the multifunctional protein $\alpha$-enolase was demonstrated by MS and confirmed by immunoblotting experiments, in HL-60 human leukaemic cells [175]. HNE caused a dose- and time-dependent reduction of the binding of plasminogen to $\alpha$-enolase. As a consequence, HNE reduced adhesion of HL-60 cells to HUVECs (human umbilical vein endothelial cells) suggesting a potential role for HNE in the control of tumour growth and invasion.

Enolase is among the six enzymes which are excessively adducted by HNE in early AD (EAD) inferior parietal lobule (IPL) compared to age-related control brain [176]. These proteins play roles in antioxidant defense (manganese superoxide dismutase), neuronal communication and neurite outgrowth (dihydropyriminidase-related protein 2 ), and energy metabolism ( $\alpha$-enolase, malate dehydrogenase, triosephosphate isomerase, and F1 ATPase, $\alpha$-subunit. These results are consistent with the hypothesis that LPO is an early event in the progression of AD. 


\subsubsection{Isomerases}

\subsection{Protein Disulfide Isomerase (PDI)}

PDI is an abundant endoplasmic reticulum (ER)-resident chaperone and oxidoreductase that catalyzes formation and rearrangement (isomerization) of disulfide bonds, thereby participating in protein folding. PDI modification by HNE or oxidized LDL (oxLDL) inhibits its enzymatic activity and potentiates both ER stress (increased mRNA expression of the stress-regulated transcription factors CHOP (C/EBP homologous protein) and apoptosis by oxLDL [177].

\subsection{Peptidyl-Prolyl Cis/Trans-Isomerase A1 (Pin1)}

Pin1, an enzyme that catalyzes the conversion of the peptide bond of pSer/pThr-Pro moieties in signaling proteins from cis to trans, is highly susceptible to HNE modification. This was demonstrated by Aluise et al., 2013 upon incubation of purified Pin1 with HNE leading to Michael adducts at the active site residues His ${ }^{157}$ and $\mathrm{Cys}^{113}$ [178]. Additionally the authors confirmed Cys ${ }^{113}$ adducts in the Pin1 active site in MDA-MB-231 breast cancer cells treated with tagged HNE (8-alkynyl-HNE) and showed that knockdown of Pin1 in MDA-MB-231 cells partially protected the cells from HNE-induced toxicity.

\subsubsection{Ligases: Glutamine Synthetase}

Glutamine synthetase is among the proteins modified by HNE in the retina as shown by Tanito et al., 2006 [179]. By analyzing HNE-modified proteins increased after intense white light exposure nine proteins including voltage-dependent anion channel, enolase $1 \alpha$, aldolase $\mathrm{C}$, crystallins $\alpha \mathrm{A}$ and $\beta \mathrm{B} 3$, heterogeneous nuclear ribonucleoprotein $\mathrm{A} 2 / \mathrm{B} 1$, albumin, and glutamine synthetase were identified. The results indicate that HNE modifications of retinal proteins are specific to a particular set of proteins rather than random events on abundant proteins.

\subsubsection{Carriers}

\subsubsection{Albumin}

Toyokuni et al., 2000 investigated the interaction of HNE with human serum albumin and found that it is rapidly quenched by human serum albumin (HSA) due to the covalent adduction to different accessible nucleophilic residues of the protein [180]. The molecular characterization of the covalent modifications led to the identification of eight Michael adducts and three Schiff bases involving nine nucleophilic sites. $\mathrm{Cys}^{34}$, His ${ }^{146}$, and Lys ${ }^{199}$ were found to be the most reactive HNE adduction sites. The latter adducted peptides were proposed as useful biomarkers of oxidative and carbonylation damage in humans [181]. In another MS investigation only those modified peptides were considered which were supported by high mass accuracy Orbitrap precursor ion measurements (high confidence hits) [182]. With HNE:HSA ratios of 1:1 and 10:1, 3 and 15 addition sites, respectively, were identified. This investigation confirmed previous work that $\mathrm{Cys}^{34}$, the only free cysteine, is the most reactive residue in HSA. 
HNE-modified HSA is highly immunogenic eliciting high titre immunogen specific antibodies as was shown for systemic lupus erythematosus (SLE), a chronic autoimmune disease, which is primarily characterized by increased levels of autoantibodies, predominantly against double stranded DNA [183]. A preferential binding of SLE autoantibodies to HNE-modified HSA as compared to native HSA or native DNA was shown and indicates a potential role of HNE-modified HSA in SLE etiopathogenesis.

\subsubsection{Hemoglobin and Myoglobin}

While hemoglobin $(\mathrm{Hb})$ can be modified by HNE in cell-free experiments, HNE-Hb adducts were not detected in erythrocytes under oxidative stress induced by gamma-irradiation [184]. In myoglobin $(\mathrm{Mb}) \mathrm{HNE}$ forms adducts with His residues and destabilizes $\mathrm{Mb}$ redox state, affecting meat colour [185]. LC-ESI-MS/MS of chicken Mb reacted with HNE identified covalent adduction of $\mathrm{His}^{64}$ and $\mathrm{His}^{93}$ at $\mathrm{pH} 7.4$.

\subsubsection{Liver and Adipocyte Fatty Acid-Binding Protein (FABP)}

FABPs have been characterized as facilitating the intracellular solubilization and transport of long-chain fatty acyl carboxylates via noncovalent interactions. Sites of HNE adduction on mouse liver recombinant FABP (L-FABP) were mapped on apo $\left(\mathrm{Lys}^{57}\right.$ and $\mathrm{Cys}^{69}$ ) and holo $\left(\mathrm{Lys}^{6}{ }^{6} \mathrm{Lys}^{31}\right.$,

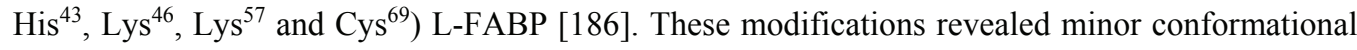
changes in global protein structure of apo and holo L-FABP by molecular modeling simulations, but apparent differences were observed within the internal binding pocket.

The FABP of adipocytes is the first protein for which the crystal structure of its HNE adduct was determined. Recent work has shown that the adipocyte FABP is covalently modified by HNE in vivo on $\mathrm{Cys}^{117}$. To evaluate HNE binding and modification, the crystal structures of adipocyte FABP covalently and noncovalently bound to HNE have been solved to $1.9 \AA$ and $2.3 \AA$ resolution, respectively [187]. While HNE in the noncovalently modified protein is coordinated similarly to a carboxylate of a fatty acid, the covalent form shows a novel coordination through a water molecule at the polar end of the lipid. Other defining features between the two structures with HNE and previously solved structures of the protein include a peptide flip between residues $\mathrm{Ala}^{36}$ and $\mathrm{Lys}^{37}$ and the rotation of the side chain of $\mathrm{Phe}^{57}$ into its closed conformation [187].

\subsubsection{Apolipoprotein B-100 (ApoB)}

The adduct formation of HNE and 4-oxo-nonenal (ONE) resp. with ApoB demonstrate that reactive aldehydes generated by LPO can differ in their biological effects, and that these differences can be mechanistically explained by the structures of the protein adducts formed [188]. In a study to understand the pathobiology of reactive lipid aldehydes the effects of HNE and ONE on the transport and secretion of very low-density lipoprotein was studied. Physiologically relevant concentrations of HNE and ONE rapidly disrupted cellular microtubules of HepG2 cells in a concentration-dependent manner. ONE reduced ApoB secretion while HNE did not significantly impair secretion. Both HNE and ONE formed adducts with ApoB protein, but HNE adducts were 
detectable as mono-adducts, while ONE adducts were present as protein-protein cross-links [188]. Interestingly another apolipoprotein, namely apolipoprotein A-I, the major HDL protein, is not modified by HNE in vitro, while MDA is adducted to lysine residues under the same conditions [189].

\subsubsection{5. $\beta$-Lactoglobulin}

No clear function has been identified for $\beta$-lactoglobulin, although it binds to several hydrophobic molecules, suggesting its role as a carrier. The strong suggestion is that the molecule exists primarily as a food source. $\beta$-Lactoglobulin was used as a model protein to study adduct formation in vitro during peroxidation of linolenic acid (LA) in the presence of $\mathrm{Fe}$ (II), and ascorbate [190]. By mass spectrometry the authors identified adducts including HNE-His Michael adducts, ONE-Lys 4-ketoamide, ONE-Lys pyrrolinone, and a Cys/His-ONE-Lys pyrrole cross-link. However, reversibly formed adducts, such as the HNE-Lys Schiff base were not present at detectable levels.

\subsubsection{Transporters and Channels}

\subsubsection{Glutamate Transport Protein}

HNE can inhibit glutamate transport in vitro and in vivo. Numerous in vitro and cell culture experiments indicate that oxidative damage decreases astrocyte glutamate transport activity. The hypothesis that LPO products impair glutamate and glucose transport in vivo has been tested by Ou et al., 2002 [191]. Their findings indicate that LPO products that irreversibly modify protein lysyl residues cause a two- to sixfold elevation in extracellular glutamate in striatum and cerebral cortex of rats undergoing microdialysis and that LPO product-evoked extracellular glutamate appeared to be derived from nonneuronal sources. These results support the hypothesis that oxidative damage leads to inhibition of glutamate transport and thereby contributes to the progression of neurodegenerative diseases. Mattson and Chan [192] showed that in AD A $\beta$ can induce membrane LPO and the formation of HNE. A $\beta$ thereby impairs the function of glutamate and glucose transporters, membrane ion-motive ATPases, and can enhance calcium influx through voltage-dependent and ligand-gated calcium channels. In sporadic amyotrophic lateral sclerosis (ALS) patients increased modification of proteins by HNE has been reported in the lumbar spinal cord compared to neurologically normal controls. Biochemical analysis revealed that one of the proteins modified by HNE was the astrocytic glutamate transporter EAAT2 [193]. The authors conclude that the function of proteins modified by HNE can be severely compromised leading to impairment of glutamate transport, and excitotoxic motor neuron degeneration in ALS.

For a homolog of HNE, 4-hydroxyhexenal, similarly the impairment of glutamate transport in astrocytes has been demonstrated [194]. 


\subsubsection{2. $\alpha$-Synuclein ( $\alpha$-Syn)}

$\alpha$-Syn is a small protein that is abundant in various regions of the brain [195]. Although the physiological function of $\alpha$-Syn is not well understood, its function is likely to involve presynaptic vesicle pool size and neurotransmitter release [196,197], and vesicle recycling [198]. One of the pathological hallmarks of Parkinson disease (PD) is the presence of intracellular inclusions called Lewy bodies that consist of aggregates of $\alpha$-Syn (for review see [199]). Xiang et al., 2013 [200] investigated posttranslational modifications (PTMs) of $\alpha$-Syn caused by oxidative stress, including modification by HNE (HNE- $\alpha$-Syn), nitration ( $\mathrm{N}-\alpha-\mathrm{Syn})$, and oxidation (O- $\alpha$-Syn), which have been reported to promote oligomerization of $\alpha$-Syn and found that modification of $\alpha$-Syn by HNE increased dopaminergic toxicity by increasing the interaction of extracellular $\alpha$-Syn with neurons. In another study [201], Bae et al., 2013 investigated the oligomerization of recombinant human $\alpha$-Syn via HNE adduct formation at the lysine and histidine residues and found that (a) HNE-induced $\alpha$-Syn oligomers are distinct from amyloid fibrils at both conformation and ultrastructure levels; (b) the HNE-induced oligomers are capable of seeding the amyloidogenesis of monomeric $\alpha$-Syn under in vitro conditions; (c) after treatment with HNE both the translocation of $\alpha$-Syn into vesicles and the release of this protein from cells were increased. The HNE- or ONE-induced $\alpha$-Syn oligomers have been characterized by Nasstrom et al., 2011 [202]. Both oligomers are rich in beta-sheet structure and have a molecular weight of about $2000 \mathrm{kDa}$. Atomic force microscopy analysis revealed that neither oligomer type polymerized into amyloid-like fibrils despite prolonged incubation. According to findings of Shibata et al., 2010 both ONE- and HNE-induced $\alpha$-Syn oligomers were cytotoxic when added exogenously to a neuroblastoma cell line, but HNE-induced $\alpha$-Syn oligomers were taken up by the cells to a significantly higher degree [203].

\subsubsection{Sarco/Endoplasmic Reticulum $\mathrm{Ca}^{2+}$-ATPase (SERCA1a)}

$\mathrm{HNE}$ has a dual effect on $\mathrm{Ca}^{2+}$ transport through sarcoplasmic reticulum membranes from rabbit fast-twitch skeletal muscle as reported by Hortigon-Vinagre et al., 2011 [204]. The authors showed that exposure of the membranes to HNE resulted in inhibition of the maximal ATPase activity and $\mathrm{Ca}^{2+}$ transport ability of SERCA1a, the $\mathrm{Ca}^{2+}$ pump in these membranes, reduced ATP binding and phosphoenzyme formation from ATP, whereas $\mathrm{Ca}^{2+}$ binding to the high-affinity sites was altered to a lower extent, and that HNE reacted with $\mathrm{Lys}^{515}$ within the nucleotide binding pocket of SERCA1a.

\subsubsection{Transient Receptor Potential Vanilloid 1 (TRPV1)}

TRPV1 is a nociceptive, $\mathrm{Ca}^{2+}$-selective ion channel activated by capsaicin, heat, and protons. The function of TRPV1 is detection and regulation of body temperature. In addition, TRPV1 provides sensing of scalding heat and pain (nociception). It has been reported that activation of TRPV1 expressed in esophageal mucosa is involved in gastroesophageal reflux disease (GERD) or in nonerosive GERD symptoms [205]. Capsaicin has been shown to significantly increase the production of HNE-modified proteins in Het1A cells, and IL-8 production in capsaicin-stimulated 
Het1A cells was enhanced by synthetic HNE treatment. By analyzing protein modifications it turned out that TRPV1 was modified by HNE [206]. It was therefore concluded that TRPV1 functions in chemokine production in esophageal epithelial cells, and this function may be regulated by HNE via posttranslational modification of TRPV1.

\subsubsection{Dopamine Transporter}

LoPachin et al., 2009 characterized the synaptosomal toxicity of HNE and evaluated the role of putative nucleophilic amino acid targets and were able to show that HNE exposure of striatal synaptosomes inhibited (3)H-dopamine membrane transport and vesicular storage which was paralleled by decreases in synaptosomal sulfhydryl content [206]. Furthermore they demonstrated that the sulfhydryl thiolate state was the HNE target, that the rate of adduct formation was $\mathrm{pH}$-dependent and that $N$-acetyl-L-cysteine, but not $N$-acetyl-L-lysine or $\beta$-alanyl-L-histidine, reduced in vitro $\mathrm{HNE}$ neurotoxicity.

\subsubsection{Receptors}

\subsubsection{Platelet-Derived Growth Factor Receptor- $\beta$ (PDGFR- $\beta$ )}

The PDGFR- $\beta$ signaling pathway regulates smooth muscle cell (SMC) migration and proliferation and plays a role in the vascular wall response to injury. HNE and oxidized low-density lipoprotein (oxLDL) induce a dual effect on PDGFR- $\beta$ signaling [207]. Short-term incubation of SMC with HNE or oxLDL induces PDGFR- $\beta$ pathway activation via the formation of PDGFR-adducts and oxidative stress increase. In contrast, long-term incubation triggers a desensitization of PDGFR to its own agonist, leading to a progressive inhibition of PDGF- $\beta$-mediated signalling and proliferation, resulting from decreased PDGF binding and inhibition of PDGFR- $\beta$ tyrosine kinase activity. PDGFR inhibition was associated with increased formation of HNE- and acrolein- PDGFR-adducts. PDGFR- $\beta$ adducts were detected in aortae of apolipoprotein-deficient mice, in hyper-cholesterolemic rabbits and in human carotid plaques. The aldehyde scavengers dinitrophenylhydrazine and hydralazine prevented both HNE- and oxLDLinduced structural modification and PDGFR- $\beta$ signaling dysfunction in cells and in vivo [207]. In conclusion, PDGFR- $\beta$ acts as a sensor for both oxidative stress and oxidized lipids in atherosclerotic areas and its progressive inhibition by HNE or acrolein may contribute to defective SMC proliferation, and decrease the stability of a vulnerable plaque.

\subsubsection{Lectin-Like Oxidized Low-Density Lipoprotein Receptor-1 (LOX-1)}

LOX-1 is an endothelial scavenger receptor that is important for the uptake of oxLDL. However, the precise structural motifs of oxLDL that are recognized by LOX-1 are unknown. By investigating the ability of BSA modified by lipid peroxidation to compete with AcLDL (acetylated low-density lipoprotein), Kumano-Kuramochi et al., 2012 showed that HNE-modified proteins most potently inhibited the uptake of AcLD, that both the modification of BSA and the oxidation of LDL resulted in the formation of HNE-histidine Michael adducts, that the HNE-histidine adduct 
inhibited the uptake of AcLDL in a dose-dependent manner, and that the HNE-histidine adduct stimulated the formation of ROS and activated extracellular-signal-regulated kinase 1/2 (ERK 1/2) and $\mathrm{NF \kappa B}$ thus initiating endothelial dysfunction and leading to atherosclerosis [208].

\subsubsection{Toll-Like Receptor 4 (TLR4)}

Toll-like receptors (TLRs) detect invading microbial pathogens and initiate immune responses as part of host defense mechanisms. They also respond to host-derived substances released from injured cells and tissues to ensure wound healing and tissue homeostasis. Dysregulation of TLRs increases the risk of chronic inflammatory diseases and immune disorders. Inflammatory events are often accompanied by oxidative stress which generates lipid peroxidation products such as HNE. Therefore, Kim et al., 2009 investigated whether HNE affects TLR activation and found that HNE blocked LPS (a TLR4 agonist)-induced activation of NFKB and IRF3 as well as expression of IFN- $\beta$, IP-10, RANTES and TNF $\alpha$ [209]. Furthermore, they were able to demonstrate that HNE suppressed both ligand-induced and ligand-independent receptor dimerization and that HNE formed adducts with cysteine residues of synthetic peptides derived from TLR4 suggesting that the reactivity of HNE with sulfhydryl moieties is implicated in the inhibition of TLR4 activation. Since inhibition of TLR4 activation by HNE also resulted in down-regulation of the phagocytic activity of macrophages it can further be concluded that HNE blocks TLR4-mediated macrophage activation and phagocytic functions.

\subsubsection{Cytoskeletal Proteins}

\subsubsection{Tau Proteins}

Tau proteins are microtubule-associated proteins found in neurons in the brain. These proteins interact with tubulin to stabilize microtubules and promote tubulin assembly into axonal microtubules. Tau has two ways of controlling microtubule stability: isoforms and phosphorylation. The formation of tau-containing neurofibrillary tangles is a major feature of $\mathrm{AD}$ and other neurodegenerative diseases. Fibers are correlated with disease severity and they have been implicated as playing a direct role in disease pathophysiology. Biochemical findings show that tau oxidative modifications are regulated by phosphorylation and that tau found in neurofibrillary tangles is oxidatively modified, suggesting that it is closely linked to the biology, not toxicity, of AD [210]. Several studies support a link between tau protein phosphorylation and adduction of tau by reactive carbonyls. The phosphorylation-dependent adduction of tau by carbonyl products resulting from LPO creates the neurofibrillary tangle-related antigen, Alz50. To determine whether epitopes of carbonyl-modified tau are major conformational changes associated with neurofibrillary tangle formation, seven distinct antibodies raised against neurofibrillary tangles have been examined that recognize unique epitopes of tau in $\mathrm{AD}$. Consistently, all seven antibodies recognize tau more strongly (4- to 34-fold) after treatment of normal tau with HNE, but only when tau is in the phosphorylated state [210]. In vitro $\mathrm{HNE}$ as well as several quinones facilitated the phosphorylated tubulin binding of tau into fibrillar polymers which have a morphology very similar to that of paired helical filaments present in the brains of AD patients [211]. These findings not 
only support the idea that oxidative stress is involved in neurofibrillary tangle formation occurring in brains of $\mathrm{AD}$ patients, but also show that HNE modifications of tau promote and contribute to the generation of the major conformational properties defining neurofibrillary tangles [210].

\subsubsection{Ankyrin}

Ankyrin is an adaptor protein in the membrane cytoskeleton of erythrocytes. The modification of ankyrin is pathologically relevant in malaria [212], however, the molecular basis for the prevalence of blood group 0 in regions where malaria is endemic remains unclear [212]. Mendez et al., 2012 [212] therefore investigated whether there are differences in carbonylated membrane proteins between the different blood groups and found that group 0 blood showed a reduced protein oxidation pattern compared to groups $\mathrm{A}, \mathrm{B}$ and $\mathrm{AB}$. By examining HNE modified proteins, ankyrins were found to be differentially carbonylated in group 0 upon malaria infection as compared to A and B groups.

\subsubsection{Spectrins}

Both and spectrin of the cytoskeleton of erythrocytes (RBC) are targets of HNE [213]. Spectrin strengthens the RBC membrane through its direct association with membrane lipids and through protein-protein interactions. Spectrin loss on the other hand reduces membrane stability and results in various types of hereditary spherocytosis. However, less is known about acquired spectrin damage. Arashiki et al., 2010 were able to show that $\alpha$ - and $\beta$-spectrin in human RBC are the primary targets of HNE, i.e., the level of HNE adducts in spectrin (particularly $\alpha$-spectrin) was increased following HNE treatment of RBC membrane ghosts [213]. In contrast, ghost preparation in the presence of MgATP reduced HNE adduct formation, with preferential $\beta$-spectrin modification and increased cross-linking of the HNE-modified spectrins resulted in selective HNE-spectrin adduct formation. These findings were interpreted in terms of preferential HNE adduction in spectrin at the interface between the skeletal proteins and lipid bilayer. A combined protocol of immune-detection, peptide enrichment, mass spectrometry, and de novo protein sequencing was applied to study the adduction sites in human $\beta$-spectrin [214]. HNE-lysine and HNE-histidine Michael adducts were detected in $\beta$-spectrin under physiological conditions.

\subsubsection{Chaperones: Heat Shock Proteins 70 and 90}

In a rat model of chronic alcohol-induced oxidative stress [91] Carbone et al., 2004 investigated the modification of both cytosolic Hsp72, the inducible variant of Hsp70, and Hsp90 by HNE. By applying mass sprectrometrical analysis they were able to show that Hsp72 treated with 10 and 100 $\mu \mathrm{M}$ HNE caused adduct formation at $\mathrm{Cys}^{267}$ in the ATPase domain of the chaperone. Cys ${ }^{572}$ was found to be a site for HNE modification in Hsp90 [90]. HNE adducted Hsp70 has been identified in neuronal necrotic cell death [215] and in ALS an age-related, fatal motor neuron degenerative disease [216]. Although multiple mechanisms contribute to the pathogenesis of motor neuron injury in ALS, it is likely that oxidative stress plays a significant role in the amplification, and possibly the initiation, of the disease. HNE levels are increased in spinal cord motor neurons of 
ALS patients, indicating that LPO is associated with the motor neuron degeneration in ALS. Three significantly HNE-modified proteins were found in the spinal cord of G93A-SOD1 transgenic mice: besides Hsp70 dihydropyrimidinase-related protein 2, and possibly $\alpha$-enolase.

HNE-modified Hsp70 is much more vulnerable to calpain cleavage [217]. The calpain-cathepsin cascade is mediating necrotic neuronal death from simpler organisms to primates. The main event of this cascade is calpain-mediated lysosomal rupture and the resultant release of lysosomal cathepsins into the cytoplasm. However, the in vivo substrate of calpain for inducing lysosomal destabilization still remains completely unknown. Recent data obtained with post-ischemic hippocampal CA1 tissues and glaucoma-suffered retina from primates suggests that Hsp70.1 might be the in vivo substrate of activated $\mu$-calpain at the lysosomal membrane of neurons. Hsp70.1 is known to stabilize lysosomal membrane by recycling damaged proteins and protect cells from oxidative stresses [217]. Sahara et al., 2010 studied the molecular interaction between activated $\mu$-calpain and the lysosomal Hsp70.1 in monkey hippocampal CA1 neurons after ischemia-reperfusion insult and found that Hsp70.1 in CA1 tissue is an in vivo substrate of activated $\mu$-calpain and that Hsp70.1 carbonylated by HNE or hydrogen peroxide is much more vulnerable to calpain cleavage [217]. These data further suggest that Hsp70.1 can become a target of the carbonylation by HNE, and Hsp70.1 is a modulator of calpain-mediated lysosomal rupture/permeabilization after ischemia-reperfusion injury.

The adduction of Hsp90 by HNE has been studied by protein-selective capture [218] in RKO colorectal cancer cells and identified His ${ }^{450}$ and $\mathrm{His}^{490}$ adducts of Hsp90 $\alpha$. Five other histidine residues were also adducted on $\mathrm{Hsp} 90 \mathrm{\beta}$ : $\mathrm{His}^{171}$, $\mathrm{His}^{442}$, $\mathrm{His}^{458}$, $\mathrm{His}^{625}$ and $\mathrm{His}^{632}$.

Both Hsp70 and Hsp90 are together with PDI, liver FABP and the protein-serine/threonine kinases ERK1 and ERK2 targets of HNE in early stages of ALD [219]. The adduction leads to inhibition of these enzymes and it was concluded that inhibition of Hsp70, Hsp90 and PDI function could be involved in initiation of the early phases of ER stress contributing to stimulation and accumulation of hepatic lipids. Likewise, impairment of L-FABP activity could also disrupt lipid transport contributing to steatosis. The modification and inhibition of Erk1/2 by HNE may in addition contribute to the decreased hepatocellular proliferation associated with ALD [219].

The electrophilic adduction of Hsp70 and Hsp90 by HNE appears to mediate in part the activation of heat shock factor 1 (HSF1), which is normally maintained in an inactive cytosolic complex [220]. This is one of the mechanisms, by which cellular responses to HNE are elicited, as was revealed by a systems analysis approach of protein modification and cellular responses induced by electrophile stress aiming to define the chemistry of protein modification and its biological consequences using lipid-derived $\alpha, \beta$-unsaturated aldehydes such as HNE as model electrophiles. In this global approach, two large data sets were analyzed: one represented the identity of proteins modified over a wide range of electrophile concentrations, and the second comprised changes in gene expression observed under similar conditions. Informatics tools showed theoretical connections based primarily on transcription factors hypothetically shared between the two data sets, downstream of adducted proteins and upstream of affected genes [220]. This technique identified a multitude of HNE targets such as several transcription factors as potential mediators of the cellular response to HNE-adducted proteins. Among these, HSF1 was confirmed as a sensitive 
and robust effector of HNE-induced changes in gene expression. Among the genes induced by HSF1, Bcl-2- associated athanogene 3 (BAG3) is notable for its actions in promoting cell survival through stabilization of antiapoptotic Bcl-2 proteins, thus having a critical role in mediating cellular protection against electrophile-induced death [220].

The ER homolog of HSP70 is glucose-regulated protein 78 (GRP78). It plays a critical role in the cellular response to ER stress by serving as a chaperone assisting protein folding and by regulating the signaling of the unfolded protein response (UPR). GRP78 revealed a marked propensity for Lys and His adduction by HNE within the ATPase domain and a relative paucity of adduct formation within the peptide-binding domain [221]. Consistent with these findings, a concomitant dose-dependent decrease in ATP-binding and ATPase activity was observed without any discernible impairment of chaperone function.

\subsubsection{Uncoupling Proteins 2 and 3 (UCP2 and UCP3)}

Most studies with HNE were focused on mitochondrial UCP2 and UCP3. Mitochondria are potent producers of cellular superoxide from complexes I and III of the electron transport chain, and mitochondrial superoxide production is a major cause of the cellular oxidative damage that may underlie degradative diseases and aging. This superoxide production is very sensitive to the proton motive force, so it can be strongly decreased by mild uncoupling of oxidative phosphorylation. Superoxide and the downstream LPO products including hydroxyalkenals such as HNE, are potent activators of proton conductance by mitochondrial uncoupling proteins such as UCP2 and UCP3, although the mechanism of activation has yet to be established. It was therefore suggested that superoxide releases iron from aconitase, leading to LPO and the release of molecules such as HNE that covalently modify and activate the proton conductance of UCPs and other proteins such as the adenine nucleotide translocase [222]. UCP2 and UCP3 do not catalyse proton leak in the absence of such acute activation. They can also catalyse export of fatty acid and other anions, although the relationship of anion transport to proton transport remains controversial [223]. The notion that HNE plays a role in the function of UCPs to minimize superoxide formation is supported by a study on plant mitochondria. HNE and a structurally related compound, trans-retinal, stimulated proton conductance in potato mitochondria, that is inhibitable by GTP, a characteristic of UCP. Proof that the effect of HNE and trans-retinal are mediated by UCPs was provided by examination of proton conductance in transgenic plants overexpressing UCP. An increase in UCP content resulted in a modest but significant decrease in the rate of superoxide production [224]. A hypothesis has been forwarded for the main function of uncoupling proteins: to cause mild uncoupling to diminish mitochondrial superoxide production, hence protecting against disease and oxidative damage at the expense of a small loss of energy. This simple feedback loop would constitute a self-limiting cycle to protect against excessive superoxide production. A more recently evolved role of UCP2 is perhaps as part of a signaling pathway to regulate insulin secretion in pancreatic beta cells [222,225,226].

Recently it was also shown that fatty acids are key players in HNE-mediated activation of UCP1 and UCP2 [227]. The molecular mechanisms of HNE action were investigated by evaluating the separate contributions of lipid and protein phases of the membrane and by comparing UCP1 and 
UCP2, which were reconstituted in planar lipid bilayers. Via this approach it was demonstrated that HNE does not directly activate either UCP1 or UCP2. Instead, it strongly potentiates the membrane conductance increase $(\mathrm{G}(\mathrm{m})$ ) mediated by different long-chain fatty acids in UCP-containing and in UCP-free membranes. This $\mathrm{G}(\mathrm{m})$ increase is concentration-dependent and exhibits a typical saturation kinetics. In addition, for the amoeba Acanthamoeba castellani it was shown that the HNE induced UCP-mediated mitochondrial uncoupling is GTP-sensitive [228].

$\mathrm{HNE}$ is able to increase the proton conductance of the inner mitochondrial membrane through effects not only on uncoupling proteins but also the adenine nucleotide translocase (ANT). To clarify the relative contribution of the two carriers to these effects mitochondria were isolated from skeletal muscle and heart of wild-type and Ucp3 knockout (Ucp3KO) mice [229]. To increase UCP3 expression, some mice were i.p. injected with LPS which did not change basal proton conductance. HNE increased the proton conductance of skeletal muscle and heart mitochondria. In skeletal muscle, this increase was lower in Ucp3KO mice and higher in LPS-treated wild-type mice, and was partially abolished by GDP (UCPs inhibitor) and completely abolished by carboxyatractylate (ANT inhibitor) or addition of both inhibitors. GDP had no effect on HNE-induced conductance in heart mitochondria, but carboxyatractylate or administration of both inhibitors had a partial effect. GDP-mediated inhibition of HNE-activated proton conductance in skeletal muscle mitochondria was not observed in Ucp3KO mice, indicating that GDP is specific for UCP3, at least in muscle. It was therefore concluded that, in skeletal muscle, HNE-induced increase in proton conductance is mediated by UCP3 (30\%) and ANT, whereas in the heart the increase is mediated by ANT and other carriers, possibly including UCP3 [229].

\subsubsection{Growth Factors: Platelet-Derived Growth Factor (PDGF)}

Jin et al., 2013 investigated breast cancer-associated changes of HNE, GSH, nitrotyrosine and halotyrosine adducts in 27 secreted proteins, for a total of 108 candidate biomarkers in plasma samples for both cases and benign controls and identified HNE-modified PDGF and GSH-modified ceruloplasmin which were significantly altered in samples from cancer patients [230]. The authors concluded that these changes may reflect redox changes in breast cancer, and that HNE-modified PDGF may be useful as plasma biomarker in breast cancer.

\subsubsection{Peptide Hormones}

\subsubsection{Insulin}

Human insulin is a target of both HNE and 4-hydroxy-hexenal (HHE) [231]. This was demonstrated by incubation of insulin in the presence of HNE or HHE and adduct analysis. The formation of covalent adducts on insulin was analyzed by mass spectrometry analysis and revealed one to five Michael adducts on insulin. Furthermore the authors report that glucose uptake in 3T3-L1 and L6C5 cells was significantly reduced after treatment with adducted insulin compared to native insulin, indicating that the formation of HNE- and HHE-Michael adducts significantly disrupted the biological activity of insulin. 


\subsubsection{Angiotensin II}

Another peptide hormone adducted by HNE is angiotensin II [232]. The octapeptide angiotensin II is the primary active hormone of the renin/angiotensin system (RAS) and has been implicated in various cardiovascular diseases. Numerous structure activity relationship studies have identified $\mathrm{Asp}^{1}, \mathrm{Arg}^{2}$, and $\mathrm{His}^{6}$ of Ang II to be critical for its biological activity and receptor binding [232]. Subsequently, it has been elaborated that the oxidative modifications on the $N$-terminus of Ang II disrupt interactions with Ang II type 1 receptor and aminopeptidase A (which cleaves the N-terminal Asp residue of Ang II to generate Ang III), which could affect the regulation of cardiovascular function [232].

\subsubsection{Extracellular Matrix Proteins: Collagen}

HNE forms adducts with type II collagen (Col II). El-Bikai et al., 2010 addressed the question whether interactions between human osteoarthritic chondrocytes and HNE-modified Col II affect cell phenotype and functions and to determine the protective role of carnosine (CAR) treatment in preventing these effects [233]. Their major findings were that Col II modification by HNE at a molar ratio (MR) approximately 1:20, strongly induced ICAM-1, $\alpha 1 \beta 1$-Integrin and metallo-proteinase-13 (MMP-13) expression as well as extracellular signal-regulated kinases 1 and $2(E R K 1 / 2)$ and NFkB-p65 phosphorylation without having an impact on cell adhesion and viability or Col II expression. In contrast, Col II modification with HNE at MR approximately 1:200, altered chondrocyte adhesion by evoking cell death and caspase-3 activity, and the inhibition of $\alpha 1 \beta 1$-integrin and Col II expression as well as ERK1/2 and NFkB-p65 phosphorylation. At the same time a release of $\mathrm{PGE}_{2}$, expression of COX-2 and p38 MAPK phosphorylation were observed. All these effects were prevented by CAR, an HNE-trapping drug (see chapter 5.1.1.2., p.20). The authors concluded that HNE-binding to Col II results in multiple abnormalities of chondrocyte phenotype and function, suggesting its contribution in osteoarthritis development [233].

\subsubsection{Histones: Histone-H2A}

Histones are DNA associated nucleoproteins, which adopt different structures under oxidative stress. A study was undertaken to test the role of HNE-modified histone-H2A (HNE-H2A) in systemic lupus erythematosus (SLE) [234]. The data revealed that HNE-mediated LPO in histone-H2A caused alteration in histidine, lysine and cysteine residues. In addition, protein carbonyl contents were also high in HNE-H2A. The specificity of autoantibodies from SLE patients were analyzed towards HNE-H2A and their results were compared with sex- and age-matched controls. SLE autoantibodies showed preferential binding to HNE-H2A in comparison with histone-H2A. In addition, HNE-H2A was also detected in SLE peripheral blood mononuclear cells suggesting a likely role of HNE-H2A in the initiation/progression of SLE [234]. 


\subsection{Reactions with Lipids}

The phospholipids phosphatidyl-ethanolamine (PE) and - to a lesser extent - phosphatidylserine, which contain a primary amino group, can undergo both Michael addition and Schiff-base formation with HNE [235]. The Schiff-base of PE partly undergoes cyclization to a pyrrole derivative, and PEs modified by HNE and other aldehydes induce monocyte adhesion to cultured endothelial cells.

LPO generates a large family of aldehyde-modified PEs (al-PEs), many of which have the potential to drive inflammation [236], i.e., gamma-ketoaldehydes (isolevuglandins, IsoLGs) form inflammatory mediators by modifying the ethanolamine headgroup of PEs. When the ability of al-PEs was tested to induce THP-1 monocyte adhesion to cultured endothelial cells, it was found that PEs modified by HNE, ONE and MDA induced adhesion with potencies similar to those of PEs modified by IsoLGs, while PEs modified by acrolein or by glucose were only partial agonists for adhesion [236].

\subsection{Reactions with Cofactors and Vitamins}

\subsubsection{Vitamin C (Ascorbic Acid)}

Of interest is the observation that HNE, like other LPO-derived electrophiles, can undergo ascorbylation. Vitamin C can form a Michael-type conjugate with HNE in vitro [237,238]. The reaction has been described as a Michael addition of an activated $\mathrm{C}-\mathrm{H}$ group of ascorbic acid to the $\mathrm{C}=\mathrm{C}$ double bond of $\mathrm{HNE}$ followed by cyclisation to produce a hemiacetal [48]. Ascorbic acid is able to promote the detoxification and elimination of HNE in human monocytic THP-1 leukemia cells [239]. In this process adduct formation of ascorbic acid with HNE is only a minor pathway. It was therefore suggested that the protective effect of ascorbate against HNE cytotoxicity is largely via modulation of multidrug resistant protein (MRP)-mediated transport of GSH-HNE conjugate metabolites.

\subsubsection{Pyridoxamine}

Also the cofactor pyridoxamine is an HNE target. In search for new scavengers of carbonyl compounds, the amino group of pyridoxamine was demonstrated to react faster with HNE than the amino group of $\mathrm{N}$-acetyl lysine, but much slower than a series of 4-ketoaldehydes with oxopentanal as model compound [240].

\subsubsection{Lipoic Acid}

The reduced form of lipoic acid contains two thiol groups which can be adducted by HNE. Both lipoic acid and its redox enzyme lipoamide dehydrogenase (LADH) are targets of HNE in AD brain. The AD brain shows increased levels of LPO products, including HNE. The levels of the HNE-modified lipoic acid in brain of subjects with AD and age-matched controls were therefore measured by Hardas et al., 2013 [241]. Lipoic acid is a key co-factor for a number of proteins 
including pyruvate dehydrogenase and $\alpha$-ketoglutarate dehydrogenase. Decreased levels of HNE-lipoic acid in the AD brain were observed in this study compared to that of age-matched controls. It was further demonstrated that both LADH levels and activities were significantly reduced in $\mathrm{AD}$ brain compared to age-matched control. The authors conclude that these data are consistent with a two-hit hypothesis of AD: oxidative stress leads to LPO that, in turn, causes oxidative dysfunction of key energy-related complexes in mitochondria, triggering neurodegeneration, and they suggest that lipoic acid supplementation could be a potential treatment for the observed loss of cellular energetics in $\mathrm{AD}$ to potentiate the antioxidant defense system to prevent or delay oxidative stress in and progression of this disorder [241].

\subsection{Reactions with Nucleic Acids}

The current knowledge on DNA damage induced by endogenously produced reactive aldehydes such as HNE, MDA, acrolein, crotonaldehyde and methylglyoxal in relation to the pathophysiology of human diseases has been reviewed by Voulgaridou et al., 2011 [242]. Minko et al., 2009 [243] gave an overview of the chemistry and biology of DNA containing $1, \mathrm{~N}^{2}$-deoxyguanosine adducts of the $\alpha, \beta$-unsaturated aldehydes HNE, acrolein, and crotonaldehyde.

HNE is genotoxic, e.g., for hepatocytes [244] and cerebral endothelial cells [245]. HNE in a concentration of $0.1 \mu \mathrm{M}$ causes elevated levels of sister chromatid exchanges in primary hepatocytes, $1 \mu \mathrm{M}$ causes elevated levels of chromosomal aberrations and at $10 \mu \mathrm{M}$ the formation of micronuclei is observed. HNE is thought to contribute to the low level of DNA adducts which are abundant both in untreated rodent and human genomes. Two pathways are considered to be responsible for the mutagenicity of HNE: one route is the formation of an adduct by direct interaction with the guanosine moiety of DNA (Figure 4). The reaction of HNE with DNA gives four diastereomeric $1, \mathrm{~N}^{2}-\gamma$-hydroxypropano adducts of deoxyguanosine; background levels of these adducts have been detected in animal tissue. Stereospecific synthesis of these four adducts at the nucleoside level have been accomplished. In addition, a versatile strategy for their site-specific incorporation into oligonucleotides has been developed [246].

Reaction of HNE with calf thymus DNA resulted in a pair of diastereomeric adducts. Background levels of the $1, \mathrm{~N}^{2}$-propane adduct of guanine (HNE-dG) in rat tissues are in the range of 18-158 adducts per $10^{9}$ nucleotides with relatively high levels in the liver [247]. The guanine adducts can form interstrand cross-links. When placed opposite $\mathrm{dC}$ in the $5^{\prime}-\mathrm{CpG}-3$ ' sequence, the $(6 \mathrm{~S}, 8 \mathrm{R}, 11 \mathrm{~S})$ diastereomer of the HNE-dG forms a $\mathrm{N}^{2}-\mathrm{dG}: \mathrm{N}^{2}-\mathrm{dG}$ interstrand cross-link [246,248]. Its structure was refined by Huang et al., 2010 and 2011 showing that the crosslink is a $\mathrm{N}^{2}-\mathrm{dG}: \mathrm{N}^{2}-\mathrm{dG}$ carbinolamine $[249,250]$. These cross-linked -enal adducts are likely to contribute to the genotoxic effects of both HNE and acrolein. These cross-linked adducts occur at levels that are similar to $1 \%-2 \%$ of the levels of the monomeric $1, \mathrm{~N}^{2}-\mathrm{dG}$ adducts in calf thymus DNA treated with either -enal.

Besides HNE also acrolein can form a cyclic adduct of deoxyguanosine (Acr-dG) in DNA. In vivo levels of Acr-dG in DNA are at least two orders of magnitude higher than those of the HNE adduct HNE-dG [251,252]. In addition to the facile reaction with acrolein, the higher levels of the acrolein adduct in vivo are due to a lower rate of repair. 
The consequences of cellular formation of LPO products, particularly HNE, in terms of genomic stability have been reviewed by Winczura et al., 2012 [253]. Elimination from DNA of LPO-induced lesions is executed by several repair systems: base excision repair (BER), direct reversal by AlkB family proteins, nucleotide excision repair (NER) and recombination. NER and recombination are involved in repair of HNE adducts to DNA bases in Escherichia coli [254]. According to the authors Acr-dG in a plasmid DNA is repaired by NER proteins, but it is repaired at a much slower rate than HNE-dG in human colon cell extracts, and the slow repair of Acr-dG is likely due to poor recognition/excision of the lesions in DNA. Using a plasmid DNA containing both adducts it was shown that the repair of Acr-dG is significantly inhibited by HNE-dG. In contrast, the repair of HNE-dG is not much affected by Acr-dG [251].

Interestingly, the 1,N-2-propane adduct of guanine in DNA can be bypassed during replication by Sulfolobus solfataricus P2 DNA polymerase IV [255].

The role of damage-specific DNA polymerases in mutagenesis induced by HNE was studied in M13mp18 phage by testing survival and mutation spectra in the lacZ $\alpha$ gene [256]. The phage was grown in uvrA(-) Escherichia coli strains, carrying one, two or all three SOS DNA polymerases. When Pol IV was the only DNA SOS polymerase in the bacterial host, survival of HNE-treated M13 DNA was similar to, but mutation frequency was lower than in the strain containing all SOS DNA polymerases. When only Pol II or Pol V were present in host bacteria, phage survival decreased dramatically. Simultaneously, mutation frequency was substantially increased, but exclusively in the strain carrying only Pol V suggesting that induction of mutations by HNE is mainly dependent on Pol V [256].

The second route for the mutagenicity of HNE putatively involves the oxidation of HNE to its epoxide. The resulting etheno adducts have been found in human lung tissue [257]. The reaction mechanism was studied by Lee et al., 2005 [258]. Analysis of the reaction between 4-hydroperoxy2-nonenal (HPNE) and 2'-deoxyguanosine (dGuo) revealed the formation of 1, $\mathrm{N}^{2}$-etheno-dGuo as well as heptanone-etheno-dGuo and trace amounts of dihydroxyheptane-etheno-dGuo. On the basis of these findings, it was concluded that HPNE, a primary product of LPO, is a major precursor to the formation of $1, \mathrm{~N}^{2}$-etheno-dGuo, and it was proposed that $1, \mathrm{~N}^{2}$-etheno-dGuo arises from the reaction of dGuo and HPNE via the intermediate formation of a cyclic hydroxy-ethano-epoxide derivative. In support of this assumption cytochrome P4502E1 appears to be responsible for the formation of HNE-etheno-DNA adducts in alcoholic liver disease [259].

Miscoding etheno-modified DNA adducts including 1,N(6)-etheno-2'-deoxyadenosine ( $\varepsilon$-dA) are excreted in urine, following elimination from tissue DNA. An ultrasensitive and specific immunoprecipitation/HPLC-fluorescence detection method was developed for quantifying $\varepsilon$-dA excreted in urine [260]. Levels in urine of Thai and European liver disease-free subjects were found to be in the range of 3-6 fmol $\varepsilon-\mathrm{dA} / \mu \mathrm{M}$ creatinine. Subjects with inflammatory cancer-prone liver diseases caused by viral infection or alcohol abuse excreted massively increased and highly variable $\varepsilon$-dA-levels. Based on this pilot study it was concluded that (i) high urinary $\varepsilon$-dA-levels, reflecting massive LPO-derived DNA damage in vivo may contribute to the development of hepatocellular carcinoma (HCC); (ii) $\varepsilon$-dA-measurements in urine and target tissues should thus be further explored as a putative risk marker to follow malignant progression of inflammatory liver 
diseases in affected patients and that (iii) etheno adducts may serve as biomarkers to assess the efficacy of (chemo-) preventive and therapeutic interventions [260].

\section{Formation of HNE in Mammalian Cells and Tissues}

High HNE levels or increases of HNE are generally an indication for accelerated LPO. However, both the high reactivity of HNE and the rapid metabolism of HNE will overlap the formation rate of HNE, i.e., even if the rates of LPO and of HNE formation are very high, the topical increasing HNE level does not fully reflect the increase of LPO and HNE formation rates due to the overlapping of formation and degradation. The dynamics of HNE turnover, which is not easy to analyze - e.g., with tracer kinetic measurements - is much higher than the simple increase rate of HNE concentration. Thus, when significant increases of HNE levels are found in organelles, cells, tissues, organs, or in whole organisms, one may assume that the total increase in LPO rate and in HNE formation rate is extremely high.

\subsection{HNE Formation in Cellular and Organ Systems}

The oxidative stress during posthypoxic reoxygenation of cell suspensions or postischemic reperfusion of organs was demonstrated by an increase of HNE levels - often paralleled by an enhancement of MDA or thiobarbituric acid-reactive substances (TBA-RS), decrease of GSH and increase of disulfides, too. Those changes were found in hepatocytes [261], renal tubular cells [262], heart [263-265], and small intestine [266]. In earlier studies, HNE increases were observed in hepatocyte suspensions under oxidative stress by $\mathrm{CCl}_{4}$ or ADP-iron [267]. An age-dependent increase of HNE in rats with traumatic brain injury was found in ipsilateral hippocampus one and seven days post injury [268]. Furthermore an increase of HNE was also found in hippocampus/parahippocampal gyrus, superior and middle temporal gyrus and cerebellum in mild cognitive impairment compared to age-matched control subjects [269]. This demonstrates that LPO occurs early in the pathogenesis of Alzheimer's disease. Similar changes in Alzheimer's disease and other neurodegenerative diseases were described by Negre-Salvayre et al., 2010 [270].

Table 1 presents a list of cellular, tissue or organ concentrations of HNE in humans and other species. Additionally, Figure 5 demonstrates human blood plasma concentrations of HNE in dependence on age of the blood donors.

HNE formation was also measured in perfused organs. Changes of perfusion conditions lead to changes in HNE formation rate. The tissue concentration of HNE only partly represents the real HNE formation rate. The dynamics of HNE formation rate was of particular interest in the postischemic reperfusion syndrome. The real flux rate of intracellular HNE formation during normoxic perfusion, during ischemia and during postischemic reperfusion was estimated on the basis of tissue HNE concentration, HNE utilization rate at defined HNE tissue concentration and HNE washout by perfusate circulation in rat small intestine [266]. Such estimations led to intestinal $\mathrm{HNE}$ formation rates of about $20 \mathrm{nmol} / \mathrm{g} \mathrm{w} . \mathrm{w} . / \mathrm{min}$ at normoxia, 20 to $40 \mathrm{nmol} / \mathrm{g} \mathrm{w} . \mathrm{w} . / \mathrm{min}$ at ischemia, $100 \mathrm{nmol} / \mathrm{g} \mathrm{w} . \mathrm{w} . / \mathrm{min}$ at $10 \mathrm{~min}$ of postischemic reperfusion, and $20 \mathrm{nmol} / \mathrm{g} \mathrm{w} . \mathrm{w} . / \mathrm{min}$ at $60 \mathrm{~min}$ of reperfusion). These data showed that the highest increase of HNE formation occured during the early reperfusion phase following ischemia. 


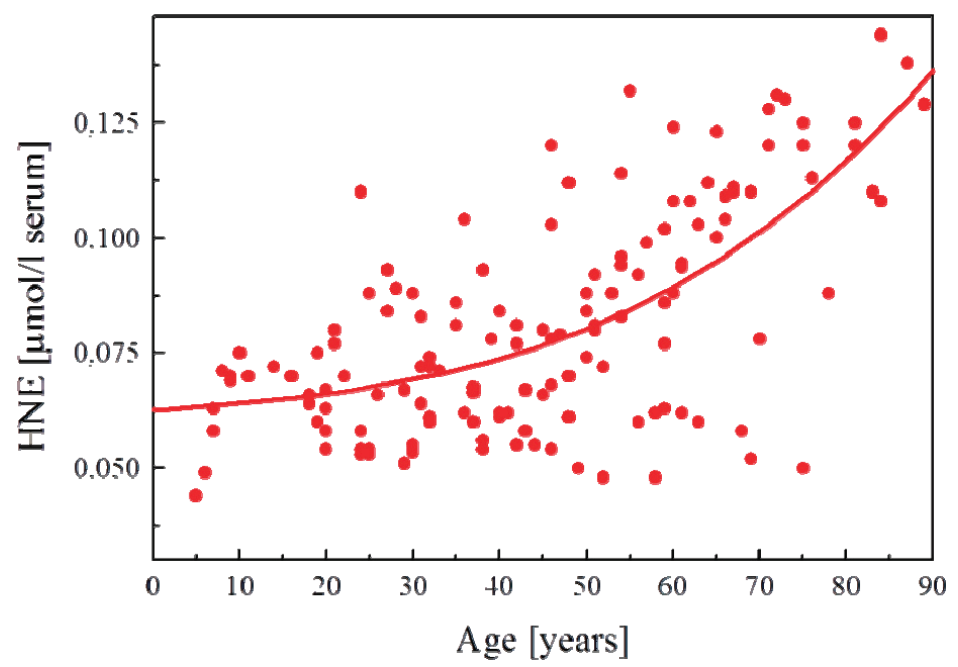

Figure 5. HNE plasma concentration in dependence on age of the blood donor (5 to 90 years).

A significant increase of HNE concentration was found in human renal tubular cells [262] and in rat hepatocytes [261,271] during postanoxic reoxygenation in comparison with preanoxic control values. In parallel to the HNE increase decreases of energy rich nucleotides, in particular of ATP, of GSH and cell viability as well as electron-microscopic morphological alterations of cells were observed. In human monocytes, HNE increased after phagocytosis of the malarial pigment hemozoin reaching $4 \mathrm{pmol} / 10^{6}$ cells at $2 \mathrm{~h}$ (approximate intracellular concentration (AIE) $8 \mu \mathrm{M}$ ), $23 \mathrm{pmol} / 10^{6}$ cells at $5 \mathrm{~h}(\mathrm{AIE} 46 \mu \mathrm{M})$, and $7.9 \mathrm{pmol} / 10^{6}$ cells (AIE $\left.16 \mu \mathrm{M}\right)$ at 12. A moderate increase in HNE, approximately $2 \mathrm{pmol} / 10^{6}$ cells (AIE $4 \mu \mathrm{M}$ ), was also observed after phagocytosis of anti-D IgG-opsonized erythrocytes. HNE in unfed controls was approximately $0.5 \mathrm{pmol} / 10^{6}$ cells (AIE $1 \mu \mathrm{M})$ [272]. The values which were measured in monocytes fed with hemozoin up to $50 \mu \mathrm{M}$ were the highest intracellular concentrations which were ever measured. In some additional studies formation and metabolism of both HNE as well as MDA were compared [14].

\subsection{HNE in the Whole Healthy Organism}

Increased HNE levels in blood serum and tissues were measured in various diseases in children and adults such as reperfusion syndrome, inflammatory diseases, rheumatological diseases (e.g., rheumatoid arthritis), lymphedema, AIDS, diabetes, and cystic fibrosis. Increased serum levels of HNE, MDA or other LPO products have also been demonstrated in several systemic autoimmune diseases in adults such as rheumatoid arthritis and systemic lupus erythematosus (SLE). Children suffering from SLE have significantly higher HNE levels (146 $\pm 14 \mathrm{nmol} / \mathrm{L})$ compared to controls $(61 \pm 10 \mathrm{nmol} / \mathrm{L})$ while children with the focal type of scleroderma (SCL) had HNE levels similar to the control group [276]. 
Table 1. HNE concentrations in cells, tissues and organs.

\begin{tabular}{|c|c|c|c|}
\hline Cells, Tissue & HNE-Concentration & Comment & Reference \\
\hline \multirow{2}{*}{$\begin{array}{l}\text { Kidney tubular } \\
\text { cells (human) }\end{array}$} & $102 \mathrm{pmol} / 10^{6}$ cells & Praeanoxic control & \multirow{2}{*}{ [262] } \\
\hline & $307 \mathrm{pmol} / 10^{6}$ cells & $30 \mathrm{~min}$ reoxygenation after $60 \mathrm{~min}$ anoxia & \\
\hline \multirow{5}{*}{$\begin{array}{c}\text { Hepatocytes } \\
\text { (rat) }\end{array}$} & $13 \pm 5 \mathrm{pmol} / 10^{6}$ cells & Freshly prepared & \multirow{3}{*}[267]{} \\
\hline & $27 \pm 18 \mathrm{pmol} / 10^{6}$ cells & $\mathrm{CCl}_{4}$-treated for $1 \mathrm{~h}$ & \\
\hline & $40 \pm 14 \mathrm{pmol} / 10^{6}$ cells & $\mathrm{ADP}-\mathrm{Fe}^{3+}$-treated for $1 \mathrm{~h}$ & \\
\hline & $0.43 \mathrm{pmol} / 5.2 \times 10^{6}$ cells & Freshly prepared & \multirow{2}{*}[271]{} \\
\hline & $1.42 \mathrm{pmol} / 5.2 \times 10^{6} \mathrm{cells}$ & $15 \mathrm{~min}$ reoxygenation after $60 \mathrm{~min}$ anoxia & \\
\hline \multirow{4}{*}{$\begin{array}{c}\text { Monocytes } \\
\text { (human) }\end{array}$} & $0.5 \mathrm{pmol} / 10^{6}$ cells $(1 \mu \mathrm{M}) *$ & Basic value & \multirow{4}{*}{ [272] } \\
\hline & 4 pmol $/ 10^{6}$ cells $(8 \mu \mathrm{M}) *$ & Hemozoin fed at $2 \mathrm{~h}$ & \\
\hline & $23 \mathrm{pmol} / 10^{6}$ cells $(46 \mu \mathrm{M}) *$ & Hemozoin fed at $5 \mathrm{~h}$ & \\
\hline & $7.9 \mathrm{pmol} / 10^{6}$ cells $(16 \mu \mathrm{M}) *$ & Hemozoin fed at $12 \mathrm{~h}$ & \\
\hline \multirow{2}{*}{$\begin{array}{l}\text { Small intestine } \\
\text { (rat) }\end{array}$} & $0.68 \mathrm{nmol} / \mathrm{g} \mathrm{w} . \mathrm{w}$. & perfusion, normoxia & \multirow{2}{*}{ [266] } \\
\hline & $3.02 \mathrm{nmol} / \mathrm{g} \mathrm{w} . \mathrm{w}$. & reperfusion after $60 \mathrm{~min}$ ischemia & \\
\hline \multirow{8}{*}{$\begin{array}{l}\text { Blood plasma } \\
\text { (human) }\end{array}$} & $0.074 \pm 0.028 \mu \mathrm{M}$ & 194 healthy woman and men & \multirow{7}{*}[273]{} \\
\hline & $0.069 \pm 0.015 \mu \mathrm{M}$ & 18 to 29 years aged & \\
\hline & $0.070 \pm 0.014 \mu \mathrm{M}$ & 30 to 39 years aged & \\
\hline & $0.072 \pm 0.020 \mu \mathrm{M}$ & 40 to 49 years aged & \\
\hline & $0.083 \pm 0.020 \mu \mathrm{M}$ & 50 to 59 years aged & \\
\hline & $0.096 \pm 0.022 \mu \mathrm{M}$ & 60 to 69 years aged & \\
\hline & $0.107 \pm 0.027 \mu \mathrm{M}$ & 70 to 84 years aged & \\
\hline & $106.3 \pm 65.8 \mathrm{ng} / \mathrm{mL}$ & $O$-pentafluorobenzyl oxime & [274] \\
\hline \multirow{5}{*}{$\begin{array}{l}\text { Umbilical cord } \\
\text { plasma } \\
\text { (human) }\end{array}$} & $0.3 \mu \mathrm{M}$ & Full-term healthy neonates & \multirow{5}{*}[275]{} \\
\hline & $0.5 \mu \mathrm{M}$ & Term neonates with acidosis & \\
\hline & $0.6 \mu \mathrm{M}$ & Term neonates with asphyxia & \\
\hline & $0.1 \mu \mathrm{M}$ & Preterm neonates (healthy) & \\
\hline & $0.4 \mu \mathrm{M}$ & Preterm neonates (asphyxia) & \\
\hline
\end{tabular}

* The approximate intracellular HNE concentration is given in parentheses.

In general, basic HNE concentrations of blood serum (for adults and children except neonates) were found to be in the range of 0.05 and $0.15 \mu \mathrm{M}$ [273]. These values were measured by means of the DTNB method and were in very good agreement with the plasma HNE concentration of $106.3 \pm 65.8 \mathrm{ng} / \mathrm{mL}$ measured by means of another assay, the $O$-pentafluorobenzyl oxime method [274]. In about 200 healthy humans of ages ranging from 18 to 84 years plasma HNE was measured together with various oxidative stress parameters, giving a mean value of $0.074 \mu \mathrm{M}$ [273]. Accelerated oxidation during ageing was demonstrated by increases of HNE, MDA, GSSG and by the slight decrease of erythrocytic GSH with age. In the group aged up to 30 years the mean HNE was $68.9 \pm 15.0$, whereas in the group aged more than 70 years the corresponding value was $107.4 \pm 27.3 \mathrm{nmol} / \mathrm{L}$ [273]. HNE levels were measured in umbilical arterial cord blood samples from healthy, acidotic, and asphyctic neonates with a gestational age ranging from 26 to 41 weeks [275]. HNE in umbilical cord plasma of full-term healthy neonates amounted to about $0.3 \mu \mathrm{M}$. After perinatal complications, HNE increased significantly in the group of term newborns with acidosis 
to about $0.5 \mu \mathrm{M}$ and in term neonates suffering from asphyxia to about $0.6 \mu \mathrm{M}$ ( $p<0.001$ in comparison with the control group of healthy term newborns). In healthy preterm neonates with a gestational age from 34 to 36 weeks the mean HNE level was $0.1 \mu \mathrm{M}$. HNE increased up to $0.4 \mu \mathrm{M}$ in preterm newborns after perinatal asphyxia $(p<0.001)$ [275].

The presence of protein-bound HNE in vivo has been assessed in various human tissues, including the human aorta with atherosclerotic lesions [277], nigral neurons in Parkinson disease [278], renal cell carcinomas [279], amyloid deposits in systemic amyloidosis [280], in brain from subjects with mild cognitive impairment [281], and trophoblast cells of preeclamptic placenta [282]. Furthermore, the demonstration of HNE protein adducts in human lung cells after ozone exposure are consistent with a potential role for HNE in the toxic effects of ozone in these cells [283].

Moreover, endogenous cyclic DNA adducts derived from HNE were associated with neurodegenerative diseases such as Alzheimer's disease. A sensitive and selective capillary liquid chromatography nanoelectrospray isotope dilution mass spectrometric method was developed to identify and quantify endogenous cyclic DNA adducts derived from HNE with 2'-deoxyguanosine (HNE-dG) in human brain tissues [284].

\subsection{Influence of Nutrition}

Parallel to endogenous formation of HNE it can also be consumed with the diet. To assess human exposure to 4-hydroxy-2-alkenals in the diet, these lipid peroxidation products were monitored in vegetable oils, fish and shellfish [285] and the Korean daily exposure to 4-hydroxy-2alkenals, excluding consumption from fried food, was calculated to be $4.3 \mu \mathrm{g} / \mathrm{day}$ ( $2.7 \mu \mathrm{g} \mathrm{HNE}$ and 1.6 $\mu \mathrm{g}$ HHE). Additionally, 4-hydroxy-2-alkenal intake from fried foods was calculated and amounted to more than $11.8 \mu \mathrm{g} /$ day. Thus the combined exposure would be therefore, $16.1 \mu \mathrm{g} /$ day corresponding to $0.3 \mu \mathrm{g} / \mathrm{kg}$ body weight/day for a $60 \mathrm{~kg}$ Korean adult [285]. It was also found that nutrition and various food constituents may have influence not only on HNE formation or intake, but also on HNE metabolism. Of course, this involves the sulfhydryl content of the food such as S-allyl-Lcysteine of garlic [286], but also other components. Dietary fibers are fermented by the gut flora to yield short chain fatty acids (SCFAs), which inhibit the growth of tumor cells, induce glutathione S-transferases, and protect cells from the genotoxic activity of HNE [287].

Beneficial effects of the probiotic VSL\#3 (a medical food that delivers a high concentration of beneficial live bacteria) on parameters of liver dysfunction in chronic liver diseases were evaluated by Loguercio et al., 2005 [288]. These effects were studied in non-alcoholic fatty liver disease, alcoholic liver cirrhosis, HCV-positive patients with chronic hepatitis without or with liver cirrhosis. Treatment with VSL\#3 improved levels of HNE and MDA except in HCV-positive patients. Furthermore, routine liver damage tests and plasma S-NO levels were improved in all groups of patients. It was concluded that manipulation of the intestinal flora should be taken into consideration as possible adjunctive therapy in some types of chronic liver disease [288].

It was further shown that mean levels of HNE and MDA in rat brain tissues were decreased in rats fed soy-protein as a dietary antioxidant [289]. 
Data on the effect of green tea on LPO products formation and parameters of the antioxidative system of liver, blood serum and central nervous tissue of healthy young rats drinking green tea for five weeks were collected by Skrzydlewska et al., 2002 [290]. The bioactive ingredients of green tea extract caused an increase in the activity of glutathione peroxidase and glutathione reductase and in the content of reduced glutathione as well as a marked decrease in lipid hydroperoxides, HNE and MDA in the liver while minor changes of the measured parameters were observed in the blood serum, but LPO products, particularly MDA, were significantly reduced. In the central nervous tissue the activity of superoxide dismutase and glutathione was found to be peroxidase while the activity of glutathione reductase and catalase were increased, and the level of lipid hydroperoxides, HNE and MDA significantly was found to be decreased after drinking green tea

Beside of food uptake the uptake of environmental toxins may increase HNE concentrations in human tissues. Among the several converging factors leading to Parkinson disease, epidemiological studies indicate a correlation between Parkinson disease with living in a rural area and/or exposure to agricultural pesticides. In this context Leiphon and Picklo, 2007 demonstrated that mitochondrial aldehyde dehydrogenases are sensitive targets of pesticide inactivation and that pesticides such as maneb and benomyl can decrease the detoxification of HNE to HNA and therefore contribute to an increase of HNE in rat brain mitochondria [291].

\section{Metabolism of HNE}

HNE is rapidly metabolized in cells. The velocity and the pattern of HNE metabolism were studied in various cell types, in subcellular organelles and in whole organisms. In all cases the overall metabolic rate of exogenously added HNE was so high, that already within a few minutes equilibrium concentrations in the nanomolar range were obtained (Figure 6). In Table 2 the maximal velocity of HNE total degradation in different biological systems is compared. From these observations it is obvious, that HNE - even at very high lipid peroxidation rates - hardly can accumulate to high levels in biological systems. The highest level ever measured to our knowledge was $6.5 \mu \mathrm{M}$ (besides the $50 \mu \mathrm{M}$ in monocytes fed with hemozoin). This value of $6.5 \mu \mathrm{M}$ was recorded in experiments with rat intestine during the postischemic reperfusion period [266].

In all experimental models investigated a variety of HNE metabolites was identified and quantified. However, one may differentiate these metabolites into primary and secondary intermediates. The primary metabolites are undergoing further metabolic conversion leading to secondary intermediates. Some of the secondary intermediates are stable end products. Possibly one may use such stable HNE products - such as mercapturic acids excreted in urine-as biomarkers for oxidative stress, specifically for LPO, also in medical diagnostics. Additionally, the HNE metabolism is an important component of the antioxidative defense system of cells because of the cytotoxic, mutagenic, and even carcinogenic effects of aldehydic products of LPO, namely of HNE. 


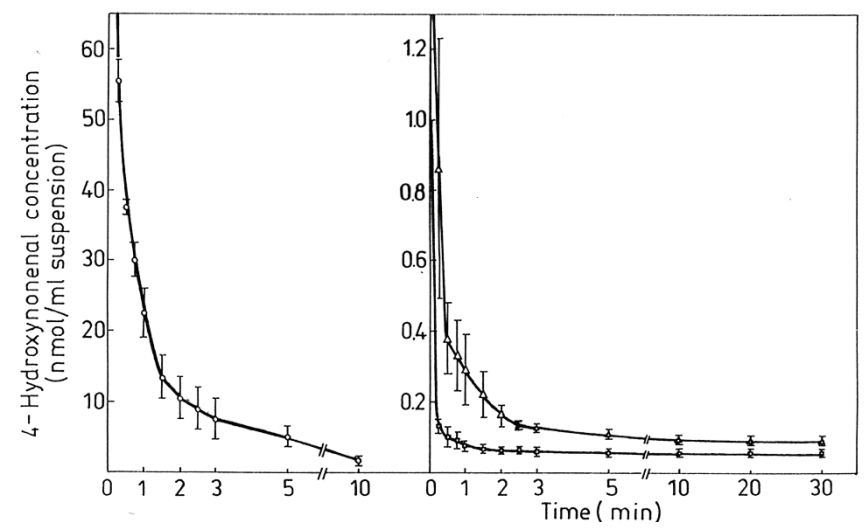

Figure 6. Degradation/metabolism of 4-HNE in rat hepatocytes. The method was the so-called direct measurement of HNE by HPLC. The remaining HNE shown in the graphs is the total of not metabolized HNE within the suspension, i.e., intracellular plus extracellular ones at the indicated time-points. The differenciation between the intracellular and extracellular compartments of HNE is not necesssary for balancing the HNE degradation/metabolism. The experimental conditions were: $37^{\circ} \mathrm{C}, \mathrm{pH} 7.4,1 \times$ $10^{6}$ hepatocytes/mL suspension; HNE was added at $100 \mu \mathrm{M}$ (o), $5 \mu \mathrm{M}(\Delta)$ or $1 \mu \mathrm{M}($ (口). Values are the mean \pm S.D.

Table 2. Maximal velocity of total HNE degradation in cells, subcellular organelles, and perfused organs.

\begin{tabular}{ccc}
\hline Biological System & Maximal Rate of HNE Catabolism & Reference \\
\hline Kidney cortex mitochondria, rat & $112.2 \mathrm{nmol} / \mathrm{mg}$ protein $/ \mathrm{min}$ & {$[292]$} \\
Hepatocytes, rat & $28.4 \mathrm{nmol} / \mathrm{mg}$ w.w./min & {$[293]$} \\
Ascites tumor cells & $9 \mathrm{nmol} / \mathrm{mg} \mathrm{w} . w . / \mathrm{min}$ & {$[264]$} \\
Thymocytes, mouse & $27.7 \mathrm{nmol} / \mathrm{mg} \mathrm{w} . \mathrm{w} . / \mathrm{min}$ & {$[294]$} \\
Synovial fibroblasts, rabbit & $27.3 \mathrm{nmol} / 10^{6} \mathrm{cell} / \mathrm{min}$ & {$[295]$} \\
Perfused kidney, rat & $160-190 \mathrm{nmol} / \mathrm{g} \mathrm{w.w.} / \mathrm{min}$ & {$[296]$} \\
Perfused intestine, rat & $22 \mathrm{nmol} / \mathrm{g} \mathrm{w} . \mathrm{w} . / \mathrm{min}$ & {$[266]$} \\
Perfused heart, rat & $50 \mathrm{nmol} / \mathrm{g} \mathrm{w} . \mathrm{w} . / \mathrm{min}$ & {$[264]$} \\
\hline
\end{tabular}

The velocity of HNE metabolism was often measured by tracer kinetic methods. Mostly [2-3H]HNE was used [266,292,293,297,298]. Sometimes only the so-called "direct HNE measurement" (free or unbound HNE) by HPLC was used detecting the release of added HNE [263,292]. In other studies, namely those investigating specific parts of HNE catabolism, glutathione consumption after adding different HNE amounts was analysed [299]. HNE added to hepatocyte suspensions $\left(10^{6}\right.$ cells $\left./ \mathrm{mL}\right)$ in concentrations of 1,5 , or even $100 \mu \mathrm{M}$ was almost completely metabolized within three minutes [300-303] (Figure 6).

Originally it was assumed that HNE metabolism is especially fast in hepatocytes [300,304-306] because the first studies on cellular HNE metabolism demonstrated that compared with liver, all other tissues possess only a low capacity to metabolize $\mathrm{HNE}$, ranging from $0 \%$ (fat pads) to $10 \%$ 
(kidney) of the activity present in liver [300]. However, later on also in other cells types a rapid HNE metabolism was found, in some cell types such as thymocytes even in a similar range as in hepatocytes. Furthermore, the first findings on the metabolism of HNE by isolated hepatocytes and by liver homogenate cytosolic fractions suggested that hepatocytes and rat liver cytosol, respectively convert HNE enzymatically mainly to the corresponding alcohol, dihydroxynonene (DHN; non-2ene-1,4-diol) via alcohol dehydrogenase. It was therefore postulated that the rapid conversion of the cytotoxic HNE and other reactive aldehydes to alcohols, which are probably less toxic, could play a role in the defense system of the liver against toxic substances arising from free radical-induced LPO [300]. This view was expanded later after the identification of a multitude of HNE products with quantitative majorities of the GSH-adduct(s) and/or hydroxynonenoic acid (HNA) and its further degradation products rather than a majority of the alcohol DHN [293].

\subsection{HNE Metabolism in Mammalian Cells and Organs}

HNE metabolism was studied in hepatocytes [293,300,302,306], hepatoma cells [307-310], ascites tumor cells [264], mucosal cells [297], synovial fibroblasts [295], thymocytes [294], vascular smooth muscle cells [311] and also in organs such as heart [264,312] and kidney [296,298]. The rapid HNE degradation found and the intracellular metabolism implicate that HNE can rapidly enter cells. The velocity of HNE entry into the cells was measured by tracer kinetics in various cell types [295]. In ascites tumor cells, the total rate of HNE metabolism by cells in the proliferating phase (early phase) and cells in the stationary phase (late phase or resting phase of tumor growth) was almost the same [264]. The formation of glutathione conjugates following the addition of HNE was higher in early phase cells when compared with cells in the late phase of tumor growth. This observation was in accordance with the increased consumption of the reduced form of glutathione (GSH). The glutathione transferase activity in tumor cells of both proliferation stages was equal, but the substrate GSH had a higher level in cells of the proliferating phase than in the cells of the resting phase. The result was a higher glutathione-adduct formation in cells of the proliferating phase. The reductive pathway of HNE metabolism was high in proliferating phase tumor cells. In contrast, the oxidative pathway was overwhelming in resting phase tumor cells. This fact corresponded with the different energetic and redox state of both phases. The cells of the resting phase showed a higher rate of uncoupling than cells during the proliferating phase, in accordance with a lower NADH/NAD ${ }^{+}$and lactate/pyruvate ratio of resting cells. The consequence of the high demand of reduced NADH by partially uncoupled mitochondria for ATP production is a lower capacity of resting cells to use NADH for HNE reduction (DHN or GSH-DHN formation). In support, addition of NADH to the homogenates of rat hepatoma cells (MH1C1) resulted in a 1.5-fold increase of aldehyde consumption [309].

In hepatoma cells, such as $\mathrm{MH1C1}$ and $\mathrm{HTC}$, the metabolic pathway for HNE removal is markedly different from hepatocytes. The results of several investigations indicate that the liver biochemical pathways involved in HNE metabolism undergo a complex variety of changes during neoplastic transformation, so that hepatoma cells with different degrees of deviation show different metabolic patterns with respect to HNE. The analysis of aldehyde enzymatic activities for example showed that MH1C1 cells have decreased levels of all enzymes involved in the catabolism of HNE 
except for NAD and NADP aldehyde dehydrogenase in the cytosol, compared to hepatocytes and to HTC cells. HNE can, however, be metabolized through a sufficiently high activity of glutathione S-transferase. NAD and NADP dependent aldehyde dehydrogenases are of great importance in HTC cells for HNE metabolism. In HTC cells the metabolic efficiency is due mainly to oxidative enzymes [310].

Comparing Chinese hamster fibroblast control cells (HA-1) with Chinese hamster fibroblast $\mathrm{H}_{2} \mathrm{O}_{2}$-resistant cell lines (OC5 and OC14) the $\mathrm{H}_{2} \mathrm{O}_{2}$-resistant cells were found to be significantly more resistant than HA-1 cells to the cytotoxicity of HNE, as determined by clonogenic cell survival. This finding was related to a significant 2-3-fold increase in the HNE degradation rate of OC5 and OC14 cells, i.e., the HNE removal from culture media containing $72 \mu \mathrm{M}$ HNE when compared with HA-1 cells [313]. Thus, the resistance appeared to be related to increased cellular HNE metabolism.

\subsection{HNE Metabolism in Subcellular Organelles}

HNE metabolism was studied in several mitochondrial suspensions. By analyzing the decrease of the HNE concentration in the first $30 \mathrm{~s}$ after treatment with $100 \mu \mathrm{M} \mathrm{HNE}$, Ullrich et al., 1994 calculated the maximal HNE detoxification rate in rat kidney cortex mitochondria to be $112.2 \mathrm{nmol} / \mathrm{mg}$ protein/min [292]. After addition of $1 \mu \mathrm{M}$ HNE the degradation of HNE approached a steady state after 2 min. The steady state for the remaining HNE level has been adjusted to about $0.1 \mu \mathrm{M}$, a range which is also known as the steady state value in human blood plasma.

HNE is metabolized by renal mitochondria to 4-hydroxynonenoic acid (HNA), 1,4dihydroxynonene (DHN), and the GSH-HNE-adduct (GSH-HNE). GSH-HNE and HNA reached the maximum value about $2 \mathrm{~min}$ after $\mathrm{HNE}$ addition to mitochondria, and thereafter these metabolites slowly decreased. Almost $10 \%$ of the radioactivity was bound to proteins. The protein bound radioactive HNE was found predominantly bound to the proteins of the intermembrane space. Lower amounts were bound to the proteins of the inner membrane and to the proteins of the matrix. The nmol HNE value per mg protein was found to be very high in the intermembrane space and almost similar for outer and inner membrane and matrix proteins [292]. In rat brain mitochondria it was found, that in situ mitochondrial HNE detoxification is affected by decrements in $\mathrm{NAD}^{+}$availability and complex I activity [314]. In investigations of HNE metabolism during diethyl-nitrosamine-induced carcinogenesis in rat liver it was established that the NAD- and NADP-dependent aldehyde dehydrogenases of the cytosolic fraction and the NADP-dependent aldehyde dehydrogenase of the microsomes show higher values in nodules and hepatoma than in normal liver [315]. Both aldehyde dehydrogenase and glutathione S-transferase activities of rat liver mitochondria are reduced in aged animals. Mitochondrial HNE oxidation by aldehyde dehydrogenase and glutathione conjugation of HNE decline at 18 and 24 months, respectively, while these enzyme activities were found to be well-preserved in dietary restriction animals throughout their life span. These findings indicate that the prevention of the age-associated decrease in HNE detoxification by dietary restriction may be an important mechanism underlying enhanced 
aldehyde elimination, thus minimizing the functional deterioration observed in mitochondria of old animals [316].

After exposure to racemic HNE, rat brain mitochondria metabolized HNE enantioselectively with a higher rate of (R)-HNE metabolism [317]. By using purified enantiomers of HNE the authors were able to demonstrate that this enantioselective metabolism of HNE was the result of higher rates of enzymatic oxidation of (R)-HNE by aldehyde dehydrogenases compared to (S)-HNE. Conjugation of HNE to glutathione was a minor metabolic pathway and was not enantioselective. These studies demonstrate that the chirality of HNE affects its mitochondrial metabolism.

\subsection{HNE Metabolism in Whole Animals and Interorgan Relationships}

The aim of many in vivo metabolic studies was the characterisation of end-products of HNE, especially in urine, in order to develop specific and non-invasive biomarkers of lipid peroxidation. When HNE is administered intravenously, it is mainly excreted into urine and bile as conjugated metabolites, in a proportion that is dependent on the administration route [118,318]. However, biliary metabolites undergo an enterohepatic cycle that limits the final excretion of faecal metabolites. Only a very low amount of metabolites is found to be bound to macromolecules.

According to Alary et al., [118,318] the main urinary metabolites are represented by two groups of compounds. The first group derives from (a) the mercapturic acid formation from 1,4 dihydroxynonene-glutathione (DHN-GSH); (b) the lactone of 4-hydroxynonanoic-GSH (HNA-lactone-GSH) and (c) HNA-GSH. The second group of metabolites is derived from the $\omega$-hydroxylation of HNA or HNA-lactone by cytochromes P450 4A, followed eventually, in the case of $\omega$-oxidized-HNA-lactone, by conjugation with GSH and subsequent mercapturic acid formation. Biliary metabolites are GSH or mercapturic acid conjugates of DHN, HNE and HNA [118,318]. Stereochemical aspects of HNE metabolism are also discussed by Alary et al. [118,318]. Experiments with injection of tritium-labeled HNE [319] and also of tritium-labeled 4-hydroxyhexenal (HHE) [320] into the vein of rats have shown that part of the radioactivity was excreted in the urine as mercapturic acid conjugates. From these experiments and studies with perfused kidneys [296] it may be concluded that the excretion of mercapturic acid conjugates as well as the excretion of DHN is the main route of HNE-product disposal from the organism. The most important studies on urinary excretion of HNE metabolites were carried out by the group of Alary, Cravedy, and Gueraud. Following intravenous administration of $\left[{ }^{3} \mathrm{H}\right] \mathrm{HNE}$ into rats, the majority of the dose appeared in urine $(67.1 \%$ after $48 \mathrm{~h}$ ) [319]. The radio-HPLC metabolic profile showed that no unchanged parent compound was detected in urine whereas at least four metabolites were present, most of them corresponding to mercapturic acid conjugates.

Two major pathways were involved in the biotransformation of HNE in vivo: the reduction/oxidation of the aldehyde group, and the conjugation to endogenous glutathione leading to mercapturic acid conjugates in urine. These end products were isolated by HPLC and identified by mass spectrometry as HNE mercapturic acid, 1,4-dihydroxynonene mercapturic acid, 4-hydroxynonenoic mercapturic acid, and the corresponding lactone. 


\subsection{Primary HNE Intermediates—Enzymatic Reactions and Quantitative Results}

Already in 1991 in the classical HNE-review of Esterbauer et al. [304] it was mentioned: the main enzymes involved in HNE metabolism are glutathione transferases, aldehyde dehydrogenases, and alcohol dehydrogenases. These are the enzymes forming the so-called primary HNE metabolites. The primary metabolites of HNE are therefore, the HNE-GSH adduct, the corresponding carboxylic acid of HNE, i.e., HNA, and the corresponding alcohol, i.e., DHN. Figures 7-9 provides examples for the analysis of HNE and HNA by HPLC (Figure 7), 1,4-DHN by HPLC plus MS with fluorimetric detection (Figure 8), and formation of HNA, HNE-GSH and DHN in hepatocytes (Figure 9). As displayed in Figure 9, after addition of a single dose of HNE to a hepatocyte suspension all three primary HNE metabolites increased very rapidly within the first minute of the experiment and reached a maximum value — in case of $100 \mu \mathrm{M} \mathrm{HNE}$ - after about 2 min, and thereafter slowly decreased. At all time points the GSH-HNE conjugate was the main product of hepatocytes followed by HNA. The concentration of DHN was considerably lower. The relative proportion of the three metabolites in hepatocytes was approximately GSH-HNE:HNA:DHN = 6:4:1, a pattern which may be quite different in other cell types. The formation of the GSH-HNE conjugate always leads to a decrease of intracellular GSH concentration. The sum of the three primary metabolites, GSH-HNE + HNA + DHN, in hepatocytes after 3 min of incubation ranged between $60 \%$ and $65 \%$ of the added HNE.

Taking into account the small fraction of the non-metabolized HNE, it follows that about $33 \%$ to $36 \%$ of the HNE was converted into other metabolites. This fraction involves secondary metabolites and HNE-modified proteins and peptides. In hepatocytes, this fraction was below $10 \%$ within the very first initial phase of about $30 \mathrm{~s}$ of incubation after addition of exogenous HNE. With increasing duration of the incubation this fraction steadily increased - while the sum of GSHHNE, HNA, and DHN decreased again - and reached a value of nearly $50 \%$ after $30 \mathrm{~min}$. These findings strongly suggest that the primary metabolites GSH-HNE conjugate, HNA, and DHN are further metabolized to secondary products [293].

As mentioned, the metabolism of HNE involves multiple pathways, including conjugation with glutathione catalyzed by glutathione S-transferases (GST) [321,322], oxidation of the aldehyde functional group to form HNA catalyzed by aldehyde dehydrogenases [323,324], and reduction by alcohol dehydrogenase to 1,4-dihydroxynonene [300]. The HNE-metabolizing aldehyde dehydrogenase isoenzymes are present in the hepatic cytosol, mitochondria, and probably also in microsomes [303,325]. The HNE-metabolizing NADH-dependent alcohol dehydrogenase is localized mainly in the hepatic cytosol [303]. The glutathione transferases are ubiquitous enzymes, being particularly rich in hepatocytes and probably other liver cells. Even though a number of rat GST isozymes show catalytic activity toward HNE, rat liver glutathione transferase $8-8$ has been demonstrated to be most effective in catalyzing the conjugation of this compound to GSH, and it has been suggested that this isozyme may be involved in the detoxification of HNE and related endogenous electrophiles [303,304,321,322].

A human acidic glutathione-S-transferase, hGST 5.8, was isolated from liver, heart, bladder, pancreas, and brain [326], and it was shown that this enzyme has an about 20-fold higher specific 
activity for HNE than for 1-chloro-2,4-dinitrobenzene, and expressed glutathione peroxidase activity toward phospholipid hydroperoxides. The hGST 5.8 was absent in lung, kidney, skeletal muscle, colon and erythrocytes [326]. Berhane et al., 1994 characterized the detoxification of base propenals (degradation products of DNA) and other unsaturated aldehyde products of free radical reactions and lipid peroxidation by human glutathione transferases [327]. They found that in general, GST A1-1 and GST M1-1, in contrast to GST P1-1, were more active with 4hydroxyalkenals than with base propenals. HNE readily reacts with GSH also in a nonenzymatic reaction. The GSH transferase-catalyzed reaction can, however, proceed about 300 to 600 times faster [304].

GST can be induced by HNE. To investigate the effect of LPO products on the expression of GSTs Fukuda et al. [328] exposed normal rat liver epithelial cells (RL34) to aldehydic compounds and found out that GST activity was induced by $\alpha, \beta$-unsaturated aldehydes, such as acrolein (1.3-fold), crotonaldehyde (1.4-fold), 4-hydroxyhexenal (HHE) (1.4-fold), and HNE 1.7-fold. The induction of GST activity by HNE was time-dependent, reaching a plateau after $16 \mathrm{~h}$. The authors concluded that the induction of GSTs by HNE may represent an important cellular defense mechanism against oxidative injury [328]. Immunoblot analysis applying polyclonal antibodies against GST isozymes demonstrated that GST-P, a well-known tumor marker, was significantly induced $16 \mathrm{~h}$ after HNE treatment [328]. In early human fibrous atherosclerotic plaques, immunohistochemical studies demonstrated marked induction of hGST A4-4 in endothelial cells overlying plaque, and in proliferating plaque vascular smooth muscle cells [329]. The authors conclude that endothelial cell mGST A4-4 can play a key role in protecting blood vessels against oxidative stress and, thus, is likely to be a critical defense mechanism against oxidants that act as atherogens. In rat aortic smooth muscle A10 cells the induction of GSH and glutathione S-transferase by 3H-1,2-dithiole-3thione (D3T, a chemoprotective chemical known to induce detoxification enzymes), and protective effects of D3T, i.e., elevated cellular defenses against HNE-mediated toxicity were characterized [330]. As results the authors report that incubation of A10 cells with D3T resulted in a marked concentration-dependent induction of both GSH and GST, that this induction exhibited a time-dependent response, and that pretreatment of A10 cells with D3T led to a dramatic decrease of HNE-induced cytotoxicity. 


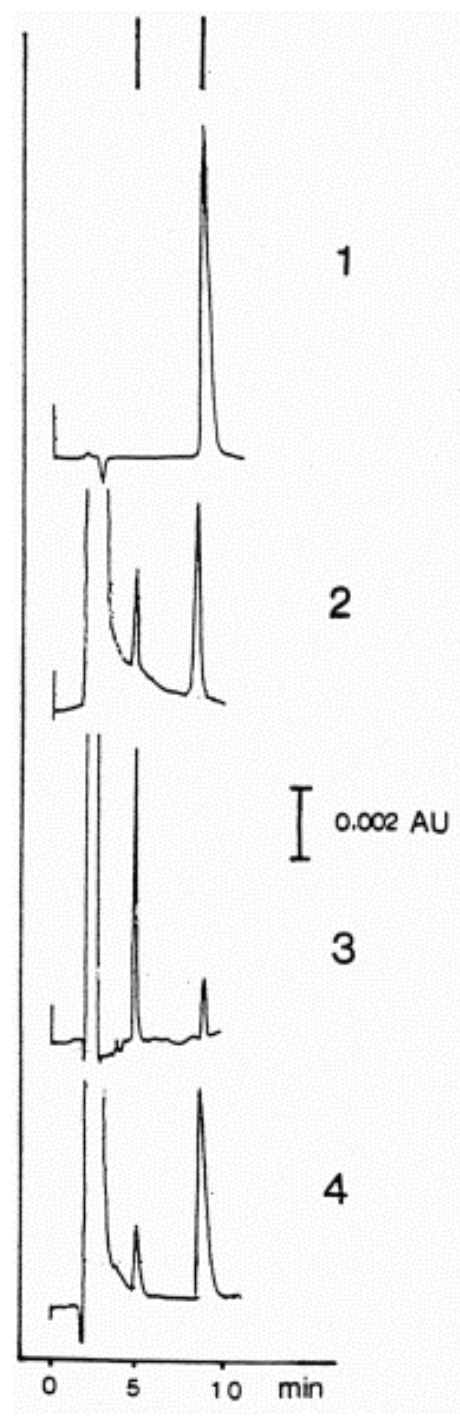

Figure 7. Identification of HNE and 4-hydroxynonenoic acid (HNA) by isocratic HPLC separation. Conditions: the eluent was $42 \%$ acetonitrile/water v/v $1 \mathrm{~mL} / \mathrm{min}$; columns were Spherisorb S5 ODS2 $250 \times 4.5 \mathrm{~mm}$; detection of both substances at $223 \mathrm{~nm}$; authentic standard of 4-HNA was produced in the system of HNE, dehydrogenase $\left(\mathrm{NAD}^{+}\right)$and aldehyde dehydrogenase; (1) HNE standard solution $30 \mu \mathrm{M}$; (2) product of oxidation of HNE: HNA $15 \mu \mathrm{M}$; (3) mixture from cellular incubation mit $\mathrm{CH}_{2} \mathrm{Cl}_{2}$ extract of $\mathrm{HNE}$ oxidation; (4) mixture formed in rat liver cells $\left(10^{6}\right.$ cells $\left./ \mathrm{mL}\right)$ after addition of $100 \mu \mathrm{M}$ HNE, the first peak representing HNA, the second peak representing HNE; for exact quantification of HNA the comparison of the HNA-peak with NADP formation in experiments on transition of HNE by means of aldehyde dehydrogenase was carried out. 


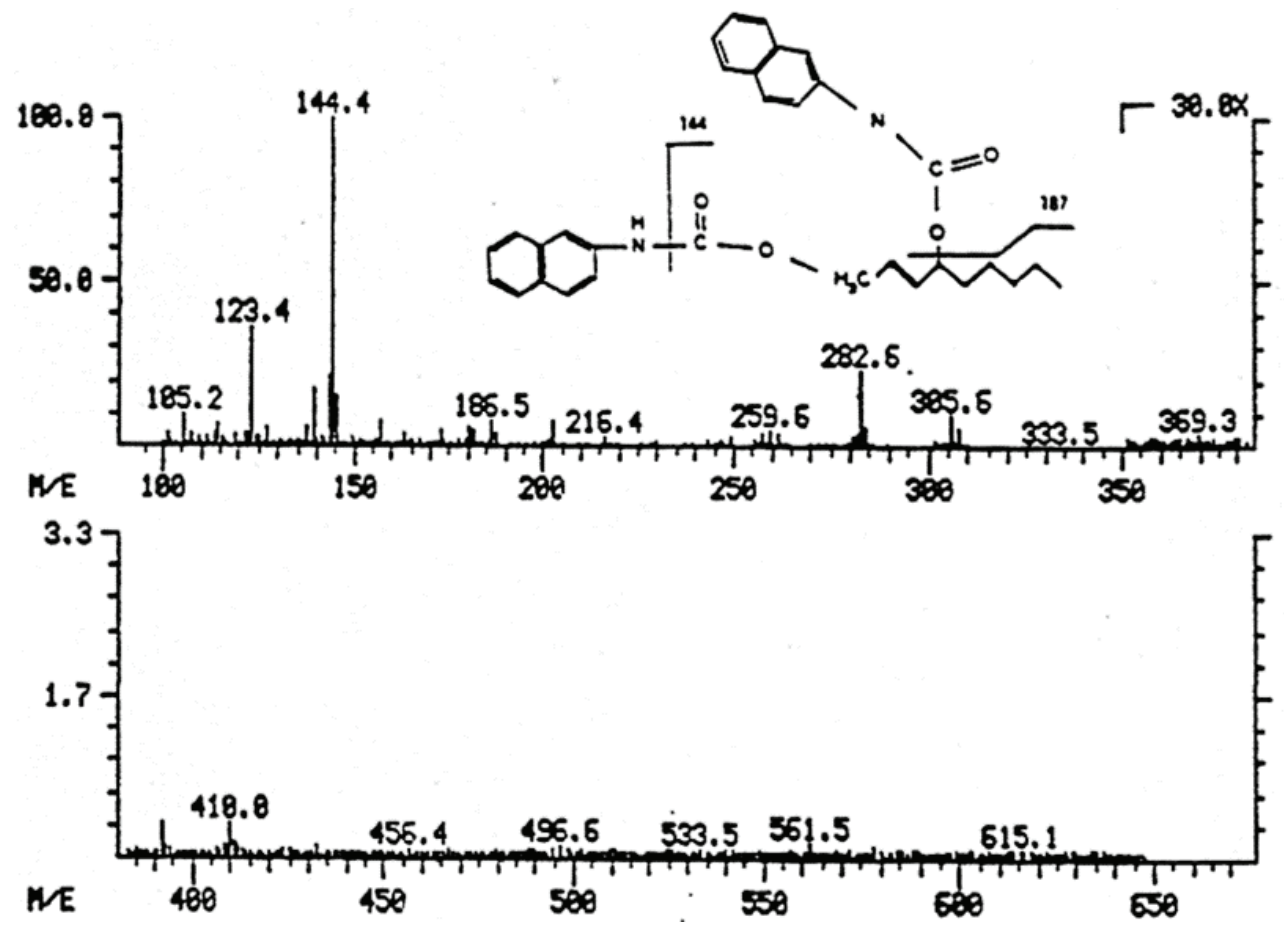

Figure 8. Mass spectrum of dihydroxynonene urethane (HPLC plus MS) with fluorimetric detection. The preparation of the reduced HNE product 1,4-DHN was carried out in the following manner: reduction of HNE with sodium boronate; extraction with dichloromethane; drying by means of sodium sulphate; purification by TLC separation, detection with $10 \%$ phosphomolybdenic acid dissolved in ethanol, extraction from TLC plates with $\mathrm{CH}_{2} \mathrm{Cl}_{2}$. Identification of 1,4-DHN: Siliylation; GC $\left(85{ }^{\circ} \mathrm{C}\right.$, with $10 \%$ min up to $150{ }^{\circ} \mathrm{C}$, $50 \mathrm{~m} \mathrm{SE30} \mathrm{column-i.d.}$ $0.32 \mathrm{~mm}$, FID); formation of urethane derivative (1,4-dinaphthyl-2,3nonenylurethane); $(305,350 \mathrm{~nm})$. Preparation of radioactively labelled 1,4-DHN: Reduction of radioactively labelled HNE with sodium boronate; extraction with dichloromethane; purification by means of TLC and elution on Bond Elut C18 columns (35\% methanol/water, v/v). Chemical ionization $\left(\mathrm{CH}_{4}\right)$. Peaks: m/e $144 \mathrm{Ar}_{-\mathrm{NH}_{3}}{ }^{+}, \mathrm{m} / \mathrm{e} 187$ Ar-NH-COOH, m/e $123(\mathrm{M}+1)-(2 \times 187) ;(\mathrm{M}+1)=497 \mathrm{~m} / \mathrm{e}[301]$. 


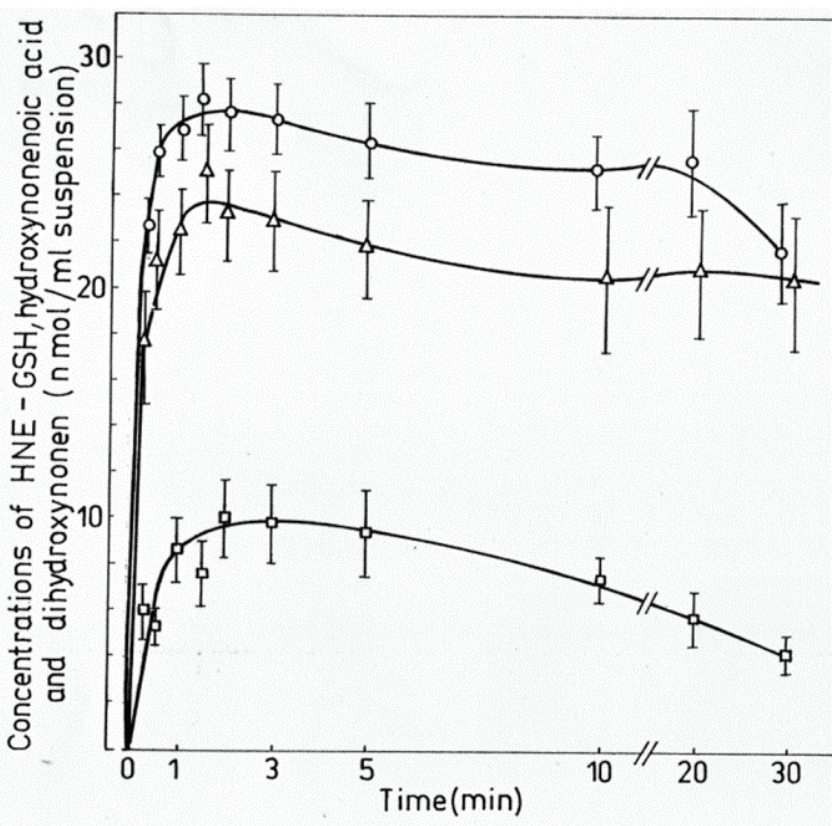

Figure 9. HNE metabolites. Formation of the so-called primary HNE metabolites HNA $(\triangle)$, HNE-GSH (o) and DHN ( $\square$ ) in rat hepatocyte suspensions after loading the cell suspensions with $100 \mu \mathrm{M}$ tritium-labelled HNE. The glutathione (GSH) adduct of HNE was quantified as follows: Measurement as isoindol derivative following the reaction of the adduct with o-phthaldialdehyde in the presence of mercaptoethanol; HPLC separation of the isoindol derivative by isocratic HPLC with $65 \%$ methanol/0.1 M sodium acetate buffer $\mathrm{pH} 6.5(\mathrm{v} / \mathrm{v})$ in the presence of an ion-pair reagent (tetraethyl-ammoniumhydroxide/TAA); fluorimetric detection at 343 and $445 \mathrm{~nm}$; columns: ODS $5 \mu \mathrm{m}, 250 \mathrm{~mm} \times 4.6 \mathrm{~mm}$ i.d.; purification of the extracts before HPLC separation on bond Elut C18 columns (methanol, water, sodium acetate buffer, hexane, methanol elutions) [293].

A redox activation of aldose reductase (AR) in the ischemic heart was shown by Kaiserova et al., 2006 [331]. Their data indicate that reactive oxygen species formed in the ischemic heart activate AR by modifying its cysteine residues to sulfenic acids. To investigate the role of AR in the late phase of ischemic preconditioning, Shinmura et al., 2002 treated conscious rabbits with six cycles of 4-min coronary occlusion/4-min reperfusion [332]. $24 \mathrm{~h}$ later they observed s a marked increase in AR protein and activity and in the myocardial content of sorbitol, a unique product of AR catalysis. AR was demonstrated to be an essential mediator of late preconditioning. Furthermore, the data suggest that myocardial ischemia/reperfusion injury is due in part to the generation of LPO products and that late preconditioning diminishes this source of injury by upregulating aldose reductase [332]. 
Aldose reductase (AR), an NADPH-dependent aldo-keto reductase, which catalyzes the reduction of glucose to sorbitol as part of the polyol pathway, is able to catalyze the reduction of a very broad range of aldehydes. HNE is an excellent substrate $(\mathrm{Km}=22 \mu \mathrm{M}$, $\mathrm{Kcat} / \mathrm{Km}=4.6 \times 10^{6} \mathrm{M}^{-1} \cdot \mathrm{min}^{-1}$ ) [332]. Therefore, it was concluded that the extrahepatic reductive metabolism of HNE involves AR [332]. ARs, present in human kidney and other tissues play an important role in the reductive metabolism of endogenous aldehydes such as HNE [333]. The kidney contributes via this pathway to a rapid urinary excretion of HNE products from the organism. It is worth to mention that AR is the only major HNE oxidoreductase of the human heart. AR has been purified from bovine retina, too. It has an apparent molecular weight of 32 kilodaltons and shares immunological and kinetic properties with the much studied aldose reductases purified from various sources. Retinal aldose reductase displays a $\mathrm{Km}$ of approximately $40 \mu \mathrm{M}$ for $\mathrm{HNE}$ and 4-hydroxyhexenal. Thus, AR was suggested to constitute a major detoxification route for these toxic aldehydes also in the retina [334]. Inhibition of aldose reductase by fidarestat suppresses several oxidative stress-induced inflammatory disorders. Fidarestat has already undergone phase III clinical trial for diabetic neuropathy and was found to be safe, though clinically not very effective [335]. It was also shown, that HNE and MDA induce the expression of the aldehyde reductase gene, which could be prevented by the carbonyl scavenger $N$-acetyl-L-cysteine (NAC) [336].

In other experiments effects of HNE addition on cellular glutathione levels were analyzed [299]. The authors studied the dose-dependent cytotoxicity of HNE after treatment of cultured fibroblasts at doses up to $50 \mathrm{nmol} / 10^{6}$ cells for $3 \mathrm{~h}$. In peroxide resistant OC14 and oxygen resistant O2R95 cells variants of HA1 cells, HNE treatment resulted in a statistically significant consumption of GSH at all doses tested. Cellular GSH levels in HA1 and O2R95 cells fell-starting from about 25 $\mathrm{nmol} \mathrm{GSH} / \mathrm{mg}$ cell protein — below $5 \mathrm{nmol} \mathrm{GSH} / \mathrm{mg}$ cell protein at HNE doses of 25 and $40 \mathrm{nmol} / 10^{6}$ cells, respectively. That argues for a very high rate of GSH-HNE adduct formation. In OC14 cells, GSH consumption - starting from about $35 \mathrm{nmol} / \mathrm{mg}$ protein — could not be detected until HNE doses exceeded $50 \mathrm{nmol} / 10^{6}$ cells and did not fall below $5 \mathrm{nmol} \mathrm{GSH} / \mathrm{mg}$ cell protein at any dose tested. In OC14 cells obviously the GSH-HNE adduct formation rate is very low [299]. This cannot be due to the capacity of glutathione-S-transferase which was even higher in OC14 cells compared to HA1 and O2R95 cells and stayed stable during the $3 \mathrm{~h}$ of HNE treatment in all three cell types, but rather to the enzymatic properties which obviously differ from one cell type to the other. These experiments also support the high stability of glutathione-S-transferase in these cultured fibroblast cell lines.

Table 3 displays the formation of primary HNE metabolites for different cells and tissues after the addition of $100 \mu \mathrm{M}$ HNE to the biological system. 
Table 3. Primary HNE metabolites in different cells and tissues after the addition of $100 \mu \mathrm{M}$ HNE to the biological system.

\begin{tabular}{cccccc}
\hline Biological System & DHN & $\begin{array}{c}\text { GSH- } \\
\text { HNE }\end{array}$ & HNA & HNE-P & Reference \\
\hline Kidney mitochondria (rat) & $9.3 \pm 1.8$ & $9.8 \pm 1.2$ & $5.9 \pm 0.7$ & $8.4 \pm 1.4$ & {$[292]$} \\
Hepatocytes (rat) & $8.1 \pm 2.1$ & $27.5 \pm 2.5$ & $25.3 \pm 5.6$ & $2.0 \pm 0.6$ & {$[293]$} \\
Enterocytes (rat) & $5.4 \pm 0.6$ & $11.0 \pm 0.5$ & $4.2 \pm 0.6$ & $1.3 \pm 0.2$ & {$[297]$} \\
Thymocytes (mouse) & 6.9 & 10.2 & 6.8 & 2.8 & {$[294]$} \\
Synovial fibroblasts (rabbit) & $0.3 \pm 0.1$ & $29.1 \pm 1.9$ & $1.0 \pm 0.3$ & $4.3 \pm 0.5$ & {$[295]$} \\
Kidney venous effluent (rat) & $24 \pm 5$ & $14 \pm 4$ & $5 \pm 1$ & n.d. & {$[296]$} \\
Ehrlich ascites cells, proliferating & 2.0 & 10.5 & 3.0 & 7.2 & {$[264]$} \\
Ehrlich ascites cells, resting & 1.5. & 8.0 & 3.8 & 1.5 & \\
\hline
\end{tabular}

HNE-P, HNE-protein; n.d., not determined. Given values represent the $\%$ of the initial HNE concentration (mean; mean \pm S.D.).

\subsection{Secondary HNE Intermediates—Enzymatic Reactions and Quantitative Results}

It is known from many studies that HNE can bind to thiol-groups of proteins. In general it is well established that HNE forms adducts with the following side chains in proteins: Cys, His and Lys. From tracer-kinetic experiments it was estimated that between $1 \%$ and $8 \%$ of exogenously added or endogenously formed HNE reacts with proteins to form HNE-protein conjugates. With that share of the totally added or formed HNE the proteins and peptides are the most important group of biomolecules targeted by HNE. In experiments studying the metabolism of exogenously added HNE always a dynamic pattern was seen: the binding of labelled HNE in the HNE-protein conjugates-pool reached a maximum - in hepatocytes $3 \mathrm{~min}$ after HNE addition - followed by a decline, underlining the fact that aldehyde-modified proteins underlie a removal of oxidatively damaged and modified proteins by the proteasomal system [337-339]. Nevertheless, the formation of HNE-protein and HNE-peptide compounds does not represent a metabolic pathway of HNE degradation. The chemical reactions leading to HNE modifications of proteins, peptides, and further biomolecules rather reflect this part of HNE "removal" which leads to damaging or signal effects by HNE. The real metabolic reactions of HNE lead to the primary and secondary HNE metabolites, which finally contribute to the detoxification and harmless removal of HNE.

Which are the real secondary metabolic products of HNE? The pool of secondary HNE intermediates contains degradation products of HNA, which is degraded via different products of $\beta$-oxidation to acetyl-CoA and, finally to carbon dioxide and water. This involves the reaction steps of the tricarboxylic acid cycle and of the respiratory chain. In experiments with ${ }^{3} \mathrm{H}$-labeled HNE the formation of ${ }^{3} \mathrm{H}$-labeled water could be shown in hepatocytes [293]. The rate of formation of radioactive water was slow, but the time curve showed not a transient maximum but a steady increase. In hepatocytes treated for $10 \mathrm{~min}$ with $100 \mu \mathrm{M}$ HNE, $14 \%$ of the radioactivity appeared in the water peak. The formation of $3 \mathrm{H}$-labeled water could be almost completely inhibited in presence of 4-pentenoic acid, an inhibitor of $\beta$-oxidation. In the presence of 4-pentenoic acid only traces of radioactive water were formed. 
Furthermore, in the presence of this inhibitor there was a significantly higher accumulation of 4-hydroxynonenoic acid (HNA) in comparison with control experiments without inhibitor. In the presence of 4-pentenoic acid HNA became the major HNE metabolite. During the first $60 \mathrm{~s}$ of incubation, the inhibitor had almost no effect on the rate of the conversion of HNE to HNA. Thereafter, however, the time profile was markedly different. In the presence of the inhibitor, HNA steadily increased, reaching $45 \mathrm{nmol} / \mathrm{mL}$ after $3 \mathrm{~min}$, whereas in the absence of the inhibitor the concentration of the carboxylic acid slowly decreased again. These experiments indicate that HNA is not an end product of HNE metabolism, but is further metabolized [293].

The oxidative pathway includes - when HNA is degraded - also tricarboxylic acid cycle products, such as aconitate, citrate, malate, fumarate, etc. In primary cultures of rabbit synovial fibroblasts $3 \mathrm{~min}$ after addition of $100 \mu \mathrm{M}$ labelled HNE the shares of radioactivity of tricarboxylic acid cycle intermediates and of water were $7.4 \%$ and $34.1 \%$, respectively [295]. The $\beta$-oxidation of HNA including the formation of tritiated water was also demonstrated in liver slices [340]. The identification of 4-hydroxy-9-carboxy-2-nonenoic acid and 4,9-dihydroxynonanoic acid demonstrated that $\omega$-oxidation significantly contributes to the biotransformation of $\mathrm{HNE}$ in liver slices [340]. Alary et al., 1998 demonstrated the existence of further secondary products generated from HNA. These are the $\omega$-oxidation product of HNA, namely 9-hydroxy-HNA, and the subsequent oxidation product of 9-OH-HNA, 9-carboxy-HNA [341]. Furthermore, 9-hydroxyDHN was identified as $\omega$-hydroxylated product of DHN [341].

The sequence of $\beta$ - and $\alpha$-oxidations in the pathway from HNA-CoA to the citric acid cycle was described in detail by Li et al., 2013 [342]. They showed that after the formation of HNA-CoA a $\beta$-oxidation takes place leading to the formation of 2-hydroxy-heptanoyl-CoA. This intermediate is converted by $\alpha$-oxidation to hexanoyl-CoA, and by three additional $\beta$-oxidations to acetyl-CoA. HNA-CoA can alternatively be phosphorylated to give 4-phospho-nonanoyl-CoA. The latter is hydrolyzed to 3-hydroxy nonanoyl-CoA, which is converted by three $\beta$-oxidations to propionyl-CoA, then to methylmalonyl-CoA and finally to succinyl-CoA as intermediate of the citric acid cycle [342].

Another group of secondary intermediates which was identified and quantified are the GSH-DHN and GSH-HNA conjugates as additional GSH-conjugates besides GSH-HNE [118]. Mercapturic acids were demonstrated to be the final products of this part of HNE metabolism (see also Chapter 6.2.). Different further secondary HNE products were postulated, such as 1-hydroxy-4-oxononene and others. In astrocytic biotransformation of HNE two novel metabolites of HNE were discovered, gamma-nonalactone and the potent pyrrole forming compound, 4-oxo-nonanal [343].

Compared to other organs, liver and kidney seem to be the most important organs for the elimination of the final products of metabolism. The importance of the kidney in the formation of HNE-mercapturic acid conjugates was demonstrated by Alary et al., 1995 [319] and Grune et al., 1997 [296]. HNE mercapturic acid, 1,4-dihydroxynonene mercapturic acid, 4-hydroxynonenoic mercapturic acid, and the corresponding lactone as HNE intermediates in urinary excretion were already mentioned above [319]. In studies with perfused kidneys it was shown, that all metabolites of HNE are also found in the urine. HNA, the HNE-mercapturic acid conjugates, and DHN had a 
higher concentration in urine than in the venous effluent of the kidney [296]. When $100 \mu \mathrm{M} \mathrm{HNE}$ was used for perfusion the highest HNE metabolite concentrations were found for DHN with $53 \mathrm{nmol} / \mathrm{mL}$ in urine and $24 \mathrm{nmol} / \mathrm{mL}$ in the venous effluent. The GSH-HNE conjugate and the products of the TCA-cycle never reached concentrations above $5 \mathrm{nmol} / \mathrm{mL}$ in urine. In the venous effluent their concentrations were about $15-17 \mathrm{nmol} / \mathrm{mL}$ which was $4-6$-fold higher than in urine. HNE-mercapturic acids seem to be stable products of HNE metabolism which are excreted from the organism. 1,4-dihydroxynonene mercapturic acid (DHN-MA), was shown by Alary et al., 1998 to be the major urinary metabolite of HNE administered to the rat [344]. It was characterized and determined to be a normal constituent of both rat and human urine. DHN-MA was excreted as a mixture of at least two stereoisomers. The 24-h urinary excretion of this compound was about $10 \mathrm{ng}$ and $5 \mu \mathrm{g}$ for rat and human, respectively. Following i.v. administration of $\mathrm{HNE}$ and $\left[4-{ }^{3} \mathrm{H}\right] \mathrm{HNE}$ to rats, besides DHN-MA 15 polar urinary metabolites accounting for about $50 \%$ of the urinary radioactivity were separated by HPLC by the same group [341]. Among them, eight major compounds and tritiated water were quantified. Most of polar metabolites were found to originate from $\omega$-oxidation of 4-hydroxy-2-nonenoic acid (HNA) to 9-hydroxy-HNA, its mercapturic acid conjugate, and two diastereoisomers of the corresponding lactone. 9-Hydroxy-HNA is a substrate for alcohol and aldehyde dehydrogenases leading to the formation and excretion of 9-carboxy-HNA and of the corresponding lactone mercapturic acid conjugate. The 9-hydoxy-HNA and the 9-carboxy-HNA were already mentioned above. The alcohol dehydrogenase metabolite 1,4-dihydroxy-2-nonene (DHN) was to a lower extent $\omega$-hydroxylated, leading to 9-hydroxy-DHN which was found to be excreted as a mercapturic acid conjugate [341].

To characterize the metabolic pathways involved in the formation of urinary HNE-MA conjugates in the rat, the metabolism of HNE-thioethers HNE-GSH, HNE-MA, and HNE-Cys by rat liver and kidney cytosolic fractions was investigated by Alary et al., 2003 [318]. The results showed that HNE-GSH is a good substrate for cytosolic incubations whereas HNE-MA and HNE-Cys are poorly metabolized. About $80 \%$ of the urinary MA conjugates originate from the primary and major HNE metabolite, namely, the hemiacetalized HNE-GSH. The direct reduction of HNE-GSH by a cytosolic aldo-keto reductase (NADPH) leads to DHN-GSH and subsequently to DHN-MA. The direct oxidation of HNE-GSH by aldehyde dehydrogenase $\left(\mathrm{NAD}^{+}\right)$leads to 4-hydroxynonenoic-lactone-GSH, the partial hydrolysis of which occurs at physiological $\mathrm{pH}$ and accounts for the corresponding HNA-GSH. Both the spontaneous- and the glutathione S-transferases-catalyzed retro-Michael cleavages of HNE-GSH and HNA-lactone-GSH are the source of HNE and HNA-lactone, respectively. HNA-lactone with both lipophilic and electrophilic properties is available for microsomal $\omega$-hydroxylation by cytochrome P450 4A enzymes and conjugation with thiol groups, and therefore is the most likely candidate for the formation of $\omega$-hydroxylated HNE-MA conjugates excreted in rat urine [318].

HNE biotransformation products were also detected in the bile of rats following a single intravenous administration of [4-(3)H]HNE. Five metabolites were present in the bile, two of them corresponded to GSH-HNE and to GSH-DHN adducts. Two further metabolites were identified as DHN and HNA-lactone mercapturic acid conjugates, whereas the fifth metabolite in the bile remained unidentified [345]. The kinetics of excretion of biliary metabolites demonstrated a rapid 
metabolism of HNE in rats: Within $4 \mathrm{~h}$ of injection, the bile accounted for $19.5 \%$ of the injected radioactivity, whereas only $3 \%$ was found in the feces within $48 \mathrm{~h}$. All rat recipients were found to have measurable levels of HNE metabolites in bile, confirming that HNE undergoes enterohepatic recirculation, and the extent of recycling was about $8 \%$ of the total dose [345]. In this context it has to be mentioned that the multidrug resistance-associated protein2 (MRP2) plays an important role in the biliary excretion of glutathione conjugates of HNE [346].

The metabolism of the two enantiomers of HNE, the (R)- and the (S)-enantiomer of its racemic mixture, was the aim of a study by Gueraud et al., 2005 [29]. The pathways were investigated in male rats after intravenous administration of the corresponding radiolabeled compounds and compared with the racemic mixture. Although the difference in the excretion rates was not statistically significant, the metabolic urinary pattern showed qualitative and quantitative differences. The level of 3-mercapturic acid-1,4-dihydroxynonene, which is considered as the major urinary metabolite of HNE, was significantly lower in the case of (S)-HNE injected rats. In order to clarify the intermediate pathways the authors performed in vitro studies using rat liver cytosolic incubations and HNE-glutathione conjugates as substrate and found large differences in the reduction and retro-Michael conversion steps of the metabolism between the conjugates originating from the two enantiomers [29]. Studies on the enantioselective metabolism of HNE by brain mitochondria were already described above [317] (see Chapter 7.2).

\section{Determination of HNE Metabolic Products and Pathways}

For the determination of HNE intermediates HPLC, HPLC-MS, and TLC were used, sometimes applying radioactively labelled compounds. In tracer kinetic experiments using tritium labelled HNE the HNE metabolites were separated by means of different methods (TLC or HPLC), and the formation of the products was simply calculated using the radioactivity of the spot or peak and dividing it by the specific radioactivity of the added HNE which can be taken as constant taking into account the overshoot of added radioactive HNE in comparison with the "low" formation of new HNE. After addition of labelled HNE aliquots of suspensions were taken. The HNE metabolizing reactions were stopped by the addition of e.g., $0.5 \mathrm{~mL}$ suspension to an equal volume of acetonitrile/acetic acid mixture $(96: 4 ; \mathrm{v} / \mathrm{v})$. After centrifugation two aliquots of the supernatant were eluted on TLC plates. The elution with hexane/diethylether $(3: 7 ; \mathrm{v} / \mathrm{v})$ allows the separation of HNE, HNA, and DHN. The elution with butanol/acetic acid/water $(4: 1: 1 ; \mathrm{v} / \mathrm{v} / \mathrm{v})$ allows the determination of GSH-adducts of HNE [294]. For quantification of HNE utilization rates the radiolabeled compounds have to be scanned by an automatic TLC linear analyzer or to be scraped off and analyzed.

Sometimes acetonitrile/acetic acid extracts were used for the determination of HNE and HNA by an HPLC-method [292,293]. The disappearance of initial HNE levels, such as 100, 10 or $1 \mu \mathrm{M}$ HNE, can be measured only by an HPLC-method (determination of remaining HNE during the whole experiment). The conditions for this "direct HNE and HNA analysis" are: C18 column, acetonitrile/water (4:6; v/v), $1 \mathrm{~mL} / \mathrm{min}, 223 \mathrm{~nm}$ detection wavelength. Quantification of the acid was based on the comparison of the hydroxynonenoic acid peak with formation of NADH in experiments in which HNE reacted with aldehyde dehydrogenase. In those experiments, the rate of 
formation of $\mathrm{NADH}$, which was observed spectrophotometrically at $340 \mathrm{~nm}$, was equal to the consumption rate of HNE (measured by HPLC at $223 \mathrm{~nm}$ ) and to the rate of formation of HNA. From the NADH formation rate (equal to the HNE consumption rate) and the peak integral of the HNA produced, one can calculate the extinction coefficient of the acid or the amount of HNA representing a defined peak area in the HPLC separation procedure [293]. HNE quantification was done by means of authentic HNE samples of known concentration, dissolved in methanol-water 80:20 (v/v) and comparison of the peak height of the standard chromatogram to sample chromatograms. A chromatogram of this direct HNE and HNA determination is shown in Figure 9.

The GSH-HNE-adduct was measured as an isoindol derivative after the reaction of the adduct existing in neutralized perchloric acid extracts with o-phthaldialdehyde in the presence of mercaptoethanol (Figure 10).

The reaction is started by addition of a sample aliquot to the o-phthaldiadehyde solution. The reaction mixture is transferred into a Hamilton syringe and the reaction is stopped by injection of the mixture into the HPLC system after $2 \mathrm{~min}$. HPLC separation is carried out in an isocratic manner with $65 \%$ methanol-0.1 M sodium acetate buffer, $\mathrm{pH} 6.5(\mathrm{v} / \mathrm{v})$ in the presence of an ion-pair reagent (tetraethylammoniumhydroxide). Isoindol is detected by means of a fluorescence detector at wavelengths of $345 \mathrm{~nm}$ and $445 \mathrm{~nm}$ for excitation and emission, respectively. The formation of enantiomer products in the reaction of GSH-HNE adduct with o-phthaldialdehyde [347] led to the appearance of a double peak of the isoindol adduct (Figure 10). The reaction product of free reduced glutathione (GSH) with o-phthaldialdehyde eluted much earlier than the isoindol adduct within the isocratic HPLC separation. Precise measurement of the adduct concentration requires an extensive preparation of the neutralized perchloric acid extract of suspensions with the aim of removing disturbing influences of amino acid derivatives after HPLC separation. For the purification of the extracts, Bond Elut C18 columns and a Baker-10 Extraction system can be recommended [293]. The columns should be rinsed with methanol and equilibrated with water. After injection of the sample the column should be rinsed with $0.1 \mathrm{M}$ sodium acetate buffer, $\mathrm{pH}$, then rinsed with hexane. The sample is then eluted with methanol, evaporated to dryness, and redissolved in a defined volume of methanol [293]. The protein precipitate is washed with physiological saline solution, then dissolved in Protosol-Tissue and Gel Solubilizer and added to toluene scintillation fluid and measured by means of a $\beta$-counter if using ${ }^{3} \mathrm{H}$-labeled HNE [292,297].

Different HPLC separations were applied in tracer kinetic experiments and in measurements of non-labelled HNE intermediates [293,312,319]. Urinary end products of HNE such as HNE mercapturic acid, 1,4-dihydroxynonene mercapturic acid, 4-hydroxynonenoic mercapturic acid, and the corresponding lactone were isolated and identified by mass spectrometry [319]. The HPLC-separation used by Srivastava et al., 1998 was combined with different detection methods: electrospray ionization MS for the detection of HNE-GSH and DHN-GSH, GC-chemical ionization MS for HNA [312]. Inhibitors were applied to modify the HPLC peak pattern. Sorbinil, an inhibitor of aldose reductase, attenuated the DHN-GSH peak, and cyanamide, an inhibitor of aldehyde dehydrogenase (ALDH), attenuated HNA formation. 
Various enzyme inhibitors were useful in studies on HNE metabolism. In investigations of HNE metabolic pathways of vascular smooth muscle cells it was shown that the formation of HNA was inhibited by cyanamide; indicating that the acid is derived from an ALDH-catalyzed pathway; while the overall rate of HNE metabolism was insensitive to inhibition of aldose reductase or aldehyde dehydrogenase. Nevetheless; inhibition of HNA formation by cyanamide led to a corresponding increase in the fraction of HNE metabolized by the GSH-linked pathway; indicating that ALDH-catalyzed oxidation competes with glutathione conjugation [311].

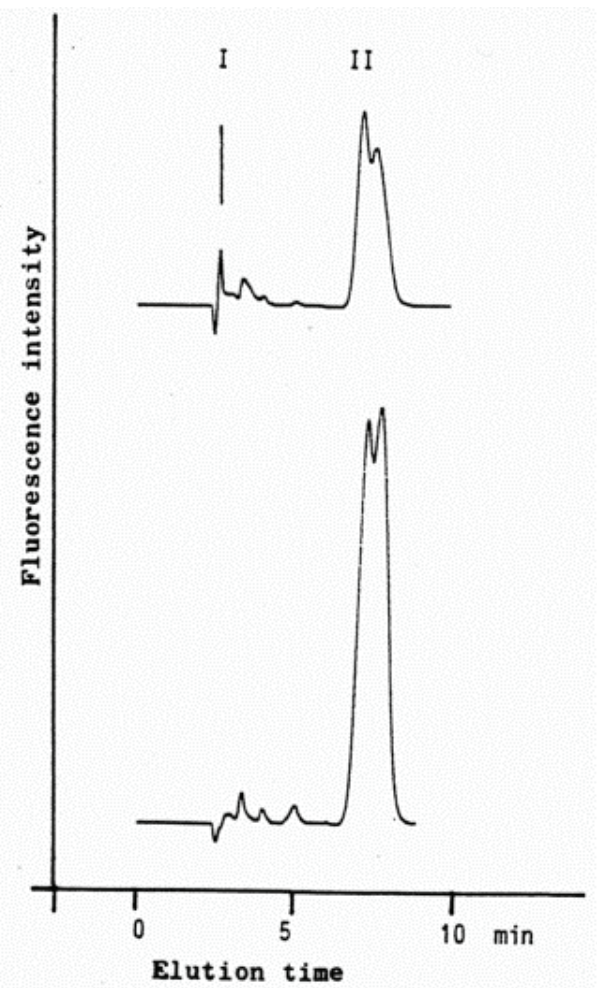

Figure 10. HPLC chromatogram of the isoindol derivative of the HNE-GSH conjugate (reaction product in presence of o-phthalaldehyde). Fluorimetric detection at $345 \mathrm{~nm}$ (excitation) and $445 \mathrm{~nm}$ (emission). Peaks: I GSH derivative; II HNE-GSH derivative; upper panel: Standard solution $(45 \mu \mathrm{M})$; lower panel: extract from hepatocyte suspension incubated in presence of $100 \mu \mathrm{M} 4-\mathrm{HNE}$ [301].

\subsection{HNE Metabolism as a Component of the Antioxidative Defense System}

The catabolism of HNE may be regarded as a very important part of the antioxidative defense system of cells and organisms. This is due to the fact, that HNE like other aldehydic products of LPO is able to exert cytotoxic, mutagenic, signal, and carcinogenic effects as described above. The degradation of HNE to less toxic intermediates diminished the reactions between HNE and biomolecules and therefore effectively contributes to the antioxidative protection of cells and 
organisms. This protective effect is the more effective the more rapidly the metabolism of HNE leads to stable HNE products, which can be easily excreted. The primary importance of HNEdegrading pathways which function both at physiological and pathophysiological HNE levels, seems to be the protection of proteins from modification by aldehydic LPO products. This should especially be valid in regions with high HNE formation rate such as in postischemic or reperfused tissues or in synovial tissue of joints during rheumatoid arthritis and other inflammatory diseases or in lymphedematous tissue, etc.

\subsection{HNE Intermediates as Potential Biomarkers of LPO}

Two groups of HNE derivatives seem to be useful as possible biomarkers in clinical chemistry and laboratory medicine. The first are the mercapturic acid conjugates including the derivatives of HNE, HNA, and DHN. These conjugates are excreted with the urine and, therefore can be analyzed from urine. The second are HNE-protein adducts of blood serum or plasma. The HNE-modified plasma proteins are already used for evaluation of rheumatic and autoimmune diseases [348]. Both body fluids - urine and serum or plasma - are easily obtained from patients. Both groups of derivatives are relatively stable. While the mercapturic acid assays are time- and equipment-consuming, the determination of HNE-modified plasma proteins is quite easy to handle. Within the mercapturic acid conjugates Peiro et al., 2005 favor the 1,4-dihydroxynonene mercapturic acid (DHN-MA) [349]. The authors compared three different urinary biomarkers of LPO during bromotrichloromethane induced oxidative stress in rats: MDA, the isoprostane 8-iso-PGF2 $\alpha$ and DHN-MA as the major HNE urinary metabolite. The rats received a single dose of $\mathrm{BrCCl}_{3}$ per os and LPO was estimated every day for a 4-day-period. All three parameters significantly increased in response to oxidative stress. DHN-MA showed the highest increase during the $24-48 \mathrm{~h}$ period after treatment.

The quantitation of the glutathione conjugate of HNE (HNE-GSH) in the human post-mortem brain was reported by Volkel et al., 2006 applying the specific and very sensitive method of electrospray ionization triple quadrupole mass spectrometry (ESI-MS-MS) [350]. Levels of HNE-GSH conjugates were determined in hippocampus, entorhinal cortex, substantia innominata, frontal and temporal cortex, as well as cerebellum from patients with Alzheimer's disease and controls matched for age, gender, post-mortem delay and storage time. The levels of HNE-GSH determined ranged between 1 and $500 \mathrm{pmol} / \mathrm{g}$ fresh weight in the brain areas examined. The brain specimen from patients with clinically and neuropathologically probable Alzheimer's disease diagnosed showed increased levels of HNE-GSH in the temporal and frontal cortex. Classification of disease severity revealed highest levels of HNE-GSH in the substantia innominata and the hippocampus, two brain regions known to be preferentially affected in Alzheimer's disease [4,9,350].

\subsection{Further Medical Applications of HNE Metabolism}

Several studies indicate that the glutathione conjugates of HNE metabolism are mediators of cell signalling and growth. A modification of the metabolic pattern, possibly by pharmacological interventions, could be of therapeutical importance [4]. Addition of HNE or the cell-permeable 
esters of HNE-GSH or DHN-GSH to cultures of rat aortic smooth muscle cells stimulated protein kinase $\mathrm{C}, \mathrm{NF \kappa B}$, and $\mathrm{AP}-1$, and increased cell growth. The mitogenic effects of HNE, but not HNE-GSH or DHN-GSH, were abolished by glutathione depletion. Inhibition of aldose reductase which catalyzes the reduction of HNE-GSH to DHN-GSH prevented protein kinase C, NFkB, and AP-1 stimulation and the increase in cell growth caused by HNE and HNE-GSH, but not DHN-GSH. Furthermore, the growth stimulating effect of DHN-GSH was enhanced in cells treated with antibodies directed against the glutathione conjugate transporters RLIP76 or the multidrug resistance protein-2 [351].

The demonstration that aldose reductase mediates endotoxin-induced inflammation and cardiomyopathy suggests that inhibition of this enzyme may be useful to attenuate maladaptive host responses and to treat acute cardiovascular dysfunction associated with endotoxic shock [335,352]. Srivastava et al., 2006 also demonstrated a contribution of aldose reductase to diabetic hyperproliferation of vascular smooth muscle cells [353]. The authors showed that aldose reductase inhibitors such as tolrestat or sorbinil or antisense aldose reductase mRNA prevented hyperproliferation of cultured rat aortic smooth muscle cells induced by high glucose levels. The results suggest that inhibition of aldose reductase prevents glucose-induced stimulation of smooth muscle cell growth. It was therefore concluded that even though inhibition of aldose reductase increases vascular oxidative stress, this approach may be useful in preventing abnormal smooth muscle cell growth in vessels of diabetic patients.

Tammali et al. [354] showed that treatment of the human colon cancer cell line Caco-2 with HNE or HNE-GSH or the aldose reductase-catalyzed product of HNE-GSH, DHN-GSH, resulted in increased cyclooxygenase-2 (COX-2) expression and prostaglandin E2 production. Inhibition of aldose reductase prevented HNE- or HNE-GSH-induced but not DHN-GSH-induced up-regulation of COX-2 and PGE2. The finding that aldose reductase inhibition by siRNAs completely arrested tumor progression in nude mice bearing human colon adenocarcinoma cells suggests that aldose reductase is an obligatory mediator of growth factor-induced up-regulation of COX-2, PGE2, and growth of Caco- 2 cells. These findings further suggest that inhibition of aldose reductase may be a novel therapeutic approach in preventing the progression of colon cancer [354].

Another oncological application of modulation of HNE metabolism was suggested in relation to the inhibition of cytosolic class 3 aldehyde dehydrogenase (ALDH3) by antisense oligonucleotides in hepatoma cells [59]. Several changes in aldehyde dehydrogenase isoenzyme expression take place in hepatoma cells. In particular cytosolic ALDH3, not expressed in normal hepatocytes, appears and increases with the degree of deviation. It has been demonstrated that cytosolic ALDH3 is important in determining the resistance of tumor cells to antitumor drugs, such as cyclophosphamide. Moreover, hepatoma-associated ALDH3 seems to be important in metabolizing aldehydes derived from LPO, and in particular the cytostatic aldehyde HNE. It was demonstrated previously that restoring endogenous LPO in hepatoma cells by enriching them with arachidonic acid causes a decrease of mRNA, protein and enzyme activity of ALDH3 and that this decrease reduces cell growth and/or causes cell death, depending on basal class 3 ALDH activity [59]. Applying antisense oligonucleotides the authors were able to demonstrate that ALDH3 mRNA and 
enzyme activity can be decreased by $90 \%$. Concomitantly cell growth was reduced by about $70 \%$ in the JM2 hepatoma cell line with high ALDH3 activity [59].

The carbonyl scavenger $N$-acetylcysteine (NAC) is a pharmaceutical drug and nutritional supplement used primarily as a mucolytic agent and in the management of paracetamol (acetaminophen) overdose. It was used in various investigations to attenuate HNE-mediated organ damage, such as in alcoholic liver damage [355]. In these investigations NAC decreased enzyme release from damaged organs, decreased inflammatory reactivity and prevented significant loss of organ GSH content. NAC exerts also significant protective effects against HNE-induced neuronal death in cerebellar granule neurons. This neuroprotective effect is due, at least in part, to preservation of mitochondrial membrane potential and intracellular GSH levels [356]. An important medical application of modification of HNE metabolism is the development of therapeutic strategies for the prevention of a number of inflammatory diseases [33].

Further studies showed that caloric restriction in mammals and in flies (Drosophila) slows the age-related and the oxidative damage-related HNE accumulation [357]. Additionally suppression of apoptosis in aged kidney-where increased HNE and MDA levels were found-by caloric restriction was observed [358]. Additionally, a decrease of oxidative damage in skeletal muscle was demonstrated after caloric restriction of rhesus monkeys [359], and a decline of aldehyde dehydrogenase and glutathione S-transferase activities as pathways of mitochondrial HNE metabolism was prevented in dietary restricted animals [316].

\section{Conclusions}

This review tries to demonstrate that there is a multitude of possible targets of HNE within a cell. Several factors can be envisaged which determine the actual reactants within a cell: The concentration of HNE which can differentially modulate cell death, growth and differentiation [360], the concentration and nucleophilicity of the target, the polarity of the microenvironment, the accessibility within the tertiary structure, and the site of formation as well as the metabolic rate of HNE.

\section{Conflicts of Interest}

The authors declare no conflict of interest.

\section{Abbreviations}

$\mathrm{A} \beta$

ACC

AcLDL

$\mathrm{ACO} 2$

Acr-dG

AD

$\mathrm{ADH}$

ADP-iron amyloid beta peptide

acetyl CoA carboxylase

acetylated low-density lipoprotein

mitochondrial aconitase

adduct of deoxyguanosine and acrolein

Alzheimer disease

alcohol dehydrogenase

adenosine diphosphate-iron 
AICAR

AIDS

AIE

AKR

ALD

ALDH

Al-PEs

ALS

AMD

AMPK

ANT

ApoB

AR

ARE

BAG3

BER

BSA

BSO

CAR

$\mathrm{CCl}_{4}$

CERAD

$\mathrm{CH}_{2} \mathrm{Cl}_{2}$

$\mathrm{CHO}$

CHOP

Col II

COX-2

CYP

$\varepsilon-\mathrm{dA}$

DHN

DHN-MA

DNA

DNA-PK

DOX

D3T

eEF-2

EGFP

EGFR

EHN

ERK1

ESI-MS-MS

FABP 5-aminoimidazole-4-carboxyamide ribonucleoside

acquired immuno-deficiency syndrome

approximate intracellular concentration (extinction)

aldo-keto reductase

alcoholic liver disease

aldehyde dehydrogenase

aldehyde-modified PEs

amyotrophic lateral sclerosis

age-related macular degeneration

AMP-activated protein kinase

adenine nucleotide translocase

apolipoprotein B-100

aldose reductase

antioxidant response element

Bcl-2- associated athanogene 3

base excision repair

bovine serum albumin

L-buthionine-S,R-sulfoximine

carnosine

carbon tetrachloride

consortium to establish a registry for Alzheimer disease

dichloromethane

Chinese-hamster ovary

C/EBP homologous protein

collagen type II

cyclooxygenase- 2

cytochrome $\mathrm{P} 450$

1,N(6)-etheno-2'-deoxyadenosine

dihydroxynonene, non-2-ene-1,4-diol

dihydroxynonene-mercapturic acid

desoxy-ribonucleic acid

DNA-dependent protein kinase

doxorubicin

3H-1,2-dithiole-3-thione, inducing detoxification enzymes

Eukaryotic Elongation factor 2

enhanced green fluorescent protein

epidermal growth factor receptor

2,3-epoxy-4-hydroxy-nonanal

extracellular signal-regulated kinase 1

electrospray ionization triple quadrupole mass spectrometry

fatty acid-binding protein 
FAK

FID

FITC

GAPDH

GC

GC/MS

GERD

GRP78

GRX

GSH

GSH-DHN

GSK3 $\beta$

GSSG

GST

HA-1

HAEC

$\mathrm{Hb}$

HCC

$\mathrm{HCV}$

HMEC-1

HHE

$\left[{ }^{3} \mathrm{H}\right] \mathrm{HNE}$

HNA

HNA-GSH

HNE

HNE-dG

HNE-H2A

$\mathrm{H} 2 \mathrm{O} 2$

HPLC

HPNE

HO-1

HSA

HU

HUVEC

ICAM-1

IsoLGs

iTRAQ

LA

LAD

$\mathrm{LADH}$

LCA $\alpha 1 \beta 1$-integrin-focal adhesion kinase

flame ionization detection

fluorescein isothiocyanate

glyceraldehyde-3-phosphate dehydrogenase

gas chromatography

gas chromatography/mass spectrometry

gastroesophageal reflux disease

glucose-regulated protein 78

glutaredoxin

glutathione

glutathione-dihydroxynonene conjugate

glycogen synthase kinase-3- $\beta$

glutathione in the oxidized form, glutathione disulfide

glutathione-S-transferase

Chinese hamster fibroblast control cells

human aortic endothelial cell

hemoglobin

hepatocellular carcinoma

hepatitis $\mathrm{C}$ virus

immortalized human microvascular endothelial cells

4-hydroxy-hexenal

tritium labeled hydroxynonenal

hydroxynonenoic acid

hydroxynonenoic acid-glutathione conjugate

4-hydroxy-nonenal

1,N-2-propane adduct of guanine and HNE

HNE-modified histone-H2A

hydrogen peroxide

high performance liquid chromatography

4-hydroperoxy-2-nonenal

heme oxygenase-1

human serum albumin

HU hydroxyurea

human umbilical vein endothelial cell

intercellular adhesion molecule-1

isolevuglandins

isobaric Tags for Relative and Absolute Quantitation

linolenic acid

late Alzheimer disease

lipoamide dehydrogenase

retinal dystrophy Leber congenital amaurosis 
LC-MS/MS

LDH

LDL

LKB1

LOX1

LPO

MAL-6

MALDI-TOF

MAPK

MBP-1

MCI

MDA

MDM2

MH1C1

MMP-13

MRP-1

NAD+

$\mathrm{NADH}$

NADP

NAFLD

NEP

NER

Nrf2

$\mathrm{OA}$

OC5 and $\mathrm{OC} 14$

ONE

oxLDL

ox-PAPC

PAF

PAPC

PCAD

PDGF

PDI

PE

PGE

Pin1

PMF

POS

PG(E2)

PtdIns(3,4,5)P-3

PTEN liquid chromatography-tandem mass spectrometry

lactate dehydrogenase

low density lipoprotein

liver kinase B1

lectin-like oxidized low-density lipoprotein receptor-1

lipid peroxidation

2,2,6,6-tetramethyl-4-maleimidopiperidin-1-oxyl

matrix-assisted laser desorption/ionization-time of flight

mitogen-activated protein kinase

c-myc binding protein-1

mild cognitive impairment

malondialdehyde

mouse double minute 2

rat hepatoma cells

matrix metalloproteinase 13

multidrug resistance protein-1

nicotinamid dinucleotide, oxidized form

nicotinamid dinucleotide, reduced form

nicotinamid dinucleotide phosphate

non-alcoholic fatty liver disease

neprilysin

nucleotide excision repair

NF-E2-related factor 2

osteoarthritic

Chinese hamster fibroblast $\mathrm{H}_{2} \mathrm{O}_{2}$-resistant cell lines

4-oxo-nonenal

oxidized low-density lipoprotein

oxidized PAPC

1-O-alkyl-2-acetyl-sn-glycero-3-phospho-choline

1-palmitoyl-2-arachidonoyl-sn-glycero-3-phospho-choline preclinical Alzheimer disease

platelet-derived growth factor

protein disulfide isomerase

phosphatidyl-ethanolamine

prostaglandin $\mathrm{E}$

peptidyl-prolyl cis/trans-isomerase A1

peptide mass fingerprinting

photoreceptor outer segment

prostaglandin E2

phosphatidylinositol-3,4,5-trisphosphate

phosphatase and tensin homolog deleted on chromosome 10 


$\begin{array}{ll}\text { RAS } & \text { rennin/angiotensin system } \\ \text { RBC } & \text { erythrocytes } \\ \text { RDH } & \text { retinol dehydrogenase } \\ \text { RLIP76 } & \text { Ral-binding GTPase activating protein } \\ \text { ROC } & \text { receiver operator curves } \\ \text { ROOH } & \text { lipid hydroperoxide } \\ \text { ROS } & \text { reactive oxygen species } \\ \text { RPE } & \text { retinal pigment epithelial } \\ \text { SAM } & \text { sterile-alpha motif } \\ \text { SCFAs } & \text { short chain fatty acids } \\ \text { SERCA1a } & \text { serco/endoplasmic reticulum Ca }{ }^{2+} \text {-ATPase } \\ \text { SIRT3 } & \text { sirtuin 3 } \\ \text { SLE } & \text { systemic lupus erythematosus } \\ \text { SMC } & \text { smooth muscle cell } \\ \text { sXBP1 } & \text { spliced X-box-binding protein-1 } \\ \alpha-S y n & \alpha \text {-synuclein } \\ \text { TBA-RS } & \text { thiobarbituric acid-reactive substances } \\ \text { TBI } & \text { traumatic brain injury } \\ \text { TLC } & \text { thin layer chromatography } \\ \text { TLR } & \text { toll-like receptor } \\ \text { TRPV1 } & \text { transient receptor potential vanilloid 1 } \\ \text { TRX } & \text { thioredoxin } \\ \text { TrxR } & \text { thioredoxin reductase } \\ \text { UCP } & \text { uncoupling protein } \\ \text { UPR } & \text { unfolded protein response } \\ \text { VSL\#3 } & \text { a medical food delivering high amount of beneficial live bacteria } \\ \text { YAP1 } & \text { Yeast Activator Protein 1 } \\ \text { ZAK kinase } & \text { sterile alpha motif and leucine zipper containing kinase (AZK) } \\ & \end{array}$

\section{References}

1. Schauenstein, E.; Esterbauer, H. Ueber die Reaktion von 9,12 Linolsaeureester in Wasser 6. Mitteilung: Chromatographische Untersuchungen ueber die Zusammensetzung des Praeparates LHPO und die Isolierung der einzelnen tumor-wirksamen Hydroperoxid-Komponenten (In Germany). Chem. Mon. 1963, 94, 11.

2. Halliwell, B.; Gutteridge, J. Free Radicals in Biology and Medicine, 4th ed.; Oxford University Press: Oxford, UK, 2007.

3. Catala, A. Lipid peroxidation of membrane phospholipids generates hydroxy-alkenals and oxidized phospholipids active in physiological and/or pathological conditions. Chem. Phys. Lipids 2009, 157, 1-11.

4. Poli, G.; Schaur, R.J.; Siems, W.G.; Leonarduzzi, G. 4-Hydroxynonenal: A membrane lipid oxidation product of medicinal interest. Med. Res. Rev. 2008, 28, 569-631. 
5. Schaur, J.R. Basic aspects of the biochemical reactivity of 4-hydroxynonenal. Mol. Asp. Med. 2003, 24, 149-159.

6. Dubinina, E.E.; Dadali, V.A. Role of 4-hydroxy-trans-2-nonenal in cell functions. Biochem. Moscow 2010, 75, 1069-1087.

7. Fritz, K.S.; Petersen, D.R. An overview of the chemistry and biology of reactive aldehydes. Free Rad. Biol. Med. 2013, 59, 85-91.

8. Zarkovic, N.; Cipak, A.; Jaganjac, M.; Borovic, S.; Zarkovic, K. Pathophysiological relevance of aldehydic protein modifications. J. Proteomics 2013, 92, 239-247.

9. Perluigi, M.; Coccia, R.; Butterfield, D.A. 4-hydroxy-2-nonenal, a reactive product of lipid peroxidation, and neurodegenerative diseases: A toxic combination illuminated by redox proteomics studies. Antioxid. Redox Signal. 2012, 17, 1590-1609.

10. Riahi, Y.; Cohen, G.; Shamni, O.; Sasson, S. Signaling and cytotoxic functions of 4hydroxyalkenals. Am. J. Physiol. Endocrinol. Metab. 2010, 299, E879-E886.

11. Forman, H.J. Reactive oxygen species and $\alpha, \beta$-unsaturated aldehydes as second messengers in signal transduction. Oxid. Nitrosative Stress Dis. 2010, 1203, 35-44.

12. Higdon, A.; Diers, A.R.; Oh, J.Y.; Landar, A.; Darley-Usmar, V.M. Cell signalling by reactive lipid species: New concepts and molecular mechanisms. Biochem. J. 2012, 442, 453-464.

13. Dwivedi, S.; Sharma, A.; Patrick, B.; Sharma, R.; Awasthi, Y.C. Role of 4-hydroxynonenal and its metabolites in signaling. Redox Rep. 2007, 12, 4-10.

14. Ayala, A.; Munoz, M.F.; Argueelles, S. Lipid peroxidation: Production, metabolism, and signaling mechanisms of malondialdehyde and 4-hydroxy-2-nonenal. Oxid. Med. Cell. Longev. 2014, doi:10.1155/2014/360438.

15. Spickett, C.M. The lipid peroxidation product 4-hydroxy-2-nonenal: Advances in chemistry and analysis. Redox Biol. 2013, 1, 145-152.

16. LoPachin, R.M.; Gavin, T.; Petersen, D.R.; Barber, D.S. Molecular mechanisms of 4-hydroxy-2-nonenal and acrolein toxicity: Nucleophilic targets and adduct formation. Chem. Res. Toxicol. 2009, 22, 1499-1508.

17. Mattson, M.P. Roles of the lipid peroxidation product 4-hydroxynonenal in obesity, the metabolic syndrome, and associated vascular and neurodegenerative disorders. Exp. Gerontol. 2009, 44, 625-633.

18. Guichardant, M.; Lagarde, M. Analysis of biomarkers from lipid peroxidation: A comparative study. Eur. J. Lipid Sci. Technol. 2009, 111, 75-82.

19. Tappel, A.L. Measurement and protection from in vivo lipid peroxidation. In Free Radicals in Biology; Pryor, W.A., Ed.; Academic Press: New York, NY, USA, 1980; pp. 1-47.

20. Aikens, J.; Dix, T.A. Perhydroxyl radical (HOO.) initiated lipid-peroxidation-The role of fatty-acid hydroperoxides. J. Biol. Chem. 1991, 266, 15091-15098.

21. Tsunada, S.; Iwakiri, R.; Noda, T.; Fujimoto, K.; Fuseler, J.; Rhoads, C.A.; Aw, T.Y. Chronic exposure to subtoxic levels of peroxidized lipids suppresses mucosal cell turnover in rat small intestine and reversal by glutathione. Dig. Dis. Sci. 2003, 48, 210-222. 
22. Bochkov, V.N.; Kadl, A.; Huber, J.; Gruber, F.; Binder, B.R.; Leitinger, N. Protective role of phospholipid oxidation products in endotoxin-induced tissue damage. Nature 2002, 419, 77-81.

23. Yin, H.; Morrow, J.D.; Porter, N.A. Identification of a novel class of endoperoxides from arachidonate autoxidation. J. Biol. Chem. 2004, 279, 3766-3776.

24. Poli, G.; Schaur, R.J. 4-Hydroxynonenal in the pathomechanisms of oxidative stress. Iubmb Life 2000, 50, 315-321.

25. Pillon, N.J.; Soulere, L.; Vella, R.E.; Croze, M.; Care, B.R.; Soula, H.A.; Doutheau, A.; Lagarde, M.; Soulage, C.O. Quantitative structure-activity relationship for 4-hydroxy-2-alkenal induced cytotoxicity in L6 muscle cells. Chem. Biol. Interact. 2010, 188, 171-180.

26. Vazdar, M.; Jurkiewicz, P.; Hof, M.; Jungwirth, P.; Cwiklik, L. Behavior of 4-hydroxynonenal in phospholipid membranes. J. Phys. Chem. B 2012, 116, 6411-6415.

27. Dabrowski, M.J.; Zolnerciks, J.K.; Balogh, L.M.; Greene, R.J.; Kavanagh, T.J.; Atkins, W.M. Stereoselective Effects of 4-hydroxynonenal in cultured mouse hepatocytes. Chem. Res. Toxicol. 2010, 23, 1601-1607.

28. Hiratsuka, A.; Saito, H.; Watabe, T. (S)-preferential cytotoxicity of 4-hydroxy-2(E)-nonenal enantiomers in rat Clone 9 cells. Toxicology 2001, 164, 199.

29. Gueraud, F.; Crouzet, F.; Alary, J.; Rao, D.; Debrauwer, L.; Laurent, F.; Cravedi, J.P. Enantioselective metabolism of (R)- and (S)-4-hydroxy-2-nonenal in rat. Biofactors 2005, 24, 97-104.

30. Sadhukhan, S.; Han, Y.; Jin, Z.; Tochtrop, G.P.; Zhang, G.-F. Glutathionylated 4-hydroxy-2-(E)-alkenal enantiomers in rat organs and their contributions toward the disposal of 4-hydroxy-2-(E)-nonenal in rat liver. Free Rad. Biol. Med. 2014, 70, 78-85.

31. Wakita, C.; Maeshima, T.; Yamazaki, A.; Shibata, T.; Ito, S.; Akagawa, M.; Ojika, M.; Yodoi, J.; Uchida, K. Stereochemical configuration of 4-hydroxy-2-nonenal-cysteine adducts and their stereoselective formation in a redox-regulated protein. J. Biol. Chem. 2009, 284, 28810-28822.

32. Komisarski, M.; Kaczmarska, Z.; Kusmierek, J.T. Practical highly enantioselective synthesis of (R)- and (S)-(E)-4-hydroxynon-2-enal. Acta Biochim. Polonica 2009, 56, 189-193.

33. Yadav, U.C.S.; Ramana, K.V. Regulation of NF-kappaB-induced inflammatory signaling by lipid peroxidation-derived aldehydes. Oxid. Med. Cell. Longevity 2013, doi:10.1155/2013/690545.

34. Singh, S.P.; Niemczyk, M.; Zimniak, L.; Zimniak, P. Fat accumulation in Caenorhabditis elegans triggered by the electrophilic lipid peroxidation product 4-Hydroxynonenal (4-HNE). Aging 2009, 1, 68-80.

35. Singh, S.P.; Niemczyk, M.; Saini, D.; Awasthi, Y.C.; Zimniak, L.; Zimniak, P. Role of the electrophilic lipid peroxidation product 4-hydroxynonenal in the development and maintenance of obesity in mice. Biochemistry 2008, 47, 3900-3911.

36. Schneider, C.; Brash, A.R. Monomeric and dimeric routes to formation of 4-hydroxynonenal during lipid peroxidation. Abstr. Papers Am. Chem. Soc. 2013, 246, Abstract 41-AGFD. 
37. Long, E.K.; Picklo, M.J., Sr. Trans-4-hydroxy-2-hexenal, a product of n-3 fatty acid peroxidation: Make some room HNE. Free Rad. Biol. Med. 2010, 49, 1-8.

38. Wang, X.; Allen, T.D.; Yang, Y.; Moore, D.R.; Huycke, M.M. Cyclooxygenase-2 generates the endogenous mutagen trans-4-hydroxy-2-nonenal in Enterococcus faecalis-infected macrophages. Cancer Prev. Res. 2013, 6, 206-216.

39. Kang, S.C.; Kim, H.-W.; Kim, K.B.; Kwack, S.J.; Ahn, I.Y.; Bae, J.Y.; Lim, S.K.; Lee, B.M. Hepatotoxicity and nephrotoxicity produced by 4-hydroxy-2-nonenal (4-HNE) following 4-week oral administration to sprague-dawley rats. J. Toxicol. Environ. Health Part A 2011, 74, 779-789.

40. Zhang, Y.; Sano, M.; Shinmura, K.; Tamaki, K.; Katsumata, Y.; Matsuhashi, T.; Morizane, S.; Ito, H.; Hishiki, T.; Endo, J.; et al. 4-Hydroxy-2-nonenal protects against cardiac ischemia-reperfusion injury via the Nrf2-dependent pathway. J. Mol. Cell. Cardiol. 2010, 49, 576-586.

41. Mannervik, B. Five decades with glutathione and the GSTome. J. Biol. Chem. 2012, 287, 6072-6083.

42. Balogh, L.M.; Atkins, W.M. Interactions of glutathione transferases with 4-hydroxynonenal. Drug Metab. Rev. 2011, 43, 165-178.

43. Rudd, L.P.; Kabler, S.L.; Morrow, C.S.; Townsend, A.J. Enhanced glutathione depletion, protein adduct formation, and cytotoxicity following exposure to 4-hydroxy-2-nonenal (HNE) in cells expressing human multidrug resistance protein-1 (MRP1) together with human glutathione S-transferase-M1 (GSTM1). Chem. Biol. Interact. 2011, 194, 113-119.

44. Shireman, L.M.; Kripps, K.A.; Balogh, L.M.; Conner, K.P.; Whittington, D.; Atkins, W.M. Glutathione transferase A4-4 resists adduction by 4-hydroxynonenal. Arch. Biochem. Biophys. 2010, 504, 182-189.

45. Zhou, S.Y.; Decker, E.A. Ability of carnosine and other skeletal muscle components to quench unsaturated aldehydic lipid oxidation products. J. Agric. Food Chem. 1999, 47, 51-55.

46. Shearn, C.T.; Smathers, R.L.; Backos, D.S.; Reigan, P.; Orlicky, D.J.; Petersen, D.R. Increased carbonylation of the lipid phosphatase PTEN contributes to Akt2 activation in a murine model of early alcohol-induced steatosis. Free Rad. Biol. Med. 2013, 65, 680-692.

47. Zhu, Q.; Zheng, Z.-P.; Cheng, K.-W.; Wu, J.-J.; Zhang, S.; Tang, Y.S.; Sze, K.-H.; Chen, J.; Chen, F.; Wang, M. Natural polyphenols as direct trapping agents of lipid peroxidation-derived acrolein and 4-hydroxy-trans-2-nonenal. Chem. Res. Toxicol. 2009, 22, 1721-1727.

48. Stevens, J.F.; Sowell, J.D.; Frei, B. Ascorbic Acid Conjugates. US Patent US20090104705A1, 2009.

49. Uchida, K. Immunochemical detection of lipid peroxidation-specific epitopes. In Biomarkers for Antioxidative Defense and Oxidative Damage; Aldini, G., Yeum, K.J., Niki, E., Russell, R.M., Eds.; Blackwell Publishing: Hoboken, NJ, USA, 2010; pp. 157-171.

50. Wakita, C.; Honda, K.; Shibata, T.; Akagawa, M.; Uchida, K. A method for detection of 4-hydroxy-2-nonenal adducts in proteins. Free Radic. Biol. Med. 2011, 51, 1-4. 
51. Zhang, B.; Shi, Z.; Duncan, D.T.; Prodduturi, N.; Marnett, L.J.; Liebler, D.C. Relating protein adduction to gene expression changes: A systems approach. Mol. Biosyst. 2011, 7, 2118-2127.

52. Dick, R.A.; Kwak, M.K.; Sutter, T.R.; Kensler, T.W. Antioxidative function and substrate specificity of $\mathrm{NAD}(\mathrm{P}) \mathrm{H}$-dependent alkenal/one oxidoreductase-A new role for leukotriene B-4 12-hydroxydehydrogenase/15-oxoprostaglandin 13-reductase. J. Biol. Chem. 2001, 276, 40803-40810.

53. Chen, H.J.C.; Chung, F.L. Epoxidation of trans-4-hydroxy-2-nonenal by fatty acid hydroperoxides and hydrogen peroxide. Chem. Res. Toxicol. 1996, 9, 306-312.

54. Perry, E.A.; Castellani, R.J.; Moreira, P.I.; Nunomura, A.; Lui, Q.; Harris, P.L.R.; Sayre, L.M.; Szweda, P.A.; Szweda, L.I.; Zhu, X.; et al. Neurofilaments are the major neuronal target of hydroxynonenal-mediated protein cross-links. Free Radic. Res. 2013, 47, 507-510.

55. Singh, I.N.; Gilmer, L.K.; Miller, D.M.; Cebak, J.E.; Wang, J.A.; Hall, E.D. Phenelzine mitochondrial functional preservation and neuroprotection after traumatic brain injury related to scavenging of the lipid peroxidation-derived aldehyde 4-hydroxy-2-nonenal. J. Cerebral Blood Flow Metab. 2013, 33, 593-599.

56. Fournet, G.; Martin, G.; Quash, G. $\alpha, \beta$-Acetylenic amino thiolester inhibitors of aldehyde dehydrogenases 1\&3: Suppressors of apoptogenic aldehyde oxidation and activators of apoptosis. Curr. Med. Chem. 2013, 20, 527-533.

57. Guo, J.-M.; Liu, A.-J.; Zang, P.; Dong, W.-Z.; Ying, L.; Wang, W.; Xu, P.; Song, X.-R.; Cai, J.; Zhang, S.-Q.; et al. ALDH2 protects against stroke by clearing 4-HNE. Cell Res. 2013, 23, 915-930.

58. Ma, H.; Guo, R.; Yu, L.; Zhang, Y.; Ren, J. Aldehyde dehydrogenase 2 (ALDH2) rescues myocardial ischaemia/reperfusion injury: Role of autophagy paradox and toxic aldehyde. Eur. Heart J. 2011, 32, 1025-1038.

59. Muzio, G.; Maggiora, M.; Paiuzzi, E.; Oraldi, M.; Canuto, R.A. Aldehyde dehydrogenases and cell proliferation. Free Radic. Biol. Med. 2012, 52, 735-746.

60. Townsend, A.J.; Leone-Kabler, S.; Haynes, R.L.; Wu, Y.; Szweda, L.; Bunting, K.D. Selective protection by stably transfected human ALDH3A1 (but not human ALDH1A1) against toxicity of aliphatic aldehydes in V79 cells. Chem. Biol. Interact. 2001, 130-132, 261-273.

61. Black, W.; Chen, Y.; Matsumoto, A.; Thompson, D.C.; Lassen, N.; Pappa, A.; Vasiliou, V. Molecular mechanisms of ALDH3A1-mediated cellular protection against 4-hydroxy-2nonenal. Free Radic. Biol. Med. 2012, 52, 1937-1944.

62. Yoval-Sanchez, B.; Rodriguez-Zavala, J.S. Differences in susceptibility to inactivation of human aldehyde dehydrogenases by lipid peroxidation byproducts. Chem. Res. Toxicol. 2012, 25, 722-729.

63. Murakami, Y.; Ohsawa, I.; Kasahara, T.; Ohta, S. Cytoprotective role of mitochondrial amyloid beta peptide-binding alcohol dehydrogenase against a cytotoxic aldehyde. Neurobiol. Aging 2009, 30, 325-329. 
64. Srivastava, S.; Vladykovskaya, E.; Barski, O.A.; Kaiserova, M.S.K.; Petrash, J.M.; Chung, S.S.; Hunt, G.; Dawn, B.; Bhatnagar, A. Aldose reductase protects against early atherosclerotic lesion formation in apolipoprotein e-null mice. Circ. Res. 2009, 105, 793-802.

65. Keith, R.J.; Haberzettl, P.; Vladykovskaya, E.; Hill, B.G.; Kaiserova, K.; Srivastava, S.; Barski, O.; Bhatnagar, A. Aldose reductase decreases endoplasmic reticulum stress in ischemic hearts. Chem. Biol. Interact. 2009, 178, 242-249.

66. Li, D.; Ferrari, M.; Ellis, E.M. Human aldo-keto reductase AKR7A2 protects against the cytotoxicity and mutagenicity of reactive aldehydes and lowers intracellular reactive oxygen species in hamster V79-4 cells. Chem. Biol. Interact. 2012, 195, 25-34.

67. Jung, K.-A.; Kwak, M.-K. Enhanced 4-hydroxynonenal resistance in KEAP1 silenced human colon cancer cells. Oxid. Med. Cell. Longevity 2013, doi:10.1155/2013/423965.

68. Lyon, R.C.; Li, D.; McGarvie, G.; Ellis, E.M. Aldo-keto reductases mediate constitutive and inducible protection against aldehyde toxicity in human neuroblastoma SH-SY5Y cells. Neurochem. Int. 2013, 62, 113-121.

69. Zhong, L.; Liu, Z.; Yan, R.; Johnson, S.; Zhao, Y.; Fang, X.; Cao, D. Aldo-keto reductase family 1 B10 protein detoxifies dietary and lipid-derived alpha, beta-unsaturated carbonyls at physiological levels. Biochem. Biophys. Res. Commun. 2009, 387, 245-250.

70. Martin, H.-J.; Maser, E. Role of human aldo-keto-reductase AKR1B10 in the protection against toxic aldehydes. Chem. Biol. Interact. 2009, 178, 145-150.

71. Endo, S.; Matsunaga, T.; Kuragano, T.; Ohno, S.; Kitade, Y.; Tajima, K.; el-Kabbani, O.; Hara, A. Properties and tissue distribution of a novel aldo-keto reductase encoding in a rat gene (Akr1b10). Arch. Biochem. Biophys. 2010, 503, 230-237.

72. Matsunaga, T.; Shinoda, Y.; Inoue, Y.; Endo, S.; el-Kabbani, O.; Hara, A. Protective effect of rat aldo-keto reductase (AKR1C15) on endothelial cell damage elicited by 4-hydroxy-2nonenal. Chem. Biol. Interact. 2011, 191, 364-370.

73. Marchette, L.D.; Thompson, D.A.; Kravtsova, M.; Ngansop, T.N.; Mandal, M.N.A.; Kasus-Jacobi, A. Retinol dehydrogenase 12 detoxifies 4-hydroxynonenal in photoreceptor cells. Free Radic. Biol. Med. 2010, 48, 16-25.

74. Amunom, I.; Dieter, L.J.; Tamasi, V.; Cai, J.; Conklin, D.J.; Srivastava, S.; Martin, M.V.; Guengerich, F.P.; Prough, R.A. Cytochromes p450 catalyze the reduction of $\alpha, \beta$-unsaturated aldehydes. Chem. Res. Toxicol. 2011, 24, 1223-1230.

75. Zhang, G.-F.; Kombu, R.S.; Kasumov, T.; Han, Y.; Sadhukhan, S.; Zhang, J.; Sayre, L.M.; Ray, D.; Gibson, K.M.; Anderson, V.A.; et al. Catabolism of 4-hydroxyacids and 4-hydroxynonenal via 4-hydroxy-4-phosphoacyl-CoAs. J. Biol. Chem. 2009, 284, 33521-33534.

76. Berthiaume, J.M.; Li, Q.; Sadhukhan, S.; Henry, F.; Tochtrop, G.P.; Brunengraber, H.; Zhang, G. Catabolism of 4-hydroxy-2(E)-nonenal (HNE) via omega oxidation in perfused rat livers. FASEB J. 2013, 27, Abstract No. 794.15.

77. Subramaniam, R.; Roediger, F.; Jordan, B.; Mattson, M.P.; Keller, J.N.; Waeg, G.; Butterfield, D.A. The lipid peroxidation product, 4-hydroxy-2-trans-nonenal, alters the conformation of cortical synaptosomal membrane proteins. J. Neurochem. 1997, 69, 1161-1169. 
78. Chen, J.J.; Yu, B.P. Alterations in mitochondrial-membrane fluidity by lipid-peroxidation products. Free Radic. Biol. Med. 1994, 17, 411-418.

79. Fleuranceau-Morel, P.; Barrier, L.; Fauconneau, B.; Piriou, A.; Huguet, F. Origin of 4-hydroxynonenal incubation-induced inhibition of dopamine transporter and $\mathrm{Na}^{+} / \mathrm{K}^{+}$ adenosine triphosphate in rat striatal synaptosomes. Neurosci. Lett. 1999, 277, 91-94.

80. Liu, Q.; Ullery, J.; Zhu, J.; Liebler, D.C.; Marnett, L.J.; Zhang, B. RNA-seq data analysis at the gene and CDS levels provides a comprehensive view of transcriptome responses induced by 4-hydroxynonenal. Mol. Biosyst. 2013, 9, 3036-3046.

81. Schreier, S.M.; Muellner, M.K.; Steinkellner, H.; Hermann, M.; Esterbauer, H.; Exner, M.; Gmeiner, B.M.K.; Kapiotis, S.; Laggner, H. Hydrogen sulfide scavenges the cytotoxic lipid oxidation product 4-HNE. Neurotox. Res. 2010, 17, 249-256.

82. Siems, W.; Grune, T. Intracellular metabolism of 4-hydroxynonenal. Mol. Asp. Med. 2003, $24,167-175$

83. Vila, A.; Tallman, K.A.; Jacobs, A.T.; Liebler, D.C.; Porter, N.A.; Marnett, L.J. Identification of protein targets of 4-hydroxynonenal using click chemistry for ex vivo biotinylation of azido and alkynyl derivatives. Chem. Res. Toxicol. 2008, 21, 432-444.

84. Tallman, K.A.; Vila, A.; Porter, N.A.; Marnett, L.J. Measuring electrophile stress. Curr. Protoc. Toxicol. 2009, 11-17.

85. Chavez, J.; Chung, W.-G.; Miranda, C.L.; Singhal, M.; Stevens, J.F.; Maier, C.S. Site-specific protein adducts of 4-hydroxy-2(E)-nonenal in human THP-1 monocytic cells: Protein carbonylation is diminished by ascorbic acid. Chem. Res. Toxicol. 2010, 23, 37-47.

86. Codreanu, S.G.; Zhang, B.; Sobecki, S.M.; Billheimer, D.D.; Liebler, D.C. Global analysis of protein damage by the lipid electrophile 4-hydroxy-2-nonenal. Mol. Cell. Proteomics 2009, 8, 670-680.

87. Petersen, D.R.; Carbone, D.; Doorn, J. Hepatocellular targets of 4-hydroxy-2-nonenal modification. In Proceedings of the 3rd International Meeting of the HNE-Club, Genova, Italy, 16-18 June 2006; Abstract No. 6.

88. Hussain, S.N.A.; Matar, G.; Barreiro, E.; Florian, M.; Divangahi, M.; Vassilakopoulos, T. Modifications of proteins by 4-hydroxy-2-nonenal in the ventilatory muscles of rats. Am. J. Physiol. Lung Cell. Mol. Physiol. 2006, 290, L996-L1003.

89. Jung, T.; Engels, M.; Kaiser, B.; Grune, T. Distribution of oxidized and HNE-modified proteins in U87 cells. Biofactors 2005, 24, 165-170.

90. Carbone, D.L.; Doorn, J.A.; Kiebler, Z.; Ickes, B.R.; Petersen, D.R. Modification of heat shock protein 90 by 4-hydroxynonenal in a rat model of chronic alcoholic liver disease. J. Pharmacol. Exp. Ther. 2005, 315, 8-15.

91. Carbone, D.L.; Doorn, J.A.; Kiebler, Z.; Sampey, B.P.; Petersen, D.R. Inhibition of Hsp72-mediated protein refolding by 4-hydroxy-2-nonenal. Chem. Res. Toxicol. 2004, 17, $1459-1467$. 
92. Legards, J.F.; des Rosiers, C. Assay of 4-hydroxynonenal (HNE) adducts with various polyaminoacids (PAA) using gas chromatography-mass spectrometry (GCMS). In Proceedings of the 3rd International Meeting of the HNE-Club, Genova, Italy, 16-18 June 2006.

93. Doorn, J.A.; Petersen, D.R. Covalent adduction of nucleophilic amino acids by 4-hydroxynonenal and 4-oxononenal. Chem. Biol. Interact. 2003, 143, 93-100.

94. Isom, A.L.; Barnes, S.; Wilson, L.; Kirk, M.; Coward, L.; Darley-Usmar, V. Modification of cytochrome c by 4-hydroxy-2-nonenal: Evidence for histidine, lysine, and arginine-aldehyde adducts. J. Am. Soc. Mass Spectrom. 2004, 15, 1136-1147.

95. Liu, Z.F.; Minkler, P.E.; Sayre, L.A. Mass spectroscopic characterization of protein modification by 4-Hydroxy-2-(E)-nonenal and 4-Oxo-2-(E)-nonenal. Chem. Res. Toxicol. 2003, 16, 901-911.

96. Aldini, G.; Dalle-Donne, I.; Vistoli, G.; Facino, R.M.; Carini, M. Covalent modification of actin by 4-hydroxy-trans-2-nonenal (HNE): LC-ESI-MS/MS evidence for Cys374 Michael adduction. J. Mass Spectrom. 2005, 40, 946-954.

97. Bennaars-Eiden, A.; Higgins, L.A.; Hertzel, A.V.; Kapphahn, R.J.; Ferrington, D.; Bernlohr, D.A. Covalent modification of epithelial fatty acid binding protein by 4-hydroxynonenal in vitro and in vivo: Evidence for a role in antioxidant biology. FASEB J. 2003, 17, Abstract No. 845.847.

98. Carbone, D.L.; Doorn, J.A.; Kiebler, Z.; Petersen, D.R. Cysteine modification by lipid peroxidation products inhibits protein disulfide isomerase. Chem. Res. Toxicol. 2005, 18, $1324-1331$

99. Asselin, C.; Bouchard, B.; Tardif, J.C.; des Rosiers, C. Circulating 4-hydroxynonenal-protein thioether adducts assessed by gas chromatography-mass spectrometry are increased with disease progression and aging in spontaneously hypertensive rats. Free Radic. Biol. Med. 2006, 41, 97-105.

100. Moreau, R.; Nguyen, B.T.; Doneanu, C.E.; Hagen, T.M. Reversal by aminoguanidine of the age-related increase in glycoxidation and lipoxidation in the cardiovascular system of Fischer 344 rats. Biochem. Pharmacol. 2005, 69, 29-40.

101. Kim, C.H.; Zou, Y.N.; Kim, D.H.; Kim, N.D.; Yu, B.P.; Chung, H.Y. Proteomic analysis of nitrated and 4-hydroxy-2-nonenal-modified serum proteins during aging. J. Gerontol. Ser. A Biol. Sci. Med. Sci. 2006, 61, 332-338.

102. Petersen, D.R.; Doorn, J.A. Reactions of 4-hydroxynonenal with proteins and cellular targets. Free Radic. Biol.Med. 2004, 37, 937-945.

103. Renner, A.; Sagstetter, M.R.; Harms, H.; Lange, V.; Gotz, M.E.; Elert, O. Formation of 4-hydroxy-2-nonenal protein adducts in the ischemic rat heart after transplantation. J. Heart Lung Transpl. 2005, 24, 730-736.

104. Galligan, J.J.; Smathers, R.L.; Fritz, K.S.; Epperson, L.E.; Hunter, L.E.; Petersen, D.R. Protein carbonylation in a murine model for early alcoholic liver disease. Chem. Res. Toxicol. 2012, 25, 1012-1021.

105. Aoyama, K.; Matsubara, K.; Kobayashi, S. Aging and oxidative stress in progressive supranuclear palsy. Eur. J. Neurol. 2006, 13, 89-92. 
106. Grune, T. HNE-modified proteins: Formation, distribution and fate. In Proceedings of the 3rd International Meeting of the HNE-Club, Genova, Italy, 2006; Abstract No. 17.

107. Carbone, D.L.; Doorn, J.A.; Petersen, D.R. 4-hydroxynonenal regulates 26S proteasomal degradation of alcohol dehydrogenase. Free Radic. Biol. Med. 2004, 37, 1430-1439.

108. Okada, K.; Wangpoengtrakul, C.; Osawa, T.; Toyokuni, S.; Tanaka, K.; Uchida, K. 4-hydroxy-2-nonenal-mediated impairment of intracellular proteolysis during oxidative stress-Identification of proteasomes as target molecules. J. Biol. Chem. 1999, 274, 23787-23793.

109. Hyun, D.H.; Lee, M.H.; Halliwell, B.; Jenner, P. Proteasomal dysfunction induced by 4-hydroxy-2,3-trans-nonenal, an end-product of lipid peroxidation: A mechanism contributing to neurodegeneration? J. Neurochem. 2002, 83, 360-370.

110. Bardag-Gorce, F.; Li, J.; French, B.A.; French, S.W. The effect of ethanol-induced CYP2E1 on proteasome activity: The role of 4-hydroxynonenal. Exp. Mol. Pathol. 2005, 78, 109-115.

111. Kessova, I.G.; Cederbaum, A.I. The effect of CYP2E1-dependent oxidant stress on activity of proteasomes in HepG2 cells. J. Pharmacol. Exp. Ther. 2005, 315, 304-312.

112. Ferrington, D.A.; Kapphahn, R.J. Catalytic site-specific inhibition of the $20 \mathrm{~S}$ proteasome by 4-hydroxynonenal. FEBS Lett. 2004, 578, 217-223.

113. Tsuchiya, Y.; Yamaguchi, M.; Chikuma, T.; Hojo, H. Degradation of glyceraldehyde-3phosphate dehydrogenase triggered by 4-hydroxy-2-nonenal and 4-hydroxy-2-hexenal. Arch. Biochem. Biophys. 2005, 438, 217-222.

114. Xu, G.Z.; Liu, Y.H.; Sayre, L.M. Independent synthesis, solution behavior, and studies on the mechanism of formation of a primary amine-derived fluorophore representing cross-linking of proteins by (E)-4-hydroxy-2-nonenal. J. Org. Chem. 1999, 64, 5732-5745.

115. Zarkovic, N. Protein-aldehydic adducts as biomarkers of oxidative stress, lipid peroxidation and oxidative homeostasis. Free Radic. Res. 2009, 43, 33.

116. Wang, J.-F.; Tan, H. Oxidative protein modification of soluble $N$-ethylmaleimide-sensitive factor attachment protein receptors. FASEB J. 2013, 27, 890-897.

117. Gallagher, E.P.; Gardner, J.L.; Barber, D.S. Several glutathione S-transferase isozymes that protect against oxidative injury are expressed in human liver mitochondria. Biochem. Pharmacol. 2006, 71, 1619-1628.

118. Alary, J.; Fernandez, Y.; Debrauwer, L.; Perdu, E.; Gueraud, F. Identification of intermediate pathways of 4-hydroxynonenal metabolism in the rat. Chem. Res. Toxicol. 2003, 16, 320-327.

119. Lucas, J.H.; Wheeler, D.G.; Guan, Z.; Suntres, Z.; Stokes, B.T. Effect of glutathione augmentation on lipid peroxidation after spinal cord injury. J. Neurotrauma 2002, 19, $763-775$.

120. Yang, Y.S.; Sharma, R.; Sharma, A.; Awasthi, S.; Awasthi, Y.C. Lipid peroxidation and cell cycle signaling: 4-Hydroxynonenal, a key molecule in stress mediated signaling. Acta Biochim. Polonica 2003, 50, 319-336. 
121. Patrick, B.; Li, J.; Jeyabal, P.V.S.; Reddy, P.; Yang, Y.S.; Sharma, R.; Sinha, M.; Luxon, B.; Zimniak, P.; Awasthi, S.; et al. Depletion of 4-hydroxynonenal in hGSTA4-transfected HLE B-3 cells results in profound changes in gene expression. Biochem. Biophys. Res. Commun. 2005, 334, 425-432.

122. Dwivedi, S.; Sharma, R.; Sharma, A.; Zimniak, P.; Ceci, J.D.; Awasthi, Y.C.; Boor, P.J. The course of $\mathrm{CCl} 4$ induced hepatotoxicity is altered in mGSTA4-4 null (-/-) mice. Toxicology 2006, 218, 58-66.

123. Engle, M.R.; Singh, S.P.; Czernik, P.J.; Gadd, D.; Montague, D.C.; Ceci, J.D.; Yang, Y.S.; Awasthi, S.; Awasthi, Y.C.; Zimniak, P. Physiological role of mGSTA4-4, a glutathione S-transferase metabolizing 4-hydroxynonenal: Generation and analysis of mGSTA4 null mouse. Toxicol. Appl. Pharmacol. 2004, 194, 296-308.

124. Knoll, N.; Ruhe, C.; Veeriah, S.; Sauer, J.; Glei, M.; Gallagher, E.P.; Pool-Zobel, B.L. Genotoxicity of 4-hydroxy-2-nonenal in human colon tumor cells is associated with cellular levels of glutathione and the modulation of glutathione S-transferase A4 expression by butyrate. Toxicol. Sci. 2005, 86, 27-35.

125. Srivastava, S.; Ramana, K.V.; Bhatnagar, A.; Srivastava, S.K. Synthesis, quantification, characterization, and signaling properties of glutathionyl conjugates of enals. Methods Enzymol. 2010, 474, 297-313.

126. Frohnert, B.I.; Long, E.K.; Hahn, W.S.; Bernlohr, D.A. Glutathionylated lipid aldehydes are products of adipocyte oxidative stress and activators of macrophage inflammation. Diabetes 2014, 63, 89-100.

127. Spite, M.; Summers, L.; Porter, T.F.; Srivastava, S.; Bhatnagar, A.; Serhan, C.N. Resolvin D1 controls inflammation initiated by glutathione-lipid conjugates formed during oxidative stress. Br. J. Pharmacol. 2009, 158, 1062-1073.

128. Aldini, G.; Facino, R.M.; Beretta, G.; Carini, M. Carnosine and related dipeptides as quenchers of reactive carbonyl species: From structural studies to therapeutic perspectives. Biofactors 2005, 24, 77-87.

129. Aldini, G.; Carini, M.; Beretta, G.; Bradamante, S.; Facino, R.M. Carnosine is a quencher of 4-hydroxy-nonenal: Through what mechanism of reaction? Biochem. Biophys. Res. Commun. 2002, 298, 699-706.

130. Barski, O.A.; Xie, Z.; Baba, S.P.; Sithu, S.D.; Agarwal, A.; Cai, J.; Bhatnagar, A.; Srivastava, S. Dietary carnosine prevents early atherosclerotic lesion formation in apolipoprotein e-null mice. Arterioscler. Thromb. Vasc. Biol. 2013, 33, 1162-1170.

131. Wang, M.; Fang, H.; Han, X. Shotgun lipidomics analysis of 4-hydroxyalkenal species directly from lipid extracts after one-step in situ derivatization. Anal. Chem. 2012, 84, 4580-4586.

132. Guiotto, A.; Calderan, A.; Ruzza, P.; Osler, A.; Rubini, C.; Jo, D.G.; Mattson, M.P.; Borin, G. Synthesis and evaluation of neuroprotective alpha,beta-unsaturated aldehyde scavenger histidyl-containing analogues of carnosine. J. Med. Chem. 2005, 48, 6156-6161.

133. Guiotto, A.; Calderan, A.; Ruzza, P.; Borin, G. Carnosine and carnosine-related antioxidants: A review. Curr. Med. Chem. 2005, 12, 2293-2315. 
134. Vistoli, G.; Orioli, M.; Pedretti, A.; Regazzoni, L.; Canevotti, R.; Negrisoli, G.; Carini, M.; Aldini, G. Design, synthesis, and evaluation of carnosine derivatives as selective and efficient sequestering agents of cytotoxic reactive carbonyl species. Chemmedchem 2009, 4, 967-975.

135. Bertinaria, M.; Rolando, B.; Giorgis, M.; Montanaro, G.; Guglielmo, S.; Buonsanti, M.F.; Carabelli, V.; Gavello, D.; Daniele, P.G.; Fruttero, R.; et al. Synthesis, physicochemical characterization, and biological activities of new carnosine derivatives stable in human serum as potential neuroprotective agents. J. Med. Chem. 2011, 54, 611-621.

136. Sugano, E.; Isago, H.; Murayama, N.; Tamai, M.; Tomita, H. Different anti-oxidant effects of thioredoxin 1 and thioredoxin 2 in retinal epithelial cells. Cell Struct. Funct. 2013, 38, 81-88.

137. Zhang, H.; Du, Y.; Zhang, X.; Lu, J.; Holmgren, A. Glutaredoxin 2 reduces both thioredoxin 2 and thioredoxin 1 and protects cells from apoptosis induced by auranofin and 4-hydroxynonenal. Antioxid. Redox Signal. 2014, 21, 669-681.

138. Tang, X.; Sayre, L.M.; Tochtrop, G.P. A mass spectrometric analysis of 4-hydroxy-2-(E)nonenal modification of cytochrome c. J. Mass Spectrom. 2011, 46, 290-297.

139. Gardner, H.W.; Deighton, N. Effect of 4-hydroxy-2(E)-nonenal on soybean lipoxygenase1. Lipids 2001, 36, 623-628.

140. Uchida, K.; Stadtman, E.R. Covalent attachment of 4-hydroxynonenal to glyceraldehyde-3phosphate dehydrogenase-A possible involvement of intramolecular and intermolecular cross-linking reaction. J. Biol. Chem. 1993, 268, 6388-6393.

141. Ishii, T.; Tatsuda, E.; Kumazawa, S.; Nakayama, T.; Uchida, K. Molecular basis of enzyme inactivation by an endogenous electrophile 4-hydroxy-2-nonenal: Identification of modification sites in glyceraldehyde-3-phosphate dehydrogenase. Biochemistry 2003, 42, 3474-3480.

142. Van Iersel, M.L.P.S.; Ploemen, J.-P.H.T.M.; lo Bello, M.; Federici, G.; van Bladeren, P.J. Interactions of $\alpha, \beta$-unsaturated aldehydes and ketones with human glutathione S-transferase P1-1. Chem. Biol. Interact. 1997, 108, 67-78.

143. Bosch-Morell, F.; Flohe, L.; Marin, N.; Romero, F.J. 4-Hydroxynonenal inhibits glutathione peroxidase: Protection by glutathione. Free Radic. Biol. Med. 1999, 26, 1383-1387.

144. Korotchkina, L.G.; Yang, H.S.; Tirosh, O.; Packer, L.; Patel, M.S. Protection by thiols of the mitochondrial complexes from 4-hydroxy-2-nonenal. Free Radic. Biol. Med. 2001, 30, 992-999.

145. Del Corso, A.; dal Monte, M.; Vilardo, P.G.; Cecconi, I.; Moschini, R.; Banditelli, S.; Cappiello, M.; Tsai, L.; Mura, U. Site-specific inactivation of aldose reductase by 4-hydroxynonenal. Arch. Biochem. Biophys. 1998, 350, 245-248.

146. Yu, M.K.; Moos, P.J.; Cassidy, P.; Wade, M.; Fitzpatrick, F.A. Conditional expression of 15-lipoxygenase-1 inhibits the selenoenzyme thioredoxin reductase-Modulation of selenoproteins by lipoxygenase enzymes. J. Biol. Chem. 2004, 279, 28028-28035.

147. Fang, J.G.; Holmgren, A. Inhibition of thioredoxin and thioredoxin reductase by 4-hydroxy-2-nonenal in vitro and in vivo. J. Am. Chem. Soc. 2006, 128, 1879-1885. 
148. Chen, Z.H.; Saito, Y.; Yoshida, Y.; Sekine, A.; Noguchi, N.; Niki, E. 4-Hydroxynonenal induces adaptive response and enhances $\mathrm{PC} 12$ cell tolerance primarily through induction of thioredoxin reductase 1 via activation of Nrf2. J. Biol. Chem. 2005, 280, 41921-41927.

149. Chen, Z.H.; Yoshida, Y.; Saito, Y.; Noguchi, N.; Niki, E. Adaptive response induced by lipid peroxidation products in cell cultures. FEBS Lett. 2006, 580, 479-483.

150. Morquette, B.; Shi, Q.; Lavigne, P.; Ranger, P.; Fernandes, J.C.; Benderdour, M. Production of lipid peroxidation products in osteoarthritic tissues-New evidence linking 4hydroxynonenal to cartilage degradation. Arthritis Rheum. 2006, 54, 271-281.

151. Ramanathan, R.; Mancini, R.A.; Suman, S.P.; Beach, C.M. Covalent binding of 4-hydroxy-2nonenal to lactate dehydrogenase decreases $\mathrm{NADH}$ formation and metmyoglobin reducing activity. J. Agric. Food Chem. 2014, 62, 2112-2117.

152. Schlisser, A.E.; Yan, J.; Hales, B.F. Teratogen-induced oxidative stress targets glyceraldehyde-3-phosphate dehydrogenase in the organogenesis stage mouse embryo. Toxicol. Sci. 2010, 118, 686-695.

153. Markesbery, W.R.; Carney, J.M. Oxidative alterations in Alzheimer's disease. Brain Pathol. 1999, 9, 133-146.

154. Butterfield, D.A.; Lauderback, C.M. Lipid peroxidation and protein oxidation in Alzheimer's disease brain: Potential causes and consequences involving amyloid $\beta$-peptide-associated free radical oxidative stress1,2. Free Radic. Biol. Med. 2002, 32, 1050-1060.

155. Markesbery, W.R.; Lovell, M.A. Four-hydroxynonenal, a product of lipid peroxidation, is increased in the brain in Alzheimer's disease. Neurobiol. Aging 1998, 19, 33-36.

156. Sultana, R.; Butterfield, D.A. Oxidatively modified GST and MRP1 in Alzheimer's disease brain: Implications for accumulation of reactive lipid peroxidation products. Neurochem. Res. 2004, 29, 2215-2220.

157. Huang, Y.; Li, W.; Kong, A.-N.T. Anti-oxidative stress regulator NF-E2-related factor 2 mediates the adaptive induction of antioxidant and detoxifying enzymes by lipid peroxidation metabolite 4-hydroxynonenal. Cell Biosci. 2012, 2, 1-7.

158. Balogh, L.M.; le Trong, I.; Kripps, K.A.; Shireman, L.M.; Stenkamp, R.E.; Zhang, W.; Mannervik, B.; Atkins, W.M. Substrate specificity combined with stereopromiscuity in glutathione transferase A4-4-dependent metabolism of 4-hydroxynonenal. Biochemistry 2010, 49, 1541-1548.

159. Calamaras, T.D.; Lee, C.; Lan, F.; Ido, Y.; Siwik, D.A.; Colucci, W.S. Post-translational modification of serine/threonine kinase LKB1 via adduction of the reactive lipid species 4-hydroxy-trans-2-nonenal (HNE) at lysine residue 97 directly inhibits kinase activity. J. Biol. Chem. 2012, 287, 42400-42406.

160. Shearn, C.T.; Backos, D.S.; Orlicky, D.J.; Smathers-McCullough, R.L.; Petersen, D.R. Identification of $5^{\prime}$ AMP-activated kinase as a target of reactive aldehydes during chronic ingestion of high concentrations of ethanol. J. Biol. Chem. 2014, 289, 15449-15462.

161. Wang, C.; Weerapana, E.; Blewett, M.M.; Cravatt, B.F. A chemoproteomic platform to quantitatively map targets of lipid-derived electrophiles. Nat. Methods 2014, 11, 79-85. 
162. Shearn, C.T.; Fritz, K.S.; Reigan, P.; Petersen, D.R. Modification of Akt2 by 4-hydroxynonenal inhibits insulin-dependent Akt signaling in HepG2 cells. Biochemistry 2011, 50, 3984-3996.

163. MacDonald, M.J.; Langberg, E.C.; Tibell, A.; Sabat, G.; Kendrick, M.A.; Szweda, L.I.; Ostenson, C.G. Identification of ATP synthase as a lipid peroxide protein adduct in pancreatic islets from humans with and without type 2 Diabetes mellitus. J. Clin. Endocrinol. Metab. 2013, 98, E727-E731.

164. Guo, J.; Prokai-Tatrai, K.; Vien, N.; Rauniyar, N.; Ughy, B.; Prokai, L. Protein targets for carbonylation by 4-hydroxy-2-nonenal in rat liver mitochondria. J. Proteomics 2011, 74, 2370-2379.

165. Shearn, C.T.; Smathers, R.L.; Stewart, B.J.; Fritz, K.S.; Galligan, J.J.; Hail, N., Jr.; Petersen, D.R. Phosphatase and tensin homolog deleted on chromosome 10 (PTEN) inhibition by 4-hydroxynonenal leads to increased Akt activation in hepatocytes. Mol. Pharmacol. 2011, 79, 941-952.

166. Fang, X.; Fu, Y.; Long, M.J.C.; Haegele, J.A.; Ge, E.J.; Parvez, S.; Aye, Y. Temporally controlled targeting of 4-hydroxynonenal to specific proteins in living cells. J. Am. Chem. Soc. 2013, 135, 14496-14499.

167. Los, G.V.; Encell, L.P.; McDougall, M.G.; Hartzell, D.D.; Karassina, N.; Zimprich, C.; Wood, M.G.; Learish, R.; Ohane, R.F.; Urh, M.; et al. HatoTag: A novel protein labeling technology for cell imaging and protein analysis. ACS Chem. Biol. 2008, 3, 373-382.

168. Fritz, K.S.; Galligan, J.J.; Smathers, R.L.; Roede, J.R.; Shearn, C.T.; Reigan, P.; Petersen, D.R. 4-hydroxynonenal inhibits SIRT3 via thiol-specific modification. Chem. Res. Toxicol. 2011, 24, 651-662.

169. Krohne, T.U.; Kaemmerer, E.; Holz, F.G.; Kopitz, J. Lipid peroxidation products reduce lysosomal protease activities in human retinal pigment epithelial cells via two different mechanisms of action. Exp. Eye Res. 2010, 90, 261-266.

170. Wang, D.S.; Iwata, N.; Hama, E.; Saido, T.C.; Dickson, K. Oxidized neprilysin in aging and Alzheimer's disease brains. Biochem. Biophys. Res. Commun. 2003, 310, 236-241.

171. Wang, R.; Wang, S.; Malter, J.S.; Wang, D.-S. Effects of HNE-modification induced by A beta on neprilysin expression and activity in SH-SY5Y cells. J. Neurochem. 2009, 108, 1072-1082.

172. Wang, R.; Malter, J.S.; Wang, D.-S. N-Acetylcysteine prevents 4-hydroxynonenal-And amyloid- $\beta$-induced modification and inactivation of neprilysin in SH-SY5Y cells. J. Alzheimers Dis. 2010, 19, 179-189.

173. Liu, Q.; Simpson, D.C.; Gronert, S. Carbonylation of mitochondrial aconitase with 4-hydroxy-2-(E)-nonenal: Localization and relative reactivity of addition sites. Biochim. Biophys. Acta Proteins Proteomics 2013, 1834, 1144-1154. 
174. Zhao, Y.; Miriyala, S.; Miao, L.; Mitov, M.; Schnell, D.; Dhar, S.K.; Cai, J.; Klein, J.B.; Sultana, R.; Butterfield, D.A.; et al. Redox proteomic identification of HNE-bound mitochondrial proteins in cardiac tissues reveals a systemic effect on energy metabolism after doxorubicin treatment. Free Radic. Biol. Med. 2014, 72, 55-65.

175. Gentile, F.; Pizzimenti, S.; Arcaro, A.; Pettazzoni, P.; Minelli, R.; D’Angelo, D.; Mamone, G.; Ferranti, P.; Toaldo, C.; Cetrangolo, G.; et al. Exposure of HL-60 human leukaemic cells to 4-hydroxynonenal promotes the formation of adduct(s) with alpha-enolase devoid of plasminogen binding activity. Biochem. J. 2009, 422, 285-294.

176. Reed, T.T.; Pierce, W.M.; Markesbery, W.R.; Butterfield, D.A. Proteomic identification of HNE-bound proteins in early Alzheimer disease: Insights into the role of lipid peroxidation in the progression of AD. Brain Res. 2009, 1274, 66-76.

177. Muller, C.; Bandemer, J.; Vindis, C.; Camare, C.; Mucher, E.; Gueraud, F.; Larroque-Cardoso, P.; Bernis, C.; Auge, N.; Salvayre, R.; et al. Protein disulfide isomerase modification and inhibition contribute to ER stress and apoptosis induced by oxidized low density lipoproteins. Antioxid. Redox Signal. 2013, 18, 731-742.

178. Aluise, C.D.; Rose, K.; Boiani, M.; Reyzer, M.L.; Manna, J.D.; Tallman, K.; Porter, N.A.; Marnett, L.J. Peptidyl-prolyl cis/trans-isomerase A1 (Pin1) is a target for modification by lipid electrophiles. Chem. Res. Toxicol. 2013, 26, 270-279.

179. Tanito, M.; Haniu, H.; Elliott, M.H.; Singh, A.K.; Matsumoto, H.; Anderson, R.E. Identification of 4-hydroxynonenal-modified retinal proteins induced by photooxidative stress prior to retinal degeneration. Free Radic. Biol. Med. 2006, 41, 1847-1859.

180. Toyokuni, S.; Yamada, S.; Kashima, M.; Ihara, Y.; Yamada, Y.; Tanaka, T.; Hiai, H.; Seino, Y.; Uchida, K. Serum 4-hydroxy-2-nonenal-modified albumin is elevated in patients with type 2 Diabetes Mellitus. Antioxid. Redox Signal. 2000, 2, 681-685.

181. Aldini, G.; Gamberoni, L.; Orioli, M.; Beretta, G.; Regazzoni, L.; Facino, R.M.; Carini, M. Mass spectrometric characterization of covalent modification of human serum albumin by 4-hydroxy-trans-2-nonenal. J. Mass Spectrom. 2006, 41, 1149-1161.

182. Liu, Q.; Simpson, D.C.; Gronert, S. The reactivity of human serum albumin towards trans-4-hydroxy-2-nonenal. J. Mass Spectrom. 2012, 47, 411-424.

183. Khatoon, F.; Alam, K.; Ali, A. Physicochemical and immunological studies on 4-hydroxynonenal modified HSA: Implications of protein damage by lipid peroxidation products in the etiopathogenesis of SLE. Hum. Immunol. 2012, 73, 1132-1139.

184. Xiang, W.; Weisbach, V.; Sticht, H.; Seebahn, A.; Bussmann, J.; Zimmermann, R.; Becker, C.-M. Oxidative stress-induced posttranslational modifications of human hemoglobin in erythrocytes. Arch. Biochem. Biophys. 2013, 529, 34-44.

185. Naveena, B.M.; Faustman, C.; Tatiyaborworntham, N.; Yin, S.; Ramanathan, R.; Mancini, R.A. Detection of 4-hydroxy-2-nonenal adducts of turkey and chicken myoglobins using mass spectrometry. Food Chem. 2010, 122, 836-840.

186. Smathers, R.L.; Fritz, K.S.; Galligan, J.J.; Shearn, C.T.; Reigan, P.; Marks, M.J.; Petersen, D.R. Characterization of 4-HNE modified L-FABP reveals alterations in structural and functional dynamics. PLOS ONE 2012, 7, e38459. 
187. Hellberg, K.; Grimsrud, P.A.; Kruse, A.C.; Banaszak, L.J.; Ohlendorf, D.H.; Bernlohr, D.A. $\mathrm{X}$-ray crystallographic analysis of adipocyte fatty acid binding protein (aP2) modified with 4-hydroxy-2-nonenal. Protein Sci. 2010, 19, 1480-1489.

188. Stewart, B.J.; Roede, J.R.; Doorn, J.A.; Petersen, D.R. Lipid aldehyde-mediated cross-linking of apolipoprotein B-100 inhibits secretion from HepG2 cells. Biochim. Biophys. Acta Mol. Cell Biol. Lipids 2009, 1791, 772-780.

189. Shao, B.; Pennathur, S.; Pagani, I.; Oda, M.N.; Witztum, J.L.; Oram, J.F.; Heinecke, J.W. Modifying apolipoprotein A-I by malondialdehyde, but not by an array of other reactive carbonyls, blocks cholesterol efflux by the ABCA1 pathway. J. Biol. Chem. 2010, 285, $18473-18484$.

190. Zhu, X.; Tang, X.; Anderson, V.E.; Sayre, L.M. Mass spectrometric characterization of protein modification by the products of nonenzymatic oxidation of linoleic acid. Chem. Res. Toxicol. 2009, 22, 1386-1397.

191. Ou, J.J.; Zhang, Y.L.; Montine, T.J. In vivo assessment of lipid peroxidation products associated with age-related neurodegenerative diseases. Exp. Neurol. 2002, 175, 363-369.

192. Mattson, M.P.; Chan, S.L. Neuronal and glial calcium signaling in Alzheimer's disease. Cell Calcium 2003, 34, 385-397.

193. Pedersen, W.A.; Fu, W.; Keller, J.N.; Markesbery, W.R.; Appel, S.; Smith, R.G.; Kasarskis, E.; Mattson, M.P. Protein modification by the lipid peroxidation product 4-hydroxynonenal in the spinal cords of amyotrophic lateral sclerosis patients. Ann. Neurol. 1998, 44, 819-824.

194. Lovell, M.A.; Bradley, M.A.; Fister, S.X. 4-Hydroxyhexenal (HHE) impairs glutamate transport in astrocyte cultures. J. Alzheimers Dis. 2012, 32, 139-146.

195. Maroteaux, L.; Campanelli, J.T.; Scheller, R.H. Synuclein: A neuron-specific protein localized to the nucleus and presynaptic nerve terminal. J. Neurosci. 1988, 8, 2804-2815.

196. Cabin, D.E.; Shimazu, K.; Murphy, D.; Cole, N.B.; Gottschalk, W.; McIlwain, K.L.; Orrison, B.; Chen, A.; Ellis, C.E.; Paylor, R.; et al. Synaptic vesicle depletion correlates with attenuated synaptic responses to prolonged repetitive stimulation in mice lacking alpha-synuclein. J. Neurosci. 2002, 22, 8797-8807.

197. Murphy, D.D.; Rueter, S.M.; Trojanowski, J.Q.; Lee, V.M. Synucleins are developmentally expressed, and alpha-synuclein regulates the size of the presynaptic vesicular pool in primary hippocampal neurons. J. Neurosci. 2000, 20, 3214-3220.

198. Lotharius, J.; Brundin, P. Impaired dopamine storage resulting from $\alpha$-synuclein mutations may contribute to the pathogenesis of Parkinson's disease. Hum. Mol. Genet. 2002, 11, 2395-2407.

199. Jomova, K.; Vondrakova, D.; Lawson, M.; Valko, M. Metals, oxidative stress and neurodegenerative disorders. Mol. Cell. Biochem. 2010, 345, 91-104.

200. Xiang, W.; Schlachetzki, J.C.M.; Helling, S.; Bussmann, J.C.; Berlinghof, M.; Schaeffer, T.E.; Marcus, K.; Winkler, J.; Klucken, J.; Becker, C.-M. Oxidative stress-induced posttranslational modifications of alpha-synuclein: Specific modification of alpha-synuclein by 4-hydroxy-2nonenal increases dopaminergic toxicity. Mol. Cell. Neurosci. 2013, 54, 71-83. 
201. Bae, E.-J.; Ho, D.-H.; Park, E.; Jung, J.W.; Cho, K.; Hong, J.H.; Lee, H.-J.; Kim, K.P.; Lee, S.-J. Lipid peroxidation product 4-hydroxy-2-nonenal promotes seeding-capable oligomer formation and cell-to-cell transfer of a-synuclein. Antioxid. Redox Signal. 2013, 18, $770-783$.

202. Nasstrom, T.; Fagerqvist, T.; Barbu, M.; Karlsson, M.; Nikolajeff, F.; Kasrayan, A.; Ekberg, M.; Lannfelt, L.; Ingelsson, M.; Bergstrom, J. The lipid peroxidation products 4-oxo-2-nonenal and 4-hydroxy-2-nonenal promote the formation of $\alpha$-synuclein oligomers with distinct biochemical, morphological, and functional properties. Free Radic. Biol. Med. 2011, 50, 428-437.

203. Shibata, N.; Inose, Y.; Toi, S.; Hiroi, A.; Yamamoto, T.; Kobayashi, M. Involvement of 4-hydroxy-2-nonenal accumulation in multiple system atrophy. Acta Histochem. Cytochem. 2010, 43, 69-75.

204. Hortigon-Vinagre, M.P.; Chardonnet, S.; Montigny, C.; Gutierrez-Martin, Y.; Champeil, P.; Henao, F. Inhibition by 4-hydroxynonenal (HNE) of $\mathrm{Ca}^{2+}$ transport by SERCA1a: Low concentrations of HNE open protein-mediated leaks in the membrane. Free Radic. Biol. Med. 2011, 50, 323-336.

205. Gunthorpe, M.J.; Benham, C.D.; Randall, A.; Davis, J.B. The diversity in the vanilloid (TRPV) receptor family of ion channels. Trends Pharmacol. Sci. 2002, 23, 183-191.

206. Kishimoto, E.; Naito, Y.; Handa, O.; Okada, H.; Mizushima, K.; Hirai, Y.; Nakabe, N.; Uchiyama, K.; Ishikawa, T.; Takagi, T.; et al. Oxidative stress-induced posttranslational modification of TRPV1 expressed in esophageal epithelial cells. Am. J. Physiol. Gastrointest. Liver Physiol. 2011, 301, G230-G238.

207. Vindis, C.; Escargueil-Blanc, I.; Elbaz, M.; Marcheix, B.; Grazide, M.H.; Uchida, K.; Salvayre, R.; Negre-Salvayre, A. Desensitization of platelet-derived growth factor receptor-beta by oxidized lipids in vascular cells and atherosclerotic lesions-Prevention by Aldehyde Scavengers. Circ. Res. 2006, 98, 785-792.

208. Kumano-Kuramochi, M.; Shimozu, Y.; Wakita, C.; Ohnishi-Kameyama, M.; Shibata, T.; Matsunaga, S.; Takano-Ishikawa, Y.; Watanabe, J.; Goto, M.; Xie, Q.; et al. Identification of 4-hydroxy-2-nonenal-histidine adducts that serve as ligands for human lectin-like oxidized LDL receptor-1. Biochem. J. 2012, 442, 171-180.

209. Kim, Y.S.; Park, Z.Y.; Kim, S.Y.; Jeong, E.; Lee, Y. Alteration of Toll-like receptor 4 activation by 4-hydroxy-2-nonenal mediated by the suppression of receptor homodimerization. Chem. Biol. Interact. 2009, 182, 59-66.

210. Liu, Q.; Smith, M.A.; Avila, J.; DeBernardis, J.; Kansal, M.; Takeda, A.; Zhu, X.W.; Nunomura, A.; Honda, K.; Moreira, P.I.; et al. Alzheimer-specific epitopes of tau represent lipid peroxidation-induced conformations. Free Radic. Biol. Med. 2005, 38, 746-754.

211. Santa-Maria, I.; Hernandez, F.; Martin, C.P.; Avila, J.; Moreno, F.J. Quinones facilitate the self-assembly of the phosphorylated tubulin binding region of tau into fibrillar polymers. Biochemistry 2004, 43, 2888-2897. 
212. Mendez, D.; Hernaez, M.L.; Kamali, A.N.; Diez, A.; Puyet, A.; Bautista, J.M. Differential carbonylation of cytoskeletal proteins in blood group $\mathrm{O}$ erythrocytes: Potential role in protection against severe malaria. Infect. Genet. Evol. 2012, 12, 1780-1787.

213. Arashiki, N.; Otsuka, Y.; Ito, D.; Yang, M.; Komatsu, T.; Sato, K.; Inaba, M. The covalent modification of spectrin in red cell membranes by the lipid peroxidation product 4-hydroxy-2-nonenal. Biochem. Biophys. Res. Commun. 2010, 391, 1543-1547.

214. Mendez, D.; Hernaez, M.L.; Diez, A.; Puyet, A.; Bautista, J.M. Combined proteomic approaches for the identification of specific amino acid residues modified by 4-hydroxy-2nonenal under physiological conditions. J. Proteome Res. 2010, 9, 5770-5781.

215. Yamashima, T. Hsp70.1 and related lysosomal factors for necrotic neuronal death. J. Neurochem. 2012, 120, 477-494.

216. Perluigi, M.; Poon, H.F.; Hensley, K.; Pierce, W.M.; Klein, J.B.; Calabrese, V.; de Marco, C.; Butterfield, D.A. Proteomic analysis of 4-hydroxy-2-nonenal-modified proteins in G93A-SOD1 transgenic mice-A model of familial amyotrophic lateral sclerosis. Free Radic. Biol. Med. 2005, 38, 960-968.

217. Sahara, S.; Yamashima, T. Calpain-mediated Hsp70.1 cleavage in hippocampal CA1 neuronal death. Biochem. Biophys. Res. Commun. 2010, 393, 806-811.

218. Connor, R.E.; Marnett, L.J.; Liebler, D.C. Protein-selective capture to analyze electrophile adduction of Hsp90 by 4-hydroxynonenal. Chem. Res. Toxicol. 2011, 24, 1275-1282.

219. Smathers, R.L.; Galligan, J.J.; Stewart, B.J.; Petersen, D.R. Overview of lipid peroxidation products and hepatic protein modification in alcoholic liver disease. Chem. Biol. Interact. 2011, 192, 107-112.

220. Jacobs, A.T.; Marnett, L.J. Systems analysis of protein modification and cellular responses induced by electrophile stress. Acc. Chem. Res. 2010, 43, 673-683.

221. Galligan, J.J.; Fritz, K.S.; Backos, D.S.; Shearn, C.T.; Smathers, R.L.; Jiang, H.; MacLean, K.N.; Reigan, P.R.; Petersen, D.R. Oxidative stress-mediated aldehyde adduction of GRP78 in a mouse model of alcoholic liver disease: Functional independence of ATPase activity and chaperone function. Free Radic. Biol. Med. 2014, 73, 411-420.

222. Brand, M.D.; Buckingham, J.A.; Esteves, T.C.; Green, K.; Lambert, A.J.; Miwa, S.; Murphy, M.P.; Pakay, J.L.; Talbot, D.A.; Echtay, K.S. Mitochondrial superoxide and aging: Uncoupling-protein activity and superoxide production. Free Radic. 2004, 203-213.

223. Esteves, T.C.; Brand, M.D. The reactions catalysed by the mitochondrial uncoupling proteins UCP2 and UCP3. Biochim. Biophys. Acta Bioenerget. 2005, 1709, 35-44.

224. Smith, A.M.O.; Ratcliffe, R.G.; Sweetlove, L.J. Activation and function of mitochondrial uncoupling protein in plants. J. Biol. Chem. 2004, 279, 51944-51952.

225. Echtay, K.S.; Esteves, T.C.; Pakay, J.L.; Jekabsons, M.B.; Lambert, A.J.; Portero-Otin, M.; Pamplona, R.; Vidal-Puig, A.J.; Wang, S.; Roebuck, S.J.; et al. A signalling role for 4-hydroxy-2-nonenal in regulation of mitochondrial uncoupling. EMBO J. 2003, 22, 4103-4110.

226. Echtay, K.S.; Pakay, J.L.; Esteves, T.C.; Brand, M.D. Hydroxynonenal and uncoupling proteins: A model for protection against oxidative damage. Biofactors 2005, 24, 119-130. 
227. Malingriaux, E.A.; Rupprecht, A.; Gille, L.; Jovanovic, O.; Jezek, P.; Jaburek, M.; Pohl, E.E. Fatty acids are key in 4-hydroxy-2-nonenal-mediated activation of uncoupling proteins 1 and 2. PLOS ONE 2013, 8, e77786.

228. Woyda-Ploszczyca, A.; Jarmuszkiewicz, W. Hydroxynonenal-stimulated activity of the uncoupling protein in Acanthamoeba castellanii mitochondria under phosphorylating conditions. Biol. Chem. 2013, 394, 649-658.

229. Aguirre, E.; Cadenas, S. GDP and carboxyatractylate inhibit 4-hydroxynonenal-activated proton conductance to differing degrees in mitochondria from skeletal muscle and heart. Biochim. Biophys. Acta Bioenerget. 2010, 1797, 1716-1726.

230. Jin, H.; Daly, D.S.; Marks, J.R.; Zangar, R.C. Oxidatively modified proteins as plasma biomarkers in breast cancer. Cancer Biomarkers 2013, 13, 193-200.

231. Pillon, N.J.; Vella, R.E.; Soulere, L.; Becchi, M.; Lagarde, M.; Soulage, C.O. Structural and functional changes in human insulin induced by the lipid peroxidation byproducts 4-hydroxy-2-nonenal and 4-hydroxy-2-hexenal. Chem. Res.Toxicol. 2011, 24, 752-762.

232. Lee, S.H.; Takahashi, R.; Goto, T.; Oe, T. Mass spectrometric characterization of modifications to angiotensin II by lipid peroxidation products, 4-oxo-2(E)-nonenal and 4-hydroxy-2(E)-nonenal. Chem. Res. Toxicol. 2010, 23, 1771-1785.

233. El-Bikai, R.; Welman, M.; Margaron, Y.; Cote, J.-F.; Macqueen, L.; Buschmann, M.D.; Fahmi, H.; Shi, Q.; Maghni, K.; Fernandes, J.C.; et al. Perturbation of adhesion molecule-mediated chondrocyte-matrix interactions by 4-hydroxynonenal binding: Implication in osteoarthritis pathogenesis. Arthritis Res. Ther. 2010, 12, doi:10.1186/ar3173.

234. Alzolibani, A.A.; al Robaee, A.A.; al-Shobaili, H.A.; Rasheed, Z. 4-Hydroxy-2-nonenal modified histone-H2A: A possible antigenic stimulus for systemic lupus erythematosus autoantibodies. Cell. Immunol. 2013, 284, 154-162.

235. Guichardant, M.; Taibi-Tronche, P.; Fay, L.B.; Lagarde, M. Covalent modifications of aminophospholipids by 4-hydroxynonenal. Free Radic. Biol. Med. 1998, 25, 1049-1056.

236. Guo, L.; Chen, Z.; Amarnath, V.; Davies, S.S. Identification of novel bioactive aldehyde-modified phosphatidylethanolamines formed by lipid peroxidation. Free Radic. Biol. Med. 2012, 53, 1226-1238.

237. Sowell, J.; Frei, B.; Stevens, J.F. Vitamin C conjugates of genotoxic lipid peroxidation products: Structural characterization and detection in human plasma. (Retracted article, see Proc. Natl. Acad. Sci. USA 2007, 104, 14543-14543) Proc. Natl. Acad. Sci. USA 2004, 101, 17964-17969.

238. Sowell, J.; Frei, B.; Stevens, J.F. Vitamin C conjugates of genotoxic lipid peroxidation products: Structural characterization and detection in human plasma. Proc. Natl. Acad. Sci. USA 2007, 104, 14543.

239. Miranda, C.L.; Reed, R.L.; Kuiper, H.C.; Alber, S.; Stevens, J.F. Ascorbic acid promotes detoxification and elimination of 4-hydroxy-2(E)-nonenal in human monocytic THP-1 cells. Chem. Res. Toxicol. 2009, 22, 863-874.

240. Amarnath, V.; Amarnath, K.; Davies, S.; Roberts, L.J. Pyridoxamine: An extremely potent scavenger of 1,4-dicarbonyls. Chem. Res. Toxicol. 2004, 17, 410-415. 
241. Hardas, S.S.; Sultana, R.; Clark, A.M.; Beckett, T.L.; Szweda, L.I.; Murphy, M.P.; Butterfield, D.A. Oxidative modification of lipoic acid by HNE in Alzheimer disease brain. Redox Biol. 2013, 1, 80-85.

242. Voulgaridou, G.-P.; Anestopoulos, I.; Franco, R.; Panayiotidis, M.I.; Pappa, A. DNA damage induced by endogenous aldehydes: Current state of knowledge. Mutat. Res. Fund. Mol. Mech. Mutagen. 2011, 711, 13-27.

243. Minko, I.G.; Kozekov, I.D.; Harris, T.A.; Rizzo, C.J.; Lloyd, R.S.; Stone, M.P. Chemistry and biology of DNA containing $1, \mathrm{~N}^{2}$-deoxyguanosine adducts of the $\alpha, \beta$-unsaturated aldehydes acrolein, crotonaldehyde, and 4-hydroxynonenal. Chem. Res. Toxicol. 2009, 22, 759-778.

244. Eckl, P.M.; Ortner, A.; Esterbauer, H. Genotoxic properties of 4-hydroxyalkenals and analogous aldehydes. Mutat. Res. Fund. Mol. Mech. Mutagen. 1993, 290, 183-192.

245. Karlhuber, G.M.; Bauer, H.C.; Eckl, P.M. Cytotoxic and genotoxic effects of 4-hydroxynonenal in cerebral endothelial cells. Mutat. Res. Fund. Mol. Mech. Mutagen. 1997, 381, 209-216.

246. Wang, H.; Kozekov, I.D.; Harris, T.M.; Rizzo, C.J. Site-specific synthesis and reactivity of oligonucleotides containing stereochemically defined $1, \mathrm{~N}^{2}$-deoxyguanosine adducts of the lipid peroxidation product trans-4-hydroxynonenal. J. Am. Chem. Soc. 2003, 125, 5687-5700.

247. Wacker, M.; Wanek, P.; Eder, E. Detection of 1, $\mathrm{N}^{2}$-propanodeoxyguanosine adducts of trans-4-hydroxy-2-nonenal after gavage of trans-4-hydroxy-2-nonenal or induction of lipid peroxidation with carbon tetrachloride in F344 rats. Chem. Biol. Interact. 2001, 137, 269-283.

248. Kozekov, I.D.; Turesky, R.J.; Alas, G.R.; Harris, C.M.; Harris, T.M.; Rizzo, C.J. Formation of deoxyguanosine cross-links from calf thymus DNA treated with acrolein and 4-hydroxy-2nonenal. Chem. Res. Toxicol. 2010, 23, 1701-1713.

249. Huang, H.; Kozekov, I.D.; Kozekova, A.; Wang, H.; Lloyd, R.S.; Rizzo, C.J.; Stone, M.P. DNA Cross-link induced by trans-4-Hydroxynonenal. Environ. Mol. Mutagen. 2010, 51, 625-634.

250. Huang, H.; Wang, H.; Kozekova, A.; Rizzo, C.J.; Stone, M.P. Formation of a $\mathrm{N}^{2}-\mathrm{dG}: \mathrm{N}^{2}-\mathrm{dG}$ carbinolamine DNA cross-link by the trans-4-Hydroxynonenal-derived $(6 \mathrm{~S}, 8 \mathrm{R}, 11 \mathrm{~S}) 1, \mathrm{~N}^{2}-\mathrm{dG}$ adduct. J. Am. Chem. Soc. 2011, 133, 16101-16110.

251. Choudhury, S.; Dyba, M.; Pan, J.; Roy, R.; Chung, F.-L. Repair kinetics of acrolein- and (E)-4-hydroxy-2-nonenal-derived DNA adducts in human colon cell extracts. Mutat. Res. Fund. Mol. Mech. Mutagen. 2013, 751, 15-23.

252. Choudhury, S.; Pan, J.; Amin, S.; Chung, F.L.; Roy, R. Repair kinetics of trans-4hydroxynonenal-induced cyclic $1, N^{2}$-propanodeoxyguanine DNA adducts by human cell nuclear extracts. Biochemistry 2004, 43, 7514-7521.

253. Winczura, A.; Zdzalik, D.; Tudek, B. Damage of DNA and proteins by major lipid peroxidation products in genome stability. Free Radic. Res. 2012, 46, 442-459. 
254. Janowska, B.; Komisarski, M.; Prorok, P.; Sokolowska, B.; Kusmierek, J.; Janion, C.; Tudek, B. Nucleotide excision repair and recombination are engaged in repair of trans-4-hydroxy-2nonenal adducts to DNA bases in Escherichia coli. Int. J. Biol. Sci. 2009, 5, 611-620.

255. Banerjee, S.; Christov, P.P.; Kozekova, A.; Rizzo, C.J.; Egli, M.; Stone, M.P. Replication bypass of the trans-4-Hydroxynonenal-derived (6S,8R,11S)-1, $N^{2}$-deoxyguanosine DNA adduct by the Sulfolobus solfataricus DNA polymerase IV. Chem. Res. Toxicol. 2012, 25, $422-435$.

256. Janowska, B.; Kurpios-Piec, D.; Prorok, P.; Szparecki, G.; Komisarski, M.; Kowalczyk, P.; Janion, C.; Tudek, B. Role of damage-specific DNA polymerases in M13 phage mutagenesis induced by a major lipid peroxidation product trans-4-hydroxy-2-nonenal. Mutat. Res. Fund. Mol. Mech. Mutagen. 2012, 729, 41-51.

257. Chen, H.J.C.; Gonzalez, F.J.; Shou, M.G.; Chung, F.L. 2,3-Epoxy-4-hydroxynonanal, a potential lipid peroxidation product for etheno adduct formation, is not a substrate of human epoxide hydrolase. Carcinogenesis 1998, 19, 939-943.

258. Lee, S.H.; Arora, J.A.; Oe, T.; Blair, I.A. 4-Hydroperoxy-2-nonenal-induced formation of 1, $N^{2}$-etheno-2'-deoxyguanosine adducts. Chem. Res. Toxicol. 2005, 18, 780-786.

259. Wang, Y.; Millonig, G.; Nair, J.; Patsenker, E.; Stickel, F.; Mueller, S.; Bartsch, H.; Seitz, H.K. Ethanol-induced cytochrome P4502E1 causes carcinogenic etheno-DNA lesions in alcoholic liver disease. Hepatology 2009, 50, 453-461.

260. Nair, J.; Srivatanakul, P.; Haas, C.; Jedpiyawongse, A.; Khuhaprema, T.; Seitz, H.K.; Bartsch, H. High urinary excretion of lipid peroxidation-derived DNA damage in patients with cancer-prone liver diseases. Mutat. Res. Fund. Mol. Mech. Mutagen. 2010, 683, 23-28.

261. Grune, T.; Muller, K.; Zollner, S.; Haseloff, R.; Blasig, I.E.; David, H.; Siems, W. Evaluation of purine nucleotide loss, lipid peroxidation and ultrastructural alterations in post-hypoxic hepatocytes. J. Physiol. Lond. 1997, 498, 511-522.

262. Grune, T.; Sommerburg, O.; Petras, T.; Siems, W.G. Postanoxic formation of aldehydic lipid-peroxidation products in human renal tubular cells. Free Radic. Biol. Med. 1995, 18, 21-27.

263. Blasig, I.E.; Grune, T.; Schonheit, K.; Rohde, E.; Jakstadt, M.; Haseloff, R.F.; Siems, W.G. 4-hydroxynonenal, a novel indicator of lipid-peroxidation for reperfusion injury of the myocardium. Am. J. Physiol. Heart Circ. Physiol. 1995, 269, H14-H22.

264. Grune, T.; Siems, W.G.; Zollner, H.; Esterbauer, H. Metabolism of 4-hydroxynonenal, a cytotoxic lipid-peroxidation product, in ehrlich mouse ascites-cells at different proliferation stages. Cancer Res. 1994, 54, 5231-5235.

265. Blasig, I.E.; Schoenheit, K.; Siems, W.G. Formation of 4-hydroxyalkenals by the reperfusion-injured rat-heart. Cell. Biochem. Mol. Asp. Reperfus. Injury 1994, 723, 462-465.

266. Siems, W.G.; Grune, T.; Esterbauer, H. 4-hydroxynonenal formation during ischemia and reperfusion of rat small-intestine. Life Sci. 1995, 57, 785-789. 
267. Poli, G.; Dianzani, M.U.; Cheeseman, K.H.; Slater, T.F.; Lang, J.; Esterbauer, H. Separation and characterization of the aldehydic products of lipid-peroxidation stimulated by carbon-tetrachloride or adp iron in isolated rat hepatocytes and rat-liver microsomal suspensions. Biochem. J. 1985, 227, 629-638.

268. Shao, C.X.; Roberts, K.N.; Markesbery, W.R.; Scheff, S.W.; Lovell, M.A. Oxidative stress in head trauma in aging. Free Radic. Biol. Med. 2006, 41, 77-85.

269. Williams, T.I.; Lynn, B.C.; Markesbery, W.R.; Lovell, M.A. Increased levels of 4-hydroxynonenal and acrolein, neurotoxic markers of lipid peroxidation, in the brain in Mild Cognitive Impairment and early Alzheimer's disease. Neurobiol. Aging 2006, 27, 1094-1099.

270. Negre-Salvayre, A.; Auge, N.; Ayala, V.; Basaga, H.; Boada, J.; Brenke, R.; Chapple, S.; Cohen, G.; Feher, J.; Grune, T.; et al. Pathological aspects of lipid peroxidation. Free Radic. Res. 2010, 44, 1125-1171.

271. Grune, T.; Siems, W.G.; Schneider, W. Accumulation of aldehydic lipid peroxidation products during postanoxic reoxygenation of isolated rat hepatocytes. Free Radic. Biol. Med. 1993, 15, 125-132.

272. Schwarzer, E.; Muller, O.; Arese, P.; Siems, W.G.; Grune, T. Increased levels of 4-hydroxynonenal in human monocytes fed with malarial pigment hemozoin-A possible clue for hemozoin toxicity. FEBS Lett. 1996, 388, 119-122.

273. Gil, L.; Siems, W.; Mazurek, B.; Gross, J.; Schroeder, P.; Voss, P.; Grune, T. Age-associated analysis of oxidative stress parameters in human plasma and erythrocytes. Free Radic. Res. 2006, 40, 495-505.

274. Selley, M.L.; Bartlett, M.R.; McGuiness, J.A.; Hapel, A.J.; Ardlie, N.G.; Lacey, M.J. Determination of the lipid-peroxidation product trans-4-hydroxy-2-nonenal in biological samples by high-performance liquid-chromatography and combined capillary column gas chromatography negative-ion chemical ionization mass-spectrometry. J. Chromatogr. Biomed. Appl. 1989, 488, 329-340.

275. Schmidt, H.; Grune, T.; Muller, R.; Siems, W.G.; Wauer, R.R. Increased levels of lipid peroxidation products malondialdehyde and 4-hydroxynonenal after perinatal hypoxia. Pediatr. Res. 1996, 40, 15-20.

276. Michel, P.; Eggert, W.; AlbrechtNebe, H.; Grune, T. Increased lipid peroxidation in children with autoimmune diseases. Acta Paediatr. 1997, 86, 609-612.

277. Uchida, K.; Itakura, K.; Kawakishi, S.; Hiai, H.; Toyokuni, S.; Stadtman, E.R. Characterization of epitopes recognized by 4-hydroxy-2-nonenal specific antibodies. Arch. Biochem. Biophys. 1995, 324, 241-248.

278. Yoritaka, A.; Hattori, N.; Uchida, K.; Tanaka, M.; Stadtman, E.R.; Mizuno, Y. Immunohistochemical detection of 4-hydroxynonenal protein adducts in Parkinson disease. Proc. Natl. Acad. Sci. USA 1996, 93, 2696-2701.

279. Okamoto, K.; Toyokuni, S.; Uchida, K.; Ogawa, O.; Takenewa, J.; Kakehi, Y.; Kinoshita, H.; Hattorinakakuki, Y.; Hiai, H.; Yoshida, O. Formation of 8-hydroxy-2'-deoxyguanosine and 4-hydroxy-2-nonenal-modified proteins in human renal-cell carcinoma. Int. J. Cancer 1994, $58,825-829$. 
280. Ando, Y.; Nyhlin, N.; Suhr, O.; Holmgren, G.; Uchida, K.; ElSahly, M.; Yamashita, T.; Terasaki, H.; Nakamura, M.; Uchino, M.; et al. Oxidative stress is found in amyloid deposits in systemic amyloidosis. Biochem. Biophys. Res. Commun. 1997, 232, 497-502.

281. Butterfield, D.A.; Reed, T.; Perluigi, M.; de Marco, C.; Coccia, R.; Cini, C.; Sultana, R. Elevated protein-bound levels of the lipid peroxidation product, 4-hydroxy-2-nonenal, in brain from persons with mild cognitive impairment. Neurosci. Lett. 2006, 397, 170-173.

282. Morikawa, S.; Kurauchi, O.; Tanaka, M.; Yoneda, M.; Uchida, K.; Itakura, A.; Furugori, K.; Mizutani, S.; Tomoda, Y. Increased mitochondrial damage by lipid peroxidation in trophoblast cells of preeclamptic placentas. Biochem. Mol. Biol. Int. 1997, 41, 767-775.

283. Hamilton, R.F.; Li, L.; Eschenbacher, W.L.; Szweda, L.; Holian, A. Potential involvement of 4-hydroxynonenal in the response of human lung cells to ozone. Am. J. Physiol. Lung Cell. Mol. Physiol. 1998, 274, L8-L16.

284. Liu, X.L.; Lovell, M.A.; Lynn, B.C. Detection and quantification of endogenous cyclic DNA adducts derived from trans-4-hydroxy-2-nonenal in human brain tissue by isotope dilution capillary liquid chromatography nanoelectrospray tandem mass spectrometry. Chem. Res. Toxicol. 2006, 19, 710-718.

285. Surh, J.; Kwon, H. Estimation of daily exposure to 4-hydroxy-2-alkenals in Korean foods containing n-3 and n-6 polyunsaturated fatty acids. Food Addit. Contam. 2005, 22, 701-708.

286. Ito, Y.; Kosuge, Y.; Sakikubo, T.; Horie, K.; Ishikawa, N.; Obokata, N.; Yokoyama, E.; Yamashina, K.; Yamamoto, M.; Saito, H.; et al. Protective effect of S-allyl-L-cysteine, a garlic compound, on amyloid beta-protein-induced cell death in nerve growth factor-differentiated PC12 cells. Neurosci. Res. 2003, 46, 119-125.

287. Glei, M.; Hofmann, T.; Kuster, K.; Hollmann, J.; Lindhauer, M.G.; Pool-Zobel, B.L. Both wheat (Triticum aestivum) bran arabinoxylans and gut flora-mediated fermentation products protect human colon cells from genotoxic activities of 4-hydroxynonenal and hydrogen peroxide. J. Agric. Food Chem. 2006, 54, 2088-2095.

288. Loguercio, C.; Federico, A.; Tuccillo, C.; Terracciano, F.; D’Auria, M.V.; de Simone, C.; Blanco, C.D. Beneficial effects of a probiotic VSL\#3 on parameters of liver dysfunction in chronic liver diseases. J. Clin. Gastroenterol. 2005, 39, 540-543.

289. Chowdhury, P.; Soulsby, M. Lipid peroxidation in rat brain is increased by simulated weightlessness and decreased by a soy-protein diet. Ann. Clin. Lab. Sci. 2002, 32, 188-192.

290. Skrzydlewska, E.; Ostrowska, J.; Farbiszewski, R.; Michalak, K. Protective effect of green tea against lipid peroxidation in the rat liver, blood serum and the brain. Phytomedicine 2002, 9, 232-238.

291. Leiphon, L.J.; Picklo, M.J., Sr. Inhibition of aldehyde detoxification in CNS mitochondria by fungicides. Neurotoxicology 2007, 28, 143-149.

292. Ullrich, O.; Grune, T.; Henke, W.; Esterbauer, H.; Siems, W.G. Identification of metabolic pathways of the lipid-peroxidation product 4-hydroxynonenal by mitochondria isolated from rat-kidney cortex. FEBS Lett. 1994, 352, 84-86.

293. Siems, W.G.; Zollner, H.; Grune, T.; Esterbauer, H. Metabolic fate of 4-hydroxynonenal in hepatocytes: 1,4-dihydroxynonene is not the main product. J. Lipid Res. 1997, 38, 612-622. 
294. Siems, W.G.; Grune, T. High capacity of secondary antioxidative pathways in thymocytes: Rapid detoxification of 4-hydroxynonenal. Int. J. Thymol. 1998, 6, 518-529.

295. Ullrich, O.; Huser, H.; Ehrlich, W.; Grune, T. Intracellular metabolism of 4-hydroxynonenal in primary cultures of rabbit synovial fibroblasts. Free Radic. Biol. Med. 1997, 22, 1153-1157.

296. Grune, T.; Siems, W.G.; Petras, T. Identification of metabolic pathways of the lipid peroxidation product 4-hydroxynonenal in in situ perfused rat kidney. J. Lipid Res. 1997, 38, $1660-1665$.

297. Grune, T.; Siems, W.; Kowalewski, J.; Zollner, H.; Esterbauer, H. Identification of metabolic pathways of the lipid-peroxidation product 4-hydroxynonenal by enterocytes of rat small-intestine. Biochem. Int. 1991, 25, 963-971.

298. Petras, T.; Siems, W.G.; Grune, T. 4-hydroxynonenal is degraded to mercapturic acid conjugate in rat-kidney. Free Radic. Biol. Med. 1995, 19, 685-688.

299. Kinter, M.; Roberts, R.J. Glutathione consumption and glutathione peroxidase inactivation in fibroblast cell lines by 4-hydroxy-2-nonenal. Free Radic. Biol. Med. 1996, 21, 457-462.

300. Esterbauer, H.; Zollner, H.; Lang, J. Metabolism of the lipid-peroxidation product 4-hydroxynonenal by isolated hepatocytes and by liver cytosolic fractions. Biochem. J. 1985, 228, 363-373.

301. Fauler, G. Investigations on the Metabolism of 4-Hydroxy-Alkenals. Ph.D. Thesis, University of Graz, Graz, Austria, 1987.

302. Siems, W.G.; Zollner, H.; Esterbauer, H. Metabolic pathways of lipid peroxidation product 4-hydroxynonenal in hepatocytes quantitative assessment of an antioxidative defense system. Free Radic. Biol. Med. 1990, 9, 110.

303. Schaur, R.J.; Zollner, H.; Esterbauer, H. Biological effects of aldehydes with particular attention to hydroxynonenal and malondialdehyde. In Membrane Lipid Peroxidation; Vigo-Pelfrey, C., Ed.; CRC Press: Boca Raton, FL, USA, 1991; Volume 3, pp. 141-163.

304. Esterbauer, H.; Schaur, R.J.; Zollner, H. Chemistry and biochemistry of 4-hydroxynonenal, malonaldehyde and related aldehydes. Free Radic. Biol. Med. 1991, 11, 81-128.

305. Siems, W.; Grune, T.; Zollner, H.; Esterbauer, H. Formation and metabolism of the lipid peroxidation product 4-hydroxynonenal in liver and small intestine. In Free Radicals: From Basic Science to Medicine; Poli, G., Albano, E., Dianzani, M.U., Eds.; Birkhäuser Verlag: Basel, Switzerland, 1993; pp. 89-101.

306. Siems, W.G.; Zollner, H.; Grune, T.; Esterbauer, H. Qualitative and quantitative-determination of metabolites of the lipid-peroxidation product 4-hydroxynonenal from hepatocytes, enterocytes and tumor-cells. Fresenius J. Anal. Chem. 1992, 343, 75-76.

307. Canuto, R.A.; Ferro, M.; Muzio, G.; Bassi, A.M.; Leonarduzzi, G.; Maggiora, M.; Adamo, D.; Poli, G.; Lindahl, R. Effects of aldehyde products of lipid peroxidation on the activity of aldehyde metabolizing enzymes in hepatomas. Adv. Exp. Med. Biol. 1993, 328, 17-25.

308. Canuto, R.A.; Muzio, G.; Maggiora, M.; Poli, G.; Biasi, F.; Dianzani, M.U.; Ferro, M.; Bassi, A.M.; Penco, S.; Marinari, U.M. Ability of different hepatoma-cells to metabolize 4-hydroxynonenal. Cell Biochem. Funct. 1993, 11, 79-86. 
309. Ferro, M.; Marinari, U.M.; Poli, G.; Dianzani, M.U.; Fauler, G.; Zollner, H.; Esterbauer, H. Metabolism of 4-hydroxynonenal by the rat hepatoma-cell line MH1C1. Cell Biochem. Funct. 1988, 6, 245-250.

310. Canuto, R.A.; Muzio, G.; Bassi, A.M.; Biocca, M.E.; Poli, G.; Esterbauer, H.; Ferro, M. Metabolism of 4 hydroxynonenal in hepatoma cell lines. In Advances in Experimental Medicine and Biology, Vol. 284. Enzymology and Molecular Biology of Carbonyl Metabolism 3, Proceedings of the Fifth International Workshop, West Lafayette, IN, USA, 12-15 June 1990; Weiner, H., Wermuth, M.B., Crabb, D.W., Eds.; Plenum Press: New York, NY, USA, 1991, pp. 75-84.

311. Srivastava, S.; Conklin, D.J.; Liu, S.Q.; Prakash, N.; Boor, P.J.; Srivastava, S.K.; Bhatnagar, A. Identification of biochemical pathways for the metabolism of oxidized low-density lipoprotein derived aldehyde-4-hydroxy trans-2-nonenal in vascular smooth muscle cells. Atherosclerosis 2001, 158, 339-350.

312. Srivastava, S.; Chandra, A.; Wang, L.F.; Seifert, W.E.; DaGue, B.B.; Ansari, N.H.; Srivastava, S.K.; Bhatnagar, A. Metabolism of the lipid peroxidation product, 4-hydroxytrans-2-nonenal, in isolated perfused rat heart. J. Biol. Chem. 1998, 273, 10893-10900.

313. Spitz, D.R.; Malcolm, R.R.; Roberts, R.J. Cytotoxicity and metabolism of 4-hydroxy-2nonenal and 2-nonenal in $\mathrm{H}_{2} \mathrm{O}_{2}$-resistant cell-lines-Do aldehydic by-products of lipidperoxidation contribute to oxidative stress. Biochem. J. 1990, 267, 453-459.

314. Meyer, M.J.; Mosely, D.E.; Amarnath, V.; Picklo, M.J. Metabohom of 4-hydroxy-trans-2nonenal by central nervous system mitochondria is dependent on age and $\mathrm{NAD}(+)$ availability. Chem. Res. Toxicol. 2004, 17, 1272-1279.

315. Canuto, R.A.; Muzio, G.; Biocca, M.E.; Dianzani, M.U. Oxidative-metabolism of 4-hydroxy2,3-nonenal during diethyl-nitrosamine-induced carcinogenesis in rat-liver. Cancer Lett. 1989, 46, 7-13.

316. Chen, J.J.; Yu, B.P. Detoxification of reactive aldehydes in mitochondria: Effects of age and dietary restriction. Aging Clin. Exp. Res. 1996, 8, 334-340.

317. Honzatko, A.; Brichac, J.; Murphy, T.C.; Reberg, A.; Kubatova, A.; Smoliakova, I.P.; Picklo, M.J. Enantioselective metabolism of trans-4-hydroxy-2-nonenal by brain mitochondria. Free Radic. Biol. Med. 2005, 39, 913-924.

318. Alary, J.; Gueraud, F.; Cravedi, J.-P. Fate of 4-hydroxynonenal in vivo: Disposition and metabolic pathways. Mol. Asp. Med. 2003, 24, 177-187.

319. Alary, J.; Bravais, F.; Cravedi, J.P.; Debrauwer, L.; Rao, D.; Bories, G. Mercapturic acid conjugates as urinary end metabolites of the lipid-peroxidation product 4-hydroxy-2-nonenal in the rat. Chem. Res. Toxicol. 1995, 8, 34-39.

320. Winter, C.K.; Segall, H.J.; Jones, A.D. Distribution of trans-4-hydroxy-2-hexenal and tandem mass-spectrometric detection of its urinary mercapturic acid in the rat. Drug Metab. Dispos. 1987, 15, 608-612.

321. Alin, P.; Danielson, U.H.; Mannervik, B. 4-hydroxyalk-2-enals are substrates for glutathione transferase. FEBS Lett. 1985, 179, 267-270. 
322. Danielson, U.H.; Esterbauer, H.; Mannervik, B. Structure-activity-relationships of 4-hydroxyalkenals in the conjugation catalyzed by mammalian glutathione transferases. Biochem. J. 1987, 247, 707-713.

323. Mitchell, D.Y.; Petersen, D.R. The oxidation of $\alpha, \beta$-unsaturated aldehydic products of lipid-peroxidation by rat-liver aldehyde dehydrogenases. Toxicol. Appl. Pharmacol. 1987, 87, 403-410.

324. Mitchell, D.Y.; Petersen, D.R. Inhibition of rat hepatic mitochondrial aldehyde dehydrogenase mediated acetaldehyde oxidation by trans-4-hydroxy-2-nonenal. Hepatology 1991, 13, 728-734.

325. Hartley, D.P.; Ruth, J.A.; Petersen, D.R. The hepatocellular metabolism of 4-hydroxynonenal by alcohol-dehydrogenase, aldehyde dehydrogenase, and glutathione-S-transferase. Arch. Biochem. Biophys. 1995, 316, 197-205.

326. Singhal, S.S.; Zimniak, P.; Awasthi, S.; Piper, J.T.; He, N.G.; Teng, J.I.; Petersen, D.R.; Awasthi, Y.C. Several closely-related glutathione-S-transferase isozymes catalyzing conjugation of 4-hydroxynonenal are differentially expressed in human tissues. Arch. Biochem. Biophys. 1994, 311, 242-250.

327. Berhane, K.; Widersten, M.; Engstrom, A.; Kozarich, J.W.; Mannervik, B. Detoxication of base propenals and other $\alpha, \beta$-unsaturated aldehyde products of radical reactions and lipid-peroxidation by human glutathione transferases. Proc. Natl. Acad. Sci. USA 1994, 91, $1480-1484$.

328. Fukuda, A.; Nakamura, Y.; Ohigashi, H.; Osawa, T.; Uchida, K. Cellular response to the redox active lipid peroxidation products: Induction of glutathione S-transferase P by 4-hydroxy2-nonenal. Biochem. Biophys. Res. Commun. 1997, 236, 505-509.

329. Yang, Y.Z.; Yang, Y.S.; Trent, M.B.; He, N.G.; Lick, S.D.; Zimniake, P.; Awasthi, Y.C.; Boor, P.J. Glutathione-S-transferase A4-4 modulates oxidative stress in endothelium: Possible role in human atherosclerosis. Atherosclerosis 2004, 173, 211-221.

330. Cao, Z.; Hardej, D.; Trombetta, L.D.; Li, Y. The role of chemically induced glutathione and glutathione S-transferase in protecting against 4-hydroxy-2-nonenal-mediated cytotoxicity in vascular smooth muscle cells. Cardiovas. Toxicol. 2003, 3, 165-177.

331. Kaiserova, K.; Srivastava, S.; Hoetker, J.D.; Awe, S.O.; Tang, X.L.; Cai, J.; Bhatnagar, A. Redox activation of aldose reductase in the ischemic heart. J. Biol. Chem. 2006, 281, 15110-15120.

332. Bander Jagt, D.L.; Kolb, N.S.; bander Jagt, T.J.; Chino, J.; Martinez, F.J.; Hunsaker, L.A.; Royer, R.E. Substrate specificity of human aldose reductase: Identification of 4-hydroxynonenal as an endogenous substrate. Biochim. Biophys. Acta 1995, 1249, 117-126.

333. Srivastava, S.K.; Ansari, N.H.; Hair, G.A.; Das, B. Aldose and aldehyde reductases in human-tissues. Biochim. Biophys. Acta 1984, 800, 220-227.

334. He, Q.; Khanna, P.; Srivastava, S.; van Kuijk, F.; Ansari, N.H. Reduction of 4-hydroxynonenal and 4-hydroxyhexenal by retinal aldose reductase. Biochem. Biophys. Res. Commun. 1998, 247, 719-722. 
335. Srivastava, S.K.; Yadav, U.C.S.; Reddy, A.B.M.; Saxena, A.; Tammali, R.; Shoeb, M.; Ansari, N.H.; Bhatnagar, A.; Petrash, M.J.; Srivastava, S.; et al. Aldose reductase inhibition suppresses oxidative stress-induced inflammatory disorders. Chem. Biol. Interact. 2011, 191, 330-338.

336. Koh, Y.H.; Park, Y.S.; Takahashi, M.; Suzuki, K.; Taniguchi, N. Aldehyde reductase gene expression by lipid peroxidation end products, MDA and HNE. Free Radic. Res. 2000, 33, 739-746.

337. Hoehn, A.; Koenig, J.; Grune, T. Protein oxidation in aging and the removal of oxidized proteins. J. Proteomics 2013, 92, 132-159.

338. Grune, T.; Davies, K.J.A. Breakdown of oxidized proteins as a part of secondary antioxidant defenses in mammalian cells. Biofactors 1997, 6, 165-172.

339. Grune, T.; Reinheckel, T.; Davies, K.J.A. Degradation of oxidized proteins in mammalian cells. FASEB J. 1997, 11, 526-534.

340. Laurent, A.; Perdu-Durand, E.; Alary, J.; Debrauwer, L.; Cravedi, J.P. Metabolism of 4-hydroxynonenal, a cytotoxic product of lipid peroxidation, in rat precision-cut liver slices. Toxicol. Lett. 2000, 114, 203-214.

341. Alary, J.; Debrauwer, L.; Fernandez, Y.; Paris, A.; Cravedi, J.P.; Dolo, L.; Rao, D.; Bories, G. Identification of novel urinary metabolites of the lipid peroxidation product 4-hydroxy-2nonenal in rats. Chem. Res. Toxicol. 1998, 11, 1368-1376.

342. Li, Q.; Sadhukhan, S.; Berthiaume, J.M.; Ibarra, R.A.; Tang, H.; Deng, S.; Hamilton, E.; Nagy, L.E.; Tochtrop, G.P.; Zhang, G.-F. 4-Hydroxy-2(E)-nonenal (HNE) catabolism and formation of HNE adducts are modulated by beta oxidation of fatty acids in the isolated rat heart. Free Rad. Biol. Med. 2013, 58, 35-44.

343. Kubatova, A.; Murphy, T.C.; Combs, C.; Picklo, M.J. Astrocytic biotransformation of trans-4-hydroxy-2-nonenal is dose-dependent. Chem. Res. Toxicol. 2006, 19, 844-851.

344. Alary, J.; Debrauwer, L.; Fernandez, Y.; Cravedi, J.P.; Rao, D.; Bories, G. 1,4dihydroxynonene mercapturic acid, the major end metabolite of exogenous 4-hydroxy-2nonenal, is a physiological component of rat and human urine. Chem. Res. Toxicol. 1998, 11, $130-135$.

345. Laurent, A.; Alary, J.; Debrauwer, L.; Cravedi, J.P. Analysis in the rat of 4-hydroxynonenal metabolites excreted in bile: Evidence of enterohepatic circulation of these byproducts of lipid peroxidation. Chem. Res. Toxicol. 1999, 12, 887-894.

346. Ji, B.; Ito, K.; Suzuki, H.; Sugiyama, Y.; Horie, T. Multidrug resistance-associated protein2 (MRP2) plays an important role in the biliary excretion of glutathione conjugates of 4-hydroxynonenal. Free Rad. Biol. Med. 2002, 33, 370-378.

347. Esterbauer, H.; Ertl, A.; Scholz, N. Reaction of cysteine with $\alpha, \beta$-unsaturated aldehydes. Tetrahedron 1976, 32, 285-289.

348. Grune, T.; Michel, P.; Sitte, N.; Eggert, W.; AlbrechtNebe, H.; Esterbauer, H.; Siems, W.G. Increased levels of 4-hydroxynonenal modified proteins in plasma of children with autoimmune diseases. Free Rad. Biol. Med. 1997, 23, 357-360. 
349. Peiro, G.; Alary, J.; Cravedi, J.P.; Rathahao, E.; Steghens, J.P.; Gueraud, F. Dihydroxynonene mercapturic acid, a urinary metabolite of 4-hydroxynonenal, as a biomarker of lipid peroxidation. Biofactors 2005, 24, 89-96.

350. Volkel, W.; Sicilia, T.; Pahler, A.; Gsell, W.; Tatschner, T.; Jellinger, K.; Leblhuber, F.; Riederer, P.; Lutz, W.K.; Gotz, M.E. Increased brain levels of 4-hydroxy-2-nonenal glutathione conjugates in severe Alzheimer's disease. Neurochem. Int. 2006, 48, 679-686.

351. Ramana, K.V.; Bhatnagar, A.; Srivastava, S.; Yadav, U.C.; Awasthi, S.; Awasthi, Y.C.; Srivastava, S.K. Mitogenic responses of vascular smooth muscle cells to lipid peroxidationderived aldehyde 4-hydroxy-trans-2-nonenal (HNE) - Role of aldose reductase-catalyzed reduction of the HNE-glutathione conjugates in regulating cell growth. J. Biol. Chem. 2006, 281, 17652-17660.

352. Ramana, K.V.; Willis, M.S.; White, M.D.; Horton, J.W.; DiMaio, J.M.; Srivastava, D.; Bhatnagar, A.; Srivastava, S.K. Endotoxin-induced cardiomyopathy and systemic inflammation in mice is prevented by aldose reductase inhibition. Circulation 2006, 114, 1838-1846.

353. Srivastava, S.; Ramana, K.V.; Tammali, R.; Srivastava, S.K.; Bhatnagar, A. Contribution of aldose reductase to diabetic hyperproliferation of vascular smooth muscle cells. Diabetes 2006, 55, 901-910.

354. Tammali, R.; Ramana, K.V.; Singhal, S.S.; Awasthi, S.; Srivastava, S.K. Aldose reductase regulates growth factor-induced cyclooxygenase-2 expression and prostaglandin E2 production in human colon cancer cells. Cancer Res. 2006, 66, 9705-9713.

355. Ronis, M.J.J.; Butura, A.; Sampey, B.P.; Shankar, K.; Prior, R.L.; Korourian, S.; Albano, E.; Ingelman-Sundberg, M.; Petersen, D.R.; Badger, T.M. Effects of $\mathrm{N}$-acetylcysteine on ethanol-induced hepatotoxicity in rats fed via total enteral nutrition. Free Rad. Biol. Med. 2005, 39, 619-630.

356. Arakawa, M.; Ushimaru, N.; Osada, N.; Oda, T.; Ishige, K.; Ito, Y. N-acetylcysteine selectively protects cerebellar granule cells from 4-hydroxynonenal-induced cell death. Neurosci. Res. 2006, 55, 255-263.

357. Zheng, J.Y.; Mutcherson, R.; Helfand, S.L. Calorie restriction delays lipid oxidative damage in Drosophila melanogaster. Aging Cell 2005, 4, 209-216.

358. Lee, J.H.; Jung, K.J.; Kim, J.W.; Kim, H.J.; Yu, B.P.; Chung, H.Y. Suppression of apoptosis by calorie restriction in aged kidney. Exp. Gerontol. 2004, 39, 1361-1368.

359. Zainal, T.A.; Oberley, T.D.; Allison, D.B.; Szweda, L.I.; Weindruch, R. Caloric restriction of rhesus monkeys lowers oxidative damage in skeletal muscle. FASEB J. 2000, 14, 1825-1836.

360. Shoeb, M.; Ansari, N.H.; Srivastava, S.K.; Ramana, K.V. 4-Hydroxynonenal in the pathogenesis and progression of human diseases. Curr. Med. Chem. 2014, 21, 230-237. 


\section{Index}

(6-4) photoproducts1 40, 163

$17 \beta$-estradiol 238, 253

2,5 DMBQ 55

20-hydroxyeicosatetraenoic acid (20-HETE) 252

\section{4,5 DMBQ 55}

4-hydroxynonenal 11,143, 168, 199, 204, $219,223,231,332,479-481,483-504$

8-oxoguanine $163,333,387$

abdominal adipose tissue 282

adaptation $38,44,63,170,198,212,216,219$, $220,229,230,232,274,326,327,329$, $335,338,368,371,377,378,385,386$

adaptive mistranslation 337

Adaptive Promiscuity 326

adaptive response 198, 203, 219, 220, 489

adipocytes 279, 280-286, 294, 295, 401, 438

adipokine 281, 284

adiponectin 220, 279, 281, 286, 295

advanced glycation endproducts (AGEs) 16, 307

AGE 139, 140, 290-293, 295, 296-310, 313, 314

Ageing 33, 146, 156, 160, 166, 190, 229, 337

Aging 17, 59, 95, 121-123, 145-147, 149, $152-154,156,158,160,168,227,267$, $272,286,306,331,332,337,379,480$, 482, 485, 489, 498, 501, 504

Albumin 117, 410, 437

alpha-synuclein 34,492

Alzheimer's disease 16-20, 31, 33-35, 247, 258, 332, 432, 451, 454, 472, 489, 490, $492,498,504$

aminotriazole 89

Amplex Red 63, 64, 81

angiotensin II 88, 165, 251, 258, 273, 282, $316,447,495$

anti-apoptotic 42, 97, 295

anti-inflammatory 97, 116, 363, 388, 389, 402-405, 414

antimycin A 67

antioxidants $86,88,93,134,144,151,152$, $154,156,163,168,173,174,179,212$, $214,216,218,220,223,225,229,242$, 268, 289, 332, 367, 372-374, 405, 487

Apolipoprotein B-100 410, 438 apoptosis $15,38,42,45,49,57-59,80,93$, $98,107,116-118,123,130,134,138,141$, $142,151,161,164,167,175,179,193,194$, $196,201,202,211,216,230,255,256,268$, $269,276,277,295,300,301,310,313-315$, $318,324,331,336,344,346,347,350,360$, $361,362,386,414,422,423,425,428,437$, 474, 482, 488, 491, 504

appressorium 53

arachidonic acid 128, 144, 151, 215, 249, $251,273,412,416,421,473$

ascorbic acid 116, 144, 152, 218, 230, 318, $352,420,448,484$

Aspergillus nidulans 47, 60

asthma $15,31,241$

atherosclerosis 20, 63, 92, 143, 252, 254, 276, $283,300,311,313,402,414,442,502$

autism 241,267

autophagy $63,127,139,150,161,171-174$, $178,192,194,340,344-347,349,350$, $356,357,360,361,422,482$

\section{BACH1 101}

BACH2 101

base excision repair (BER) 140, 450

Beta cell dysfunction 288

Bilirubin 97, 104, 105, 114, 116, 118, 119

biliverdin reductase 97,116

biomarker analysis 14

BK channels 235, 237-241, 243-257, 262$265,267,272,273,277$

blood flow 251, 252, 253, 280, 282-285

bottom-up 3, 4, 6, 7, 9, 27

BRL3A 100-107, 109-113

BVR 97, 99, 100

Ca-homeostasis 249

calcium bilirubinate 108

calcium gradient 121, 124, 143

calcium-activated potassium channels 262 264, 266-269, 272, 274

calpain 444

Candida albicans 50, 327, 337, 346, 347, $354,360,364,366,379-387$

Cap1 369-372, 377, 378, 382, 383

carbon monoxide 97, 117, 245, 249, 269, 270,271 
carbonylation 14, 19, 24, 27, 29, 30, 33, 34, $136,157,158,417,437,444,481,484$, $485,490,494$

cardiovascular disease 34, 93, 94, 161, 298, 312

carnosine 420, 429, 430, 447, 475, 481, 487, 488

carotid body 246,271

catalase $39,84,87,89,90,91,132,137,143$, $144,154,157,214,217,223,289,366-$ $368,371,372,382,455$

Cathepsin C 347

caveolae 251, 273

cell metabolism 113, 238, 242, 243, 324

cell signaling 58, 59, 94, 141, 150, 219, 356, 358,413

Cellobiose dehydrogenase 53

cellular signaling 45, 242, 257, 268, 308, 354

cellular stress $2,175,181,335,419$

cellulose $53,55,61,62$

ceramide $148,249,273,346$

cerebellar ataxia 241

cerebral arterial muscle cells 252, 274

ceruloplasmin $87,93,189,446$

cGMP 244, 249, 252, 254, 255, 259, 264

channel gating 237, 254, 264

channelopathies 255,267

chaperonine $41,42,45$

charybdotoxin $239,246,247,249,250,253$, $265,273,276$

chelerythrine 252

Chemical tagging 5

chloramine-T 242, 250, 251

chlorotyrosine $3,11,12,16,20$

c-myc 43, 183, 428, 477

cobalt chloride 281

cochlea 235

collagen 122, 123, 130, 139, 142, 145, 146, $162,298,299,300,312,313,315,348$, $432,447,475$

combinatorial stress 367,371

Conditional promiscuity 324

conductance 237, 238, 246, 251, 254, 259, $260,262-275,277,343,350,445,446$, 495

CoQ10 121, 126, 130, 131, 134, 144

corn stover 53, 54

cornified envelope 121, 124, 129, 143, 147, 149,152

coupling state 65

CRBP 107, 108
CREB 43, 222, 223, 226, 259, 357

$\mathrm{Cu} / \mathrm{ZnSOD} 89,381$

cyclobutane pyrimidine dimers 140,163

cysteine oxidation 14, 19, 20, 27, 28, 375

cystic fibrosis $241,355,359,452$

cytochrome c $53,86,92,104,125,126,176$, 199, 253, 295-297, 426, 430, 485, 488

cytochrome p450 84, 150

cytochrome P450 2A 105

cytochrome P450 family 125, 127, 144, 424

cytokines $88,95,97,175,190,196,209,292$, $344,365,388,393,395,399,401,402$, 403

de novo sequencing 9, 10, 19, 26, 27

Diabetes 17, 93, 119, 161, 196, 225, 273, 276, 285-288, 290, 297, 304, 306-316, 487, 490, 491, 504

diabetes mellitus 17, 33, 135, 177, 273, 279, 281, 284, 287, 291, 304, 306, 310, 311

diabetic complications 161, 287, 288, 291, 292, 297-304, 306, 311

diabetic nephropathy 299, 302, 307

diabetic retinopathy $258,298,300,301,311$

diagnostics $20,36,145,455$

dithiothreitol (DTT) 245, 248

DMSO 66-68, 102, 103, 105, 106, 108-110, 112,392

DNA damage $86,92,140,142,144,152$, $162,164,165,167,219,230,268,322$, $328,366,369,376-378,387,429,449$, 450, 496, 497

DNA Damage Checkpoint 376

DOCK proteins 347

double strand breaks 140

dslo 245

DTNB 242, 243, 245, 248, 259, 453

EGF 43

electrical tuning 235

electron spin resonance spectroscopy 89

electron transfer system capacity 63

endoplasmic reticulum $42,46,58,97,118$ $120,125-127,144,150,158,241,283$, $342,437,478,483$

Endoplasmic reticulum stress 119, 158

Enzyme Promiscuity 317

Epichloe festucae 46, 53, 61

epilepsy 235, 241, 262, 267

ER 40, 42, 43, 48, 58, 61, 97, 98, 99, 100, $106-109,113,119,121,126-128,137$, $141,158,161,162,259,346,437,444$, 445,491 
ER stress 58, 97, 107, 108, 109, 119, 161, 437, 444, 445, 491

estrogen 222, 223, 238, 253, 263, 274

ETosis 340, 341, 344, 347, 349-353

evolution $37,39,58,60,90,263,278,317$, $320,322,326,329,330,333,337,339$

exercise 212-216, 218, 220-234, 270, 282, 388

Extracellular Matrix 411, 447

extracellular SOD 87

Extracellular Traps 340

extrinsic aging 121, 122

fat $132,137,218,279,280-282,284,285$, $291,407,418,429,456$

female sterile 47

Fenton reaction $54,55,85,86,92,121,128$, $133,140,144,151,171,177,178,180$, $289,318,345$

Ferric reductase 60

filaggrin 124, 125

flavonoids 332, 394, 402, 406-408

force generation $212,215,217,223$

fragmentation 1, 3, 5-8, 10-12, 102, 103, 135, 201

functional food 403

gallstones 108, 119

genome stability 496

GGLG loop 41

glia cells 246

glibenclamide 258

Gloeophyllum trabeum 55, 62

glucose regulated protein 78 (GRP78) 107, 108

glucose transporter 282

glutathione 2, 38, 39, 44, 45, 89, 121, 126, $129,130,132,133,137,143,144,152$, $154,180,181,205,208,209,214,217$, $218,222,223,228,229,234,242,245$, 246, 248, 257, 269, 270, 271, 281, 286, $289,319,320,332,333,335,366,370$, $372,382,418,420,423,427,428,429$, $431,432,454,455,456,457,458,459$, $460,461,464,465,468,469,470-472$, $474,476,479,481,486,487-489,500$ 504

glutathione peroxidase $39,44,89,133,144$, 154, 214, 217, 223, 286, 320, 333, 366, $370,372,427,455,461,488,500$

Glutathione-S-Transferase 410, 432

Gpx3 44, 370-372, 378, 382

GRP78 107, 108, 111, 445, 474, 494
$\mathrm{H}_{2} \mathrm{O}_{2}$ flux 63, 64, 68-75, 77

$\mathrm{H}_{2} \mathrm{O}_{2} / \mathrm{O}$ flux ratio $68,70,73,75$

$\mathrm{H}_{2} \mathrm{~S} 244,245,255,259,262,264,269,425$

Haber-Weiss reaction 92, 128, 365

hair cells 235, 240, 264

Haskap 388, 389, 395, 399, 405

Heat Shock Proteins 411, 443

HEK 293T cells 64, 66-73, 75

HEK cells 70, 245, 246, 250

heme 48, 54, 86, 97-102, 104, 114, 116, 117, $128,131,132,143,169,170,172,175-$ $179,182,185,186,188,191,192,197$, $198,200,201,207,237,248,433,476$

Heme Degradation 97

Heme Oxygenase 97, 110-112

hemeoxygenase- 1257

hemicellulose 53, 55

hemin 97, 98, 100-106, 109, 110, 112, 113, 196, 248

hemoxygenase- 2249

HIF 85, 87, 89, 94, 210, 233, 281, 283

High-Resolution Respirometry 63, 77, 81

histidine kinase 374, 385

HNE 177, 181, 185, 199, 204, 219, 223, 409, $411,412,413,417-474,476,480-486$, 489-491, 493, 496, 503, 504

HO- 197, 99-101, 103, 105, 107, 108, 111, $117,119,143,257,407,433,474$

HO-2 100, 249, 259

HOC 86, 90, 96, 343, 345, 350, 351, 362, 366

Hog1 369, 372-375, 378, 381, 384-386

homeostasis 38, 56-58, 98, 133, 135, 145, $148,156,169,170,173,176,188,189$, $192,195,197,199,200,202,203,206$, $207,210,218,234,245,247,249,252$, $253,268,271,318,331,385,426,428$, 442,486

hslo 245, 265, 269

HSP32 97

hydrogen peroxide $1,15,25,36,37,39,43-$ $45,47,49,58,59,63,79,80,81,82,85$, $91,92,96,127,128,131-133,135,141$, $151,158,165,194,203,204,208,217$, $225,226,228,245,249,270,288,318$, $336,345,359,362,364,365,380-382$, $384,386,387,421,428,433,444,476$, 482, 499

hydroxyl radical 37, 54, 62, 84, 91, 93, 128, $138,140,141,150,158,199,289,318$, $331,345,362,413$

hyperoxidation 45 
hyperpolarised buds 369,376

hypertension $95,210,241,252,255,262$, $276,283,312$

hyphal growth $37,46,52$

hypochlorite anion 47

hypochlorous acid 2, 86, 91, 212, 342, 343, $345,350,358,361,362,366$

hypoxia $29,85,97,119,165,170,183,189$, $210,246,248,252,253,256,270,272$, 274, 275, 277, 279, 280-286, 387, 498

Hypoxia-inducible factor 210

IL6 107, 108, 111

IL-6 88, 175, 215, 281, 283, 388, 390, 393, $395,399-403$

IMR 46, 49, 50, 51, 52

inflammasome 88,95

inflammation $32,87,88,119,122,132,133$, $153,162,169,170,175,189,190,256$, 279, 283, 284-286, 292-295, 300, 301, $309,314,341,350,351,357,359,360$, $388,389,393,399,401-407,429,431$, $448,473,487,504$

inflammatory response $88,95,107,119,120$, 283, 292, 307, 309, 402, 408

innate immune defense 340

innate immune system $84,86,90$

innate immunity $35,88,354,355,359$

iNOS 32, 88, 116, 220, 223, 296, 297, 303, $345,366,395,402,403,407$

insulin $85,87,94,96,119,143,166,214$, $216,220,221,226,232,279-288,292-$ 297, 302, 304, 306, 307, 309-311, 314, $396,406,434,445,446,490,495$

insulin resistance 220, 279, 280-284, 286, 287, 288, 292-295, 302, 304, 307, 309, $310,396,406,434$

integral membrane reductases 51

interleukin $6107,108,292,300$

interstitium 280, 281

intrinsic aging 121,122

iron metabolism 169, 177, 179, 181-183, 185, 187-192, 196, 198, 202, 203, 205, 207, 209, 336

ischemia $33-35,61,63,80,119,127,191$, $230,247-249,253,254,256,274-277$, $419,423,427,444,451,453,464,481$, 497

Ischemic Heart Disease 300

ischemic preconditioning 219, 249, 272, 464

isobaric tags for relative and absolute quantification 13, 21
Isolated Mitochondria 63

JC-1 102, 103, 110

label-dependent approaches 11

Label-free approaches 7

laccase 53-55

LC-MS/MS 2, 7, 17-19, 21, 32, 391, 395, 427,474

LEAK respiration $65,67,68,70,74,77$

leptin 119, 271, 279, 281, 295

lipid peroxidation $27,85,86,93,130,131$, $133,134,143,144,152,159,160,168$, $173,174,176,178,181,192,195,204$, $215,216,218,219,224,230,290,291$, $304,318,320,332,362,409,412-415$, $418,426,428-430,441,442,454,455$, $459,461,477,479-483,485,486,489$, 491-504

lipid vacuole 280

Lipofuscin 135, 137, 158-160, 192

lipolytic signaling 281

lipopolysaccharide 6, 22, 95, 109, 209, 388$390,400,401,405,407$

lipoprotein 20, 21, 35, 140, 162, 208, 255, $281,285,438,441,474,477,501$

lipoprotein lipase 281, 285

lipoxygenase 128, 144, 151, 153, 249, 421, $430,431,488$

liquid chromatography $21,24,31,32,118$, 290, 291, 298, 303-305, 307, 388, 390, $391,427,454,476,477,499$

Lolium perenne 53

Lonicera caerulea 388, 389, 405, 406

loricrin 124, 125, 130, 148

lysophosphatidylcholine 255

macrophage 6, 46, 47, 96, 159, 198, 206, 209, $279,283,286,301,352,354,358,362$, $368,369,372,377,382,388,392,396$, $401,405,407,429,442,487$

Magnaporthe grisea 50, 53

MAPK 17, 44, 215, 219, 221, 224, 344, 345, $346,347,348,350,359,362,373,375$, $385,418,447,474$

MAPK/ERK 344, 346, 347, 348, 350

Mascot 7, 8, 9

mass spectrometry $1-3,7,13,15,16,21-28$, $30-32,34,118,147,291,304,305,307$, $427,439,443,446,459,470,472,475$, 476, 477, 485, 491, 499

matrix metalloproteinases 142, 283

membrane potential $64,80,81,102,240$, $248,255,275,430,474$ 
membrane resting potential $235,236,240$, 246, 251, 257

mercapturic acid 459, 467-470, 472, 475, 500, 501, 503, 504

Metabolic Inaccuracy 328

metabolic network 317, 323, 329, 330

metabolic side products 317,323

metabolic syndrome $284,286,325,388,401$, 405, 413, 479

Metabolism 151, 169, 190, 209, 232, 281, $285,306,310,311,317,321,330,406$, $411,412,455,457,458,459,471,472$, 497, 500, 501, 503

Metabolite Damage 321

metabolite repair 329, 332, 333

Methionine oxidation 20, 34, 93, 250, 332

Methionine sulfoxide 157, 244, 260, 332

methylglyoxal 161, 290, 291, 293-295, 298$300,305,306,310-312,423,449$

Michael adducts 11, 420, 437, 439, 441, 443, 446

mitochondria $19,33,34,37,40,45,60,63-$ $65,68,72,73,75,76,78-80,82,83,86-$ $89,91,94,95,97-105,113,114,117,120$, $121,124-129,131,132,138,145,151$, $154,171,172,173,176-180,191,192$, $198,199,202,204,213,214,221,225-$ $227,232,241,248,253,256,268,272$, $275,323,326,337,342,349,350,361$, $373,374,423,428,430,434,436,445$, 446, 449, 455-460, 466, 469, 486, 490, $495,499,501$

mitochondrial electron transport chain 125 , 144, 225, 296

mitochondrial permeability transition pore 88,277

mitochondrial ROS 64, 80, 87, 88, 96, 223, $350,352,361,403$

MitoQ 88

mitoTEMPO 88

Mkc1 373

MMPs 122

MnSOD 46, 64, 89, 176, 234, 289

mode of action 364,402

monocyte 95, 196, 227, 283, 292, 301, 308, $314,315,448$

monooxygenase 84,150

mouse brain $19,30,33,64,70,72,74,75,80$, $117,187,271$

mouse heart 34, 64, 75, 80, 204, 436

MPO 91, 340, 343, 345, 346, 348-351, 366
MPTP 250, 260

MSRA 242, 244, 260

mtAOX 88

mtBK 248, 253, 254, 256, 260

mTOR 343, 344, 345, 347, 349, 357, 360, 422

mtROS 64, 88, 403

MTSEA 242, 243, 260

MTT assay 106, 107

multiple reaction monitoring $12,22,28$

muscle $17,19,33,80-83,96,118,165,174$, 196, 212-218, 220, 221, 223-235, 239, $240,245,248,249,251,252,255,257$, $266,268-270,273,274,278,279,281-$ 283, 293-295, 308-310, 402, 417, 425, $429,440,441,446,457,461,471,473$, 474, 478, 480, 481, 495, 501, 502, 504

Myostatin 215, 227

n-6 polyunsaturated fatty acids 499

NADPH 15, 37-39, 42-48, 50-53, 55, 56, $59-61,84,88,91,95,111,112,121,125-$ $128,132,143,144,151,179,181,184$, $213,214,218,224,226,227,254,292$, $303,308,309,320,322,328,334,338$, $340,343-352,355-359,361,362,365$, $369,372,379,380,423,424,465,468$

NADPH oxidase 43, 45-48, 50-52, 59-61, $91,95,144,226,227,292,308,309,340$, $343-352,355,357-359,361,362,365$, $369,372,379,380$

Narrow-Window Extracted Ion

Chromatograms 12

nBK channels 241

$N$-chlorosuccinimid 242, 260

NER 140, 450, 474

Nernst equation 38

NETs 340, 341, 342, 345, 346, 349, 352-355, 359

Neurospora crassa 47, 50, 61

Neutral loss scanning 11

neutrophil elastase $342,343,345$

neutrophils 93, 122, 213, 214, 226, 340-343, $345-350,352-365,367-369,375,380$, $381,387,388$

new technologies 21

NF-кB 87, 89, 96, 123, 141, 175, 196, 217, 219-221, 224, 227, 231, 283, 308, 353, $363,395,401,402$

nitrated amino acids 86 
nitric oxide $29,37,84,86,91,94,95,119$, $127,152,178,182,207-209,220,223$, 242, 245, 249, 269, 276, 282, 283, 288, $296,303,311,314,315,318,331,345$, $351,366,390,392,395,401-403,406$, 408

nitric oxide synthase $84,91,95,209,220,223$, $283,296,303,314,315,345,366,392$, 395,403

Nitrotyrosine 160

NLRP3 88, 95

NO synthase 254, 260, 301

non-canonical metabolites 319, 323, 330

Non-Enzymatic Reactions 317

NOS 84, 91, 182, 183, 260

NOX1 48, 251, 344

NOX2 46, 344, 349, 350, 358

NOX4 95, 344

NS11021 253, 254

NS-1619 253

nucleotide excision repair (NER) 140, 450

obesity $33,204,225,282,283,284,285,286$, 293, 388, 407, 413, 418, 479, 480

obstructive sleep apnea 32, 281, 283

$\mathrm{OH}$ radicals $85,176,180$

ornithine decarboxylase 256

oxidative burst 213,367

oxidative phosphorylation $63,65,81,96$, $343,350,445$

oxidative post-translational modification 22

oxidative stress $1,12,13,19,20,24,28,33$, $34,37-40,42,43,45,47,51,54-58,61$, $63,80,81,84,86,87,90,94,108,116-$ $118,121,123,125,135,136,139,140$, $142,143,145,147,150,153,155,156-$ $159,162,167,169-171,173-175,177-$ $179,181,184,185,189,191,192,194$, 196-198, 201-205, 207-209, 212, 215, $217,219,220,222,224-231,234,235$, $241,245,246,248,250,251,254-258$, $268,269,275,276,278,279,281,283$, 284, 286, 287, 288, 290-292, 295, 306, $307,309,311,313,314,318,320,322$, $326,328,331,332,337,338,350,357$, $361,364,366-369,371-375,377-383$, $387,405,407,409,413,417,418,422$, $423,425,427-431,433,434,438,440$ $443,447,449,451,453,455,461,465$, $472,473,480,485-487,489,492,498$, 501-503
OXPHOS 63, 65, 67, 68, 70-73, 75, 77, 79, 81-83, 343, 349-351

oxygen radicals 54, 56, 127, 132, 163

oxygen transport 280

patch clamp 236, 248, 257

Pathology 199, 240

paxilline 238, 247, 254, 271

PCMB 242

PCR 108, 111, 115

PDGF 315, 411, 441, 446, 474

PDI 40, 42, 43, 46, 127, 150, 410, 425, 426, $437,444,474$

pentosidine 290, 291, 298, 305, 306, 312

Permeabilized Cells 63

peroxiredoxin $15,30,37,38,40,43-45,58-$ $60,155,158,384,386$

peroxisomes $40,125,127,138,150,173,342$ peroxynitrite $1,20,37,40,84,86,90,91$, $182,207,318,331,332,345,366$

PGC-1 $\alpha$ 215, 220-224, 227, 232-234

PGI2 synthase 251

Phanerochaete chrysosporium 53, 54, 62 phenotypic variability 329

phorbol ester 252, 309

phorbol-12-myristate-13-acetate 252

phospholipase A2 213, 214, 215, 224, 227

phosphotyrosine phosphatase 43, 45

photoaging 122, 128-131, 142, 144, 151, 152,163

PKC 88, 90, 141, 249, 252, 253, 260, 293, $303,309,343,347,360$

plBK 256, 260

Podospora anserina 51, 61

Polyamines 255, 256, 262

Polyphenols 388, 389, 391

pores 94, 174, 236, 256

Postia placenta 55, 62

postprandial 282, 285

preconditioning 230, 247, 256, 259, 268, $272,274,276,277,464$

precursor ion scanning 11

pressure 144, 235, 240, 250, 253, 255, 279, $291,304,329,358,426$

programmed cell death $38,142,166,366$

prostacyclin 2 (PGI2) 251

prostacyclin synthase 87

proteasome 137, 138, 139, 158, 159, 174, $182,195,206,207,370,427,486$

protein carbonyls $16,19,33,135,144,160$, 221

protein damage $16,157,181,327,484,491$ 
Protein Disulfide Isomerase 42, 410, 437

protein kinase C 88, 116, 130, 165, 181, 205, 252, 264, 274, 293, 309, 315, 343, 347, 428, 473

Proteomics 22, 24-34, 36, 147, 157, 204, $383,385,479,484,490,503$

protoporphyrin IX 100, 110, 178

putrescine 255,328

Rad53 369, 376, 378, 387

radical scavengers $88,332,402$

Rboh 52

RCK-domains 238

reactive nitrogen species $1,31,35,84,127$, 182,186

reactive oxygen and nitrogen species 29, 84, 94, 227, 228, 267, 380

reactive oxygen species (ROS) 51, 63, 121, $169,212,214,283,288,318,364,365$

receptor for advanced glycation endproducts (RAGE) 316

redox biology 21, 304, 331

redox homeostasis 133

Redox Modification/Regulation 245

redox potential $32,37,320$

redox sensor 204, 254, 273

redox-buffer 38

Redox-Sensitive Antioxidant Proteins 375

reoxygenation $279,451,452,453,498$

reperfusion $17,19,33,34,35,61,80,119$, $127,150,191,212,219,230,250,251$, $253,254,256,274,275,276,277,419$, $422,423,427,444,451-453,455,464$, 481, 482, 497

Reporter Ion-Based Methodologies 10 residual oxygen consumption $66,77,79$ response regulator $371,373,374,384-387$ reversed electron flow 71

RONS 37, 84-87, 89, 90, 433

ROS 37-40, 42-44, 46-48, 51-53, 55-57, 59-61, 63, 68, 71, 79-81, 84-90, 95, 96, $121-123,125-144,150,152,156,165$, 169, 178-181, 184-186, 212-224, 226, $227,234,241-243,245,247-251,253-$ $258,260,268,272,275,276,283,288$, $289,292,295,301,302,304,318,319$, $326,327,331,340,342-354,356,357$, $360,364-372,374-376,379,384,403$, 423, 430, 431, 442, 474

ROS-ETosis interaction 353

rotenone 34, 65, 67, 70, 73, 74, 77, 79, 113

S. pombe 42, 43, 44, 52, 369, 372, 374, 375
Schiff-Base 409, 421

Schizosaccharomyces pombe 366

search engines $3,7,8,9$

Sho 1 374, 386

signaling cascade $88,196,251$

singlet oxygen $37,128,131,212,218,243$, $342,345,350,351,362$

skeletal muscle 33, 212, 216, 218, 219, 282, 294, 446

skin 116, 121-159, 161-166, 168, 198, 297, $300,306,328,338,364,406,423$

Skn7 371, 372, 378, 384

Slo binding proteins 240

Slobs 239, 240

SOD 45, 46, 51, 77, 79, 86-91, 121, 126, $131,140,177,214,217-220,222-224$, $343,345,349,366,368$

spermidine $127,255,328,338$

spermine $255,328,338$

sphingomyelin 250

spindle assembly checkpoint 377,387

spore germination 46,47

SPRRs 124, 125, 130, 136

Ssk1 373, 374, 378, 385

stratum corneum 123, 124, 129, 132, 136, $144,149,152,154,157$

stress $31,32,34,37,38,40,42,44,56-58$, $62,63,84,86,93,95,96,97,107-109$, $113,116-119,121,128,134,135,137$, $140,142,146,150,157,158,160,162$, $164,166,167,169-171,175,176,178$, $179,181-185,198,201,217,219,220$, $225,230,231,234,235,238,241,247$, 256-258, 263, 273, 276, 277, 279, 281, 283, 286-288, 293, 295, 296, 304, 309, $314,317,318,320,323,325,326-332$, $335,336,344,357,361,364,366,367$, $369,371-375,377,378,380-387,395$, $405,407,413,418,420,422,425-430$, 432, 434, 437, 441, 443, 444, 449, 451, 472, 483, 484, 486, 491-494, 498, 499

stress defense $38-40,44,45$

Stress-axis regulated exon 260

stretch 240, 266

stretch sensitivity 240

stretch-activated BK channels 240

STREX 238, 240, 260, 266

stroke 219, 241, 247, 248, 256, 272, 402, 403, 482

subcellular compartments $38,40,322$ 
substrate specificity $317,320,322,323,326$, 330,482

substrate-uncoupler-inhibitor titration 64,77 , 79

Sulcatone 257

sulfenic acid 24, 41, 42, 133

superoxide dismutase $34,39,45,46,48,64$, $77,79,84,86,91,93,94,126,131,140$, $153,154,176,205,214,217,223,224$, $229,234,243,247,326,343,345,364$ $366,381,436,455$

superoxide radical $37,56,84,131,268,345$

sXBP1 107, 108, 474

tempol 254

tetraethylammonium TEA 238, 250, 252, $261,263,470$

TGF- $\beta 196$

thaliana 52

thioredoxin $29,39-42,45,59,60,134-136$, $157,214,218,224,229,268,320,321$, $367,370,371,375,376,386,387,418$, $478,488,489$

thioredoxin peroxidase 59,375

Tissue Homogenate 63

TNF 89, 175, 213-215, 224, 281, 283, 309, $341,348,388,390,393,395,399-403$, 407

top-down 3-7, 9, 22, 25, 27, 29

toxicity $20,34,40,85,92,97,98,101,113$, $159,208,228,256,308,331,368,417$, $422,423,430,437,440,441,442,461$, $479,482,483,492,498$ transcription factor 44, 96, 122, 139, 142, 143, 146, 217, 219, 221, 222, 224, 292, 296, 299, 308, 369-371, 373, 382-385, 402, 414

Translational fidelity 326

trypsin 4, 23, 109, 112, 427

tumor necrosis factor $89,158,162,196,213$, $214,224,281,293,341,407$

tunnel proteins 236

Ubiquinone 131

uncoupling 65, 220, 223, 224, 227, 231, 445, 446, 457, 478, 494, 495

uncoupling proteins 220,223, 445, 446, 494, 495

underground metabolism 322, 339

unfolded protein response 42, 58, 137, 445, 478

unspecificity 329

UVA, UVB 129

vascular remodeling 255

vasoconstriction $87,249,255,276$

VEGF 89, 96, 222, 224, 233, 301, 304

Vertebrobasilar insufficiency 248, 261

vitamin C 126, 130, 133, 134, 152, 220, 289

vitamin E 129, 130, 133, 134, 152, 224

voltage clamp 236

voltage sensor $241,251,257$

white adipose tissue 279, 280, 281, 283, 284, 295

X-Box binding protein 1 107, 108

XBP1 107, 108, 111, 119

XBP1 splice variant 107

Ybp1 370-372, 378, 382, 384

Ypd1 374, 386

ZnIXPP 102, 103, 104 
MDPI AG

St. Alban-Anlage 66

4052 Basel, Switzerland

Tel. +41616837734

Fax +41613028918

http://www.mdpi.com

Biomolecules Editorial Office

E-mail: biomolecules@mdpi.com

http://www.mdpi.com/journal/biomolecules 

MDPI AG

St. Alban-Anlage 66

4052 Basel

Switzerland

Tel: +41 616837734

Fax: +41 613028918 